\title{
On Stereocontrol in Organocatalytic a-Chlorinations of Aldehydes
}

\author{
Sebastian Ponath ${ }^{\S \ddagger}$, Chetan Joshi ${ }^{\ddagger}$, Amy T. Merrill ${ }^{\dagger}$, Volker Schmidts $^{\times}$, Kim Greis ${ }^{\S \#}$, Maike Let- \\ tow $^{\S \#}$, Manuela Weber ${ }^{\S}$, Simon Steinhauer ${ }^{\S}$, Kevin Pagel $^{\S \# *}$, Christina Thiele ${ }^{\star *}$, Dean J. Tan- \\ tillo $^{{ }^{*}}$, Mathew J. Vetticatt ${ }^{\star *}$, Mathias Christmann ${ }^{\S *}$ \\ SInstitute of Chemistry and Biochemistry, Freie Universität Berlin, 14195 Berlin, Germany \\ ‡Department of Chemistry, Binghamton University, Binghamton, New York 13902, United States \\ tDepartment of Chemistry, University of California - Davis, Davis, California 95616, United States \\ ${ }^{\times}$Clemens Schöpf Institute of Organic Chemistry and Biochemistry, Technische Universität Darmstadt, 64287 \\ Darmstadt, Germany \\ "Fritz Haber Institute of the Max Planck Society, 14195 Berlin, Germany
}

KEYWORDS: Organocatalysis, Chlorination, Reaction Mechanism, Computational Chemistry, Kinetic Isotope Effect

\begin{abstract}
A comprehensive analysis of the organocatalytic $\alpha$-chlorination of aldehydes with $N$-chloroimides and different catalysts is presented. For this reaction, alternate mechanisms were proposed that differ in the role of resting state intermediates and the rationalization of the observed enantioselectivity. This manuscript aims at resolving these fundamental questions on the basis of rigorous structural characterization of intermediates (configuration and conformation), NMR studies, ion mobility-mass spectrometry, concentration profiles, isotope studies, and DFT calculations.
\end{abstract}

\section{- INTRODUCTION}

The development of novel concepts to exert stereochemical control over chemical reactions constitutes a fundamental challenge. ${ }^{1}$ Toward this goal, catalytic strategies that avoid expensive catalysts, toxic reagents and byproducts are considered among the most desirable. In order to improve on the selectivity and efficiency of a given transformation, ${ }^{2}$ it is important to align experimental results with consistent models.

Enantioselective organocatalytic reactions ${ }^{3}$ via enamines commence with a condensation of a carbonyl compound and a chiral amine catalyst. In the selectivity-determining step (Scheme 1), the electrophile reacts preferentially with either of the two diastereotopic faces of the most reactive enamine conformer. The relative rates of formation $\left(\mathrm{k}_{\mathrm{ma}}\right.$ jor $/ \mathrm{k}_{\text {minor }}$ ) of the diastereomeric iminium intermediates $\mathbf{I}$ (d.r.) and the enantiomeric ratio (e.r.) of the hydrolysis products $\mathbf{P}$ are usually rationalized by the relative energies of the respective transition states of the enamine-electrophile reaction. In a study on enantioselective fluorinations published in 2005, Jorgensen 4 showed for the first time that processes downstream to the formations of the stereogenic center may result in a product e.r. that is different from the d.r. of the two iminium intermediates (d.r. $\neq$ e.r.).

In 2012, Blackmond, Bures, and Armstrong5,6 investigated two reactions catalyzed by Jørgensen-Hayashi diarylprolinol silyl ethers: ${ }^{7,8}$ the addition of aldehydes to nitroolefins, and the $\alpha$-chlorination of isovaleraldehyde.
They arrived at the conclusion that "selectivity in these examples is rationalized not by comparison of transition states for formation of the stereogenic center but by the relative stability and reactivity of equilibrated downstream intermediates and in the separate branches of a competitive reaction network".

Scheme 1. Stereopredictive Models

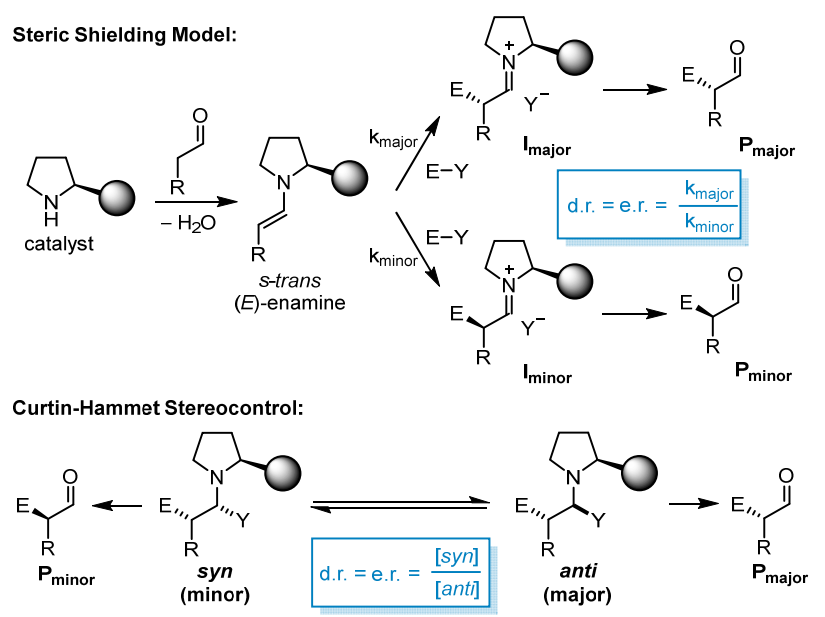

It was further suggested that their concept coined "CurtinHammett stereocontrol" may "represent a general phenomenon for amine catalysts lacking an acidic directing proton”. This projection is no exaggeration because if true, the design of enantioselective catalysts in these cases 
should be augmenting the relative thermodynamic stability of intermediates rather than relative energies of transition states. In the following study, we will validate both models ${ }^{9}$ against new experimental data for the $\alpha$-chlorination of aldehydes with different $N$-chloroimides, catalysts and substrates. To facilitate the reading, we will commence by framing the mechanistic discussion in the historical context.

\section{- BACKGROUND}

\section{Asymmetric Aldehyde Chlorinations}

Over the last years, chiral $\alpha$-haloaldehydes ${ }^{10,11}$ have emerged as important building blocks. The first direct asymmetric $\alpha$-chlorinations of aldehydes ${ }^{12}$ were independently developed by two groups (Scheme 2). In the chlorination of octanal (1), MacMillan ${ }^{13}$ and co-workers employed 1.2 equiv of Lectka's chloroketone $(2)^{14}$ at $-30{ }^{\circ} \mathrm{C}$ in acetone in the presence of $5 \mathrm{~mol} \%$ of imidazolidinone $3 \mathbf{a}$ $(\mathrm{X}=\mathrm{Y}=\mathrm{Me})$ to give chloroaldehyde $(S)_{-4}$ in good yield and high enantiomeric excess.

\section{Scheme 2. $\alpha$-Chlorination of Aldehydes (2004-2009)}

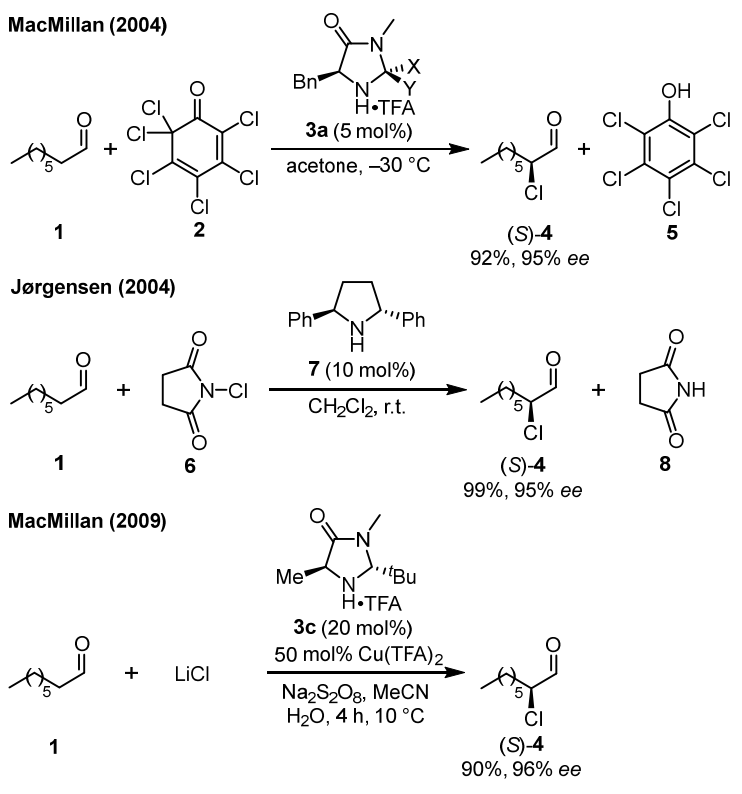

They noted that under these conditions, "product epimerization, formation of 2,2-dichlorooctanal, or octanal aldol dimerization were comprehensively suppressed". As the byproduct, pentachlorophenol (5), is a probable human carcinogen, alternative chlorinating agents were considered. Using $\mathrm{N}$-chlorosuccinimide (6, NCS) as an alternative $\mathrm{Cl}^{+}$source at $4{ }^{\circ} \mathrm{C}$, MacMillan observed almost racemic product. By monitoring the chlorination at $23{ }^{\circ} \mathrm{C}$, Brown (MacMillan group) noticed an erosion of the product ee over time. ${ }^{15}$ Taken together with the concomitant formation of 2,2-dichlorooctanal, this behavior was ascribed to the reversible formation of a chloroenamine intermediate subsequent to the formation of the stereogenic center. Racemization of the chiral chloroaldehyde can be effected by catalysts $3 \mathbf{a}$ and $3 \mathbf{b}\left(\mathrm{X}={ }^{t} \mathrm{Bu}, \mathrm{Y}=\mathrm{H}\right)$ in or without the presence of a chlorinating reagent. ${ }^{16}$ It is suppressed effectively at $-30{ }^{\circ} \mathrm{C}$. Interestingly, Jørgensen et al. reported that $\mathrm{C}_{2}{ }^{-}$ symmetric $(R, R)$-2,5-diphenylpyrrolidine (7) (10 mol\%) catalyzed the chlorination of aldehydes with NCS (1.3 equiv) at room temperature without noticeable racemization. ${ }^{17}$ In order to inhibit chloroenamine formation at room temperature, MacMillan designed imidazolidinone catalyst $3 \mathbf{c}$ with loosely $\mathrm{C}_{2}$-like symmetry. ${ }^{18}$ This catalyst and Lectka's chloroketone constitute a highly effective combination for chlorinations at elevated temperatures ${ }^{15}$ and subsequent follow-up transformations. ${ }^{19}$ In 2009, MacMillan demonstrated that $3 \mathrm{c}$ can be used for the SOMO chlorination of aldehydes..$^{18}$ We started our own investigations in 2011 by initially using the MacMillan's SOMO protocol $^{2 \mathrm{O}}$ but later moved to the combination of $3 \mathrm{C} \mathrm{(20} \mathrm{mol \% )}$ and NCS in acetonitrile ${ }^{21}$ due to side reactions ${ }^{22}$ of radical intermediates in the SOMO chlorination of terpene aldehydes.

\section{Chlorinations with Jørgensen-Hayashi-type Catalysts}

The 2-substituted pyrrolidine scaffold 9 was first employed in enamine chlorinations of isovaleraldehyde 10 by Jørgensen et al. in 2004 (Scheme 3). Using 9a (20 mol\%) in 1,2dichloroethane afforded $(S)$-2-chloroisovaler-aldehyde 12 with moderate enantioselectivity. (e.r. 82:18). ${ }^{17}$ The corresponding diarylprolinol silyl ethers $9 b$ and $9 c \quad(R=H$, $\mathrm{X}=\mathrm{OTMS}$ ) had not been disclosed by that time. ${ }^{8}$ In 2012 , Blackmond et al. studied the $\alpha$-chlorination of isovaleraldehyde with NCS in $\mathrm{CDCl}_{3}$ using these two catalysts achieving enantioselectivities in the same ballpark. ${ }^{6}$ With the aid of reaction calorimetry, they showed that "a rapid initial rate corresponding to ca. one turnover of the catalyst was followed by a slower zero-order regime, demonstrating that the reaction does not depend on the concentration of either of the two reactants." The observation of two sets of NMR signals at $-54{ }^{\circ} \mathrm{C}$ featuring EXSY cross peaks led the authors to suggest that those correspond to two interconverting diastereomeric aminals 11 as resting states of the catalytic system. The two species were proposed to be constitutionally "analogous to a species reported by Jørgensen using pyrrolidine" and diastereomeric at $C_{1}$. Surprisingly, no attempt was reported to directly determine the structure, yet the major species was assigned to be the anti-configurated. An inductive reasoning for the structural assignment implies an enamine-NCS reaction occurring with "near perfect facial selectivity" which was derived from the observation of "only two out of four possible diastereomers". In the mechanistic proposal (Scheme 3), stereoselective enamine (13)/NCS reaction followed by reversible addition of succinimide to the iminium carbon of the chloroiminium ion affords two aminals 11, "which equilibrate between one of the two possible syn and one of the two possible anti diastereomers". ${ }^{23}$ Two separate stereospecific E2 eliminations proceeding at identical rates $\left(\mathrm{k}_{\mathrm{syn}}\right.$ $\approx \mathrm{k}_{\text {anti }}$ ) would provide an $E$-chloroenamine (E-15) from the major anti-aminal and a Z-chloroenamine (Z-15) from the minor syn-aminal. A stereospecific protonation/hydrolysis which, was not further detailed, would lead to the chloroaldehydes in an enantiomeric ratio identical to the ratio of the observed aminals. ${ }^{24}$ The apparent correlation between the ratio of two rapidly equilibrating species (cause) and 
the ratio of two product enantiomers (effect) led the authors to formalize a new concept for stereochemical control in organocatalysis entitled the "Curtin-Hammett paradigm".

Scheme 3. On-Cycle Mechanism for Chlorinations with Monosubstituted Pyrrolidine Catalysts
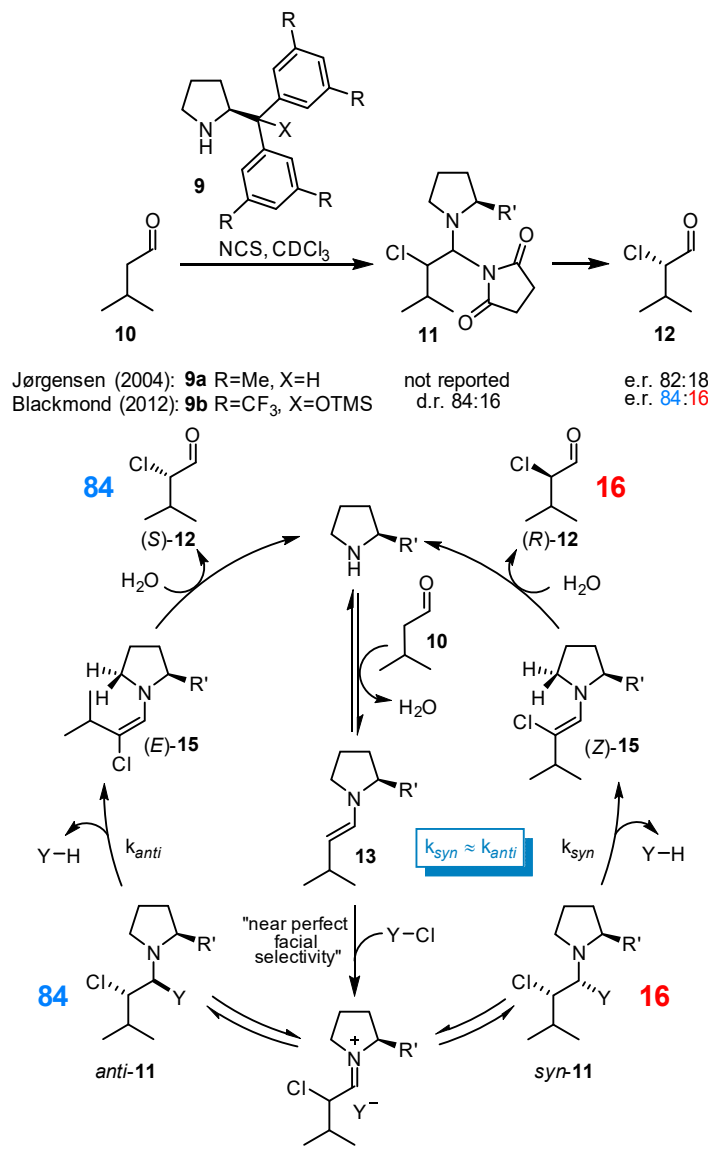

\section{- RESULTS AND DISCUSSION}

Chlorinations with Imidazolidinone Catalysts

In 2018, our group reported an investigation of the $\alpha$-chlorination of aldehyde 16 using catalyst 3c.TFA ( $20 \mathrm{~mol} \%)$ with different $\mathrm{N}$-chloroimides in acetonitrile. ${ }^{25}$ The aim was to improve on the catalytic efficiency and to decrease the catalyst loading. We quickly realized that the main obstacle for good turnover is catalyst accumulation within stable aminals similar to those previously proposed. Gratifyingly, we were able to isolate aminal intermediates 18 in pure form and to obtain several X-ray crystal structures for the first time (Scheme 4). Each of the isolated species was obtained as a single diastereomer with syn-configuration. With $N$-chlorophthalimide (NCP) as the chlorinating reagent, opening of the phthalimide moiety was observed to give 18a upon reductive quenching/work-up. Merging the absolute configuration of the chloroaldehyde product 19 and the aminal $\mathbf{1 8}$, we concluded that both compounds would be derived from iminium ion precursors possessing identical configurations at the chlorine substituted stereogenic center $\left(C_{2}\right)$
Scheme 4. Isolation of Aminal Intermediates Using MacMillan-Type Catalyst 3c

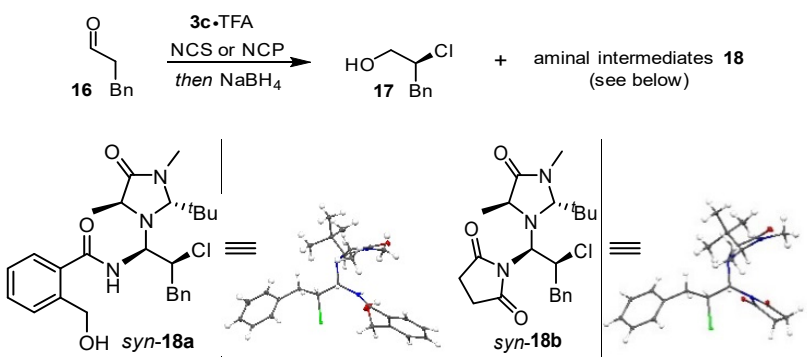

In our mechanistic proposal (Scheme 5), high facial selec tivity for the depicted chloroiminium ion 19 was rationalized by the standard steric shielding model. In contrast to the previously discussed on-cycle model, we placed the aminal species syn-18 and anti-18 off the catalytic cycle. As a major consequence of the model, neither kinetic nor thermodynamic ratios of aminal $C_{1}$ epimers affect the enantiomeric ratio of the chloroaldehyde. Yet, the accumulation of the catalysts within stable aminals is the major negative influence for diminished catalytic efficiency.

Scheme 5. Chlorination Using MacMillan-Type Catalyst $3 \mathrm{c}$ with Off-Cycle Aminal Intermediates

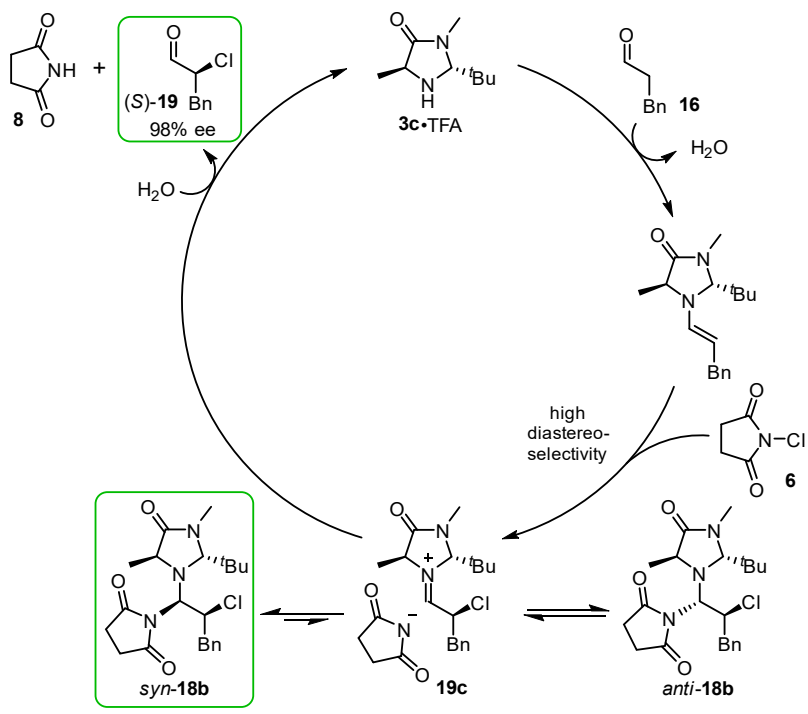

The problem was overcome by using $N$-chloro-4-nitrophthalimide as NCS surrogate. The on-cycle model proposes the stereochemical outcome to be thermodynamically controlled, i.e. the formation of the major enantiomer must proceed through the most stable aminal. In that sense, the on-cycle mechanism predicts a formal inversion of the initially generated stereogenic center for the $(2 S)$-syn-aminal to give the $(2 R)$-chloroaldehyde product under the premise that chlorination and protonation occur with the same sense of stereocontrol imposed by the catalyst. Importantly, due to the $\mathrm{C}_{2}$-like symmetry of the catalyst, a $180^{\circ}$ rotation along the enamine and the chloroenamine $C$ $N$ bond axis does not change the facial shielding significantly. Summarizing the stereochemical considerations, only the off-cycle mechanism aligns the configuration of 
the most stable aminal with the observed $(S)$-configuration of the product, whereas the on-cycle model predicts the $(R)$-enantiomer as the major product.

In case of the first and second generation MacMillan catalysts $3 \mathbf{a}$ and $\mathbf{3} \mathbf{b}$, similar aminals were proposed. ${ }^{26,27,28}$ The syn/anti ratio of respective aminals had been correlated to the enantiomeric ratio, and the anti-diastereomer was suggested to lead to the major product enantiomer. It is somewhat puzzling that to the best of our knowledge not a single aminal structure was established directly either by NMR or by X-ray crystallography. Repeating the experiments described for chlorination of isovaleraldehyde using catalysts $\mathbf{3} \mathbf{a}$ and $\mathbf{3} \mathbf{b}$ and NCS as the chlorinating reagent, we have been able to isolate most of the respective stable aminals which are separable chromatographically with no subsequent equilibration being observed. For each of those aminals, single crystals were grown and analyzed.

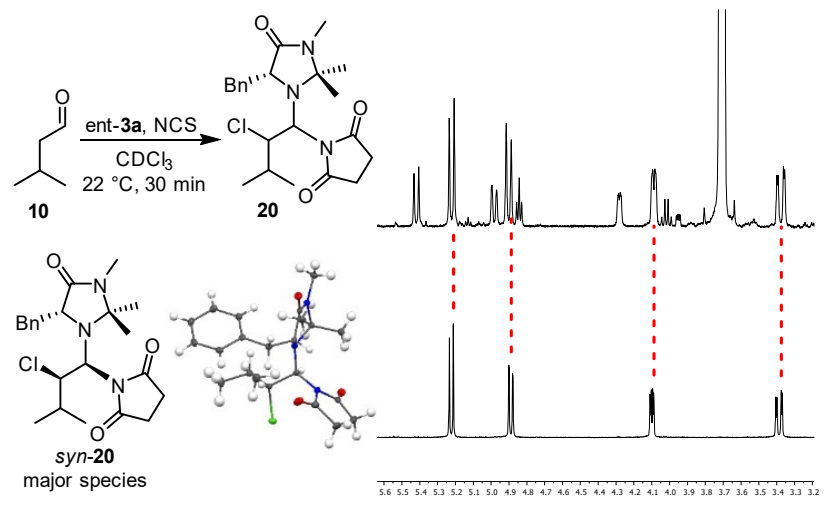

Figure 1. ${ }^{1} \mathrm{H}-\mathrm{NMR}$ spectrum of the reaction (in accordance with the literature ${ }^{28}$ ) (top). Crystal structure and ${ }^{1} \mathrm{H}-\mathrm{NMR}$ spectrum of the isolated aminal bearing MacMillan-catalyst za (bottom).

The obtained crystal structures reveal two surprising findings: first, the major aminal diastereomers syn-2o and syn$\mathbf{2 1}$ for the imidazolidinone catalysts $3 \mathbf{a}$ and $\mathbf{3} \mathbf{b}$ possess the syn- and not anti-configuration; second, in case of catalyst $\mathbf{3 b}$, we also isolated a third aminal, clearly visible in the crude NMR data that was previously overlooked (see SI for details). This third aminal also bears syn-configuration and is present in higher concentrations than the minor aminal in Blackmond's assignment (Figures 1 and 2).

The conformations of the diastereomeric aminals are reflective of steric and stereoelectronic interactions between the catalyst and the chloroaminal substructures and within the chloroaminal substructure itself. In all crystal structures, both syn- and anti-aminals adopt an antiperiplanar orientation of $\mathrm{C}_{1}-\mathrm{H}$ and $\mathrm{C}_{2}-\mathrm{H}$ (Scheme 6). Such an arrangement constitutes the only staggered conformation along the $\mathrm{C}_{1}-\mathrm{C}_{2}$ axis where each non-hydrogen substituent $(\mathrm{N}$, $\mathrm{Cl}, \mathrm{R}$ ) possesses only one synclinal interaction with another non-hydrogen substituent. A similar conformational preference in solution is indicated by the observation of large $3 J_{\mathrm{C}_{1}-\mathrm{H}, \mathrm{C} 2-\mathrm{H}}$ coupling constants $(10-11 \mathrm{~Hz})$.

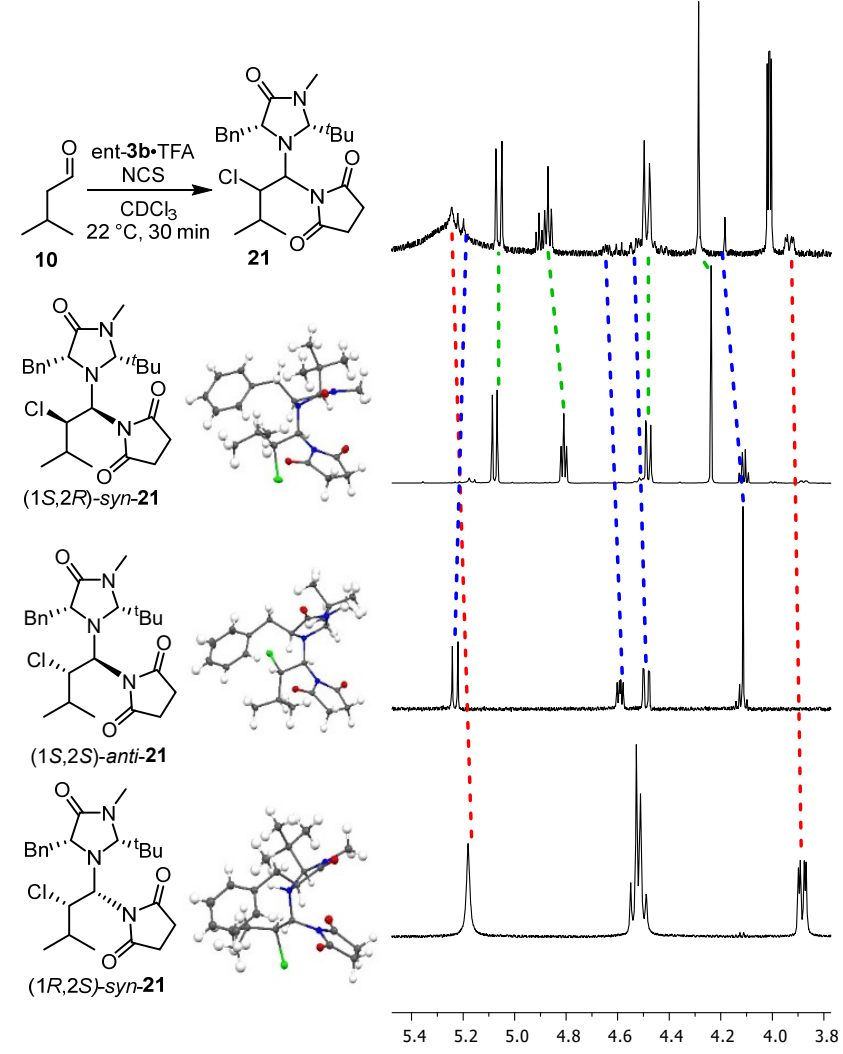

Figure 2. ${ }^{1} \mathrm{H}-\mathrm{NMR}$ spectrum of the reaction (in accordance with the literature ${ }^{27,28}$ ) (top). Crystal structures and ${ }^{1} \mathrm{H}-\mathrm{NMR}$ spectra of different isolated aminal diastereoisomers bearing MacMillan-catalyst 3b (bottom).

The stability of the aminals can be rationalized by a synergistic interplay of anomeric stabilization and gauche effects. In the syn-diastereomer, the two best $\sigma \rightarrow \sigma^{*}$ acceptors $(\mathrm{C}-\mathrm{Cl}, \mathrm{C}-\mathrm{N}$-imide) are in a gauche orientation whereas in the anti-diastereomer, the succinimide and the chlorine substituent are antiperiplanar. As previously noted by others, ${ }^{29}$ the catalyst's aminal nitrogen lone pair prefers to be cis to the bulkiest substituent (to minimize strain) and antiperiplanar to a $\mathrm{C}-\mathrm{C}$ or a $\mathrm{C}-\mathrm{X}(\mathrm{X}=\mathrm{O}, \mathrm{N}, \mathrm{S})$ bond (allowing for $n \rightarrow \sigma^{*}$ stabilization).

Curtin-Hammett conditions refer to situations where each of two rapidly interconverting conformational isomers reacts irreversibly to a different product. The Curtin-Hammett principle ${ }^{30}$ issues a warning not to relate a conformer ratio directly to the product ratio if the rate of product formation is small relative to the rate of interconversion. Diastereomers must not be treated as individual reactive conformers but as an ensemble of conformations. Diastereomer stability is dominated by the lowest energy conformers but not necessarily by reactive conformations relevant to an ensuing transformation (Scheme 6) let alone the standard free energies $\left(\Delta \Delta \mathrm{G}^{\ddagger}\right)$ of the respective transition states. The "Curtin-Hammett paradigm for stereocontrol" suggests a quantitative relationship between the ratio of two equilibrating diastereomeric aminals and the ratio of their respective E2 elimination products. However, the rel- 
ative stability of aminal conformations with $\mathrm{C}_{2}-\mathrm{H}$ and succinimide in an antiperiplanar orientation (the basis for the stereospecificity of the E2 elimination) is not directly related to the lowest energy conformation. It is also worth noting that the rate of E2 eliminations is expected to be sensitive towards the size of the substituents and other parameters (base, solvent).

Scheme 6. Lowest Energy and Reactive Conformations of Syn- and Anti-Aminals

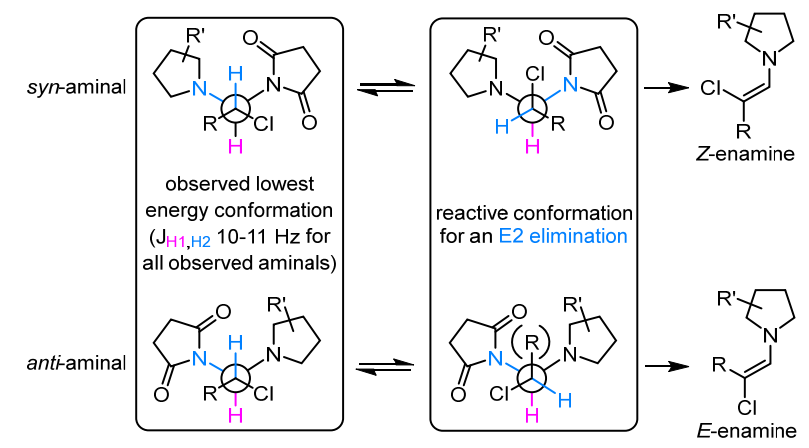

\section{Kinetic Isotope Effects and DFT Calculations}

In order to evaluate the first irreversible step in the catalytic cycle, we performed the reaction of 16 and 6 using $20 \mathrm{~mol} \%$ 3c $^{\mathrm{c}}$ TFA for determination of ${ }^{13} \mathrm{C}$ kinetic isotope effects (KIEs) at natural abundance. ${ }^{31}$ By using 6 as a limiting reagent, the reaction was taken to $77 \%$ conversion of $\mathbf{1 6}$. The unreacted 16 was re-isolated from the reaction mixture as the corresponding alcohol following a reductive workup (see SI for full experimental details). The ${ }^{13} \mathrm{C}$ isotopic composition of this re-isolated sample was compared to that of a standard sample of reduced $\mathbf{1 6}$ (taken from the same batch used for the high conversion reaction) using quantitative NMR methodology. From the relative isotopic composition and the fractional conversion, ${ }^{13} \mathrm{C}$ KIEs for 16 were determined in a standard way. ${ }^{31}$ The resulting ${ }^{13} \mathrm{C}$ KIE measurements, from duplicate runs of this experiment, are shown in Figure $3 \mathrm{~A}$. There is a normal KIE of $\sim 1.015$ on the former carbonyl carbon and unity KIEs on all other carbon atoms of 16. The qualitative interpretation of the experimental KIEs is that only the carbonyl carbon is involved in the first irreversible step for $\mathbf{1 6}$ in the catalytic cycle. There are several steps in the catalytic cycle that involve the carbonyl carbon atom and the exact step that accounts for the experimental KIE is not immediately clear. For the quantitative interpretation of experimental KIEs, we modeled the key steps in the catalytic cycle using $\mathrm{B}_{3} \mathrm{LYP} / 6-31+\mathrm{G}^{* *}$ with a PCM solvent model for acetonitrile as executed in Gaussian '16. ${ }^{32,33,34,35}$ High-level single point energy calculations that include dispersion corrections were performed for all stationary points using $\mathrm{B}_{3} \mathrm{LYP}-\mathrm{D}_{3}(\mathrm{BJ})^{36} / 6-311+\mathrm{G}^{* *} \mathrm{PCM}$ (acetonitrile). The reported Gibbs free energies were obtained by adding the free energy correction from the optimizations to the high-level single point energy calculations. The free energies were corrected using Grimme's quasi rigid rotor -harmonic oscillator ( $\mathrm{RRRHO}$ ) approach, which raises vibrational frequencies that are below $100 \mathrm{~cm}^{-1}$ to
$100 \mathrm{~cm}^{-1} .37$ This approach is routinely utilized and is wellestablished for evaluating reactivity and selectivity in similar catalytic systems. ${ }^{38,39}$ The reaction coordinate diagram for the reaction of $\mathbf{1 6}$ and $\mathbf{6}$ catalyzed by $3 c$.TFA leading up to the formation of the chloroiminium ion Int4 is shown in Figure $3 \mathrm{~B}$. The trifluoroacetate counterion of the catalyst salt is included in these calculated structures to either act as a proton shuttle or to stabilize positively charged transition structures/intermediates. The reported free energy barriers $\left(\Delta \mathrm{G}^{\ddagger}\right)$ are computed with respect to the sum of the energies of neutral 3c, trifluoroacetic acid, 6, and 16. The computed free energy profile reveals that the transition structures (TSs) for initial formation of the carbinol amine intermediate Int $1\left(\mathrm{TS} 1, \Delta \mathrm{G}^{\ddagger}=10.2 \mathrm{kcal} \mathrm{mol}^{-1}\right)$, loss of water to form the iminium ion Int2 $\left(\mathrm{TS}_{2}, \Delta \mathrm{G}^{\ddagger}=10.1 \mathrm{kcal} \mathrm{mol}^{-1}\right.$ ), and deprotonation of Int2 to form the enamine Int3 (TS $_{3}$, $\left.\Delta \mathrm{G}^{\ddagger}=10.4 \mathrm{kcal} \mathrm{mol}^{-1}\right)$ all lie within $0.7 \mathrm{kcal} \mathrm{mol}^{-1}$ of each other. The TS for chlorination of Int3 by $\mathbf{6}\left(\mathrm{TS}_{4}\right)$ has a $\Delta \mathrm{G}^{\ddagger}$ of $9.3 \mathrm{kcal} \mathrm{mol}^{-1}$ and this step is an exergonic process resulting in a $\alpha$-chloroiminium ion intermediate Int4 that is $25.1 \mathrm{kcal} \mathrm{mol}^{-1}$ below separated starting materials. The experimental ${ }^{13} \mathrm{C}$ KIE determined for $\mathbf{1 6}$ reports on TS for the first irreversible step in the catalytic cycle for $\mathbf{1 6}$. Since the $\Delta \mathrm{G}^{\ddagger}$ for $\mathrm{TS}_{1}-\mathrm{TS}_{4}$ lie within $1.1 \mathrm{kcal} \mathrm{mol}^{-1}$ of each other, the prediction of ${ }^{13} \mathrm{C}$ KIEs for the interpretation of our experimental KIE values is complicated - each of these TSs contribute to the observed KIE, with the highest energy transition structure $\left(\mathrm{TS}_{3}\right)$ contributing the most followed by $\mathrm{TS}_{1}, \mathrm{TS}_{2}$, and $\mathrm{TS}_{4}$. Figure ${ }_{4} \mathrm{C}$ shows the predicted ${ }^{13} \mathrm{C}$ KIEs for TS1-4 assuming each to be the sole first irreversible step for 16. The percentage contribution of each TS (based on relative $\Delta \mathrm{G}^{\ddagger}$ ) to the observed KIEs is also indicated in parentheses. Based on the predicted ${ }^{13} \mathrm{C}$ KIE values for TS1-4, the energy-weighted predicted ${ }^{13} \mathrm{C}$ KIEs for $\mathrm{C}_{1}$ and $\mathrm{C}_{2}$ of 16 are computed (Figure $3 \mathrm{C}$, last row). Comparison of experimental (Figure $3 \mathrm{~A}$ ) and energy-weighted predicted ${ }^{13} \mathrm{C}$ KIEs reveals a good agreement between the two - lending support to the computed reaction coordinate diagram. ${ }^{40} \mathrm{Next}$, we computed the steps from the chloroiminium ion intermediate Int 4 a to the final $\alpha$-chloroaldehyde product 19 (Int4a is a complex between the chloroiminium ion-TFA salt and a molecule of water and differs from Int 4 which is a complex between the chloroiminium ion-TFA salt and a molecule of succinimide). In the resulting reaction coordinate diagram (Figure $4 \mathrm{~A}$ ), the reported free energy barriers $\left(\Delta \mathrm{G}^{\ddagger}\right)$ are computed with respect to Int4a (the global minimum on this reaction coordinate). The hydrolysis of Int4a proceeds via two lower barrier steps - TS6 $\left(\Delta \mathrm{G}^{\ddagger}=8.8 \mathrm{kcal} \mathrm{mol}^{-1}\right)$ and $\mathrm{TS}_{7}\left(\Delta \mathrm{G}^{\ddagger}=8.3 \mathrm{kcal} \mathrm{mol}^{-1}\right)$ - to deliver the product aldehyde 19 and regenerate $3 \mathrm{c}$ and trifluoroacetic acid. Based on the observation of syn- and anti-aminal intermediates in this reaction (Figure 6 and 7), we also computed transition structures for the conversion of Int 4 to syn-18b $\left(\mathrm{TS}_{5}\right)$ and anti-18b ( $\mathrm{TS}_{5}$ '). The transition structures $\mathrm{TS}_{5}$ and TS 5 ' face a free energy barrier of 11.1 and $8.9 \mathrm{kcal} \mathrm{mol}^{-1}$ (relative to Int4). The syn-aminal syn-18b was found to be thermodynamically favored over the anti- 


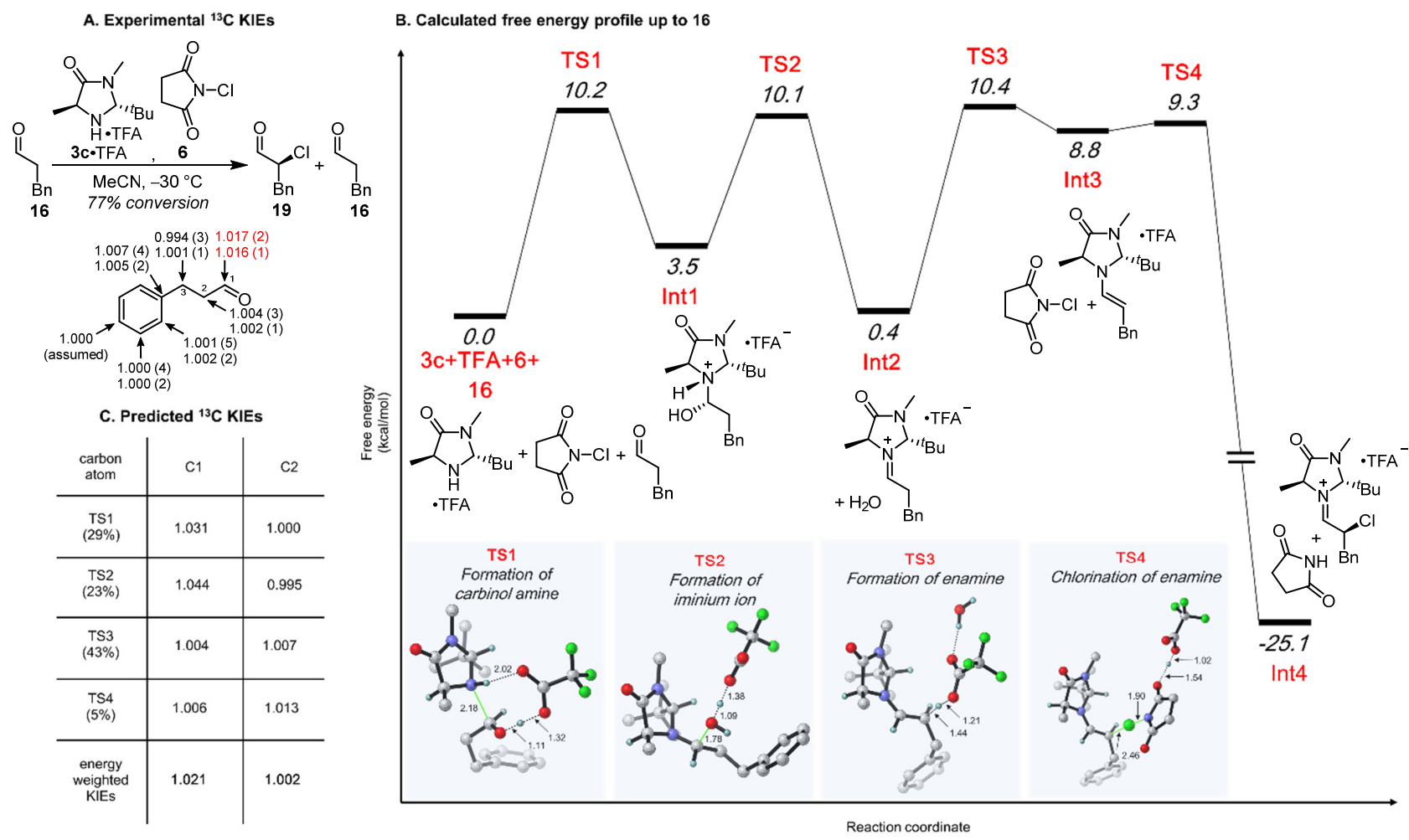

FIGURE 3. Experimental ${ }^{13} \mathrm{C}$ KIE studies and supporting DFT calculations. (A) Experimental ${ }^{13} \mathrm{C}$ KIEs for the $\alpha$-chlorination of 16 with the MacMillan Catalyst 3c-TFA. (B) Reaction coordinate diagram for the reaction of $\mathbf{1 6}$ and $\mathbf{6}$ catalyzed by $\mathbf{3}^{\bullet} \cdot{ }^{\circ}$ TFA up to the formation of the chloroiminium ion Int 4 calculated at the B3LYP-D $3(B J) / 6-311+G^{* *} P C M$ (acetonitrile)//B3 $L Y P / 6-31+G^{* *} P C M$ (acetonitrile) level of theory. Some hydrogen atoms have been removed for clarity in the $3 \mathrm{D}$ representation of the key transition structures. Key bond-forming and bond-breaking distances are in angstroms (Å). (C) Predicted KIEs at $\mathrm{C}_{1}$ (aldehyde-carbon) and C2 ( $\alpha$-carbon) of 16 for TS1-4 along with energy-weighted predictions for comparison to experimental KIEs.

aminal anti-18b by $1.7 \mathrm{kcal} \mathrm{mol}^{-1}$. These computed free energies are consistent with the experimental observation of anti-18b in the initial 5 minutes of the reaction (kinetic aminal) and subsequent accumulation of syn-18b (thermodynamic aminal) (Figure 7).Our calculations suggest that syn-18b is a parasitic off-cycle intermediate that has only one path to product $\mathbf{1 9}$ - by re-entering the catalytic cycle via $\mathrm{TS}_{5}\left(\Delta \mathrm{G}^{\ddagger}=15.3 \mathrm{kcal} \mathrm{mol}^{-1}\right)$ to form Int 4 followed by the previously described hydrolysis via TS6 and TS7. If this pathway is operational for the conversion of syn-18b to $\mathbf{1 9}$, re-entry of the syn-18b into the catalytic cycle via $\mathrm{TS}_{5}$ represents the largest change in free energy in the conversion of syn-18b to 19 (see Supporting Information for a discussion and supporting calculations for the conversion of anti$\mathbf{1 8 b}$ to 19). Alternatively, syn-18b could be an on-cycle intermediate (Curtin-Hammett paradigm ${ }^{6}$ ) and could undergo an E2-elimination to yield a chloroenamine intermediate. Protonation and subsequent hydrolysis of this chloroenamine intermediate would deliver the product and regenerate the catalyst. In order to evaluate this alternative mechanism, we explored transition state models for the formation of the chloroenamine intermediate utilizing either trifluoroacetate or succinimide anion leaving group as the base that effects the deprotonation with concomitant loss of the succinimide anion. All attempts to locate this TS resulted in an E1-type structure where the succinimide anion is completely dissociated and the base deprotonates a structure resembling a chloroiminium ion (Figure $4 \mathrm{~A}$, TSE1; $\Delta \mathrm{G}^{\ddagger}=28.2 \mathrm{kcal} \mathrm{mol}^{-1}$ with respect to syn-18b). This is because the incipient carbocation, that forms as the succinimide leaving group departs, is stabilized by the lone pair of electrons on the catalyst nitrogen before deprotonation can occur. Based on this result we hypothesized that disengaging this lone pair from the reaction coordinate by protonation, could result in the identification of an E2elimination TS. ${ }^{41}$ Accordingly, we explored the $\alpha$-deprotonation of $N$-protonated syn-18b (with concomitant loss of the succinimide anion) using trifluoroacetate as the base to generate the protonated chloroenamine intermediate. These explorations resulted in the identification of TS-E2 $\left(\Delta \mathrm{G}^{\ddagger}=50.3 \mathrm{kcal} \mathrm{mol}^{-1}\right.$ with respect to $\left.s y n-\mathbf{1 8 b}\right)$, for the direct conversion of $N$-protonated syn-18b to the protonated chloroenamine intermediate. Clearly, the barriers for the E2-elimination and E1-elimination pathways are significantly higher than the pathway involving re-entry of syn$\mathbf{1 8 b}$ into the catalytic cycle as Int4 (Figure $4 \mathrm{D}, \mathrm{TS}_{5}$; $\Delta \mathrm{G}^{\ddagger}=\mathbf{1 4 . 2} \mathrm{kcal} \mathrm{mol}^{-1}$ with respect to syn-18b). In order to gain experimental evidence on the catalytic role of syn-18b, we envisioned that determination of ${ }^{13} \mathrm{C}$ KIEs for the stoichiometric conversion of syn-18b to product 19 could probe the first irreversible step in the pathway for this reaction (Figure $4 \mathrm{~B}$ ). If the reaction proceeded via turnoverlimiting re-entry into the catalytic cycle, a normal ${ }^{13} \mathrm{C} \mathrm{KIE}$ would be expected only on $C_{1}$. However, if either the E1- or 


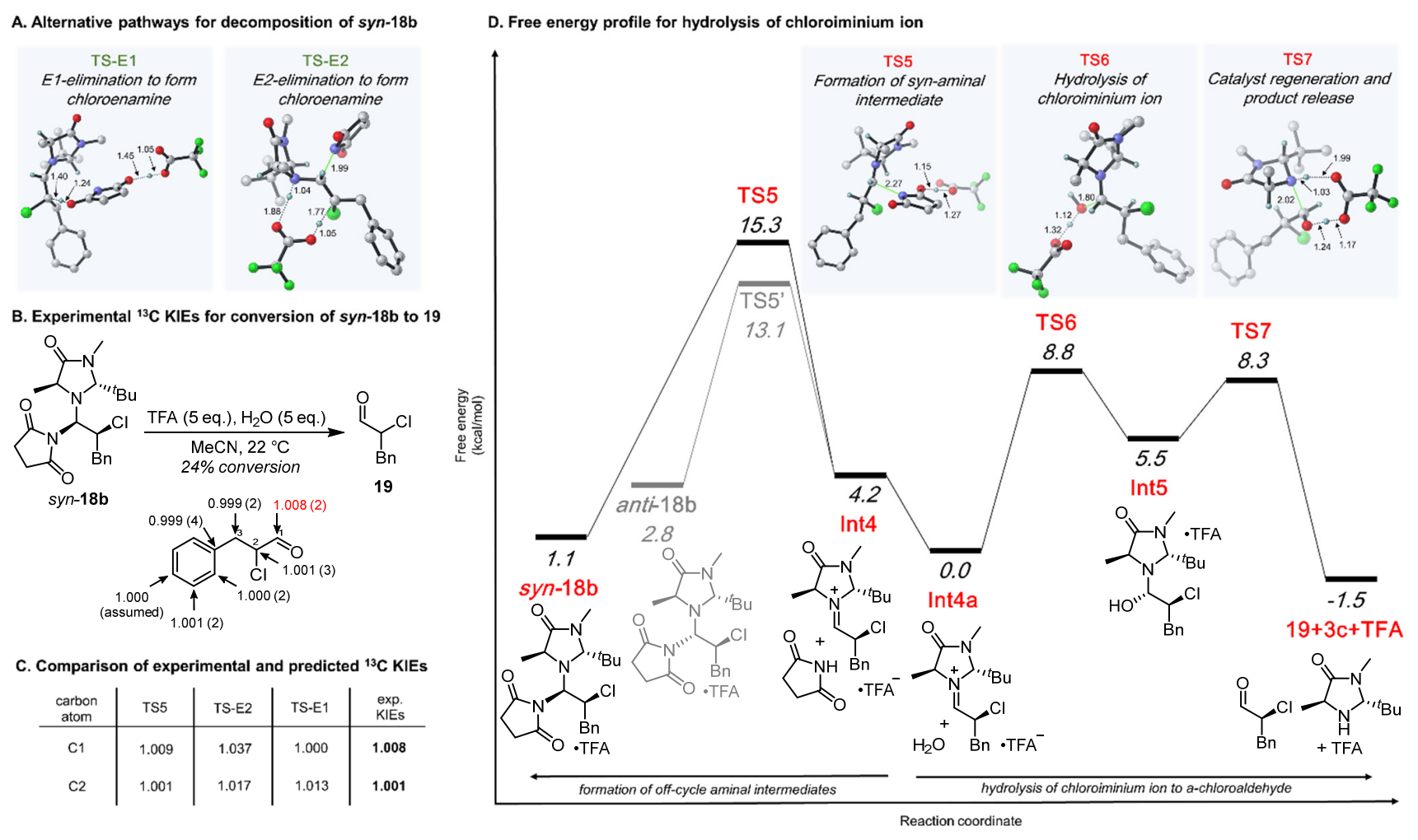

FIGURE 4. Hydrolysis of chloroiminium ion and investigation of the role of experimentally observed aminal intermediates. (A) Calculated transition structures for alternative pathways for re-entry of syn-18b (as a chloroenamine) into the catalytic cycle. (B) Experimental ${ }^{13} \mathrm{C}$ KIEs determined for the stoichiometric conversion of syn-18b to 19. (C) Comparison of experimental ${ }^{13} \mathrm{C}$ KIEs for the conversion of syn-18b to $\mathbf{1 9}$ with the predicted ${ }^{3} \mathrm{C}$ KIE for the rate-determining step in each pathway that converts syn-18b to 19. (D) Reaction coordinate diagram for the conversion of the chloroiminium ion Int 4 to the final product 19 calculated at the $\mathrm{B}_{3} \mathrm{LYP}-\mathrm{D}_{3}(\mathrm{BJ}) / 6-311+\mathrm{G}^{* *} \mathrm{PCM}$ (acetonitrile)// B3LYP/6-31+G** PCM(acetonitrile) level of theory. Also shown is the calculated pathway for the parasitic off-cycle aminal intermediates syn-18b and anti-18b. Some hydrogen atoms have been removed for clarity in the 3 D representation of the key transition structures. Key bond-forming and bond-breaking distances are in angstroms ( $\AA$ ).

E2-Elimination pathway is operational, a normal ${ }^{13} \mathrm{C} \mathrm{KIE}$ would be expected on $\mathrm{C}_{2}$ or both $\mathrm{C}_{1}$ and $\mathrm{C}_{2}$, respectively. The conversion of syn-18b to 19 was initiated using 5 equivalents each of trifluoroacetic acid and water (Figure ${ }_{4} \mathrm{~B}$ ). The ${ }^{13} \mathrm{C}$ KIEs were determined by product analysis ${ }^{42}-$ by comparison of samples of reduced 19 isolated from $24 \%$ and $100 \%$ conversion reactions. The key result from this experiment was a 1.008 (2) KIE on $\mathrm{C}_{1}$ and a 1.001 (4) KIE on $\mathrm{C}_{2}$ (Figure $4 \mathrm{~B}$ ) - in qualitative agreement with a mechanism involving turnover-limiting re-entry of syn-18b into the catalytic cycle. A comparison of this experimental KIE and theoretical KIEs for TS5, TS-E2, and TS-E1 reveals that the experimental values are in excellent agreement with predicted values for $\mathrm{TS}_{5}$ and in poor agreement with those for TS-E2 and TS-E1 (Figure $4 \mathrm{C}$ ). Thus, evaluation of the reaction energetics and KIEs for the conversion of syn-18b to 19 supports the role of $s y n-\mathbf{1 8 b}$ as a parasitic, off-cycle intermediate that can re-enter the catalytic cycle via turnover-limiting formation of Int4. Finally, from the computed reaction coordinate diagram shown in Figure $3 \mathrm{~B}$, the chlorination of the enamine intermediate $\left(\mathrm{TS}_{4}\right)$ is irreversible, and the enantioselectivity-determining step of the reaction. Accordingly, we performed a thorough confor- mational search for the formation of both $R$ and $S$ chloroiminium ion via chlorination of the $S i$ - and $R e$ - faces, respectively, of all possible enamine geometries. The $\Delta \Delta \mathrm{G}^{\ddagger}$ between the lowest energy transition structures leading to $R$ and $S$ chloroiminium ion is $2.9 \mathrm{kcal} \mathrm{mol}^{-1}$ (favoring $S$ ) - a value that is qualitatively consistent with the $>90 \%$ ee observed for this reaction. The full details of these explorations are included in the Supporting Information. Thus, our experimental KIE results along with a comprehensive DFT analysis of the entire reaction coordinate supports a scenario where the enantioselectivity-determining step is the initial formation of the chloroiminium ion and not any downstream processes.

\section{Deuterium Incorporation Studies}

In a deuterium incorporation study, aldehyde $\mathbf{1 6}$ was subjected to a chlorination reaction with the MacMillan catalysts $\mathbf{3} \mathbf{b}$ and $\mathbf{3} \mathbf{c}$ and the Jørgensen-Hayashi catalyst $\mathbf{9 c}$ and NCS at two different temperatures. The obtained chloroaldehyde was reduced to alcohol 22 (to avoid racemization) and submitted to NMR measurements. At $-30{ }^{\circ} \mathrm{C}$, all catalysts showed little to no incorporation of deuterium. At $20{ }^{\circ} \mathrm{C}$, the MacMillan catalyst $\mathbf{3} \mathbf{b}$ shows significant deuterium incorporation (Figure 5). This observation is in line 
with the racemization MacMillan observed for $\mathbf{3} \mathbf{b}$ but not for $3 c$ (vide supra). Our results also rule out the protonation of fleeting chloroenamine intermediates due to the absence of significant deuterium incorporation at low temperature. While the intermediacy of chloroenamine intermediates cannot be studied by these experiments, their presence is not unlikely as chlorination might account for the formation of significant amounts dichloroaldehyde products for catalysts $\mathbf{9 c}, \mathbf{3} \mathbf{a}$, and $\mathbf{3} \mathbf{b}$.
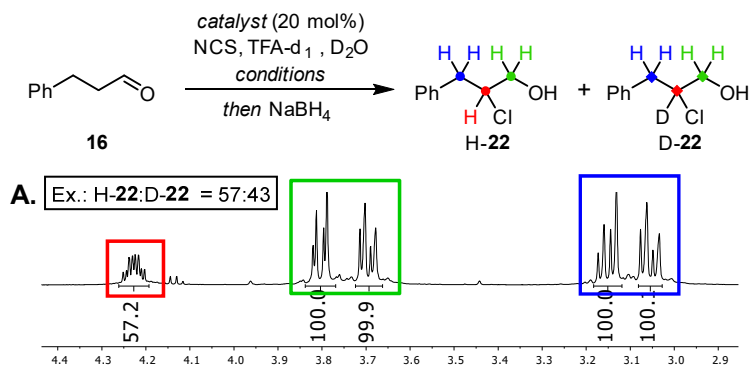

B.

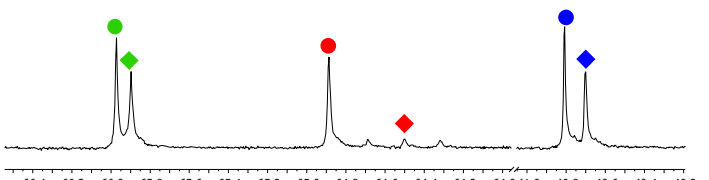

C.

\begin{tabular}{|c|c|c|c|c|}
\hline catalyst & solvent & $\mathrm{T}\left[{ }^{\circ} \mathrm{C}\right]$ & $t$ & $\mathrm{H}-22: \mathrm{D}-22$ \\
\hline & $\mathrm{MeCN}$ & 20 & $18 \mathrm{~h}$ & $90: 10$ \\
\hline & $\mathrm{MeCN}$ & -30 & $18 \mathrm{~h}$ & 100:0 \\
\hline & $\mathrm{MeCN}$ & 20 & $18 \mathrm{~h}$ & 57:34 \\
\hline & $\mathrm{MeCN}$ & -30 & $18 \mathrm{~h}$ & 95:5 \\
\hline & DCM & 20 & $18 \mathrm{~h}$ & 83:17 \\
\hline & DCM & -30 & $18 \mathrm{~h}$ & 89:11 \\
\hline & DCM & 20 & $5 \mathrm{~min}$ & $97: 3^{\mathrm{a}}$ \\
\hline
\end{tabular}

Figure 5. Deuterium incorporation of $\mathbf{1 6}$ with different catalysts and temperatures with exemplary ${ }^{1} \mathrm{H}-\mathrm{NMR}(\mathrm{A})$ and ${ }^{13} \mathrm{C}$ NMR (B) spectra; C) Quantification of deuterium incorporation under different conditions.

\section{NMR Reaction Progress}

We interrogated concentration profiles in order to assess whether the two mechanisms are kinetically distinguishable. Gratifyingly, aldehyde $\mathbf{1 6}$ (the substrate), the chloroaldehyde product 19 and the corresponding crystallographically characterized aminal syn-18b possess separate, nonoverlapping NMR resonances (see SI). Monitoring their concentrations over time, it is apparent that the initial rate of chloroaldehyde formation exceeds the build-up of the aminal (Figure 6A). This observation eliminates the possibility of the catalyst being turned over through the thermodynamically most stable aminal. The alternate off-cycle mechanism provides a scenario where the rate of iminium hydrolysis exceeds that of aminal formation. From the iminium ion intermediate, part of the catalyst is diverted into a stable aminal resting state. To identify these subtleties, it is essential to follow individual concentrations over time (NMR spectroscopy) rather than observing a single value for the whole system, for example, the amount of energy released (reaction calorimetry).
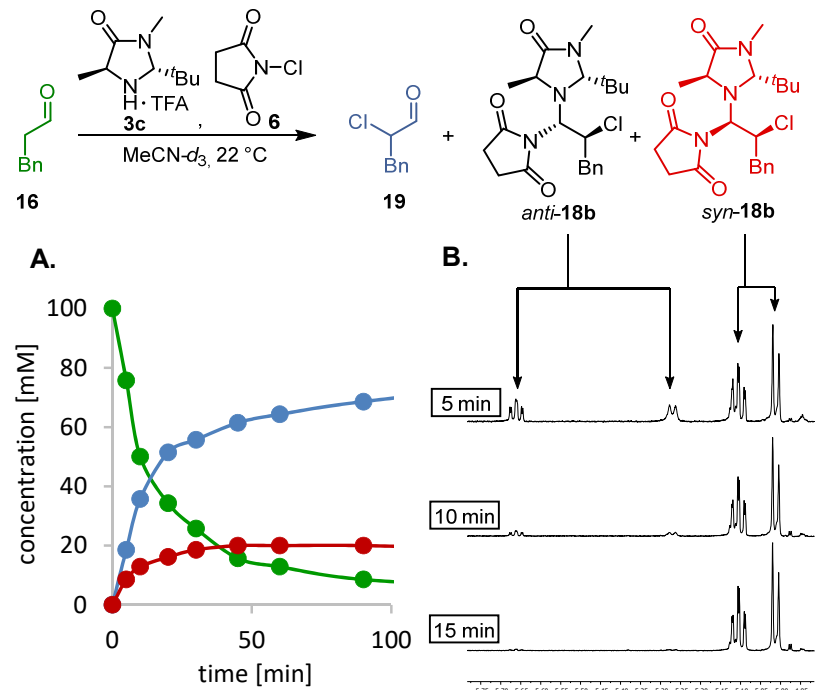

Figure 6. $\alpha$-Chlorination of aldehyde 16 with catalyst $3 \mathrm{c}$ and NCS (6) with concentration/time profile (A) and ${ }^{1} \mathrm{H}-\mathrm{NMR}$ spectra with characteristic aminal signals for the first $15 \mathrm{~min}$ of the reaction (B).

Caution is due when arguing based on the presence or absence of intermediates in the NMR spectrum. Fleeting species, e.g. enamines ${ }^{43}$ and iminium ions, ${ }^{44}$ are difficult to observe and reversibly forming aminal diastereomers are certainly no exception. When reanalyzing our data for this reaction, we noted additional, previously overlooked resonances in the NMR spectrum at $\mathrm{t}=5 \mathrm{~min}$ (Figure 6B) (quickly disappearing thereafter). The possibility of the corresponding species to be the elusive aminal anti-18b is supported by comparison of the observed and computed ${ }^{1} \mathrm{H}$ chemical shifts (Figure 7 ).
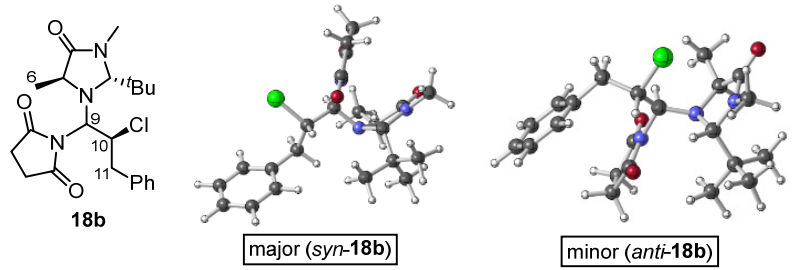

\begin{tabular}{c|ccc|ccc} 
Nuc.\# & Calc. $\delta$ & Exp. $\delta$ & $\begin{array}{l}\text { Abs. } \\
\text { Dev. }\end{array}$ & Calc. $\delta$ & Exp. $\delta$ & $\begin{array}{l}\text { Abs. } \\
\text { Dev. }\end{array}$ \\
\hline $\mathrm{H} 6$ & 1.55 & 1.47 & 0.08 & 1.51 & 1.50 & 0.01 \\
$\mathrm{H} 9$ & 4.81 & 5.01 & 0.20 & 5.15 & 5.27 & 0.12 \\
$\mathrm{H} 10$ & 5.20 & 5.10 & 0.10 & 5.94 & 5.66 & 0.28 \\
$\mathrm{H} 11 \mathrm{a}$ & 4.09 & 3.96 & 0.13 & 3.47 & 3.18 & 0.29 \\
${ }^{t} \mathrm{Bu}$ & 1.08 & 1.10 & 0.02 & 0.77 & 0.91 & 0.14 \\
\hline MAD & & & 0.11 & & & 0.17
\end{tabular}

Figure 7. Calculated syn-18b and anti-18b lowest energy conformations of the aminal $\mathbf{1 8 b}$ (top). Key ${ }^{1} \mathrm{H}$ chemical shifts calculated at $\mathrm{PCM}(\mathrm{ACN})-\mathrm{mPW} 1 \mathrm{PW} 91 / 6-311+\mathrm{G}(2 \mathrm{~d}, \mathrm{p}) / / \mathrm{PCM}$ 
$(A C N)-B_{3} L Y P-D_{3}(B J) / 6-31 G+(d, p)$ level of theory (bottom) (for additional details see $\mathrm{SI}$ ).

This hypothesis is in line with the calculated reaction path as the thermodynamically more stable aminal syn-18b accumulates in favor of anti-18b. While minor anti-18b will continue to be formed at an (unknown) kinetic ratio, its concentration quickly becomes too small to be observed by NMR as the amount of available catalyst diminishes rapidly into the syn-18b aminal reservoir. It is worth noting that under Curtin-Hammett conditions, the equilibrium distribution of the equilibrating species and the product distribution must be time-invariant. 45

\section{The Role of Chloroenamine Intermediates}

The DFT calculations for catalyst $3 \mathbf{c}$ demonstrated that chloroenamine formation - either by iminium deprotonation or by E2 elimination from the aminal intermediate - is energetically disfavored compared to the alternative hydrolysis pathway. This assessment is supported by the notable lack of evidence of protonation (no deuterium incorporation) and the absence of dichlorination products. For catalyst 3a, erosion of the ee was shown upon complete turnover of the substrate in a chlorination reaction..$^{15}$ In a direct comparison, MacMillan et al. ${ }^{18}$ also showed that in the absence of NCS, catalyst $\mathbf{3} \mathbf{b}$ effects rapid racemization of chloroaldehydes. This result suggests that chloroenamines are accessible even in the absence of chloroaminals, i.e. from the iminium ions by deprotonation. During our efforts to separate aminals from reaction mixtures, we also have been able to characterize the first dichloroaminal $24 \mathbf{a}$ from catalyst 3a, which we suspect to be derived from the corresponding dichloroiminium intermediate (Scheme 7). In case of the Jørgensen-Hayashi catalyst or unsubstituted pyrrolidine, it was shown that chloroenamine formation is competing ${ }^{46}$ with the hydrolysis thus leading to the observation of significant amounts of dichlorination byproduct in both cases. This problem can be overcome by accelerating the hydrolysis step via intramolecular (proline ${ }^{47}$ ) or intermolecular assistance (2,6-lutidine and $\left.\mathrm{H}_{2} \mathrm{O}^{46}\right)$.

\section{Scheme 7. Chloroenamines can competitively lead to racemization and dichlorination}

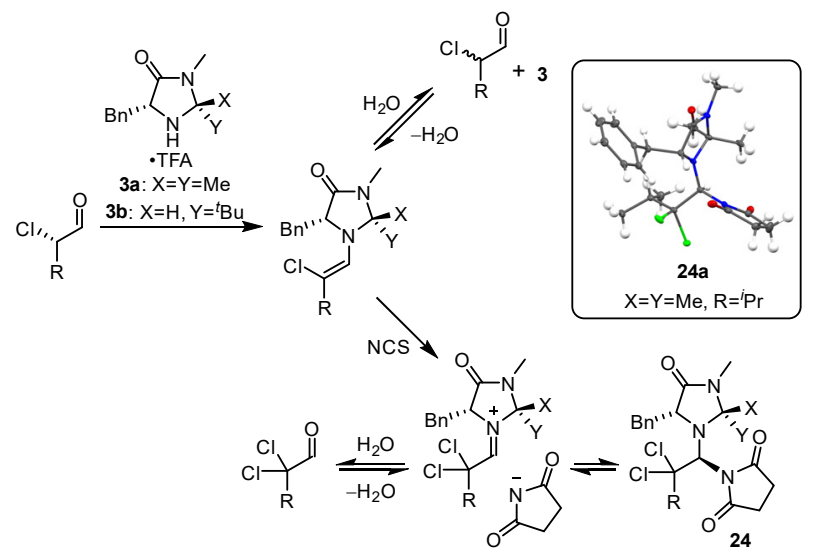

Ion Mobility-Mass Spectrometry Studies

Aminals can be subjected to electrospray ionization followed by collision-induced dissociation. For each aminal, diastereomeric iminium ions can be formed as $E$ - and Zisomers. In a feasibility study, we aimed to find out whether iminium ions derived from two isolated C2-epimeric aminals would be separable due their different ion mobilities in a drift gas (He).

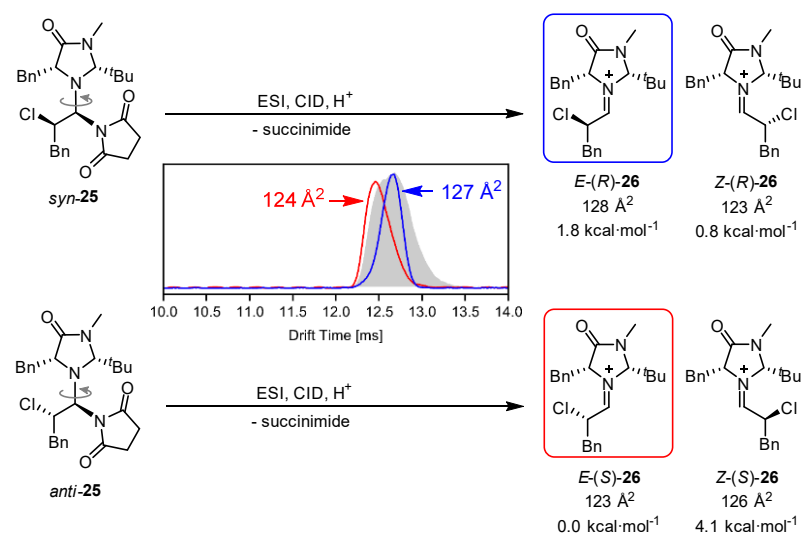

Figure 8. Separate ionization of the diastereomeric aminals syn-25 and anti-25 with arrival time distributions and experimental CCSs of the generated diastereomeric iminium ions (blue and red traces). The trace of the mixture of both diastereomers is depicted in grey. The four possible chloroiminium ions and their calculated CCSs and relative free energies are shown on the right.

Toward this end, instrument-independent collision cross sections (CCSs) were determined by ion mobility-mass spectrometry experiments on a custom-built instrument. ${ }^{48}$ The arrival time distributions (ATDs) of the selectively dissociated aminals are represented in Figure 8 . The conformational space of the iminium ions $\mathbf{2 6}$ was sampled using a generic algorithm ${ }^{49}$ and promising candidate structures were reoptimized at the PBEoD $3 / 6-311+G(d, p)$ level of theory..$^{\circ}$ Computed CCSs were obtained using the trajectory method..$^{\circ}$ The combined approach was successfully used to make a prediction whether $E$ - or $Z$ - iminium ions are formed in the decomposition process of the two diastereomeric aminals syn-25 and anti-25. The ATD traces of the selectively dissociated aminals suggest that the dissociation of each aminal leads to the corresponding $E$-iminium ion. The comparison of the computed and the experimental CCS value suggests the $E$-iminium to be formed for both epimers (see SI for details). Future research will involve spectroscopic investigation (e.g., IR spectroscopy) of the separate iminium ions and following the iminium ion distribution over the course of the reaction.

Aminals with Jørgensen-Hayashi-Type Catalysts

In light of the observations described above for the MacMillan catalysts, we decided to reinvestigate the JørgensenHayashi system for which we have not been able to obtain crystals of sufficient quality. Toward this end, we prepared a reaction mixture using isovaleraldehyde, catalyst $\mathbf{9 b}$ and $N$-chlorophthalimide (NCP) as previously described. ${ }^{6}$ In order to obtain cleaner spectra than those reported, we removed all volatile constituents by evaporation. In the ${ }^{1} \mathrm{H}-$ NMR spectrum, 11 showed rather broad resonances at room temperature which split up at around $-20{ }^{\circ} \mathrm{C}$ and at 
$-54^{\circ} \mathrm{C}$ two sharp signal sets with a 3:1 ratio were observed. The presence of EXSY exchange peaks between the two signal groups indicated two rapidly interconverting species. These observations had been previously interpreted as $\mathbf{1 1}$ being a mixture of interconverting diastereomers at the aminal carbon with the anti-diastereomer as the major and the syn-diastereomer as the minor constituent. ${ }^{6}$ Interestingly, in both species the large $3 \mathrm{~J}_{\mathrm{H}-\mathrm{H}}$ coupling indicates an antiperiplanar arrangement of the $\mathrm{H}$-atoms along the $\mathrm{Cl}^{\prime}$ $\mathrm{C}_{2}$ ' bond. In order to elucidate the structures of the two species from NOE data, we aimed for minimizing exchange contributions to the NOE. This was largely achieved by changing the solvent from $\mathrm{CDCl}_{3}$ to $\mathrm{CD}_{2} \mathrm{Cl}_{2}$, which allows cooling to $-83^{\circ} \mathrm{C}$. We managed to assign most signals of both species and obtained a rather large body of distance data from the NOE of the major species and far less of the minor (see SI). The challenge now is to find out, why two signal sets are obtained and whether we can assign the two signal sets to possible structures of species. It is conceivable that these are interconverting diastereoisomers (syn and anti) as proposed previously by Blackmond et al. ${ }^{6}$ (Scheme 9). An alternative explanation would involve interconverting conformational isomers. For the major species, a comparison of distances with calculated structures allowed the assignment of syn-configuration (Figure 9). This is in contrast to what has been claimed previously. ${ }^{6}$ The key question now is whether the minor species is a diastereomer or a conformational isomer. For both species, only small difference in the distances were observed indicating a very similar relative orientation of the pyrrolidine ring to the chlorinated substrate; the antiperiplanar arrangement of $\mathrm{Hr}^{\prime}$ and $\mathrm{H}_{2}$ ' is conserved (see above). While our recently published pure shift ROE experiment ( $\mathrm{F} 1$ PSYCHE EASY ROESY ${ }^{51}$ ) afforded additional distance data, still no safe assignment of the minor species was possible. From the $33_{\mathrm{H}-\mathrm{H}}$ coupling data in the pyrrolidine ring, it is not unlikely that multiple envelope conformations are present. The "averaged" pucker of the pyrrolidine is different for the major and minor species, which we consider unlikely for the ap arrangement of $\mathrm{H}_{1}$ ' and $\mathrm{H}_{2}$ ' in syn vs. anti-diastereoisomers. Thus we considered hindered rotation as being a plausible cause. From J-coupling analysis (see above and data in the SI) it is quite unlikely, that the pyrrolidine puckers are the reason for the separate signal sets; thus we focused our attention to the orientation of the diarylsilylether group. This hypothesis seems plausible as a structure search in the CCDC data base for Jørgensen-Hayashi catalysts with the pyrrolidine nitrogen bound to stereogenic $\mathrm{sp}^{3}$ hybridized carbon reveals striking similarities concerning the catalyst structure (see SI)..$^{22}$ Each structure in the database had the pyrrolidine ring in an envelope conformation with the $\gamma$-carbon tilted out of plane toward the diarylsilylether moiety and the nitrogen lone pair (down conformation). For the orientation of the diarylsilylether group, two distinct conformational minima are observed. In 7/10 crystal structures, the $\mathrm{N}$ - and $\mathrm{O}$-substituent at the exocyclic $\mathrm{C}-\mathrm{C}$ bond of the catalyst possess an antiperiplanar ( $a p)$ relationship whereas in three cases an exosynclinal (exo-sc) conformation was adopted.
As NOE data was not entirely conclusive on the nature of the minor species and the process of the two interconverting aminal species $\mathbf{1 4}$ at $-54^{\circ} \mathrm{C}$, a calculation of the NMR shifts for several conformational isomers and stereoisomers was performed (see SI for details). When the predicted conformers for $\mathbf{1 4}$ were separated into the ap and exo-sc classifications the ap chemical shifts corresponded to the major product (mean average deviation (MAD): $1.4 \mathrm{ppm}^{13} \mathrm{C}$, o.10 ppm ${ }^{1} \mathrm{H}$ ) and the exo-sc chemical shifts were in excellent agreement with the minor product (MAD: 1.5 ppm ${ }^{13} \mathrm{C}$, $0.13 \mathrm{ppm}{ }^{1} \mathrm{H}$ ) for key chemical shifts (Figure 9).
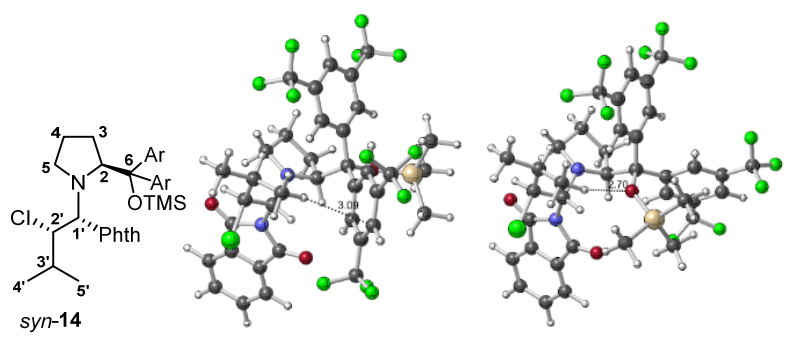

\begin{tabular}{|c|c|c|c|c|c|c|}
\hline \multirow[b]{2}{*}{ Nuc.\# } & \multicolumn{3}{|c|}{\begin{tabular}{|l|} 
major (syn-ap-14) \\
\end{tabular}} & \multicolumn{3}{|c|}{ minor (syn-sc-exo-14) } \\
\hline & Calc. $\delta$ & Exp. $\delta$ & $\begin{array}{l}\text { Abs. } \\
\text { Dev. }\end{array}$ & Calc. $\delta$ & Exp. $\delta$ & $\begin{array}{l}\text { Abs. } \\
\text { Dev. }\end{array}$ \\
\hline C1' & 75.2 & 76.3 & 1.1 & 74.6 & 76.1 & 1.5 \\
\hline $\mathrm{C} 2$ & 67.9 & 68.6 & 0.7 & 67.1 & 68.6 & 1.5 \\
\hline C2' & 72.9 & 65.6 & 7.3 & 74.1 & 66.8 & 7.3 \\
\hline C3' & 32.2 & 29.3 & 2.9 & 32.1 & 29.0 & 3.1 \\
\hline $\mathrm{C} 4$ & 25.2 & 24.3 & 0.9 & 25.4 & 24.1 & 1.3 \\
\hline C4' & 19.9 & 21.3 & 1.4 & 19.7 & 21.4 & 1.7 \\
\hline $\mathrm{C} 5$ & 48.4 & 48.8 & 0.4 & 47.9 & 48.5 & 0.6 \\
\hline C5' & 12.3 & 12.2 & 0.1 & 16.2 & 15.7 & 0.5 \\
\hline $\mathrm{C} 6$ & 82.7 & 82.4 & 0.3 & 84.3 & 84.2 & 0.1 \\
\hline MAD & & & 1.4 & & & 1.5 \\
\hline $\mathrm{H} 1^{\prime}$ & 5.24 & 5.16 & 0.08 & 6.04 & 5.81 & 0.23 \\
\hline $\mathrm{H} 2$ & 4.80 & 4.60 & 0.20 & 4.24 & 4.15 & 0.09 \\
\hline $\mathrm{H} 2^{\prime}$ & 5.25 & 4.98 & 0.27 & 5.28 & 5.05 & 0.23 \\
\hline H3' & 2.39 & 2.13 & 0.26 & 2.67 & 2.35 & 0.32 \\
\hline $\mathrm{H} 3 \mathrm{a}$ & 1.76 & 1.71 & 0.05 & 1.81 & 1.82 & 0.01 \\
\hline $\mathrm{H} 3 \mathrm{~b}$ & 1.80 & 1.71 & 0.09 & 1.32 & 1.38 & 0.06 \\
\hline $\mathrm{H} 4^{\prime}$ & 1.07 & 0.95 & 0.12 & 1.20 & 1.06 & 0.14 \\
\hline $\mathrm{H} 4 \mathrm{a}$ & 1.18 & 1.20 & 0.02 & 1.25 & 1.28 & 0.03 \\
\hline $\mathrm{H} 4 \mathrm{~b}$ & 0.08 & 0.09 & 0.01 & 0.63 & 0.63 & 0.00 \\
\hline H5' & 0.05 & -0.01 & 0.06 & 1.50 & 1.28 & 0.22 \\
\hline $\mathrm{H} 5 \mathrm{a}$ & 3.37 & 3.29 & 0.08 & 3.44 & 3.27 & 0.17 \\
\hline $\mathrm{H} 5 \mathrm{~b}$ & 1.97 & 1.97 & 0.00 & 2.16 & 2.16 & 0.00 \\
\hline MAD & & & 0.10 & & & 0.13 \\
\hline
\end{tabular}

Figure 9. Calculcated ap (top left) and exo-sc (top right) conformations of the Jørgensen-Hayashi aminal syn-14 $\left(\mathrm{R}=\mathrm{C}\left(3,5^{-}\right.\right.$ $\left.\mathrm{CF}_{3}-\mathrm{C}_{6} \mathrm{H}_{3}\right)_{2} \mathrm{OTMS}$ ). Bottom: key chemical ${ }^{1} \mathrm{H}$ and ${ }^{13} \mathrm{C}$ shifts. ${ }^{1} \mathrm{H}$ and ${ }_{13} \mathrm{C}$ chemical shifts calculated with the $\mathrm{mPW}_{1} \mathrm{PW}_{91} / 6-$ $311+\mathrm{G}(2 \mathrm{~d}, \mathrm{p})\left(\mathrm{GIAO}\right.$; PCM, $\left.\mathrm{CHCl}_{3}, 219.15 \mathrm{~K}\right) / / \mathrm{B}_{3} \mathrm{LYP}-\mathrm{D}_{3}(\mathrm{BJ}) / 6-$ ${ }_{31} \mathrm{G}+(\mathrm{d}, \mathrm{p})(219.15 \mathrm{~K})$ level of theory (indicative atoms are highlighted in bold).

From the integration of the exchange signals in $1 \mathrm{D}$ NOE series a barrier of $12.8(4) \mathrm{kcal} \mathrm{mol}^{-1}$ can be determined at $-54{ }^{\circ} \mathrm{C}$. Investigation into the rotational barrier from the $a p$ 
to the exo-sc for syn-14 using DFT calculations (see SI for additional details), revealed the barrier of rotation to be $\sim 13.9 \mathrm{kcal} \mathrm{mol}^{-1}$, within the error expected for this method to compare to the experimental $12.8 \mathrm{kcal} \mathrm{mol}^{-1}$ barrier between the major and minor products at $-54{ }^{\circ} \mathrm{C}$. Further separation of the conformers into concave and convex pyrrolidine ring puckering, led to worse predicted chemical shifts for both the major and minor products, suggesting an averaging of the ring puckering in the NMR solution (barrier of $\sim 1 \mathrm{kcal} \mathrm{mol}^{-1}$ for the major (ap) product). The $\mathrm{H}_{5}$ ' proton shift appears to be diagnostic of ap and exo-sc conformations with the shift at -0.o1 ppm for the major product and at $1.28 \mathrm{ppm}$ for the minor product. In the lowest energy conformer calculated for the ap product, a $\mathrm{C}-\mathrm{H} \cdots \pi$ interaction can be seen shielding this methyl group proton, which does not occur in the exo-sc product (Figure 9).

Additionally, NMR calculations to determine the major and minor products bearing a succinimide (aminal 11) substituent instead of phthalimide (aminal 14) found by Blackmond and co workers ${ }^{6}$ at $-54 \mathrm{C}$ were performed. The experimental chemical shifts were compared to the syn-11 ap and exo-sc, anti( $\left.\mathrm{C}_{1}^{\prime}\right)$ ap and exo-sc, and anti( $\left.\mathrm{C}_{2}^{\prime}\right)-11$ ap calculated chemical shifts. The syn-ap-11 calculated chemical shifts were in good agreement with the major product, while the syn-exo-sc-11 produced some shifts above the expected deviation (<0.3 ppm for ${ }^{1} \mathrm{H}$ shifts and $\leq 3.1 \mathrm{ppm}$ for ${ }^{13} \mathrm{C}$ shifts - with the exception of $7.3 \mathrm{ppm}$ for $\mathrm{C} 2$ '- $\mathrm{Cl}$ ). ${ }^{53}$ The anti $\mathrm{C}_{1}$ ' and $\mathrm{C}_{2}$ ' aminals were found to have greater mean average deviations (MADs) for the ${ }^{1} \mathrm{H}$ and ${ }^{13} \mathrm{C}$ chemical shifts when compared to the minor product chemical shifts than the syn-exo-sc-11 aminal. These results, along with the agreement of syn-exo-sc-14 calculated chemical shifts with the minor product experimental chemical shifts, suggest further analysis of the minor product experimental chemical shifts for $\mathbf{1 1}$ at $-54{ }^{\circ} \mathrm{C}$ may be needed to confirm the chemical shift assignment.

The computationally and NMR spectroscopically derived $\left(2 S, 1^{\prime} R, 2^{\prime} S\right)$-configuration and the conformation of the synaminal 14 share several structural features with the crystallographically determined aminals of the MacMillan catalysts including an antiperiplanar orientation of $\mathrm{C}_{1}$ '- $\mathrm{H}$ and $\mathrm{C}_{2}$ '- $\mathrm{H}$ and the lone pair of aminal nitrogen from the catalysts being antiperiplanar to the imide nitrogen. The stabilization energy of this $\mathrm{n}_{\mathrm{N}(\mathrm{cat})} \rightarrow \sigma^{*}{ }_{\mathrm{C}-\mathrm{N} \text { (imide) }}$ interaction (Scheme 8) is predicted to be $16.3 \mathrm{kcal} \mathrm{mol}^{-1}$ for the ap product and $16.0 \mathrm{kcal} \mathrm{mol}^{-1}$ for exo-sc product. This interaction is even stronger when the succinimide substituent is present 17.5 (ap) and 17.2 (exo-sc) $\mathrm{kcal} \mathrm{mol}^{-1}$; and it decreases to $\sim 8.0 \mathrm{kcal} \mathrm{mol}^{-1}$ in the lowest energy conformer of the anti-aminal (see SI for details). ${ }^{54}$

In summary, only the syn-diastereomer $\left(2 S, 1^{\prime} R, 2^{\prime} S\right)-14$ allows for an optimal synergistic interplay of stereoelectronic effects and minimized steric interactions.

As additional and independent supporting evidence for the structural assignment, aminal $\mathbf{1 4}$ was reduced and the resulting alcohol was converted to the crystalline dinitrobenzoate 15. After a thorough isolation process only one diastereomer could be isolated. Crystal structure analysis confirmed the syn-configuration of aminal 15 (Scheme 8).
Scheme 8. Stereochemical Assignment of Aminal 14

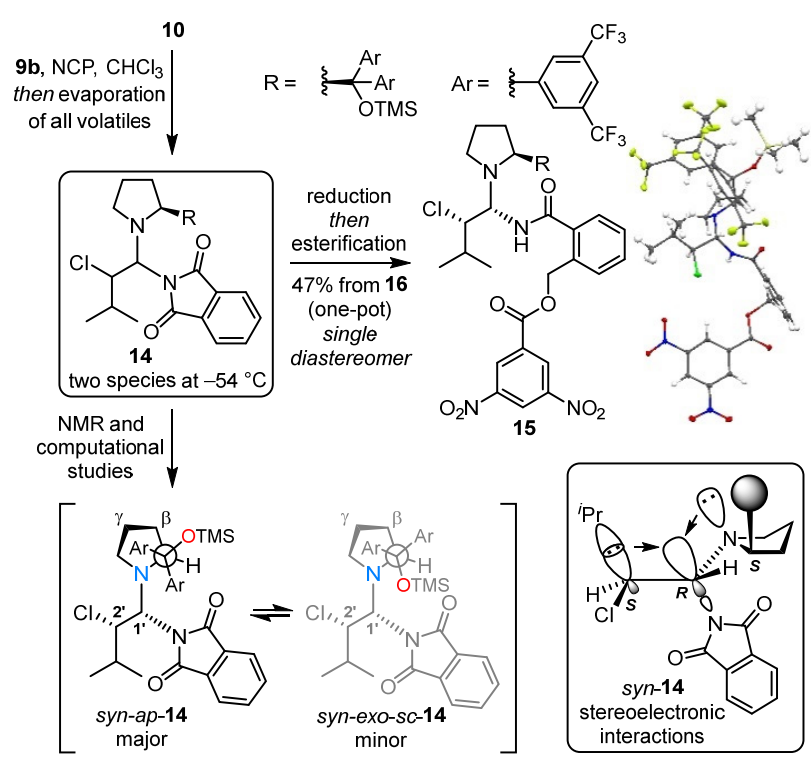

When substituting isovaleraldehyde with propanal, the analogous aminal could be obtained along with the chloroaldehyde being formed in a 57:43 enantiomeric ratio. NMR spectroscopic analysis of the aminal at $-54^{\circ} \mathrm{C}$ showed the presence two separate sets of sharp signals in a 68:32 ratio. Decomposition of the aminal into the corresponding chloroaldehyde was promoted by addition of 2 equivalents of water and TFA. Determination of the enantiomeric excess revealed high enantiomeric excess for the chloroaldehyde product (1-120 min: 95-93\% ee). Additionally, almost no deuterium incorporation was observed when the decomposition of the propanal-derived aminal or aminal 14 was promoted by $\mathrm{D}_{2} \mathrm{O}$ with or without TFA- $\mathrm{d}_{1}$. These observations suggest that only one out of four possible aminal diastereomers is present in sufficient concentration to be detected and isolated. Thus, aminal accumulation solely depends on thermodynamic stability.

\section{- CONCLUSION}

A comparative analysis of the Curtin-Hammett model for stereocontrol and the steric shielding model showed only the latter to be consistent with experimental evidence. Using crystallography, NMR spectroscopy and computational NMR shift prediction, we have elucidated the configuration and conformation of several aminal intermediates of different catalysts in the organocatalytic $\alpha$-chlorination of aldehydes. Their relative stability was rationalized by minimizing steric interactions and maximizing stabilizing stereoelectronic effects.

In the case of the Jørgensen-Hayashi catalyst $\mathbf{9 b}$, the underlying assumption of two equilibrating $\mathrm{C}_{1}$-aminal diastereomers 14 with the anti-diastereomer being the major and the syn-diastereomer being the minor species ${ }^{6}$ was shown to be incorrect. Instead, the two interconverting species, found upon cooling, are proposed to be conformational isomers of the syn-diastereomer (Scheme 9). Moreover, the suggested formation of the $\mathrm{C}_{2}$-stereogenic center 
by chloroenamine protonation was excluded by the absence of deuterium incorporation under the reaction conditions.

\section{Scheme 9. Structural Revision of Aminal of 14}

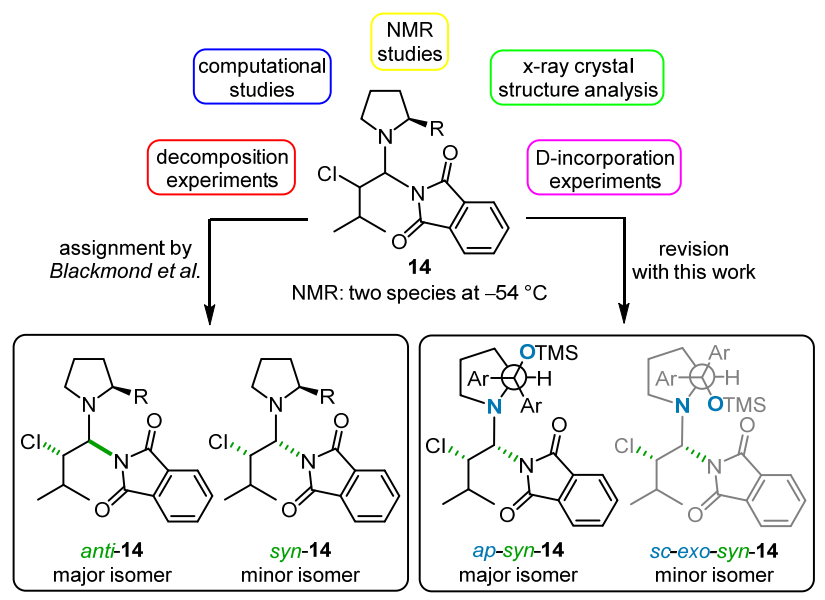

In the case of the MacMillan catalyst 3c, a combination of ${ }_{3}{ }^{3} \mathrm{C}$ KIE studies and DFT calculations demonstrated enamine chlorination not to be the first irreversible step. It also provided complementary evidence to rule out decomposition of the aminal intermediates by an E2 elimination. The calculated transition structures for the enamine chlorination not only correctly predict the stereochemical outcome but also demonstrate that enamine chlorination is faster than (re-)protonation. This in an important finding as it shows that enamine formation in this case is not reversible, i.e. it is not thermodynamically controlled. This observation is supported by the chlorination product lacking significant amounts of deuterium incorporation. With respect to the $\mathrm{C}_{1}$ diastereomeric aminals, DFT calculations predicted the anti-aminal as the kinetic and the syn-aminal as the thermodynamic intermediate.

Following the concentration/time profile by NMR spectroscopy, we were able to identify the previously overlooked anti-aminal being formed within the first 5 minutes of the reaction. It quickly disappears due to accumulation of the catalyst in the syn-aminal resting state. The structure of the anti-aminal was determined by NMR shift computations and the method was validated against the crystallographically characterized syn-aminal diastereomer. These observations rule out a Curtin-Hammett scenario for two reasons. First, Curtin-Hammett conditions require the ratio of the two interconverting intermediates to be time-invariant. Secondly, the dominant conformational minima of the two diastereomeric aminals are unrelated to the reactive conformations for an $\mathrm{E}_{2}$ elimination let alone the respective transition states.

Additionally, we were able to isolate and characterize most of the relevant aminals for the MacMillan catalyst za and 3b by crystal structure analysis for the first time and to reevaluate the incorrect assignment made in the literature. We also isolated and characterized an intermediate relevant to formation of dihalogenation byproducts. Similar to the fluorination reaction described by Jørgensen, formation of dichlorinated intermediates also could change the initial stereoisomeric ratio of the monochlorination product.

Regarding the iminium intermediates, we have for the first time been able to separate diastereomeric iminium ions in an organocatalytic reaction by ion mobility-mass spectrometry. Future research will involve spectroscopic characterization of the separated iminium ions.

These results highlight the added value of combined computational work and experimentation. They also demonstrate the importance of a careful characterization of reaction intermediates to the understanding of complex reaction networks. Understanding and countering the stabilizing interactions within stable intermediates may provide clues to the design of more efficient catalytic systems.

\section{ASSOCIATED CONTENT}

Supporting Information. Experimental procedures, computational details, characterization data of all compounds, NMR spectra, crystallographic data, HPLC and GC traces. This material is available free of charge via the Internet at http://pubs.acs.org.

Accession Codes. 2041073-2041078, 2043370, 2045387 and 2049431 contain supplementary crystallographic data for this paper. These data can be obtained free of charge via www.ccdc.cam.ac.uk/data_request/cif, or by emailing data_request@ccdc.cam.ac.uk, or by contacting The Cambridge Crystallographic Data Centre, 12Union Road, Cambridge CB2 1EZ, UK; fax: +44 1223336033.

\section{AUTHOR INFORMATION}

\section{Corresponding Authors}

Mathias Christmann - Institute of Chemistry and Biochemistry, Freie Universität Berlin, 14195 Berlin, Germany; ORCiD: oooo-ooo1-9313-2392; Email: mathias.christmann@fu-berlin.de

Mathew J. Vetticatt - Department of Chemistry, Binghamton University, Binghamton, New York 13902, United States; ORCiD: oooo-0001-5709-0885; Email: vetticatt@binghamton.edu

Dean J. Tantillo - Department of Chemistry, University of California - Davis, Davis, California 95616, United States; ORCiD: oooo-0oo2-2992-8844; Email: djtantillo@ucdavis.edu

Christina Thiele - Clemens Schöpf Institute of Organic Chemistry and Biochemistry, Technische Universität Darmstadt, 64287 Darmstadt, Germany; ORCiD: oooo-ooo1-7876536X; Email: cthiele@thielelab.de

Kevin Pagel - Institute of Chemistry and Biochemistry, Freie Universität Berlin, 14195 Berlin, Germany; Fritz Haber Institute of the Max Planck Society, 14195 Berlin, Germany; ORCiD: oooo-ooo1-8054-4718; Email: kevin.pagel@fu-berlin.de

\section{Authors}

Sebastian Ponath - Institute of Chemistry and Biochemistry, Freie Universität Berlin, 14195 Berlin, Germany; ORCiD: ooooooo3-2763-0443; 
Chetan Joshi - Department of Chemistry, Binghamton University, Binghamton, New York 13902, United States;

Amy T. Merrill - Department of Chemistry, University of California - Davis, Davis, California 95616, United States; ORCiD: 0ooo-0o03-4801-1721;

Volker Schmidts - Clemens Schöpf Institute of Organic Chemistry and Biochemistry, Technische Universität Darmstadt, 64287 Darmstadt, Germany; ORCiD: oooo-0oo2-7195$312 X$;

Kim Greis - Institute of Chemistry and Biochemistry, Freie Universität Berlin, 14195 Berlin, Germany; Fritz Haber Institute of the Max Planck Society, 14195 Berlin, Germany; ORCiD: oooo-0002-9107-2282;

Maike Lettow - Institute of Chemistry and Biochemistry, Freie Universität Berlin, 14195 Berlin, Germany; Fritz Haber Institute of the Max Planck Society, 14195 Berlin, Germany;

Manuela Weber - Institute of Chemistry and Biochemistry, Freie Universität Berlin, 14195 Berlin, Germany

Simon Steinhauer- Institute of Chemistry and Biochemistry, Freie Universität Berlin, 14195 Berlin, Germany; ORCiD: ooooooo1-7420-1153;

\section{Author Contributions}

§These authors contributed equally.

\section{Notes}

The authors declare no competing financial interest.

\section{ACKNOWLEDGMENT}

K.G. thanks the Fonds National de la Recherche (FNR), Luxembourg for funding for the project GlycoCat (13549747). C.M.T. thanks the DFG for funding the project TH1115/12-1. D. J. T. is thankful for the support from the US National Science Foundation (CHE-1856416 and the XSEDE program (CHE030089)). We thank Patrick Voßnacker (FU Berlin) for crystallographic support.

\section{REFERENCES}

(1) Carreira, E. M.; Kvaerno, L. Classics in Stereoselective Synthesis; Wiley-VCH, Weinheim, 2009.

(2) Carlson, R.; Carlson, J. E. Design and optimization in organic synthesis, 2nd rev. and enl. ed.; Elsevier, Amsterdam, San Diego, CA, 2005.

(3) List, B.; Maruoka, K. Science of Synthesis, Asymmetric Organocatalysis Vol. 1; Georg Thieme, Stuttgart, New York, 2012.

(4) Marigo, M.; Fielenbach, D.; Braunton, A.; Kjaersgaard, A.; Jørgensen, K. A. Angew. Chem. Int. Ed. 2005, 44, 3703-3706.

(5) Burés, J.; Armstrong, A.; Blackmond, D. G. Pure Appl. Chem. 2013, 85, 1919-1934.

(6) a) Burés, J.; Armstrong, A.; Blackmond, D. G. J. Am. Chem. Soc. 2012, 134, 6741-6750; b) Burés, J.; Armstrong, A.; Blackmond, D. G. J. Am. Chem. Soc. 2012, 134, 14264.

(7) a) Reyes-Rodríguez, G. J.; Rezayee, N. M.; Vidal-Albalat, A.; Jørgensen, K. A. Chem. Rev. 2019, 119, 4221-426o; b) Marigo, M.; Wabnitz, T. C.; Fielenbach, D.; Jørgensen, K. A. Angew. Chem. Int. Ed. 2005, 44, 794-797.

(8) Hayashi, Y.; Gotoh, H.; Hayashi, T.; Shoji, M. Angew. Chem. Int. Ed. 2005, 44, 4212-4215.
(9) Földes, T.; Madarász, Á.; Révész, Á.; Dobi, Z.; Varga, S.; Hamza, A.; Nagy, P. R.; Pihko, P. M.; Pápai, I. J. Am. Chem. Soc. 2017, 139, 17052-17063.

(10) Oestreich, M. Angew. Chem. Int. Ed. 2005, 44, 2324-2327.

(11) Britton, R.; Kang, B. Nat. Prod. Rep. 2013, 30, 227-236.

(12) Shibatomi, K.; Narayama, A. Asian J. Org. Chem. 2013, 2, 812823.

(13) Brochu, M. P.; Brown, S. P.; MacMillan, D. W. C. J. Am. Chem. Soc. 2004, 126, 4108-4109.

(14) Wack, H.; Taggi, A. E.; Hafez, A. M.; Drury, W. J.; Lectka, T. J. Am. Chem. Soc. 2001, 123, 1531-1532.

(15) Brown S. P. Iminium and Enamine Activation: Methods for Enantioselective Organocatalysis; California Institute of Technology, Pasadena, California, 2005.

(16) Duan, X.-H.; Mayr, H. Org. Lett. 2010, 12, 2238-2241.

(17) Halland, N.; Braunton, A.; Bachmann, S.; Marigo, M.; Jørgensen, K. A. J. Am. Chem. Soc. 2004, 126, 4790-4791.

(18) Amatore, M.; Beeson, T. D.; Brown, S. P.; MacMillan, D. W. C. Angew. Chem. Int. Ed. 2009, 48, 5121-5124.

(19) a) Bourboula, A.; Limnios, D.; Kokotou, M. G.; Mountanea, O. G.; Kokotos, G. Molecules 2019, 24, 2081; b) Mountanea, O. G.; Limnios, D.; Kokotou, M. G.; Bourboula, A.; Kokotos, G. Eur. J. Org. Chem. 2019, 2010-2019; c) Kaplaneris, N.; Spyropoulos, C.; Kokotou, M. G.; Kokotos, C. G. Org. Lett. 2016, 18, 5800-5803.

(20) Winter, P.; Vaxelaire, C.; Heinz, C.; Christmann, M. Chem. Commun. 2011, 47, 394-396.

(21) a) Winter, P.; Swatschek, J.; Willot, M.; Radtke, L.; Olbrisch, T.; Schäfer, A.; Christmann, M. Chem. Commun. 2011, 47, 1220012202; b) Winter, P.; Hiller, W.; Christmann, M. Angew. Chem. Int. Ed. 2012, 51, 3396-3400; c) Swatschek, J.; Grothues, L.; Bauer, J. O.; Strohmann, C.; Christmann, M. J. Org. Chem. 2014, 79, 976-983.

(22) Comito, R. J.; Finelli, F. G.; MacMillan, D. W. C. J. Am. Chem. Soc. 2013, 135, 9358-9361.

(23) The succinimide anion/iminium ion pair must be considered inert toward water as direct hydrolysis of the iminium ion (a.k.a. the "classical" pathway) or proton transfer to the succinimide anion would bypass the aminal intermediate.

(24) A publication on organocatalytic selenylations ${ }^{27}$ suggested that an exclusive stereospecific reaction of either enamine with water (pKa 14) may "lead selectively and irreversibly" to the two enantiomeric products via iminium ions "being counter-balanced by a hydroxy group [sic]".

(25) Ponath, S.; Menger, M.; Grothues, L.; Weber, M.; Lentz, D.; Strohmann, C.; Christmann, M. Angew. Chem. Int. Ed. 2018, 57, $11683-11687$.

(26) Burés, J.; Armstrong, A.; Blackmond, D. G. Acc. Chem. Res. 2016, 49, 214-222.

(27) a) Burés, J.; Dingwall, P.; Armstrong, A.; Blackmond, D. G. Angew. Chem. Int. Ed. 2014, 53, 8700-8704.

(28) Wiest, J. The Role of Charged and Uncharged Intermediates in Organocatalysis, MS Thesis, 2012, Universität Basel (conducted in the Blackmond group at The Scripps Research Institute).

(29) a) Alamillo-Ferrer, C.; Nielsen, C. D.-T.; Salzano, A.; Companyó, X.; Di Sanza, R.; Spivey, A. C.; Rzepa, H. S.; Burés, J. J. Org. Chem. 2021, 86, 4326-4335; b) Seebach, D.; Sun, X.; Ebert, M.-O.; Schweizer, W. B.; Purkayastha, N.; Beck, A. K.; Duschmalé, J.; Wennemers, H.; Mukaiyama, T.; Benohoud, M.; Hayashi, Y.; Reiher, M. Helv. Chim. Acta 2013, 96, 799-852; c) Seebach, D.; Grošelj, U.; Badine, D. M.; Schweizer, W. B.; Beck, A. K. Helv. Chim. Acta 2008, 91, 1999-2034. 
(30) a) Seeman, J. I. Chem. Rev. 1983, 83, 83-134; b) Seeman, J. I. J. Chem. Educ. 1986, 63, 42-48; c) Chakraborty, S.; Saha, C. Reson 2016, 21, 151-171.

(31) Singleton, D. A.; Thomas, A. A. J. Am. Chem. Soc. 1995, 117, 9357-9358.

(32) a) Becke, A. D. J. Chem. Phys. 1993, 98, 5648-5652; b) Stephens, P. J.; Devlin, F. J.; Chabalowski, C. F.; Frisch, M. J. J. Phys. Chem. 1994, 98, 11623-11627; c) Lee, C.; Yang, W.; Parr, R. G. Phys. Rev. B 1988, 37, 785-789.

(33) Hariharan, P. C.; Pople, J. A. Theoret. Chim. Acta 1973, 28, 213-222.

(34) Tomasi, J.; Mennucci, B.; Cammi, R. Chem. Rev. 2005, 105, 2999-3093.

(35) Frisch, M. J.; Trucks, G. W.; Schlegel, H. B.; Scuseria, G. E.; Robb, M. A.; Cheeseman, J. R.; Scalmani, G.; Barone, V.; Petersson, G. A.; Nakatsuji, H.; Li, X.; Caricato, M.; Marenich, A. V.; Bloino, J.; Janesko, B. G.; Gomperts, R.; Mennucci, B.; Hratchian, H. P.; Ortiz, J. V.; Izmaylov, A. F.; Sonnenberg, J. L.; Williams-Young, D.; Ding, F.; Lipparini, F.; Egidi, F.; Goings, J.; Peng, B.; Petrone, A.; Henderson, T.; Ranasinghe, D.; Zakrzewski, V. G.; Gao, J.; Rega, N.; Zheng, G.; Liang, W.; Hada, M.; Ehara, M.; Toyota, K.; Fukuda, R.; Hasegawa, J.; Ishida, M.; Nakajima, T.; Honda, Y.; Kitao, O.; Nakai, H.; Vreven, T.; Throssell, K.; Montgomery, J. A., Jr.; Peralta, J. E.; Ogliaro, F.; Bearpark, M. J.; Heyd, J. J.; Brothers, E. N.; Kudin, K. N.; Staroverov, V. N.; Keith, T. A.; Kobayashi, R.; Normand, J.; Raghavachari, K.; Rendell, A. P.; Burant, J. C.; Iyengar, S. S.; Tomasi, J.; Cossi, M.; Millam, J. M.; Klene, M.; Adamo, C.; Cammi, R.; Ochterski, J. W.; Martin, R. L.; Morokuma, K.; Farkas, O.; Foresman, J. B.; Fox, D. J. Gaussian 16, Revision C.o1; Gaussian, Inc., Wallingford CT, 2016.

(36) a) Grimme, S.; Antony, J.; Ehrlich, S.; Krieg, H. J. Chem. Phys. 2010, 132, 154104; b) Grimme, S.; Ehrlich, S.; Goerigk, L. J. Comput. Chem. 2011, 32, 1456-1465.

(37) Grimme, S. Chem. Eur. J. 2012, 18, 9955-9964.

(38) a) Zhu, H.; Clemente, F. R.; Houk, K. N.; Meyer, M. P. J. Am. Chem. Soc. 2009, 131, 1632-1633; b) Xu, H.; Zuend, S. J.; Woll, M. G.; Tao, Y.; Jacobsen, E. N. Science 2010, 327, 986-990; c) Klausen, R. S.; Kennedy, C. R.; Hyde, A. M.; Jacobsen, E. N. J. Am. Chem. Soc. 2017, 139, 12299-12309; d) Roytman, V. A.; Karugu, R. W.; Hong, Y.; Hirschi, J. S.; Vetticatt, M. J. Chem. Eur. J. 2018, 24, 8098-8102.

(39) Ashley, M. A.; Hirschi, J. S.; Izzo, J. A.; Vetticatt, M. J. J. Am. Chem. Soc. 2016, 138, 1756-1759.

(40) Considering that there are errors ( $\left.0.5 \mathrm{kcal} \mathrm{mol}^{-1}\right)$ associated with the computed energetics of the reaction coordinate, the slight discrepancy between experimental and predicted KIEs for the carbonyl carbon is not significant.

(41) See reference (39) for a similar E2-elimination step in an $\alpha$ amination reaction catalyzed by L-proline.

(42) a) Frantz, D. E.; Singleton, D. A.; Snyder, J. P. J. Am. Chem. Soc. 1997, 119, 3383-3384; b) Singleton, D. A.; Schulmeier, B. E. J. Am. Chem. Soc. 1999, 121, 9313-9317; c) Frantz, D. E.; Singleton, D. A. J. Am. Chem. Soc. 200o, 122, 3288-3295; d) Bandar, J. S.; Sauer,
G. S.; Wulff, W. D.; Lambert, T. H.; Vetticatt, M. J. J. Am. Chem. Soc. 2014, 136, 10700-10707.

(43) a) Schmid, M. B.; Zeitler, K.; Gschwind, R. M. Angew. Chem. Int. Ed. 2010, 49, 4997-5003; b) Schmid, M. B.; Zeitler, K.; Gschwind, R. M. Chem. Sci. 2011, 2, 1793-1803.

(44) Mayr, H.; Ofial, A. R.; Würthwein, E.-U.; Aust, N. C. J. Am. Chem. Soc. 1997, 119, 12727-12733.

(45) Seeman, J. I.; Farone, W. A. J. Org. Chem. 1978, 43, 18541864 .

(46) Jimeno, C.; Cao, L.; Renaud, P. J. Org. Chem. 2016, 81, 12511255 .

(47) Hayes, M. D.; Rodríguez-Alvarado, M.; Brenner-Moyer, S. E. Tetrahedron Lett. 2015, 56, 4718-4720.

(48) a) Warnke, S.; Helden, G. von; Pagel, K. Proteomics 2015, 15, 2804-2812; b) Hoffmann, W.; Langenhan, J.; Huhmann, S.; Moschner, J.; Chang, R.; Accorsi, M.; Seo, J.; Rademann, J.; Meijer, G.; Koksch, B.; Bowers, M. T.; Helden, G. von; Pagel, K. Angew. Chem. Int. Ed. 2019, 58, 8216-8220.

(49) Supady, A.; Blum, V.; Baldauf, C. J. Chem. Inf. Model. 2015, 55, 2338-2348.

(50) a) Adamo, C.; Barone, V. J. Chem. Phys. 1999, 110, 6158-6170; b) Mesleh, M. F.; Hunter, J. M.; Shvartsburg; Schatz G. C.; Jarrold, M. F. J. Phys. Chem. 1996, 100, 16082-16086.

(51) a) Ilgen, J.; Kaltschnee, L.; Thiele, C. M. Magn. Reson. Chem. 2018, 56, 918-933; b) Ilgen, J.; Nowag, J.; Kaltschnee, L.; Schmidts, V.; Thiele, C. M. J. Magn. Reson. 2021, 324, 106900.

(52) a) Seebach, D.; Sun, X.; Sparr, C.; Ebert, M.-O.; Schweizer, W. B.; Beck, A. K. Helv. Chim. Acta 2012, 95, 1064-1078; b) Seebach, D.; Sun, X.; Ebert, M.-O.; W. Schweizer, W. B.; Purkayastha, N.; Beck, A. K.; Duschmalé, J.; Wennemers, H.; Mukaiyama, T.; Benohoud, M.; Hayashi, Y.; Reiher, M. Helv. Chim. Acta 2013, 96, 799-852; c) Cassani, C.; Melchiorre, P. Org. Lett. 2012, 14, 55905593; d) Weber, A. K.; Schachtner, J.; Fichtler, R.; Leermann, T. M.; Neudörfl, J. M.; von Wangelin, A. J. Org. Biomol. Chem. 2014, 12, 5267-5277; e) Gurubrahamam, R.; Chen, Y. M.; Huang, W.-Y.; Chan, Y.-T.; Chang, H.-K.; Tsai, M.-K.; Chen, K. Org. Lett. 2o16, 18, 3046-3049; f) Boeckman Junior, R. K.; Wang, H.; Rugg, K. W.; Genung, N. E.; Chen, K.; Ryder, T. R. Org. Lett. 2o16, 18, 6136-6139. (53) Lodewyk, M. W.; Siebert, M. R.; Tantillo, D. J. Chem. Rev. 2012, 112, 1839-1862.

(54) References NBO method: (B3LYP-D3 (BJ)/6-31g(d,p), io(3/32=2), 219.15 K, with a Fine grid); a) Foster, J. P.; Weinhold, F. J. Am. Chem. Soc. 1980,102, 7211-7218; b) Reed, A. E.; Weinhold, F. J. Chem. Phys. 1983, 78, 4066-4073; c) Reed, A. E.; Weinstock, R. B.; Weinhold, F. J. Chem. Phys. 1985, 83, 735-746; d) Reed, A. E.; Weinhold, F. J. Chem. Phys. 1985, 83, 1736-1740; e) J. E. Carpenter, Extension of Lewis structure concepts to open-shell and excitedstate molecular species, Ph.D. thesis, University of Wisconsin, Madison, WI, 1987; f) Carpenter, J. E.; Weinhold, F. J. Mol. Struct. (Theochem). 1988, 139, 41-62; g) Reed, A. E.; Curtiss, L. A.; Weinhold, F. Chem. Rev. 1988, 88, 899-926; h) Weinhold, F. and Carpenter, J. E. in The Structure of Small Molecules and Ions, Naaman, E. R.; Vager, Z. Plenum, 1988, 227-236. 


\title{
Supporting Information
}

\section{On Stereocontrol in Organocatalytic $\alpha$-Chlorinations of Aldehydes}

\author{
Sebastian Ponath ${ }^{\S \ddagger}$, Chetan Joshi ${ }^{\ddagger}$, Amy T. Merrill ${ }^{\dagger}$, Volker Schmidts ${ }^{\star}$, Kim Greis ${ }^{\S}$, Maike \\ Lettow $^{\S \#}$, Manuela Weber ${ }^{\S}$, Simon Steinhauer ${ }^{\S}$, Kevin Pagel ${ }^{\S *}$, Christina Thiele ${ }^{\star \star}$, Dean J. \\ Tantillo $^{\dagger \star}$, Mathew J. Vetticatt ${ }^{\ddagger \star}$, Mathias Christmann ${ }^{\S}$ \\ §Institute of Chemistry and Biochemistry, Freie Universität Berlin, 14195 Berlin, Germany \\ ‡Department of Chemistry, Binghamton University, Binghamton, New York 13902, United States \\ tDepartment of Chemistry, University of California - Davis, Davis, California 95616, United States \\ ${ }^{\times}$Clemens Schöpf Institute of Organic Chemistry and Biochemistry, Technische Universität Darmstadt, \\ 64287 Darmstadt, Germany \\ \#Fritz Haber Institute of the Max Planck Society, 14195 Berlin, Germany
}




\section{TABLE OF CONTENTS}

1. GENERAL INFORMATION 1

2. CHLORINATIONS WITH MACMILLAN-TYPE CATALYSTS .................................................. 4

2.1. Aminals Derived from $1^{\text {st }}, 2^{\text {nd }}$ and $3^{\text {rd }}$ Generation MacMillan Catalysts ...................................... 4

2.2. NMR Reaction Progress (3rd Generation MacMillan Catalyst).....................................................22

2.3. KIEs and DFT Calculations ( $3^{\text {rd }}$ Generation MacMillan Catalyst) .............................................28

2.4. Ion Mobility-Mass Spectrometry with Aminals ( $2^{\text {nd }}$ Generation MacMillan Catalyst) ................... 111

3. CHLORINATIONS WITH THE JØRGENSEN-HAYASHI-TYPE CATALYST ................................ 119

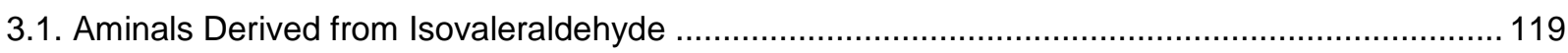

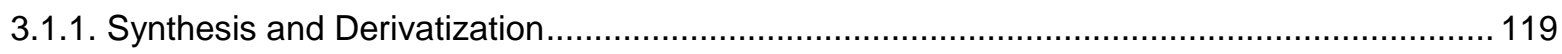

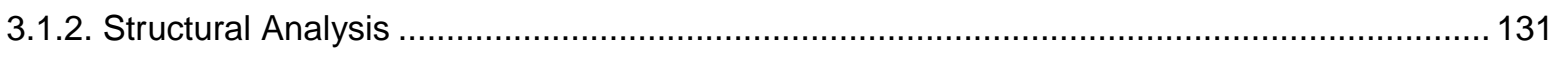

3.1.3. Decomposition and Deuterium Incorporation Studies .................................................... 169

3.2. Aminals Derived from Propanal ....................................................................................... 178

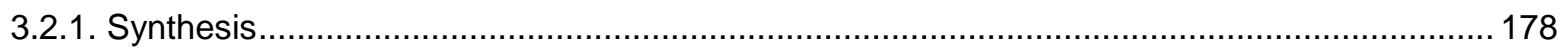

3.2.2. Decomposition and Deuterium Incorporation Studies ................................................... 184

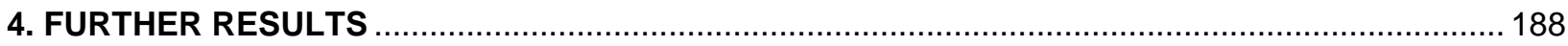

5. REFERENCES

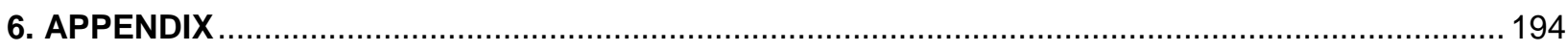

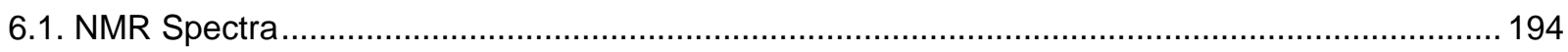

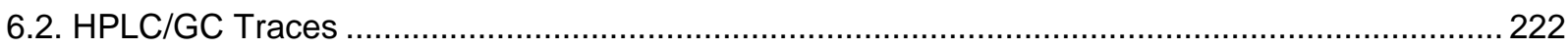

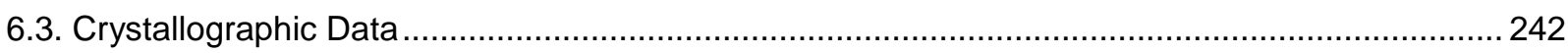




\section{GENERAL INFORMATION}

Anhydrous solvents were provided by purification with MBraun SPS-800 solvent system using solvents of HPLC grade purchased from Fischer Scientific. Solvents for extraction, crystallization and flash column chromatography were purchased in technical grade and distilled under reduced pressure prior to use. Unless otherwise indicated, all starting materials and reagents were purchased from commercial distributors and used without further purification. Products were purified by flash column chromatography on silica gel $60 \mathrm{M}$ (0.040-0.063 mm, 230-400 mesh, Macherey-Nagel). TLC-analysis was performed on silica gel coated aluminum plates ALUGRAM $^{\circledR}$ Xtra SIL G/UV 254 purchased from Macherey-Nagel. Products were detected by UV light at $254 \mathrm{~nm}$ and by using staining reagents based on $\mathrm{KMnO}_{4}$, anisaldehyde, molybdophosphoric acid and cerium sulfate. ${ }^{1} \mathrm{H}-\mathrm{NMR}$ and ${ }^{13} \mathrm{C}-\mathrm{NMR}$ spectral data were recorded on Bruker (AC 500, AVIII 700) and JEOL (ECX 400, Eclipse 500, ECZ 600) spectrometers. The chemical shifts $(\delta)$ are listed in parts per million (ppm) and are reported relative to the corresponding residual solvent signal $\left(\mathrm{CDCl}_{3}: \delta_{\mathrm{H}}=7.26 \mathrm{ppm}, \delta_{\mathrm{c}}=77.16 \mathrm{ppm}, \mathrm{CD}_{2} \mathrm{Cl}_{2}: \delta_{\mathrm{H}}=5.32 \mathrm{ppm}, \delta_{\mathrm{c}}=53.84 \mathrm{ppm}, \mathrm{DMSOd}_{6}: \delta_{\mathrm{H}}=2.50 \mathrm{ppm}\right.$, $\delta_{\mathrm{C}}=39.52 \mathrm{ppm}, \mathrm{CD}_{3} \mathrm{OD}: \delta_{\mathrm{H}}=3.31 \mathrm{ppm}, \delta_{\mathrm{C}}=49.00 \mathrm{ppm}, \mathrm{CD}_{3} \mathrm{CN}: \delta_{\mathrm{H}}=1.94 \mathrm{ppm}, \delta_{\mathrm{c}}=118.26, \mathrm{DMFd}_{7}: \delta_{\mathrm{H}}$ $\left.=8.03 \mathrm{ppm}, \delta_{\mathrm{C}}=163.15 \mathrm{ppm}, \mathrm{C}_{6} \mathrm{D}_{6}: \delta_{\mathrm{H}}=7.16 \mathrm{ppm}, \delta_{\mathrm{C}}=128.06 \mathrm{ppm}\right)$. Integrals are in accordance with assignments; coupling constants $(\mathcal{J}$ ) are given in $\mathrm{Hz}$. Multiplicity is indicated as follows: $s$ (singlet), $d$ (doublet), $\mathrm{t}$ (triplet), q (quartet), $\mathrm{m}$ (multiplet), dd (doublet of doublet), etc. ${ }^{13} \mathrm{CNMR}$ spectra are ${ }^{1} \mathrm{H}$ broadband decoupled. For detailed peak assignments 2D spectra were measured (COSY, HMQC, HMBC). IR spectra were measured with a Jasco spectrometer (FT/IR-4100) equipped with an ATR unit. High resolution mass spectra were measured with an Agilent $6210 \mathrm{ESI}-\mathrm{TOF}(10 \mu \mathrm{L} / \mathrm{min}, 1.0 \mathrm{bar}, 4 \mathrm{kV})$ instrument. Melting points were determined by digital melting point apparatus (Stuart SMP30) and are uncorrected. Optical rotations values were determined with a Jasco P-2000 polarimeter at the temperatures given. Diastereomeric ratios were determined by ${ }^{1} \mathrm{HNMR}$. Enantiomeric ratios were determined by chiral HPLC (Agilent Series 1200 with DAD) or by GC (Agilent 7890B) on a chiral column. The specific conditions are given in each case.

\section{NMR Methods for NOE and J-Coupling Analysis}

NMR spectra for ${ }^{1} \mathrm{H}-{ }^{1} \mathrm{H}$ NOE and J-coupling analysis were recorded on a Bruker AVIII $600 \mathrm{MHz}$ spectrometer equipped with a $5 \mathrm{~mm}$ triple-resonance broadband inverse probe $\left({ }^{1} \mathrm{H} /{ }^{31} \mathrm{P}-\mathrm{BB}-{ }^{2} \mathrm{H}\right)$ with $\mathrm{z}$ gradient $\left(0.494 \mathrm{~T} \cdot \mathrm{m}^{-1}\right.$ maximum gradient strength). Sample temperature was regulated between $190 \mathrm{~K}$ and $300 \mathrm{~K}$ using a liquid nitrogen evaporator. Experiments were recorded and analyzed with TopSpin 3.5pl7. Assignment spectra at $190 \mathrm{~K}$ were recorded using the standard library sequences for $\mathrm{HSQC},{ }^{1} \mathrm{HMBC}^{2}$ and EASY-ROESY ${ }^{3}$. Selective 1D NOE measurements supplemented with a Thrippleton-Keeler filter ${ }^{4}$ to suppress unwanted zero-quantum contributions of $J$-coupled nuclei were performed using the standard library selnogpzs sequence with a spectral width of 12 ppm and 65536 complex points with 32 scans. Sufficient relaxation was ensured by setting the recovery delay to $15 \mathrm{~s}$, which is larger than $5^{\star} T_{1}$ for every signal as determined by an inversion recovery experiment. The carrier frequency offset was set to $4.7 \mathrm{ppm}$ and the selective pulse offset to the respective signal. The RSnob ${ }^{5}$ shape was used for the selective pulse with bandwidths of 15,25 or $50 \mathrm{~Hz}$, depending on the required selectivity. A mixing time series of 8 independent time points was acquired between 50 and $400 \mathrm{~ms}$ in $50 \mathrm{~ms}$ steps for each selected resonance. Spectra were processed by zero-filling once and apodization with exponential broadening with $0.1 \mathrm{~Hz}$. Manual phase correction and automatic baseline correction were applied for each spectrum before integrating the series using the intser command. Additionally, a 2D gradient-selected F1-PSYCHE-EASYROESY ${ }^{6}$ was acquired with spectral widths of 12 ppm in both dimensions and 4096 and 512 complex points, respectively, with 8 scans. The recovery delay was not sufficient for full relaxation to equilibrium. PSYCHE7 
homonuclear decoupling in the indirect dimension was applied with a $30 \mathrm{~ms}$ element consisting of two low power chirp pulses with $25^{\circ}$ flip angle, $10 \mathrm{kHz}$ sweep width and $15 \mathrm{~ms}$ durations each. The pair of spinlocks were set to a tilt angle of $45^{\circ}$ and an RF field of $6500 \mathrm{~Hz}$. Gradient strengths were set to the values given in the original publication. The spectrum was processed by zero-filling once in the indirect dimension and applying a $90^{\circ}$ shifted squared sine bell apodization. While the quantification may show larger deviations due to the insufficient recovery delay, volume integrals were nevertheless collected for all diagonal peaks, where they were sufficiently resolved by the PSYCHE homodecoupling, and all associated cross-peaks. Integrals were normalized using the PANIC ${ }^{8}$ approach: within each spectrum, the cross-peak integrals were divided by the integrals of the selected peak for the 1D spectra or the corresponding diagonal peak at the same chemical shift in $\mathrm{F} 1$ for the $2 \mathrm{D}$ spectrum. The normalized peak integrals are subjected to a linear fit versus the mixing time. The slope of this fit gives the cross-relaxation rate for NOE cross-peaks or the exchange rate for cross-peaks generated by chemical exchange. The fit usually shows (very) high correlation coefficients (Pearson $\mathrm{R}>0.9$ ), with only two exceptions: in some spectra the very last mixing time data point $(400 \mathrm{~ms})$ apparently no longer fulfilled the initial rate approximation and had to be excluded from the fitting process to achieve a sufficiently high correlation coefficient. Secondly, for the NOE from the neighboring nuclei 1' and 2' in the aminal part, the fit showed larger deviations resulting in lower apparent cross-relaxation rates, presumably due to insufficient suppression of $J$-coupling contributions and/or strongcoupling effects between these two nuclei. ${ }^{9}$ The individual cross-relaxation rates $\sigma_{I S}$ were converted to NOE derived distances $r_{I S}$ by internal calibration with the cross-relaxation rate of the diastereotopic proton pair $\mathrm{H} 5 \mathrm{a} / \mathrm{b}$ (denoted " $r e f$ ", set to $1.76 \AA$ ) using equation (1). For methyl groups, the three internuclear distances to individual protons were averaged using " $r^{-3}$ averaging" as described in equation (2). ${ }^{10,11}$ Conformational averaging for different conformers was performed using " $r^{-6}$ averaging" using equation (3), ${ }^{10}$ where $p_{i}$ are the individual conformer populations of the $M$ total conformations. Uncertainties were estimated by Gaussian error propagation. ${ }^{11}$ In cases where both protons could be selected individually by the selective pulse, the NOE derived distances were averaged and the larger value between the deviations of the individual NOE distances to the average distance or the individual estimated uncertainties are used as an error estimate for the NOE distance. While in some cases the error from Gaussian error propagation is as low as $0.01 \mathrm{~A}$, we assume a reasonable estimate to be at least $0.1 \AA$ in $1 \mathrm{D}$ NOE spectra and $0.4 \AA$ in the semiquantitative PSYCHE-EASY ROESY spectra.

$$
\begin{aligned}
& r_{I S}=r_{r e f}\left(\sigma_{r e f} / \sigma_{I S}\right)^{-1 / 6} \\
& r_{I S, a v e}^{\text {Methyl }}=\left(\sum_{i=1}^{3} \frac{1}{3} \times r_{I S, i}^{-3}\right)^{-1 / 3} \\
& r_{I S, \text { ave }}=\left(\sum_{i=1}^{M} \mathrm{p}_{i} \times r_{I S, i}^{-6}\right)^{-1 / 6}
\end{aligned}
$$

$J$-coupling constants were determined either by lineshape analysis in the 1D spectrum or by a 2D (nonpure-shift) version of the PSYCHEDELIC ${ }^{12}$ experiment with spectral widths of $7211 \mathrm{~Hz}$ and $50 \mathrm{~Hz}$ over 16384 and 128 complex points with 16 scans. As for the 1D sel. NOE, the carrier frequency offset was set to $4.7 \mathrm{ppm}$ and the selective pulse offset to the respective signal. The RSnob shape was used for the selective pulse with bandwidths of 15,25 or $50 \mathrm{~Hz}$, depending on the required selectivity. Spectra were processed by zero-filling to 512 points and apodization with exponential broadening with $1 \mathrm{~Hz}$. Manual phase correction was applied before tilting the spectrum with a shear transformation $\left(45^{\circ}\right)$. Coupling constants were extracted from the peak maxima along the column with the highest sensitivity of the multiplet. 


\section{Computational NMR Methods}

The NMR chemical shifts for syn-11, iminium ion derived from syn-11, anti(C1')-11, anti(C2')-11, and syn14 were calculated using the GIAO method with PCM(chloroform)-mPW1PW91/6-311+G(2d,p)// B3LYP$\mathrm{D} 3(\mathrm{BJ}) / 6-31 \mathrm{G}+(\mathrm{d}, \mathrm{p})^{13}$ at $219.15 \mathrm{~K}$ using Gaussian16 ${ }^{14}$ Revision C.01. Conformational searching was performed using Grimme's CREST tool in XTB, using version 2.9 (syn-11; anti(C1')-11; anti(C2')-11; syn14) and version 2.10 .2 (iminium ion derived from syn-11) with the gfn-ff method and chloroform as the solvent (force constant of 1.0). ${ }^{15,16}$ Conformers generated from CREST were optimized with B3LYP$\mathrm{D} 3(\mathrm{BJ}) / 3-21$ and then any conformers within $5 \mathrm{kcal} \mathrm{mol}^{-1}$ from the lowest free energy conformer were reoptimized with $\mathrm{B} 3 \mathrm{LYP}-\mathrm{D} 3(\mathrm{BJ}) / 6-31 \mathrm{G}+(\mathrm{d}, \mathrm{p})$. If more than 100 conformers were generated from CREST, only the conformers within $5 \mathrm{kcal} \mathrm{mol}^{-1}$ from the conformer search were used for DFT optimization. Any conformers within $3 \mathrm{kcal} \mathrm{mol}^{-1}$ of the lowest energy conformer at this level of theory were reoptimized with the same level of theory at the lower. Unique conformers from this third optimization were used for the NMR calculations. The scaling factors used to convert the isotropics from the NMR calculations to chemical shifts were a slope $=-1.0719$, intercept $=31.8733$ for ${ }^{1} \mathrm{H}$ shifts and slope $=-1.0420$, intercept $=186.3567$ for ${ }^{13} \mathrm{C}$ shifts. ${ }^{17,18}$ The NMR chemical shifts were calculated by averaging the conformer chemical shifts with weights, or percentages, for each conformer determined by minimizing the deviation between the experimental and theoretical shifts using the excel solver function. The barrier of rotation between syn-14 ap and exo-sc was calculated with optimizing using modredundant and the PCM(chloroform-B3LYP/3-21G $(219.15 \mathrm{~K})$ level of theory using Gaussian16, Revision C.01 for the 11, 2, 33, 56 atom dihedral angle with 36 steps of $10^{\circ}$. The $\mathrm{j}$-coupling values $(\mathrm{Hz})$ were calculated using the fermi contact $(\mathrm{FC})$ values from reoptimizing relevant conformers from the NMR calculations with $B 3 L Y P / 6-31 G(d)$ and then calculating coupling using B3LYP/6-31G(d,p) nmr= (FCOnly,Atoms=H,MixedH). The FC values were scaled to $\mathrm{j}$ coupling constants $(\mathrm{Hz})$ values were scaled by 0.9155 as recommended on CHESHIRE. 17,18 The NMR calculations for $\mathbf{1 8 b}$ were performed using the GIAO method with PCM(acetonitrile)-mPW1PW91/6$311+\mathrm{G}(2 \mathrm{~d}, \mathrm{p})$ from the lowest energy conformer from the mechanistic calculations (Section \#) using Gaussian16, Revision C.01. The scaling factors used were slope $=-1.0681$ and intercept $=31.7773$ for ${ }^{1} \mathrm{H}$ shifts and slope $=-1.0502$ and intercept $=186.9263$ for ${ }^{13} \mathrm{C}$ shifts. ${ }^{17,18}$ The NBO calculations ${ }^{19}$ were performed with B3LYP-D3(BJ)/6-31G+(d,p) with the keywords: $p o p=(f u l l, n b o)$ int=finegrid, iop $(3 / 32=2)$, scf=tight, temperature=219.15 with Gaussian16, Revision A.03. Molecular images were generated using CylView. ${ }^{20}$ The optimized geometries for $\mathbf{1 1}$ and $\mathbf{1 4}$ are deposited in ioChem-BD (doi:10.19061/iochem-bd-6-83; http://dx.doi.org/10.19061/iochem-bd-6-83)..$^{21}$ 


\section{CHLORINATIONS WITH MACMILLAN-TYPE CATALYSTS}

\subsection{Aminals Derived from $1^{\text {st }}, 2^{\text {nd }}$ and $3^{\text {rd }}$ Generation MacMillan Catalysts}

Motivated by the isolation and X-ray crystal structure analysis of several aminals derived from the $3^{\text {rd }}$ generation MacMillan catalyst $3 c,{ }^{22}$ we aimed at extending the structure determination to the $1^{\text {st }}$ and $2^{\text {nd }}$ generation MacMillan catalysts $\mathbf{3 a}$ and $\mathbf{3 b}$. The ${ }^{1} \mathrm{H}-\mathrm{NMR}$ spectra of the isolated and crystallographically characterized aminals were compared to the NMR spectra recorded during the reactions and our stereochemical assignments were compared to those reported in the literature. ${ }^{23,24}$

Aminals Derived from Isovaleraldehyde, NCS and the $1^{\text {st }}$ Generation MacMillan Catalyst ent-3a (NMR Experiment)

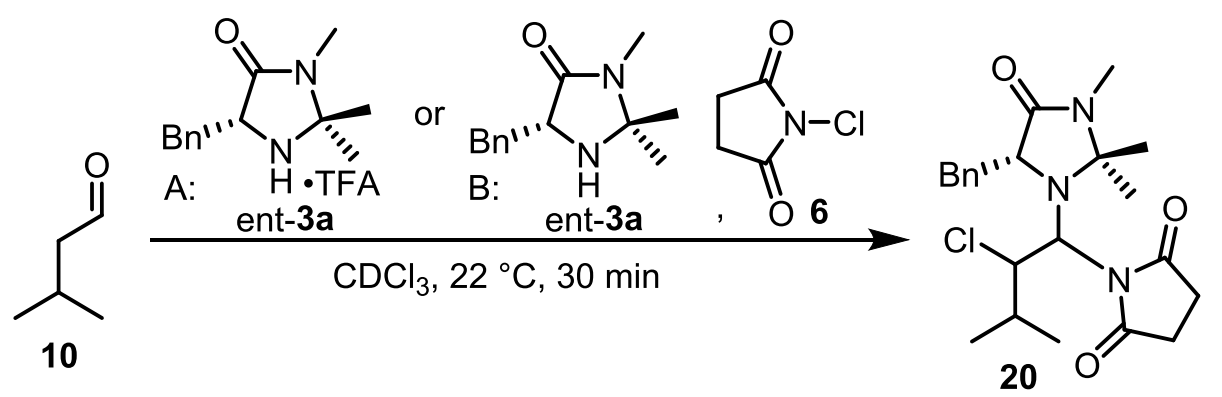

The TFA-salt of catalyst ent-3a $(6.64 \mathrm{mg}, 20.0 \mu \mathrm{mol}, 20 \mathrm{~mol} \%)$ or catalyst ent-3a as the free base $(4.36 \mathrm{mg}$, $20.0 \mu \mathrm{mol}, 20 \mathrm{~mol} \%$ or $21.8 \mathrm{mg}, 100 \mu \mathrm{mol}, 100 \mathrm{~mol} \%$ ) and NCS (16.0 mg, $120 \mu \mathrm{mol}, 1.2$ equiv) were added successively to an NMR-tube containing a solution of isovaleraldehyde (10.8 $\mu \mathrm{L}, 100 \mu \mathrm{mol}, 1.0$ equiv) in $\mathrm{CDCl}_{3}(0.70 \mathrm{~mL})$ at $22^{\circ} \mathrm{C} .{ }^{1} \mathrm{H}-\mathrm{NMR}$ spectra were recorded after $30 \mathrm{~min}$. The ratio of aminal diastereoisomers is 20:1 for the TFA-salt of the catalyst and 2:1 for the free base. The diastereomeric ratios and the ${ }^{1} \mathrm{H}-\mathrm{NMR}$ spectrum for the reaction with the free base of the catalyst is in agreement with the literature. ${ }^{25}$ 


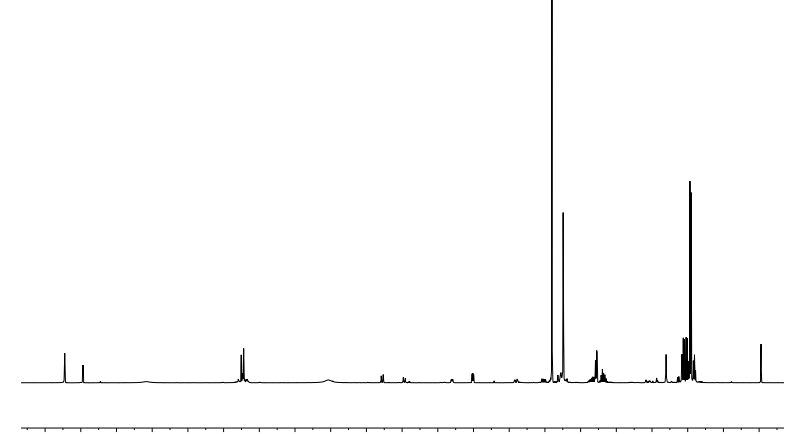

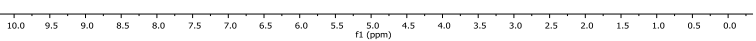

$20 \mathrm{~mol} \%$ catalyst ent-3a as a free base

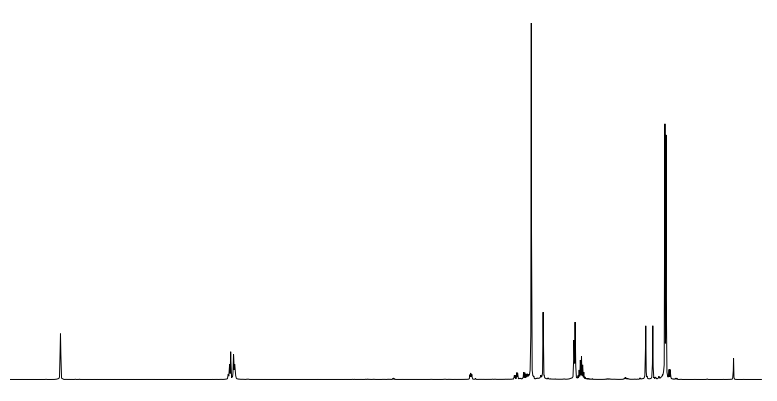

100 mol\% catalyst ent-3a as free base

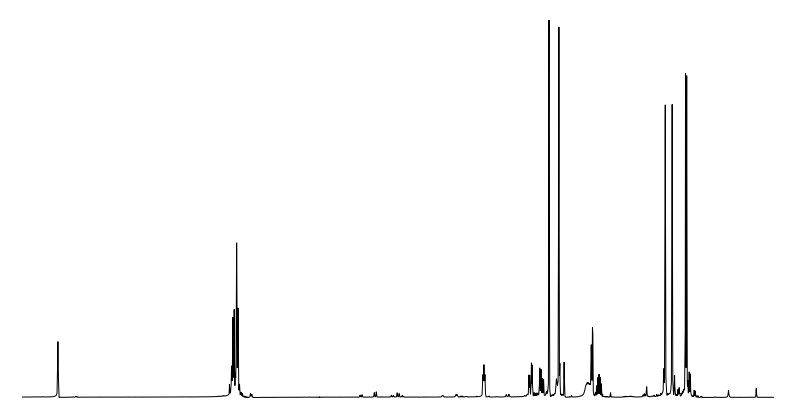

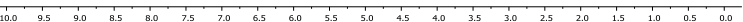

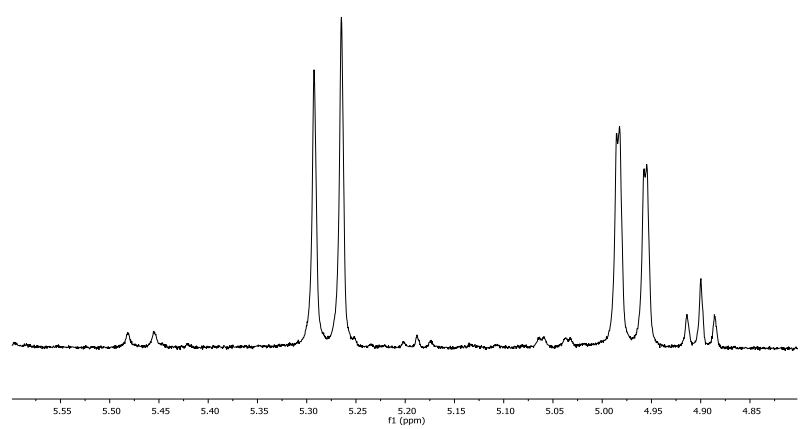

20 mol\% catalyst ent-3a as a free base (zoom)

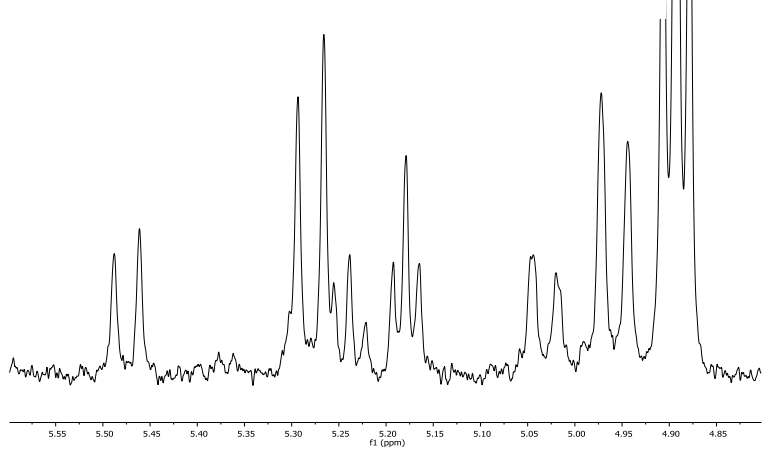

$100 \mathrm{~mol} \%$ catalyst ent-3a as free base (zoom)

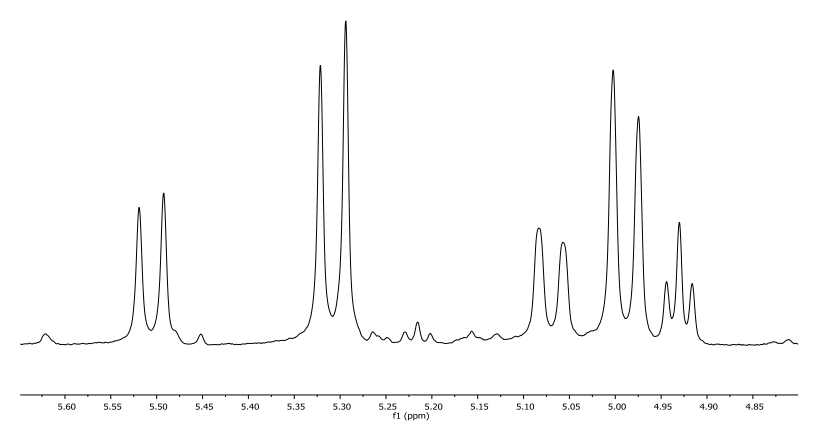

Figure SI-1. ${ }^{1} \mathrm{H}-\mathrm{NMR}$ spectra of the $\alpha$-chlorination reaction (isovaleraldehyde, NCS, catalyst ent-3a or ent3a・TFA) after $30 \mathrm{~min}$. 


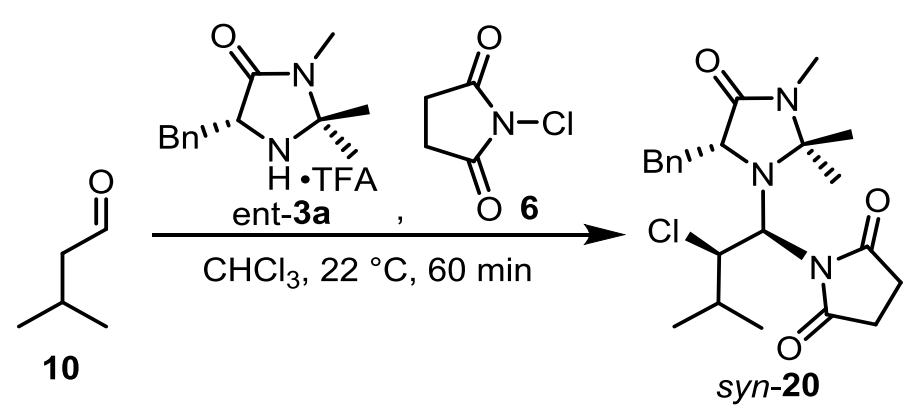

The TFA-salt of catalyst ent-3a $(0.770 \mathrm{~g}, 2.32 \mathrm{mmol}, 20 \mathrm{~mol} \%)$ and NCS (1.55 g, $11.6 \mathrm{mmol}, 1.0$ equiv) were added successively to a solution of isovaleraldehyde $\left(1.25 \mathrm{~mL}, 11.6 \mathrm{mmol}, 1.0\right.$ equiv) in $\mathrm{CHCl}_{3}(46$ $\mathrm{mL})$. The reaction was stirred for $60 \mathrm{~min}$ and quenched with aqueous saturated $\mathrm{NaHCO}_{3}(50 \mathrm{~mL})$. The layers were separated and the aqueous phase was extracted with $\mathrm{CH}_{2} \mathrm{Cl}_{2}(3 \times 50 \mathrm{~mL})$. The combined organic phases were dried over $\mathrm{NaSO}_{4}$, the solvent was removed under reduced pressure and the crude product was purified by column chromatography $\left(\mathrm{SiO}_{2}\right.$, pentane/Et $2 \mathrm{O} / \mathrm{EtOAc}, 1: 1: 1$ to $0: 1: 1$ to $\left.0: 0: 1\right)$. Aminal syn20 was obtained as a colorless solid $(0.664 \mathrm{~g}, 1.58 \mathrm{mmol}, 68 \%)$. The absolute configuration was determined by $\mathrm{X}$-ray crystal structure analysis.

The ${ }^{1} \mathrm{H}-\mathrm{NMR}$-spectrum of the major species is consistent with that reported literature, which was tentatively assigned the syn-coniguration. ${ }^{23,25}$

$\mathbf{m p}=107^{\circ} \mathrm{C}$

$[\alpha]_{D}^{26}=-40.8^{\circ}(c=1.00$, dichloromethane $)$

${ }^{1} \mathrm{H}-\mathrm{NMR}\left(\mathrm{CDCl}_{3}, 600 \mathrm{MHz}\right) \delta=7.26-7.13(\mathrm{~m}, 5 \mathrm{H}), 5.29(\mathrm{~d}, J=11.0 \mathrm{~Hz}, 1 \mathrm{H}), 4.96(\mathrm{~d}, J=11.2 \mathrm{~Hz}, 1 \mathrm{H})$, 4.17 (dd, $J=7.0,3.0 \mathrm{~Hz}, 1 \mathrm{H}$ ), 3.46 (dd, $J=14.8,2.9 \mathrm{~Hz}, 1 \mathrm{H}$ ), 3.06 (dd, $J=14.8,7.1 \mathrm{~Hz}, 1 \mathrm{H}$ ), $2.78-2.71$ (m, 7H), 2.34 (hept, $J=6.1 \mathrm{~Hz}, 1 \mathrm{H}), 1.26(\mathrm{~s}, 3 \mathrm{H}), 1.10(\mathrm{~s}, 3 \mathrm{H}), 0.95$ (d, J=6.8 Hz, 3H), 0.91 (d, $J=6.5 \mathrm{~Hz}$, 3H) ppm.

${ }^{13} \mathrm{C}-\mathrm{NMR}\left(\mathrm{CDCl}_{3}, 151 \mathrm{MHz}\right) \delta=179.4,177.6,170.8,137.7,129.8,128.1,126.4,79.9,67.9,67.3,60.7$, $39.7,28.6,28.4,27.8,27.1,25.4,23.1,21.4,15.1$ ppm.

IR (ATR): $\tilde{v}=2969,2930,1773,1704,1364,1291,1173,911,818,728 \mathrm{~cm}^{-1}$.

HRMS (ESI, pos. mode): $\mathrm{m} / \mathrm{z}$ calculated for $\mathrm{C}_{22} \mathrm{H}_{31} \mathrm{ClN}_{3} \mathrm{O}_{3}[\mathrm{M}+\mathrm{H}]^{+}: 420.2049$, found 420.2044; $\mathrm{m} / \mathrm{z}$ calculated for $\mathrm{C}_{22} \mathrm{H}_{30} \mathrm{CIN}_{3} \mathrm{NaO}_{3}[\mathrm{M}+\mathrm{Na}]^{+}: 442.1868$, found $442.1865 ; \mathrm{m} / z$ calculated for $\mathrm{C}_{22} \mathrm{H}_{30} \mathrm{ClKN}_{3} \mathrm{O}_{3}$ $[\mathrm{M}+\mathrm{K}]^{+}:$458.1608, found 458.1598. 

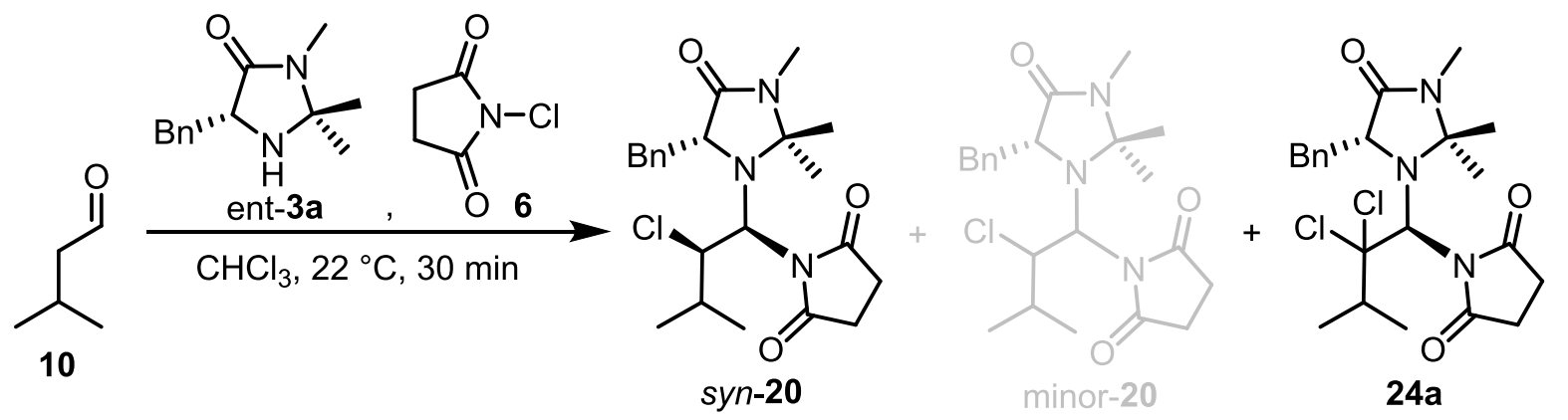

Catalyst ent-3a (2.23 g, $10.2 \mathrm{mmol}, 100 \mathrm{~mol} \%)$ and NCS (1.64 g, $12.3 \mathrm{mmol}, 1.2$ equiv) were added successively to a solution of isovaleraldehyde $\left(1.10 \mathrm{~mL}, 10.2 \mathrm{mmol}, 1.0\right.$ equiv) in $\mathrm{CHCl}_{3}(20 \mathrm{~mL})$ and the reaction was stirred for $30 \mathrm{~min}$ at $22^{\circ} \mathrm{C}$. The solvent was removed under reduced pressure and the crude product was purified by column chromatography $\left(\mathrm{SiO}_{2}, \mathrm{EtOAc}\right)$. Aminal syn-20 was obtained as a colorless solid (1.94 g, $4.62 \mathrm{mmol}, 45 \%)$. The ${ }^{1} \mathrm{H}-\mathrm{NMR}$ spectrum of a mixed fraction showed the existence of the diastereomeric aminal minor-20. After a second chromatographic separation attempt $\left(\mathrm{SiO}_{2}, \mathrm{Et}_{2} \mathrm{O}\right.$ then EtOAc), aminal minor-20 was not detectable anymore. Gratifyingly, a previously unknown dichloroaminal 24a (96.0 mg, $0.211 \mathrm{mmol}, 2 \%$ ) could be obtained as a colorless solid. The absolute configuration was confirmed by X-ray crystal structure analysis.

The ${ }^{1} \mathrm{H}$-NMR-spectrum of aminal syn-20 is in accordance with the previous experiment.

\section{Aminal 24a:}

$\mathbf{m p}=116{ }^{\circ} \mathrm{C}$

$[\alpha]_{D}^{26}=-53.9^{\circ}(c=0.85$, dichloromethane $)$

${ }^{1} \mathrm{H}-\mathrm{NMR}\left(\mathrm{CDCl}_{3}, 600 \mathrm{MHz}\right) \delta=7.31-7.15(\mathrm{~m}, 5 \mathrm{H}), 6.01(\mathrm{~s}, 1 \mathrm{H}), 5.74(\mathrm{dd}, J=8.0,2.7 \mathrm{~Hz}, 1 \mathrm{H}), 3.54-3.45$ (m, 1H), $3.05(\mathrm{dd}, J=14.4,7.9 \mathrm{~Hz}, 1 \mathrm{H}), 2.96(\mathrm{dt}, J=13.2,6.4 \mathrm{~Hz}, 1 \mathrm{H}), 2.87-2.70(\mathrm{~m}, 4 \mathrm{H}), 2.70(\mathrm{~s}, 3 \mathrm{H})$, $1.34(\mathrm{~d}, J=6.6 \mathrm{~Hz}, 3 \mathrm{H}), 1.31(\mathrm{~s}, 3 \mathrm{H}), 1.22(\mathrm{~d}, J=6.3 \mathrm{~Hz}, 3 \mathrm{H}), 1.02(\mathrm{~s}, 3 \mathrm{H}) \mathrm{ppm}$.

${ }^{13} \mathrm{C}-\mathrm{NMR}\left(\mathrm{CDCl}_{3}, 151 \mathrm{MHz}\right) \delta=178.1,176.8,172.1,138.3,129.7,128.2,126.5,101.0,80.6,73.5,62.2$, $41.3,39.5,28.6,28.2,28.1,25.4,24.7,19.2,18.7 \mathrm{ppm}$.

IR (ATR): $\tilde{v}=3058,2978,1777,1710,1431,1398,1309,1266,1181,1141,947,818,768,732 \mathrm{~cm}^{-1}$.

HRMS (ESI, pos. mode): $\mathrm{m} / \mathrm{z}$ calculated for $\mathrm{C}_{22} \mathrm{H}_{30} \mathrm{Cl}_{2} \mathrm{~N}_{3} \mathrm{O}_{3}[\mathrm{M}+\mathrm{H}]^{+}:$454.1659, found 454.1666; $\mathrm{m} / \mathrm{z}$ calculated for $\mathrm{C}_{22} \mathrm{H}_{29} \mathrm{Cl}_{2} \mathrm{~N}_{3} \mathrm{NaO}_{3}[\mathrm{M}+\mathrm{Na}]^{+}: 476.1478$, found $476.1489 ; \mathrm{m} / z$ calculated for $\mathrm{C}_{22} \mathrm{H}_{29} \mathrm{Cl}_{2} \mathrm{KN}_{3} \mathrm{O}_{3}$ $[\mathrm{M}+\mathrm{K}]^{+}$: 492.1218, found 492.1231 . 


\section{Assignment of Aminal Diastereoisomers Derived from the $1^{\text {st }}$ Generation MacMillan Catalyst}

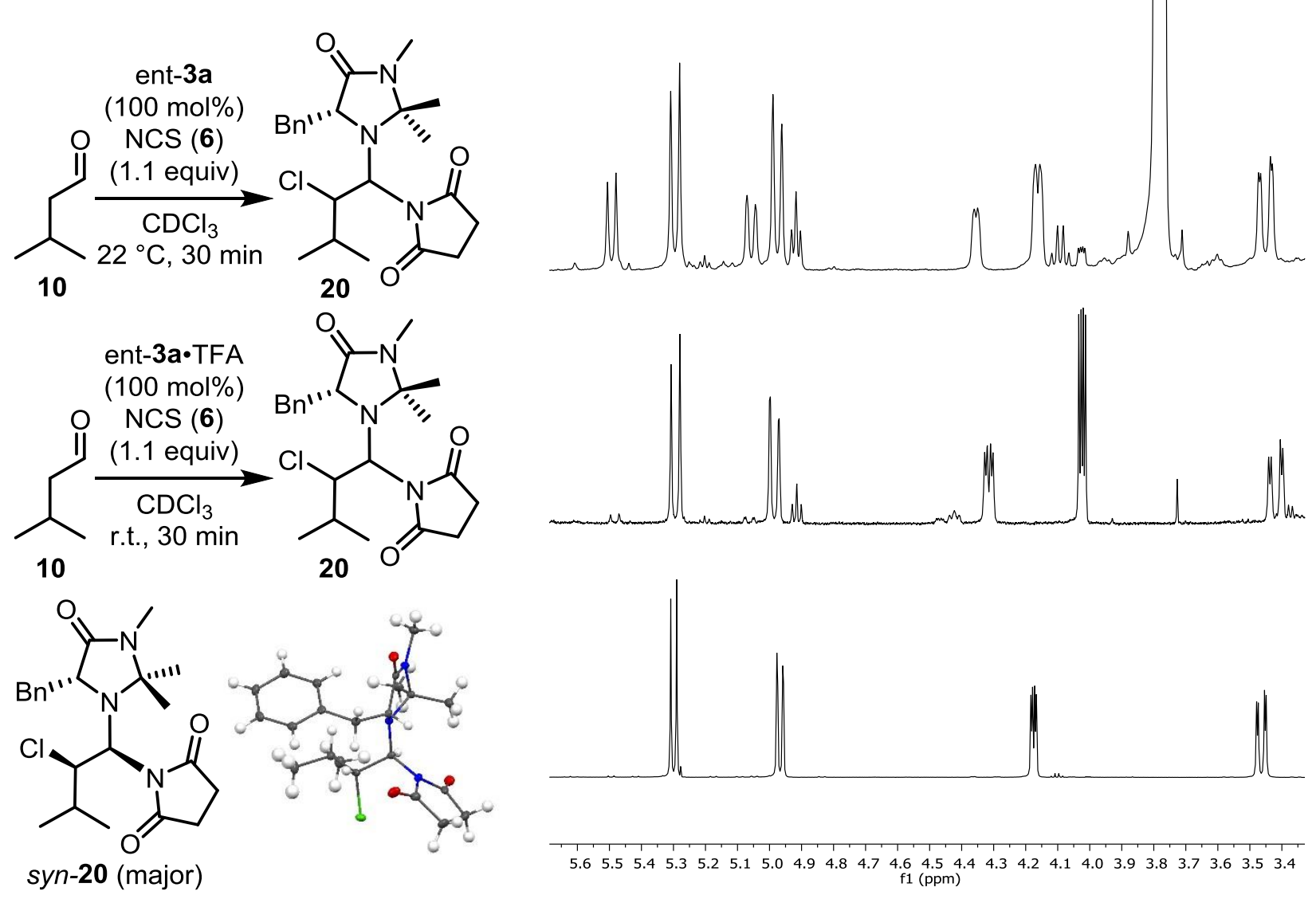

Figure SI-2. ${ }^{1} \mathrm{H}-\mathrm{NMR}$ spectrum of the reaction with $100 \mathrm{~mol} \%$ ent-3a after $30 \mathrm{~min}$ (according to Blackmond et al. ${ }^{23,25}$ (top). ${ }^{1} \mathrm{H}-\mathrm{NMR}$ spectrum of the reaction with $100 \mathrm{~mol} \%$ ent-3a・TFA after $30 \mathrm{~min}$ (middle). Crystal structure and ${ }^{1} \mathrm{H}-\mathrm{NMR}$ spectrum of the isolated aminal syn-20 (bottom).

In their publication "Explaining Anomalies in Enamine Catalysis: "Downstream Species" as a New Paradigm for Stereocontrol"23 the authors correlated species described as "major" and "minor" for the $1^{\text {st }}$ generation MacMillan catalyst to the major and minor product enantiomer. According to their model, the major diastereoisomer of the corresponding aminal was suggested to possess the anti-configuration whereas the minor diastereoisomer was suggested to possess the syn-configuration. We were able to isolate and characterize the major species by X-ray analysis and determined it to possess the syn-configuration. Moreover, the authors correlated the enantiomeric ratio of the product ( $\alpha$-chloroaldehyde) to the diastereomeric ratio of the aminals. ${ }^{23}$ The e.r.-value of the $\alpha$-chloroaldehyde (40:60) was cited from the original MacMillan publication ${ }^{26}$ and the d.r.-value (33:67) of the aminal refers to experiments done by the authors ${ }^{25}$. These two values however are not directly comparable, since MacMillan and co-workers used the TFA-salt and Blackmond and co-workers used the free base of the catalyst to generate a 33:67 ratio of diastereomers. The different diastereomeric ratios of the aminals are a consequence of the presence or absence of TFA as apparent in the ${ }^{1} \mathrm{H}$-HNMR spectra shown above. Therefore, a correlation between enantiomeric ratio of the product and diastereomeric ratio of the aminal incorrectly compares two different situations. 


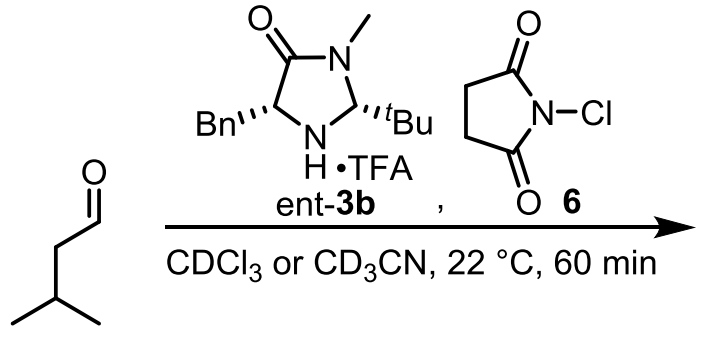

10

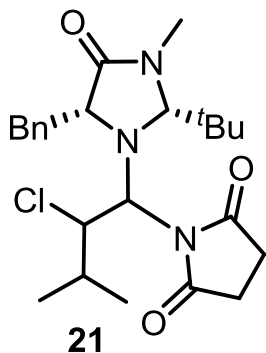

21

The TFA-salt of catalyst ent-3b (7.20 mg, $20.0 \mu \mathrm{mol}, 20 \mathrm{~mol} \%)$ and NCS (13.3 mg, $100 \mu \mathrm{mol}, 1.0$ equiv) were added successively to an NMR-tube containing a solution of isovaleraldehyde $(10.8 \mu \mathrm{L}, 100 \mu \mathrm{mol}, 1.0$ equiv) in $\mathrm{CDCl}_{3}$ or $\mathrm{CD}_{3} \mathrm{CN}(0.70 \mathrm{~mL})$ at $22{ }^{\circ} \mathrm{C}$. ${ }^{1} \mathrm{H}-\mathrm{NMR}$ spectra were recorded after $60 \mathrm{~min}$. The diastereomeric ratio is approximately 9:1:4 in $\mathrm{CDCl}_{3}$ and 3:2 in $\mathrm{CD}_{3} \mathrm{CN}$. The ${ }^{1} \mathrm{H}-\mathrm{NMR}$ spectrum $\left(\mathrm{CDCl}_{3}\right)$ is in accordance with that the literature. ${ }^{24,25}$

$\mathrm{CDCl}_{3}:$

$\mathrm{CDCl}_{3}(\mathrm{zoOm})$ :

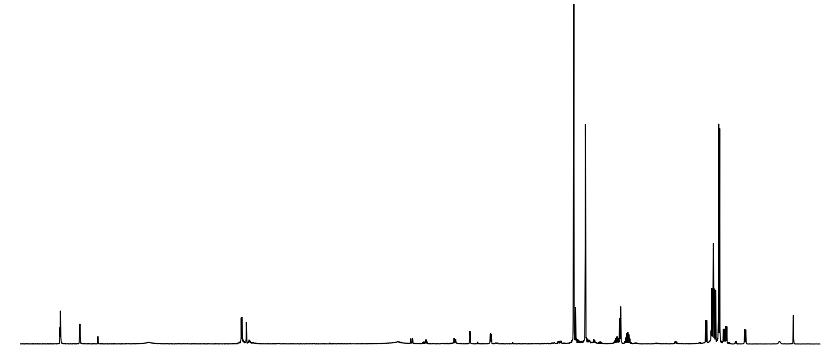

$\mathrm{CD}_{3} \mathrm{CN}$ :

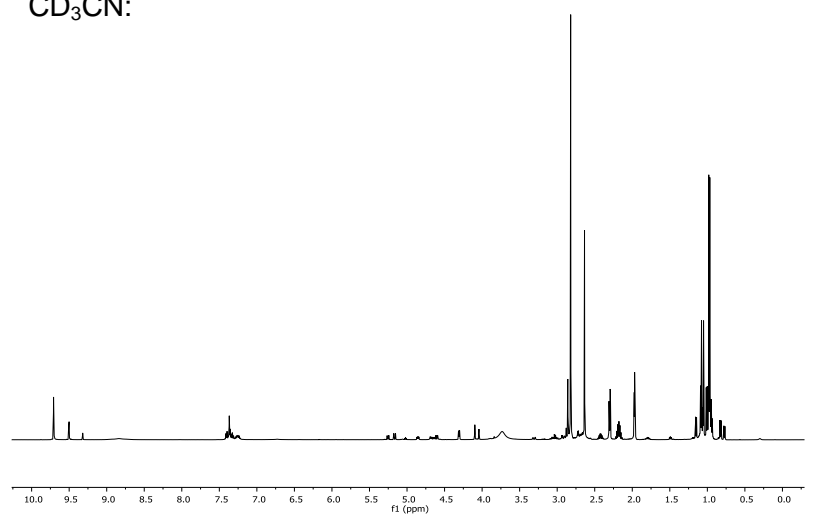

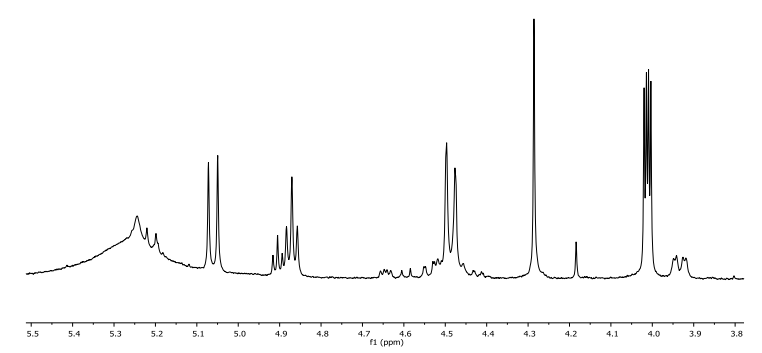

$\mathrm{CD}_{3} \mathrm{CN}(\mathrm{zoom})$ :

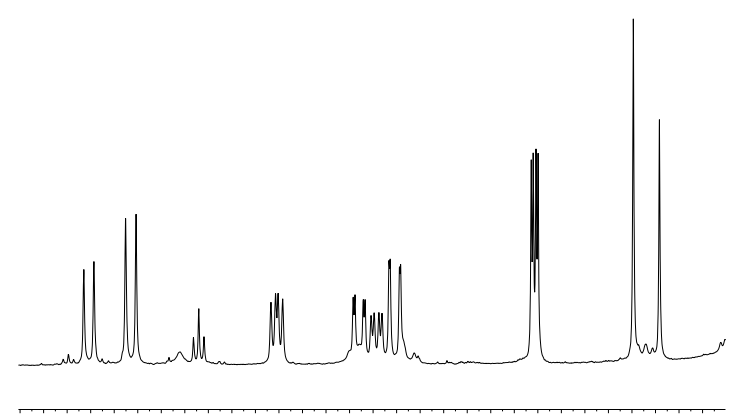

Figure SI-3. ${ }^{1} \mathrm{H}-\mathrm{NMR}$ spectra of the $\alpha$-chlorination reaction (isovaleraldehyde, NCS, catalyst ent-3b•TFA) in different solvents after $60 \mathrm{~min}$. 
1-((1S,2R)-1-((2R,5R)-5-benzyl-2-(tert-butyl)-3-methyl-4-oxoimidazolidin-1-yl)-2-chloro-3-methylbutyl)pyrrolidine-2,5-dione ((1S,2R)-syn-21),

1-((1S,2S)-1-((2R,5R)-5-benzyl-2-(tert-butyl)-3-methyl-4-oxoimidazolidin-1-yl)-2-chloro-3-methylbutyl)pyrrolidine-2,5-dione ((1S,2S)-anti-21),

1-((1R,2S)-1-((2R,5R)-5-benzyl-2-(tert-butyl)-3-methyl-4-oxoimidazolidin-1-yl)-2-chloro-3-methylbutyl)pyrrolidine-2,5-dione ((1R,2S)-syn-21)

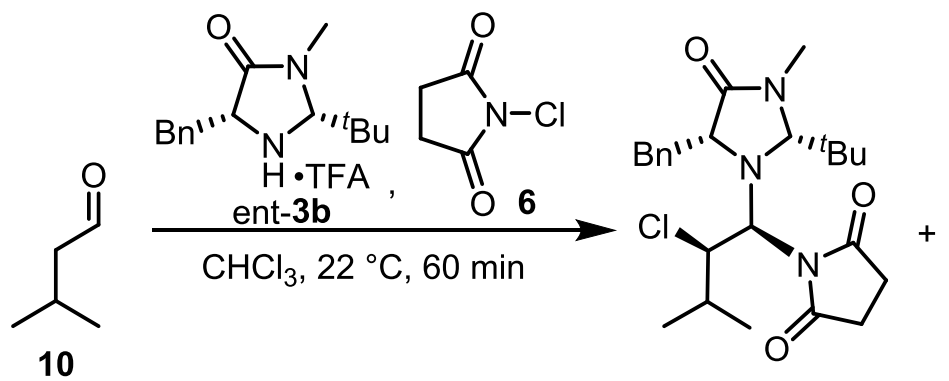

$(1 S, 2 R)-s y n-21$<smiles>CC(C)[C@H](Cl)[C@@H](N1C(=O)CC[C@H]1Br)N1C(=O)[C@@H](Br)C(=O)N1C</smiles>

(1S,2S)-anti-21<smiles>CC(C)[C@H](Cl)[C@@H](N1C(=O)CCC1=O)N1[C@H](Br)[C@@H](Br)C(=O)N1C</smiles>

$(1 R, 2 S)-s y n-21$

The TFA-salt of catalyst ent-3b (1.26 g, $3.48 \mathrm{mmol}, 20 \mathrm{~mol} \%$ ) and NCS (2.33 g, $17.4 \mathrm{mmol}, 1.0$ equiv) were added successively to a solution of isovaleraldehyde $\left(1.88 \mathrm{~mL}, 17.4 \mathrm{mmol}, 1.0\right.$ equiv) in $\mathrm{CHCl}_{3}(70 \mathrm{~mL})$. The reaction was stirred for $60 \mathrm{~min}$ and quenched with aqueous saturated $\mathrm{NaHCO}_{3}(50 \mathrm{~mL})$. The aqueous phase was extracted with dichloromethane $(3 \times 50 \mathrm{~mL})$, the combined organic phases were dried over $\mathrm{NaSO}_{4}$, the solvent was removed under reduced pressure and the crude product was purified by column chromatography $\left(\mathrm{SiO}_{2}\right.$, pentane/Et $2 \mathrm{O} / \mathrm{EtOAc}, 1: 1: 1$ to $0: 1: 1$ to $\left.0: 0: 1\right)$. Pure aminal $(1 S, 2 R)$-syn-21 was obtained as a colorless solid $(0.807 \mathrm{~g}, 1.80 \mathrm{mmol}, 52 \%)$. Separation of another fraction from the column chromatography by chiral HPLC enabled further separation of aminal (1S,2R)-syn-21 (0.339 g, 0.757 mmol, 22\%), (1S,2S)-anti-21 (93.0 mg, $0.208 \mathrm{mmol}, 6 \%)$ and (1R,2S)-syn-21 (0.271 g, $0.605 \mathrm{mmol}, 17 \%)$. The absolute configuration of the three aminals was confirmed by $\mathrm{X}$-ray crystal structure analysis.

\section{Aminal (1S,2R)-syn-21:}

The spectroscopic data $\left({ }^{1} \mathrm{H}\right.$ - and $\left.{ }^{13} \mathrm{C}-\mathrm{NMR}\right)$ are in accordance with the major aminal from the literature..$^{24,25}$

$m p=104^{\circ} \mathrm{C}$

$[\alpha]_{D}^{26}=-32.1^{\circ}\left(c=1.00, \mathrm{CH}_{2} \mathrm{Cl}_{2}\right)$

${ }^{1} \mathrm{H}-\mathrm{NMR}\left(\mathrm{CDCl}_{3}, 600 \mathrm{MHz}\right) \delta=7.37-7.31(\mathrm{~m}, 4 \mathrm{H}), 7.24-7.20(\mathrm{~m}, 1 \mathrm{H}), 5.08(\mathrm{~d}, J=11.1 \mathrm{~Hz}, 1 \mathrm{H}), 4.81$ (t, $1 \mathrm{H}$ ), 4.48 (dd, $J=11.2,1.0 \mathrm{~Hz}, 1 \mathrm{H}), 4.24(\mathrm{~s}, 1 \mathrm{H}), 3.12$ (dd, $J=14.9,6.5 \mathrm{~Hz}, 1 \mathrm{H}), 2.88(\mathrm{~s}, 3 \mathrm{H}), 2.85$ (dd, $J$ $=14.6,6.9 \mathrm{~Hz}, 1 \mathrm{H}), 2.75-2.65(\mathrm{~m}, 4 \mathrm{H}), 2.36(\mathrm{~h}, J=7.2,6.6 \mathrm{~Hz}, 1 \mathrm{H}), 1.04(\mathrm{~s}, 9 \mathrm{H}), 0.88(\mathrm{~d}, J=6.5 \mathrm{~Hz}, 3 \mathrm{H})$, $0.61(\mathrm{~d}, J=6.7 \mathrm{~Hz}, 3 \mathrm{H}) \mathrm{ppm}$.

${ }^{13} \mathrm{C}-\mathrm{NMR}\left(\mathrm{CDCl}_{3}, 151 \mathrm{MHz}\right) \delta=179.6,179.2,173.9,138.6,129.3,128.8,126.8,88.5,76.7,65.4,61.8$, $41.1,37.0,31.7,29.1,28.2,27.9,26.9,21.2,15.8$ ppm.

IR (ATR): $\tilde{v}=2959,2924,2871,1774,1702,1456,1328,1172,1136,820,751 \mathrm{~cm}^{-1}$.

HRMS (ESI, pos. mode): $\mathrm{m} / \mathrm{z}$ calculated for $\mathrm{C}_{24} \mathrm{H}_{35} \mathrm{ClN}_{3} \mathrm{O}_{3}[\mathrm{M}+\mathrm{H}]^{+}: 448.2362$, found 448.2373; $\mathrm{m} / \mathrm{z}$ calculated for $\mathrm{C}_{24} \mathrm{H}_{34} \mathrm{CIN}_{3} \mathrm{NaO}_{3}[\mathrm{M}+\mathrm{Na}]^{+}: 470.2181$, found $470.2188 ; \mathrm{m} / z$ calculated for $\mathrm{C}_{24} \mathrm{H}_{34} \mathrm{ClKN}_{3} \mathrm{O}_{3}$ $[\mathrm{M}+\mathrm{K}]^{+}:$486.1921, found 486.1930. 


\section{Aminal (1S,2S)-anti-21:}

The spectroscopic data $\left({ }^{1} \mathrm{H}\right.$ - and $\left.{ }^{13} \mathrm{C}-\mathrm{NMR}\right)$ are in accordance with the minor aminal from the literature. ${ }^{24,25}$

$m p=86^{\circ} \mathrm{C}$

$[\alpha]_{D}^{26}=-6.5^{\circ}(c=0.40$, dichloromethane $)$

${ }^{1} \mathrm{H}-\mathrm{NMR}\left(\mathrm{CDCl}_{3}, 500 \mathrm{MHz}\right) \delta=7.43-7.40(\mathrm{~m}, 2 \mathrm{H}), 7.33-7.29(\mathrm{~m}, 2 \mathrm{H}), 7.23-7.19(\mathrm{~m}, 1 \mathrm{H}), 5.23(\mathrm{~d}, J=$ $10.8 \mathrm{~Hz}, 1 \mathrm{H}$ ), 4.59 (dd, $J=7.2,4.6 \mathrm{~Hz}, 1 \mathrm{H}$ ), 4.49 (dd, $J=10.8,2.0 \mathrm{~Hz}, 1 \mathrm{H}$ ), 4.11 (s, 1H), 3.23 (dd, $J=14.5$, $4.5 \mathrm{~Hz}, 1 \mathrm{H}), 3.12(\mathrm{dd}, J=14.4,7.3 \mathrm{~Hz}, 1 \mathrm{H}), 2.88(\mathrm{~s}, 3 \mathrm{H}), 2.78-2.53(\mathrm{~m}, 4 \mathrm{H}), 1.61-1.49(\mathrm{~m}, 1 \mathrm{H}), 1.08(\mathrm{~s}$, $9 \mathrm{H}), 0.89(\mathrm{~d}, J=6.6 \mathrm{~Hz}, 3 \mathrm{H}), 0.76(\mathrm{~d}, J=6.6 \mathrm{~Hz}, 3 \mathrm{H}) \mathrm{ppm}$.

${ }^{13} \mathrm{C}-\mathrm{NMR}\left(\mathrm{CDCl}_{3}, 151 \mathrm{MHz}\right) \delta=179.6,178.9,174.1,139.4,129.6,128.4,126.4,86.2,75.4,66.2,61.7$, $41.2,38.2,31.9,29.8,28.6,28.0,26.3,21.3,14.6$ ppm.

IR (ATR): $\tilde{v}=2964,2925,2874,1772,1701,1455,1364,1317,1153,819,753,720 \mathrm{~cm}^{-1}$.

HRMS (ESI, pos. mode): $\mathrm{m} / \mathrm{z}$ calculated for $\mathrm{C}_{24} \mathrm{H}_{35} \mathrm{CIN}_{3} \mathrm{O}_{3}[\mathrm{M}+\mathrm{H}]^{+}: 448.2362$, found 448.2352; $\mathrm{m} / \mathrm{z}$ calculated for $\mathrm{C}_{24} \mathrm{H}_{34} \mathrm{CIN}_{3} \mathrm{NaO}_{3}[\mathrm{M}+\mathrm{Na}]^{+}:$470.2181, found 470.2163; m/z calculated for $\mathrm{C}_{24} \mathrm{H}_{34} \mathrm{ClKN}_{3} \mathrm{O}_{3}$ $[\mathrm{M}+\mathrm{K}]^{+}$: 486.1921, found 486.1904.

\section{Aminal (1R,2S)-syn-21:}

$\mathbf{m p}=136^{\circ} \mathrm{C}$

$[\alpha]_{D}^{26}=-13.1^{\circ}(c=1.00$, dichloromethane $)$

${ }^{1} \mathrm{H}-\mathrm{NMR}\left(\mathrm{CDCl}_{3}, 600 \mathrm{MHz}\right) \delta=7.35-7.31(\mathrm{~m}, 4 \mathrm{H}), 7.24-7.19(\mathrm{~m}, 1 \mathrm{H}), 5.17(\mathrm{~s}, 7 \mathrm{H}), 4.50(\mathrm{~s}, 1 \mathrm{H}), 4.50$ (dd, $J=18.0 \mathrm{~Hz}, 11.4 \mathrm{~Hz}, 1 \mathrm{H}$ ), 3.87 (dd, $J=11.2,2.9 \mathrm{~Hz}, 1 \mathrm{H}$ ), 3.20 (dd, $J=13.9,3.3 \mathrm{~Hz}, 1 \mathrm{H}$ ), 2.90 (s, 3H), $2.81(\mathrm{dd}, J=13.8,11.6 \mathrm{~Hz}, 1 \mathrm{H}), 2.78-2.53(\mathrm{~m}, 4 \mathrm{H}), 2.10(\mathrm{~h}, J=11.8,6.5,6.1 \mathrm{~Hz}, 1 \mathrm{H}), 1.04(\mathrm{~s}, 9 \mathrm{H}), 0.88$ (d, $J=6.8 \mathrm{~Hz}, 3 \mathrm{H}), 0.16(\mathrm{~s}, 3 \mathrm{H}) \mathrm{ppm}$.

${ }^{13} \mathrm{C}-N M R\left(\mathrm{CDCl}_{3}, 151 \mathrm{MHz}\right) \delta=179.7,178.6,173.9,138.3,129.6,129.1,127.0,85.9,77.4,67.4,65.6$, $38.6,37.2,32.2,29.7,28.3,28.1,28.0,20.8,14.8$ ppm.

IR (ATR): $\tilde{v}=2964,2927,2827,1776,1704,1456,1329,1216,1175,1091,908,750,735 \mathrm{~cm}^{-1}$.

HRMS (ESI, pos. mode): $\mathrm{m} / \mathrm{z}$ calculated for $\mathrm{C}_{24} \mathrm{H}_{35} \mathrm{CIN}_{3} \mathrm{O}_{3}[\mathrm{M}+\mathrm{H}]^{+}: 448.2362$, found 448.2362; $\mathrm{m} / \mathrm{z}$ calculated for $\mathrm{C}_{24} \mathrm{H}_{34} \mathrm{CIN}_{3} \mathrm{NaO}_{3}[\mathrm{M}+\mathrm{Na}]^{+}: 470.2181$, found $470.2172 ; \mathrm{m} / z$ calculated for $\mathrm{C}_{24} \mathrm{H}_{34} \mathrm{ClKN}_{3} \mathrm{O}_{3}$ $[\mathrm{M}+\mathrm{K}]^{+}$: 486.1921, found 486.1911. 


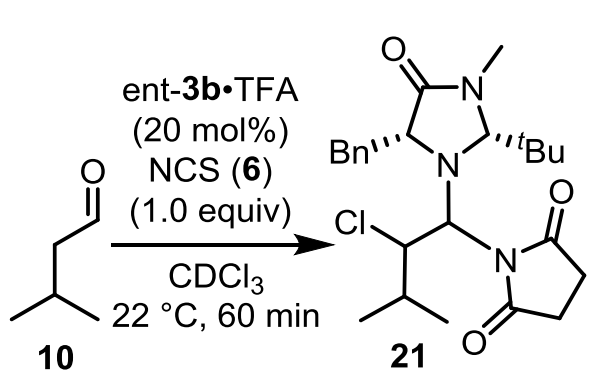<smiles>CC(C)[C@H](Cl)[C@@H](N1C(=O)CCC1=O)N1C(=O)N(C)C(=O)[C@H]1Br</smiles>

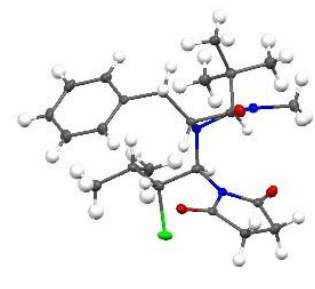

$(1 S, 2 R)-s y n-21$<smiles>CC(C)[C@H](Cl)[C@@H](N1C(=O)CCC1=O)N1C(=O)N(C)C(=O)[C@H]1Br</smiles>

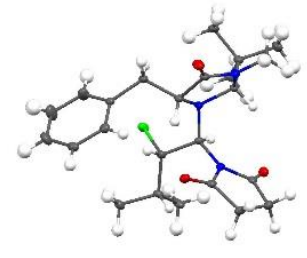

(1S,2S)-anti-21<smiles>CC(C)[C@H](Cl)[C@H](N1C(=O)CCC1=O)N1C(=O)N(C)C(=O)[C@H]1Br</smiles>
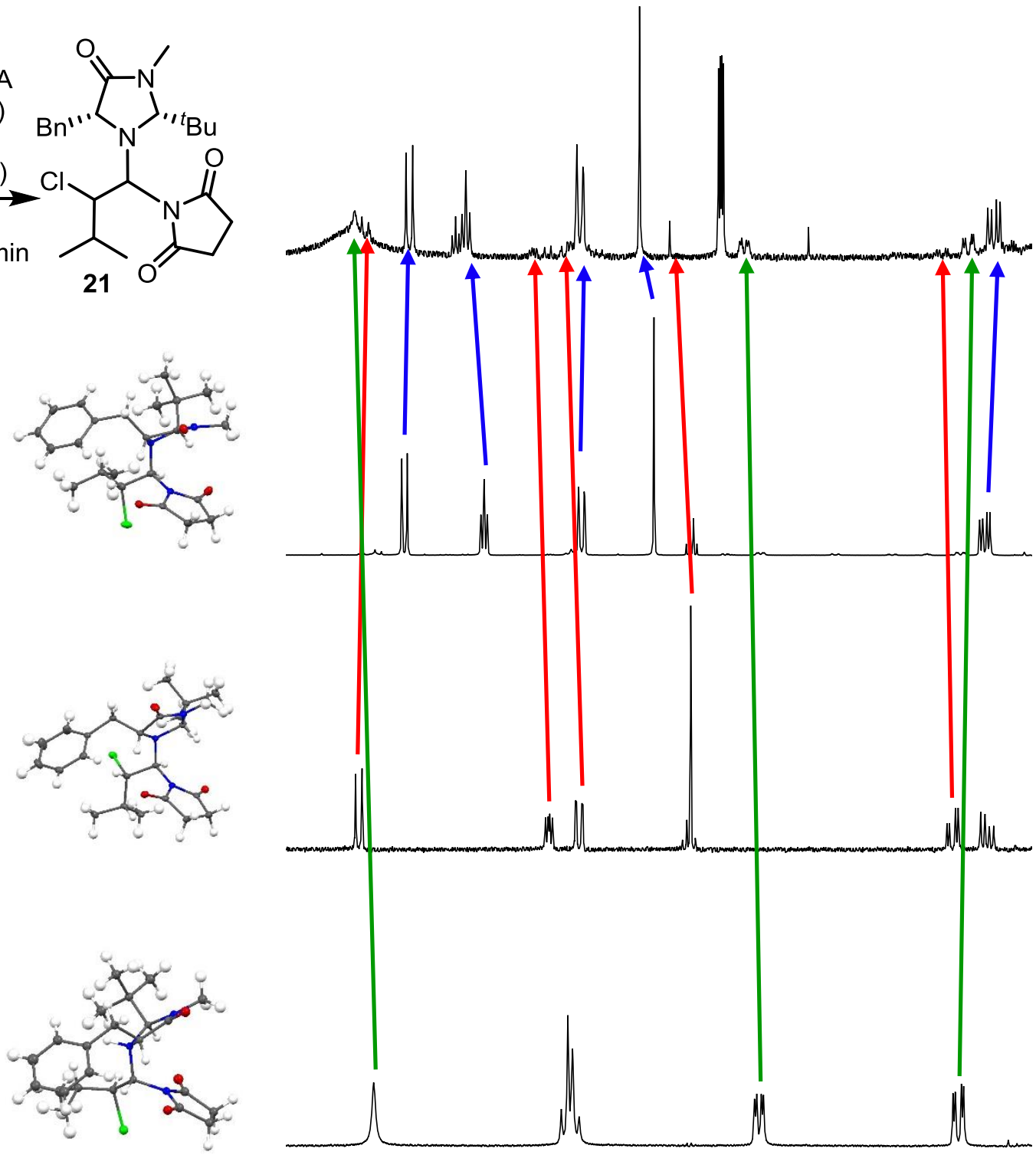

$(1 R, 2 S)-s y n-21$

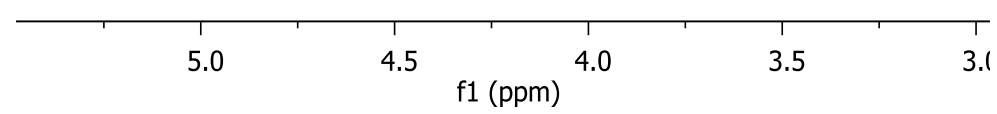

Figure SI-4. ${ }^{1} \mathrm{H}-\mathrm{NMR}$ spectrum of the reaction with $20 \mathrm{~mol} \%$ ent-3b-TFA after $60 \mathrm{~min}$ (according to Blackmond et al. ${ }^{24,25}$ (top). Crystal structures and ${ }^{1} \mathrm{H}-\mathrm{NMR}$ spectra of the isolated diastereomeric aminals 21 (below).

In their publication "Rationalization of an Unusual Solvent-Induced Inversion of Enantiomeric Excess in Organocatalytic Selenylation of Aldehydes"24,25 the authors discussed two aminal diastereomers for the $2^{\text {nd }}$ generation MacMillan catalyst but overlooked a third aminal diastereomer (1R,2S)-syn-21) indicated by the green arrows. Their rationale is based on the existence of only two out of four possible aminal species. Isolation and subsequent $\mathrm{X}$-ray crystal structure analysis reveals syn-configuration for the major aminal (1S,2R)-syn-21 and anti-configuration for minor aminal (1S,2S)-anti-21. The third, previously overlooked aminal $(1 R, 2 S)$-syn-21 also has the syn-configuration. It is actually present in higher concentration than the species which was named "minor". 


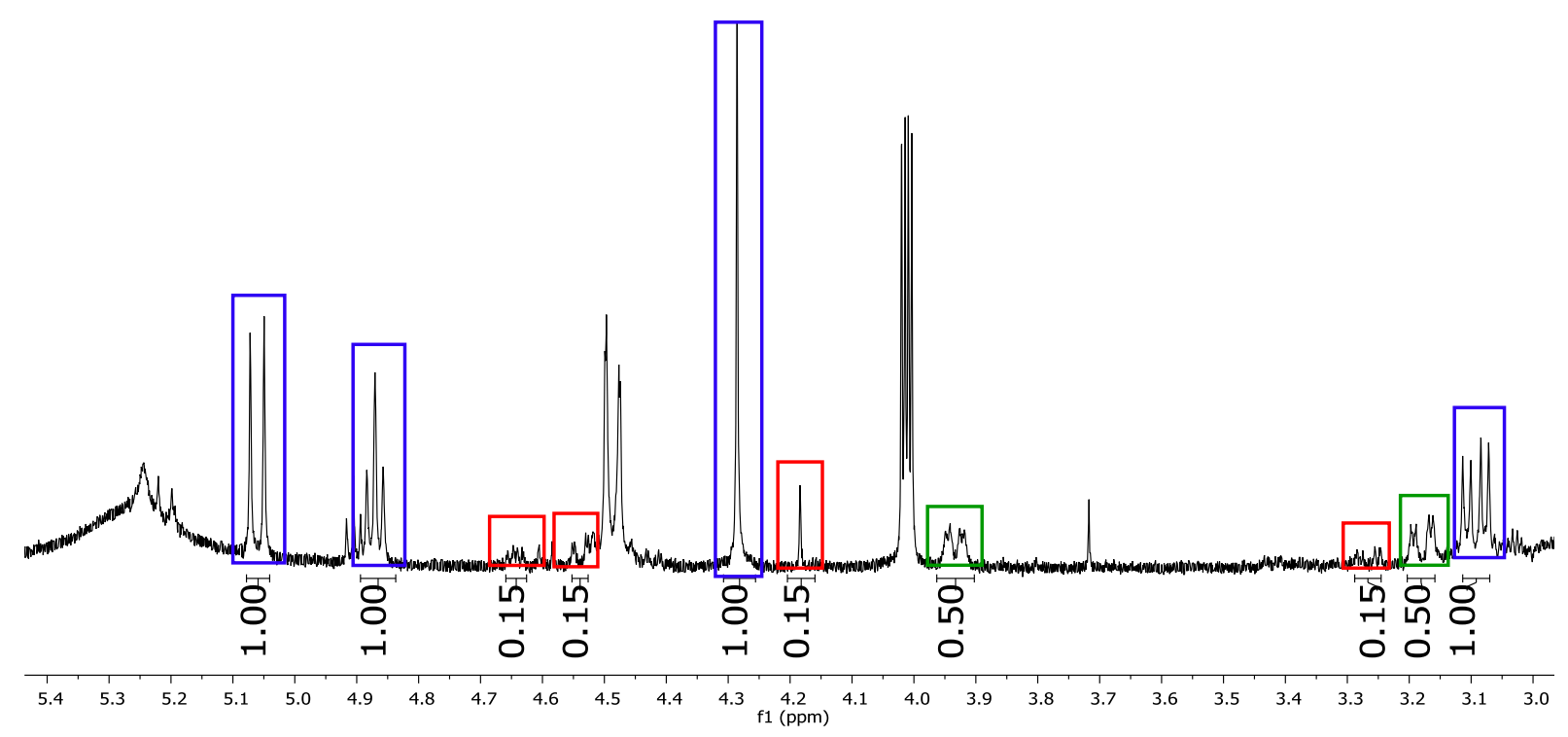

Figure SI-5. ${ }^{1} \mathrm{H}$-NMR spectrum of the reaction with $20 \mathrm{~mol} \%$ ent-3b-TFA after $60 \mathrm{~min}$ with integrated signals (blue: major aminal $(1 S, 2 R)$-syn-21; red: minor aminal $(1 S, 2 S)$-anti-21; green: previously overlooked aminal (1R,2S)-syn-21 (according to Blackmond et al. ${ }^{24,25}$ 
Since the quality of the crystallographic data for aminal $(1 S, 2 R)$-syn-21 was not optimal and to confirm the previous results and conclusions, the same experiments and isolation procedures were repeated using hydrocinnamic aldehyde instead of isovaleraldehyde.

Aminals Derived from Hydrocinnamic Aldehyde, NCS and the $2^{\text {nd }}$ Generation MacMillan Catalyst ent-3b (NMR experiment)

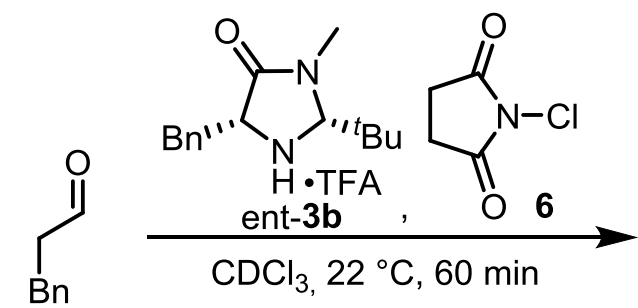

16<smiles>CN1C(=O)[C@@H](Br)N(C(C(Cl)Br)N2C(=O)CCC2=O)[C@@H]1Br</smiles>

The TFA-salt of catalyst ent-3b (7.20 mg, $20.0 \mu \mathrm{mol}, 20 \mathrm{~mol} \%$ ) and NCS (13.3 mg, $100 \mu \mathrm{mol}, 1.0$ equiv) were added successively to an NMR-tube containing a solution of hydrocinnamic aldehyde (13.4 $\mu \mathrm{L}, 100$ $\mu$ mol, 1.0 equiv) in $\mathrm{CDCl}_{3}(0.70 \mathrm{~mL})$ at $22{ }^{\circ} \mathrm{C}$. The ${ }^{1} \mathrm{H}$-NMR spectrum was recorded after $60 \mathrm{~min}$. The diastereomeric ratio is approximately $5: 1: 3$.

$\mathrm{CDCl}_{3}$ :

$\mathrm{CDCl}_{3}$ (zoom):
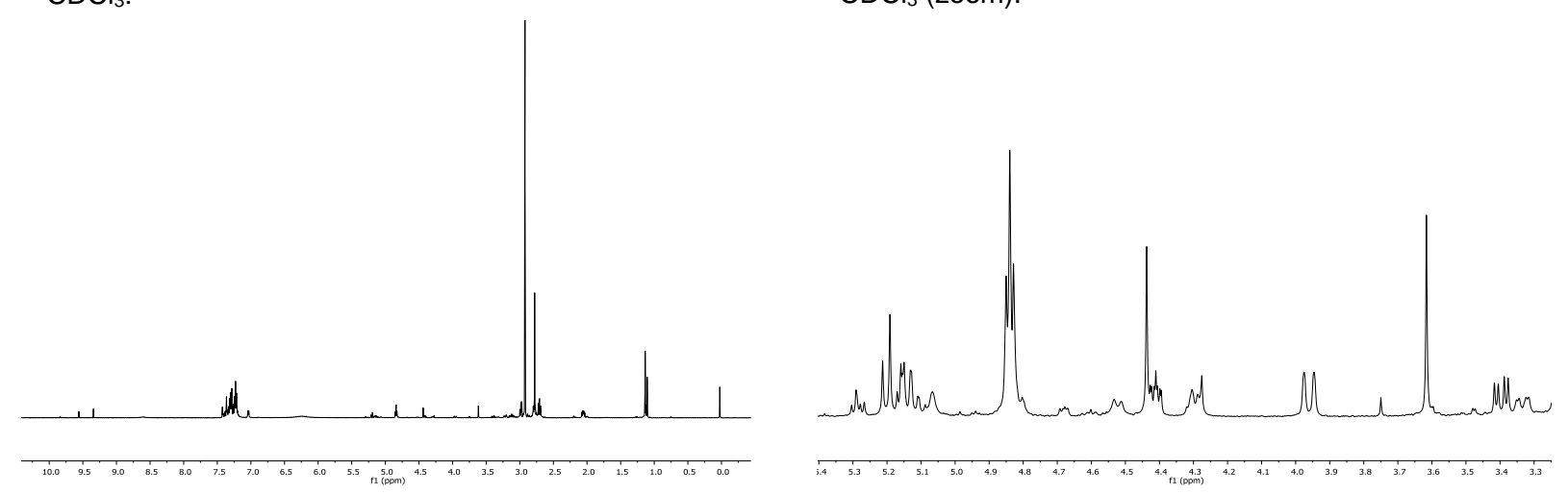

Figure SI-6. ${ }^{1} \mathrm{H}-\mathrm{NMR}$ spectra of the a-chlorination reaction (hydrocinnamic aldehyde, NCS, catalyst ent3b-TFA) after $60 \mathrm{~min}$. 
1-((1S,2R)-1-((2R,5R)-5-benzyl-2-(tert-butyl)-3-methyl-4-oxoimidazolidin-1-yl)-2-chloro-3-phenylpropyl)pyrrolidine-2,5-dione ((1S,2R)-syn-25),

1-((1S,2S)-1-((2R,5R)-5-benzyl-2-(tert-butyl)-3-methyl-4-oxoimidazolidin-1-yl)-2-chloro-3-phenylpropyl)pyrrolidine-2,5-dione ((1S,2S)-anti-25), 1-((1R,2S)-1-((2R,5R)-5-benzyl-2-(tert-butyl)-3-methyl-4-oxoimidazolidin-1-yl)-2-chloro-3-phenylpropyl)pyrrolidine-2,5-dione ((1R,2S)-syn-25)

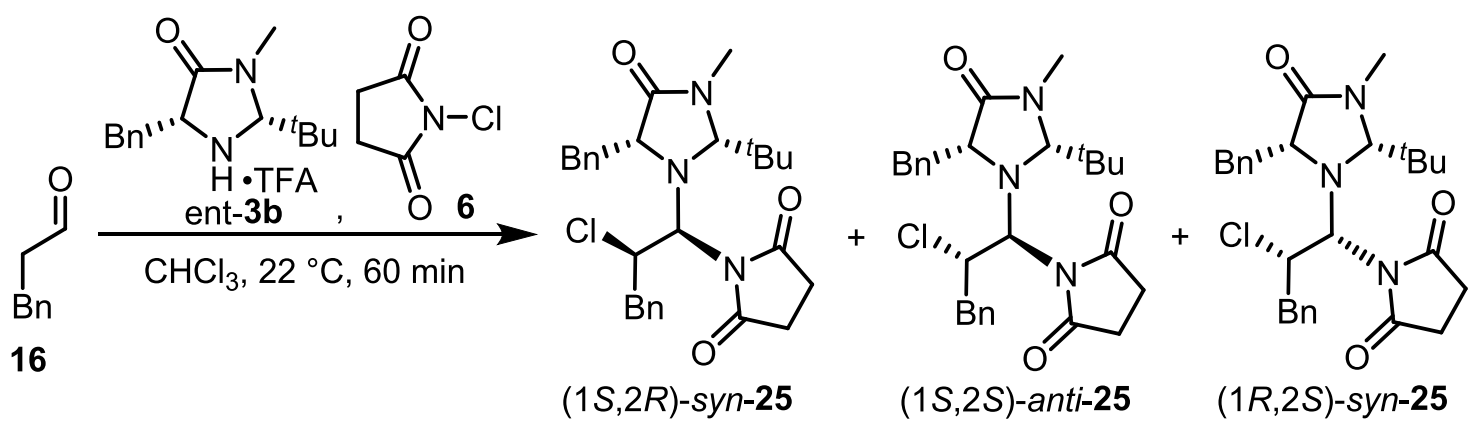

The TFA-salt of catalyst ent-3b (0.837 g, $2.32 \mathrm{mmol}, 20 \mathrm{~mol} \%)$ and NCS (1.55 g, $11.6 \mathrm{mmol}, 1.0$ equiv) were added successively to a solution of hydrocinnamic aldehyde (1.54 mL, $11.6 \mathrm{mmol}, 1.0$ equiv) in $\mathrm{CHCl}_{3}$ $(46 \mathrm{~mL})$. The reaction was stirred for $60 \mathrm{~min}$ and quenched with aqueous saturated $\mathrm{NaHCO}_{3}(50 \mathrm{~mL})$. The aqueous phase was extracted with dichloromethane $(3 \times 50 \mathrm{~mL})$, the combined organic phases were dried over $\mathrm{NaSO}_{4}$, the solvent was removed under reduced pressure and the crude product was purified by column chromatography $\left(\mathrm{SiO}_{2}\right.$, pentane/Et $2 \mathrm{O} / \mathrm{EtOAc}, 1: 1: 1$ to $0: 1: 1$ to $\left.0: 0: 1\right)$. Aminal $(1 S, 2 R)$-syn-25 was obtained as a colorless solid $(0.381 \mathrm{~g}, 0.768 \mathrm{mmol}, 33 \%)$. Separation of another fraction from the column chromatography by chiral HPLC enabled further separation of aminal (1S,2S)-anti-25 (57.0 mg, 0.115 mmol, 5\%) and aminal (1R,2S)-syn-25 (0.137 g, $0.272 \mathrm{mmol}, 12 \%)$. The absolute configuration of the three aminals was determined by $\mathrm{X}$-ray crystal structure analysis.

Aminal (1S,2R)-syn-25:

$m p=158^{\circ} \mathrm{C}$

$[\alpha]_{D}^{26}=-1.96^{\circ}(c=1.00$, dichloromethane $)$

${ }^{1} \mathrm{H}-\mathrm{NMR}\left(\mathrm{CDCl}_{3}, 600 \mathrm{MHz}\right) \delta=7.39-7.36(\mathrm{~m}, 2 \mathrm{H}), 7.33-7.28(\mathrm{~m}, 2 \mathrm{H}), 7.26-7.18(\mathrm{~m}, 4 \mathrm{H}), 7.00-6.97$ (m, 2H), $5.18(\mathrm{~d}, J=10.7 \mathrm{~Hz}, 1 \mathrm{H}), 5.08$ (td, $J=10.8,1.9 \mathrm{~Hz}, 1 \mathrm{H}), 4.75$ (dd, $J=7.6,5.7 \mathrm{~Hz}, 1 \mathrm{H}$ ), 4.33 (s, 1H), $3.93(\mathrm{dd}, J=14.7,1.4 \mathrm{~Hz}, 1 \mathrm{H}), 3.18-3.09(\mathrm{~m}, 2 \mathrm{H}), 2.88(\mathrm{~s}, 3 \mathrm{H}), 2.77-2.70(\mathrm{~m}, 5 \mathrm{H}), 1.09(\mathrm{~s}, 9 \mathrm{H})$ ppm.

${ }^{13} \mathrm{C}-\mathrm{NMR}\left(\mathrm{CDCl}_{3}, 151 \mathrm{MHz}\right) \delta=179.5,173.4,138.1,137.0,129.4,129.1,128.8,128.5,127.0,126.8,87.8$, $79.1,61.2,59.6,42.3,41.6,37.7,31.9,29.7,28.1,26.7$ ppm.

IR (ATR): $\tilde{v}=2957,2925,1774,1702,1455,1328,1253,1148,1061,749 \mathrm{~cm}^{-1}$.

HRMS (ESI, pos. mode): $\mathrm{m} / \mathrm{z}$ calculated for $\mathrm{C}_{28} \mathrm{H}_{35} \mathrm{CIN}_{3} \mathrm{O}_{3}[\mathrm{M}+\mathrm{H}]^{+}:$496.2362, found 496.2350; $\mathrm{m} / \mathrm{z}$ calculated for $\mathrm{C}_{28} \mathrm{H}_{34} \mathrm{CIN}_{3} \mathrm{NaO}_{3}[\mathrm{M}+\mathrm{Na}]^{+}: 518.2181$, found $518.2160 ; \mathrm{m} / z$ calculated for $\mathrm{C}_{28} \mathrm{H}_{34} \mathrm{ClKN}_{3} \mathrm{O}_{3}$ $[\mathrm{M}+\mathrm{K}]^{+}:$534.1921, found 534.1899. 


\section{Aminal (1S,2S)-anti-25:}

$\mathbf{m p}=150^{\circ} \mathrm{C}$

$[\alpha]_{D}^{26}=-18.43^{\circ}(c=0.45$, dichloromethane $)$

${ }^{1} \mathrm{H}-\mathrm{NMR}\left(\mathrm{CDCl}_{3}, 700 \mathrm{MHz}\right) \delta=7.41-7.38(\mathrm{~m}, 2 \mathrm{H}), 7.32-7.24(\mathrm{~m}, 5 \mathrm{H}), 7.24-7.19(\mathrm{~m}, 1 \mathrm{H}), 7.03-7.01$ $(\mathrm{m}, 2 \mathrm{H}), 5.29(\mathrm{~d}, J=10.5 \mathrm{~Hz}, 1 \mathrm{H}), 4.75$ (td, $J=10.2,3.1 \mathrm{~Hz}, 1 \mathrm{H}), 4.62$ (dd, $J=6.7,4.8 \mathrm{~Hz}, 1 \mathrm{H}), 4.19$ (s, $1 \mathrm{H}), 3.20-3.13(\mathrm{~m}, 2 \mathrm{H}), 2.92(\mathrm{~s}, 3 \mathrm{H}), 2.85(\mathrm{dd}, J=14.3,3.1 \mathrm{~Hz}, 1 \mathrm{H}), 2.76-2.66(\mathrm{~m}, 4 \mathrm{H}), 2.64(\mathrm{dd}, J=$ $14.3,10.0 \mathrm{~Hz}, 1 \mathrm{H}), 1.11(\mathrm{~s}, 9 \mathrm{H}) \mathrm{ppm}$.

${ }^{13} \mathrm{C}-\mathrm{NMR}\left(\mathrm{CDCl}_{3}, 151 \mathrm{MHz}\right) \delta=179.2,174.1,139.3,136.6,129.5,129.1,128.5,128.4,127.1,126.4,86.2$, $76.9,61.8,60.3,41.2,41.0,38.2,31.9,29.8,28.3,26.3$ ppm.

IR $(A T R): \tilde{v}=2955,2925,1772,1702,1455,1363,1255,1156,1079,794,750 \mathrm{~cm}^{-1}$.

HRMS (ESI, pos. mode): $\mathrm{m} / \mathrm{z}$ calculated for $\mathrm{C}_{28} \mathrm{H}_{35} \mathrm{CIN}_{3} \mathrm{O}_{3}[\mathrm{M}+\mathrm{H}]^{+}: 496.2362$, found $496.2381 ; \mathrm{m} / \mathrm{z}$ calculated for $\mathrm{C}_{28} \mathrm{H}_{34} \mathrm{CIN}_{3} \mathrm{NaO}_{3}[\mathrm{M}+\mathrm{Na}]^{+}: 518.2181$, found $518.2191 ; \mathrm{m} / z$ calculated for $\mathrm{C}_{28} \mathrm{H}_{34} \mathrm{ClKN}_{3} \mathrm{O}_{3}$ $[\mathrm{M}+\mathrm{K}]^{+}:$534.1921, found 534.1935.

\section{Aminal (1R,2S)-syn-25:}

$\mathbf{m p}=178^{\circ} \mathrm{C}$

$[\alpha]_{D}^{26}=-55.64^{\circ}(c=1.45$, dichloromethane $)$

${ }^{1} \mathrm{H}-\mathrm{NMR}\left(\mathrm{CDCl}_{3}, 700 \mathrm{MHz}\right) \delta=7.45-7.39(\mathrm{~m}, 4 \mathrm{H}), 7.33-7.19(\mathrm{~m}, 4 \mathrm{H}), 7.06-7.03(\mathrm{~m}, 2 \mathrm{H}), 4.98(\mathrm{~s}, 1 \mathrm{H})$, $4.85(\mathrm{t}, J=11.0 \mathrm{~Hz}, 1 \mathrm{H}), 4.54(\mathrm{~d}, J=10.0 \mathrm{~Hz}, 1 \mathrm{H}), 4.24$ (dd, $J=11.6,4.3 \mathrm{~Hz}, 1 \mathrm{H}), 3.35$ (dd, $J=14.1,4.2$ $\mathrm{Hz}, 1 \mathrm{H}), 3.23(\mathrm{~d}, J=15.9 \mathrm{~Hz}, 1 \mathrm{H}), 2.96(\mathrm{~s}, 3 \mathrm{H}), 2.88$ (dd, $J=14.1,11.5 \mathrm{~Hz}, 1 \mathrm{H}), 2.85-2.58(\mathrm{~m}, 4 \mathrm{H}), 1.72$ $(\mathrm{s}, 1 \mathrm{H}), 1.10(\mathrm{~s}, 9 \mathrm{H}) \mathrm{ppm}$.

${ }^{13} \mathrm{C}-\mathrm{NMR}\left(\mathrm{CDCl}_{3}, 176 \mathrm{MHz}\right) \delta=179.3,178.2,173.5,138.2,137.2,129.8,128.9,128.7,128.5,126.9,126.8$, $86.2,80.3,67.2,58.9,41.0,38.6,37.2,32.2,28.1,28.0,27.8$ ppm.

IR (ATR): $\tilde{v}=3030,2958,1705,1329,1254,1161,1094,740 \mathrm{~cm}^{-1}$.

HRMS (ESI, pos. mode): $\mathrm{m} / \mathrm{z}$ calculated for $\mathrm{C}_{28} \mathrm{H}_{34} \mathrm{CIN}_{3} \mathrm{NaO}_{3}[\mathrm{M}+\mathrm{Na}]^{+}: 518.2181$, found $518.2184 ; \mathrm{m} / \mathrm{z}$ calculated for $\mathrm{C}_{28} \mathrm{H}_{34} \mathrm{ClKN}_{3} \mathrm{O}_{3}[\mathrm{M}+\mathrm{K}]^{+}:$534.1921, found 534.1920. 
Assignment of Aminal Diastereoisomers Derived from the $2^{\text {nd }}$ Generation MacMillan Catalyst (Hydrocinnamic Aldehyde as the Substrate)

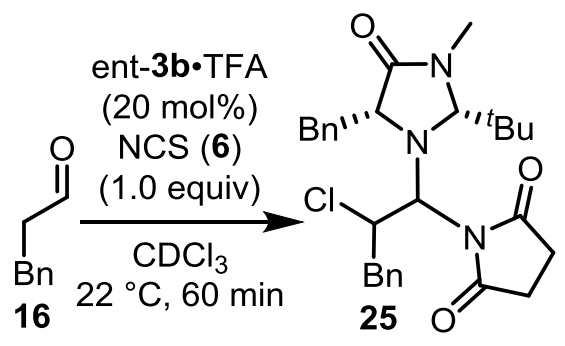<smiles>CN1C(=O)[C@@H](Br)N([C@H]([C@H](Cl)Br)N2C(=O)CCC2=O)[C@@H]1Br</smiles>

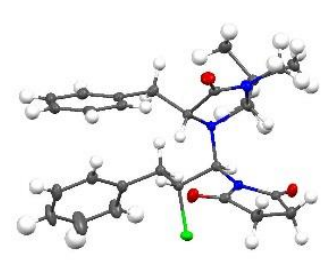

$(1 S, 2 R)-s y n-25$<smiles>CN1C(=O)[C@@H](Br)N([C@H]([C@H](Cl)Br)N2C(=O)CCC2=O)[C@@H]1Br</smiles>

$(1 S, 2 S)-a n t i-25$

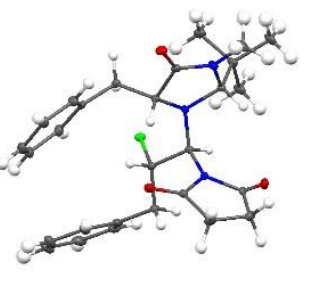<smiles>CN1C(=O)[C@@H](Br)N([C@H]([C@H](Cl)Br)N2C(=O)CCC2=O)[C@@H]1Br</smiles>
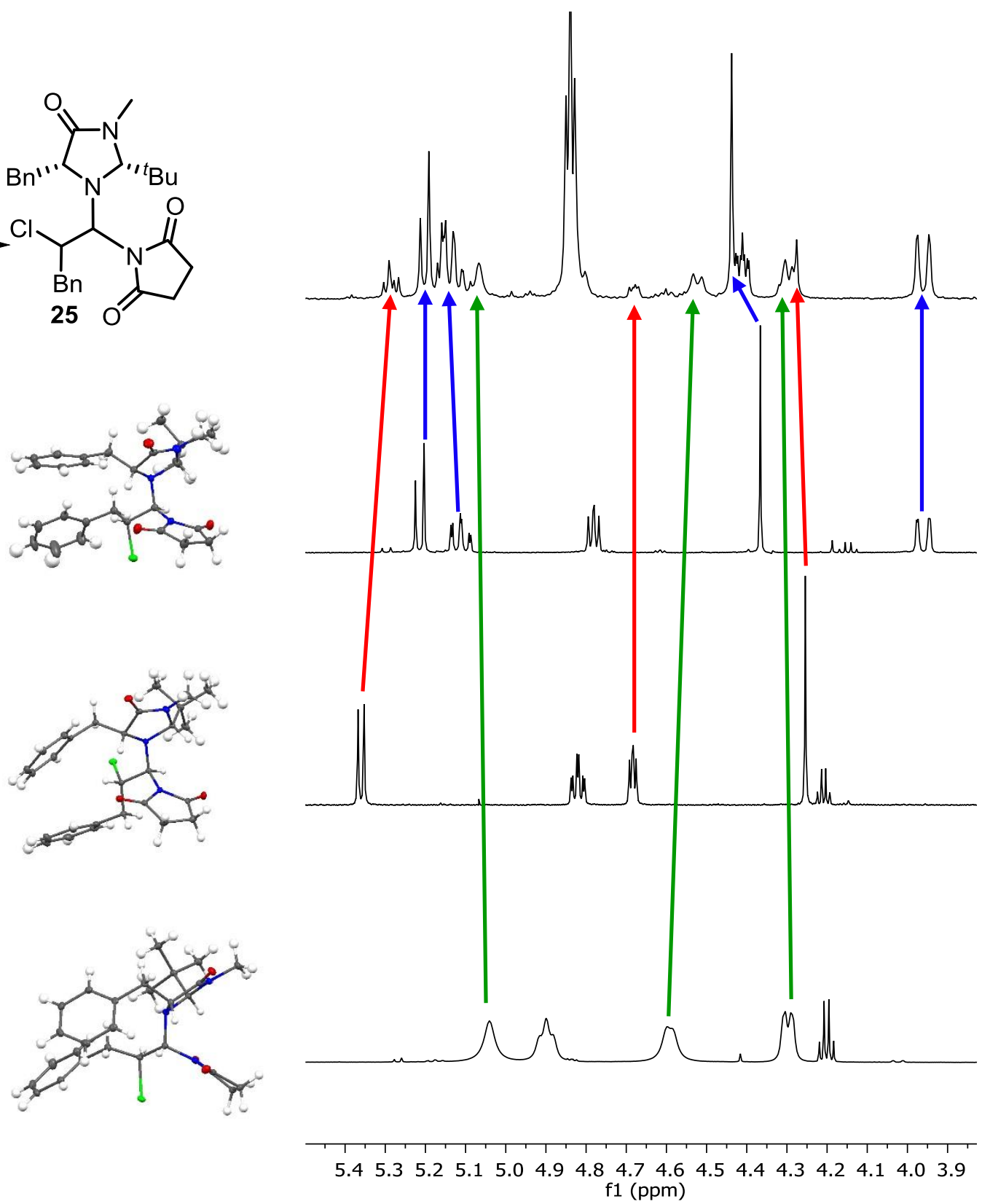

Figure SI-7. ${ }^{1} \mathrm{H}-\mathrm{NMR}$ spectrum of the reaction with $20 \mathrm{~mol} \%$ ent-3b-TFA after $60 \mathrm{~min}$ (top). Crystal structures and ${ }^{1} \mathrm{H}-\mathrm{NMR}$ spectra of the isolated diastereomeric aminals $\mathbf{2 1}$ (below).

As expected, three diastereomeric species can be detected in the ${ }^{1} \mathrm{H}-\mathrm{NMR}$ spectrum. All of them could be isolated and fully characterized. The absolute configurations of the three aminals were determined by $X$ ray crystal structure analysis and are in accordance with the previous experiment (isovaleraldehyde as the substrate). 


\section{Decomposition of Aminal syn-18b (Derived from $3^{\text {rd }}$ Generation MacMillan Catalyst)}

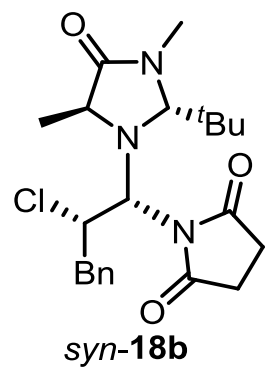

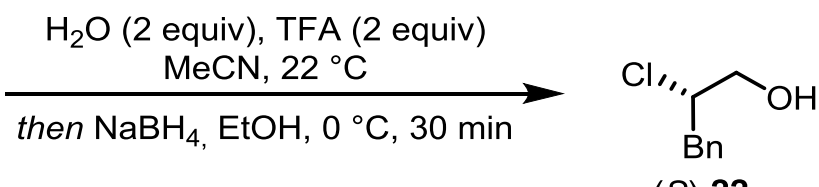

syn-18b

(S)-22

Aminal syn-18b was synthesized according to the literature procedure. ${ }^{22} \mathrm{H}_{2} \mathrm{O}(85.8 \mu \mathrm{L}, 4.76 \mathrm{mmol}, 2.0$ equiv) and TFA (364 $\mu \mathrm{L}, 4.76 \mathrm{mmol}, 2.0$ equiv) were added to a solution of aminal syn-18b (1.00 g, 2.38 mmol, 1.0 equiv) in $\mathrm{MeCN}(30 \mathrm{~mL})$ at $22{ }^{\circ} \mathrm{C}$. Aliquots $(6 \mathrm{~mL})$ were taken from the reaction mixture after defined points of time (5 min, $10 \mathrm{~min}, 60 \mathrm{~min}$ ) and added to a solution of $\mathrm{NaBH}_{4}$ (75.1 mg, $1.98 \mathrm{mmol}, 5.0$ equiv) in $\mathrm{EtOH}(2.0 \mathrm{~mL})$. After $30 \mathrm{~min}$ the reactions were quenched by the addition of aqueous saturated $\mathrm{NH}_{4} \mathrm{Cl}$. The aqueous phase was extracted with EtOAc $(3 \times 5 \mathrm{~mL})$, the combined organic phases were dried over $\mathrm{Na}_{2} \mathrm{SO}_{4}$ and the solvent was removed under reduced pressure. The crude products were purified by column chromatography $\left(\mathrm{SiO}_{2}\right.$, pentane/EtOAc 10:1 to 5:1) and the enantiomeric excess was determined by chiral HPLC. The absolute configuration was determined by comparison of the optical rotation with the literature.

The ${ }^{1} \mathrm{H}-\mathrm{NMR}$ spectrum is in accordance with the literature. ${ }^{22}$

${ }^{1} \mathrm{H}-\mathrm{NMR}\left(\mathrm{CDCl}_{3}, 400 \mathrm{MHz}\right) \delta=7.35-7.30(\mathrm{~m}, 2 \mathrm{H}), 7.29-7.22(\mathrm{~m}, 3 \mathrm{H}), 4.26-4.20(\mathrm{~m}, 1 \mathrm{H}), 3.81$ (ddd, J $=12.1,7.2,3.6 \mathrm{~Hz}, 1 \mathrm{H}), 3.69(\mathrm{dt}, J=12.1,6.1 \mathrm{~Hz}, 1 \mathrm{H}), 3.14(\mathrm{dd}, J=14.0,7.0 \mathrm{~Hz}, 1 \mathrm{H}), 3.06(\mathrm{dd}, J=14.0$, $7.4 \mathrm{~Hz}, 1 \mathrm{H}), 2.03$ (t, $J=6.7 \mathrm{~Hz}, 1 \mathrm{H}) \mathrm{ppm}$.

1. aliquot (5 $\mathrm{min}): 95 \%$ ee

2. aliquot (10 $\mathrm{min}): 95 \%$ ee

3. aliquot (60 $\mathrm{min}): 94 \%$ ee

Sample: $[\alpha]_{D}^{24}=-11.4^{\circ}(c=1.00$, dichloromethane $)$

(S)-2-chloro-3-phenylpropan-1-ol (95\% ee): $[\alpha]_{D}^{24}=-13.8^{\circ}(c=1.00$, dichloromethane)

$(R)$-2-chloro-3-phenylpropan-1-ol (93\% ee): $[\alpha]_{D}^{24}=+14.0^{\circ}(c=1.00$, dichloromethane)

(S)-2-chloro-3-phenylpropan-1-ol (95\% ee): $[\alpha]_{D}=-21.7^{\circ}\left(c=1.00, \mathrm{CHCl}_{3}\right)^{27}$ 

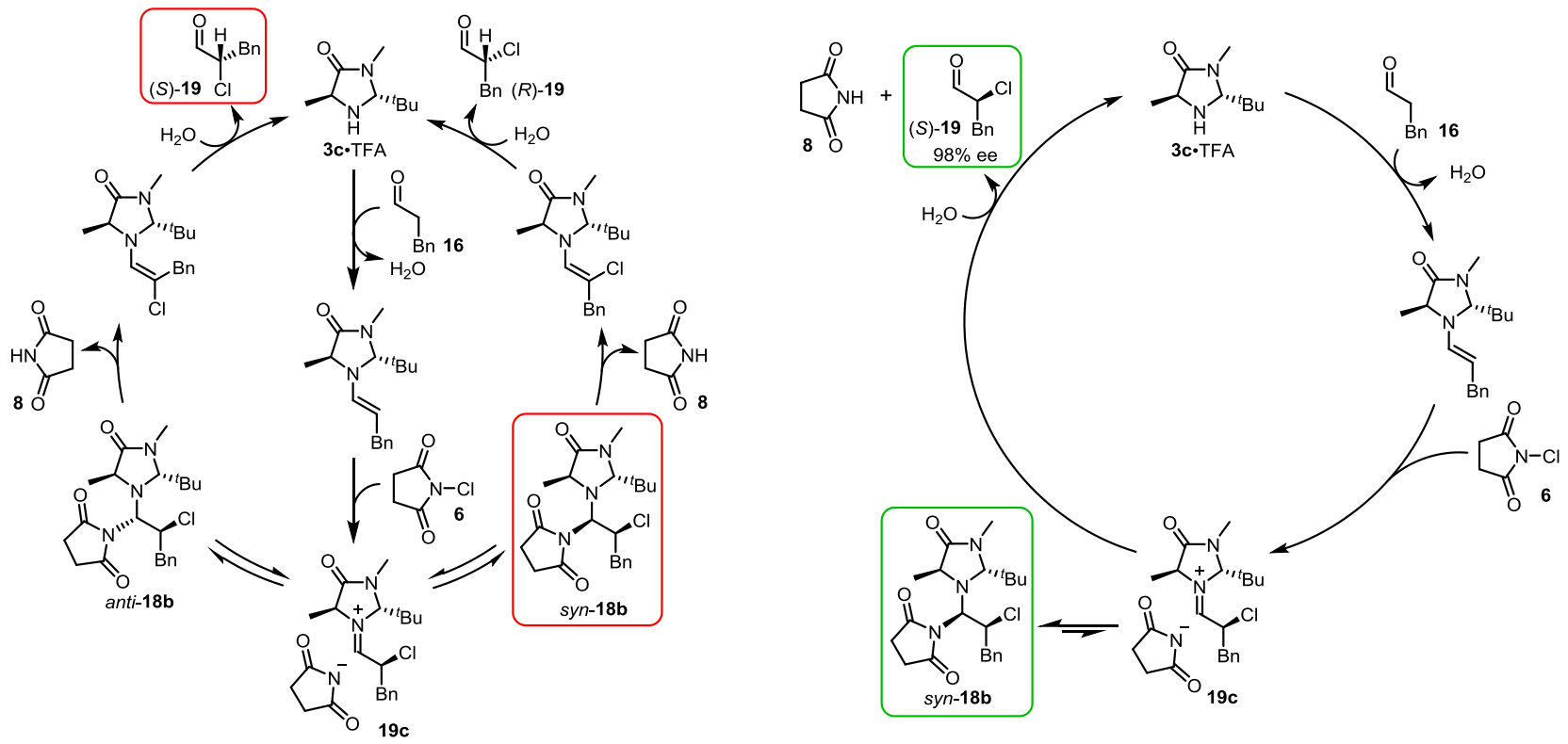

Figure SI-8. Only the off-cycle mechanism (right) can rationalize the observed enenatioselectivity $((S)$ chloroaldehyde) of the $\alpha$-chlorination reaction and the decomposition pathway. The on-cycle mechanism ${ }^{28}$ (left) predicts the formation of the wrong enantiomer of the chloroaldeyhde $((R)$-chloroaldehyde).

As observed for most of the aminals derived from the $1^{\text {st }}$ and $2^{\text {nd }}$ generation MacMillan catalyst, aminal syn$18 \mathrm{~b}$ is bench-stable and can be isolated and characterized. ${ }^{22}$ According to the catalytic cycle of the oncycle scenario ${ }^{28}$ aminal syn-18b should generate the $(R)$-enantiomer of the chloroaldehyde product. Decomposition under acidic conditions (TFA and water are also present in the catalytic reaction) clearly shows that aminal syn-18b was converted almost exclusively (95\% ee) to the (S)-chloroaldehyde product. This experimental outcome is in accordance with our proposed decomposition pathway via a chloroiminium ion. 


\section{(S)-2-chloro-3-phenylpropan-1-ol ((S)-22)}

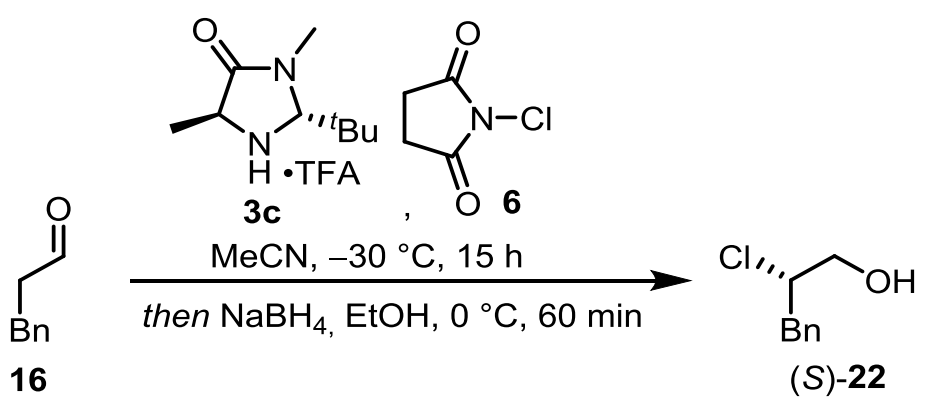

Hydrocinnamic aldehyde $(0.266 \mathrm{~mL}, 2.00 \mathrm{mmol}, 1.0$ equiv) was dissolved in $\mathrm{MeCN}(8.0 \mathrm{~mL})$ and cooled to $-30{ }^{\circ} \mathrm{C}$. Catalyst $3 \mathrm{c}(0.114 \mathrm{~g}, 0.400 \mathrm{mmol}, 20 \mathrm{~mol} \%)$ and NCS $(0.320 \mathrm{~g}, 2.40 \mathrm{mmol}, 1.2$ equiv) were added subsequently and the reaction mixture was stirred for $15 \mathrm{~h}$ at the same temperature. After the reaction was warmed to $0{ }^{\circ} \mathrm{C}, \mathrm{MeOH}(3.0 \mathrm{~mL})$ and $\mathrm{NaBH}_{4}(0.189 \mathrm{~g}, 5.00 \mathrm{mmol}, 2.5$ equiv) were added and the mixture was stirred for $60 \mathrm{~min}$ at the same temperature. The reaction was quenched with aqueous saturated $\mathrm{NH}_{4} \mathrm{Cl}$ $(10 \mathrm{~mL})$, the aqueous phase was extracted with dichloromethane $(3 \times 15 \mathrm{~mL})$ and the combined organic phases were dried over $\mathrm{NaSO}_{4}$. The solvent was removed under reduced pressure and the crude product was purified by column chromatography (Silica, pentane/EtOAc 10:1). Pure chloroalcohol (S)-22 (0.248 g, $1.45 \mathrm{mmol}, 73 \%, 95 \%$ ee) was obtained as a colorless oil.

${ }^{1} \mathrm{H}-\mathrm{NMR}$ is in accordance with the literature. ${ }^{22}$

$[\alpha]_{D}^{24}=-13.8^{\circ}(c=1.00$, dichloromethane $)$

${ }^{1} \mathrm{H}-\mathrm{NMR}\left(\mathrm{CDCl}_{3}, 400 \mathrm{MHz}\right) \delta=7.35-7.30(\mathrm{~m}, 2 \mathrm{H}), 7.29-7.22(\mathrm{~m}, 3 \mathrm{H}), 4.26-4.20(\mathrm{~m}, 1 \mathrm{H}), 3.81$ (ddd, J $=12.1,7.2,3.6 \mathrm{~Hz}, 1 \mathrm{H}), 3.69(\mathrm{dt}, J=12.1,6.1 \mathrm{~Hz}, 1 \mathrm{H}), 3.14(\mathrm{dd}, J=14.0,7.0 \mathrm{~Hz}, 1 \mathrm{H}), 3.06(\mathrm{dd}, J=14.0$, $7.4 \mathrm{~Hz}, 1 \mathrm{H}), 2.03(\mathrm{t}, J=6.7 \mathrm{~Hz}, 1 \mathrm{H}) \mathrm{ppm}$. 


\section{(R)-2-chloro-3-phenylpropan-1-ol ((R)-22)}

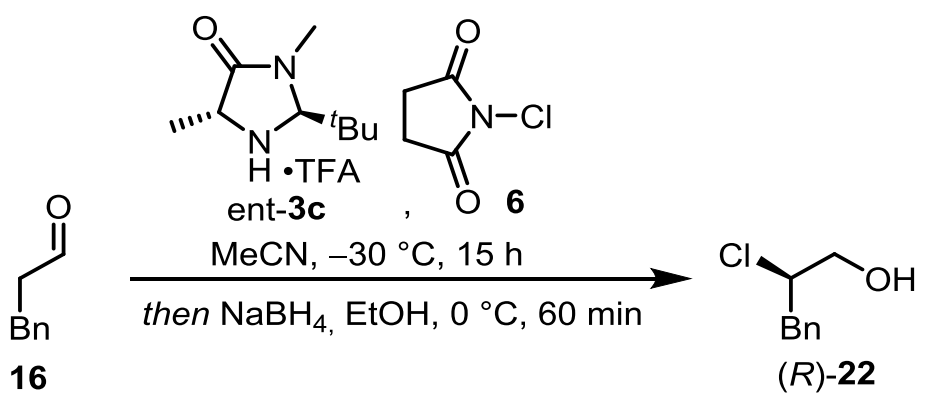

Hydrocinnamic aldehyde $(0.266 \mathrm{~mL}, 2.00 \mathrm{mmol}, 1.0$ equiv) was dissolved in $\mathrm{MeCN}(8.0 \mathrm{~mL})$ and cooled to $-30{ }^{\circ} \mathrm{C}$. Catalyst ent-3c $(0.114 \mathrm{~g}, 0.400 \mathrm{mmol}, 20 \mathrm{~mol} \%)$ and NCS (0.320 g, $2.40 \mathrm{mmol}, 1.2$ equiv) were added subsequently and the reaction mixture was stirred for $15 \mathrm{~h}$ at the same temperature. After the reaction was warmed to $0{ }^{\circ} \mathrm{C}, \mathrm{MeOH}(3.0 \mathrm{~mL})$ and $\mathrm{NaBH}_{4}(0.189 \mathrm{~g}, 5.00 \mathrm{mmol}, 2.5$ equiv) were added and the mixture was stirred for $60 \mathrm{~min}$ at the same temperature. The reaction was quenched with aqueous saturated $\mathrm{NH}_{4} \mathrm{Cl}(10 \mathrm{~mL})$, the aqueous phase was extracted with dichloromethane $(3 \times 15 \mathrm{~mL})$ and the combined organic phases were dried over $\mathrm{NaSO}_{4}$. The solvent was removed under reduced pressure and the crude product was purified by column chromatography (Silica, pentane/EtOAc 10:1). Pure chloroalcohol $(R)-22(0.229 \mathrm{~g}, 1.34 \mathrm{mmol}, 67 \%, 93 \%$ ee) was obtained as a colorless oil.

${ }^{1} \mathrm{H}-\mathrm{NMR}$ is in accordance with the literature. ${ }^{22}$

$[\alpha]_{D}^{24}=+14.0^{\circ}(c=1.00$, dichloromethane $)$

${ }^{1} \mathrm{H}-\mathrm{NMR}\left(\mathrm{CDCl}_{3}, 400 \mathrm{MHz}\right) \delta=7.35-7.30(\mathrm{~m}, 2 \mathrm{H}), 7.29-7.22(\mathrm{~m}, 3 \mathrm{H}), 4.26-4.20(\mathrm{~m}, 1 \mathrm{H}), 3.81$ (ddd, J $=12.1,7.2,3.6 \mathrm{~Hz}, 1 \mathrm{H}), 3.69(\mathrm{dt}, J=12.1,6.1 \mathrm{~Hz}, 1 \mathrm{H}), 3.14(\mathrm{dd}, J=14.0,7.0 \mathrm{~Hz}, 1 \mathrm{H}), 3.06(\mathrm{dd}, J=14.0$, $7.4 \mathrm{~Hz}, 1 \mathrm{H}), 2.03(\mathrm{t}, J=6.7 \mathrm{~Hz}, 1 \mathrm{H}) \mathrm{ppm}$. 


\subsection{NMR reaction progress (3rd generation MacMillan catalyst)}

${ }^{1} \mathrm{H}-\mathrm{NMR}$ reaction progress<smiles>CC1C(=O)N(C)[C@@H]([13CH3])N1C(C(Cl)Br)N1C(=O)CCC1=O</smiles>
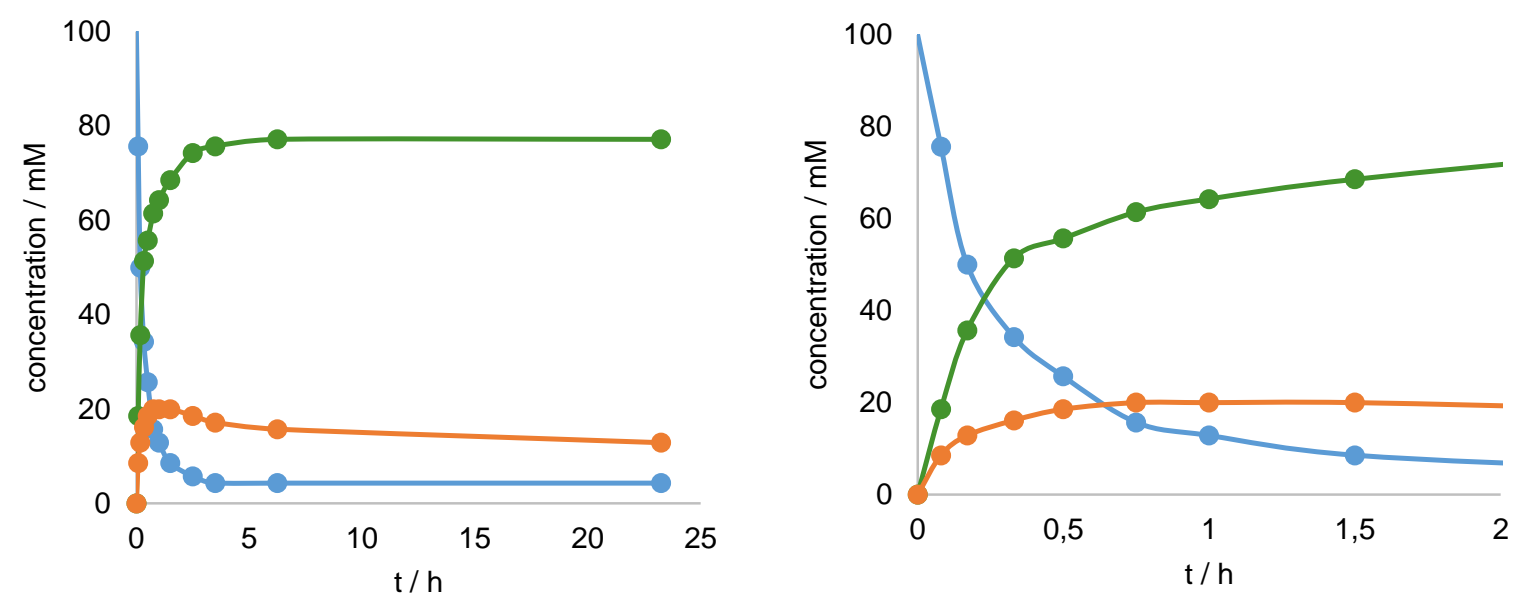

Figure SI-9. ${ }^{1} \mathrm{H}-\mathrm{NMR}$ reaction progress of the $\alpha$-chlorination reaction (hydrocinnamic aldehyde, NCS, catalyst $3 \mathrm{c} \cdot$ TFA) according to our previous publication. ${ }^{22}$

Table SI-1. Experimental data of the reaction progress analysis. ${ }^{22}$

\begin{tabular}{c|c|cc|cc|cc}
$\mathrm{t}[\mathrm{min}]$ & $\mathrm{t}[\mathrm{h}]$ & \multicolumn{2}{|c|}{ aldehyde } & \multicolumn{2}{|c}{ Cl-aldehyde } & \multicolumn{2}{|c}{ aminal } \\
\hline 0 & 0 & 0,7 & 100,03 & 0 & 0 & 0 \\
5 & 0,08 & 0,53 & 75,74 & 0,13 & 18,58 & 0,06 & 8,57 \\
10 & 0,17 & 0,35 & 50,02 & 0,25 & 35,73 & 0,09 & 12,86 \\
20 & 0,33 & 0,24 & 34,3 & 0,36 & 51,44 & 0,11 & 16,15 \\
30 & 0,5 & 0,18 & 25,72 & 0,39 & 55,73 & 0,13 & 18,58 \\
45 & 0,75 & 0,11 & 15,72 & 0,43 & 61,45 & 0,14 & 20,01 \\
60 & 1 & 0,09 & 12,86 & 0,45 & 64,31 & 0,14 & 20,01 \\
90 & 1,5 & 0,06 & 8,57 & 0,48 & 68,59 & 0,14 & 20,01 \\
150 & 2,5 & 0,04 & 5,72 & 0,52 & 74,31 & 0,13 & 18,58 \\
210 & 3,5 & 0,03 & 4,29 & 0,53 & 75,74 & 0,12 & 17,15 \\
375 & 6,25 & 0,03 & 4,29 & 0,54 & 77,17 & 0,11 & 15,72 \\
1395 & 23,25 & 0,03 & 4,29 & 0,54 & 77,17 & 0,09 & 12,86
\end{tabular}

We interrogated concentration profiles in order to assess whether the two mechanisms (steric shielding model and Curtin-Hammett scenario) are kinetically distinguishable. Gratifyingly, aldehyde 16, the chloroaldehyde product 19 and the corresponding crystallographically characterized aminal syn-18b possess separate, non-overlapping NMR resonances. By monitoring their concentrations over time ${ }^{22}$ it is apparent that the initial rate of chloroaldehyde formation exceeds the build-up of the aminal. This observation eliminates the possibility of the catalyst being turned over through the thermodynamically most 
stable aminal (Curtin-Hammett scenario). The alternate off-cycle mechanism provides a scenario where the rate of iminium hydrolysis exceeds that of aminal formation. From the iminium ion intermediate, part of the catalyst is diverted into a stable aminal resting state. To further illustrate the absence of an on-cyclemechanism, the first three ${ }^{1} \mathrm{H}$-NMR-spectra of the previously considered reaction are shown below. The aminal concentration at no point exceeds the product concentration, not even in the initial phase (5 min) of the reaction. This observation strongly supports an off-cycle-scenario, in which the product can be formed without going through the aminal intermediate. When reanalyzing our data for this reaction, we noted additional, previously overlooked resonances in the NMR spectrum at $t=5$ min (quickly disappearing thereafter). The possibility of the corresponding species to be the elusive aminal anti-18b is supported by comparison of the observed and computed ${ }^{1} \mathrm{H}$ chemical shifts.

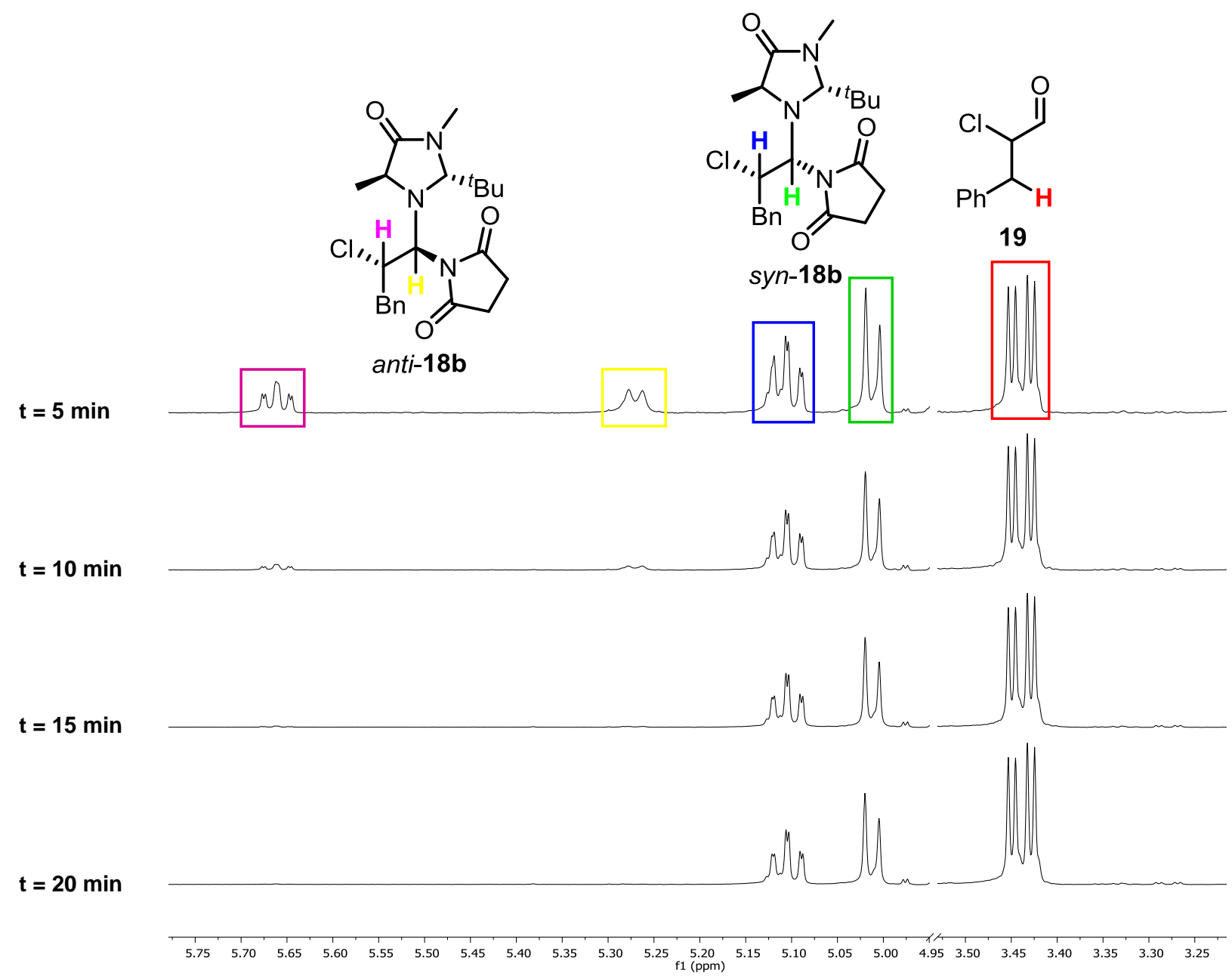

Figure SI-10. ${ }^{1} \mathrm{H}$-NMR spectra of the initial phase of the $\alpha$-chlorination reaction (hydrocinnamic aldehyde, NCS, catalyst 3c・TFA).

\section{NMR Calculations for syn-18b and anti-18b}

${ }^{1} \mathrm{H}$ and ${ }^{13} \mathrm{C}$ NMR calculations were performed to compare to the major and minor sppecies at $\mathrm{t}=5 \mathrm{~min}$. The key ${ }^{1} \mathrm{H}$ chemical shifts for syn-18b and anti-18b were in good agreement $(<0.3$ ppm absolute deviation between experimental and calculated chemical shifts $)^{17,18}$ with the experimental shifts for the major and minor products. 
Table SI-2. Calculated ${ }^{13} \mathrm{C}$ NMR $\delta$ 's for syn-18b.<smiles></smiles>

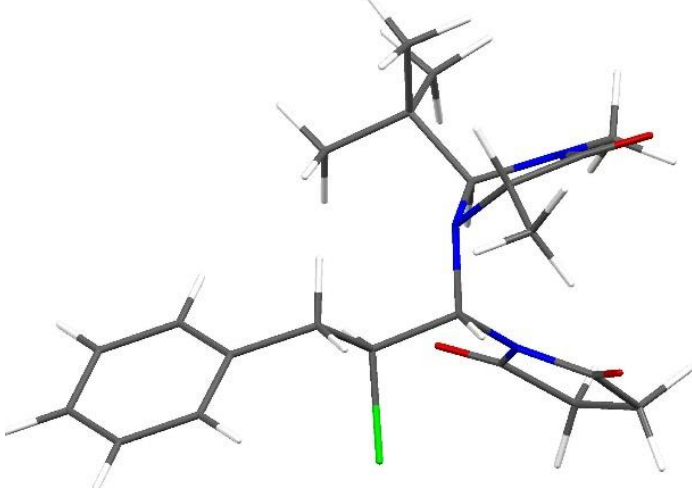

\begin{tabular}{|llll|}
\hline Exp. \# & Comp \# & $\begin{array}{l}\text { Comp. } \\
\text { Isotropic }\end{array}$ & Comp. \\
\hline C5 & 1 & 98.9366 & 83.8 \\
C2 & 2 & 3.6610 & 174.5 \\
C3 & 3 & 123.1277 & 60.7 \\
C4 & 7 & 153.8751 & 31.5 \\
C1' & 11 & 113.1924 & 70.2 \\
C2' & 13 & 111.3355 & 72.0 \\
C3' & 14 & 142.8361 & 42.0 \\
& & & \\
C4' & 17 & 175.0080 & 11.3 \\
C5' & 22 & 143.6420 & 41.2 \\
& 26 & -4.5320 & 182.3 \\
& 27 & -2.1380 & 180.0 \\
C5" & 28 & 155.0907 & 30.3 \\
C2" & 29 & 155.6905 & 29.7 \\
C6 & 34 & 158.5958 & 27.0 \\
& 38 & 156.9911 & 28.5 \\
& 42 & 163.1739 & 22.6 \\
& 49 & 41.0137 & 138.9 \\
& 50 & 50.0409 & 130.3 \\
& 51 & 53.6789 & 126.9 \\
& 52 & 52.7727 & 127.7 \\
& 54 & 52.5161 & 128.0 \\
& 56 & 54.4196 & 126.2 \\
\hline
\end{tabular}


Table SI-3. Comparison of calculated and experimental ${ }^{1} \mathrm{H}$ NMR $\delta$ 's for syn-18b.

\begin{tabular}{|c|c|c|c|c|c|c|c|}
\hline $\begin{array}{l}\text { Exp. } \\
\text { C\# }\end{array}$ & $\begin{array}{l}\text { Comp. } \\
\text { C\# }\end{array}$ & Comp. H\# & $\begin{array}{l}\text { Comp. } \\
\text { Isotropic }\end{array}$ & Comp. $\delta$ & $\begin{array}{l}\text { Avg. } \\
\text { Comp. } \delta\end{array}$ & $\begin{array}{l}\text { Exp. } \\
\delta\end{array}$ & Abs. Dev. \\
\hline 8 & 7 & 8 & 28.57 & 3.00 & & & \\
\hline 8 & 7 & 9 & 29.26 & 2.36 & & & \\
\hline 8 & 7 & 10 & 29.12 & 2.49 & & & \\
\hline 9 & 11 & 12 & 26.64 & 4.81 & 4.81 & 5.01 & 0.20 \\
\hline 11 & 14 & 15 & 29.18 & 2.43 & 2.43 & & \\
\hline 10 & 13 & 16 & 26.22 & 5.20 & 5.20 & 5.10 & 0.10 \\
\hline 6 & 17 & 18 & 30.32 & 1.37 & & & \\
\hline 6 & 17 & 19 & 30.54 & 1.16 & & & \\
\hline 6 & 17 & 20 & 29.51 & 2.13 & 1.55 & 1.47 & 0.08 \\
\hline 3 & 3 & 21 & 27.65 & 3.87 & & & \\
\hline 5 & 1 & 23 & 27.27 & 4.22 & & & \\
\hline 15 & 28 & 30 & 28.99 & 2.61 & & & \\
\hline 16 & 29 & 31 & 28.98 & 2.62 & & & \\
\hline $\mathrm{tBu}$ & 34 & 35 & 30.82 & 0.89 & & & \\
\hline $\mathrm{tBu}$ & 34 & 36 & 30.65 & 1.05 & & & \\
\hline tBu & 34 & 37 & 30.79 & 0.93 & & & \\
\hline $\mathrm{tBu}$ & 38 & 39 & 30.29 & 1.39 & & & \\
\hline $\mathrm{tBu}$ & 38 & 40 & 30.02 & 1.64 & & & \\
\hline tBu & 38 & 41 & 30.58 & 1.12 & & & \\
\hline $\mathrm{tBu}$ & 42 & 43 & 30.76 & 0.95 & & & \\
\hline $\mathrm{tBu}$ & 42 & H32 & 26.54 & 0.80 & & & \\
\hline $\mathrm{tBu}$ & 42 & 45 & 30.75 & 0.96 & 1.08 & 1.10 & 0.02 \\
\hline 16 & 29 & 46 & 29.03 & 2.57 & & & \\
\hline 15 & 28 & 47 & 28.96 & 2.64 & & & \\
\hline \multirow[t]{7}{*}{11} & 14 & 48 & 27.41 & 4.09 & 4.09 & 3.96 & 0.13 \\
\hline & 50 & 53 & 24.03 & 7.25 & & & \\
\hline & 51 & 55 & 23.81 & 7.46 & & & \\
\hline & 52 & 57 & 23.95 & 7.33 & & & \\
\hline & 54 & 58 & 23.92 & 7.36 & & & \\
\hline & 56 & 59 & 23.99 & 7.29 & & & \\
\hline & & & & & & MAD & 0.11 \\
\hline
\end{tabular}


Table SI-4. Calculated ${ }^{13} \mathrm{C}$ NMR $\delta$ 's for anti-18b<smiles>CC(C)[10BH]N1[C@H]([C@@H](Cl)Br)[C@@H](C)C(=O)N1C</smiles>

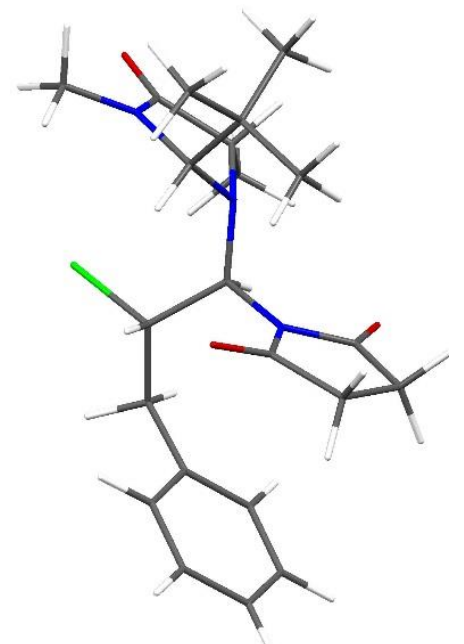

\begin{tabular}{|llll|}
\hline & & Comp. & Comp. \\
Exp. \# & Comp \# & Isotropic & ठ \\
\hline C5 & C & 97.8311 & 84.8 \\
C2 & C & 2.7976 & 175.3 \\
C3 & C & 121.5410 & 62.3 \\
C4 & C & 152.4037 & 32.9 \\
C1' & C & 112.1019 & 71.2 \\
C2' & C & 116.7850 & 66.8 \\
C3' & C & 137.7947 & 46.8 \\
C4' & C & 173.1138 & 13.2 \\
C5' & C & 141.3341 & 43.4 \\
& C & -0.9819 & 178.9 \\
& C & -3.1036 & 180.9 \\
C5" & C & 155.3086 & 30.1 \\
C2" & C & 156.1731 & 29.3 \\
C6 & C & 158.5219 & 27.0 \\
& C & 159.5979 & 26.0 \\
& C & 162.7703 & 23.0 \\
& C & 41.9613 & 138.0 \\
& C & 50.8312 & 129.6 \\
& C & 52.3459 & 128.1 \\
& C & 52.4331 & 128.1 \\
& C & 52.4293 & 128.1 \\
& C & 53.9564 & 126.6 \\
\hline
\end{tabular}


Table SI-5. Comparison of calculated and experimental ${ }^{1} \mathrm{H}$ NMR $\delta$ 's for anti-18b.

\begin{tabular}{|c|c|c|c|c|c|c|c|}
\hline $\begin{array}{l}\text { Exp. } \\
\text { C\# }\end{array}$ & $\begin{array}{l}\text { Comp. } \\
\text { C\# }\end{array}$ & Comp. H\# & Comp. Isotropic & Comp. $\delta$ & $\begin{array}{l}\text { Avg. } \\
\text { Comp. } \delta\end{array}$ & $\begin{array}{l}\text { Exp. } \\
\delta\end{array}$ & Abs. Dev. \\
\hline 8 & 7 & 8 & 28.48 & 3.09 & & & \\
\hline 8 & 7 & 9 & 29.02 & 2.58 & & & \\
\hline 8 & 7 & 10 & 28.98 & 2.62 & & & \\
\hline 9 & 11 & 12 & 26.27 & 5.15 & 5.15 & 5.27 & 0.12 \\
\hline 11 & 14 & 15 & 28.67 & 2.91 & & & \\
\hline 11 & 14 & 16 & 28.07 & 3.47 & 3.47 & 3.18 & 0.29 \\
\hline 10 & 13 & 17 & 25.43 & 5.94 & 5.94 & 5.66 & 0.28 \\
\hline 6 & 18 & 19 & 30.11 & 1.56 & & & \\
\hline 6 & 18 & 20 & 30.13 & 1.54 & & & \\
\hline 6 & 18 & 21 & 30.26 & 1.42 & 1.51 & 1.50 & 0.01 \\
\hline 3 & 3 & 22 & 27.68 & 3.83 & 3.83 & 3.91 & 0.08 \\
\hline 5 & 1 & 24 & 27.05 & 4.42 & 4.42 & 4.46 & 0.04 \\
\hline 15 & 29 & 31 & 29.62 & 2.02 & & & \\
\hline 16 & 30 & 32 & 29.70 & 1.94 & & & \\
\hline $\mathrm{tBu}$ & 35 & 36 & 30.98 & 0.75 & & & \\
\hline $\mathrm{tBu}$ & 35 & 37 & 30.64 & 1.06 & & & \\
\hline $\mathrm{tBu}$ & 35 & 38 & 30.79 & 0.92 & & & \\
\hline $\mathrm{tBu}$ & 39 & 40 & 30.88 & 0.84 & & & \\
\hline $\mathrm{tBu}$ & 39 & 41 & 31.10 & 0.63 & & & \\
\hline $\mathrm{tBu}$ & 39 & 42 & 31.21 & 0.53 & & & \\
\hline $\mathrm{tBu}$ & 43 & 44 & 26.54 & 0.86 & & & \\
\hline $\mathrm{tBu}$ & 43 & 45 & 31.17 & 0.57 & & & \\
\hline tBu & 43 & 46 & 30.97 & 0.75 & 0.77 & 0.91 & 0.14 \\
\hline 16 & 30 & 47 & 30.59 & 1.11 & & & \\
\hline \multirow[t]{7}{*}{15} & 29 & 48 & 29.30 & 2.32 & & & \\
\hline & 50 & 53 & 24.02 & 7.26 & & & \\
\hline & 51 & 55 & 24.26 & 7.04 & & & \\
\hline & 52 & 57 & 23.95 & 7.33 & & & \\
\hline & 54 & 58 & 24.08 & 7.21 & & & \\
\hline & 56 & 59 & 24.07 & 7.21 & & & \\
\hline & & & & & & MAD & 0.14 \\
\hline
\end{tabular}




\subsection{KIEs and DFT Calculations ( $3^{\text {rd }}$ Generation MacMillan Catalyst)}

\section{KIE-experiment (1)}

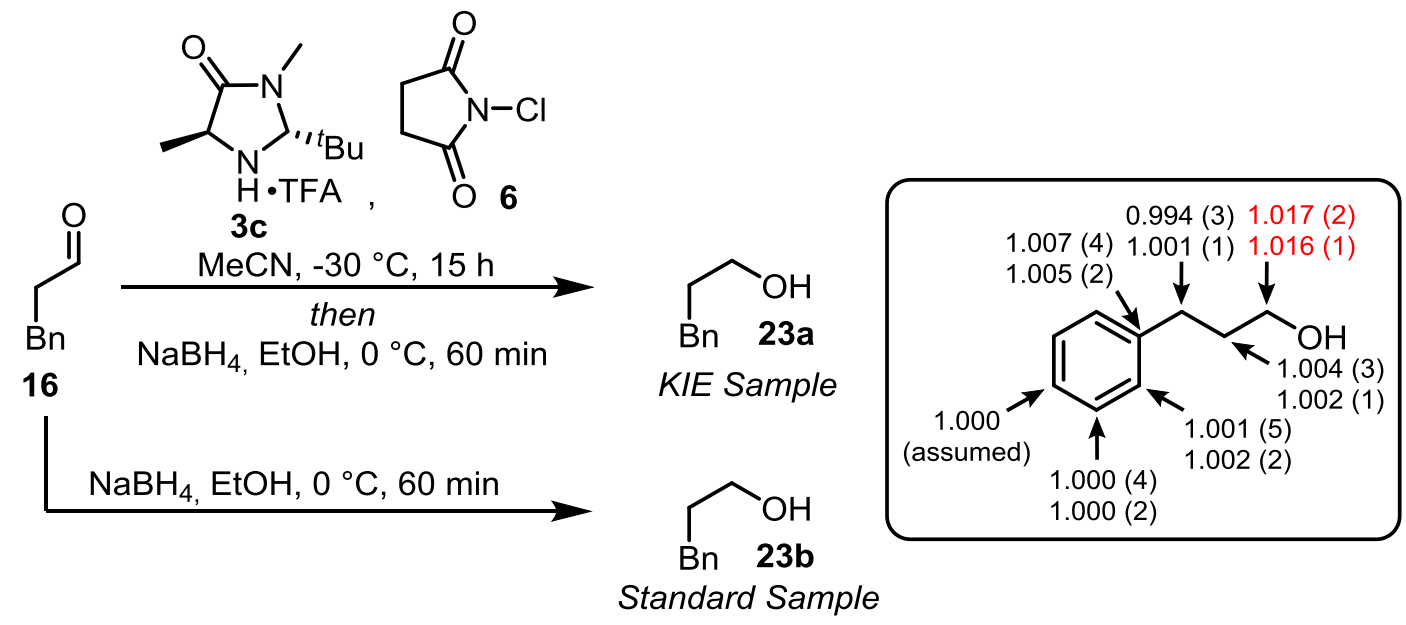

KIE Sample (KIE1\&2): Distilled hydrocinnamic aldehyde (1.61 g, $12.0 \mathrm{mmol}, 1.0$ equiv) was dissolved in $\mathrm{MeCN}(40 \mathrm{~mL})$ in a $50 \mathrm{~mL}$ graduated flask and cooled to $-30^{\circ} \mathrm{C}$. Catalyst $3 \mathrm{c}(0.682 \mathrm{~g}, 2.40 \mathrm{mmol}, 20 \mathrm{~mol} \%)$ and NCS (1.20 g, $9.00 \mathrm{mmol}, 0.75$ equiv) were added successively, the volume was adjusted to $50 \mathrm{~mL}$ by adding MeCN and the solution was stirred for 15 hours at $-30^{\circ} \mathrm{C}$. EtOH $(15 \mathrm{~mL})$ and $\mathrm{NaBH}_{4}(1.13 \mathrm{~g}, 30.0$ $\mathrm{mmol}, 2.5$ equiv) were added successively and the reaction mixture was stirred for 1 hour at $0{ }^{\circ} \mathrm{C}$. The reaction was quenched with aqueous saturated $\mathrm{NH}_{4} \mathrm{Cl}(30 \mathrm{~mL})$, extracted with $\mathrm{EtOAc}(6 \times 50 \mathrm{~mL})$ and dried over $\mathrm{Na}_{2} \mathrm{SO}_{4}$. The combined organic phases were transferred into a $500 \mathrm{~mL}$ graduated flask and the volume was adjusted to $500 \mathrm{~mL}$ by adding EtOAc. $10.0 \mathrm{~mL}$ were removed from the graduated flask and the solvent was removed under reduced pressure. The crude mixture was taken up with $2 \times 0.50 \mathrm{~mL} \mathrm{MeCNd}_{3}$ and transferred into an NMR-tube containing $10.0 \mathrm{mg}$ of the internal standard (1,3,5-trimethoxybenzene). The solvent of the remaining organic phase was removed under reduced pressure and the reduced starting material was purified by column chromatography (Silica, 10:1 to 5:1 pentane/EtOAc).

Standard Sample (STD1\&2): $\mathrm{NaBH}_{4}(0.284 \mathrm{~g}, 7.50 \mathrm{mmol}, 2.5$ equiv) was added to a solution of distilled hydrocinnamic aldehyde (from the same batch as in the KIE-experiment) $(0.403 \mathrm{~g}, 3.00 \mathrm{mmol}, 1.0$ equiv) in $\mathrm{EtOH}(3.5 \mathrm{~mL})$ at $0{ }^{\circ} \mathrm{C}$. The reaction mixture was stirred for $60 \mathrm{~min}$ at $0{ }^{\circ} \mathrm{C}$ and subsequently quenched with aqueous saturated $\mathrm{NH}_{4} \mathrm{Cl}(5 \mathrm{~mL})$. The aqueous phase was extracted with EtOAc $(6 \times 5 \mathrm{~mL})$, the combined organic phases were dried over $\mathrm{Na}_{2} \mathrm{SO}_{4}$ and the solvent was removed under reduced pressure. The crude product was purified by column chromatography (Silica, 10:1 to 5:1 pentane/EtOAc).

This experiment was done in duplicate.

KIE1: $\mathrm{m}=0.349 \mathrm{~g}(2.56 \mathrm{mmol}, 21 \%)$

STD1: $\mathrm{m}=0.387 \mathrm{~g}(2.84 \mathrm{mmol}, 95 \%)$

KIE2: $m=0.189 \mathrm{~g}(1.39 \mathrm{mmol}, 12 \%)$

STD2: $\mathrm{m}=0.378 \mathrm{~g}(2.78 \mathrm{mmol}, 93 \%)$

${ }^{1} \mathrm{H}$-NMR spectra are in accordance with the literature. ${ }^{29}$

${ }^{1} \mathrm{H}-\mathrm{NMR}\left(\mathrm{CDCl}_{3}, 400 \mathrm{MHz}\right) \delta=7.32-7.27(\mathrm{~m}, 2 \mathrm{H}), 7.23-7.17(\mathrm{~m}, 3 \mathrm{H}), 3.68(\mathrm{t}, J=6.4 \mathrm{~Hz}, 2 \mathrm{H}), 2.76-$ $2.67(\mathrm{~m}, 2 \mathrm{H}), 2.00-1.80(\mathrm{~m}, 2 \mathrm{H}), 1.41-1.34(\mathrm{~s}, 1 \mathrm{H}) \mathrm{ppm}$. 
Calculation of the conversion:

Mass (internal standard): $m_{\text {is }}=0.010 \mathrm{~g}$

Amount of substance (internal standard): $\mathrm{n}_{\text {is }}=0.060 \mathrm{mmol}$

${ }^{1} \mathrm{H}-\mathrm{NMR}$ integration (internal standard): $\mathrm{l}_{\text {is }}=3.00 \mathrm{H}$

Integration (reduced starting material (3.52 ppm)): $I_{\text {rsm }}=1.85 \mathrm{H}: 2=0.93 \mathrm{H}$

Integration (reduced starting material $(1.78 \mathrm{ppm})$ ): $\operatorname{Irsm}_{\mathrm{rsm}}=1.85 \mathrm{H}: 2=0.93 \mathrm{H}$

Amount of substance (reduced starting material): $n_{\mathrm{sm}}=0.060 \mathrm{mmol}^{*} 0.93{ }^{*} 50=2.767 \mathrm{mmol}$

Amount of substance (starting material): $\mathrm{n}_{\mathrm{sm}}=12.00 \mathrm{mmol}-(12.00 \mathrm{mmol} / 50)=11.76 \mathrm{mmol}$

(1/50 of the volume was removed before reductive work-up for H-NMR-analysis!)

Conversion: $2.767 \mathrm{mmol} / 11.76 \mathrm{mmol}-1){ }^{*}(-100)=77 \%$

KIE1 $=77 \%$ conversion

KIE2 $=77 \%$ conversion<smiles>COc1ccc(OC)cc1</smiles>

internal standard $\delta=6.10 \mathrm{ppm}\left(\mathrm{MeCNd}_{3}\right)$

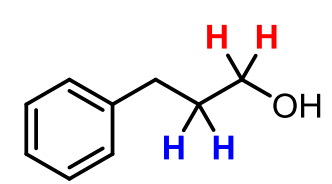

reduced starting material

$\delta=3.52 \mathrm{ppm}\left(\mathrm{MeCNd}_{3}\right)$

$\delta=1.78 \mathrm{ppm}\left(\mathrm{MeCNd}_{3}\right)$

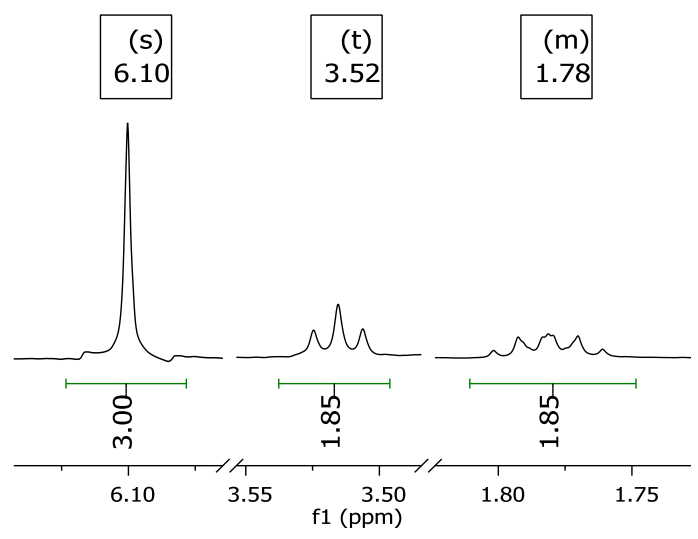

Figure SI-11. Cutout from the ${ }^{1} \mathrm{H}-\mathrm{NMR}$ spectrum of the quenched reaction (for determination of the conversion).

Experimental ${ }^{13} \mathrm{C}$ KIEs for the system were determined from analysis of starting material 3-phenylpropanal using NMR methodology at natural abundance. Two independent reactions of starting aldehyde were taken to $77 \pm 2$ conversion. The unreacted 3-phenylpropanal was reduced in situ to 3-phenylpropanol and reisolated for NMR analysis. The reisolated samples KIE1 and KIE2 were compared to two separate standard samples STD1 and STD2, respectively. The standard samples were prepared by reducing 3phenylpropanal taken from the same batch used for the KIE experiments. Experimental ${ }^{13} \mathrm{C}$ KIEs were measured from relative isotopic composition of the two samples and fractional conversion in a standard way. The isotope effects were then averaged, and a 95\% confidence range (C) was calculated using standard method.

$$
C=\frac{t_{p, v} S}{\sqrt{n}}
$$

$\mathbf{S}$ is the standard deviation, $\mathbf{n}$ is the number of measurements, and $\mathbf{t}_{\mathbf{p}, \mathbf{v}}$ is distribution function corresponding to confidence level $\mathbf{n}-1$. 


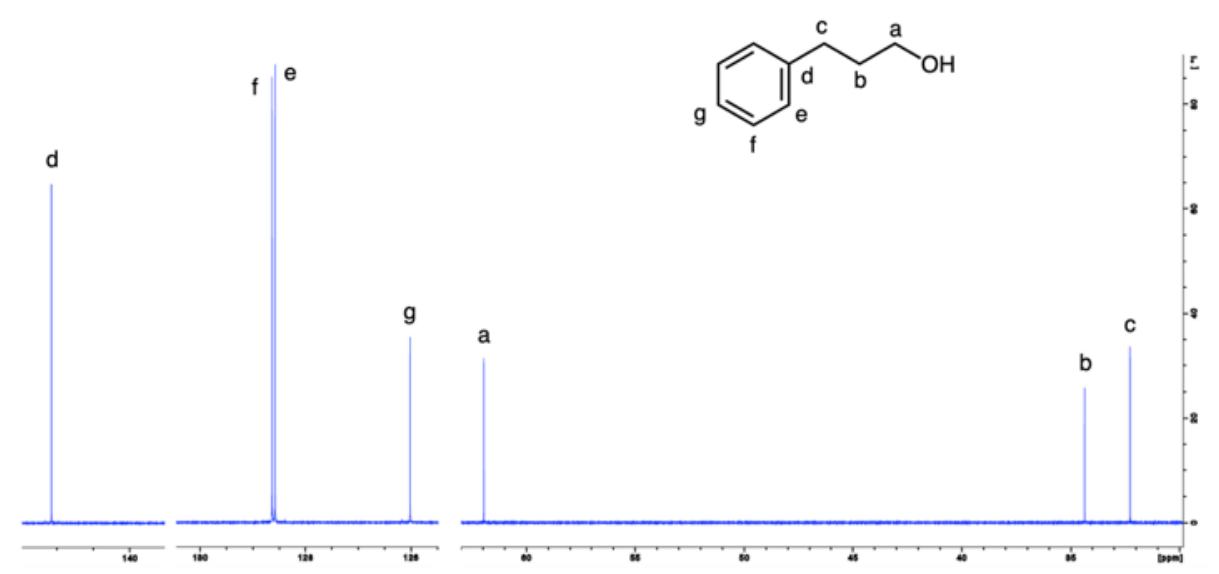

Figure SI-12. ${ }^{13} \mathrm{C}-\mathrm{NMR}$ spectrum of the reduced and reisolated starting material (3-phenylpropanol).

\section{STD1/KIE1}

The sample for determination of ${ }^{13} \mathrm{C}$ KIEs was prepared from $160 \mathrm{mg}$ of the reisolated sample KIE1, which was quantitatively transferred to a new $5 \mathrm{~mm}$ NMR tube using HPLC grade deuterated chloroform $\left(\mathrm{CDCl}_{3}\right)$ to a height of $5 \mathrm{~cm}$. Likewise, $160 \mathrm{mg}$ of standard sample STD1 was also prepared quantitatively in a $5 \mathrm{~mm}$ NMR tube using HPLC grade deuterated chloroform $\left(\mathrm{CDCl}_{3}\right)$ to a height of $5 \mathrm{~cm}$. For quantitative measurement, ${ }^{13} \mathrm{C}$ spectra were recorded at $150 \mathrm{MHz}$ using inverse gated decoupling with an acquisition time of $10 \mathrm{~s}$, delay time $36 \mathrm{~s}$ (5 times T1 of slowest relaxing peak) and 128 transients for acquiring 6 fids for both the samples. A constant integration area was defined for each individual peak which is equal to 10 times the peak-widths at half-height for each peak. The para carbon of 3-phenylpropanol was taken as the standard carbon which was set to an integration value of 1000.0000. All the integrals for STD1 and KIE1 are listed in the tables below.

\section{STD2/KIE2}

Similar to the procedure described above, samples for KIE2 and STD2 were each prepared using $268 \mathrm{mg}$. For quantitative measurement, ${ }^{13} \mathrm{C}$ spectra were recorded at $150 \mathrm{MHz}$ using inverse gated decoupling with an acquisition time of $10 \mathrm{~s}$, delay time $30 \mathrm{~s}$ ( 5 times T1 of slowest relaxing peak) and 128 transients for acquiring 6 fids for both the samples. The spectra were processed as described for the above sample. All the integrals for STD2 and KIE2 are listed in the tables below.

Table SI-6. Standard Sample (STD1 160mg/CDCl 3 ).

\begin{tabular}{|c|c|c|c|c|c|c|c|c|c|}
\hline peak & fid1 & fid2 & fid3 & fid4 & fid5 & fid6 & average & stddev & $\begin{array}{l}\text { cuts }=10 \quad X \\
\text { avg hh }\end{array}$ \\
\hline 1 & 1000.9305 & 1003.0809 & 1002.0465 & 1009.6958 & 996.4917 & 1006.0323 & 1003.0463 & 4.5072 & $1.5,1.5$ \\
\hline 2 & 2025.1360 & 2035.2550 & 2018.4216 & 2036.3256 & 2025.5118 & 2036.9713 & 2029.6036 & 7.6570 & 2,2 \\
\hline 3 & 2072.8509 & 2068.8465 & 2075.8805 & 2064.7618 & 2080.6641 & 2067.4642 & 2071.7447 & 5.8884 & 2,2 \\
\hline 4 & 1000.0000 & 1000.0000 & 1000.0000 & 1000.0000 & 1000.0000 & 1000.0000 & 1000.0000 & 0.0000 & 2,2 \\
\hline 5 & 1025.5784 & 1026.0890 & 1024.6846 & 1025.4123 & 1027.1945 & 1023.1769 & 1025.3560 & 1.3542 & $3.5,3.5$ \\
\hline 6 & 1041.1890 & 1042.1708 & 1038.5628 & 1038.6082 & 1040.9270 & 1040.9304 & 1040.3980 & 1.4767 & 4,4 \\
\hline 7 & 996.7377 & 999.2351 & 996.4748 & 1000.5757 & 996.1794 & 1000.3555 & 998.2597 & 2.0267 & 3,3 \\
\hline
\end{tabular}


Table SI-7. Reaction taken to $77 \%$ conversion (KIE1 160mg/CDCl 3 ).

\begin{tabular}{|c|c|c|c|c|c|c|c|c|c|c|}
\hline peak & fid1 & fid2 & fid3 & fid4 & fid5 & fid6 & average & stddev & KIE & $\begin{array}{l}95 \% \\
\text { conf }\end{array}$ \\
\hline 1 & 1013.6563 & 1013.2420 & 1013.8523 & 1014.0950 & 1013.5953 & 1012.7271 & 1013.5280 & 0.4839 & 1.007 & 0.004 \\
\hline 2 & 2024.8861 & 2037.0148 & 2029.6127 & 2040.8483 & 2026.1498 & 2044.3620 & 2033.8123 & 8.0881 & 1.001 & 0.005 \\
\hline 3 & 2076.6163 & 2074.6922 & 2075.3353 & 2057.5687 & 2074.9792 & 2062.1561 & 2070.2246 & 8.1830 & 1.000 & 0.004 \\
\hline 4 & 1000.0000 & 1000.0000 & 1000.0000 & 1000.0000 & 1000.0000 & 1000.0000 & 1000.0000 & 0.0000 & 1.000 & 0.000 \\
\hline 5 & 1050.1213 & 1052.8462 & 1055.0797 & 1047.5008 & 1052.0531 & 1050.8890 & 1051.4150 & 2.5751 & 1.017 & 0.002 \\
\hline 6 & 1044.5877 & 1050.5003 & 1049.9389 & 1044.8451 & 1042.4711 & 1047.6090 & 1046.6587 & 3.2104 & 1.004 & 0.003 \\
\hline 7 & 992.6145 & 992.3526 & 990.7697 & 985.5134 & 992.9584 & 985.9063 & 990.0192 & 3.4235 & 0.994 & 0.003 \\
\hline
\end{tabular}

Table SI-8. Standard Sample (STD2 268mg/CDCl 3 ).

\begin{tabular}{|l|l|l|l|l|l|l|l|l|l|}
\hline peak & fid1 & fid2 & fid3 & fid4 & fid5 & fid6 & average & stddev $\begin{array}{l}\text { cuts=10 } \\
\text { avg hh }\end{array}$ \\
\hline 1 & 1013.1913 & 1015.2475 & 1017.2836 & 1012.6850 & 1014.4221 & 1016.6279 & 1014.9096 & 1.8353 & $2.5,2.5$ \\
\hline 2 & 2057.1301 & 2050.2951 & 2057.6368 & 2052.1572 & 2051.3216 & 2060.7208 & 2054.8769 & 4.1923 & 4,4 \\
\hline 3 & 2100.1524 & 2100.3305 & 2098.9928 & 2101.5752 & 2103.3006 & 2091.8123 & 2099.3606 & 3.9776 & 4,4 \\
\hline 4 & 1000.0000 & 1000.0000 & 1000.0000 & 1000.0000 & 1000.0000 & 1000.0000 & 1000.0000 & 0.0000 & 4,4 \\
\hline 5 & 1015.9678 & 1014.7441 & 1015.3965 & 1015.7316 & 1014.9892 & 1014.8254 & 1015.2758 & 0.5038 & 5,5 \\
\hline 6 & 1032.2505 & 1032.3731 & 1034.0529 & 1031.8139 & 1032.2724 & 1031.1377 & 1032.3168 & 0.9661 & 6,6 \\
\hline 7 & 988.9633 & 988.2676 & 989.7361 & 987.9015 & 989.4858 & 988.0125 & 988.7278 & 0.7814 & 4,4 \\
\hline
\end{tabular}

Table SI-9. Reaction taken to $77 \%$ conversion (KIE2 268mg/CDCl 3 ).

\begin{tabular}{|c|c|c|c|c|c|c|c|c|c|c|}
\hline peak & fid 1 & fid2 & fid3 & fid4 & fid5 & fid6 & average & stddev & KIE & $\begin{array}{l}95 \% \\
\text { conf }\end{array}$ \\
\hline 1 & 1022.9126 & 1022.9606 & 1021.5724 & 1024.8493 & 1020.9465 & 1021.8850 & 1022.5211 & 1.3821 & 1.005 & 0.002 \\
\hline 2 & 2060.4713 & 2063.4748 & 2057.1022 & 2056.2644 & 2063.5671 & 2060.5035 & 2060.2306 & 3.0759 & 1.002 & 0.002 \\
\hline 3 & 2102.8840 & 2094.4883 & 2099.5798 & 2104.5966 & 2095.5660 & 2096.5294 & 2098.9407 & 4.1225 & 1.000 & 0.002 \\
\hline 4 & 1000.0000 & 1000.0000 & 1000.0000 & 1000.0000 & 1000.0000 & 1000.0000 & 1000.0000 & 0.0000 & 1.000 & 0.000 \\
\hline 5 & 1037.2121 & 1038.4920 & 1038.6580 & 1040.5217 & 1039.7760 & 1038.1707 & 1038.8051 & 1.1789 & 1.016 & 0.001 \\
\hline 6 & 1035.0012 & 1035.9482 & 1036.6547 & 1035.4925 & 1036.0883 & 1034.6687 & 1035.6423 & 0.7347 & 1.002 & 0.001 \\
\hline 7 & 991.4310 & 989.5594 & 988.8080 & 992.5506 & 991.0334 & 989.8827 & 990.5442 & 1.3765 & 1.001 & 0.001 \\
\hline
\end{tabular}




\section{KIE-experiment (2)}
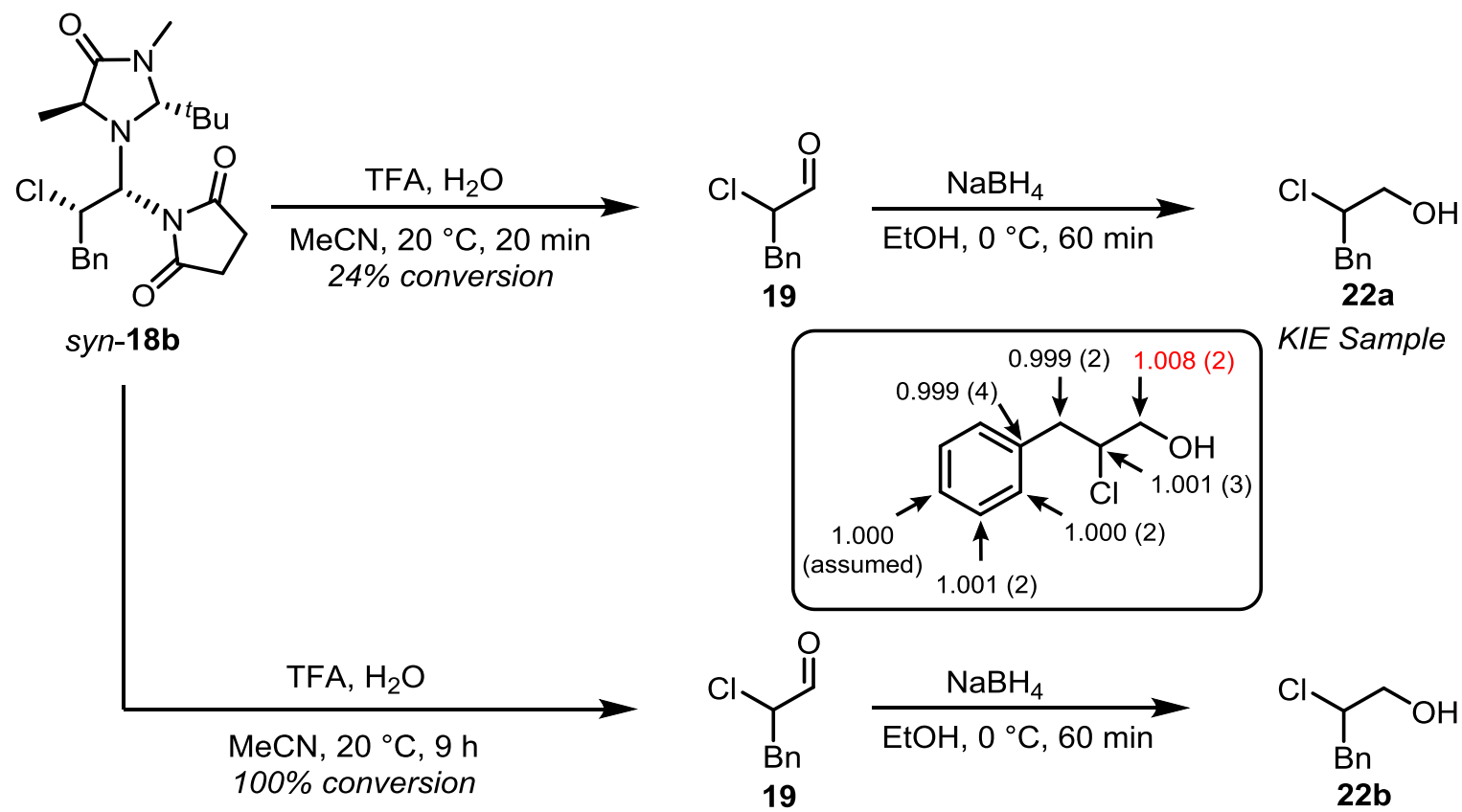

Standard sample

KIE-Sample: Aminal syn-18b was synthesized according to the literature procedure. ${ }^{22}$ The exact time (20 min) and suitable conditions (equivalents of $\mathrm{H}_{2} \mathrm{O}$ and TFA, concentration) for $20-30 \%$ conversion were defined by a previously performed kinetic ${ }^{1} \mathrm{H}$-NMR-experiment using 1,3,5-trimethoxybenzene as an internal standard. Aminal syn-18b (3.36 g, 8.00 mmol, 1.0 equiv) was dissolved in MeCN (200 mL), $\mathrm{H}_{2} \mathrm{O}$ ( $0.722 \mathrm{~mL}, 40.0 \mathrm{mmol}, 5.0$ equiv) and TFA (3.06 mL, $40.0 \mathrm{mmol}, 5.0$ equiv) were added subsequently and the solution was stirred for $20 \mathrm{~min}$ at $20{ }^{\circ} \mathrm{C}$. After quenching with saturated aqueous $\mathrm{NaHCO}_{3}(200 \mathrm{~mL})$, the aqueous phase was extracted with EtOAc $(3 \times 200 \mathrm{~mL})$ three times. The combined organic phases were dried over $\mathrm{NaSO}_{4}$ and the exact volume of the organic phase was adjusted to $1200 \mathrm{ml} .12 \mathrm{~mL}$ were taken from the solution and added to flask containing 1,3,5-trimethoxybenzene (6.73 mg, $40.0 \mu \mathrm{mol}$ ) as an internal standard. The solvents were removed under reduced pressure and the residue was taken up by $1.0 \mathrm{~mL}$ of $\mathrm{MeCNd}_{3}$. 24\% conversion was calculated from the ${ }^{1} \mathrm{H}-\mathrm{NMR}$ spectra. The solvents from the combined organic phases were removed under reduced pressure and the product was obtained after column chromatography (5:1 pentane/EtOAc). The pure a-chloro aldehyde $(0.434 \mathrm{~g}, 2.57 \mathrm{mmol}, 1.0 \mathrm{equiv})$ was dissolved in $\mathrm{EtOH}(20.0 \mathrm{~mL})$ and cooled to $0{ }^{\circ} \mathrm{C} . \mathrm{NaBH}_{4}(0.243 \mathrm{~g}, 6.43 \mathrm{mmol}, 2.5$ equiv) was added and the reaction mixture was stirred for $60 \mathrm{~min}$ at the same temperature. After quenching with saturated aqueous $\mathrm{NH}_{4} \mathrm{Cl}$, the aqueous phase was extracted with EtOAc $(3 \times 20 \mathrm{~mL})$. The combined organic phases were dried over $\mathrm{NaSO}_{4}$ and the solvents were removed under reduced pressure. The pure $\beta$-chloroalcohol $(0.350 \mathrm{~g}, 2.05 \mathrm{mmol}, 80 \%)$ was obtained after column chromatography (5:1 pentane/EtOAc).

Standard Sample: Aminal syn-18b (from the same batch as KIE-experiment) $(0.840 \mathrm{mg}, 2.00 \mathrm{mmol}$, 1.0 equiv) was dissolved in $\mathrm{MeCN}(50 \mathrm{~mL}), \mathrm{H}_{2} \mathrm{O}(0.180 \mathrm{~mL})$ and TFA $(0.765 \mathrm{~mL})$ were added subsequently and the solution was stirred at $20^{\circ} \mathrm{C}$ until TLC showed complete conversion of the aminal (9 h). After quenching with saturated aqueous $\mathrm{NaHCO}_{3}(40 \mathrm{~mL})$, the aqueous phase was extracted with $\mathrm{EtOAc}(3 \times 50$ $\mathrm{mL}$ ). The combined organic phases were dried over $\mathrm{NaSO}_{4}$ and the solvents were removed under reduced pressure. ${ }^{1} \mathrm{H}-\mathrm{NMR}$ of the crude sample confirmed complete conversion of the aminal. The product was obtained after column chromatography (5:1 pentane/EtOAc). The pure a-chloro aldehyde $(0.301 \mathrm{~g}$, $1.79 \mathrm{mmol}, 1.0$ equiv) was dissolved in $\mathrm{EtOH}(15 \mathrm{~mL})$ and cooled to $0{ }^{\circ} \mathrm{C}$. $\mathrm{NaBH}_{4}(0.169 \mathrm{~g}, 4.46 \mathrm{mmol}$, 2.5 equiv) was added and the reaction mixture was stirred for $60 \mathrm{~min}$ at the same temperature. After quenching with saturated aqueous $\mathrm{NH}_{4} \mathrm{Cl}(10 \mathrm{~mL})$, the aqueous phase was extracted with EtOAc 
$(3 \times 20 \mathrm{~mL})$. The combined organic phases were dried over $\mathrm{NaSO}_{4}$ and the solvents were removed under reduced pressure. The pure $\beta$-chloro alcohol $(0.240 \mathrm{~g}, 1.41 \mathrm{mmol}, 79 \%)$ was obtained after column chromatography (5:1 pentane/EtOAc).

${ }^{1} \mathrm{H}-\mathrm{NMRs}$ are in accordance with the literature. ${ }^{22}$

Product KIEs were performed by isolating the product 2-chloro-3-phenylpropanal from a reaction of synaminal (syn-18b) taken to $24 \%$ conversion and reduced insitu to 2-chloro-3-phenylpropanol. The standard sample was prepared by isolating the product taken to $100 \%$ conversion and reduced in situ Experimental ${ }^{13} \mathrm{C}$ KIEs were measured from changes in relative isotopic composition and fractional conversion.

\section{STD/KIE}

Similar to the procedure described for determination of ${ }^{13} \mathrm{C} \mathrm{KIEs} \mathrm{for} \mathrm{3-phenylpropananol,} \mathrm{low} \mathrm{conversion}$ and $100 \%$ conversion samples of 2-chloro-3-phenylpropanol were each prepared using $231 \mathrm{mg}$. For quantitative measurement, ${ }^{13} \mathrm{C}$ spectra were recorded at $150 \mathrm{MHz}$ using inverse gated decoupling with an acquisition time of $10 \mathrm{~s}$, delay time $30 \mathrm{~s}$ (5 times T1 of slowest relaxing peak) and 128 transients for acquiring 6 fids for both the samples. The spectra were processed as described for the previous samples. A representative spectrum along with integration for both samples are shown in the table below. This experiment was not performed in duplicate due the challenges associated with synthesizing a larger quantities of syn-18b required for the experiment.

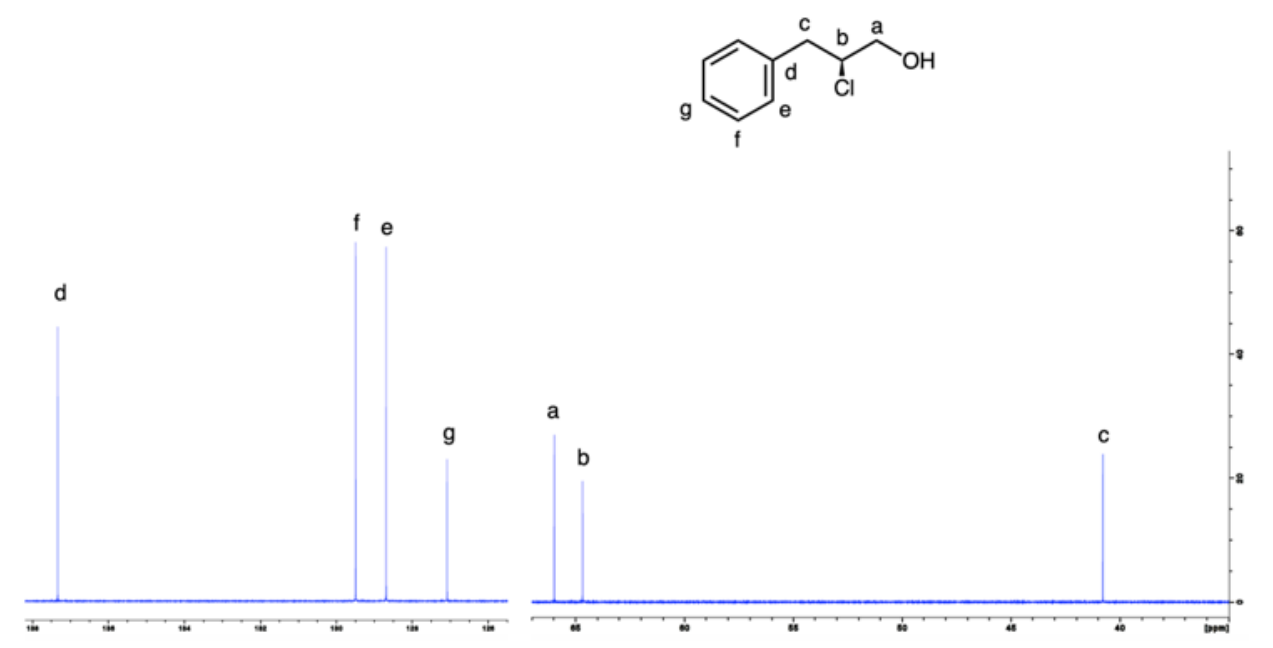

Figure SI-13. ${ }^{13} \mathrm{C}-\mathrm{NMR}$ spectrum of the reduced decomposition product of syn-18b (2-chloro-3phenylpropanol).

Table SI-10. Standard Sample (STD 231mg/CDCl 3 ).

\begin{tabular}{|l|l|l|l|l|l|l|l|l|l|l|}
\hline peak & fid1 & fid2 & fid3 & fid4 & fid5 & fid6 & average & stddev & $\begin{array}{l}\text { cuts } \\
\text { hh }\end{array}$ \\
\hline 1 & 1026.0648 & 1026.8439 & 1026.4918 & 1024.7271 & 1028.6749 & 1025.2016 & 1026.3340 & 1.3930 & 2,2 \\
\hline 2 & 2002.9656 & 2004.9762 & 2001.9009 & 2000.6530 & 2004.8393 & 2002.2518 & 2002.9311 & 1.7051 & $2.5,2.5$ \\
\hline 3 & 1999.7604 & 2000.0493 & 2000.2890 & 1998.7059 & 1999.6764 & 1997.1237 & 1999.2675 & 1.1812 & $2.5,2.5$ \\
\hline 4 & 1000.0000 & 1000.0000 & 1000.0000 & 1000.0000 & 1000.0000 & 1000.0000 & 1000.0000 & 0.0000 & 3,3 & \\
\hline 5 & 994.7805 & 991.0943 & 994.3898 & 995.1336 & 991.5581 & 992.6222 & 993.2631 & 1.7374 & 3,3 \\
\hline 6 & 1046.7547 & 1043.5144 & 1045.1485 & 1037.3534 & 1045.1662 & 1045.3339 & 1043.8785 & 3.3577 & 10,10 \\
\hline 7 & 997.2385 & 998.3051 & 1000.9168 & 999.3783 & 998.0785 & 996.2951 & 998.3687 & 1.6234 & 4,4 \\
\hline
\end{tabular}


Table SI-11. Reaction taken to $24 \%$ conversion (KIE $231 \mathrm{mg} / \mathrm{CDCl}_{3}$ ).

\begin{tabular}{|l|l|l|l|l|l|l|l|l|l|l|}
\hline peak & fid1 & fid2 & fid3 & fid4 & fid5 & fid6 & average & stddev & KIE & $\begin{array}{l}\mathbf{9 5 \%} \\
\text { conf }\end{array}$ \\
\hline 1 & 1021.2407 & 1023.3972 & 1024.4986 & 1027.7109 & 1033.4467 & 1030.6276 & 1026.8203 & 4.6355 & $\mathbf{0 . 9 9 9}$ & $\mathbf{0 . 0 0 4}$ \\
\hline $\mathbf{2}$ & 1997.0448 & 2001.1775 & 2004.2681 & 2004.6367 & 2011.8553 & 2003.1204 & 2003.6838 & 4.8723 & $\mathbf{1 . 0 0 0}$ & $\mathbf{0 . 0 0 2}$ \\
\hline $\mathbf{3}$ & 1994.1101 & 1997.2838 & 1999.9637 & 1994.5238 & 2005.8038 & 1998.4097 & 1998.3492 & 4.2877 & $\mathbf{1 . 0 0 1}$ & $\mathbf{0 . 0 0 2}$ \\
\hline $\mathbf{4}$ & 1000.0000 & 1000.0000 & 1000.0000 & 1000.0000 & 1000.0000 & 1000.0000 & 1000.0000 & 0.0000 & $\mathbf{1 . 0 0 0}$ & $\mathbf{0 . 0 0 0}$ \\
\hline $\mathbf{5}$ & 984.5027 & 985.8564 & 986.8568 & 986.6361 & 988.6683 & 987.7684 & 986.7148 & 1.4548 & $\mathbf{1 . 0 0 8}$ & $\mathbf{0 . 0 0 2}$ \\
\hline $\mathbf{6}$ & 1043.8129 & 1042.6836 & 1040.2764 & 1043.1687 & 1045.8196 & 1043.1081 & 1043.1449 & 1.7913 & $\mathbf{1 . 0 0 1}$ & $\mathbf{0 . 0 0 3}$ \\
\hline $\mathbf{7}$ & 997.5046 & 998.9728 & 1000.1525 & 999.9749 & 1002.1993 & 998.2256 & 999.5050 & 1.6626 & $\mathbf{0 . 9 9 9}$ & $\mathbf{0 . 0 0 2}$ \\
\hline & & & & & & & & & &
\end{tabular}




\section{Theoretical Studies}

General Information:

All the DFT calculations presented in the manuscript were performed using B3LYP/6-31+G**PCM (solvent $=$ acetonitrile) level of theory as implemented in Gaussian16. All the starting materials and intermediates were verified as true minima (no imaginary frequencies) whereas all the transition states for the system were characterized by one imaginary frequency. Single point energy calculations were performed separately using B3LYP functional with Grimme's dispersion correction with Becke-Johnson damping (D3BJ) and a triple- $\zeta$ basis set $\left(6-311+G^{* *}\right)$ and a PCM solvent model for acetonitrile was also applied to all single point geometry optimizations. Gibbs free energies were estimated at reaction temperature $243 \mathrm{~K}$ and were corrected using Grimme's quasi-RRHO approach. Each intermediate along the reaction coordinate were located by performing Paton's quick reaction coordinate ( $Q R C)$ procedure from the respective transition states. A thorough conformation search was done for all the optimized transition state and intermediate geometries to identify the lowest energy geometry for each stationary point. Cartesian coordinates of all computed starting materials, intermediates, and transition states are included in the section below. The lowest energy conformation for each transition state reported in the manuscript is highlighted in red. The higher energy conformations for each transition state are also included along with their relative energies.

\section{Exploring the origins of enantioselectivity:}

From the computed reaction coordinate diagram shown in Figure 3 of the manuscript, the chlorination of the enamine intermediate (TS4) is irreversible, and the enantioselectivity-determining step of the reaction. Accordingly, we modeled the approach of NCS (6) to either face of the two possible (E)-enamine intermediates. After a thorough conformational search, the lowest-energy TS for each distinct approach was identified. The approach of 6 from the Re-face of enamine that is syn-to the $t$-butyl group of the catalyst 3c was found to be the lowest-energy TS leading to the formation of the (S)-enantiomer of 19 (TS4-S1). In contrast, the TS for the approach of $\mathbf{6}$ to the Si-face of the enamine that is anti to the $t$-butyl group of $\mathbf{3 c}$ (TS4-R1) was identified as the lowest energy TS for the formation of the $(R)$-enantiomer of 19 . The $\Delta \Delta \mathrm{G}^{\ddagger}$ between these two TSs is $2.9 \mathrm{kcal} \mathrm{mol}^{-1}$ favoring TS4-S1 - a value that is qualitatively consistent with the $>90 \%$ ee observed for this reaction. 
TS4-S,

$\Delta \mathrm{G}^{\ddagger}=9.3 \mathrm{kcal} / \mathrm{mol}$

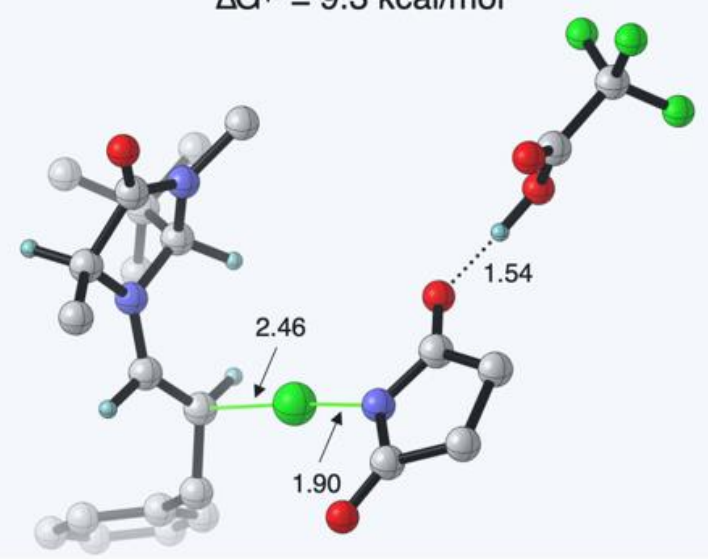

TS4- $R_{1}$

$\Delta \mathrm{G}^{\ddagger}=12.2 \mathrm{kcal} / \mathrm{mol}$

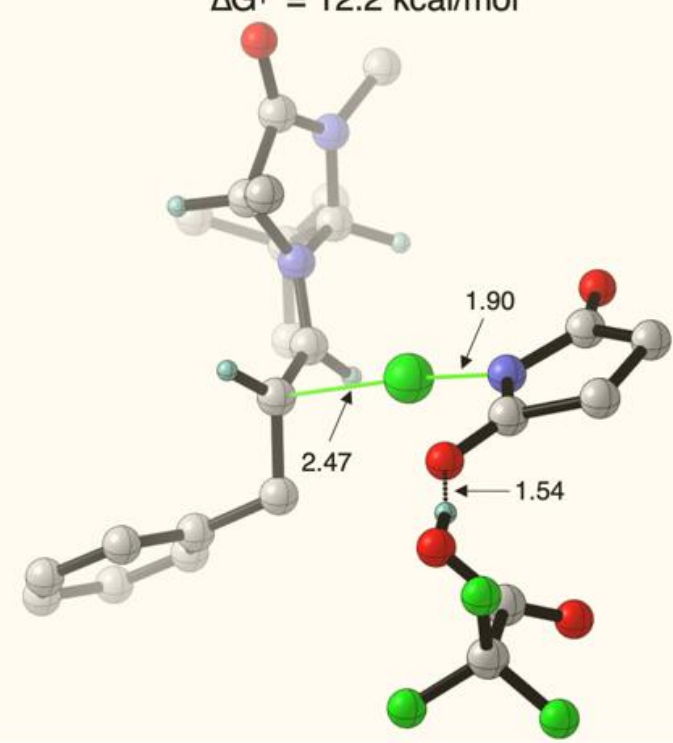

TS4-S,

$\Delta \mathrm{G}^{\ddagger}=10.7 \mathrm{kcal} / \mathrm{mol}$

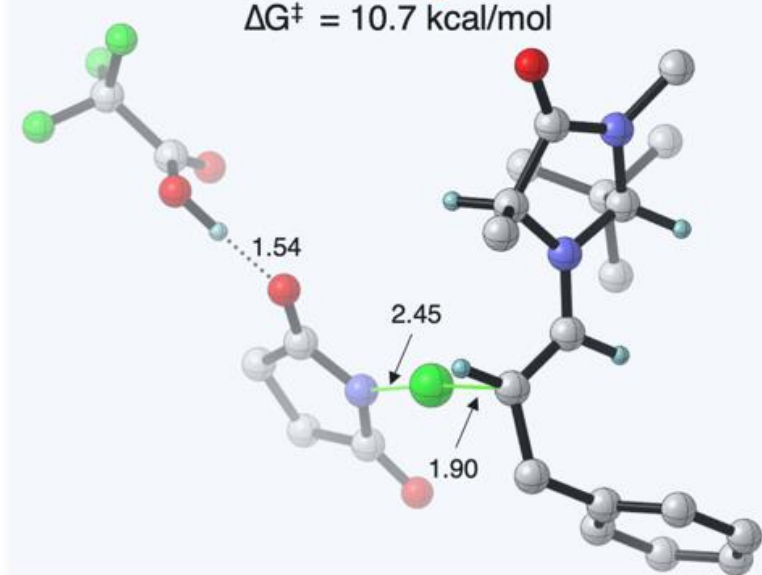

TS4- $R_{2}$ $\Delta \mathrm{G}^{\ddagger}=12.7 \mathrm{kcal} / \mathrm{mol}$

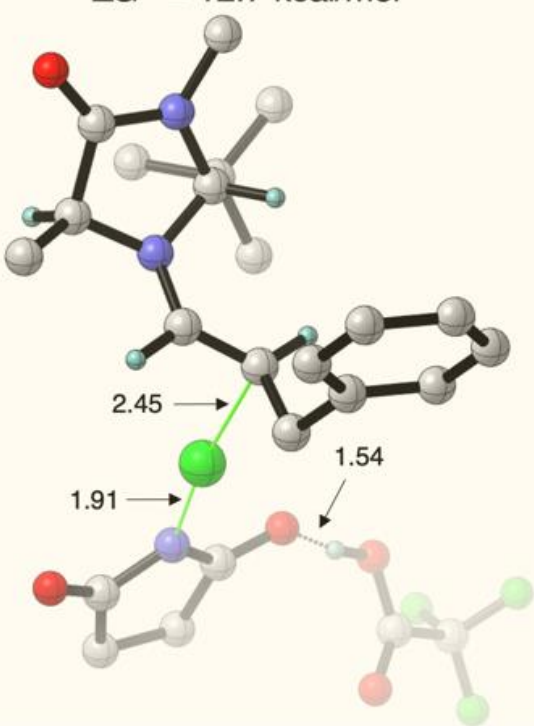

Figure SI-14. Lowest energy transition states for the front and back approach of 6 to the two distinct $(E)$ enamine intermediates calculated at the B3LYP-D3(BJ)/6-311+G** PCM(acetonitrile)// B3LYP/6-31+G** PCM(acetonitrile) level of theory. Some hydrogen atoms have been removed for clarity in the 3D representation of the key transition structures. Key bond-forming and bond-breaking distances are in angstroms $(\AA)$.

Computed pathways for the formation of all diastereomers of the aminal intermediate:

In the manuscript, we have described the formation of syn-18b and anti-18b from the Z-chloroiminium ion (Int4). We have also computed the formation of diastereomeric aminals (syn-18b' and anti-18b') from the E-chloroiminium ion with the same $S$-configuration at the $\alpha$-carbon (Int4'). The reaction coordinate diagram for the formation of all four aminal diastereomers from the respective enamine intermediates (Int3 and Int3') is shown in the Figure below. It is important to note that even though Int4' is lower in energy that Int4, the barrier for its formation from Int3' is $1.4 \mathrm{kcal} \mathrm{mol}^{-1}$ higher in energy than the barrier for formation of Int4 from Int3 (see Figure below). If Int4' forms, then it faces only a $6.2 \mathrm{kcal} \mathrm{mol}^{-1}$ barrier to form anti-18b' via $\mathrm{TS}_{\text {syn-18b}} \cdot$ In contrast, formation of syn-18b' from Int4' faces a much higher barrier of $17.2 \mathrm{kcal} \mathrm{mol}^{-1}$ via $\mathrm{TS}_{\text {anti-18b' }}$. 


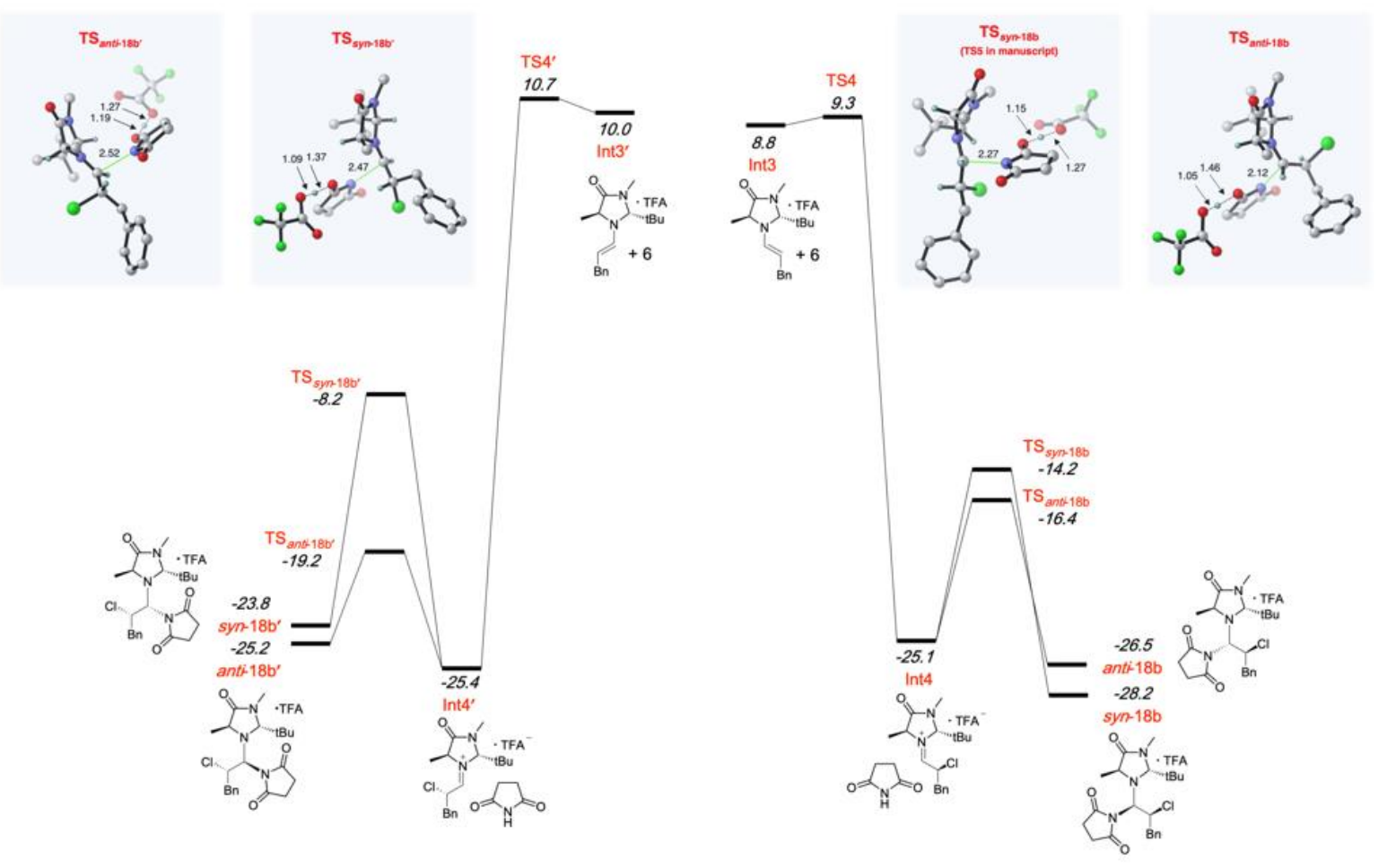

Figure SI-15. Reaction coordinate diagram for the formation of diastereomeric aminals from the respective enamines calculated at the B3LYP-D3(BJ)/6-311+G** $P C M($ acetonitrile)// B3LYP/6-31+G** $\mathrm{PCM}$ (acetonitrile) level of theory. Some hydrogen atoms have been removed for clarity in the 3D representation of the key transition structures. Key bond-forming and bond-breaking distances are in angstroms $(\AA)$.

In the manuscript, we show the pathway that converts Int4 to syn-18b and anti-18b via TS5 and TS5'. These transition structures are $\mathrm{TS}_{s y n-18 \mathrm{~b}}$ and $\mathrm{TS}_{a n t-18 \mathrm{~b}}$ respectively in the Figure above. We implied that syn18b and anti-18b re-entered the catalytic cycle as the Z-chloroiminium ion (Int4) via these same transition states (principle of microscopic reversibility). However, there is a possibility of a $\mathrm{C}-\mathrm{N}$ bond-rotation event that converts syn-18b to syn-18b' and anti-18b to anti-18b' and subsequent re-entry of these intermediates into the catalytic cycle as the E-chloroiminium intermediate (Int4') via TS $_{\text {syn-18b' }}$ and TS $_{a n t i 18 b^{\prime}}$. This possibility is unlikely for the syn-aminal since $\mathrm{TS}_{\text {syn-18b }}$ ' is significantly higher in energy that $\mathrm{TS}_{s y n-18 \mathbf{b}}$. On the other hand, such a pathway is more than likely for the anti-aminal since $\mathrm{TS}_{\text {anti-18b }}$ is lower in energy that

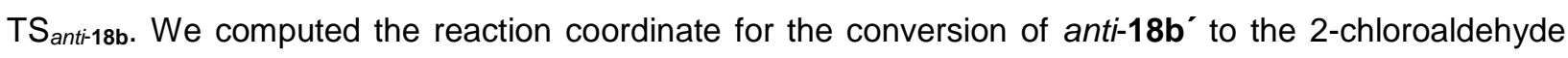
product via the three sequential transition states TS $_{a n t-18 b^{\prime}}$. TS6', and TS7 corresponding to re-entry into the catalytic cycle as the E-chloroiminium, hydrolysis, and catalyst regeneration and product release (Figure below). Incidentally, the highest energy barrier for this reaction pathway is re-entry into the catalytic cycle

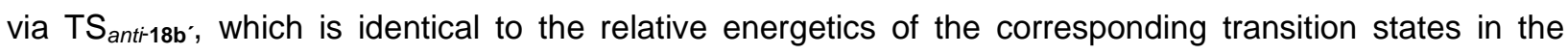
conversion of syn-18b to the product 19 described in Figure 4 of the manuscript. 


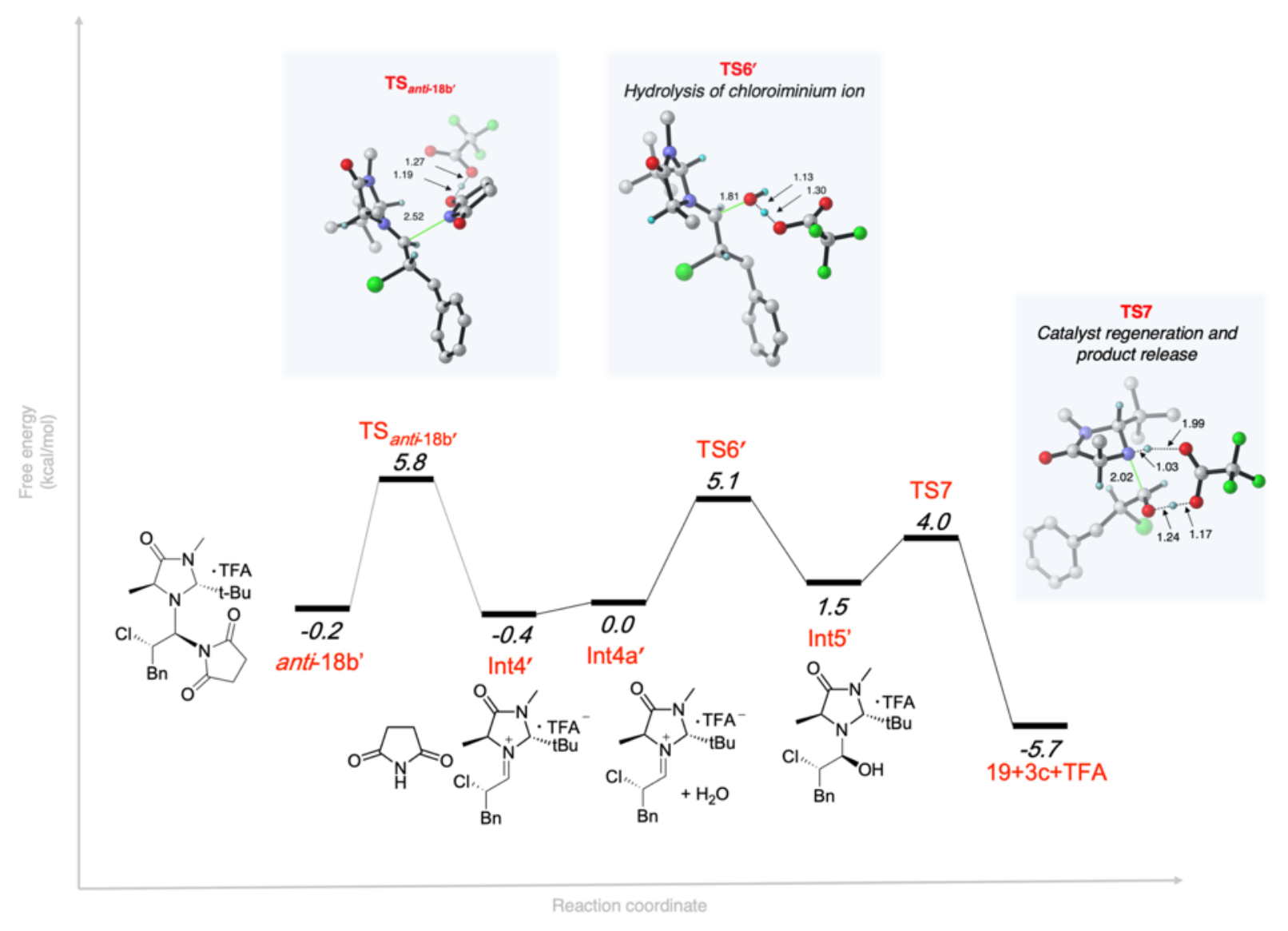

Figure SI-16. Reaction coordinate diagram for the conversion of anti-18b' to 19 calculated at the B3LYP$\mathrm{D} 3(\mathrm{BJ}) / 6-311+\mathrm{G}^{* *} \mathrm{PCM}$ (acetonitrile)// B3LYP/6-31+G** $\mathrm{PCM}$ (acetonitrile) level of theory. Some hydrogen atoms have been removed for clarity in the $3 \mathrm{D}$ representation of the key transition structures. Key bondforming and bond-breaking distances are in angstroms $(\AA)$. 
Table SI-12. Coordinates and energies of computed Trifluoroacetic acid (tfa.log) structures.

Free Energy $=-526.81566$

Zero-point Energy $=-526.79175$

Potential Energy $=-526.83013$

\section{Starting Materials}

3-Phenylpropanal (sm.log)

Free Energy $=-424.09683$

Zero-point Energy $=-424.07017$

Potential Energy $=-424.23595$

Single-Point Energy B3LYP-D3(BJ)/6-311+G** PCM = 424.35584

Free Energy B3LYP-D3(BJ)/6-311+G** PCM (extrapolated free energy from qRRHO) $=-424.21671$

Charge $=0$ Multiplicity $=1$

$\begin{array}{lrrr}\mathrm{C} & -3.3413160 & -0.0005570 & 0.5403040 \\ \mathrm{H} & -3.9288280 & -0.0002450 & 1.4807970 \\ \mathrm{C} & -1.8491150 & -0.0005340 & 0.7243600 \\ \mathrm{H} & -1.6071760 & 0.8704580 & 1.3516390 \\ \mathrm{H} & -1.6070840 & -0.8719850 & 1.3509560 \\ \mathrm{C} & -1.0313240 & 0.0000370 & -0.5758450 \\ \mathrm{H} & -1.3069810 & -0.8785130 & -1.1687130 \\ \mathrm{H} & -1.3071090 & 0.8790120 & -1.1680190 \\ \mathrm{C} & 0.4615430 & 0.0000500 & -0.3184150 \\ \mathrm{C} & 1.1687220 & 1.2054270 & -0.1841310 \\ \mathrm{C} & 1.1688060 & -1.2053300 & -0.1845390 \\ \mathrm{C} & 2.5419580 & 1.2082530 & 0.0802440 \\ \mathrm{H} & 0.6406430 & 2.149560 & -0.2924210 \\ \mathrm{C} & 2.5420360 & -1.2081510 & 0.0798330 \\ \mathrm{H} & 0.6407890 & -2.1498560 & -0.2931560 \\ \mathrm{C} & 3.2340200 & 0.0000540 & 0.2141230 \\ \mathrm{H} & 3.0706490 & 2.1524890 & 0.1765460 \\ \mathrm{H} & 3.0707990 & -2.1523810 & 0.1758140 \\ \mathrm{H} & 4.3012090 & 0.0000500 & 0.4160980 \\ \mathrm{O} & -3.9173610 & 0.0006900 & -0.5356430\end{array}$

Catalyst (cat.log)

Free Energy $=-538.35806$

Zero-point Energy $=-538.32923$

Potential Energy $=-538.59681$

Single-Point Energy B3LYP-D3(BJ)/6-311+G** PCM = -

538.76234

Free Energy B3LYP-D3(BJ)/6-311+G** PCM (extrapolated free energy from $\mathrm{qRRHO})=-538.52359$

Charge $=0$ Multiplicity $=1$

$\begin{array}{lrrr}\mathrm{C} & -0.4544180 & -0.1166990 & -0.6994830 \\ \mathrm{~N} & 0.4266560 & 1.0008460 & -0.2914750 \\ \mathrm{C} & 1.5852240 & 0.5777350 & 0.2652530 \\ \mathrm{O} & 2.4887570 & 1.3031530 & 0.7022350 \\ \mathrm{C} & 1.5745020 & -0.9521890 & 0.2523210 \\ \mathrm{~N} & 0.4181660 & -1.3045010 & -0.5947510 \\ \mathrm{H} & 0.7590160 & -1.5344140 & -1.5245490 \\ \mathrm{C} & 2.8849960 & -1.5589870 & -0.2398010 \\ \mathrm{H} & 3.7062150 & -1.2645140 & 0.4186440 \\ \mathrm{H} & 2.8194820 & -2.6512640 & -0.2414640 \\ \mathrm{H} & 3.1204180 & -1.2213170 & -1.2554950 \\ \mathrm{H} & 1.3979930 & -1.2887160 & 1.2835090 \\ \mathrm{C} & 0.1807200 & 2.4085020 & -0.5718670 \\ \mathrm{H} & -0.2985190 & 2.9212640 & 0.2681390 \\ \mathrm{H} & -0.4580440 & 2.4993570 & -1.4525720 \\ \mathrm{H} & 1.1386980 & 2.8949920 & -0.7695520 \\ \mathrm{H} & -0.7422380 & 0.0344340 & -1.7475790 \\ \mathrm{C} & -1.7730610 & -0.2694120 & 0.1258260 \\ \mathrm{C} & -2.6574980 & 0.9869790 & -0.0160930 \\ \mathrm{H} & -2.8146340 & 1.2546050 & -1.0678020 \\ \mathrm{H} & -3.6416030 & 0.7942700 & 0.4248400 \\ \mathrm{H} & -2.2356580 & 1.8533340 & 0.4996520 \\ \mathrm{C} & -2.5421370 & -1.4694220 & -0.4650670 \\ \mathrm{H} & -2.7842300 & -1.3035630 & -1.5222530 \\ \mathrm{H} & -3.4853780 & -1.6125740 & 0.0733430 \\ \mathrm{H} & -1.9560190 & -2.3888790 & -0.3889400 \\ \mathrm{C} & -1.4809210 & -0.5254740 & 1.6153320 \\ \mathrm{H} & -0.9261150 & -1.4578230 & 1.7573130 \\ \mathrm{H} & -2.4212300 & -0.6094630 & 2.1713200 \\ \mathrm{H} & -0.9064070 & 0.2944330 & 2.0606280 \\ & & & \end{array}$

Single-Point Energy B3LYP-D3(BJ)/6-311+G** PCM = 526.98128

Free Energy B3LYP-D3(BJ)/6-311+G** PCM (extrapolated free energy from qRRHO) $=-526.96680$ Charge $=0$ Multiplicity $=1$

$\begin{array}{lrrr}\text { C } & 0.9502640 & -0.1544060 & -0.0000050 \\ \mathrm{C} & -0.5942250 & 0.0015770 & 0.0000060 \\ \mathrm{O} & 1.4971790 & -1.2288920 & -0.0000170 \\ \mathrm{O} & 1.5236480 & 1.0442750 & -0.0000080 \\ \mathrm{H} & 2.4938330 & 0.9458880 & -0.0000090 \\ \mathrm{~F} & -1.0055400 & 0.6793790 & 1.0919440 \\ \mathrm{~F} & -1.1885270 & -1.1982590 & -0.0002140 \\ \mathrm{~F} & -1.0055640 & 0.6797710 & -1.0917070\end{array}$

Water (h2o.log)

Free Energy = -76.43466

Zero-point Energy = -76.42090

Potential Energy $=-76.44210$

Single-Point Energy B3LYP-D3(BJ)/6-311+G** PCM = 76.46677

Free Energy B3LYP-D3(BJ)/6-311+G** PCM (extrapolated free energy from $q R R H O)=-76.45933$

Charge $=0$ Multiplicity $=1$

$\begin{array}{rrrr}\mathrm{O} & -0.0000000 & -0.0000000 & 0.1177180 \\ \mathrm{H} & -0.0000000 & 0.7669030 & -0.4708720 \\ \mathrm{H} & -0.0000000 & -0.7669030 & -0.4708720\end{array}$

N-chlorosuccinimide (suc.log)

Free Energy $=-820.19336$

Zero-point Energy $=-820.16822$

Potential Energy = -820.24947

Single-Point Energy B3LYP-D3(BJ)/6-311+G** PCM = 820.38399

Free Energy B3LYP-D3(BJ)/6-311+G** PCM (extrapolated free energy from qRRHO) $=-820.32788$

Charge $=0$ Multiplicity $=1$

$\begin{array}{lrrr}\mathrm{C} & -0.4323230 & 1.1871590 & 0.0000130 \\ \mathrm{C} & -1.8907470 & 0.7700950 & 0.0001270 \\ \mathrm{C} & -1.8907470 & -0.7700950 & 0.0000880 \\ \mathrm{C} & -0.4323230 & -1.1871590 & -0.0000720 \\ \mathrm{~N} & 0.3190470 & -0.0000000 & -0.0000370 \\ \mathrm{H} & -2.3726500 & 1.1995430 & 0.8824860 \\ \mathrm{H} & -2.3728100 & -1.1995470 & -0.8821820 \\ \mathrm{H} & -2.3726010 & -1.1995720 & 0.8824610 \\ \mathrm{H} & -2.3727600 & 1.1995770 & -0.8821560 \\ \mathrm{O} & 0.0417140 & -2.3036950 & -0.0001500 \\ \mathrm{O} & 0.0417140 & 2.3036950 & 0.0001580 \\ \mathrm{Cl} & 2.0274650 & -0.0000000 & -0.0000790\end{array}$

Succinimide (suc-h.log)

Free Energy $=-360.63967$

Zero-point Energy $=-360.61626$

Potential Energy $=-360.70798$

Single-Point Energy B3LYP-D3(BJ)/6-311+G** PCM = 360.81019

Free Energy B3LYP-D3(BJ)/6-311+G** PCM (extrapolated free energy from qRRHO) $=-360.74188$ Charge $=0$ Multiplicity $=1$

$\begin{array}{rrrr}\mathrm{C} & -1.1670680 & 0.2088320 & -0.0006280 \\ \mathrm{C} & -0.7696220 & -1.2596600 & 0.0000790 \\ \mathrm{C} & 0.7696220 & -1.2596600 & 0.0000120 \\ \mathrm{C} & 1.1670680 & 0.2088320 & -0.0005610 \\ \mathrm{~N} & 0.0000000 & 0.9594370 & -0.0000830 \\ \mathrm{H} & -1.2020090 & -1.7408940 & 0.8816570 \\ \mathrm{H} & 1.2020600 & -1.7415660 & -0.8811660 \\ \mathrm{H} & 1.2020730 & -1.7409440 & 0.8815300 \\ \mathrm{H} & -1.2021240 & -1.7416150 & -0.8810400 \\ \mathrm{O} & 2.2926660 & 0.6803470 & 0.0002360 \\ \mathrm{O} & -2.2926660 & 0.6803470 & 0.0004760 \\ \mathrm{H} & 0.0000000 & 1.9733530 & 0.0004920\end{array}$


Intermediates

Int1.log

Potential Energy $=-1489.67565$

Zero-point Energy $=-1489.19554$

Free Energy $=-1489.23816$

Single-Point Energy B3LYP-D3(BJ)/6-311+G** PCM = 1490.13898

Free Energy B3LYP-D3(BJ)/6-311+G** PCM (extrapolated free energy from $q R R H O)=-1489.70148$

Charge $=0$ Multiplicity $=1$

\begin{tabular}{|c|c|c|}
\hline 1.9462360 & -0.4304000 & 1.7290860 \\
\hline 2.3898140 & -1.2580300 & \\
\hline 1.5830110 & .8318660 & \\
\hline 3.0381520 & 6197670 & \\
\hline 0.7674580 & 0.1622330 & 0.963375 \\
\hline 0.1653840 & .1293300 & \\
\hline-0.4028390 & 7375180 & 195131 \\
\hline .0625910 & 0.5568670 & -0.01193 \\
\hline-1.5240140 & 2.6278790 & 3692 \\
\hline-2.4769980 & 1.8901530 & \\
\hline-3.7216940 & 2.6623270 & -0552543 \\
\hline-3.3819940 & 3.4704220 & -1.58865 \\
\hline-4.7076170 & & 89162 \\
\hline-4.2547680 & & 13197 \\
\hline-2.5666420 & & \\
\hline 3.7589450 & & \\
\hline 3.77 & & \\
\hline 4.84 & & \\
\hline 3.39 & & \\
\hline 5.520 & & \\
\hline 5.1 & & \\
\hline 4.06 & & -0.8 \\
\hline & & \\
\hline 5.1 & & \\
\hline 6.3 & & \\
\hline & & -1 \\
\hline & & \\
\hline & & \\
\hline-0.3 & & \\
\hline-0.69 & & 2 \\
\hline & & \\
\hline 10 & & \\
\hline & & \\
\hline & & \\
\hline-2.47 & & 1.32381 \\
\hline & & $-0.475 t$ \\
\hline & & -1 \\
\hline & & \\
\hline & & \\
\hline & & \\
\hline 0.80 & & \\
\hline & & \\
\hline-0.34 & & \\
\hline & & \\
\hline & & \\
\hline & & \\
\hline & & \\
\hline & & \\
\hline & & \\
\hline 1.40 & & \\
\hline 0.6525450 & & -0.30899 \\
\hline & & \\
\hline & & \\
\hline-1.3324190 & -1.3964190 & 2.91241 \\
\hline-0.6244830 & -0.8030320 & 3.49102 \\
\hline & & \\
\hline & & \\
\hline 5058 & -0.26597 & \\
\hline
\end{tabular}

Int2.log

Potential Energy $=-1489.67471$

Zero-point Energy $=-1489.20052$

Free Energy $=-1489.24669$

Single-Point Energy B3LYP-D3(BJ)/6-311+G** PCM = 1490.13451
Free Energy B3LYP-D3(BJ)/6-311+G** PCM (extrapolated free energy from qRRHO) $=-1489.70649$ Charge $=0$ Multiplicity $=1$

\begin{tabular}{|c|c|c|c|}
\hline $\mathrm{C}$ & -0.9076670 & -1.4510240 & -0.3872190 \\
\hline $\mathrm{C}$ & -0.7053740 & -2.8638920 & 1.5019160 \\
\hline $\mathrm{C}$ & 0.7227300 & -2.6529040 & 0.9841170 \\
\hline $\mathrm{N}$ & 0.5181550 & -1.4692480 & 0.0906370 \\
\hline $\mathrm{N}$ & -1.5554800 & -2.1514540 & 0.7253680 \\
\hline $\mathrm{O}$ & -1.0033980 & -3.5500940 & 2.4755070 \\
\hline $\mathrm{C}$ & -2.9582150 & -1.9579010 & 1.0920940 \\
\hline $\mathrm{H}$ & -3.5989610 & -2.7370730 & 0.6714540 \\
\hline $\mathrm{H}$ & -3.0323810 & -1.9934810 & 2.1804440 \\
\hline $\mathrm{H}$ & -3.2843840 & -0.9790670 & 0.7399530 \\
\hline $\mathrm{C}$ & 1.3809230 & -0.5192300 & -0.0314960 \\
\hline $\mathrm{C}$ & 1.2192450 & 0.7867870 & -0.7149230 \\
\hline $\mathrm{C}$ & 2.4250840 & 1.1515040 & -1.6268150 \\
\hline $\mathrm{H}$ & 2.4994840 & 0.4246100 & -2.4409870 \\
\hline $\mathrm{H}$ & 2.1759710 & 2.1138820 & -2.0854520 \\
\hline $\mathrm{C}$ & 1.7463740 & -2.4673650 & 2.0931350 \\
\hline $\mathrm{H}$ & 1.6535160 & -3.3229180 & 2.7662070 \\
\hline $\mathrm{H}$ & 2.7672300 & -2.4599330 & 1.7041890 \\
\hline $\mathrm{H}$ & 1.5515820 & -1.5515990 & 2.6568910 \\
\hline $\mathrm{H}$ & 0.9956780 & -3.5047370 & 0.3536320 \\
\hline $\mathrm{C}$ & -1.1152890 & -2.1068240 & -1.7988310 \\
\hline $\mathrm{H}$ & -1.2533120 & -0.4145590 & -0.3978180 \\
\hline $\mathrm{C}$ & -2.6229500 & -2.0259360 & -2.1323180 \\
\hline $\mathrm{H}$ & -2.7730020 & -2.3302500 & -3.1725630 \\
\hline $\mathrm{H}$ & -3.2195590 & -2.6943650 & -1.5080200 \\
\hline $\mathrm{H}$ & -3.0105980 & -1.0074450 & -2.0241620 \\
\hline $\mathrm{C}$ & -0.3458890 & -1.3109870 & -2.8717890 \\
\hline $\mathrm{H}$ & -0.6860510 & -0.2724570 & -2.9338690 \\
\hline $\mathrm{H}$ & 0.7353230 & -1.3221330 & -2.7055950 \\
\hline $\mathrm{H}$ & -0.5233210 & -1.7697240 & -3.8490820 \\
\hline $\mathrm{C}$ & -0.6677540 & -3.5802940 & -1.8427280 \\
\hline $\mathrm{H}$ & -1.1401380 & -4.1777900 & -1.0567630 \\
\hline $\mathrm{H}$ & -0.9632340 & -4.0144800 & -2.8027090 \\
\hline $\mathrm{H}$ & 0.4190780 & -3.6853190 & -1.7664190 \\
\hline $\mathrm{H}$ & 1.1649890 & 1.5129720 & 0.1086330 \\
\hline $\mathrm{H}$ & 0.2856240 & 0.8612390 & -1.2682000 \\
\hline $\mathrm{C}$ & 3.7537620 & 1.2537760 & -0.9029550 \\
\hline C & 4.0146940 & 2.3280740 & -0.0361460 \\
\hline $\mathrm{C}$ & 4.7496640 & 0.2823880 & -1.0826630 \\
\hline $\mathrm{C}$ & 5.2355070 & 2.4255990 & 0.6357360 \\
\hline $\mathrm{H}$ & 3.2608890 & 3.0982630 & 0.1094100 \\
\hline $\mathrm{C}$ & 5.9742900 & 0.3767590 & -0.4120960 \\
\hline $\mathrm{H}$ & 4.5694440 & -0.5514930 & -1.7568160 \\
\hline $\mathrm{C}$ & 6.2199550 & 1.4481700 & 0.4506480 \\
\hline $\mathrm{H}$ & 5.4200860 & 3.2658580 & 1.2988600 \\
\hline $\mathrm{H}$ & 6.7333200 & -0.3846850 & -0.5667840 \\
\hline $\mathrm{H}$ & 7.1700720 & 1.5245520 & 0.9711210 \\
\hline $\mathrm{H}$ & 2.3367140 & -0.6964060 & 0.4541960 \\
\hline $\mathrm{O}$ & 0.2055980 & 0.6727320 & 2.5360000 \\
\hline $\mathrm{H}$ & -0.5650500 & 1.0245000 & 2.0250470 \\
\hline $\mathrm{H}$ & 0.3612240 & 1.3062950 & 3.2479440 \\
\hline $\mathrm{O}$ & -1.8272060 & 1.3783850 & 0.8585520 \\
\hline $\mathrm{C}$ & -2.4962880 & 2.4448910 & 0.9188010 \\
\hline $\mathrm{C}$ & -3.5454270 & 2.5697760 & -0.2410770 \\
\hline $\mathrm{O}$ & -2.4417150 & 3.3874580 & 1.7255190 \\
\hline $\mathrm{F}$ & -4.3615340 & 3.6413520 & -0.1226540 \\
\hline $\mathrm{F}$ & -4.3489880 & 1.4744090 & -0.3167630 \\
\hline & -2.9249700 & 2.6827500 & -1.4496620 \\
\hline
\end{tabular}

Int3.log

Potential Energy $=-2233.46906$

Zero-point Energy $=-2232.93826$

Free Energy $=-2232.98928$

Single-Point Energy B3LYP-D3(BJ)/6-311+G** PCM = 2234.04146

Free Energy B3LYP-D3(BJ)/6-311+G** PCM (extrapolated free energy from qRRHO) $=-2233.56168$

Charge $=0$ Multiplicity $=1$

$\begin{array}{llll}\mathrm{C} & -1.1664720 & 2.4657730 & -0.1544700 \\ \mathrm{C} & -0.8513740 & 2.2752420 & 2.1757360 \\ \mathrm{C} & -2.3119350 & 1.9105080 & 1.9023010 \\ \mathrm{~N} & -2.3392020 & 1.7958790 & 0.4289940 \\ \mathrm{~N} & -0.2484460 & 2.5334850 & 0.9948030\end{array}$




\begin{tabular}{|c|c|c|c|}
\hline $\mathrm{O}$ & -0.3144210 & 2.3101580 & 3.2878670 \\
\hline $\mathrm{C}$ & 1.1866930 & 2.7691910 & 0.9011040 \\
\hline $\mathrm{H}$ & 1.6843780 & 2.1758760 & 1.6709860 \\
\hline $\mathrm{H}$ & 1.5438040 & 2.4572960 & -0.0813760 \\
\hline $\mathrm{H}$ & 1.4423890 & 3.8218890 & 1.0576150 \\
\hline $\mathrm{C}$ & -2.9811220 & 0.7477450 & -0.2013560 \\
\hline $\mathrm{H}$ & -3.7572350 & 0.3006010 & 0.4096050 \\
\hline $\mathrm{C}$ & -2.7937670 & 0.2608730 & -1.4531300 \\
\hline $\mathrm{C}$ & -3.6703920 & -0.7961890 & -2.1066510 \\
\hline $\mathrm{H}$ & -3.0300030 & -1.6017840 & -2.4904950 \\
\hline $\mathrm{H}$ & -2.0634830 & 0.7088280 & -2.1199590 \\
\hline $\mathrm{C}$ & -2.7364480 & 0.6738020 & 2.6927140 \\
\hline $\mathrm{H}$ & -2.5204360 & 0.8572640 & 3.7484130 \\
\hline $\mathrm{H}$ & -3.8080210 & 0.4825230 & 2.5981590 \\
\hline $\mathrm{H}$ & -2.1859090 & -0.2162640 & 2.3742630 \\
\hline $\mathrm{H}$ & -2.9320000 & 2.7584290 & 2.2277080 \\
\hline $\mathrm{C}$ & -1.4940740 & 3.8573210 & -0.8060200 \\
\hline $\mathrm{H}$ & -0.7157310 & 1.8236720 & -0.9210120 \\
\hline $\mathrm{Cl}$ & -0.6328290 & -1.4406910 & -0.4240500 \\
\hline $\mathrm{N}$ & 0.6830970 & -2.5138660 & -0.0345290 \\
\hline $\mathrm{C}$ & 0.4805070 & -3.7822890 & 0.5498390 \\
\hline $\mathrm{C}$ & 2.0047690 & -2.1910920 & -0.2610340 \\
\hline $\mathrm{C}$ & 1.8549980 & -4.3933150 & 0.7470550 \\
\hline $\mathrm{C}$ & 2.8523010 & -3.3582650 & 0.1963350 \\
\hline $\mathrm{H}$ & 1.9936620 & -4.5954540 & 1.8126050 \\
\hline $\mathrm{H}$ & 3.4239780 & -3.7275400 & -0.6600470 \\
\hline $\mathrm{O}$ & 2.3748020 & -1.1276540 & -0.7528730 \\
\hline $\mathrm{O}$ & -0.6062310 & -4.2424560 & 0.8226390 \\
\hline $\mathrm{C}$ & -0.2292940 & 4.4427310 & -1.4710590 \\
\hline $\mathrm{H}$ & -0.5034150 & 5.3241060 & -2.0605060 \\
\hline $\mathrm{H}$ & 0.5191650 & 4.7617330 & -0.7420670 \\
\hline $\mathrm{H}$ & 0.2379380 & 3.7224030 & -2.1528340 \\
\hline $\mathrm{C}$ & -2.5584360 & 3.6566090 & -1.9043720 \\
\hline $\mathrm{H}$ & -2.1929380 & 3.0079730 & -2.7075700 \\
\hline $\mathrm{H}$ & -3.4771210 & 3.2217720 & -1.5022470 \\
\hline $\mathrm{H}$ & -2.8069850 & 4.6255750 & -2.3503730 \\
\hline $\mathrm{C}$ & -2.0324260 & 4.8503100 & 0.2406480 \\
\hline $\mathrm{H}$ & -1.3193820 & 5.0112630 & 1.0559370 \\
\hline $\mathrm{H}$ & -2.2170010 & 5.8205370 & -0.2325960 \\
\hline $\mathrm{H}$ & -2.9796260 & 4.5072470 & 0.6686220 \\
\hline $\mathrm{H}$ & 3.5687840 & -2.9985350 & 0.9397540 \\
\hline $\mathrm{H}$ & 1.8919410 & -5.3503410 & 0.2203270 \\
\hline $\mathrm{H}$ & 3.8924930 & -0.6320820 & -0.9117720 \\
\hline $\mathrm{O}$ & 4.8011550 & -0.2227150 & -1.0884920 \\
\hline $\mathrm{C}$ & 5.6235100 & -0.4107390 & -0.0808380 \\
\hline $\mathrm{O}$ & 5.4112800 & -0.9962000 & 0.9590350 \\
\hline $\mathrm{C}$ & 7.0013900 & 0.2419270 & -0.3793350 \\
\hline $\mathrm{F}$ & 6.8699910 & 1.5709780 & -0.5864840 \\
\hline $\mathrm{F}$ & 7.5587580 & -0.2956490 & -1.4872250 \\
\hline $\mathrm{F}$ & 7.8512910 & 0.0625310 & 0.6426210 \\
\hline $\mathrm{H}$ & -4.1410040 & -0.3493710 & -2.9941210 \\
\hline $\mathrm{C}$ & -4.7545800 & -1.3986480 & -1.2351620 \\
\hline $\mathrm{C}$ & -6.0382910 & -0.8318760 & -1.1939430 \\
\hline $\mathrm{C}$ & -4.5036130 & -2.5347980 & -0.4500470 \\
\hline $\mathrm{C}$ & -7.0428470 & -1.3805660 & -0.3904470 \\
\hline $\mathrm{H}$ & -6.2535360 & 0.0446210 & -1.8007330 \\
\hline $\mathrm{C}$ & -5.5043350 & -3.0882180 & 0.3552080 \\
\hline $\mathrm{H}$ & -3.5175480 & -2.9913900 & -0.4718620 \\
\hline $\mathrm{C}$ & -6.7784160 & -2.5119950 & 0.3884050 \\
\hline $\mathrm{H}$ & -8.0306510 & -0.9282820 & -0.3769950 \\
\hline $\mathrm{H}$ & -5.2898060 & -3.9706590 & 0.9516830 \\
\hline $\mathrm{H}$ & -7.5578880 & -2.9427040 & 1.0103600 \\
\hline
\end{tabular}

Int4.log

Potential Energy = -2233.52559

Zero-point Energy = -2232.99216

Free Energy = -2233.04199

Single-Point Energy B3LYP-D3(BJ)/6-311+G** PCM = -

2234.09921

Free Energy B3LYP-D3(BJ)/6-311+G** PCM (extrapolated free energy from $\mathrm{qRRHO})=-2233.61562$

Charge $=0$ Multiplicity $=1$

$\begin{array}{llll}\mathrm{C} & 0.9402390 & 2.2301840 & 0.5755130 \\ \mathrm{C} & 0.5027330 & 4.1457900 & -0.7397400 \\ \mathrm{C} & 1.9868650 & 3.8132210 & -0.9631080 \\ \mathrm{~N} & 2.0249080 & 2.4045230 & -0.4527940\end{array}$

\begin{tabular}{|c|c|c|c|}
\hline $\mathrm{N}$ & -0.0284770 & 3.2119810 & 0.0836410 \\
\hline $\mathrm{O}$ & -0.0837880 & 5.0894640 & -1.2598720 \\
\hline $\mathrm{C}$ & -1.4705080 & 3.1036440 & 0.3140480 \\
\hline $\mathrm{H}$ & -1.9822690 & 3.4779800 & -0.5743480 \\
\hline $\mathrm{H}$ & -1.7275290 & 2.0537610 & 0.4670710 \\
\hline $\mathrm{H}$ & -1.7870090 & 3.6961130 & 1.1761920 \\
\hline $\mathrm{C}$ & 2.7454050 & 1.4817430 & -0.9874700 \\
\hline $\mathrm{H}$ & 3.4500860 & 1.8019600 & -1.7499830 \\
\hline $\mathrm{C}$ & 2.6813470 & 0.0069470 & -0.7343940 \\
\hline $\mathrm{C}$ & 4.0810820 & -0.5922070 & -0.5054300 \\
\hline $\mathrm{H}$ & 4.7390100 & -0.2847360 & -1.3239030 \\
\hline $\mathrm{H}$ & 4.4684180 & -0.1297800 & 0.4103860 \\
\hline $\mathrm{H}$ & 1.9639690 & -0.2813880 & 0.0330220 \\
\hline $\mathrm{C}$ & 2.4441960 & 4.0079270 & -2.3990230 \\
\hline $\mathrm{H}$ & 2.2023740 & 5.0366690 & -2.6757610 \\
\hline $\mathrm{H}$ & 3.5239480 & 3.8811440 & -2.5036970 \\
\hline $\mathrm{H}$ & 1.9227810 & 3.3364590 & -3.0864960 \\
\hline $\mathrm{H}$ & 2.5907930 & 4.4261560 & -0.2877630 \\
\hline $\mathrm{C}$ & 1.4341060 & 2.4492160 & 2.0519330 \\
\hline $\mathrm{H}$ & 0.5295620 & 1.2191770 & 0.4936670 \\
\hline $\mathrm{Cl}$ & 1.9440840 & -0.5927850 & -2.3085880 \\
\hline $\mathrm{C}$ & 0.2090740 & 2.2460370 & 2.9736820 \\
\hline $\mathrm{H}$ & 0.5486150 & 2.2349770 & 4.0137550 \\
\hline $\mathrm{H}$ & -0.5172130 & 3.0558250 & 2.8760750 \\
\hline $\mathrm{H}$ & -0.2924930 & 1.2945410 & 2.7735490 \\
\hline $\mathrm{C}$ & 2.4841840 & 1.3786940 & 2.4044470 \\
\hline $\mathrm{H}$ & 2.0792960 & 0.3693310 & 2.2897890 \\
\hline $\mathrm{H}$ & 3.3947320 & 1.4774860 & 1.8050190 \\
\hline $\mathrm{H}$ & 2.7812480 & 1.4993960 & 3.4504460 \\
\hline $\mathrm{C}$ & 2.0232770 & & 2.2981650 \\
\hline $\mathrm{H}$ & 1.3376190 & 4.6469180 & 1.9929090 \\
\hline $\mathrm{H}$ & 2.2057970 & 3.9736950 & 3.3700080 \\
\hline $\mathrm{H}$ & 2.9847450 & 3.9922280 & 1.7947840 \\
\hline $\mathrm{N}$ & -4.6968210 & -0.5822140 & -0.5044950 \\
\hline $\mathrm{C}$ & & -1.1676640 & -1.6866090 \\
\hline $\mathrm{C}$ & -5.7182360 & 0.0131040 & 0.2131920 \\
\hline $\mathrm{C}$ & -6.6157670 & -0.9585470 & -1.8197010 \\
\hline $\mathrm{C}$ & -7.0173420 & -0.1761770 & -0.5586030 \\
\hline $\mathrm{H}$ & -7.1030100 & -1.9345370 & -1.8984060 \\
\hline $\mathrm{H}$ & -7.7299820 & -0.7113950 & 0.0752450 \\
\hline $\mathrm{H}$ & -7.4467460 & 0.8059610 & -0.7755470 \\
\hline $\mathrm{H}$ & -6.8168200 & -0.4171750 & -2.7484660 \\
\hline $\mathrm{O}$ & -5.5737010 & 0.5869480 & 1.2843430 \\
\hline $\mathrm{O}$ & -4.3766780 & -1.7480110 & -2.4727230 \\
\hline $\mathrm{C}$ & & & -0.3592940 \\
\hline $\mathrm{C}$ & 4.5129340 & -2.9137850 & -1.4226040 \\
\hline $\mathrm{C}$ & 3.7082090 & -2.7091430 & 0.8446030 \\
\hline $\mathrm{C}$ & 4.5360160 & -4.3057020 & -1.2904990 \\
\hline $\mathrm{H}$ & 4.8249520 & -2.4566540 & -2.3575660 \\
\hline $\mathrm{C}$ & & & 0.9784350 \\
\hline $\mathrm{H}$ & 3.3966170 & -2.0960600 & 1.6859870 \\
\hline $\mathrm{C}$ & 4.1406900 & -4.9026520 & -0.0900150 \\
\hline $\mathrm{H}$ & 4.8642030 & -4.9201600 & -2.1237840 \\
\hline $\mathrm{H}$ & 3.4261290 & -4.5544150 & 1.9175990 \\
\hline $\mathrm{H}$ & 4.1590860 & -5.9834430 & 0.0147770 \\
\hline $\mathrm{H}$ & -3.7112260 & -0.5937930 & -0.1843440 \\
\hline $\mathrm{O}$ & 0.0996000 & -0.9122890 & 0.9713150 \\
\hline $\mathrm{C}$ & -1.1159290 & -1.1300350 & 0.7838390 \\
\hline $\mathrm{O}$ & -1.9890280 & -0.4009980 & 0.2577920 \\
\hline $\mathrm{C}$ & -1.5906120 & -2.5334120 & 1.2983540 \\
\hline $\mathrm{F}$ & -0.9371750 & -3.5386520 & 0.6568490 \\
\hline $\mathrm{F}$ & -2.9146170 & -2.7571000 & 1.1266560 \\
\hline $\mathrm{F}$ & -1.3378790 & -2.6842920 & 2.6260660 \\
\hline
\end{tabular}

Int4a.log

Potential Energy $=-1949.26126$

Zero-point Energy = -1948.79604

Free Energy $=-1948.84194$

Single-Point Energy B3LYP-D3(BJ)/6-311+G** PCM = 1949.75917

Free Energy B3LYP-D3(BJ)/6-311+G** PCM (extrapolated free energy from $\mathrm{qRRHO})=-1949.33986$ Charge $=0$ Multiplicity $=1$
C $\quad-2.9040680$ $-0.4194010$
0.2350550
C $\quad-2.6325550-2.6725610$ $-0.4206930$
C $\quad-1.2574720 \quad-2.0274910 \quad-0.6080670$ 


\begin{tabular}{|c|c|c|c|}
\hline $\mathrm{N}$ & -1.4376240 & -0.7549740 & 0.1673800 \\
\hline $\mathrm{N}$ & -3.4767610 & -1.7587520 & 0.1195280 \\
\hline $\mathrm{O}$ & -2.8985910 & -3.8425840 & -0.6762100 \\
\hline $\mathrm{C}$ & -4.7923660 & -2.1362520 & 0.6348710 \\
\hline $\mathrm{H}$ & -5.5494840 & -2.1479020 & -0.1532200 \\
\hline $\mathrm{H}$ & -4.7169250 & -3.1372150 & 1.0635260 \\
\hline $\mathrm{H}$ & -5.0911810 & -1.4325230 & 1.4123930 \\
\hline $\mathrm{C}$ & -0.4784210 & -0.1969400 & 0.8226760 \\
\hline $\mathrm{C}$ & -0.6089920 & 0.8921270 & 1.8509710 \\
\hline $\mathrm{C}$ & 0.6110340 & 1.8250560 & 1.9247100 \\
\hline $\mathrm{H}$ & 0.5205620 & 2.3889040 & 2.8568570 \\
\hline $\mathrm{H}$ & 1.5157700 & 1.2140160 & 1.9877750 \\
\hline $\mathrm{C}$ & -0.1269740 & -2.9228550 & -0.1213980 \\
\hline $\mathrm{H}$ & -0.2065090 & -3.8660360 & -0.6668420 \\
\hline $\mathrm{H}$ & 0.8500250 & -2.4865910 & -0.3300750 \\
\hline $\mathrm{H}$ & -0.2131470 & -3.1336140 & 0.9484260 \\
\hline $\mathrm{H}$ & -1.0885910 & -1.7528200 & -1.6541730 \\
\hline $\mathrm{C}$ & -3.3771390 & 0.6021360 & -0.8629000 \\
\hline $\mathrm{H}$ & -3.1201270 & -0.0123440 & 1.2258540 \\
\hline $\mathrm{C}$ & -4.8976800 & 0.8140910 & -0.6800610 \\
\hline $\mathrm{H}$ & -5.2204640 & 1.6237170 & -1.3413180 \\
\hline $\mathrm{H}$ & -5.4755720 & -0.0730440 & -0.9462230 \\
\hline $\mathrm{H}$ & -5.1512320 & 1.1046410 & 0.3452410 \\
\hline $\mathrm{C}$ & -2.6791020 & 1.9586820 & -0.6596500 \\
\hline $\mathrm{H}$ & -2.9102910 & 2.3953910 & 0.3175530 \\
\hline $\mathrm{H}$ & -1.5939840 & 1.8966420 & -0.7758730 \\
\hline $\mathrm{H}$ & -3.0406400 & 2.6604550 & -1.4168920 \\
\hline $\mathrm{C}$ & -3.1018020 & 0.0942390 & -2.2904840 \\
\hline $\mathrm{H}$ & -3.5533630 & -0.8857840 & -2.4731620 \\
\hline $\mathrm{H}$ & -3.5448580 & 0.7933110 & -3.0060820 \\
\hline $\mathrm{H}$ & -2.0316130 & 0.0402470 & -2.5099730 \\
\hline $\mathrm{H}$ & -1.5386970 & 1.4502730 & 1.7886430 \\
\hline $\mathrm{C}$ & 0.6941620 & 2.7826230 & 0.7489030 \\
\hline $\mathrm{C}$ & 0.0575970 & 4.0321350 & 0.8053310 \\
\hline $\mathrm{C}$ & 1.4137910 & 2.4405040 & -0.4062140 \\
\hline $\mathrm{C}$ & 0.1303800 & 4.9182230 & -0.2723730 \\
\hline $\mathrm{H}$ & -0.4925490 & 4.3154130 & 1.6991690 \\
\hline $\mathrm{C}$ & 1.4861780 & 3.3258760 & -1.4865580 \\
\hline $\mathrm{H}$ & 1.9268520 & 1.4844260 & -0.4538080 \\
\hline $\mathrm{C}$ & 0.8434730 & 4.5656320 & -1.4234280 \\
\hline $\mathrm{H}$ & -0.3638680 & 5.8834290 & -0.2113780 \\
\hline $\mathrm{H}$ & 2.0507550 & 3.0486270 & -2.3720580 \\
\hline $\mathrm{H}$ & 0.9033330 & 5.2549600 & -2.2605080 \\
\hline $\mathrm{Cl}$ & -0.7270040 & -0.0834880 & 3.4123120 \\
\hline $\mathrm{H}$ & 0.5305050 & -0.5953730 & 0.6739480 \\
\hline $\mathrm{O}$ & 0.6578420 & -0.6755290 & -2.7444460 \\
\hline $\mathrm{H}$ & 1.5411640 & -0.7915010 & -2.3165700 \\
\hline $\mathrm{O}$ & 3.1296830 & -1.0929550 & -1.6112530 \\
\hline $\mathrm{C}$ & 3.3478300 & -1.0444570 & -0.3760050 \\
\hline $\mathrm{C}$ & 4.8433580 & -1.3228350 & 0.0009640 \\
\hline $\mathrm{O}$ & 2.5720920 & -0.8066350 & 0.5724530 \\
\hline $\mathrm{F}$ & 5.2605550 & -2.5262780 & -0.4752800 \\
\hline $\mathrm{F}$ & 5.6662370 & -0.3807570 & -0.5359510 \\
\hline $\mathrm{F}$ & 5.0757760 & -1.3294200 & 1.3330070 \\
\hline $\mathrm{H}$ & 0.6851830 & 0.1942080 & -3.1631330 \\
\hline
\end{tabular}

syn-18b (syn-aminal).log

Potential Energy $=-2233.52383$

Zero-point Energy = -2232.98813

Free Energy = -2233.03620

Single-Point Energy B3LYP-D3(BJ)/6-311+G** PCM = 2234.10822

Free Energy B3LYP-D3(BJ)/6-311+G** PCM (extrapolated free energy from $q R R H O)=-2233.62060$

Charge $=0$ Multiplicity $=1$

$\begin{array}{llll}\mathrm{C} & -0.4491690 & -1.7975970 & 1.1574570 \\ \mathrm{C} & -1.5239110 & -3.7805250 & 0.4830270 \\ \mathrm{C} & -2.5342340 & -2.6316080 & 0.3980450 \\ \mathrm{~N} & -1.6701200 & -1.4104740 & 0.4124130 \\ \mathrm{~N} & -0.3499070 & -3.2517390 & 0.8952080 \\ \mathrm{O} & -1.7378290 & -4.9712520 & 0.2287250 \\ \mathrm{C} & 0.8717840 & -4.0428360 & 0.9685090 \\ \mathrm{H} & 0.8645170 & -4.7706060 & 0.1536310 \\ \mathrm{H} & 1.7374070 & -3.3879500 & 0.8578660 \\ \mathrm{H} & 0.9563130 & -4.5866740 & 1.9145590 \\ \mathrm{C} & -1.5216920 & -0.6556080 & -0.8301570\end{array}$

\begin{tabular}{|c|c|c|c|}
\hline $\mathrm{H}$ & -2.3139000 & -0.9715680 & -1.5076500 \\
\hline $\mathrm{C}$ & -1.7364110 & 0.8661820 & -0.5967830 \\
\hline $\mathrm{C}$ & -3.1391240 & 1.1523050 & -0.0449680 \\
\hline $\mathrm{H}$ & -3.8827000 & 0.7665050 & -0.7502160 \\
\hline $\mathrm{H}$ & -0.9583350 & 1.2858360 & 0.0367750 \\
\hline $\mathrm{C}$ & -3.5586120 & -2.7996610 & -0.7177210 \\
\hline $\mathrm{H}$ & -4.1242620 & -3.7111330 & -0.5079500 \\
\hline $\mathrm{H}$ & -4.2673270 & -1.9664040 & -0.7298210 \\
\hline $\mathrm{H}$ & -3.1033820 & -2.9052280 & -1.7037040 \\
\hline $\mathrm{H}$ & -3.0927780 & -2.6355340 & 1.3413190 \\
\hline $\mathrm{C}$ & -0.4513420 & -1.4065050 & 2.6785170 \\
\hline $\mathrm{H}$ & 0.4236440 & -1.3086810 & 0.7267240 \\
\hline $\mathrm{Cl}$ & -1.5383760 & 1.7378200 & -2.2092710 \\
\hline $\mathrm{N}$ & -0.2798400 & -0.9287740 & -1.6166500 \\
\hline $\mathrm{C}$ & 0.9561520 & -0.3321510 & -1.4665370 \\
\hline $\mathrm{C}$ & -0.3161310 & -1.7563270 & -2.7650000 \\
\hline $\mathrm{C}$ & 1.8883350 & -0.7973030 & -2.5603030 \\
\hline $\mathrm{C}$ & 1.0667240 & -1.7992320 & -3.3776140 \\
\hline $\mathrm{H}$ & 2.1970110 & 0.0771420 & -3.1414570 \\
\hline $\mathrm{H}$ & 1.4467220 & -2.8227150 & -3.3067670 \\
\hline $\mathrm{O}$ & -1.3207750 & -2.3135010 & -3.1599950 \\
\hline $\mathrm{O}$ & 1.2308430 & 0.4576540 & -0.5585490 \\
\hline $\mathrm{C}$ & 0.8860890 & -1.8467700 & 3.3155760 \\
\hline $\mathrm{H}$ & 0.9605100 & -1.4320220 & 4.3261300 \\
\hline $\mathrm{H}$ & 0.9654550 & -2.9328430 & 3.4048180 \\
\hline $\mathrm{H}$ & 1.7485210 & -1.4826930 & 2.7453810 \\
\hline $\mathrm{C}$ & -0.5447920 & 0.1280410 & 2.7809370 \\
\hline $\mathrm{H}$ & 0.2601080 & 0.6199700 & 2.2235140 \\
\hline $\mathrm{H}$ & -1.5031820 & 0.4900370 & 2.4026260 \\
\hline $\mathrm{H}$ & -0.4622630 & 0.4359540 & 3.8284710 \\
\hline $\mathrm{C}$ & -1.6105730 & -2.0368110 & 3.4719460 \\
\hline $\mathrm{H}$ & -1.6252030 & -3.1278980 & 3.3781310 \\
\hline $\mathrm{H}$ & -1.4941480 & -1.8017190 & 4.5355030 \\
\hline $\mathrm{H}$ & -2.5804130 & -1.6415740 & 3.1558010 \\
\hline $\mathrm{H}$ & 0.9936730 & -1.5477370 & -4.4382980 \\
\hline $\mathrm{H}$ & 2.7876280 & -1.2198940 & -2.1045250 \\
\hline $\mathrm{H}$ & -3.2356830 & 0.5615430 & 0.8719940 \\
\hline $\mathrm{C}$ & -3.4281280 & 2.6117000 & 0.2486430 \\
\hline $\mathrm{C}$ & -4.4150020 & 3.2993150 & -0.4719660 \\
\hline $\mathrm{C}$ & -2.7375500 & 3.3006700 & 1.2588490 \\
\hline $\mathrm{C}$ & -4.7041960 & 4.6401400 & -0.1970360 \\
\hline $\mathrm{H}$ & -4.9626210 & 2.7818060 & -1.2553470 \\
\hline $\mathrm{C}$ & -3.0199140 & 4.6410840 & 1.5344820 \\
\hline $\mathrm{H}$ & -1.9755200 & 2.7890740 & 1.8406390 \\
\hline $\mathrm{C}$ & -4.0054240 & 5.3167740 & 0.8065030 \\
\hline $\mathrm{H}$ & -5.4734770 & 5.1529660 & -0.7673900 \\
\hline $\mathrm{H}$ & -2.4742500 & 5.1552840 & 2.3205740 \\
\hline $\mathrm{H}$ & -4.2273670 & 6.3578550 & 1.0221380 \\
\hline $\mathrm{H}$ & 2.6555720 & 1.1278530 & -0.3040150 \\
\hline $\mathrm{O}$ & 3.4957000 & 1.6485780 & -0.0774810 \\
\hline $\mathrm{C}$ & 4.5546770 & 0.8726600 & -0.0322030 \\
\hline $\mathrm{C}$ & 5.8198960 & 1.7052090 & 0.3140990 \\
\hline $\mathrm{F}$ & 6.0443260 & 2.6500860 & -0.6263830 \\
\hline $\mathrm{F}$ & 6.9074390 & 0.9232660 & 0.3850580 \\
\hline $\mathrm{F}$ & 5.6764600 & 2.3319390 & 1.5027410 \\
\hline $\mathrm{O}$ & 4.6161190 & -0.3226640 & -0.2250980 \\
\hline
\end{tabular}

Int5.log

Potential Energy = -1949.26139

Zero-point Energy = -1948.79334

Free Energy = -1948.83756

Single-Point Energy B3LYP-D3(BJ)/6-311+G** PCM = 1949.75481

Free Energy B3LYP-D3(BJ)/6-311+G** PCM (extrapolated free energy from qRRHO) $=-1949.33098$

Charge $=0$ Multiplicity $=1$

$\begin{array}{llrr}\mathrm{C} & 2.8479570 & -0.4198890 & 0.2974400 \\ \mathrm{C} & 3.1788680 & -2.0851900 & -1.3420500 \\ \mathrm{C} & 1.7708930 & -2.2976070 & -0.7866200 \\ \mathrm{~N} & 1.5497880 & -1.0639370 & 0.0043330 \\ \mathrm{~N} & 3.7174470 & -1.0041560 & -0.7385140 \\ \mathrm{O} & 3.7192410 & -2.7693720 & -2.2175730 \\ \mathrm{C} & 4.9482250 & -0.3944440 & -1.2321830 \\ \mathrm{H} & 5.8410300 & -0.8700730 & -0.8165410 \\ \mathrm{H} & 4.9758230 & -0.4998760 & -2.3191860 \\ \mathrm{H} & 4.9574550 & 0.6650230 & -0.9739010\end{array}$




\begin{tabular}{|c|c|c|c|}
\hline $\mathrm{C}$ & 0.2907740 & -0.4074070 & -0.0013390 \\
\hline $\mathrm{C}$ & 0.2888550 & 1.1310430 & -0.1127780 \\
\hline $\mathrm{C}$ & -1.1139280 & 1.7421000 & 0.0170570 \\
\hline $\mathrm{H}$ & -1.7602340 & 1.3272430 & -0.7631280 \\
\hline $\mathrm{H}$ & -1.5075070 & 1.4056490 & 0.9815060 \\
\hline $\mathrm{C}$ & 0.7798260 & -2.5898450 & -1.9130310 \\
\hline $\mathrm{H}$ & 1.1464220 & -3.4635690 & -2.4574550 \\
\hline $\mathrm{H}$ & -0.2158730 & -2.8261270 & -1.5296960 \\
\hline $\mathrm{H}$ & 0.7045620 & -1.7555190 & -2.6169870 \\
\hline $\mathrm{H}$ & 1.7989960 & -3.1750510 & -0.1239160 \\
\hline $\mathrm{C}$ & 3.3730440 & -0.6330290 & 1.7646110 \\
\hline $\mathrm{H}$ & 2.7947160 & 0.6585110 & 0.1191560 \\
\hline $\mathrm{C}$ & 4.8176950 & -0.1036950 & 1.8969710 \\
\hline $\mathrm{H}$ & 5.1080340 & -0.1055460 & 2.9527070 \\
\hline $\mathrm{H}$ & 5.5372040 & -0.7273040 & 1.3614860 \\
\hline $\mathrm{H}$ & 4.9106770 & 0.9260690 & 1.5335750 \\
\hline $\mathrm{C}$ & 2.4938300 & 0.1870880 & 2.7312480 \\
\hline $\mathrm{H}$ & 2.5490060 & 1.2587960 & 2.5051630 \\
\hline $\mathrm{H}$ & 1.4432880 & -0.1105950 & 2.7060870 \\
\hline $\mathrm{H}$ & 2.8493720 & 0.0509060 & 3.7577440 \\
\hline $\mathrm{C}$ & 3.3463780 & -2.1192400 & 2.1630480 \\
\hline $\mathrm{H}$ & 3.9600680 & -2.7275570 & 1.4899480 \\
\hline $\mathrm{H}$ & 3.7492100 & -2.2399940 & 3.1741220 \\
\hline $\mathrm{H}$ & 2.3292020 & -2.5227180 & 2.1622510 \\
\hline $\mathrm{H}$ & 0.9591250 & 1.5815620 & 0.6189800 \\
\hline $\mathrm{C}$ & -1.1448710 & 3.2569880 & -0.0296560 \\
\hline $\mathrm{C}$ & -0.6636340 & 4.0147090 & 1.0501740 \\
\hline $\mathrm{C}$ & -1.6670460 & 3.9325440 & -1.1415380 \\
\hline $\mathrm{C}$ & -0.6924050 & 5.4113320 & 1.0140520 \\
\hline $\mathrm{H}$ & -0.2700580 & 3.5110630 & 1.9300370 \\
\hline $\mathrm{C}$ & -1.7014550 & 5.3304200 & -1.1804510 \\
\hline $\mathrm{H}$ & -2.0501870 & 3.3611940 & -1.9828680 \\
\hline $\mathrm{C}$ & -1.2113670 & 6.0745520 & -0.1034320 \\
\hline $\mathrm{H}$ & -0.3169830 & 5.9802880 & 1.8598650 \\
\hline $\mathrm{H}$ & -2.1112780 & 5.8351730 & -2.0506910 \\
\hline $\mathrm{H}$ & -1.2378360 & 7.1599870 & -0.1311740 \\
\hline $\mathrm{Cl}$ & 1.0022510 & 1.5561170 & -1.7515770 \\
\hline $\mathrm{H}$ & -0.3244410 & -0.8018520 & -0.8102060 \\
\hline $\mathrm{O}$ & -0.4673670 & -0.7159980 & 1.2419540 \\
\hline $\mathrm{H}$ & -2.0000610 & -1.1423450 & 1.1659450 \\
\hline $\mathrm{H}$ & 0.0218740 & -1.4334340 & 1.6737380 \\
\hline $\mathrm{O}$ & -2.9808750 & -1.4074570 & 1.2305400 \\
\hline $\mathrm{C}$ & -3.4575800 & -1.8027600 & 0.0724130 \\
\hline $\mathrm{C}$ & -4.9484380 & -2.2165940 & 0.2141020 \\
\hline $\mathrm{O}$ & -2.8808970 & -1.8647910 & -0.9923350 \\
\hline $\mathrm{F}$ & -5.0818620 & -3.2388260 & 1.0887070 \\
\hline $\mathrm{F}$ & -5.6949440 & -1.1853330 & 0.6677750 \\
\hline $\mathrm{F}$ & -5.4548690 & -2.6094800 & -0.9641920 \\
\hline
\end{tabular}

product.log $(S-(19))$

Potential Energy $=-883.82667$

Zero-point Energy $=-883.66972$

Free Energy = -883.69775

Single-Point Energy B3LYP-D3(BJ)/6-311+G** PCM = 883.98063

Free Energy B3LYP-D3(BJ)/6-311+G** PCM (extrapolated free energy from $q$ RRHO) $=-883.85171$ Charge $=0$ Multiplicity $=1$

$\begin{array}{lccc}\mathrm{C} & 1.5543360 & -0.1401000 & -0.2522360 \\ \mathrm{C} & 0.5788650 & -0.8150130 & 0.7258110 \\ \mathrm{H} & 1.3676790 & -0.4306580 & -1.2862890 \\ \mathrm{Cl} & 3.2770780 & -0.6606210 & 0.1189520 \\ \mathrm{H} & 0.7501140 & -1.8944180 & 0.6703410 \\ \mathrm{C} & 1.5643120 & 1.3750860 & -0.1387350 \\ \mathrm{H} & 1.8473870 & 1.7745930 & 0.8535280 \\ \mathrm{O} & 1.2837260 & 2.1117370 & -1.0628590 \\ \mathrm{H} & 0.8173130 & -0.4989870 & 1.7465030 \\ \mathrm{C} & -0.8671920 & -0.4916620 & 0.3938040 \\ \mathrm{C} & -1.5760040 & 0.4711890 & 1.1273470 \\ \mathrm{C} & -1.5186940 & -1.1475590 & -0.6630480 \\ \mathrm{C} & -2.9044020 & 0.7751410 & 0.8117550 \\ \mathrm{H} & -1.0912900 & 0.9815950 & 1.9557640 \\ \mathrm{C} & -2.8451130 & -0.8455530 & -0.9812380 \\ \mathrm{H} & -0.9884110 & -1.9045210 & -1.2357550 \\ \mathrm{C} & -3.5419630 & 0.1187320 & -0.2446900 \\ \mathrm{H} & -3.4389460 & 1.5206960 & 1.3931920\end{array}$

$\begin{array}{llll}\mathrm{H} & -3.3349500 & -1.3653710 & -1.7995070 \\ \mathrm{H} & -4.5739020 & 0.3521650 & -0.4897160\end{array}$

anti-18b.log

Potential Energy $=-2233.51758$

Zero-point Energy = -2232.98167

Free Energy = -2233.02959

Single-Point Energy B3LYP-D3(BJ)/6-311+G** PCM = -

2234.10584

Free Energy B3LYP-D3(BJ)/6-311+G** PCM (extrapolated

free energy from qRRHO) $=-2233.61785$

Charge $=0$ Multiplicity $=1$

$\begin{array}{llll}\text { C } & 2.9544110 & -1.3243090 & -0.1002390\end{array}$

$\begin{array}{llll}\text { C } & 2.6737200 & -1.0248020 & -2.4146860\end{array}$

C $\quad 1.2861080 \quad-0.9717320 \quad-1.7662800$

$\mathrm{N} \quad 1.5980950 \quad-0.7775800 \quad-0.3211500$

$\mathrm{N} \quad 3.5820770 \quad-1.2046860 \quad-1.4325050$

$\begin{array}{llll}\mathrm{O} & 2.9203350 & -0.9234620 & -3.6224130\end{array}$

$\begin{array}{llll}\mathrm{C} & 5.0134190 & -1.1134380 & -1.6842030\end{array}$

$\begin{array}{llll}\mathrm{H} & 5.1836440 & -0.3621570 & -2.4593090\end{array}$

H $\quad 5.5253020 \quad-0.8071010 \quad-0.7707000$

$\begin{array}{llll}\mathrm{H} & 5.4351900 & -2.0639150 & -2.0263420\end{array}$

$\begin{array}{llll}\text { C } & 1.1601280 & 0.4374230 & 0.3404310\end{array}$

$\begin{array}{llll}\mathrm{H} & 0.3598170 & 0.8508330 & -0.2698600\end{array}$

$\begin{array}{llll}\mathrm{C} & 2.1727410 & 1.5931060 & 0.6006320\end{array}$

$\begin{array}{llll}\mathrm{C} & 1.5711680 & 2.8738550 & 1.2239240\end{array}$

$\begin{array}{llll}\mathrm{H} & 2.9882220 & 1.2656280 & 1.2385760\end{array}$

$\begin{array}{llll}\text { C } & 0.3481590 & 0.0148920 & -2.4544380\end{array}$

$\mathrm{H} \quad 0.2497460 \quad-0.3008260 \quad-3.4963020$

$\mathrm{H} \quad-0.6456830 \quad-0.0052230 \quad-2.0011390$

$\begin{array}{llll}\mathrm{H} & 0.7316020 & 1.0374070 & -2.4508190\end{array}$

H $\quad 0.8403080 \quad-1.9676590 \quad-1.8862410$

$\begin{array}{llll}\text { C } & 2.9920850 & -2.7955900 & 0.4603130\end{array}$

$\begin{array}{llll}\mathrm{H} & 3.5033230 & -0.6969110 & 0.6093060\end{array}$

$\begin{array}{llll}\mathrm{Cl} & 2.9889070 & 2.0185560 & -0.9878270\end{array}$

$\begin{array}{llll}\mathrm{N} & 0.4561460 & 0.1057430 & 1.6282660\end{array}$

$\begin{array}{llll}\mathrm{C} & -0.8689050 & -0.2899060 & 1.6061870\end{array}$

$\begin{array}{llll}\mathrm{C} & 1.0027610 & 0.0243020 & 2.9227410\end{array}$

$\begin{array}{llll}\mathrm{C} & -1.3205100 & -0.6577570 & 3.0003200\end{array}$

C $\quad-0.0875620 \quad-0.4183520 \quad 3.8791330$

$\mathrm{H} \quad-2.1800740 \quad-0.0388340 \quad 3.2724590$

$\mathrm{H} \quad 0.2508230 \quad-1.3118100 \quad 4.4098930$

$\begin{array}{llll}\mathrm{O} & 2.1594890 & 0.2819190 & 3.2006630\end{array}$

$\begin{array}{llll}\text { O } & -1.5423060 & -0.3195560 & 0.5749050\end{array}$

$\begin{array}{llll}\text { C } & 4.4627100 & -3.2144140 & 0.6854470\end{array}$

$\mathrm{H} \quad 4.4913280 \quad-4.1686520 \quad 1.2217670$

$\begin{array}{llll}\mathrm{H} & 5.0041870 & -3.3548050 & -0.2529580\end{array}$

$\mathrm{H} \quad 5.0057940 \quad-2.4792860 \quad 1.2909420$

$\begin{array}{llll}\text { C } & 2.2740340 & -2.8443600 & 1.8201050\end{array}$

$\begin{array}{llll}\mathrm{H} & 2.7394210 & -2.1719470 & 2.5471310\end{array}$

$\mathrm{H} \quad 1.2181620 \quad-2.5809810 \quad 1.7167860$

$\begin{array}{llll}\mathrm{H} & 2.3244460 & -3.8599290 & 2.2264840\end{array}$

C $2.3239680 \quad-3.8080880 \quad-0.4893400$

$\mathrm{H} \quad 2.7638430 \quad-3.7836250 \quad-1.4915270$

$\mathrm{H} \quad 2.4608660 \quad-4.8210290 \quad-0.0954640$

$\mathrm{H} \quad 1.2471240 \quad-3.6338460 \quad-0.5724800$

$\mathrm{H} \quad-0.2359960 \quad 0.3689890 \quad 4.6234600$

H $\quad-1.6550560 \quad-1.6994000 \quad 2.9974680$

$\mathrm{H} \quad 1.2757670 \quad 2.6240190 \quad 2.2471820$

$\mathrm{H} \quad 2.3980390 \quad 3.5847990 \quad 1.3163060$

C $\quad 0.4065630 \quad 3.5349510-0.5084340$

$\begin{array}{llll}\text { C } & 0.6201740 & 4.4235060 & -0.5591730\end{array}$

$\begin{array}{llll}\text { C } & -0.9140560 & 3.3119760 & 0.9288940\end{array}$

$\begin{array}{llll}\mathrm{C} & -0.4534840 & 5.0446110 & -1.2028800\end{array}$

$\mathrm{H} \quad 1.6335620 \quad 4.6367890 \quad-0.8838850$

$\begin{array}{llll}\text { C } & -1.9919960 & 3.9311240 & 0.2883140\end{array}$

$\mathrm{H} \quad-1.1052070 \quad 2.6642240 \quad 1.7796120$

C $\quad-1.7651810 \quad 4.7961960 \quad-0.7853020$

$\begin{array}{llll}\mathrm{H} & -0.2640870 & 5.7284080 & -2.0254310\end{array}$

$\begin{array}{llll}\mathrm{H} & -3.0042010 & 3.7429140 & 0.6345360\end{array}$

$\mathrm{H} \quad-2.5995260 \quad 5.2811400 \quad-1.2835530$

$\mathrm{H} \quad-3.0867530 \quad-0.7157070 \quad 0.4958220$

$\begin{array}{llll}\text { O } & -4.0733650 & -0.9489840 & 0.4995030\end{array}$

C $\quad-4.5537260 \quad-1.1134290 \quad-0.7126060$

O $\quad-3.9714420 \quad-1.0230510 \quad-1.7711010$

$\begin{array}{llll}\text { C } & -6.0670080 & -1.4618580 & -0.6463300\end{array}$ 


$\begin{array}{llll}\text { F } & -6.2729370 & -2.5907890 & 0.0682100 \\ \text { F } & -6.5734600 & -1.6497260 & -1.8741570 \\ \text { F } & -6.7646690 & -0.4665610 & -0.0540270\end{array}$

Int $3^{\prime} \cdot \log$

Potential Energy = -2233.46721

Zero-point Energy = -2232.93678

Free Energy $=-2232.98830$

Single-Point Energy B3LYP-D3(BJ)/6-311+G** PCM = 2234.03863

Free Energy B3LYP-D3(BJ)/6-311+G** PCM (extrapolated free energy from $q R R H O)=-2233.55972$

Charge $=0$ Multiplicity $=1$

$\begin{array}{lrrr}\mathrm{C} & -2.6266560 & 2.4503800 & 0.7784270 \\ \mathrm{C} & -1.5223420 & 3.9157680 & -0.7036390 \\ \mathrm{C} & -1.4995120 & 2.5479870 & -1.3843830 \\ \mathrm{~N} & -2.3190760 & 1.7439350 & -0.4755820 \\ \mathrm{~N} & -2.2467700 & 3.8256180 & 0.4340010 \\ \mathrm{O} & -1.0229690 & 4.9494330 & -1.1588150 \\ \mathrm{C} & -2.6800680 & 5.0203450 & 1.1497690\end{array}$

$\begin{array}{llll}\text { C } & -2.6800680 & 5.0203450 & 1.1497690\end{array}$

$\mathrm{H} \quad-2.8929100 \quad 5.8029220 \quad 0.4180380$

H $\quad-3.5873750 \quad 4.7972780 \quad 1.7119570$

$\mathrm{H} \quad-1.9141910 \quad 5.3914730 \quad 1.8372380$

C $\quad-2.8890640 \quad 0.5278930 \quad-0.7748120$

$\mathrm{H} \quad-3.7156960 \quad 0.2693140 \quad-0.1181140$

C $\quad-2.5480320 \quad-0.3347000 \quad-1.7643380$

$\begin{array}{llll}\text { C } & -3.3463400 & -1.5718740 & -2.1430910\end{array}$

$\mathrm{H} \quad-2.6565380 \quad-2.4200540 \quad-2.2465020$

$\mathrm{H} \quad-0.4627090 \quad 2.1815710 \quad-1.4321660$

$\begin{array}{llll}\text { C } & -1.8870200 & 1.8911850 & 2.0517060\end{array}$

$\mathrm{H} \quad-3.7091810 \quad 2.4089470 \quad 0.9711340$

$\mathrm{H}-1.7300520 \quad-0.0962950 \quad-2.4377340$

Cl $\quad-0.2363270 \quad-1.6516930 \quad-0.4739190$

$\begin{array}{llll}\mathrm{N} & 1.1471420 & -2.5417150 & 0.0763850\end{array}$

C $\quad 2.4420110 \quad-2.1007200 \quad-0.1074630$

$\begin{array}{llll}\text { C } & 2.4506000 & -4.2249380 & 1.0669680\end{array}$

C $3.3713980 \quad-3.1349220 \quad 0.4890930$

$\mathrm{H} \quad 2.6145650 \quad-5.2083090 \quad 0.6189150$

$\mathrm{H} \quad 4.0294480 \quad-3.5060380 \quad-0.3019760$

$\mathrm{H} \quad 4.0006990 \quad-2.6479780 \quad 1.2388360$

$\mathrm{H}-2.5447700-4.3343510-2.1507180$

$\begin{array}{llll}\mathrm{O} & 2.7320410 & -1.0465780 & -0.6668220\end{array}$

$\begin{array}{llll}\text { O } & -0.0138360 & -4.3256460 & 1.0012080\end{array}$

$\begin{array}{llll}\mathrm{C} & -2.0489010 & 2.7048910 & -2.8121990\end{array}$

$\begin{array}{llll}\mathrm{H} & -1.9815400 & 1.7816430 & -3.3867170\end{array}$

$\begin{array}{llll}\mathrm{H} & -3.0954690 & 3.0247850 & -2.7883540\end{array}$

$\mathrm{H} \quad-1.4596170 \quad 3.4710050 \quad-3.3225100$

$\begin{array}{llll}\mathrm{C} & -2.2691950 & 2.7253540 & 3.2945580\end{array}$

$\mathrm{H} \quad-1.8821820 \quad 2.2333710 \quad 4.1930320$

$\begin{array}{llll}\mathrm{H} & -1.8471390 & 3.7320160 & 3.2716330\end{array}$

$\begin{array}{llll}\mathrm{H} & -3.3566490 & 2.8076790 & 3.4083120\end{array}$

C $\quad-0.3611380 \quad 1.9234840 \quad 1.8549500$

$\begin{array}{llll}\mathrm{H} & 0.0052410 & 2.9433680 & 1.6973150\end{array}$

$\mathrm{H} \quad 0.1375190 \quad 1.5274920 \quad 2.7461530$

$\begin{array}{llll}\mathrm{H} & -0.0552940 & 1.3089460 & 1.0025880\end{array}$

$\begin{array}{llll}\mathrm{C} & -2.3435480 & 0.4409220 & 2.3163490\end{array}$

$\begin{array}{llll}\mathrm{H} & -3.4336810 & 0.3733900 & 2.4148440\end{array}$

$\begin{array}{llll}\mathrm{H} & -2.0249580 & -0.2464270 & 1.5305160\end{array}$

$\mathrm{H} \quad-1.9061550 \quad 0.0909070 \quad 3.2574030$

$\begin{array}{llll}\mathrm{H} & -3.7735670 & -1.4198290 & -3.1449540\end{array}$

C $\quad-4.4630630 \quad-1.9580020 \quad-1.1934430$

$\begin{array}{llll}\text { C } & -4.2095650 & -2.7655950 & -0.0733220\end{array}$

$\begin{array}{llll}\text { C } & -5.7776370 & -1.5172080 & -1.4116650\end{array}$

C $\quad-5.2380530 \quad-3.1213360 \quad 0.8046880$

$\mathrm{H}-3.1989240 \quad-3.1217310 \quad 0.1094840$

C $\quad-6.8105050 \quad-1.8690560 \quad-0.5363640$

$\mathrm{H} \quad-5.9948610 \quad-0.8965530 \quad-2.2779170$

C $\quad-6.5434660 \quad-2.6727920 \quad 0.5765620$

$\mathrm{H} \quad-5.0208980 \quad-3.7518150 \quad 1.6625460$

$\mathrm{H} \quad-7.8216360 \quad-1.5197980 \quad-0.7265350$

$\mathrm{H} \quad-7.3442960 \quad-2.9510480 \quad 1.2556610$

$\mathrm{H} \quad 4.2053280 \quad-0.4257260 \quad-0.8220110$

O $\quad 5.0824190 \quad 0.0408130 \quad-1.0131430$

$\begin{array}{llll}\text { C } & 5.8752080 & 0.0342720 & 0.0349300\end{array}$

$\begin{array}{llll}\text { C } & 7.2094610 & 0.7594330 & -0.2931800\end{array}$

$\begin{array}{lllc}\mathrm{O} & 5.6676980 & -0.4490210 & 1.1269470 \\ \mathrm{~F} & 8.0263500 & 0.7706310 & 0.7706260 \\ \mathrm{~F} & 6.9854230 & 2.0395260 & -0.6632370 \\ \mathrm{~F} & 7.8535440 & 0.1423900 & -1.3090260\end{array}$

syn-18b'.log

Potential Energy = -2233.51380

Zero-point Energy = -2232.97812

Free Energy = -2233.02594

Single-Point Energy B3LYP-D3(BJ)/6-311+G** PCM = -

2234.10139

Free Energy B3LYP-D3(BJ)/6-311+G** PCM (extrapolated

free energy from $q R R H O)=-2233.61352$

Charge $=0$ Multiplicity $=1$

$\begin{array}{llll}\text { C } & -1.8564840 & 1.5162690 & 0.5390720\end{array}$

C $\quad-1.0558940 \quad 2.6485280 \quad-1.3582430$

$\begin{array}{llll}\mathrm{C} & -0.1829640 & 1.4019620 & -1.2095780\end{array}$

$\mathrm{N} \quad-0.9067160 \quad 0.6342080 \quad-0.1733550$

$\mathrm{N} \quad-2.0258090 \quad 2.6159430 \quad-0.4207840$

$\begin{array}{llll}\text { O } & -0.9212940 & 3.5198530 & -2.2252900\end{array}$

C $\quad-3.1410830 \quad 3.5545640 \quad-0.4437960$

$\mathrm{H} \quad-3.4066970 \quad 3.7491130 \quad-1.4854550$

$\begin{array}{llll}\mathrm{H} & -3.9969730 & 3.1142900 & 0.0687400\end{array}$

H $\quad-2.8902920 \quad 4.5085310 \quad 0.0301960$

C $\quad-1.0612310 \quad-0.7812760 \quad-0.0359600$

$\mathrm{H} \quad-1.8517260 \quad-0.9152110 \quad 0.7038930$

C $\quad-1.5783720 \quad-1.5790380 \quad-1.2979850$

C $\quad-2.8512570 \quad-2.4065590 \quad-1.0185230$

$\mathrm{H} \quad-3.0719650 \quad-2.9743660 \quad-1.9274720$

$\mathrm{H} \quad-2.6487100 \quad-3.1254320 \quad-0.2211320$

$\mathrm{H} \quad 0.7991750 \quad 1.7077950 \quad-0.8262900$

$\begin{array}{llll}\text { C } & -1.3904810 & 2.0009280 & 1.9626020\end{array}$

$\mathrm{H} \quad-2.8191090 \quad 1.0018620 \quad 0.6615780$

$\mathrm{H} \quad-1.7776430 \quad-0.8680320 \quad-2.0928030$

$\begin{array}{llll}\mathrm{Cl} & -0.3275230 & -2.7195670 & -2.0195620\end{array}$

$\begin{array}{llll}\mathrm{N} & 0.0848200 & -1.4904980 & 0.6317360\end{array}$

$\begin{array}{llll}\mathrm{C} & 1.4339680 & -1.3187860 & 0.4183320\end{array}$

C $\quad-0.1690210 \quad-2.4815330 \quad 1.6010800$

$\begin{array}{llll}\text { C } & 2.2146990 & -2.2500960 & 1.3207470\end{array}$

$\begin{array}{llll}\text { C } & 1.1506810 & -3.0198380 & 2.1098960\end{array}$

$\mathrm{H} \quad 2.8814030 \quad-1.6518450 \quad 1.9479080$

$\mathrm{H} \quad 1.2054580 \quad-2.8544120 \quad 3.1894060$

$\mathrm{H} \quad 1.1792360 \quad-4.0995380 \quad 1.9408560$

$\mathrm{H} \quad 2.8423160 \quad-2.8932250 \quad 0.6971150$

O $\quad-1.2894440 \quad-2.8115390 \quad 1.9446560$

$\begin{array}{llll}\text { O } & 1.9138040 & -0.5184570 & -0.3868330\end{array}$

$\begin{array}{llll}\text { C } & 0.0511510 & 0.7837330 & -2.5922080\end{array}$

$\mathrm{H} \quad 0.6112370 \quad-0.1475030 \quad-2.5244570$

$\mathrm{H} \quad-0.8787650 \quad 0.6189110 \quad-3.1435800$

$\begin{array}{llll}\mathrm{H} & 0.6483590 & 1.4966790 & -3.1666690\end{array}$

$\begin{array}{llll}\text { C } & -2.4786610 & 2.8953560 & 2.5975720\end{array}$

$\mathrm{H} \quad-2.2209090 \quad 3.0933550 \quad 3.6432880$

$\begin{array}{llll}\mathrm{H} & -2.5698340 & 3.8628330 & 2.0997370\end{array}$

$\begin{array}{llll}\mathrm{H} & -3.4604050 & 2.4073440 & 2.5879680\end{array}$

$\begin{array}{llll}\text { C } & -0.0648890 & 2.7789510 & 1.8839380\end{array}$

$\begin{array}{llll}\mathrm{H} & -0.1531690 & 3.6705170 & 1.2544100\end{array}$

$\begin{array}{llll}\mathrm{H} & 0.2311490 & 3.1102370 & 2.8851610\end{array}$

$\mathrm{H} \quad 0.7420380 \quad 2.1532840 \quad 1.4901630$

$\begin{array}{llll}\text { C } & -1.2053860 & 0.7767440 & 2.8805530\end{array}$

$\mathrm{H} \quad-2.1085340 \quad 0.1571530 \quad 2.9235220$

$\begin{array}{llll}\mathrm{H} & -0.3682180 & 0.1546080 & 2.5592900\end{array}$

$\mathrm{H} \quad-0.9889660 \quad 1.1118390 \quad 3.9001410$

$\begin{array}{llll}\text { C } & -4.0529870 & -1.5514520 & -0.6573110\end{array}$

$\begin{array}{llll}\text { C } & -4.5709550 & -1.5493350 & 0.6468020\end{array}$

$\begin{array}{llll}\text { C } & -4.6867750 & -0.7619670 & -1.6317890\end{array}$

$\begin{array}{llll}\text { C } & -5.6921380 & -0.7773260 & 0.9717230\end{array}$

$\mathrm{H} \quad-4.0977860 \quad-2.1612420 \quad 1.4099460$

$\begin{array}{llll}\mathrm{C} & -5.8037200 & 0.0125290 & -1.3099850\end{array}$

$\begin{array}{llll}\mathrm{H} & -4.3115380 & -0.7612590 & -2.6524120\end{array}$

$\begin{array}{llll}\mathrm{C} & -6.3102680 & 0.0075480 & -0.0051020\end{array}$

$\begin{array}{llll}\mathrm{H} & -6.0808200 & -0.7928670 & 1.9859130\end{array}$

$\mathrm{H} \quad-6.2821950 \quad 0.6137970 \quad-2.0777230$

$\begin{array}{llll}\mathrm{H} & -7.1812400 & 0.6063630 & 0.2446450\end{array}$

$\begin{array}{llll}\mathrm{H} & 3.4502220 & -0.2769000 & -0.6037500\end{array}$

$\begin{array}{llll}\text { O } & 4.4160170 & -0.0882660 & -0.8688960\end{array}$

$\begin{array}{llll}\text { C } & 5.1693500 & 0.1863350 & 0.1700780\end{array}$ 


$\begin{array}{lrrr}\mathrm{O} & 4.8473470 & 0.2370470 & 1.3382510 \\ \mathrm{C} & 6.6331210 & 0.4522780 & -0.2786510 \\ \mathrm{~F} & 7.1571450 & -0.6422470 & -0.8755220 \\ \mathrm{~F} & 7.4107460 & 0.7653100 & 0.7688980 \\ \mathrm{~F} & 6.6924280 & 1.4722900 & -1.1630920\end{array}$

anti-18b'.log

Potential Energy $=-2233.51605$

Zero-point Energy = -2232.98059

Free Energy $=-2233.02877$

Single-Point Energy B3LYP-D3(BJ)/6-311+G** PCM = 2234.10308

Free Energy B3LYP-D3(BJ)/6-311+G** PCM (extrapolated free energy from qRRHO) $=-2233.61580$

Charge $=0$ Multiplicity $=1$

\begin{tabular}{|c|c|c|c|}
\hline $\mathrm{C}$ & 0.9018130 & -1.7271780 & -0.9590970 \\
\hline $\mathrm{C}$ & 2.4756080 & -3.2785000 & -0.1497600 \\
\hline $\mathrm{C}$ & 3.0426390 & -1.8960290 & 0.1790040 \\
\hline $\mathrm{N}$ & 1.9167800 & -1.0052690 & -0.1549570 \\
\hline $\mathrm{N}$ & 1.2443790 & -3.1291900 & -0.6785010 \\
\hline $\mathrm{O}$ & 3.0338980 & -4.3558530 & 0.0873170 \\
\hline $\mathrm{C}$ & 0.3585820 & -4.2708350 & -0.8738650 \\
\hline $\mathrm{H}$ & 0.4963440 & -4.9633590 & -0.0401170 \\
\hline $\mathrm{H}$ & -0.6762420 & -3.9277280 & -0.8893740 \\
\hline $\mathrm{H}$ & 0.5722030 & -4.8069730 & -1.8036170 \\
\hline $\mathrm{C}$ & 1.5887340 & 0.2349000 & 0.4577310 \\
\hline $\mathrm{H}$ & 0.8024540 & 0.6880210 & -0.1443410 \\
\hline $\mathrm{C}$ & 2.7428850 & 1.2705370 & 0.5925640 \\
\hline $\mathrm{C}$ & 2.2931770 & 2.7013300 & 0.9653110 \\
\hline $\mathrm{H}$ & 3.8953800 & -1.6988840 & -0.4877090 \\
\hline $\mathrm{C}$ & 0.8984730 & -1.3844450 & -2.4960980 \\
\hline $\mathrm{H}$ & -0.0999910 & -1.5210680 & -0.5714640 \\
\hline $\mathrm{H}$ & 3.4602480 & 0.9458330 & 1.3377040 \\
\hline $\mathrm{Cl}$ & 3.7155140 & 1.3128120 & -0.9603400 \\
\hline $\mathrm{N}$ & 0.8896240 & 0.1186550 & 1.8360340 \\
\hline $\mathrm{C}$ & -0.4775180 & -0.0786710 & 1.8911410 \\
\hline $\mathrm{C}$ & 1.4383610 & 0.2043890 & 3.1366820 \\
\hline $\mathrm{C}$ & -0.9379850 & -0.2106690 & 3.3231400 \\
\hline $\mathrm{C}$ & 0.3393300 & -0.0530850 & 4.1501510 \\
\hline $\mathrm{H}$ & -1.4254750 & -1.1817840 & 3.4484310 \\
\hline $\mathrm{H}$ & 0.5984260 & -0.9502620 & 4.7198330 \\
\hline $\mathrm{H}$ & 0.3062870 & 0.7840970 & 4.8517100 \\
\hline $\mathrm{H}$ & -1.6868140 & 0.5603480 & 3.5259430 \\
\hline $\mathrm{O}$ & 2.5997200 & 0.4617410 & 3.3900420 \\
\hline $\mathrm{O}$ & -1.1957140 & -0.1241950 & 0.8879040 \\
\hline $\mathrm{C}$ & 3.5652060 & -1.9190910 & 1.6186660 \\
\hline $\mathrm{H}$ & 4.0298040 & -0.9785590 & 1.9114540 \\
\hline $\mathrm{H}$ & 2.7745400 & -2.1596200 & 2.3342630 \\
\hline $\mathrm{H}$ & 4.3238850 & -2.7037970 & 1.6778550 \\
\hline $\mathrm{C}$ & -0.2001170 & -2.2026000 & -3.2121960 \\
\hline $\mathrm{H}$ & -0.3065260 & -1.8417970 & -4.2407180 \\
\hline $\mathrm{H}$ & 0.0367120 & -3.2670730 & -3.2679150 \\
\hline $\mathrm{H}$ & -1.1747160 & -2.0894060 & -2.7227090 \\
\hline $\mathrm{C}$ & 2.2628350 & -1.6837230 & -3.1419500 \\
\hline $\mathrm{H}$ & 2.5285200 & -2.7427290 & -3.0547750 \\
\hline $\mathrm{H}$ & 2.2294550 & -1.4401900 & -4.2096240 \\
\hline $\mathrm{H}$ & 3.0573670 & -1.0849130 & -2.6881600 \\
\hline $\mathrm{C}$ & 0.5531480 & 0.1073580 & -2.6889910 \\
\hline $\mathrm{H}$ & -0.3975130 & 0.3669720 & -2.2088240 \\
\hline $\mathrm{H}$ & 1.3348850 & 0.7640070 & -2.3033150 \\
\hline $\mathrm{H}$ & 0.4480280 & 0.3201950 & -3.7583010 \\
\hline $\mathrm{H}$ & 1.8484580 & 2.6465940 & 1.9637900 \\
\hline $\mathrm{H}$ & 3.2072590 & 3.2922480 & 1.0751620 \\
\hline $\mathrm{C}$ & 1.3379770 & 3.4111440 & 0.0244960 \\
\hline $\mathrm{C}$ & -0.0499260 & 3.3584040 & 0.2285570 \\
\hline $\mathrm{C}$ & 1.8218200 & 4.1743640 & -1.0510960 \\
\hline $\mathrm{C}$ & -0.9315460 & 4.0268550 & -0.6267690 \\
\hline $\mathrm{H}$ & -0.4502530 & 2.8041740 & 1.0727480 \\
\hline $\mathrm{C}$ & 0.9452390 & 4.8453150 & -1.9079790 \\
\hline $\mathrm{H}$ & 2.8926520 & 4.2496740 & -1.2140470 \\
\hline $\mathrm{C}$ & -0.4362110 & 4.7700890 & -1.7015760 \\
\hline $\mathrm{H}$ & -2.0013360 & 3.9721500 & -0.4470400 \\
\hline $\mathrm{H}$ & 1.3418590 & 5.4314920 & -2.7320840 \\
\hline $\mathrm{H}$ & -1.1181100 & 5.2937830 & -2.3651760 \\
\hline $\mathrm{H}$ & -2.7786970 & -0.3152100 & 0.9029630 \\
\hline $\mathrm{O}$ & -3.7789470 & -0.4726670 & 0.9595420 \\
\hline
\end{tabular}

$\begin{array}{lrrr}\text { C } & -4.3987220 & -0.1648870 & -0.1577250 \\ \text { O } & -3.9247170 & 0.2621700 & -1.1875490 \\ \text { C } & -5.9241100 & -0.4246520 & -0.0130270 \\ \text { F } & -6.1675200 & -1.7218550 & 0.2795830 \\ \text { F } & -6.5747430 & -0.1260870 & -1.1474420 \\ \text { F } & -6.4488920 & 0.3273980 & 0.9804430\end{array}$

Int4'.log

Potential Energy $=-2233.52515$

Zero-point Energy = -2232.99167

Free Energy $=-2233.04178$

Single-Point Energy B3LYP-D3(BJ)/6-311+G** PCM = 2234.09953

Free Energy B3LYP-D3(BJ)/6-311+G** PCM (extrapolated free energy from $q R R H O)=-2233.61616$

Charge $=0$ Multiplicity $=1$

\begin{tabular}{|c|c|c|c|}
\hline & & & \\
\hline $\mathrm{C}$ & 0.5057100 & 2.0118400 & -0.5734890 \\
\hline $\mathrm{C}$ & 0.3276840 & 3.3585180 & 1.3565140 \\
\hline & -1.0855050 & 2.7958810 & 1.1348800 \\
\hline & -0.8166260 & 1.7463180 & 0.1044920 \\
\hline & 1.1647370 & 2.8349480 & 0.4340460 \\
\hline & 0.6298980 & 4.1230640 & 2.2678620 \\
\hline & 2.6145810 & 3.0071770 & 0.5321140 \\
\hline $\mathrm{H}$ & 2.8852540 & 3.0151490 & 1.5896410 \\
\hline & 3.1158550 & 2.1749730 & 0.0372260 \\
\hline & 2.9390320 & 3.9491610 & 0.0818730 \\
\hline$C$ & -1.5083000 & 0.7082520 & -0.2162790 \\
\hline 11 & -1.0354380 & 0.0051190 & -0.913419 \\
\hline $\mathrm{C}$ & -2.8825480 & 0.3440270 & 0.2850680 \\
\hline $\mathrm{C}$ & -3.3183950 & -1.0261410 & -0.25573 \\
\hline $\mathrm{H}$ & -1.7141450 & 3.5636590 & 0.671347 \\
\hline $\mathrm{C}$ & 0.3429650 & 2.6831150 & -1.987417 \\
\hline $\mathrm{H}$ & 1.0172930 & 1.0476930 & -0.6744390 \\
\hline $\mathrm{H}$ & -2.9127650 & 0.3481070 & \\
\hline $\mathrm{Cl}$ & -4.0387350 & 1.6686030 & -0.2374 \\
\hline $\mathrm{N}$ & 4.1818220 & -1.1429250 & 0.894054 \\
\hline $\mathrm{C}$ & 5.1854980 & -0.2369080 & \\
\hline $\mathrm{C}$ & 4.4671940 & -1.9680830 & 1.967681 \\
\hline $\mathrm{C}$ & 6.3226990 & -0.4512430 & \\
\hline $\mathrm{C}$ & 5.8474520 & -1.6016200 & 2.496374 \\
\hline $\mathrm{H}$ & 6.4992380 & 0.4816390 & 2.136392 \\
\hline $\mathrm{H}$ & 5.7598100 & -1.3191690 & 3.54 \\
\hline $\mathrm{H}$ & 6.4903840 & -2.4849620 & 2.447702 \\
\hline $\mathrm{H}$ & 7.2358910 & -0.6838740 & \\
\hline $\mathrm{O}$ & 3.7176470 & -2.8341710 & 2.39726 \\
\hline $\mathrm{O}$ & 5.1367910 & 0.5887320 & -0.298096 \\
\hline $\mathrm{C}$ & -1.6968020 & 2.3327890 & 2.455462 \\
\hline $\mathrm{H}$ & -2.7738770 & 2.1839050 & 2.38539 \\
\hline $\mathrm{H}$ & -1.2109470 & 1.4286240 & 2.831287 \\
\hline $\mathrm{H}$ & -1.5208150 & 3.1359420 & 3.17486 \\
\hline $\mathrm{C}$ & 1.7610040 & 2.8547710 & -2.581174 \\
\hline $\mathrm{H}$ & 1.6713370 & 3.1987690 & -3.615812 \\
\hline $\mathrm{H}$ & 2.3504620 & 3.5985690 & -2.041810 \\
\hline $\mathrm{H}$ & 2.3141190 & 1.9098220 & \\
\hline $\mathrm{C}$ & -0.3487320 & 4.0560700 & -1.90520 \\
\hline $\mathrm{H}$ & 0.1737970 & 4.7412470 & -1.23049 \\
\hline $\mathrm{H}$ & -0.3536470 & 4.5155160 & -2.89830 \\
\hline $\mathrm{H}$ & -1.3930290 & 3.9735300 & -1.58733 \\
\hline $\mathrm{C}$ & -0.4549220 & 1.7575590 & \\
\hline $\mathrm{H}$ & -0.0329230 & 0.7485720 & -2.96908 \\
\hline $\mathrm{H}$ & -1.5111910 & 1.6845070 & -2.65068 \\
\hline $\mathrm{H}$ & -0.4257660 & 2.1741260 & -3.93961 \\
\hline $\mathrm{H}$ & -2.5049960 & -1.7207440 & -0.011313 \\
\hline $\mathrm{H}$ & -3.3780880 & -0.9798880 & \\
\hline 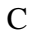 & -4.6195300 & -1.5323630 & 0.330467 \\
\hline $\mathrm{C}$ & -5.8015790 & -1.5146080 & -0.42284 \\
\hline & -4.6613630 & -2.0372810 & 1.63984 \\
\hline & -7.0023260 & -1.9818210 & 0.121278 \\
\hline $\mathrm{H}$ & -5.7830340 & -1.1361010 & -1.44125 \\
\hline 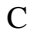 & -5.8598210 & -2.5017960 & 2.18753 \\
\hline & -3.7509370 & -2.0771790 & 2.233341 \\
\hline & -7.0352610 & -2.4738830 & 1.429148 \\
\hline & -7.9083690 & -1.9622140 & -0.477379 \\
\hline & -5.8739030 & -2.8915890 & 3.201210 \\
\hline & -7.9665830 & -2.8384240 & 1.852532 \\
\hline & -0.1177520 & -1.6251080 & -1.686312 \\
\hline
\end{tabular}




$\begin{array}{llll}\text { H } & 3.2990100 & -1.1972780 & 0.3561010 \\ \text { C } & 1.0348540 & -1.7967120 & -1.2376970 \\ \text { O } & 1.7120970 & -1.0605120 & -0.4795400 \\ \text { C } & 1.7065260 & -3.1343570 & -1.7007020 \\ \text { F } & 1.0135460 & -4.2109070 & -1.2423040 \\ \text { F } & 2.9815280 & -3.2776250 & -1.2715580 \\ \text { F } & 1.7351460 & -3.2311330 & -3.0554200\end{array}$

Int $4 a^{\prime} \cdot \log$

Potential Energy = -1949.25759

Zero-point Energy = -1948.79233

Free Energy = -1948.83881

Single-Point Energy B3LYP-D3(BJ)/6-311+G** PCM = 1949.75178

Free Energy B3LYP-D3(BJ)/6-311+G** PCM (extrapolated free energy from $q R R H O)=-1949.33300$ Charge $=0$ Multiplicity $=1$

\begin{tabular}{|c|c|c|c|}
\hline $\mathrm{C}$ & 3.3793040 & -0.3438340 & -0.6281640 \\
\hline $\mathrm{C}$ & 3.0655760 & -1.7154410 & 1.2670310 \\
\hline $\mathrm{C}$ & 2.0713280 & -0.5588050 & 1.4469340 \\
\hline $\mathrm{N}$ & 2.1267590 & 0.0767900 & 0.0955720 \\
\hline $\mathrm{N}$ & 3.7079030 & -1.5715620 & 0.0841130 \\
\hline $\mathrm{O}$ & 3.2075540 & -2.6371710 & 2.0632940 \\
\hline $\mathrm{C}$ & 4.5314220 & -2.6463780 & -0.4699910 \\
\hline $\mathrm{H}$ & 4.0957420 & -3.5999170 & -0.1666090 \\
\hline $\mathrm{H}$ & 4.5296550 & -2.5780160 & -1.5581430 \\
\hline $\mathrm{H}$ & 5.5601560 & -2.5981440 & -0.1046600 \\
\hline $\mathrm{C}$ & 1.2463370 & 0.7983050 & -0.5085210 \\
\hline $\mathrm{H}$ & 1.4831790 & 1.0792040 & -1.5273280 \\
\hline $\mathrm{C}$ & -0.0153530 & 1.3863510 & 0.0716440 \\
\hline $\mathrm{C}$ & -0.8112710 & 2.1515590 & -0.9940500 \\
\hline $\mathrm{H}$ & -0.1991810 & 2.9712710 & -1.3824930 \\
\hline $\mathrm{H}$ & 2.4811850 & 0.1582300 & 2.1664910 \\
\hline $\mathrm{C}$ & 4.4970840 & 0.7601150 & -0.6261380 \\
\hline $\mathrm{H}$ & 3.0958370 & -0.5743460 & -1.6597580 \\
\hline $\mathrm{H}$ & -0.6306090 & 0.6118450 & 0.5263510 \\
\hline $\mathrm{Cl}$ & 0.4814650 & 2.4992290 & 1.4445480 \\
\hline $\mathrm{C}$ & 0.7142870 & -1.0725110 & 1.9225470 \\
\hline $\mathrm{H}$ & 0.0921170 & -0.2741970 & 2.3267010 \\
\hline $\mathrm{H}$ & 0.1770710 & -1.6039630 & 1.1334990 \\
\hline $\mathrm{H}$ & 0.9171910 & -1.7758980 & 2.7336140 \\
\hline $\mathrm{C}$ & 5.7146760 & 0.2024240 & -1.3989070 \\
\hline $\mathrm{H}$ & 6.4513870 & 1.0019410 & -1.5196940 \\
\hline $\mathrm{H}$ & 6.2062700 & -0.6138700 & -0.8665150 \\
\hline $\mathrm{H}$ & 5.4385350 & -0.1465920 & -2.3998790 \\
\hline $\mathrm{C}$ & 4.9252890 & 1.1383090 & 0.8036840 \\
\hline $\mathrm{H}$ & 5.2627510 & 0.2666420 & 1.3727740 \\
\hline $\mathrm{H}$ & 5.7620170 & 1.8415450 & 0.7547120 \\
\hline $\mathrm{H}$ & 4.1218280 & 1.6338760 & 1.3579490 \\
\hline $\mathrm{C}$ & 4.0029380 & 2.0184970 & -1.3694640 \\
\hline $\mathrm{H}$ & 3.6566680 & 1.7884750 & -2.3836170 \\
\hline $\mathrm{H}$ & 3.2070660 & 2.5427280 & -0.8326210 \\
\hline $\mathrm{H}$ & 4.8347470 & 2.7229210 & -1.4617320 \\
\hline $\mathrm{H}$ & -0.9668460 & 1.4411140 & -1.8146340 \\
\hline $\mathrm{C}$ & -2.1478430 & 2.6722430 & -0.5071500 \\
\hline $\mathrm{C}$ & -2.3787170 & 4.0488380 & -0.3837030 \\
\hline $\mathrm{C}$ & -3.1831380 & 1.7791330 & -0.1860950 \\
\hline $\mathrm{C}$ & -3.6168370 & 4.5283550 & 0.0572630 \\
\hline $\mathrm{H}$ & -1.5877290 & 4.7508080 & -0.6342430 \\
\hline $\mathrm{C}$ & -4.4186690 & 2.2564510 & 0.2584840 \\
\hline $\mathrm{H}$ & -3.0231550 & 0.7078980 & -0.2857540 \\
\hline $\mathrm{C}$ & -4.6396370 & 3.6328990 & 0.3816820 \\
\hline $\mathrm{H}$ & -3.7793100 & 5.5986310 & 0.1463910 \\
\hline $\mathrm{H}$ & -5.2101650 & 1.5537520 & 0.5027270 \\
\hline $\mathrm{H}$ & -5.6016240 & 4.0029630 & 0.7242030 \\
\hline $\mathrm{O}$ & 0.1323700 & -1.0197810 & -1.9114570 \\
\hline $\mathrm{H}$ & -0.7084270 & -1.3127920 & -1.4605150 \\
\hline $\mathrm{O}$ & -2.0214000 & -1.6607310 & -0.4697650 \\
\hline $\mathrm{C}$ & -2.8574200 & -2.4955140 & -0.9143120 \\
\hline $\mathrm{O}$ & -2.8958700 & -3.0930550 & -2.0015370 \\
\hline $\mathrm{C}$ & -4.0140370 & -2.7960410 & 0.1011900 \\
\hline $\mathrm{F}$ & -4.6918040 & -1.6631090 & 0.4324370 \\
\hline $\mathrm{F}$ & -3.5308240 & -3.3170380 & 1.2624030 \\
\hline $\mathrm{F}$ & -4.9305710 & -3.6737670 & -0.3663180 \\
\hline $\mathrm{H}$ & -0.0882440 & -0.9181980 & -2.8470760 \\
\hline
\end{tabular}

Int $5^{\prime} \cdot \log$

Potential Energy = -1949.26099

Zero-point Energy = -1948.79335

Free Energy = -1948.83855

Single-Point Energy B3LYP-D3(BJ)/6-311+G** PCM = 1949.75299

Free Energy B3LYP-D3(BJ)/6-311+G** PCM (extrapolated free energy from $\mathrm{qRRHO})=-1949.33054$

Charge $=0$ Multiplicity $=1$

$\begin{array}{llll}\text { C } & 3.1423700 & -0.4627680 & -0.5876620\end{array}$

C $\quad 2.9921210 \quad-1.5649160 \quad 1.4859430$

C $\quad 2.1576970 \quad-0.2863180 \quad 1.6051260$

$\begin{array}{llll}\mathrm{N} & 2.0736570 & 0.1905140 & 0.2039530\end{array}$

$\mathrm{N} \quad 3.4923300 \quad-1.6309230 \quad 0.2328640$

$\begin{array}{llll}\mathrm{O} & 3.1703980 & -2.3979020 & 2.3809440\end{array}$

$\begin{array}{llll}\text { C } & 4.2071650 & -2.8073760 & -0.2464530\end{array}$

$\mathrm{H} \quad 3.7892620 \quad-3.6889490 \quad 0.2444790$

$\begin{array}{llll}\mathrm{H} & 4.0751140 & -2.8992690 & -1.3256940\end{array}$

$\begin{array}{llll}\mathrm{H} & 5.2768250 & -2.7614000 & -0.0201730\end{array}$

$\begin{array}{llll}\text { C } & 0.8585870 & 0.4326410 & -0.4897690\end{array}$

$\begin{array}{llll}\mathrm{H} & 1.1289170 & 0.7414440 & -1.5018270\end{array}$

$\begin{array}{llll}\text { C } & -0.1027130 & 1.5014070 & 0.0885170\end{array}$

$\begin{array}{llll}\mathrm{C} & -1.0741420 & 2.0110830 & -0.9896530\end{array}$

$\begin{array}{llll}\mathrm{H} & -0.5034910 & 2.5569490 & -1.7480220\end{array}$

$\begin{array}{llll}\mathrm{H} & 2.7526370 & 0.4336970 & 2.1851800\end{array}$

$\begin{array}{llll}\mathrm{C} & 4.3400090 & 0.4921030 & -0.9247020\end{array}$

$\begin{array}{llll}\mathrm{H} & 2.7382860 & -0.8231920 & -1.5436500\end{array}$

$\mathrm{H} \quad-0.6570520 \quad 1.0883880 \quad 0.9294940$

$\begin{array}{llll}\mathrm{Cl} & 0.8293510 & 2.9065140 & 0.7952750\end{array}$

$\begin{array}{llll}\text { C } & 0.8714750 & -0.5461420 & 2.3909940\end{array}$

$\begin{array}{llll}\mathrm{H} & 0.3586270 & 0.3833330 & 2.6444180\end{array}$

H $\quad 0.1875730 \quad-1.2113390 \quad 1.8614600$

$\mathrm{H} \quad 1.1583290 \quad-1.0288010 \quad 3.3289790$

C $\quad 5.3866790 \quad-0.2467700 \quad-1.7861810$

$\begin{array}{llll}\mathrm{H} & 6.1276800 & 0.4709600 & -2.1537010\end{array}$

$\mathrm{H} \quad 5.9280450 \quad-1.0101580 \quad-1.2225320$

$\begin{array}{llll}\mathrm{H} & 4.9283600 & -0.7234670 & -2.6607870\end{array}$

$\begin{array}{llll}\mathrm{C} & 5.0109370 & 1.0273390 & 0.3528200\end{array}$

$\begin{array}{llll}\mathrm{H} & 5.3934650 & 0.2149320 & 0.9800920\end{array}$

$\begin{array}{llll}\mathrm{H} & 5.8600520 & 1.6656520 & 0.0857520\end{array}$

$\begin{array}{llll}\mathrm{H} & 4.3166790 & 1.6300500 & 0.9455750\end{array}$

$\begin{array}{llll}\text { C } & 3.7922260 & 1.6745430 & -1.7507400\end{array}$

$\mathrm{H} \quad 3.3130630 \quad 1.3313720 \quad-2.6761220$

$\begin{array}{llll}\mathrm{H} & 3.0688460 & 2.2622880 & -1.1800780\end{array}$

$\begin{array}{llll}\mathrm{H} & 4.6158060 & 2.3377890 & -2.0356930\end{array}$

$\mathrm{H} \quad-1.4886810 \quad 1.1219530 \quad-1.4780330$

$\begin{array}{llll}\text { C } & -2.2063340 & 2.8713970 & -0.4668650\end{array}$

C $\quad-2.2318620 \quad 4.2527590 \quad-0.7052430$

$\begin{array}{llll}\text { C } & -3.2628110 & 2.2970000 & 0.2587190\end{array}$

$\begin{array}{llll}\mathrm{C} & -3.2803140 & 5.0446410 & -0.2249160\end{array}$

$\mathrm{H} \quad-1.4262070 \quad 4.7128840 \quad-1.2712090$

C $\quad-4.3102960 \quad 3.0845900 \quad 0.7433830$

$\begin{array}{llll}\mathrm{H} & -3.2718190 & 1.2249810 & 0.4405740\end{array}$

$\begin{array}{llll}\text { C } & -4.3220010 & 4.4631320 & 0.5034780\end{array}$

$\mathrm{H} \quad-3.2819480 \quad 6.1130930 \quad-0.4210960$

$\begin{array}{llll}\mathrm{H} & -5.1194000 & 2.6215280 & 1.3010730\end{array}$

$\mathrm{H} \quad-5.1375710 \quad 5.0758540 \quad 0.8764310$

$\mathrm{O} \quad-0.0120260 \quad-0.7576120 \quad-0.6406340$

$\mathrm{H} \quad-1.4565980 \quad-1.3224630 \quad-0.1375430$

$\begin{array}{llll}\mathrm{O} & -2.3365630 & -1.7312320 & 0.1332700\end{array}$

C $\quad-2.6225620 \quad-2.7602200 \quad-0.6360220$

O $\quad-1.9677270 \quad-3.2072360 \quad-1.5521230$

C $\quad-3.9846380 \quad-3.3826390 \quad-0.2247640$

$\begin{array}{llll}\text { F } & -4.9855390 & -2.4886020 & -0.3857620\end{array}$

$\begin{array}{llll}\mathrm{F} & -4.9855390 & -2.4886020 & -0.3857620 \\ \mathrm{~F} & -3.9724210 & -3.7606370 & 1.0718730\end{array}$

F $\quad-4.2650080 \quad-4.4608080 \quad-0.9709960$

$\mathrm{H} \quad 0.2688110 \quad-1.2766220 \quad-1.4085040$ 


\section{Transition States}

Formation of carbinol amine (TS1)

$\begin{array}{cc}\text { Conformation } & \begin{array}{c}\text { Extrapolated } \\ \text { name }\end{array} \\ \begin{array}{c}\text { Free energy } \\ (\mathrm{kcal} / \mathrm{mol})\end{array}\end{array}$

$\begin{array}{cc}\text { TS1-lowest } & 0.0 \\ 1 & 0.9 \\ 2 & 0.1 \\ 3 & 1.5 \\ 4 & 2.3 \\ 5 & 1.4 \\ 6 & 1.5 \\ 7 & 0.5\end{array}$

TS1-lowest.log

Potential Energy = -1489.66362

Zero-point Energy = -1489.18887

Free Energy = -1489.23186

Single-Point Energy B3LYP-D3(BJ)/6-311+G** PCM = 1490.12258

Free Energy B3LYP-D3(BJ)/6-311+G** PCM (extrapolated free energy from $\mathrm{qRRHO})=-1489.69083$

Nimag $=1(-162.6618)$

Charge $=0$ Multiplicity $=1$

\begin{tabular}{|c|c|c|c|}
\hline 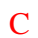 & 1.8517090 & -0.8511400 & 1.6926950 \\
\hline $\mathrm{H}$ & 2.0070440 & -1.7005300 & 1.0261210 \\
\hline & 1.4013680 & -1.2130030 & 2.6208840 \\
\hline & 3.2250410 & -0.2001630 & 2.0381640 \\
\hline & 0.9361530 & 0.1470150 & 1.0546780 \\
\hline & 0.4214710 & 1.0438850 & 1.8105020 \\
\hline & -0.0740900 & 1.8766850 & 1.2616730 \\
\hline & 1.0578790 & 0.3821560 & -0.0013810 \\
\hline & -0.6648440 & 2.8873570 & 0.6484180 \\
\hline & -1.7764790 & 2.5928830 & 0.0968280 \\
\hline & -2.4661420 & 3.8293810 & -0.5562970 \\
\hline & -1.7045350 & 4.3270220 & -1.5635700 \\
\hline & -3.6739420 & 3.5343710 & -1.0747810 \\
\hline & -2.6448290 & 4.8269570 & 0.3433280 \\
\hline & -2.3375590 & 1.4956530 & 0.0125340 \\
\hline & 3.7872370 & -0.9518640 & 2.6022390 \\
\hline & 4.0319780 & 0.2445030 & 0.8336160 \\
\hline & 4.7148170 & -0.6966460 & 0.0456070 \\
\hline $\mathrm{C}$ & 4.1130900 & 1.5989250 & 0.4760850 \\
\hline & 5.4509620 & -0.2970820 & -1.0728450 \\
\hline & 4.6757730 & -1.7500000 & 0.3134110 \\
\hline & 4.8487960 & 2.0035020 & -0.64318 \\
\hline $\mathrm{H}$ & 3.6020130 & 2.3446700 & 1.0800220 \\
\hline & 5.5186850 & 1.0561710 & -1.4225760 \\
\hline & 5.9752980 & -1.0401360 & -1.6670750 \\
\hline & 4.9001820 & 3.0573670 & -0.9016440 \\
\hline & & & -2.2901460 \\
\hline $\mathrm{H}$ & 3.0559370 & 0.6454640 & 2.7114060 \\
\hline & -0.8125710 & -1.0379910 & 0.534558 \\
\hline 0 & -1.2744170 & -1.9646060 & 1.6063180 \\
\hline $\mathrm{C}$ & -1.2471790 & -1.6343010 & -0.779525 \\
\hline C & -2.4527290 & & \\
\hline $\mathrm{H}$ & -0.4871050 & -2.6962800 & 1.819046 \\
\hline & -1.5765160 & -0.8042420 & -1.412960 \\
\hline $\mathrm{O}$ & -3.2962320 & -3.3773790 & 1.538034 \\
\hline $\mathrm{N}$ & -2.4280920 & -2.4223760 & -0.373375 \\
\hline $\mathrm{C}$ & -3.5480770 & -2.7692240 & -1.242741 \\
\hline & -3.4840610 & -3.7987480 & -1.606987 \\
\hline & -4.4743010 & -2.6615600 & -0.673672 \\
\hline & -3.5688350 & -2.0892630 & -2.095258 \\
\hline & -0.1805590 & -2.4460100 & -1.584170 \\
\hline & -0.8325990 & -2.9522400 & -2.892842 \\
\hline & -1.5888910 & -3.7185880 & -2.71262 \\
\hline
\end{tabular}

$\begin{array}{lrrr}\mathrm{H} & -1.2928600 & -2.1352340 & -3.4600700 \\ \mathrm{H} & -0.0615370 & -3.4008130 & -3.5269190 \\ \mathrm{C} & 0.9802190 & -1.5134500 & -1.9876800 \\ \mathrm{H} & 0.6135150 & -0.5892060 & -2.4491230 \\ \mathrm{H} & 1.6204850 & -1.2506450 & -1.1449520 \\ \mathrm{H} & 1.6167270 & -2.0179160 & -2.7214270 \\ \mathrm{C} & 0.3584270 & -3.6590820 & -0.8036040 \\ \mathrm{H} & -0.4461780 & -4.3447160 & -0.5187960 \\ \mathrm{H} & 1.0597230 & -4.2178330 & -1.4320280 \\ \mathrm{H} & 0.8974220 & -3.3659480 & 0.1013920 \\ \mathrm{C} & -1.6886230 & -1.2641990 & 2.8982030 \\ \mathrm{H} & -0.8400980 & -0.7734750 & 3.3776320 \\ \mathrm{H} & -2.4619780 & -0.5130660 & 2.7077140 \\ \mathrm{H} & -2.0961040 & -2.0077730 & 3.5874050 \\ \mathrm{H} & -1.3774290 & -0.1821550 & 0.6063230\end{array}$

1.log

Potential Energy $=-1489.66305$

Zero-point Energy = -1489.18786

Free Energy = -1489.23113

Single-Point Energy B3LYP-D3(BJ)/6-311+G** PCM = 1490.12134

Free Energy B3LYP-D3(BJ)/6-311+G** PCM (extrapolated free energy from $q$ RRHO) $=-1489.68941$

Nimag $=1(-133.0078)$

Charge $=0$ Multiplicity $=1$

C 1.3952340

$-2.0585190-0.2956850$

$\begin{array}{llll}\mathrm{H} & 0.6543910 & -2.7384950 & -0.7173550\end{array}$

H $\quad 1.5549830 \quad-2.3234810 \quad 0.7525740$

$\begin{array}{llll}\mathrm{C} & 2.7223600 & -2.2428810 & -1.0968820\end{array}$

$\begin{array}{llll}\text { C } & 0.8997970 & -0.6508130 & -0.3909580\end{array}$

$\begin{array}{llll}\text { O } & 1.3984420 & 0.2147100 & 0.4164610\end{array}$

$\mathrm{H} \quad 1.1683160 \quad 1.2481300 \quad 0.1533250$

$\mathrm{H} \quad 0.5363960 \quad-0.2916880 \quad-1.3530400$

$\begin{array}{llll}\text { O } & 0.9013840 & 2.5506950 & -0.1393410\end{array}$

C $\quad-0.3032820 \quad 2.9011140 \quad 0.0743550$

$\begin{array}{llll}\text { C } & -0.5365840 & 4.4259900 & -0.1568090\end{array}$

F $\quad-0.1999490 \quad 4.7857890 \quad-1.4207210$

F $\quad-1.8194550 \quad 4.7914340 \quad 0.0351080$

F $\quad 0.2243440 \quad 5.1668250 \quad 0.6875980$

$\begin{array}{llll}\text { O } & -1.2666370 & 2.2105270 & 0.4283730\end{array}$

$\begin{array}{llll}\mathrm{H} & 2.8918930 & -3.3241100 & -1.1457100\end{array}$

$\begin{array}{llll}\mathrm{N} & -1.1276380 & -0.7687640 & 0.3082940\end{array}$

C $\quad-1.3055380 \quad-1.5724850 \quad 1.5509810$

$\begin{array}{llll}\text { C } & -2.4312110 & -0.8364550 & -0.4483670\end{array}$

C $\quad-2.8214460 \quad-1.5322140 \quad 1.7740390$

$\mathrm{H} \quad-1.0095870 \quad-2.6095680 \quad 1.3581450$

$\begin{array}{llll}\mathrm{H} & -2.5984200 & 0.1555130 & -0.8803050\end{array}$

$\begin{array}{llll}\mathrm{O} & -3.4079200 & -1.8478030 & 2.8120990\end{array}$

$\begin{array}{llll}\mathrm{N} & -3.3967640 & -1.0496250 & 0.6482010\end{array}$

$\begin{array}{llll}\text { C } & -4.8041900 & -0.6638800 & 0.6185940\end{array}$

$\begin{array}{llll}\mathrm{H} & -5.4600800 & -1.5120210 & 0.4016090\end{array}$

$\mathrm{H} \quad-5.0726100 \quad-0.2620670 \quad 1.5982070$

$\begin{array}{llll}\mathrm{H} & -4.9529060 & 0.1066340 & -0.1388470\end{array}$

$\begin{array}{llll}\mathrm{C} & -2.5387860 & -1.8794290 & -1.6086610\end{array}$

$\begin{array}{llll}\text { C } & -3.9394450 & -1.7492500 & -2.2536400\end{array}$

$\mathrm{H} \quad-4.7378150 \quad-2.0848270 \quad-1.5891580$

$\mathrm{H} \quad-4.1510560 \quad-0.7185810 \quad-2.5600750$

$\begin{array}{llll}\mathrm{H} & -3.9800800 & -2.3751560 & -3.1504490\end{array}$

$\begin{array}{llll}\mathrm{C} & -1.5046070 & -1.5463420 & -2.7040070\end{array}$

$\begin{array}{llll}\mathrm{H} & -1.5451900 & -0.4889660 & -2.9901390\end{array}$

$\begin{array}{llll}\mathrm{H} & -0.4842290 & -1.7870390 & -2.4039890\end{array}$

$\mathrm{H} \quad-1.7176530 \quad-2.1381650 \quad-3.5997510$

$\begin{array}{llll}\text { C } & -2.3545080 & -3.3304070 & -1.1269920\end{array}$

$\mathrm{H} \quad-3.0841800 \quad-3.5945350 \quad-0.3548720$

$\mathrm{H} \quad-2.5005830 \quad-4.0171980 \quad-1.9671150$

$\mathrm{H} \quad-1.3524710 \quad-3.5143660 \quad-0.7299250$

$\begin{array}{llll}\text { C } & -0.5396960 & -1.0353980 & 2.7574440\end{array}$

$\mathrm{H} \quad 0.5391090 \quad-1.1023900 \quad 2.6092910$

H $\quad-0.7993970 \quad 0.0094530 \quad 2.9558850$

$\begin{array}{llll}\mathrm{H} & -0.8087180 & -1.6271620 & 3.6357470\end{array}$

$\mathrm{H} \quad-1.0704100 \quad 0.2198650 \quad 0.5867710$

$\begin{array}{llll}\mathrm{H} & 2.5724970 & -1.9048860 & -2.1277910\end{array}$

C $\quad 3.9415430 \quad-1.5664760 \quad-0.5046010$

C $4.5174920 \quad-0.4455590 \quad-1.1196000$

$\begin{array}{llll}\text { C } & 4.5319200 & -2.0612450 & 0.6699130\end{array}$ 


$\begin{array}{lrrr}\mathrm{C} & 5.6483880 & 0.1720030 & -0.5743510 \\ \mathrm{H} & 4.0795290 & -0.0518050 & -2.0335340 \\ \mathrm{C} & 5.6601160 & -1.4472440 & 1.2192630 \\ \mathrm{H} & 4.1094150 & -2.9371500 & 1.1568430 \\ \mathrm{C} & 6.2224610 & -0.3258920 & 0.5986110 \\ \mathrm{H} & 6.0789280 & 1.0392140 & -1.0670180 \\ \mathrm{H} & 6.1030400 & -1.8465780 & 2.1273130 \\ \mathrm{H} & 7.1009810 & 0.1509690 & 1.0234210\end{array}$

$2 . \log$

Potential Energy = -1489.66496

Zero-point Energy = -1489.19036

Free Energy $=-1489.23392$

Single-Point Energy B3LYP-D3(BJ)/6-311+G** PCM = 1490.12166

Free Energy B3LYP-D3(BJ)/6-311+G** PCM (extrapolated free energy from qRRHO) $=-1489.69061$

Nimag $=1(-159.5359)$

Charge $=0$ Multiplicity $=1$

C $\quad-1.9653850$

$\mathrm{H}-2.2411440-0.1988790$

$\mathrm{H} \quad-2.1445120 \quad-0.5648650 \quad 1.1787030$

C $\quad-2.8470350 \quad-1.8908900 \quad-0.4008790$

C $\quad-0.5229010 \quad-1.0335570 \quad-0.1203970$

$\begin{array}{llll}\text { O } & 0.0367360 & -1.8601330 & 0.6826190\end{array}$

$\mathrm{H} \quad 1.0298210 \quad-2.2281050 \quad 0.3435010$

$\mathrm{H} \quad-0.1213740 \quad-0.9566050 \quad-1.1303810$

$\begin{array}{llll}\text { O } & 2.2120980 & -2.6975850 & -0.0244520\end{array}$

$\begin{array}{llll}\text { C } & 3.1570820 & -1.8426210 & 0.0234930\end{array}$

$\begin{array}{llll}\text { C } & 4.5447200 & -2.4646840 & -0.3217580\end{array}$

$\begin{array}{llll}\mathrm{F} & 4.5342030 & -3.0264980 & -1.5560110 \\ \mathrm{~F} & 5.5386610 & -1.5555300 & -0.3030570\end{array}$

$\begin{array}{llll}\text { F } & 5.5386610 & -1.5555300 & -0.3030570\end{array}$

F $\quad 4.8723890 \quad-3.4403360 \quad 0.5616880$

O $3.1057220 \quad-0.6393620 \quad 0.2981610$

$\mathrm{H} \quad-2.5724640 \quad-2.7984990 \quad 0.1452100$

$\begin{array}{llll}\mathrm{N} & 0.4916070 & 0.8218670 & 0.3783950\end{array}$

$\begin{array}{llll}\text { C } & 0.0210830 & 1.4512440 & 1.6441730\end{array}$

$\begin{array}{llll}\text { C } & 1.0179580 & 1.9310480 & -0.4958790\end{array}$

$\begin{array}{llll}\text { C } & 0.8347450 & 2.7483000 & 1.7117850\end{array}$

$\begin{array}{llll}\mathrm{H} & -1.0395140 & 1.7097530 & 1.5494400\end{array}$

$\mathrm{H} \quad 1.9127700 \quad 1.5458770 \quad-0.9956940$

O $\quad 0.9590800-3.4734240-2.7019940$

$\begin{array}{llll}\mathrm{N} & 1.4256300 & 2.9337000 & 0.5087110\end{array}$

$\begin{array}{llll}\text { C } & 2.4563230 & 3.9488180 & 0.3158100\end{array}$

$\begin{array}{llll}\mathrm{H} & 2.0310410 & 4.9303660 & 0.0868400\end{array}$

$\mathrm{H} \quad 3.0373150 \quad 4.0309630 \quad 1.2372090$

$\begin{array}{llll}\mathrm{H} & 3.1154660 & 3.6457920 & -0.4985480\end{array}$

C $\quad 0.0622600 \quad 2.4852040 \quad-1.6026320$

$\begin{array}{llll}\text { C } & 0.8123240 & 3.5825990 & -2.3948410\end{array}$

$\mathrm{H} \quad 0.9933910 \quad 4.4785980 \quad-1.7981010$

$\begin{array}{llll}\mathrm{H} & 1.7723400 & 3.2206810 & -2.7801410\end{array}$

$\begin{array}{llll}\mathrm{H} & 0.2043280 & 3.8831080 & -3.2538160\end{array}$

$\begin{array}{llll}\text { C } & -0.2800470 & 1.3605290 & -2.6019440\end{array}$

$\begin{array}{llll}\mathrm{H} & 0.6199480 & 0.8382900 & -2.9466160\end{array}$

$\begin{array}{llll}\mathrm{H} & -0.9699960 & 0.6257350 & -2.1856990\end{array}$

$\begin{array}{llll}\mathrm{H} & -0.7691490 & 1.7898930 & -3.4819870\end{array}$

C $\quad-1.2290100 \quad 3.0931700 \quad-1.0243210$

$\mathrm{H} \quad-1.0129680 \quad 3.9051040 \quad-0.3225060$

$\begin{array}{llll}\mathrm{H} & -1.8332580 & 3.5114700 & -1.8359840\end{array}$

$\begin{array}{llll}\mathrm{H} & -1.8458440 & 2.3506050 & -0.5110750\end{array}$

$\begin{array}{llll}\text { C } & 0.2293250 & 0.5859890 & 2.8846950\end{array}$

$\begin{array}{llll}\mathrm{H} & -0.3797080 & -0.3185910 & 2.8485190\end{array}$

$\begin{array}{llll}\mathrm{H} & 1.2793060 & 0.2939310 & 2.9886820\end{array}$

$\begin{array}{llll}\mathrm{H} & -0.0545900 & 1.1629750 & 3.7680840\end{array}$

$\begin{array}{llll}\mathrm{H} & 1.3318110 & 0.2729610 & 0.6003710\end{array}$

$\mathrm{H} \quad-2.6324740-2.0687120-1.4604280$

C $\quad-4.3223640 \quad-1.6003290 \quad-0.2190490$

$\begin{array}{llll}\text { C } & -5.0609430 & -0.9706910 & -1.2326460\end{array}$

$\begin{array}{llll}\text { C } & -4.9755740 & -1.9366720 & 0.9767510\end{array}$

C $\quad-6.4176120 \quad-0.6818430 \quad-1.0564020$

$\mathrm{H} \quad-4.5726070 \quad-0.7097480 \quad-2.1685520$

C $\quad-6.3319600 \quad-1.6495970 \quad 1.1570440$

$\mathrm{H} \quad-4.4201830 \quad-2.4318310 \quad 1.7696800$

C $\quad-7.0575310 \quad-1.0197750 \quad 0.1404570$

$\mathrm{H} \quad-6.9738930 \quad-0.1981590 \quad-1.8543470$

$\mathrm{H} \quad-6.8215800 \quad-1.9217310 \quad 2.0878040$
$\mathrm{H}$

$-8.1120450 \quad-0.7989810$

0.2778100

3.log

Potential Energy $=-1489.66343$

Zero-point Energy = -1489.18829

Free Energy $=-1489.23175$

Single-Point Energy B3LYP-D3(BJ)/6-311+G** PCM = 1490.12013

Free Energy B3LYP-D3(BJ)/6-311+G** PCM (extrapolated free energy from $\mathrm{qRRHO})=-1489.68845$

Nimag $=1(-134.5445)$

Charge $=0$ Multiplicity $=1$

$\begin{array}{llll}\text { C } & 0.1158290 & 1.5392610 & 1.6073560\end{array}$

$\begin{array}{llll}\text { C } & 1.0535020 & 1.9694490 & -0.5663780\end{array}$

$\begin{array}{llll}\mathrm{N} & 0.5247230 & 0.8806700 & 0.3344090\end{array}$

$\begin{array}{lrrr}\mathrm{H} & -0.9273680 & 1.8642190 & 1.5300780\end{array}$

$\mathrm{H} \quad 1.9136050 \quad 1.5587500 \quad-1.1056700$

$\begin{array}{llll}\text { C } & 1.0069150 & 2.7861640 & 1.6512620\end{array}$

$\begin{array}{llll}\mathrm{O} & 1.2218000 & 3.4885800 & 2.6418120\end{array}$

$\begin{array}{llll}\mathrm{N} & 1.5450560 & 2.9536430 & 0.4207820\end{array}$

$\begin{array}{llll}\text { C } & 2.6384300 & 3.8931390 & 0.1888380\end{array}$

$\begin{array}{llll}\mathrm{H} & 3.2476740 & 3.5395480 & -0.6441380\end{array}$

$\begin{array}{llll}\mathrm{H} & 2.2774370 & 4.9002950 & -0.0365010\end{array}$

$\mathrm{H} \quad 3.2526940 \quad 3.9387560 \quad 1.0909630$

$\begin{array}{llll}\text { C } & 0.2879640 & 0.6636660 & 2.8452930\end{array}$

$\mathrm{H} \quad 0.1017610 \quad 1.2715150 \quad 3.7339240$

$\mathrm{H} \quad-0.4207850 \quad-0.1679470 \quad 2.8532270$

$\begin{array}{llll}\mathrm{H} & 1.3043170 & 0.2629820 & 2.9109990\end{array}$

C $\quad 0.0664120 \quad 2.5514000 \quad-1.6273220$

$\begin{array}{llll}\mathrm{C} & -1.2722050 & 2.9985010 & -1.0113870\end{array}$

$\mathrm{H} \quad-1.8943810 \quad 3.4595720 \quad-1.7854940$

$\begin{array}{llll}\mathrm{H} & -1.8380170 & 2.1618160 & -0.5955010\end{array}$

$\begin{array}{llll}\mathrm{H} & -1.1242470 & 3.7456830 & -0.2244040\end{array}$

$\begin{array}{llll}\text { C } & 0.7197470 & 3.7750080 & -2.3101550\end{array}$

$\begin{array}{llll}\mathrm{H} & 1.7203950 & 3.5448820 & -2.6925890\end{array}$

$\mathrm{H} \quad 0.1038910 \quad 4.0775210 \quad-3.1630600$

$\begin{array}{llll}\mathrm{H} & 0.7914970 & 4.6339780 & -1.6390470\end{array}$

$\begin{array}{llll}\mathrm{C} & -0.1682280 & 1.4909240 & -2.7222320\end{array}$

$\begin{array}{llll}\mathrm{H} & -0.8412390 & 1.8946020 & -3.4857400\end{array}$

$\begin{array}{llll}\mathrm{H} & 0.7736790 & 1.2247730 & -3.2161180\end{array}$

$\mathrm{H} \quad-0.6082060 \quad 0.5724040 \quad-2.3362380$

$\begin{array}{llll}\mathrm{C} & -1.9709030 & -0.6179270 & -0.0808880\end{array}$

$\begin{array}{llll}\mathrm{H} & -2.1935420 & 0.0568850 & 0.7484680\end{array}$

$\begin{array}{llll}\mathrm{H} & -2.2433430 & -0.1318410 & -1.0198380\end{array}$

C $\quad-2.8099060 \quad-1.9206870 \quad 0.0889800$

$\mathrm{H} \quad-2.5064330 \quad-2.4288810 \quad 1.0108070$

$\begin{array}{llll}\mathrm{H} & -2.5875930 & -2.5941300 & -0.7445000\end{array}$

C $\quad-4.2939570 \quad-1.6210990 \quad 0.1331700$

$\begin{array}{llll}\text { C } & -4.9401730 & -1.3711560 & 1.3534460\end{array}$

C $\quad-5.0480160 \quad-1.5663330 \quad-1.0492530$

C $\quad-6.3055840 \quad-1.0725170 \quad 1.3926090$

$\begin{array}{llll}\mathrm{H} & -4.3725720 & -1.4163920 & 2.2798190\end{array}$

C $\quad-6.4134370 \quad-1.2679730 \quad-1.0144090$

$\begin{array}{llll}\mathrm{H} & -4.5646570 & -1.7643240 & -2.0029570\end{array}$

$\begin{array}{llll}\mathrm{C} & -7.0466970 & -1.0189630 & 0.2076770\end{array}$

$\begin{array}{llll}\mathrm{H} & -6.7896870 & -0.8867430 & 2.3471900\end{array}$

$\mathrm{H} \quad-6.9817740 \quad-1.2346870 \quad-1.9396030$

$\begin{array}{llll}\mathrm{H} & -8.1080450 & -0.7902490 & 0.2366440\end{array}$

$\begin{array}{llll}\mathrm{C} & -0.5188640 & -0.9695350 & -0.0842710\end{array}$

O $-0.0044950 \quad-1.4388050 \quad-1.1606430$

$\begin{array}{llll}\mathrm{H} & 0.9783100 & -1.8940480 & -1.0063830\end{array}$

$\mathrm{H} \quad-0.0781410 \quad-1.2928620 \quad 0.8601760$

$\begin{array}{llll}\text { O } & 2.1835600 & -2.4978020 & -0.8542280\end{array}$

$\begin{array}{llll}\text { C } & 3.0198630 & -1.9252290 & -0.0855850\end{array}$

C $4.3939740 \quad-2.6617440 \quad-0.0366060$

$\begin{array}{llll}\text { F } & 4.2445780 & -3.9552450 & 0.3411570\end{array}$

$\begin{array}{llll}\text { F } & 5.2596090 & -2.0903500 & 0.8234370\end{array}$

F $\quad 4.9828430 \quad-2.6652880 \quad-1.2593220$

$\begin{array}{llll}\mathrm{H} & 1.3577620 & 0.3139080 & 0.5441370\end{array}$

$\begin{array}{llll}\text { O } & 2.8862970 & -0.9005130 & 0.5951460\end{array}$

4.log

Potential Energy $=-1489.66173$

Zero-point Energy = -1489.18580

Free Energy = -1489.22874 
Free Energy B3LYP-D3(BJ)/6-311+G** PCM (extrapolated

free energy from $\mathrm{qRRHO})=-1489.68718$

Nimag $=1(-121.2814)$

Charge $=0$ Multiplicity $=1$

$\begin{array}{llrr}\text { C } & -1.9362220 & -1.2364370 & 1.6801090 \\ \text { C } & -2.1796150 & -1.0106530 & -0.7029140\end{array}$

$\begin{array}{llll}\text { C } & -2.1796150 & -1.0106530 & -0.7029140\end{array}$

$\mathrm{N} \quad-1.2733680 \quad-0.6907640 \quad 0.4615470$

$\mathrm{H} \quad-1.6324250 \quad-2.2789660 \quad 1.8237220$

$\mathrm{H} \quad-2.1490240 \quad-0.1541110 \quad-1.3843020$

C $\quad-3.4213220 \quad-1.2158550 \quad 1.2978550$

O $-4.3713680 \quad-1.3083160 \quad 2.0782900$

$\mathrm{N} \quad-3.5012210 \quad-1.0378190 \quad-0.0417000$

C $\quad-4.7660950 \quad-0.7094380 \quad-0.6932850$

$\mathrm{H} \quad-4.5680000 \quad-0.1213330 \quad-1.5905630$

$\mathrm{H} \quad-5.3329470 \quad-1.6023130 \quad-0.9704380$

H $\quad-5.3681280 \quad-0.1172740 \quad-0.0004970$

C $\quad-1.6542950 \quad-0.4426060 \quad 2.9523350$

$\mathrm{H} \quad-2.2940510 \quad-0.8231750 \quad 3.7521610$

$\mathrm{H} \quad-0.6155040 \quad-0.5513770 \quad 3.2734460$

$\mathrm{H} \quad-1.8731220 \quad 0.6208580 \quad 2.8151330$

$\begin{array}{llll}\text { C } & -1.8451250 & -2.2889170 & -1.5360590\end{array}$

C $\quad-1.6683590 \quad-3.5429540 \quad-0.6599880$

$\mathrm{H} \quad-1.5115150 \quad-4.4163480 \quad-1.3014640$

$\mathrm{H} \quad-0.8030050 \quad-3.4674180 \quad 0.0023290$

$\mathrm{H} \quad-2.5580740 \quad-3.7405890 \quad-0.0527110$

$\begin{array}{llll}\text { C } & -2.9939460 & -2.5542530 & -2.5363390\end{array}$

$\mathrm{H} \quad-3.2299200 \quad-1.6673810 \quad-3.1348620$

$\mathrm{H} \quad-2.6871920 \quad-3.3450400 \quad-3.2282410$

$\mathrm{H} \quad-3.9057640 \quad-2.8922280 \quad-2.0386560$

$\begin{array}{llll}\text { C } & -0.5713420 & -2.0260140 & -2.3645790\end{array}$

$\mathrm{H} \quad-0.3424100 \quad-2.9062890 \quad-2.9740740$

$\mathrm{H} \quad-0.7150750 \quad-1.1784090 \quad-3.0448160$

$\begin{array}{llll}\mathrm{H} & 0.2985450 & -1.8079400 & -1.7468610\end{array}$

C $\quad 1.3528600 \quad-1.8902930 \quad 0.9390880$

$\begin{array}{llll}\mathrm{H} & 0.6522400 & -2.3752630 & 1.6219580\end{array}$

$\begin{array}{llll}\mathrm{H} & 1.4412220 & -2.4965700 & 0.0353980\end{array}$

$\begin{array}{llll}\text { C } & 2.7329700 & -1.8084870 & 1.6644330\end{array}$

$\begin{array}{llll}\mathrm{H} & 2.6439480 & -1.1521750 & 2.5366990\end{array}$

C $\quad 0.8550440 \quad-0.5233020 \quad 0.5974070$

$\begin{array}{llll}\text { O } & 1.2402560 & 0.0186690 & -0.5020880\end{array}$

$\mathrm{H} \quad 1.0395020 \quad 1.0786750 \quad-0.5401520$

$\begin{array}{llll}\mathrm{H} & 0.6412490 & 0.1473350 & 1.4312650\end{array}$

$\begin{array}{llll}\mathrm{O} & 0.8261190 & 2.4459400 & -0.6095520\end{array}$

$\begin{array}{llll}\text { C } & -0.2825750 & 2.8814930 & -0.1691350\end{array}$

$\begin{array}{llll}\text { C } & -0.4347940 & 4.4251500 & -0.3373100\end{array}$

$\begin{array}{llll}\text { F } & 0.5196980 & 5.0843330 & 0.3674700\end{array}$

$\begin{array}{llll}\text { F } & -1.6305060 & 4.8810610 & 0.0857210\end{array}$

F $\quad-0.3003110 \quad 4.7927030 \quad-1.6357530$

$\mathrm{H} \quad-1.3240700 \quad 0.3360380 \quad 0.5288940$

$\begin{array}{llll}\text { O } & -1.2156550 & 2.2637620 & 0.3625670\end{array}$

C $\quad 3.8960150 \quad-1.3657270 \quad 0.8005320$

$\begin{array}{llll}\text { C } & 4.4900060 & -0.1076740 & 0.9767820\end{array}$

$\begin{array}{llll}\text { C } & 4.4137930 & -2.2163540 & -0.1900370\end{array}$

$\begin{array}{llll}\text { C } & 5.5676450 & 0.2963630 & 0.1811120\end{array}$

$\mathrm{H} \quad 4.1084510 \quad 0.5621850 \quad 1.7435190$

C $\quad 5.4885730 \quad-1.8169100 \quad-0.9881540$

$\begin{array}{llll}\mathrm{H} & 3.9768520 & -3.2016240 & -0.3354080\end{array}$

$\begin{array}{llll}\mathrm{C} & 6.0691910 & -0.5565260 & -0.8059420\end{array}$

$\begin{array}{llll}\mathrm{H} & 6.0133710 & 1.2750970 & 0.3344290\end{array}$

H $\quad 5.8757580 \quad-2.4902910 \quad-1.7476500$

$\mathrm{H} \quad 6.9061800 \quad-0.2457170 \quad-1.4243980$

$\begin{array}{llll}\mathrm{H} & 2.9258150 & -2.8152190 & 2.0510700\end{array}$

$5 . \log$

Potential Energy = -1489.66220

Zero-point Energy = -1489.18672

Free Energy = -1489.22968

Single-Point Energy B3LYP-D3(BJ)/6-311+G** PCM = -

1490.12110

Free Energy B3LYP-D3(BJ)/6-311+G** PCM (extrapolated free energy from $q$ RRHO) $=-1489.68857$

Nimag $=1(-135.1105)$

Charge $=0$ Multiplicity $=1$

C $\quad-0.9506580 \quad-1.5101310 \quad 1.4119860$

C $\quad-2.5037390$

$\mathrm{N}-1.0486660$

$\mathrm{H} \quad-0.7896810$

H -2.7331220

C -2.3502470

O $\quad-2.6633830$

$\mathrm{N} \quad-3.1685550$

C $\quad-4.4998500$

$\mathrm{H}-4.7744930$

$\mathrm{H} \quad-5.2580780$

H -4.4727980

C 0.1417270

H 0.0366650

H 1.1402380

H 0.0544960

C $\quad-2.9442430$

C -2.5017090

H -2.8917740

H -1.4147910

H $\quad-2.8931870$

C $\quad-4.4854180$

H -4.8741780

H -4.7800730

H -4.9737760

C $\quad-2.3756420$

H -2.6929920

H $\quad-2.7520950$

H -1.2876400

C 1.1096270

H 0.9421260

H 0.4780020

C 2.6018870

C 0.7744810

O 0.5354070

H $\quad 0.5373670$

H 1.1943880

O $\quad 0.5660670$

C $\quad 0.0367510$

0.0843970

1.3567100

$-0.4232090$

$-0.6245640$

$-0.8638080$

$-0.4892300$

2.7407200

3.6090200

3.8457250

4.3228660

4.7644570

3.3122710

5.2436250

4.1617100

5.4658750

4.9362440

5.7868860

6.1816290

$-0.6472750$

$-0.6657880$

$-2.5542920$

0.3635400

$-1.3896660$

$-1.6939950$

$-0.8520290$

$-0.3613880$

0.4465040

$-1.1471120$

0.0218490

$-1.0743970$

$-1.6482310$

$-1.2643080$

$-0.0114280$

$-1.6476250$

$-3.0936030$

$-3.7585470$

$-3.2018790$

$-3.4492130$

$-1.6271670$

$-0.6094870$

$-2.1954390$

$-2.0904220$

$-1.1673930$

$-1.8494600$

$-0.1681500$

$-1.1201150$

$-1.9785120$

$-2.6234780$

$-2.3180930$

$-2.0914680$

$-0.5620810$

0.2544600

1.3037050

$-0.1456740$

2.6142610

2.9356870

4.4720070

4.9382530

4.8080860

5.1462430

0.3040120

2.2145280

$-3.1237140$

$-1.7690440$

$-2.6765800$

$-0.5612880$

$-2.3824600$

$-3.6241060$

$-0.2624630$

0.1498480

$-1.1716340$

$-3.0993380$

0.6778320

$-0.9427710$

$-1.4457030$

$-0.2115040$

0.1871530

1.1223230

$-0.5648450$

2.0265770

3.1799850

1.0923590

1.4371840

0.7574600

1.3809760

2.4597300

2.3850460

3.3090040

1.9844590

2.6306490

$-1.3270330$

$-1.0359880$

$-1.8136380$

$-1.0301350$

$-0.0769160$

$-1.4479910$

$-1.5652340$

$-2.3359620$

$-0.5876100$

$-2.6776960$

$-3.4732130$

$-2.9262840$

$-2.6874470$

$-1.3281990$

$-0.4630040$

$-2.1515030$

$-1.7694210$

$-0.9875260$

$-1.9458940$

$-1.6310900$

$-0.0716440$

$-1.2937820$

$-0.1825340$

0.0824530

0.0371050

1.2844310

$-0.8580260$

0.4774330

0.6743470

$-2.1082340$

$-0.6823450$

0.3633890

$-0.6925260$

1.3741940

0.3832510

0.3178150

$-1.4989880$

1.3557890

2.1721110

0.2904900

2.1399240

H 2.7653780

$-2.6375060$

6.log

Potential Energy = -1489.66343

Zero-point Energy = -1489.18829

Free Energy = -1489.23176

Single-Point Energy B3LYP-D3(BJ)/6-311+G** PCM = -

1490.12013

Free Energy B3LYP-D3(BJ)/6-311+G** PCM (extrapolated

free energy from $q R R H O)=-1489.68846$

Nimag $=1(-134.5974)$

Charge $=0$ Multiplicity $=1$

$\begin{array}{lrrr}\mathrm{C} & 0.1157540 & 1.5393690 & 1.6073830 \\ \mathrm{C} & 1.0536400 & 1.9692590 & -0.5663350 \\ \mathrm{~N} & 0.5247270 & 0.8806120 & 0.3345420 \\ \mathrm{H} & -0.9274310 & 1.8643370 & 1.5299920 \\ \mathrm{H} & 1.9137670 & 1.5584550 & -1.1055090 \\ \mathrm{C} & 1.0068650 & 2.7862540 & 1.6512020 \\ \mathrm{O} & 1.2216510 & 3.4888220 & 2.6416660 \\ \mathrm{~N} & 1.5451580 & 2.9535300 & 0.4207660\end{array}$




\begin{tabular}{|c|c|c|c|}
\hline $\mathrm{C}$ & 2.6385000 & 3.8930590 & 0.1887990 \\
\hline $\mathrm{H}$ & 3.2474980 & 3.5396860 & -0.6444420 \\
\hline $\mathrm{H}$ & 2.2774610 & 4.9002850 & -0.0361610 \\
\hline $\mathrm{H}$ & 3.2530230 & 3.9384170 & 1.0907630 \\
\hline $\mathrm{C}$ & 0.2877830 & 0.6639120 & 2.8454320 \\
\hline $\mathrm{H}$ & 0.1015020 & 1.2718600 & 3.7339790 \\
\hline $\mathrm{H}$ & -0.4209670 & -0.1677000 & 2.8533890 \\
\hline $\mathrm{H}$ & 1.3041280 & 0.2632310 & 2.9112670 \\
\hline $\mathrm{C}$ & 0.0666680 & 2.5511730 & -1.6274130 \\
\hline $\mathrm{C}$ & -1.2719390 & 2.9984770 & -1.0116030 \\
\hline $\mathrm{H}$ & -1.8940340 & 3.4594990 & -1.7858040 \\
\hline $\mathrm{H}$ & -1.8378420 & 2.1618930 & -0.5956380 \\
\hline $\mathrm{H}$ & -1.1239610 & 3.7457500 & -0.2247090 \\
\hline $\mathrm{C}$ & 0.7201710 & 3.7746320 & -2.3103540 \\
\hline $\mathrm{H}$ & 1.7207950 & 3.5443410 & -2.6927540 \\
\hline $\mathrm{H}$ & 0.1043660 & 4.0771370 & -3.1632980 \\
\hline $\mathrm{H}$ & 0.7920230 & 4.6336610 & -1.6393340 \\
\hline $\mathrm{C}$ & -0.1680350 & 1.4906050 & -2.7222190 \\
\hline $\mathrm{H}$ & -0.8408670 & 1.8943270 & -3.4858620 \\
\hline $\mathrm{H}$ & 0.7738800 & 1.2242030 & -3.2159540 \\
\hline $\mathrm{H}$ & -0.6082510 & 0.5722240 & -2.3361670 \\
\hline $\mathrm{C}$ & -1.9709290 & -0.6178790 & -0.0807230 \\
\hline $\mathrm{H}$ & -2.1935190 & 0.0570010 & 0.7485900 \\
\hline $\mathrm{H}$ & -2.2433960 & -0.1318520 & -1.0196970 \\
\hline $\mathrm{C}$ & -2.8099620 & -1.9206000 & 0.0892750 \\
\hline $\mathrm{H}$ & -2.5065970 & -2.4286370 & 1.0112230 \\
\hline $\mathrm{H}$ & -2.5875650 & -2.5941880 & -0.7440670 \\
\hline $\mathrm{C}$ & -4.2940160 & -1.6209920 & 0.1332460 \\
\hline $\mathrm{C}$ & -4.9404880 & -1.3713760 & 1.3534510 \\
\hline $\mathrm{C}$ & -5.0478240 & -1.5658850 & -1.0493240 \\
\hline $\mathrm{C}$ & -6.3059040 & -1.0727200 & 1.3924020 \\
\hline $\mathrm{H}$ & -4.3730880 & -1.4168800 & 2.2799320 \\
\hline $\mathrm{C}$ & -6.4132430 & -1.2675090 & -1.0146910 \\
\hline $\mathrm{H}$ & -4.5642650 & -1.7636250 & -2.0029800 \\
\hline $\mathrm{C}$ & -7.0467620 & -1.0188240 & 0.2073290 \\
\hline $\mathrm{H}$ & -6.7902070 & -0.8872000 & 2.3469320 \\
\hline $\mathrm{H}$ & -6.9813820 & -1.2339590 & -1.9399980 \\
\hline $\mathrm{H}$ & -8.1081110 & -0.7900970 & 0.2361310 \\
\hline $\mathrm{C}$ & -0.5188900 & -0.9695120 & -0.0841700 \\
\hline $\mathrm{O}$ & -0.0046290 & -1.4388200 & -1.1605870 \\
\hline $\mathrm{H}$ & 0.9782970 & -1.8939170 & -1.0065410 \\
\hline $\mathrm{H}$ & -0.0781190 & -1.2928540 & 0.8602500 \\
\hline $\mathrm{O}$ & 2.1838700 & -2.4973260 & -0.8549180 \\
\hline $\mathrm{C}$ & 3.0197720 & -1.9252860 & -0.0854450 \\
\hline $\mathrm{C}$ & 4.3940020 & -2.6615880 & -0.0367050 \\
\hline $\mathrm{F}$ & 4.2448790 & -3.9542560 & 0.3441700 \\
\hline $\mathrm{F}$ & 5.2607060 & -2.0883670 & 0.8210350 \\
\hline $\mathrm{F}$ & 4.9814230 & -2.6679720 & -1.2600600 \\
\hline $\mathrm{H}$ & 1.3576910 & 0.3138010 & 0.5444440 \\
\hline $\mathrm{O}$ & 2.8856000 & -0.9014580 & 0.5965050 \\
\hline
\end{tabular}

$\begin{array}{lccc}\mathrm{H} & -1.9199100 & 0.0267720 & -0.7809090 \\ \mathrm{C} & -2.2804870 & -1.8205580 & 0.3333950 \\ \mathrm{H} & -2.1256540 & -1.3865840 & 1.3251090 \\ \mathrm{H} & -1.8623840 & -2.8310260 & 0.3590680 \\ \mathrm{~N} & 0.5157080 & 0.8691710 & 0.3759510 \\ \mathrm{C} & 3.6959380 & -1.1549220 & 0.0828330 \\ \mathrm{C} & 5.2039670 & -1.5382140 & -0.0180230 \\ \mathrm{O} & 3.4047620 & 0.0100360 & 0.3679100 \\ \mathrm{H} & 1.5287650 & 0.7000970 & 0.3539300 \\ \mathrm{O} & 2.9260650 & -2.1445230 & -0.1550350 \\ \mathrm{H} & 1.6455390 & -1.9312690 & -0.0082900 \\ \mathrm{C} & 0.3494040 & 2.6362720 & -1.5179040 \\ \mathrm{C} & 0.3498410 & 4.1772530 & -1.6821340 \\ \mathrm{H} & 0.6386990 & 4.4264430 & -2.7079490 \\ \mathrm{H} & -0.6285710 & 4.6263820 & -1.5042260 \\ \mathrm{H} & 1.0725610 & 4.6516440 & -1.0092660 \\ \mathrm{C} & -0.7491520 & 2.0362820 & -2.4146110 \\ \mathrm{H} & -0.6541690 & 2.4481880 & -3.4245660 \\ \mathrm{H} & -1.7545870 & 2.2726660 & -2.0550440 \\ \mathrm{C} & 1.7364350 & 2.1403570 & -1.9878610 \\ \mathrm{H} & 1.9010690 & 2.4547000 & -3.0229800 \\ \mathrm{H} & 2.5416890 & 2.5653120 & -1.3785330 \\ \mathrm{H} & 1.8318970 & 1.0518800 & -1.9587410 \\ \mathrm{C} & 0.9692440 & 1.1336870 & 2.8657260 \\ \mathrm{H} & 0.4784380 & 1.0320320 & 3.8375760 \\ \mathrm{H} & 1.8476810 & 0.4834370 & 2.8502160 \\ \mathrm{H} & 1.3004680 & 2.1705970 & 2.7548640 \\ \mathrm{H} & -0.6679660 & 0.9519230 & -2.5058830 \\ \mathrm{~F} & 5.5025580 & -2.0222100 & -1.2490470 \\ \mathrm{~F} & 6.0194600 & -0.4907310 & 0.2105480 \\ \mathrm{~F} & 5.5215480 & -2.5021540 & 0.8816280 \\ \mathrm{C} & -3.7668930 & -1.8834840 & 0.0415970 \\ \mathrm{C} & -4.6391300 & -0.9069140 & 0.5473950 \\ \mathrm{C} & -4.2991390 & -2.9063790 & -0.7584330 \\ \mathrm{C} & -6.0067430 & -0.9487310 & 0.2590680 \\ \mathrm{H} & -4.2446260 & -0.1122770 & 1.1762920 \\ \mathrm{H} & -5.6661800 & -2.9519270 & -1.0495990 \\ \mathrm{H} & -3.6397390 & -3.6765340 & -1.1519150 \\ \mathrm{H} & -6.5249980 & -1.9716000 & -0.5420200 \\ \mathrm{H} & -6.05958580 & -0.1861660 & 0.6634840 \\ \mathrm{H} & -3.7543950 & -1.6673160 \\ \mathrm{H} & -2.0071920 & -0.7641970 \\ \mathrm{H} & -1.4267620 & -1.7250610\end{array}$

Formation of iminium ion (TS2)

$\begin{array}{cc}\begin{array}{c}\text { Conformation } \\ \text { name }\end{array} & \begin{array}{c}\text { Extrapolated } \\ \text { Free energy }\end{array} \\ & (\mathrm{kcal} / \mathrm{mol})\end{array}$

$7 . \log$

Potential Energy = -1489.66169

Zero-point Energy = -1489.18761

Free Energy $=-1489.23092$

Single-Point Energy B3LYP-D3(BJ)/6-311+G** PCM = -

1490.12078

Free Energy B3LYP-D3(BJ)/6-311+G** PCM (extrapolated

kcal/mol)

free energy from $q R R H O)=-1489.69002$

Nimag $=1(-225.2892)$

Charge $=0$ Multiplicity $=1$

$\begin{array}{lrrr}\mathrm{C} & -1.2272110 & 1.6296880 & 1.7244310 \\ \mathrm{C} & 0.2093770 & 2.2830240 & -0.0051090 \\ \mathrm{C} & -0.0097490 & 0.7165340 & 1.7547960 \\ \mathrm{H} & 0.9349330 & 2.9336880 & 0.5117010 \\ \mathrm{H} & -0.3052540 & -0.3203680 & 1.9252010 \\ \mathrm{C} & -0.0322600 & -0.9759410 & -0.6285860 \\ \mathrm{H} & 0.5214760 & -0.6141840 & -1.4939590 \\ \mathrm{O} & 0.5365800 & -1.8271480 & 0.1386550 \\ \mathrm{~N} & -1.1067290 & 2.4547060 & 0.6437900 \\ \mathrm{C} & -1.9444290 & 3.6440330 & 0.5351690 \\ \mathrm{H} & -2.3907120 & 3.7290280 & -0.4566380 \\ \mathrm{H} & -2.7414310 & 3.5444480 & 1.2724860 \\ \mathrm{H} & -1.3688160 & 4.5495920 & 0.7535220 \\ \mathrm{O} & -2.1363140 & 1.6398050 & 2.5557440 \\ \mathrm{C} & -1.5343390 & -0.9932450 & -0.7322160\end{array}$

$\begin{array}{cc}\text { TS2-lowest } & 0.0 \\ 1 & 2.2 \\ 2 & 2.1 \\ 3 & 1.2 \\ 4 & 0.6 \\ 5 & 0.5 \\ 6 & 0.6 \\ 7 & 1.4 \\ 8 & 1.9 \\ 9 & 4.1 \\ 10 & 1.4 \\ 11 & 1.9 \\ 12 & 1.1 \\ 13 & 1.4\end{array}$




$\begin{array}{ll}14 & 1.0 \\ 15 & -0.4 \\ 16 & -0.1 \\ 17 & -0.2 \\ 18 & 1.5 \\ 19 & 1.1 \\ 20 & 1.5 \\ 21 & 5.8 \\ 22 & 6.1 \\ 23 & 7.1 \\ 24 & 4.7 \\ 25 & 7.4 \\ 26 & 5.1\end{array}$

TS2-lowest.log (Enamine 2)

Potential Energy $=-1489.66107$

Zero-point Energy = -1489.18625

Free Energy $=-1489.22898$

Single-Point Energy B3LYP-D3(BJ)/6-311+G** PCM = -

1490.12306

Free Energy B3LYP-D3(BJ)/6-311+G** PCM (extrapolated

free energy from $\mathrm{qRRHO})=-1489.69097$

Nimag $=1\left(-269.9625 \mathrm{~cm}^{-1}\right)$

Charge $=0$ Multiplicity $=1$

\begin{tabular}{|c|c|c|c|}
\hline $\mathrm{C}$ & -2.1887330 & -0.0465630 & -0.4475410 \\
\hline $\mathrm{C}$ & -2.7481900 & -0.1260620 & 1.8444790 \\
\hline $\mathrm{C}$ & -2.5697810 & -1.5728380 & 1.3734760 \\
\hline $\mathrm{N}$ & -1.8347970 & -1.3904070 & 0.0881530 \\
\hline $\mathrm{N}$ & -2.4932770 & 0.6877860 & 0.7939620 \\
\hline $\mathrm{O}$ & -3.0679970 & 0.2296140 & 2.9811960 \\
\hline $\mathrm{C}$ & -2.4508120 & 2.1389360 & 0.9339600 \\
\hline $\mathrm{H}$ & -3.4414530 & 2.5905240 & 0.8241220 \\
\hline $\mathrm{H}$ & -2.0710280 & 2.3770210 & 1.9296920 \\
\hline $\mathrm{H}$ & -1.7767760 & 2.5548760 & 0.1848880 \\
\hline $\mathrm{C}$ & -0.5887650 & -1.9120650 & -0.0662850 \\
\hline $\mathrm{C}$ & 0.0851510 & -1.9191980 & -1.4268600 \\
\hline $\mathrm{C}$ & 1.3082870 & -2.8621740 & -1.5373900 \\
\hline $\mathrm{H}$ & 1.0196350 & -3.8580710 & -1.1825680 \\
\hline $\mathrm{H}$ & 1.5179980 & -2.9669310 & -2.6075520 \\
\hline $\mathrm{C}$ & -1.9418990 & -2.4809050 & 2.4228550 \\
\hline $\mathrm{H}$ & -2.5830430 & -2.4508140 & 3.3072180 \\
\hline $\mathrm{H}$ & -1.9014060 & -3.5178500 & 2.0782260 \\
\hline $\mathrm{H}$ & -0.9425260 & -2.1512050 & 2.7131070 \\
\hline $\mathrm{H}$ & -3.5650420 & -1.9656920 & 1.1381160 \\
\hline $\mathrm{C}$ & -3.3463480 & -0.0415220 & -1.5080320 \\
\hline $\mathrm{H}$ & -1.3045690 & 0.4017240 & -0.9087200 \\
\hline $\mathrm{C}$ & -3.5273770 & 1.4006170 & -2.0344580 \\
\hline $\mathrm{H}$ & -4.2194910 & 1.3912200 & -2.8825200 \\
\hline $\mathrm{H}$ & -3.9480180 & 2.0697210 & -1.2805420 \\
\hline $\mathrm{H}$ & -2.5801040 & 1.8262400 & -2.3846700 \\
\hline $\mathrm{C}$ & -2.9527870 & -0.9365580 & -2.6992030 \\
\hline $\mathrm{H}$ & -2.0250170 & -0.5983930 & \\
\hline $\mathrm{H}$ & -2.8358390 & -1.9811100 & -2.3962620 \\
\hline $\mathrm{H}$ & -3.7413090 & -0.8980890 & -3.4576200 \\
\hline $\mathrm{C}$ & -4.6861930 & -0.5387460 & -0.9323100 \\
\hline $\mathrm{H}$ & -4.9824260 & 0.0185130 & -0.0378730 \\
\hline $\mathrm{H}$ & -5.4740820 & -0.4007800 & -1.6800410 \\
\hline $\mathrm{H}$ & -4.6553220 & -1.6052750 & -0.6898860 \\
\hline $\mathrm{H}$ & 0.3472340 & -0.9044970 & -1.7402720 \\
\hline $\mathrm{H}$ & -0.6648520 & -2.2851630 & -2.1325360 \\
\hline $\mathrm{C}$ & 2.5802210 & -2.4220080 & -0.8369560 \\
\hline $\mathrm{C}$ & 3.3477900 & -1.3619480 & -1.3497680 \\
\hline $\mathrm{C}$ & 3.0313450 & -3.0683030 & 0.3262110 \\
\hline $\mathrm{C}$ & 4.5224230 & -0.9541730 & -0.7148850 \\
\hline $\mathrm{H}$ & 3.0243870 & -0.8554700 & -2.2557540 \\
\hline $\mathrm{C}$ & 4.2083930 & -2.6594510 & 0.9673840 \\
\hline $\mathrm{H}$ & 2.4677770 & -3.9077660 & 0.7266830 \\
\hline $\mathrm{C}$ & 4.9562100 & -1.6002100 & 0.4491520 \\
\hline $\mathrm{H}$ & 5.1016210 & -0.1340300 & -1.1292890 \\
\hline
\end{tabular}

$\begin{array}{lrrr}\mathrm{H} & 4.5383210 & -3.1742420 & 1.8649700 \\ \mathrm{H} & 5.8699630 & -1.2823890 & 0.9422360 \\ \mathrm{H} & -0.4808910 & -2.8478780 & 0.4729440 \\ \mathrm{O} & 0.5171030 & -0.9860990 & 0.9686830 \\ \mathrm{H} & 0.7168780 & 0.0101890 & 0.5838690 \\ \mathrm{H} & 1.3729980 & -1.4509040 & 1.0249260 \\ \mathrm{O} & 0.8953060 & 1.2539410 & 0.0146890 \\ \mathrm{C} & 1.6225310 & 2.0897860 & 0.6466230 \\ \mathrm{C} & 1.8136060 & 3.4209040 & -0.1497850 \\ \mathrm{O} & 2.1733030 & 1.9846670 & 1.7435760 \\ \mathrm{~F} & 2.5259700 & 4.3409930 & 0.5321730 \\ \mathrm{~F} & 0.6211220 & 3.9909260 & -0.4618580 \\ \mathrm{~F} & 2.4663250 & 3.1983890 & -1.3209710\end{array}$

$1 . \log$

Potential Energy $=-1489.66249$

Zero-point Energy = -1489.18824

Free Energy $=-1489.23172$

Single-Point Energy B3LYP-D3(BJ)/6-311+G** PCM = 1490.11830

Free Energy B3LYP-D3(BJ)/6-311+G** PCM (extrapolated free energy from $\mathrm{qRRHO})=-1489.68753$

Nimag $=1\left(-309.6177 \mathrm{~cm}^{-1}\right)$

Charge $=0$ Multiplicity $=1$

$\begin{array}{llll}\text { C } & 2.9163420 & -0.0892340 & -0.3998050\end{array}$

$\begin{array}{llll}\text { C } & 3.0941390 & -2.3930880 & 0.0742480\end{array}$

C $\quad 2.4340840 \quad-1.7323080 \quad 1.2886810$

$\mathrm{N} \quad 2.0021580 \quad-0.4196300 \quad 0.7273880$

$\mathrm{N} \quad 3.3212000 \quad-1.4310090 \quad-0.8507370$

$\begin{array}{llll}\text { O } & 3.3681970 & -3.5895760 & -0.0373540\end{array}$

C $\quad 3.8155230 \quad-1.7444070 \quad-2.1865760$

$\mathrm{H} \quad 4.9087010 \quad-1.7654930 \quad-2.2261400$

$\mathrm{H} \quad 3.4399180 \quad-2.7298620 \quad-2.4695140$

$\mathrm{H} \quad 3.4464850 \quad-1.0010640 \quad-2.8951280$

$\begin{array}{llll}\text { C } & 0.6885600 & -0.0846140 & 0.6966300\end{array}$

$\begin{array}{llll}\text { C } & 0.2516700 & 1.3123210 & 0.3111540\end{array}$

$\begin{array}{llll}\text { C } & -1.2719890 & 1.5398080 & 0.4004290\end{array}$

$\mathrm{H} \quad-1.7892110 \quad 0.9284120 \quad-0.3444370$

$\mathrm{H} \quad-1.6227930 \quad 1.2106010 \quad 1.3853150$

C $\quad 1.3732150 \quad-2.6012100 \quad 1.9507580$

$\mathrm{H} \quad 1.8609770 \quad-3.5253290 \quad 2.2701570$

H $\quad 0.9615480 \quad-2.1191500 \quad 2.8416240$

$\mathrm{H} \quad 0.5626630 \quad-2.8657380 \quad 1.2693160$

$\begin{array}{llll}\mathrm{H} & 3.2211080 & -1.5386160 & 2.0255120\end{array}$

$\begin{array}{llll}\mathrm{C} & 4.1050930 & 0.8649880 & -0.0247340\end{array}$

$\begin{array}{llll}\mathrm{H} & 2.3443270 & 0.3911040 & -1.1987510\end{array}$

$\begin{array}{llll}\mathrm{C} & 4.8940600 & 1.2007950 & -1.3105310\end{array}$

$\mathrm{H} \quad 5.6309560 \quad 1.9792970 \quad-1.0890220$

H $\quad 5.4405250 \quad 0.3394880 \quad-1.7014760$

$\begin{array}{llll}\mathrm{H} & 4.2377730 & 1.5826090 & -2.1010160\end{array}$

$\begin{array}{llll}\text { C } & 3.5403810 & 2.1800430 & 0.5472630\end{array}$

H $2.8848500 \quad 2.6874560 \quad-0.1682940$

$\mathrm{H} \quad 2.9855850 \quad 2.0104930 \quad 1.4744730$

$\begin{array}{llll}\mathrm{H} & 4.3669960 & 2.8607140 & 0.7746210\end{array}$

$\begin{array}{llll}\mathrm{C} & 5.0646310 & 0.2414890 & 1.0068460\end{array}$

$\begin{array}{llll}\mathrm{H} & 5.4427090 & -0.7326880 & 0.6808710\end{array}$

$\mathrm{H} \quad 5.9285980 \quad 0.9006440 \quad 1.1403730$

$\mathrm{H} \quad 4.5920740 \quad 0.1272190 \quad 1.9869770$

$\begin{array}{llll}\mathrm{H} & 0.6176640 & 1.5868750 & -0.6833930\end{array}$

$\begin{array}{llll}\mathrm{H} & 0.7473270 & 1.9838980 & 1.0200450\end{array}$

$\begin{array}{llll}\text { C } & -1.6343500 & 2.9972760 & 0.1938430\end{array}$

$\begin{array}{llll}\mathrm{C} & -1.6670560 & 3.8897400 & 1.2767530\end{array}$

$\begin{array}{lllr}\text { C } & -1.9235370 & 3.4902750 & -1.0878270\end{array}$

$\begin{array}{llll}\text { C } & -1.9765150 & 5.2396480 & 1.0851320\end{array}$

$\begin{array}{llll}\mathrm{H} & -1.4551670 & 3.5239490 & 2.2787680\end{array}$

$\begin{array}{llll}\mathrm{C} & -2.2327660 & 4.8398060 & -1.2847030\end{array}$

$\mathrm{H} \quad-1.9127450 \quad 2.8116840 \quad-1.9374520$

$\begin{array}{llll}\text { C } & -2.2592710 & 5.7196580 & -0.1979470\end{array}$

$\mathrm{H} \quad-2.0014380 \quad 5.9134190 \quad 1.9369710$

$\mathrm{H} \quad-2.4575730 \quad 5.2012740 \quad-2.2841980$

$\begin{array}{llll}\mathrm{H} & -2.5031470 & 6.7673200 & -0.3483300\end{array}$

$\mathrm{H} \quad 0.1435240 \quad-0.4855490 \quad 1.5453420$

$\begin{array}{llll}\mathrm{O} & -0.1256740 & -1.1394490 & -0.5045090\end{array}$

$\mathrm{H} \quad-0.2425590 \quad-0.6952790 \quad-1.3596100$

$\mathrm{H} \quad-1.1104150 \quad-1.5302140 \quad-0.1983810$

$\begin{array}{llll}\text { O } & -2.2760230 & -2.0600690 & 0.2166960\end{array}$ 


$\begin{array}{llll}\mathrm{C} & -3.2632010 & -1.8081300 & -0.5529800 \\ \mathrm{O} & -3.2887880 & -1.1654220 & -1.6042330 \\ \mathrm{C} & -4.5957220 & -2.4280770 & -0.0264160 \\ \mathrm{~F} & -4.5002500 & -3.7777020 & 0.0850540 \\ \mathrm{~F} & -4.9075450 & -1.9467770 & 1.2044070 \\ \mathrm{~F} & -5.6448950 & -2.1683600 & -0.8325580\end{array}$

$2 . \log$

Potential Energy $=-1489.65965$

Zero-point Energy $=-1489.18505$

Free Energy $=-1489.22863$

Single-Point Energy B3LYP-D3(BJ)/6-311+G** PCM = 1490.11864

Free Energy B3LYP-D3(BJ)/6-311+G** PCM (extrapolated free energy from $\mathrm{qRRHO})=-1489.68763$

Nimag $=1\left(-222.1627 \mathrm{~cm}^{-1}\right)$

Charge $=0$ Multiplicity $=1$

$\begin{array}{lrrr}\mathrm{C} & -2.7695910 & -0.1669450 & 0.3649140 \\ \mathrm{C} & -1.8990570 & -2.3524920 & 0.1672370 \\ \mathrm{C} & -1.8496940 & -1.7152730 & -1.2252570 \\ \mathrm{~N} & -1.9970380 & -0.2667750 & -0.9043290 \\ \mathrm{~N} & -2.4034680 & -1.4329180 & 1.0237490\end{array}$

$\begin{array}{llll}-2.4034680 & -1.4329180 & 1.0237490\end{array}$

$\begin{array}{llll}\mathrm{O} & -1.5444360 & -3.4978390 & 0.4532300\end{array}$

$\begin{array}{llll}\text { C } & -2.4519230 & -1.6621950 & 2.4629530\end{array}$

$\mathrm{H} \quad-3.3818170 \quad-2.1494890 \quad 2.7712660$

$\begin{array}{llll}\mathrm{H} & -1.6152830 & -2.3086270 & 2.7354160\end{array}$

$\begin{array}{llll}\mathrm{H} & -2.3562610 & -0.7096780 & 2.9868740\end{array}$

$\begin{array}{llll}\text { C } & -1.0130300 & 0.6208940 & -1.1885420\end{array}$

$\begin{array}{llll}\text { C } & -1.2980500 & 2.1089270 & -1.1563150\end{array}$

$\begin{array}{llll}\text { C } & -0.1438890 & 3.0265960 & -1.6216880\end{array}$

$\begin{array}{llll}\mathrm{H} & 0.3126100 & 2.6059020 & -2.5241690\end{array}$

$\begin{array}{llll}\mathrm{H} & -0.6074980 & 3.9714640 & -1.9280310\end{array}$

$\begin{array}{llll}\text { C } & -0.6510510 & -2.1376540 & -2.0620440\end{array}$

$\begin{array}{llll}\mathrm{H} & -0.7053200 & -3.2226760 & -2.1815740\end{array}$

$\begin{array}{llll}\mathrm{H} & -0.6915760 & -1.6925980 & -3.0603280\end{array}$

$\mathrm{H} \quad 0.3064380 \quad-1.9016300 \quad-1.5941070$

$\begin{array}{llll}\mathrm{H} & -2.7555600 & -2.0250490 & -1.7576560\end{array}$

$\begin{array}{llll}\text { C } & -4.3134790 & 0.0589970 & 0.1957800\end{array}$

$\begin{array}{llll}\mathrm{H} & -2.3682800 & 0.6613860 & 0.9553270\end{array}$

$\begin{array}{llll}\text { C } & -4.9335480 & 0.2671260 & 1.5963520\end{array}$

$\begin{array}{llll}\mathrm{H} & -5.9781290 & 0.5756100 & 1.4872680\end{array}$

$\begin{array}{llll}\mathrm{H} & -4.9262780 & -0.6463810 & 2.1952730\end{array}$

$\begin{array}{llll}\mathrm{H} & -4.4131240 & 1.0522860 & 2.1567470\end{array}$

$\begin{array}{llll}\text { C } & -4.5579460 & 1.3372450 & -0.6295750\end{array}$

$\begin{array}{llll}\mathrm{H} & -4.1190150 & 2.2202950 & -0.1536740\end{array}$

$\begin{array}{llll}\mathrm{H} & -4.1556180 & 1.2461050 & -1.6424990\end{array}$

$\begin{array}{llll}\mathrm{H} & -5.6351200 & 1.5126960 & -0.7146350\end{array}$

$\begin{array}{lrrr}\text { C } & -5.0186590 & -1.1239000 & -0.4955230\end{array}$

$\begin{array}{llll}\mathrm{H} & -4.8184070 & -2.0741660 & 0.0093590\end{array}$

$\begin{array}{llll}\mathrm{H} & -6.1012630 & -0.9619520 & -0.4725170\end{array}$

$\begin{array}{llll}\mathrm{H} & -4.7293430 & -1.2146290 & -1.5467950\end{array}$

$\begin{array}{llll}\mathrm{H} & -1.6711790 & 2.4188530 & -0.1762330\end{array}$

$\begin{array}{llll}\mathrm{H} & -2.1282030 & 2.2440450 & -1.8566190\end{array}$

$\begin{array}{llll}\text { C } & 0.9407990 & 3.3394800 & -0.6035480\end{array}$

$\begin{array}{llll}\text { C } & 0.6268950 & 3.9646150 & 0.6172480\end{array}$

$\begin{array}{llll}\text { C } & 2.2893330 & 3.0576850 & -0.8783500\end{array}$

$\begin{array}{llll}\text { C } & 1.6288830 & 4.2899790 & 1.5356390\end{array}$

$\begin{array}{llll}\mathrm{H} & -0.4065750 & 4.2099770 & 0.8488780\end{array}$

$\begin{array}{llll}\text { C } & 3.2946180 & 3.3851860 & 0.0375430\end{array}$

$\begin{array}{llll}\mathrm{H} & 2.5536510 & 2.5778210 & -1.8170590\end{array}$

$\begin{array}{llll}\text { C } & 2.9671930 & 4.0014750 & 1.2483160\end{array}$

$\begin{array}{llll}\mathrm{H} & 1.3641490 & 4.7734070 & 2.4714780\end{array}$

$\begin{array}{llll}\mathrm{H} & 4.3303380 & 3.1566640 & -0.1961360\end{array}$

$\begin{array}{llll}\mathrm{H} & 3.7459980 & 4.2548510 & 1.9613430\end{array}$

$\begin{array}{llll}\mathrm{H} & -0.4100740 & 0.3146890 & -2.0377020\end{array}$

$\begin{array}{llll}\mathrm{O} & 0.2498020 & 0.4462580 & 0.0892450\end{array}$

$\begin{array}{llll}\mathrm{H} & 0.6285480 & 1.3179610 & 0.3048790\end{array}$

$\begin{array}{llll}\mathrm{H} & 1.0869670 & -0.1913560 & -0.1366250\end{array}$

$\begin{array}{llll}\mathrm{O} & 2.1777580 & -1.0070040 & -0.4604330\end{array}$

$\begin{array}{llll}\text { C } & 2.9763500 & -1.3174150 & 0.4831580\end{array}$

$\begin{array}{llll}\mathrm{O} & 2.9531000 & -1.0028320 & 1.6750300\end{array}$

$\begin{array}{llll}\text { C } & 4.1438700 & -2.2311930 & -0.0130220\end{array}$

$\begin{array}{llll}\text { F } & 3.6741190 & -3.3713620 & -0.5819850\end{array}$

$\begin{array}{llll}\text { F } & 4.8959010 & -1.5990060 & -0.9524160\end{array}$

$\begin{array}{llll}\text { F } & 4.9800720 & -2.6000110 & 0.9797440\end{array}$
3. $\log$

Potential Energy = -1489.66151

Zero-point Energy = -1489.18751

Free Energy = -1489.23110

Single-Point Energy B3LYP-D3(BJ)/6-311+G** PCM = 1490.11948

Free Energy B3LYP-D3(BJ)/6-311+G** PCM (extrapolated free energy from $q R R H O)=-1489.68907$

Nimag $=1\left(-324.0648 \mathrm{~cm}^{-1}\right)$ Charge $=0$ Multiplicity $=1$

$\begin{array}{llll}\text { C } & 3.0112410 & -0.4737330 & -0.4844450\end{array}$

$\begin{array}{llll}\mathrm{C} & 2.6207980 & -1.9757880 & 1.2915410\end{array}$

$\begin{array}{llll}\text { C } & 2.0312580 & -0.6235450 & 1.7049870\end{array}$

$\begin{array}{llll}\mathrm{N} & 1.9536360 & 0.0910360 & 0.3977810\end{array}$

$\begin{array}{llll}\mathrm{N} & 3.1374380 & -1.8411710 & 0.0474330\end{array}$

O $\quad 2.6222740 \quad-3.0029010 \quad 1.9730450$

C $\quad 3.6489220 \quad-2.9822410 \quad-0.7033290$

H $4.7051020 \quad-3.1737100 \quad-0.4916100$

$\mathrm{H} \quad 3.0735890 \quad-3.8660870 \quad-0.4202920$

$\mathrm{H} \quad 3.5261120 \quad-2.8001350 \quad-1.7722060$

C $\quad 0.7514200 \quad 0.4792220 \quad-0.0986660$

C $\quad 0.6657720 \quad 1.3456090 \quad-1.3378940$

$\begin{array}{llll}\text { C } & -0.7540190 & 1.8698410 & -1.6509830\end{array}$

$\mathrm{H} \quad-0.6964970 \quad 2.3740370 \quad-2.6216200$

$\mathrm{H} \quad-1.4461780 \quad 1.0322470 \quad-1.7761580$

$\begin{array}{llll}\text { C } & 0.7520730 & -0.7442590 & 2.5223790\end{array}$

$\mathrm{H} \quad 0.9923840 \quad-1.3174110 \quad 3.4211090$

$\begin{array}{llll}\mathrm{H} & 0.3885100 & 0.2359790 & 2.8418420\end{array}$

$\begin{array}{llll}\mathrm{H} & -0.0398160 & -1.2720120 & 1.9880260\end{array}$

H $\quad 2.7793920 \quad-0.1134720 \quad 2.3213340$

C $4.3500180 \quad 0.3449520 \quad-0.5140560$

$\begin{array}{llll}\mathrm{H} & 2.6343380 & -0.5295950 & -1.5098870\end{array}$

C $\quad 5.3114770 \quad-0.3167720 \quad-1.5268280$

$\mathrm{H} \quad 6.1850980 \quad 0.3271040 \quad-1.6695390$

$\mathrm{H} \quad 5.6766020 \quad-1.2867710 \quad-1.1817280$

$\mathrm{H} \quad 4.8382480 \quad-0.4534000 \quad-2.5058560$

$\begin{array}{llll}\text { C } & 4.0581100 & 1.7790190 & -0.9973600\end{array}$

$\mathrm{H} \quad 3.6063460 \quad 1.7884280 \quad-1.9951590$

$\begin{array}{llll}\mathrm{H} & 3.3982810 & 2.3102380 & -0.3054070\end{array}$

$\begin{array}{llll}\mathrm{H} & 4.9963450 & 2.3397690 & -1.0570230\end{array}$

$\begin{array}{llll}\mathrm{C} & 5.0377360 & 0.4115470 & 0.8629530\end{array}$

$\begin{array}{llll}\mathrm{H} & 5.2061720 & -0.5833960 & 1.2872790\end{array}$

$\begin{array}{llll}\mathrm{H} & 6.0159060 & 0.8914190 & 0.7548370\end{array}$

H $4.4629260 \quad 1.0084320 \quad 1.5773530$

$\begin{array}{llll}\mathrm{H} & 1.0647980 & 0.8193970 & -2.2111550\end{array}$

$\begin{array}{llll}\mathrm{H} & 1.3255260 & 2.2008780 & -1.1661760\end{array}$

$\begin{array}{llll}\text { C } & -1.2977930 & 2.8358020 & -0.6142460\end{array}$

$\begin{array}{llll}\text { C } & -2.2861990 & 2.4403630 & 0.3000610\end{array}$

$\begin{array}{llll}\text { C } & -0.8143290 & 4.1536470 & -0.5479490\end{array}$

$\begin{array}{llll}\text { C } & -2.7751350 & 3.3354720 & 1.2590260\end{array}$

$\begin{array}{llll}\mathrm{H} & -2.6779170 & 1.4271490 & 0.2605910\end{array}$

$\begin{array}{llll}\text { C } & -1.3000650 & 5.0494080 & 0.4076540\end{array}$

$\mathrm{H} \quad-0.0574450 \quad 4.4842950 \quad-1.2556610$

$\begin{array}{llll}\mathrm{C} & -2.2832110 & 4.6419770 & 1.3169840\end{array}$

$\mathrm{H} \quad-3.5416390 \quad 3.0100040 \quad 1.9567860$

$\begin{array}{llll}\mathrm{H} & -0.9166330 & 6.0654080 & 0.4389170\end{array}$

$\begin{array}{llll}\mathrm{H} & -2.6637740 & 5.3381570 & 2.0587570\end{array}$

$\begin{array}{llll}\mathrm{H} & 0.0631210 & 0.7750910 & 0.6862780\end{array}$

$\begin{array}{llll}\mathrm{O} & -0.1580410 & -0.9898530 & -0.5597260\end{array}$

$\mathrm{H} \quad-0.0893720 \quad-1.1797920 \quad-1.5090540$

$\begin{array}{llll}\mathrm{H} & -1.2276000 & -1.0552580 & -0.2813260\end{array}$

$\begin{array}{llll}\text { O } & -2.5023720 & -1.1546130 & 0.1139630\end{array}$

C $\quad-3.2453450 \quad-1.8294730 \quad-0.6769120$

$\begin{array}{llll}\text { O } & -2.9598880 & -2.3687830 & -1.7467420\end{array}$

C $\quad-4.7120760 \quad-1.9421230 \quad-0.1535690$

$\begin{array}{llll}\text { F } & -4.7555040 & -2.5254800 & 1.0712460\end{array}$

$\begin{array}{llll}\text { F } & -5.2840190 & -0.7164070 & -0.0326940\end{array}$

F $\quad-5.5003800 \quad-2.6703790 \quad-0.9696810$

4.log

Potential Energy = -1489.66303

Zero-point Energy = -1489.18910

Free Energy = -1489.23259

Single-Point Energy B3LYP-D3(BJ)/6-311+G** PCM = 1490.12040 
Free Energy B3LYP-D3(BJ)/6-311+G** PCM (extrapolated free energy from qRRHO) $=-1489.68996$

Nimag $=1\left(-326.9458 \mathrm{~cm}^{-1}\right)$

Charge $=0$ Multiplicity $=1$

\begin{tabular}{|c|c|c|c|}
\hline $\mathrm{C}$ & 1.9498940 & -0.6852460 & 0.6724980 \\
\hline $\mathrm{C}$ & 3.7033260 & -0.3305070 & -0.8676560 \\
\hline $\mathrm{C}$ & 2.9514240 & -1.5854720 & -1.3217480 \\
\hline $\mathrm{N}$ & 1.6733950 & -1.4726930 & -0.5610700 \\
\hline $\mathrm{N}$ & 3.0863740 & 0.1462170 & 0.2384880 \\
\hline $\mathrm{O}$ & 4.6946510 & 0.1560680 & -1.4162020 \\
\hline $\mathrm{C}$ & 3.4664490 & 1.4146260 & 0.8499540 \\
\hline $\mathrm{H}$ & 4.2667080 & 1.2922290 & 1.5859150 \\
\hline $\mathrm{H}$ & 3.8199250 & 2.0832420 & 0.0623780 \\
\hline $\mathrm{H}$ & 2.5959840 & 1.8587800 & 1.3346670 \\
\hline $\mathrm{C}$ & 0.4915310 & -1.3590850 & -1.2212330 \\
\hline $\mathrm{C}$ & -0.8204140 & -1.4594610 & -0.4723920 \\
\hline $\mathrm{C}$ & -2.0679270 & -1.3427020 & -1.3729370 \\
\hline $\mathrm{H}$ & -2.1503700 & -0.3253670 & -1.7675130 \\
\hline $\mathrm{H}$ & -1.9571730 & -2.0170060 & -2.2306780 \\
\hline $\mathrm{C}$ & 2.8615840 & -1.7253330 & -2.8355860 \\
\hline $\mathrm{H}$ & 3.8830200 & -1.7479590 & -3.2230060 \\
\hline $\mathrm{H}$ & 2.3778890 & -2.6635720 & -3.1206780 \\
\hline $\mathrm{H}$ & 2.3396390 & -0.8868340 & -3.3006720 \\
\hline $\mathrm{H}$ & 3.4966530 & -2.4530430 & -0.9340080 \\
\hline $\mathrm{C}$ & 2.2201560 & -1.5479400 & 1.9550480 \\
\hline $\mathrm{H}$ & 1.1002040 & -0.0287720 & 0.8800320 \\
\hline $\mathrm{C}$ & 2.3915150 & -0.6000660 & 3.1636310 \\
\hline $\mathrm{H}$ & 2.4259030 & -1.1912780 & 4.0842710 \\
\hline $\mathrm{H}$ & 3.3183670 & -0.0241370 & 3.1137000 \\
\hline $\mathrm{H}$ & 1.5533230 & 0.1004770 & 3.2514750 \\
\hline $\mathrm{C}$ & 1.0010490 & -2.4515790 & 2.2257070 \\
\hline $\mathrm{H}$ & 0.0858590 & -1.8685330 & 2.3729280 \\
\hline $\mathrm{H}$ & 0.8378140 & -3.1625740 & 1.4109210 \\
\hline $\mathrm{H}$ & 1.1734760 & -3.0279490 & 3.1402830 \\
\hline $\mathrm{C}$ & 3.4766770 & -2.4297430 & 1.8239170 \\
\hline $\mathrm{H}$ & 4.3644200 & -1.8449620 & 1.5623190 \\
\hline $\mathrm{H}$ & 3.6769630 & -2.9184760 & 2.7830100 \\
\hline $\mathrm{H}$ & 3.3434070 & -3.2213810 & 1.0804460 \\
\hline $\mathrm{H}$ & -0.8743300 & -0.7274900 & 0.3377300 \\
\hline $\mathrm{H}$ & -0.8170310 & -2.4511620 & -0.0071680 \\
\hline $\mathrm{C}$ & -3.3390300 & -1.6853770 & -0.6208780 \\
\hline $\mathrm{C}$ & -3.7990870 & -3.0097650 & -0.5543000 \\
\hline $\mathrm{C}$ & -4.0698690 & -0.6888690 & 0.0439370 \\
\hline $\mathrm{C}$ & -4.9568510 & -3.3317150 & 0.1603620 \\
\hline $\mathrm{H}$ & -3.2506110 & -3.7945200 & -1.0701520 \\
\hline $\mathrm{C}$ & -5.2280040 & -1.0062500 & 0.7605160 \\
\hline $\mathrm{H}$ & -3.7328940 & 0.3437730 & -0.0035190 \\
\hline $\mathrm{C}$ & -5.6751080 & -2.3299370 & 0.8215540 \\
\hline $\mathrm{H}$ & -5.2994760 & -4.3620190 & 0.1961250 \\
\hline $\mathrm{H}$ & -5.7817580 & -0.2199050 & 1.2656730 \\
\hline $\mathrm{H}$ & -6.5765220 & -2.5777320 & 1.3744840 \\
\hline $\mathrm{H}$ & 0.4935220 & -1.9113080 & -2.1556880 \\
\hline $\mathrm{O}$ & 0.4585630 & 0.2559860 & -1.9622620 \\
\hline $\mathrm{H}$ & 0.1490030 & 1.0588990 & -1.2715310 \\
\hline $\mathrm{H}$ & -0.1114070 & 0.2834180 & -2.7479220 \\
\hline $\mathrm{O}$ & -0.1404720 & 1.9824470 & -0.3388710 \\
\hline $\mathrm{C}$ & -0.8462150 & 2.9706510 & -0.7355600 \\
\hline $\mathrm{C}$ & -1.1394870 & 3.9824360 & 0.4174190 \\
\hline $\mathrm{O}$ & -1.3019890 & 3.2080180 & -1.8544740 \\
\hline $\mathrm{F}$ & -1.8582110 & 5.0462620 & 0.0058850 \\
\hline $\mathrm{F}$ & 0.0093440 & 4.4606090 & 0.9594990 \\
\hline $\mathrm{F}$ & -1.8383140 & 3.3907220 & 1.4207500 \\
\hline
\end{tabular}

$5 . \log$

Potential Energy $=-1489.66201$

Zero-point Energy = -1489.18806

Free Energy = -1489.23178

Single-Point Energy B3LYP-D3(BJ)/6-311+G** PCM = -

1490.12032

Free Energy B3LYP-D3(BJ)/6-311+G** PCM (extrapolated

free energy from $\mathrm{qRRHO})=-1489.69009$

Nimag $=1\left(-338.0783 \mathrm{~cm}^{-1}\right)$

Charge $=0$ Multiplicity $=1$

$\begin{array}{lrrr}\text { C } & -0.6678660 & 2.0195690 & 0.4497310 \\ \text { C } & -0.6493540 & 2.8554140 & -1.7572660 \\ \text { C } & 0.8029790 & 2.5509570 & -1.3768490\end{array}$

\begin{tabular}{|c|c|c|c|}
\hline $\mathrm{N}$ & 0.6328230 & 1.6693650 & -0.1857940 \\
\hline $\mathrm{N}$ & -1.4328840 & 2.5201910 & -0.7057340 \\
\hline $\mathrm{O}$ & -1.0332190 & 3.3231880 & -2.8315370 \\
\hline $\mathrm{C}$ & -2.8888900 & 2.5535000 & -0.7856480 \\
\hline $\mathrm{H}$ & -3.2920060 & 3.5372500 & -0.5270820 \\
\hline $\mathrm{H}$ & -3.1828790 & 2.3173560 & -1.8104100 \\
\hline $\mathrm{H}$ & -3.3072070 & 1.8058500 & -0.1101890 \\
\hline $\mathrm{C}$ & 1.0733200 & 0.3840990 & -0.2197110 \\
\hline $\mathrm{C}$ & 1.0737550 & -0.4595920 & 1.0381770 \\
\hline $\mathrm{C}$ & 1.7316680 & -1.8487110 & 0.8883360 \\
\hline $\mathrm{H}$ & 1.6081660 & -2.3550020 & 1.8527860 \\
\hline $\mathrm{H}$ & 1.1824840 & -2.4527460 & 0.1598720 \\
\hline $\mathrm{C}$ & 1.6373960 & 2.0202500 & -2.5350360 \\
\hline $\mathrm{H}$ & 1.6280230 & 2.7798830 & -3.3203950 \\
\hline $\mathrm{H}$ & 2.6778390 & 1.8645010 & -2.2374180 \\
\hline $\mathrm{H}$ & 1.2324120 & 1.0964410 & -2.9526980 \\
\hline $\mathrm{H}$ & 1.2565240 & 3.4883870 & -1.0365820 \\
\hline $\mathrm{C}$ & -0.5648210 & 3.0226660 & 1.6521100 \\
\hline $\mathrm{H}$ & -1.1471910 & 1.1070180 & 0.8157560 \\
\hline $\mathrm{C}$ & -1.9726970 & 3.2111160 & 2.2611550 \\
\hline $\mathrm{H}$ & -1.8907840 & 3.7838090 & 3.1904050 \\
\hline $\mathrm{H}$ & -2.6426090 & 3.7633680 & 1.5981470 \\
\hline $\mathrm{H}$ & -2.4412190 & 2.2507100 & 2.5046930 \\
\hline $\mathrm{C}$ & 0.3498120 & 2.4227210 & 2.7379900 \\
\hline $\mathrm{H}$ & -0.0271170 & 1.4613460 & 3.1028500 \\
\hline $\mathrm{H}$ & 1.3714210 & 2.2851260 & 2.3724480 \\
\hline $\mathrm{H}$ & 0.3921910 & 3.1044920 & 3.5934400 \\
\hline $\mathrm{C}$ & -0.0092160 & 4.3973250 & 1.2338410 \\
\hline $\mathrm{H}$ & -0.5857620 & 4.8424630 & 0.4166490 \\
\hline $\mathrm{H}$ & -0.0642880 & 5.0834170 & 2.0853590 \\
\hline $\mathrm{H}$ & 1.0414610 & 4.3385840 & 0.9343120 \\
\hline $\mathrm{H}$ & 0.0579940 & -0.5823160 & 1.4230950 \\
\hline $\mathrm{H}$ & 1.6268180 & 0.1146490 & 1.7872030 \\
\hline $\mathrm{C}$ & 3.2062110 & -1.8192050 & 0.5253400 \\
\hline $\mathrm{C}$ & 3.6700910 & -2.3813670 & -0.6734640 \\
\hline $\mathrm{C}$ & 4.1469920 & -1.2428990 & 1.3965260 \\
\hline $\mathrm{C}$ & 5.0309630 & -2.3617600 & -1.0006790 \\
\hline $\mathrm{H}$ & 2.9643340 & -2.8474010 & -1.3567630 \\
\hline $\mathrm{C}$ & 5.5058860 & -1.2203430 & 1.0742960 \\
\hline $\mathrm{H}$ & 3.8174510 & -0.8152050 & 2.3404280 \\
\hline $\mathrm{C}$ & 5.9534840 & -1.7783740 & -0.1288600 \\
\hline $\mathrm{H}$ & 5.3661560 & -2.8038110 & -1.9346460 \\
\hline $\mathrm{H}$ & 6.2155950 & -0.7722770 & 1.7640160 \\
\hline $\mathrm{H}$ & 7.0100910 & -1.7622690 & -0.3796240 \\
\hline $\mathrm{H}$ & 1.9753850 & 0.2793060 & -0.8143100 \\
\hline $\mathrm{O}$ & 0.0003580 & -0.5014050 & -1.3285240 \\
\hline $\mathrm{H}$ & -0.9032860 & -0.9328190 & -0.8604480 \\
\hline $\mathrm{H}$ & 0.4599550 & -1.2122210 & -1.8032520 \\
\hline $\mathrm{O}$ & -2.0262060 & -1.3672060 & -0.2691220 \\
\hline $\mathrm{C}$ & -2.2886720 & -2.5991100 & -0.4849310 \\
\hline $\mathrm{C}$ & -3.5932620 & -3.0598410 & 0.2384820 \\
\hline $\mathrm{O}$ & -1.6647700 & -3.4237890 & -1.1536310 \\
\hline $\mathrm{F}$ & -3.4615510 & -2.9613780 & 1.5871270 \\
\hline $\mathrm{F}$ & -3.9165290 & -4.3392850 & -0.0375260 \\
\hline $\mathrm{F}$ & -4.6547530 & -2.2926230 & -0.1157890 \\
\hline
\end{tabular}

$6 . \log$

Potential Energy = -1489.66303

Zero-point Energy = -1489.18910

Free Energy = -1489.23259

Single-Point Energy B3LYP-D3(BJ)/6-311+G** PCM = -

1490.12040

Free Energy B3LYP-D3(BJ)/6-311+G** PCM (extrapolated

free energy from $\mathrm{qRRHO})=-1489.68996$

Nimag $=1\left(-327.0302 \mathrm{~cm}^{-1}\right)$

Charge $=0$ Multiplicity $=1$

$\begin{array}{llll}\mathrm{C} & 1.9497960 & -0.6855510 & 0.6724740 \\ \mathrm{C} & 3.7032480 & -0.3311680 & -0.8677400 \\ \mathrm{C} & 2.9509950 & -1.5859030 & -1.3218810 \\ \mathrm{~N} & 1.6730390 & -1.4728540 & -0.5611250 \\ \mathrm{~N} & 3.0864730 & 0.1456470 & 0.2384630 \\ \mathrm{O} & 4.6946800 & 0.1551780 & -1.4162980 \\ \mathrm{C} & 3.4669310 & 1.4138960 & 0.8500180 \\ \mathrm{H} & 4.2670550 & 1.2911890 & 1.5860750 \\ \mathrm{H} & 3.8207500 & 2.0824020 & 0.0625040 \\ \mathrm{H} & 2.5965680 & 1.8583490 & 1.3346420\end{array}$




\begin{tabular}{|c|c|c|c|}
\hline $\mathrm{C}$ & 0.4911560 & -1.3589270 & -1.2212230 \\
\hline $\mathrm{C}$ & -0.8207720 & -1.4590850 & -0.4723180 \\
\hline $\mathrm{C}$ & -2.0683000 & -1.3420470 & -1.3728110 \\
\hline $\mathrm{H}$ & -2.1507160 & -0.3246080 & -1.7671270 \\
\hline $\mathrm{H}$ & -1.9575820 & -2.0161370 & -2.2307270 \\
\hline $\mathrm{C}$ & 2.8610390 & -1.7256130 & -2.8357280 \\
\hline $\mathrm{H}$ & 3.8824490 & -1.7484500 & -3.2232050 \\
\hline $\mathrm{H}$ & 2.3771040 & -2.6637110 & -3.1208790 \\
\hline $\mathrm{H}$ & 2.3392690 & -0.8869490 & -3.3007110 \\
\hline $\mathrm{H}$ & 3.4960260 & -2.4536450 & -0.9342430 \\
\hline $\mathrm{C}$ & 2.2198970 & -1.5483790 & 1.9549690 \\
\hline $\mathrm{H}$ & 1.1002840 & -0.0288700 & 0.8800860 \\
\hline $\mathrm{C}$ & 2.3914810 & -0.6006110 & 3.1636030 \\
\hline $\mathrm{H}$ & 2.4257320 & -1.1918800 & 4.0842130 \\
\hline $\mathrm{H}$ & 3.3184690 & -0.0248990 & 3.1136990 \\
\hline $\mathrm{H}$ & 1.5534540 & 0.1001270 & 3.2514830 \\
\hline $\mathrm{C}$ & 1.0006030 & -2.4517720 & 2.2256040 \\
\hline $\mathrm{H}$ & 0.0855480 & -1.8685310 & 2.3728910 \\
\hline $\mathrm{H}$ & 0.8371860 & -3.1626770 & 1.4107780 \\
\hline $\mathrm{H}$ & 1.1729320 & -3.0282370 & 3.1401390 \\
\hline $\mathrm{C}$ & 3.4762280 & -2.4304430 & 1.8237590 \\
\hline $\mathrm{H}$ & 4.3640890 & -1.8458350 & 1.5621760 \\
\hline $\mathrm{H}$ & 3.6764310 & -2.9192770 & 2.7828180 \\
\hline $\mathrm{H}$ & 3.3427750 & -3.2220070 & 1.0802420 \\
\hline $\mathrm{H}$ & -0.8745120 & -0.7271380 & 0.3378380 \\
\hline $\mathrm{H}$ & -0.8175590 & -2.4508040 & -0.0071350 \\
\hline $\mathrm{C}$ & -3.3394040 & -1.6848840 & -0.6208270 \\
\hline $\mathrm{C}$ & -3.7993220 & -3.0093270 & -0.5543480 \\
\hline $\mathrm{C}$ & -4.0703780 & -0.6884940 & 0.0440130 \\
\hline $\mathrm{C}$ & -4.9570830 & -3.3314430 & 0.1602420 \\
\hline $\mathrm{H}$ & -3.2507340 & -3.7939930 & -1.0702170 \\
\hline $\mathrm{C}$ & -5.2285120 & -1.0060420 & 0.7605210 \\
\hline $\mathrm{H}$ & -3.7335110 & 0.3441870 & -0.0033670 \\
\hline $\mathrm{C}$ & -5.6754770 & -2.3297800 & 0.8214600 \\
\hline $\mathrm{H}$ & -5.2995990 & -4.3617860 & 0.1959290 \\
\hline $\mathrm{H}$ & -5.7823730 & -0.2197880 & 1.2657020 \\
\hline $\mathrm{H}$ & -6.5768900 & -2.5777040 & 1.3743350 \\
\hline $\mathrm{H}$ & 0.4929690 & -1.9111120 & -2.1557010 \\
\hline $\mathrm{O}$ & 0.4584900 & 0.2561280 & -1.9621050 \\
\hline $\mathrm{H}$ & 0.1491980 & 1.0591180 & -1.2712900 \\
\hline $\mathrm{H}$ & -0.1115580 & 0.2837410 & -2.7477020 \\
\hline $\mathrm{O}$ & -0.1399530 & 1.9827030 & -0.3387040 \\
\hline $\mathrm{C}$ & -0.8456320 & 2.9709460 & -0.7354260 \\
\hline $\mathrm{C}$ & -1.1386590 & 3.9828970 & 0.4174670 \\
\hline $\mathrm{O}$ & -1.3015190 & 3.2082090 & -1.8543140 \\
\hline $\mathrm{F}$ & -1.8570940 & 5.0468780 & 0.0058340 \\
\hline $\mathrm{F}$ & 0.0102750 & 4.4608080 & 0.9595520 \\
\hline $\mathrm{F}$ & -1.8376730 & 3.3914270 & 1.4208130 \\
\hline
\end{tabular}

7.log

Potential Energy = -1489.66171

Zero-point Energy = -1489.18784

Free Energy = -1489.23117

Single-Point Energy B3LYP-D3(BJ)/6-311+G** PCM = 1490.11933

Free Energy B3LYP-D3(BJ)/6-311+G** PCM (extrapolated

free energy from qRRHO) $=-1489.68879$

Nimag $=1\left(-319.4351 \mathrm{~cm}^{-1}\right)$

Charge $=0$ Multiplicity $=1$

$\begin{array}{lccc}\mathrm{C} & 1.4178620 & -1.2262770 & 0.6745040 \\ \mathrm{C} & 3.2013550 & -1.5723010 & -0.8345990 \\ \mathrm{C} & 2.0640050 & -2.5107840 & -1.2536040 \\ \mathrm{~N} & 0.8979530 & -1.9239230 & -0.5341140 \\ \mathrm{~N} & 2.7712060 & -0.8499690 & 0.2258590 \\ \mathrm{O} & 4.3059410 & -1.4900100 & -1.3755540 \\ \mathrm{C} & 3.5648920 & 0.2322330 & 0.7976190 \\ \mathrm{H} & 4.2719440 & -0.1307950 & 1.5495610 \\ \mathrm{H} & 4.1295870 & 0.7062580 & -0.0077490 \\ \mathrm{H} & 2.8999670 & 0.9677010 & 1.2518420 \\ \mathrm{C} & -0.1909630 & -1.4704780 & -1.2082150 \\ \mathrm{C} & -1.4743980 & -1.1403080 & -0.4750600 \\ \mathrm{C} & -2.6317890 & -0.7250630 & -1.4075760 \\ \mathrm{H} & -2.3741560 & 0.2059880 & -1.9178280 \\ \mathrm{H} & -2.7571940 & -1.4923420 & -2.1808990 \\ \mathrm{C} & 1.9486850 & -2.7019800 & -2.7599030 \\ \mathrm{H} & 2.8989160 & -3.1115830 & -3.1103500\end{array}$

$\begin{array}{lrrr}\mathrm{H} & 1.1628360 & -3.4179270 & -3.0130320 \\ \mathrm{H} & 1.7759640 & -1.7720760 & -3.3080950 \\ \mathrm{H} & 2.2687700 & -3.4920550 & -0.8119550 \\ \mathrm{C} & 1.3804030 & -2.0697370 & 1.9983800 \\ \mathrm{H} & 0.8397500 & -0.3124340 & 0.8356320 \\ \mathrm{C} & 1.8397880 & -1.1705070 & 3.1689520 \\ \mathrm{H} & 1.6739230 & -1.6968970 & 4.1142580 \\ \mathrm{H} & 2.9032760 & -0.9270210 & 3.1166300 \\ \mathrm{H} & 1.2719740 & -0.2340450 & 3.2077080 \\ \mathrm{C} & -0.0674410 & -2.5158510 & 2.2810980 \\ \mathrm{H} & -0.7459950 & -1.6620020 & 2.3781060 \\ \mathrm{H} & -0.4450240 & -3.1791610 & 1.4979110 \\ \mathrm{H} & -0.0973210 & -3.0677840 & 3.2259900 \\ \mathrm{C} & 2.2834150 & -3.3167630 & 1.9373110 \\ \mathrm{H} & 3.3147590 & -3.0669500 & 1.6687210 \\ \mathrm{H} & 2.3081080 & -3.7925580 & 2.9230800 \\ \mathrm{H} & 1.9048270 & -4.0614110 & 1.2306500 \\ \mathrm{H} & -1.3083760 & -0.3714840 & 0.2852860 \\ \mathrm{H} & -1.7585510 & -2.0545680 & 0.0552120 \\ \mathrm{C} & -3.9323980 & -0.5528230 & -0.6488360 \\ \mathrm{C} & -4.7996200 & -1.6384110 & -0.4495570 \\ \mathrm{C} & -4.2884410 & 0.6936340 & -0.1105740 \\ \mathrm{C} & -5.9891170 & -1.4851710 & 0.2693720 \\ \mathrm{H} & -4.5451730 & -2.6102790 & -0.8661970 \\ \mathrm{C} & -5.4764720 & 0.8519850 & 0.6098290 \\ \mathrm{H} & -3.6331990 & 1.5482010 & -0.2616560 \\ \mathrm{C} & -6.3311560 & -0.2381670 & 0.8027820 \\ \mathrm{H} & -6.6491730 & -2.3368670 & 0.4081230 \\ \mathrm{H} & -5.7356480 & 1.8261260 & 1.0150310 \\ \mathrm{H} & -7.2563050 & -0.1162320 & 1.3586400 \\ \mathrm{H} & -0.3571540 & -2.0162580 & -2.1316540 \\ \mathrm{O} & 0.1084320 & 0.1041850 & -1.9885060 \\ \mathrm{H} & 0.2524590 & 0.9417600 & -1.2896100 \\ \mathrm{H} & 0.8689460 & 0.0654900 & -2.5881710 \\ \mathrm{O} & 0.3481200 & 1.9180930 & -0.3629320 \\ \mathrm{C} & 0.8361630 & 3.0291990 & -0.7619620 \\ \mathrm{C} & 0.8725280 & 4.0962700 & 0.3781170 \\ & 1.2568120 & 3.3462410 & -1.8747470 \\ \mathrm{~F} & 1.5950590 & 3.6581350 & 1.4410990 \\ & -0.3775910 & 4.3711270 & 0.8318970\end{array}$

8. $\log$

Potential Energy = -1489.66281

Zero-point Energy = -1489.18914

Free Energy $=-1489.23327$

Single-Point Energy B3LYP-D3(BJ)/6-311+G** PCM = 1490.11742

Free Energy B3LYP-D3(BJ)/6-311+G** PCM (extrapolated

free energy from $\mathrm{qRRHO})=-1489.68788$

Nimag $=1\left(-301.9079 \mathrm{~cm}^{-1}\right)$

Charge $=0$ Multiplicity $=1$

$\begin{array}{lrrr}\mathrm{C} & 2.8651430 & -0.4922060 & 0.2683590 \\ \mathrm{C} & 3.2823360 & -2.0585370 & -1.4520040 \\ \mathrm{C} & 1.7835630 & -2.1426610 & -1.1535730 \\ \mathrm{~N} & 1.5779300 & -0.9171800 & -0.3408840 \\ \mathrm{~N} & 3.8234450 & -1.0974800 & -0.6693640 \\ \mathrm{O} & 3.8825970 & -2.7326000 & -2.2912850 \\ \mathrm{C} & 5.1801200 & -0.6066500 & -0.8983240 \\ \mathrm{H} & 5.9303530 & -1.2211030 & -0.3935500 \\ \mathrm{H} & 5.3793770 & -0.6287680 & -1.9719730 \\ \mathrm{H} & 5.2593130 & 0.4197160 & -0.5386430 \\ \mathrm{C} & 0.4521800 & -0.1676890 & -0.4155720 \\ \mathrm{C} & 0.5144400 & 1.3280920 & -0.1898490 \\ \mathrm{C} & -0.8298460 & 2.0490660 & -0.4179590 \\ \mathrm{H} & -1.2221620 & 1.7766390 & -1.4046890 \\ \mathrm{H} & -1.5589180 & 1.7113870 & 0.3237270 \\ \mathrm{C} & 0.9614520 & -2.2432030 & -2.4351110 \\ \mathrm{H} & 1.3283750 & -3.1053040 & -2.9970900 \\ \mathrm{H} & -0.0988210 & -2.4087300 & -2.2287290 \\ \mathrm{H} & 1.0752260 & -1.3528560 & -3.0610170 \\ \mathrm{H} & 1.6031170 & -3.0388050 & -0.5461120 \\ \mathrm{C} & 3.0755840 & -0.9145040 & 1.7668060 \\ \mathrm{H} & 2.9557420 & 0.5964730 & 0.2042090 \\ \mathrm{C} & 4.5216400 & -0.5811040 & 2.1954280 \\ \mathrm{H} & 4.6168710 & -0.7270460 & 3.2760740\end{array}$




$\begin{array}{lrrr}\mathrm{H} & 5.2567070 & -1.2297720 & 1.7137370 \\ \mathrm{H} & 4.7813660 & 0.4616700 & 1.9811640 \\ \mathrm{C} & 2.1249780 & -0.0987000 & 2.6672330 \\ \mathrm{H} & 2.3331210 & 0.9750300 & 2.5946070 \\ \mathrm{H} & 1.0724450 & -0.2644210 & 2.4284140 \\ \mathrm{H} & 2.2772320 & -0.3904230 & 3.7113740 \\ \mathrm{C} & 2.8271640 & -2.4191090 & 1.9783460 \\ \mathrm{H} & 3.4697290 & -3.0321390 & 1.3378280 \\ \mathrm{H} & 3.0526770 & -2.6837850 & 3.0163370 \\ \mathrm{H} & 1.7842710 & -2.6943710 & 1.7936140 \\ \mathrm{H} & 1.2479060 & 1.7125240 & -0.9103610 \\ \mathrm{H} & 0.8905830 & 1.5553510 & 0.8093320 \\ \mathrm{C} & -0.6806880 & 3.5549060 & -0.3288660 \\ \mathrm{C} & -0.8714320 & 4.2250700 & 0.8893230 \\ \mathrm{C} & -0.3241780 & 4.3092340 & -1.4578050 \\ \mathrm{C} & -0.7071520 & 5.6109590 & 0.9797360 \\ \mathrm{H} & -1.1561120 & 3.6586480 & 1.7728630 \\ \mathrm{C} & -0.1591380 & 5.6949600 & -1.3726600 \\ \mathrm{H} & -0.1806690 & 3.8088910 & -2.4127010 \\ \mathrm{C} & -0.3491440 & 6.3508060 & -0.1517920 \\ \mathrm{H} & -0.8632200 & 6.1114920 & 1.9312360 \\ \mathrm{H} & 0.1123790 & 6.2613220 & -2.2591110 \\ \mathrm{H} & -0.2246160 & 7.4276820 & -0.0843650 \\ \mathrm{H} & -0.1806830 & -0.4535230 & -1.2494940 \\ \mathrm{O} & -0.6818800 & -0.6674930 & 0.8398350 \\ \mathrm{H} & -1.7546010 & -0.6605710 & 0.5400490 \\ \mathrm{H} & -0.4843870 & -1.5977110 & 1.0390780 \\ \mathrm{O} & -3.0468740 & -0.6593970 & 0.2160810 \\ \mathrm{C} & -3.5873260 & -1.8065140 & 0.3768880 \\ \mathrm{O} & -3.0775040 & -2.8587520 & 0.7652300 \\ \mathrm{C} & -5.1054620 & -1.8014020 & 0.0152990 \\ \mathrm{~F} & -5.7924510 & -0.9317990 & 0.7993650 \\ \mathrm{~F} & -5.3032540 & -1.4207810 & -1.2724430 \\ \mathrm{~F} & -5.6785560 & -3.0122900 & 0.1657040\end{array}$

$9 . \log$

Potential Energy $=-1489.65736$

Zero-point Energy $=-1489.18316$

Free Energy $=-1489.22653$

Single-Point Energy B3LYP-D3(BJ)/6-311+G** PCM = 1490.11533

Free Energy B3LYP-D3(BJ)/6-311+G** PCM (extrapolated free energy from qRRHO) $=-1489.68449$

Nimag $=1\left(-259.3944 \mathrm{~cm}^{-1}\right)$

Charge $=0$ Multiplicity $=1$

$\begin{array}{lrrr}\mathrm{C} & 3.0482640 & 0.2436420 & -0.0441230 \\ \mathrm{C} & 3.4644160 & -1.9819580 & -0.7227370 \\ \mathrm{C} & 1.9581610 & -1.8897830 & -0.4686920 \\ \mathrm{~N} & 1.7637570 & -0.4195740 & -0.3912150 \\ \mathrm{~N} & 4.0106260 & -0.7612020 & -0.5200690 \\ \mathrm{O} & 4.0670680 & -2.9932880 & -1.0868310 \\ \mathrm{C} & 5.3830840 & -0.4664820 & -0.9244820 \\ \mathrm{H} & 6.1078560 & -0.7529900 & -0.1577510 \\ \mathrm{H} & 5.6030410 & -1.0266670 & -1.8359390 \\ \mathrm{H} & 5.4799960 & 0.6008170 & -1.1258950 \\ \mathrm{C} & 0.6545870 & 0.1961500 & -0.8653320 \\ \mathrm{C} & 0.7441060 & 1.6047450 & -1.4130420 \\ \mathrm{C} & -0.5620650 & 2.1680300 & -2.0237720 \\ \mathrm{H} & -0.2627110 & 2.8253500 & -2.8486440 \\ \mathrm{H} & -1.1374460 & 1.3568530 & -2.4831300 \\ \mathrm{C} & 1.1622470 & -2.6150730 & -1.5496900 \\ \mathrm{H} & 1.5196910 & -3.6468000 & -1.5874320 \\ \mathrm{H} & 0.0942190 & -2.6417970 & -1.3207940 \\ \mathrm{H} & 1.3122410 & -2.1646520 & -2.5355790 \\ \mathrm{H} & 1.7400970 & -2.3533490 & 0.5019120 \\ \mathrm{C} & 3.2217230 & 0.6335620 & 1.4678860 \\ \mathrm{H} & 3.1637300 & 1.1496430 & -0.6465170 \\ \mathrm{C} & 4.6643930 & 1.1328510 & 1.7059490 \\ \mathrm{H} & 4.7353030 & 1.5542550 & 2.7136380 \\ \mathrm{H} & 5.3978000 & 0.3264100 & 1.6385020 \\ \mathrm{H} & 4.9464180 & 1.9220210 & 0.9999530 \\ \mathrm{C} & 2.2699410 & 1.7978050 & 1.8117390 \\ \mathrm{H} & 2.5000130 & 2.6888600 & 1.2168060 \\ \mathrm{H} & 1.2173640 & 1.5493820 & 1.6611490 \\ \mathrm{H} & 2.3932900 & 2.0675660 & 2.8653050 \\ \mathrm{C} & 2.9483320 & -0.5590900 & 2.4026120 \\ & & & \\ & & & \end{array}$

$\begin{array}{rrrr}\mathrm{H} & 3.5963100 & -1.4113130 & 2.1734960 \\ \mathrm{H} & 3.1482290 & -0.2650680 & 3.4378170 \\ \mathrm{H} & 1.9070620 & -0.8933160 & 2.3579250 \\ \mathrm{H} & 1.4933000 & 1.5389680 & -2.2124790 \\ \mathrm{H} & 1.1397260 & 2.2929000 & -0.6649820 \\ \mathrm{C} & -1.4565940 & 2.9656670 & -1.0911530 \\ \mathrm{C} & -2.7926740 & 2.5983210 & -0.8801170 \\ \mathrm{C} & -0.9790790 & 4.1271470 & -0.4616160 \\ \mathrm{C} & -3.6279450 & 3.3597950 & -0.0550930 \\ \mathrm{H} & -3.1823350 & 1.7032410 & -1.3570420 \\ \mathrm{C} & -1.8075600 & 4.8896910 & 0.3656200 \\ \mathrm{H} & 0.0481230 & 4.4459400 & -0.6229630 \\ \mathrm{C} & -3.1378290 & 4.5076540 & 0.5735390 \\ \mathrm{H} & -4.6593140 & 3.0533660 & 0.0960450 \\ \mathrm{H} & -1.4167570 & 5.7844800 & 0.8421830 \\ \mathrm{H} & -3.7838090 & 5.1003250 & 1.2148030 \\ \mathrm{H} & 0.0419400 & -0.4601420 & -1.4746960 \\ \mathrm{O} & -0.5507630 & 0.3432800 & 0.4279420 \\ \mathrm{H} & -1.4788490 & -0.1801210 & 0.1740350 \\ \mathrm{H} & -0.1969480 & -0.1503970 & 1.1863850 \\ \mathrm{O} & -2.6169520 & -0.8721740 & -0.1298980 \\ \mathrm{C} & -2.7952510 & -1.8678820 & 0.6479280 \\ \mathrm{O} & -2.1015080 & -2.2544040 & 1.5918840 \\ \mathrm{C} & -4.0937260 & -2.6644900 & 0.3057590 \\ \mathrm{~F} & -5.1959210 & -1.8814690 & 0.4333880 \\ \mathrm{~F} & -4.0717520 & -3.1167570 & -0.9742550 \\ \mathrm{~F} & -4.2734600 & -3.7370440 & 1.1031990\end{array}$

$10 . \log$

Potential Energy $=-1489.66178$

Zero-point Energy $=-1489.18774$

Free Energy $=-1489.23108$

Single-Point Energy B3LYP-D3(BJ)/6-311+G** PCM = 1490.11946

Free Energy B3LYP-D3(BJ)/6-311+G** PCM (extrapolated free energy from qRRHO) $=-1489.68876$

Nimag $=1\left(-331.0284 \mathrm{~cm}^{-1}\right)$

Charge $=0$ Multiplicity $=1$

$\begin{array}{lrrr}\mathrm{C} & -3.0935230 & -0.3488320 & -0.4440750 \\ \mathrm{C} & -3.4353370 & 0.0880690 & 1.8531470 \\ \mathrm{C} & -1.9704560 & -0.3317880 & 1.7100630 \\ \mathrm{~N} & -1.7811600 & -0.2371540 & 0.2412340 \\ \mathrm{~N} & -3.9986140 & 0.1052170 & 0.6237800 \\ \mathrm{O} & -3.9926600 & 0.3907780 & 2.9102330 \\ \mathrm{C} & -5.3130970 & 0.7003000 & 0.3972550 \\ \mathrm{H} & -6.1237550 & -0.0071230 & 0.5917340 \\ \mathrm{H} & -5.4283200 & 1.5530220 & 1.0700100 \\ \mathrm{H} & -5.3808180 & 1.0448490 & -0.6350980 \\ \mathrm{C} & -0.6228760 & 0.1871470 & -0.3245560 \\ \mathrm{C} & -0.6483790 & 0.9181020 & -1.6520460 \\ \mathrm{C} & 0.7003070 & 1.5548320 & -2.0554010 \\ \mathrm{H} & 1.4760740 & 0.7859740 & -2.1121260 \\ \mathrm{H} & 0.5737270 & 1.9419700 & -3.0725200 \\ \mathrm{C} & -1.0493420 & 0.5348470 & 2.5634580 \\ \mathrm{H} & -1.4039160 & 0.4783670 & 3.5953500 \\ \mathrm{H} & -0.0182080 & 0.1727570 & 2.5461480 \\ \mathrm{H} & -1.0716450 & 1.5831040 & 2.2505800 \\ \mathrm{H} & -1.8779540 & -1.3746650 & 2.0399930 \\ \mathrm{C} & -3.4402470 & -1.7683450 & -1.0202260 \\ \mathrm{H} & -3.1369780 & 0.3706210 & -1.2673680 \\ \mathrm{C} & -4.8988700 & -1.7726690 & -1.5285930 \\ \mathrm{H} & -5.0859850 & -2.7040550 & -2.0723690 \\ \mathrm{H} & -5.6227320 & -1.7217910 & -0.7121630 \\ \mathrm{H} & -5.0929250 & -0.9445330 & -2.2195930 \\ \mathrm{C} & -2.5266940 & -2.0665370 & -2.2269330 \\ \mathrm{H} & -2.6745210 & -1.3326270 & -3.0277950 \\ \mathrm{H} & -1.4682490 & -2.0788150 & -1.9591180 \\ \mathrm{H} & -2.7766530 & -3.0508580 & -2.6358490 \\ \mathrm{C} & -3.2769440 & -2.8723860 & 0.0401850 \\ \mathrm{H} & -3.8989550 & -2.6854640 & 0.9217490 \\ \mathrm{H} & -3.5877900 & -3.8336150 & -0.3815400 \\ \mathrm{H} & -2.2371570 & -2.9808300 & 0.3632940 \\ \mathrm{H} & -1.3978530 & 1.7134800 & -1.5602730 \\ \mathrm{H} & -0.9837270 & 0.2467880 & -2.4446040 \\ \mathrm{C} & 1.1532600 & 2.6808780 & -1.1439030 \\ \mathrm{C} & 0.5076620 & 3.9287110 & -1.1802430\end{array}$




$\begin{array}{lccc}\mathrm{C} & 2.2177090 & 2.5064780 & -0.2463700 \\ \mathrm{C} & 0.9096440 & 4.9702660 & -0.3405600 \\ \mathrm{H} & -0.3112810 & 4.0896750 & -1.8778230 \\ \mathrm{C} & 2.6235850 & 3.5481440 & 0.5964410 \\ \mathrm{H} & 2.7342900 & 1.5508860 & -0.2116250 \\ \mathrm{C} & 1.9702900 & 4.7825340 & 0.5535580 \\ \mathrm{H} & 0.4002300 & 5.9286980 & -0.3877270 \\ \mathrm{H} & 3.4513710 & 3.3932430 & 1.2828920 \\ \mathrm{H} & 2.2855660 & 5.5922270 & 1.2052960 \\ \mathrm{H} & 0.0522920 & 0.6268900 & 0.4021310 \\ \mathrm{O} & 0.3785640 & -1.1959370 & -0.7083210 \\ \mathrm{H} & 1.4916940 & -1.0793800 & -0.6182840 \\ \mathrm{H} & 0.1481330 & -1.8915130 & -0.0700530 \\ \mathrm{O} & 2.8048640 & -0.9987640 & -0.5349500 \\ \mathrm{C} & 3.2963710 & -1.9134310 & 0.2127620 \\ \mathrm{O} & 2.7142030 & -2.7994270 & 0.8389160 \\ \mathrm{C} & 4.8523130 & -1.8342660 & 0.2963420 \\ \mathrm{~F} & 5.4162260 & -1.9632080 & -0.9313160 \\ \mathrm{~F} & 5.2558390 & -0.6397620 & 0.7987060 \\ \mathrm{~F} & 5.3782050 & -2.7958360 & 1.0807000\end{array}$

11.log

Potential Energy = -1489.66281

Zero-point Energy = -1489.18914

Free Energy $=-1489.23327$

Single-Point Energy B3LYP-D3(BJ)/6-311+G** PCM = 1490.11742

Free Energy B3LYP-D3(BJ)/6-311+G** PCM (extrapolated free energy from qRRHO) $=-1489.68788$

Nimag $=1\left(-301.8792 \mathrm{~cm}^{-1}\right)$

Charge $=0$ Multiplicity $=1$

$\begin{array}{lrrr}\mathrm{C} & 2.8650320 & -0.4926000 & 0.2684990 \\ \mathrm{C} & 3.2821400 & -2.0586630 & -1.4521230 \\ \mathrm{C} & 1.7833850 & -2.1428240 & -1.1536030 \\ \mathrm{~N} & 1.5777950 & -0.9174120 & -0.3407940 \\ \mathrm{~N} & 3.8233160 & -1.0977640 & -0.6693210 \\ \mathrm{O} & 3.8823220 & -2.7325890 & -2.2915680 \\ \mathrm{C} & 5.1799130 & -0.6067470 & -0.8983450 \\ \mathrm{H} & 5.9302200 & -1.2204870 & -0.3928220 \\ \mathrm{H} & 5.3794230 & -0.6298170 & -1.9719220 \\ \mathrm{H} & 5.2587010 & 0.4199890 & -0.5396000 \\ \mathrm{C} & 0.4521560 & -0.1677620 & -0.4155270 \\ \mathrm{C} & 0.5145970 & 1.3279940 & -0.1896900 \\ \mathrm{C} & -0.8295090 & 2.0491880 & -0.4181810 \\ \mathrm{H} & -1.2215310 & 1.7768950 & -1.4050650 \\ \mathrm{H} & -1.5588840 & 1.7115630 & 0.3232340 \\ \mathrm{C} & 0.9611760 & -2.2433030 & -2.4350770 \\ \mathrm{H} & 1.3280230 & -3.1054070 & -2.9970990 \\ \mathrm{H} & -0.0990860 & -2.4087910 & -2.2286130 \\ \mathrm{H} & 1.0749360 & -1.3529530 & -3.0609820 \\ \mathrm{H} & 1.6030060 & -3.0390030 & -0.5461780 \\ \mathrm{C} & 3.0754270 & -0.9150980 & 1.7668900 \\ \mathrm{H} & 2.9557030 & 0.5960790 & 0.2045220 \\ \mathrm{C} & 4.5215120 & -0.5818620 & 2.1955300 \\ \mathrm{H} & 4.6167130 & -0.7277570 & 3.2761860 \\ \mathrm{H} & -0.3481590 & 6.3508270 & -0.1515310 \\ \mathrm{H} & 5.2564980 & -1.2306500 & 1.7138800 \\ \mathrm{H} & 4.7813620 & 0.4608710 & 1.9812040 \\ \mathrm{C} & 2.1248910 & -0.0992850 & 2.6673860 \\ \mathrm{H} & 2.3332280 & 0.9744210 & 2.5949600 \\ \mathrm{H} & 1.0723500 & -0.2647830 & 2.4284470 \\ \mathrm{H} & 2.2770100 & -0.3912140 & 3.7114900 \\ \mathrm{C} & 2.8268500 & -2.4196970 & 1.9782820 \\ \mathrm{H} & 3.4693120 & -3.0327320 & 1.3376630 \\ \mathrm{H} & 3.0523870 & -2.6845090 & 3.0162330 \\ \mathrm{H} & 1.7839120 & -2.6948180 & 1.7935810 \\ \mathrm{H} & 1.2483430 & 1.7123780 & -0.9099400 \\ \mathrm{C} & 0.8904580 & 1.5551180 & 0.8096290 \\ \mathrm{C} & -0.6801390 & 3.5549980 & -0.3289270 \\ \mathrm{H} & -0.3231400 & 4.3093510 & -1.4576940 \\ \mathrm{H} & -1.1562120 & 3.6586610 & 1.7726600 \\ \mathrm{H} & -0.1578840 & 5.6950420 & -1.3723910 \\ \mathrm{H} & & & \\ \mathrm{H} & & & \\ \mathrm{H} & & & \end{array}$

$\begin{array}{lrrr}\text { H } & 0.1140150 & 6.2614240 & -2.2587120 \\ \text { H } & -0.2234620 & 7.4276750 & -0.0839810 \\ \text { H } & -0.1806920 & -0.4534610 & -1.2495050 \\ \text { O } & -0.6820350 & -0.6674720 & 0.8397780 \\ \text { H } & -1.7547380 & -0.6604760 & 0.5399670 \\ \text { H } & -0.4845840 & -1.5976860 & 1.0390900 \\ \text { O } & -3.0470200 & -0.6591170 & 0.2160180 \\ \text { C } & -3.5876450 & -1.8061480 & 0.3768550 \\ \text { O } & -3.0779780 & -2.8584560 & 0.7652060 \\ \text { C } & -5.1057700 & -1.8008050 & 0.0152200 \\ \text { F } & -5.7925650 & -0.9306060 & 0.7987870 \\ \text { F } & -5.3033720 & -1.4207960 & -1.2727390 \\ \text { F } & -5.6792060 & -3.0114550 & 0.1662120\end{array}$

12.log

Potential Energy = -1489.66213

Zero-point Energy = -1489.18799

Free Energy $=-1489.23136$

Single-Point Energy B3LYP-D3(BJ)/6-311+G** PCM = 1490.12005

Free Energy B3LYP-D3(BJ)/6-311+G** PCM (extrapolated free energy from $\mathrm{qRRHO})=-1489.68928$

Nimag $=1\left(-310.6199 \mathrm{~cm}^{-1}\right)$

Charge $=0$ Multiplicity $=1$

$\begin{array}{llll}\text { C } & -2.8903140 & -0.6469700 & -0.5149400\end{array}$

$\begin{array}{llll}\mathrm{C} & -3.5303720 & 0.2473580 & 1.5773630\end{array}$

C $\quad-2.0718030 \quad-0.1926140 \quad 1.7245390$

$\begin{array}{llll}\mathrm{N} & -1.6817550 & -0.3732850 & 0.3048840\end{array}$

$\begin{array}{llll}\mathrm{N} & -3.9211430 & 0.0116440 & 0.3036690\end{array}$

$\begin{array}{llll}\text { O } & -4.2180760 & 0.7582360 & 2.4634720\end{array}$

$\begin{array}{llll}\text { C } & -5.1774820 & 0.5445240 & -0.2171280\end{array}$

$\begin{array}{llll}\mathrm{H} & -6.0245080 & -0.1109680 & 0.0024070\end{array}$

$\begin{array}{llll}\mathrm{H} & -5.3612260 & 1.5156180 & 0.2478380\end{array}$

$\mathrm{H} \quad-5.0969960 \quad 0.6725620 \quad-1.2970750$

$\begin{array}{llll}\text { C } & -0.4325170 & -0.1293050 & -0.1547310\end{array}$

$\begin{array}{llll}\text { C } & -0.1997140 & 0.2977170 & -1.5904420\end{array}$

$\begin{array}{llll}\text { C } & -0.6051660 & 1.7687080 & -1.8551870\end{array}$

$\mathrm{H} \quad-0.5117540 \quad 1.9254300 \quad-2.9363410$

$\mathrm{H} \quad-1.6620160 \quad 1.9188220 \quad-1.6124340$

$\begin{array}{llll}\mathrm{C} & -1.2482260 & 0.8113380 & 2.5244550\end{array}$

$\mathrm{H} \quad-1.7419920 \quad 0.9525250 \quad 3.4888950$

$\begin{array}{llll}\mathrm{H} & -0.2376370 & 0.4435080 & 2.7200380\end{array}$

$\begin{array}{llll}\mathrm{H} & -1.1889590 & 1.7810280 & 2.0224820\end{array}$

$\begin{array}{llll}\mathrm{H} & -2.0564450 & -1.1568870 & 2.2491380\end{array}$

C $\quad-3.1846000 \quad-2.1596560 \quad-0.8249230$

$\mathrm{H} \quad-2.8121110 \quad-0.1163990 \quad-1.4685130$

$\begin{array}{llll}\mathrm{C} & -4.5702860 & -2.2803770 & -1.4970600\end{array}$

$\mathrm{H} \quad-4.7052660 \quad-3.3057640 \quad-1.8554980$

$\begin{array}{llll}\mathrm{H} & -5.3875350 & -2.0677960 & -0.8041190\end{array}$

$\mathrm{H} \quad-4.6639980 \quad-1.6155160 \quad-2.3630500$

C $\quad-2.1352180 \quad-2.6939350 \quad-1.8215670$

$\begin{array}{llll}\mathrm{H} & -2.1670500 & -2.1409210 & -2.7677910\end{array}$

$\mathrm{H} \quad-1.1204760 \quad-2.6470900 \quad-1.4219790$

$\begin{array}{llll}\mathrm{H} & -2.3563850 & -3.7416490 & -2.0504030\end{array}$

$\begin{array}{llll}\mathrm{C} & -3.1719450 & -3.0235420 & 0.4496200\end{array}$

$\mathrm{H} \quad-3.8857350 \quad-2.6600320 \quad 1.1961920$

$\mathrm{H} \quad-3.4591800 \quad-4.0492840 \quad 0.1967430$

$\mathrm{H} \quad-2.1780630 \quad-3.0680800 \quad 0.9046890$

$\begin{array}{llll}\mathrm{H} & -0.7318720 & -0.3595860 & -2.2795040\end{array}$

$\begin{array}{llll}\mathrm{H} & 0.8646090 & 0.1751370 & -1.8034990\end{array}$

$\begin{array}{llll}\text { C } & 0.2292100 & 2.8058820 & -1.1247520\end{array}$

$\begin{array}{llll}\mathrm{C} & -0.3592080 & 3.6865100 & -0.2052980\end{array}$

$\begin{array}{llll}\text { C } & 1.6071190 & 2.9256540 & -1.3730580\end{array}$

$\begin{array}{llll}\text { C } & 0.4042320 & 4.6560070 & 0.4550500\end{array}$

$\begin{array}{llll}\mathrm{H} & -1.4261420 & 3.6183430 & -0.0076410\end{array}$

$\begin{array}{llll}\text { C } & 2.3739190 & 3.8903270 & -0.7150840\end{array}$

$\begin{array}{llll}\mathrm{H} & 2.0856110 & 2.2651630 & -2.0921280\end{array}$

$\begin{array}{llll}\text { C } & 1.7747410 & 4.7594910 & 0.2039770\end{array}$

$\mathrm{H} \quad-0.0734160 \quad 5.3276820 \quad 1.1627580$

$\mathrm{H} \quad 3.4373650 \quad 3.9667740 \quad-0.9233380$

$\begin{array}{llll}\mathrm{H} & 2.3701370 & 5.5107410 & 0.7145550\end{array}$

$\begin{array}{llll}\mathrm{H} & 2.3701370 & 5.5107410 & 0.7145550 \\ \mathrm{H} & 0.1660430 & 0.4259420 & 0.5600690\end{array}$

O $\quad 0.5238830 \quad-1.5994870 \quad-0.1121890$

$\mathrm{H} \quad 1.6233220 \quad-1.4303830 \quad-0.2344690$

$\mathrm{H} \quad 0.4015280 \quad-2.0097130 \quad 0.7597120$

$\begin{array}{llll}\text { O } & 2.9146110 & -1.2328350 & -0.4405690\end{array}$ 


$\begin{array}{llll}\text { C } & 3.6602130 & -1.6752210 & 0.4992500 \\ \text { O } & 3.3420540 & -2.2408950 & 1.5456160 \\ \text { C } & 5.1745690 & -1.4220960 & 0.2180400 \\ \text { F } & 5.5551520 & -1.9580210 & -0.9688460 \\ \text { F } & 5.4422700 & -0.0918030 & 0.1602850 \\ \text { F } & 5.9734380 & -1.9486950 & 1.1677840\end{array}$

13.log

Potential Energy $=-1489.66178$

Zero-point Energy = -1489.18774

Free Energy $=-1489.23108$

Single-Point Energy B3LYP-D3(BJ)/6-311+G** PCM = 1490.11946

Free Energy B3LYP-D3(BJ)/6-311+G** PCM (extrapolated free energy from $q R R H O)=-1489.68876$

Nimag $=1\left(-330.9669 \mathrm{~cm}^{-1}\right)$

Charge $=0$ Multiplicity $=1$

$\begin{array}{llll}\text { C } & 3.0935500 & -0.3485140 & 0.4439820\end{array}$

C $\quad 3.4350370 \quad 0.0877620 \quad-1.8534080$

$\begin{array}{llll}\mathrm{C} & 1.9701980 & -0.3321370 & -1.7100250\end{array}$

$\mathrm{N} \quad 1.7810920 \quad-0.2371240 \quad-0.2411940$

$\mathrm{N} \quad 3.9984600 \quad 0.1053250 \quad-0.6241160$

$\begin{array}{llll}\text { O } & 3.9922150 & 0.3901960 & -2.9106480\end{array}$

C $\quad 5.3129340 \quad 0.7005680 \quad-0.3979590$

$\begin{array}{llll}\mathrm{H} & 6.1236130 & -0.0068010 & -0.5925510\end{array}$

$\mathrm{H} \quad \begin{array}{llll}5.4279210 & 1.5532270 & -1.0708370\end{array}$

$\begin{array}{llll}\mathrm{H} & 5.3808630 & 1.0452400 & 0.6343370\end{array}$

C $\quad 0.6228350 \quad 0.1871480 \quad 0.3246450$

$\begin{array}{llll}\text { C } & 0.6483910 & 0.9182950 & 1.6520230\end{array}$

$\begin{array}{llll}\text { C } & -0.7003040 & 1.5549860 & 2.0554170\end{array}$

$\mathrm{H} \quad-1.4760150 \quad 0.7860850 \quad 2.1123190$

$\mathrm{H} \quad-0.5736450 \quad 1.9422630 \quad 3.0724730$

$\begin{array}{llll}\text { C } & 1.0489460 & 0.5342530 & -2.5635230\end{array}$

$\mathrm{H} \quad 1.4033620 \quad 0.4774910 \quad-3.5954540$

H $\quad 0.0178170 \quad 0.1721690 \quad-2.5459580$

$\mathrm{H} \quad 1.0712890 \quad 1.5825960 \quad-2.2509380$

$\mathrm{H} \quad 1.8776960-1.3751040-2.0396700$

$\begin{array}{llll}\mathrm{C} & 3.4405120 & -1.7678390 & 1.0204620\end{array}$

$\begin{array}{llll}\mathrm{H} & 3.1370350 & 0.3711650 & 1.2670760\end{array}$

$\begin{array}{llll}\text { C } & 4.8991660 & -1.7718040 & 1.5287470\end{array}$

$\mathrm{H} \quad 5.0864860 \quad-2.7030730 \quad 2.0726530$

$\begin{array}{llll}\mathrm{H} & 5.6229770 & -1.7209130 & 0.7122740\end{array}$

H $\quad 5.0931010 \quad-0.9435290 \quad 2.2196150$

$\begin{array}{llll}\text { C } & 2.5270870 & -2.0659030 & 2.2272980\end{array}$

$\mathrm{H} \quad 2.6746980 \quad-1.3316710 \quad 3.0279030$

$\mathrm{H} \quad 1.4686330 \quad-2.0786520 \quad 1.9595300$

$\begin{array}{llll}\mathrm{H} & 2.7773940 & -3.0499960 & 2.6365520\end{array}$

$\begin{array}{llll}\text { C } & 3.2773310 & -2.8721640 & -0.0396710\end{array}$

$\begin{array}{llll}\mathrm{H} & 3.8993130 & -2.6853850 & -0.9212870\end{array}$

$\mathrm{H} \quad 3.5883010 \quad-3.8332500 \quad 0.3822920$

$\begin{array}{llll}\mathrm{H} & 2.2375560 & -2.9808190 & -0.3627420\end{array}$

$\begin{array}{llll}\mathrm{H} & 1.3977970 & 1.7137180 & 1.5600750\end{array}$

$\begin{array}{llll}\mathrm{H} & 0.9838590 & 0.2471180 & 2.4446430\end{array}$

$\begin{array}{llll}\mathrm{C} & -1.1534130 & 2.6808920 & 1.1438270\end{array}$

C $\quad-0.5079980 \quad 3.9288220 \quad 1.1800890$

$\begin{array}{llll}\text { C } & -2.2178200 & 2.5062730 & 0.2462880\end{array}$

C $\quad-0.9101120 \quad 4.9702580 \quad 0.3403210$

$\mathrm{H} \quad 0.3109050 \quad 4.0899520 \quad 1.8776790$

$\begin{array}{llll}\text { C } & -2.6238290 & 3.5478180 & -0.5966090\end{array}$

$\mathrm{H} \quad-2.7342790 \quad 1.5506120 \quad 0.2116140$

$\begin{array}{llll}\text { C } & -1.9707120 & 4.7823060 & -0.5538050\end{array}$

$\begin{array}{llll}\mathrm{H} & -0.4008390 & 5.9287680 & 0.3874300\end{array}$

$\mathrm{H} \quad-3.4515820 \quad 3.3927490 \quad-1.2830610$

$\begin{array}{llll}\mathrm{H} & -2.2860930 & 5.5919050 & -1.2056080\end{array}$

$\mathrm{H} \quad-0.0524770 \quad 0.6266730 \quad-0.4020410$

$\begin{array}{llll}\mathrm{O} & -0.3784600 & -1.1960140 & 0.7087670\end{array}$

$\mathrm{H} \quad-1.4915860 \quad-1.0794940 \quad 0.6188600$

$\mathrm{H} \quad-0.1481600 \quad-1.8916120 \quad 0.0704760$

$\begin{array}{llll}\mathrm{O} & -2.8048140 & -0.9989940 & 0.5355310\end{array}$

C $\quad-3.2961330 \quad-1.9135090 \quad-0.2124810$

$\begin{array}{llll}\text { O } & -2.7137880 & -2.7992780 & -0.8387920\end{array}$

$\begin{array}{llll}\mathrm{C} & -4.8520650 & -1.8344450 & -0.2963200\end{array}$

F $\quad-5.4162050 \quad-1.9631320 \quad 0.9312520$

F $\quad-5.2555280 \quad-0.6400580 \quad-0.7990310$

F $\quad-5.3777820 \quad-2.7962010 \quad-1.0805700$ 14.log

Potential Energy $=-1489.66326$

Zero-point Energy = -1489.19008

Free Energy = -1489.23415

Single-Point Energy B3LYP-D3(BJ)/6-311+G** PCM = 1490.11854

Free Energy B3LYP-D3(BJ)/6-311+G** PCM (extrapolated free energy from $\mathrm{qRRHO})=-1489.68943$

Nimag $=1\left(-378.5909 \mathrm{~cm}^{-1}\right)$ Charge $=0$ Multiplicity $=1$

$\begin{array}{llll}\text { C } & -1.2140390 & 1.6419790 & 0.4122200\end{array}$

$\begin{array}{llll}\mathrm{C} & -1.1311840 & 2.7783480 & -1.6503700\end{array}$

$\begin{array}{llll}\text { C } & 0.2929720 & 2.2678690 & -1.4011770\end{array}$

$\mathrm{N} \quad 0.0927420 \quad 1.3673840 \quad-0.2403640$

$\mathrm{N} \quad-1.9330390 \quad 2.3470900 \quad-0.6518730$

O $\quad-1.4855890 \quad 3.4448390 \quad-2.6252990$

C $\quad-3.3825270 \quad 2.5024470 \quad-0.7158030$

$\mathrm{H} \quad-3.6915490 \quad 2.4169630 \quad-1.7595630$

$\mathrm{H} \quad-3.8571920 \quad 1.7118850 \quad-0.1335370$

$\begin{array}{llll}\mathrm{H} & -3.7090070 & 3.4753160 & -0.3367380\end{array}$

$\begin{array}{llll}\text { C } & 0.7376550 & 0.1984100 & 0.0069190\end{array}$

$\mathrm{H} \quad 0.4142720 \quad-0.2384160 \quad 0.9474100$

$\begin{array}{llll}\text { C } & 2.2390470 & 0.0932050 & -0.2132410\end{array}$

C $2.8822060 \quad-1.0752030 \quad 0.5653970$

$\mathrm{H} \quad 2.6429640 \quad-0.9714090 \quad 1.6296360$

$\mathrm{H} \quad 0.9133920 \quad 3.1225550 \quad-1.1006470$

$\begin{array}{llll}\text { C } & -1.0884940 & 2.4426890 & 1.7570580\end{array}$

$\mathrm{H} \quad-1.7236560 \quad 0.6938000 \quad 0.6182570$

$\mathrm{H} \quad 2.4660480 \quad-0.0020980 \quad-1.2762420$

$\begin{array}{llll}\text { C } & 0.8685670 & 1.6697130 & -2.6876890\end{array}$

$\mathrm{H} \quad 1.9449780 \quad 1.5107920 \quad-2.6188410$

$\begin{array}{llll}\mathrm{H} & 0.3797040 & 0.7288190 & -2.9486030\end{array}$

$\begin{array}{llll}\mathrm{H} & 0.6902630 & 2.3925720 & -3.4878940\end{array}$

$\begin{array}{llll}\text { C } & -2.4921340 & 2.6364900 & 2.3725230\end{array}$

$\begin{array}{llll}\mathrm{H} & -2.3915260 & 3.0619660 & 3.3761700\end{array}$

$\mathrm{H} \quad-3.1120180 \quad 3.3226610 \quad 1.7916210$

$\begin{array}{llll}\mathrm{H} & -3.0265510 & 1.6844590 & 2.4696650\end{array}$

$\begin{array}{llll}\mathrm{C} & -0.4265150 & 3.8151050 & 1.5376880\end{array}$

$\begin{array}{llll}\mathrm{H} & -0.9891470 & 4.4303260 & 0.8280660\end{array}$

$\begin{array}{llll}\mathrm{H} & -0.3857640 & 4.3601270 & 2.4864780\end{array}$

$\mathrm{H} \quad 0.6008590 \quad 3.7133940 \quad 1.1738620$

$\begin{array}{llll}\mathrm{C} & -0.2453970 & 1.6267110 & 2.7595710\end{array}$

$\mathrm{H} \quad-0.6644440 \quad 0.6272710 \quad 2.9266460$

$\begin{array}{llll}\mathrm{H} & 0.7943440 & 1.5238500 & 2.4363630\end{array}$

$\mathrm{H} \quad-0.2340050 \quad 2.1408390 \quad 3.7259120$

O $\quad 0.0955260 \quad-1.0241680 \quad-1.0458000$

$\begin{array}{llll}\mathrm{H} & -0.9744650 & -1.2758530 & -0.7932920\end{array}$

$\mathrm{H} \quad 0.5955700 \quad-1.8531720 \quad-0.9564000$

O $\quad-2.2284180 \quad-1.5161810 \quad-0.5095180$

C $\quad-2.4620310 \quad-2.7351820 \quad-0.1957170$

$\begin{array}{llll}\text { O } & -1.6834850 & -3.6860520 & -0.1391460\end{array}$

$\begin{array}{llll}\text { C } & -3.9628220 & -2.9706050 & 0.1607560\end{array}$

F $\quad-4.7781470 \quad-2.5962060 \quad-0.8563290$

F $\quad-4.2330790 \quad-4.2618780 \quad 0.4355540$

$\begin{array}{llll}\mathrm{F} & -4.3217100 & -2.2436220 & 1.2503250\end{array}$

$\begin{array}{llll}\mathrm{H} & 2.6802960 & 1.0367060 & 0.1266030\end{array}$

$\begin{array}{llll}\mathrm{H} & 2.4537860 & -2.0296920 & 0.2368740\end{array}$

C $4.3858750 \quad-1.1204550 \quad 0.3826400$

$\begin{array}{llll}\text { C } & 4.9585980 & -1.8301260 & -0.6841530\end{array}$

$\begin{array}{llll}\text { C } & 5.2361810 & -0.4321680 & 1.2617090\end{array}$

$\begin{array}{llll}\mathrm{C} & 6.3440140 & -1.8489290 & -0.8714710\end{array}$

$\mathrm{H} \quad 4.3158770 \quad-2.3764520 \quad-1.3704340$

C $\quad 6.6223440 \quad-0.4480250 \quad 1.0783210$

$\begin{array}{llll}\mathrm{H} & 4.8111160 & 0.1160900 & 2.0991310\end{array}$

C $7.1809900 \quad-1.1563670 \quad 0.0096060$

$\mathrm{H} \quad 6.7684720 \quad-2.4073610 \quad-1.7010110$

$\begin{array}{llll}\mathrm{H} & 7.2641170 & 0.0878220 & 1.7719890\end{array}$

H $\quad 8.2576590 \quad-1.1726550 \quad-0.1323980$

15.log

Potential Energy = -1489.66309

Zero-point Energy = -1489.18922

Free Energy = -1489.23270

Single-Point Energy B3LYP-D3(BJ)/6-311+G** PCM = 1490.12199 
Free Energy B3LYP-D3(BJ)/6-311+G** PCM (extrapolated free energy from qRRHO) $=-1489.69160$ Nimag $=1\left(-291.0837 \mathrm{~cm}^{-1}\right)$

Charge $=0$ Multiplicity $=1$

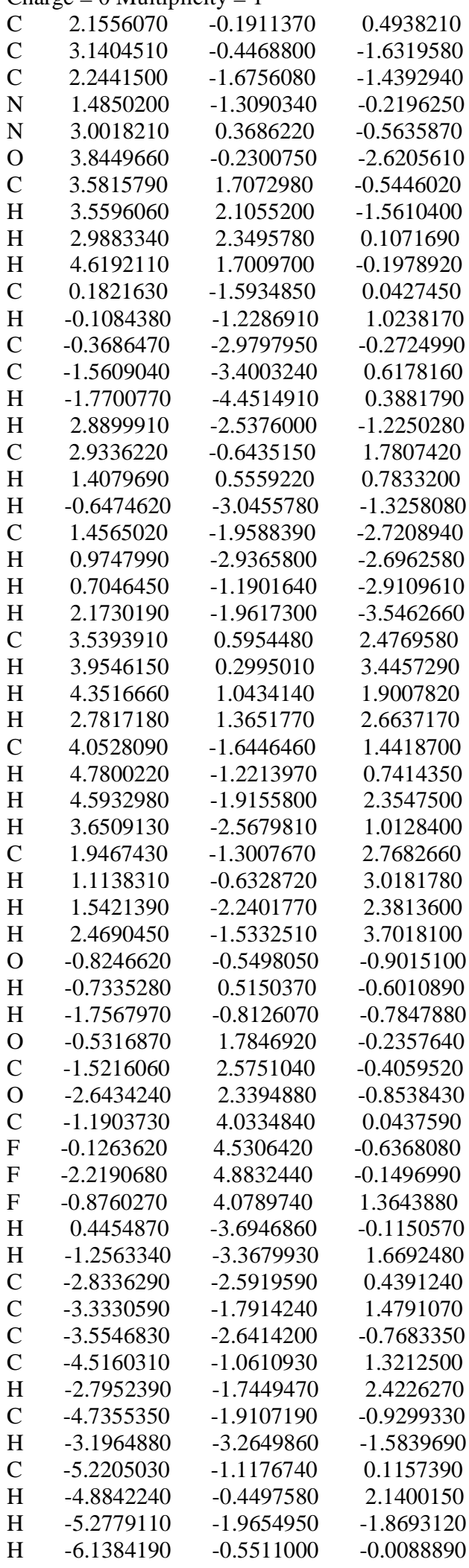

16.log

Potential Energy $=-1489.66225$

Zero-point Energy $=-1489.18884$

Free Energy $=-1489.23238$

Single-Point Energy B3LYP-D3(BJ)/6-311+G** PCM = -

1490.12098

Free Energy B3LYP-D3(BJ)/6-311+G** PCM (extrapolated

free energy from $\mathrm{qRRHO})=-1489.69110$

Nimag $=1\left(-361.3958 \mathrm{~cm}^{-1}\right)$

Charge $=0$ Multiplicity $=1$

$\begin{array}{lrrr}\text { C } & -0.2826440 & 1.6543360 & 0.6961040 \\ \text { C } & -0.8995090 & 3.4405790 & -0.7097270 \\ \text { C } & 0.3779920 & 2.8519770 & -1.3213680\end{array}$

\begin{tabular}{|c|c|c|c|}
\hline N & 0.5375240 & 1.5984350 & -0.5428510 \\
\hline $\mathrm{N}$ & -1.2536260 & 2.6999010 & 0.3629430 \\
\hline $\mathrm{O}$ & -1.5150200 & 4.4133860 & -1.1517730 \\
\hline C & -2.5320930 & 2.8961280 & 1.0384480 \\
\hline $\mathrm{H}$ & -3.2771770 & 3.1764780 & 0.2910750 \\
\hline $\mathrm{H}$ & -2.8353470 & 1.9645410 & 1.5172410 \\
\hline $\mathrm{H}$ & -2.4815760 & 3.6883230 & 1.7911700 \\
\hline $\mathrm{C}$ & 0.9706560 & 0.3964840 & -1.0047680 \\
\hline $\mathrm{H}$ & 1.0047860 & -0.3424270 & -0.2091530 \\
\hline $\mathrm{C}$ & 2.1690260 & 0.3238370 & -1.9358550 \\
\hline C & 2.7779630 & -1.0887970 & -2.0978680 \\
\hline $\mathrm{H}$ & 2.0514140 & -1.7699070 & -2.5557640 \\
\hline $\mathrm{H}$ & 1.2085250 & 3.5322920 & -1.0915120 \\
\hline $\mathrm{C}$ & 0.5568170 & 1.9322650 & 1.9928570 \\
\hline $\mathrm{H}$ & -0.8126530 & 0.7034360 & 0.8231740 \\
\hline $\mathrm{H}$ & 1.9097380 & 0.7103170 & -2.9219130 \\
\hline $\mathrm{C}$ & 0.2319540 & 2.7523980 & -2.8422250 \\
\hline $\mathrm{H}$ & 1.1899460 & 2.5859480 & -3.3353690 \\
\hline $\mathrm{H}$ & -0.4670100 & 1.9646570 & -3.1303670 \\
\hline $\mathrm{H}$ & -0.1576120 & 3.7123850 & -3.1906610 \\
\hline $\mathrm{C}$ & -0.3795190 & 1.9367010 & 3.2216290 \\
\hline $\mathrm{H}$ & 0.2231440 & 1.9764410 & 4.1347280 \\
\hline $\mathrm{H}$ & -1.0455070 & 2.8021350 & 3.2360410 \\
\hline $\mathrm{H}$ & -0.9906450 & 1.0280320 & 3.2689510 \\
\hline $\mathrm{C}$ & 1.3011930 & 3.2770640 & 1.9084530 \\
\hline $\mathrm{H}$ & 0.6123110 & 4.1157380 & 1.7640280 \\
\hline $\mathrm{H}$ & 1.8456920 & 3.4549950 & 2.8416020 \\
\hline $\mathrm{H}$ & 2.0340490 & 3.2819000 & 1.0955160 \\
\hline $\mathrm{C}$ & 1.5803310 & 0.7946270 & 2.1859410 \\
\hline $\mathrm{H}$ & 1.0936210 & -0.1866020 & 2.2318720 \\
\hline $\mathrm{H}$ & 2.3323010 & 0.7758620 & 1.3924200 \\
\hline $\mathrm{H}$ & 2.1105770 & 0.9406900 & 3.1324170 \\
\hline $\mathrm{O}$ & -0.3083620 & -0.3211870 & -1.9387240 \\
\hline $\mathrm{H}$ & -1.1697440 & -0.6460000 & -1.2950890 \\
\hline $\mathrm{H}$ & -0.0031540 & -1.1168810 & -2.4058980 \\
\hline $\mathrm{O}$ & -2.1835330 & -0.9729720 & -0.5252020 \\
\hline $\mathrm{C}$ & -2.5548070 & -2.1913150 & -0.6495840 \\
\hline $\mathrm{O}$ & -2.1030010 & -3.0712750 & -1.3814970 \\
\hline $\mathrm{C}$ & -3.7525770 & -2.5382430 & 0.2887560 \\
\hline $\mathrm{F}$ & -4.8305530 & -1.7599440 & 0.0168340 \\
\hline F & -4.1466900 & -3.8216880 & 0.1719090 \\
\hline $\mathrm{F}$ & -3.4296860 & -2.3340020 & 1.5913430 \\
\hline $\mathrm{H}$ & 2.9272210 & 0.9963820 & -1.5195460 \\
\hline $\mathrm{H}$ & 3.5935570 & -0.9950930 & -2.8232270 \\
\hline $\mathrm{C}$ & 3.3096010 & -1.7047970 & -0.8180420 \\
\hline C & 4.4985100 & -1.2287430 & -0.2398280 \\
\hline C & 2.6357160 & -2.7594310 & -0.1840040 \\
\hline $\mathrm{C}$ & 4.9960830 & -1.7868410 & 0.9400590 \\
\hline $\mathrm{H}$ & 5.0436250 & -0.4201970 & -0.7214500 \\
\hline C & 3.1303720 & -3.3214240 & 0.9986330 \\
\hline $\mathrm{H}$ & 1.7207400 & -3.1519260 & -0.6212900 \\
\hline C & 4.3117330 & -2.8358320 & 1.5650410 \\
\hline $\mathrm{H}$ & 5.9196060 & -1.4076020 & 1.3683960 \\
\hline $\mathrm{H}$ & 2.5937520 & -4.1387760 & 1.4719290 \\
\hline $\mathrm{H}$ & 4.6994320 & -3.2722450 & 2.4809240 \\
\hline
\end{tabular}

17. $\log$

Potential Energy $=-1489.66308$

Zero-point Energy $=-1489.18914$

Free Energy $=-1489.23251$

Single-Point Energy B3LYP-D3(BJ)/6-311+G** PCM = -

1490.12191

Free Energy B3LYP-D3(BJ)/6-311+G** PCM (extrapolated

free energy from $q R R H O)=-1489.69134$

Nimag $=1\left(-290.5330 \mathrm{~cm}^{-1}\right)$

Charge $=0$ Multiplicity $=1$

$\begin{array}{lrrr}\mathrm{C} & -2.1568900 & -0.1633950 & -0.4805990 \\ \mathrm{C} & -3.1344230 & -0.4490860 & 1.6446970 \\ \mathrm{C} & -2.2536260 & -1.6841930 & 1.4232750 \\ \mathrm{~N} & -1.4958600 & -1.3026680 & 0.2073770 \\ \mathrm{~N} & -2.9920150 & 0.3852600 & 0.5915060 \\ \mathrm{O} & -3.8309500 & -0.2430910 & 2.6412660 \\ \mathrm{C} & -3.5540640 & 1.7316490 & 0.6028710 \\ \mathrm{H} & -3.5170670 & 2.1109870 & 1.6261150 \\ \mathrm{H} & -2.9588080 & 2.3779870 & -0.0430040 \\ \mathrm{H} & -4.5949270 & 1.7454270 & 0.2662690\end{array}$




\begin{tabular}{|c|c|c|c|}
\hline C & -0.1963520 & -1.5942890 & -0.0654330 \\
\hline $\mathrm{H}$ & 0.0930650 & -1.2133900 & -1.0406880 \\
\hline $\mathrm{C}$ & 0.3412410 & -2.9928870 & 0.2172890 \\
\hline $\mathrm{C}$ & 1.5324410 & -3.4025630 & -0.6798270 \\
\hline $\mathrm{H}$ & 1.7325220 & -4.4604360 & -0.4743360 \\
\hline $\mathrm{H}$ & -2.9108150 & -2.5339660 & 1.1950660 \\
\hline $\mathrm{C}$ & -2.9454820 & -0.5822410 & -1.7722720 \\
\hline $\mathrm{H}$ & -1.4021940 & 0.5805900 & -0.7595430 \\
\hline $\mathrm{H}$ & 0.6162480 & -3.0880700 & 1.2694500 \\
\hline $\mathrm{C}$ & -1.4637100 & -2.0023640 & 2.6952400 \\
\hline $\mathrm{H}$ & -0.9939100 & -2.9851090 & 2.6488020 \\
\hline $\mathrm{H}$ & -0.7020370 & -1.2464950 & 2.8971370 \\
\hline $\mathrm{H}$ & -2.1764580 & -2.0136230 & 3.5237990 \\
\hline $\mathrm{C}$ & -3.5514460 & 0.6746890 & -2.4352620 \\
\hline $\mathrm{H}$ & -3.9714040 & 0.4034670 & -3.4092270 \\
\hline $\mathrm{H}$ & -4.3604090 & 1.1095650 & -1.8445500 \\
\hline $\mathrm{H}$ & -2.7933170 & 1.4476150 & -2.6062990 \\
\hline $\mathrm{C}$ & -4.0662500 & -1.5868360 & -1.4493550 \\
\hline $\mathrm{H}$ & -4.7860380 & -1.1778990 & -0.7328760 \\
\hline $\mathrm{H}$ & -4.6152380 & -1.8326970 & -2.3642580 \\
\hline $\mathrm{H}$ & -3.6649370 & -2.5221250 & -1.0464910 \\
\hline $\mathrm{C}$ & -1.9671230 & -1.2196790 & -2.7811420 \\
\hline $\mathrm{H}$ & -1.1345880 & -0.5479580 & -3.0219900 \\
\hline $\mathrm{H}$ & -1.5614850 & -2.1681740 & -2.4180250 \\
\hline $\mathrm{H}$ & -2.4964500 & -1.4305930 & -3.7158570 \\
\hline $\mathrm{O}$ & 0.8226500 & -0.5778080 & 0.8921830 \\
\hline $\mathrm{H}$ & 0.7884350 & 0.4791440 & 0.5519370 \\
\hline $\mathrm{H}$ & 1.7453420 & -0.8842340 & 0.8113060 \\
\hline $\mathrm{O}$ & 0.6542370 & 1.7266910 & 0.0968050 \\
\hline $\mathrm{C}$ & 1.4967400 & 2.5857890 & 0.5283260 \\
\hline $\mathrm{O}$ & 2.4290960 & 2.4419420 & 1.3182590 \\
\hline $\mathrm{C}$ & 1.2460530 & 4.0035740 & -0.0765760 \\
\hline $\mathrm{F}$ & 0.0117090 & 4.4655460 & 0.2503780 \\
\hline $\mathrm{F}$ & 2.1396660 & 4.9170010 & 0.3530950 \\
\hline $\mathrm{F}$ & 1.3172730 & 3.9805180 & -1.4323260 \\
\hline $\mathrm{H}$ & -0.4792030 & -3.6958700 & 0.0403230 \\
\hline $\mathrm{H}$ & 1.2311190 & -3.3428370 & -1.7310130 \\
\hline $\mathrm{C}$ & 2.8101080 & -2.6076760 & -0.4775100 \\
\hline $\mathrm{C}$ & 3.3139570 & -1.7782580 & -1.4924310 \\
\hline $\mathrm{C}$ & 3.5284730 & -2.6947580 & 0.7294550 \\
\hline $\mathrm{C}$ & 4.4979300 & -1.0553830 & -1.3102130 \\
\hline $\mathrm{H}$ & 2.7783110 & -1.7022530 & -2.4352150 \\
\hline $\mathrm{C}$ & 4.7106180 & -1.9716680 & 0.9153560 \\
\hline $\mathrm{H}$ & 3.1672140 & -3.3415280 & 1.5254610 \\
\hline $\mathrm{C}$ & 5.1994190 & -1.1488100 & -0.1051090 \\
\hline $\mathrm{H}$ & 4.8694260 & -0.4209850 & -2.1097280 \\
\hline $\mathrm{H}$ & 5.2507550 & -2.0556760 & 1.8538470 \\
\hline $\mathrm{H}$ & 6.1182210 & -0.5881470 & 0.0382890 \\
\hline
\end{tabular}

18.log

Potential Energy $=-1489.66019$

Zero-point Energy = -1489.18653

Free Energy = -1489.22984

Single-Point Energy B3LYP-D3(BJ)/6-311+G** PCM = 1490.11899

Free Energy B3LYP-D3(BJ)/6-311+G** PCM (extrapolated

free energy from qRRHO) $=-1489.68863$

Nimag $=1\left(-392.7208 \mathrm{~cm}^{-1}\right)$

Charge $=0$ Multiplicity $=1$

$\begin{array}{lrrr}\mathrm{C} & -2.8509660 & -0.1189270 & 0.5924330 \\ \mathrm{C} & -2.6438470 & -2.2067690 & -0.4783960 \\ \mathrm{C} & -2.0375180 & -1.1791810 & -1.4414520 \\ \mathrm{~N} & -1.9560870 & 0.0295210 & -0.5841970 \\ \mathrm{~N} & -3.0235780 & -1.5726330 & 0.6533040 \\ \mathrm{O} & -2.7410090 & -3.4160790 & -0.6976450 \\ \mathrm{C} & -3.4670080 & -2.3088540 & 1.8319330 \\ \mathrm{H} & -2.9247530 & -3.2557560 & 1.8698720 \\ \mathrm{H} & -3.2417630 & -1.7284480 & 2.7277280 \\ \mathrm{H} & -4.5391600 & -2.5247090 & 1.8030430 \\ \mathrm{C} & -0.9581320 & 0.9485100 & -0.5287500 \\ \mathrm{H} & -1.2380320 & 1.7828450 & 0.1072070 \\ \mathrm{C} & -0.2411590 & 1.3821020 & -1.8012260 \\ \mathrm{C} & 0.2813470 & 2.8379720 & -1.7467280 \\ \mathrm{H} & 0.6403220 & 3.0825810 & -2.7523730 \\ \mathrm{H} & -2.7664470 & -0.9980800 & -2.2424370 \\ \mathrm{C} & -4.1879450 & 0.6965480 & 0.4812050\end{array}$

$\begin{array}{crrr}\mathrm{H} & -2.3150490 & 0.2127970 & 1.4892080 \\ \mathrm{H} & 0.5802450 & 0.7007460 & -2.0298410 \\ \mathrm{C} & -0.7559100 & -1.7256030 & -2.0692080 \\ \mathrm{H} & -0.4122730 & -1.1080610 & -2.8993880 \\ \mathrm{H} & 0.0506340 & -1.8373430 & -1.3422550 \\ \mathrm{H} & -0.9942330 & -2.7155050 & -2.4672690 \\ \mathrm{C} & -4.9954720 & 0.5355290 & 1.7882740 \\ \mathrm{H} & -5.8527880 & 1.2161530 & 1.7707930 \\ \mathrm{H} & -5.3882320 & -0.4755780 & 1.9148080 \\ \mathrm{H} & -4.3931140 & 0.7855410 & 2.6692650 \\ \mathrm{C} & -5.0404200 & 0.2283500 & -0.7122960 \\ \mathrm{H} & -5.2943710 & -0.8340920 & -0.6402140 \\ \mathrm{H} & -5.9795080 & 0.7908520 & -0.7363440 \\ \mathrm{H} & -4.5305860 & 0.4022450 & -1.6651410 \\ \mathrm{C} & -3.8596250 & 2.1947980 & 0.3112590 \\ \mathrm{H} & -3.2362160 & 2.5722230 & 1.1305520 \\ \mathrm{H} & -3.3572470 & 2.3991400 & -0.6385300 \\ \mathrm{H} & -4.7894200 & 2.7724870 & 0.3178200 \\ \mathrm{O} & 0.2798580 & 0.3939260 & 0.5637880 \\ \mathrm{H} & 1.0893110 & -0.3223170 & 0.2274600 \\ \mathrm{H} & 0.7290740 & 1.1775000 & 0.9265170 \\ \mathrm{O} & 2.0134440 & -1.1562600 & -0.1512960 \\ \mathrm{C} & 2.9003880 & -1.4512960 & 0.7237960 \\ \mathrm{O} & 3.0147020 & -1.0642580 & 1.8850630 \\ \mathrm{C} & 3.9564480 & -2.4551270 & 0.1628790 \\ \mathrm{~F} & 4.5759020 & -1.9563490 & -0.9368370 \\ \mathrm{~F} & 4.9166570 & -2.7512130 & 1.0613670 \\ \mathrm{~F} & 3.3734420 & -3.6255450 & -0.2024310 \\ \mathrm{H} & -0.9583810 & 1.3092890 & -2.6253360 \\ \mathrm{H} & -0.5584990 & 3.5115010 & -1.5427110 \\ \mathrm{C} & 1.3922950 & 3.0931200 & -0.7464010 \\ \mathrm{C} & 1.1371610 & 3.7433820 & 0.4721430 \\ \mathrm{C} & 2.7079040 & 2.6795990 & -1.0176280 \\ \mathrm{C} & 2.1631400 & 3.9630770 & 1.4003830 \\ \mathrm{H} & 0.1324260 & 4.0961320 & 0.6921120 \\ \mathrm{C} & 3.7340510 & 2.9002250 & -0.0963610 \\ \mathrm{H} & 2.9301420 & 2.1839760 & -1.9595070 \\ & 3.4645330 & 3.5406980 & 1.1189860 \\ & 1.9429830 & 4.4689120 & 2.3360080 \\ \mathrm{H} & 4.7444340 & 2.5749500 & -0.3271640 \\ & & 3.7122190 & 1.8351200\end{array}$

$19 . \log$

Potential Energy = -1489.66231

Zero-point Energy = -1489.18932

Free Energy = -1489.23334

Single-Point Energy B3LYP-D3(BJ)/6-311+G** PCM = 1490.11822

Free Energy B3LYP-D3(BJ)/6-311+G** PCM (extrapolated

free energy from $\mathrm{qRRHO})=-1489.68925$

Nimag $=1\left(-406.5721 \mathrm{~cm}^{-1}\right)$

Charge $=0$ Multiplicity $=1$

$\begin{array}{lrrr}\mathrm{C} & 3.3139270 & -0.2198350 & -0.5508950 \\ \mathrm{C} & 3.3116440 & -1.3128700 & 1.5352840 \\ \mathrm{C} & 2.1613940 & -0.3013600 & 1.5971430 \\ \mathrm{~N} & 2.0808420 & 0.1560300 & 0.1884660 \\ \mathrm{~N} & 3.8669720 & -1.2717520 & 0.3039050 \\ \mathrm{O} & 3.6449860 & -2.0640800 & 2.4538880 \\ \mathrm{C} & 4.8512580 & -2.2608790 & -0.1227090 \\ \mathrm{H} & 4.6121460 & -3.2118290 & 0.3575500 \\ \mathrm{H} & 4.7990360 & -2.3805760 & -1.2056180 \\ \mathrm{H} & 5.8692250 & -1.9769150 & 0.1596130 \\ \mathrm{C} & 0.9619750 & 0.4568180 & -0.5132440 \\ \mathrm{H} & 1.2059120 & 0.7973830 & -1.5147950 \\ \mathrm{C} & -0.1620720 & 1.2556710 & 0.1228170 \\ \mathrm{C} & -1.1301690 & 1.8498940 & -0.9215420 \\ \mathrm{H} & 2.4749730 & 0.5353030 & 2.2348190 \\ \mathrm{C} & 4.2817570 & 0.9896640 & -0.8092750 \\ \mathrm{H} & 3.0346820 & -0.6548400 & -1.5182420 \\ \mathrm{H} & -0.7217390 & 0.6504080 & 0.8366440 \\ \mathrm{C} & 0.9258030 & -0.9570980 & 2.2179830 \\ \mathrm{H} & 0.1621150 & -0.2265120 & 2.4841430 \\ \mathrm{H} & 0.4926190 & -1.7153850 & 1.5619650 \\ \mathrm{H} & 1.2508300 & -1.4471340 & 3.1392960 \\ \mathrm{C} & 5.4973880 & 0.5099840 & -1.6329860 \\ \mathrm{H} & 6.0940670 & 1.3766850 & -1.9349350\end{array}$




$\begin{array}{lrrr}\mathrm{H} & 6.1538380 & -0.1511720 & -1.0634890 \\ \mathrm{H} & 5.1877700 & -0.0114320 & -2.5461010 \\ \mathrm{C} & 4.7661260 & 1.6122820 & 0.5128420 \\ \mathrm{H} & 5.2935810 & 0.8840480 & 1.1372660 \\ \mathrm{H} & 5.4620020 & 2.4305250 & 0.3009270 \\ \mathrm{H} & 3.9348200 & 2.0299920 & 1.0895990 \\ \mathrm{C} & 3.5484280 & 2.0658330 & -1.6375930 \\ \mathrm{H} & 3.1631010 & 1.6628510 & -2.5817430 \\ \mathrm{H} & 2.7219800 & 2.5203750 & -1.0839790 \\ \mathrm{H} & 4.2494020 & 2.8681520 & -1.8888570 \\ \mathrm{O} & 0.1943470 & -1.0374940 & -1.0350620 \\ \mathrm{H} & -0.7945880 & -1.3560620 & -0.5873080 \\ \mathrm{H} & 0.0921130 & -1.0479800 & -2.0001180 \\ \mathrm{O} & -1.9023870 & -1.7625540 & -0.0411190 \\ \mathrm{C} & -2.7314960 & -2.2767180 & -0.8699970 \\ \mathrm{O} & -2.6267120 & -2.4199370 & -2.0874350 \\ \mathrm{C} & -4.0363550 & -2.7645480 & -0.1667290 \\ \mathrm{~F} & -4.6763540 & -1.7350090 & 0.4443360 \\ \mathrm{~F} & -4.9097870 & -3.3230450 & -1.0278180 \\ \mathrm{~F} & -3.7646610 & -3.6900080 & 0.7875730 \\ \mathrm{H} & 0.3067010 & 2.0680490 & 0.6904850 \\ \mathrm{H} & -0.5585780 & 2.4458360 & -1.6425130 \\ \mathrm{H} & -1.6045850 & 1.0366880 & -1.4807250 \\ \mathrm{C} & -2.1969300 & 2.7160390 & -0.2828200 \\ \mathrm{C} & -3.3952750 & 2.1534620 & 0.1841940 \\ \mathrm{C} & -1.9994630 & 4.0962640 & -0.1225070 \\ \mathrm{C} & -4.3687880 & 2.9473430 & 0.7980620 \\ \mathrm{H} & -3.5687960 & 1.0870530 & 0.0637760 \\ \mathrm{C} & -2.9705470 & 4.8943780 & 0.4903760 \\ \mathrm{H} & -1.0806230 & 4.5510750 & -0.4851850 \\ \mathrm{C} & -4.1592360 & 4.3215730 & 0.9539010 \\ \mathrm{H} & -5.2910720 & 2.4934800 & 1.1497350 \\ \mathrm{H} & -2.8002450 & 5.9616380 & 0.6010120 \\ \mathrm{H} & -4.9162490 & 4.9400500 & 1.4273560 \\ & & & \end{array}$

20.log

Potential Energy = -1489.66019

Zero-point Energy = -1489.18653

Free Energy $=-1489.22984$

Single-Point Energy B3LYP-D3(BJ)/6-311+G** PCM = 1490.11898

Free Energy B3LYP-D3(BJ)/6-311+G** PCM (extrapolated free energy from $\mathrm{qRRHO})=-1489.68863$

Nimag $=1\left(-392.8019 \mathrm{~cm}^{-1}\right)$

Charge $=0$ Multiplicity $=1$

$\begin{array}{lrrr}\mathrm{C} & -2.8507190 & -0.1190700 & 0.5924780 \\ \mathrm{C} & -2.6436850 & -2.2066600 & -0.4788590 \\ \mathrm{C} & -2.0374180 & -1.1788570 & -1.4417230 \\ \mathrm{~N} & -1.9559650 & 0.0296610 & -0.5842120 \\ \mathrm{~N} & -3.0232760 & -1.5727940 & 0.6530380 \\ \mathrm{O} & -2.7408990 & -3.4159140 & -0.6983950 \\ \mathrm{C} & -3.4666120 & -2.3092920 & 1.8315290 \\ \mathrm{H} & -2.9243860 & -3.2562240 & 1.8691720 \\ \mathrm{H} & -3.2412480 & -1.7291200 & 2.7274460 \\ \mathrm{H} & -4.5387750 & -2.5251030 & 1.8027000 \\ \mathrm{C} & -0.9580950 & 0.9487320 & -0.5287180 \\ \mathrm{H} & -1.2379930 & 1.7829440 & 0.1073980 \\ \mathrm{C} & -0.2412510 & 1.3825630 & -1.8011850 \\ \mathrm{C} & 0.2811030 & 2.8384800 & -1.7465260 \\ \mathrm{H} & -2.7663700 & -0.9975980 & -2.2426490 \\ \mathrm{C} & -4.1877460 & 0.6963760 & 0.4815780 \\ \mathrm{H} & -2.3147160 & 0.2124730 & 1.4892670 \\ \mathrm{H} & 0.5802170 & 0.7013260 & -2.0299240 \\ \mathrm{C} & -0.7558170 & -1.7251380 & -2.0696230 \\ \mathrm{H} & -0.4121980 & -1.1074150 & -2.8996760 \\ \mathrm{H} & 0.0507380 & -1.8370280 & -1.3427060 \\ \mathrm{H} & -0.9941400 & -2.7149550 & -2.4678960 \\ \mathrm{C} & -4.9950700 & 0.5351100 & 1.7887430 \\ \mathrm{H} & -5.8524140 & 1.2157060 & 1.7715050 \\ \mathrm{H} & -5.3877750 & -0.4760310 & 1.9151730 \\ \mathrm{H} & -4.3925860 & 0.7850000 & 2.6696830 \\ \mathrm{C} & -5.0403760 & 0.2283370 & -0.7118730 \\ \mathrm{H} & -5.2943020 & -0.8341190 & -0.6399110 \\ \mathrm{H} & -5.9794750 & 0.7908290 & -0.7357140 \\ \mathrm{H} & -4.5306740 & 0.4023750 & -1.6647630 \\ \mathrm{C} & -3.8595200 & 2.1946680 & 0.3118340\end{array}$

$\begin{array}{lrrr}\mathrm{H} & -3.2359860 & 2.5719790 & 1.1310840 \\ \mathrm{H} & -3.3573180 & 2.3991990 & -0.6380080 \\ \mathrm{H} & -4.7893400 & 2.7723140 & 0.3186600 \\ \mathrm{O} & 0.2800350 & 0.3940950 & 0.5636610 \\ \mathrm{H} & 1.0893790 & -0.3223020 & 0.2273510 \\ \mathrm{H} & 0.7293540 & 1.1776420 & 0.9263190 \\ \mathrm{O} & 2.0133670 & -1.1564140 & -0.1513330 \\ \mathrm{C} & 2.9001330 & -1.4517050 & 0.7238570 \\ \mathrm{O} & 3.0143760 & -1.0647750 & 1.8851660 \\ \mathrm{C} & 3.9561000 & -2.4556610 & 0.1629850 \\ \mathrm{~F} & 4.5763040 & -1.9565150 & -0.9361500 \\ \mathrm{~F} & 4.9157370 & -2.7525550 & 1.0618180 \\ \mathrm{~F} & 3.3728840 & -3.6256780 & -0.2032470 \\ \mathrm{H} & -0.9585070 & 1.3097720 & -2.6252680 \\ \mathrm{H} & 0.6399120 & 3.0832940 & -2.7521810 \\ \mathrm{H} & -0.5587830 & 3.5118880 & -1.5422820 \\ \mathrm{C} & 1.3921650 & 3.0935810 & -0.7463130 \\ \mathrm{C} & 1.1371530 & 3.7437010 & 0.4723320 \\ \mathrm{C} & 2.7077650 & 2.6801690 & -1.0177570 \\ \mathrm{C} & 2.1632440 & 3.9633750 & 1.4004530 \\ \mathrm{H} & 0.1324220 & 4.0963520 & 0.6924760 \\ \mathrm{C} & 3.7340220 & 2.9007730 & -0.0966070 \\ \mathrm{H} & 2.9299090 & 2.1846550 & -1.9597150 \\ \mathrm{C} & 3.4646260 & 3.5411130 & 1.1188370 \\ \mathrm{H} & 1.9431800 & 4.4691010 & 2.3361600 \\ \mathrm{H} & 4.7443950 & 2.5755840 & -0.3275770 \\ \mathrm{H} & 4.2627920 & 3.7126180 & 1.8348790\end{array}$

21.log

Potential Energy = -1489.65495

Zero-point Energy = -1489.18085

Free Energy = -1489.22400

Single-Point Energy B3LYP-D3(BJ)/6-311+G** PCM = 1490.11262

Free Energy B3LYP-D3(BJ)/6-311+G** PCM (extrapolated free energy from qRRHO) $=-1489.68166$

Nimag $=1\left(-330.9198 \mathrm{~cm}^{-1}\right)$

Charge $=0$ Multiplicity $=1$

$\begin{array}{lccc}\mathrm{C} & 2.7836090 & -1.4664520 & -0.1699070 \\ \mathrm{C} & 2.8317280 & -0.4095240 & 1.9397200 \\ \mathrm{C} & 1.5180930 & 0.0340310 & 1.2940840 \\ \mathrm{~N} & 1.4377840 & -0.8874680 & 0.1351920 \\ \mathrm{~N} & 3.4491010 & -1.2938040 & 1.1230670 \\ \mathrm{O} & 3.2286750 & -0.0643470 & 3.0537400 \\ \mathrm{C} & 4.5380950 & -2.1362370 & 1.6145920 \\ \mathrm{H} & 4.3367830 & -2.3875910 & 2.6583360 \\ \mathrm{H} & 4.5798680 & -3.0526030 & 1.0255840 \\ \mathrm{H} & 5.5060020 & -1.6316220 & 1.5599500 \\ \mathrm{C} & 0.3589430 & -1.1643800 & -0.6319060 \\ \mathrm{C} & -1.0393860 & -1.3671830 & -0.0668200 \\ \mathrm{C} & -2.0076050 & -1.9764890 & -1.1038860 \\ \mathrm{H} & -2.1126760 & -1.2903650 & -1.9489430 \\ \mathrm{H} & 1.6146620 & 1.0674090 & 0.9336090 \\ \mathrm{C} & 3.5715730 & -0.8389470 & -1.3786910 \\ \mathrm{H} & 2.6588970 & -2.5383010 & -0.3656820 \\ \mathrm{H} & -0.9366920 & -2.0576640 & 0.7766630 \\ \mathrm{C} & 0.3978400 & -0.0200960 & 2.3367590 \\ \mathrm{H} & -0.5196000 & 0.4478840 & 1.9815630 \\ \mathrm{H} & 0.1861720 & -1.0444300 & 2.6540250 \\ \mathrm{H} & 0.7446530 & 0.5423400 & 3.2073850 \\ \mathrm{C} & 5.0218340 & -1.3756270 & -1.3756410 \\ \mathrm{H} & 5.5233470 & -1.0498300 & -2.2922130 \\ \mathrm{H} & 5.6019680 & -0.9923310 & -0.5341460 \\ \mathrm{H} & 5.0543380 & -2.4703880 & -1.3563270 \\ \mathrm{C} & 3.6211380 & 0.6971660 & -1.2888200 \\ \mathrm{H} & 4.1283180 & 1.0246010 & -0.3752330 \\ \mathrm{H} & 4.1822990 & 1.0995930 & -2.1380240 \\ \mathrm{H} & 2.6303470 & 1.1612420 & -1.2989320 \\ \mathrm{C} & 2.9446590 & -1.2981520 & -2.7149060 \\ \mathrm{H} & 2.9213050 & -2.3918080 & -2.7841970 \\ \mathrm{H} & 1.9285150 & -0.9381650 & -2.8869620 \\ \mathrm{H} & -1.5519750 & -0.9273490 & -3.5460430 \\ \mathrm{H} & -3.369136400 & -0.4369660 & 0.3238830 \\ \mathrm{H} & -2.9052430 & -1.4934650 \\ \mathrm{H} & -2.2678030 & -0.5063770 \\ \mathrm{H} & -3.4966490 & 0.1195550\end{array}$




$\begin{array}{lrrr}\text { C } & -4.3917060 & -1.3070800 & -0.5456560 \\ \mathrm{C} & -4.8791880 & -3.7589440 & 0.6933070 \\ \mathrm{H} & -2.8543300 & -4.2569410 & 0.1527450 \\ \mathrm{C} & -5.6412960 & -1.5644270 & 0.0267790 \\ \mathrm{H} & -4.2098440 & -0.3519110 & -1.0324650 \\ \mathrm{C} & -5.8890770 & -2.7921260 & 0.6493570 \\ \mathrm{H} & -5.0633480 & -4.7181660 & 1.1690310 \\ \mathrm{H} & -6.4205880 & -0.8087740 & -0.0176470 \\ \mathrm{H} & -6.8602510 & -2.9951380 & 1.0913610 \\ \mathrm{H} & 0.6012660 & -1.9113420 & -1.3836600 \\ \mathrm{O} & -0.0150290 & 0.1494610 & -1.7681940 \\ \mathrm{H} & -0.5375690 & 1.0187570 & -1.3192900 \\ \mathrm{H} & 0.8042930 & 0.4843740 & -2.1636460 \\ \mathrm{O} & -1.2453960 & 2.0494480 & -0.8454200 \\ \mathrm{C} & -0.5612310 & 3.0081200 & -0.3496840 \\ \mathrm{O} & 0.6580700 & 3.1025340 & -0.2016170 \\ \mathrm{C} & -1.4620590 & 4.1976380 & 0.1096140 \\ \mathrm{~F} & -2.1345890 & 4.7337430 & -0.9414170 \\ \mathrm{~F} & -0.7557170 & 5.1941050 & 0.6804630 \\ \mathrm{~F} & -2.3900360 & 3.7945780 & 1.0140680\end{array}$

22.log

Potential Energy $=-1489.65593$

Zero-point Energy = -1489.18221

Free Energy = -1489.22598

Single-Point Energy B3LYP-D3(BJ)/6-311+G** PCM = 1490.11120

Free Energy B3LYP-D3(BJ)/6-311+G** PCM (extrapolated free energy from qRRHO) $=-1489.68125$

Nimag $=1\left(-297.0900 \mathrm{~cm}^{-1}\right)$

Charge $=0$ Multiplicity $=1$

\begin{tabular}{|c|c|c|c|}
\hline $\mathrm{C}$ & -3.1260970 & -0.3858910 & -0.7275710 \\
\hline $\mathrm{C}$ & -4.2680010 & 0.9950400 & 0.8050760 \\
\hline & -2.8075580 & 0.9818010 & 1.2684260 \\
\hline N & -2.1382050 & 0.3838820 & 0.0885860 \\
\hline & -4.3703010 & 0.2859820 & -0.3408020 \\
\hline J & -5.1837310 & 1.6050900 & 1.3595050 \\
\hline & -5.5566060 & 0.3890970 & -1.1884710 \\
\hline & -5.9312360 & 1.4138720 & -1.1368390 \\
\hline & -5.2849590 & 0.1569180 & -2.2184910 \\
\hline $\mathrm{H}$ & -6.3522220 & -0.2881520 & -0.867887 \\
\hline $\mathrm{C}$ & -0.8233600 & 0.3886520 & -0.2167930 \\
\hline & 0.0646570 & 1.6046270 & 0.0022760 \\
\hline E & 1.4234440 & 1.4957730 & -0.7209620 \\
\hline $\mathrm{H}$ & 1.9845670 & 0.6404330 & -0.3347100 \\
\hline $\mathrm{H}$ & -2.7145650 & 0.3040990 & 2.1278460 \\
\hline $\mathrm{C}$ & -3.1565350 & -1.9461630 & -0.5310180 \\
\hline $\mathrm{H}$ & -2.9258750 & -0.1804110 & -1.785833 \\
\hline $\mathrm{H}$ & -0.4799500 & 2.4654140 & -0.3994450 \\
\hline & -2.3971660 & 2.3896350 & 1.7060920 \\
\hline $\mathrm{H}$ & -1.4305290 & 2.4016610 & 2.2088380 \\
\hline $\mathrm{H}$ & -2.3825850 & 3.0896560 & 0.8668880 \\
\hline $\mathrm{H}$ & -3.1498730 & 2.7316580 & 2.4208760 \\
\hline $\mathrm{C}$ & -4.4183160 & -2.5176040 & -1.2163860 \\
\hline $\mathrm{H}$ & -4.3649410 & -3.6106890 & -1.202723 \\
\hline $\mathrm{H}$ & -5.3360330 & -2.2301250 & -0.698740 \\
\hline $\mathrm{H}$ & -4.4942940 & -2.2068600 & -2.264431 \\
\hline $\mathrm{C}$ & -3.1790660 & -2.3329660 & 0.9588760 \\
\hline $\mathrm{H}$ & -4.0447760 & -1.9028280 & 1.474212 \\
\hline $\mathrm{H}$ & -3.2528140 & -3.4215220 & 1.053211 \\
\hline $\mathrm{H}$ & -2.2664890 & -2.0163420 & 1.470108 \\
\hline $\mathrm{C}$ & -1.9350340 & -2.5846600 & -1.227556 \\
\hline $\mathrm{H}$ & -1.8760850 & -2.2882810 & -2.281716 \\
\hline $\mathrm{H}$ & -0.9942840 & -2.3362720 & -0.737958 \\
\hline $\mathrm{H}$ & -2.0330700 & -3.6747520 & -1.199264 \\
\hline $\mathrm{H}$ & 0.2263430 & 1.8004290 & 1.0643410 \\
\hline $\mathrm{H}$ & 1.2454710 & 1.3072060 & -1.7862130 \\
\hline $\mathrm{C}$ & 2.2478580 & 2.7572950 & -0.5602150 \\
\hline $\mathrm{C}$ & 2.1234780 & 3.8188840 & -1.4699490 \\
\hline $\mathrm{C}$ & 3.1360200 & 2.9011170 & 0.5170990 \\
\hline $\mathrm{C}$ & 2.8625180 & 4.9946460 & -1.3067890 \\
\hline 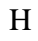 & 1.4469770 & 3.7224740 & -2.3160030 \\
\hline 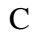 & 3.8768710 & 4.0750420 & 0.6849160 \\
\hline 11 & 3.2521410 & 2.0858370 & 1.2272960 \\
\hline & 3.7416340 & 5.1269760 & -0.2269660 \\
\hline & 2.7550530 & 5.8031960 & -2.024397 \\
\hline
\end{tabular}

$\begin{array}{cccc}\text { H } & 4.5617320 & 4.1652680 & 1.5234120 \\ \text { H } & 4.3189860 & 6.0382010 & -0.1002470 \\ \text { H } & -0.6450800 & -0.0868260 & -1.1784200 \\ \text { O } & 0.0075320 & -0.8582640 & 0.7478410 \\ \text { H } & 0.8980800 & -1.3655960 & 0.3286360 \\ \text { H } & 0.2631080 & -0.4924740 & 1.6097470 \\ \text { O } & 1.9343400 & -2.0331700 & -0.1933640 \\ \text { C } & 2.8591220 & -2.3056860 & 0.6449780 \\ \text { O } & 2.9319900 & -2.0320200 & 1.8437930 \\ \text { C } & 4.0318870 & -3.1007970 & -0.0108690 \\ \text { F } & 4.5716830 & -2.4157700 & -1.0509880 \\ \text { F } & 3.6018870 & -4.2932170 & -0.4980910 \\ \text { F } & 5.0316740 & -3.3617040 & 0.8553060\end{array}$

23.log

Potential Energy $=-1489.65068$

Zero-point Energy = -1489.17631

Free Energy = -1489.21947

Single-Point Energy B3LYP-D3(BJ)/6-311+G** PCM = 1490.11087

Free Energy B3LYP-D3(BJ)/6-311+G** PCM (extrapolated free energy from $\mathrm{qRRHO})=-1489.67967$

Nimag $=1\left(-280.3329 \mathrm{~cm}^{-1}\right)$

Charge $=0$ Multiplicity $=1$

\begin{tabular}{|c|c|c|c|}
\hline $\mathrm{C}$ & -2.8367730 & -1.0145370 & -0.0039030 \\
\hline $\mathrm{C}$ & -3.3021610 & 1.0374540 & -1.0736320 \\
\hline $\mathrm{C}$ & -1.7779870 & 0.9659920 & -0.9746880 \\
\hline $\mathrm{N}$ & -1.5783980 & -0.4511600 & -0.5862440 \\
\hline $\mathrm{N}$ & -3.8291050 & -0.1125360 & -0.5930170 \\
\hline $\mathrm{O}$ & -3.9401320 & 1.9671540 & -1.5695950 \\
\hline $\mathrm{C}$ & -5.2206470 & -0.4720010 & -0.8598700 \\
\hline $\mathrm{H}$ & -5.4882090 & -0.1036580 & -1.8527250 \\
\hline $\mathrm{H}$ & -5.3234360 & -1.5572230 & -0.8386020 \\
\hline $\mathrm{H}$ & -5.9066610 & -0.0334720 & -0.1307890 \\
\hline $\mathrm{C}$ & -0.4353250 & -1.1697490 & -0.6385420 \\
\hline $\mathrm{C}$ & 0.5255010 & -1.1174480 & -1.8242490 \\
\hline $\mathrm{C}$ & 1.3345460 & -2.4207190 & -2.0296550 \\
\hline $\mathrm{H}$ & 0.6382300 & -3.2664550 & -2.0643560 \\
\hline $\mathrm{H}$ & -1.4311780 & 1.6165790 & -0.1598950 \\
\hline $\mathrm{C}$ & -2.9179120 & -1.1123690 & 1.5637870 \\
\hline $\mathrm{H}$ & -2.9820920 & -2.0205590 & -0.4150130 \\
\hline $\mathrm{H}$ & -0.0914990 & -0.9795820 & -2.7150820 \\
\hline $\mathrm{C}$ & -1.1550850 & 1.4530130 & -2.2851570 \\
\hline $\mathrm{H}$ & -0.0691710 & 1.5218720 & -2.2244550 \\
\hline $\mathrm{H}$ & -1.4353510 & 0.8203450 & -3.1312710 \\
\hline $\mathrm{H}$ & -1.5457050 & 2.4571440 & -2.4683730 \\
\hline $\mathrm{C}$ & -4.3627840 & -1.4799310 & 1.9740780 \\
\hline $\mathrm{H}$ & -4.3851810 & -1.6898810 & 3.0479440 \\
\hline $\mathrm{H}$ & -5.0645520 & -0.6648010 & 1.7876950 \\
\hline $\mathrm{H}$ & -4.7201640 & -2.3771690 & 1.4569670 \\
\hline $\mathrm{C}$ & -2.5428170 & 0.2194330 & 2.2385010 \\
\hline $\mathrm{H}$ & -3.2194860 & 1.0239730 & 1.9324030 \\
\hline $\mathrm{H}$ & -2.6234450 & 0.1182900 & 3.3253520 \\
\hline $\mathrm{H}$ & -1.5223850 & 0.5441370 & 2.0134470 \\
\hline $\mathrm{C}$ & -2.0175650 & -2.2617930 & 2.0702360 \\
\hline $\mathrm{H}$ & -2.2913520 & -3.2139710 & 1.6016670 \\
\hline $\mathrm{H}$ & -0.9501490 & -2.1078990 & 1.9013720 \\
\hline $\mathrm{H}$ & -2.1496540 & -2.3754910 & 3.1503810 \\
\hline $\mathrm{H}$ & 1.1948190 & -0.2569150 & -1.7635830 \\
\hline $\mathrm{H}$ & 1.7768640 & -2.3488710 & -3.0303640 \\
\hline $\mathrm{C}$ & 2.4363380 & -2.7125590 & -1.0298500 \\
\hline $\mathrm{C}$ & 3.6025590 & -1.9304610 & -1.0024660 \\
\hline $\mathrm{C}$ & 2.3334510 & -3.7844290 & -0.1318550 \\
\hline $\mathrm{C}$ & 4.6275340 & -2.2006240 & -0.0928780 \\
\hline $\mathrm{H}$ & 3.7113350 & -1.1030190 & -1.6996630 \\
\hline $\mathrm{C}$ & 3.3576680 & -4.0606340 & 0.7810860 \\
\hline $\mathrm{H}$ & 1.4458620 & -4.4123830 & -0.1464980 \\
\hline $\mathrm{C}$ & 4.5077950 & -3.2672710 & 0.8057480 \\
\hline $\mathrm{H}$ & 5.5212510 & -1.5828350 & -0.0883850 \\
\hline $\mathrm{H}$ & 3.2562840 & -4.8959040 & 1.4684540 \\
\hline $\mathrm{H}$ & 5.3053180 & -3.4797530 & 1.5118500 \\
\hline $\mathrm{H}$ & -0.6012360 & -2.1857820 & -0.2903450 \\
\hline $\mathrm{O}$ & 0.6962800 & -0.6996370 & 0.6644390 \\
\hline $\mathrm{H}$ & 1.2310040 & 0.2302480 & 0.4766850 \\
\hline $\mathrm{H}$ & 0.2061740 & -0.5737040 & 1.4919320 \\
\hline $\mathrm{O}$ & 1.9715760 & 1.3650930 & 0.2466370 \\
\hline
\end{tabular}




$\begin{array}{llll}\text { C } & 1.4605470 & 2.4463410 & 0.6897930 \\ \text { O } & 0.3801050 & 2.6260340 & 1.2571550 \\ \text { C } & 2.3818920 & 3.6828560 & 0.4418540 \\ \text { F } & 3.5915430 & 3.5235160 & 1.0373710 \\ \text { F } & 1.8540730 & 4.8302940 & 0.9147650 \\ \text { F } & 2.6111600 & 3.8650880 & -0.8844740\end{array}$

24.log

Potential Energy $=-1489.65446$

Zero-point Energy = -1489.18064

Free Energy $=-1489.22390$

Single-Point Energy B3LYP-D3(BJ)/6-311+G** PCM = 1490.11397

Free Energy B3LYP-D3(BJ)/6-311+G** PCM (extrapolated free energy from $q R R H O)=-1489.68341$

Nimag $=1\left(-331.0632 \mathrm{~cm}^{-1}\right)$

Charge $=0$ Multiplicity $=1$

C $\quad-1.2994360 \quad 2.0527380$

C $\quad 0.1922520 \quad 2.8433220 \quad-1.5229660$

$\begin{array}{llll}\mathrm{C} & 0.6664490 & 1.4271090 & -1.1922750\end{array}$

$\mathrm{N} \quad-0.4733880 \quad 0.9176870 \quad-0.3920810$

$\mathrm{N} \quad-0.9388050 \quad 3.1039230 \quad-0.8287580$

$\begin{array}{llll}\text { O } & 0.7219130 & 3.5980040 & -2.3403050\end{array}$

C $\quad-1.7960430 \quad 4.2332540 \quad-1.1847940$

$\mathrm{H}-1.7725200-43569070-22698510$

$\mathrm{H} \quad-2.8182370 \quad 4.0249660 \quad-0.8682300$

$\mathrm{H} \quad-1.4618550 \quad 5.1655430 \quad-0.7225060$

C $\quad-0.7621490 \quad-0.3661380 \quad-0.0754380$

$\begin{array}{llll}\text { C } & -0.6141920 & -1.5173730 & -1.0583240\end{array}$

$\begin{array}{llll}\text { C } & -1.2642110 & -2.8333620 & -0.5722210\end{array}$

$\mathrm{H} \quad-1.0555090 \quad-3.5879910 \quad-1.3389640$

$\begin{array}{llll}\mathrm{H} & 1.5637410 & 1.4775030 & -0.5604480\end{array}$

C $\quad-1.0853200 \quad 2.4616210 \quad 1.6290910$

$\mathrm{H} \quad-2.3568200 \quad 1.7953540 \quad-0.0106120$

$\begin{array}{llll}\mathrm{H} & -1.0995030 & -1.1962630 & -1.9854060\end{array}$

$\begin{array}{llll}\mathrm{C} & 1.0215110 & 0.6983960 & -2.4916550\end{array}$

$\begin{array}{llll}\mathrm{H} & 1.5259890 & -0.2497660 & -2.3090410\end{array}$

$\begin{array}{llll}\mathrm{H} & 0.1413290 & 0.5298190 & -3.1173900\end{array}$

$\begin{array}{llll}\mathrm{H} & 1.7117920 & 1.3439350 & -3.0406620\end{array}$

$\begin{array}{llll}\mathrm{C} & -1.8126650 & 3.7981080 & 1.9048970\end{array}$

$\mathrm{H} \quad-1.7862490 \quad 4.0021310 \quad 2.9798610$

$\mathrm{H} \quad-1.3325900-4.6402150-1.4028240$

$\mathrm{H} \quad-2.8654400 \quad 3.7625450 \quad 1.6042230$

$\begin{array}{llll}\text { C } & 0.4070950 & 2.6425090 & 1.9613360\end{array}$

$\mathrm{H} \quad 0.8552700 \quad 3.4354260 \quad 1.3534820$

$\mathrm{H} \quad 0.5198080 \quad 2.9307150 \quad 3.0112210$

$\mathrm{H} \quad 0.9962390-1.7341670-1.8036130$

C $\quad-1.7391480 \quad 1.4150320 \quad 2.5593540$

$\begin{array}{llll}\mathrm{H} & -2.8072620 & 1.3061400 & 2.3397690\end{array}$

$\begin{array}{llll}\mathrm{H} & -1.2932830 & 0.4202020 & 2.5068690\end{array}$

H $\quad-1.6470040 \quad 1.7455200 \quad 3.5983170$

$\mathrm{H} \quad 0.4339740 \quad-1.7070820 \quad-1.2919060$

$\mathrm{H} \quad-0.7701410 \quad-3.1682720 \quad 0.3436180$

$\begin{array}{llll}\text { C } & -2.7616660 & -2.7494530 & -0.3448290\end{array}$

C $\quad-3.3007540 \quad-2.7797560 \quad 0.9503120$

C $\quad-3.6462660 \quad-2.6382860 \quad-1.4314000$

C $\quad-4.6826160 \quad-2.6969620 \quad 1.1580750$

$\mathrm{H} \quad-2.6346600 \quad-2.8759290 \quad 1.8041780$

C $\quad-5.0260180 \quad-2.5548330 \quad-1.2293890$

$\mathrm{H} \quad-3.2525680 \quad-2.6256840 \quad-2.4451390$

C $\quad-5.5498420 \quad-2.5823750 \quad 0.0685190$

$\mathrm{H} \quad-5.0780350 \quad-2.7248830 \quad 2.1695430$

$\mathrm{H} \quad-5.6922750 \quad-2.4743300 \quad-2.0837320$

$-2.5203930$

$\mathrm{H} \quad-1.6947940 \quad-0.4253770 \quad 0.4798020$

$\begin{array}{llll}\mathrm{O} & 0.2811770 & -0.9966660 & 1.2118330\end{array}$

$\mathrm{H} \quad 1.3002480 \quad-1.2928230 \quad 0.8848590$

$\mathrm{H} \quad 0.3886920 \quad-0.3283220 \quad 1.9057670$

$\begin{array}{llll}\text { O } & 2.5031790 & -1.7423490 & 0.5202240\end{array}$

C $\quad 3.4411210 \quad-0.8742810 \quad 0.5336810$

$\begin{array}{llll}\text { O } & 3.3886960 & 0.3276600 & 0.7976980\end{array}$

C $\quad 4.8231340 \quad-1.4953040 \quad 0.1565360$

$\begin{array}{llll}\text { F } & 5.1943600 & -2.4409000 & 1.0578550\end{array}$

F $\quad 5.8100150 \quad-0.5778200 \quad 0.1122150$

$\begin{array}{llll}\text { F } & 4.7803460 & -2.0970830 & -1.0591070\end{array}$ 25.log

Potential Energy = -1489.65026

Zero-point Energy = -1489.17613

Free Energy = -1489.21937

Single-Point Energy B3LYP-D3(BJ)/6-311+G** PCM = 1490.11007

Free Energy B3LYP-D3(BJ)/6-311+G** PCM (extrapolated free energy from $q R R H O)=-1489.67919$

Nimag $=1\left(-236.1868 \mathrm{~cm}^{-1}\right)$ Charge $=0$ Multiplicity $=1$

$\begin{array}{llll}\text { C } & 2.9655790 & -0.7450410 & 0.6106690\end{array}$

$\begin{array}{llll}\mathrm{C} & 4.4321080 & 0.7061370 & -0.5284450\end{array}$

C $\quad 3.0214750 \quad 1.2467400 \quad-0.7881910$

$\begin{array}{llll}\mathrm{N} & 2.2396610 & 0.5069990 & 0.2333050\end{array}$

$\begin{array}{llll}\mathrm{N} & 4.3451060 & -0.3543190 & 0.3043430\end{array}$

$\begin{array}{llll}\text { O } & 5.4741260 & 1.1958540 & -0.9670610\end{array}$

C $\quad 5.5249760 \quad-0.8816780 \quad 0.9865860$

$\mathrm{H} \quad 6.2083350 \quad-0.0527500 \quad 1.1832920$

$\mathrm{H} \quad 5.2227530 \quad-1.3319140 \quad 1.9329440$

H $\quad 6.0493770 \quad-1.6299140 \quad 0.3871820$

C $\quad 1.0213930 \quad 0.8014080 \quad 0.7349330$

$\begin{array}{llll}\text { C } & 0.6384340 & 2.2166790 & 1.1385650\end{array}$

$\begin{array}{llll}\text { C } & -0.7581190 & 2.3781870 & 1.7796020\end{array}$

$\mathrm{H} \quad-0.9990470 \quad 1.4958420 \quad 2.3824200$

$\begin{array}{llll}\mathrm{H} & 2.6999500 & 0.9250300 & -1.7875580\end{array}$

$\begin{array}{llll}\text { C } & 2.5065830 & -2.0757000 & -0.0899820\end{array}$

$\mathrm{H} \quad 2.8711870 \quad-0.8794080 \quad 1.6944240$

$\begin{array}{llll}\mathrm{H} & 1.3939700 & 2.4908450 & 1.8840140\end{array}$

C $\quad 3.0390790 \quad 2.7757330 \quad-0.7580580$

$\begin{array}{llll}\mathrm{H} & 2.1183460 & 3.2047510 & -1.1544030\end{array}$

$\begin{array}{llll}\mathrm{H} & 3.2286320 & 3.1668860 & 0.2443520\end{array}$

$\begin{array}{llll}\mathrm{H} & 3.8591140 & 3.0930490 & -1.4074760\end{array}$

C $\quad 3.5745190 \quad-3.1690130 \quad 0.1368200$

$\mathrm{H} \quad 3.1849640 \quad-4.1250250 \quad-0.2269840$

$\mathrm{H} \quad 4.4973810 \quad-2.9632810 \quad-0.4101870$

$\mathrm{H} \quad 3.8161000 \quad-3.2970830 \quad 1.1977950$

$\begin{array}{llll}\mathrm{C} & 2.3024020 & -1.8859650 & -1.6041390\end{array}$

$\mathrm{H} \quad 3.2211330 \quad-1.5486300 \quad-2.0963400$

$\mathrm{H} \quad 2.0246950 \quad-2.8432810 \quad-2.0575520$

H $\quad 1.5021840 \quad-1.1720600 \quad-1.8164090$

C $\quad 1.2006860 \quad-2.5729550 \quad 0.5630510$

$\begin{array}{llll}\mathrm{H} & 1.3201040 & -2.6972810 & 1.6459840\end{array}$

$\mathrm{H} \quad 0.3511680 \quad-1.9158500 \quad 0.3818990$

$\begin{array}{llll}\mathrm{H} & 0.9364670 & -3.5508610 & 0.1473550\end{array}$

$\begin{array}{llll}\mathrm{H} & 0.7586960 & 2.9180310 & 0.3154440\end{array}$

$\begin{array}{llll}\mathrm{H} & -0.6806330 & 3.2116740 & 2.4885040\end{array}$

$\begin{array}{llll}\mathrm{C} & -1.9123610 & 2.6769210 & 0.8344640\end{array}$

$\begin{array}{llll}\mathrm{C} & -1.8218470 & 3.6928950 & -0.1331350\end{array}$

$\begin{array}{llll}\text { C } & -3.1279210 & 1.9855930 & 0.9553580\end{array}$

$\begin{array}{llll}\text { C } & -2.9075200 & 3.9985270 & -0.9601450\end{array}$

$\mathrm{H} \quad-0.9043430 \quad 4.2661790 \quad-0.2380510$

$\begin{array}{llll}\text { C } & -4.2173290 & 2.2914820 & 0.1341800\end{array}$

$\mathrm{H} \quad-3.2201130 \quad 1.1959480 \quad 1.6954270$

$\begin{array}{llll}\text { C } & -4.1105810 & 3.2981950 & -0.8298360\end{array}$

$\begin{array}{llll}\mathrm{H} & -2.8131800 & 4.7880780 & -1.7002090\end{array}$

$\begin{array}{llll}\mathrm{H} & -5.1463430 & 1.7396710 & 0.2458090\end{array}$

$\mathrm{H} \quad-4.9543790 \quad 3.5348540 \quad-1.4712570$

$\mathrm{H} \quad 0.7140450 \quad 0.0686800 \quad 1.4776190$

$\begin{array}{llll}\mathrm{O} & -0.2215510 & 0.3850130 & -0.4906190\end{array}$

$\mathrm{H} \quad-1.0233320 \quad-0.2825890 \quad-0.1737430$

$\begin{array}{llll}\mathrm{H} & -0.6430620 & 1.1944170 & -0.8252770\end{array}$

$\begin{array}{llll}\mathrm{O} & -1.9813860 & -1.1609470 & 0.2652910\end{array}$

$\begin{array}{llll}\text { C } & -2.8160470 & -1.5887280 & -0.5979480\end{array}$

$\begin{array}{llll}\text { O } & -2.9087590 & -1.3359580 & -1.8008490\end{array}$

$\begin{array}{llll}\text { C } & -3.8506340 & -2.5778900 & 0.0297410\end{array}$

$\begin{array}{llll}\text { F } & -4.5566410 & -1.9857090 & 1.0279140\end{array}$

F $\quad-3.2326250 \quad-3.6610840 \quad 0.5688000$

F $\quad-4.7458720 \quad-3.0391650 \quad-0.8679560$

26.log

Potential Energy = -1489.65542

Zero-point Energy = -1489.18140

Free Energy $=-1489.22461$

Single-Point Energy B3LYP-D3(BJ)/6-311+G** PCM = 1490.11360 
Free Energy B3LYP-D3(BJ)/6-311+G** PCM (extrapolated free energy from qRRHO) $=-1489.68278$

Nimag $=1\left(-305.1265 \mathrm{~cm}^{-1}\right)$

Charge $=0$ Multiplicity $=1$

$\begin{array}{lll}\mathrm{C} & -2.6594770 & 0.3627560 \\ \mathrm{C} & -4.2758100 & 0.6552950 \\ \mathrm{C} & -2.9187240 & 0.7163630 \\ \mathrm{~N} & -2.0180240 & 0.2048410 \\ \mathrm{~N} & -4.0744490 & 0.3832110\end{array}$

$\mathrm{N} \quad-4.0744490 \quad 0.3832110$

$\begin{array}{lll}\mathrm{O} & -5.3714230 & 0.7754100\end{array}$

C $\quad-5.1728560 \quad-0.0572890$

$\mathrm{H} \quad-5.8521990 \quad-0.6717240$

$\mathrm{H} \quad-4.7727950 \quad-0.6541940$

$\mathrm{H} \quad-5.7363230 \quad 0.7834110$

C $\quad-0.7497720 \quad-0.2450180$

$\begin{array}{lll}\text { C } & -0.3046190 & -1.1100200\end{array}$

C $1.0543550 \quad-1.8112140$

H $1.2926120 \quad-2.3376640$

$\mathrm{H} \quad-2.6781050 \quad 1.7664330$

C $\quad-2.2165580 \quad 1.5991840$

$\begin{array}{lll}\mathrm{H} & -2.4633750 & -0.5465570\end{array}$

$\mathrm{H}-1.0767310$

C $\quad-2.9992220$

$-1.8729200$

$-0.0479650$

0.1050020

H -2.1170780

H $\quad-3.1557290$

H -3.8625940

C -3.1864350

$-1.1182510$

0.3461160

1.7557790

2.5229440

2.0760540

0.8292750

2.9010920

3.1098630

3.7440050

2.8661740

1.3472940

0.4008310

1.3428750

2.1481350

$-0.5338740$

$-1.0662890$

$-2.7980890$

$-2.5098690$

$-4.0252420$

$-3.4194550$

$-1.5693480$

$-4.9350850$

$-4.2746670$

$-4.6340440$

$-3.1777590$

$-5.8802560$

$-5.3419200$

$-0.6084600$

1.1356330

1.0421710

1.4379030

0.9658580

1.5887680

2.2229370

1.4909890

0.1986330

2.0486450

2.1163020

0.7733560

$-0.9180470$

$-1.6264930$

$-0.5669730$

0.3901150

$-1.4692310$

1.2477360

0.6526200

2.0677310

1.6602630

$-0.6933940$

$-1.8639070$

$-1.6392890$

$-2.5702210$

$-1.8410500$

1.6391320

1.3538520

$-2.0028390$

$-2.9501080$

$-3.5712920$

$-2.7938280$

$-3.4922410$

2.8321240

3.5081950

2.5167870

3.4093380

0.8176270

0.3987350

1.4654940

0.0052040

2.2284900

2.7808910

1.4680130

2.9340290

$-2.7905720$

$-1.4918430$

$-0.4864730$

0.7085210

$-0.5913620$

1.7728040

0.8030810

0.4686850

$-1.5130770$

1.6571740

2.6886140

0.3657520

2.4809890

0.2589350

$-0.8083270$

$-0.4225650$

$-1.7289230$

0.0944950

$-0.5808790$

$-1.6297590$

0.0741950

0.2164190

1.3122650

$-0.6485550$

Formation of enamine (TS3)

\section{Conformation Extrapolated name \\ Free energy}

TS3-lowest

0.0

$\begin{array}{ll}1 & 1.2 \\ 2 & 0.5\end{array}$

\begin{tabular}{|c|c|}
\hline 3 & 1.3 \\
\hline 4 & 0.9 \\
\hline 5 & 1.6 \\
\hline 6 & 1.3 \\
\hline 7 & 3.2 \\
\hline 8 & 2.3 \\
\hline 9 & 2.6 \\
\hline 10 & 2.7 \\
\hline 11 & 2.2 \\
\hline 12 & 3.4 \\
\hline 13 & 3.1 \\
\hline 14 & 2.7 \\
\hline 15 & 2.4 \\
\hline 16 & 2.1 \\
\hline 17 & 3.2 \\
\hline 18 & 2.4 \\
\hline 19 & 2.0 \\
\hline 20 & 1.8 \\
\hline 21 & 1.7 \\
\hline 22 & 2.1 \\
\hline 23 & 1.6 \\
\hline 24 & 1.8 \\
\hline
\end{tabular}

TS3-lowest.log (Enamine 2)

Potential Energy = -1489.65579

Zero-point Energy $=-1489.18747$

Free Energy = -1489.23284

Single-Point Energy B3LYP-D3(BJ)/6-311+G** PCM = 1490.11344

Free Energy B3LYP-D3(BJ)/6-311+G** PCM (extrapolated free energy from $q R R H O)=-1489.69048$

Nimag $=1\left(-1187.7406 \mathrm{~cm}^{-1}\right)$

Charge $=0$ Multiplicity $=1$

$\begin{array}{lrrr}\mathrm{C} & -0.1574210 & 1.7542590 & 0.3996040 \\ \mathrm{C} & -1.3320550 & 2.8990120 & -1.3018630 \\ \mathrm{C} & -0.0728660 & 2.3291580 & -1.9627140 \\ \mathrm{~N} & 0.3945820 & 1.3747860 & -0.9257660 \\ \mathrm{~N} & -1.3482980 & 2.5141450 & -0.0053190 \\ \mathrm{O} & -2.1909620 & 3.5738310 & -1.8717520 \\ \mathrm{C} & -2.5095980 & 2.7435460 & 0.8492150 \\ \mathrm{H} & -3.4076800 & 2.6833550 & 0.2311380 \\ \mathrm{H} & -2.5520570 & 1.9734170 & 1.6201480 \\ \mathrm{H} & -2.4800390 & 3.7289410 & 1.3230100 \\ \mathrm{C} & 0.9173820 & 0.1811110 & -1.2147440 \\ \mathrm{C} & 1.0484730 & -0.9477280 & -0.4019030 \\ \mathrm{C} & 2.0034900 & -2.0666860 & -0.8350700 \\ \mathrm{C} & -0.3612370 & 1.7340590 & -3.3362680 \\ \mathrm{H} & -0.8513680 & 2.5040260 & -3.9369780 \\ \mathrm{H} & 0.5563790 & 1.4420260 & -3.8522550 \\ \mathrm{H} & -1.0291510 & 0.8702780 & -3.2690510 \\ \mathrm{H} & 0.6564210 & 3.1414950 & -2.0677920 \\ \mathrm{C} & 0.8500190 & 2.5471490 & 1.3056370 \\ \mathrm{H} & -0.4803160 & 0.8499870 & 0.9236530 \\ \mathrm{C} & 0.1647760 & 2.8799520 & 2.6495070 \\ \mathrm{H} & 0.9083580 & 3.2918250 & 3.3393230 \\ \mathrm{H} & -0.6254200 & 3.6261000 & 2.5408500 \\ \mathrm{H} & -0.2626970 & 1.9869650 & 3.1191230 \\ \mathrm{C} & 2.0777410 & 1.6630800 & 1.6045950 \\ \mathrm{H} & 1.7997300 & 0.7555850 & 2.1505320 \\ \mathrm{H} & 2.6091810 & 1.3720320 & 0.6946270 \\ \mathrm{H} & 2.7778460 & 2.2222560 & 2.2336280 \\ \mathrm{C} & 1.3197570 & 3.8521460 & 0.6356490 \\ \mathrm{H} & 0.4798250 & 4.4979330 & 0.3599250 \\ & & & \end{array}$




$\begin{array}{lrrr}\mathrm{H} & 1.9483690 & 4.4130820 & 1.3346460 \\ \mathrm{H} & 1.9221090 & 3.6566850 & -0.2570300 \\ \mathrm{H} & 1.1981000 & 0.0736060 & -2.2594320 \\ \mathrm{H} & -0.2430860 & -1.5221250 & -0.6572260 \\ \mathrm{H} & 0.9706550 & -0.8154030 & 0.6754280 \\ \mathrm{O} & -1.2610430 & -2.1561810 & -0.8289860 \\ \mathrm{C} & -2.1793160 & -1.9079390 & 0.0354620 \\ \mathrm{O} & -2.1485280 & -1.1296760 & 0.9854510 \\ \mathrm{C} & -3.4663560 & -2.7467530 & -0.2311360 \\ \mathrm{~F} & -3.2025320 & -4.0721870 & -0.1611240 \\ \mathrm{~F} & -3.9644160 & -2.4934630 & -1.4633050 \\ \mathrm{~F} & -4.4354460 & -2.4761230 & 0.6638120 \\ \mathrm{H} & 2.0067640 & -2.1345120 & -1.9293480 \\ \mathrm{H} & -3.3245770 & -0.6518550 & 2.3728570 \\ \mathrm{O} & -3.7938960 & -0.2767860 & 3.1423840 \\ \mathrm{H} & -4.6103530 & -0.7864980 & 3.2209610 \\ \mathrm{H} & 1.6142110 & -3.0216520 & -0.4643030 \\ \mathrm{C} & 3.4264910 & -1.8885320 & -0.3310520 \\ \mathrm{C} & 3.8032040 & -2.3726340 & 0.9313230 \\ \mathrm{C} & 4.3901070 & -1.2223570 & -1.1040280 \\ \mathrm{C} & 5.1026130 & -2.1879600 & 1.4141390 \\ \mathrm{H} & 3.0738010 & -2.9012680 & 1.5407440 \\ \mathrm{C} & 5.6915520 & -1.0370630 & -0.6271110 \\ \mathrm{H} & 4.1234540 & -0.8496190 & -2.0902350 \\ \mathrm{C} & 6.0522300 & -1.5176850 & 0.6361200 \\ \mathrm{H} & 5.3737700 & -2.5720210 & 2.3936740 \\ \mathrm{H} & 6.4236190 & -0.5228690 & -1.2437360 \\ \mathrm{H} & 7.0632140 & -1.3766510 & 1.0073250\end{array}$

$1 . \log$

Potential Energy $=-1489.65425$

Zero-point Energy $=-1489.18574$

Free Energy $=-1489.23052$

Single-Point Energy B3LYP-D3(BJ)/6-311+G** PCM = -

1490.11230

Free Energy B3LYP-D3(BJ)/6-311+G** PCM (extrapolated free energy from qRRHO) $=-1489.68857$

Nimag $=1\left(-1184.5882 \mathrm{~cm}^{-1}\right)$

Charge $=0$ Multiplicity $=1$

$\begin{array}{lrrr}\mathrm{C} & -2.1995800 & -0.6142750 & -0.4140810 \\ \mathrm{C} & -3.4781750 & 0.5370270 & 1.2061690 \\ \mathrm{C} & -2.8341940 & -0.6468350 & 1.9347400 \\ \mathrm{~N} & -1.8205050 & -1.0833510 & 0.9425240 \\ \mathrm{~N} & -3.0595710 & 0.5293860 & -0.0801630 \\ \mathrm{O} & -4.2314890 & 1.3644400 & 1.7217140 \\ \mathrm{C} & -3.3525130 & 1.6336070 & -0.9893400 \\ \mathrm{H} & -3.3871960 & 2.5567040 & -0.4072410 \\ \mathrm{H} & -2.5618400 & 1.7096650 & -1.7366250 \\ \mathrm{H} & -4.3152640 & 1.5046960 & -1.4922570 \\ \mathrm{C} & -0.6069380 & -1.5251660 & 1.2804200 \\ \mathrm{C} & 0.5526290 & -1.6020410 & 0.5059200 \\ \mathrm{C} & 1.6791980 & -2.5287680 & 0.9839770 \\ \mathrm{H} & 1.4273160 & -3.5651560 & 0.7201920 \\ \mathrm{C} & -2.2972410 & -0.2578130 & 3.3076120 \\ \mathrm{H} & -3.1153710 & 0.1997900 & 3.8690340 \\ \mathrm{H} & -1.9532280 & -1.1292130 & 3.8696260 \\ \mathrm{H} & -1.4818030 & 0.4673640 & 3.2303670 \\ \mathrm{H} & -3.5932910 & -1.4296960 & 2.0529520 \\ \mathrm{C} & -2.8859690 & -1.7169040 & -1.2967570 \\ \mathrm{H} & -1.3077780 & -0.2440420 & -0.9279140 \\ \mathrm{C} & -3.2253200 & -1.1160230 & -2.6789220 \\ \mathrm{H} & -3.5546830 & -1.9171600 & -3.3483390 \\ \mathrm{H} & -4.0339460 & -0.3835950 & -2.6273720 \\ \mathrm{H} & -2.3530190 & -0.6383740 & -3.1388900 \\ \mathrm{C} & -1.9048080 & -2.8871840 & -1.5153570 \\ \mathrm{H} & -1.0018530 & -2.5661840 & -2.0450560 \\ \mathrm{H} & -1.6078170 & -3.3580270 & -0.5741590 \\ \mathrm{H} & -2.3907230 & -3.6521010 & -2.1296240 \\ \mathrm{C} & -4.1729800 & -2.2557400 & -0.6442030 \\ \mathrm{H} & -4.8895580 & -1.4569750 & -0.4274680 \\ \mathrm{H} & -4.6610040 & -2.9566130 & -1.3290760 \\ \mathrm{H} & -3.9619670 & -2.8004870 & 0.2813860 \\ \mathrm{H} & -0.5165410 & -1.7812550 & 2.3332640 \\ \mathrm{H} & 0.9957340 & -0.2431380 & 0.7622300 \\ \mathrm{H} & 0.4493110 & -1.5427120 & -0.5756870 \\ \mathrm{O} & 1.4463780 & 0.8603840 & 0.9428050 \\ & & & \end{array}$

$\begin{array}{cccc}\mathrm{C} & 1.1773420 & 1.6969510 & 0.0050670 \\ \mathrm{O} & 0.5343670 & 1.5143820 & -1.0258470 \\ \mathrm{C} & 1.7812050 & 3.1046700 & 0.2954000 \\ \mathrm{~F} & 1.5002420 & 3.9828990 & -0.6860850 \\ \mathrm{~F} & 3.1271700 & 3.0404060 & 0.4148750 \\ \mathrm{~F} & 1.2916850 & 3.6117670 & 1.4507760 \\ \mathrm{H} & 1.7401610 & -2.4923760 & 2.0777460 \\ \mathrm{C} & 3.0371140 & -2.1980740 & 0.3922060 \\ \mathrm{C} & 3.3270960 & -2.5122570 & -0.9455620 \\ \mathrm{C} & 4.0327890 & -1.5781210 & 1.1609950 \\ \mathrm{C} & 4.5724690 & -2.2072000 & -1.5021060 \\ \mathrm{H} & 2.5740990 & -3.0056270 & -1.5558950 \\ \mathrm{C} & 5.2824270 & -1.2739950 & 0.6104170 \\ \mathrm{H} & 3.8293970 & -1.3310590 & 2.2000380 \\ \mathrm{C} & 5.5559970 & -1.5855960 & -0.7247450 \\ \mathrm{H} & 4.7765900 & -2.4597910 & -2.5389170 \\ \mathrm{H} & 6.0401870 & -0.7957930 & 1.2248990 \\ \mathrm{H} & 6.5257800 & -1.3512600 & -1.1541790 \\ \mathrm{H} & 0.0036500 & 2.5699450 & -2.4867800 \\ \mathrm{O} & -0.3829370 & 2.9644340 & -3.2918480 \\ \mathrm{H} & 0.0394720 & 3.8278970 & -3.3845810\end{array}$

$2 . \log$

Potential Energy $=-1489.65426$

Zero-point Energy $=-1489.18578$

Free Energy $=-1489.23079$

Single-Point Energy B3LYP-D3(BJ)/6-311+G** PCM = -

1490.11317

Free Energy B3LYP-D3(BJ)/6-311+G** PCM (extrapolated free energy from $\mathrm{qRRHO})=-1489.68969$

Nimag $=1\left(-1170.4086 \mathrm{~cm}^{-1}\right)$

Charge $=0$ Multiplicity $=1$

$\begin{array}{lrrr}\mathrm{C} & -1.5988630 & -1.4802660 & -0.2662370 \\ \mathrm{C} & -1.7836370 & -1.7350240 & 2.0758960 \\ \mathrm{C} & -0.3720050 & -2.2012210 & 1.7061200 \\ \mathrm{~N} & -0.2510530 & -1.6934900 & 0.3168800 \\ \mathrm{~N} & -2.4033040 & -1.3087550 & 0.9516200 \\ \mathrm{O} & -2.2573110 & -1.7322150 & 3.2134500 \\ \mathrm{C} & -3.6980110 & -0.6359000 & 0.9967630 \\ \mathrm{H} & -3.7573780 & -0.0682040 & 1.9275360 \\ \mathrm{H} & -3.7817870 & 0.0489080 & 0.1521650 \\ \mathrm{H} & -4.5293050 & -1.3463230 & 0.9705110 \\ \mathrm{C} & 0.8802550 & -1.1854430 & -0.1809500 \\ \mathrm{C} & 1.0555300 & -0.3468300 & -1.2815550 \\ \mathrm{C} & 2.4390800 & -0.1990160 & -1.9391710 \\ \mathrm{H} & 2.5383100 & -0.9548380 & -2.7286760 \\ \mathrm{C} & 0.6785340 & -1.7010260 & 2.6912290 \\ \mathrm{H} & 0.3745280 & -2.0142270 & 3.6929540 \\ \mathrm{H} & 1.6607990 & -2.1344970 & 2.4896790 \\ \mathrm{H} & 0.7556540 & -0.6098940 & 2.6777190 \\ \mathrm{H} & -0.3654850 & -3.2981280 & 1.6988690 \\ \mathrm{C} & -2.0743790 & -2.6428560 & -1.2084850 \\ \mathrm{H} & -1.6036170 & -0.5396510 & -0.8245750 \\ \mathrm{C} & -3.4764480 & -2.3024900 & -1.7605670 \\ \mathrm{H} & -3.7427830 & -3.0262650 & -2.5375250 \\ \mathrm{H} & -4.2503870 & -2.3528850 & -0.9914560 \\ \mathrm{H} & -3.5024000 & -1.3054150 & -2.2142700 \\ \mathrm{C} & -1.1088140 & -2.7634690 & -2.4056260 \\ \mathrm{H} & -1.0921020 & -1.8485710 & -3.0070710 \\ \mathrm{H} & -0.0869160 & -2.9914860 & -2.0901740 \\ \mathrm{H} & -1.4445820 & -3.5772920 & -3.0563730 \\ \mathrm{C} & -2.1306790 & -3.9934270 & -0.4700780 \\ \mathrm{H} & -2.7714200 & -3.9500120 & 0.4165280 \\ \mathrm{H} & -2.5459530 & -4.7552810 & -1.1376450 \\ \mathrm{H} & -1.1349860 & -4.3337370 & -0.1689310 \\ \mathrm{H} & 1.7576570 & -1.4101130 & 0.4187240 \\ \mathrm{H} & 0.8364150 & 0.9176020 & -0.6076940 \\ \mathrm{H} & 0.2386470 & -0.2719870 & -1.9965740 \\ \mathrm{O} & 0.7219440 & 2.0371430 & -0.1840330 \\ \mathrm{C} & -0.4606350 & 2.5268470 & -0.3105380 \\ \mathrm{O} & -1.4587960 & 2.0051850 & -0.7997700 \\ \mathrm{C} & -0.5494690 & 3.9742770 & 0.2619370 \\ \mathrm{~F} & -1.7831550 & 4.4943590 & 0.1182180 \\ \mathrm{~F} & 0.3189740 & 4.7990000 & -0.3669700 \\ \mathrm{~F} & -0.2483990 & 3.9912360 & 1.5809470 \\ \mathrm{H} & -3.2350130 & 2.5415230 & -1.1201900 \\ & & & \\ & & & \end{array}$




$\begin{array}{lrrr}\mathrm{O} & -4.1777790 & 2.6414080 & -1.3526460 \\ \mathrm{H} & -4.4031180 & 3.5536130 & -1.1296900 \\ \mathrm{H} & 2.4659490 & 0.7732520 & -2.4452820 \\ \mathrm{C} & 3.6276570 & -0.3076260 & -1.0029900 \\ \mathrm{C} & 4.0272370 & 0.7830460 & -0.2129840 \\ \mathrm{C} & 4.3564480 & -1.5030680 & -0.9045150 \\ \mathrm{C} & 5.1210860 & 0.6809850 & 0.6511350 \\ \mathrm{H} & 3.4792150 & 1.7193280 & -0.2779700 \\ \mathrm{C} & 5.4518440 & -1.6104470 & -0.0406480 \\ \mathrm{H} & 4.0674760 & -2.3561010 & -1.5140360 \\ \mathrm{C} & 5.8373280 & -0.5176950 & 0.7413890 \\ \mathrm{H} & 5.4167070 & 1.5381540 & 1.2496550 \\ \mathrm{H} & 6.0036030 & -2.5445160 & 0.0171640 \\ \mathrm{H} & 6.6891610 & -0.5968570 & 1.4106160\end{array}$

$3 . \log$

Potential Energy $=-1489.65556$

Zero-point Energy = -1489.18711

Free Energy $=-1489.23272$

Single-Point Energy B3LYP-D3(BJ)/6-311+G** PCM = -

1490.11126

Free Energy B3LYP-D3(BJ)/6-311+G** PCM (extrapolated free energy from $\mathrm{qRRHO})=-1489.68842$

Nimag $=1\left(-1179.1869 \mathrm{~cm}^{-1}\right)$

Charge $=0$ Multiplicity $=1$

\begin{tabular}{|c|c|c|c|}
\hline $\mathrm{C}$ & -1.2013630 & -1.7339540 & 0.7129140 \\
\hline $\mathrm{C}$ & -1.1177050 & -3.6126720 & -0.7172050 \\
\hline $\mathrm{C}$ & -1.6616950 & -2.4566270 & -1.5624440 \\
\hline $\mathrm{N}$ & -1.3552170 & -1.2910960 & -0.6945270 \\
\hline $\mathrm{N}$ & -0.8414180 & -3.1449970 & 0.5223580 \\
\hline $\mathrm{O}$ & -0.9435410 & -4.7650760 & -1.1149070 \\
\hline $\mathrm{C}$ & -0.1351030 & -3.9562770 & 1.5092570 \\
\hline $\mathrm{H}$ & 0.5758460 & -4.5970220 & 0.9837740 \\
\hline $\mathrm{H}$ & 0.4067080 & -3.3027000 & 2.1939280 \\
\hline $\mathrm{H}$ & -0.8177720 & -4.5904200 & 2.0819360 \\
\hline $\mathrm{C}$ & -0.9329980 & -0.1174070 & -1.1696340 \\
\hline $\mathrm{C}$ & -0.2532820 & 0.9110000 & -0.5113590 \\
\hline $\mathrm{C}$ & -0.2219150 & 2.3048410 & -1.1502730 \\
\hline $\mathrm{C}$ & -1.0529080 & -2.4260030 & -2.9596460 \\
\hline $\mathrm{H}$ & -1.2217150 & -3.4032960 & -3.4182280 \\
\hline $\mathrm{H}$ & -1.5298130 & -1.6738430 & -3.5924480 \\
\hline $\mathrm{H}$ & 0.0247330 & -2.2407850 & -2.9261350 \\
\hline $\mathrm{H}$ & -2.7487850 & -2.5748150 & -1.6452470 \\
\hline $\mathrm{C}$ & -2.4738460 & -1.4916820 & 1.6004530 \\
\hline $\mathrm{H}$ & -0.3529830 & -1.2099080 & 1.1640930 \\
\hline $\mathrm{C}$ & -2.1918740 & -1.9937660 & 3.0338400 \\
\hline $\mathrm{H}$ & -3.0137570 & -1.6880500 & 3.6889990 \\
\hline $\mathrm{H}$ & -2.1218890 & -3.0824990 & 3.0879670 \\
\hline $\mathrm{H}$ & -1.2690660 & -1.5650560 & 3.4407560 \\
\hline $\mathrm{C}$ & -2.7673400 & 0.0204600 & 1.6744130 \\
\hline $\mathrm{H}$ & -1.9411110 & 0.5713910 & 2.1355860 \\
\hline $\mathrm{H}$ & -2.9653120 & 0.4522930 & 0.6898340 \\
\hline $\mathrm{H}$ & -3.6556430 & 0.1834210 & 2.2930590 \\
\hline $\mathrm{C}$ & -3.7100840 & -2.2192170 & 1.0393240 \\
\hline $\mathrm{H}$ & -3.5369620 & -3.2939580 & 0.9237300 \\
\hline $\mathrm{H}$ & -4.5485540 & -2.0954220 & 1.7321010 \\
\hline $\mathrm{H}$ & -4.0222660 & -1.8058470 & 0.0752090 \\
\hline $\mathrm{H}$ & -1.0795080 & -0.0068720 & -2.2411350 \\
\hline $\mathrm{H}$ & 1.0767140 & 0.4409540 & -0.7566020 \\
\hline $\mathrm{H}$ & -0.2609710 & 0.9172030 & 0.5766600 \\
\hline $\mathrm{O}$ & 2.2264260 & 0.1224080 & -0.9825350 \\
\hline $\mathrm{C}$ & 3.0862490 & 0.5276060 & -0.1172970 \\
\hline $\mathrm{O}$ & 2.9027490 & 1.1998250 & 0.8937020 \\
\hline $\mathrm{C}$ & 4.5306140 & 0.0664240 & -0.4823350 \\
\hline $\mathrm{F}$ & 4.9194450 & 0.5939970 & -1.6669020 \\
\hline $\mathrm{F}$ & 4.6013680 & -1.2800490 & -0.5923490 \\
\hline $\mathrm{F}$ & 5.4288530 & 0.4466250 & 0.4465890 \\
\hline $\mathrm{H}$ & -0.1868390 & 2.1993680 & -2.2411350 \\
\hline $\mathrm{H}$ & 4.0005810 & 1.8736770 & 2.2649270 \\
\hline $\mathrm{O}$ & 4.4322160 & 2.2804310 & 3.0406110 \\
\hline $\mathrm{H}$ & 5.3759960 & 2.1094750 & 2.9279480 \\
\hline $\mathrm{H}$ & 0.7070710 & 2.8039420 & -0.8531760 \\
\hline $\mathrm{C}$ & -1.3978130 & 3.1862780 & -0.7620130 \\
\hline $\mathrm{C}$ & -1.3167730 & 4.0402370 & 0.3482670 \\
\hline $\mathrm{C}$ & -2.5955710 & 3.1549750 & -1.4933140 \\
\hline $\mathrm{C}$ & -2.4043920 & 4.8351340 & 0.7251110 \\
\hline
\end{tabular}

$\begin{array}{lrrr}\mathrm{H} & -0.3934630 & 4.0857480 & 0.9210760 \\ \mathrm{C} & -3.6849090 & 3.9487960 & -1.1224510 \\ \mathrm{H} & -2.6763060 & 2.5079940 & -2.3638220 \\ \mathrm{C} & -3.5937260 & 4.7913580 & -0.0090850 \\ \mathrm{H} & -2.3200960 & 5.4908600 & 1.5873210 \\ \mathrm{H} & -4.6013250 & 3.9133450 & -1.7050760 \\ \mathrm{H} & -4.4382700 & 5.4105850 & 0.2791300\end{array}$

4. $\log$

Potential Energy $=-1489.65510$

Zero-point Energy = -1489.18668

Free Energy = -1489.23265

Single-Point Energy B3LYP-D3(BJ)/6-311+G** PCM = 1490.11153

Free Energy B3LYP-D3(BJ)/6-311+G** PCM (extrapolated

free energy from $\mathrm{qRRHO})=-1489.68908$

Nimag $=1\left(-1209.0288 \mathrm{~cm}^{-1}\right)$

Charge $=0$ Multiplicity $=1$

$\begin{array}{llll}\text { C } & -0.9401270 & 2.0526560 & -0.5268090\end{array}$

$\begin{array}{llll}\text { C } & 0.3886130 & 3.3236300 & 0.9566900\end{array}$

$\begin{array}{llll}\mathrm{C} & -0.1653610 & 2.1532730 & 1.7752950\end{array}$

$\begin{array}{llll}\mathrm{N} & -0.6769740 & 1.2637380 & 0.7014760\end{array}$

$\begin{array}{llll}\mathrm{N} & -0.0408920 & 3.1954900 & -0.3204950\end{array}$

$\begin{array}{llll}\mathrm{O} & 1.1181890 & 4.2148540 & 1.3922600\end{array}$

$\begin{array}{llll}\text { C } & 0.4607460 & 4.0539170 & -1.3893150\end{array}$

$\mathrm{H} \quad 1.5006310 \quad 4.3046510 \quad-1.1704420$

$\begin{array}{llll}\mathrm{H} & 0.4111530 & 3.5177530 & -2.3378700\end{array}$

$\begin{array}{llll}\mathrm{H} & -0.1111640 & 4.9825910 & -1.4708000\end{array}$

$\begin{array}{llll}\text { C } & -0.5576810 & -0.0644260 & 0.7360230\end{array}$

$\begin{array}{llll}\text { C } & -0.6176540 & -0.9867070 & -0.3126710\end{array}$

$\begin{array}{llll}\text { C } & -0.8340940 & -2.4685790 & 0.0174670\end{array}$

$\begin{array}{llll}\text { C } & 0.8810920 & 1.5455200 & 2.7018550\end{array}$

$\mathrm{H} \quad 1.2888760 \quad 2.3510730 \quad 3.3173300$

$\mathrm{H} \quad 0.4434890 \quad 0.8020010 \quad 3.3721690$

$\begin{array}{llll}\mathrm{H} & 1.7001700 & 1.0868330 & 2.1405010\end{array}$

$\mathrm{H} \quad-1.0062910 \quad 2.5233950 \quad 2.3739360$

$\begin{array}{llll}\text { C } & -2.4505060 & 2.4295700 & -0.7319600\end{array}$

$\mathrm{H} \quad-0.5972790 \quad 1.4852250 \quad-1.3977260$

$\begin{array}{llll}\mathrm{C} & -2.5941270 & 3.2355070 & -2.0418900\end{array}$

$\mathrm{H} \quad-3.6570160 \quad 3.3640340 \quad-2.2698150$

$\mathrm{H} \quad-2.1555060 \quad 4.2330780 \quad-1.9673410$

$\begin{array}{llll}\mathrm{H} & -2.1347720 & 2.7161540 & -2.8905860\end{array}$

$\begin{array}{llll}\mathrm{C} & -3.2877350 & 1.1416900 & -0.8691740\end{array}$

$\begin{array}{llll}\mathrm{H} & -2.9851030 & 0.5511160 & -1.7400470\end{array}$

$\begin{array}{llll}\mathrm{H} & -3.2210720 & 0.5085460 & 0.0195050\end{array}$

$\mathrm{H} \quad-4.3398170 \quad 1.4112360 \quad-1.0063200$

$\begin{array}{llll}\text { C } & -2.9965140 & 3.2634160 & 0.4424360\end{array}$

$\mathrm{H} \quad-2.4052730 \quad 4.1687730 \quad 0.6133830$

$\begin{array}{llll}\mathrm{H} & -4.0205180 & 3.5784390 & 0.2177970\end{array}$

$\mathrm{H} \quad-3.0314100 \quad 2.6843880 \quad 1.3704840$

H $\quad-0.2922000 \quad-0.4491670 \quad 1.7171800$

$\mathrm{H} \quad 0.7609080 \quad-0.9484930 \quad-0.6953280$

$\mathrm{H} \quad-1.0675190 \quad-0.6669840 \quad-1.2511800$

$\begin{array}{llll}\mathrm{O} & 1.8543190 & -0.9981530 & -1.2297570\end{array}$

$\begin{array}{llll}\mathrm{C} & 2.8279000 & -1.2106700 & -0.4182040\end{array}$

$\begin{array}{llll}\mathrm{O} & 2.7911430 & -1.3840490 & 0.7972820\end{array}$

C $4.2004500 \quad-1.2363090 \quad-1.1579500$

F $\quad 4.4275660 \quad-0.0685590 \quad-1.8028250$

$\begin{array}{llll}\text { F } & 4.2344690 & -2.2258170 & -2.0808260\end{array}$

F $\quad 5.2254450 \quad-1.4375190 \quad-0.3075860$

$\begin{array}{llll}\mathrm{H} & -0.3339620 & -2.7017070 & 0.9648200\end{array}$

$\mathrm{H} \quad 4.0669490 \quad-1.7398580 \quad 2.1323530$

$\begin{array}{llll}\mathrm{O} & 4.5993070 & -1.9299470 & 2.9286040\end{array}$

$\begin{array}{llll}\mathrm{H} & 5.5119000 & -1.9916970 & 2.6188180\end{array}$

$\mathrm{H} \quad-0.3431410 \quad-3.0744070 \quad-0.7521910$

$\begin{array}{llll}\text { C } & -2.2973650 & -2.8718430 & 0.0994090\end{array}$

C $\quad-2.9636860 \quad-3.3814290 \quad-1.0251860$

C $\quad-3.0204420 \quad-2.7271390 \quad 1.2939810$

C $\quad-4.3174380 \quad-3.7288100 \quad-0.9634140$

$\mathrm{H} \quad-2.4177470 \quad-3.5104260 \quad-1.9569190$

$\begin{array}{llll}\text { C } & -4.3726400 & -3.0748770 & 1.3620920\end{array}$

$\mathrm{H} \quad-2.5212060 \quad-2.3434240 \quad 2.1807630$

$\begin{array}{llll}\text { C } & -5.0275300 & -3.5753780 & 0.2312850\end{array}$

$\mathrm{H} \quad-4.8139590 \quad-4.1229270 \quad-1.8458230$

$\mathrm{H} \quad-4.9127270 \quad-2.9597100 \quad 2.2978010$

H $\quad-6.0777180 \quad-3.8477680 \quad 0.2829440$ 
5.log

Potential Energy = -1489.65358

Zero-point Energy = -1489.18548

Free Energy = -1489.23092

Single-Point Energy B3LYP-D3(BJ)/6-311+G** PCM = 1490.11057

Free Energy B3LYP-D3(BJ)/6-311+G** PCM (extrapolated free energy from qRRHO) $=-1489.68791$

Nimag $=1\left(-1208.5109 \mathrm{~cm}^{-1}\right)$

Charge $=0$ Multiplicity $=$

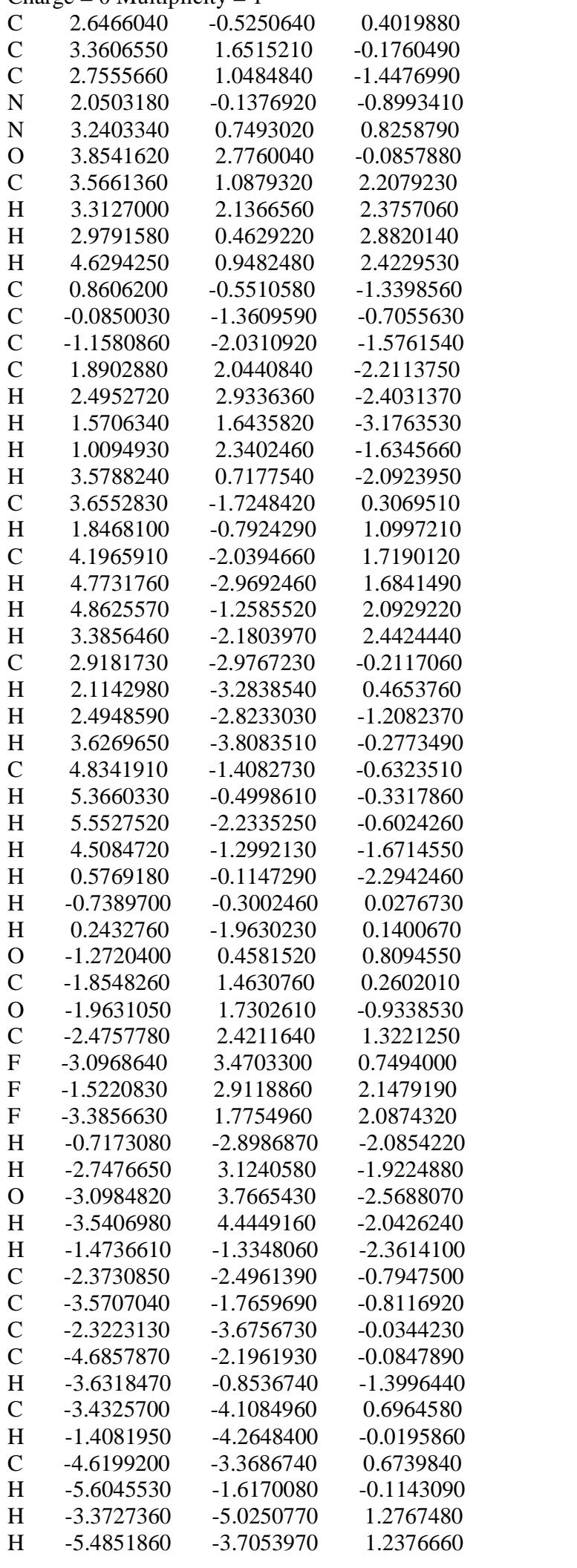

6.log

Potential Energy = -1489.65300

Zero-point Energy = -1489.18458

Free Energy = -1489.22976

Single-Point Energy B3LYP-D3(BJ)/6-311+G** PCM = 1490.11164
Free Energy B3LYP-D3(BJ)/6-311+G** PCM (extrapolated

free energy from $q R R H O)=-1489.68841$

Nimag $=1\left(-1198.2314 \mathrm{~cm}^{-1}\right)$

Charge $=0$ Multiplicity $=$

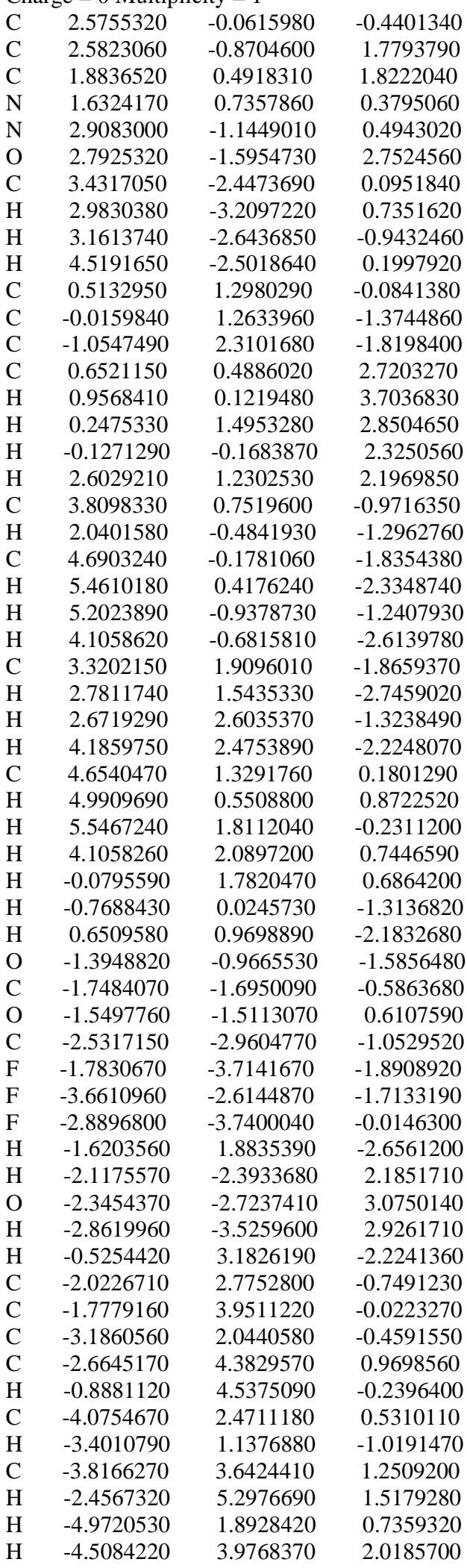

7.log

Potential Energy = -1489.65094

Zero-point Energy = -1489.18265

Free Energy $=-1489.22755$

Single-Point Energy B3LYP-D3(BJ)/6-311+G** PCM = -

1490.10884

Free Energy B3LYP-D3(BJ)/6-311+G** PCM (extrapolated free energy from $q R R H O)=-1489.68545$

Nimag $=1\left(-1148.9197 \mathrm{~cm}^{-1}\right)$

Charge $=0$ Multiplicity $=1$

$\begin{array}{llll}\mathrm{C} & 2.8422840 & 0.0222060 & 0.7162370 \\ \mathrm{C} & 4.7535410 & 0.4491740 & -0.6002260 \\ \mathrm{C} & 3.5987170 & 0.2055680 & -1.5792960\end{array}$




\begin{tabular}{|c|c|c|c|}
\hline $\mathrm{N}$ & 2.4243840 & 0.3170220 & -0.6760040 \\
\hline $\mathrm{N}$ & 4.2637490 & 0.3942470 & 0.6594600 \\
\hline $\mathrm{O}$ & 5.9212320 & 0.6778020 & -0.9188760 \\
\hline $\mathrm{C}$ & 5.0768560 & 0.7372370 & 1.8211630 \\
\hline $\mathrm{H}$ & 5.7753310 & 1.5245400 & 1.5309220 \\
\hline $\mathrm{H}$ & 4.4308060 & 1.1045510 & 2.6198360 \\
\hline $\mathrm{H}$ & 5.6510860 & -0.1188510 & 2.1876020 \\
\hline $\mathrm{C}$ & 1.3234260 & 1.0017670 & -1.0022530 \\
\hline $\mathrm{C}$ & 0.1863610 & 1.2912790 & -0.2452910 \\
\hline $\mathrm{C}$ & -0.6028700 & 2.5532530 & -0.6462910 \\
\hline $\mathrm{H}$ & -0.6765920 & 2.6006750 & -1.7389510 \\
\hline $\mathrm{C}$ & 3.6370330 & 1.1633880 & -2.7637400 \\
\hline $\mathrm{H}$ & 4.6136580 & 1.0562370 & -3.2415710 \\
\hline $\mathrm{H}$ & 2.8737240 & 0.9207550 & -3.5068710 \\
\hline $\mathrm{H}$ & 3.5215230 & 2.2051120 & -2.4497760 \\
\hline $\mathrm{H}$ & 3.6737870 & -0.8230900 & -1.9510580 \\
\hline $\mathrm{C}$ & 2.5748010 & -1.4488440 & 1.1951550 \\
\hline $\mathrm{H}$ & 2.3147110 & 0.7086670 & 1.3866550 \\
\hline $\mathrm{C}$ & 2.9421890 & -1.5591440 & 2.6920130 \\
\hline $\mathrm{H}$ & 2.6179500 & -2.5335440 & 3.0710170 \\
\hline $\mathrm{H}$ & 4.0180340 & -1.4858640 & 2.8658610 \\
\hline $\mathrm{H}$ & 2.4413090 & -0.7889960 & 3.2895160 \\
\hline $\mathrm{C}$ & 1.0763340 & -1.7679250 & 1.0437300 \\
\hline $\mathrm{H}$ & 0.4503260 & -1.0903650 & 1.6318300 \\
\hline $\mathrm{H}$ & 0.7566220 & -1.7199660 & -0.0002470 \\
\hline $\mathrm{H}$ & 0.8849280 & -2.7841750 & 1.4026040 \\
\hline $\mathrm{C}$ & 3.3919460 & -2.4793760 & 0.3927300 \\
\hline $\mathrm{H}$ & 4.4660740 & -2.2723040 & 0.4274220 \\
\hline $\mathrm{H}$ & 3.2358050 & -3.4750160 & 0.8202500 \\
\hline $\mathrm{H}$ & 3.0761540 & -2.5252290 & -0.6540480 \\
\hline $\mathrm{H}$ & 1.3092880 & 1.3237100 & -2.0404650 \\
\hline $\mathrm{H}$ & -0.6742560 & 0.2125650 & -0.7324520 \\
\hline $\mathrm{H}$ & -0.0357570 & 3.4409800 & -0.3354630 \\
\hline $\mathrm{C}$ & -1.9917630 & 2.6410960 & -0.0428930 \\
\hline $\mathrm{C}$ & -3.1391380 & 2.4397270 & -0.8237520 \\
\hline $\mathrm{C}$ & -2.1571980 & 2.9447590 & 1.3182950 \\
\hline $\mathrm{C}$ & -4.4172890 & 2.5311090 & -0.2618940 \\
\hline $\mathrm{H}$ & -3.0328730 & 2.2112770 & -1.8812810 \\
\hline $\mathrm{C}$ & -3.4312060 & 3.0343810 & 1.8855480 \\
\hline $\mathrm{H}$ & -1.2812450 & 3.1194040 & 1.9388850 \\
\hline $\mathrm{C}$ & -4.5677730 & 2.8266600 & 1.0960940 \\
\hline $\mathrm{H}$ & -5.2927390 & 2.3734770 & -0.8857830 \\
\hline $\mathrm{H}$ & -3.5367240 & 3.2712400 & 2.9406080 \\
\hline $\mathrm{H}$ & -5.5590060 & 2.8989660 & 1.5342720 \\
\hline $\mathrm{H}$ & 0.2242210 & 1.0976300 & 0.8266450 \\
\hline $\mathrm{O}$ & -1.4139080 & -0.5805730 & -1.2306070 \\
\hline $\mathrm{C}$ & -2.2596010 & -1.1566930 & -0.4496740 \\
\hline $\mathrm{O}$ & -2.4032180 & -1.0307770 & 0.7612160 \\
\hline $\mathrm{C}$ & -3.1801650 & -2.1393420 & -1.2363710 \\
\hline $\mathrm{F}$ & -2.4529820 & -3.1242980 & -1.8146040 \\
\hline $\mathrm{F}$ & -4.0905400 & -2.7237740 & -0.4341880 \\
\hline $\mathrm{F}$ & -3.8529650 & -1.4981810 & -2.2193480 \\
\hline $\mathrm{H}$ & -3.5178250 & -1.8093330 & 2.0648750 \\
\hline $\mathrm{O}$ & -4.0032980 & -2.1377200 & 2.8457500 \\
\hline $\mathrm{H}$ & -4.7222410 & -2.6771930 & 2.4926620 \\
\hline
\end{tabular}

\begin{tabular}{|c|c|c|c|}
\hline C +2 & 0.8281670 & 0.0637500 & -1.0752860 \\
\hline $\mathrm{C}$ & -0.1395070 & 0.7476050 & -0.3367150 \\
\hline $\mathrm{C}$ & -0.5812380 & 2.1346820 & -0.8285620 \\
\hline $\mathrm{H}$ & -1.6087310 & 2.3129490 & -0.4933410 \\
\hline $\mathrm{C}$ & 3.0231210 & -0.6614760 & -2.8511080 \\
\hline $\mathrm{H}$ & 3.8985240 & -1.1150490 & -3.3218030 \\
\hline $\mathrm{H}$ & 2.1989430 & -0.7061030 & -3.5670860 \\
\hline $\mathrm{H}$ & 3.2561200 & 0.3840090 & -2.6291640 \\
\hline $\mathrm{H}$ & 2.4473790 & -2.4809550 & -1.8678260 \\
\hline $\mathrm{C}$ & 1.2989120 & -2.4590000 & 1.3228660 \\
\hline $\mathrm{H}$ & 1.7632380 & -0.3266120 & 1.3303650 \\
\hline $\mathrm{C}$ & 1.7131220 & -2.6074920 & 2.8039190 \\
\hline $\mathrm{H}$ & 1.1006830 & -3.3850410 & 3.2713580 \\
\hline $\mathrm{H}$ & 2.7576210 & -2.9058080 & 2.9180610 \\
\hline $\mathrm{H}$ & 1.5534070 & -1.6798060 & 3.3650630 \\
\hline $\mathrm{C}$ & -0.2270850 & -2.2535660 & 1.2714460 \\
\hline $\mathrm{H}$ & -0.5393840 & -1.3734680 & 1.8430290 \\
\hline $\mathrm{H}$ & -0.5916600 & -2.1525950 & 0.2464170 \\
\hline $\mathrm{H}$ & -0.7226360 & -3.1236040 & 1.7138420 \\
\hline $\mathrm{C}$ & 1.6579480 & -3.7499180 & 0.5627120 \\
\hline $\mathrm{H}$ & 2.7386800 & -3.9198530 & 0.5266680 \\
\hline $\mathrm{H}$ & 1.2084430 & -4.6069790 & 1.0743330 \\
\hline $\mathrm{H}$ & 1.2673450 & -3.7427690 & -0.4595490 \\
\hline $\mathrm{H}$ & 0.8769210 & 0.2825280 & -2.1387240 \\
\hline $\mathrm{H}$ & -1.3099290 & -0.0159080 & -0.7043150 \\
\hline $\mathrm{H}$ & -0.6000400 & 2.1404930 & -1.9247410 \\
\hline $\mathrm{C}$ & 0.3041390 & 3.2640330 & -0.3282580 \\
\hline $\mathrm{C}$ & 0.0548430 & 3.8708200 & 0.9125790 \\
\hline $\mathrm{C}$ & 1.4006310 & 3.7122480 & -1.0810850 \\
\hline $\mathrm{C}$ & 0.8826100 & 4.8901480 & 1.3938500 \\
\hline $\mathrm{H}$ & -0.7962310 & 3.5446270 & 1.5059090 \\
\hline $\mathrm{C}$ & 2.2301240 & 4.7326210 & -0.6056040 \\
\hline $\mathrm{H}$ & 1.6054480 & 3.2636160 & -2.0503440 \\
\hline $\mathrm{C}$ & 1.9751450 & 5.3243380 & 0.6361130 \\
\hline $\mathrm{H}$ & 0.6708970 & 5.3477120 & 2.3563010 \\
\hline $\mathrm{H}$ & 3.0708760 & 5.0679430 & -1.2066140 \\
\hline $\mathrm{H}$ & 2.6172200 & 6.1183730 & 1.0061990 \\
\hline $\mathrm{H}$ & -0.1246400 & 0.6369480 & 0.7472690 \\
\hline $\mathrm{O}$ & -2.3198040 & -0.5629090 & -1.0544830 \\
\hline $\mathrm{C}$ & -3.3533630 & -0.3299340 & -0.3242540 \\
\hline $\mathrm{O}$ & -3.4474100 & 0.3880210 & 0.6664760 \\
\hline $\mathrm{C}$ & -4.6035270 & -1.1086410 & -0.8346910 \\
\hline $\mathrm{F}$ & -4.3978880 & -2.4452180 & -0.7653970 \\
\hline $\mathrm{F}$ & -5.7004560 & -0.8263920 & -0.1086870 \\
\hline $\mathrm{F}$ & -4.8755300 & -0.8041130 & -2.1246060 \\
\hline $\mathrm{H}$ & -4.8319060 & 0.8289030 & 1.8819560 \\
\hline $\mathrm{O}$ & -5.4731140 & 1.1096570 & 2.5622250 \\
\hline $\mathrm{H}$ & -4.9873710 & 1.7187150 & 3.1331980 \\
\hline
\end{tabular}

$8 . \log$

Potential Energy = -1489.65382

Zero-point Energy = -1489.18543

Free Energy = -1489.23089

Single-Point Energy B3LYP-D3(BJ)/6-311+G** PCM = -

1490.10971

Free Energy B3LYP-D3(BJ)/6-311+G** PCM (extrapolated

free energy from $\mathrm{qRRHO})=-1489.68677$

Nimag $=1\left(-1155.2441 \mathrm{~cm}^{-1}\right)$

Charge $=0$ Multiplicity $=1$

$\begin{array}{llll}\mathrm{C} & 2.0155920 & -1.1994680 & 0.7191720 \\ \mathrm{C} & 3.9205020 & -1.5198830 & -0.6376280 \\ \mathrm{C} & 2.7184950 & -1.4563950 & -1.5873390 \\ \mathrm{~N} & 1.6721310 & -0.8987490 & -0.6924380 \\ \mathrm{~N} & 3.4781120 & -1.3184850 & 0.6248120 \\ \mathrm{O} & 5.0899840 & -1.7008210 & -0.9792260 \\ \mathrm{C} & 4.3965730 & -1.1458380 & 1.7456710 \\ \mathrm{H} & 5.2838310 & -0.6201650 & 1.3869230 \\ \mathrm{H} & 3.9132600 & -0.5492520 & 2.5203160 \\ \mathrm{H} & 4.7083920 & -2.1040890 & 2.1711670\end{array}$

0.7191720

.6376280

0.6924380

.6248120

7456710

2.5203160

2.1711670

9.log

Potential Energy $=-1489.65129$

Zero-point Energy = -1489.18284

Free Energy = -1489.22819

Single-Point Energy B3LYP-D3(BJ)/6-311+G** PCM = 1490.10949

Free Energy B3LYP-D3(BJ)/6-311+G** PCM (extrapolated

free energy from $q R R H O)=-1489.68639$

Nimag $=1\left(-1151.9620 \mathrm{~cm}^{-1}\right)$

Charge $=0$ Multiplicity $=1$

$\begin{array}{lrrr}\mathrm{C} & 2.4854800 & -1.1826820 & 0.4863830 \\ \mathrm{C} & 4.3367050 & -0.0291300 & -0.4153150 \\ \mathrm{C} & 3.0962660 & 0.5068580 & -1.1393590 \\ \mathrm{~N} & 2.0078240 & 0.0067150 & -0.2605160 \\ \mathrm{~N} & 3.9336460 & -0.9257030 & 0.5139370 \\ \mathrm{O} & 5.4975780 & 0.3203630 & -0.6344670 \\ \mathrm{C} & 4.8588640 & -1.4953200 & 1.4881700 \\ \mathrm{H} & 5.5984990 & -0.7348560 & 1.7468600 \\ \mathrm{H} & 4.3090470 & -1.7823280 & 2.3854100 \\ \mathrm{H} & 5.3835930 & -2.3698690 & 1.0925990 \\ \mathrm{C} & 0.9766440 & 0.7711920 & 0.1179670 \\ \mathrm{C} & -0.0654470 & 0.4835860 & 0.9985280 \\ \mathrm{C} & -0.8098080 & 1.6321840 & 1.7121070 \\ \mathrm{H} & -0.3292650 & 1.8005460 & 2.6837060 \\ \mathrm{C} & 3.1592200 & 2.0142460 & -1.3526810 \\ \mathrm{H} & 4.0825690 & 2.2311410 & -1.8949660 \\ \mathrm{H} & 2.3239710 & 2.3730850 & -1.9588770\end{array}$




\begin{tabular}{|c|c|c|c|}
\hline $\mathrm{H}$ & 3.1818440 & 2.5603510 & -0.4051120 \\
\hline $\mathrm{H}$ & 3.0290650 & 0.0129700 & -2.1159190 \\
\hline $\mathrm{C}$ & 2.0848770 & -2.5666320 & -0.1362290 \\
\hline $\mathrm{H}$ & 2.0904780 & -1.1313600 & 1.5065390 \\
\hline $\mathrm{C}$ & 2.5967460 & -3.6964470 & 0.7849830 \\
\hline $\mathrm{H}$ & 2.1857650 & -4.6518030 & 0.4435840 \\
\hline $\mathrm{H}$ & 3.6850160 & -3.7878800 & 0.7694050 \\
\hline $\mathrm{H}$ & 2.2753600 & -3.5509690 & 1.8226100 \\
\hline $\mathrm{C}$ & 0.5498850 & -2.6679440 & -0.2099230 \\
\hline $\mathrm{H}$ & 0.0885030 & -2.5918480 & 0.7801310 \\
\hline $\mathrm{H}$ & 0.1212700 & -1.8985820 & -0.8563270 \\
\hline $\mathrm{H}$ & 0.2720460 & -3.6417370 & -0.6257170 \\
\hline $\mathrm{C}$ & 2.6689790 & -2.7586720 & -1.5488580 \\
\hline $\mathrm{H}$ & 3.7576030 & -2.6451500 & -1.5636850 \\
\hline $\mathrm{H}$ & 2.4403180 & -3.7701230 & -1.8996940 \\
\hline $\mathrm{H}$ & 2.2314310 & -2.0605560 & -2.2691840 \\
\hline $\mathrm{H}$ & 0.9318610 & 1.7242390 & -0.4001670 \\
\hline $\mathrm{H}$ & -1.0747930 & 0.0294610 & 0.0625280 \\
\hline $\mathrm{H}$ & -1.8277070 & 1.2895270 & 1.9323850 \\
\hline $\mathrm{C}$ & -0.8723130 & 2.9519740 & 0.9665600 \\
\hline $\mathrm{C}$ & 0.0084740 & 3.9965150 & 1.2891820 \\
\hline $\mathrm{C}$ & -1.8113900 & 3.1661440 & -0.0558050 \\
\hline $\mathrm{C}$ & -0.0430110 & 5.2189640 & 0.6112940 \\
\hline $\mathrm{H}$ & 0.7381970 & 3.8531990 & 2.0826680 \\
\hline $\mathrm{C}$ & -1.8668480 & 4.3860070 & -0.7364650 \\
\hline $\mathrm{H}$ & -2.5061740 & 2.3735610 & -0.3190860 \\
\hline $\mathrm{C}$ & -0.9817190 & 5.4175230 & -0.4056710 \\
\hline $\mathrm{H}$ & 0.6460360 & 6.0145210 & 0.8806630 \\
\hline $\mathrm{H}$ & -2.6045660 & 4.5318410 & -1.5205800 \\
\hline $\mathrm{H}$ & -1.0265490 & 6.3667510 & -0.9316820 \\
\hline $\mathrm{H}$ & 0.0295870 & -0.4082780 & 1.6179600 \\
\hline $\mathrm{O}$ & -1.9742050 & -0.2999010 & -0.6577600 \\
\hline $\mathrm{C}$ & -2.9233130 & -0.9549510 & -0.0854320 \\
\hline $\mathrm{O}$ & -3.0418730 & -1.2569540 & 1.0977330 \\
\hline $\mathrm{C}$ & -4.0249200 & -1.3754920 & -1.1045700 \\
\hline $\mathrm{F}$ & -5.0171130 & -2.0648450 & -0.5129250 \\
\hline $\mathrm{F}$ & -4.5754650 & -0.2896970 & -1.6963250 \\
\hline $\mathrm{F}$ & -3.5085960 & -2.1557010 & -2.0823600 \\
\hline $\mathrm{H}$ & -4.3186440 & -2.1996040 & 2.1345320 \\
\hline $\mathrm{O}$ & -4.9114780 & -2.6681500 & 2.7522020 \\
\hline $\mathrm{H}$ & -4.4944810 & -2.5815630 & 3.6191120 \\
\hline
\end{tabular}

$10 . \log$

Potential Energy $=-1489.65071$

Zero-point Energy = -1489.18202

Free Energy = -1489.22688

Single-Point Energy B3LYP-D3(BJ)/6-311+G** PCM = 1490.10994

Free Energy B3LYP-D3(BJ)/6-311+G** PCM (extrapolated free energy from qRRHO) $=-1489.68610$

Nimag $=1\left(-1179.5848 \mathrm{~cm}^{-1}\right)$

Charge $=0$ Multiplicity $=1$

$\begin{array}{lrrr}\mathrm{C} & -2.7589840 & -0.3587960 & -0.6907580 \\ \mathrm{C} & -3.8148220 & 0.7923480 & 1.0785090 \\ \mathrm{C} & -2.3273100 & 0.7110010 & 1.4399430 \\ \mathrm{~N} & -1.7320350 & 0.3222720 & 0.1355180 \\ \mathrm{~N} & -3.9887320 & 0.2309490 & -0.1399210 \\ \mathrm{O} & -4.6921740 & 1.3035980 & 1.7761100 \\ \mathrm{C} & -5.2662060 & 0.2923320 & -0.8420980 \\ \mathrm{H} & -5.7482810 & 1.2407210 & -0.5964490 \\ \mathrm{H} & -5.0916720 & 0.2447170 & -1.9178960 \\ \mathrm{H} & -5.9347380 & -0.5227240 & -0.5496270 \\ \mathrm{C} & -0.6312020 & 0.9005670 & -0.3575370 \\ \mathrm{C} & -0.0070480 & 0.7113550 & -1.5894580 \\ \mathrm{C} & 0.8679780 & 1.8313230 & -2.1939570 \\ \mathrm{H} & 0.2273250 & 2.4699930 & -2.8150730 \\ \mathrm{C} & -1.8127000 & 2.0021810 & 2.0638380 \\ \mathrm{H} & -2.4382510 & 2.2197170 & 2.9327800 \\ \mathrm{H} & -0.7816990 & 1.9011880 & 2.4110460 \\ \mathrm{H} & -1.8821280 & 2.8460500 & 1.3713080 \\ \mathrm{H} & -2.1931240 & -0.1073940 & 2.1569460 \\ \mathrm{C} & -2.7184160 & -1.9275920 & -0.6502060 \\ \mathrm{H} & -2.6366330 & -0.0334930 & -1.7292190 \\ \mathrm{C} & -3.7805410 & -2.4804460 & -1.6265860 \\ \mathrm{H} & -3.6527180 & -3.5635190 & -1.7206380 \\ \mathrm{H} & -4.8009250 & -2.3038980 & -1.2793800\end{array}$

$\begin{array}{lrrr}\mathrm{H} & -3.6751440 & -2.0474620 & -2.6278150 \\ \mathrm{C} & -1.3350210 & -2.4138930 & -1.1209570 \\ \mathrm{H} & -1.1144950 & -2.0921290 & -2.1438900 \\ \mathrm{H} & -0.5397770 & -2.0609760 & -0.4608550 \\ \mathrm{H} & -1.3137030 & -3.5083200 & -1.1100990 \\ \mathrm{C} & -2.9893270 & -2.4790760 & 0.7626590 \\ \mathrm{H} & -3.9409500 & -2.1223530 & 1.1692460 \\ \mathrm{H} & -3.0400170 & -3.5718320 & 0.7201580 \\ \mathrm{H} & -2.1881950 & -2.2202670 & 1.4616340 \\ \mathrm{H} & -0.1351210 & 1.5590380 & 0.3485940 \\ \mathrm{H} & 1.0108490 & -0.2724590 & -1.2412030 \\ \mathrm{H} & 1.5874750 & 1.3654770 & -2.8767210 \\ \mathrm{C} & 1.6142800 & 2.7053130 & -1.2041690 \\ \mathrm{C} & 1.0547400 & 3.9099280 & -0.7483710 \\ \mathrm{C} & 2.8833730 & 2.3396570 & -0.7275730 \\ \mathrm{C} & 1.7371930 & 4.7224720 & 0.1625190 \\ \mathrm{H} & 0.0782000 & 4.2175630 & -1.1153160 \\ \mathrm{C} & 3.5700830 & 3.1483780 & 0.1831250 \\ \mathrm{H} & 3.3407130 & 1.4190240 & -1.0795430 \\ \mathrm{C} & 2.9980760 & 4.3426690 & 0.6332800 \\ \mathrm{H} & 1.2866120 & 5.6519350 & 0.4991610 \\ \mathrm{H} & 4.5530950 & 2.8484220 & 0.5353010 \\ \mathrm{H} & 3.5317340 & 4.9734400 & 1.3382860 \\ \mathrm{H} & -0.5530880 & 0.1499450 & -2.3480810 \\ \mathrm{O} & 1.9359010 & -1.0332650 & -1.2343550 \\ \mathrm{C} & 2.2550580 & -1.4810050 & -0.0708910 \\ \mathrm{O} & 1.7196790 & -1.2568950 & 1.0103110 \\ \mathrm{C} & 3.4910510 & -2.4285550 & -0.1400090 \\ \mathrm{~F} & 3.8324900 & -2.8941330 & 1.0765410 \\ \mathrm{~F} & 3.2378720 & -3.4967520 & -0.9310370 \\ \mathrm{~F} & 4.5671260 & -1.7870760 & -0.6503830 \\ \mathrm{H} & 2.0375450 & -1.7874030 & 2.7911810 \\ \mathrm{O} & 2.0736810 & -1.9703710 & 3.7493960 \\ \mathrm{H} & 2.8368770 & -2.5493950 & 3.8713870\end{array}$

11.log

Potential Energy $=-1489.65354$

Zero-point Energy = -1489.18515

Free Energy $=-1489.23091$

Single-Point Energy B3LYP-D3(BJ)/6-311+G** PCM = 1490.10954

Free Energy B3LYP-D3(BJ)/6-311+G** PCM (extrapolated free energy from $\mathrm{qRRHO})=-1489.68691$

Nimag $=1\left(-1180.7665 \mathrm{~cm}^{-1}\right)$

Charge $=0$ Multiplicity $=1$

$\begin{array}{lrrr}\mathrm{C} & -1.9986950 & -1.4229600 & -0.5600410 \\ \mathrm{C} & -2.9907190 & -1.9988850 & 1.5034270 \\ \mathrm{C} & -1.5235830 & -1.6723660 & 1.8053490 \\ \mathrm{~N} & -1.1155190 & -0.9892290 & 0.5506270 \\ \mathrm{~N} & -3.2109190 & -1.7951420 & 0.1843860 \\ \mathrm{O} & -3.8255450 & -2.3630350 & 2.3326280 \\ \mathrm{C} & -4.5515340 & -1.8510430 & -0.3898370 \\ \mathrm{H} & -5.2587920 & -1.4629030 & 0.3460060 \\ \mathrm{H} & -4.5873120 & -1.2309390 & -1.2861550 \\ \mathrm{H} & -4.8439630 & -2.8729440 & -0.6479760 \\ \mathrm{C} & -0.3808400 & 0.1262000 & 0.5392600 \\ \mathrm{C} & 0.0110900 & 0.9264010 & -0.5355630 \\ \mathrm{C} & 0.3609330 & 2.3966680 & -0.2576890 \\ \mathrm{H} & 1.0847410 & 2.7338360 & -1.0079470 \\ \mathrm{C} & -1.3618610 & -0.8754080 & 3.0939770 \\ \mathrm{H} & -1.8366910 & -1.4460860 & 3.8955930 \\ \mathrm{H} & -0.3104390 & -0.7404870 & 3.3587130 \\ \mathrm{H} & -1.8497430 & 0.1017300 & 3.0326730 \\ \mathrm{H} & -0.9743410 & -2.6164650 & 1.8991720 \\ \mathrm{C} & -1.4161310 & -2.5653590 & -1.4652340 \\ \mathrm{H} & -2.2142410 & -0.5532980 & -1.1897520 \\ \mathrm{C} & -2.4284820 & -2.8758640 & -2.5903300 \\ \mathrm{H} & -1.9633190 & -3.5517900 & -3.3148350 \\ \mathrm{H} & -3.3283200 & -3.3701570 & -2.2174650 \\ \mathrm{H} & -2.7277240 & -1.9696360 & -3.1292480 \\ \mathrm{C} & -0.1071530 & -2.0833310 & -2.1190510 \\ \mathrm{H} & -0.2650940 & -1.1972040 & -2.7425660 \\ \mathrm{H} & 0.6617720 & -1.8548820 & -1.3774810 \\ \mathrm{H} & 0.2841040 & -2.8740270 & -2.7671430 \\ \mathrm{H} & -1.1345830 & -3.8516980 & -0.6658170 \\ & -2.0241580 & -4.2132160 & -0.1403020 \\ & & & \\ & & \end{array}$




$\begin{array}{crrr}\mathrm{H} & -0.8169860 & -4.6419980 & -1.3535430 \\ \mathrm{H} & -0.3276490 & -3.7115790 & 0.0600480 \\ \mathrm{H} & 0.0193970 & 0.3909400 & 1.5139290 \\ \mathrm{H} & 1.3427960 & 0.4085420 & -0.7573400 \\ \mathrm{H} & 0.8593640 & 2.4699380 & 0.7156010 \\ \mathrm{C} & -0.8442260 & 3.3220970 & -0.2869700 \\ \mathrm{C} & -1.2564990 & 3.9198040 & -1.4879320 \\ \mathrm{C} & -1.5834430 & 3.5856930 & 0.8768730 \\ \mathrm{C} & -2.3805130 & 4.7511550 & -1.5291190 \\ \mathrm{H} & -0.6905990 & 3.7358270 & -2.3983020 \\ \mathrm{C} & -2.7063880 & 4.4179660 & 0.8418210 \\ \mathrm{H} & -1.2760680 & 3.1412540 & 1.8206310 \\ \mathrm{C} & -3.1108820 & 5.0024570 & -0.3632280 \\ \mathrm{H} & -2.6812470 & 5.2054550 & -2.4691580 \\ \mathrm{H} & -3.2613830 & 4.6132620 & 1.7552400 \\ \mathrm{H} & -3.9818360 & 5.6508160 & -0.3917710 \\ \mathrm{H} & -0.4611820 & 0.7500280 & -1.5018810 \\ \mathrm{O} & 2.4597270 & 0.1193330 & -1.1005300 \\ \mathrm{C} & 3.3036730 & 0.0773210 & -0.1305810 \\ \mathrm{O} & 3.1156770 & 0.3106820 & 1.0600200 \\ \mathrm{C} & 4.7245460 & -0.3363490 & -0.6210950 \\ \mathrm{~F} & 5.6135470 & -0.3677410 & 0.3899550 \\ \mathrm{~F} & 4.7029600 & -1.5658520 & -1.1859170 \\ \mathrm{~F} & 5.1899690 & 0.5315540 & -1.5487610 \\ \mathrm{H} & 4.1783810 & 0.2740210 & 2.6138850 \\ \mathrm{O} & 4.5877200 & 0.2900150 & 3.5001150 \\ \mathrm{H} & 5.5284430 & 0.1299500 & 3.3516050\end{array}$

12.log

Potential Energy = -1489.65137

Zero-point Energy = -1489.18299

Free Energy = -1489.22808

Single-Point Energy B3LYP-D3(BJ)/6-311+G** PCM = 1490.10840

Free Energy B3LYP-D3(BJ)/6-311+G** PCM (extrapolated free energy from $\mathrm{qRRHO})=-1489.68511$

Nimag $=1\left(-1166.0594 \mathrm{~cm}^{-1}\right)$

Charge $=0$ Multiplicity $=1$

$\begin{array}{lrrr}\mathrm{C} & -2.9115690 & -0.6317250 & 0.3494180 \\ \mathrm{C} & -4.3509180 & 0.5274750 & -1.1199090 \\ \mathrm{C} & -2.9918330 & 1.2327820 & -1.1976470 \\ \mathrm{~N} & -2.1067990 & 0.2275280 & -0.5543430 \\ \mathrm{~N} & -4.2352880 & -0.5303100 & -0.2843120 \\ \mathrm{O} & -5.3639510 & 0.8609110 & -1.7361660 \\ \mathrm{C} & -5.3015500 & -1.5164980 & -0.1384630 \\ \mathrm{H} & -5.8053890 & -1.6238900 & -1.1012210 \\ \mathrm{H} & -4.8708180 & -2.4757370 & 0.1517000 \\ \mathrm{H} & -6.0407480 & -1.2139810 & 0.6086870 \\ \mathrm{C} & -0.9156210 & -0.1136550 & -1.0555320 \\ \mathrm{C} & -0.0069710 & -1.0788470 & -0.6198500 \\ \mathrm{C} & 0.9657450 & -1.6566590 & -1.6635130 \\ \mathrm{H} & 1.3420290 & -0.8448860 & -2.2958210 \\ \mathrm{C} & -2.6329830 & 1.6416840 & -2.6207510 \\ \mathrm{H} & -3.4462910 & 2.2661130 & -2.9978850 \\ \mathrm{H} & -1.7149760 & 2.2334420 & -2.6532510 \\ \mathrm{H} & -2.5311830 & 0.7755820 & -3.2812790 \\ \mathrm{H} & -3.0342260 & 2.1304740 & -0.5700120 \\ \mathrm{C} & -2.8828860 & -0.2179230 & 1.8628610 \\ \mathrm{H} & -2.5470610 & -1.6606430 & 0.2615100 \\ \mathrm{C} & -3.7530750 & -1.2077460 & 2.6689900 \\ \mathrm{H} & -3.6080600 & -1.0236710 & 3.7382090 \\ \mathrm{H} & -4.8190950 & -1.0907110 & 2.4618330 \\ \mathrm{H} & -3.4725890 & -2.2488400 & 2.4728700 \\ \mathrm{C} & -1.4387390 & -0.3115960 & 2.3905150 \\ \mathrm{H} & -1.0379240 & -1.3269490 & 2.3020420 \\ \mathrm{H} & -0.7659730 & 0.3746430 & 1.8716430 \\ \mathrm{H} & -1.4252990 & -0.0464620 & 3.4525950 \\ \mathrm{C} & -3.4073250 & 1.2137750 & 2.0822960 \\ \mathrm{H} & -4.4162130 & 1.3486690 & 1.6794230 \\ \mathrm{H} & -3.4533350 & 1.4211310 & 3.1562150 \\ \mathrm{H} & -2.7452560 & 1.9626110 & 1.6366580 \\ & -0.5968480 & 0.5151310 & -1.8824950 \\ & 0.8530900 & -0.1932880 & 0.1615880 \\ \mathrm{C} & 2.1356530 & -2.4125160 & -1.0610830 \\ & & -1.9057940 & -1.1302570\end{array}$

$\begin{array}{lccc}\mathrm{C} & 1.9348210 & -3.6509960 & -0.4292470 \\ \mathrm{C} & 4.5175750 & -2.6097510 & -0.5784630 \\ \mathrm{H} & 3.6190050 & -0.9527230 & -1.6220200 \\ \mathrm{C} & 3.0052690 & -4.3567300 & 0.1266590 \\ \mathrm{H} & 0.9329860 & -4.0710440 & -0.3773250 \\ \mathrm{C} & 4.3027370 & -3.8371720 & 0.0547560 \\ \mathrm{H} & 5.5212090 & -2.1990080 & -0.6453050 \\ \mathrm{H} & 2.8278300 & -5.3135720 & 0.6098170 \\ \mathrm{H} & 5.1363860 & -4.3859070 & 0.4833670 \\ \mathrm{H} & -0.3441190 & -1.7908280 & 0.1331640 \\ \mathrm{O} & 1.6059750 & 0.4143070 & 0.8691060 \\ \mathrm{C} & 2.1943510 & 1.3948080 & 0.2802730 \\ \mathrm{O} & 2.1139580 & 1.7435190 & -0.8943030 \\ \mathrm{C} & 3.1006330 & 2.1963690 & 1.2630770 \\ \mathrm{~F} & 3.7558630 & 3.1939200 & 0.6389200 \\ \mathrm{~F} & 2.3632140 & 2.7488510 & 2.2548090 \\ \mathrm{~F} & 4.0259960 & 1.3959550 & 1.8389250 \\ \mathrm{H} & 2.8809260 & 3.1120330 & -1.9350250 \\ \mathrm{O} & 3.1853600 & 3.7640610 & -2.5948840 \\ \mathrm{H} & 3.8755110 & 4.2742460 & -2.1521010\end{array}$

13.log

Potential Energy $=-1489.65086$

Zero-point Energy = -1489.18293

Free Energy $=-1489.22874$

Single-Point Energy B3LYP-D3(BJ)/6-311+G** PCM = 1490.10774

Free Energy B3LYP-D3(BJ)/6-311+G** PCM (extrapolated free energy from qRRHO) $=-1489.68561$

Nimag $=1\left(-1175.9065 \mathrm{~cm}^{-1}\right)$

Charge $=0$ Multiplicity $=1$

$\begin{array}{lccc}\mathrm{C} & 3.1581390 & 0.1724770 & 0.5004580 \\ \mathrm{C} & 3.5154660 & 0.2860960 & -1.8270180 \\ \mathrm{C} & 2.2784190 & -0.6004070 & -1.6528610 \\ \mathrm{~N} & 2.0049990 & -0.4450310 & -0.2086340 \\ \mathrm{~N} & 3.8882160 & 0.7665370 & -0.6187210 \\ \mathrm{O} & 4.0362650 & 0.5664360 & -2.9068380 \\ \mathrm{C} & 4.8688790 & 1.8417140 & -0.4988340 \\ \mathrm{H} & 4.7315320 & 2.5272330 & -1.3375850 \\ \mathrm{H} & 4.7037810 & 2.3783530 & 0.4357680 \\ \mathrm{H} & 5.8955290 & 1.4658310 & -0.5227510 \\ \mathrm{C} & 0.8881240 & -0.7751150 & 0.4431770 \\ \mathrm{C} & -0.2731740 & -1.3896950 & -0.0270410 \\ \mathrm{C} & -1.1851510 & -2.0624460 & 1.0145110 \\ \mathrm{H} & -1.2872280 & -1.4066490 & 1.8860450 \\ \mathrm{C} & 1.1787180 & -0.1133400 & -2.6040410 \\ \mathrm{H} & 1.6179480 & -0.0394500 & -3.6018600 \\ \mathrm{H} & 0.3368370 & -0.8020330 & -2.6560120 \\ \mathrm{H} & 0.8128600 & 0.8738770 & -2.3097760 \\ \mathrm{H} & 2.5342680 & -1.6433430 & -1.8802860 \\ \mathrm{C} & 4.0056060 & -0.8463920 & 1.3508500 \\ \mathrm{H} & 2.7876950 & 0.9630440 & 1.1634930 \\ \mathrm{C} & 5.1660180 & -0.0862630 & 2.0323680 \\ \mathrm{H} & 5.6730430 & -0.7580290 & 2.7319410 \\ \mathrm{H} & 5.9150540 & 0.2608740 & 1.3182650 \\ \mathrm{H} & 4.8054210 & 0.7755950 & 2.6054340 \\ \mathrm{C} & 3.1384750 & -1.4649640 & 2.4679050 \\ \mathrm{H} & -2.5627730 & -2.4132600 & 0.4836910 \\ \mathrm{H} & 2.6686790 & -0.6984620 & 3.0951850 \\ \mathrm{H} & 2.3596010 & -2.1264210 & 2.0782020 \\ \mathrm{H} & 3.7763410 & -2.0722660 & 3.1175780 \\ \mathrm{C} & 4.5732480 & -1.9728130 & 0.4679170 \\ \mathrm{H} & 5.2126350 & -1.5834100 & -0.3305720 \\ \mathrm{H} & 5.1831360 & -2.6452560 & 1.0795920 \\ \mathrm{H} & 3.7773240 & -2.5742570 & 0.0167630 \\ \mathrm{H} & 0.8859250 & -0.4469210 & 1.4800640 \\ \mathrm{H} & -0.2079830 & -1.9186370 & -0.9769720 \\ \mathrm{C} & -1.7001040 & 0.6919240 & -0.8465450 \\ \mathrm{H} & -2.0014860 & 1.5818530 & 0.0315960 \\ \mathrm{H} & -2.8508900 & 2.7346980 & -0.5848550 \\ \mathrm{H} & -3.9948660 & 2.2600870 & -1.1284240 \\ \mathrm{H} & -1653690 & 3.3698150 & -1.5638460 \\ \mathrm{H} & -1.7110910 & 0.8990480\end{array}$




$\begin{array}{llll}\mathrm{C} & -2.7252570 & -3.4635030 & -0.4349340 \\ \mathrm{C} & -4.9712510 & -2.0412720 & 0.4073260 \\ \mathrm{H} & -3.5999560 & -0.8998180 & 1.6152010 \\ \mathrm{C} & -3.9885510 & -3.7955610 & -0.9318570 \\ \mathrm{H} & -1.8563660 & -4.0312910 & -0.7598600 \\ \mathrm{C} & -5.1180130 & -3.0837440 & -0.5122480 \\ \mathrm{H} & -5.8416070 & -1.4851390 & 0.7444670 \\ \mathrm{H} & -4.0919040 & -4.6124420 & -1.6407170 \\ \mathrm{H} & -6.1011390 & -3.3424780 & -0.8947770 \\ \mathrm{H} & -1.0171690 & -0.1940660 & -0.4210170 \\ \mathrm{H} & -2.0238650 & 2.8047190 & 2.6442980 \\ \mathrm{O} & -2.0921160 & 3.3226880 & 3.4691490 \\ \mathrm{H} & -2.6994580 & 4.0461860 & 3.2683520\end{array}$

14.log

Potential Energy = -1489.65001

Zero-point Energy = -1489.18150

Free Energy = -1489.22641

Single-Point Energy B3LYP-D3(BJ)/6-311+G** PCM = 1490.10979

Free Energy B3LYP-D3(BJ)/6-311+G** PCM (extrapolated free energy from $\mathrm{qRRHO})=-1489.68619$

Nimag $=1\left(-1181.5008 \mathrm{~cm}^{-1}\right)$

Charge $=0$ Multiplicity $=1$

\begin{tabular}{|c|c|c|c|}
\hline C & -2.5365300 & -0.2219870 & 0.5204660 \\
\hline $\mathrm{C}$ & -2.7903430 & -2.2793990 & -0.5991670 \\
\hline $\mathrm{C}$ & -1.8958920 & -1.4328110 & -1.5102460 \\
\hline & -1.6189180 & -0.2630750 & -0.6498550 \\
\hline $\mathrm{N}$ & -3.0134180 & -1.6041490 & 0.551091 \\
\hline $\mathrm{O}$ & -3.1779470 & -3.4202880 & -0.853175 \\
\hline & -3.6127270 & -2.2568250 & 1.7114510 \\
\hline $\mathrm{H}$ & -3.2481560 & -3.2852330 & 1.753945 \\
\hline $\mathrm{H}$ & -3.3118830 & -1.7303320 & 2.617447 \\
\hline $\mathrm{H}$ & -4.7045430 & -2.2785830 & 1.652487 \\
\hline C & -0.6701210 & 0.6620720 & -0.817766 \\
\hline $\mathrm{C}$ & 0.2527520 & 0.8052780 & -1.8527310 \\
\hline$C$ & 0.9477330 & 2.1634850 & -2.0881440 \\
\hline $\mathrm{C}$ & -0.6823000 & -2.2739100 & -1.924282 \\
\hline $\mathrm{H}$ & -1.0605860 & -3.2165720 & -2.32699 \\
\hline $\mathrm{H}$ & -0.0833720 & -1.7939930 & \\
\hline $\mathrm{H}$ & -0.0489940 & -2.4947150 & -1.061280 \\
\hline $\mathrm{H}$ & -2.4580340 & -1.1243430 & \\
\hline $\mathrm{C}$ & -3.6864450 & 0.8470010 & 0.399131 \\
\hline $\mathrm{H}$ & -1.9517060 & -0.0101240 & 1.423296 \\
\hline $\mathrm{C}$ & -4.5518410 & 0.8002460 & \\
\hline $\mathrm{H}$ & -5.2600640 & 1.6345140 & 1.6638200 \\
\hline $\mathrm{H}$ & -5.1370340 & -0.1180220 & \\
\hline $\mathrm{H}$ & -3.9429220 & 0.9012130 & 2.5846880 \\
\hline $\mathrm{C}$ & -3.0883670 & 2.2650360 & 0.2954620 \\
\hline $\mathrm{H}$ & -2.4065530 & 2.4852740 & 1.1247910 \\
\hline $\mathrm{H}$ & -2.5578770 & 2.4269500 & -0.646719 \\
\hline $\mathrm{H}$ & -3.9000000 & 2.9982100 & 0.3356200 \\
\hline $\mathrm{C}$ & -4.5705430 & 0.5795320 & -0.832795 \\
\hline $\mathrm{H}$ & -5.0350620 & -0.4108190 & -0.796045 \\
\hline $\mathrm{H}$ & -5.3762200 & 1.3195910 & 72448 \\
\hline $\mathrm{H}$ & -4.0033880 & 0.6651800 & -1.765433 \\
\hline $\mathrm{H}$ & -0.5908840 & 1.3505630 & 0.0198940 \\
\hline $\mathrm{H}$ & 0.0320640 & 0.2837130 & -2.7837320 \\
\hline $\mathrm{O}$ & 2.3857980 & -0.6233270 & -1.253790 \\
\hline $\mathrm{C}$ & 2.5273830 & -1.2151880 & -0.1193870 \\
\hline $\mathrm{O}$ & 1.7372680 & -1.2786640 & 0.8171780 \\
\hline $\mathrm{C}$ & 3.9149150 & -1.9174490 & -0.0096120 \\
\hline $\mathrm{F}$ & 4.0629130 & -2.5501850 & 1.1682760 \\
\hline $\mathrm{F}$ & 4.9211740 & -1.0194210 & -0.1232540 \\
\hline $\mathrm{F}$ & 4.0732890 & -2.8348180 & -0.9917210 \\
\hline $\mathrm{H}$ & 0.3177690 & 2.7681300 & -2.7528230 \\
\hline $\mathrm{H}$ & 1.3580620 & -0.0278140 & -1.3872180 \\
\hline $\mathrm{H}$ & 1.7881650 & -2.0258960 & 2.5606280 \\
\hline $\mathrm{O}$ & 1.7228000 & -2.3694170 & 3.4717770 \\
\hline $\mathrm{H}$ & 0.8725030 & -2.0525560 & 3.8025590 \\
\hline $\mathbf{H}$ & 1.8743710 & 1.9706590 & -2.6410320 \\
\hline $\mathrm{C}$ & 1.2702530 & 2.9761800 & -0.8486670 \\
\hline$C$ & 0.4357430 & 4.0298190 & -0.4441030 \\
\hline $\mathrm{C}$ & 2.4173290 & 2.7065150 & -0.0836510 \\
\hline & 0.7296530 & 4.7868150 & 0.6952190 \\
\hline
\end{tabular}

$\mathrm{H} \quad-0.4485600 \quad 4.2658870 \quad-1.0310320$

$\begin{array}{lccc}\mathrm{C} & 2.7154330 & 3.4590390 & 1.0556660 \\ \mathrm{H} & 3.0868860 & 1.9073110 & -0.3891920 \\ \mathrm{C} & 1.8703550 & 4.5016920 & 1.4511420 \\ \mathrm{H} & 0.0707420 & 5.5997530 & 0.9871190 \\ \mathrm{H} & 3.6103550 & 3.2357670 & 1.6297530 \\ \mathrm{H} & 2.1028990 & 5.0893900 & 2.3344470\end{array}$

15.log

Potential Energy $=-1489.65263$

Zero-point Energy = -1489.18455

Free Energy $=-1489.23051$

Single-Point Energy B3LYP-D3(BJ)/6-311+G** PCM = 1490.10882

Free Energy B3LYP-D3(BJ)/6-311+G** PCM (extrapolated free energy from $\mathrm{qRRHO})=-1489.68670$

Nimag $=1\left(-1202.9716 \mathrm{~cm}^{-1}\right)$

Charge $=0$ Multiplicity $=1$

$\begin{array}{llll}\text { C } & -1.6477470 & -1.9980930 & 0.6194240\end{array}$

C $\quad-1.5440690 \quad-2.9808100 \quad-1.5207660$

$\begin{array}{llll}\text { C } & -1.1989170 & -1.5043720 & -1.7393170\end{array}$

$\mathrm{N} \quad-1.0784370 \quad-1.0195600 \quad-0.3473450$

$\mathrm{N} \quad-1.6758590 \quad-3.2122820 \quad-0.1948260$

$\begin{array}{llll}\text { O } & -1.6167740 & -3.8274110 & -2.4116610\end{array}$

C $\quad-1.7362100 \quad-4.5714110 \quad 0.3360410$

H $\quad-1.0787190 \quad-5.2042590 \quad-0.2636530$

$\mathrm{H} \quad-1.3906640 \quad-4.5707290 \quad 1.3700960$

$\begin{array}{llll}\mathrm{H} & -2.7479080 & -4.9841570 & 0.2940910\end{array}$

$\begin{array}{llll}\text { C } & -0.5088200 & 0.1132940 & 0.0675460\end{array}$

C $\quad 0.0591230 \quad 1.1499880 \quad-0.6763660$

$\begin{array}{llll}\text { C } & 0.2753980 & 2.5085160 & 0.0076170\end{array}$

C $\quad 0.0675770 \quad-1.4136910 \quad-2.5995180$

$\mathrm{H} \quad-0.0864130 \quad-2.0499220 \quad-3.4744350$

$\mathrm{H} \quad 0.2640120 \quad-0.4014730 \quad-2.9494860$

$\mathrm{H} \quad 0.9414500 \quad-1.7787600 \quad-2.0531320$

$\mathrm{H} \quad-2.0321000 \quad-1.0000220 \quad-2.2450000$

C $\quad-3.0497850 \quad-1.5806580 \quad 1.1995300$

$\mathrm{H} \quad-0.9461080 \quad-2.1266870 \quad 1.4519030$

$\begin{array}{llll}\text { C } & -3.5427320 & -2.6834720 & 2.1635280\end{array}$

$\mathrm{H} \quad-4.4432040 \quad-2.3335580 \quad 2.6777880$

$\mathrm{H} \quad-3.8061300 \quad-3.6060790 \quad 1.6428630$

$\mathrm{H} \quad-2.7952810 \quad-2.9170850 \quad 2.9303050$

$\begin{array}{llll}\text { C } & -2.9207840 & -0.2763970 & 2.0134990\end{array}$

$\mathrm{H} \quad-2.1557190 \quad-0.3553940 \quad 2.7948500$

$\begin{array}{llll}\mathrm{H} & -2.6946130 & 0.5898970 & 1.3862130\end{array}$

$\begin{array}{llll}\mathrm{H} & -3.8743550 & -0.0698900 & 2.5093940\end{array}$

C $\quad-4.0819640 \quad-1.3785300 \quad 0.0751310$

$\mathrm{H} \quad-4.2182560 \quad-2.2858750 \quad-0.5217900$

$\mathrm{H} \quad-5.0529010 \quad-1.1229480 \quad 0.5113230$

$\mathrm{H} \quad-3.7986880 \quad-0.5588090 \quad-0.5930630$

$\mathrm{H} \quad-0.4252090 \quad 0.1794490 \quad 1.1498550$

$\mathrm{H} \quad-0.2109090 \quad 1.2156640 \quad-1.7294310$

$\begin{array}{llll}\text { O } & 2.5863500 & 0.4708880 & -1.0042180\end{array}$

C $\quad 3.2817210 \quad 0.2139730 \quad 0.0464920$

$\begin{array}{llll}\mathrm{O} & 2.9166190 & 0.1741690 & 1.2180810\end{array}$

$\begin{array}{llll}\mathrm{C} & 4.7730010 & -0.0736520 & -0.3067530\end{array}$

$\begin{array}{llll}\text { F } & 5.5043800 & -0.3453040 & 0.7910350\end{array}$

F $\quad 5.3390590 \quad 0.9899850 \quad-0.9226320$

F $\quad 4.8785840 \quad-1.1333300 \quad-1.1415330$

$\begin{array}{llll}\mathrm{H} & 1.4213470 & 0.6886840 & -0.7775840\end{array}$

$\begin{array}{llll}\mathrm{H} & 3.7448250 & -0.2009390 & 2.8652140\end{array}$

$\begin{array}{llll}\mathrm{O} & 4.0202790 & -0.3792480 & 3.7848260\end{array}$

$\begin{array}{llll}\mathrm{H} & 4.9764060 & -0.5094480 & 3.7460940\end{array}$

$\begin{array}{llll}\mathrm{H} & 1.1353630 & 3.0013500 & -0.4610710\end{array}$

$\begin{array}{llll}\mathrm{H} & 0.5439410 & 2.3465540 & 1.0573820\end{array}$

$\begin{array}{llll}\text { C } & -0.9251220 & 3.4363380 & -0.0748040\end{array}$

$\begin{array}{llll}\text { C } & -1.7871760 & 3.6057020 & 1.0190760\end{array}$

C $\quad-1.2042340 \quad 4.1392230 \quad-1.2582050$

C $\quad-2.9024720 \quad 4.4461650 \quad 0.9329610$

$\mathrm{H} \quad-1.5810690 \quad 3.0816780 \quad 1.9492740$

C $\quad-2.3181840 \quad 4.9783410 \quad-1.3502850$

$\begin{array}{llll}\mathrm{H} & -0.5414010 & 4.0319190 & -2.1138170\end{array}$

C $\quad-3.1736740 \quad 5.1337950 \quad-0.2537940$

$\mathrm{H} \quad-3.5546970 \quad 4.5659850 \quad 1.7936350$

$\begin{array}{llll}\mathrm{H} & -2.5147010 & 5.5146270 & -2.2745610\end{array}$

$\mathrm{H} \quad-4.0377890 \quad 5.7882730 \quad-0.3223260$ 
$16 . \log$

Potential Energy $=-1489.65301$

Zero-point Energy = -1489.18503

Free Energy = -1489.23095

Single-Point Energy B3LYP-D3(BJ)/6-311+G** PCM = 1490.10925

Free Energy B3LYP-D3(BJ)/6-311+G** PCM (extrapolated free energy from $\mathrm{qRRHO})=-1489.68719$

Nimag $=1\left(-1172.7140 \mathrm{~cm}^{-1}\right)$ Charge $=0$ Multiplicity $=1$

\begin{tabular}{|c|c|c|c|}
\hline $\mathrm{C}$ & 2.7467140 & -1.4089580 & -0.5812360 \\
\hline $\mathrm{C}$ & 2.2282760 & -2.6018590 & 1.3847600 \\
\hline $\mathrm{C}$ & 1.3111580 & -1.3749960 & 1.4057380 \\
\hline $\mathrm{N}$ & 1.5485490 & -0.8050750 & 0.0623380 \\
\hline $\mathrm{N}$ & 2.9130310 & -2.6217610 & 0.2184620 \\
\hline $\mathrm{O}$ & 2.2728250 & -3.4640330 & 2.2624080 \\
\hline $\mathrm{C}$ & 3.6603010 & -3.8033780 & -0.2030700 \\
\hline $\mathrm{H}$ & 3.1141790 & -4.6900790 & 0.1253740 \\
\hline $\mathrm{H}$ & 3.7399670 & -3.8113470 & -1.2904720 \\
\hline $\mathrm{H}$ & 4.6628910 & -3.8327530 & 0.2325360 \\
\hline $\mathrm{C}$ & 0.8014310 & 0.0994820 & -0.5742460 \\
\hline $\mathrm{C}$ & -0.3325000 & 0.7844350 & -0.1323790 \\
\hline $\mathrm{C}$ & -0.7647770 & 2.0513040 & -0.8857710 \\
\hline $\mathrm{C}$ & -0.1221780 & -1.8347780 & 1.7008640 \\
\hline $\mathrm{H}$ & -0.0848900 & -2.4682010 & 2.5903910 \\
\hline $\mathrm{H}$ & -0.7938440 & -1.0032030 & 1.9081480 \\
\hline $\mathrm{H}$ & -0.5245570 & -2.4230830 & 0.8716640 \\
\hline $\mathrm{H}$ & 1.6462040 & -0.6739300 & 2.1806860 \\
\hline $\mathrm{C}$ & 4.0088790 & -0.4688450 & -0.5926550 \\
\hline $\mathrm{H}$ & 2.4983420 & -1.6776940 & -1.6147750 \\
\hline $\mathrm{C}$ & 5.1774240 & -1.2060590 & -1.2852130 \\
\hline $\mathrm{H}$ & 6.0106220 & -0.5095100 & -1.4206910 \\
\hline $\mathrm{H}$ & 5.5513650 & -2.0450740 & -0.6955870 \\
\hline $\mathrm{H}$ & 4.8929620 & -1.5762240 & -2.2768980 \\
\hline $\mathrm{C}$ & 3.7156450 & 0.8058630 & -1.4108110 \\
\hline $\mathrm{H}$ & 3.3641380 & 0.5712940 & -2.4224200 \\
\hline $\mathrm{H}$ & 2.9835220 & 1.4572400 & -0.9261970 \\
\hline $\mathrm{H}$ & 4.6390560 & 1.3846600 & -1.5120210 \\
\hline $\mathrm{C}$ & 4.4217750 & -0.0712890 & 0.8362470 \\
\hline $\mathrm{H}$ & 4.6590340 & -0.9451870 & 1.4511770 \\
\hline $\mathrm{H}$ & 5.3176530 & 0.5565050 & 0.7969020 \\
\hline $\mathrm{H}$ & 3.6396790 & 0.5078590 & 1.3378110 \\
\hline $\mathrm{H}$ & 1.1041490 & 0.2539710 & -1.6078820 \\
\hline $\mathrm{H}$ & -0.5065050 & 0.8205400 & 0.9418460 \\
\hline $\mathrm{O}$ & -2.2573070 & -0.8261510 & -0.9604750 \\
\hline $\mathrm{C}$ & -3.3787680 & -0.6749780 & -0.3487510 \\
\hline $\mathrm{O}$ & -3.6511320 & 0.0798590 & 0.5797690 \\
\hline $\mathrm{C}$ & -4.4804890 & -1.6124700 & -0.9299230 \\
\hline $\mathrm{F}$ & -5.6542160 & -1.4649790 & -0.2864090 \\
\hline $\mathrm{F}$ & -4.1172150 & -2.9116420 & -0.8265820 \\
\hline $\mathrm{F}$ & -4.6965110 & -1.3512420 & -2.2400250 \\
\hline $\mathrm{H}$ & -1.3590980 & -0.1347660 & -0.5563110 \\
\hline $\mathrm{H}$ & -5.1986510 & 0.4475830 & 1.5903740 \\
\hline $\mathrm{O}$ & -5.9097640 & 0.7447770 & 2.1896000 \\
\hline $\mathrm{H}$ & -6.6845520 & 0.2217600 & 1.9470980 \\
\hline $\mathrm{H}$ & -1.8535210 & 2.1503630 & -0.8049120 \\
\hline $\mathrm{H}$ & -0.5397480 & 1.9350180 & -1.9520050 \\
\hline $\mathrm{C}$ & -0.1185730 & 3.3242110 & -0.3649290 \\
\hline $\mathrm{C}$ & 0.9631540 & 3.9155410 & -1.0346590 \\
\hline $\mathrm{C}$ & -0.5872910 & 3.9321410 & 0.8110600 \\
\hline $\mathrm{C}$ & 1.5673730 & 5.0767970 & -0.5406400 \\
\hline $\mathrm{H}$ & 1.3327340 & 3.4684010 & -1.9543300 \\
\hline $\mathrm{C}$ & 0.0140080 & 5.0910450 & 1.3099190 \\
\hline $\mathrm{H}$ & -1.4328640 & 3.4967700 & 1.3385220 \\
\hline $\mathrm{C}$ & 1.0962700 & 5.6675950 & 0.6357120 \\
\hline $\mathrm{H}$ & 2.4014730 & 5.5204150 & -1.0773130 \\
\hline $\mathrm{H}$ & -0.3656890 & 5.5466030 & 2.2202990 \\
\hline $\mathrm{H}$ & 1.5624070 & 6.5702450 & 1.0200060 \\
\hline
\end{tabular}

17.log

Potential Energy = -1489.65085

Zero-point Energy = -1489.18269

Free Energy = -1489.22774

Single-Point Energy B3LYP-D3(BJ)/6-311+G** PCM = 1490.10845
Free Energy B3LYP-D3(BJ)/6-311+G** PCM (extrapolated free energy from $\mathrm{qRRHO})=-1489.68534$

Nimag $=1\left(-1147.5580 \mathrm{~cm}^{-1}\right)$

Charge $=0$ Multiplicity $=1$

\begin{tabular}{|c|c|c|c|}
\hline $\mathrm{C}$ & 3.5330880 & -0.0058360 & -0.6121920 \\
\hline $\mathrm{C}$ & 3.4042920 & -1.5510720 & 1.1651460 \\
\hline $\mathrm{C}$ & 2.1670000 & -0.6633110 & 1.3167540 \\
\hline $\mathrm{N}$ & 2.2241460 & 0.1414470 & 0.0799990 \\
\hline $\mathrm{N}$ & 4.0498390 & -1.2170440 & 0.0238690 \\
\hline $\mathrm{O}$ & 3.7104130 & -2.4712070 & 1.9238290 \\
\hline $\mathrm{C}$ & 5.1040350 & -2.0645950 & -0.5259480 \\
\hline $\mathrm{H}$ & 4.8407930 & -3.1070850 & -0.3355250 \\
\hline $\mathrm{H}$ & 5.1746210 & -1.9016020 & -1.6015570 \\
\hline $\mathrm{H}$ & 6.0753850 & -1.8629230 & -0.0657820 \\
\hline $\mathrm{C}$ & 1.2585060 & 0.9189690 & -0.4153500 \\
\hline $\mathrm{C}$ & 0.0040750 & 1.2295390 & 0.1121410 \\
\hline $\mathrm{C}$ & -0.6801900 & 2.5016600 & -0.4236840 \\
\hline $\mathrm{C}$ & 0.9332060 & -1.5621560 & 1.4679520 \\
\hline $\mathrm{H}$ & 1.1250150 & -2.2492410 & 2.2956640 \\
\hline $\mathrm{H}$ & 0.0286770 & -1.0010850 & 1.6963530 \\
\hline $\mathrm{H}$ & 0.7676990 & -2.1500800 & 0.5604580 \\
\hline $\mathrm{H}$ & 2.2724200 & -0.0173880 & 2.1977250 \\
\hline $\mathrm{C}$ & 4.4785010 & 1.2459160 & -0.4658250 \\
\hline $\mathrm{H}$ & 3.3556690 & -0.1930250 & -1.6780840 \\
\hline $\mathrm{C}$ & 5.7923350 & 0.9737280 & -1.2332010 \\
\hline $\mathrm{H}$ & 6.3946020 & 1.8873520 & -1.2489600 \\
\hline $\mathrm{H}$ & 6.3982160 & 0.1964190 & -0.7642530 \\
\hline $\mathrm{H}$ & 5.6017480 & 0.6868780 & -2.2737480 \\
\hline $\mathrm{C}$ & 3.8264460 & 2.4937150 & -1.0988310 \\
\hline $\mathrm{H}$ & 3.5283040 & 2.3192670 & -2.1392990 \\
\hline $\mathrm{H}$ & 2.9560810 & 2.8446560 & -0.5375970 \\
\hline $\mathrm{H}$ & 4.5539830 & 3.3113290 & -1.1007910 \\
\hline $\mathrm{C}$ & 4.7922550 & 1.5349320 & 1.0136060 \\
\hline $\mathrm{H}$ & 5.2878460 & 0.6886500 & 1.4994620 \\
\hline $\mathrm{H}$ & 5.4654890 & 2.3952220 & 1.0838360 \\
\hline $\mathrm{H}$ & 3.8877510 & 1.7816020 & 1.5793250 \\
\hline $\mathrm{H}$ & 1.4895410 & 1.3101230 & -1.4036240 \\
\hline $\mathrm{H}$ & -0.1485480 & 1.0481110 & 1.1755110 \\
\hline $\mathrm{O}$ & -1.4300380 & -0.6631700 & -1.0719940 \\
\hline $\mathrm{C}$ & -2.3365110 & -1.2559980 & -0.3768570 \\
\hline $\mathrm{O}$ & -2.5887900 & -1.1418700 & 0.8178750 \\
\hline $\mathrm{C}$ & -3.1760540 & -2.2354290 & -1.2525860 \\
\hline $\mathrm{F}$ & -4.1224150 & -2.8660820 & -0.5312930 \\
\hline $\mathrm{F}$ & -2.3887130 & -3.1834410 & -1.8120140 \\
\hline $\mathrm{F}$ & -3.7984220 & -1.5765010 & -2.2571310 \\
\hline $\mathrm{H}$ & -0.7708910 & 0.1444240 & -0.4946570 \\
\hline $\mathrm{H}$ & -3.8375050 & -1.9224240 & 1.9959970 \\
\hline $\mathrm{O}$ & -4.4098770 & -2.2485250 & 2.7164970 \\
\hline $\mathrm{H}$ & -5.0593930 & -2.8207520 & 2.2883490 \\
\hline $\mathrm{H}$ & -0.5701560 & 2.5419800 & -1.5134300 \\
\hline $\mathrm{H}$ & -0.1608390 & 3.3833920 & -0.0241830 \\
\hline $\mathrm{C}$ & -2.1495040 & 2.6107280 & -0.0619800 \\
\hline $\mathrm{C}$ & -2.5352880 & 2.9603150 & 1.2423960 \\
\hline $\mathrm{C}$ & -3.1538180 & 2.3761690 & -1.0123710 \\
\hline $\mathrm{C}$ & -3.8849800 & 3.0631050 & 1.5902130 \\
\hline $\mathrm{H}$ & -1.7723600 & 3.1599900 & 1.9914110 \\
\hline $\mathrm{C}$ & -4.5065700 & 2.4805500 & -0.6706840 \\
\hline $\mathrm{H}$ & -2.8759570 & 2.1113390 & -2.0294260 \\
\hline $\mathrm{C}$ & -4.8770540 & 2.8222580 & 0.6332900 \\
\hline $\mathrm{H}$ & -4.1615510 & 3.3356670 & 2.6049140 \\
\hline $\mathrm{H}$ & -5.2682750 & 2.2969790 & -1.4234110 \\
\hline $\mathrm{H}$ & -5.9266050 & 2.9046680 & 0.9005920 \\
\hline
\end{tabular}

18.log

Potential Energy $=-1489.65101$

Zero-point Energy $=-1489.18265$

Free Energy = -1489.22799

Single-Point Energy B3LYP-D3(BJ)/6-311+G** PCM = -

1490.10962

Free Energy B3LYP-D3(BJ)/6-311+G** PCM (extrapolated

free energy from qRRHO) $=-1489.68661$

Nimag $=1\left(-1145.3557 \mathrm{~cm}^{-1}\right)$

Charge $=0$ Multiplicity $=1$

$\begin{array}{llll}\mathrm{C} & 2.9284020 & -0.6227170 & -0.5075450 \\ \mathrm{C} & 2.4269210 & -2.8838830 & -0.0762700 \\ \mathrm{C} & 1.4389360 & -2.0810820 & 0.7765820\end{array}$




\begin{tabular}{|c|c|c|c|}
\hline $\mathrm{N}$ & 1.6736970 & -0.7070710 & 0.2856970 \\
\hline $\mathrm{N}$ & 3.1434740 & -2.0300050 & -0.8420370 \\
\hline $\mathrm{O}$ & 2.4995460 & -4.1128390 & -0.1041030 \\
\hline $\mathrm{C}$ & 3.9804540 & -2.5097040 & -1.9377680 \\
\hline $\mathrm{H}$ & 3.4810840 & -3.3610450 & -2.4049940 \\
\hline $\mathrm{H}$ & 4.1019960 & -1.7147530 & -2.6740070 \\
\hline $\mathrm{H}$ & 4.9657030 & -2.8335980 & -1.5906550 \\
\hline $\mathrm{C}$ & 0.8937840 & 0.3642590 & 0.4615460 \\
\hline $\mathrm{C}$ & -0.2865120 & 0.4885830 & 1.1923340 \\
\hline $\mathrm{C}$ & -0.7905420 & 1.8840990 & 1.6132300 \\
\hline $\mathrm{C}$ & 0.0290370 & -2.6454760 & 0.5645950 \\
\hline $\mathrm{H}$ & 0.0834520 & -3.7260330 & 0.7174250 \\
\hline $\mathrm{H}$ & -0.6933700 & -2.2420490 & 1.2725750 \\
\hline $\mathrm{H}$ & -0.3207610 & -2.4557640 & -0.4537480 \\
\hline $\mathrm{H}$ & 1.7156860 & -2.1589890 & 1.8359690 \\
\hline $\mathrm{C}$ & 4.1301280 & 0.0426600 & 0.2618280 \\
\hline $\mathrm{H}$ & 2.7338670 & -0.0492330 & -1.4216430 \\
\hline $\mathrm{C}$ & 5.3622200 & 0.0828790 & -0.6703710 \\
\hline $\mathrm{H}$ & 6.1533210 & 0.6711770 & -0.1951020 \\
\hline $\mathrm{H}$ & 5.7717050 & -0.9100460 & -0.8654490 \\
\hline $\mathrm{H}$ & 5.1292920 & 0.5580950 & -1.6301820 \\
\hline $\mathrm{C}$ & 3.7827100 & 1.4984200 & 0.6356070 \\
\hline $\mathrm{H}$ & 3.4802720 & 2.0858580 & -0.2387410 \\
\hline $\mathrm{H}$ & 2.9930110 & 1.5599790 & 1.3893220 \\
\hline $\mathrm{H}$ & 4.6694120 & 1.9785320 & 1.0613000 \\
\hline $\mathrm{C}$ & 4.4757440 & -0.7393270 & 1.5424020 \\
\hline $\mathrm{H}$ & 4.7486190 & -1.7772760 & 1.3274420 \\
\hline $\mathrm{H}$ & 5.3317870 & -0.2698510 & 2.0375410 \\
\hline $\mathrm{H}$ & 3.6451020 & -0.7366630 & 2.2556900 \\
\hline $\mathrm{H}$ & 1.2203950 & 1.2292670 & -0.1105590 \\
\hline $\mathrm{H}$ & -0.4853820 & -0.2745320 & 1.9435090 \\
\hline $\mathrm{O}$ & -2.1475700 & -0.1737230 & -0.5689700 \\
\hline $\mathrm{C}$ & -3.2174630 & -0.6481130 & -0.0319240 \\
\hline $\mathrm{O}$ & -3.4540700 & -0.8523000 & 1.1541860 \\
\hline $\mathrm{C}$ & -4.3009240 & -0.9726300 & -1.1043310 \\
\hline $\mathrm{F}$ & -5.4102680 & -1.4974430 & -0.5507090 \\
\hline $\mathrm{F}$ & -3.8363080 & -1.8607380 & -2.0124930 \\
\hline $\mathrm{F}$ & -4.6661800 & 0.1449470 & -1.7742330 \\
\hline $\mathrm{H}$ & -1.2748400 & 0.0895040 & 0.2006730 \\
\hline $\mathrm{H}$ & -4.9363650 & -1.4951300 & 2.1339430 \\
\hline $\mathrm{O}$ & -5.6087120 & -1.8020590 & 2.7713870 \\
\hline $\mathrm{H}$ & -6.3958150 & -1.9892780 & 2.2440210 \\
\hline $\mathrm{H}$ & -0.3687510 & 2.1264900 & 2.5965570 \\
\hline $\mathrm{H}$ & -1.8755290 & 1.8170680 & 1.7592370 \\
\hline $\mathrm{C}$ & -0.4847630 & 3.0235380 & 0.6592280 \\
\hline $\mathrm{C}$ & -1.1996940 & 3.1800660 & -0.5405920 \\
\hline $\mathrm{C}$ & 0.5147630 & 3.9605460 & 0.9632300 \\
\hline $\mathrm{C}$ & -0.9207920 & 4.2383410 & -1.4097210 \\
\hline $\mathrm{H}$ & -1.9816000 & 2.4696720 & -0.7943370 \\
\hline $\mathrm{C}$ & 0.7970490 & 5.0224450 & 0.0968520 \\
\hline $\mathrm{H}$ & 1.0739410 & 3.8618930 & 1.8905010 \\
\hline $\mathrm{C}$ & 0.0799520 & 5.1642710 & -1.0943480 \\
\hline $\mathrm{H}$ & -1.4888440 & 4.3430680 & -2.3299320 \\
\hline $\mathrm{H}$ & 1.5728900 & 5.7376260 & 0.3554740 \\
\hline $\mathrm{H}$ & 0.2943360 & 5.9889720 & -1.7679140 \\
\hline
\end{tabular}

19.log

Potential Energy $=-1489.65248$

Zero-point Energy = -1489.184748

Free Energy = -1489.230437

Single-Point Energy B3LYP-D3(BJ)/6-311+G** PCM = -

1490.10942

Free Energy B3LYP-D3(BJ)/6-311+G** PCM (extrapolated

free energy from $\mathrm{qRRHO})=-1489.68737$

Nimag $=1\left(-1194.8229 \mathrm{~cm}^{-1}\right)$

Charge $=0$ Multiplicity $=1$

$\begin{array}{llll}\mathrm{C} & 3.3290180 & -0.4374140 & -0.7853580 \\ \mathrm{C} & 3.7998740 & -0.8514930 & 1.4893910 \\ \mathrm{C} & 2.2832100 & -1.0154950 & 1.3573420 \\ \mathrm{~N} & 2.1046080 & -0.9445340 & -0.1091280 \\ \mathrm{~N} & 4.3290320 & -0.6286650 & 0.2646700 \\ \mathrm{O} & 4.4339170 & -0.9725760 & 2.5378790 \\ \mathrm{C} & 5.7696060 & -0.7047330 & 0.0370570 \\ \mathrm{H} & 6.1791340 & -1.4843560 & 0.6827380 \\ \mathrm{H} & 5.9569840 & -0.9641930 & -1.0052260 \\ \mathrm{H} & 6.2722180 & 0.2379580 & 0.2700630\end{array}$

\begin{tabular}{|c|c|c|c|}
\hline $\mathrm{C}$ & 1.0419450 & -1.3527420 & -0.8052030 \\
\hline $\mathrm{C}$ & -0.2632970 & -1.5834680 & -0.3641930 \\
\hline $\mathrm{C}$ & -1.1584210 & -2.5001910 & -1.2114380 \\
\hline $\mathrm{H}$ & -0.8606560 & -3.5442980 & -1.0462600 \\
\hline $\mathrm{C}$ & 1.8626750 & -2.3372490 & 2.0126510 \\
\hline $\mathrm{H}$ & 2.2952770 & -2.3600540 & 3.0156310 \\
\hline $\mathrm{H}$ & 0.7823730 & -2.4338250 & 2.1097870 \\
\hline $\mathrm{H}$ & 2.2468150 & -3.1908140 & 1.4465170 \\
\hline $\mathrm{H}$ & 1.7801820 & -0.1737750 & 1.8488090 \\
\hline $\mathrm{C}$ & 3.2083580 & 1.0413240 & -1.3057600 \\
\hline $\mathrm{H}$ & 3.5590160 & -1.0900420 & -1.6366510 \\
\hline $\mathrm{C}$ & 4.5476790 & 1.4637040 & -1.9502730 \\
\hline $\mathrm{H}$ & 4.4199160 & 2.4363970 & -2.4354830 \\
\hline $\mathrm{H}$ & 5.3490080 & 1.5717350 & -1.2168880 \\
\hline $\mathrm{H}$ & 4.8716140 & 0.7526620 & -2.7189110 \\
\hline $\mathrm{C}$ & 2.1212790 & 1.1239120 & -2.3981660 \\
\hline $\mathrm{H}$ & 2.3037450 & 0.4082760 & -3.2086100 \\
\hline $\mathrm{H}$ & 1.1150970 & 0.9619690 & -2.0048320 \\
\hline $\mathrm{H}$ & 2.1336740 & 2.1260280 & -2.8383360 \\
\hline $\mathrm{C}$ & 2.8610200 & 2.0074270 & -0.1587120 \\
\hline $\mathrm{H}$ & 3.6205520 & 1.9918120 & 0.6296240 \\
\hline $\mathrm{H}$ & 2.8072350 & 3.0305360 & -0.5442800 \\
\hline $\mathrm{H}$ & 1.8889870 & 1.7746090 & 0.2874730 \\
\hline $\mathrm{H}$ & 1.2261200 & -1.4323290 & -1.8754600 \\
\hline $\mathrm{H}$ & -0.7575650 & -0.2480010 & -0.5696920 \\
\hline $\mathrm{H}$ & -0.9865860 & -2.2923730 & -2.2741610 \\
\hline $\mathrm{C}$ & -2.6379740 & -2.3679820 & -0.9015290 \\
\hline $\mathrm{C}$ & -3.1790780 & -2.9646770 & 0.2488140 \\
\hline $\mathrm{C}$ & -3.4984210 & -1.6554380 & -1.7494050 \\
\hline $\mathrm{C}$ & -4.5389380 & -2.8450290 & 0.5491760 \\
\hline $\mathrm{H}$ & -2.5315460 & -3.5330510 & 0.9126310 \\
\hline $\mathrm{C}$ & -4.8609500 & -1.5355370 & -1.4557710 \\
\hline $\mathrm{H}$ & -3.0998220 & -1.1909560 & -2.6478390 \\
\hline $\mathrm{C}$ & -5.3857730 & -2.1283570 & -0.3034210 \\
\hline $\mathrm{H}$ & -4.9373820 & -3.3152100 & 1.4439160 \\
\hline $\mathrm{H}$ & -5.5103990 & -0.9813800 & -2.1277770 \\
\hline $\mathrm{H}$ & -6.4435640 & -2.0372650 & -0.0740790 \\
\hline $\mathrm{H}$ & -0.4343380 & -1.6416050 & 0.7084240 \\
\hline $\mathrm{O}$ & -1.2453100 & 0.8441770 & -0.7666890 \\
\hline $\mathrm{C}$ & -1.7486280 & 1.4142180 & 0.2694390 \\
\hline $\mathrm{O}$ & -1.7922430 & 1.0099340 & 1.4279260 \\
\hline $\mathrm{C}$ & -2.3652030 & 2.8028730 & -0.0827890 \\
\hline $\mathrm{F}$ & -2.8931950 & 3.4070510 & 0.9992720 \\
\hline $\mathrm{F}$ & -1.4287740 & 3.6335620 & -0.5977400 \\
\hline $\mathrm{F}$ & -3.3499670 & 2.6784230 & -1.0024370 \\
\hline $\mathrm{H}$ & -2.4531100 & 1.6864460 & 3.0531970 \\
\hline $\mathrm{O}$ & -2.7320370 & 1.9060100 & 3.9629180 \\
\hline $\mathrm{H}$ & -3.2235370 & 2.7338800 & 3.8876480 \\
\hline
\end{tabular}

20.log

Potential Energy = -1489.65454

Zero-point Energy = -1489.18650

Free Energy = -1489.23215

Single-Point Energy B3LYP-D3(BJ)/6-311+G** PCM = 1490.11007

Free Energy B3LYP-D3(BJ)/6-311+G** PCM (extrapolated

free energy from $q R R H O)=-1489.68768$

Nimag $=1\left(-1183.6187 \mathrm{~cm}^{-1}\right)$

Charge $=0$ Multiplicity $=1$

$\begin{array}{lrrr}\mathrm{C} & -2.9257910 & -0.9239940 & -0.5253150 \\ \mathrm{C} & -3.4250940 & -0.5082010 & 1.7427910 \\ \mathrm{C} & -1.9775470 & -0.0440860 & 1.5588550 \\ \mathrm{~N} & -1.8195760 & -0.1439780 & 0.0922040 \\ \mathrm{~N} & -3.9222500 & -0.8919610 & 0.5447350 \\ \mathrm{O} & -4.0486040 & -0.4696020 & 2.8037360 \\ \mathrm{C} & -5.3523870 & -1.1213400 & 0.3570240 \\ \mathrm{H} & -5.8993600 & -0.4113640 & 0.9808170 \\ \mathrm{H} & -5.6103230 & -0.9554100 & -0.6892040 \\ \mathrm{H} & -5.6468730 & -2.1343760 & 0.6443720 \\ \mathrm{C} & -0.8697680 & 0.4262290 & -0.6504850 \\ \mathrm{C} & 0.3727240 & 0.9285460 & -0.2551350 \\ \mathrm{C} & 1.0929990 & 1.9297090 & -1.1652190 \\ \mathrm{H} & 0.8878470 & 1.6766900 & -2.2122650 \\ \mathrm{C} & -1.8233010 & 1.3640750 & 2.1469820 \\ \mathrm{H} & -2.2313180 & 1.3442510 & 3.1603170 \\ \mathrm{H} & -0.7840460 & 1.6833350 & 2.2078790\end{array}$




$\begin{array}{lrrr}\mathrm{H} & -2.3851920 & 2.0941950 & 1.5574330 \\ \mathrm{H} & -1.3018420 & -0.7425730 & 2.0681240 \\ \mathrm{C} & -2.5118000 & -2.3701720 & -0.9836760 \\ \mathrm{H} & -3.3021370 & -0.3742730 & -1.3968320 \\ \mathrm{C} & -3.7506360 & -3.0942270 & -1.5570030 \\ \mathrm{H} & -3.4355740 & -4.0418430 & -2.0047750 \\ \mathrm{H} & -4.4895200 & -3.3300600 & -0.7887960 \\ \mathrm{H} & -4.2389510 & -2.5059420 & -2.3423540 \\ \mathrm{C} & -1.4622180 & -2.2827610 & -2.1120950 \\ \mathrm{H} & -1.8097850 & -1.6586580 & -2.9439480 \\ \mathrm{H} & -0.4991990 & -1.9019940 & -1.7642480 \\ \mathrm{H} & -1.2818520 & -3.2865630 & -2.5095410 \\ \mathrm{C} & -1.9371650 & -3.1824440 & 0.1911070 \\ \mathrm{H} & -2.6570050 & -3.2782410 & 1.0104040 \\ \mathrm{H} & -1.6885620 & -4.1930240 & -0.1484080 \\ \mathrm{H} & -1.0185440 & -2.7343450 & 0.5830640 \\ \mathrm{H} & -1.0822350 & 0.4077290 & -1.7179480 \\ \mathrm{H} & 1.1427890 & -0.2591250 & -0.4392820 \\ \mathrm{H} & 2.1729430 & 1.8193630 & -1.0182480 \\ \mathrm{C} & 0.7059620 & 3.3767720 & -0.9065650 \\ \mathrm{C} & -0.3606050 & 3.9742820 & -1.5960260 \\ \mathrm{C} & 1.3971160 & 4.1422870 & 0.0451230 \\ \mathrm{C} & -0.7316270 & 5.2975220 & -1.3381580 \\ \mathrm{H} & -0.9028560 & 3.4024160 & -2.3455920 \\ \mathrm{C} & 1.0285380 & 5.4654770 & 0.3087290 \\ \mathrm{H} & 2.2328640 & 3.6998310 & 0.5821570 \\ \mathrm{C} & -0.0390110 & 6.0479650 & -0.3819260 \\ \mathrm{H} & -1.5570160 & 5.7428600 & -1.8867350 \\ \mathrm{H} & 1.5779320 & 6.0411900 & 1.0484570 \\ \mathrm{H} & -0.3245970 & 7.0765910 & -0.1816080 \\ \mathrm{H} & 0.5478320 & 1.0719170 & 0.8089590 \\ \mathrm{O} & 1.8602080 & -1.2264300 & -0.5984020 \\ \mathrm{C} & 2.9891400 & -1.1721880 & 0.0142410 \\ \mathrm{O} & 3.4442570 & -0.2686480 & 0.7096940 \\ \mathrm{C} & 3.8280130 & -2.4672480 & -0.2107690 \\ \mathrm{~F} & 5.0138130 & -2.4137880 & 0.4258370 \\ \mathrm{~F} & 3.1684760 & -3.5577910 & 0.2439940 \\ \mathrm{~F} & 4.0788560 & -2.6606880 & -1.5264190 \\ \mathrm{H} & 5.0382740 & -0.0077060 & 1.6766790 \\ & 5.8013080 & 0.2679330 & 2.2199670 \\ & 6.4462000 & -0.4461300 & 2.1370100\end{array}$

21.log

Potential Energy $=-1489.65274$

Zero-point Energy = -1489.18458

Free Energy = -1489.23013

Single-Point Energy B3LYP-D3(BJ)/6-311+G** PCM = 1490.11031

Free Energy B3LYP-D3(BJ)/6-311+G** PCM (extrapolated free energy from $\mathrm{qRRHO})=-1489.68769$

Nimag $=1\left(-1176.9071 \mathrm{~cm}^{-1}\right)$

Charge $=0$ Multiplicity $=1$

$\begin{array}{lrrr}\mathrm{C} & 2.9590250 & -0.0971970 & 0.3830970 \\ \mathrm{C} & 3.5438430 & -1.8075490 & -1.1324680 \\ \mathrm{C} & 2.0946440 & -1.4478880 & -1.4719530 \\ \mathrm{~N} & 1.8828100 & -0.2504280 & -0.6316800 \\ \mathrm{~N} & 3.9952780 & -0.9666350 & -0.1740900 \\ \mathrm{O} & 4.2045710 & -2.6805620 & -1.6961160 \\ \mathrm{C} & 5.4146110 & -0.8931800 & 0.1613460 \\ \mathrm{H} & 5.9918900 & -1.0551800 & -0.7512780 \\ \mathrm{H} & 5.6412440 & 0.0958690 & 0.5603480 \\ \mathrm{H} & 5.7035140 & -1.6523640 & 0.8933840 \\ \mathrm{C} & 0.9180770 & 0.6629160 & -0.7678990 \\ \mathrm{C} & -0.2995430 & 0.5486050 & -1.4396420 \\ \mathrm{C} & -1.0670050 & 1.8013290 & -1.8956300 \\ \mathrm{H} & -2.1244650 & 1.5275160 & -1.9879860 \\ \mathrm{C} & 1.9764400 & -1.2259490 & -2.9855080 \\ \mathrm{H} & 2.4280340 & -2.0884910 & -3.4812960 \\ \mathrm{H} & 0.9433390 & -1.1477070 & -3.3209930 \\ \mathrm{H} & 2.5178140 & -0.3251350 & -3.2888870 \\ \mathrm{H} & 1.4320640 & -2.2640920 & -1.1575740 \\ \mathrm{C} & 2.5201100 & -0.4686920 & 1.8468030 \\ \mathrm{H} & 3.3056950 & 0.9436330 & 0.3757360 \\ \mathrm{C} & 3.7240520 & -0.2880950 & 2.7986440 \\ \mathrm{H} & 3.3838880 & -0.4050440 & 3.8323720 \\ \mathrm{H} & 4.5056590 & -1.0313400 & 2.6300990\end{array}$

\begin{tabular}{lrrr}
$\mathrm{H}$ & 4.1674440 & 0.7103810 & 2.7092390 \\
$\mathrm{C}$ & 1.4066020 & 0.4895840 & 2.3194520 \\
$\mathrm{H}$ & 1.7067860 & 1.5399750 & 2.2290220 \\
$\mathrm{H}$ & 0.4685380 & 0.3443380 & 1.7789650 \\
$\mathrm{H}$ & 1.1981610 & 0.2992980 & 3.3770270 \\
$\mathrm{C}$ & 2.0141890 & -1.9205420 & 1.9239130 \\
$\mathrm{H}$ & 2.7816650 & -2.6360660 & 1.6117190 \\
$\mathrm{H}$ & 1.7414320 & -2.1598290 & 2.9567420 \\
$\mathrm{H}$ & 1.1226420 & -2.0723450 & 1.3071390 \\
$\mathrm{H}$ & 1.0962770 & 1.5741250 & -0.2011910 \\
$\mathrm{H}$ & -1.0838020 & 0.0137790 & -0.3639200 \\
$\mathrm{H}$ & -0.7333170 & 2.0703260 & -2.9057760 \\
$\mathrm{C}$ & -0.9388130 & 3.0158560 & -0.9958160 \\
$\mathrm{C}$ & -1.7187110 & 3.1407010 & 0.1656970 \\
$\mathrm{C}$ & -0.0375510 & 4.0464190 & -1.3056870 \\
$\mathrm{C}$ & -1.5999930 & 4.2604740 & 0.9938270 \\
$\mathrm{H}$ & -2.4274210 & 2.3573990 & 0.4209550 \\
$\mathrm{C}$ & 0.0858470 & 5.1687710 & -0.4796390 \\
$\mathrm{H}$ & 0.5686840 & 3.9724480 & -2.2055300 \\
$\mathrm{C}$ & -0.6954920 & 5.2789950 & 0.6744450 \\
$\mathrm{H}$ & -2.2171140 & 4.3396780 & 1.8844640 \\
$\mathrm{H}$ & 0.7874070 & 5.9561340 & -0.7406880 \\
$\mathrm{H}$ & -0.6049750 & 6.1510170 & 1.3156390 \\
$\mathrm{H}$ & -0.4232870 & -0.2970180 & -2.1127070 \\
$\mathrm{O}$ & -1.8046220 & -0.4504170 & 0.4903130 \\
$\mathrm{C}$ & -2.8346120 & -1.0800060 & 0.0466230 \\
$\mathrm{O}$ & -3.1973540 & -1.2392030 & -1.1151350 \\
$\mathrm{C}$ & -3.6800730 & -1.6875380 & 1.2073340 \\
$\mathrm{~F}$ & -4.7648510 & -2.3408200 & 0.7484330 \\
$\mathrm{~F}$ & -2.9493890 & -2.5661320 & 1.9318880 \\
$\mathrm{~F}$ & -4.1112940 & -0.7210830 & 2.0505600 \\
$\mathrm{H}$ & -4.6252940 & -2.1398300 & -1.9507730 \\
$\mathrm{O}$ & -5.2978760 & -2.5613080 & -2.5192880 \\
$\mathrm{H}$ & -5.9209500 & -2.9798680 & -1.9116150 \\
& & & \\
\hline
\end{tabular}

22.log

Potential Energy $=-1489.65177$

Zero-point Energy = -1489.18389

Free Energy $=-1489.22932$

Single-Point Energy B3LYP-D3(BJ)/6-311+G** PCM = 1490.10954

Free Energy B3LYP-D3(BJ)/6-311+G** PCM (extrapolated

free energy from qRRHO) $=-1489.68709$

Nimag $=1\left(-1199.7506 \mathrm{~cm}^{-1}\right)$

Charge $=0$ Multiplicity $=1$

$\begin{array}{lrrr}\mathrm{C} & -3.2328400 & 0.0939410 & 0.8033320 \\ \mathrm{C} & -4.1367980 & -1.3447230 & -0.8332130 \\ \mathrm{C} & -2.6256020 & -1.5903040 & -0.8726920 \\ \mathrm{~N} & -2.1704560 & -0.8228460 & 0.3066430 \\ \mathrm{~N} & -4.4185080 & -0.4799010 & 0.1677420 \\ \mathrm{O} & -4.9613830 & -1.9039320 & -1.5564820 \\ \mathrm{C} & -5.7897330 & -0.2816580 & 0.6315090 \\ \mathrm{H} & -6.3169310 & -1.2358700 & 0.5671330 \\ \mathrm{H} & -5.7754100 & 0.0526190 & 1.6690380 \\ \mathrm{H} & -6.3257380 & 0.4526260 & 0.0244110 \\ \mathrm{C} & -0.9962430 & -0.9366710 & 0.9302350 \\ \mathrm{C} & 0.1895020 & -1.5169620 & 0.4733690 \\ \mathrm{C} & 1.2250000 & -1.9657100 & 1.5160260 \\ \mathrm{H} & 0.8453880 & -2.8518640 & 2.0423140 \\ \mathrm{C} & -2.3633260 & -3.1012640 & -0.8276880 \\ \mathrm{H} & -2.9989080 & -3.5670860 & -1.5845460 \\ \mathrm{H} & -1.3293160 & -3.3570420 & -1.0537430 \\ \mathrm{H} & -2.6261410 & -3.5127660 & 0.1511070 \\ \mathrm{H} & -2.2091040 & -1.1626350 & -1.7933930 \\ \mathrm{C} & -2.9816880 & 1.6080110 & 0.4629230 \\ \mathrm{H} & -3.3107240 & -0.0115790 & 1.8923280 \\ \mathrm{C} & -4.2024920 & 2.4413900 & 0.9124630 \\ \mathrm{H} & -3.9645620 & 3.5050930 & 0.8134910 \\ \mathrm{H} & -5.0857370 & 2.2491270 & 0.3001570 \\ \mathrm{H} & -4.4577990 & 2.2581530 & 1.9623160 \\ \mathrm{C} & -1.7576950 & 2.1208450 & 1.2496160 \\ \mathrm{H} & -1.8720470 & 1.9566020 & 2.3276560 \\ \mathrm{H} & -0.8203120 & 1.6625240 & 0.9276800 \\ \mathrm{H} & -1.6552200 & 3.1988920 & 1.0907820 \\ \mathrm{C} & -2.7521460 & 1.8084010 & -1.0461170 \\ \mathrm{H} & -3.6049710 & 1.4569010 & -1.6361000\end{array}$




$\begin{array}{lrrr}\mathrm{H} & -2.6231140 & 2.8743770 & -1.2590640 \\ \mathrm{H} & -1.8504320 & 1.2938130 & -1.3926350 \\ \mathrm{H} & -0.9670120 & -0.4371920 & 1.8971960 \\ \mathrm{H} & 0.7494440 & -0.3636470 & -0.1957210 \\ \mathrm{H} & 1.3456240 & -1.1811680 & 2.2715680 \\ \mathrm{C} & 2.5775170 & -2.3082450 & 0.9188360 \\ \mathrm{C} & 2.7514540 & -3.4980970 & 0.1927180 \\ \mathrm{C} & 3.6826140 & -1.4601490 & 1.0811370 \\ \mathrm{C} & 3.9906800 & -3.8267770 & -0.3635130 \\ \mathrm{H} & 1.9112720 & -4.1773320 & 0.0674550 \\ \mathrm{C} & 4.9264360 & -1.7860830 & 0.5290280 \\ \mathrm{H} & 3.5685050 & -0.5369730 & 1.6427610 \\ \mathrm{C} & 5.0844850 & -2.9698550 & -0.1974130 \\ \mathrm{H} & 4.1037690 & -4.7530320 & -0.9200340 \\ \mathrm{H} & 5.7700660 & -1.1158190 & 0.6689550 \\ \mathrm{H} & 6.0494960 & -3.2251110 & -0.6257140 \\ \mathrm{H} & 0.1312060 & -2.1503850 & -0.4094110 \\ \mathrm{O} & 1.2672250 & 0.4377030 & -0.9368700 \\ \mathrm{C} & 1.8656010 & 1.4175680 & -0.3583950 \\ \mathrm{O} & 1.9489950 & 1.6670710 & 0.8407940 \\ \mathrm{C} & 2.5403330 & 2.3697870 & -1.3920500 \\ \mathrm{~F} & 3.1817980 & 3.3895770 & -0.7895560 \\ \mathrm{~F} & 3.4454170 & 1.7065500 & -2.1473460 \\ \mathrm{~F} & 1.6208760 & 2.9033250 & -2.2298550 \\ \mathrm{H} & 2.7540260 & 3.0254720 & 1.8655230 \\ \mathrm{O} & 3.1079470 & 3.6511020 & 2.5263520 \\ \mathrm{H} & 3.5819860 & 4.3212370 & 2.0175850 \\ & & & \end{array}$

23.log

Potential Energy $=-1489.65395$

Zero-point Energy = -1489.18594

Free Energy = -1489.23165

Single-Point Energy B3LYP-D3(BJ)/6-311+G** PCM = 1490.11023

Free Energy B3LYP-D3(BJ)/6-311+G** PCM (extrapolated free energy from $\mathrm{qRRHO})=-1489.68794$

Nimag $=1\left(-1207.5758 \mathrm{~cm}^{-1}\right)$

Charge $=0$ Multiplicity $=1$

$\begin{array}{lccc}\mathrm{C} & 1.8357380 & -2.0017510 & 0.8069500 \\ \mathrm{C} & 3.2612390 & -2.0519450 & -1.0725350 \\ \mathrm{C} & 2.2607000 & -0.9287750 & -1.3583270 \\ \mathrm{~N} & 1.5467820 & -0.8322580 & -0.0669380 \\ \mathrm{~N} & 3.0381570 & -2.5376380 & 0.1700720 \\ \mathrm{O} & 4.1659360 & -2.4004230 & -1.8313980 \\ \mathrm{C} & 4.0096400 & -3.4084200 & 0.8274000 \\ \mathrm{H} & 5.0120070 & -3.0892190 & 0.5342750 \\ \mathrm{H} & 3.9036990 & -3.3161270 & 1.9085440 \\ \mathrm{H} & 3.8814260 & -4.4557790 & 0.5413710 \\ \mathrm{C} & 0.7797000 & 0.1768180 & 0.3472300 \\ \mathrm{C} & 0.1721890 & 1.1854340 & -0.4060800 \\ \mathrm{C} & -0.2569870 & 2.4800870 & 0.2935070 \\ \mathrm{H} & -0.5978740 & 2.2447300 & 1.3079380 \\ \mathrm{C} & 3.0304950 & 0.3285560 & -1.7830760 \\ \mathrm{H} & 3.7138460 & 0.0413340 & -2.5857520 \\ \mathrm{H} & 2.3781730 & 1.1146490 & -2.1601280 \\ \mathrm{H} & 3.6168730 & 0.7242930 & -0.9488370 \\ \mathrm{H} & 1.5749790 & -1.2407980 & -2.1559810 \\ \mathrm{C} & 0.6485920 & -3.0269580 & 0.9167230 \\ \mathrm{H} & 2.0809830 & -1.6398470 & 1.8128040 \\ \mathrm{C} & 1.0963080 & -4.2328330 & 1.7727860 \\ \mathrm{H} & 0.2301740 & -4.8719230 & 1.9704280 \\ \mathrm{H} & 1.8431550 & -4.8478760 & 1.2670460 \\ \mathrm{H} & 1.5007870 & -3.9180670 & 2.7415800 \\ \mathrm{C} & -0.5478230 & -2.3681120 & 1.6347250 \\ \mathrm{H} & -0.2621040 & -1.9583780 & 2.6106780 \\ \mathrm{H} & -1.0091300 & -1.5727170 & 1.0450830 \\ \mathrm{H} & -1.3204850 & -3.1233410 & 1.8092100 \\ \mathrm{C} & 0.2088970 & -3.5183310 & -0.4743260 \\ \mathrm{H} & 1.0307040 & -4.0013540 & -1.0127850 \\ \mathrm{H} & -0.5923850 & -4.2563180 & -0.3665310 \\ & -0.1799250 & -2.7005760 & -1.0894290 \\ \mathrm{H} & 0.5450150 & 0.1393070 & 1.4095230 \\ \mathrm{H} & -1.0702970 & 0.5419070 & -0.6942430 \\ \mathrm{H} & 1.740403300 & 3.5303950 & 0.3516550 \\ \mathrm{H} & & 3.5764970 & 1.4277690\end{array}$

$\begin{array}{lrrr}\mathrm{C} & 0.9900860 & 4.4666540 & -0.6829350 \\ \mathrm{C} & 2.7656900 & 4.5262920 & 1.4669640 \\ \mathrm{H} & 1.6363380 & 2.8672810 & 2.2457060 \\ \mathrm{C} & 2.0155690 & 5.4172760 & -0.6501140 \\ \mathrm{H} & 0.2960660 & 4.4535340 & -1.5201680 \\ \mathrm{C} & 2.9086120 & 5.4497000 & 0.4256110 \\ \mathrm{H} & 3.4486110 & 4.5480270 & 2.3117550 \\ \mathrm{H} & 2.1127730 & 6.1340650 & -1.4608280 \\ \mathrm{H} & 3.7034340 & 6.1893830 & 0.4553360 \\ \mathrm{H} & 0.5011930 & 1.3137340 & -1.4357250 \\ \mathrm{O} & -2.1161300 & 0.1271370 & -1.1587260 \\ \mathrm{C} & -3.1228480 & 0.1635420 & -0.3599430 \\ \mathrm{O} & -3.1623690 & 0.5298190 & 0.8112390 \\ \mathrm{C} & -4.4234860 & -0.3493360 & -1.0504420 \\ \mathrm{~F} & -5.4862640 & -0.2910900 & -0.2250280 \\ \mathrm{~F} & -4.7162360 & 0.3880460 & -2.1463440 \\ \mathrm{~F} & -4.2846830 & -1.6355170 & -1.4483620 \\ \mathrm{H} & -4.4922510 & 0.6113850 & 2.1394900 \\ \mathrm{O} & -5.0632820 & 0.7021580 & 2.9263090 \\ \mathrm{H} & -5.9476390 & 0.4493480 & 2.6320100\end{array}$

24.log

Potential Energy = -1489.65274

Zero-point Energy = -1489.18457

Free Energy = -1489.23008

Single-Point Energy B3LYP-D3(BJ)/6-311+G** PCM = 1490.11033

Free Energy B3LYP-D3(BJ)/6-311+G** PCM (extrapolated free energy from qRRHO) $=-1489.68767$

Nimag $=1\left(-1177.1316 \mathrm{~cm}^{-1}\right)$

Charge $=0$ Multiplicity $=1$

\begin{tabular}{|c|c|c|c|}
\hline $\mathrm{C}$ & 2.9618060 & -0.0865770 & 0.3642210 \\
\hline $\mathrm{C}$ & 3.5449600 & -1.7715100 & -1.1802500 \\
\hline $\mathrm{C}$ & 2.0928870 & -1.4117810 & -1.5070080 \\
\hline $\mathrm{N}$ & 1.8814780 & -0.2275070 & -0.6481950 \\
\hline $\mathrm{N}$ & 3.9980810 & -0.9442650 & -0.2108650 \\
\hline $\mathrm{O}$ & 4.2059500 & -2.6331860 & -1.7607690 \\
\hline $\mathrm{C}$ & 5.4188520 & -0.8710010 & 0.1186110 \\
\hline $\mathrm{H}$ & 5.9921740 & -1.0148280 & -0.7995470 \\
\hline $\mathrm{H}$ & 5.6436580 & 0.1118480 & 0.5335870 \\
\hline $\mathrm{H}$ & 5.7143450 & -1.6416300 & 0.8358910 \\
\hline $\mathrm{C}$ & 0.9113860 & 0.6828860 & -0.7642310 \\
\hline $\mathrm{C}$ & -0.3093480 & 0.5737050 & -1.4313550 \\
\hline $\mathrm{C}$ & -1.0876200 & 1.8297760 & -1.8583990 \\
\hline $\mathrm{H}$ & -2.1435150 & 1.5499780 & -1.9493270 \\
\hline $\mathrm{C}$ & 1.9657300 & -1.1686240 & -3.0165530 \\
\hline $\mathrm{H}$ & 2.4194020 & -2.0215190 & -3.5269350 \\
\hline $\mathrm{H}$ & 0.9305020 & -1.0912490 & -3.3456550 \\
\hline $\mathrm{H}$ & 2.5007360 & -0.2606640 & -3.3097000 \\
\hline $\mathrm{H}$ & 1.4346260 & -2.2346490 & -1.2009180 \\
\hline $\mathrm{C}$ & 2.5303060 & -0.4820040 & 1.8238860 \\
\hline $\mathrm{H}$ & 3.3055510 & 0.9552160 & 0.3713500 \\
\hline $\mathrm{C}$ & 3.7388810 & -0.3174100 & 2.7727180 \\
\hline $\mathrm{H}$ & 3.4037430 & -0.4525860 & 3.8058660 \\
\hline $\mathrm{H}$ & 4.5199460 & -1.0572290 & 2.5874240 \\
\hline $\mathrm{H}$ & 4.1813320 & 0.6827240 & 2.6987670 \\
\hline $\mathrm{C}$ & 1.4195950 & 0.4687400 & 2.3178670 \\
\hline $\mathrm{H}$ & 1.7197490 & 1.5203380 & 2.2425140 \\
\hline $\mathrm{H}$ & 0.4785630 & 0.3324030 & 1.7802980 \\
\hline $\mathrm{H}$ & 1.2167910 & 0.2615880 & 3.3733670 \\
\hline $\mathrm{C}$ & 2.0243950 & -1.9348380 & 1.8796590 \\
\hline $\mathrm{H}$ & 2.7898190 & -2.6452050 & 1.5510070 \\
\hline $\mathrm{H}$ & 1.7577890 & -2.1912940 & 2.9099760 \\
\hline $\mathrm{H}$ & 1.1291080 & -2.0760200 & 1.2658340 \\
\hline $\mathrm{H}$ & 1.0875040 & 1.5852070 & -0.1828920 \\
\hline $\mathrm{H}$ & -1.0797110 & 0.0076730 & -0.3615410 \\
\hline $\mathrm{H}$ & -0.7617680 & 2.1204570 & -2.8651090 \\
\hline $\mathrm{C}$ & -0.9618600 & 3.0275200 & -0.9361170 \\
\hline $\mathrm{C}$ & -1.7374220 & 3.1261560 & 0.2308040 \\
\hline $\mathrm{C}$ & -0.0672400 & 4.0685930 & -1.2298300 \\
\hline $\mathrm{C}$ & -1.6211380 & 4.2305440 & 1.0796890 \\
\hline $\mathrm{H}$ & -2.4416990 & 2.3349760 & 0.4738190 \\
\hline $\mathrm{C}$ & 0.0537570 & 5.1756310 & -0.3830470 \\
\hline $\mathrm{H}$ & 0.5355870 & 4.0150360 & -2.1334130 \\
\hline $\mathrm{C}$ & -0.7232930 & 5.2597220 & 0.7761290 \\
\hline $\mathrm{H}$ & -2.2350250 & 4.2895290 & 1.9741130 \\
\hline
\end{tabular}




$\begin{array}{lrrr}\mathrm{H} & 0.7500840 & 5.9715370 & -0.6319190 \\ \mathrm{H} & -0.6346890 & 6.1198750 & 1.4334130 \\ \mathrm{H} & -0.4309980 & -0.2590190 & -2.1208050 \\ \mathrm{O} & -1.7743690 & -0.5028890 & 0.4879370 \\ \mathrm{C} & -2.8387380 & -1.0749220 & 0.0475940 \\ \mathrm{O} & -3.2737010 & -1.1113690 & -1.0996330 \\ \mathrm{C} & -3.6117900 & -1.8066920 & 1.1868750 \\ \mathrm{~F} & -4.7825030 & -2.3121880 & 0.7532730 \\ \mathrm{~F} & -2.8787870 & -2.8345850 & 1.6769500 \\ \mathrm{~F} & -3.8828750 & -0.9718060 & 2.2152340 \\ \mathrm{H} & -4.7583180 & -1.9102490 & -1.9397790 \\ \mathrm{O} & -5.4641260 & -2.2646170 & -2.5137660 \\ \mathrm{H} & -6.0593720 & -2.7436240 & -1.9230980\end{array}$

\section{Chlorination of enamine (TS4)}

\section{(S)-TS-front}

\section{Conformation Extrapolated name Free energy}

$\begin{array}{cc}\text { TS4-Sf-lowest } & \mathbf{0 . 0} \\ 1 & 0.5 \\ 2 & 1.0 \\ 3 & 0.2 \\ 4 & 1.4 \\ 5 & 0.6 \\ 6 & 0.6 \\ 7 & 0.9\end{array}$

TS-Sf-lowest.log

Potential Energy = -2233.46980

Zero-point Energy = -2232.93920

Free Energy $=-2232.98976$

Single-Point Energy B3LYP-D3(BJ)/6-311+G** PCM = 2234.04089

Free Energy B3LYP-D3(BJ)/6-311+G** PCM (extrapolated free energy from qRRHO) $=-2233.56086$

Nimag $=1\left(-171.2674 \mathrm{~cm}^{-1}\right)$

Charge $=0$ Multiplicity $=1$

$\begin{array}{lrrr}\mathrm{C} & 1.4362000 & -2.0260730 & 0.1852510 \\ \mathrm{C} & 0.6589640 & -2.5454550 & 2.3535050 \\ \mathrm{C} & 1.9872400 & -1.8056210 & 2.5386940 \\ \mathrm{~N} & 2.2158010 & -1.2590210 & 1.1802660 \\ \mathrm{~N} & 0.3820540 & -2.6041080 & 1.0309380 \\ \mathrm{O} & -0.0429360 & -2.9908790 & 3.2634590 \\ \mathrm{C} & -0.9020110 & -3.0898600 & 0.5364720 \\ \mathrm{H} & -1.6744240 & -2.8186980 & 1.2590040 \\ \mathrm{H} & -1.1237620 & -2.6181050 & -0.4216270 \\ \mathrm{H} & -0.9089440 & -4.1766620 & 0.4121940 \\ \mathrm{C} & 2.6962700 & -0.0165110 & 0.9672230 \\ \mathrm{H} & 3.1743400 & 0.4192710 & 1.8393750 \\ \mathrm{C} & 2.6298390 & 0.7631100 & -0.1734300 \\ \mathrm{C} & 3.4840250 & 2.0109890 & -0.3095340 \\ \mathrm{H} & 3.7139220 & 2.4055100 & 0.6867900 \\ \mathrm{H} & 2.9099480 & 2.7835200 & -0.8322730 \\ \mathrm{H} & 2.2665530 & 0.3375310 & -1.1023570 \\ \mathrm{C} & 1.9217000 & -0.7861010 & 3.6715830 \\ \mathrm{H} & 1.5900990 & -1.3082500 & 4.5724590 \\ \mathrm{H} & 2.9013980 & -0.3496510 & 3.8798890 \\ \mathrm{H} & 1.2096700 & 0.0145820 & 3.4518760 \\ \mathrm{H} & 2.7596910 & -2.5493130 & 2.7731390 \\ \mathrm{C} & 2.2850320 & -3.0774390 & -0.6150130 \\ \mathrm{H} & 0.9755380 & -1.3327920 & -0.5265310 \\ \mathrm{Cl} & 0.4689130 & 1.7273330 & 0.4829900 \\ & & & \\ & & & \\ & & \end{array}$

\begin{tabular}{|c|c|c|c|}
\hline $\mathrm{N}$ & -1.2008020 & 2.5821520 & 0.7876870 \\
\hline $\mathrm{C}$ & -1.3399020 & 3.7131330 & 1.5954140 \\
\hline & -2.3732320 & 2.1463290 & 0.2431010 \\
\hline & -2.8144340 & 4.0976710 & 1.6004990 \\
\hline & -3.4990880 & 3.0598870 & 0.6961410 \\
\hline & -3.1780470 & 4.0783990 & 2.6314920 \\
\hline & -3.9716900 & 3.5004970 & \\
\hline & -2.4718490 & 1.1604460 & -0.498630 \\
\hline 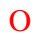 & -0.4271040 & 4.2681930 & 2.179470 \\
\hline & 1.3752820 & -3.8038310 & -1.629930 \\
\hline & 1.9936850 & -4.4149550 & -2.295210 \\
\hline $\mathrm{H}$ & 0.6614060 & -4.4734930 & -1.145062 \\
\hline $\mathrm{H}$ & 0.8183980 & -3.0962140 & -2.2546 \\
\hline $\mathrm{C}$ & 3.3899880 & -2.3456000 & -1.4034 \\
\hline $\mathrm{H}$ & 2.9707990 & -1.6475540 & \\
\hline $\mathrm{H}$ & 4.0669580 & -1.7934900 & -0.7462 \\
\hline $\mathrm{H}$ & 3.9855440 & -3.0805060 & -1.9546 \\
\hline $\mathrm{C}$ & 2.9351940 & -4.1129440 & 0.3214 \\
\hline $\mathrm{H}$ & 2.1929360 & -4.6324590 & 0.9360 \\
\hline $\mathrm{H}$ & 3.4537290 & -4.8694300 & -0.276 \\
\hline $\mathrm{H}$ & 3.6781470 & -3.6526550 & 0.9803 \\
\hline $\mathrm{H}$ & -4.2550230 & 2.4590660 & 1.2094 \\
\hline $\mathrm{H}$ & -2.9121420 & 5.1228650 & 1.233 \\
\hline $\mathrm{C}$ & 4.7781290 & 1.7606790 & -1.0718 \\
\hline $\mathrm{C}$ & 4.8343980 & 1.9359080 & -2.462770 \\
\hline $\mathrm{C}$ & 5.9369270 & 1.3332880 & -0.405020 \\
\hline $\mathrm{C}$ & 6.0135370 & 1.6837950 & -3.171777 \\
\hline $\mathrm{H}$ & 3.9493190 & 2.2762250 & -2.995277 \\
\hline $\mathrm{C}$ & 7.1187870 & 1.0828490 & -1.108425 \\
\hline $\mathrm{H}$ & 5.9164780 & 1.1995340 & 0.674002 \\
\hline $\mathrm{C}$ & 7.1605770 & 1.2552410 & -2.496360 \\
\hline $\mathrm{H}$ & 6.0367420 & 1.8274260 & -4.248480 \\
\hline $\mathrm{H}$ & 8.0066210 & 0.7579240 & -0.573011 \\
\hline $\mathrm{H}$ & 8.0785510 & 1.0629090 & -3.044175 \\
\hline $\mathrm{H}$ & -3.7921740 & 0.5960690 & -1.064375 \\
\hline $\mathrm{O}$ & -4.5875760 & 0.1596840 & -1.541360 \\
\hline $\mathrm{C}$ & -5.5791260 & -0.0979560 & -0.723358 \\
\hline $\mathrm{O}$ & -5.6488720 & 0.1226780 & 0.467720 \\
\hline $\mathrm{C}$ & -6.7532220 & -0.7683980 & -1.489784 \\
\hline $\mathrm{F}$ & -6.3628880 & -1.9411010 & \\
\hline & -7.1952980 & 0.0269540 & -2.489795 \\
\hline & -7.7868040 & -1.0179020 & -0.671335 \\
\hline
\end{tabular}

$1 . \log$

Potential Energy = -2233.46971

Zero-point Energy = -2232.93890

Free Energy $=-2232.98921$

Single-Point Energy B3LYP-D3(BJ)/6-311+G** PCM = 2234.04053

Free Energy B3LYP-D3(BJ)/6-311+G** PCM (extrapolated free energy from $\mathrm{qRRHO})=-2233.56003$

Nimag $=1\left(-171.7683 \mathrm{~cm}^{-1}\right)$

Charge $=0$ Multiplicity $=1$

$\begin{array}{lrrr}\mathrm{C} & -3.6684520 & -1.2846770 & 0.0439140 \\ \mathrm{C} & -3.1339440 & -2.8612820 & -1.6305480 \\ \mathrm{C} & -2.9388380 & -1.4799660 & -2.2628740 \\ \mathrm{~N} & -2.9850500 & -0.5982410 & -1.0729360 \\ \mathrm{~N} & -3.4985410 & -2.6935340 & -0.3390530 \\ \mathrm{O} & -2.9658140 & -3.9391640 & -2.2042870 \\ \mathrm{C} & -3.5938430 & -3.8190540 & 0.5844800 \\ \mathrm{H} & -2.8382370 & -4.5570070 & 0.3076520 \\ \mathrm{H} & -3.4027260 & -3.4714640 & 1.6004680 \\ \mathrm{H} & -4.5767530 & -4.2978240 & 0.5472560 \\ \mathrm{C} & -2.1605030 & 0.4586480 & -0.9178040 \\ \mathrm{H} & -1.6905320 & 0.7812090 & -1.8419910 \\ \mathrm{C} & -1.8223210 & 1.1420680 & 0.2357150 \\ \mathrm{C} & -1.1164920 & 2.4843520 & 0.1620650 \\ \mathrm{H} & -0.5429530 & 2.5430960 & -0.7700860 \\ \mathrm{H} & -0.3958140 & 2.5566160 & 0.9835840 \\ \mathrm{H} & -2.3425670 & 0.9332850 & 1.1640620 \\ \mathrm{C} & -1.6676740 & -1.4089620 & -3.1034460 \\ \mathrm{H} & -1.7067390 & -2.2170420 & -3.8380570 \\ \mathrm{H} & -1.5959210 & -0.4648870 & -3.6487410 \\ \mathrm{H} & -0.7717180 & -1.5398480 & -2.4898910 \\ \mathrm{H} & -3.8036920 & -1.2774690 & -2.9078000 \\ \mathrm{C} & -5.1603820 & -0.8375750 & 0.2497230\end{array}$




$\begin{array}{lrrr}\mathrm{H} & -3.1164690 & -1.1035410 & 0.9725750 \\ \mathrm{Cl} & 0.0755730 & -0.3545370 & 0.6944540 \\ \mathrm{~N} & 1.5740650 & -1.3810250 & 1.2446500 \\ \mathrm{C} & 2.8640780 & -0.9720530 & 1.0693310 \\ \mathrm{C} & 1.4567550 & -2.6250500 & 1.8692570 \\ \mathrm{C} & 3.7991320 & -2.0318770 & 1.6256780 \\ \mathrm{C} & 2.8650860 & -3.1361220 & 2.1468190 \\ \mathrm{H} & 4.4705540 & -2.3625960 & 0.8284330 \\ \mathrm{H} & 2.9681890 & -3.3202260 & 3.2195200 \\ \mathrm{O} & 0.4034320 & -3.1768090 & 2.1303680 \\ \mathrm{O} & 3.1889180 & 0.0976490 & 0.5386820 \\ \mathrm{C} & -5.7570470 & -1.5887310 & 1.4602600 \\ \mathrm{H} & -6.7360890 & -1.1625780 & 1.7019930 \\ \mathrm{H} & -5.9074070 & -2.6518710 & 1.2595060 \\ \mathrm{H} & -5.1250830 & -1.4892690 & 2.3501780 \\ \mathrm{C} & -5.2029910 & 0.6726930 & 0.5585660 \\ \mathrm{H} & -4.6681250 & 0.9126690 & 1.4833470 \\ \mathrm{H} & -4.7788860 & 1.2703530 & -0.2525180 \\ \mathrm{H} & -6.2443400 & 0.9830880 & 0.6925900 \\ \mathrm{C} & -6.0149210 & -1.1202460 & -1.0000870 \\ \mathrm{H} & -5.9872710 & -2.1767110 & -1.2857080 \\ \mathrm{H} & -7.0588290 & -0.8634470 & -0.7928520 \\ \mathrm{H} & -5.6943830 & -0.5164430 & -1.8548300 \\ \mathrm{H} & 2.9983050 & -4.0915050 & 1.6318550 \\ \mathrm{H} & 4.4150930 & -1.5815920 & 2.4096780 \\ \mathrm{C} & -2.0765920 & 3.6631450 & 0.2459770 \\ \mathrm{C} & -2.3922390 & 4.2428020 & 1.4840310 \\ \mathrm{C} & -2.6791270 & 4.1853430 & -0.9091200 \\ \mathrm{C} & -3.2919500 & 5.3105240 & 1.5690170 \\ \mathrm{H} & -1.9269620 & 3.8583670 & 2.3887120 \\ \mathrm{C} & -3.5765370 & 5.2542600 & -0.8301860 \\ \mathrm{H} & -2.4407000 & 3.7553670 & -1.8790830 \\ \mathrm{C} & -3.8883170 & 5.8197040 & 0.411580 \\ \mathrm{H} & -3.5216100 & 5.7466870 & 2.5372550 \\ \mathrm{H} & -4.0280650 & 5.6476450 & -1.7367530 \\ \mathrm{H} & -4.5839200 & 6.6515250 & 0.4742240 \\ \mathrm{H} & 4.6400180 & 0.5562630 & 0.2808210 \\ \mathrm{O} & 5.5511380 & 0.9935370 & 0.1096450 \\ \mathrm{C} & 6.3794550 & 0.1925400 & -0.5153900 \\ \mathrm{O} & 6.1896900 & -0.9479880 & -0.8835480 \\ \mathrm{C} & 7.7403280 & 0.9030150 & -0.7578160 \\ \mathrm{~F} & 8.2996620 & 1.2878020 & 0.4115320 \\ \mathrm{~F} & 8.6064930 & 0.0914900 & -1.3837460 \\ \mathrm{~F} & 7.5783660 & 2.0081600 & -1.5198910 \\ & & & \end{array}$

$\begin{array}{cccc}\mathrm{C} & -5.3056810 & -1.2061500 & -0.1958200 \\ \mathrm{H} & -3.4660920 & -0.4595990 & 0.7127990 \\ \mathrm{Cl} & -0.4588620 & 0.6652590 & 0.3627440 \\ \mathrm{~N} & 0.9475810 & 0.4923970 & 1.6026870 \\ \mathrm{C} & 2.2560700 & 0.3560530 & 1.2366210 \\ \mathrm{C} & 0.7472150 & 0.5238770 & 2.9855990 \\ \mathrm{C} & 3.1122930 & 0.2815920 & 2.4885040 \\ \mathrm{C} & 2.1095970 & 0.3747720 & 3.6503370 \\ \mathrm{H} & 3.6829460 & -0.6508850 & 2.4712050 \\ \mathrm{H} & 2.2796910 & 1.2358750 & 4.3020250 \\ \mathrm{O} & -0.3349360 & 0.6474210 & 3.5288400 \\ \mathrm{O} & 2.6484770 & 0.3065740 & 0.0646700 \\ \mathrm{C} & -6.0507950 & -1.2070520 & 1.1569640 \\ \mathrm{H} & -7.1097410 & -0.9876390 & 0.9863340 \\ \mathrm{H} & -5.9976320 & -2.1741710 & 1.6619650 \\ \mathrm{H} & -5.6596430 & -0.4399380 & 1.8350970 \\ \mathrm{C} & -5.6311700 & 0.1173390 & -0.9181900 \\ \mathrm{H} & -5.3589230 & 0.9877870 & -0.3121200 \\ \mathrm{H} & -5.1226210 & 0.1933590 & -1.8831280 \\ \mathrm{H} & -6.7089750 & 0.1708090 & -1.1031470 \\ \mathrm{C} & -5.8019640 & -2.3836980 & -1.0555470 \\ \mathrm{H} & -5.5617200 & -3.3511810 & -0.6030570 \\ \mathrm{H} & -6.8909000 & -2.3288760 & -1.1554380 \\ \mathrm{H} & -5.3799250 & -2.3536320 & -2.0649510 \\ \mathrm{H} & 2.0959750 & -0.5195130 & 4.2794210 \\ \mathrm{H} & 3.8300110 & 1.1073040 & 2.4710170 \\ \mathrm{H} & 4.1144480 & 0.0888980 & -0.3798640 \\ \mathrm{O} & 5.0452210 & 0.0058580 & -0.7987800 \\ \mathrm{C} & 5.7380100 & -0.9802450 & -0.2824840 \\ \mathrm{O} & 5.4095180 & -1.7524050 & 0.5938620 \\ \mathrm{C} & 7.1364070 & -1.0694720 & -0.9546840 \\ \mathrm{~F} & 7.8324010 & 0.0756200 & -0.7733370 \\ \mathrm{~F} & 7.8575600 & -2.0780460 & -0.4416560 \\ \mathrm{~F} & 7.0203220 & -1.2743060 & -2.2859970 \\ \mathrm{H} & -2.7323200 & 2.5690050 & -2.7113840 \\ \mathrm{C} & -1.5332080 & 3.5590400 & -1.2531360 \\ \mathrm{C} & -2.5125380 & 4.3185530 & -0.5928070 \\ \mathrm{C} & -0.2029150 & 3.9987030 & -1.2017000 \\ \mathrm{C} & -2.1704710 & 5.4771020 & 0.1103750 \\ \mathrm{H} & -3.5533530 & 4.0055340 & -0.6331790 \\ \mathrm{C} & 0.1445290 & 5.1604160 & -0.5036630 \\ \mathrm{H} & 0.5685970 & 3.4268470 & -1.7107320 \\ \mathrm{C} & -0.8382740 & 5.9026070 & 0.1578690 \\ \mathrm{H} & -2.9437210 & 6.0502890 & 0.6143400 \\ \mathrm{H} & 1.1815390 & 5.4836250 & -0.4778760 \\ \mathrm{H} & -0.5709840 & 6.8047820 & 0.7005110\end{array}$

$2 . \log$

Potential Energy $=-2233.46737$

Zero-point Energy $=-2232.93698$

Free Energy $=-2232.98725$

Single-Point Energy B3LYP-D3(BJ)/6-311+G** PCM = 2234.03933

Free Energy B3LYP-D3(BJ)/6-311+G** PCM (extrapolated free energy from $\mathrm{qRRHO})=-2233.55921$

Nimag $=1\left(-167.5942 \mathrm{~cm}^{-1}\right)$

Charge $=0$ Multiplicity $=1$

$\begin{array}{lrrr}\mathrm{C} & -3.7582770 & -1.2903020 & 0.0612160 \\ \mathrm{C} & -2.6282090 & -3.3491590 & -0.1830060 \\ \mathrm{C} & -2.4951300 & -2.6227220 & -1.5246840 \\ \mathrm{~N} & -2.9446640 & -1.2565110 & -1.1712110 \\ \mathrm{~N} & -3.3054780 & -2.5488370 & 0.6713530 \\ \mathrm{O} & -2.1764780 & -4.4674510 & 0.0727590 \\ \mathrm{C} & -3.4510990 & -2.8829010 & 2.0839360 \\ \mathrm{H} & -2.5559740 & -3.4194740 & 2.4049950 \\ \mathrm{H} & -3.5495660 & -1.9654210 & 2.6655410 \\ \mathrm{H} & -4.3215950 & -3.5197630 & 2.2672320 \\ \mathrm{C} & -2.3370910 & -0.1428660 & -1.6402840 \\ \mathrm{H} & -1.7237270 & -0.3211680 & -2.5186430 \\ \mathrm{C} & -2.3848910 & 1.1471720 & -1.1532920 \\ \mathrm{C} & -1.9172930 & 2.3062050 & -2.0198820 \\ \mathrm{H} & -1.0772150 & 1.9837600 & -2.6448560 \\ \mathrm{H} & -3.0629580 & 1.4002420 & -0.3459600 \\ \mathrm{C} & -1.0892480 & -2.7408880 & -2.1053500 \\ \mathrm{H} & -0.8380130 & -3.8029610 & -2.1613090 \\ \mathrm{H} & -1.0345800 & -2.3312140 & -3.1168600 \\ \mathrm{H} & -0.3491290 & -2.2411590 & -1.4740110 \\ \mathrm{H} & -3.2074580 & -3.0775090 & -2.2256660\end{array}$

$3 . \log$

Potential Energy = -2233.46751

Zero-point Energy $=-2232.93690$

Free Energy $=-2232.98708$

Single-Point Energy B3LYP-D3(BJ)/6-311+G** PCM = 2234.04096

Free Energy B3LYP-D3(BJ)/6-311+G** PCM (extrapolated

free energy from $\mathrm{qRRHO})=-2233.56052$

Nimag $=1\left(-185.2852 \mathrm{~cm}^{-1}\right)$

Charge $=0$ Multiplicity $=1$

$\begin{array}{lrrr}\mathrm{C} & 4.1883060 & -0.5942920 & -0.1173580 \\ \mathrm{C} & 3.8784760 & -1.1507810 & 2.1561150 \\ \mathrm{C} & 3.5007990 & 0.3270880 & 2.0199240 \\ \mathrm{~N} & 3.4264230 & 0.4820260 & 0.5489490 \\ \mathrm{~N} & 4.2126800 & -1.6218250 & 0.9332250 \\ \mathrm{O} & 3.8595620 & -1.7994760 & 3.2042620 \\ \mathrm{C} & 4.4555190 & -3.0407760 & 0.6963100 \\ \mathrm{H} & 3.8036710 & -3.6175260 & 1.3556340 \\ \mathrm{H} & 4.2203530 & -3.2799180 & -0.3415020 \\ \mathrm{H} & 5.4930890 & -3.3193510 & 0.9031320 \\ \mathrm{C} & 2.4785970 & 1.2282170 & -0.0587720 \\ \mathrm{H} & 1.9863450 & 1.9304810 & 0.6053870 \\ \mathrm{C} & 2.0566760 & 1.1885710 & -1.3731780 \\ \mathrm{C} & 1.2203700 & 2.2940210 & -2.0057160 \\ \mathrm{H} & 0.4867730 & 1.8314010 & -2.6764420 \\ \mathrm{H} & 2.5954070 & 0.5796870 & -2.0904700 \\ \mathrm{C} & 2.2315120 & 0.6678470 & 2.7949610 \\ \mathrm{H} & 2.3769340 & 0.3546660 & 3.8317380 \\ \mathrm{H} & 2.0346480 & 1.7424710 & 2.7949010\end{array}$




\begin{tabular}{|c|c|c|c|}
\hline $\mathrm{H}$ & 1.3612070 & 0.1425800 & 2.3912820 \\
\hline $\mathrm{H}$ & 4.3312890 & 0.9264020 & 2.4154520 \\
\hline $\mathrm{C}$ & 5.6058360 & -0.1469850 & -0.6275030 \\
\hline $\mathrm{H}$ & 3.6122030 & -0.9752390 & -0.9676110 \\
\hline $\mathrm{Cl}$ & 0.4071210 & -0.5897190 & -0.9327190 \\
\hline $\mathrm{N}$ & -0.9070010 & -1.9556400 & -0.8533110 \\
\hline $\mathrm{C}$ & -2.2433120 & -1.6950980 & -0.7623890 \\
\hline $\mathrm{C}$ & -0.6000060 & -3.3169400 & -0.9140380 \\
\hline $\mathrm{C}$ & -3.0036540 & -3.0101370 & -0.7489200 \\
\hline $\mathrm{C}$ & -1.9118680 & -4.0888190 & -0.8398800 \\
\hline $\mathrm{H}$ & -3.5998200 & -3.0634710 & 0.1662620 \\
\hline $\mathrm{H}$ & -1.9996480 & -4.7199460 & -1.7282390 \\
\hline $\mathrm{O}$ & 0.5243860 & -3.7740500 & -1.0084380 \\
\hline $\mathrm{O}$ & -2.7276790 & -0.5578660 & -0.7031980 \\
\hline $\mathrm{C}$ & 6.2962370 & -1.3349560 & -1.3328370 \\
\hline $\mathrm{H}$ & 7.2102860 & -0.9823460 & -1.8215390 \\
\hline $\mathrm{H}$ & 6.5858410 & -2.1234850 & -0.6346910 \\
\hline $\mathrm{H}$ & 5.6569620 & -1.7745910 & -2.1069850 \\
\hline $\mathrm{C}$ & 5.4443760 & 0.9896650 & -1.6577550 \\
\hline $\mathrm{H}$ & 4.8757600 & 0.6644600 & -2.5353570 \\
\hline $\mathrm{H}$ & 4.9501010 & 1.8653290 & -1.2285500 \\
\hline $\mathrm{H}$ & 6.4341220 & 1.3023110 & -2.0062140 \\
\hline $\mathrm{C}$ & 6.4934980 & 0.3465150 & 0.5305170 \\
\hline $\mathrm{H}$ & 6.6114410 & -0.4136630 & 1.3094850 \\
\hline $\mathrm{H}$ & 7.4919800 & 0.5832970 & 0.1489380 \\
\hline $\mathrm{H}$ & 6.0942190 & 1.2578310 & 0.9865380 \\
\hline $\mathrm{H}$ & -1.8823840 & -4.7468080 & 0.0329320 \\
\hline $\mathrm{H}$ & -3.6966200 & -3.0244700 & -1.5953440 \\
\hline $\mathrm{H}$ & -4.2322830 & -0.2478210 & -0.5574580 \\
\hline $\mathrm{O}$ & -5.2041920 & 0.0770120 & -0.5262050 \\
\hline $\mathrm{C}$ & -5.8759540 & -0.4537370 & 0.4665300 \\
\hline $\mathrm{O}$ & -5.4869730 & -1.2437450 & 1.3013030 \\
\hline $\mathrm{C}$ & -7.3421750 & 0.0618870 & 0.4648040 \\
\hline $\mathrm{F}$ & -7.9755770 & -0.2992870 & -0.6745780 \\
\hline $\mathrm{F}$ & -8.0339860 & -0.4394450 & 1.4995160 \\
\hline $\mathrm{F}$ & -7.3840980 & 1.4102930 & 0.5488230 \\
\hline $\mathrm{H}$ & 1.8847310 & 2.8838230 & -2.6526390 \\
\hline $\mathrm{C}$ & 0.5110260 & 3.2357420 & -1.0511320 \\
\hline $\mathrm{C}$ & -0.7552280 & 2.9240800 & -0.5300640 \\
\hline $\mathrm{C}$ & 1.1077490 & 4.4489580 & -0.6731060 \\
\hline $\mathrm{C}$ & -1.4041060 & 3.7996080 & 0.3463260 \\
\hline $\mathrm{H}$ & -1.2357390 & 1.9911900 & -0.8111460 \\
\hline $\mathrm{C}$ & 0.4626350 & 5.3270830 & 0.2037950 \\
\hline $\mathrm{H}$ & 2.0842520 & 4.7111390 & -1.0737000 \\
\hline $\mathrm{C}$ & -0.7972250 & 5.0041030 & 0.7174190 \\
\hline $\mathrm{H}$ & -2.3855250 & 3.5424920 & 0.7351010 \\
\hline $\mathrm{H}$ & 0.9412330 & 6.2624820 & 0.4799900 \\
\hline $\mathrm{H}$ & -1.3029920 & 5.6856610 & 1.3953180 \\
\hline
\end{tabular}

4.log

Potential Energy $=-2233.46733$

Zero-point Energy $=-2232.93678$

Free Energy $=-2232.98692$

Single-Point Energy B3LYP-D3(BJ)/6-311+G** PCM = 2234.03906

Free Energy B3LYP-D3(BJ)/6-311+G** PCM (extrapolated free energy from $q R R H O)=-2233.55865$

Nimag $=1\left(-169.1557 \mathrm{~cm}^{-1}\right)$

Charge $=0$ Multiplicity $=1$

$\begin{array}{llll}\mathrm{C} & 2.3315200 & -2.1027860 & -0.5619230 \\ \mathrm{C} & 2.2387070 & -3.1470730 & 1.5532530 \\ \mathrm{C} & 3.4184580 & -2.1721090 & 1.6073580 \\ \mathrm{~N} & 3.2012690 & -1.3704900 & 0.3811250 \\ \mathrm{~N} & 1.6461990 & -3.0365800 & 0.3426470 \\ \mathrm{O} & 1.8832200 & -3.8897320 & 2.4709260 \\ \mathrm{C} & 0.3886080 & -3.7106670 & 0.0388320 \\ \mathrm{H} & -0.2096270 & -3.7521820 & 0.9513070 \\ \mathrm{H} & -0.1521440 & -3.1446890 & -0.7206940 \\ \mathrm{H} & 0.5468520 & -4.7322810 & -0.3192110 \\ \mathrm{C} & 3.3978360 & -0.0330410 & 0.3386070 \\ \mathrm{H} & 4.0277540 & 0.3384410 & 1.1416030 \\ \mathrm{C} & 2.9031380 & 0.8916660 & -0.5581840 \\ \mathrm{C} & 3.5209430 & 2.2783460 & -0.6446620 \\ \mathrm{H} & 3.8577810 & 2.5942750 & 0.3488150 \\ \mathrm{H} & 2.3507570 & 0.5614480 & -1.4309200\end{array}$

\begin{tabular}{|c|c|c|c|}
\hline $\mathrm{C}$ & 3.4557230 & -1.3954720 & 2.9205180 \\
\hline $\mathrm{H}$ & 3.4479790 & -2.1198850 & 3.7387680 \\
\hline $\mathrm{H}$ & 4.3660680 & -0.7982920 & 3.0116430 \\
\hline $\mathrm{H}$ & 2.5848790 & -0.7421020 & 3.0251070 \\
\hline $\mathrm{H}$ & 4.3438680 & -2.7552940 & 1.5121720 \\
\hline $\mathrm{C}$ & 3.1112390 & -2.7874540 & -1.7416700 \\
\hline $\mathrm{H}$ & 1.5905280 & -1.4153090 & -0.9841910 \\
\hline $\mathrm{Cl}$ & 0.8819810 & 1.1958520 & 0.8787330 \\
\hline $\mathrm{N}$ & -0.7154320 & 1.5425790 & 1.8148560 \\
\hline $\mathrm{C}$ & -0.7413320 & 2.1682860 & 3.0645770 \\
\hline $\mathrm{C}$ & -1.9517230 & 1.2320780 & 1.3259730 \\
\hline $\mathrm{C}$ & -2.2000130 & 2.2936200 & 3.4854020 \\
\hline $\mathrm{C}$ & -3.0026220 & 1.6687260 & 2.3322340 \\
\hline $\mathrm{H}$ & -2.3370770 & 1.7745770 & 4.4378270 \\
\hline $\mathrm{H}$ & -3.6857230 & 2.3722520 & 1.8477680 \\
\hline $\mathrm{O}$ & -2.1506510 & 0.6838830 & 0.2353700 \\
\hline $\mathrm{O}$ & 0.2428160 & 2.5350500 & 3.6794220 \\
\hline $\mathrm{C}$ & 2.1056600 & -3.4793470 & -2.6880620 \\
\hline $\mathrm{H}$ & 2.6293470 & -3.8139260 & -3.5894260 \\
\hline $\mathrm{H}$ & 1.6448130 & -4.3602730 & -2.2355380 \\
\hline $\mathrm{H}$ & 1.3095770 & -2.7950880 & -3.0034620 \\
\hline $\mathrm{C}$ & 3.8631590 & -1.7103630 & -2.5503470 \\
\hline $\mathrm{H}$ & 3.1758280 & -0.9850530 & -2.9982170 \\
\hline $\mathrm{H}$ & 4.5877260 & -1.1685180 & -1.9364530 \\
\hline $\mathrm{H}$ & 4.4106430 & -2.1912080 & -3.3675700 \\
\hline $\mathrm{C}$ & 4.1241330 & -3.8249230 & -1.2222470 \\
\hline $\mathrm{H}$ & 3.6443560 & -4.5946060 & -0.6090940 \\
\hline $\mathrm{H}$ & 4.5980510 & -4.3287180 & -2.0709270 \\
\hline $\mathrm{H}$ & 4.9198960 & -3.3549130 & -0.6359400 \\
\hline $\mathrm{H}$ & -3.5867580 & 0.7948250 & 2.6344040 \\
\hline $\mathrm{H}$ & -2.4289170 & 3.3504560 & 3.6472890 \\
\hline $\mathrm{H}$ & -3.5447110 & 0.3194480 & -0.3362230 \\
\hline $\mathrm{O}$ & -4.4265390 & 0.1355780 & -0.8220400 \\
\hline $\mathrm{C}$ & -5.0199380 & -0.9518760 & -0.3915060 \\
\hline $\mathrm{O}$ & -4.6506440 & -1.7218310 & 0.4697350 \\
\hline $\mathrm{C}$ & -6.3572960 & -1.1735390 & -1.1521000 \\
\hline $\mathrm{F}$ & -6.1470400 & -1.2793560 & -2.4833810 \\
\hline $\mathrm{F}$ & -7.2027030 & -0.1372190 & -0.9503450 \\
\hline $\mathrm{F}$ & -6.9699500 & -2.2937630 & -0.7392860 \\
\hline $\mathrm{H}$ & 4.4248440 & 2.2131120 & -1.2693980 \\
\hline $\mathrm{C}$ & 2.6165400 & 3.3422430 & -1.2410680 \\
\hline $\mathrm{C}$ & 2.2649410 & 3.2989250 & -2.5999490 \\
\hline $\mathrm{C}$ & 2.1291660 & 4.4002870 & -0.4608750 \\
\hline $\mathrm{C}$ & 1.4388470 & 4.2773500 & -3.1602500 \\
\hline $\mathrm{H}$ & 2.6449680 & 2.4969470 & -3.2286620 \\
\hline $\mathrm{C}$ & 1.3061900 & 5.3853520 & -1.0176290 \\
\hline $\mathrm{H}$ & 2.3941980 & 4.4540990 & 0.5918820 \\
\hline $\mathrm{C}$ & 0.9551470 & 5.3255670 & -2.3694860 \\
\hline $\mathrm{H}$ & 1.1789060 & 4.2250070 & -4.2138720 \\
\hline $\mathrm{H}$ & 0.9407340 & 6.1971300 & -0.3947480 \\
\hline $\mathrm{H}$ & 0.3161190 & 6.0889550 & -2.8038810 \\
\hline
\end{tabular}

$5 . \log$

Potential Energy $=-2233.46755$

Zero-point Energy = -2232.93690

Free Energy = -2232.98702

Single-Point Energy B3LYP-D3(BJ)/6-311+G** PCM = 2234.04042

Free Energy B3LYP-D3(BJ)/6-311+G** PCM (extrapolated

free energy from $\mathrm{qRRHO})=-2233.55989$

Nimag $=1\left(-182.4978 \mathrm{~cm}^{-1}\right)$

Charge $=0$ Multiplicity $=1$

$\begin{array}{lrrr}\mathrm{C} & -1.8954910 & 2.4654130 & -0.1473520 \\ \mathrm{C} & -2.4465080 & 2.5194600 & 2.1477600 \\ \mathrm{C} & -3.5036680 & 1.6414560 & 1.4713940 \\ \mathrm{~N} & -2.8977740 & 1.4181920 & 0.1383960 \\ \mathrm{~N} & -1.5567540 & 2.9171320 & 1.2097250 \\ \mathrm{O} & -2.4091360 & 2.7967130 & 3.3483490 \\ \mathrm{C} & -0.3303870 & 3.6235060 & 1.5637370 \\ \mathrm{H} & 0.0054000 & 3.2645020 & 2.5387600 \\ \mathrm{H} & 0.4374120 & 3.4127050 & 0.8181500 \\ \mathrm{H} & -0.4830430 & 4.7050210 & 1.6259610 \\ \mathrm{C} & -2.9475610 & 0.2264450 & -0.4958300 \\ \mathrm{H} & -3.7074990 & -0.4422300 & -0.1061660 \\ \mathrm{C} & -2.1558650 & -0.2368410 & -1.5282260 \\ \mathrm{C} & -2.5159530 & -1.4591580 & -2.3634370\end{array}$




\begin{tabular}{|c|c|c|c|}
\hline $\mathrm{H}$ & -1.5925380 & -2.0026160 & -2.5950300 \\
\hline $\mathrm{H}$ & -1.4638300 & 0.4394710 & -2.0172750 \\
\hline $\mathrm{C}$ & -3.8159240 & 0.3894130 & 2.2849650 \\
\hline $\mathrm{H}$ & -4.1003920 & 0.7056680 & 3.2915570 \\
\hline $\mathrm{H}$ & -4.6538630 & -0.1688090 & 1.8609110 \\
\hline $\mathrm{H}$ & -2.9457300 & -0.2687580 & 2.3598130 \\
\hline $\mathrm{H}$ & -4.4206340 & 2.2361940 & 1.3673570 \\
\hline $\mathrm{C}$ & -2.4134340 & 3.5981870 & -1.1048680 \\
\hline $\mathrm{H}$ & -1.0081520 & 2.0064930 & -0.5963350 \\
\hline $\mathrm{Cl}$ & -0.4156240 & -1.0628910 & 0.0157620 \\
\hline $\mathrm{N}$ & 1.0554580 & -1.7356210 & 1.0069190 \\
\hline $\mathrm{C}$ & 0.9047710 & -2.4619160 & 2.1912860 \\
\hline $\mathrm{C}$ & 2.3545090 & -1.5605350 & 0.6291690 \\
\hline $\mathrm{C}$ & 2.2983210 & -2.8256120 & 2.6888120 \\
\hline $\mathrm{C}$ & 3.2602340 & -2.2352950 & 1.6447800 \\
\hline $\mathrm{H}$ & 2.4348770 & -2.4029780 & 3.6879930 \\
\hline $\mathrm{H}$ & 3.8599570 & -2.9935780 & 1.1326680 \\
\hline $\mathrm{O}$ & 2.7085210 & -0.9459070 & -0.3851650 \\
\hline $\mathrm{O}$ & -0.1622890 & -2.7376270 & 2.7079140 \\
\hline $\mathrm{C}$ & -1.2736110 & 4.6067590 & -1.3674340 \\
\hline $\mathrm{H}$ & -1.5810860 & 5.3021900 & -2.1550980 \\
\hline $\mathrm{H}$ & -1.0328160 & 5.2032460 & -0.4845970 \\
\hline $\mathrm{H}$ & -0.3600180 & 4.1056060 & -1.7073690 \\
\hline $\mathrm{C}$ & -2.8213270 & 2.9776600 & -2.4569360 \\
\hline $\mathrm{H}$ & -1.9720750 & 2.5003080 & -2.9570790 \\
\hline $\mathrm{H}$ & -3.6188800 & 2.2382250 & -2.3448110 \\
\hline $\mathrm{H}$ & -3.1892620 & 3.7681420 & -3.1191300 \\
\hline $\mathrm{C}$ & -3.6238630 & 4.3378370 & -0.5049710 \\
\hline $\mathrm{H}$ & -3.3967220 & 4.7663760 & 0.4765180 \\
\hline $\mathrm{H}$ & -3.9106920 & 5.1626800 & -1.1652690 \\
\hline $\mathrm{H}$ & -4.4932370 & & -0.4084250 \\
\hline $\mathrm{H}$ & 3.9490090 & -1.4902400 & 2.0525720 \\
\hline $\mathrm{H}$ & 2.3683420 & -3.9130990 & 2.7764500 \\
\hline $\mathrm{H}$ & 4.1768120 & -0.6857380 & -0.7844390 \\
\hline $\mathrm{O}$ & 5.1050350 & -0.4999680 & -1.1778690 \\
\hline $\mathrm{C}$ & 5.9092430 & 0.0666600 & -0.3113950 \\
\hline $\mathrm{O}$ & 5.6811260 & 0.3675700 & 0.8417500 \\
\hline $\mathrm{C}$ & 7.2980440 & 0.3338390 & -0.9557670 \\
\hline $\mathrm{F}$ & 7.1839570 & 1.1411450 & -2.0342400 \\
\hline $\mathrm{F}$ & 7.8691810 & -0.8215910 & -1.3651070 \\
\hline $\mathrm{F}$ & 8.1319770 & 0.9220430 & -0.0844920 \\
\hline $\mathrm{C}$ & -3.5280870 & -2.4156830 & -1.7628530 \\
\hline $\mathrm{C}$ & -3.1215700 & -3.4830520 & -0.9467190 \\
\hline $\mathrm{C}$ & -4.8995220 & -2.2577460 & -2.0189570 \\
\hline $\mathrm{C}$ & -4.0581870 & -4.3641850 & -0.3974220 \\
\hline $\mathrm{H}$ & -2.0641620 & -3.6262680 & -0.7429340 \\
\hline $\mathrm{C}$ & -5.8404060 & -3.1354730 & -1.4711020 \\
\hline $\mathrm{H}$ & -5.2329840 & -1.4433750 & -2.6579670 \\
\hline $\mathrm{C}$ & -5.4218870 & -4.1923870 & -0.6564740 \\
\hline $\mathrm{H}$ & -3.7220210 & -5.1863310 & 0.2281730 \\
\hline $\mathrm{H}$ & -6.8964730 & -2.9966370 & -1.6849340 \\
\hline $\mathrm{H}$ & -6.1499360 & -4.8781210 & -0.2328000 \\
\hline $\mathrm{H}$ & -2.8999740 & -1.0999900 & -3.3287060 \\
\hline
\end{tabular}

\begin{tabular}{|c|c|c|c|}
\hline $\mathrm{C}$ & 2.4816200 & 0.7604280 & -0.2634450 \\
\hline $\mathrm{C}$ & 3.2844710 & 2.0057710 & -0.5947880 \\
\hline $\mathrm{H}$ & 3.6370040 & 2.4697750 & 0.3334060 \\
\hline $\mathrm{H}$ & 2.6297400 & 2.7341880 & -1.0850930 \\
\hline $\mathrm{H}$ & 1.9986110 & 0.2738240 & -1.1036760 \\
\hline $\mathrm{C}$ & 2.3453660 & -0.5498460 & 3.7317600 \\
\hline $\mathrm{H}$ & 2.1455110 & -1.0165650 & 4.6994110 \\
\hline $\mathrm{H}$ & 3.3410920 & -0.1023330 & 3.7762970 \\
\hline $\mathrm{H}$ & 1.6036240 & 0.2366230 & 3.5655830 \\
\hline $\mathrm{H}$ & 3.0670280 & -2.3628690 & 2.8329900 \\
\hline $\mathrm{C}$ & 2.1387220 & -3.1044010 & -0.4137870 \\
\hline $\mathrm{H}$ & 0.8332100 & -1.3619450 & -0.2558050 \\
\hline $\mathrm{Cl}$ & 0.4146430 & 1.7537730 & 0.6256440 \\
\hline $\mathrm{N}$ & -1.2047800 & 2.6239330 & 1.0939920 \\
\hline $\mathrm{C}$ & -1.2435580 & 3.8560640 & 1.7510980 \\
\hline $\mathrm{C}$ & -2.4366310 & 2.1137700 & 0.8043730 \\
\hline $\mathrm{C}$ & -2.7082240 & 4.2312820 & 1.9409610 \\
\hline $\mathrm{C}$ & -3.5001310 & 3.0757400 & 1.3068350 \\
\hline $\mathrm{H}$ & -2.9037780 & 4.3526370 & 3.0098740 \\
\hline $\mathrm{H}$ & -4.1211890 & 3.3884170 & 0.4624850 \\
\hline $\mathrm{O}$ & -2.6252310 & 1.0372570 & 0.2243800 \\
\hline $\mathrm{O}$ & -0.2647480 & 4.4910920 & 2.0984270 \\
\hline $\mathrm{C}$ & 1.1094350 & -3.8821040 & -1.2631480 \\
\hline $\mathrm{H}$ & 1.6400410 & -4.5327380 & -1.9657880 \\
\hline $\mathrm{H}$ & 0.4632980 & -4.5199400 & -0.6558920 \\
\hline $\mathrm{H}$ & 0.4769630 & -3.2073350 & -1.8513050 \\
\hline $\mathrm{C}$ & 3.1315550 & -2.4217270 & -1.3763380 \\
\hline $\mathrm{H}$ & 2.6199090 & -1.7647560 & -2.0874490 \\
\hline $\mathrm{H}$ & 3.8843410 & -1.8356920 & -0.8425800 \\
\hline $\mathrm{H}$ & 3.6547810 & -3.1885730 & -1.9567520 \\
\hline $\mathrm{C}$ & 2.9066870 & -4.0884670 & 0.4883160 \\
\hline $\mathrm{H}$ & 2.2508190 & -4.5732620 & 1.2187170 \\
\hline $\mathrm{H}$ & 3.3508370 & -4.8766900 & -0.1281520 \\
\hline $\mathrm{H}$ & 3.7235670 & -3.5940070 & 1.0231170 \\
\hline $\mathrm{H}$ & -4.1462300 & 2.5512260 & 2.0165030 \\
\hline $\mathrm{H}$ & -2.8897900 & 5.1955210 & 1.4586970 \\
\hline $\mathrm{C}$ & 4.4703020 & 1.7196880 & -1.5059130 \\
\hline $\mathrm{C}$ & 4.3315710 & 1.7802630 & -2.9008330 \\
\hline $\mathrm{C}$ & 5.7218490 & 1.3730790 & -0.9735710 \\
\hline $\mathrm{C}$ & 5.4108830 & 1.4944170 & -3.7433460 \\
\hline $\mathrm{H}$ & 3.3720330 & 2.0573410 & -3.3312680 \\
\hline $\mathrm{C}$ & 6.8046510 & 1.0893940 & -1.8111300 \\
\hline $\mathrm{H}$ & 5.8522220 & 1.3289230 & 0.1051290 \\
\hline $\mathrm{C}$ & 6.6520820 & 1.1468460 & -3.2007470 \\
\hline $\mathrm{H}$ & 5.2832590 & 1.5488650 & -4.8209370 \\
\hline $\mathrm{H}$ & 7.7668150 & 0.8283910 & -1.3791320 \\
\hline $\mathrm{H}$ & 7.4927940 & 0.9287080 & -3.8529590 \\
\hline $\mathrm{H}$ & -4.0145400 & 0.4493170 & -0.1154240 \\
\hline $\mathrm{O}$ & -4.9256270 & 0.0070650 & -0.2683420 \\
\hline $\mathrm{C}$ & -5.1716100 & -0.2196350 & -1.5363600 \\
\hline $\mathrm{O}$ & -4.4712630 & 0.0226760 & -2.4963950 \\
\hline $\mathrm{C}$ & -6.5656130 & -0.8870050 & -1.7031680 \\
\hline $\mathrm{F}$ & -7.5419330 & -0.1027060 & -1.1933220 \\
\hline $\mathrm{F}$ & -6.6121780 & -2.0742710 & -1.0571920 \\
\hline $\mathrm{F}$ & -6.8459540 & -1.1085250 & -2.9966490 \\
\hline
\end{tabular}

$6 . \log$

Potential Energy $=-2233.46970$

Zero-point Energy = -2232.93905

Free Energy = -2232.98951

Single-Point Energy B3LYP-D3(BJ)/6-311+G** PCM = 2234.04006

Free Energy B3LYP-D3(BJ)/6-311+G** PCM (extrapolated free energy from $\mathrm{qRRHO})=-2233.55987$

Nimag $=1\left(-169.4774 \mathrm{~cm}^{-1}\right)$

Charge $=0$ Multiplicity $=1$

$\begin{array}{lccc}\mathrm{C} & 1.3950860 & -2.0067280 & 0.4283510 \\ \mathrm{C} & 0.9290820 & -2.3898500 & 2.7111870 \\ \mathrm{C} & 2.2618500 & -1.6362050 & 2.6642300 \\ \mathrm{~N} & 2.2935500 & -1.1746490 & 1.2565240 \\ \mathrm{~N} & 0.4738700 & -2.5331540 & 1.4457330 \\ \mathrm{O} & 0.3645460 & -2.7796200 & 3.7352760 \\ \mathrm{C} & -0.8556250 & -3.0632200 & 1.1627250 \\ \mathrm{H} & -1.5286490 & -2.7526120 & 1.9644640 \\ \mathrm{H} & -1.2129920 & -2.6583030 & 0.2151990 \\ \mathrm{H} & -0.8587130 & -4.1559960 & 1.1107260 \\ \mathrm{C} & 2.7184810 & 0.0565190 & 0.9028700 \\ \mathrm{H} & 3.3063360 & 0.5501230 & 1.6708350\end{array}$

Potential Energy $=-2233.46969$

Zero-point Energy = -2232.93889

Free Energy = -2232.98902

Single-Point Energy B3LYP-D3(BJ)/6-311+G** PCM = 2234.04007

Free Energy B3LYP-D3(BJ)/6-311+G** PCM (extrapolated free energy from $\mathrm{qRRHO})=-2233.55940$

Nimag $=1\left(-169.2657 \mathrm{~cm}^{-1}\right)$

Charge $=0$ Multiplicity $=1$

$\begin{array}{llll}\mathrm{C} & 1.2996140 & -1.9352750 & 0.6324310 \\ \mathrm{C} & 0.8454910 & -2.0783730 & 2.9448770 \\ \mathrm{C} & 2.1951360 & -1.3657420 & 2.8145450 \\ \mathrm{~N} & 2.2263070 & -1.0484250 & 1.3674750 \\ \mathrm{~N} & 0.3752800 & -2.3351570 & 1.7031670 \\ \mathrm{O} & 0.2806820 & -2.3493760 & 4.0065110 \\ \mathrm{C} & -0.9729830 & -2.8479740 & 1.4832690 \\ \mathrm{H} & -1.6266920 & -2.4412530 & 2.2575250 \\ \mathrm{H} & -1.3292360 & -2.5240510 & 0.5046630\end{array}$




$\begin{array}{lrrr}\mathrm{H} & -1.0101160 & -3.9399670 & 1.5361330 \\ \mathrm{C} & 2.6817160 & 0.1287730 & 0.8893260 \\ \mathrm{H} & 3.2897080 & 0.6797960 & 1.6005110 \\ \mathrm{C} & 2.4539930 & 0.7190740 & -0.3400200 \\ \mathrm{C} & 3.2881840 & 1.9015090 & -0.7995490 \\ \mathrm{H} & 3.6639790 & 2.4444070 & 0.0752150 \\ \mathrm{H} & 2.6492810 & 2.5969230 & -1.3544130 \\ \mathrm{H} & 1.9490470 & 0.1656530 & -1.1241720 \\ \mathrm{C} & 2.3095850 & -0.1794730 & 3.7667990 \\ \mathrm{H} & 2.1062350 & -0.5418190 & 4.7775200 \\ \mathrm{H} & 3.3147540 & 0.2486050 & 3.7613670 \\ \mathrm{H} & 1.5837030 & 0.6021890 & 3.5251810 \\ \mathrm{H} & 2.9850590 & -2.0900320 & 3.0515190 \\ \mathrm{C} & 2.0088670 & -3.1294120 & -0.1008830 \\ \mathrm{H} & 0.7466410 & -1.3475560 & -0.1080210 \\ \mathrm{Cl} & 0.4247100 & 1.8592730 & 0.4627130 \\ \mathrm{~N} & -1.1594390 & 2.8222390 & 0.8609130 \\ \mathrm{C} & -1.1525850 & 4.0630080 & 1.5028280 \\ \mathrm{C} & -2.4072310 & 2.3762320 & 0.5347240 \\ \mathrm{H}\end{array}$

$(R)$-TS-front

$\begin{array}{cc}\begin{array}{c}\text { Conformation } \\ \text { name }\end{array} & \begin{array}{c}\text { Extrapolated } \\ \text { Free energy } \\ (\mathrm{kcal} / \mathrm{mol})\end{array}\end{array}$

$\begin{array}{cc}\text { TS4-Rf-lowest } & 0.0 \\ 1 & 0.9 \\ 2 & 0.7 \\ 3 & 0.5 \\ 4 & 1.7 \\ 5 & 1.0\end{array}$

6

0.3

TS4-Rf-lowest.log

Potential Energy $=-2233.46593$

Zero-point Energy = -2232.93534

Free Energy = -2232.98602

Single-Point Energy B3LYP-D3(BJ)/6-311+G** PCM = 2234.03614

Free Energy B3LYP-D3(BJ)/6-311+G** PCM (extrapolated

free energy from $\mathrm{qRRHO})=-2233.55623$

Nimag $=1\left(-187.5221 \mathrm{~cm}^{-1}\right)$

Charge $=0$ Multiplicity $=1$

C $\quad 4.4000250 \quad-1.2425990 \quad-0.3$

$\begin{array}{llll}\text { C } & 3.2823380 & -0.8511910 & 1.7804990\end{array}$

$\mathrm{N} \quad 3.2966190 \quad-0.5371380 \quad 0.3392030$

$\begin{array}{llll}\mathrm{N} & 4.7121860 & -2.2984330 & 0.6045560\end{array}$

$\begin{array}{llll}\mathrm{O} & 4.4118610 & -2.7583960 & 2.8391880\end{array}$

C $\quad 5.4119630 \quad-3.5398940 \quad 0.2888250$

$\mathrm{H} \quad 4.9318380 \quad-4.3547750 \quad 0.8348710$

$\mathrm{H} \quad 5.3395200 \quad-3.7323540 \quad-0.7818300$

$\mathrm{H} \quad 6.4660880 \quad-3.5032030 \quad 0.5781510$

$\begin{array}{llll}\text { C } & 2.4552490 & 0.2707560 & -0.3356960\end{array}$

$\begin{array}{llll}\mathrm{H} & 2.6290590 & 0.2687660 & -1.4081080\end{array}$

$\begin{array}{llll}\text { C } & 1.4126760 & 1.0519050 & 0.1282470\end{array}$

$\begin{array}{llll}\text { C } & 0.8313370 & 2.1594600 & -0.7365040\end{array}$

$\begin{array}{llll}\mathrm{H} & -0.2476540 & 2.2259870 & -0.5591040\end{array}$

$\mathrm{H} \quad 1.2584990 \quad 1.1601240 \quad 1.1962360$

$\begin{array}{llll}\mathrm{Cl} & -0.2804220 & -0.6748720 & -0.3578210\end{array}$

$\mathrm{N} \quad-1.7415300 \quad-1.8426890 \quad-0.6698720$

$\begin{array}{llll}\text { C } & -3.0299030 & -1.5339690 & -0.3440030\end{array}$

$\begin{array}{llll}\text { C } & -1.5948840 & -3.0997180 & -1.2613000\end{array}$

$\begin{array}{llll}\text { C } & -3.9305470 & -2.6928160 & -0.7353360\end{array}$

C $\quad-2.9787460 \quad-3.7310010 \quad-1.3516370$

$\mathrm{H} \quad-4.4456250 \quad-3.0559920 \quad 0.1588480$

$\mathrm{H} \quad-2.9678620 \quad-4.6819150 \quad-0.8121960$

$\mathrm{H} \quad-3.1972920 \quad-3.9473700 \quad-2.4009530$

$\begin{array}{llll}\mathrm{H} & -4.6932290 & -2.3277820 & -1.4288260\end{array}$

$\begin{array}{llll}\mathrm{O} & -0.5371680 & -3.5770760 & -1.6297210\end{array}$

$\begin{array}{llll}\text { O } & -3.3781910 & -0.4715290 & 0.1866930\end{array}$

$\begin{array}{llll}\text { C } & 1.9305190 & -1.2417980 & 2.3917860\end{array}$

$\mathrm{H} \quad 2.1311490 \quad-1.6632810 \quad 3.3799310$

$\begin{array}{llll}\mathrm{H} & 1.2650980 & -0.3889660 & 2.5171320\end{array}$

H $\quad 1.4268290 \quad-1.9971680 \quad 1.7839970$

$\begin{array}{llll}\mathrm{H} & 3.7189960 & -0.0231740 & 2.3553590\end{array}$

$\begin{array}{llll}\text { C } & 5.6337740 & -0.3312190 & -0.7216980\end{array}$

$\mathrm{H} \quad 4.0126390 \quad-1.6900540 \quad-1.2831520$

$\begin{array}{llll}\text { C } & 6.7020710 & -1.1901250 & -1.4357510\end{array}$

$\mathrm{H} \quad 7.4936410 \quad-0.5365650 \quad-1.8155420$

H $7.1738590 \quad-1.9127300 \quad-0.7673170$

$\begin{array}{llll}\mathrm{H} & 6.2822170 & -1.7306060 & -2.2919510\end{array}$

C $\quad 5.2093590 \quad 0.7844970 \quad-1.6996710$

$\begin{array}{llll}\mathrm{H} & 4.7142980 & 0.3819690 & -2.5912450\end{array}$

$\mathrm{H} \quad 4.5491620 \quad 1.5202220 \quad-1.2336770$

$\begin{array}{llll}\mathrm{H} & 6.1016010 & 1.3219240 & -2.0363850\end{array}$

$\begin{array}{llll}\text { C } & 6.2432420 & 0.3078930 & 0.5393680\end{array}$

H $\quad 6.5846220 \quad-0.4464230 \quad 1.2553320$

$\begin{array}{llll}\mathrm{H} & 7.1115210 & 0.9137530 & 0.2606720\end{array}$

$\begin{array}{llll}\mathrm{H} & 5.5305390 & 0.9698650 & 1.0418080\end{array}$

$\mathrm{H} \quad-4.8350680 \quad-0.0901000 \quad 0.5245130$

$\begin{array}{llll}\text { O } & -5.7464270 & 0.2488840 & 0.8492910\end{array}$

$\begin{array}{llll}\text { C } & -6.6711790 & 0.1597230 & -0.0754620\end{array}$

$\begin{array}{llll}\mathrm{O} & -6.5650670 & -0.2636830 & -1.2076310\end{array}$

$\begin{array}{llll}\text { C } & -8.0293330 & 0.6888240 & 0.4640650\end{array}$

$\begin{array}{llll}\text { F } & -7.9277010 & 1.9818730 & 0.8455330\end{array}$

F $\quad-8.9878780 \quad 0.6069150 \quad-0.4714170$

F $\quad-8.4328190 \quad-0.0265920 \quad 1.5383770$

$\begin{array}{llll}\mathrm{H} & 0.9639280 & 1.9087710 & -1.7946990\end{array}$

$\begin{array}{llll}\text { C } & 1.4610620 & 3.5154190 & -0.4492710\end{array}$

$\begin{array}{llll}\text { C } & 1.0409270 & 4.2777710 & 0.6524450\end{array}$

$\begin{array}{llll}\text { C } & 2.4806960 & 4.0282750 & -1.2652020\end{array}$

C $\quad 1.6300410 \quad 5.5129400 \quad 0.9379380$

$\mathrm{H} \quad 0.2432920 \quad 3.9033180 \quad 1.2899060$

C $\quad 3.0712420 \quad 5.2651350 \quad-0.9857490$

$\begin{array}{llll}\mathrm{H} & 2.8120050 & 3.4587940 & -2.1300990\end{array}$

$\begin{array}{llll}\text { C } & 2.6496010 & 6.0110580 & 0.1193690\end{array}$ 


$\begin{array}{llllllll}\mathrm{H} & 1.2893030 & 6.0881620 & 1.7943710 & \mathrm{O} & 4.6588170 & 1.8799650 & 0.4834820 \\ \mathrm{H} & 3.8561480 & 5.6461990 & -1.6331910 & \mathrm{C} & 6.0608560 & 1.9834860 & -1.4829980 \\ \mathrm{H} & 3.1057850 & 6.9723950 & 0.3371990 & \mathrm{~F} & 5.5526130 & 2.3384090 & -2.6849450 \\ & & & & \mathrm{~F} & 6.5979500 & 3.0775880 & -0.9214390 \\ 1 . \log & & & & \mathrm{F} & 7.0569140 & 1.0960850 & -1.7034010\end{array}$

Potential Energy $=-2233.46286$

Zero-point Energy = -2232.93195

Free Energy $=-2232.98172$

Single-Point Energy B3LYP-D3(BJ)/6-311+G** PCM = 2234.03590

Free Energy B3LYP-D3(BJ)/6-311+G** PCM (extrapolated free energy from $\mathrm{qRRHO})=-2233.55476$

Nimag $=1\left(-208.1776 \mathrm{~cm}^{-1}\right)$

Charge $=0$ Multiplicity $=1$

$\begin{array}{llrr}\text { Charge }=0 \text { Multiplicity }=1 & \\ \mathrm{C} & -2.5843210 & 2.0334160 & -0.0069720 \\ \mathrm{C} & -2.5821120 & 2.5607910 & 2.2868460\end{array}$

$2 . \log$

Potential Energy = -2233.46590

Zero-point Energy = -2232.93524

Free Energy $=-2232.98560$

Single-Point Energy B3LYP-D3(BJ)/6-311+G** PCM = 2234.03546

Free Energy B3LYP-D3(BJ)/6-311+G** PCM (extrapolated

free energy from $\mathrm{qRRHO})=-2233.55515$

Nimag $=1\left(-185.9689 \mathrm{~cm}^{-1}\right)$

Charge $=0$ Multiplicity $=1$

$\begin{array}{llll}\mathrm{C} & -2.9226860 & 1.0687680 & 2.2118540\end{array}$

$\begin{array}{llll}\mathrm{N} & -2.6792770 & 0.7813400 & 0.7849360\end{array}$

$\mathrm{N} \quad-2.2926970 \quad 3.0084830 \quad 1.0447690$

$\begin{array}{llll}\mathrm{O} & -2.5153950 & 3.2209110 & 3.3252550\end{array}$

$\begin{array}{llll}\mathrm{C} & -1.6792330 & 4.3152890 & 0.8303970\end{array}$

$\mathrm{H} \quad-0.9761600 \quad 4.5035220 \quad 1.6446230$

$\mathrm{H} \quad-1.1397020 \quad 4.3095740 \quad-0.1170140$

$\mathrm{H} \quad-2.4189970 \quad 5.1208750 \quad 0.8192480$

C $\quad-2.5630360 \quad-0.4230960 \quad 0.1879840$

$\mathrm{H} \quad-2.3267310 \quad-0.3555180 \quad-0.8693490$

$\begin{array}{llll}\text { C } & -2.6692240 & -1.6897150 & 0.7303460\end{array}$

C $\quad-2.8922710 \quad-2.9393700 \quad-0.1178430$

$\mathrm{H} \quad-3.9416150 \quad-3.2358450 \quad 0.0160930$

H $\quad-2.2973280 \quad-3.7579180 \quad 0.3039740$

$\begin{array}{llll}\mathrm{H} & -2.9952970 & -1.7976610 & 1.7587640\end{array}$

$\mathrm{Cl} \quad-0.2520810 \quad-1.8798750 \quad 1.1905940$

$\begin{array}{llll}\mathrm{N} & 1.5513880 & -2.2587060 & 1.6481990\end{array}$

C $2.6188000 \quad-1.6692550 \quad 1.0362110$

$\begin{array}{llll}\text { C } & 1.9049880 & -3.1703490 & 2.6453420\end{array}$

C $\quad 3.9000400 \quad-2.2080000 \quad 1.6505560$

$\begin{array}{llll}\text { C } & 3.4264490 & -3.2051660 & 2.7207260\end{array}$

$\mathrm{H} \quad 4.5025060 \quad-2.6702330 \quad 0.8633460$

$\begin{array}{llll}\mathrm{H} & 3.7642430 & -4.2286750 & 2.5370690\end{array}$

$\mathrm{H} \quad 3.7345250 \quad-2.9305870 \quad 3.7332170$

$\mathrm{H} \quad 4.4748000 \quad-1.3725400 \quad 2.0602810$

$\begin{array}{llll}\mathrm{O} & 1.1153750 & -3.8083350 & 3.3175690\end{array}$

$\begin{array}{llll}\text { O } & 2.5275850 & -0.8339820 & 0.1278520\end{array}$

$\begin{array}{llll}\text { C } & -2.0603430 & 0.3214770 & 3.2367250\end{array}$

$\begin{array}{llll}\mathrm{H} & -2.2023060 & 0.8174120 & 4.2002270\end{array}$

$\mathrm{H} \quad-2.3512480 \quad-0.7220030 \quad 3.3484030$

$\begin{array}{llll}\mathrm{H} & -1.0018550 & 0.3678790 & 2.9713090\end{array}$

$\begin{array}{llll}\mathrm{H} & -3.9842900 & 0.9200160 & 2.4527850\end{array}$

$\begin{array}{llll}\mathrm{C} & -3.8714370 & 2.3799110 & -0.8479500\end{array}$

$\mathrm{H} \quad-1.7298120 \quad 1.9615550 \quad-0.6907740$

C $\quad-3.6289030 \quad 3.6975600 \quad-1.6186960$

$\mathrm{H} \quad-4.4587680 \quad 3.8663240 \quad-2.3122130$

$\mathrm{H} \quad-3.5782470 \quad 4.5658450 \quad-0.9591060$

$\begin{array}{llll}\mathrm{H} & -2.7076790 & 3.6564890 & -2.2112410\end{array}$

C $\quad-4.1427160 \quad 1.2779420 \quad-1.8923940$

$\mathrm{H} \quad-3.2698630 \quad 1.0921420 \quad-2.5288510$

$\begin{array}{llll}\mathrm{H} & -4.4473190 & 0.3336240 & -1.4346820\end{array}$

$\begin{array}{llll}\mathrm{H} & -4.9611960 & 1.5982860 & -2.5449750\end{array}$

$\begin{array}{llll}\text { C } & -5.1071610 & 2.5315110 & 0.0578000\end{array}$

$\mathrm{H} \quad-4.9729910 \quad 3.3200200 \quad 0.8050030$

$\begin{array}{llll}\mathrm{H} & -5.9768250 & 2.7998270 & -0.5507730\end{array}$

$\begin{array}{llll}\mathrm{H} & -5.3463260 & 1.5966220 & 0.5748700\end{array}$

$\begin{array}{llll}\text { C } & -2.6136960 & -2.8280040 & -1.6052570\end{array}$

$\begin{array}{llll}\text { C } & -1.3214530 & -3.0148390 & -2.1226900\end{array}$

C $\quad-3.6573260 \quad-2.5612420 \quad-2.5055500$

$\begin{array}{llll}\mathrm{C} & -1.0783870 & -2.9285620 & -3.4968680\end{array}$

$\mathrm{H} \quad-0.5017090 \quad-3.2334570 \quad-1.4449150$

$\begin{array}{llll}\text { C } & -3.4197890 & -2.4745670 & -3.8810220\end{array}$

$\mathrm{H} \quad-4.6676110 \quad-2.4273490 \quad-2.1262930$

C $\quad-2.1270880 \quad-2.6566040 \quad-4.3816850$

$\begin{array}{llll}\mathrm{H} & -0.0715910 & -3.0803170 & -3.8759670\end{array}$

$\mathrm{H} \quad-4.2439610 \quad-2.2709350 \quad-4.5589300$

$\begin{array}{llll}\mathrm{H} & -1.9397790 & -2.5938870 & -5.4497600\end{array}$

H $3.7252460 \quad-0.1400000 \quad-0.5623340$

$\begin{array}{llll}\mathrm{O} & 4.4610530 & 0.2905460 & -1.1300920\end{array}$

$\begin{array}{llll}\mathrm{C} & 4.9584850 & 1.3695140 & -0.5751840\end{array}$
C $\quad 1.1550040 \quad 2.5438430$

$-0.1961330$

$2.5353320-2.5514090$

$\begin{array}{llll}\mathrm{C} & 1.9157310 & 1.2859100 & -2.1508830\end{array}$

$\begin{array}{llll}\mathrm{N} & 1.7031090 & 1.2563890 & -0.6916680\end{array}$

$\begin{array}{llll}\mathrm{N} & 0.6377590 & 3.1238870 & -1.4363430\end{array}$

$\begin{array}{llll}\mathrm{O} & 0.9132840 & 2.8975160 & -3.7098390\end{array}$

$\begin{array}{llll}\mathrm{C} & -0.3733170 & 4.1740460 & -1.5130640\end{array}$

$\mathrm{H} \quad-1.0466950 \quad 3.9435480 \quad-2.3413940$

$\mathrm{H} \quad-0.9418950 \quad 4.1971180 \quad-0.5830920$

$\mathrm{H} \quad 0.0685050 \quad 5.1587110 \quad-1.6903890$

$\begin{array}{llll}\text { C } & 1.9468610 & 0.2363010 & 0.1548110\end{array}$

$\begin{array}{llll}\mathrm{H} & 1.6268760 & 0.4394610 & 1.1728580\end{array}$

$\begin{array}{llll}\text { C } & 2.5004900 & -1.0065790 & -0.0910560\end{array}$

$\begin{array}{llll}\text { C } & 3.0186490 & -1.8631180 & 1.0535660\end{array}$

$\mathrm{H} \quad 2.8174920 \quad-2.9175410 \quad 0.8346830$

$\mathrm{H} \quad 2.9203470 \quad-1.2230150 \quad-1.0672810$

$\begin{array}{llll}\mathrm{Cl} & 0.2988060 & -2.0946630 & -0.3529930\end{array}$

$\mathrm{N} \quad-1.2685800 \quad-3.1352170 \quad-0.5747340$

$\begin{array}{llll}\text { C } & -2.5225670 & -2.6556310 & -0.3303050\end{array}$

C $\quad-1.2456090 \quad-4.4630630 \quad-1.0074390$

$\begin{array}{llll}\text { C } & -3.5346300 & -3.7533900 & -0.6130270\end{array}$

$\begin{array}{llll}\mathrm{C} & -2.6868230 & -4.9547350 & -1.0628520\end{array}$

$\mathrm{H} \quad-4.1110470 \quad-3.9457640 \quad 0.2964570$

$\mathrm{H} \quad-2.7872550 \quad-5.8237760 \quad-0.4070710$

$\mathrm{H} \quad-2.9076240 \quad-5.2813390 \quad-2.0826450$

$\mathrm{H} \quad-4.2324250 \quad-3.4033420 \quad-1.3791750$

O $-0.2380800 \quad-5.0880710 \quad-1.2836680$

$\begin{array}{llll}\text { O } & -2.7642770 & -1.5065780 & 0.0593500\end{array}$

$\begin{array}{llll}\text { C } & 1.3813730 & 0.0923370 & -2.9532330\end{array}$

$\begin{array}{llll}\mathrm{H} & 1.4027480 & 0.3750300 & -4.0086370\end{array}$

$\begin{array}{llll}\mathrm{H} & 1.9912110 & -0.8015520 & -2.8306620\end{array}$

$\mathrm{H} \quad 0.3513360 \quad-0.1408250 \quad-2.6730860$

$\begin{array}{llll}\mathrm{H} & 2.9803720 & 1.4331080 & -2.3783190\end{array}$

$\begin{array}{llll}\mathrm{C} & 2.2033630 & 3.4598250 & 0.5424720\end{array}$

$\begin{array}{llll}\mathrm{H} & 0.3246230 & 2.3398660 & 0.4909430\end{array}$

$\begin{array}{llll}\mathrm{C} & 1.5043010 & 4.7616870 & 0.9956500\end{array}$

$\begin{array}{llll}\mathrm{H} & 2.1871170 & 5.3332990 & 1.6321030\end{array}$

$\begin{array}{llll}\mathrm{H} & 1.2313800 & 5.4045040 & 0.1567530\end{array}$

$\begin{array}{llll}\mathrm{H} & 0.6025130 & 4.5544300 & 1.5832880\end{array}$

C $\quad 2.7321820 \quad 2.7561120 \quad 1.8098590$

$\begin{array}{llll}\mathrm{H} & 1.9175180 & 2.4371210 & 2.4706860\end{array}$

$\begin{array}{llll}\mathrm{H} & 3.3560720 & 1.8893740 & 1.5779840\end{array}$

$\begin{array}{llll}\mathrm{H} & 3.3547070 & 3.4580500 & 2.3737410\end{array}$

$\begin{array}{llll}\mathrm{C} & 3.3889610 & 3.8027930 & -0.3777710\end{array}$

$\begin{array}{llll}\mathrm{H} & 3.0654450 & 4.3278510 & -1.2821160\end{array}$

$\mathrm{H} \quad 4.0869710 \quad 4.4592030 \quad 0.1518880$

$\begin{array}{llll}\mathrm{H} & 3.9434930 & 2.9063340 & -0.6734900\end{array}$

$\mathrm{H} \quad-4.1784070 \quad-0.9384690 \quad 0.3313930$

$\begin{array}{llll}\mathrm{O} & -5.1271040 & -0.5877290 & 0.4899330\end{array}$

$\begin{array}{llll}\text { C } & -5.1431090 & 0.6237500 & 0.9918000\end{array}$

$\begin{array}{llll}\mathrm{O} & -4.2037510 & 1.3355620 & 1.2774030\end{array}$

C $\quad-6.6082680 \quad 1.1002110 \quad 1.1996930$

$\begin{array}{llll}\text { F } & -7.2758880 & 0.2675640 & 2.0297900\end{array}$

F $\quad-6.6469250 \quad 2.3322560 \quad 1.7303120$

$\begin{array}{llll}\text { F } & -7.2760790 & 1.1316240 & 0.0240190\end{array}$

$\begin{array}{llll}\mathrm{H} & 2.4726570 & -1.6196800 & 1.9716140\end{array}$

$\begin{array}{llll}\text { C } & 4.5128930 & -1.6862680 & 1.2851020\end{array}$

$\begin{array}{llll}\text { C } & 5.4439310 & -2.3632170 & 0.4811630\end{array}$

$\begin{array}{llll}\text { C } & 4.9946960 & -0.8372640 & 2.2928140\end{array}$

$\begin{array}{llll}\text { C } & 6.8179160 & -2.1887530 & 0.6707070\end{array}$ 


$\begin{array}{llll}\mathrm{H} & 5.0904430 & -3.0355870 & -0.2971710 \\ \mathrm{C} & 6.3685420 & -0.6616950 & 2.4887330 \\ \mathrm{H} & 4.2900800 & -0.3135260 & 2.9341820 \\ \mathrm{C} & 7.2856690 & -1.3350670 & 1.6757450 \\ \mathrm{H} & 7.5218790 & -2.7231750 & 0.0388150 \\ \mathrm{H} & 6.7206250 & -0.0031010 & 3.2778920 \\ \mathrm{H} & 8.3528570 & -1.2012630 & 1.8271800\end{array}$

3.log

Potential Energy $=-2233.46576$

Zero-point Energy = -2232.93514

Free Energy $=-2232.98568$

Single-Point Energy B3LYP-D3(BJ)/6-311+G** PCM = 2234.03546

Free Energy B3LYP-D3(BJ)/6-311+G** PCM (extrapolated free energy from $\mathrm{qRRHO})=-2233.55538$

Nimag $=1\left(-187.8362 \mathrm{~cm}^{-1}\right)$

Charge $=0$ Multiplicity $=1$

$\begin{array}{lllr}\mathrm{C} & 4.4464930 & -1.0152420 & -0.1966280 \\ \mathrm{C} & 4.1597430 & -1.7940070 & 2.0074730\end{array}$

$\begin{array}{llll}\text { C } & 4.1597430 & -1.7940070 & 2.0074730\end{array}$

$\begin{array}{llll}\text { C } & 3.1261750 & -0.6768850 & 1.8335430\end{array}$

$\begin{array}{llll}\mathrm{N} & 3.2267720 & -0.4036630 & 0.3875120\end{array}$

$\begin{array}{llll}\mathrm{N} & 4.7781540 & -2.0094900 & 0.8247140\end{array}$

$\begin{array}{llll}\mathrm{O} & 4.3411700 & -2.4359480 & 3.0433880\end{array}$

C $\quad 5.6182820 \quad-3.1836730 \quad 0.6097270$

$\mathrm{H} \quad 5.1745910 \quad-4.0273070 \quad 1.1427910$

$\mathrm{H} \quad 5.6557330 \quad-3.4108060 \quad-0.4559130$

H $\quad 6.6357560 \quad-3.0353770 \quad 0.9825940$

$\begin{array}{llll}\text { C } & 2.3687880 & 0.2953190 & -0.3815640\end{array}$

$\begin{array}{llll}\mathrm{H} & 2.6300630 & 0.2784630 & -1.4358770\end{array}$

$\begin{array}{llll}\mathrm{C} & 1.2201730 & 0.9818870 & -0.0329070\end{array}$

$\begin{array}{llll}\text { C } & 0.6052800 & 1.9941870 & -0.9867860\end{array}$

$\begin{array}{llll}\mathrm{H} & -0.4863310 & 1.9471690 & -0.9079840\end{array}$

$\mathrm{H} \quad 0.9682880 \quad 1.1096700 \quad 1.0141720$

$\begin{array}{llll}\mathrm{Cl} & -0.2551720 & -0.9209390 & -0.5682280\end{array}$

$\mathrm{N} \quad-1.5698980 \quad-2.2390650 \quad-0.9273380$

C $\quad-2.9075660-2.0258560-0.7632520$

C $\quad-1.2532330 \quad-3.5244110 \quad-1.3728410$

$\begin{array}{llll}\text { C } & -3.6586610 & -3.2952840 & -1.1285110\end{array}$

C $\quad-2.5601990 \quad-4.2901470 \quad-1.5383050$

$\mathrm{H} \quad-4.2395920 \quad-3.6241490 \quad-0.2620530$

$\mathrm{H}-2.5275460-\quad-5.1819940-0.9068170$

$\mathrm{H} \quad-2.6437960 \quad-4.6217270 \quad-2.5768330$

$\mathrm{H} \quad-4.3638460 \quad-3.0717510 \quad-1.9340400$

O $-0.1249520 \quad-3.9303670 \quad-1.5837000$

$\begin{array}{llll}\text { O } & -3.3998050 & -0.9587480 & -0.3762060\end{array}$

$\begin{array}{llll}\text { C } & 1.7725230 & -1.1780960 & 2.3527030\end{array}$

$\mathrm{H} \quad 1.9323270 \quad-1.5507290 \quad 3.3675630$

$\begin{array}{llll}\mathrm{H} & 1.0228890 & -0.3894310 & 23967100\end{array}$

H $\quad 1.3914470 \quad-1.9946330 \quad 1.7349610$

$\begin{array}{llll}\mathrm{H} & 3.4353170 & 0.2053850 & 2.4103920\end{array}$

$\begin{array}{llll}\text { C } & 5.6126400 & 0.0032540 & -0.4905470\end{array}$

$\mathrm{H} \quad 4.1805260 \quad-1.5217010 \quad-1.1326220$

C $\quad 6.8136650 \quad-0.7654060 \quad-1.0868190$

$\begin{array}{llll}\mathrm{H} & 7.5669710 & -0.0478140 & -1.4267920\end{array}$

$\begin{array}{llll}\mathrm{H} & 7.2965910 & -1.4187340 & -0.3577940\end{array}$

$\begin{array}{llll}\mathrm{H} & 6.5189470 & -1.3688500 & -1.9532210\end{array}$

C $\quad 5.1654380 \quad 1.0444200 \quad-1.5379240$

$\begin{array}{llll}\mathrm{H} & 4.7891360 & 0.5703400 & -2.4521180\end{array}$

$\mathrm{H} \quad 4.3997230 \quad 1.7224300 \quad-1.1529360$

$\begin{array}{llll}\mathrm{H} & 6.0262130 & 1.6593060 & -1.8194140\end{array}$

$\begin{array}{llll}\mathrm{C} & 6.0506070 & 0.7332320 & 0.7922340\end{array}$

$\begin{array}{llll}\mathrm{H} & 6.3995240 & 0.0358240 & 1.5604050\end{array}$

$\begin{array}{llll}\mathrm{H} & 6.8785530 & 1.4121700 & 0.5637490\end{array}$

$\begin{array}{llll}\mathrm{H} & 5.2387360 & 1.3368740 & 1.2107390\end{array}$

$\begin{array}{llll}\mathrm{H} & -4.9137670 & -0.7098520 & -0.1767190\end{array}$

$\begin{array}{llll}\mathrm{O} & -5.9234920 & -0.5798290 & -0.0655270\end{array}$

$\begin{array}{llll}\text { C } & -6.2302190 & 0.5680890 & 0.4895910\end{array}$

$\begin{array}{llll}\text { O } & -5.4857500 & 1.4496420 & 0.8627270\end{array}$

$\begin{array}{llll}\text { C } & -7.7722620 & 0.6986960 & 0.6370740\end{array}$

F $\quad-8.2683760 \quad-0.2919220 \quad 1.4127230$

F $\quad-8.1085570 \quad 1.8707090 \quad 1.1975300$

F $\quad-8.3820570 \quad 0.6222920 \quad-0.5674100$

$\begin{array}{llll}\mathrm{H} & 0.8607640 & 1.7293050 & -2.0187010\end{array}$

C $\quad 1.0569840 \quad 3.4189250 \quad-0.6979660$

C $0.4505920 \quad 4.1655720 \quad 0.3248260$

$\begin{array}{lrrr}\mathrm{C} & 2.0927220 & 4.0139740 & -1.4338960 \\ \mathrm{C} & 0.8739670 & 5.4665080 & 0.6121500 \\ \mathrm{H} & -0.3626610 & 3.7266330 & 0.8981920 \\ \mathrm{C} & 2.5179640 & 5.3165050 & -1.1525170 \\ \mathrm{H} & 2.5666810 & 3.4566290 & -2.2381800 \\ \mathrm{C} & 1.9113590 & 6.0468850 & -0.1257880 \\ \mathrm{H} & 0.3901700 & 6.0281840 & 1.4064410 \\ \mathrm{H} & 3.3185940 & 5.7604550 & -1.7376660 \\ \mathrm{H} & 2.2387390 & 7.0591250 & 0.0931640\end{array}$

4. $\log$

Potential Energy = -2233.46324

Zero-point Energy = -2232.93284

Free Energy = -2232.98280

Single-Point Energy B3LYP-D3(BJ)/6-311+G** PCM = 2234.03401

Free Energy B3LYP-D3(BJ)/6-311+G** PCM (extrapolated free energy from $q R R H O)=-2233.55357$

Nimag $=1\left(-191.0590 \mathrm{~cm}^{-1}\right)$

Charge $=0$ Multiplicity $=1$

\begin{tabular}{|c|c|c|c|}
\hline $\mathrm{C}$ & 2.7482410 & -2.6180430 & -0.2848630 \\
\hline $\mathrm{C}$ & 3.7041980 & -2.3771060 & 1.8540380 \\
\hline $\mathrm{C}$ & 3.7316070 & -0.9763590 & 1.2348760 \\
\hline $\mathrm{N}$ & 2.9300200 & -1.1777050 & 0.0152660 \\
\hline $\mathrm{N}$ & 3.0525600 & -3.2153350 & 1.0165620 \\
\hline $\mathrm{O}$ & 4.1449640 & -2.6691520 & 2.9672500 \\
\hline $\mathrm{C}$ & 2.6345370 & -4.5460280 & 1.4466470 \\
\hline $\mathrm{H}$ & 2.3305730 & -4.4904010 & 2.4941710 \\
\hline $\mathrm{H}$ & 1.7867580 & -4.8711580 & 0.8430460 \\
\hline $\mathrm{H}$ & 3.4412020 & -5.2794570 & 1.3591740 \\
\hline $\mathrm{C}$ & 2.4100290 & -0.2314630 & -0.7964080 \\
\hline $\mathrm{H}$ & 1.7922580 & -0.6451400 & -1.5884270 \\
\hline $\mathrm{C}$ & 2.5333960 & 1.1415800 & -0.7538350 \\
\hline $\mathrm{C}$ & 2.1981110 & 1.9686980 & -1.9878030 \\
\hline $\mathrm{H}$ & 3.0459210 & 1.8987310 & -2.6865470 \\
\hline $\mathrm{H}$ & 3.2059760 & 1.6008030 & -0.0377060 \\
\hline $\mathrm{Cl}$ & 0.3671970 & 1.3204150 & 0.5078240 \\
\hline $\mathrm{N}$ & -1.2326160 & 1.6623610 & 1.4310920 \\
\hline $\mathrm{C}$ & -2.4430780 & 1.1719230 & 1.0322090 \\
\hline $\mathrm{C}$ & -1.2949690 & 2.4619560 & 2.5765930 \\
\hline $\mathrm{C}$ & -3.5121470 & 1.6578560 & 1.9954380 \\
\hline $\mathrm{C}$ & -2.7514410 & 2.5169520 & 3.0188700 \\
\hline $\mathrm{H}$ & -4.2661930 & 2.2172640 & 1.4343950 \\
\hline $\mathrm{H}$ & -3.0748880 & 3.5612930 & 3.0304010 \\
\hline $\mathrm{H}$ & -2.8231710 & 2.1359260 & 4.0410930 \\
\hline $\mathrm{H}$ & -4.0070280 & 0.7899370 & 2.4404450 \\
\hline $\mathrm{O}$ & -0.3391140 & 3.0027020 & 3.1003010 \\
\hline $\mathrm{O}$ & -2.6087930 & 0.4504630 & 0.0416160 \\
\hline $\mathrm{C}$ & 3.1886800 & 0.0258350 & 2.2613620 \\
\hline $\mathrm{H}$ & 3.7609780 & -0.1083300 & 3.1826870 \\
\hline $\mathrm{H}$ & 3.3054960 & 1.0593670 & 1.9382250 \\
\hline $\mathrm{H}$ & 2.1331620 & -0.1602640 & 2.4733150 \\
\hline $\mathrm{H}$ & 4.7653330 & -0.7080560 & 0.9753490 \\
\hline $\mathrm{C}$ & 3.6506520 & -3.1628360 & -1.4573100 \\
\hline $\mathrm{H}$ & 1.6972870 & -2.8034440 & -0.5397170 \\
\hline $\mathrm{C}$ & 3.3711660 & -4.6705940 & -1.6502170 \\
\hline $\mathrm{H}$ & 3.8823500 & -5.0190010 & -2.5532010 \\
\hline $\mathrm{H}$ & 3.7395720 & -5.2737390 & -0.8182510 \\
\hline $\mathrm{H}$ & 2.3013870 & -4.8718410 & -1.7787070 \\
\hline $\mathrm{C}$ & 3.2974500 & -2.4549470 & -2.7828370 \\
\hline $\mathrm{H}$ & 2.2276710 & -2.5249990 & -3.0125440 \\
\hline $\mathrm{H}$ & 3.5882100 & -1.4011290 & -2.7852250 \\
\hline $\mathrm{H}$ & 3.8397140 & -2.9396470 & -3.6009230 \\
\hline $\mathrm{C}$ & 5.1450860 & -2.9500670 & -1.1548410 \\
\hline $\mathrm{H}$ & 5.4510650 & -3.4549120 & -0.2330110 \\
\hline $\mathrm{H}$ & 5.7471990 & -3.3621590 & -1.9711520 \\
\hline $\mathrm{H}$ & 5.3926390 & -1.8870210 & -1.0699430 \\
\hline $\mathrm{H}$ & -3.9702520 & -0.1166810 & -0.4368190 \\
\hline $\mathrm{O}$ & -4.8293780 & -0.4536870 & -0.8790290 \\
\hline $\mathrm{C}$ & -5.3609860 & -1.4665390 & -0.2375030 \\
\hline $\mathrm{O}$ & -4.9623690 & -2.0147840 & 0.7682570 \\
\hline $\mathrm{C}$ & -6.6590940 & -1.9359790 & -0.9519220 \\
\hline $\mathrm{F}$ & -6.3930190 & -2.3483900 & -2.2120680 \\
\hline $\mathrm{F}$ & -7.2323130 & -2.9558890 & -0.2947760 \\
\hline $\mathrm{F}$ & -7.5600310 & -0.9308570 & -1.0296960 \\
\hline
\end{tabular}




$\begin{array}{lrrr}\mathrm{C} & 1.9380230 & 3.4407040 & -1.7215850 \\ \mathrm{C} & 2.9676750 & 4.2724910 & -1.2521120 \\ \mathrm{C} & 0.6805980 & 4.0116760 & -1.9638870 \\ \mathrm{C} & 2.7439520 & 5.6322270 & -1.0187430 \\ \mathrm{H} & 3.9560760 & 3.8557740 & -1.0726900 \\ \mathrm{C} & 0.4522790 & 5.3734220 & -1.7372730 \\ \mathrm{H} & -0.1284130 & 3.3859240 & -2.3317980 \\ \mathrm{C} & 1.4829300 & 6.1885990 & -1.2605400 \\ \mathrm{H} & 3.5546800 & 6.2574440 & -0.6550540 \\ \mathrm{H} & -0.5299680 & 5.7947640 & -1.9327560 \\ \mathrm{H} & 1.3079480 & 7.2458350 & -1.0832060\end{array}$

$5 . \log$

Potential Energy = -2233.46325

Zero-point Energy = -2232.93305

Free Energy $=-2232.98350$

Single-Point Energy B3LYP-D3(BJ)/6-311+G** PCM = 2234.03446

Free Energy B3LYP-D3(BJ)/6-311+G** PCM (extrapolated free energy from qRRHO) $=-2233.55471$

Nimag $=1\left(-192.4734 \mathrm{~cm}^{-1}\right)$

Charge $=0$ Multiplicity $=1$

$\begin{array}{llrr}\text { C } & 3.0240340 & -2.4589160 & -0.3299190 \\ \text { C } & 3.9688390 & -2.1618410 & 1.8070340\end{array}$

$\begin{array}{llll}\text { C } & 3.9688390 & -2.1618410 & 1.8070340\end{array}$

$\begin{array}{llll}\text { C } & 3.8716210 & -0.7566610 & 1.2051170\end{array}$

$\mathrm{N} \quad 3.0819700 \quad-1.0117790 \quad-0.0125360$

$\mathrm{N} \quad 3.3886730 \quad-3.0434540 \quad 0.9616540$

$\begin{array}{llll}\mathrm{O} & 4.4378100 & -2.4280400 & 2.9151470\end{array}$

$\begin{array}{llll}\text { C } & 3.0843850 & -4.4085310 & 1.3793450\end{array}$

$\mathrm{H} \quad 2.7845240 \quad-4.3890550 \quad 2.4293380$

H $2.2616990 \quad-4.7952890 \quad 0.7773280$

$\begin{array}{llll}\mathrm{H} & 3.9476070 & -5.0724950 & 1.2785430\end{array}$

C $\quad 2.4749780 \quad-0.1047690 \quad-0.8081680$

$\begin{array}{llll}\mathrm{H} & 1.8917740 & -0.5611110 & -1.6028200\end{array}$

$\begin{array}{llll}\text { C } & 2.4751760 & 1.2733500 & -0.7468860\end{array}$

C $\quad 2.0627920-2.0837810-1.9684920$

$\mathrm{H} \quad 3.1079820 \quad 1.7807880 \quad-0.0271300$

$\begin{array}{llll}\mathrm{Cl} & 0.3100390 & 1.2414760 & 0.5219470\end{array}$

$\begin{array}{llll}\mathrm{N} & -1.3102800 & 1.4304960 & 1.4563660\end{array}$

C $\quad-2.4990500 \quad 0.9403130 \quad 0.9970680$

C $\quad-1.4094610-2.1007770-2.6795350$

C $\quad-3.5938470 \quad 1.2956010 \quad 1.9873310$

C $\quad-2.8673000 \quad 2.0434650 \quad 3.1172000$

$\mathrm{H} \quad-4.3412350 \quad 1.9096840 \quad 1.4759300$

$\mathrm{H} \quad-3.2317010 \quad 3.0630980 \quad 3.2672990$

$\mathrm{H} \quad-2.9223560 \quad 1.5263820 \quad 4.0792770$

$\mathrm{H} \quad-4.0891490 \quad 0.3765510 \quad 2.3120920$

O $-0.4782150-2.6218460 \quad 3.2642610$

$\begin{array}{llll}\mathrm{O} & -2.6306750 & 0.3131540 & -0.0610720\end{array}$

$\begin{array}{llll}\text { C } & 3.2513650 & 0.1824790 & 2.2472200\end{array}$

$\begin{array}{llll}\mathrm{H} & 3.8399840 & 0.0880160 & 3.1631390\end{array}$

$\mathrm{H} \quad 3.2754840 \quad 1.2260390 \quad 1.9363830$

$\mathrm{H} \quad 2.2177050-0.0972000-2.4638250$

$\begin{array}{llll}\mathrm{H} & 4.8765570 & -0.3972630 & 0.9426370\end{array}$

$\begin{array}{llll}\text { C } & 3.9615610 & -2.9077970 & -1.5151870\end{array}$

H $\quad 1.9913670 \quad-2.7324860 \quad-0.5800500$

C $\quad 3.8229110 \quad-4.4335460 \quad-1.7191010$

$\begin{array}{llll}\mathrm{H} & 4.3586330 & -4.7251110 & -2.6279600\end{array}$

$\mathrm{H} \quad 4.2519770 \quad-5.0057860 \quad-0.8943960$

$\mathrm{H} \quad 2.7761220 \quad-4.7339270 \quad-1.8434920$

$\begin{array}{llll}\text { C } & 3.5299760 & -2.2253750 & -2.8308700\end{array}$

$\mathrm{H} \quad 2.4686220 \quad-2.3922540 \quad-3.0496840$

$\begin{array}{llll}\mathrm{H} & 3.7220630 & -1.1491910 & -2.8272930\end{array}$

$\mathrm{H} \quad 4.1053450 \quad-2.6515100 \quad-3.6588790$

$\begin{array}{llll}\mathrm{C} & 5.4325400 & -2.5588480 & -1.2243060\end{array}$

$\mathrm{H} \quad 5.7923800 \quad-3.0389380 \quad-0.3087280$

$\begin{array}{llll}\mathrm{H} & 6.0628250 & -2.9082650 & -2.0485600\end{array}$

H $\quad 5.5810580 \quad-1.4779210 \quad-1.1341860$

$\mathrm{H} \quad-3.9517100 \quad-0.3173870 \quad-0.5603260$

O $\quad-4.7675720 \quad-0.7318900 \quad-1.0204680$

C $\quad-5.4597600 \quad-1.4981270 \quad-0.2126430$

$\begin{array}{llll}\text { O } & -5.2480540 & -1.7443010 & 0.9564440\end{array}$

$\begin{array}{llll}\text { C } & -6.6793690 & -2.1083080 & -0.9580010\end{array}$

F $\quad-6.2781240 \quad-2.8496300 \quad-2.0150780$

F $\quad-7.3975520 \quad-2.8991350 \quad-0.1461040$

$\begin{array}{lrrr}\mathrm{F} & -7.4959960 & -1.1361550 & -1.4232820 \\ \mathrm{H} & 1.2478990 & 1.5743570 & -2.4940640 \\ \mathrm{H} & 2.9129550 & 2.1037150 & -2.6676370 \\ \mathrm{C} & 1.6651750 & 3.5207770 & -1.6813560 \\ \mathrm{C} & 2.6091430 & 4.4380000 & -1.1913860 \\ \mathrm{C} & 0.3612160 & 3.9749810 & -1.9247590 \\ \mathrm{C} & 2.2568990 & 5.7667840 & -0.9388730 \\ \mathrm{H} & 3.6313940 & 4.1136210 & -1.0108790 \\ \mathrm{C} & 0.0042170 & 5.3053420 & -1.6788510 \\ \mathrm{H} & -0.3827560 & 3.2817060 & -2.3085700 \\ \mathrm{C} & 0.9505990 & 6.2059530 & -1.1815580 \\ \mathrm{H} & 3.0028770 & 6.4595910 & -0.5594380 \\ \mathrm{H} & -1.0121270 & 5.6354840 & -1.8753660 \\ \mathrm{H} & 0.6758430 & 7.2390510 & -0.9891160\end{array}$

$6 . \log$

Potential Energy $=-2233.46284$

Zero-point Energy = -2232.93211

Free Energy = -2232.98229

Single-Point Energy B3LYP-D3(BJ)/6-311+G** PCM = 2234.03622

Free Energy B3LYP-D3(BJ)/6-311+G** PCM (extrapolated free energy from $q$ RRHO) $=-2233.55567$

Nimag $=1\left(-207.1104 \mathrm{~cm}^{-1}\right)$

Charge $=0$ Multiplicity $=1$

$\begin{array}{llll}\text { C } & -4.5370210 & 0.5806250 & -0.2161190\end{array}$

C $\quad-4.3850850 \quad 2.6059480 \quad 0.9738200$

$\begin{array}{llll}\mathrm{C} & -3.3864980 & 1.6602270 & 1.6496020\end{array}$

$\mathrm{N} \quad-3.3821360 \quad 0.5179380 \quad 0.7148350$

$\begin{array}{llll}\mathrm{N} & -4.9022520 & 1.9941700 & -0.1147890\end{array}$

$\begin{array}{llll}\mathrm{O} & -4.6202250 & 3.7614740 & 1.3316000\end{array}$

$\begin{array}{llll}\text { C } & -5.6803100 & 2.7323980 & -1.1046720\end{array}$

$\mathrm{H} \quad-5.2479700 \quad 3.7299140 \quad-1.2087040$

$\begin{array}{llll}\mathrm{H} & -5.6281780 & 2.2158300 & -2.0633490\end{array}$

$\begin{array}{llll}\mathrm{H} & -6.7279420 & 2.8388700 & -0.8087330\end{array}$

C $\quad-2.4974600 \quad-0.4981730 \quad 0.6474160$

$\begin{array}{llll}\mathrm{H} & -2.6917500 & -1.1810390 & -0.1737470\end{array}$

C $\quad-1.3936440 \quad-0.7614400 \quad 1.4366790$

C $\quad-0.7187530 \quad-2.1300910 \quad 1.4877250$

$\begin{array}{llll}\mathrm{H} & -1.0037260 & -2.5934410 & 2.4421410\end{array}$

$\mathrm{H} \quad-1.2208210 \quad-0.1577910 \quad 2.3205380$

$\begin{array}{llll}\mathrm{Cl} & 0.1589790 & 0.4505080 & -0.0441080\end{array}$

$\mathrm{N} \quad 1.5399860 \quad 1.3187960 \quad-1.0215580$

$\begin{array}{llll}\text { C } & 2.8553220 & 1.2623780 & -0.6647980\end{array}$

$\begin{array}{llll}\text { C } & 1.3013350 & 2.0630690 & -2.1789610\end{array}$

C $\quad 3.6748750 \quad 2.0596600 \quad-1.6648930$

$\begin{array}{llll}\text { C } & 2.6437190 & 2.5857550 & -2.6765170\end{array}$

$\mathrm{H} \quad 4.2026970 \quad 2.8559760 \quad-1.1317700$

$\mathrm{H} \quad 2.6011800 \quad 3.6772480 \quad-2.7231760$

$\mathrm{H} \quad 2.8086910 \quad 2.2163120 \quad-3.6923490$

$\mathrm{H} \quad 4.4291990 \quad 1.4021720 \quad-2.1059600$

$\begin{array}{llll}\mathrm{O} & 0.2055080 & 2.2428460 & -2.6785280\end{array}$

$\begin{array}{llll}\text { O } & 3.2823120 & 0.6514370 & 0.3234370\end{array}$

$\begin{array}{llll}\mathrm{C} & -2.0637210 & 2.4100310 & 1.8511180\end{array}$

$\begin{array}{llll}\mathrm{H} & -2.2981080 & 3.3438340 & 2.3683290\end{array}$

$\begin{array}{llll}\mathrm{H} & -1.3561540 & 1.8540120 & 2.4645940\end{array}$

H $\quad-1.5934290 \quad 2.6476090 \quad 0.8944320$

$\begin{array}{llll}\mathrm{H} & -3.7792060 & 1.3481860 & 2.6271320\end{array}$

$\begin{array}{llll}\text { C } & -5.7183460 & -0.4026530 & 0.1332750\end{array}$

$\mathrm{H} \quad-4.1895410 \quad 0.3620570 \quad-1.2331750$

$\begin{array}{llll}\text { C } & -6.8369400 & -0.2393130 & -0.9208270\end{array}$

$\begin{array}{llll}\mathrm{H} & -7.5930240 & -1.0154690 & -0.7657680\end{array}$

H $\quad-7.3447550 \quad 0.7242160 \quad-0.8484630$

$\begin{array}{llll}\mathrm{H} & -6.4516150 & -0.3538390 & -1.9405890\end{array}$

C $\quad-5.2360490 \quad-1.8662740 \quad 0.0698540$

$\begin{array}{llll}\mathrm{H} & -4.7749690 & -2.1051210 & -0.8954000\end{array}$

$\mathrm{H} \quad-4.5259800 \quad-2.1077260 \quad 0.8645410$

$\begin{array}{llll}\mathrm{H} & -6.0967780 & -2.5311650 & 0.1941410\end{array}$

C $\quad-6.2851580 \quad-0.1192610 \quad 1.5364320$

$\begin{array}{llll}\mathrm{H} & -6.6611270 & 0.9046980 & 1.6271940\end{array}$

$\begin{array}{llll}\mathrm{H} & -7.1221630 & -0.7959530 & 1.7369040\end{array}$

$\begin{array}{llll}\mathrm{H} & -5.5356850 & -0.2870820 & 2.3166990\end{array}$

$\begin{array}{llll}\mathrm{H} & 4.7672430 & 0.5084410 & 0.7098470\end{array}$

$\begin{array}{llll}\text { O } & 5.7070170 & 0.3854460 & 1.1014800\end{array}$

C $\quad 6.5692750 \quad-0.0352380 \quad 0.2084280$

$\begin{array}{llll}\text { O } & 6.3812850 & -0.2711270 & -0.9669480\end{array}$ 


$\begin{array}{lccc}\mathrm{C} & 7.9720400 & -0.2146700 & 0.8532430 \\ \mathrm{~F} & 7.9277630 & -1.1092020 & 1.8660250 \\ \mathrm{~F} & 8.8673960 & -0.6510230 & -0.0459710 \\ \mathrm{~F} & 8.4241260 & 0.9558330 & 1.3575830 \\ \mathrm{H} & 0.3660390 & -1.9820140 & 1.5428060 \\ \mathrm{C} & -1.0388870 & -3.1045560 & 0.3694880 \\ \mathrm{C} & -2.0358450 & -4.0781700 & 0.5397390 \\ \mathrm{C} & -0.3353370 & -3.0796600 & -0.8458530 \\ \mathrm{C} & -2.3301070 & -4.9953520 & -0.4741680 \\ \mathrm{H} & -2.5823290 & -4.1233130 & 1.4787520 \\ \mathrm{C} & -0.6262090 & -3.9937440 & -1.8630840 \\ \mathrm{H} & 0.4484200 & -2.3428250 & -0.9938370 \\ \mathrm{C} & -1.6263670 & -4.9546880 & -1.6816110 \\ \mathrm{H} & -3.1032110 & -5.7424730 & -0.3179390 \\ \mathrm{H} & -0.0668930 & -3.9590550 & -2.7938550 \\ \mathrm{H} & -1.8496500 & -5.6679510 & -2.4697800\end{array}$

\section{(S)-TS-back}

\section{Conformation Extrapolated name}

$\begin{array}{cc}\text { TS4-Sb-lowest } & 0.0 \\ 1 & 1.9 \\ 2 & 2.4 \\ 3 & 1.5\end{array}$

TS4-Sb-lowest.log

Potential Energy = -2233.46543 Zero-point Energy = -2232.93478

Free Energy $=-2232.98492$

Single-Point Energy B3LYP-D3(BJ)/6-311+G** PCM = 2234.03911

Free Energy B3LYP-D3(BJ)/6-311+G** PCM (extrapolated free energy from $q R R H O)=-2233.55860$

Nimag $=1\left(-192.3533 \mathrm{~cm}^{-1}\right)$ Charge $=0$ Multiplicity $=1$

$\begin{array}{lccc}\mathrm{C} & -2.3955980 & 2.4865830 & 0.7785440 \\ \mathrm{C} & -1.3161090 & 3.9298080 & -0.7440620 \\ \mathrm{C} & -1.2860590 & 2.5499090 & -1.4048870 \\ \mathrm{~N} & -2.1288780 & 1.7612910 & -0.4884320 \\ \mathrm{~N} & -2.0238940 & 3.8527700 & 0.4051680 \\ \mathrm{O} & -0.8334860 & 4.9563420 & -1.2256140 \\ \mathrm{C} & -2.4697650 & 5.0583040 & 1.0982740 \\ \mathrm{H} & -2.7242770 & 5.8123630 & 0.3502560 \\ \mathrm{H} & -3.3540380 & 4.8277010 & 1.6926350 \\ \mathrm{H} & -1.6940600 & 5.4670470 & 1.7516100 \\ \mathrm{C} & -2.6982290 & 0.5677980 & -0.7476880 \\ \mathrm{H} & -3.4593230 & 0.2822410 & -0.0271560 \\ \mathrm{C} & -2.4136100 & -0.3207910 & -1.7691190 \\ \mathrm{C} & -3.3688660 & -1.4401540 & -2.1674150 \\ \mathrm{H} & -2.7750740 & -2.3116890 & -2.4663760 \\ \mathrm{H} & -0.2561020 & 2.1688400 & -1.4130720 \\ \mathrm{C} & -1.6181800 & 1.9288640 & 2.0275870 \\ \mathrm{H} & -3.4712880 & 2.4454240 & 0.9939480 \\ \mathrm{H} & -1.7252400 & -0.0240820 & -2.5508670 \\ \mathrm{Cl} & -0.5771380 & -1.4955010 & -0.6593680 \\ \mathrm{~N} & 0.8929820 & -2.5142660 & -0.0143360 \\ \mathrm{C} & 0.7425780 & -3.7398220 & 0.6388080 \\ \mathrm{C} & 2.1919690 & -2.1242340 & -0.1612330 \\ \mathrm{C} & 2.1351470 & -4.2464640 & 0.9949440 \\ \mathrm{C} & 3.0981290 & -3.1802700 & 0.4479110 \\ \mathrm{H} & 2.2779830 & -5.2318660 & 0.5435670 \\ \mathrm{H} & 3.7653410 & -3.5609750 & -0.3311580 \\ \mathrm{H} & 3.7209060 & -2.7119440 & 1.2149570 \\ \mathrm{H} & 2.1967860 & -4.3666180 & 2.0800840 \\ \mathrm{O} & 2.5456680 & -1.0728230 & -0.7101840 \\ \mathrm{O} & -0.3237020 & -4.2799460 & 0.8701340 \\ \mathrm{C} & -1.8059920 & 2.6823530 & -2.8442200 \\ \mathrm{H} & -1.6431490 & 1.7826820 & -3.4359480 \\ \mathrm{H} & -2.8734920 & 2.9219470 & -2.8496240 \\ \mathrm{H} & -1.2611350 & 3.5017680 & -3.3192460 \\ \mathrm{C} & -1.9164000 & 2.8175840 & 3.2557540\end{array}$

$\begin{array}{lrrr}\mathrm{H} & -1.5008290 & 2.3426200 & 4.1500960 \\ \mathrm{H} & -1.4632770 & 3.8076220 & 3.1759510 \\ \mathrm{H} & -2.9936040 & 2.9385930 & 3.4185590 \\ \mathrm{C} & -0.1014040 & 1.9016520 & 1.7675220 \\ \mathrm{H} & 0.2899430 & 2.9004980 & 1.5479830 \\ \mathrm{H} & 0.4181270 & 1.5335870 & 2.6583270 \\ \mathrm{H} & 0.1539060 & 1.2348940 & 0.9383360 \\ \mathrm{C} & -2.1151350 & 0.5056540 & 2.3586800 \\ \mathrm{H} & -3.2025610 & 0.4795630 & 2.4975070 \\ \mathrm{H} & -1.8424520 & -0.2235510 & 1.5935280 \\ \mathrm{H} & -1.6569250 & 0.1761380 & 3.2968280 \\ \mathrm{H} & -3.9018680 & -1.1174210 & -3.0726540 \\ \mathrm{C} & -4.3904750 & -1.8564790 & -1.1262420 \\ \mathrm{C} & -4.0747600 & -2.8023100 & -0.1375760 \\ \mathrm{C} & -5.6822120 & -1.3067430 & -1.1348160 \\ \mathrm{C} & -5.0226080 & -3.1852910 & 0.8165080 \\ \mathrm{H} & -3.0818570 & -3.2424330 & -0.1159040 \\ \mathrm{C} & -6.6331480 & -1.6858910 & -0.1817540 \\ \mathrm{H} & -5.9475790 & -0.5794590 & -1.8986480 \\ \mathrm{C} & -6.3053450 & -2.6274690 & 0.7987210 \\ \mathrm{H} & -4.7601950 & -3.9226330 & 1.5701170 \\ \mathrm{H} & -7.6281580 & -1.2505360 & -0.2093230 \\ \mathrm{H} & -7.0428310 & -2.9275640 & 1.5375850 \\ \mathrm{H} & 3.9990760 & -0.5681260 & -0.8127540 \\ \mathrm{O} & 4.9180340 & -0.1483500 & -0.9877360 \\ \mathrm{C} & 5.6569310 & -0.0982780 & 0.0937080 \\ \mathrm{C} & 7.0254430 & 0.5709370 & -0.2147380 \\ \mathrm{O} & 5.3852560 & -0.4903180 & 1.2095120 \\ \mathrm{~F} & 7.8150200 & 0.5823730 & 0.8703240 \\ \mathrm{~F} & 6.8529280 & 1.8500810 & -0.6187910 \\ \mathrm{~F} & 7.6781810 & -0.0869450 & -1.1987410\end{array}$

$1 . \log$

Potential Energy = -2233.46774

Zero-point Energy = -2232.93705

Free Energy = -2232.98699

Single-Point Energy B3LYP-D3(BJ)/6-311+G** PCM = 2234.03861

Free Energy B3LYP-D3(BJ)/6-311+G** PCM (extrapolated

free energy from $\mathrm{qRRHO})=-2233.55785$

Nimag $=1\left(-170.9527 \mathrm{~cm}^{-1}\right)$

Charge $=0$ Multiplicity $=1$

\begin{tabular}{lccc}
$\mathrm{C}$ & -1.8852200 & 2.5765870 & 1.2115560 \\
$\mathrm{C}$ & -1.6050980 & 3.4927530 & -0.9449790 \\
$\mathrm{C}$ & -1.7219820 & 1.9825740 & -1.1607040 \\
$\mathrm{~N}$ & -2.0737010 & 1.5139850 & 0.1919760 \\
$\mathrm{~N}$ & -1.8021620 & 3.7677550 & 0.3640070 \\
$\mathrm{O}$ & -1.4330760 & 4.3241830 & -1.8379390 \\
$\mathrm{C}$ & -2.0397650 & 5.1348930 & 0.8188200 \\
$\mathrm{H}$ & -2.6154150 & 5.6576070 & 0.0518930 \\
$\mathrm{H}$ & -2.6116760 & 5.1133230 & 1.7466490 \\
$\mathrm{H}$ & -1.1071300 & 5.6813010 & 0.9837480 \\
$\mathrm{C}$ & -2.5947170 & 0.3140520 & 0.5128910 \\
$\mathrm{H}$ & -2.9837310 & 0.2655960 & 1.5273000 \\
$\mathrm{C}$ & -2.6634770 & -0.8381500 & -0.2506600 \\
$\mathrm{C}$ & -3.5960680 & -1.9682770 & 0.1496610 \\
$\mathrm{H}$ & -3.1347190 & -2.9264490 & -0.1113500 \\
$\mathrm{H}$ & -3.7266590 & -1.9634870 & 1.2381460 \\
$\mathrm{H}$ & -0.7490840 & 1.5789840 & -1.4714650 \\
$\mathrm{C}$ & -0.6397750 & 2.3701280 & 2.1510190 \\
$\mathrm{H}$ & -2.7852940 & 2.6346200 & 1.8375400 \\
$\mathrm{H}$ & -2.3684890 & -0.8105830 & -1.2926950 \\
$\mathrm{Cl}$ & -0.4914990 & -1.7713120 & 0.3938080 \\
$\mathrm{~N}$ & 1.1433030 & -2.6604090 & 0.7426650 \\
$\mathrm{C}$ & 1.2343040 & -3.7956370 & 1.5521520 \\
$\mathrm{C}$ & 2.3398460 & -2.2527310 & 0.2272970 \\
$\mathrm{C}$ & 2.6986370 & -4.2143880 & 1.5948450 \\
$\mathrm{C}$ & 3.4309800 & -3.1925460 & 0.7100300 \\
$\mathrm{H}$ & 2.7816030 & -5.2413450 & 1.2290350 \\
$\mathrm{H}$ & 3.9168140 & -3.6434890 & -0.1602910 \\
$\mathrm{H}$ & 4.1864690 & -2.6093790 & 1.2439530 \\
$\mathrm{H}$ & 3.0356890 & -4.2044890 & 2.6349580 \\
$\mathrm{O}$ & 2.4807750 & -1.2711490 & -0.5123460 \\
$\mathrm{O}$ & 0.2938900 & -4.3296240 & 2.1110260 \\
$\mathrm{C}$ & -2.7691030 & 1.7212200 & -2.2533860 \\
$\mathrm{H}$ & -2.7769410 & 0.6859380 & -2.5908060 \\
& & & \\
\hline
\end{tabular}




$\begin{array}{lrrr}\mathrm{H} & -3.7704130 & 1.9870800 & -1.9020550 \\ \mathrm{H} & -2.5189640 & 2.3535250 & -3.1087340 \\ \mathrm{C} & -0.5152300 & 3.5760430 & 3.1088980 \\ \mathrm{H} & 0.2554950 & 3.3609310 & 3.8557500 \\ \mathrm{H} & -0.2184550 & 4.4914940 & 2.5936030 \\ \mathrm{H} & -1.4506650 & 3.7656820 & 3.6478240 \\ \mathrm{C} & 0.6539950 & 2.2288520 & 1.3294870 \\ \mathrm{H} & 0.8418580 & 3.1151560 & 0.7145770 \\ \mathrm{H} & 1.5077400 & 2.1098680 & 2.0045940 \\ \mathrm{H} & 0.6250290 & 1.3502440 & 0.6780460 \\ \mathrm{C} & -0.8430240 & 1.1121050 & 3.0219520 \\ \mathrm{H} & -1.7850350 & 1.1552800 & 3.5817080 \\ \mathrm{H} & -0.8234560 & 0.1894140 & 2.4390320 \\ \mathrm{H} & -0.0306970 & 1.0488280 & 3.7532240 \\ \mathrm{C} & -4.9566250 & -1.8776400 & -0.5279670 \\ \mathrm{C} & -5.9989950 & -1.1307930 & 0.0428020 \\ \mathrm{C} & -5.1909250 & -2.5242280 & -1.7509510 \\ \mathrm{C} & -7.2401440 & -1.0282070 & -0.5922030 \\ \mathrm{H} & -5.8401480 & -0.6286430 & 0.9943030 \\ \mathrm{C} & -6.4299140 & -2.4222110 & -2.3922330 \\ \mathrm{H} & -4.3982050 & -3.1155290 & -2.2033820 \\ \mathrm{C} & -7.4594630 & -1.6724840 & -1.8148050 \\ \mathrm{H} & -8.0358860 & -0.4496980 & -0.1310780 \\ \mathrm{H} & -6.5913500 & -2.9319140 & -3.3380830 \\ \mathrm{H} & -8.4236650 & -1.5950280 & -2.3088320 \\ \mathrm{H} & 3.8312090 & -0.7171300 & -1.0224150 \\ \mathrm{O} & 4.6511420 & -0.2855950 & -1.4591250 \\ \mathrm{C} & 5.6108760 & -0.0562610 & -0.5959110 \\ \mathrm{O} & 5.6263960 & -0.2971460 & 0.5932020 \\ \mathrm{C} & 6.8261200 & 0.6091770 & -1.3000530 \\ \mathrm{~F} & 6.4792470 & 1.8029400 & -1.8322250 \\ \mathrm{~F} & 7.8319400 & 0.8172670 & -0.4364790 \\ \mathrm{~F} & 7.2913840 & -0.1689930 & -2.3028370\end{array}$

2.log

Potential Energy $=-2233.46485$

Zero-point Energy = -2232.93457

Free Energy = -2232.98474

Single-Point Energy B3LYP-D3(BJ)/6-311+G** PCM = 2234.03723

Free Energy B3LYP-D3(BJ)/6-311+G** PCM (extrapolated free energy from $\mathrm{qRRHO})=-2233.55711$

Nimag $=1\left(-171.8028 \mathrm{~cm}^{-1}\right)$

Charge $=0$ Multiplicity $=1$

$\begin{array}{lccc}\mathrm{C} & -2.7058680 & 3.0154530 & 0.6276940 \\ \mathrm{C} & -1.8811130 & 3.7965180 & -1.4413000 \\ \mathrm{C} & -1.9170200 & 2.2731130 & -1.5712490 \\ \mathrm{~N} & -2.5903330 & 1.8808380 & -0.3214810 \\ \mathrm{~N} & -2.4226090 & 4.1501540 & -0.2540050 \\ \mathrm{O} & -1.4918530 & 4.5736960 & -2.3151280 \\ \mathrm{C} & -2.8014910 & 5.5340440 & 0.0175810 \\ \mathrm{H} & -3.1744500 & 5.9778370 & -0.9081480 \\ \mathrm{H} & -3.5909720 & 5.5517160 & 0.7690210 \\ \mathrm{H} & -1.9556600 & 6.1303670 & 0.3706090 \\ \mathrm{C} & -3.1477100 & 0.6794350 & -0.0599520 \\ \mathrm{H} & -3.7954200 & 0.6824560 & 0.8139220 \\ \mathrm{C} & -2.9881560 & -0.5168990 & -0.7311370 \\ \mathrm{C} & -3.9937280 & -1.6392060 & -0.5170510 \\ \mathrm{H} & -4.3015620 & -1.6611780 & 0.5344890 \\ \mathrm{H} & -4.9005250 & -1.4058200 & -1.0957840 \\ \mathrm{H} & -0.8917990 & 1.8807690 & -1.6040410 \\ \mathrm{C} & -1.7561670 & 2.9254220 & 1.8793070 \\ \mathrm{H} & -3.7422300 & 3.0792560 & 0.9854010 \\ \mathrm{H} & -2.4203300 & -0.5450690 & -1.6532300 \\ \mathrm{Cl} & -0.9680190 & -1.2229210 & 0.5375410 \\ \mathrm{~N} & 0.5877030 & -1.8890280 & 1.3528590 \\ \mathrm{C} & 0.5680300 & -2.6744550 & 2.5094870 \\ \mathrm{C} & 1.8414160 & -1.6568590 & 0.8628440 \\ \mathrm{C} & 2.0112280 & -3.0218040 & 2.8517570 \\ \mathrm{C} & 2.8541230 & -2.3588540 & 1.7498760 \\ \mathrm{H} & 2.1140180 & -4.1098580 & 2.8798160 \\ \mathrm{H} & 3.4118360 & -3.0776490 & 1.1421560 \\ \mathrm{H} & 3.5678170 & -1.6198360 & 2.1241040 \\ \mathrm{H} & 2.2346870 & -2.6415540 & 3.8523500 \\ \mathrm{O} & 2.0785310 & -0.9803870 & -0.1447560 \\ \mathrm{O} & -0.4373480 & -3.0010550 & 3.1121990\end{array}$

$\begin{array}{lrrr}\mathrm{C} & -2.6569520 & 1.9112220 & -2.8685320 \\ \mathrm{H} & -2.5856000 & 0.8525200 & -3.1133250 \\ \mathrm{H} & -3.7133950 & 2.1878530 & -2.8005930 \\ \mathrm{H} & -2.1985990 & 2.4779830 & -3.6825020 \\ \mathrm{C} & -1.9106450 & 4.2021860 & 2.7358800 \\ \mathrm{H} & -1.3662340 & 4.0694240 & 3.6762110 \\ \mathrm{H} & -1.4985940 & 5.0869160 & 2.2468490 \\ \mathrm{H} & -2.9589220 & 4.3991320 & 2.9887990 \\ \mathrm{C} & -0.2872460 & 2.7711010 & 1.4466650 \\ \mathrm{H} & 0.0463530 & 3.6185750 & 0.8386330 \\ \mathrm{H} & 0.3554040 & 2.7285790 & 2.3322150 \\ \mathrm{H} & -0.1288030 & 1.8492440 & 0.8791010 \\ \mathrm{C} & -2.1694420 & 1.7293900 & 2.7631340 \\ \mathrm{H} & -3.2287440 & 1.7814810 & 3.0421000 \\ \mathrm{H} & -1.9799080 & 0.7671060 & 2.2844990 \\ \mathrm{H} & -1.5855070 & 1.7499860 & 3.6890280 \\ \mathrm{C} & -3.5179310 & -3.0181110 & -0.9373960 \\ \mathrm{C} & -3.2595130 & -3.3032360 & -2.2880760 \\ \mathrm{C} & -3.3525920 & -4.0450890 & 0.0025000 \\ \mathrm{C} & -2.8352680 & -4.5740310 & -2.6861440 \\ \mathrm{H} & -3.3964510 & -2.5271430 & -3.0375770 \\ \mathrm{C} & -2.9330340 & -5.3206520 & -0.3908330 \\ \mathrm{H} & -3.5509050 & -3.8454600 & 1.0524250 \\ \mathrm{C} & -2.6698580 & -5.5891850 & -1.7371680 \\ \mathrm{H} & -2.6405460 & -4.7731820 & -3.7363370 \\ \mathrm{H} & -2.8118150 & -6.1015390 & 0.3548520 \\ \mathrm{H} & -2.3435480 & -6.5784020 & -2.0450910 \\ \mathrm{H} & 3.4846340 & -0.5998850 & -0.6684180 \\ \mathrm{O} & 4.3475510 & -0.3149500 & -1.1398130 \\ \mathrm{C} & 5.2296730 & 0.1802760 & -0.3057800 \\ \mathrm{O} & 5.1357230 & 0.3139860 & 0.8964810 \\ \mathrm{C} & 6.5119960 & 0.6143270 & -1.0691440 \\ \mathrm{~F} & 6.2277470 & 1.5668820 & -1.9857080 \\ \mathrm{~F} & 7.4326260 & 1.1102860 & -0.2282180 \\ \mathrm{~F} & 7.0642440 & -0.4331310 & -1.7217370\end{array}$

3.log

Potential Energy $=-2233.46543$

Zero-point Energy = -2232.93491

Free Energy $=-2232.98531$

Single-Point Energy B3LYP-D3(BJ)/6-311+G** PCM = 2234.03863

Free Energy B3LYP-D3(BJ)/6-311+G** PCM (extrapolated free energy from $q R R H O)=-2233.55852$

Nimag $=1\left(-190.9753 \mathrm{~cm}^{-1}\right)$

Charge $=0$ Multiplicity $=1$

$\begin{array}{lccc}\mathrm{C} & -3.7783670 & -0.3556430 & 1.4307710 \\ \mathrm{C} & -5.5319040 & 0.9399670 & 0.5300570 \\ \mathrm{C} & -4.5051520 & 0.8098680 & -0.5974540 \\ \mathrm{~N} & -3.5493990 & -0.1532610 & -0.0213310 \\ \mathrm{~N} & -5.1249230 & 0.1984310 & 1.5845880 \\ \mathrm{O} & -6.5926070 & 1.5624790 & 0.4534660 \\ \mathrm{C} & -6.0338280 & -0.1106920 & 2.6850990 \\ \mathrm{H} & -7.0376160 & -0.2451630 & 2.2761610 \\ \mathrm{H} & -5.7147660 & -1.0342610 & 3.1686120 \\ \mathrm{H} & -6.0673800 & 0.6908200 & 3.4281010 \\ \mathrm{C} & -2.6275470 & -0.8701160 & -0.6939810 \\ \mathrm{H} & -2.2022530 & -1.6857150 & -0.1159730 \\ \mathrm{C} & -2.1528860 & -0.6753540 & -1.9787980 \\ \mathrm{C} & -1.4118530 & -1.7614940 & -2.7501900 \\ \mathrm{H} & -0.6387170 & -1.2846260 & -3.3636260 \\ \mathrm{H} & -4.0239930 & 1.7817560 & -0.7703030 \\ \mathrm{C} & -2.6997920 & 0.3124040 & 2.3611140 \\ \mathrm{H} & -3.7988860 & -1.4326750 & 1.6424910 \\ \mathrm{H} & -2.6187670 & 0.0754620 & -2.6051770 \\ \mathrm{Cl} & -0.4011950 & 0.9407510 & -1.4227980 \\ \mathrm{~N} & 0.9772170 & 2.2364860 & -1.2374980 \\ \mathrm{C} & 0.8642230 & 3.5373580 & -1.7327390 \\ \mathrm{C} & 2.1701300 & 1.9802970 & -0.6268790 \\ \mathrm{C} & 2.1590610 & 4.2706300 & -1.4038050 \\ \mathrm{C} & 3.0278930 & 3.2339150 & -0.6726880 \\ \mathrm{H} & 1.9214550 & 5.1439270 & -0.7902370 \\ \mathrm{H} & 3.2836630 & 3.5254180 & 0.3499950 \\ \mathrm{H} & 3.9611830 & 3.0048060 & -1.1949460 \\ \mathrm{H} & 2.6063740 & 4.6305030 & -2.3342530 \\ \mathrm{O} & 2.4761440 & 0.8917700 & -0.1247690\end{array}$




$\begin{array}{lrrr}\mathrm{O} & -0.1084200 & 3.9782560 & -2.3174940 \\ \mathrm{C} & -5.2299520 & 0.3521020 & -1.8721190 \\ \mathrm{H} & -4.6024440 & 0.4139090 & -2.7601200 \\ \mathrm{H} & -5.5919560 & -0.6746850 & -1.7645910 \\ \mathrm{H} & -6.0885930 & 1.0116550 & -2.0192300 \\ \mathrm{C} & -3.1023040 & 0.1129540 & 3.8393230 \\ \mathrm{H} & -2.2786010 & 0.4406840 & 4.4814040 \\ \mathrm{H} & -3.9804640 & 0.6996030 & 4.1160260 \\ \mathrm{H} & -3.2992310 & -0.9403820 & 4.0697430 \\ \mathrm{C} & -2.5681550 & 1.8168630 & 2.0633320 \\ \mathrm{H} & -3.5159530 & 2.3440500 & 2.2147610 \\ \mathrm{H} & -1.8323550 & 2.2628540 & 2.7405690 \\ \mathrm{H} & -2.2260370 & 1.9974810 & 1.0397390 \\ \mathrm{C} & -1.3360180 & -0.3805600 & 2.1575160 \\ \mathrm{H} & -1.4063050 & -1.4636370 & 2.3134640 \\ \mathrm{H} & -0.9142000 & -0.1963460 & 1.1678360 \\ \mathrm{H} & -0.6227200 & 0.0103330 & 2.8903460 \\ \mathrm{H} & -2.1220120 & -2.2089050 & -3.4596270 \\ \mathrm{C} & -0.7897640 & -2.8703940 & -1.9232950 \\ \mathrm{C} & 0.5001350 & -2.7376310 & -1.3852320 \\ \mathrm{C} & -1.4921620 & -4.0621650 & -1.6839380 \\ \mathrm{C} & 1.0703550 & -3.7643510 & -0.6263500 \\ \mathrm{H} & 1.0619400 & -1.8253880 & -1.5642640 \\ \mathrm{C} & -0.9266890 & -5.0917320 & -0.9247800 \\ \mathrm{H} & -2.4887120 & -4.1871040 & -2.1011280 \\ \mathrm{C} & 0.3581460 & -4.9453350 & -0.3924520 \\ \mathrm{H} & 2.0718800 & -3.6435580 & -0.2230510 \\ \mathrm{H} & -1.4865550 & -6.0071890 & -0.7550620 \\ \mathrm{H} & 0.8019860 & -5.7448710 & 0.1936970 \\ \mathrm{H} & 3.8284510 & 0.5742090 & 0.5543120 \\ \mathrm{O} & 4.6564440 & 0.3192020 & 1.1008010 \\ \mathrm{C} & 5.6489640 & -0.0924380 & 0.3491570 \\ \mathrm{C} & 6.8745280 & -0.4633750 & 1.2304460 \\ \mathrm{O} & 5.6885440 & -0.1951230 & -0.8587350 \\ \mathrm{~F} & 7.8968810 & -0.9020480 & 0.4800070 \\ & 6.5590760 & -1.4359020 & 2.1151410 \\ & 7.3038690 & 0.6090100 & 1.9340450\end{array}$

\section{$(R)$-TS-back}

\section{Conformation Extrapolated name \\ (kcal/mol)}

TS4-Rb-lowest

0.0

$\begin{array}{ll}1 & 0.6 \\ 2 & 0.9 \\ 3 & 1.7\end{array}$

TS4-Rb-lowest.log

Potential Energy = -2233.46612

Zero-point Energy = -2232.93524

Free Energy = -2232.98573

Single-Point Energy B3LYP-D3(BJ)/6-311+G** PCM = 2234.03577

Free Energy B3LYP-D3(BJ)/6-311+G** PCM (extrapolated free energy from $q R R H O)=-2233.55538$

Nimag $=1\left(-203.2629 \mathrm{~cm}^{-1}\right)$

Charge $=0$ Multiplicity $=1$

$\begin{array}{lrrr}\mathrm{C} & -3.3008510 & -1.1285990 & -1.2914060 \\ \mathrm{C} & -5.2086170 & -2.0593700 & -0.2577480 \\ \mathrm{C} & -4.1217700 & -1.9819430 & 0.8201900 \\ \mathrm{~N} & -3.1343610 & -1.0759070 & 0.1821480 \\ \mathrm{~N} & -4.7156180 & -1.5244490 & -1.3974470 \\ \mathrm{O} & -6.3450400 & -2.5092930 & -0.0981590 \\ \mathrm{C} & -5.5671230 & -1.2645690 & -2.5540690 \\ \mathrm{H} & -6.5544980 & -0.9647180 & -2.1962530 \\ \mathrm{H} & -5.1374910 & -0.4550950 & -3.1452800 \\ \mathrm{H} & -5.6812330 & -2.1493110 & -3.1868930 \\ \mathrm{C} & -2.6007950 & -0.0235120 & 0.8367350 \\ \mathrm{H} & -2.8642470 & 0.0193710 & 1.8887950 \\ \mathrm{C} & -1.7481330 & 0.9609320 & 0.3653790 \\ \mathrm{C} & -1.6451990 & 2.2967620 & 1.0842670\end{array}$

\begin{tabular}{|c|c|c|c|}
\hline $\mathrm{H}$ & -0.6224410 & 2.6771120 & 0.9940490 \\
\hline $\mathrm{H}$ & -1.8405000 & 2.1532950 & 2.1529280 \\
\hline $\mathrm{H}$ & -1.4573530 & 0.9631370 & -0.6806990 \\
\hline $\mathrm{C}$ & -4.6837830 & -1.5467210 & 2.1688460 \\
\hline $\mathrm{H}$ & -5.4704360 & -2.2546630 & 2.4400500 \\
\hline $\mathrm{H}$ & -3.9230020 & -1.5757000 & 2.9528070 \\
\hline $\mathrm{H}$ & -5.1247410 & -0.5465510 & 2.1268060 \\
\hline $\mathrm{H}$ & -3.6774990 & -2.9784400 & 0.9320750 \\
\hline $\mathrm{C}$ & -2.3083690 & -2.0806760 & -2.0456340 \\
\hline $\mathrm{H}$ & -3.1822950 & -0.1150950 & -1.6896070 \\
\hline $\mathrm{Cl}$ & 0.2191250 & -0.0853760 & 1.3725210 \\
\hline $\mathrm{N}$ & 1.8748670 & -0.6814830 & 2.1135310 \\
\hline $\mathrm{C}$ & 3.0460280 & -0.6931600 & 1.4153300 \\
\hline $\mathrm{C}$ & 2.0030770 & -1.1167490 & 3.4339620 \\
\hline $\mathrm{C}$ & 4.1566500 & -1.2059950 & 2.3162300 \\
\hline $\mathrm{C}$ & 3.4657930 & -1.4796240 & 3.6618390 \\
\hline $\mathrm{H}$ & 4.9438360 & -0.4489110 & 2.3708200 \\
\hline $\mathrm{H}$ & 3.8555830 & -0.8668030 & 4.4792740 \\
\hline $\mathrm{O}$ & 1.0907140 & -1.1834540 & 4.2380170 \\
\hline $\mathrm{O}$ & 3.1564830 & -0.3373420 & 0.2345770 \\
\hline $\mathrm{C}$ & -2.6353330 & -2.0632550 & -3.5552670 \\
\hline $\mathrm{H}$ & -1.8480350 & -2.5959910 & -4.0981740 \\
\hline $\mathrm{H}$ & -3.5803090 & -2.5611870 & -3.7836290 \\
\hline $\mathrm{H}$ & -2.6748620 & -1.0421860 & -3.9515630 \\
\hline $\mathrm{C}$ & -2.3905470 & -3.5283320 & -1.5262170 \\
\hline $\mathrm{H}$ & -1.7435840 & -4.1691420 & -2.1340200 \\
\hline $\mathrm{H}$ & -2.0463880 & -3.6075500 & -0.4904420 \\
\hline $\mathrm{H}$ & -3.4062130 & -3.9316970 & -1.5935060 \\
\hline $\mathrm{C}$ & -0.8708420 & -1.5589130 & -1.8645760 \\
\hline $\mathrm{H}$ & -0.7525780 & -0.5501000 & -2.2757140 \\
\hline $\mathrm{H}$ & -0.5699240 & -1.5440250 & -0.8153560 \\
\hline $\mathrm{H}$ & -0.1769230 & -2.2137130 & -2.4016630 \\
\hline $\mathrm{H}$ & 3.5248150 & -2.5256460 & 3.9742890 \\
\hline $\mathrm{H}$ & 4.5919550 & -2.1019600 & 1.8637310 \\
\hline $\mathrm{C}$ & -2.6092740 & 3.3330440 & 0.5230770 \\
\hline $\mathrm{C}$ & -3.9029410 & 3.4755300 & 1.0482320 \\
\hline $\mathrm{C}$ & -2.2282040 & 4.1570940 & -0.5469370 \\
\hline $\mathrm{C}$ & -4.7945890 & 4.4117340 & 0.5160230 \\
\hline $\mathrm{H}$ & -4.2143040 & 2.8530440 & 1.8837550 \\
\hline $\mathrm{C}$ & -3.1173850 & 5.0930340 & -1.0849640 \\
\hline $\mathrm{H}$ & -1.2261690 & 4.0680600 & -0.9601020 \\
\hline $\mathrm{C}$ & -4.4052800 & 5.2228960 & -0.5553700 \\
\hline $\mathrm{H}$ & -5.7899050 & 4.5103920 & 0.9403690 \\
\hline $\mathrm{H}$ & -2.8022950 & 5.7228770 & -1.9122760 \\
\hline $\mathrm{H}$ & -5.0961730 & 5.9518630 & -0.9688890 \\
\hline $\mathrm{H}$ & 4.4803670 & -0.2698870 & -0.5474300 \\
\hline $\mathrm{O}$ & 5.2819090 & -0.2286310 & -1.1867560 \\
\hline $\mathrm{C}$ & 6.2986220 & 0.4182710 & -0.6714190 \\
\hline $\mathrm{O}$ & 6.3866630 & 0.9320900 & 0.4244020 \\
\hline $\mathrm{C}$ & 7.4785950 & 0.4672500 & -1.6819120 \\
\hline $\mathrm{F}$ & 7.1144790 & 1.1043420 & -2.8177230 \\
\hline $\mathrm{F}$ & 8.5339270 & 1.1146620 & -1.1642670 \\
\hline $\mathrm{F}$ & 7.8786260 & -0.7794740 & -2.0194450 \\
\hline
\end{tabular}

$1 . \log$

Potential Energy = -2233.46607

Zero-point Energy = -2232.93504

Free Energy $=-2232.98523$

Single-Point Energy B3LYP-D3(BJ)/6-311+G** PCM = 2234.03525

Free Energy B3LYP-D3(BJ)/6-311+G** PCM (extrapolated

free energy from $q R R H O)=-2233.55440$

Nimag $=1\left(-203.4857 \mathrm{~cm}^{-1}\right)$

Charge $=0$ Multiplicity $=1$

$\begin{array}{llll}\mathrm{C} & -3.2966830 & -0.8747570 & -1.4396090 \\ \mathrm{C} & -5.2671500 & -1.8516490 & -0.5813030 \\ \mathrm{C} & -4.2042890 & -1.9768120 & 0.5157690 \\ \mathrm{~N} & -3.1623870 & -1.0383910 & 0.0290560 \\ \mathrm{~N} & -4.7250260 & -1.1838230 & -1.6244170 \\ \mathrm{O} & -6.4252730 & -2.2672610 & -0.5071230 \\ \mathrm{C} & -5.5380460 & -0.7245360 & -2.7462660 \\ \mathrm{H} & -6.5199940 & -0.4353080 & -2.3655850 \\ \mathrm{H} & -5.0623340 & 0.1411190 & -3.2083660 \\ \mathrm{H} & -5.6745530 & -1.5043010 & -3.5009780 \\ \mathrm{C} & -2.5964370 & -0.1163530 & 0.8357730 \\ \mathrm{H} & -2.8817150 & -0.2110850 & 1.8787430\end{array}$




\begin{tabular}{|c|c|c|c|}
\hline $\mathrm{C}$ & -1.6902180 & 0.8834270 & 0.5241440 \\
\hline $\mathrm{C}$ & -1.5427480 & 2.0964180 & 1.4286310 \\
\hline $\mathrm{H}$ & -0.4999200 & 2.4297500 & 1.4201340 \\
\hline $\mathrm{H}$ & -1.7802110 & 1.8144610 & 2.4606280 \\
\hline $\mathrm{H}$ & -1.3757580 & 1.0211110 & -0.5059020 \\
\hline $\mathrm{C}$ & -4.7808620 & -1.7061380 & 1.9009100 \\
\hline $\mathrm{H}$ & -5.6070470 & -2.4043450 & 2.0542780 \\
\hline $\mathrm{H}$ & -4.0438440 & -1.8818010 & 2.6883500 \\
\hline $\mathrm{H}$ & -5.1730670 & -0.6889330 & 1.9904080 \\
\hline $\mathrm{H}$ & -3.8075620 & -2.9990700 & 0.4955700 \\
\hline $\mathrm{C}$ & -2.3309450 & -1.7569920 & -2.3051800 \\
\hline $\mathrm{H}$ & -3.1223800 & 0.1777640 & -1.6879310 \\
\hline $\mathrm{Cl}$ & 0.2107420 & -0.3895880 & 1.3914400 \\
\hline $\mathrm{N}$ & 1.8272150 & -1.1668340 & 2.0423010 \\
\hline $\mathrm{C}$ & 3.0253380 & -1.0405600 & 1.4036480 \\
\hline $\mathrm{C}$ & 1.8928980 & -1.9135590 & 3.2202550 \\
\hline $\mathrm{C}$ & 4.0916980 & -1.7750780 & 2.1995480 \\
\hline $\mathrm{C}$ & 3.3384310 & -2.3597840 & 3.4054900 \\
\hline $\mathrm{H}$ & 4.8749340 & -1.0651570 & 2.4807630 \\
\hline $\mathrm{H}$ & 3.7022210 & -1.9841410 & 4.3656750 \\
\hline $\mathrm{O}$ & 0.9467860 & -2.1509110 & 3.9492890 \\
\hline $\mathrm{O}$ & 3.1887520 & -0.4207110 & 0.3446390 \\
\hline $\mathrm{C}$ & -2.6253090 & -1.5140430 & -3.8020210 \\
\hline $\mathrm{H}$ & -1.8485390 & -1.9980100 & -4.4026560 \\
\hline $\mathrm{H}$ & -3.5843130 & -1.9345840 & -4.1126260 \\
\hline $\mathrm{H}$ & -2.6161710 & -0.4466820 & -4.0507910 \\
\hline $\mathrm{C}$ & -2.4859500 & -3.2576960 & -1.9952190 \\
\hline $\mathrm{H}$ & -1.8530390 & -3.8360900 & -2.6761310 \\
\hline $\mathrm{H}$ & -2.1690720 & -3.4958380 & -0.9751580 \\
\hline $\mathrm{H}$ & -3.5159770 & -3.6015750 & -2.1352250 \\
\hline $\mathrm{C}$ & -0.8764960 & -1.3311820 & -2.0320760 \\
\hline $\mathrm{H}$ & -0.7070500 & -0.2810140 & -2.2953210 \\
\hline $\mathrm{H}$ & -0.5965620 & -1.4771390 & -0.9871390 \\
\hline $\mathrm{H}$ & -0.2005620 & -1.9347580 & -2.6464640 \\
\hline $\mathrm{H}$ & 3.3667690 & -3.4521250 & 3.4440750 \\
\hline $\mathrm{H}$ & 4.5488310 & -2.5370340 & 1.5618090 \\
\hline $\mathrm{C}$ & -2.4337560 & 3.2529350 & 0.9959330 \\
\hline $\mathrm{C}$ & -3.7549830 & 3.3593370 & 1.4577440 \\
\hline $\mathrm{C}$ & -1.9556970 & 4.2293570 & 0.1089420 \\
\hline $\mathrm{C}$ & -4.5782580 & 4.4096580 & 1.0416160 \\
\hline $\mathrm{H}$ & -4.1424020 & 2.6175570 & 2.1522320 \\
\hline $\mathrm{C}$ & -2.7764010 & 5.2806150 & -0.3129460 \\
\hline $\mathrm{H}$ & -0.9315970 & 4.1687790 & -0.2519420 \\
\hline $\mathrm{C}$ & -4.0919720 & 5.3738660 & 0.1517850 \\
\hline $\mathrm{H}$ & -5.5963000 & 4.4773530 & 1.4150740 \\
\hline $\mathrm{H}$ & -2.3863410 & 6.0274770 & -0.9988570 \\
\hline $\mathrm{H}$ & -4.7299250 & 6.1914670 & -0.1711260 \\
\hline $\mathrm{H}$ & 4.5374210 & -0.2996810 & -0.3961310 \\
\hline $\mathrm{O}$ & 5.3771070 & -0.2739740 & -0.9838440 \\
\hline $\mathrm{C}$ & 6.1586870 & 0.7410680 & -0.7049120 \\
\hline $\mathrm{O}$ & 5.9988500 & 1.6111220 & 0.1249910 \\
\hline $\mathrm{C}$ & 7.4204920 & 0.7148700 & -1.6125220 \\
\hline $\mathrm{F}$ & 7.0802630 & 0.7407870 & -2.9205090 \\
\hline $\mathrm{F}$ & 8.2148870 & 1.7690000 & -1.3704050 \\
\hline $\mathrm{F}$ & 8.1422950 & -0.4089450 & -1.3974070 \\
\hline
\end{tabular}

$2 . \log$

Potential Energy $=-2233.46318$

Zero-point Energy = -2232.93256

Free Energy = -2232.98266

Single-Point Energy B3LYP-D3(BJ)/6-311+G** PCM = 2234.03449

Free Energy B3LYP-D3(BJ)/6-311+G** PCM (extrapolated free energy from $\mathrm{qRRHO})=-2233.55397$

Nimag $=1\left(-210.8518 \mathrm{~cm}^{-1}\right)$

Charge $=0$ Multiplicity $=1$

$\begin{array}{llll}\mathrm{C} & -3.9907910 & -1.0164820 & -0.9464790 \\ \mathrm{C} & -5.9962170 & -0.9961520 & 0.2988210 \\ \mathrm{C} & -4.8967050 & -0.4768100 & 1.2316440 \\ \mathrm{~N} & -3.8000060 & -0.1938080 & 0.2728710 \\ \mathrm{~N} & -5.4481600 & -1.2314190 & -0.9144640 \\ \mathrm{O} & -7.1820660 & -1.1446750 & 0.6016960 \\ \mathrm{C} & -6.2710620 & -1.5317320 & -2.0820460 \\ \mathrm{H} & -7.2012990 & -0.9645640 & -2.0049490 \\ \mathrm{H} & -5.7406850 & -1.2308210 & -2.9862270 \\ \mathrm{H} & -6.5178450 & -2.5950880 & -2.1486560\end{array}$

\begin{tabular}{|c|c|c|}
\hline-3.1225930 & 0.9763750 & 0.2865730 \\
\hline-3.3782970 & 1.6223050 & 1.1205470 \\
\hline-2.1483350 & 1.4337220 & -0.5812480 \\
\hline-1.8804550 & 2.9282130 & -0.6977060 \\
\hline-1.9665750 & 3.3972020 & 0.2886820 \\
\hline-2.6767860 & 3.3692440 & -1.3159290 \\
\hline-1.8572040 & 0.8248880 & -1.4314950 \\
\hline-5.3813240 & 0.6884370 & 2.0873380 \\
\hline-6.2562310 & 0.3446970 & 2.6441410 \\
\hline-4.6252320 & 1.0013240 & 2.8115670 \\
\hline-5.6800120 & 1.5477860 & 1.4797850 \\
\hline-4.5943370 & -1.2954310 & 1.8962330 \\
\hline-3.1484230 & -2.3382090 & -1.0034560 \\
\hline-3.7386020 & -0.4059480 & -1.8205200 \\
\hline-0.3945840 & 0.8091800 & 1.0480730 \\
\hline 1.0963940 & 0.4412040 & 2.1667260 \\
\hline 2.2361870 & -0.1587690 & 1.7168030 \\
\hline 1.1354130 & 0.7803270 & 3.5209200 \\
\hline 3.2160670 & -0.2890720 & 2.8699630 \\
\hline 2.4931070 & 0.3526750 & 4.0651710 \\
\hline 4.1500890 & 0.2099730 & 2.5978950 \\
\hline 3.0069650 & 1.2356330 & 4.4553080 \\
\hline 0.2308090 & 1.3221250 & 4.1300980 \\
\hline 2.4128690 & -0.5295290 & 0.5493110 \\
\hline-3.5113930 & -3.1202040 & -2.2850770 \\
\hline-2.8103160 & -3.9519850 & -2.4074430 \\
\hline & -3.5464730 & \\
\hline-3.4362230 & -2.4924450 & -3.1803990 \\
\hline-3.3982150 & -3.2338310 & 0.2244330 \\
\hline-2.85 & -4.1760450 & 0.1036 \\
\hline-3.0394480 & -2.7657290 & 1.1462440 \\
\hline & & 0.3428780 \\
\hline-1.6520550 & -1.9807570 & -1.0733950 \\
\hline-1.4195240 & -1.3900360 & -1.9667260 \\
\hline-1.32 & -1.4251060 & -0.1936990 \\
\hline-1.0612600 & -2.9009830 & -1.1279260 \\
\hline 2.33 & -0.3365170 & 4.8994850 \\
\hline 3.4407840 & -1.3492340 & 3.0206210 \\
\hline-0.5472160 & 3.2917590 & -1.3263400 \\
\hline-0.2878870 & 2.9876050 & -2.6727010 \\
\hline 0.4422450 & 3.9620320 & -0.5933380 \\
\hline 0.9301090 & 3.3313990 & -3.2657070 \\
\hline-1.0468480 & 2.4831980 & -3.2663330 \\
\hline 1.6614970 & 4.3129870 & -1.1831730 \\
\hline 0.2587530 & 4.2103610 & 0.4487220 \\
\hline 1.9112960 & 3.9958640 & -2.5214870 \\
\hline 1.1102180 & 3.0864770 & -4.3087700 \\
\hline 2.4140640 & 4.8323390 & -0.5963060 \\
\hline 2.8573500 & 4.2665490 & -2.9813900 \\
\hline 3.7121550 & -1.1494270 & -0.0000130 \\
\hline 4.4927890 & -1.6179090 & -0.4722120 \\
\hline 5.6426100 & -1.0348500 & -0.2361470 \\
\hline 5.8655410 & -0.0605940 & 0.4520500 \\
\hline 6.7877000 & -1.7725740 & -0.9845790 \\
\hline 6.5838100 & -1.7475440 & -2.3211420 \\
\hline 7.9762220 & -1.2011600 & -0.7363460 \\
\hline 6.8597750 & -3.0678710 & -0.6035340 \\
\hline
\end{tabular}

3. $\log$

Potential Energy $=-2233.46277$

Zero-point Energy = -2232.93196

Free Energy = -2232.98065

Single-Point Energy B3LYP-D3(BJ)/6-311+G** PCM = 2234.03485

Free Energy B3LYP-D3(BJ)/6-311+G** PCM (extrapolated free energy from $\mathrm{qRRHO})=-2233.55273$

Nimag $=1\left(-221.9132 \mathrm{~cm}^{-1}\right)$ Charge $=0$ Multiplicity $=1$

$\begin{array}{lrrr}\mathrm{C} & -3.1584530 & -2.2484970 & -0.4667830 \\ \mathrm{C} & -5.2630690 & -1.9280350 & 0.5547800 \\ \mathrm{C} & -4.3275320 & -0.8621820 & 1.1349350 \\ \mathrm{~N} & -3.1880880 & -0.9150100 & 0.1850450 \\ \mathrm{~N} & -4.5767060 & -2.6375010 & -0.3690790 \\ \mathrm{O} & -6.4464170 & -2.0874420 & 0.8615140 \\ \mathrm{C} & -5.2418430 & -3.5903310 & -1.2524250 \\ \mathrm{H} & -6.2349070 & -3.2043230 & -1.4928770\end{array}$




\begin{tabular}{|c|c|c|c|}
\hline $\mathrm{H}$ & -4.6645720 & -3.6958980 & -2.1715000 \\
\hline $\mathrm{H}$ & -5.3555390 & -4.5729650 & -0.7858910 \\
\hline $\mathrm{C}$ & -2.6773370 & 0.2028990 & -0.3766570 \\
\hline $\mathrm{H}$ & -3.1008660 & 1.1234910 & 0.0083110 \\
\hline $\mathrm{C}$ & -1.6766090 & 0.3180890 & -1.3254730 \\
\hline $\mathrm{C}$ & -1.5293870 & 1.5430730 & -2.2238310 \\
\hline $\mathrm{H}$ & -1.8807260 & 1.2486410 & -3.2224600 \\
\hline $\mathrm{H}$ & -0.4638230 & 1.7714490 & -2.3382760 \\
\hline $\mathrm{H}$ & -1.2251020 & -0.5828600 & -1.7292440 \\
\hline $\mathrm{C}$ & -5.0273120 & 0.4822110 & 1.2992050 \\
\hline $\mathrm{H}$ & -5.8959000 & 0.3224580 & 1.9423680 \\
\hline $\mathrm{H}$ & -4.3815450 & 1.2179960 & 1.7846090 \\
\hline $\mathrm{H}$ & -5.3796140 & 0.8824430 & 0.3441760 \\
\hline $\mathrm{H}$ & -3.9891020 & -1.2013830 & 2.1215450 \\
\hline $\mathrm{C}$ & -2.1647990 & -3.2869250 & 0.1615200 \\
\hline $\mathrm{H}$ & -2.8972720 & -2.1117940 & -1.5219750 \\
\hline $\mathrm{Cl}$ & 0.0097550 & 0.8107780 & 0.3758520 \\
\hline $\mathrm{N}$ & 1.4984790 & 1.2249670 & 1.5067340 \\
\hline $\mathrm{C}$ & 2.7564720 & 0.7480430 & 1.2873610 \\
\hline $\mathrm{C}$ & 1.3965270 & 2.0466720 & 2.6305190 \\
\hline $\mathrm{C}$ & 3.6859910 & 1.2822890 & 2.3648960 \\
\hline $\mathrm{C}$ & 2.7809120 & 2.1426100 & 3.2617760 \\
\hline $\mathrm{H}$ & 4.4899080 & 1.8502890 & 1.8878110 \\
\hline $\mathrm{H}$ & 3.0798050 & 3.1937480 & 3.2959630 \\
\hline $\mathrm{O}$ & 0.3706550 & 2.5779810 & 3.0160790 \\
\hline $\mathrm{O}$ & 3.0641840 & -0.0004890 & 0.3503580 \\
\hline $\mathrm{C}$ & -2.3038080 & -4.6361870 & -0.5777280 \\
\hline $\mathrm{H}$ & -1.5031270 & -5.3073130 & -0.2508840 \\
\hline $\mathrm{H}$ & -3.2519570 & -5.1342290 & -0.3632460 \\
\hline $\mathrm{H}$ & -2.2101900 & -4.5174710 & -1.6633030 \\
\hline $\mathrm{C}$ & -2.4291710 & -3.5027560 & 1.6634520 \\
\hline $\mathrm{H}$ & -1.7672890 & -4.2896840 & 2.0393980 \\
\hline $\mathrm{H}$ & -2.2233800 & -2.5987180 & 2.2447270 \\
\hline $\mathrm{H}$ & -3.4584740 & -3.8217330 & 1.8574710 \\
\hline $\mathrm{C}$ & -0.7211550 & -2.7874760 & -0.0316820 \\
\hline $\mathrm{H}$ & -0.4717600 & -2.6742790 & -1.0929130 \\
\hline $\mathrm{H}$ & -0.5476010 & -1.8340640 & 0.4702870 \\
\hline $\mathrm{H}$ & -0.0243450 & -3.5188970 & 0.3906360 \\
\hline $\mathrm{H}$ & 2.7234570 & 1.7801760 & 4.2917380 \\
\hline $\mathrm{H}$ & 4.1432450 & 0.4390180 & 2.8903300 \\
\hline $\mathrm{C}$ & -2.2765520 & 2.7941960 & -1.8016890 \\
\hline $\mathrm{C}$ & -1.6607010 & 3.7816580 & -1.0175820 \\
\hline $\mathrm{C}$ & -3.6059770 & 2.9980930 & -2.2064230 \\
\hline $\mathrm{C}$ & -2.3545550 & 4.9368080 & -0.6420560 \\
\hline $\mathrm{H}$ & -0.6304040 & 3.6466580 & -0.7015280 \\
\hline $\mathrm{C}$ & -4.3041870 & 4.1500770 & -1.8329200 \\
\hline $\mathrm{H}$ & -4.0962450 & 2.2498570 & -2.8249730 \\
\hline $\mathrm{C}$ & -3.6796720 & 5.1243430 & -1.0469960 \\
\hline $\mathrm{H}$ & -1.8577600 & 5.6907440 & -0.0378680 \\
\hline $\mathrm{H}$ & -5.3308330 & 4.2888080 & -2.1600430 \\
\hline $\mathrm{H}$ & -4.2183090 & 6.0223960 & -0.7586340 \\
\hline $\mathrm{H}$ & 4.4771560 & -0.5550870 & 0.0883270 \\
\hline $\mathrm{O}$ & 5.3606920 & -1.0414950 & -0.0983450 \\
\hline $\mathrm{C}$ & 6.2184790 & -0.2964520 & -0.7523380 \\
\hline $\mathrm{O}$ & 6.0850070 & 0.8453300 & -1.1397110 \\
\hline $\mathrm{C}$ & 7.5368390 & -1.0820150 & -0.9993610 \\
\hline $\mathrm{F}$ & 7.3024910 & -2.2200340 & -1.6903770 \\
\hline $\mathrm{F}$ & 8.4162630 & -0.3473280 & -1.6977570 \\
\hline $\mathrm{F}$ & 8.1183410 & -1.4248810 & 0.1729530 \\
\hline
\end{tabular}

Formation of syn-aminal syn-18b (TS5)

$\begin{array}{cc}\begin{array}{c}\text { Conformation } \\ \text { name }\end{array} & \begin{array}{c}\text { Extrapolated } \\ \text { Free energy }\end{array}\end{array}$

$\begin{array}{cc}\text { TS5-int-lowest } & 0.0 \\ 1 & 6.0 \\ 2 & 0.4 \\ 3 & 2.5 \\ 4 & 2.9\end{array}$

TS5-int-lowest.log

Potential Energy = -2233.49662
Zero-point Energy = -2232.96689

Free Energy $=-2233.01536$

Single-Point Energy B3LYP-D3(BJ)/6-311+G** PCM = -

2234.07919

Free Energy B3LYP-D3(BJ)/6-311+G** PCM (extrapolated free energy from $q R R H O)=-2233.59792$

Nimag $=1\left(-242.8971 \mathrm{~cm}^{-1}\right)$

Charge $=0$ Multiplicity $=1$

\begin{tabular}{|c|c|c|c|}
\hline $\mathrm{C}$ & -0.0676900 & 2.1356700 & -0.6441190 \\
\hline $\mathrm{C}$ & 0.9788090 & 3.1016740 & 1.2401920 \\
\hline $\mathrm{C}$ & -0.5069930 & 2.9616280 & 1.5893770 \\
\hline $\mathrm{N}$ & -0.9509820 & 1.9741380 & 0.5565620 \\
\hline $\mathrm{N}$ & 1.1690310 & 2.5910120 & 0.0026550 \\
\hline $\mathrm{O}$ & 1.8373290 & 3.5880490 & 1.9750120 \\
\hline $\mathrm{C}$ & 2.4970760 & 2.4401650 & -0.5832410 \\
\hline $\mathrm{H}$ & 3.2135170 & 2.3313810 & 0.2325880 \\
\hline $\mathrm{H}$ & 2.5237350 & 1.5438740 & -1.2048540 \\
\hline $\mathrm{H}$ & 2.7799400 & 3.3122370 & -1.1802000 \\
\hline $\mathrm{C}$ & -1.6942840 & 0.9372200 & 0.8608640 \\
\hline $\mathrm{H}$ & -2.1639830 & 0.9970090 & 1.8353970 \\
\hline $\mathrm{C}$ & -2.4458780 & 0.0269650 & -0.0963270 \\
\hline $\mathrm{C}$ & -3.3817480 & -0.9441160 & 0.6443950 \\
\hline $\mathrm{H}$ & -2.7845330 & -1.6901020 & 1.1700190 \\
\hline $\mathrm{H}$ & -3.9068770 & -0.3570510 & 1.4072770 \\
\hline $\mathrm{H}$ & -3.0491590 & 0.6961920 & -0.7150180 \\
\hline $\mathrm{C}$ & -0.7655650 & 2.6180150 & 3.0469230 \\
\hline $\mathrm{H}$ & -0.2965590 & 3.4011620 & 3.6471960 \\
\hline $\mathrm{H}$ & -1.8332430 & 2.6174410 & 3.2788630 \\
\hline $\mathrm{H}$ & -0.3272610 & 1.6609880 & 3.3316340 \\
\hline $\mathrm{H}$ & -0.9998720 & 3.9116160 & 1.3569160 \\
\hline $\mathrm{C}$ & -0.6157420 & 3.1043120 & -1.7578170 \\
\hline $\mathrm{H}$ & 0.0974660 & 1.1490490 & -1.0755460 \\
\hline $\mathrm{Cl}$ & -1.4136870 & -0.8828310 & -1.2879210 \\
\hline $\mathrm{C}$ & 0.2834820 & 2.9306420 & -3.0045730 \\
\hline $\mathrm{H}$ & -0.1396280 & 3.5043420 & -3.8349190 \\
\hline $\mathrm{H}$ & 1.2995430 & 3.2964250 & -2.8415670 \\
\hline $\mathrm{H}$ & 0.3395630 & 1.8825890 & -3.3176700 \\
\hline $\mathrm{C}$ & -2.0544100 & 2.7154100 & -2.1509580 \\
\hline $\mathrm{H}$ & -2.1112020 & 1.6905540 & -2.5296750 \\
\hline $\mathrm{H}$ & -2.7515780 & 2.8350020 & -1.3156950 \\
\hline $\mathrm{H}$ & -2.3965060 & 3.3763980 & -2.9532310 \\
\hline $\mathrm{C}$ & -0.6004470 & 4.5850190 & -1.3291630 \\
\hline $\mathrm{H}$ & 0.3842370 & 4.9041600 & -0.9752460 \\
\hline $\mathrm{H}$ & -0.8561500 & 5.2076520 & -2.1922510 \\
\hline $\mathrm{H}$ & -1.3401610 & 4.8007420 & -0.5522300 \\
\hline $\mathrm{N}$ & -0.3978570 & -0.6315820 & 1.8649200 \\
\hline $\mathrm{C}$ & -0.7766850 & -1.1686660 & 3.0935660 \\
\hline $\mathrm{C}$ & 0.8306060 & -1.0403030 & 1.5649360 \\
\hline $\mathrm{C}$ & 0.3165750 & -2.0852860 & 3.6416530 \\
\hline $\mathrm{C}$ & 1.4286050 & -1.9865420 & 2.5908720 \\
\hline $\mathrm{H}$ & -0.0848200 & -3.0963210 & 3.7555430 \\
\hline $\mathrm{H}$ & 1.6711590 & -2.9410090 & 2.1140590 \\
\hline $\mathrm{H}$ & 2.3630620 & -1.5690780 & 2.9778770 \\
\hline $\mathrm{H}$ & 0.6206480 & -1.7372640 & 4.6326570 \\
\hline $\mathrm{O}$ & 1.4394860 & -0.6535150 & 0.5116560 \\
\hline $\mathrm{O}$ & -1.8443010 & -0.9175730 & 3.6424710 \\
\hline $\mathrm{H}$ & 2.4918950 & -1.0858790 & 0.3430170 \\
\hline $\mathrm{O}$ & 3.6545720 & -1.5644430 & 0.1757170 \\
\hline $\mathrm{C}$ & 4.1898400 & -1.3628880 & -0.9718070 \\
\hline $\mathrm{C}$ & 5.6122050 & -1.9990250 & -1.0639670 \\
\hline $\mathrm{O}$ & 3.7456330 & -0.7647180 & -1.9484080 \\
\hline $\mathrm{F}$ & 6.4442030 & -1.4802660 & -0.1254900 \\
\hline $\mathrm{F}$ & 5.5650180 & -3.3399090 & -0.8600690 \\
\hline $\mathrm{F}$ & 6.1895210 & -1.7974740 & -2.2649060 \\
\hline $\mathrm{C}$ & -4.3991650 & -1.6148080 & -0.2566480 \\
\hline $\mathrm{C}$ & -4.2834460 & -2.9728860 & -0.5841800 \\
\hline $\mathrm{C}$ & -5.4881580 & -0.8905870 & -0.7674960 \\
\hline $\mathrm{C}$ & -5.2268660 & -3.5932020 & -1.4097180 \\
\hline $\mathrm{H}$ & -3.4509800 & -3.5495840 & -0.1901910 \\
\hline $\mathrm{C}$ & -6.4311560 & -1.5055800 & -1.5950360 \\
\hline $\mathrm{H}$ & -5.6072120 & 0.1594780 & -0.5103080 \\
\hline $\mathrm{C}$ & -6.3020670 & -2.8603420 & -1.9201860 \\
\hline $\mathrm{H}$ & -5.1209280 & -4.6468120 & -1.6516680 \\
\hline $\mathrm{H}$ & -7.2683870 & -0.9299920 & -1.9792050 \\
\hline $\mathrm{H}$ & -7.0362030 & -3.3405350 & -2.5605070 \\
\hline
\end{tabular}


$1 . \log$

Potential Energy $=-2233.48496$

Zero-point Energy $=-2232.95406$

Free Energy $=-2233.00229$

Single-Point Energy B3LYP-D3(BJ)/6-311+G** PCM = 2234.07105

Free Energy B3LYP-D3(BJ)/6-311+G** PCM (extrapolated free energy from $q R R H O)=-2233.58838$

Nimag $=1\left(-101.8706 \mathrm{~cm}^{-1}\right)$ Charge $=0$ Multiplicity $=1$

\begin{tabular}{|c|c|c|c|}
\hline C & -0.0106870 & 2.4907310 & -0.4376370 \\
\hline C & 0.2278160 & 2.9209930 & 1.8731100 \\
\hline $\mathrm{C}$ & -1.2757390 & 2.9052890 & 1.5746230 \\
\hline $\mathrm{N}$ & -1.2982800 & 2.1796660 & 0.2681380 \\
\hline $\mathrm{N}$ & 0.8815700 & 2.6473780 & 0.7203170 \\
\hline $\mathrm{O}$ & 0.7354740 & 3.1427780 & 2.9716370 \\
\hline $\mathrm{C}$ & 2.3304100 & 2.4804990 & 0.6638020 \\
\hline $\mathrm{H}$ & 2.6648120 & 2.1164680 & 1.6368010 \\
\hline $\mathrm{H}$ & 2.5872920 & 1.7457870 & -0.1006790 \\
\hline $\mathrm{H}$ & 2.8398480 & 3.4258870 & 0.4535010 \\
\hline C & -2.0723070 & 1.1379720 & 0.0490340 \\
\hline $\mathrm{H}$ & -2.8608890 & 1.0032940 & 0.7807140 \\
\hline $\mathrm{C}$ & -2.4184770 & 0.5864320 & -1.3307070 \\
\hline C & -3.7019110 & -0.2802240 & -1.4140390 \\
\hline $\mathrm{H}$ & -4.4454720 & 0.2145910 & -0.7783600 \\
\hline $\mathrm{H}$ & -4.0542820 & -0.1513040 & -2.4427020 \\
\hline $\mathrm{H}$ & -2.6536750 & 1.4886440 & -1.8984220 \\
\hline C & -2.1385950 & 2.3733380 & 2.7040690 \\
\hline $\mathrm{H}$ & -1.9397210 & 2.9993540 & 3.5772540 \\
\hline $\mathrm{H}$ & -3.2024320 & 2.4597560 & 2.4686050 \\
\hline $\mathrm{H}$ & -1.9108740 & 1.3404560 & 2.9632030 \\
\hline $\mathrm{H}$ & -1.5808930 & 3.9353060 & 1.3617420 \\
\hline $\mathrm{C}$ & -0.0410040 & 3.7264970 & -1.4126750 \\
\hline $\mathrm{H}$ & 0.2870370 & 1.6048200 & -0.9971630 \\
\hline $\mathrm{Cl}$ & -1.0450820 & -0.1541010 & -2.2584830 \\
\hline $\mathrm{C}$ & 1.2661130 & 3.6896900 & -2.2400150 \\
\hline $\mathrm{H}$ & 1.2264660 & 4.4672850 & -3.0090340 \\
\hline $\mathrm{H}$ & 2.1524350 & 3.8799850 & -1.6308330 \\
\hline $\mathrm{H}$ & 1.3954150 & 2.7264540 & -2.7449950 \\
\hline C & -1.2262820 & 3.6129790 & -2.3913350 \\
\hline $\mathrm{H}$ & -1.1699180 & 2.7030320 & -2.9965030 \\
\hline $\mathrm{H}$ & -2.1887250 & 3.6480110 & -1.8707510 \\
\hline $\mathrm{H}$ & -1.2022700 & 4.4615950 & -3.0819850 \\
\hline C & -0.1316300 & 5.0793390 & -0.6790390 \\
\hline $\mathrm{H}$ & 0.6424320 & 5.1894560 & 0.0860750 \\
\hline $\mathrm{H}$ & 0.0075460 & 5.8868170 & -1.4047110 \\
\hline $\mathrm{H}$ & -1.1098010 & 5.2357390 & -0.2145750 \\
\hline $\mathrm{N}$ & -1.1139780 & -0.6278450 & 1.0797740 \\
\hline $\mathrm{C}$ & -1.7910530 & -1.2411960 & 2.1367090 \\
\hline C & 0.1167650 & -1.1198340 & 1.0117440 \\
\hline C & -0.9380270 & -2.3467230 & 2.7543530 \\
\hline C & 0.3922140 & -2.2354450 & 2.0023130 \\
\hline $\mathrm{H}$ & -1.4392100 & -3.3075140 & 2.6039190 \\
\hline $\mathrm{H}$ & 0.6757970 & -3.1443340 & 1.4634900 \\
\hline $\mathrm{H}$ & 1.2355540 & -1.9600270 & 2.6427460 \\
\hline $\mathrm{H}$ & -0.8488910 & -2.1842280 & 3.8315430 \\
\hline $\mathrm{O}$ & 0.9893500 & -0.6705680 & 0.1905560 \\
\hline $\mathrm{O}$ & -2.9060750 & -0.9031140 & 2.5134950 \\
\hline $\mathrm{H}$ & 1.9902380 & -1.1695170 & 0.2266670 \\
\hline $\mathrm{O}$ & 3.1535260 & -1.7760390 & 0.2709330 \\
\hline C & 4.1032370 & -1.2430160 & -0.3996990 \\
\hline C & 5.4309260 & -2.0554690 & -0.2715260 \\
\hline $\mathrm{O}$ & 4.1011350 & -0.2270230 & -1.0936050 \\
\hline $\mathrm{F}$ & 5.8339460 & -2.1392190 & 1.0223750 \\
\hline $\mathrm{F}$ & 5.2755800 & -3.3260430 & -0.7243680 \\
\hline $\mathrm{F}$ & 6.4453920 & -1.5058180 & -0.9691460 \\
\hline $\mathrm{C}$ & -3.6811230 & -1.7670060 & -1.1067940 \\
\hline $\mathrm{C}$ & -4.2671320 & -2.2631730 & 0.0661790 \\
\hline C & -3.1858400 & -2.6900760 & -2.0444480 \\
\hline $\mathrm{C}$ & -4.3257870 & -3.6380280 & 0.3166990 \\
\hline $\mathrm{H}$ & -4.6750240 & -1.5708210 & 0.7947880 \\
\hline C & -3.2369220 & -4.0638180 & -1.7956780 \\
\hline $\mathrm{H}$ & -2.7678240 & -2.3347750 & -2.9806610 \\
\hline C & -3.8041910 & -4.5439470 & -0.6103770 \\
\hline $\mathrm{H}$ & -4.7837150 & -3.9976820 & 1.2338120 \\
\hline $\mathrm{H}$ & -2.8456240 & -4.7583930 & -2.5336240 \\
\hline
\end{tabular}

2.log

Potential Energy $=-2233.49716$

Zero-point Energy $=-2232.96578$

Free Energy $=-2233.01358$

Single-Point Energy B3LYP-D3(BJ)/6-311+G** PCM = 2234.08080

Free Energy B3LYP-D3(BJ)/6-311+G** PCM (extrapolated free energy from qRRHO) $=-2233.59722$

Nimag $=1\left(-41.5476 \mathrm{~cm}^{-1}\right)$

Charge $=0$ Multiplicity $=1$

$\begin{array}{llll}\text { C } & -0.2079630 & 2.0054930 & 0.0927230\end{array}$

C $\quad 0.2336690 \quad 1.6796300 \quad 2.3910850$

C $\quad-1.2249670 \quad 1.2658580 \quad 2.1687090$

$\mathrm{N} \quad-1.2420470 \quad 1.0896830 \quad 0.6819630$

$\mathrm{N} \quad 0.7512260 \quad 2.0605390 \quad 1.2012560$

$\begin{array}{llll}\text { O } & 0.8200500 & 1.6530350 & 3.4715570\end{array}$

C $\quad 2.1754370 \quad 2.3338310 \quad 1.0310920$

$\begin{array}{llll}\mathrm{H} & 2.7229610 & 1.7397450 & 1.7644590\end{array}$

$\mathrm{H} \quad 2.4855200 \quad 2.0373530 \quad 0.0285540$

$\mathrm{H} \quad 2.4084040 \quad 3.3900490 \quad 1.1919530$

C $\quad-1.8114260 \quad 0.0648760 \quad 0.1111190$

$\begin{array}{llll}\mathrm{H} & -2.4566640 & -0.5140270 & 0.7589900\end{array}$

C $\quad-2.0629700 \quad-0.1502940 \quad-1.3692320$

C $\quad-3.0049530 \quad-1.3410270 \quad-1.6331140$

$\mathrm{H} \quad-3.0293390 \quad-1.4901980 \quad-2.7159990$

$\mathrm{H} \quad-2.5888080 \quad-2.2407270 \quad-1.1767560$

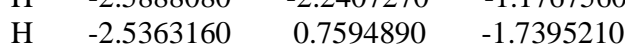

C $\quad-1.6667590 \quad 0.0829670 \quad 3.0141540$

$\mathrm{H} \quad-1.4802460 \quad 0.3492260 \quad 4.0572100$

$\mathrm{H} \quad-2.7366170 \quad-0.1110050 \quad 2.9072590$

$\mathrm{H} \quad-1.1032980 \quad-0.8209730 \quad 2.7804590$

$\begin{array}{llll}\mathrm{H} & -1.8605040 & 2.1276850 & 2.3968520\end{array}$

$\begin{array}{llll}\text { C } & -0.7439880 & 3.4084070 & -0.3733680\end{array}$

$\mathrm{H} \quad 0.2493010 \quad 1.4936390 \quad-0.7546610$

$\begin{array}{llll}\mathrm{Cl} & -0.5614480 & -0.3093210 & -2.3907240\end{array}$

$\begin{array}{llll}\text { C } & 0.4313480 & 4.1504000 & -1.0519320\end{array}$

$\mathrm{H} \quad 0.0558580 \quad 5.0732780 \quad-1.5043210$

$\begin{array}{llll}\mathrm{H} & 1.2118260 & 4.4311920 & -0.3418930\end{array}$

$\mathrm{H} \quad 0.8849040 \quad 3.5506130 \quad-1.8483690$

$\begin{array}{llll}\mathrm{C} & -1.8634140 & 3.2365380 & -1.4183050\end{array}$

$\mathrm{H} \quad-1.5253300 \quad 2.6644420 \quad-2.2884000$

$\begin{array}{llll}\mathrm{H} & -2.7526590 & 2.7630160 & -0.9909670\end{array}$

$\begin{array}{llll}\mathrm{H} & -2.1698940 & 4.2231180 & -1.7789880\end{array}$

$\begin{array}{llll}\mathrm{C} & -1.2760370 & 4.2663930 & 0.7906410\end{array}$

$\begin{array}{llll}\mathrm{H} & -0.5454950 & 4.3652660 & 1.5992110\end{array}$

$\begin{array}{llll}\mathrm{H} & -1.4928330 & 5.2734050 & 0.4209180\end{array}$

$\begin{array}{llll}\mathrm{H} & -2.2098390 & 3.8708160 & 1.2017360\end{array}$

$\mathrm{N} \quad-0.3852940 \quad-1.8578010 \quad 0.4756280$

$\begin{array}{llll}\mathrm{C} & -0.8543680 & -3.0948900 & 0.8726380\end{array}$

$\begin{array}{llll}\text { C } & 0.9553810 & -1.8789870 & 0.4420200\end{array}$

C $\quad 0.2968100 \quad-4.0774160 \quad 1.1038010$

$\begin{array}{llll}\text { C } & 1.5429090 & -3.2397990 & 0.8027220\end{array}$

$\mathrm{H} \quad 0.1760400 \quad-4.9380370 \quad 0.4392860$

$\begin{array}{llll}\mathrm{H} & 2.1335090 & -3.6178540 & -0.0377040\end{array}$

$\mathrm{H} \quad 2.2197850 \quad-3.1354290 \quad 1.6562660$

$\mathrm{H} \quad 0.2568890 \quad-4.4483060 \quad 2.1321790$

$\begin{array}{llll}\mathrm{O} & 1.6585160 & -0.8705390 & 0.1495960\end{array}$

$\begin{array}{llll}\mathrm{O} & -2.0475620 & -3.3668060 & 1.0228970\end{array}$

$\begin{array}{llll}\mathrm{H} & 2.9908630 & -0.9936730 & 0.0928700\end{array}$

$\begin{array}{llll}\mathrm{O} & 4.0898510 & -1.1087500 & 0.0847470\end{array}$

$\begin{array}{llll}\text { C } & 4.7145330 & -0.3105810 & -0.7252880\end{array}$

$\begin{array}{llll}\text { C } & 6.2504860 & -0.5618770 & -0.6743310\end{array}$

$\begin{array}{llll}\mathrm{O} & 4.2597940 & 0.5474750 & -1.4631090\end{array}$

$\begin{array}{llll}\text { F } & 6.7351370 & -0.3715400 & 0.5760140\end{array}$

F $6.5478190 \quad-1.8329230 \quad-1.0362130$

$\begin{array}{llll}\text { F } & 6.9179330 & 0.2634070 & -1.4997520\end{array}$

C $\quad-4.4122270 \quad-1.0819270 \quad-1.1209010$

$\begin{array}{llll}\text { C } & -5.2650740 & -0.1997290 & -1.8054900\end{array}$

$\begin{array}{llll}\text { C } & -4.8887090 & -1.7204870 & 0.0342090\end{array}$

$\begin{array}{llll}\text { C } & -6.5609250 & 0.0432280 & -1.3444490\end{array}$

$\begin{array}{llll}\mathrm{H} & -4.9183150 & 0.2921150 & -2.7114300\end{array}$

C $\quad-6.1876820 \quad-1.4791690 \quad 0.4962020$

$\begin{array}{llll}\mathrm{H} & -4.2376830 & -2.4099610 & 0.5645290\end{array}$

$\begin{array}{llll}\text { C } & -7.0260750 & -0.5961730 & -0.1894670\end{array}$ 


$\begin{array}{lrrrrrrr}\mathrm{H} & -7.2083420 & 0.7247450 & -1.8887040 & \mathrm{H} & 2.1407110 & 2.7176290 & -2.0337330 \\ \mathrm{H} & -6.5429990 & -1.9844700 & 1.3898350 & \mathrm{C} & 3.1077510 & 5.4479360 & -0.2449210 \\ \mathrm{H} & -8.0346600 & -0.4104220 & 0.1681380 & \mathrm{H} & 2.9859560 & 5.6201490 & 1.9031640 \\ & & & & \mathrm{H} & 3.1122110 & 4.9666940 & -2.3500950 \\ \text { 3.log } & & & \mathrm{H} & 3.5410510 & 6.4341100 & -0.3838930\end{array}$

Potential Energy $=-2233.49340$

Zero-point Energy = -2232.96070

Free Energy = -2233.00849

Single-Point Energy B3LYP-D3(BJ)/6-311+G** PCM = 2234.07881

Free Energy B3LYP-D3(BJ)/6-311+G** PCM (extrapolated free energy from $\mathrm{qRRHO})=-2233.59390$

Nimag $=1\left(-57.5343 \mathrm{~cm}^{-1}\right)$

Charge $=0$ Multiplicity $=1$

C $\quad-2.9809140 \quad 0.1760540$

C $\quad-3.2989800 \quad-2.1426870$

C $\quad-1.8699800 \quad-1.9682390$

$\mathrm{N} \quad-1.6479400 \quad-0.5092420$

$\mathrm{N} \quad-3.8573470 \quad-0.9199850$

O $\quad-3.8275400 \quad-3.2214260$

C $\quad-5.1434840 \quad-0.7206130$

$\begin{array}{lll}\mathrm{H} & -5.9760910 & -0.7768430\end{array}$

$\mathrm{H} \quad-5.2682080 \quad-1.5029260$

$\mathrm{H} \quad-5.1479590 \quad 0.2519350$

C $\quad-0.5216150 \quad-0.0552300$

$\begin{array}{lrr}\mathrm{C} & -0.5216150 & -0.0552300 \\ \mathrm{H} & 0.3050640 & -0.7521950\end{array}$

$\begin{array}{lll}\text { C } & -0.0713320 & 1.3920670\end{array}$

C $\quad 1.3899750 \quad 1.5074310$

$\mathrm{H} \quad 1.4623290 \quad 1.1963390$

H $\quad 1.9583230 \quad 0.7841620$

$\mathrm{H} \quad-0.1485410 \quad 1.7952440$

C $\quad-0.8794700 \quad-2.9434100$

$\mathrm{H} \quad-1.2629120 \quad-3.9488480$

$\mathrm{H} \quad 0.1049620 \quad-2.8633470$

H $\quad-0.7879960 \quad-2.8032900$

$\begin{array}{lll}\mathrm{H} & -1.8850300 & -2.1090050\end{array}$

$\begin{array}{lll}\text { C } & -3.3769310 & 0.8029190\end{array}$

$\mathrm{H} \quad-2.9789870 \quad 0.9491390$

$\mathrm{Cl}-1.1382720 \quad 2.4835280$

C $\quad-4.6811680 \quad 1.6073020$

$\mathrm{H} \quad-4.9174350 \quad 2.1550960$

H $\quad-5.5358030 \quad 0.9651340$

$\mathrm{H} \quad-4.5774220 \quad 2.3399010$

$\begin{array}{lll}\text { C } & -2.2918980 & 1.7804250\end{array}$

$\begin{array}{lll}\mathrm{H} & -2.1121260 & 2.5873320\end{array}$

$\mathrm{H}-1.3503460$

H -2.6265730

C $\quad-3.6136590$

H -4.3386570

$\mathrm{H} \quad-4.0132230$

H -2.6865920

$\mathrm{N} \quad-0.4299630$

C $\quad-1.5746620$

C $\quad 0.5817410$

C $\quad-1.3034760$

C 0.1735090

$\begin{array}{ll}\mathrm{H} & -1.9754690\end{array}$

H 0.3555520

H 0.8034610

H $\quad-1.5273090$

O 1.7499330

O $\quad-2.6709330$

H 2.6082350

O 3.3933050

C 3.2188310

C 4.4425440

O 2.2691860

F 4.6269260

F $\quad 4.2770510$

F $\quad 5.5736170$

C 1.9857420

C 2.2391460

C 2.3117060

C 2.7961020

$\begin{array}{ll}\mathrm{H} & 2.0001380\end{array}$

C 2.8648710
$-0.7843200$

$-0.4827470$

$-1.0069420$

$-0.7503780$

$-0.3590240$

$-0.2130040$

0.2996650

$-0.4076050$

1.0501380

0.7921500

$-0.2599430$

$-0.3210400$

$-0.1578110$

0.3068300

1.3496520

$-0.2888760$

$-1.1698570$

$-0.3907870$

$-0.5803230$

$-0.8552610$

0.6871560

$-2.0930120$

$-2.1736920$

$-0.0170180$

0.8376360

$-1.9614660$

$-2.8789220$

$-1.7380580$

$-1.1536000$

$-2.6652510$

$-1.9484200$

$-2.8837390$

$\begin{array}{rr}2.2438700 & -3.5984620 \\ -0.2591650 & -3.2653480\end{array}$

$-1.0174520 \quad-2.9552440$

$0.2309100-4.1588330$

$-0.7580930 \quad-3.5639300$

$-0.5585310 \quad 2.0319830$

$-0.4464490 \quad 2.8024210$

$-1.0257320 \quad 2.7925970$

$-0.8398770 \quad 4.2578970$

$-1.2351470 \quad 4.2487710$

$-1.6545110 \quad 4.5437740$

$-2.2778450 \quad 4.5267570$

$0.6119770 \quad 4.8911750$

$0.0126010 \quad 4.9064390$

$-1.2709540 \quad 2.4055770$

$-0.0850540 \quad 2.3761520$

$-1.6864800 \quad 1.2727640$

$-2.0690860 \quad 0.7136350$

$-2.0144780 \quad-0.5766370$

$-2.6184870-1.3209350$

$-1.5718540-1.1957940$

$-3.9120560-0.9738300$

$-2.5665270 \quad-2.6518330$

$-1.9441730-1.0147920$

$2.8875180 \quad 0.1156510$

$3.7196440 \quad 1.2149850$

$3.3537670-1.1681600$

$4.9904970 \quad 1.0386130$

$3.3710030 \quad 2.2160900$

$4.6240520-1.3494130$ 4.log

Potential Energy = -2233.49469

Zero-point Energy = -2232.96320

Free Energy $=-2233.01182$

Single-Point Energy B3LYP-D3(BJ)/6-311+G** PCM = 2234.07622

Free Energy B3LYP-D3(BJ)/6-311+G** PCM (extrapolated

free energy from $\mathrm{qRRHO})=-2233.59335$

Nimag $=1\left(-78.2152 \mathrm{~cm}^{-1}\right)$

Charge $=0$ Multiplicity $=1$

$\begin{array}{llll}\text { C } & 0.6256560 & 3.3962350 & -0.7103440\end{array}$

C $\quad 0.8831690 \quad 2.3336090 \quad-1.7881450$

$\begin{array}{llll}\mathrm{N} & 1.5994580 & 1.2984390 & -0.9784650\end{array}$

$\mathrm{N} \quad 1.4503170 \quad 3.1413770 \quad 0.3324070$

O $\quad-0.1945290 \quad 4.3080640 \quad-0.7912610$

$\begin{array}{llll}\text { C } & 1.3869530 & 3.8940940 & 1.5809500\end{array}$

$\mathrm{H} \quad 0.3570050 \quad 4.2249200 \quad 1.7255840$

$\mathrm{H} \quad 1.6762370 \quad 3.2438970 \quad 2.4070350$

$\mathrm{H} \quad 2.0360420 \quad 4.7748400 \quad 1.5585560$

C $\quad 1.1375770 \quad 0.0810020 \quad-0.8797500$

$\begin{array}{llll}\mathrm{H} & 0.3705870 & -0.1890720 & -1.5964970\end{array}$

C $\quad 1.8168330 \quad-1.0857280 \quad-0.2045950$

C $\quad 2.8152490 \quad-1.7367740 \quad-1.1991160$

$\mathrm{H} \quad 2.2626690 \quad-2.0503620 \quad-2.0895390$

H $\quad 2.2895350 \quad-0.8019810 \quad 0.7343670$

$\begin{array}{llll}\text { C } & -0.3564400 & 1.8927540 & -2.5459530\end{array}$

$\mathrm{H} \quad-0.7793650 \quad 2.7870270 \quad-3.0102610$

$\mathrm{H} \quad-0.1019480 \quad 1.1926440 \quad-3.3464990$

$\mathrm{H} \quad-1.1147270 \quad 1.4530210 \quad-1.8947970$

$\begin{array}{llll}\mathrm{H} & 1.6125680 & 2.7367350 & -2.4972620\end{array}$

$\begin{array}{llll}\text { C } & 3.8405550 & 2.4612300 & -0.2484910\end{array}$

$\begin{array}{llll}\mathrm{H} & 2.3721040 & 1.3769430 & 0.9791850\end{array}$

$\begin{array}{llll}\mathrm{Cl} & 0.5697870 & -2.3397740 & 0.2310160\end{array}$

$\begin{array}{llll}\mathrm{N} & -0.5894940 & 0.4465120 & 0.8170910\end{array}$

$\begin{array}{llll}\text { C } & -1.9213530 & 0.3539430 & 0.7114470\end{array}$

$\begin{array}{llll}\text { C } & -0.2397960 & 0.5121820 & 2.1491370\end{array}$

$\begin{array}{llll}\text { C } & -2.6362800 & 0.3916360 & 2.0576720\end{array}$

$\begin{array}{llll}\text { C } & -1.4758850 & 0.5033840 & 3.0513820\end{array}$

$\begin{array}{llll}\mathrm{H} & -3.3242250 & 1.2424070 & 2.0872100\end{array}$

$\mathrm{H} \quad-1.4120040 \quad-0.3389140 \quad 3.7465230$

$\begin{array}{llll}\mathrm{O} & 0.9246270 & 0.5651810 & 2.5576620\end{array}$

$\begin{array}{llll}\text { O } & -2.5211560 & 0.2413200 & -0.3971940\end{array}$

$\begin{array}{llll}\mathrm{C} & 4.5116610 & 2.8644510 & 1.0871130\end{array}$

$\begin{array}{llll}\mathrm{H} & 5.5671190 & 3.0883630 & 0.9049010\end{array}$

$\mathrm{H} \quad 4.0621880 \quad 3.7558950 \quad 1.5298790$

$\mathrm{H} \quad 4.4638700 \quad 2.0532470 \quad 1.8214720$

$\begin{array}{llll}\mathrm{C} & 4.6388140 & 1.2909290 & -0.8458470\end{array}$

$\begin{array}{llll}\mathrm{H} & 4.6724730 & 0.4314740 & -0.1699930\end{array}$

$\begin{array}{llll}\mathrm{H} & 4.2353030 & 0.9773960 & -1.8129800\end{array}$

H $\quad 5.6728600 \quad 1.6081920 \quad-1.0115580$

C $\quad 3.9084720 \quad 3.6556150 \quad-1.2216790$

$\begin{array}{llll}\mathrm{H} & 3.3117820 & 4.5056110 & -0.8790300\end{array}$

$\mathrm{H} \quad 4.9470500 \quad 3.9924840 \quad-1.2956780$

$\begin{array}{llll}\mathrm{H} & 3.5897860 & 3.3885940 & -2.2335090\end{array}$

$\mathrm{H} \quad-1.4966610 \quad 1.4204740 \quad 3.6475720$

$\begin{array}{llll}\mathrm{H} & -3.2335150 & -0.5173000 & 2.1792010\end{array}$

$\begin{array}{llll}\mathrm{H} & 3.5190090 & -0.9654680 & -1.5180200\end{array}$

C $\quad 3.5768330 \quad-2.9165840 \quad-0.6248940$

$\begin{array}{llll}\text { C } & 3.4357690 & -4.1903850 & -1.1923610\end{array}$

$\begin{array}{llll}\mathrm{C} & 4.4555960 & -2.7563360 & 0.4577940\end{array}$

C $4.1540900 \quad-5.2808880 \quad-0.6915740$

H $2.7597720 \quad-4.3311500 \quad-2.0314180$

$\begin{array}{llll}\mathrm{C} & 5.1716810 & -3.8440850 & 0.9634570\end{array}$

$\begin{array}{llll}\mathrm{H} & 4.5890230 & -1.7786760 & 0.9134650\end{array}$

$\begin{array}{llll}\text { C } & 5.0230670 & -5.1113450 & 0.3896970\end{array}$

$\begin{array}{llll}\mathrm{H} & 4.0314930 & -6.2600230 & -1.1453770\end{array}$

$\mathrm{H} \quad 5.8470810 \quad-3.7005300 \quad 1.8018030$

$\mathrm{H} \quad 5.5805960 \quad-5.9569640 \quad 0.7814420$

$\begin{array}{llll}\mathrm{H} & -3.8704250 & 0.1329330 & -0.4348510\end{array}$ 


$\begin{array}{llcc}\mathrm{O} & -4.9587500 & 0.1233960 & -0.5683730 \\ \mathrm{C} & -5.5384690 & -0.9701480 & -0.1750460 \\ \mathrm{C} & -7.0767990 & -0.8933330 & -0.4008290 \\ \mathrm{~F} & -7.6175240 & 0.1415650 & 0.2853010 \\ \mathrm{~F} & -7.6949860 & -2.0170480 & 0.0019000 \\ \mathrm{~F} & -7.3687020 & -0.7101810 & -1.7103760 \\ \mathrm{O} & -5.0397640 & -1.9679260 & 0.3169070\end{array}$

Formation of anti-aminal anti-18b (TS5')

$\begin{array}{cc}\begin{array}{c}\text { Conformation } \\ \text { name }\end{array} & \begin{array}{c}\text { Extrapolated } \\ \text { Free energ } \\ \text { (kcal/mol) }\end{array} \\ \text { TS-lowest } & 0.0 \\ 1 & 3.2 \\ 2 & 0.0\end{array}$

TS5'-lowest.log

Potential Energy $=-2233.50047$

Zero-point Energy = -2232.96722

Free Energy $=-2233.01545$

Single-Point Energy B3LYP-D3(BJ)/6-311+G** PCM = 2234.08648

Free Energy B3LYP-D3(BJ)/6-311+G** PCM (extrapolated

free energy from qRRHO) $=-2233.60146$

Nimag $=1(-94.9987)$

Charge $=0$ Multiplicity $=1$

\begin{tabular}{|c|c|c|c|}
\hline $\mathrm{C}$ & 2.9618650 & -1.5642330 & -0.2622350 \\
\hline $\mathrm{C}$ & 1.9922820 & -2.1236090 & -2.3428050 \\
\hline $\mathrm{C}$ & 0.9290250 & -1.3190890 & -1.5939730 \\
\hline $\mathrm{N}$ & 1.7342770 & -0.7355930 & -0.4815950 \\
\hline $\mathrm{N}$ & 3.1171150 & -2.1622620 & -1.5928540 \\
\hline $\mathrm{O}$ & 1.8550800 & -2.6041590 & -3.4678160 \\
\hline $\mathrm{C}$ & 4.3976650 & -2.5832650 & -2.1588660 \\
\hline $\mathrm{H}$ & 4.4278680 & -2.2777080 & -3.2067860 \\
\hline $\mathrm{H}$ & 5.2085030 & -2.0941960 & -1.6177920 \\
\hline $\mathrm{H}$ & 4.5352060 & -3.6659710 & -2.1067180 \\
\hline $\mathrm{C}$ & 1.4269200 & 0.4300670 & 0.0622570 \\
\hline $\mathrm{H}$ & 0.6002530 & 0.9299270 & -0.4232240 \\
\hline $\mathrm{C}$ & 2.4364540 & 1.3628500 & 0.7138100 \\
\hline $\mathrm{C}$ & 1.8746620 & 2.7225910 & 1.1774350 \\
\hline $\mathrm{H}$ & 2.7314510 & 3.2909240 & 1.5504360 \\
\hline $\mathrm{H}$ & 2.9514930 & 0.8885630 & 1.5452460 \\
\hline $\mathrm{C}$ & 0.2614040 & -0.3061880 & -2.5195270 \\
\hline $\mathrm{H}$ & -0.1302860 & -0.8552580 & -3.3791900 \\
\hline $\mathrm{H}$ & -0.5738430 & 0.1925060 & -2.0270040 \\
\hline $\mathrm{H}$ & 0.9779180 & 0.4362040 & -2.8832910 \\
\hline $\mathrm{H}$ & 0.1713550 & -1.9931910 & -1.1814810 \\
\hline $\mathrm{C}$ & 2.8688980 & -2.6071120 & 0.9084130 \\
\hline $\mathrm{H}$ & 3.8084330 & -0.9000150 & -0.0738760 \\
\hline $\mathrm{Cl}$ & 3.7578040 & 1.6704260 & -0.5487560 \\
\hline $\mathrm{N}$ & 0.1311670 & 0.1736090 & 1.7237170 \\
\hline $\mathrm{C}$ & -1.1200310 & -0.2409550 & 1.4214170 \\
\hline $\mathrm{C}$ & 0.2566440 & 0.3419070 & 3.0976380 \\
\hline $\mathrm{C}$ & -1.9896290 & -0.4076160 & 2.6582920 \\
\hline $\mathrm{C}$ & -1.0521050 & -0.0122430 & 3.8021390 \\
\hline $\mathrm{H}$ & -2.8715850 & 0.2330960 & 2.5664310 \\
\hline $\mathrm{H}$ & -0.8664710 & -0.8203380 & 4.5155240 \\
\hline $\mathrm{O}$ & 1.2792640 & 0.7235780 & 3.6605220 \\
\hline $\mathrm{O}$ & -1.5075170 & -0.4593300 & 0.2492970 \\
\hline $\mathrm{C}$ & 4.0194670 & -3.6286020 & 0.7742980 \\
\hline $\mathrm{H}$ & 4.0383040 & -4.2560540 & 1.6708830 \\
\hline $\mathrm{H}$ & 3.8857250 & -4.2898350 & -0.0849360 \\
\hline $\mathrm{H}$ & 4.9973250 & -3.1416100 & 0.6949950 \\
\hline $\mathrm{C}$ & 3.0538370 & -1.8693820 & 2.2474720 \\
\hline $\mathrm{H}$ & 4.0280560 & -1.3682800 & 2.2919970 \\
\hline $\mathrm{H}$ & 2.2768980 & -1.1286960 & 2.4307600 \\
\hline $\mathrm{H}$ & 3.0176200 & -2.5901400 & 3.0707380 \\
\hline $\mathrm{C}$ & 1.5296000 & -3.3659840 & 0.9012280 \\
\hline $\mathrm{H}$ & 1.3683380 & -3.8975460 & -0.0426050 \\
\hline $\mathrm{H}$ & 1.5326400 & -4.1156480 & 1.6988620 \\
\hline $\mathrm{H}$ & 0.6819370 & -2.6985830 & 1.0790990 \\
\hline $\mathrm{H}$ & -1.3972550 & 0.8560160 & 4.3707130 \\
\hline $\mathrm{H}$ & -2.3408810 & -1.4424970 & 2.7158300 \\
\hline
\end{tabular}

$\begin{array}{lrrr}\mathrm{H} & -2.8788490 & -0.8574420 & -0.0785830 \\ \mathrm{O} & -3.8022470 & -1.2140170 & -0.4229040 \\ \mathrm{C} & -4.8026960 & -0.4295210 & -0.1229830 \\ \mathrm{O} & -4.7862480 & 0.6192520 & 0.4911390 \\ \mathrm{C} & -6.1364630 & -1.0142400 & -0.6685480 \\ \mathrm{~F} & -6.0933630 & -1.1462850 & -2.0142360 \\ \mathrm{~F} & -7.1779900 & -0.2228900 & -0.3654010 \\ \mathrm{~F} & -6.3786850 & -2.2378710 & -0.1443530 \\ \mathrm{H} & 1.2427280 & 2.5180100 & 2.0426600 \\ \mathrm{C} & 1.1132750 & 3.5486910 & 0.1581350 \\ \mathrm{C} & -0.2815680 & 3.4344780 & 0.0395100 \\ \mathrm{C} & 1.7762660 & 4.4776060 & -0.6606930 \\ \mathrm{C} & -0.9918290 & 4.2063230 & -0.8854140 \\ \mathrm{H} & -0.8207070 & 2.7476510 & 0.6857630 \\ \mathrm{C} & 1.0701840 & 5.2523220 & -1.5850480 \\ \mathrm{H} & 2.8511330 & 4.6010890 & -0.5685870 \\ \mathrm{C} & -0.3167810 & 5.1158630 & -1.7041680 \\ \mathrm{H} & -2.0705320 & 4.1018340 & -0.9579460 \\ \mathrm{H} & 1.6026380 & 5.9666000 & -2.2065540 \\ \mathrm{H} & -0.8664000 & 5.7199450 & -2.4201120\end{array}$

$1 . \log$

Potential Energy $=-2233.49345$

Zero-point Energy = -2232.96020

Free Energy $=-2233.00817$

Single-Point Energy B3LYP-D3(BJ)/6-311+G** PCM = 2234.08163

Free Energy B3LYP-D3(BJ)/6-311+G** PCM (extrapolated

free energy from $\mathrm{qRRHO})=-2233.59635$

Nimag $=1(-107.7322)$

Charge $=0$ Multiplicity $=1$

\begin{tabular}{|c|c|c|c|}
\hline $\mathrm{C}$ & 3.3205930 & -1.2017400 & 0.1074410 \\
\hline $\mathrm{C}$ & 2.4327860 & -2.9175490 & -1.2524370 \\
\hline $\mathrm{C}$ & 1.2917090 & -1.9249120 & -1.0382270 \\
\hline $\mathrm{N}$ & 2.0242210 & -0.7422980 & -0.4913900 \\
\hline $\mathrm{N}$ & 3.5452170 & -2.4323800 & -0.6591540 \\
\hline $\mathrm{O}$ & 2.3546530 & -3.9578160 & -1.9075160 \\
\hline $\mathrm{C}$ & 4.8675570 & -2.9845730 & -0.9476130 \\
\hline $\mathrm{H}$ & 4.8799230 & -3.3257100 & -1.9849090 \\
\hline $\mathrm{H}$ & 5.6190400 & -2.2051840 & -0.8157470 \\
\hline $\mathrm{H}$ & 5.1121070 & -3.8293630 & -0.2991830 \\
\hline $\mathrm{C}$ & 1.5094600 & 0.4760500 & -0.6021020 \\
\hline $\mathrm{H}$ & 0.6113970 & 0.4761210 & -1.2046550 \\
\hline $\mathrm{C}$ & 2.2589560 & 1.8136230 & -0.6744720 \\
\hline $\mathrm{C}$ & 1.5625820 & 2.8073060 & -1.6416520 \\
\hline $\mathrm{H}$ & 1.5540310 & 2.3514190 & -2.6371800 \\
\hline $\mathrm{H}$ & 2.2197340 & 3.6793160 & -1.6991960 \\
\hline $\mathrm{H}$ & 2.4000360 & 2.2434650 & 0.3142060 \\
\hline $\mathrm{C}$ & 0.5334220 & -1.6788290 & -2.3395020 \\
\hline $\mathrm{H}$ & 0.2324680 & -2.6523390 & -2.7342750 \\
\hline $\mathrm{H}$ & -0.3695110 & -1.0909650 & -2.1710000 \\
\hline $\mathrm{H}$ & 1.1663980 & -1.1862230 & -3.0838070 \\
\hline $\mathrm{H}$ & 0.5996180 & -2.3078710 & -0.2808330 \\
\hline $\mathrm{C}$ & 3.3139700 & -1.4000550 & 1.6653620 \\
\hline $\mathrm{H}$ & 4.1006470 & -0.4844200 & -0.1430650 \\
\hline $\mathrm{Cl}$ & 3.9598750 & 1.6151750 & -1.3599130 \\
\hline $\mathrm{N}$ & 0.2659280 & 0.9410490 & 1.0015120 \\
\hline $\mathrm{C}$ & -0.9402640 & 0.3315930 & 1.0192650 \\
\hline $\mathrm{C}$ & 0.3380440 & 1.8862920 & 2.0164540 \\
\hline $\mathrm{C}$ & -1.8226930 & 0.8315280 & 2.1526480 \\
\hline $\mathrm{C}$ & -0.9563370 & 1.8987930 & 2.8270900 \\
\hline $\mathrm{H}$ & -2.7588900 & 1.2159730 & 1.7380680 \\
\hline $\mathrm{H}$ & -0.7262230 & 1.6805600 & 3.8738280 \\
\hline $\mathrm{O}$ & 1.3117720 & 2.6035410 & 2.2333650 \\
\hline $\mathrm{O}$ & -1.2761550 & -0.5495660 & 0.1932860 \\
\hline $\mathrm{C}$ & 4.5395620 & -2.2451060 & 2.0774140 \\
\hline $\mathrm{H}$ & 4.6124560 & -2.2471680 & 3.1696110 \\
\hline $\mathrm{H}$ & 4.4513270 & -3.2847570 & 1.7530070 \\
\hline $\mathrm{H}$ & 5.4766430 & -1.8329760 & 1.6879890 \\
\hline $\mathrm{C}$ & 3.4569240 & -0.0188090 & 2.3319860 \\
\hline $\mathrm{H}$ & 4.3992650 & 0.4604100 & 2.0423880 \\
\hline $\mathrm{H}$ & 2.6409810 & 0.6584650 & 2.0850590 \\
\hline $\mathrm{H}$ & 3.4656490 & -0.1353980 & 3.4207660 \\
\hline $\mathrm{C}$ & 2.0407600 & -2.1091650 & 2.1598060 \\
\hline $\mathrm{H}$ & 1.9201440 & -3.0946380 & 1.6973020 \\
\hline
\end{tabular}




$\begin{array}{rrrr}\text { H } & 1.1433700 & -1.5164880 & 1.9665010 \\ \mathrm{H} & -1.3900200 & 2.9019000 & 2.7858190 \\ \mathrm{H} & -2.0744340 & -0.0071490 & 2.8092560 \\ \mathrm{C} & 0.1620810 & 3.2695220 & -1.2853190 \\ \mathrm{C} & -0.0358470 & 4.2963680 & -0.3484940 \\ \mathrm{C} & -0.9577620 & 2.7359200 & -1.9404330 \\ \mathrm{C} & -1.3205300 & 4.7604590 & -0.0571770 \\ \mathrm{H} & 0.8208450 & 4.7365200 & 0.1539810 \\ \mathrm{C} & -2.2462960 & 3.1965130 & -1.6503740 \\ \mathrm{H} & -0.8244970 & 1.9657150 & -2.6961030 \\ \mathrm{C} & -2.4316370 & 4.2086220 & -0.7049690 \\ \mathrm{H} & -1.4536070 & 5.5571260 & 0.6691790 \\ \mathrm{H} & -3.1001470 & 2.7705000 & -2.1689850 \\ \mathrm{H} & -3.4305420 & 4.5715810 & -0.4813640 \\ \mathrm{H} & -2.5894480 & -1.2027000 & 0.1962010 \\ \mathrm{O} & -3.4533530 & -1.7934380 & 0.1488990 \\ \mathrm{C} & -4.5486990 & -1.0980270 & -0.0026280 \\ \mathrm{O} & -4.6762920 & 0.1076170 & -0.0868010 \\ \mathrm{C} & -5.7860980 & -2.0376070 & -0.0710760 \\ \mathrm{~F} & -5.6939740 & -2.8821210 & -1.1242240 \\ \mathrm{~F} & -6.9246690 & -1.3379870 & -0.2025550 \\ \mathrm{~F} & -5.8881130 & -2.7891260 & 1.0491910\end{array}$

2.log

Potential Energy $=-2233.50217$

Zero-point Energy = -2232.96933

Free Energy $=-2233.01848$

Single-Point Energy B3LYP-D3(BJ)/6-311+G** PCM = 2234.08509

Free Energy B3LYP-D3(BJ)/6-311+G** PCM (extrapolated free energy from $\mathrm{qRRHO})=-2233.60140$

Nimag $=1(-86.7932)$

Charge $=0$ Multiplicity $=1$

\begin{tabular}{|c|c|c|c|}
\hline $\mathrm{C}$ & -1.7531000 & 2.5492890 & 0.2229870 \\
\hline $\mathrm{C}$ & -0.2696500 & 3.6869190 & -1.2227940 \\
\hline $\mathrm{C}$ & 0.3534760 & 2.3092670 & -0.9936490 \\
\hline $\mathrm{N}$ & -0.7866820 & 1.5731760 & -0.3732690 \\
\hline $\mathrm{N}$ & -1.4651000 & 3.7343240 & -0.5918680 \\
\hline $\mathrm{O}$ & 0.2185850 & 4.5779720 & -1.9173640 \\
\hline $\mathrm{C}$ & -2.4355150 & 4.7907220 & -0.8742090 \\
\hline $\mathrm{H}$ & -2.3424510 & 5.0715390 & -1.9253260 \\
\hline $\mathrm{H}$ & -3.4418870 & 4.4132860 & -0.6898860 \\
\hline $\mathrm{H}$ & -2.2684160 & 5.6781550 & -0.2591230 \\
\hline $\mathrm{C}$ & -0.9253970 & 0.2682310 & -0.5093920 \\
\hline $\mathrm{H}$ & -0.1868970 & -0.1840050 & -1.1574790 \\
\hline $\mathrm{C}$ & -2.2540690 & -0.4740520 & -0.4555020 \\
\hline $\mathrm{C}$ & -2.1089420 & -1.9590650 & -0.8194550 \\
\hline $\mathrm{H}$ & -2.7695420 & -0.3400940 & 0.4921190 \\
\hline $\mathrm{C}$ & 0.8749770 & 1.7230060 & -2.3025140 \\
\hline $\mathrm{H}$ & 1.5646280 & 2.4510920 & -2.7363560 \\
\hline $\mathrm{H}$ & 1.4240190 & 0.7965130 & -2.1320100 \\
\hline $\mathrm{H}$ & 0.0628060 & 1.5516990 & -3.0152830 \\
\hline $\mathrm{H}$ & 1.1734650 & 2.3834140 & -0.2718320 \\
\hline $\mathrm{C}$ & -1.6081390 & 2.7752630 & 1.7702480 \\
\hline $\mathrm{H}$ & -2.7702960 & 2.2128760 & 0.0098600 \\
\hline $\mathrm{Cl}$ & -3.3442240 & 0.3237000 & -1.7222850 \\
\hline $\mathrm{N}$ & 0.0802780 & -0.7760010 & 1.0758870 \\
\hline $\mathrm{C}$ & 1.4289140 & -0.7764700 & 1.0010440 \\
\hline $\mathrm{C}$ & -0.3354700 & -1.5472280 & 2.1526210 \\
\hline $\mathrm{C}$ & 2.0717140 & -1.5916880 & 2.1135900 \\
\hline $\mathrm{C}$ & 0.8690410 & -2.1035140 & 2.9114400 \\
\hline $\mathrm{H}$ & 2.6783430 & -2.3874210 & 1.6709350 \\
\hline $\mathrm{H}$ & 0.8416020 & -1.7376210 & 3.9420280 \\
\hline $\mathrm{O}$ & -1.5106270 & -1.7510880 & 2.4470670 \\
\hline $\mathrm{O}$ & 2.0685060 & -0.1686570 & 0.1088180 \\
\hline $\mathrm{C}$ & -2.3792820 & 4.0514230 & 2.1726960 \\
\hline $\mathrm{H}$ & -2.4005090 & 4.1187520 & 3.2648620 \\
\hline $\mathrm{H}$ & -1.8998250 & 4.9587710 & 1.7982320 \\
\hline $\mathrm{H}$ & -3.4179740 & 4.0329470 & 1.8255530 \\
\hline $\mathrm{C}$ & -2.2525720 & 1.5878220 & 2.5093860 \\
\hline $\mathrm{H}$ & -3.3159600 & 1.4960650 & 2.2582350 \\
\hline $\mathrm{H}$ & -1.7629070 & 0.6392860 & 2.2929180 \\
\hline $\mathrm{H}$ & -2.1838070 & 1.7495750 & 3.5900360 \\
\hline $\mathrm{C}$ & -0.1365920 & 2.9288620 & 2.1953700 \\
\hline $\mathrm{H}$ & 0.3510580 & 3.7633970 & 1.6803750 \\
\hline $\mathrm{H}$ & -0.0889880 & 3.1383110 & 3.2687180 \\
\hline
\end{tabular}

$\begin{array}{llll}\mathrm{H} & 0.4371600 & 2.0162180 & 2.0116470 \\ \mathrm{H} & 0.7977470 & -3.1942250 & 2.9474270 \\ \mathrm{H} & 2.7437650 & -0.9506880 & 2.6922750 \\ \mathrm{H} & 3.5214700 & -0.2174080 & 0.0043700 \\ \mathrm{O} & 4.5606300 & -0.1250710 & -0.1207960 \\ \mathrm{C} & 5.1531930 & -1.2628860 & -0.3647370 \\ \mathrm{O} & 4.6611580 & -2.3711290 & -0.4512790 \\ \mathrm{C} & 6.6827310 & -1.0533480 & -0.5524100 \\ \mathrm{~F} & 6.9327330 & -0.2432540 & -1.6071630 \\ \mathrm{~F} & 7.3133950 & -2.2187690 & -0.7699150 \\ \mathrm{~F} & 7.2338110 & -0.4817020 & 0.5428010 \\ \mathrm{H} & -1.3897980 & -2.3886370 & -0.1189740 \\ \mathrm{H} & -1.6715780 & -2.0317040 & -1.8201040 \\ \mathrm{C} & -3.4036870 & -2.7458750 & -0.7619280 \\ \mathrm{C} & -3.9852200 & -3.2526140 & -1.9324060 \\ \mathrm{C} & -4.0328480 & -2.9990150 & 0.4679490 \\ \mathrm{C} & -5.1728930 & -3.9901040 & -1.8810080 \\ \mathrm{H} & -3.5066700 & -3.0700950 & -2.8910650 \\ \mathrm{C} & -5.2218830 & -3.7312940 & 0.5214840 \\ \mathrm{H} & -3.5807200 & -2.6276560 & 1.3834310 \\ \mathrm{C} & -5.7967970 & -4.2291170 & -0.6532520 \\ \mathrm{H} & -5.6080870 & -4.3755430 & -2.7986780 \\ \mathrm{H} & -5.6956230 & -3.9189770 & 1.4810140 \\ \mathrm{H} & -6.7191920 & -4.8011120 & -0.6107420\end{array}$

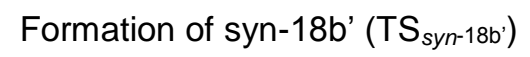

\section{Conformation Extrapolated name \\ Free energy \\ (kcal/mol)}

TS-lowest $\quad 0.0$

1.4

TS-lowest.log

Potential Energy = -2233.49084

Zero-point Energy = -2232.95906

Free Energy $=-2233.00727$

Single-Point Energy B3LYP-D3(BJ)/6-311+G** PCM = 2234.07235

Free Energy B3LYP-D3(BJ)/6-311+G** PCM (extrapolated free energy from $q R R H O)=-2233.58878$

Nimag $=1(-58.6095)$

Charge $=0$ Multiplicity $=1$

$\begin{array}{lrrr}\mathrm{C} & 3.3758330 & -1.2079090 & -0.6598080 \\ \mathrm{C} & 2.2233830 & -1.2548260 & -2.7199250 \\ \mathrm{C} & 1.1945980 & -0.7703580 & -1.6951220 \\ \mathrm{~N} & 2.0766130 & -0.4323860 & -0.5336930 \\ \mathrm{~N} & 3.4199070 & -1.4046260 & -2.1065300 \\ \mathrm{O} & 1.9987620 & -1.4196840 & -3.9164320 \\ \mathrm{C} & 4.6561960 & -1.5404920 & -2.8776180 \\ \mathrm{H} & 4.5565080 & -0.9521490 & -3.7918840 \\ \mathrm{H} & 5.4905030 & -1.1564650 & -2.2899860 \\ \mathrm{H} & 4.8567750 & -2.5793350 & -3.1491310 \\ \mathrm{C} & 1.8576010 & 0.3433720 & 0.4912330 \\ \mathrm{H} & 2.6334280 & 0.3328340 & 1.2500660 \\ \mathrm{C} & 0.9705990 & 1.5679850 & 0.5216520 \\ \mathrm{C} & 1.6926170 & 2.7073230 & -0.2538520 \\ \mathrm{H} & 2.6649340 & 2.8820700 & 0.2163040 \\ \mathrm{H} & 0.5671310 & -1.6032150 & -1.3595520 \\ \mathrm{C} & 3.4792960 & -2.5207660 & 0.1901660 \\ \mathrm{H} & 4.1831310 & -0.5308780 & -0.3647720 \\ \mathrm{H} & -0.0266880 & 1.3763210 & 0.1380870 \\ \mathrm{Cl} & 0.7416250 & 2.1101380 & 2.2421080 \\ \mathrm{~N} & 0.3458150 & -0.9495720 & 1.9479720 \\ \mathrm{C} & -0.8834150 & -1.2372330 & 1.5052380 \\ \mathrm{C} & 0.4088030 & -1.1919290 & 3.3102950 \\ \mathrm{C} & -1.8158260 & -1.7580470 & 2.5916780 \\ \mathrm{C} & -0.9242390 & -1.7354600 & 3.8361900 \\ \mathrm{H} & -2.1734200 & -2.7571470 & 2.3232850 \\ \mathrm{H} & -0.7641300 & -2.7236810 & 4.2774500 \\ \mathrm{H} & -1.2949130 & -1.0781100 & 4.6281690 \\ \mathrm{H} & -2.6915140 & -1.1056370 & 2.6652910 \\ \mathrm{O} & 1.4046430 & -1.0000090 & 4.0070730 \\ \mathrm{O} & -1.2336810 & -1.0847430 & 0.2966370 \\ & & & \end{array}$




$\begin{array}{lrrr}\mathrm{C} & 0.2864820 & 0.2898610 & -2.3072060 \\ \mathrm{H} & -0.5374900 & 0.5448310 & -1.6436340 \\ \mathrm{H} & 0.8288170 & 1.1827370 & -2.6211600 \\ \mathrm{H} & -0.1413610 & -0.1638080 & -3.2047130 \\ \mathrm{C} & 4.7223430 & -3.3090410 & -0.2819260 \\ \mathrm{H} & 4.8827140 & -4.1474640 & 0.4025230 \\ \mathrm{H} & 4.5925920 & -3.7250990 & -1.2833100 \\ \mathrm{H} & 5.6308640 & -2.6971490 & -0.2686770 \\ \mathrm{C} & 2.2335450 & -3.4108810 & 0.0324270 \\ \mathrm{H} & 2.0530900 & -3.6769900 & -1.0146220 \\ \mathrm{H} & 2.3863960 & -4.3450310 & 0.5821430 \\ \mathrm{H} & 1.3408200 & -2.9308570 & 0.4409640 \\ \mathrm{C} & 3.6936990 & -2.1587390 & 1.6742370 \\ \mathrm{H} & 4.5764080 & -1.5210300 & 1.8041810 \\ \mathrm{H} & 2.8324110 & -1.6663490 & 2.1260570 \\ \mathrm{H} & 3.8697450 & -3.0778310 & 2.2425990 \\ \mathrm{H} & 1.8936980 & 2.3439270 & -1.2653200 \\ \mathrm{C} & 0.9121700 & 4.0056700 & -0.3178440 \\ \mathrm{C} & 1.3882170 & 5.1506510 & 0.3358320 \\ \mathrm{C} & -0.2812630 & 4.0965470 & -1.0514140 \\ \mathrm{C} & 0.6884660 & 6.3592960 & 0.2631730 \\ \mathrm{H} & 2.3122390 & 5.0975380 & 0.9051180 \\ \mathrm{C} & -0.9848560 & 5.3013390 & -1.1229720 \\ \mathrm{H} & -0.6656380 & 3.2271210 & -1.5776620 \\ \mathrm{C} & -0.5021280 & 6.4374370 & -0.4645900 \\ \mathrm{H} & 1.0738070 & 7.2360210 & 0.7755570 \\ \mathrm{H} & -1.9058990 & 5.3530210 & -1.6961870 \\ \mathrm{H} & -1.0477760 & 7.3746630 & -0.5217350 \\ \mathrm{H} & -2.5066280 & -1.3861940 & -0.1142360 \\ \mathrm{O} & -3.4572070 & -1.6772830 & -0.5552050 \\ \mathrm{C} & -4.4428740 & -0.8758100 & -0.2794600 \\ \mathrm{O} & -4.4425340 & 0.1304530 & 0.4074990 \\ \mathrm{C} & -5.7523160 & -1.3528970 & -0.9726190 \\ \mathrm{~F} & -6.0649080 & -2.6194650 & -0.6119930 \\ \mathrm{~F} & -5.6187140 & -1.3340360 & -2.3204680 \\ & -6.7959300 & -0.5679840 & -0.6547000\end{array}$

$1 . \log$

Potential Energy $=-2233.49102$

Zero-point Energy = -2232.95979

Free Energy $=-2233.00858$

Single-Point Energy B3LYP-D3(BJ)/6-311+G** PCM = 2234.07121

Free Energy B3LYP-D3(BJ)/6-311+G** PCM (extrapolated free energy from $\mathrm{qRRHO})=-2233.58877$

Nimag $=1(-61.9068)$

Charge $=0$ Multiplicity $=1$

$\begin{array}{lccc}\mathrm{C} & -1.8130170 & -2.4931970 & -1.1402690 \\ \mathrm{C} & -3.8246230 & -2.7245180 & 0.0727710 \\ \mathrm{C} & -3.0569120 & -1.6039910 & 0.7782400 \\ \mathrm{~N} & -2.0022920 & -1.2892390 & -0.2360220 \\ \mathrm{~N} & -3.1279240 & -3.1172680 & -1.0187790 \\ \mathrm{O} & -4.9254770 & -3.1473700 & 0.4179770 \\ \mathrm{C} & -3.7491450 & -3.9274280 & -2.0669600 \\ \mathrm{H} & -4.8054300 & -3.6580460 & -2.1271030 \\ \mathrm{H} & -3.2664680 & -3.7124190 & -3.0210900 \\ \mathrm{H} & -3.6714160 & -4.9968830 & -1.8588220 \\ \mathrm{C} & -1.2660640 & -0.2217790 & -0.3820060 \\ \mathrm{H} & -0.4972460 & -0.2971870 & -1.1440820 \\ \mathrm{C} & -1.6536880 & 1.1972650 & -0.0267710 \\ \mathrm{C} & -2.7005070 & 1.6923450 & -1.0645390 \\ \mathrm{H} & -2.2633010 & 1.6063350 & -2.0637200 \\ \mathrm{H} & -2.5227240 & -1.9908450 & 1.6530020 \\ \mathrm{C} & -0.6024710 & -3.4291430 & -0.7987290 \\ \mathrm{H} & -1.6886300 & -2.1133340 & -2.1586160 \\ \mathrm{H} & -2.0067920 & 1.2936300 & 0.9955180 \\ \mathrm{Cl} & -0.1893940 & 2.2705920 & -0.1457840 \\ \mathrm{~N} & 0.3949080 & -0.3482360 & 1.3969580 \\ \mathrm{C} & -0.0490660 & -0.2869960 & 2.7011350 \\ \mathrm{C} & 1.7258500 & -0.1716540 & 1.3714040 \\ \mathrm{C} & 1.1111470 & -0.0766010 & 3.6769500 \\ \mathrm{C} & 2.3343000 & 0.0029300 & 2.7590160 \\ \mathrm{H} & 1.1464170 & -0.9117540 & 4.3827990 \\ \mathrm{H} & 3.0729720 & -0.7840240 & 2.9403270 \\ \mathrm{H} & 2.8596970 & 0.9618190 & 2.8097700 \\ \mathrm{H} & 0.9373890 & 0.8353620 & 4.2556860\end{array}$

$\begin{array}{lrrr}\mathrm{O} & 2.4012120 & -0.1538000 & 0.3052730 \\ \mathrm{O} & -1.2334650 & -0.3869600 & 3.0343500 \\ \mathrm{C} & -4.0141380 & -0.5180310 & 1.2549270 \\ \mathrm{H} & -3.5054780 & 0.2183840 & 1.8738090 \\ \mathrm{H} & -4.5554090 & -0.0409260 & 0.4367680 \\ \mathrm{H} & -4.7521090 & -1.0230550 & 1.8832440 \\ \mathrm{C} & -0.7446210 & -4.7294210 & -1.6221700 \\ \mathrm{H} & 0.1681660 & -5.3202900 & -1.4998680 \\ \mathrm{H} & -1.5800690 & -5.3449890 & -1.2818670 \\ \mathrm{H} & -0.8650420 & -4.5304850 & -2.6926380 \\ \mathrm{C} & -0.5534600 & -3.7882710 & 0.6970860 \\ \mathrm{H} & -1.4770350 & -4.2744720 & 1.0291170 \\ \mathrm{H} & 0.2647320 & -4.4942600 & 0.8713890 \\ \mathrm{H} & -0.3673120 & -2.9082600 & 1.3176850 \\ \mathrm{C} & 0.7125190 & -2.7477950 & -1.2299550 \\ \mathrm{H} & 0.6913490 & -2.4803750 & -2.2932380 \\ \mathrm{H} & 0.9458030 & -1.8571090 & -0.6471910 \\ \mathrm{H} & 1.5410920 & -3.4489100 & -1.0866880 \\ \mathrm{H} & -3.5495590 & 1.0042980 & -1.0329590 \\ \mathrm{C} & -3.1794360 & 3.1126060 & -0.8350750 \\ \mathrm{C} & -2.8914600 & 4.1155150 & -1.7711090 \\ \mathrm{C} & -3.9398020 & 3.4467890 & 0.2966450 \\ \mathrm{C} & -3.3489700 & 5.4235270 & -1.5827210 \\ \mathrm{H} & -2.3058790 & 3.8722950 & -2.6535510 \\ \mathrm{C} & -4.3948410 & 4.7534250 & 0.4903200 \\ \mathrm{H} & -4.1864030 & 2.6863200 & 1.0325170 \\ \mathrm{C} & -4.1002580 & 5.7470050 & -0.4494780 \\ \mathrm{H} & -3.1166170 & 6.1864660 & -2.3200240 \\ \mathrm{H} & -4.9827210 & 4.9932890 & 1.3715000 \\ \mathrm{H} & -4.4558900 & 6.7622370 & -0.3003350 \\ \mathrm{H} & 3.7538890 & 0.0246360 & 0.3358730 \\ \mathrm{O} & 4.8380730 & 0.1351660 & 0.3763710 \\ \mathrm{C} & 5.3811670 & 0.4974920 & -0.7473270 \\ \mathrm{C} & 6.9262320 & 0.6188720 & -0.5995170 \\ & 4.8478370 & 0.7308190 & -1.8176920 \\ \mathrm{~F} & 7.5060890 & 1.0015960 & -1.7503050 \\ & 7.2561790 & 1.5271120 & 0.3490590\end{array}$

Formation of anti-anti18b' (TS ${ }_{a n t i-18 b ')}$

TS-lowest.log

Potential Energy = -2233.50709

Zero-point Energy = -2232.97791

Free Energy $=-2233.02678$

Single-Point Energy B3LYP-D3(BJ)/6-311+G** PCM = 2234.08656

Free Energy B3LYP-D3(BJ)/6-311+G** PCM (extrapolated free energy from qRRHO) $=-2233.60624$

Nimag $=1(-494.2657)$

Charge $=0$ Multiplicity $=1$

$\begin{array}{lccc}\mathrm{C} & 0.7553950 & 2.0075520 & -0.1593370 \\ \mathrm{C} & 0.2995140 & 2.8729210 & 1.9872940 \\ \mathrm{C} & -1.0680540 & 2.4503560 & 1.4277240 \\ \mathrm{~N} & -0.6532540 & 1.6570390 & 0.2335520 \\ \mathrm{~N} & 1.2637350 & 2.5507530 & 1.0957680 \\ \mathrm{O} & 0.4695730 & 3.3815280 & 3.0920910 \\ \mathrm{C} & 2.6837520 & 2.6033130 & 1.4462810 \\ \mathrm{H} & 2.7816970 & 2.3576760 & 2.5055510 \\ \mathrm{H} & 3.2295660 & 1.8696990 & 0.8514970 \\ \mathrm{H} & 3.1043600 & 3.5983810 & 1.2792520 \\ \mathrm{C} & -1.2604740 & 0.6747920 & -0.3513370 \\ \mathrm{H} & -0.7053660 & 0.1912140 & -1.1459570 \\ \mathrm{C} & -2.7142960 & 0.2818740 & -0.2167870 \\ \mathrm{C} & -3.0654790 & -0.8567410 & -1.1849440 \\ \mathrm{H} & -1.6004830 & 3.3397430 & 1.0742580 \\ \mathrm{C} & 0.8302640 & 2.9761920 & -1.3923690 \\ \mathrm{H} & 1.2719540 & 1.0727950 & -0.3981280 \\ \mathrm{H} & -2.9483800 & 0.0030550 & 0.8081800 \\ \mathrm{Cl} & -3.7337960 & 1.7689450 & -0.5820500 \\ \mathrm{~N} & -0.4735560 & -1.4067320 & 0.8263090 \\ \mathrm{C} & 0.7597970 & -1.8201470 & 0.5668020 \\ \mathrm{C} & -0.9996430 & -2.1714740 & 1.8588800 \\ \mathrm{C} & 1.2274440 & -2.9758000 & 1.4358890 \\ \mathrm{C} & 0.0053300 & -3.2274680 & 2.3275200\end{array}$




\begin{tabular}{|c|c|c|c|}
\hline $\mathrm{H}$ & 2.1242030 & -2.6839770 & 1.9911980 \\
\hline $\mathrm{H}$ & 0.2069320 & -3.0922170 & 3.3939080 \\
\hline $\mathrm{H}$ & -0.4286110 & -4.2232040 & 2.1982200 \\
\hline $\mathrm{H}$ & 1.4977250 & -3.8292530 & 0.8066020 \\
\hline $\mathrm{O}$ & -2.1255430 & -2.0065410 & 2.3245710 \\
\hline $\mathrm{O}$ & 1.4776180 & -1.2734230 & -0.3415940 \\
\hline $\mathrm{C}$ & -1.8926690 & 1.7263430 & 2.4888510 \\
\hline $\mathrm{H}$ & -2.9451290 & 1.6599210 & 2.2139220 \\
\hline $\mathrm{H}$ & -1.4994630 & 0.7293090 & 2.6978530 \\
\hline $\mathrm{H}$ & -1.8233980 & 2.3236400 & 3.4010400 \\
\hline $\mathrm{C}$ & 2.3214220 & 3.2690830 & -1.6760050 \\
\hline $\mathrm{H}$ & 2.4017200 & 3.8195800 & -2.6180780 \\
\hline $\mathrm{H}$ & 2.7760740 & 3.8863470 & -0.8986310 \\
\hline $\mathrm{H}$ & 2.9057750 & 2.3484020 & -1.7769920 \\
\hline $\mathrm{C}$ & 0.0879850 & 4.2999610 & -1.1340690 \\
\hline $\mathrm{H}$ & 0.4625840 & 4.8109670 & -0.2414560 \\
\hline $\mathrm{H}$ & 0.2410070 & 4.9719670 & -1.9840850 \\
\hline $\mathrm{H}$ & -0.9915810 & 4.1524690 & -1.0304690 \\
\hline $\mathrm{C}$ & 0.2381520 & 2.2841240 & -2.6378060 \\
\hline $\mathrm{H}$ & 0.7211940 & 1.3211860 & -2.8387200 \\
\hline $\mathrm{H}$ & -0.8423220 & 2.1299950 & -2.5625300 \\
\hline $\mathrm{H}$ & 0.4048610 & 2.9218760 & -3.5110210 \\
\hline $\mathrm{H}$ & -2.3519090 & -1.6596160 & -0.9676150 \\
\hline $\mathrm{H}$ & -2.8820800 & -0.5251930 & -2.2115970 \\
\hline $\mathrm{C}$ & -4.4822070 & -1.3758820 & -1.0485500 \\
\hline $\mathrm{C}$ & -5.4078690 & -1.2068570 & -2.0871710 \\
\hline $\mathrm{C}$ & -4.8855820 & -2.0543560 & 0.1133120 \\
\hline $\mathrm{C}$ & -6.7122220 & -1.6990960 & -1.9701700 \\
\hline $\mathrm{H}$ & -5.1080000 & -0.6881560 & -2.9940340 \\
\hline $\mathrm{C}$ & -6.1890880 & -2.5430530 & 0.2336870 \\
\hline $\mathrm{H}$ & -4.1749090 & -2.2010720 & 0.9231240 \\
\hline $\mathrm{C}$ & -7.1071300 & -2.3673020 & -0.8079500 \\
\hline $\mathrm{H}$ & -7.4158670 & -1.5597180 & -2.7858850 \\
\hline $\mathrm{H}$ & -6.4862490 & -3.0664100 & 1.1381300 \\
\hline $\mathrm{H}$ & -8.1191260 & -2.7505810 & -0.7147530 \\
\hline $\mathrm{H}$ & 2.6018430 & -1.6573800 & -0.4675820 \\
\hline $\mathrm{O}$ & 3.7335270 & -2.0611650 & -0.6549070 \\
\hline $\mathrm{C}$ & 4.6670350 & -1.1894870 & -0.5031280 \\
\hline $\mathrm{C}$ & 6.0746670 & -1.8103610 & -0.7591650 \\
\hline $\mathrm{O}$ & 4.5837530 & -0.0031650 & -0.2038500 \\
\hline $\mathrm{F}$ & 6.1826360 & -2.2617720 & -2.0340160 \\
\hline $\mathrm{F}$ & 6.3051030 & -2.8645830 & 0.0617720 \\
\hline $\mathrm{F}$ & 7.0677870 & -0.9225030 & -0.5616410 \\
\hline
\end{tabular}

$1 . \log$

Potential Energy $=-2233.50099$

Zero-point Energy = -2232.97161

Free Energy $=-2233.02034$

Single-Point Energy B3LYP-D3(BJ)/6-311+G** PCM = -

2234.08469

Free Energy B3LYP-D3(BJ)/6-311+G** PCM (extrapolated

free energy from $\mathrm{qRRHO})=-2233.60403$

Nimag $=1(-389.2397)$

Charge $=0$ Multiplicity $=1$

$\begin{array}{lrrr}\mathrm{C} & -1.1561550 & 1.4775340 & -1.2816290 \\ \mathrm{C} & -2.7760180 & 2.9260920 & -0.3623500 \\ \mathrm{C} & -3.1981720 & 1.5178010 & 0.0829510 \\ \mathrm{~N} & -1.9823030 & 0.7295480 & -0.2737740 \\ \mathrm{~N} & -1.6173240 & 2.8439240 & -1.0544130 \\ \mathrm{O} & -3.3867600 & 3.9558020 & -0.0876860 \\ \mathrm{C} & -0.8588390 & 4.0399240 & -1.4172450 \\ \mathrm{H} & 0.1940090 & 3.7797770 & -1.5269370 \\ \mathrm{H} & -1.2213630 & 4.4824740 & -2.3488290 \\ \mathrm{H} & -0.9692440 & 4.7730640 & -0.6159710 \\ \mathrm{C} & -1.5144300 & -0.3538880 & 0.2667590 \\ \mathrm{H} & -0.5661160 & -0.6950600 & -0.1273800 \\ \mathrm{C} & -2.2664960 & -1.3156440 & 1.1566980 \\ \mathrm{C} & -1.4431560 & -2.5427760 & 1.5937880 \\ \mathrm{H} & -2.1162160 & -3.1687290 & 2.1863620 \\ \mathrm{H} & -0.6842880 & -2.1566050 & 2.2798250 \\ \mathrm{~N} & -0.0491940 & 0.3793210 & 2.0910860 \\ \mathrm{C} & 1.1522630 & 0.8726510 & 1.7977880 \\ \mathrm{C} & -0.2696240 & 0.4893950 & 3.4558820 \\ \mathrm{C} & 1.9168260 & 1.3861640 & 3.0089610 \\ \mathrm{C} & 0.9317430 & 1.1303760 & 4.1556190 \\ \mathrm{H} & 2.8593000 & 0.8391060 & 3.1097970\end{array}$

\begin{tabular}{|c|c|c|c|}
\hline $\mathrm{H}$ & 1.3162690 & 0.4458430 & 4.9171570 \\
\hline $\mathrm{H}$ & 0.6108510 & 2.0431950 & 4.6658400 \\
\hline & 2.1668220 & 2.4422680 & 2.8695210 \\
\hline & -1.3006160 & 0.1173000 & 4.0145120 \\
\hline & 1.6027450 & 0.9048410 & 0.6060810 \\
\hline & -3.6319310 & 1.5169140 & 1.5468160 \\
\hline & -4.2944960 & 2.3759140 & 1.6768140 \\
\hline & -4.1960600 & 0.6209250 & 1.8054400 \\
\hline & -2.7829050 & 1.6292050 & 2.2239110 \\
\hline & -4.0212260 & 1.1749050 & -0.553378 \\
\hline & -1.3308770 & 0.9480280 & -2.749296 \\
\hline & -0.1075350 & 1.3812960 & -0.981185 \\
\hline & -3.7820070 & -1.8347810 & 0.24689 \\
\hline & -2.6225610 & -0.8107010 & 2.05116 \\
\hline & -0.8657360 & -0.5201380 & -2.836193 \\
\hline & 0.1588000 & -0.6472740 & -2.469336 \\
\hline & -1.5235940 & -1.2040940 & -2.292560 \\
\hline & -0.8786020 & -0.8352290 & -3.883891 \\
\hline & -0.4182100 & 1.7885860 & -3.671719 \\
\hline & -0.7646140 & 2.8189860 & -3.772860 \\
\hline & 0.6180570 & 1.8011540 & -3.3168370 \\
\hline & -0.4199180 & 1.3453900 & -4.672004 \\
\hline & -2.7884920 & 1.0570540 & -3.232853 \\
\hline & -3.1661260 & 2.0822700 & -3.166838 \\
\hline & -2.8445530 & 0.7544890 & -4.283049 \\
\hline & -3.4576410 & 0.3975190 & -2.671555 \\
\hline & -0.7798090 & -3.3664270 & 0.507659 \\
\hline & 0.5610970 & -3.1364440 & 0.158937 \\
\hline & -1.4726610 & -4.3979320 & -0.147380 \\
\hline & 1.1875140 & -3.8988460 & -0.832070 \\
\hline & 1.1266130 & -2.3649470 & 0.675458 \\
\hline & -0.8493880 & -5.1624790 & -1.137183 \\
\hline & -2.5022760 & -4.6097840 & 0.124792 \\
\hline & 0.4821490 & -4.9126170 & -1.485981 \\
\hline & 2.2258620 & -3.7049100 & -1.084691 \\
\hline & -1.4013610 & -5.9575810 & -1.630148 \\
\hline & 0.9674970 & -5.5094220 & -2.252665 \\
\hline & 2.7458930 & 1.4011750 & 0.4130400 \\
\hline & 3.7336280 & 1.9633310 & 0.2171470 \\
\hline & 4.7854380 & 1.3795160 & -0.2556840 \\
\hline & 4.6232730 & -0.1441130 & -0.560168 \\
\hline & 3.6437630 & -0.3552530 & -1.4737500 \\
\hline & 5.7529090 & -0.6859170 & -1.0440570 \\
\hline & 4.2870850 & -0.8311490 & 0.5599900 \\
\hline & 5.8648710 & 1.9056760 & -0.479902 \\
\hline
\end{tabular}

\section{E2-Mechanism (TS-E2)}

\section{Conformation Extrapolated name \\ Free energy \\ (kcal/mol)}

TS-E2

0.0

1

0.9

TS-E2.log

Potential Energy $=-2233.42985$

Zero-point Energy = -2232.89629

Free Energy $=-2232.94300$

Single-Point Energy B3LYP-D3(BJ)/6-311+G** PCM = 2234.02725

Free Energy B3LYP-D3(BJ)/6-311+G** PCM (extrapolated free energy from qRRHO) $=-2233.54041$

Nimag $=1\left(-338.6068 \mathrm{~cm}^{-1}\right)$

Charge $=0$ Multiplicity $=1$

$\begin{array}{lrrr}\mathrm{C} & -2.2726820 & -0.9271960 & -0.7481570 \\ \mathrm{C} & -3.1142270 & -1.2022830 & 1.4434540 \\ \mathrm{C} & -1.6178270 & -1.4975780 & 1.5356850 \\ \mathrm{~N} & -1.0817470 & -0.8216420 & 0.2523880 \\ \mathrm{~N} & -3.4090540 & -0.8714840 & 0.1665630 \\ \mathrm{O} & -3.9001980 & -1.2481600 & 2.3867650 \\ \mathrm{C} & -4.7162290 & -0.3178230 & -0.1867410 \\ \mathrm{H} & -5.0405680 & 0.3502890 & 0.6140570 \\ \mathrm{H} & -4.6305640 & 0.2445990 & -1.1154850 \\ \mathrm{H} & -5.4634790 & -1.1059000 & -0.3093810\end{array}$




\begin{tabular}{|c|c|c|c|}
\hline $\mathrm{C}$ & -0.4888800 & 0.5420700 & 0.4951520 \\
\hline $\mathrm{H}$ & -0.4783400 & 0.7825330 & 1.5482030 \\
\hline $\mathrm{C}$ & 0.6685680 & 0.9533080 & -0.1725320 \\
\hline $\mathrm{C}$ & 1.2007610 & 2.3320760 & 0.2112530 \\
\hline $\mathrm{H}$ & 0.6130110 & 3.0947730 & -0.3191040 \\
\hline $\mathrm{H}$ & 1.8647630 & -0.2221270 & 0.3789040 \\
\hline $\mathrm{C}$ & -0.9881730 & -1.1159980 & 2.8668330 \\
\hline $\mathrm{H}$ & -1.4143400 & -1.7937190 & 3.6111600 \\
\hline $\mathrm{H}$ & 0.0936260 & -1.2666010 & 2.8631620 \\
\hline $\mathrm{H}$ & -1.2262470 & -0.0953020 & 3.1691910 \\
\hline $\mathrm{H}$ & -1.4609540 & -2.5640220 & 1.3665690 \\
\hline $\mathrm{C}$ & -2.2450480 & -2.1968370 & -1.6700770 \\
\hline $\mathrm{H}$ & -2.2403510 & -0.0303830 & -1.3699270 \\
\hline $\mathrm{Cl}$ & 0.8090770 & 0.6159590 & -1.9388910 \\
\hline $\mathrm{N}$ & -2.0358710 & 1.7908020 & 0.3421700 \\
\hline $\mathrm{C}$ & -2.5292130 & 2.4522740 & -0.7622140 \\
\hline $\mathrm{C}$ & -2.2567880 & 2.5230590 & 1.4880050 \\
\hline $\mathrm{C}$ & -3.1928190 & 3.7657990 & -0.3470270 \\
\hline $\mathrm{C}$ & -3.0390260 & 3.7962760 & 1.1766840 \\
\hline $\mathrm{H}$ & -2.6849810 & 4.5931490 & -0.8520690 \\
\hline $\mathrm{H}$ & -3.9935210 & 3.7665700 & 1.7110230 \\
\hline $\mathrm{O}$ & -1.8664190 & 2.1888550 & 2.6107570 \\
\hline $\mathrm{O}$ & -2.4671140 & 2.0358290 & -1.9209940 \\
\hline $\mathrm{C}$ & -3.5459110 & -2.1541910 & -2.5095850 \\
\hline $\mathrm{H}$ & -3.4937160 & -2.9353800 & -3.2734280 \\
\hline $\mathrm{H}$ & -4.4351700 & -2.3466970 & -1.9057710 \\
\hline $\mathrm{H}$ & -3.6698140 & -1.1959010 & -3.0246730 \\
\hline $\mathrm{C}$ & -1.0587800 & -2.1344200 & -2.6532160 \\
\hline $\mathrm{H}$ & -1.0862160 & -1.2231250 & -3.2565110 \\
\hline $\mathrm{H}$ & -0.0884820 & -2.1877220 & -2.1552460 \\
\hline $\mathrm{H}$ & -1.1221990 & -2.9909290 & -3.3317260 \\
\hline $\mathrm{C}$ & -2.2061980 & -3.5351400 & -0.9050420 \\
\hline $\mathrm{H}$ & -3.0082540 & -3.6123060 & -0.1642640 \\
\hline $\mathrm{H}$ & -2.3478390 & -4.3517440 & -1.6195900 \\
\hline $\mathrm{H}$ & -1.2433310 & -3.7113590 & -0.4157050 \\
\hline $\mathrm{H}$ & -2.4824810 & 4.6611420 & 1.5492080 \\
\hline $\mathrm{H}$ & -4.2321450 & 3.7640100 & -0.6874780 \\
\hline $\mathrm{H}$ & 0.9942020 & 2.4671940 & 1.2792650 \\
\hline $\mathrm{C}$ & 2.6774590 & 2.5838060 & -0.0375620 \\
\hline $\mathrm{C}$ & 3.1145450 & 3.1916480 & -1.2238080 \\
\hline $\mathrm{C}$ & 3.6345840 & 2.2424070 & 0.9302760 \\
\hline $\mathrm{C}$ & 4.4732970 & 3.4370180 & -1.4462210 \\
\hline $\mathrm{H}$ & 2.3859310 & 3.4785840 & -1.9771280 \\
\hline $\mathrm{C}$ & 4.9935410 & 2.4871090 & 0.7140550 \\
\hline $\mathrm{H}$ & 3.3142480 & 1.7859200 & 1.8635970 \\
\hline $\mathrm{C}$ & 5.4180660 & 3.0832520 & -0.4780510 \\
\hline $\mathrm{H}$ & 4.7914240 & 3.9086710 & -2.3717270 \\
\hline $\mathrm{H}$ & 5.7181140 & 2.2180010 & 1.4774640 \\
\hline $\mathrm{H}$ & 6.4734040 & 3.2770320 & -0.6468880 \\
\hline $\mathrm{H}$ & -0.2941070 & -1.4078670 & -0.0853860 \\
\hline $\mathrm{O}$ & 1.2065520 & -2.5374070 & -0.0206420 \\
\hline $\mathrm{C}$ & 2.3055770 & -2.1190380 & 0.3109540 \\
\hline $\mathrm{O}$ & 2.6543340 & -0.8886610 & 0.5511390 \\
\hline $\mathrm{C}$ & 3.4997560 & -3.0943460 & 0.4978530 \\
\hline $\mathrm{F}$ & 3.9810410 & -3.0183410 & 1.7567560 \\
\hline $\mathrm{F}$ & 4.5020160 & -2.7846810 & -0.3510950 \\
\hline $\mathrm{F}$ & 3.1299900 & -4.3615970 & 0.2662250 \\
\hline
\end{tabular}

$1 . \log$

Potential Energy = -2233.43162

Zero-point Energy = -2232.89817

Free Energy = -2232.94495

Single-Point Energy B3LYP-D3(BJ)/6-311+G** PCM = 2234.02560

Free Energy B3LYP-D3(BJ)/6-311+G** PCM (extrapolated free energy from $q R R H O)=-2233.53893$

Nimag $=1\left(-323.8199 \mathrm{~cm}^{-1}\right)$

Charge $=0$ Multiplicity $=1$

$\begin{array}{llll}\mathrm{C} & -0.8868030 & -2.2718910 & -0.0544120 \\ \mathrm{C} & -1.4009180 & -1.7870380 & 2.2002150 \\ \mathrm{C} & -0.0587240 & -1.1098600 & 1.9276830 \\ \mathrm{~N} & -0.0445590 & -1.0360190 & 0.3834360 \\ \mathrm{~N} & -1.8257100 & -2.3735510 & 1.0575630 \\ \mathrm{O} & -1.9851120 & -1.7831920 & 3.2806250 \\ \mathrm{C} & -3.1807220 & -2.9097540 & 0.9381560 \\ \mathrm{H} & -3.8650170 & -2.2519030 & 1.4779420\end{array}$

\begin{tabular}{|c|c|c|c|}
\hline $\mathrm{H}$ & -3.4642540 & -2.9428840 & -0.1133960 \\
\hline $\mathrm{H}$ & -3.2547290 & -3.9143110 & 1.3623180 \\
\hline $\mathrm{C}$ & -0.4393850 & 0.3142990 & -0.1668660 \\
\hline $\mathrm{H}$ & -0.6478580 & 1.0227210 & 0.6218580 \\
\hline $\mathrm{C}$ & 0.2701650 & 0.8605970 & -1.2360620 \\
\hline $\mathrm{C}$ & -0.0476120 & 2.2698960 & -1.7346830 \\
\hline $\mathrm{H}$ & 0.7686350 & 2.5695480 & -2.4024650 \\
\hline $\mathrm{H}$ & 1.9137280 & 0.9895550 & -0.6359380 \\
\hline $\mathrm{C}$ & 0.1655660 & 0.1761880 & 2.7071130 \\
\hline $\mathrm{H}$ & 0.2582210 & -0.1134280 & 3.7570900 \\
\hline $\mathrm{H}$ & 1.0959430 & 0.6677640 & 2.4149840 \\
\hline $\mathrm{H}$ & -0.6737250 & 0.8670630 & 2.6229150 \\
\hline $\mathrm{H}$ & 0.7409650 & -1.8109210 & 2.1713760 \\
\hline $\mathrm{C}$ & -0.0535120 & -3.5770610 & -0.3184400 \\
\hline $\mathrm{H}$ & -1.4034650 & -1.9871710 & -0.9724210 \\
\hline $\mathrm{Cl}$ & 0.6898770 & -0.2082340 & -2.6288440 \\
\hline $\mathrm{N}$ & -2.4218580 & 0.1529340 & -0.4051890 \\
\hline $\mathrm{C}$ & -3.1311150 & -0.1712350 & -1.5400340 \\
\hline $\mathrm{C}$ & -3.1797360 & 0.9272400 & 0.4442170 \\
\hline $\mathrm{C}$ & -4.5245200 & 0.4590920 & -1.4975550 \\
\hline $\mathrm{C}$ & -4.5697120 & 1.1703790 & -0.1411620 \\
\hline $\mathrm{H}$ & -4.6301790 & 1.1392340 & -2.3485170 \\
\hline $\mathrm{H}$ & -5.3205920 & 0.7597570 & 0.5406520 \\
\hline $\mathrm{O}$ & -2.7837080 & 1.3557890 & 1.5309980 \\
\hline $\mathrm{O}$ & -2.7149820 & -0.8932980 & -2.4485210 \\
\hline $\mathrm{C}$ & -1.0788010 & -4.6821220 & -0.6747450 \\
\hline $\mathrm{H}$ & -0.5337260 & -5.5660970 & -1.0182210 \\
\hline $\mathrm{H}$ & -1.6798330 & -4.9836770 & 0.1854410 \\
\hline $\mathrm{H}$ & -1.7495410 & -4.3729150 & -1.4831150 \\
\hline $\mathrm{C}$ & 0.8763820 & & -1.5356530 \\
\hline $\mathrm{H}$ & 0.3174500 & -3.1151450 & -2.4302660 \\
\hline $\mathrm{H}$ & 1.6623820 & -2.6621520 & -1.3693880 \\
\hline $\mathrm{H}$ & 1.3697090 & -4.3576840 & -1.7373180 \\
\hline $\mathrm{C}$ & 0.7752130 & -4.0567500 & 0.8904120 \\
\hline $\mathrm{H}$ & & & 1.7954040 \\
\hline $\mathrm{H}$ & 1.1852050 & -5.0455430 & 0.6632180 \\
\hline $\mathrm{H}$ & 1.6290120 & -3.4050090 & 1.0995250 \\
\hline $\mathrm{H}$ & -4.7470330 & 2.2476460 & -0.2110190 \\
\hline $\mathrm{H}$ & -5.2774960 & -0.3258020 & -1.6114860 \\
\hline $\mathrm{H}$ & -0.9508390 & & -2.3588900 \\
\hline $\mathrm{C}$ & -0.2337290 & 3.3376430 & -0.6688680 \\
\hline $\mathrm{C}$ & 0.8354710 & 3.7614010 & 0.1383650 \\
\hline $\mathrm{C}$ & -1.4745840 & 3.9723210 & -0.5153810 \\
\hline $\mathrm{C}$ & 0.6626880 & 4.7784550 & 1.0805470 \\
\hline $\mathrm{H}$ & & & 0.0253010 \\
\hline $\mathrm{C}$ & -1.6509090 & 4.9951240 & 0.4223590 \\
\hline $\mathrm{H}$ & -2.3099170 & 3.6689680 & -1.1408800 \\
\hline $\mathrm{C}$ & -0.5827580 & 5.3992660 & 1.2270030 \\
\hline $\mathrm{H}$ & 1.5030520 & 5.0917500 & 1.6935320 \\
\hline $\mathrm{H}$ & -2.6212980 & 5.4729630 & 0.5220490 \\
\hline $\mathrm{H}$ & -0.7158720 & 6.1925150 & 1.9568080 \\
\hline $\mathrm{H}$ & 0.9413660 & -1.1742460 & 0.0954950 \\
\hline $\mathrm{O}$ & 2.8404570 & -1.0719010 & 0.2444320 \\
\hline $\mathrm{C}$ & 3.4415600 & -0.0449040 & -0.0284180 \\
\hline $\mathrm{O}$ & 2.9456650 & 1.0719020 & -0.4808280 \\
\hline $\mathrm{C}$ & 4.9820090 & 0.0451330 & 0.1440610 \\
\hline $\mathrm{F}$ & 5.3101470 & 1.0562670 & 0.9751630 \\
\hline $\mathrm{F}$ & 5.5761940 & 0.2736870 & -1.0462160 \\
\hline $\mathrm{F}$ & 5.4783190 & -1.0922890 & 0.6495350 \\
\hline
\end{tabular}

E1-Mechanism (TS-E1) $\begin{array}{cc}\begin{array}{c}\text { Conformation } \\ \text { name }\end{array} & \begin{array}{c}\text { Extrapolated } \\ \text { Free energy } \\ (\mathrm{kcal} / \mathrm{mol})\end{array}\end{array}$

TS-E1

12.8

2

1.9

3

1.1

TS-E1.log

Potential Energy = -2233.47717

Zero-point Energy = -2232.94991 
Free Energy $=-2232.99863$

Single-Point Energy B3LYP-D3(BJ)/6-311+G** PCM = 2234.05418

Free Energy B3LYP-D3(BJ)/6-311+G** PCM (extrapolated free energy from $q$ RRHO) $=-2233.57563$

Nimag $=1\left(-1285.1321 \mathrm{~cm}^{-1}\right)$

Charge $=0$ Multiplicity $=1$

\begin{tabular}{|c|c|c|c|}
\hline C & 1.3689690 & -2.0491260 & -0.7237330 \\
\hline & 0.2940610 & -3.3944340 & 0.8866180 \\
\hline & 1.7298810 & -3.1901280 & 1.3841540 \\
\hline & 2.1691450 & -2.0459990 & 0.5295190 \\
\hline & 0.1338850 & -2.6826180 & -0.2520010 \\
\hline & -0.5550960 & -4.0831730 & \\
\hline & -1.1408350 & -2.5872250 & -0.952390 \\
\hline & -1.9280990 & -2.8401450 & -0.241256 \\
\hline & -1.2896690 & -1.5642440 & -1.303430 \\
\hline H & -1.1961960 & -3.2836280 & \\
\hline & 2.9679790 & -1.1028390 & 0.997643 \\
\hline $\mathrm{H}$ & 3.4076700 & -1.3738900 & 1.9513660 \\
\hline C & 3.2284560 & 0.2503300 & 0.6268280 \\
\hline $\mathrm{C}$ & 3.2459730 & 0.8283380 & -0.7930200 \\
\hline $\mathrm{H}$ & 4.1085340 & 0.4142400 & -1.3252960 \\
\hline $\mathrm{H}$ & 2.1888830 & 0.8220090 & \\
\hline $\mathrm{C}$ & 1.8005340 & -2.9720910 & 2.8892 \\
\hline $\mathrm{H}$ & 1.2788340 & -3.8063340 & \\
\hline $\mathrm{H}$ & 2.8293400 & -2.9722260 & 3.2563 \\
\hline $\mathrm{H}$ & 1.3064450 & -2.0425920 & \\
\hline $\mathrm{H}$ & 2.3164230 & -4.0765110 & \\
\hline $\mathrm{C}$ & 2.0504700 & -2.7997610 & -1.9409670 \\
\hline $\mathrm{H}$ & 1.1454130 & -1.0175760 & -0.997144 \\
\hline $\mathrm{Cl}$ & 4.6860040 & 0.8378580 & 1.575064 \\
\hline $\mathrm{N}$ & -0.2254080 & & \\
\hline $\mathrm{C}$ & 0.1632540 & 1.3678640 & 1.7152120 \\
\hline $\mathrm{C}$ & -1.5733250 & 1.0696170 & 0.4316080 \\
\hline $\mathrm{C}$ & -0.9562680 & 1.9254890 & \\
\hline $\mathrm{C}$ & -2.1792970 & 1.7175390 & 1.6731320 \\
\hline $\mathrm{H}$ & -1.0049260 & & \\
\hline $\mathrm{H}$ & -2.6806470 & 2.64984 & 1.3962690 \\
\hline $\mathrm{O}$ & -2.2267910 & 0.7360390 & -0.581073 \\
\hline $\mathrm{O}$ & 1.3718970 & 1.3753500 & 2.1200210 \\
\hline $\mathrm{C}$ & 1.3838910 & -2.2876840 & -3.2387590 \\
\hline $\mathrm{H}$ & 1.8557690 & -2.7644920 & -4.103608 \\
\hline $\mathrm{H}$ & 0.3160900 & -2.5163670 & -3.280523 \\
\hline $\mathrm{H}$ & 1.5038430 & -1.2045840 & -3.349395 \\
\hline $\mathrm{C}$ & 3.5585040 & -2.4895170 & -2.0172610 \\
\hline $\mathrm{H}$ & 3.7553220 & -1.4258320 & -2.163485 \\
\hline $\mathrm{H}$ & 4.0954050 & -2.8256200 & -1.125487 \\
\hline $\mathrm{H}$ & 3.9839250 & -3.0156030 & -2.8775160 \\
\hline $\mathrm{C}$ & 1.8765220 & -4.3299780 & -1.8482440 \\
\hline $\mathrm{H}$ & 0.8268810 & -4.6314920 & -1.801119 \\
\hline $\mathrm{H}$ & 2.3110070 & -4.7903090 & -2.740959 \\
\hline $\mathrm{H}$ & & -4.7553420 & -0.9848690 \\
\hline $\mathrm{H}$ & -2.9353200 & 1.0530800 & 2.1014620 \\
\hline $\mathrm{H}$ & -0.7544860 & 2.9742630 & 2.8100480 \\
\hline $\mathrm{H}$ & 2.3465750 & 0.4842140 & -1.3074080 \\
\hline $\mathrm{C}$ & 3.3025620 & 2.3444030 & -0.8993940 \\
\hline $\mathrm{C}$ & 2.1335940 & 3.1181160 & -0.8448390 \\
\hline $\mathrm{C}$ & 4.5271610 & 2.9951650 & -1.1112790 \\
\hline $\mathrm{C}$ & 2.1897600 & 4.5088590 & -0.9763820 \\
\hline $\mathrm{H}$ & 1.1755760 & 2.6264670 & -0.7058750 \\
\hline $\mathrm{C}$ & 4.5876810 & 4.3856470 & -1.2435030 \\
\hline $\mathrm{H}$ & 5.4407550 & 2.4103080 & -1.1739670 \\
\hline $\mathrm{C}$ & 3.4178190 & 5.1483580 & -1.1728800 \\
\hline $\mathrm{H}$ & 1.2733060 & 5.0907430 & -0.9331470 \\
\hline $\mathrm{H}$ & 5.5462390 & 4.8701520 & -1.4062560 \\
\hline $\mathrm{H}$ & 3.4616830 & 6.2285110 & -1.2784510 \\
\hline $\mathrm{H}$ & -3.6659990 & 0.8996880 & -0.680107 \\
\hline $\mathrm{O}$ & -4.6886710 & 1.0347100 & -0.8914340 \\
\hline $\mathrm{C}$ & -5.4755860 & 0.4245080 & -0.0474010 \\
\hline $\mathrm{C}$ & -6.9665160 & 0.6578860 & -0.4234770 \\
\hline $\mathrm{O}$ & -5.1755860 & -0.2460590 & 0.9214110 \\
\hline $\mathrm{F}$ & -7.2290440 & 0.2096930 & -1.6725800 \\
\hline $\mathrm{F}$ & -7.2728440 & 1.9756860 & -0.3883260 \\
\hline & -7.7912890 & 0.0212940 & 0.4239030 \\
\hline
\end{tabular}

$1 . \log$
Potential Energy $=-2233.47575$

Zero-point Energy = -2232.94890

Free Energy = -2232.99826

Single-Point Energy B3LYP-D3(BJ)/6-311+G** PCM = 2234.04871

Free Energy B3LYP-D3(BJ)/6-311+G** PCM (extrapolated

free energy from $\mathrm{qRRHO})=-2233.57122$

Nimag $=1\left(-1295.0076 \mathrm{~cm}^{-1}\right)$

Charge $=0$ Multiplicity $=1$

$\begin{array}{llll}\text { C } & 2.9849450 & 2.0380300 & 0.358105\end{array}$

$\begin{array}{llll}\text { C } & 1.0843850 & 3.4271840 & 0.4489930\end{array}$

$\begin{array}{llll}\text { C } & 1.1370300 & 2.8444990 & -0.9701130\end{array}$

$\begin{array}{llll}\mathrm{N} & 2.0778810 & 1.7007670 & -0.7689890\end{array}$

$\mathrm{N} \quad 2.1058080 \quad 2.8931610 \quad 1.1611230$

$\begin{array}{llll}\text { O } & 0.2491830 & 4.2326480 & 0.8546230\end{array}$

$\begin{array}{llll}\text { C } & 2.3191630 & 3.1715970 & 2.5751710\end{array}$

$\mathrm{H} \quad 1.3661850 \quad 3.4870400 \quad 3.0015500$

$\mathrm{H} \quad 2.6597170 \quad 2.2647160 \quad 3.0799680$

$\mathrm{H} \quad 3.0524280 \quad 3.9692090 \quad 2.7301560$

$\begin{array}{llll}\text { C } & 1.8723700 & 0.5214660 & -1.3295430\end{array}$

$\mathrm{H} \quad 1.0939470 \quad 0.5508840 \quad-2.0835690$

C $\quad 2.3221410 \quad-0.7985250 \quad-1.0265770$

C $3.7060190 \quad-1.1857200 \quad-0.4870360$

$\mathrm{H} \quad 4.4365310 \quad-1.0610340 \quad-1.2939470$

$\mathrm{H} \quad 1.3645590 \quad-1.1144050 \quad-0.0623030$

C $\quad-0.2354590 \quad 2.5050250 \quad-1.5299010$

$\mathrm{H} \quad-0.8563000 \quad 3.3989880 \quad-1.4313600$

$\begin{array}{llll}\mathrm{H} & -0.1863080 & 2.2499890 & -2.5915130\end{array}$

$\mathrm{H} \quad-0.7079870 \quad 1.6849930 \quad-0.9828250$

$\begin{array}{llll}\mathrm{H} & 1.6229840 & 3.5805750 & -1.6205290\end{array}$

C $4.3513130 \quad 2.7177300 \quad-0.0657190$

$\begin{array}{llll}\mathrm{H} & 3.1967710 & 1.1307240 & 0.9235510\end{array}$

$\mathrm{Cl} \quad 1.8623020 \quad-1.9095260 \quad-2.4091090$

$\mathrm{N} \quad-1.1868470 \quad-0.8494400 \quad-0.3862090$

$\begin{array}{llll}\text { C } & -0.6202230 & -1.3798330 & 0.7014370\end{array}$

$\begin{array}{llll}\text { C } & -2.5372430 & -0.9004080 & -0.2159170\end{array}$

$\begin{array}{llll}\text { C } & -1.5988880 & -1.8475150 & 1.7629530\end{array}$

C $\quad-2.9487630 \quad-1.5247310 \quad 1.1146080$

$\mathrm{H} \quad-1.4465710 \quad-2.9117360 \quad 1.9651650$

$\mathrm{H} \quad-3.5553180 \quad-0.8138680 \quad 1.6829020$

O $\quad-3.3426740 \quad-0.4740160 \quad-1.0733930$

$\begin{array}{llll}\text { O } & 0.6370460 & -1.5008050 & 0.8723940\end{array}$

$\begin{array}{llll}\mathrm{C} & 5.3550630 & 2.5054060 & 1.0909660\end{array}$

$\begin{array}{llll}\mathrm{H} & 6.3217570 & 2.9410740 & 0.8203490\end{array}$

$\begin{array}{llll}\mathrm{H} & 5.0293330 & 2.9818150 & 2.0195010\end{array}$

H $\quad 5.5159890 \quad 1.4408600 \quad 1.2930640$

$\begin{array}{llll}\mathrm{C} & 4.9238930 & 2.0614100 & -1.3379810\end{array}$

$\mathrm{H} \quad 5.1282960 \quad 0.9974930 \quad-1.2009150$

H $\quad 4.2573420 \quad 2.1794560 \quad-2.1971200$

$\mathrm{H} \quad 5.8748730 \quad 2.5424270 \quad-1.5868230$

C $\quad 4.1943650 \quad 4.2306370 \quad-0.3248420$

$\begin{array}{llll}\mathrm{H} & 3.7907840 & 4.7638430 & 0.5399310\end{array}$

$\mathrm{H} \quad 5.1794120 \quad 4.6549100 \quad-0.5422380$

$\begin{array}{llll}\mathrm{H} & 3.5583380 & 4.4434790 & -1.1889970\end{array}$

$\mathrm{H} \quad-3.5654720 \quad-2.4098430 \quad 0.9307000$

$\begin{array}{llll}\mathrm{H} & -1.4176160 & -1.3069370 & 2.6969210\end{array}$

$\begin{array}{llll}\mathrm{H} & 3.9792590 & -0.4822290 & 0.3010170\end{array}$

C $\quad 3.8400890 \quad-2.5912310 \quad 0.0768730$

$\begin{array}{llll}\text { C } & 4.3698600 & -3.6257050 & -0.7081860\end{array}$

$\begin{array}{llll}\text { C } & 3.4935500 & -2.8703920 & 1.4073900\end{array}$

$\begin{array}{llll}\text { C } & 4.5319200 & -4.9124370 & -0.1854090\end{array}$

$\mathrm{H} \quad 4.6596230 \quad-3.4232720 \quad-1.7355810$

C $\quad 3.6550470 \quad-4.1544420 \quad 1.9348460$

$\mathrm{H} \quad 3.0924930 \quad-2.0801010 \quad 2.0353270$

C $4.1722450 \quad-5.1815480 \quad 1.1385350$

$\mathrm{H} \quad 4.9432530 \quad-5.7000390 \quad-0.8102900$

$\mathrm{H} \quad 3.3820720 \quad-4.3504800 \quad 2.9678910$

$\mathrm{H} \quad 4.3007230 \quad-6.1791630 \quad 1.5484130$

$\mathrm{H} \quad-4.7732380 \quad-0.5065760 \quad-0.8896170$

$\begin{array}{llll}\mathrm{O} & -5.8286780 & -0.5484920 & -0.8972330\end{array}$

$\begin{array}{llll}\text { C } & -6.3819040 & 0.0987880 & 0.0911780\end{array}$

$\begin{array}{llll}\text { C } & -7.9319930 & -0.0013930 & 0.0105390\end{array}$

$\begin{array}{llll}\mathrm{O} & -5.8445390 & 0.7172260 & 0.9900350\end{array}$

F $\quad-8.3380330 \quad-1.2916890 \quad 0.0438180$

F $\quad-8.3928150 \quad 0.5429590 \quad-1.1395120$

$\begin{array}{llll}\text { F } & -8.5192780 & 0.6407180 & 1.0335780\end{array}$ 
2.log

Potential Energy = -2233.47519

Zero-point Energy = -2232.94829

Free Energy = -2232.99732

Single-Point Energy B3LYP-D3(BJ)/6-311+G** PCM = 2234.05049

Free Energy B3LYP-D3(BJ)/6-311+G** PCM (extrapolated free energy from qRRHO) $=-2233.57262$

Nimag $=1\left(-1259.1695 \mathrm{~cm}^{-1}\right)$

Charge $=0$ Multiplicity $=1$

$\begin{array}{lccc}\mathrm{C} & 2.0498260 & 1.8758320 & 0.4238910 \\ \mathrm{C} & 0.0898470 & 2.7268940 & -0.5656430 \\ \mathrm{C} & 0.8114320 & 1.9101900 & -1.6486550 \\ \mathrm{~N} & 1.7480020 & 1.1008190 & -0.8094870 \\ \mathrm{~N} & 0.7880520 & 2.6055080 & 0.5892540 \\ \mathrm{O} & -0.9474910 & 3.3645870 & -0.7321720 \\ \mathrm{C} & 0.3496220 & 3.1930280 & 1.8484260 \\ \mathrm{H} & -0.7335960 & 3.3119600 & 1.8036720\end{array}$

$\mathrm{H} \quad 0.6069940 \quad 2.5242600 \quad 2.6727260$

$\begin{array}{llll}\mathrm{H} & 0.8018440 & 4.1743190 & 2.0233420\end{array}$

$\begin{array}{llll}\text { C } & 1.8763960 & -0.2040580 & -0.9813270\end{array}$

$\mathrm{H} \quad 1.4159670 \quad-0.5521190 \quad-1.8990550$

$\begin{array}{llll}\text { C } & 2.3209290 & -1.2435930 & -0.1108110\end{array}$

$\begin{array}{llll}\text { C } & 3.5172410 & -1.2388880 & 0.8371880\end{array}$

$\mathrm{H} \quad 3.5758340 \quad-0.2560830 \quad 1.3164360$

$\begin{array}{llll}\mathrm{H} & 1.2678590 & -1.3163430 & 0.7554230\end{array}$

$\begin{array}{llll}\text { C } & -0.1448790 & 1.1362520 & -2.5425030\end{array}$

$\mathrm{H} \quad-0.8460440 \quad 1.8587240 \quad-2.9672670$

$\mathrm{H} \quad 0.3779460 \quad 0.6546450 \quad-3.3728120$

$\begin{array}{llll}\mathrm{H} & -0.7121350 & 0.3914160 & -1.9776500\end{array}$

$\begin{array}{llll}\mathrm{H} & 1.4055250 & 2.5981900 & -2.2597040\end{array}$

$\begin{array}{llll}\text { C } & 3.3291090 & 2.8029110 & 0.3525920\end{array}$

$\mathrm{H} \quad 2.1631080 \quad 1.1746830 \quad 1.2524840$

$\begin{array}{llll}\mathrm{Cl} & 2.1894250 & -2.8589800 & -0.9594130\end{array}$

$\mathrm{N} \quad-1.2182530 \quad-1.2356040 \quad 0.1639400$

$\begin{array}{llll}\mathrm{C} & -0.7657930 & -1.4451750 & 1.4038700\end{array}$

$\begin{array}{llll}\text { C } & -2.5793740 & -1.2549920 & 0.2115160\end{array}$

$\begin{array}{llll}\text { C } & -1.8472490 & -1.6338310 & 2.4514440\end{array}$

$\begin{array}{llll}\text { C } & -3.1244870 & -1.5012090 & 1.6158950\end{array}$

$\mathrm{H} \quad-1.7315600 \quad-2.6102200 \quad 2.9313360$

$\mathrm{H} \quad-3.7623090 \quad-0.6640850 \quad 1.9141150$

$\begin{array}{llll}\text { O } & -3.2939820 & -1.0860100 & -0.8015390\end{array}$

$\begin{array}{llll}\text { O } & 0.4689340 & -1.4876430 & 1.7174500\end{array}$

$\begin{array}{llll}\text { C } & 3.7780970 & 3.0867240 & 1.8047530\end{array}$

$\begin{array}{llll}\mathrm{H} & 4.6862080 & 3.6972180 & 1.7931150\end{array}$

H 3.0235260

$\begin{array}{llll}\mathrm{H} & 4.0078530 & 2.1601060 & 2.3421790\end{array}$

C $\quad 4.4782070 \quad 2.0918710-0.3855850$

$\mathrm{H} \quad 4.7763940 \quad 1.1614260 \quad 0.1015070$

$\begin{array}{llll}\mathrm{H} & 4.2204150 & 1.8698280 & -1.4251490\end{array}$

$\begin{array}{llll}\mathrm{H} & 5.3557850 & 2.7458250 & -0.3933170\end{array}$

C $3.0454220 \quad 4.1433240 \quad-0.3569430$

$\mathrm{H} \quad 2.2347530 \quad 4.7045810 \quad 0.1151190$

$\begin{array}{llll}\mathrm{H} & 3.9454870 & 4.7639750 & -0.3090640\end{array}$

$\begin{array}{llll}\mathrm{H} & 2.8046110 & 4.0133090 & -1.4160480\end{array}$

$\mathrm{H} \quad-3.7432990 \quad-2.4035470 \quad 1.6188660$

$\mathrm{H} \quad-1.7465020 \quad-0.8718270 \quad 3.2300600$

$\begin{array}{llll}\mathrm{H} & 3.2983220 & -1.9387400 & 1.6499940\end{array}$

C $\quad 4.8820340 \quad-1.5938300 \quad 0.2572290$

$\begin{array}{llll}\text { C } & 5.8415780 & -2.1572390 & 1.1130100\end{array}$

$\begin{array}{llll}\text { C } & 5.2379270 & -1.3529710 & -1.0762240\end{array}$

$\begin{array}{llll}\text { C } & 7.1262940 & -2.4595460 & 0.6537150\end{array}$

$\mathrm{H} \quad 5.5807170 \quad-2.3614580 \quad 2.1486140$

C $\quad 6.5207500 \quad-1.6577430 \quad-1.5418490$

$\begin{array}{llll}\mathrm{H} & 4.5133680 & -0.9327910 & -1.7672410\end{array}$

$\begin{array}{llll}\text { C } & 7.4711140 & -2.2101930 & -0.6784170\end{array}$

$\mathrm{H} \quad 7.8526650 \quad-2.8948380 \quad 1.3340110$

$\mathrm{H} \quad 6.7735390 \quad-1.4661550 \quad-2.5807220$

H $\quad 8.4664980 \quad-2.4495280 \quad-1.0409000$

$\mathrm{H} \quad-4.7354010 \quad-1.0677030 \quad-0.7406680$

$\mathrm{O} \quad-5.7880650 \quad-1.1082210 \quad-0.8207020$

$\begin{array}{llll}\text { C } & -6.3969050 & -0.2008100 & -0.1078870\end{array}$

C $\quad-7.9392760 \quad-0.3233670 \quad-0.2666330$

$\begin{array}{llll}\text { O } & -5.9120320 & 0.6510470 & 0.6120710\end{array}$

F $\quad-8.3746760 \quad-1.5244730 \quad 0.1811780$ $\begin{array}{llll}\text { F } & -8.3078930 & -0.2120650 & -1.5631600\end{array}$

F $\quad-8.5813520 \quad 0.6308000 \quad 0.4271480$

$3 . \log$

Potential Energy $=-2233.47279$

Zero-point Energy = -2232.94589

Free Energy = -2232.99537

Single-Point Energy B3LYP-D3(BJ)/6-311+G** PCM = -

2234.05134

Free Energy B3LYP-D3(BJ)/6-311+G** PCM (extrapolated

free energy from $\mathrm{qRRHO})=-2233.57392$

Nimag $=1\left(-1303.8422 \mathrm{~cm}^{-1}\right)$

Charge $=0$ Multiplicity $=1$

C $\quad-3.6113070 \quad 1.2122550 \quad 0.1742270$

$\begin{array}{llll}\text { C } & -2.4974060 & 2.5515250 & -1.4138140\end{array}$

$\begin{array}{llll}\mathrm{C} & -1.4539120 & 2.2071300 & -0.3462540\end{array}$

$\mathrm{N} \quad-2.1440510 \quad 1.1051440 \quad 0.3846140$

$\mathrm{N} \quad-3.6542310 \quad 1.9193400 \quad-1.1078660$

$\begin{array}{llll}\mathrm{O} & -2.2928160 & 3.2599570 & -2.3986260\end{array}$

C $\quad-4.7942750 \quad 1.9124360 \quad-2.0199670$

$\mathrm{H} \quad-4.4148550 \quad 1.8880300 \quad-3.0432730$

$\mathrm{H} \quad-5.3970870 \quad 1.0222020 \quad-1.8384180$

$\mathrm{H} \quad-5.4176640 \quad 2.8032340 \quad-1.9009070$

C $\quad-1.4780230 \quad 0.1811510 \quad 1.0580480$

$\mathrm{H} \quad-0.4598440 \quad 0.4672810 \quad 1.3133660$

C $\quad-1.7722930 \quad-1.1811050 \quad 1.3571830$

C $\quad-3.0548830 \quad-1.9764760 \quad 1.1340950$

$\mathrm{H} \quad-2.8220540 \quad-2.9968470 \quad 1.4635910$

$\begin{array}{llll}\mathrm{H} & -0.7495980 & -1.7895450 & 0.5933580\end{array}$

$\begin{array}{llll}\text { C } & -0.1118010 & 1.8275950 & -0.9616940\end{array}$

$\mathrm{H} \quad 0.2202980 \quad 2.6617790 \quad-1.5844860$

H $\quad 0.6472090 \quad 1.6455580 \quad-0.1992440$

$\begin{array}{llll}\mathrm{H} & -0.2042490 & 0.9373690 & -1.5902760\end{array}$

$\begin{array}{llll}\mathrm{H} & -1.3300190 & 3.0696080 & 0.3184460\end{array}$

$\begin{array}{llll}\mathrm{C} & -4.3502390 & 1.9520030 & 1.3550200\end{array}$

$\mathrm{H} \quad-4.0441800 \quad 0.2193210 \quad 0.0550350$

$\begin{array}{llll}\mathrm{Cl} & -1.0783390 & -1.5991500 & 2.9993370\end{array}$

$\begin{array}{llll}\mathrm{N} & 1.6605580 & -0.9517030 & 0.6751260\end{array}$

$\begin{array}{llll}\text { C } & 1.2855760 & -2.0942030 & 0.0968160\end{array}$

$\begin{array}{llll}\text { C } & 3.0132320 & -0.8445620 & 0.5401860\end{array}$

$\begin{array}{llll}\text { C } & 2.4142910 & -2.8980960 & -0.5183470\end{array}$

$\begin{array}{llll}\mathrm{C} & 3.6332980 & -2.0255890 & -0.2011780\end{array}$

$\mathrm{H} \quad 2.4511140 \quad-3.8932430 \quad-0.0653540$

$\mathrm{H} \quad 4.1631430 \quad-1.6658200 \quad-1.0878040$

$\begin{array}{llll}\text { O } & 3.6601560 & 0.1287310 & 0.9842660\end{array}$

$\begin{array}{llll}\text { O } & 0.0791500 & -2.5101670 & 0.0425230\end{array}$

$\begin{array}{llll}\mathrm{C} & -5.8661420 & 1.9443530 & 1.0506060\end{array}$

$\mathrm{H} \quad-6.4082290 \quad 2.3186720 \quad 1.9245070$

$\begin{array}{llll}\mathrm{H} & -6.1241720 & 2.5894660 & 0.2080940\end{array}$

$\begin{array}{llll}\mathrm{H} & -6.2339120 & 0.9341360 & 0.8403400\end{array}$

$\begin{array}{llll}\text { C } & -4.1258830 & 1.1939620 & 2.6778110\end{array}$

$\begin{array}{llll}\mathrm{H} & -4.5183980 & 0.1737170 & 2.6364570\end{array}$

$\mathrm{H} \quad-3.0711090 \quad 1.1538750 \quad 2.9643490$

$\begin{array}{llll}\mathrm{H} & -4.6596910 & 1.7122290 & 3.4803980\end{array}$

$\begin{array}{llll}\mathrm{C} & -3.8748850 & 3.4074710 & 1.5255330\end{array}$

$\begin{array}{llll}\mathrm{H} & -3.9587270 & 3.9818470 & 0.5977760\end{array}$

$\begin{array}{llll}\mathrm{H} & -4.5013780 & 3.9034320 & 2.2735320\end{array}$

$\mathrm{H} \quad-2.8426540 \quad 3.4635160 \quad 1.8845320$

$\begin{array}{llll}\mathrm{H} & 4.3674070 & -2.5200690 & 0.4422190\end{array}$

$\begin{array}{llll}\mathrm{H} & 2.2332700 & -3.0319470 & -1.5890970\end{array}$

$\begin{array}{llll}\mathrm{H} & -3.8469440 & -1.6359880 & 1.8107060\end{array}$

$\begin{array}{llll}\text { C } & -3.6161960 & -2.0743910 & -0.2816020\end{array}$

$\begin{array}{llll}\text { C } & -4.9904930 & -2.3338250 & -0.4266730\end{array}$

$\begin{array}{llll}\text { C } & -2.8310390 & -1.9917020 & -1.4414610\end{array}$

C $\quad-5.5640920 \quad-2.5074540 \quad-1.6880920$

$\begin{array}{llll}\mathrm{H} & -5.6173290 & -2.4003680 & 0.4591290\end{array}$

$\begin{array}{llll}\mathrm{C} & -3.4053160 & -2.1581620 & -2.7069680\end{array}$

$\begin{array}{llll}\mathrm{H} & -1.7655090 & -1.8051000 & -1.3724070\end{array}$

$\begin{array}{llll}\text { C } & -4.7715980 & -2.4180150 & -2.8371270\end{array}$

$\mathrm{H} \quad-6.6283620 \quad-2.7077850 \quad-1.7719370$

$\mathrm{H} \quad-2.7779990 \quad-2.0859530 \quad-3.5908490$

$\mathrm{H} \quad-5.2142220 \quad-2.5474250 \quad-3.8202870$

$\begin{array}{llll}\mathrm{H} & 5.0942590 & 0.2672730 & 0.8143140\end{array}$

$\begin{array}{llll}\text { O } & 6.1335020 & 0.4388930 & 0.8125370\end{array}$

C $\quad 6.6477480 \quad 0.4860620 \quad-0.3860950$

$\begin{array}{llll}\text { C } & 8.1788560 & 0.7499210 & -0.3142150\end{array}$ 


$\begin{array}{lrrr}\mathrm{O} & 6.0891410 & 0.3512460 & -1.4577040 \\ \mathrm{~F} & 8.8076550 & -0.2336760 & 0.3703100 \\ \mathrm{~F} & 8.4414450 & 1.9186180 & 0.3145690 \\ \mathrm{~F} & 8.7236720 & 0.8106880 & -1.5401960\end{array}$

Hydrolysis of chloroiminium ion (TS6)

\section{Conformation Extrapolated name}

\section{Free energy}

$\begin{array}{cc}\text { TS6-lowest } & 0.0 \\ 1 & 3.7 \\ 2 & 5.6 \\ 3 & 4.5 \\ 4 & 4.0 \\ 5 & 2.6 \\ 6 & 2.0 \\ 7 & 1.6 \\ 8 & 1.1 \\ 9 & 2.2 \\ 10 & 2.6\end{array}$

TS6-lowest.log

Potential Energy $=-1949.25117$

Zero-point Energy = -1948.78680

Free Energy $=-1948.83082$

Single-Point Energy B3LYP-D3(BJ)/6-311+G** PCM = 1949.74621

Free Energy B3LYP-D3(BJ)/6-311+G** PCM (extrapolated free energy from $\mathrm{qRRHO})=-1949.32585$

Nimag $=1\left(-334.7303 \mathrm{~cm}^{-1}\right)$

Charge $=0$ Multiplicity $=1$

$\begin{array}{lrrr}\mathrm{C} & 2.8955910 & -0.0482040 & 0.1680960 \\ \mathrm{C} & 3.3827080 & -1.9184980 & -1.1956040 \\ \mathrm{C} & 1.9931950 & -2.1754130 & -0.6106640 \\ \mathrm{~N} & 1.6500630 & -0.8319040 & -0.0725290 \\ \mathrm{~N} & 3.8134410 & -0.7085280 & -0.7701720 \\ \mathrm{O} & 3.9865730 & -2.6754010 & -1.9565940 \\ \mathrm{C} & 4.9835810 & -0.0681420 & -1.3674970 \\ \mathrm{H} & 5.9142360 & -0.3904180 & -0.8932950 \\ \mathrm{H} & 5.0215260 & -0.3365020 & -2.4254350 \\ \mathrm{H} & 4.8913790 & 1.0142570 & -1.2740780 \\ \mathrm{C} & 0.3887410 & -0.3649380 & -0.0606740 \\ \mathrm{C} & 0.1073720 & 1.1334310 & -0.1578130 \\ \mathrm{C} & -1.3636050 & 1.5186740 & 0.0527000 \\ \mathrm{H} & -1.9841560 & 0.9700550 & -0.6619880 \\ \mathrm{H} & -1.6355370 & 1.1772550 & 1.0552300 \\ \mathrm{C} & 1.0347960 & -2.7372850 & -1.6557870 \\ \mathrm{H} & 1.4825630 & -3.6488260 & -2.0586000 \\ \mathrm{H} & 0.0663930 & -2.9980050 & -1.2235440 \\ \mathrm{H} & 0.8823480 & -2.0347010 & -2.4804220 \\ \mathrm{H} & 2.0900890 & -2.8953500 & 0.2117130 \\ \mathrm{C} & 3.4022340 & -0.0169090 & 1.6545150 \\ \mathrm{H} & 2.7442570 & 0.9815200 & -0.1670940 \\ \mathrm{C} & 4.8005240 & 0.6392610 & 1.7010610 \\ \mathrm{H} & 5.0824460 & 0.8027360 & 2.7458240 \\ \mathrm{H} & 5.5691920 & 0.0069530 & 1.2516840 \\ \mathrm{H} & 4.8125070 & 1.6144620 & 1.2017180 \\ \mathrm{C} & 2.4570170 & 0.8579320 & 2.5045900 \\ \mathrm{H} & 2.4396960 & 1.8923230 & 2.1418340 \\ \mathrm{H} & 1.4316250 & 0.4833980 & 2.5301740 \\ \mathrm{H} & 2.8194450 & 0.8834030 & 3.5370200 \\ \mathrm{C} & 3.4983560 & -1.4298860 & 2.2579750 \\ \mathrm{H} & 4.1547930 & -2.0786350 & 1.6690970 \\ \mathrm{H} & 3.9174040 & -1.3691500 & 3.2671700 \\ \mathrm{H} & 2.5206820 & -1.9141480 & 2.3442290 \\ \mathrm{H} & 0.7495260 & 1.7083960 & 0.5061280 \\ \mathrm{C} & -1.6284650 & 3.0082380 & -0.0467960 \\ \mathrm{C} & -1.2175730 & 3.8732190 & 0.9799540\end{array}$

$\begin{array}{lrrr}\text { C } & -2.3010370 & 3.5493270 & -1.1511980 \\ \text { C } & -1.4610340 & 5.2467510 & 0.8984580 \\ \text { H } & -0.7111170 & 3.4708650 & 1.8542360 \\ \text { C } & -2.5508290 & 4.9230530 & -1.2345340 \\ \text { H } & -2.6327820 & 2.8924230 & -1.9507320 \\ \text { C } & -2.1285540 & 5.7765510 & -0.2111940 \\ \text { H } & -1.1371220 & 5.9003740 & 1.7033080 \\ \text { H } & -3.0750960 & 5.3236820 & -2.0974100 \\ \text { H } & -2.3223280 & 6.8433720 & -0.2739470 \\ \text { Cl } & 0.6329890 & 1.6004930 & -1.8595830 \\ \text { H } & -0.3149590 & -0.9651320 & -0.6304690 \\ \text { O } & -0.3816310 & -0.7214830 & 1.5286200 \\ \text { H } & -1.4001990 & -1.1730380 & 1.4189980 \\ \text { H } & 0.1793360 & -1.3944580 & 1.9462030 \\ \text { O } & -2.6177710 & -1.6904950 & 1.3615540 \\ \text { C } & -3.0128880 & -2.0296770 & 0.1949010 \\ \text { C } & -4.4366550 & -2.6675900 & 0.2143890 \\ \text { O } & -2.4311580 & -1.9202830 & -0.8863440 \\ \text { F } & -4.4504550 & -3.8032910 & 0.9579780 \\ \text { F } & -5.3480060 & -1.8221440 & 0.7573920 \\ \text { F } & -4.8760320 & -2.9912920 & -1.0179130\end{array}$

$1 . \log$

Potential Energy $=-1949.24688$

Zero-point Energy = -1948.78171

Free Energy = -1948.82596

Single-Point Energy B3LYP-D3(BJ)/6-311+G** PCM = -

1949.74093

Free Energy B3LYP-D3(BJ)/6-311+G** PCM (extrapolated free energy from $q R R H O)=-1949.32002$

Nimag $=1\left(-222.3064 \mathrm{~cm}^{-1}\right)$

Charge $=0$ Multiplicity $=1$

\begin{tabular}{|c|c|c|c|}
\hline $\mathrm{C}$ & 3.0208430 & -0.1564760 & -0.3326480 \\
\hline $\mathrm{C}$ & 3.1710770 & -2.4505080 & 0.1991190 \\
\hline $\mathrm{C}$ & 2.3387470 & -1.7907600 & 1.3046420 \\
\hline $\mathrm{N}$ & 1.9540580 & -0.4993170 & 0.6548990 \\
\hline $\mathrm{N}$ & 3.4969950 & -1.4971020 & -0.7045160 \\
\hline $\mathrm{O}$ & 3.4782950 & -3.6414590 & 0.1470840 \\
\hline $\mathrm{C}$ & 4.1596550 & -1.8173120 & -1.9646690 \\
\hline $\mathrm{H}$ & 5.2488800 & -1.8254910 & -1.8643290 \\
\hline $\mathrm{H}$ & 3.8326560 & -2.8093020 & -2.2825050 \\
\hline $\mathrm{H}$ & 3.8746640 & -1.0841330 & -2.7208260 \\
\hline $\mathrm{C}$ & 0.6660350 & -0.1588130 & 0.5356160 \\
\hline $\mathrm{C}$ & 0.1764590 & 1.2384760 & 0.1499530 \\
\hline $\mathrm{C}$ & -1.2988920 & 1.4475700 & 0.5316560 \\
\hline $\mathrm{H}$ & -1.9314590 & 0.7816770 & -0.0621500 \\
\hline $\mathrm{H}$ & -1.3911670 & 1.1348680 & 1.5795980 \\
\hline $\mathrm{C}$ & 1.2203870 & -2.6786750 & 1.8308400 \\
\hline $\mathrm{H}$ & 1.6858710 & -3.5930820 & 2.2063740 \\
\hline $\mathrm{H}$ & 0.6935940 & -2.2100730 & 2.6663900 \\
\hline $\mathrm{H}$ & 0.5058540 & -2.9577900 & 1.0545510 \\
\hline $\mathrm{H}$ & 3.0121430 & -1.5551160 & 2.1350460 \\
\hline $\mathrm{C}$ & 4.1332430 & 0.8105020 & 0.2084680 \\
\hline $\mathrm{H}$ & 2.5605610 & 0.3109390 & -1.2034170 \\
\hline $\mathrm{C}$ & 5.0865100 & 1.1468360 & -0.9614250 \\
\hline $\mathrm{H}$ & 5.7781930 & 1.9344450 & -0.6468720 \\
\hline $\mathrm{H}$ & 5.6897250 & 0.2882190 & -1.2650900 \\
\hline $\mathrm{H}$ & 4.5414120 & 1.5148480 & -1.8378080 \\
\hline $\mathrm{C}$ & 3.4841760 & 2.1226040 & 0.6912930 \\
\hline $\mathrm{H}$ & 2.9192600 & 2.6158190 & -0.1067560 \\
\hline $\mathrm{H}$ & 2.8267270 & 1.9556680 & 1.5500300 \\
\hline $\mathrm{H}$ & 4.2667610 & 2.8169710 & 1.0124960 \\
\hline $\mathrm{C}$ & 4.9527010 & 0.2031550 & 1.3631570 \\
\hline $\mathrm{H}$ & 5.3777320 & -0.7702360 & 1.0990350 \\
\hline $\mathrm{H}$ & 5.7870630 & 0.8708500 & 1.6006720 \\
\hline $\mathrm{H}$ & 4.3591150 & 0.0950540 & 2.2758340 \\
\hline $\mathrm{H}$ & 0.8025100 & 1.9552700 & 0.6811980 \\
\hline $\mathrm{C}$ & -1.7722000 & 2.8813410 & 0.3998400 \\
\hline $\mathrm{C}$ & -1.3470770 & 3.8606940 & 1.3116490 \\
\hline $\mathrm{C}$ & -2.6546460 & 3.2536400 & -0.6237390 \\
\hline $\mathrm{C}$ & -1.7839230 & 5.1827580 & 1.1956740 \\
\hline $\mathrm{H}$ & -0.6768810 & 3.5889210 & 2.1239640 \\
\hline $\mathrm{C}$ & -3.0964840 & 4.5755080 & -0.7413250 \\
\hline $\mathrm{H}$ & -2.9998130 & 2.5051310 & -1.3315270 \\
\hline $\mathrm{C}$ & -2.6602420 & 5.5445530 & 0.1666020 \\
\hline $\mathrm{H}$ & -1.4464960 & 5.9268450 & 1.9114150 \\
\hline
\end{tabular}




$\begin{array}{cccc}\mathrm{H} & -3.7813710 & 4.8451910 & -1.5400890 \\ \mathrm{H} & -3.0035870 & 6.5710800 & 0.0776080 \\ \mathrm{H} & 0.0547610 & -0.5798070 & 1.3258100 \\ \mathrm{O} & -0.1160670 & -1.2443160 & -0.7628840 \\ \mathrm{H} & -0.2406990 & -0.7564940 & -1.5941480 \\ \mathrm{H} & -1.0555830 & -1.6722160 & -0.4765930 \\ \mathrm{O} & -2.2140850 & -2.3383180 & -0.0336620 \\ \mathrm{C} & -3.3264630 & -1.8800520 & -0.4501430 \\ \mathrm{O} & -3.5552820 & -0.9059090 & -1.1729060 \\ \mathrm{C} & -4.5429530 & -2.7096330 & 0.0738980 \\ \mathrm{~F} & -4.4369140 & -4.0189620 & -0.2694920 \\ \mathrm{~F} & -4.6195250 & -2.6590390 & 1.4301720 \\ \mathrm{~F} & -5.7248240 & -2.2692010 & -0.4055400 \\ \mathrm{Cl} & 0.4281750 & 1.6405450 & -1.6216870\end{array}$

2.log

Potential Energy = -1949.24102

Zero-point Energy = -1948.77561

Free Energy = -1948.81978

Single-Point Energy B3LYP-D3(BJ)/6-311+G** PCM = -

1949.73822

Free Energy B3LYP-D3(BJ)/6-311+G** PCM (extrapolated

free energy from $\mathrm{qRRHO})=-1949.31697$

Nimag $=1\left(-192.4248 \mathrm{~cm}^{-1}\right)$

Charge $=0$ Multiplicity $=1$

\begin{tabular}{|c|c|c|c|}
\hline $\mathrm{C}$ & 2.8894970 & 0.4179300 & 0.3334400 \\
\hline $\mathrm{C}$ & 1.9558430 & 2.4592440 & -0.3999840 \\
\hline $\mathrm{C}$ & 1.7478320 & 1.4390170 & -1.5255110 \\
\hline $\mathrm{N}$ & 1.9463970 & 0.1504670 & -0.7949480 \\
\hline $\mathrm{N}$ & 2.5694180 & 1.8239840 & 0.6266920 \\
\hline $\mathrm{O}$ & 1.6198690 & 3.6430760 & -0.4302890 \\
\hline $\mathrm{C}$ & 2.7918360 & 2.4695610 & 1.9160210 \\
\hline $\mathrm{H}$ & 3.7414650 & 3.0121030 & 1.9444520 \\
\hline $\mathrm{H}$ & 1.9807310 & 3.1797810 & 2.0874890 \\
\hline $\mathrm{H}$ & 2.7844320 & 1.7167250 & 2.7058150 \\
\hline $\mathrm{C}$ & 0.9881180 & -0.7810200 & -0.7533630 \\
\hline $\mathrm{C}$ & 1.2649510 & -2.2372670 & -0.3661630 \\
\hline $\mathrm{C}$ & 0.2700700 & -3.2468120 & -0.9773750 \\
\hline $\mathrm{H}$ & 0.3833770 & -3.1507590 & -2.0636930 \\
\hline $\mathrm{H}$ & 0.6469790 & -4.2403780 & -0.7169870 \\
\hline $\mathrm{C}$ & 0.4550880 & 1.6231910 & -2.3043350 \\
\hline $\mathrm{H}$ & 0.4854960 & 2.6240780 & -2.7422440 \\
\hline $\mathrm{H}$ & 0.3830250 & 0.9072500 & -3.1277170 \\
\hline $\mathrm{H}$ & -0.4374990 & 1.5607890 & -1.6797890 \\
\hline $\mathrm{H}$ & 2.5841280 & 1.5426380 & -2.2243130 \\
\hline $\mathrm{C}$ & 4.4055310 & 0.1416630 & 0.0270390 \\
\hline $\mathrm{H}$ & 2.5936490 & -0.1983500 & 1.1833380 \\
\hline $\mathrm{C}$ & 5.1935200 & 0.3079790 & 1.3473810 \\
\hline $\mathrm{H}$ & 6.2282490 & -0.0129210 & 1.1920110 \\
\hline $\mathrm{H}$ & 5.2235470 & 1.3459740 & 1.6862210 \\
\hline $\mathrm{H}$ & 4.7725200 & -0.3062330 & 2.1510700 \\
\hline $\mathrm{C}$ & 4.5844710 & -1.3108130 & -0.4568240 \\
\hline $\mathrm{H}$ & 4.2292000 & -2.0344590 & 0.2842180 \\
\hline $\mathrm{H}$ & 4.0734040 & -1.4852690 & -1.4088790 \\
\hline $\mathrm{H}$ & 5.6485190 & -1.5091650 & -0.6190590 \\
\hline $\mathrm{C}$ & 4.9952690 & 1.0951550 & -1.0304710 \\
\hline $\mathrm{H}$ & 4.8283590 & 2.1467100 & -0.7775010 \\
\hline $\mathrm{H}$ & 6.0774120 & 0.9402260 & -1.0881030 \\
\hline $\mathrm{H}$ & 4.5916740 & 0.9035200 & -2.0291240 \\
\hline $\mathrm{H}$ & 2.2465620 & -2.4731730 & -0.7744000 \\
\hline $\mathrm{C}$ & -1.1977920 & -3.1367630 & -0.6131450 \\
\hline $\mathrm{C}$ & -1.6901780 & -3.6668010 & 0.5920030 \\
\hline $\mathrm{C}$ & -2.1074970 & -2.5447310 & -1.5034050 \\
\hline $\mathrm{C}$ & -3.0496080 & -3.5831160 & 0.9068390 \\
\hline $\mathrm{H}$ & -1.0111460 & -4.1593760 & 1.2810550 \\
\hline $\mathrm{C}$ & -3.4681320 & -2.4641760 & -1.1930640 \\
\hline $\mathrm{H}$ & -1.7536540 & -2.1532530 & -2.4538320 \\
\hline $\mathrm{C}$ & -3.9428200 & -2.9790660 & 0.0163960 \\
\hline $\mathrm{H}$ & -3.4107550 & -3.9992230 & 1.8428030 \\
\hline $\mathrm{H}$ & -4.1543180 & -2.0031770 & -1.8973560 \\
\hline $\mathrm{H}$ & -4.9996240 & -2.9190220 & 0.2590010 \\
\hline $\mathrm{H}$ & 0.3030960 & -0.7143960 & -1.5913040 \\
\hline $\mathrm{O}$ & -0.2605370 & -0.2465070 & 0.5422210 \\
\hline $\mathrm{H}$ & -0.6111020 & -0.9975070 & 1.0487370 \\
\hline $\mathrm{H}$ & -1.0955650 & 0.3295850 & 0.2269110 \\
\hline $\mathrm{O}$ & -2.1910560 & 1.1610540 & -0.1716250 \\
\hline
\end{tabular}

$\begin{array}{lccc}\text { C } & -3.0805160 & 1.3804530 & 0.7112190 \\ \text { O } & -3.1585750 & 0.9743680 & 1.8743230 \\ \text { C } & -4.2183640 & 2.3154250 & 0.1853990 \\ \text { F } & -3.7302810 & 3.5353450 & -0.1625320 \\ \text { F } & -4.8156700 & 1.7974860 & -0.9195600 \\ \text { F } & -5.1910690 & 2.5251690 & 1.0974720 \\ \text { Cl } & 1.4641170 & -2.5203600 & 1.4324080\end{array}$

$3 . \log$

Potential Energy $=-1949.24357$

Zero-point Energy = -1948.77902

Free Energy = -1948.82349

Single-Point Energy B3LYP-D3(BJ)/6-311+G** PCM = 1949.73882

Free Energy B3LYP-D3(BJ)/6-311+G** PCM (extrapolated

free energy from $\mathrm{qRRHO})=-1949.31874$

Nimag $=1\left(-270.5133 \mathrm{~cm}^{-1}\right)$

Charge $=0$ Multiplicity $=1$

$\begin{array}{llll}\text { C } & -1.0258770 & -1.7574150 & -0.5882850\end{array}$

$\begin{array}{llll}\text { C } & -2.5649860 & -2.3536380 & 1.1003070\end{array}$

C $\quad-1.1685940 \quad-2.6872120 \quad 1.6360520$

$\begin{array}{llll}\mathrm{N} & -0.2976520 & -1.9019590 & 0.7074530\end{array}$

$\begin{array}{llll}\mathrm{N} & -2.4216590 & -1.8005640 & -0.1259950\end{array}$

$\begin{array}{llll}\mathrm{O} & -3.6259040 & -2.5546840 & 1.6927850\end{array}$

$\begin{array}{llll}\text { C } & -3.5519460 & -1.2590890 & -0.8716840\end{array}$

$\mathrm{H} \quad-4.0326460 \quad-2.0191450 \quad-1.4949580$

$\mathrm{H} \quad-4.2838410 \quad-0.8824680 \quad-0.1551640$

$\mathrm{H} \quad-3.2093260 \quad-0.4357180 \quad-1.4991950$

C $\quad 0.5927710 \quad-1.0273360 \quad 1.1904650$

$\begin{array}{llll}\text { C } & 1.7553820 & -0.4376040 & 0.3920710\end{array}$

$\begin{array}{llll}\mathrm{C} & 2.8097950 & 0.2101550 & 1.3102420\end{array}$

$\begin{array}{llll}\mathrm{H} & 2.4040620 & 1.1214370 & 1.7583460\end{array}$

$\begin{array}{llll}\mathrm{H} & 2.9964970 & -0.4990320 & 2.1259030\end{array}$

C $\quad-1.0157840 \quad-2.4335390 \quad 3.1292590$

$\mathrm{H} \quad-1.7885120 \quad-3.0167270 \quad 3.6359260$

$\mathrm{H} \quad-0.0464630 \quad-2.7799600 \quad 3.4978110$

$\mathrm{H} \quad-1.1534250 \quad-1.3813000 \quad 3.3851330$

$\mathrm{H} \quad-0.9830910 \quad-3.7495530 \quad 1.4444520$

$\begin{array}{llll}\text { C } & -0.6641210 & -2.8335330 & -1.6760540\end{array}$

$\mathrm{H} \quad-0.8191030 \quad-0.7689580 \quad-0.9999490$

$\begin{array}{llll}\mathrm{C} & -1.3379500 & -2.4165650 & -3.0040180\end{array}$

$\mathrm{H} \quad-0.9894480 \quad-3.0733930 \quad-3.8071530$

$\mathrm{H} \quad-2.4261660 \quad-2.5016850 \quad-2.9651640$

$\mathrm{H} \quad-1.0816740 \quad-1.3879640 \quad-3.2810550$

C $\quad 0.8605180 \quad-2.8579410 \quad-1.9019750$

$\mathrm{H} \quad 1.2405750 \quad-1.8840450 \quad-2.2257070$

$\mathrm{H} \quad 1.3964690 \quad-3.1740110 \quad-1.0018810$

$\mathrm{H} \quad 1.0963460 \quad-3.5787670 \quad-2.6911660$

C $\quad-1.1329990 \quad-4.2516540 \quad-1.2965740$

$\mathrm{H} \quad-2.2003500 \quad-4.2845640 \quad-1.0574080$

$\mathrm{H} \quad-0.9661320 \quad-4.9233450 \quad-2.1447860$

$\begin{array}{llll}\mathrm{H} & -0.5700270 & -4.6585360 & -0.4511880\end{array}$

$\begin{array}{llll}\mathrm{H} & 2.2119870 & -1.2757470 & -0.1375880\end{array}$

$\begin{array}{llll}\text { C } & 4.1206840 & 0.5205640 & 0.6156410\end{array}$

$\begin{array}{llll}\mathrm{C} & 5.0060650 & -0.5147710 & 0.2764800\end{array}$

$\begin{array}{llll}\mathrm{C} & 4.4806480 & 1.8406750 & 0.3121540\end{array}$

$\begin{array}{llll}\text { C } & 6.2172150 & -0.2379420 & -0.3625780\end{array}$

$\begin{array}{llll}\mathrm{H} & 4.7526000 & -1.5438700 & 0.5202600\end{array}$

$\begin{array}{llll}\mathrm{C} & 5.6939210 & 2.1219210 & -0.3244250\end{array}$

$\mathrm{H} \quad 3.8098290 \quad 2.6539250 \quad 0.5754930$

$\begin{array}{llll}\mathrm{C} & 6.5644660 & 1.0830850 & -0.6659500\end{array}$

$\mathrm{H} \quad 6.8913260 \quad-1.0510600 \quad-0.6158380$

$\begin{array}{llll}\mathrm{H} & 5.9570950 & 3.1510770 & -0.5509040\end{array}$

$\begin{array}{llll}\mathrm{H} & 7.5075280 & 1.3000350 & -1.1589600\end{array}$

$\mathrm{H} \quad 0.9192290 \quad-1.2943370 \quad 2.1892300$

$\begin{array}{llll}\mathrm{O} & -0.3467970 & 0.4513560 & 1.8041540\end{array}$

$\mathrm{H} \quad-1.0017590 \quad 0.9784800 \quad 1.1236980$

$\begin{array}{llll}\text { O } & -1.9211670 & 1.6449010 & 0.3228360\end{array}$

$\begin{array}{llll}\text { C } & -1.8718290 & 2.9096320 & 0.4609950\end{array}$

$\begin{array}{llll}\mathrm{O} & -1.1483910 & 3.5917820 & 1.1927050\end{array}$

$\begin{array}{llll}\text { C } & -2.8964960 & 3.6489300 & -0.4579750\end{array}$

F $\quad-2.6502070 \quad 3.3956360 \quad-1.7701490$

$\begin{array}{llll}\text { F } & -4.1684320 & 3.2443360 & -0.2071050\end{array}$

$\begin{array}{llll}\text { F } & -2.8691780 & 4.9887960 & -0.3016830\end{array}$

$\mathrm{Cl} \quad 1.2651020 \quad 0.7311390 \quad-0.9157250$

$\begin{array}{llll}\mathrm{H} & 0.1999970 & 1.1214450 & 2.2446370\end{array}$ 
4.log

Potential Energy $=-1949.24205$

Zero-point Energy = -1948.77664

Free Energy = -1948.82065

Single-Point Energy B3LYP-D3(BJ)/6-311+G** PCM = 1949.74090

Free Energy B3LYP-D3(BJ)/6-311+G** PCM (extrapolated free energy from qRRHO) $=-1949.31950$

Nimag $=1\left(-226.1030 \mathrm{~cm}^{-1}\right)$

Charge $=0$ Multiplicity $=1$

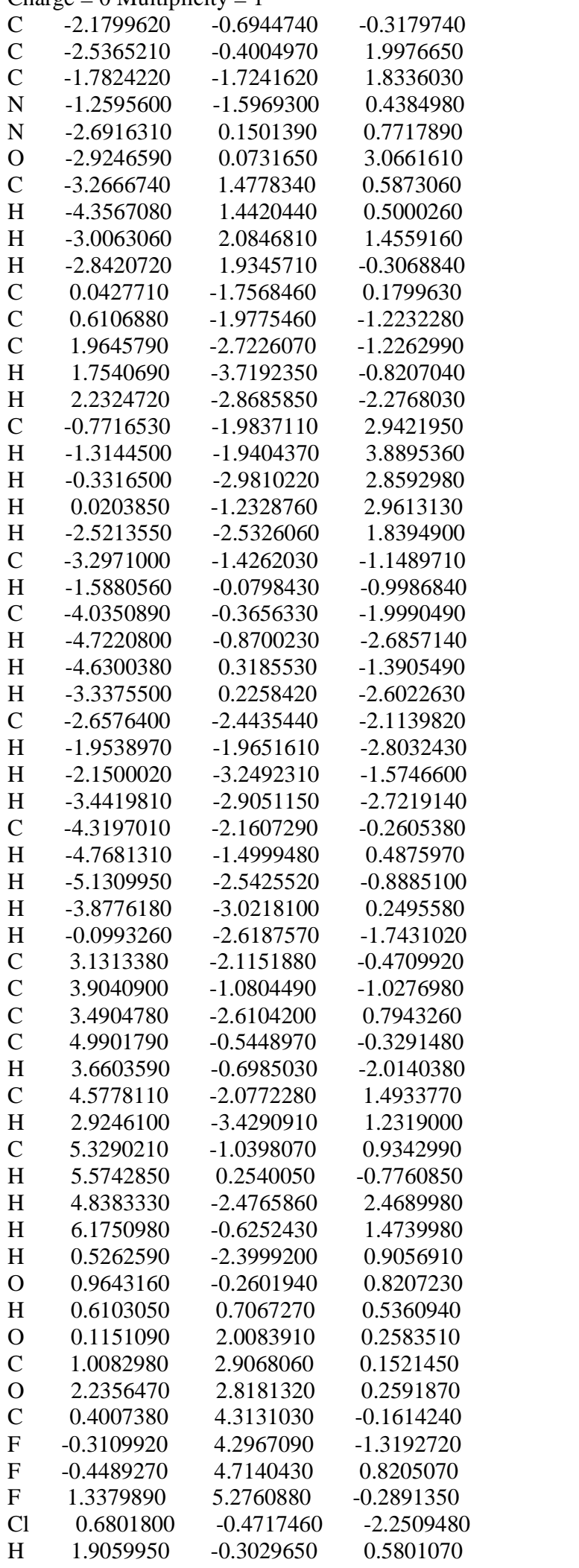

5.log

Potential Energy $=-1949.24618$

Zero-point Energy = -1948.78104

Free Energy = -1948.82472

Single-Point Energy B3LYP-D3(BJ)/6-311+G** PCM = 1949.74322
Free Energy B3LYP-D3(BJ)/6-311+G** PCM (extrapolated free energy from qRRHO) $=-1949.32175$

Nimag $=1\left(-261.7286 \mathrm{~cm}^{-1}\right)$

Charge $=0$ Multiplicity $=$

\begin{tabular}{|c|c|c|c|}
\hline & & & \\
\hline $\mathrm{C}$ & 2.9130930 & -0.0045410 & -0.0920550 \\
\hline $\mathrm{C}$ & 3.3528360 & 2.3167710 & 0.0171920 \\
\hline & 1.8725980 & 2.1869440 & -0.3446230 \\
\hline & 1.6263550 & 0.7482990 & -0.0568310 \\
\hline N & 3.8649320 & 1.0768240 & 0.1937540 \\
\hline & 3.9603010 & 3.3781240 & 0.1616750 \\
\hline & 5.1741910 & 0.8773790 & 0.8121020 \\
\hline $\mathrm{H}$ & 5.9855310 & 0.9279140 & 0.0815060 \\
\hline $\mathrm{H}$ & 5.3272350 & 1.6613040 & 1.5568300 \\
\hline $\mathrm{H}$ & 5.1966090 & -0.0955330 & 1.3039560 \\
\hline $\mathrm{C}$ & 0.4398440 & 0.3006290 & 0.3898440 \\
\hline $\mathrm{C}$ & 0.3506340 & -0.9335190 & 1.2841040 \\
\hline $\mathrm{C}$ & -1.0789220 & -1.4551230 & 1.5609090 \\
\hline $\mathrm{H}$ & -1.1249700 & -1.7122400 & 2.6226890 \\
\hline $\mathrm{H}$ & -1.8014300 & -0.6489030 & 1.4104100 \\
\hline $\mathrm{C}$ & 1.0145490 & 3.1766990 & 0.4371680 \\
\hline $\mathrm{H}$ & 1.3990460 & 4.1788810 & 0.2336520 \\
\hline $\mathrm{H}$ & -0.0321160 & 3.1456320 & 5200 \\
\hline $\mathrm{H}$ & 1.0752710 & 2.9964620 & 1.5145210 \\
\hline $\mathrm{H}$ & 1.7561740 & 2.3802580 & -1.4183320 \\
\hline $\mathrm{C}$ & 3.2042930 & -0.7875680 & -1.4225410 \\
\hline $\mathrm{H}$ & 2.9403480 & -0.7143830 & 0.7397360 \\
\hline $\mathrm{C}$ & 4.6610840 & -1.3023960 & -1.40 \\
\hline $\mathrm{H}$ & 4.8111240 & -1.9806740 & -2.2474530 \\
\hline $\mathrm{H}$ & 5.3863890 & -0.4919740 & \\
\hline $\mathrm{H}$ & 4.8851950 & -1.8630110 & -0.4 \\
\hline $\mathrm{C}$ & 2.2824660 & -2.0218270 & -1.50 \\
\hline $\mathrm{H}$ & 2.4637610 & -2.7092110 & \\
\hline $\mathrm{H}$ & 1.2207910 & -1.7672840 & -1.516650 \\
\hline $\mathrm{H}$ & & & -2.429430 \\
\hline $\mathrm{C}$ & 3.0131610 & 0.0998790 & -2.6 \\
\hline $\mathrm{H}$ & 3.6448250 & 0.9932310 & -2.6 \\
\hline $\mathrm{H}$ & 3.2938820 & -0.4617060 & -3.5 \\
\hline $\mathrm{H}$ & 1.9740190 & 0.4174260 & -2.7 \\
\hline $\mathrm{H}$ & 0.9896640 & -1.7379410 & \\
\hline $\mathrm{C}$ & -1.4712070 & -2.6872470 & 0.7 \\
\hline $\mathrm{C}$ & -2.5808210 & -2.6721500 & -0.0 \\
\hline $\mathrm{C}$ & -0.7622570 & -3.8893330 & 0.9 \\
\hline $\mathrm{C}$ & -2.9690720 & -3.8255940 & -0.784801 \\
\hline $\mathrm{H}$ & -3.1415990 & -1.7518920 & -0.229766 \\
\hline $\mathrm{C}$ & -1.1430430 & -5.0419480 & 0.2 \\
\hline $\mathrm{H}$ & 0.0884920 & -3.9334050 & 1.593955 \\
\hline $\mathrm{C}$ & -2.2495100 & -5.0137030 & -0.630066 \\
\hline $\mathrm{H}$ & -3.8321780 & -3.7925870 & -1.443654 \\
\hline $\mathrm{H}$ & -0.5814350 & -5.9618350 & 0.361315 \\
\hline $\mathrm{H}$ & -2.5491100 & -5.9094480 & -1.166242 \\
\hline $\mathrm{Cl}$ & 1.1214350 & -0.3854150 & 2.869648 \\
\hline $\mathrm{H}$ & -0.2478580 & 1.0856020 & 0.6917810 \\
\hline $\mathrm{O}$ & -0.5755970 & -0.2415570 & -0.993233 \\
\hline $\mathrm{H}$ & -1.5668580 & 0.2344320 & -0.992402 \\
\hline $\mathrm{H}$ & -0.1198490 & 0.0683590 & -1.792108 \\
\hline $\mathrm{O}$ & -2.8076090 & 0.7835340 & -1.050597 \\
\hline $\mathrm{C}$ & -3.0748940 & 1.6554160 & -0.158729 \\
\hline $\mathrm{C}$ & -4.4958080 & 2.2745060 & -0.340456 \\
\hline $\mathrm{O}$ & -2.3806380 & 2.0434650 & 0.784824 \\
\hline $\mathrm{F}$ & -4.6188110 & 2.8718620 & -1.552930 \\
\hline & -5.4578980 & 1.3198830 & -0.265135 \\
\hline & -4.7820250 & 3.2038370 & 0.5930370 \\
\hline
\end{tabular}

6.log

Potential Energy = -1949.24682

Zero-point Energy $=-1948.78252$

Free Energy $=-1948.82707$

Single-Point Energy B3LYP-D3(BJ)/6-311+G** PCM = 1949.74236

Free Energy B3LYP-D3(BJ)/6-311+G** PCM (extrapolated free energy from $q R R H O)=-1949.32261$

Nimag $=1\left(-303.8552 \mathrm{~cm}^{-1}\right)$

Charge $=0$ Multiplicity $=1$

$\begin{array}{llll}\mathrm{C} & -2.9405970 & 0.4737770 & 0.2440760 \\ \mathrm{C} & -2.8819030 & 0.9851190 & -2.0648600 \\ \mathrm{C} & -1.4254360 & 0.9857490 & -1.5957550\end{array}$ 


\begin{tabular}{|c|c|c|c|}
\hline $\mathrm{N}$ & -1.5491580 & 0.3158870 & -0.2733930 \\
\hline $\mathrm{N}$ & -3.6717200 & 0.6514360 & -1.0177150 \\
\hline $\mathrm{O}$ & -3.2600850 & 1.2129770 & -3.2138920 \\
\hline $\mathrm{C}$ & -5.0774910 & 0.2991690 & -1.2102990 \\
\hline $\mathrm{H}$ & -5.7249970 & 1.1795660 & -1.2077020 \\
\hline $\mathrm{H}$ & -5.1781350 & -0.2040870 & -2.1742520 \\
\hline $\mathrm{H}$ & -5.3925340 & -0.3782380 & -0.4161100 \\
\hline $\mathrm{C}$ & -0.5742100 & -0.4546230 & 0.2289240 \\
\hline $\mathrm{C}$ & -0.8674160 & -1.5843140 & 1.2128260 \\
\hline $\mathrm{C}$ & 0.3627620 & -2.3397080 & 1.7580610 \\
\hline $\mathrm{H}$ & 0.9676050 & -1.5992500 & 2.2889220 \\
\hline $\mathrm{H}$ & -0.0200850 & -3.0316920 & 2.5136000 \\
\hline $\mathrm{C}$ & -0.5054750 & 0.3051960 & -2.6035740 \\
\hline $\mathrm{H}$ & -0.6446610 & 0.8041970 & -3.5654110 \\
\hline $\mathrm{H}$ & 0.5470140 & 0.4054010 & -2.3282680 \\
\hline $\mathrm{H}$ & -0.7523290 & -0.7532820 & -2.7267160 \\
\hline $\mathrm{H}$ & -1.0999440 & 2.0241290 & -1.4574770 \\
\hline $\mathrm{C}$ & -3.1550860 & 1.6315330 & 1.2849350 \\
\hline $\mathrm{H}$ & -3.2615610 & -0.4656410 & 0.7011370 \\
\hline $\mathrm{C}$ & -4.6705490 & 1.8369850 & 1.5075120 \\
\hline $\mathrm{H}$ & -4.8163350 & 2.5326570 & 2.3395840 \\
\hline $\mathrm{H}$ & -5.1606000 & 2.2685560 & 0.6322180 \\
\hline $\mathrm{H}$ & -5.1775210 & 0.9019580 & 1.7689590 \\
\hline $\mathrm{C}$ & -2.5486170 & 1.2230880 & 2.6441360 \\
\hline $\mathrm{H}$ & -3.0248730 & 0.3157780 & 3.0333130 \\
\hline $\mathrm{H}$ & -1.4705930 & 1.0531990 & 2.6074490 \\
\hline $\mathrm{H}$ & -2.7213780 & 2.0205290 & 3.3731920 \\
\hline $\mathrm{C}$ & -2.5458800 & 2.9619800 & 0.8069530 \\
\hline $\mathrm{H}$ & -2.9685190 & 3.2786060 & -0.1519560 \\
\hline $\mathrm{H}$ & -2.7652420 & 3.7475590 & 1.5364070 \\
\hline $\mathrm{H}$ & -1.4571490 & 2.9156740 & 0.7026590 \\
\hline $\mathrm{H}$ & -1.4478900 & -1.2163930 & 2.0561320 \\
\hline $\mathrm{C}$ & 1.2161230 & -3.1035780 & 0.7618300 \\
\hline $\mathrm{C}$ & 0.9597080 & -4.4603290 & 0.5037550 \\
\hline $\mathrm{C}$ & 2.2972220 & -2.4934280 & 0.1056830 \\
\hline $\mathrm{C}$ & 1.7448200 & -5.1815540 & -0.3993050 \\
\hline $\mathrm{H}$ & 0.1425850 & -4.9573620 & 1.0184440 \\
\hline $\mathrm{C}$ & 3.0834560 & -3.2121020 & -0.8014060 \\
\hline $\mathrm{H}$ & 2.5473840 & -1.4572380 & 0.3112530 \\
\hline $\mathrm{C}$ & 2.8078370 & -4.5573710 & -1.0602520 \\
\hline $\mathrm{H}$ & 1.5295440 & -6.2305130 & -0.5817590 \\
\hline $\mathrm{H}$ & 3.9160800 & -2.7204000 & -1.2963160 \\
\hline $\mathrm{H}$ & 3.4208790 & -5.1169240 & -1.7607500 \\
\hline $\mathrm{Cl}$ & -1.9853350 & -2.7594940 & 0.3438450 \\
\hline $\mathrm{H}$ & 0.1990080 & -0.6979050 & -0.4906090 \\
\hline $\mathrm{O}$ & 0.5248390 & 0.5406790 & 1.2881700 \\
\hline $\mathrm{H}$ & 1.5629640 & 0.6852280 & 0.9160610 \\
\hline $\mathrm{H}$ & 0.1557160 & 1.4394350 & 1.2851160 \\
\hline $\mathrm{O}$ & 2.8104600 & 0.9215060 & 0.5067550 \\
\hline $\mathrm{C}$ & 3.0177190 & 2.1739100 & 0.3503520 \\
\hline $\mathrm{C}$ & 4.4692900 & 2.4793060 & -0.1331610 \\
\hline $\mathrm{O}$ & 2.2419550 & 3.1146800 & 0.5252570 \\
\hline $\mathrm{F}$ & 5.3907580 & 1.9894600 & 0.7336870 \\
\hline $\mathrm{F}$ & 4.7106760 & 1.9067380 & -1.3402620 \\
\hline $\mathrm{F}$ & 4.7049420 & 3.7999160 & -0.2631600 \\
\hline
\end{tabular}

7.log

Potential Energy = -1949.24800

Zero-point Energy = -1948.78443

Free Energy = -1948.82873

Single-Point Energy B3LYP-D3(BJ)/6-311+G** PCM = -

1949.74262

Free Energy B3LYP-D3(BJ)/6-311+G** PCM (extrapolated

free energy from $\mathrm{qRRHO})=-1949.32334$

Nimag $=1\left(-448.0910 \mathrm{~cm}^{-1}\right)$

Charge $=0$ Multiplicity $=1$

$\begin{array}{llll}\mathrm{C} & 2.9079170 & -0.0286740 & 0.1623210 \\ \mathrm{C} & 3.3687160 & -1.9005530 & -1.2049060 \\ \mathrm{C} & 1.9605740 & -2.1174130 & -0.6485620 \\ \mathrm{~N} & 1.6475950 & -0.7660510 & -0.1228810 \\ \mathrm{~N} & 3.8233570 & -0.7026090 & -0.7696180 \\ \mathrm{O} & 3.9663290 & -2.6700060 & -1.9588610 \\ \mathrm{C} & 5.0104370 & -0.0825980 & -1.3544190 \\ \mathrm{H} & 5.9320870 & -0.4294580 & -0.8800600 \\ \mathrm{H} & 5.0477990 & -0.3393980 & -2.4153630 \\ \mathrm{H} & 4.9415650 & 1.0005520 & -1.2489830\end{array}$

\begin{tabular}{|c|c|c|c|}
\hline $\mathrm{C}$ & 0.3890350 & -0.3032600 & -0.0596060 \\
\hline $\mathrm{C}$ & 0.0963090 & 1.1970090 & -0.1365290 \\
\hline $\mathrm{C}$ & -1.3923660 & 1.5545160 & -0.0024100 \\
\hline $\mathrm{H}$ & -1.9542800 & 1.0247860 & -0.7770780 \\
\hline $\mathrm{H}$ & -1.7395460 & 1.1681400 & 0.9602490 \\
\hline $\mathrm{C}$ & 1.0114650 & -2.6471080 & -1.7197060 \\
\hline $\mathrm{H}$ & 1.4448690 & -3.5658120 & -2.1221630 \\
\hline $\mathrm{H}$ & 0.0274360 & -2.8872250 & -1.3117190 \\
\hline $\mathrm{H}$ & 0.8946040 & -1.9323700 & -2.5399460 \\
\hline $\mathrm{H}$ & 2.0109550 & -2.8390500 & 0.1763870 \\
\hline $\mathrm{C}$ & 3.3937610 & -0.0476440 & 1.6584360 \\
\hline $\mathrm{H}$ & 2.7976700 & 1.0128120 & -0.1543490 \\
\hline $\mathrm{C}$ & 4.8425050 & 0.4841860 & 1.7301580 \\
\hline $\mathrm{H}$ & 5.1257640 & 0.6063630 & 2.7802100 \\
\hline $\mathrm{H}$ & 5.5565040 & -0.2077990 & 1.2785590 \\
\hline $\mathrm{H}$ & 4.9468800 & 1.4615210 & 1.2462040 \\
\hline $\mathrm{C}$ & 2.5185520 & 0.9010700 & 2.5011440 \\
\hline $\mathrm{H}$ & 2.5288260 & 1.9229800 & 2.1054120 \\
\hline $\mathrm{H}$ & 1.4846640 & 0.5600320 & 2.5722860 \\
\hline $\mathrm{H}$ & 2.9047510 & 0.9426080 & 3.5240590 \\
\hline $\mathrm{C}$ & 3.3483450 & -1.4642510 & 2.2594310 \\
\hline $\mathrm{H}$ & 3.9659480 & -2.1658700 & 1.6890880 \\
\hline $\mathrm{H}$ & 3.7427710 & -1.4403870 & 3.2804280 \\
\hline $\mathrm{H}$ & 2.3260380 & -1.8503080 & 2.3077100 \\
\hline $\mathrm{H}$ & 0.6960140 & 1.7668180 & 0.5718530 \\
\hline $\mathrm{C}$ & -1.6765610 & 3.0426860 & -0.0620890 \\
\hline $\mathrm{C}$ & -1.3082810 & 3.8813120 & 1.0018030 \\
\hline $\mathrm{C}$ & -2.3275110 & 3.6067870 & -1.1677540 \\
\hline $\mathrm{C}$ & -1.5735050 & 5.2523810 & 0.9563390 \\
\hline $\mathrm{H}$ & -0.8173510 & 3.4608110 & 1.8763840 \\
\hline $\mathrm{C}$ & -2.5989850 & 4.9781270 & -1.2151830 \\
\hline $\mathrm{H}$ & -2.6249200 & 2.9701040 & -1.9966220 \\
\hline $\mathrm{C}$ & -2.2199510 & 5.8056510 & -0.1543780 \\
\hline $\mathrm{H}$ & -1.2824920 & 5.8856990 & 1.7894470 \\
\hline $\mathrm{H}$ & -3.1058840 & 5.3970480 & -2.0797100 \\
\hline $\mathrm{H}$ & -2.4304160 & 6.8705560 & -0.1893160 \\
\hline $\mathrm{Cl}$ & 0.6923570 & 1.7302100 & -1.7946690 \\
\hline $\mathrm{H}$ & -0.3206270 & -0.8911660 & -0.6348510 \\
\hline $\mathrm{O}$ & -0.3374150 & -0.8133460 & 1.4866310 \\
\hline $\mathrm{H}$ & -1.3709470 & -1.2809390 & 1.3952960 \\
\hline $\mathrm{H}$ & -0.3429530 & -0.1420810 & 2.1864500 \\
\hline $\mathrm{O}$ & -2.5296390 & -1.8511860 & 1.3516070 \\
\hline $\mathrm{C}$ & -2.9676010 & -2.1039460 & 0.1762180 \\
\hline $\mathrm{C}$ & -4.3733550 & -2.7790850 & 0.2050100 \\
\hline $\mathrm{O}$ & -2.4373600 & -1.8859350 & -0.9134890 \\
\hline $\mathrm{F}$ & -4.3432900 & -3.9443670 & 0.8988200 \\
\hline $\mathrm{F}$ & -5.2876460 & -1.9769450 & 0.8065240 \\
\hline $\mathrm{F}$ & -4.8362640 & -3.0590540 & -1.0289010 \\
\hline
\end{tabular}

$8 . \log$

Potential Energy $=-1949.24828$

Zero-point Energy = -1948.78306

Free Energy = -1948.82687

Single-Point Energy B3LYP-D3(BJ)/6-311+G** PCM = 1949.74551

Free Energy B3LYP-D3(BJ)/6-311+G** PCM (extrapolated

free energy from $\mathrm{qRRHO})=-1949.32411$

Nimag $=1\left(-221.1495 \mathrm{~cm}^{-1}\right)$

Charge $=0$ Multiplicity $=1$

$\begin{array}{lrrr}\mathrm{C} & -2.7520840 & 0.0407560 & 0.3317730 \\ \mathrm{C} & -2.9423950 & 2.2811700 & -0.3972320 \\ \mathrm{C} & -1.4443950 & 1.9729000 & -0.3778850 \\ \mathrm{~N} & -1.4544570 & 0.4930800 & -0.2443250 \\ \mathrm{~N} & -3.6196960 & 1.1606140 & -0.0575620 \\ \mathrm{O} & -3.4430200 & 3.3602300 & -0.7171940 \\ \mathrm{C} & -5.0618980 & 1.0528570 & -0.2684670 \\ \mathrm{H} & -5.6321140 & 1.4602920 & 0.5702050 \\ \mathrm{H} & -5.3217270 & 1.6122140 & -1.1698030 \\ \mathrm{H} & -5.3300520 & 0.0048340 & -0.4058150 \\ \mathrm{C} & -0.4720850 & -0.2775020 & -0.7309370 \\ \mathrm{C} & -0.7578620 & -1.6950240 & -1.2367380 \\ \mathrm{C} & 0.4531090 & -2.4765850 & -1.7917240 \\ \mathrm{H} & 0.0428870 & -3.2423630 & -2.4561450 \\ \mathrm{H} & 1.0544420 & -1.8089630 & -2.4161450 \\ \mathrm{C} & -0.7551050 & 2.5157850 & -1.6273950 \\ \mathrm{H} & -0.9575430 & 3.5883630 & -1.6775630\end{array}$




\begin{tabular}{|c|c|c|c|}
\hline $\mathrm{H}$ & 0.3275260 & 2.3783790 & -1.5911680 \\
\hline $\mathrm{H}$ & -1.1503060 & 2.0475530 & -2.5339390 \\
\hline $\mathrm{H}$ & -0.9969290 & 2.4292640 & 0.5137080 \\
\hline $\mathrm{C}$ & -2.7456920 & -0.2620930 & 1.8744650 \\
\hline $\mathrm{H}$ & -3.0883120 & -0.8563390 & -0.1961120 \\
\hline $\mathrm{C}$ & -4.2010170 & -0.4210900 & 2.3676120 \\
\hline $\mathrm{H}$ & -4.1873680 & -0.7766740 & 3.4026310 \\
\hline $\mathrm{H}$ & -4.7476110 & 0.5244970 & 2.3553730 \\
\hline $\mathrm{H}$ & -4.7582880 & -1.1561430 & 1.7763100 \\
\hline $\mathrm{C}$ & -2.0200030 & -1.5982810 & 2.1338040 \\
\hline $\mathrm{H}$ & -2.4996190 & -2.4236640 & 1.5936160 \\
\hline $\mathrm{H}$ & -0.9654180 & -1.5541270 & 1.8595070 \\
\hline $\mathrm{H}$ & -2.0726690 & -1.8367890 & 3.2010590 \\
\hline $\mathrm{C}$ & -2.0666010 & 0.8640110 & 2.6750150 \\
\hline $\mathrm{H}$ & -2.5543730 & 1.8305890 & 2.5096810 \\
\hline $\mathrm{H}$ & -2.1369080 & 0.6438640 & 3.7451400 \\
\hline $\mathrm{H}$ & -1.0056180 & 0.9555210 & 2.4266350 \\
\hline $\mathrm{H}$ & -1.2996630 & -2.2840890 & -0.4990710 \\
\hline $\mathrm{C}$ & 1.3315240 & -3.1674400 & -0.7607330 \\
\hline $\mathrm{C}$ & 2.6907980 & -2.8338140 & -0.6410010 \\
\hline $\mathrm{C}$ & 0.8166880 & -4.1916750 & 0.0536830 \\
\hline $\mathrm{C}$ & 3.5134090 & -3.4975110 & 0.2777680 \\
\hline $\mathrm{H}$ & 3.1107070 & -2.0575950 & -1.2754600 \\
\hline $\mathrm{C}$ & 1.6352660 & -4.8520130 & 0.9711460 \\
\hline $\mathrm{H}$ & -0.2266410 & -4.4827500 & -0.0364080 \\
\hline $\mathrm{C}$ & 2.9867260 & -4.5052530 & 1.0876980 \\
\hline $\mathrm{H}$ & 4.5617920 & -3.2254820 & 0.3538620 \\
\hline $\mathrm{H}$ & 1.2204940 & -5.6409720 & 1.5913840 \\
\hline $\mathrm{H}$ & 3.6221890 & -5.0209210 & 1.8012160 \\
\hline $\mathrm{Cl}$ & -1.9373770 & -1.4656400 & -2.6332270 \\
\hline $\mathrm{H}$ & 0.2284320 & 0.2620050 & -1.3607740 \\
\hline $\mathrm{O}$ & 0.6984830 & -0.5791320 & 0.6048750 \\
\hline $\mathrm{H}$ & 1.3553260 & 0.2774960 & 0.7299370 \\
\hline $\mathrm{H}$ & 1.2797140 & -1.3447370 & 0.4289420 \\
\hline $\mathrm{O}$ & 2.2248110 & 1.3233300 & 0.9756600 \\
\hline $\mathrm{C}$ & 2.8150090 & 1.7834240 & -0.0560900 \\
\hline $\mathrm{C}$ & 3.8855480 & 2.8628690 & 0.3018100 \\
\hline $\mathrm{O}$ & 2.6474090 & 1.4987070 & -1.2453620 \\
\hline $\mathrm{F}$ & 3.3212480 & 3.9195310 & 0.9417010 \\
\hline $\mathrm{F}$ & 4.8367240 & 2.3577130 & 1.1285530 \\
\hline $\mathrm{F}$ & 4.5205880 & 3.3492800 & -0.7842530 \\
\hline
\end{tabular}

$9 . \log$

Potential Energy $=-1949.24741$

Zero-point Energy = -1948.78399

Free Energy = -1948.82843

Single-Point Energy B3LYP-D3(BJ)/6-311+G** PCM = 1949.74140

Free Energy B3LYP-D3(BJ)/6-311+G** PCM (extrapolated free energy from qRRHO) $=-1949.32242$

Nimag $=1\left(-442.2547 \mathrm{~cm}^{-1}\right)$

Charge $=0$ Multiplicity $=1$

$\begin{array}{lrrr}\mathrm{C} & 2.7191600 & -0.2041100 & 0.7638350 \\ \mathrm{C} & 4.5393150 & -0.4094500 & -0.7304020 \\ \mathrm{C} & 3.2870380 & -0.7790120 & -1.5292260 \\ \mathrm{~N} & 2.2186010 & -0.2645920 & -0.6368800 \\ \mathrm{~N} & 4.1609790 & -0.0529110 & 0.5187420 \\ \mathrm{O} & 5.6902090 & -0.4035680 & -1.1688430 \\ \mathrm{C} & 5.0897000 & 0.6031930 & 1.4366960 \\ \mathrm{H} & 5.6944710 & -0.1171470 & 1.9936510 \\ \mathrm{H} & 5.7578550 & 1.2418880 & 0.8548840 \\ \mathrm{H} & 4.5282460 & 1.2174800 & 2.1412870 \\ \mathrm{C} & 1.0497860 & 0.1802760 & -1.1105650 \\ \mathrm{C} & 0.2225040 & 1.2251810 & -0.3623110 \\ \mathrm{C} & -1.0819220 & 1.6098090 & -1.0771300 \\ \mathrm{H} & -0.8377930 & 2.0020550 & -2.0691950 \\ \mathrm{H} & -1.6548270 & 0.6911020 & -1.2229420 \\ \mathrm{C} & 3.3176910 & -0.1930320 & -2.9368540 \\ \mathrm{H} & 4.2447510 & -0.5220110 & -3.4121450 \\ \mathrm{H} & 2.4869260 & -0.5555120 & -3.5472160 \\ \mathrm{H} & 3.3087010 & 0.9007960 & -2.9208440 \\ \mathrm{H} & 3.2213820 & -1.8718660 & -1.5968500 \\ \mathrm{C} & 2.3445550 & -1.4249090 & 1.6782770 \\ \mathrm{H} & 2.3495210 & 0.7092380 & 1.2379090 \\ \mathrm{C} & 3.1501070 & -1.3364480 & 2.9932920 \\ \mathrm{H} & 2.7791680 & -2.0952670 & 3.6893110\end{array}$

$\begin{array}{lccc}\mathrm{H} & 4.2142740 & -1.5282020 & 2.8390160 \\ \mathrm{H} & 3.0365970 & -0.3612300 & 3.4798250 \\ \mathrm{C} & 0.8489760 & -1.3486350 & 2.0427710 \\ \mathrm{H} & 0.6065240 & -0.4077350 & 2.5507890 \\ \mathrm{H} & 0.2035610 & -1.4609390 & 1.1715990 \\ \mathrm{H} & 0.6011230 & -2.1622770 & 2.7317340 \\ \mathrm{C} & 2.6415290 & -2.7723860 & 0.9956000 \\ \mathrm{H} & 3.6954250 & -2.8610240 & 0.7113130 \\ \mathrm{H} & 2.4233130 & -3.5885240 & 1.6919460 \\ \mathrm{H} & 2.0195340 & -2.9207890 & 0.1081810 \\ \mathrm{H} & 0.0328310 & 0.9272360 & 0.6675390 \\ \mathrm{C} & -1.9341110 & 2.6037630 & -0.3125270 \\ \mathrm{C} & -2.6262310 & 2.2044080 & 0.8420910 \\ \mathrm{C} & -2.0637910 & 3.9292710 & -0.7492050 \\ \mathrm{C} & -3.4185540 & 3.1122510 & 1.5495010 \\ \mathrm{H} & -2.5550530 & 1.1745880 & 1.1833240 \\ \mathrm{C} & -2.8597340 & 4.8396890 & -0.0461910 \\ \mathrm{H} & -1.5403700 & 4.2519990 & -1.6451920 \\ \mathrm{C} & -3.5370750 & 4.4345100 & 1.1074780 \\ \mathrm{H} & -3.9487220 & 2.7853240 & 2.4394210 \\ \mathrm{H} & -2.9493680 & 5.8625250 & -0.4007170 \\ \mathrm{H} & -4.1561360 & 5.1398330 & 1.6542000 \\ \mathrm{Cl} & 1.2926670 & 2.7199000 & -0.2376770 \\ \mathrm{H} & 1.0602780 & 0.3494780 & -2.1816800 \\ \mathrm{O} & -0.0940980 & -1.2212090 & -1.2173520 \\ \mathrm{H} & -1.0842360 & -1.3713070 & -0.6763750 \\ \mathrm{H} & -0.2685590 & -1.4000370 & -2.1554150 \\ \mathrm{O} & -2.1971090 & -1.5884870 & -0.0599540 \\ \mathrm{C} & -3.0439210 & -2.2483560 & -0.7601000 \\ \mathrm{C} & -4.3666850 & -2.5177610 & 0.0216830 \\ \mathrm{O} & -2.9413380 & -2.6622300 & -1.9133620 \\ \mathrm{~F} & -4.1281640 & -3.1660360 & 1.1887880 \\ \mathrm{~F} & -4.9941230 & -1.3541380 & 0.3297630 \\ \mathrm{~F} & -5.2369520 & -3.2677160 & -0.6823350 \\ & & & \end{array}$

$10 . \log$

Potential Energy $=-1949.24591$

Zero-point Energy = -1948.78201

Free Energy $=-1948.82618$

Single-Point Energy B3LYP-D3(BJ)/6-311+G** PCM = 1949.74144

Free Energy B3LYP-D3(BJ)/6-311+G** PCM (extrapolated

free energy from $\mathrm{qRRHO})=-1949.32170$

Nimag $=1\left(-480.8318 \mathrm{~cm}^{-1}\right)$

Charge $=0$ Multiplicity $=1$

$\begin{array}{lrrr}\mathrm{C} & -2.7588980 & -0.9998410 & -0.4688040 \\ \mathrm{C} & -4.0750940 & -0.1463160 & 1.3005930 \\ \mathrm{C} & -2.6173310 & 0.0506980 & 1.7218570 \\ \mathrm{~N} & -1.9155980 & -0.1633540 & 0.4306890 \\ \mathrm{~N} & -4.0944050 & -0.6870590 & 0.0607950 \\ \mathrm{O} & -5.0595890 & 0.1724430 & 1.9684750 \\ \mathrm{C} & -5.3263020 & -0.7439100 & -0.7235560 \\ \mathrm{H} & -5.9172790 & -1.6347300 & -0.4958340 \\ \mathrm{H} & -5.9250670 & 0.1395320 & -0.4916000 \\ \mathrm{H} & -5.0789990 & -0.7420730 & -1.7854880 \\ \mathrm{C} & -0.7534060 & 0.4303280 & 0.1428800 \\ \mathrm{C} & -0.3340510 & 0.7367420 & -1.2920280 \\ \mathrm{C} & 1.0309720 & 1.4347470 & -1.4615320 \\ \mathrm{H} & 1.8008310 & 0.7012580 & -1.2102090 \\ \mathrm{H} & 1.1360220 & 1.6355690 & -2.5315880 \\ \mathrm{C} & -2.3968440 & 1.4096950 & 2.3784540 \\ \mathrm{H} & -3.1045950 & 1.4961120 & 3.2062730 \\ \mathrm{H} & -1.3902680 & 1.5062580 & 2.7925700 \\ \mathrm{H} & -2.5824350 & 2.2299890 & 1.6789670 \\ \mathrm{H} & -2.3461140 & -0.7414290 & 2.4307560 \\ \mathrm{C} & -2.4185860 & -2.5334610 & -0.4988370 \\ \mathrm{H} & -2.6899590 & -0.6104470 & -1.4880270 \\ \mathrm{C} & -3.5427690 & -3.2837490 & -1.2469370 \\ \mathrm{H} & -3.2338690 & -4.3221150 & -1.4024100 \\ \mathrm{H} & -4.4758340 & -3.3026860 & -0.6793140 \\ \mathrm{H} & -3.7410070 & -2.8493240 & -2.2330470 \\ \mathrm{C} & -1.1094210 & -2.7527310 & -1.2842360 \\ \mathrm{H} & -1.1871640 & -2.3644030 & -2.3069480 \\ \mathrm{H} & -0.2531070 & -2.2940500 & -0.7885340 \\ \mathrm{H} & -0.9086340 & -3.8262950 & -1.3597700 \\ \mathrm{C} & -2.2787080 & -3.1220070 & 0.9166820\end{array}$




$\begin{array}{cccc}\mathrm{H} & -3.1879310 & -2.9757700 & 1.5094400 \\ \mathrm{H} & -2.1066150 & -4.2009600 & 0.8469300 \\ \mathrm{H} & -1.4303620 & -2.6873880 & 1.4528750 \\ \mathrm{H} & -0.3497050 & -0.1607460 & -1.9075220 \\ \mathrm{C} & 1.2595780 & 2.7116390 & -0.6742950 \\ \mathrm{C} & 0.9303120 & 3.9625380 & -1.2214220 \\ \mathrm{C} & 1.8394010 & 2.6762270 & 0.6045850 \\ \mathrm{C} & 1.1538880 & 5.1411760 & -0.5044240 \\ \mathrm{H} & 0.5029750 & 4.0149090 & -2.2183490 \\ \mathrm{C} & 2.0635900 & 3.8533130 & 1.3256700 \\ \mathrm{H} & 2.1336010 & 1.7237870 & 1.0362520 \\ \mathrm{C} & 1.7172220 & 5.0902570 & 0.7746930 \\ \mathrm{H} & 0.8937810 & 6.0981570 & -0.9476660 \\ \mathrm{H} & 2.5156070 & 3.8019530 & 2.3120030 \\ \mathrm{H} & 1.8942790 & 6.0059040 & 1.3312770 \\ \mathrm{Cl} & -1.6559370 & 1.8111550 & -1.9963600 \\ \mathrm{H} & -0.4911890 & 1.2201740 & 0.8369330 \\ \mathrm{O} & 0.5996500 & -0.6804320 & 0.6471830 \\ \mathrm{H} & 1.6280550 & -0.9291430 & 0.2162200 \\ \mathrm{H} & 0.6982530 & -0.6068590 & 1.6091220 \\ \mathrm{O} & 2.7653220 & -1.2725730 & -0.2645030 \\ \mathrm{C} & 3.6354640 & -1.5504090 & 0.6354870 \\ \mathrm{C} & 5.0010960 & -1.9778990 & 0.0144600 \\ \mathrm{O} & 3.5215070 & -1.5211930 & 1.8589030 \\ \mathrm{~F} & 4.8612370 & -3.0838800 & -0.7590820 \\ \mathrm{~F} & 5.5096920 & -0.9981260 & -0.7740550 \\ \mathrm{~F} & 5.9270600 & -2.2591190 & 0.9518270\end{array}$

Hydrolysis of chloroiminium ion (TS6')

\section{Conformation Extrapolated name Free energy} (kcal/mol)

$\begin{array}{cc}\text { TS6'-lowest } & 0.0 \\ 1 & 0.3 \\ 2 & 0.4 \\ 3 & 1.3 \\ 4 & 1.4 \\ 5 & 7.8\end{array}$

TS6'-lowest.log

Potential Energy = -1949.24993

Zero-point Energy = -1948.78615

Free Energy = -1948.83066

Single-Point Energy B3LYP-D3(BJ)/6-311+G** PCM = 1949.74410

Free Energy B3LYP-D3(BJ)/6-311+G** PCM (extrapolated free energy from qRRHO) $=-1949.32483$

Nimag $=1\left(-394.5465 \mathrm{~cm}^{-1}\right)$

Charge $=0$ Multiplicity $=1$

$\begin{array}{lcrr}\mathrm{C} & 3.3143630 & -0.3728650 & -0.6322180 \\ \mathrm{C} & 3.2125240 & -1.5659510 & 1.3980330 \\ \mathrm{C} & 2.1441210 & -0.4717540 & 1.5097160 \\ \mathrm{~N} & 2.0901440 & 0.0360690 & 0.1147710 \\ \mathrm{~N} & 3.7866740 & -1.4968120 & 0.1760890 \\ \mathrm{O} & 3.4749480 & -2.3887490 & 2.2755060 \\ \mathrm{C} & 4.6968430 & -2.5351590 & -0.2987110 \\ \mathrm{H} & 4.3712660 & -3.4922370 & 0.1136690 \\ \mathrm{H} & 4.6585420 & -2.5796420 & -1.3876470 \\ \mathrm{H} & 5.7273660 & -2.3525940 & 0.0185900 \\ \mathrm{C} & 1.0189370 & 0.4459970 & -0.5736820 \\ \mathrm{H} & 1.2718860 & 0.7991990 & -1.5674850 \\ \mathrm{C} & -0.1277950 & 1.2342110 & 0.0631440 \\ \mathrm{C} & -1.0601490 & 1.8436440 & -0.9957120 \\ \mathrm{H} & -0.4865610 & 2.5379880 & -1.6176840 \\ \mathrm{H} & 2.5302590 & 0.3220860 & 2.1602310 \\ \mathrm{C} & 4.3516930 & 0.7887180 & -0.8216370 \\ \mathrm{H} & 3.0173970 & -0.7425430 & -1.6204870 \\ \mathrm{H} & -0.6905590 & 0.6171060 & 0.7587660 \\ \mathrm{Cl} & 0.5938430 & 2.5687080 & 1.0954060 \\ \mathrm{C} & 0.8632590 & -1.0367110 & 2.1229130 \\ \mathrm{H} & 0.1755740 & -0.2500000 & 2.4352360\end{array}$

$\begin{array}{lccc}\mathrm{H} & 0.3536080 & -1.7290030 & 1.4495120 \\ \mathrm{H} & 1.1564220 & -1.5885800 & 3.0194560 \\ \mathrm{C} & 5.5659580 & 0.2606790 & -1.6174770 \\ \mathrm{H} & 6.2166290 & 1.1010970 & -1.8788510 \\ \mathrm{H} & 6.1665320 & -0.4462110 & -1.0413150 \\ \mathrm{H} & 5.2607990 & -0.2232420 & -2.5523080 \\ \mathrm{C} & 4.8261780 & 1.3480660 & 0.5320460 \\ \mathrm{H} & 5.2812280 & 0.5723990 & 1.1564280 \\ \mathrm{H} & 5.5830090 & 2.1208340 & 0.3634240 \\ \mathrm{H} & 4.0048160 & 1.8103470 & 1.0881120 \\ \mathrm{C} & 3.7060300 & 1.9234310 & -1.6448980 \\ \mathrm{H} & 3.3310080 & 1.5645590 & -2.6108170 \\ \mathrm{H} & 2.8895930 & 2.4124660 & -1.1067240 \\ \mathrm{H} & 4.4590870 & 2.6897270 & -1.8535900 \\ \mathrm{H} & -1.3835220 & 1.0220180 & -1.6440890 \\ \mathrm{C} & -2.2810110 & 2.5367470 & -0.4243860 \\ \mathrm{C} & -2.4289480 & 3.9267900 & -0.5240990 \\ \mathrm{C} & -3.2947930 & 1.7937010 & 0.2017410 \\ \mathrm{C} & -3.5604550 & 4.5649000 & -0.0048560 \\ \mathrm{H} & -1.6555240 & 4.5148890 & -1.0110050 \\ \mathrm{C} & -4.4239530 & 2.4285480 & 0.7251000 \\ \mathrm{H} & -3.2042640 & 0.7128080 & 0.2755500 \\ \mathrm{C} & -4.5603050 & 3.8175490 & 0.6237400 \\ \mathrm{H} & -3.6583100 & 5.6431720 & -0.0922200 \\ \mathrm{H} & -5.1991690 & 1.8385410 & 1.2055730 \\ \mathrm{H} & -5.4396750 & 4.3108730 & 1.0273950 \\ \mathrm{O} & 0.1195270 & -1.0138210 & -1.1678240 \\ \mathrm{H} & -0.8530080 & -1.3550020 & -0.7044250 \\ \mathrm{O} & -1.9392930 & -1.7639340 & -0.1166430 \\ \mathrm{C} & -2.7419960 & -2.4054470 & -0.8816290 \\ \mathrm{O} & -2.6442510 & -2.6474990 & -2.0831510 \\ \mathrm{C} & -3.9949680 & -2.9254450 & -0.1102420 \\ \mathrm{~F} & -4.6747740 & -1.9043110 & 0.4697490 \\ \mathrm{~F} & -3.6378220 & -3.7824230 & 0.8797350 \\ \mathrm{~F} & -4.8601210 & -3.5780190 & -0.9112280 \\ \mathrm{H} & -0.0036890 & -0.9769930 & -2.1299370 \\ & & & \end{array}$

$1 . \log$

Potential Energy $=-1949.24780$

Zero-point Energy = -1948.78402

Free Energy = -1948.82865

Single-Point Energy B3LYP-D3(BJ)/6-311+G** PCM = 1949.74365

Free Energy B3LYP-D3(BJ)/6-311+G** PCM (extrapolated

free energy from $\mathrm{qRRHO})=-1949.32449$

Nimag $=1\left(-416.3740 \mathrm{~cm}^{-1}\right)$

Charge $=0$ Multiplicity $=1$

$\begin{array}{lrrr}\mathrm{C} & 2.4085910 & -1.2682910 & -0.7753590 \\ \mathrm{C} & 1.8237410 & -3.2259470 & 0.3986370 \\ \mathrm{C} & 1.2459380 & -2.0655920 & 1.2177850 \\ \mathrm{~N} & 1.4125200 & -0.9247690 & 0.2801390 \\ \mathrm{~N} & 2.4051040 & -2.7299470 & -0.7161690 \\ \mathrm{O} & 1.7384640 & -4.4161160 & 0.7023400 \\ \mathrm{C} & 2.8645740 & -3.6046480 & -1.7910630 \\ \mathrm{H} & 2.1963810 & -4.4666190 & -1.8416930 \\ \mathrm{H} & 2.8290250 & -3.0639740 & -2.7375090 \\ \mathrm{H} & 3.8830810 & -3.9640470 & -1.6195730 \\ \mathrm{C} & 0.5769410 & 0.1005620 & 0.0743890 \\ \mathrm{H} & 0.9545620 & 0.8094490 & -0.6546790 \\ \mathrm{C} & -0.2127310 & 0.7610050 & 1.2045720 \\ \mathrm{C} & -0.9287320 & 2.0704680 & 0.8090080 \\ \mathrm{H} & 1.8917130 & -1.9038330 & 2.0891530 \\ \mathrm{C} & 3.8117260 & -0.6019720 & -0.5577460 \\ \mathrm{H} & 2.0172360 & -0.9492180 & -1.7480330 \\ \mathrm{H} & -0.9588090 & 0.0779200 & 1.6012990 \\ \mathrm{Cl} & 0.9299800 & 1.0520030 & 2.6117440 \\ \mathrm{C} & -0.1633820 & -2.4072350 & 1.7013250 \\ \mathrm{H} & -0.5072360 & -1.7259000 & 2.4803630 \\ \mathrm{H} & -0.8850210 & -2.4303240 & 0.8820730 \\ \mathrm{H} & -0.1146250 & -3.4070020 & 2.1399310 \\ \mathrm{C} & 4.7518600 & -1.0109970 & -1.7134930 \\ \mathrm{H} & 5.6766170 & -0.4293010 & -1.6469010 \\ \mathrm{H} & 5.0296730 & -2.0662390 & -1.6717690 \\ \mathrm{H} & 4.3042430 & -0.8080960 & -2.6931860 \\ \mathrm{C} & 4.4340590 & -1.0219860 & 0.7863570 \\ \mathrm{H} & 4.5459410 & -2.1085090 & 0.8615360\end{array}$




$\begin{array}{lccc}\mathrm{H} & 5.4315960 & -0.5811980 & 0.8813120 \\ \mathrm{H} & 3.8387140 & -0.6700930 & 1.6345050 \\ \mathrm{C} & 3.6599670 & 0.9330980 & -0.5938750 \\ \mathrm{H} & 3.1972880 & 1.2756090 & -1.5270450 \\ \mathrm{H} & 3.0769270 & 1.3115530 & 0.2497090 \\ \mathrm{H} & 4.6508060 & 1.3942090 & -0.5342400 \\ \mathrm{H} & -1.7321960 & 1.7871540 & 0.1223100 \\ \mathrm{O} & -0.7977950 & -0.4820210 & -0.9598230 \\ \mathrm{H} & -1.8548720 & -0.5849260 & -0.5641110 \\ \mathrm{O} & -3.0320940 & -0.7513090 & -0.0518260 \\ \mathrm{C} & -4.0045460 & -0.5508810 & -0.8613790 \\ \mathrm{O} & -3.9757840 & -0.2134940 & -2.0432090 \\ \mathrm{C} & -5.3884060 & -0.7935710 & -0.1824690 \\ \mathrm{~F} & -5.5467530 & -0.0064180 & 0.9110420 \\ \mathrm{~F} & -5.5093480 & -2.0812240 & 0.2295730 \\ \mathrm{~F} & -6.4206280 & -0.5399710 & -1.0108070 \\ \mathrm{H} & -0.8313520 & -0.0053840 & -1.8040330 \\ \mathrm{H} & -1.4169280 & 2.4377180 & 1.7162530 \\ \mathrm{C} & -0.0911210 & 3.1701530 & 0.1854960 \\ \mathrm{C} & 0.6317820 & 4.0736720 & 0.9823320 \\ \mathrm{C} & -0.0507990 & 3.3328760 & -1.2086140 \\ \mathrm{C} & 1.3907520 & 5.0926430 & 0.4009740 \\ \mathrm{H} & 0.5936090 & 3.9848550 & 2.0635570 \\ \mathrm{C} & 0.7083190 & 4.3509880 & -1.7945860 \\ \mathrm{H} & -0.6349420 & 2.6733620 & -1.8454350 \\ \mathrm{C} & 1.4362050 & 5.2317290 & -0.9902930 \\ \mathrm{H} & 1.9402540 & 5.7822620 & 1.0352860 \\ \mathrm{H} & 0.7217940 & 4.4588310 & -2.8752310 \\ \mathrm{H} & 2.0232990 & 6.0262750 & -1.4413900 \\ & & & \\ & & & \\ & & \end{array}$

$\begin{array}{lccc}\mathrm{H} & -1.3944220 & 1.1637050 & -0.6054160 \\ \mathrm{O} & -2.4852520 & 1.3041200 & 0.0985040 \\ \mathrm{C} & -3.4636540 & 1.8039220 & -0.5592290 \\ \mathrm{O} & -3.5125610 & 2.1476670 & -1.7392100 \\ \mathrm{C} & -4.7409190 & 1.9649360 & 0.3227800 \\ \mathrm{~F} & -4.4989300 & 2.7514960 & 1.4015840 \\ \mathrm{~F} & -5.1652690 & 0.7632210 & 0.7903610 \\ \mathrm{~F} & -5.7687720 & 2.5168910 & -0.3517550 \\ \mathrm{H} & -0.6113200 & 1.1205340 & -2.1184720 \\ \mathrm{H} & -0.8072130 & -2.5990920 & -2.7167870 \\ \mathrm{H} & -1.5667080 & -1.1875120 & -2.0094260 \\ \mathrm{C} & -1.1782820 & -2.7640980 & -0.6040710 \\ \mathrm{C} & -0.8047380 & -4.1135170 & -0.4930050 \\ \mathrm{C} & -1.9363070 & -2.1921560 & 0.4308100 \\ \mathrm{C} & -1.1599170 & -4.8648680 & 0.6303010 \\ \mathrm{H} & -0.2399070 & -4.5810580 & -1.2939400 \\ \mathrm{C} & -2.2918850 & -2.9421510 & 1.5568060 \\ \mathrm{H} & -2.2669770 & -1.1596110 & 0.3535120 \\ \mathrm{C} & -1.9016110 & -4.2798590 & 1.6620490 \\ \mathrm{H} & -0.8636500 & -5.9078640 & 0.6957260 \\ \mathrm{H} & -2.8805280 & -2.4814500 & 2.3449550 \\ \mathrm{H} & -2.1812770 & -4.8644930 & 2.5336140\end{array}$

$2 . \log$

Potential Energy = -1949.24751

Zero-point Energy = -1948.78376

Free Energy $=-1948.82843$

Single-Point Energy B3LYP-D3(BJ)/6-311+G** PCM = 1949.74337

Free Energy B3LYP-D3(BJ)/6-311+G** PCM (extrapolated free energy from qRRHO) $=-1949.32429$

Nimag $=1\left(-381.0451 \mathrm{~cm}^{-1}\right)$

Charge $=0$ Multiplicity $=1$

$\begin{array}{lccc}\text { Charge }=\text { C Muliplity }=1 & \\ \mathrm{C} & 2.1176430 & 0.9731310 & 0.9323700 \\ \mathrm{C} & 3.4741420 & 2.0704710 & -0.6512730 \\ \mathrm{C} & 2.9562280 & 0.8109220 & -1.3558230 \\ \mathrm{~N} & 1.9210180 & 0.3366280 & -0.4023190 \\ \mathrm{~N} & 2.9276180 & 2.1399490 & 0.5825520 \\ \mathrm{O} & 4.2364400 & 2.8965920 & -1.1538200 \\ \mathrm{C} & 3.0703450 & 3.3316330 & 1.4140100 \\ \mathrm{H} & 3.0879320 & 4.2058710 & 0.7602430 \\ \mathrm{H} & 2.2190100 & 3.4043340 & 2.0916260 \\ \mathrm{H} & 3.9953030 & 3.3154590 & 1.9972570 \\ \mathrm{C} & 0.7416970 & -0.2323160 & -0.6831590 \\ \mathrm{H} & 0.1823960 & -0.4968330 & 0.2072320 \\ \mathrm{C} & 0.5574570 & -1.2317880 & -1.8265640 \\ \mathrm{C} & -0.8068230 & -1.9555910 & -1.8325960 \\ \mathrm{H} & 3.7680970 & 0.0749140 & -1.3947380 \\ \mathrm{C} & 2.7642150 & 0.0179260 & 1.9960390 \\ \mathrm{H} & 1.1456450 & 1.3114200 & 1.3091390 \\ \mathrm{H} & 0.6866500 & -0.7441760 & -2.7898130 \\ \mathrm{Cl} & 1.9304330 & -2.4460640 & -1.7602710 \\ \mathrm{C} & 2.5255170 & 1.1497470 & -2.7833510 \\ \mathrm{H} & 2.3578600 & 0.2582720 & -3.3884890 \\ \mathrm{H} & 1.6393100 & 1.7885790 & -2.8021460 \\ \mathrm{H} & 3.3488950 & 1.7037600 & -3.2407800 \\ \mathrm{C} & 2.8857650 & 0.7670830 & 3.3416990 \\ \mathrm{H} & 3.1938830 & 0.0610490 & 4.1192120 \\ \mathrm{H} & 3.6341220 & 1.5616770 & 3.3117140 \\ \mathrm{H} & 1.9288480 & 1.2024210 & 3.6514610 \\ \mathrm{C} & 4.1547340 & -0.4674320 & 1.5473760 \\ \mathrm{H} & 4.8409920 & 0.3671710 & 1.3711880 \\ \mathrm{H} & 4.5932000 & -1.0945110 & 2.3302940 \\ \mathrm{H} & 4.0970220 & -1.0729800 & 0.6376220 \\ \mathrm{C} & 1.8448630 & -1.2012690 & 2.2172750 \\ \mathrm{H} & 0.8329100 & -0.9017060 & 2.5138630 \\ \mathrm{H} & 1.7800110 & -1.8371080 & 1.3305330 \\ \mathrm{H} & 2.2515150 & -1.8160040 & 3.0263570 \\ \mathrm{O} & -0.4240640 & 1.0640550 & -1.1678400\end{array}$

$3 . \log$

Potential Energy = -1949.249631

Zero-point Energy = -1948.78593

Free Energy = -1948.83085

Single-Point Energy B3LYP-D3(BJ)/6-311+G** PCM = 1949.74168

Free Energy B3LYP-D3(BJ)/6-311+G** PCM (extrapolated

free energy from $\mathrm{qRRHO})=-1949.32290$

Nimag $=1\left(-355.7261 \mathrm{~cm}^{-1}\right)$

Charge $=0$ Multiplicity $=1$

\begin{tabular}{|c|c|c|c|}
\hline $\mathrm{C}$ & 2.8627180 & 0.7576730 & 0.3601370 \\
\hline $\mathrm{C}$ & 3.8037000 & 0.0081630 & -1.6670530 \\
\hline $\mathrm{C}$ & 2.7100560 & -1.0092370 & -1.3209250 \\
\hline $\mathrm{N}$ & 2.0088240 & -0.3272390 & -0.2044480 \\
\hline $\mathrm{N}$ & 3.8004560 & 0.9902260 & -0.7381290 \\
\hline $\mathrm{O}$ & 4.5361930 & -0.0533380 & -2.6546330 \\
\hline $\mathrm{C}$ & 4.5912050 & 2.2056460 & -0.9097940 \\
\hline $\mathrm{H}$ & 4.6401110 & 2.4349030 & -1.9760520 \\
\hline $\mathrm{H}$ & 4.1066910 & 3.0299970 & -0.3852160 \\
\hline $\mathrm{H}$ & 5.6108690 & 2.0876690 & -0.5326430 \\
\hline $\mathrm{C}$ & 0.7004050 & -0.3313200 & 0.0729530 \\
\hline $\mathrm{H}$ & 0.4616890 & 0.2365400 & 0.9654660 \\
\hline $\mathrm{C}$ & -0.1886180 & -1.5667240 & -0.0999830 \\
\hline $\mathrm{C}$ & -1.5379380 & -1.4018820 & 0.6188140 \\
\hline $\mathrm{H}$ & -1.3566120 & -1.3227040 & 1.6950990 \\
\hline $\mathrm{H}$ & 3.1884440 & -1.9183920 & -0.9377380 \\
\hline $\mathrm{C}$ & 3.5293970 & 0.3842840 & 1.7306160 \\
\hline $\mathrm{H}$ & 2.2464590 & 1.6527940 & 0.5023330 \\
\hline $\mathrm{H}$ & -0.3403050 & -1.8010320 & -1.1514950 \\
\hline $\mathrm{Cl}$ & 0.6995020 & -3.0251330 & 0.5694680 \\
\hline $\mathrm{C}$ & 1.9031250 & -1.3628170 & -2.5700510 \\
\hline $\mathrm{H}$ & 1.2499090 & -2.2215080 & -2.4123140 \\
\hline $\mathrm{H}$ & 1.3230290 & -0.5128180 & -2.9379180 \\
\hline $\mathrm{H}$ & 2.6213360 & -1.6373650 & -3.3464710 \\
\hline $\mathrm{C}$ & 4.3518600 & 1.5902680 & 2.2369190 \\
\hline $\mathrm{H}$ & 4.6934270 & 1.3895980 & 3.2572090 \\
\hline $\mathrm{H}$ & 5.2399470 & 1.7751040 & 1.6291370 \\
\hline $\mathrm{H}$ & 3.7522360 & 2.5072980 & 2.2639070 \\
\hline $\mathrm{C}$ & 4.4444030 & -0.8464710 & 1.5957800 \\
\hline $\mathrm{H}$ & 5.2366540 & -0.6865240 & 0.8573910 \\
\hline $\mathrm{H}$ & 4.9266770 & -1.0509980 & 2.5569970 \\
\hline $\mathrm{H}$ & 3.8794650 & -1.7412670 & 1.3166680 \\
\hline $\mathrm{C}$ & 2.4311140 & 0.0924170 & 2.7750980 \\
\hline $\mathrm{H}$ & 1.7368080 & 0.9340000 & 2.8839650 \\
\hline $\mathrm{H}$ & 1.8610780 & -0.8094060 & 2.5362400 \\
\hline $\mathrm{H}$ & 2.8980780 & -0.0711340 & 3.7513340 \\
\hline $\mathrm{H}$ & -1.9589970 & -0.4443490 & 0.2944510 \\
\hline $\mathrm{C}$ & -2.5315700 & -2.5088740 & 0.3309370 \\
\hline $\mathrm{C}$ & -2.8320240 & -3.4774470 & 1.2985700 \\
\hline $\mathrm{C}$ & -3.1798050 & -2.5759020 & -0.9126460 \\
\hline $\mathrm{C}$ & -3.7518720 & -4.4961720 & 1.0292500 \\
\hline $\mathrm{H}$ & -2.3452260 & -3.4346460 & 2.2691480 \\
\hline
\end{tabular}

C $\quad-4.0964180 \quad-3.5941250 \quad-1.1868480$ 


$\begin{array}{lllr}\mathrm{H} & -2.9739220 & -1.8228230 & -1.6697920 \\ \mathrm{C} & -4.3846090 & -4.5591210 & -0.2155260 \\ \mathrm{H} & -3.9730550 & -5.2373160 & 1.7918800 \\ \mathrm{H} & -4.5899390 & -3.6293290 & -2.1537930 \\ \mathrm{H} & -5.0997860 & -5.3489190 & -0.4259280 \\ \mathrm{O} & -0.1375420 & 0.8610760 & -1.0069230 \\ \mathrm{H} & -0.8752150 & 1.5495950 & -0.5174920 \\ \mathrm{O} & -1.6760590 & 2.3684370 & 0.1231140 \\ \mathrm{C} & -2.6199060 & 2.8650380 & -0.5837380 \\ \mathrm{O} & -2.8859150 & 2.6809300 & -1.7707570 \\ \mathrm{C} & -3.5270410 & 3.8269470 & 0.2462300 \\ \mathrm{~F} & -2.8055420 & 4.8386050 & 0.7916730 \\ \mathrm{~F} & -4.1287280 & 3.1676830 & 1.2695740 \\ \mathrm{~F} & -4.5028620 & 4.3877000 & -0.4954100 \\ \mathrm{H} & -0.5446520 & 0.4745950 & -1.7986640\end{array}$

4.log

Potential Energy $=-1949.247187$

Zero-point Energy = -1948.78263

Free Energy = -1948.82673

Single-Point Energy B3LYP-D3(BJ)/6-311+G** PCM = 1949.74308

Free Energy B3LYP-D3(BJ)/6-311+G** PCM (extrapolated free energy from $\mathrm{qRRHO})=-1949.32262$

Nimag $=1\left(-310.9690 \mathrm{~cm}^{-1}\right)$

Charge $=0$ Multiplicity $=1$

\begin{tabular}{|c|c|c|c|}
\hline $\mathrm{C}$ & -2.6986250 & 0.4657670 & -0.8877210 \\
\hline $\mathrm{C}$ & -2.3567930 & 2.3382520 & 0.5025160 \\
\hline $\mathrm{C}$ & -1.9179440 & 1.1233180 & 1.3285200 \\
\hline $\mathrm{N}$ & -1.8854940 & 0.0566510 & 0.2936240 \\
\hline $\mathrm{N}$ & -2.7302300 & 1.9200200 & -0.7280910 \\
\hline $\mathrm{O}$ & -2.3435540 & 3.5045270 & 0.8973940 \\
\hline $\mathrm{C}$ & -3.0111670 & 2.8663030 & -1.8036490 \\
\hline $\mathrm{H}$ & -2.3672810 & 3.7377620 & -1.6700490 \\
\hline $\mathrm{H}$ & -2.7911020 & 2.3991840 & -2.7643800 \\
\hline $\mathrm{H}$ & -4.0527940 & 3.1994440 & -1.7950520 \\
\hline $\mathrm{C}$ & -1.0165880 & -0.9531120 & 0.1518680 \\
\hline $\mathrm{H}$ & -1.2934790 & -1.6204180 & -0.6568350 \\
\hline $\mathrm{C}$ & -0.3934530 & -1.6937070 & 1.3412590 \\
\hline $\mathrm{C}$ & 0.1620690 & -3.0917230 & 0.9846510 \\
\hline $\mathrm{H}$ & -2.7164440 & 0.8886570 & 2.0424190 \\
\hline $\mathrm{C}$ & -4.1054930 & -0.2248560 & -0.9574270 \\
\hline $\mathrm{H}$ & -2.1412680 & 0.2183090 & -1.7980030 \\
\hline $\mathrm{H}$ & 0.3652930 & -1.0850420 & 1.8282190 \\
\hline $\mathrm{Cl}$ & -1.6958140 & -1.9515560 & 2.6134520 \\
\hline $\mathrm{C}$ & -0.6400660 & 1.4293320 & 2.1056200 \\
\hline $\mathrm{H}$ & -0.4217640 & 0.6610240 & 2.8482080 \\
\hline $\mathrm{H}$ & 0.2235440 & 1.5722240 & 1.4542250 \\
\hline $\mathrm{H}$ & -0.8154470 & 2.3643390 & 2.6439920 \\
\hline $\mathrm{C}$ & -4.8367240 & 0.2469510 & -2.2338950 \\
\hline $\mathrm{H}$ & -5.7522400 & -0.3392810 & -2.3614890 \\
\hline $\mathrm{H}$ & -5.1299580 & 1.2975840 & -2.1819800 \\
\hline $\mathrm{H}$ & -4.2237180 & 0.1011380 & -3.1306690 \\
\hline $\mathrm{C}$ & -4.9624010 & 0.1039090 & 0.2786890 \\
\hline $\mathrm{H}$ & -5.1086150 & 1.1822290 & 0.3983170 \\
\hline $\mathrm{H}$ & -5.9517270 & -0.3520770 & 0.1698240 \\
\hline $\mathrm{H}$ & -4.5182570 & -0.2946700 & 1.1959600 \\
\hline $\mathrm{C}$ & -3.9206450 & -1.7540110 & -1.0571620 \\
\hline $\mathrm{H}$ & -3.2945270 & -2.0348170 & -1.9126130 \\
\hline $\mathrm{H}$ & -3.4882100 & -2.1780730 & -0.1466490 \\
\hline $\mathrm{H}$ & -4.8973400 & -2.2265950 & -1.2007150 \\
\hline $\mathrm{O}$ & 0.4309350 & -0.3485620 & -0.7378910 \\
\hline $\mathrm{H}$ & 1.2179940 & 0.3012090 & -0.2936140 \\
\hline $\mathrm{O}$ & 2.1562380 & 1.0911600 & 0.2196490 \\
\hline $\mathrm{C}$ & 3.0262320 & 1.5476110 & -0.5988100 \\
\hline $\mathrm{O}$ & 3.1461220 & 1.3530810 & -1.8077530 \\
\hline $\mathrm{C}$ & 4.0566810 & 2.4835630 & 0.1095810 \\
\hline $\mathrm{F}$ & 3.4408090 & 3.5552600 & 0.6715820 \\
\hline $\mathrm{F}$ & 4.9918920 & 2.9606690 & -0.7365390 \\
\hline $\mathrm{F}$ & 4.7115850 & 1.8323100 & 1.1046640 \\
\hline $\mathrm{H}$ & 0.8826560 & -1.0953540 & -1.1693810 \\
\hline $\mathrm{H}$ & 0.3844720 & -3.5827400 & 1.9360120 \\
\hline $\mathrm{H}$ & -0.6347650 & -3.6703770 & 0.5071440 \\
\hline $\mathrm{C}$ & 1.4110940 & -3.1102730 & 0.1225150 \\
\hline $\mathrm{C}$ & 1.3509910 & -3.4956290 & -1.2267300 \\
\hline $\mathrm{C}$ & 2.6605230 & -2.7755120 & 0.6706840 \\
\hline
\end{tabular}

$\begin{array}{lllr}\mathrm{C} & 2.5090760 & -3.5321820 & -2.0136230 \\ \mathrm{H} & 0.3982110 & -3.7857520 & -1.6625820 \\ \mathrm{C} & 3.8161080 & -2.8123630 & -0.1120960 \\ \mathrm{H} & 2.7304780 & -2.4903420 & 1.7173200 \\ \mathrm{C} & 3.7437330 & -3.1882240 & -1.4585270 \\ \mathrm{H} & 2.4423780 & -3.8348650 & -3.0543210 \\ \mathrm{H} & 4.7735190 & -2.5515570 & 0.3291730 \\ \mathrm{H} & 4.6431540 & -3.2176770 & -2.0660220\end{array}$

$5 . \log$

Potential Energy = -1949.23941

Zero-point Energy = -1948.77437

Free Energy = -1948.81900

Single-Point Energy B3LYP-D3(BJ)/6-311+G** PCM = 1949.73291

Free Energy B3LYP-D3(BJ)/6-311+G** PCM (extrapolated free energy from qRRHO) $=-1949.31249$

Nimag $=1\left(-186.9810 \mathrm{~cm}^{-1}\right)$

Charge $=0$ Multiplicity $=1$

$\begin{array}{llll}\text { C } & 3.1181100 & 0.5354530 & -0.8234870\end{array}$

$\begin{array}{llll}\text { C } & 4.3992260 & -0.8366830 & 0.6059300\end{array}$

C $2.9543720 \quad-1.0417580 \quad 1.0668240$

$\begin{array}{llll}\mathrm{N} & 2.1941190 & -0.3111330 & 0.0165670\end{array}$

$\mathrm{N} \quad 4.4048120 \quad-0.0854250 \quad-0.5166800$

$\begin{array}{llll}\text { O } & 5.3819840 & -1.3696430 & 1.1200890\end{array}$

C $\quad 5.5626730 \quad-0.0867920 \quad-1.4112300$

$\mathrm{H} \quad 5.9905320 \quad-1.0916980 \quad-1.4185320$

$\mathrm{H} \quad 5.2380640 \quad 0.1737550 \quad-2.4185060$

$\begin{array}{llll}\mathrm{H} & 6.3335550 & 0.6171530 & -1.0898670\end{array}$

$\begin{array}{llll}\text { C } & 0.8743770 & -0.2896400 & -0.1859570\end{array}$

$\begin{array}{llll}\mathrm{C} & -0.0494010 & -1.4899800 & 0.0760330\end{array}$

C $\quad-1.3163060 \quad-1.4094250 \quad-0.7931530$

$\mathrm{H} \quad-1.8953630 \quad-0.5245470 \quad-0.5159560$

$\begin{array}{llll}\mathrm{H} & 2.7905050 & -0.5683310 & 2.0415960\end{array}$

$\begin{array}{llll}\mathrm{C} & 3.0905050 & 2.0882560 & -0.5626190\end{array}$

$\mathrm{H} \quad 2.8709100 \quad 0.3548470 \quad-1.8761930$

$\begin{array}{llll}\mathrm{H} & 0.5065240 & -2.3946070 & -0.1675040\end{array}$

$\begin{array}{lllr}\mathrm{Cl} & -0.5043900 & -1.6974070 & 1.8415520\end{array}$

$\begin{array}{llll}\text { C } & 2.7336070 & -2.5584810 & 1.1697460\end{array}$

H $1.7889200 \quad-2.8167410 \quad 1.6422850$

$\mathrm{H} \quad 2.8050570 \quad-3.0347130 \quad 0.1874920$

$\begin{array}{llll}\mathrm{H} & 3.5367650 & -2.9532980 & 1.7965040\end{array}$

$\begin{array}{llll}\mathrm{C} & 4.3162510 & 2.7349400 & -1.2494700\end{array}$

$\begin{array}{llll}\mathrm{H} & 4.2180430 & 3.8228320 & -1.1852220\end{array}$

$\mathrm{H} \quad 5.2551630 \quad 2.4649230 \quad-0.7624950$

$\mathrm{H} \quad 4.3811060 \quad 2.4757380 \quad-2.3119040$

$\begin{array}{llll}\text { C } & 3.1442450 & 2.4075990 & 0.9423250\end{array}$

$\mathrm{H} \quad 4.0432050 \quad 1.9888800 \quad 1.4079520$

$\begin{array}{llll}\mathrm{H} & 3.1818600 & 3.4927590 & 1.0836440\end{array}$

$\begin{array}{llll}\mathrm{H} & 2.2630820 & 2.0339960 & 1.4678440\end{array}$

C $\quad 1.8400570 \quad 2.7166820 \quad-1.2158340$

$\begin{array}{llll}\mathrm{H} & 1.7784070 & 2.4597640 & -2.2800030\end{array}$

$\mathrm{H} \quad 0.9109700 \quad 2.4288540 \quad-0.7287550$

$\mathrm{H} \quad 1.9097270 \quad 3.8068810 \quad-1.1463480$

$\mathrm{H} \quad-0.9679460 \quad-1.2561510 \quad-1.8226710$

$\begin{array}{llll}\text { C } & -2.1835550 & -2.6506700 & -0.7443110\end{array}$

$\begin{array}{llll}\text { C } & -3.4143510 & -2.6407240 & -0.0734770\end{array}$

C $\quad-1.7749140 \quad-3.8312320 \quad-1.3847630$

$\begin{array}{llll}\mathrm{C} & -4.2168030 & -3.7856200 & -0.0350460\end{array}$

$\mathrm{H} \quad-3.7481260 \quad-1.7323880 \quad 0.4206310$

$\begin{array}{llll}\text { C } & -2.5722810 & -4.9778990 & -1.3458280\end{array}$

$\mathrm{H} \quad-0.8312320 \quad-3.8540450 \quad-1.9249910$

$\begin{array}{llll}\text { C } & -3.7967520 & -4.9583730 & -0.6690570\end{array}$

$\mathrm{H} \quad-5.1682380 \quad-3.7589660 \quad 0.4882400$

$\mathrm{H} \quad-2.2415790 \quad-5.8820340 \quad-1.8487940$

$\mathrm{H} \quad-4.4193510 \quad-5.8478220 \quad-0.6414460$

$\mathrm{H} \quad 0.6121250 \quad 0.2142600 \quad-1.1111280$

O $\quad 0.0749520 \quad 1.0253110 \quad 0.8481290$

$\begin{array}{llll}\mathrm{H} & -0.8164190 & 1.4617730 & 0.4348660\end{array}$

$\begin{array}{llll}\mathrm{H} & -0.1720190 & 0.6380890 & 1.7055100\end{array}$

$\begin{array}{llll}\mathrm{O} & -1.9303770 & 2.0646620 & -0.1663760\end{array}$

C $\quad-2.8245870 \quad 2.4913420 \quad 0.6348910$

$\begin{array}{llll}\text { C } & -4.0053950 & 3.1889250 & -0.1142660\end{array}$

$\begin{array}{llll}\text { O } & -2.8748540 & 2.4261550 & 1.8656720\end{array}$

F $\quad-4.6102350 \quad 2.3366770 \quad-0.9824610$

$\begin{array}{llll}\text { F } & -3.5695330 & 4.2517870 & -0.8393840\end{array}$ 
Catalyst regeneration and product release (TS7)

\section{Conformation Extrapolated name \\ Free energy \\ (kcal/mol)}

$\begin{array}{cc}\text { TS7-lowest } & 0.0 \\ 1 & 3.6 \\ 2 & 3.6 \\ 3 & 4.5 \\ 4 & 2.3 \\ 5 & 5.3 \\ 6 & 3.8 \\ 7 & 5.6 \\ 8 & 1.1 \\ 9 & 0.7 \\ 10 & 1.6\end{array}$

TS7-lowest.log

Potential Energy $=-1949.25101$

Zero-point Energy = -1948.78659

Free Energy = -1948.83046

Single-Point Energy B3LYP-D3(BJ)/6-311+G** PCM = 1949.74709

Free Energy B3LYP-D3(BJ)/6-311+G** PCM (extrapolated

free energy from $q R R H O)=-1949.32653$

Nimag $=1\left(-492.3687 \mathrm{~cm}^{-1}\right)$

Charge $=0$ Multiplicity $=1$

$\begin{array}{lrrr}\mathrm{C} & 1.0639590 & 1.7565980 & -1.7950990 \\ \mathrm{C} & -0.2708930 & 2.3394820 & 0.0328890 \\ \mathrm{C} & -0.1808220 & 0.8788080 & -1.8252270 \\ \mathrm{H} & -0.9995880 & 3.0098360 & -0.4502400 \\ \mathrm{H} & 0.0659700 & -0.1538370 & -2.0768710 \\ \mathrm{C} & -0.1489250 & -0.8124890 & 0.4540330 \\ \mathrm{H} & -0.6715330 & -0.5341840 & 1.3706490 \\ \mathrm{O} & -0.6908270 & -1.6602060 & -0.3341430 \\ \mathrm{~N} & 1.0289200 & 2.5007140 & -0.6487910 \\ \mathrm{C} & 1.9203040 & 3.6473820 & -0.4984670 \\ \mathrm{H} & 2.3730770 & 3.6700700 & 0.4930590 \\ \mathrm{H} & 2.7081110 & 3.5396320 & -1.2441460 \\ \mathrm{H} & 1.3868740 & 4.5863310 & -0.6790740 \\ \mathrm{O} & 1.9286660 & 1.7983340 & -2.6690720 \\ \mathrm{C} & 1.3778500 & -0.8602110 & 0.5916570 \\ \mathrm{H} & 1.7864850 & 0.1287560 & 0.7839120 \\ \mathrm{C} & 2.1135610 & -1.5683490 & -0.5498780 \\ \mathrm{H} & 1.8706510 & -1.0305140 & -1.4725620 \\ \mathrm{H} & 1.7078280 & -2.5763580 & -0.6604390 \\ \mathrm{~N} & -0.6271330 & 0.9464870 & -0.4067470 \\ \mathrm{C} & -3.8247910 & -1.0629700 & -0.2117750 \\ \mathrm{C} & -5.3295320 & -1.4568300 & -0.1564260 \\ \mathrm{O} & -3.5182530 & 0.1205400 & -0.3345990 \\ \mathrm{H} & -1.6437700 & 0.8025400 & -0.3472500 \\ \mathrm{O} & -3.0553030 & -2.0889970 & -0.1176320 \\ \mathrm{H} & -1.9104120 & -1.8428750 & -0.2039220 \\ \mathrm{C} & -0.3851580 & 2.6589670 & 1.5545680 \\ \mathrm{C} & -0.3428680 & 4.1973580 & 1.7431830 \\ \mathrm{H} & -0.6213520 & 4.4353270 & 2.7742350 \\ \mathrm{H} & 0.6471800 & 4.6218070 & 1.5700880 \\ \mathrm{H} & -1.0544310 & 4.7031880 & 1.0816820 \\ \mathrm{C} & 0.7004860 & 2.0169540 & 2.4374130 \\ \mathrm{H} & 0.6293040 & 2.4311810 & 3.4481500 \\ \mathrm{H} & 1.7109970 & 2.2210180 & 2.0719910 \\ & -1.7832970 & 2.1937080 & 2.0250640 \\ -1.9293570 & 2.4928870 & 3.0671670 \\ \mathrm{H} & -1.9110970 & 1.1090200 & 1.9795190 \\ \mathrm{H} & -1.2108860 & 1.3949030 & -2.8426400 \\ & & & \\ \mathrm{H} & & & \\ \mathrm{H} & & & \end{array}$

$\begin{array}{lccc}\mathrm{H} & -2.1022840 & 0.7631120 & -2.8231080 \\ \mathrm{H} & -1.5090980 & 2.4285260 & -2.6452470 \\ \mathrm{H} & 0.5802510 & 0.9365340 & 2.5342450 \\ \mathrm{Cl} & 1.6440390 & -1.7747320 & 2.1772690 \\ \mathrm{~F} & -5.6133560 & -2.1331630 & 0.9811550 \\ \mathrm{~F} & -6.1342890 & -0.3805610 & -0.2026320 \\ \mathrm{~F} & -5.6549450 & -2.2581010 & -1.1988180 \\ \mathrm{C} & 3.6209790 & -1.6130600 & -0.3902230 \\ \mathrm{C} & 4.3981510 & -0.4602750 & -0.5857570 \\ \mathrm{C} & 4.2701170 & -2.8116730 & -0.0623630 \\ \mathrm{C} & 5.7876570 & -0.5018580 & -0.4426900 \\ \mathrm{H} & 3.9181180 & 0.4742910 & -0.8648520 \\ \mathrm{C} & 5.6608930 & -2.8581950 & 0.0785730 \\ \mathrm{H} & 3.6842230 & -3.7152580 & 0.0830470 \\ \mathrm{C} & 6.4240590 & -1.7019910 & -0.1081280 \\ \mathrm{H} & 6.3725040 & 0.3998660 & -0.6003930 \\ \mathrm{H} & 6.1453790 & -3.7968230 & 0.3320110 \\ \mathrm{H} & 7.5042880 & -1.7362130 & -0.0005890\end{array}$

$1 . \log$

Potential Energy $=-1949.24594$

Zero-point Energy = -1948.78197

Free Energy $=-1948.82590$

Single-Point Energy B3LYP-D3(BJ)/6-311+G** PCM = 1949.74085

Free Energy B3LYP-D3(BJ)/6-311+G** PCM (extrapolated free energy from qRRHO) $=-1949.32081$

Nimag $=1\left(-506.0454 \mathrm{~cm}^{-1}\right)$

Charge $=0$ Multiplicity $=1$

$\begin{array}{llll}\text { C } & -0.4872840 & 2.5446190 & 1.7234570\end{array}$

$\begin{array}{llll}\mathrm{C} & 1.0762730 & 2.2660040 & 0.0038530\end{array}$

C $\quad 0.1217160 \quad 1.1589380 \quad 1.8817700$

$\begin{array}{llll}\mathrm{H} & 2.0289420 & 2.5006800 & 0.5057360\end{array}$

H $\quad-0.6537840 \quad 0.4297950 \quad 2.1172460$

$\begin{array}{llll}\text { C } & -0.3080020 & -0.6205850 & -0.3207610\end{array}$

$\mathrm{H} \quad 0.0157800 \quad-0.3655960 \quad-1.3310290$

O $\quad 0.2235360 \quad-1.6211500 \quad 0.2839970$

$\mathrm{N} \quad 0.0248150 \quad 3.1081160 \quad 0.5952660$

C $\quad-0.1561850 \quad 4.5334570 \quad 0.3445280$

$\mathrm{H} \quad-0.5049350 \quad 4.7192850 \quad-0.6718900$

$\mathrm{H} \quad-0.9123850 \quad 4.8860940 \quad 1.0460720$

$\mathrm{H} \quad 0.7734970 \quad 5.0846920 \quad 0.5186860$

$\begin{array}{llll}\mathrm{O} & -1.2706190 & 3.0666710 & 2.5170300\end{array}$

$\begin{array}{llll}\text { C } & -1.8077000 & -0.5146900 & -0.0544560\end{array}$

$\mathrm{H} \quad-1.9958980 \quad-0.5702960 \quad 1.0177990$

C $\quad-2.5096700 \quad-1.7012040 \quad-0.7606600$

$\mathrm{H} \quad-1.9825670 \quad-2.6038020 \quad-0.4352370$

$\mathrm{H} \quad-2.3657290 \quad-1.6077240 \quad-1.8417360$

$\begin{array}{llll}\mathrm{N} & 0.6829530 & 0.8962630 & 0.5233480\end{array}$

$\begin{array}{llll}\text { C } & 3.3518350 & -1.7996570 & 0.1976880\end{array}$

$\begin{array}{llll}\text { C } & 4.7337570 & -2.4920670 & 0.0195940\end{array}$

$\begin{array}{llll}\text { O } & 3.2919670 & -0.7046010 & 0.7510930\end{array}$

H $\quad 1.5453660 \quad 0.3433990 \quad 0.6139270$

$\begin{array}{llll}\mathrm{O} & 2.3902640 & -2.5034540 & -0.2885910\end{array}$

$\begin{array}{llll}\mathrm{H} & 1.3345030 & -2.0376040 & -0.0569430\end{array}$

$\begin{array}{llll}\mathrm{C} & 1.3892290 & 2.3986890 & -1.5201030\end{array}$

$\begin{array}{llll}\mathrm{C} & 2.1495840 & 3.7304700 & -1.7535410\end{array}$

$\begin{array}{llll}\mathrm{H} & 2.5334120 & 3.7429820 & -2.7782020\end{array}$

$\begin{array}{llll}\mathrm{H} & 1.5172560 & 4.6112730 & -1.6358790\end{array}$

$\mathrm{H} \quad 3.0057910 \quad 3.8286690 \quad-1.0772270$

$\begin{array}{llll}\mathrm{C} & 0.1587540 & 2.3583410 & -2.4432950\end{array}$

$\begin{array}{llll}\mathrm{H} & 0.4815200 & 2.5520000 & -3.4716370\end{array}$

$\begin{array}{llll}\mathrm{H} & -0.5838910 & 3.1148360 & -2.1785410\end{array}$

$\begin{array}{llll}\mathrm{C} & 2.3704160 & 1.2675120 & -1.9059270\end{array}$

$\begin{array}{llll}\mathrm{H} & 2.7017290 & 1.4180130 & -2.9375620\end{array}$

$\begin{array}{llll}\mathrm{H} & 3.2588010 & 1.2674330 & -1.2662390\end{array}$

$\begin{array}{llll}\mathrm{H} & 1.9177530 & 0.2743840 & -1.8586530\end{array}$

$\begin{array}{llll}\text { C } & 1.1699940 & 1.1363750 & 3.0050450\end{array}$

$\begin{array}{llll}\mathrm{H} & 0.6849770 & 1.3973950 & 3.9492260\end{array}$

$\mathrm{H} \quad 1.5986200 \quad 0.1356160 \quad 3.0988020$

$\begin{array}{llll}\mathrm{H} & 1.9797150 & 1.8497220 & 2.8256400\end{array}$

$\mathrm{H} \quad-0.3442650 \quad 1.3908570 \quad-2.4340150$

$\begin{array}{llll}\mathrm{Cl} & -2.5273120 & 1.0581990 & -0.6082370\end{array}$

$\begin{array}{llll}\text { F } & 4.9879130 & -2.7377960 & -1.2873290\end{array}$

F $\quad 5.7401080 \quad-1.7382280 \quad 0.4961600$

$\begin{array}{llll}\text { F } & 4.7655200 & -3.6790310 & 0.6708520\end{array}$ 


$\begin{array}{lccc}\mathrm{C} & -3.9838620 & -1.8268520 & -0.4351590 \\ \mathrm{C} & -4.9621170 & -1.5094110 & -1.3875890 \\ \mathrm{C} & -4.3968780 & -2.2813720 & 0.8275070 \\ \mathrm{C} & -6.3222590 & -1.6359250 & -1.0863690 \\ \mathrm{H} & -4.6588070 & -1.1625290 & -2.3717580 \\ \mathrm{C} & -5.7544120 & -2.4055210 & 1.1335800 \\ \mathrm{H} & -3.6531530 & -2.5471540 & 1.5751160 \\ \mathrm{C} & -6.7225150 & -2.0817910 & 0.1764440 \\ \mathrm{H} & -7.0658010 & -1.3867800 & -1.8380340 \\ \mathrm{H} & -6.0555300 & -2.7606740 & 2.1149610 \\ \mathrm{H} & -7.7780640 & -2.1811940 & 0.4120840\end{array}$

$2 . \log$

Potential Energy $=-1949.24440$

Zero-point Energy $=-1948.77977$

Free Energy = -1948.82337

Single-Point Energy B3LYP-D3(BJ)/6-311+G** PCM = 1949.74179

Free Energy B3LYP-D3(BJ)/6-311+G** PCM (extrapolated free energy from $\mathrm{qRRHO})=-1949.32076$

Nimag $=1\left(-500.3399 \mathrm{~cm}^{-1}\right)$ Charge $=0$ Multiplicity $=1$

\begin{tabular}{|c|c|c|c|}
\hline $\mathrm{C}$ & -0.8663320 & -3.3403780 & -1.0437420 \\
\hline $\mathrm{C}$ & 0.1349870 & -2.2737740 & 0.7848040 \\
\hline $\mathrm{C}$ & 0.0726130 & -2.2784330 & -1.5966060 \\
\hline $\mathrm{H}$ & 1.1083300 & -2.7843610 & 0.8642510 \\
\hline $\mathrm{H}$ & -0.4068830 & -1.7442420 & -2.4170640 \\
\hline $\mathrm{C}$ & -0.5309800 & 0.4263900 & -0.8299190 \\
\hline $\mathrm{H}$ & -0.6076720 & 0.7018920 & 0.2214320 \\
\hline $\mathrm{O}$ & 0.3927900 & 0.9424720 & -1.5579610 \\
\hline $\mathrm{N}$ & -0.8614910 & -3.2485480 & 0.3141580 \\
\hline $\mathrm{C}$ & -1.4395300 & -4.3188200 & 1.1191670 \\
\hline $\mathrm{H}$ & -2.1163530 & -3.9268540 & 1.8792780 \\
\hline $\mathrm{H}$ & -2.0048500 & -4.9573890 & 0.4401760 \\
\hline $\mathrm{H}$ & -0.6572060 & -4.9156670 & 1.5988190 \\
\hline $\mathrm{O}$ & -1.4793790 & -4.1637100 & -1.7236550 \\
\hline $\mathrm{C}$ & -1.8457870 & 0.2817290 & -1.5893160 \\
\hline $\mathrm{H}$ & -1.6606870 & -0.2223320 & -2.5377240 \\
\hline $\mathrm{C}$ & -2.4340900 & 1.6802750 & -1.9203900 \\
\hline $\mathrm{H}$ & -1.7038590 & 2.1616400 & -2.5782890 \\
\hline $\mathrm{N}$ & 0.2591420 & -1.3694190 & -0.4270920 \\
\hline $\mathrm{C}$ & 3.2919530 & 0.9235950 & -0.3629900 \\
\hline $\mathrm{C}$ & 4.6795040 & 1.5161040 & 0.0172690 \\
\hline $\mathrm{O}$ & 3.1314240 & -0.2941950 & -0.3527040 \\
\hline $\mathrm{H}$ & 1.2187860 & -0.9997250 & -0.4347920 \\
\hline $\mathrm{O}$ & 2.4357760 & 1.8405000 & -0.6506470 \\
\hline $\mathrm{H}$ & 1.4179440 & 1.3968250 & -1.0379920 \\
\hline $\mathrm{C}$ & -0.0714030 & -1.5933200 & 2.1748960 \\
\hline $\mathrm{C}$ & 0.2341610 & -2.6327520 & 3.2851260 \\
\hline $\mathrm{H}$ & 0.2761220 & -2.1169310 & 4.2492800 \\
\hline $\mathrm{H}$ & -0.5282130 & -3.4081260 & 3.3681820 \\
\hline $\mathrm{H}$ & 1.2032110 & -3.1182130 & 3.1259630 \\
\hline $\mathrm{C}$ & -1.4775050 & -1.0136570 & 2.4092600 \\
\hline $\mathrm{H}$ & -1.5420420 & -0.6454520 & 3.4385090 \\
\hline $\mathrm{H}$ & -2.2614450 & -1.7625930 & 2.2731410 \\
\hline $\mathrm{C}$ & 0.9907020 & -0.4793760 & 2.3255830 \\
\hline $\mathrm{H}$ & 0.9377040 & -0.0696060 & 3.3384800 \\
\hline $\mathrm{H}$ & 2.0035710 & -0.8662160 & 2.1744670 \\
\hline $\mathrm{H}$ & 0.8402230 & 0.3548980 & 1.6367140 \\
\hline $\mathrm{C}$ & 1.3762340 & -2.9035110 & -2.1170710 \\
\hline $\mathrm{H}$ & 1.1393990 & -3.5907750 & -2.9334300 \\
\hline $\mathrm{H}$ & 2.0399660 & -2.1242610 & -2.4995920 \\
\hline $\mathrm{H}$ & 1.9030450 & -3.4628020 & -1.3383970 \\
\hline $\mathrm{H}$ & -1.7070370 & -0.1784240 & 1.7470680 \\
\hline $\mathrm{Cl}$ & -3.0731040 & -0.7399810 & -0.7219070 \\
\hline $\mathrm{F}$ & 4.5757030 & 2.3354400 & 1.0905160 \\
\hline $\mathrm{F}$ & 5.5710690 & 0.5565600 & 0.3212620 \\
\hline $\mathrm{F}$ & 5.1912580 & 2.2443810 & -1.0029040 \\
\hline $\mathrm{C}$ & -2.7517330 & 2.5878710 & -0.7477990 \\
\hline $\mathrm{C}$ & -1.7878690 & 3.4787270 & -0.2477660 \\
\hline $\mathrm{C}$ & -4.0240040 & 2.5784060 & -0.1546740 \\
\hline $\mathrm{C}$ & -2.0800710 & 4.3225810 & 0.8279310 \\
\hline $\mathrm{H}$ & -0.8046500 & 3.5205300 & -0.7088580 \\
\hline $\mathrm{C}$ & -4.3202500 & 3.4215570 & 0.9204500 \\
\hline $\mathrm{H}$ & -4.7893930 & 1.9112540 & -0.5404580 \\
\hline $\mathrm{C}$ & -3.3473510 & 4.2939930 & 1.4182720 \\
\hline
\end{tabular}

$\begin{array}{llll}\mathrm{H} & -1.3209400 & 5.0057370 & 1.1980010 \\ \mathrm{H} & -5.3116830 & 3.4004510 & 1.3638640 \\ \mathrm{H} & -3.5775460 & 4.9518970 & 2.2512240 \\ \mathrm{H} & -3.3352780 & 1.5030900 & -2.5143420\end{array}$

3.log

Potential Energy $=-1949.24375$

Zero-point Energy = -1948.77976

Free Energy = -1948.82349

Single-Point Energy B3LYP-D3(BJ)/6-311+G** PCM = 1949.73962

Free Energy B3LYP-D3(BJ)/6-311+G** PCM (extrapolated free energy from $q$ RRHO) $=-1949.31936$

Nimag $=1\left(-491.2565 \mathrm{~cm}^{-1}\right)$ Charge $=0$ Multiplicity $=1$

\begin{tabular}{|c|c|c|c|}
\hline $\mathrm{C}$ & -2.9135440 & -1.3243350 & 1.6240160 \\
\hline $\mathrm{C}$ & -2.7165180 & 0.3464560 & -0.0061310 \\
\hline $\mathrm{C}$ & -1.5584750 & -0.6610650 & 1.8181770 \\
\hline $\mathrm{H}$ & -3.0123460 & 1.2622580 & 0.5311450 \\
\hline $\mathrm{H}$ & -0.7946290 & -1.4194170 & 1.9938590 \\
\hline $\mathrm{C}$ & 0.3072860 & -0.8350170 & -0.3847590 \\
\hline $\mathrm{H}$ & 0.0295450 & -0.4679820 & -1.3725930 \\
\hline $\mathrm{O}$ & 1.2410800 & -0.2502170 & 0.2727870 \\
\hline $\mathrm{N}$ & -3.5042300 & -0.7755950 & 0.5279240 \\
\hline $\mathrm{C}$ & -4.9217240 & -1.0039940 & 0.2715990 \\
\hline $\mathrm{H}$ & -5.0955640 & -1.3314330 & -0.7543060 \\
\hline $\mathrm{H}$ & -5.2455610 & -1.7918750 & 0.9518780 \\
\hline $\mathrm{H}$ & -5.5064080 & -0.1002540 & 0.4707210 \\
\hline $\mathrm{O}$ & -3.3963840 & -2.1761480 & 2.3710240 \\
\hline $\mathrm{C}$ & 0.3229550 & -2.3443950 & -0.2038150 \\
\hline $\mathrm{H}$ & 0.4770870 & -2.5887190 & 0.8472130 \\
\hline $\mathrm{C}$ & 1.4623590 & -2.9673970 & -1.0634920 \\
\hline $\mathrm{H}$ & 1.3414410 & -2.6386670 & -2.1004660 \\
\hline $\mathrm{N}$ & -1.3308390 & 0.0080390 & 0.5041160 \\
\hline $\mathrm{C}$ & 1.1362100 & 2.8807350 & 0.3133440 \\
\hline $\mathrm{C}$ & 1.7070240 & 4.3254860 & 0.2055460 \\
\hline $\mathrm{O}$ & 0.0590520 & 2.7050300 & 0.8843280 \\
\hline $\mathrm{H}$ & -0.8367290 & 0.8977750 & 0.6546250 \\
\hline $\mathrm{O}$ & 1.9006950 & 2.0111520 & -0.2358670 \\
\hline $\mathrm{H}$ & 1.5293940 & 0.8459250 & -0.0214940 \\
\hline $\mathrm{C}$ & -2.8524760 & 0.7074530 & -1.5194080 \\
\hline $\mathrm{C}$ & -4.2254630 & 1.3917940 & -1.7485770 \\
\hline $\mathrm{H}$ & -4.2442720 & 1.8166920 & -2.7568770 \\
\hline $\mathrm{H}$ & -5.0664190 & 0.7013110 & -1.6748990 \\
\hline $\mathrm{H}$ & -4.3899070 & 2.2113220 & -1.0403820 \\
\hline $\mathrm{C}$ & -2.7252820 & -0.4890280 & -2.4784950 \\
\hline $\mathrm{H}$ & -2.9249660 & -0.1488720 & -3.5000450 \\
\hline $\mathrm{H}$ & -3.4390000 & -1.2831040 & -2.2452890 \\
\hline $\mathrm{C}$ & -1.7807510 & 1.7696260 & -1.8571260 \\
\hline $\mathrm{H}$ & -1.9345630 & 2.1196520 & -2.8821190 \\
\hline $\mathrm{H}$ & -1.8501170 & 2.6373220 & -1.1932740 \\
\hline $\mathrm{H}$ & -0.7605240 & 1.3831940 & -1.8021930 \\
\hline $\mathrm{C}$ & -1.5784650 & 0.3017130 & 3.0157010 \\
\hline $\mathrm{H}$ & -1.8072840 & -0.2628600 & 3.9234000 \\
\hline $\mathrm{H}$ & -0.5998020 & 0.7727300 & 3.1358970 \\
\hline $\mathrm{H}$ & -2.3328990 & 1.0852880 & 2.8982320 \\
\hline $\mathrm{H}$ & -1.7293500 & -0.9334010 & -2.4695590 \\
\hline $\mathrm{Cl}$ & -1.2391730 & -3.1337400 & -0.6890370 \\
\hline $\mathrm{F}$ & 1.9087570 & 4.6735640 & -1.0881790 \\
\hline $\mathrm{F}$ & 0.8836570 & 5.2413600 & 0.7479860 \\
\hline $\mathrm{F}$ & 2.8998330 & 4.4217190 & 0.8417020 \\
\hline $\mathrm{H}$ & 1.2858470 & -4.0470800 & -1.0490460 \\
\hline $\mathrm{C}$ & 2.8696280 & -2.6839530 & -0.5774840 \\
\hline $\mathrm{C}$ & 3.7244290 & -1.8391360 & -1.2988820 \\
\hline $\mathrm{C}$ & 3.3560990 & -3.2970990 & 0.5879760 \\
\hline $\mathrm{C}$ & 5.0322920 & -1.6031090 & -0.8635670 \\
\hline $\mathrm{H}$ & 3.3662940 & -1.3622310 & -2.2076680 \\
\hline $\mathrm{C}$ & 4.6602230 & -3.0600070 & 1.0291120 \\
\hline $\mathrm{H}$ & 2.7145450 & -3.9703430 & 1.1517030 \\
\hline $\mathrm{C}$ & 5.5030420 & -2.2099880 & 0.3043560 \\
\hline $\mathrm{H}$ & 5.6805030 & -0.9464180 & -1.4366750 \\
\hline $\mathrm{H}$ & 5.0199460 & -3.5433250 & 1.9329340 \\
\hline $\mathrm{H}$ & 6.5183240 & -2.0277820 & 0.6443020 \\
\hline
\end{tabular}

4.log

Potential Energy = -1949.24967 
Zero-point Energy = -1948.78455

Free Energy $=-1948.82828$

Single-Point Energy B3LYP-D3(BJ)/6-311+G** PCM = -

1949.74430

Free Energy B3LYP-D3(BJ)/6-311+G** PCM (extrapolated

free energy from $q$ RRHO) $=-1949.32292$

Nimag $=1\left(-353.0256 \mathrm{~cm}^{-1}\right)$

Charge $=0$ Multiplicity $=1$

\begin{tabular}{|c|c|c|c|}
\hline $\mathrm{C}$ & -0.2350390 & -0.9723700 & -0.4731210 \\
\hline $\mathrm{H}$ & 0.2935820 & -0.8131030 & -1.4123540 \\
\hline $\mathrm{O}$ & 0.2216980 & -1.8435150 & 0.3476350 \\
\hline $\mathrm{C}$ & -1.7506830 & -0.8167670 & -0.5376460 \\
\hline $\mathrm{H}$ & -2.0272890 & 0.1872770 & -0.8486700 \\
\hline $\mathrm{C}$ & 3.3816840 & -1.6531910 & 0.1521120 \\
\hline $\mathrm{C}$ & 4.8358420 & -2.1969680 & 0.0250670 \\
\hline $\mathrm{O}$ & 3.2168810 & -0.4504010 & 0.3742200 \\
\hline $\mathrm{O}$ & 2.5021340 & -2.5672250 & -0.0025820 \\
\hline $\mathrm{H}$ & 1.3090400 & -2.1661570 & 0.1625800 \\
\hline $\mathrm{Cl}$ & -2.2184270 & -1.8774470 & -1.9747610 \\
\hline $\mathrm{F}$ & 5.0444000 & -2.7332470 & -1.2023480 \\
\hline $\mathrm{F}$ & 5.7603500 & -1.2361920 & 0.2088820 \\
\hline $\mathrm{F}$ & 5.0728320 & -3.1701410 & 0.9373090 \\
\hline $\mathrm{N}$ & 0.5324180 & 0.7975310 & 0.2406800 \\
\hline $\mathrm{C}$ & 0.0159300 & 1.3490050 & 1.5325950 \\
\hline $\mathrm{C}$ & 1.0426340 & 1.9752410 & -0.5712650 \\
\hline $\mathrm{C}$ & 0.7963720 & 2.6615130 & 1.6762370 \\
\hline $\mathrm{H}$ & -1.0495820 & 1.5780350 & 1.4350350 \\
\hline $\mathrm{H}$ & 1.9510520 & 1.6344390 & -1.0782280 \\
\hline $\mathrm{H}$ & 1.4031120 & 0.2953860 & 0.4699680 \\
\hline $\mathrm{O}$ & 0.8855720 & 3.3338550 & 2.7043540 \\
\hline $\mathrm{N}$ & 1.4170040 & 2.9151410 & 0.5002810 \\
\hline $\mathrm{C}$ & 2.4701980 & 3.9234210 & 0.4010910 \\
\hline $\mathrm{H}$ & 2.0662880 & 4.9260310 & 0.2369350 \\
\hline $\mathrm{H}$ & 3.0346960 & 3.9261560 & 1.3361890 \\
\hline $\mathrm{H}$ & 3.1387930 & 3.6675780 & -0.4214420 \\
\hline $\mathrm{C}$ & 0.2577120 & 0.4368700 & 2.7342540 \\
\hline $\mathrm{H}$ & -0.2783420 & -0.5081200 & 2.6415730 \\
\hline $\mathrm{H}$ & 1.3237060 & 0.2183860 & 2.8500810 \\
\hline $\mathrm{H}$ & -0.0867590 & 0.9486180 & 3.6358290 \\
\hline $\mathrm{C}$ & 0.0963690 & 2.5869260 & -1.6552420 \\
\hline $\mathrm{C}$ & 0.8102440 & 3.8055770 & -2.2890420 \\
\hline $\mathrm{H}$ & 0.2223310 & 4.1564840 & -3.1425820 \\
\hline $\mathrm{H}$ & 0.9019770 & 4.6417260 & -1.5935280 \\
\hline $\mathrm{H}$ & 1.8079910 & 3.5470000 & -2.6602580 \\
\hline $\mathrm{C}$ & -0.1310850 & 1.5674030 & -2.7909960 \\
\hline $\mathrm{H}$ & 0.8187070 & 1.1775740 & -3.1745990 \\
\hline $\mathrm{H}$ & -0.7545250 & 0.7230620 & -2.4974360 \\
\hline $\mathrm{H}$ & -0.6447510 & 2.0592970 & -3.6226550 \\
\hline $\mathrm{C}$ & -1.2472690 & 3.0649950 & -1.0753030 \\
\hline $\mathrm{H}$ & -1.1027240 & 3.8107250 & -0.2871030 \\
\hline $\mathrm{H}$ & -1.8414810 & 3.5334950 & -1.8661660 \\
\hline $\mathrm{H}$ & -1.8463880 & 2.2479260 & -0.6653510 \\
\hline $\mathrm{C}$ & -2.5054800 & -1.2508130 & 0.7213050 \\
\hline $\mathrm{H}$ & -2.2202970 & -2.2755280 & 0.9692800 \\
\hline $\mathrm{H}$ & -2.1439760 & -0.6165010 & 1.5376080 \\
\hline $\mathrm{C}$ & -4.0137050 & -1.1259060 & 0.6260050 \\
\hline $\mathrm{C}$ & -4.8151100 & -2.2661800 & 0.4722390 \\
\hline $\mathrm{C}$ & -4.6394830 & 0.1278300 & 0.7092090 \\
\hline $\mathrm{C}$ & -6.2070920 & -2.1580260 & 0.3921580 \\
\hline $\mathrm{H}$ & -4.3477990 & -3.2455750 & 0.4160790 \\
\hline $\mathrm{C}$ & -6.0300020 & 0.2406550 & 0.6265370 \\
\hline $\mathrm{H}$ & -4.0406690 & 1.0240090 & 0.8503240 \\
\hline $\mathrm{C}$ & -6.8189220 & -0.9032910 & 0.4661150 \\
\hline $\mathrm{H}$ & -6.8107490 & -3.0533350 & 0.2744080 \\
\hline $\mathrm{H}$ & -6.4959470 & 1.2194250 & 0.6952560 \\
\hline $\mathrm{H}$ & -7.8999140 & -0.8174260 & 0.4059730 \\
\hline
\end{tabular}

$5 . \log$

Potential Energy $=-1949.24583$

Zero-point Energy = -1948.77984

Free Energy $=-1948.82356$

Single-Point Energy B3LYP-D3(BJ)/6-311+G** PCM = 1949.74041

Free Energy B3LYP-D3(BJ)/6-311+G** PCM (extrapolated free energy from $q R R H O)=-1949.31813$

Nimag $=1\left(-182.7738 \mathrm{~cm}^{-1}\right)$
Charge $=0$ Multiplicity $=1$

$\begin{array}{llrr}\mathrm{C} & -0.4263140 & -0.7666210 & -0.3001490 \\ \mathrm{H} & -0.1255300 & -1.2251390 & 0.6439450\end{array}$

O $\quad 0.1220020 \quad-1.2109160 \quad-1.3699740$

C $\quad-1.8984960 \quad-0.3937960 \quad-0.3160830$

$\begin{array}{llll}\text { C } & -2.7102910 & -1.6160730 & 0.1901840\end{array}$

$\mathrm{H} \quad-2.5565940 \quad-2.4493500 \quad-0.5016470$

$\begin{array}{llll}\mathrm{H} & -2.2782530 & -1.9106530 & 1.1541600\end{array}$

$\begin{array}{llll}\text { C } & 2.8965280 & -2.1112280 & 0.0191990\end{array}$

C $\quad 4.2033880 \quad-2.9515910 \quad 0.1476470$

O $2.6878930 \quad-1.2268180 \quad 0.8598960$

O $\quad 2.1852890 \quad-2.4569360 \quad-0.9776660$

H $\quad 1.0624870 \quad-1.7597240 \quad-1.1754870$

F $\quad 3.9265770 \quad-4.2750010 \quad 0.2407800$

F $\quad 4.9308560 \quad-2.6154520 \quad 1.2301380$

$\begin{array}{llll}\text { F } & 4.9942070 & -2.7804980 & -0.9410210\end{array}$

$\begin{array}{llll}\text { C } & -4.1893320 & -1.3418860 & 0.3715860\end{array}$

$\begin{array}{llll}\text { C } & -4.6460630 & -0.5921910 & 1.4669770\end{array}$

C $\quad-5.1286550 \quad-1.8428010 \quad-0.5404020$

C $\quad-6.0092050 \quad-0.3388760 \quad 1.6398060$

$\mathrm{H} \quad-3.9350620 \quad-0.2105470 \quad 2.1958700$

C $\quad-6.4942100 \quad-1.5944760 \quad-0.3682010$

$\mathrm{H} \quad-4.7913190 \quad-2.4317410 \quad-1.3888610$

C $\quad-6.9381580 \quad-0.8390000 \quad 0.7208460$

H $\quad-6.3454120 \quad 0.2411870 \quad 2.4943420$

$\mathrm{H} \quad-7.2080740 \quad-1.9921320 \quad-1.0837600$

$\mathrm{H} \quad-7.9982860 \quad-0.6460270 \quad 0.8564180$

$\mathrm{H} \quad-2.0835440 \quad 0.4603930 \quad 0.3344050$

$\mathrm{Cl} \quad-2.4707480 \quad 0.1024800 \quad-1.9629800$

$\begin{array}{llll}\mathrm{N} & 0.6753100 & 0.8485570 & 0.3522160\end{array}$

C $\quad 0.1436060 \quad 1.5363480 \quad 1.5679230$

$\begin{array}{llll}\text { C } & 1.5201210 & 1.8712290 & -0.3914650\end{array}$

$\begin{array}{llll}\text { C } & 1.2012360 & 2.6162770 & 1.8311170\end{array}$

$\mathrm{H} \quad-0.8032990 \quad 2.0281410 \quad 1.3229480$

$\begin{array}{llll}\mathrm{H} & 2.3824010 & 1.3345900 & -0.8001050\end{array}$

$\begin{array}{llll}\mathrm{O} & 1.3406770 & 3.2448820 & 2.8810260\end{array}$

$\begin{array}{llll}\text { C } & 3.2489280 & 3.4391950 & 0.7564580\end{array}$

$\begin{array}{llll}\mathrm{H} & 3.1224210 & 4.5014770 & 0.5328920\end{array}$

$\begin{array}{llll}\mathrm{H} & 3.6800150 & 3.3421340 & 1.7551900\end{array}$

$\begin{array}{llll}\mathrm{H} & 3.9300390 & 3.0003910 & 0.0262380\end{array}$

$\begin{array}{llll}\mathrm{N} & 1.9803960 & 2.7143930 & 0.7292800\end{array}$

$\begin{array}{llll}\mathrm{H} & 1.3849230 & 0.1774860 & 0.6825000\end{array}$

$\begin{array}{llll}\mathrm{C} & -0.0396580 & 0.6226470 & 2.7770100\end{array}$

$\begin{array}{llll}\mathrm{H} & -0.2687100 & 1.2430330 & 3.6466330\end{array}$

$\mathrm{H} \quad-0.8660220 \quad-0.0787480 \quad 2.6432760$

$\begin{array}{llll}\mathrm{H} & 0.8726410 & 0.0578670 & 2.9922280\end{array}$

$\begin{array}{llll}\mathrm{C} & 0.8503490 & 2.6557080 & -1.5622020\end{array}$

$\begin{array}{llll}\mathrm{C} & 1.7749790 & 3.8222520 & -1.9818640\end{array}$

$\mathrm{H} \quad 1.8091280 \quad 4.6145370 \quad-1.2304290$

$\begin{array}{llll}\mathrm{H} & 2.7974890 & 3.4853980 & -2.1843380\end{array}$

$\begin{array}{llll}\mathrm{H} & 1.3869270 & 4.2632560 & -2.9053790\end{array}$

C $\quad-0.5182660 \quad 3.2450140 \quad-1.1753870$

$\mathrm{H} \quad-0.9087330 \quad 3.8363640 \quad-2.0100240$

$\begin{array}{llll}\mathrm{H} & -1.2574830 & 2.4715340 & -0.9579620\end{array}$

$\mathrm{H} \quad-0.4388520 \quad 3.9145330 \quad-0.3122080$

$\begin{array}{llll}\text { C } & 0.7257160 & 1.7159240 & -2.7785460\end{array}$

$\mathrm{H} \quad 0.1230170 \quad 0.8337230 \quad-2.5719470$

$\mathrm{H} \quad 0.2615610 \quad 2.2544860-3.6112950$

$\mathrm{H} \quad 1.7160200 \quad 1.3812530 \quad-3.1094650$

$6 . \log$

Potential Energy $=-1949.24520$

Zero-point Energy = -1948.77947

Free Energy $=-1948.82349$

Single-Point Energy B3LYP-D3(BJ)/6-311+G** PCM = 1949.74218

Free Energy B3LYP-D3(BJ)/6-311+G** PCM (extrapolated free energy from $q R R H O)=-1949.32047$

Nimag $=1\left(-184.3497 \mathrm{~cm}^{-1}\right)$

Charge $=0$ Multiplicity $=1$

$\begin{array}{lrrr}\mathrm{C} & 0.7139820 & -0.3444080 & -1.0328300 \\ \mathrm{H} & 1.1607050 & 0.2641110 & -0.2446750 \\ \mathrm{O} & 0.1745010 & 0.2686700 & -2.0208860 \\ \mathrm{C} & 1.4989440 & -1.6141770 & -1.2925640 \\ \mathrm{C} & 2.9902150 & -1.2552330 & -1.5667990 \\ \mathrm{H} & 3.0289940 & -0.5430540 & -2.3963320\end{array}$




\begin{tabular}{|c|c|c|c|}
\hline $\mathrm{C}$ & -0.6336890 & 2.9344980 & -0.3845530 \\
\hline $\mathrm{C}$ & -0.9798820 & 4.4469490 & -0.2338760 \\
\hline $\mathrm{O}$ & -0.6765710 & 2.2228750 & 0.6273310 \\
\hline $\mathrm{O}$ & -0.3415570 & 2.6140260 & -1.5810210 \\
\hline $\mathrm{H}$ & -0.0640200 & 1.3257380 & -1.7972240 \\
\hline $\mathrm{F}$ & 0.0446560 & 5.2271940 & -0.6564210 \\
\hline $\mathrm{F}$ & -1.2498430 & 4.7920110 & 1.0396640 \\
\hline $\mathrm{F}$ & -2.0661670 & 4.7687800 & -0.9797120 \\
\hline $\mathrm{H}$ & 1.4341980 & -2.2911070 & -0.4413460 \\
\hline $\mathrm{Cl}$ & 0.8724320 & -2.5388000 & -2.7241510 \\
\hline $\mathrm{N}$ & -0.7753960 & -0.7079250 & 0.3613030 \\
\hline $\mathrm{C}$ & -0.3565940 & -1.4767910 & 1.5731260 \\
\hline $\mathrm{C}$ & -2.2759000 & -0.9105740 & 0.2128410 \\
\hline $\mathrm{C}$ & -1.6422120 & -1.5178300 & 2.4088140 \\
\hline $\mathrm{H}$ & -0.0907440 & -2.4984460 & 1.2829840 \\
\hline $\mathrm{H}$ & -2.6930430 & 0.0426090 & -0.1274340 \\
\hline $\mathrm{O}$ & -1.7137200 & -1.8106420 & 3.6032280 \\
\hline $\mathrm{C}$ & -3.9657770 & -0.7583660 & 2.1843450 \\
\hline $\mathrm{H}$ & -4.6202530 & -1.6258140 & 2.3016600 \\
\hline $\mathrm{H}$ & -3.8033360 & -0.3091940 & 3.1666070 \\
\hline $\mathrm{H}$ & -4.4518140 & -0.0317080 & 1.5320780 \\
\hline $\mathrm{N}$ & -2.6666820 & -1.1186880 & 1.6205320 \\
\hline $\mathrm{H}$ & -0.7146040 & 0.2892960 & 0.6143910 \\
\hline $\mathrm{C}$ & 0.7886630 & -0.8343910 & 2.3516020 \\
\hline $\mathrm{H}$ & 0.9252720 & -1.3859120 & 3.2847560 \\
\hline $\mathrm{H}$ & 1.7311070 & -0.8691160 & 1.8017790 \\
\hline $\mathrm{H}$ & 0.5613410 & 0.2066040 & 2.6014610 \\
\hline $\mathrm{C}$ & -2.7668450 & -2.0239320 & -0.7639550 \\
\hline $\mathrm{C}$ & -4.2795100 & -2.2596000 & -0.5445260 \\
\hline $\mathrm{H}$ & -4.4880200 & -2.7492250 & 0.4095530 \\
\hline $\mathrm{H}$ & -4.8540150 & -1.3283180 & -0.5947840 \\
\hline $\mathrm{H}$ & -4.6509950 & -2.9161530 & -1.3375730 \\
\hline $\mathrm{C}$ & -2.0387140 & -3.3626440 & -0.5453170 \\
\hline $\mathrm{H}$ & -2.4605280 & -4.1201490 & -1.2140120 \\
\hline $\mathrm{H}$ & -0.9721060 & -3.2962140 & -0.7673530 \\
\hline $\mathrm{H}$ & -2.1670220 & -3.7266290 & 0.4797440 \\
\hline $\mathrm{C}$ & -2.5925200 & -1.5232120 & -2.2121600 \\
\hline $\mathrm{H}$ & -1.5607420 & -1.2760240 & -2.4537310 \\
\hline $\mathrm{H}$ & -2.9271890 & -2.2972810 & -2.9106310 \\
\hline $\mathrm{H}$ & -3.2055440 & -0.6308350 & -2.3860990 \\
\hline $\mathrm{H}$ & 3.4633260 & -2.1792750 & -1.9095500 \\
\hline $\mathrm{C}$ & 3.7394860 & -0.7181530 & -0.3622390 \\
\hline $\mathrm{C}$ & 3.9569610 & 0.6583600 & -0.1975490 \\
\hline $\mathrm{C}$ & 4.2510010 & -1.6005490 & 0.6031610 \\
\hline $\mathrm{C}$ & 4.6575450 & 1.1436880 & 0.9112190 \\
\hline $\mathrm{H}$ & 3.5867250 & 1.3561650 & -0.9444200 \\
\hline $\mathrm{C}$ & 4.9516490 & -1.1186370 & 1.7118450 \\
\hline $\mathrm{H}$ & 4.1078470 & -2.6716250 & 0.4827900 \\
\hline $\mathrm{C}$ & 5.1543470 & 0.2563400 & 1.8703780 \\
\hline $\mathrm{H}$ & 4.8183770 & 2.2121130 & 1.0208060 \\
\hline $\mathrm{H}$ & 5.3425990 & -1.8158260 & 2.4470770 \\
\hline $\mathrm{H}$ & 5.7007660 & 0.6318370 & 2.7304190 \\
\hline
\end{tabular}

7.log

Potential Energy $=-1949.24378$

Zero-point Energy $=-1948.77757$

Free Energy $=-1948.82128$

Single-Point Energy B3LYP-D3(BJ)/6-311+G** PCM = 1949.74017

Free Energy B3LYP-D3(BJ)/6-311+G** PCM (extrapolated free energy from $\mathrm{qRRHO})=-1949.31767$

Nimag $=1\left(-166.7933 \mathrm{~cm}^{-1}\right)$

Charge $=0$ Multiplicity $=1$

$\begin{array}{rrrr}\text { C } & 0.6752510 & -0.6500430 & 0.1739330 \\ \text { H } & 0.7524660 & 0.0164450 & 1.0346350 \\ \text { O } & 0.9418270 & -0.1531300 & -0.9781810 \\ \text { C } & 1.1078590 & -2.0690590 & 0.4975860 \\ \text { C } & 2.3932850 & -2.0672730 & 1.3750050 \\ \text { C } & 0.0470380 & 2.8675910 & -0.2387100 \\ \text { C } & 0.0695870 & 4.4207630 & -0.3729760 \\ \text { O } & -0.7258530 & 2.3668250 & 0.5899550 \\ \text { O } & 0.8548910 & 2.2926090 & -1.0342230 \\ \text { H } & 0.8842690 & 0.9437210 & -0.9913280 \\ \text { F } & 1.3206460 & 4.9104360 & -0.1958450 \\ \text { F } & -0.7312860 & 5.0307070 & 0.5219870 \\ \text { F } & -0.3409940 & 4.8012930 & -1.6089070\end{array}$

$\begin{array}{lrrr}\mathrm{H} & 0.3316210 & -2.5759320 & 1.0695100 \\ \mathrm{Cl} & 1.3425340 & -3.0769260 & -0.9922950 \\ \mathrm{~N} & -1.3491250 & -0.4953750 & 0.4685840 \\ \mathrm{C} & -1.8539370 & -1.0804500 & 1.7477600 \\ \mathrm{C} & -2.5180530 & -0.5038380 & -0.5044690 \\ \mathrm{C} & -3.3533010 & -0.7598440 & 1.6943160 \\ \mathrm{H} & -1.7295270 & -2.1680070 & 1.7252370 \\ \mathrm{H} & -2.4501200 & 0.4170960 & -1.0922010 \\ \mathrm{O} & -4.1298460 & -0.7960150 & 2.6492880 \\ \mathrm{C} & -4.9220910 & 0.2719630 & 0.1123960 \\ \mathrm{H} & -5.7045770 & -0.4461960 & -0.1447110 \\ \mathrm{H} & -5.2440850 & 0.8398520 & 0.9877850 \\ \mathrm{H} & -4.7758300 & 0.9545830 & -0.7260550 \\ \mathrm{~N} & -3.6538350 & -0.3809090 & 0.4302040 \\ \mathrm{H} & -1.2112200 & 0.5124210 & 0.6410390 \\ \mathrm{C} & -1.1978270 & -0.5060550 & 3.0005800 \\ \mathrm{H} & -1.7371390 & -0.8768600 & 3.8752000 \\ \mathrm{H} & -0.1550470 & -0.8163270 & 3.0967720 \\ \mathrm{H} & -1.2433390 & 0.5872650 & 3.0089200 \\ \mathrm{C} & -2.6246200 & -1.6975640 & -1.5042260 \\ \mathrm{C} & -4.0150670 & -1.6651450 & -2.1803630 \\ \mathrm{H} & -4.8168420 & -1.9379010 & -1.4901590 \\ \mathrm{H} & -4.2406760 & -0.6838090 & -2.6114720 \\ \mathrm{H} & -4.0253310 & -2.3922660 & -2.9983330 \\ \mathrm{C} & -2.4588090 & -3.0654050 & -0.8176430 \\ \mathrm{H} & -2.6088240 & -3.8622280 & -1.5533240 \\ \mathrm{H} & -1.4600760 & -3.2017980 & -0.3981150 \\ \mathrm{H} & -3.2006050 & -3.2115760 & -0.0252960 \\ \mathrm{C} & -1.5739780 & -1.5058000 & -2.6169670 \\ \mathrm{H} & -0.5546100 & -1.4746180 & -2.2369860 \\ \mathrm{H} & -1.6426040 & -2.3325370 & -3.3316150 \\ \mathrm{H} & -1.7602350 & -0.5751660 & -3.1660400 \\ \mathrm{H} & 2.6130250 & -3.1168820 & 1.5899040 \\ \mathrm{H} & 2.1166040 & -1.6063460 & 2.3302920 \\ \mathrm{C} & 3.6184810 & -1.3754110 & 0.8116440 \\ \mathrm{C} & 4.5142710 & -2.0620070 & -0.0234000 \\ \mathrm{C} & 3.8999310 & -0.0398910 & 1.1401470 \\ \mathrm{C} & 5.6476800 & -1.4238870 & -0.5347400 \\ \mathrm{H} & 4.3274120 & -3.1027050 & -0.2701960 \\ \mathrm{C} & 5.0325510 & 0.6017180 & 0.6302610 \\ \mathrm{H} & 3.2379930 & 0.5029280 & 1.8104190 \\ \mathrm{C} & 5.9082880 & -0.0879690 & -0.2131530 \\ \mathrm{H} & 6.3293840 & -1.9723900 & -1.1783560 \\ \mathrm{H} & 5.2322290 & 1.6351730 & 0.8980480 \\ \mathrm{H} & 6.7906450 & 0.4071950 & -0.6077890\end{array}$

$8 . \log$

Potential Energy $=-1949.25022$

Zero-point Energy $=-1948.785715$

Free Energy $=-1948.82956$

Single-Point Energy B3LYP-D3(BJ)/6-311+G** PCM = 1949.74546

Free Energy B3LYP-D3(BJ)/6-311+G** PCM (extrapolated free energy from $\mathrm{qRRHO})=-1949.32480$

Nimag $=1\left(-381.4409 \mathrm{~cm}^{-1}\right)$ Charge $=0$ Multiplicity $=1$

$\begin{array}{lrrr}\mathrm{C} & 0.5113790 & 2.3894900 & -1.8330060 \\ \mathrm{C} & -0.4357110 & 2.3747420 & 0.3008610 \\ \mathrm{C} & -0.6177670 & 1.3674950 & -1.8331020 \\ \mathrm{H} & -1.3019540 & 3.0344680 & 0.1323670 \\ \mathrm{H} & -0.3165630 & 0.4604450 & -2.3572620 \\ \mathrm{C} & 0.0135680 & -0.7607400 & -0.0582360 \\ \mathrm{H} & -0.2790290 & -0.7283410 & 0.9957960 \\ \mathrm{O} & -0.6626630 & -1.4858070 & -0.8595100 \\ \mathrm{~N} & 0.6742930 & 2.8427850 & -0.5521700 \\ \mathrm{C} & 1.4759140 & 4.0371800 & -0.3040440 \\ \mathrm{H} & 2.1263050 & 3.9047380 & 0.5610270 \\ \mathrm{H} & 2.0932140 & 4.1968150 & -1.1882330 \\ \mathrm{H} & 0.8404300 & 4.9161070 & -0.1525930 \\ \mathrm{O} & 1.1352120 & 2.7640380 & -2.8243990 \\ \mathrm{C} & 1.5321930 & -0.6995040 & -0.2302080 \\ \mathrm{C} & 2.1565290 & -1.8598560 & 0.5839970 \\ \mathrm{H} & 1.7599960 & -2.8040380 & 0.1989230 \\ \mathrm{H} & 1.7952040 & -1.7565330 & 1.6146950 \\ \mathrm{~N} & -0.7604260 & 1.0723340 & -0.3779680 \\ \mathrm{C} & -3.7217120 & -1.2779530 & 0.0419550\end{array}$




$\begin{array}{lrrr}\mathrm{C} & -5.1433020 & -1.8425490 & 0.3269950 \\ \mathrm{O} & -3.5313730 & -0.0668710 & 0.0939920 \\ \mathrm{H} & -1.7289560 & 0.8000970 & -0.1671330 \\ \mathrm{O} & -2.8798140 & -2.2149840 & -0.2291290 \\ \mathrm{H} & -1.8270460 & -1.8326380 & -0.4976910 \\ \mathrm{C} & -0.2551260 & 2.3478960 & 1.8483900 \\ \mathrm{C} & -0.2880900 & 3.8034570 & 2.3814370 \\ \mathrm{H} & -0.3667560 & 3.7788920 & 3.4725630 \\ \mathrm{H} & 0.6112580 & 4.3697100 & 2.1353440 \\ \mathrm{H} & -1.1552770 & 4.3519990 & 1.9982040 \\ \mathrm{C} & 1.0352580 & 1.6651210 & 2.3352760 \\ \mathrm{H} & 1.1591830 & 1.8498380 & 3.4069570 \\ \mathrm{H} & 1.9266570 & 2.0474040 & 1.8300860 \\ \mathrm{C} & -1.4854310 & 1.6292560 & 2.4498420 \\ \mathrm{H} & -1.4337880 & 1.6793220 & 3.5414850 \\ \mathrm{H} & -2.4207920 & 2.1067920 & 2.1382750 \\ \mathrm{H} & -1.5390050 & 0.5723350 & 2.1762990 \\ \mathrm{C} & -1.8877010 & 1.9295770 & -2.4907840 \\ \mathrm{H} & -1.6726950 & 2.1645640 & -3.5366070 \\ \mathrm{H} & -2.6853540 & 1.1829690 & -2.4606870 \\ \mathrm{H} & -2.2425030 & 2.8415650 & -2.0013210 \\ \mathrm{H} & 1.0019670 & 0.5816810 & 2.2071570 \\ \mathrm{~F} & -5.1180430 & -2.7243360 & 1.3535200 \\ \mathrm{~F} & -6.0148630 & -0.8697530 & 0.6450270 \\ \mathrm{~F} & -5.6288670 & -2.4922470 & -0.7571310 \\ \mathrm{C} & 3.6720180 & -1.8899080 & 0.5857330 \\ \mathrm{C} & 4.4092590 & -1.0116910 & 1.3957050 \\ \mathrm{C} & 4.3679640 & -2.8097270 & -0.2119970 \\ \mathrm{C} & 5.8065070 & -1.0424910 & 1.3971830 \\ \mathrm{H} & 3.8929710 & -0.3038610 & 2.0388660 \\ \mathrm{C} & 5.7659000 & -2.8458850 & -0.2108090 \\ \mathrm{H} & 3.8126000 & -3.5032080 & -0.8373770 \\ \mathrm{C} & 6.4900080 & -1.9595810 & 0.5918470 \\ \mathrm{H} & 6.3594900 & -0.3564130 & 2.0323220 \\ \mathrm{H} & 6.2863720 & -3.5667080 & -0.8348330 \\ \mathrm{H} & 7.5757400 & -1.9867970 & 0.5952690 \\ \mathrm{H} & 1.9227920 & 0.2586540 & 0.1060780 \\ \mathrm{Cl} & 2.0213880 & -0.8309190 & -1.9799760\end{array}$

$9 . \log$

Potential Energy = -1949.24902

Zero-point Energy $=-1948.78464$

Free Energy $=-1948.82845$

Single-Point Energy B3LYP-D3(BJ)/6-311+G** PCM = -

1949.74601

Free Energy B3LYP-D3(BJ)/6-311+G** PCM (extrapolated

free energy from $\mathrm{qRRHO})=-1949.32543$

Nimag $=1\left(-453.2927 \mathrm{~cm}^{-1}\right)$

Charge $=0$ Multiplicity $=1$

$\begin{array}{lrrr}\mathrm{C} & -1.5214730 & -3.1195530 & -0.6560270 \\ \mathrm{C} & -0.9135380 & -1.9030380 & 1.2401530 \\ \mathrm{C} & -0.1401490 & -2.5299160 & -0.9057060 \\ \mathrm{H} & -0.2814690 & -2.6478690 & 1.7503390 \\ \mathrm{H} & -0.0684390 & -2.1323290 & -1.9177700 \\ \mathrm{C} & -0.2336920 & 0.3209230 & -0.9920280 \\ \mathrm{H} & -0.0678050 & 0.9114280 & -0.0871150 \\ \mathrm{O} & 0.7256320 & 0.1814740 & -1.8224700 \\ \mathrm{~N} & -1.9887090 & -2.6209230 & 0.5286920 \\ \mathrm{C} & -3.1491770 & -3.2365470 & 1.1645650 \\ \mathrm{H} & -3.8366170 & -2.4826820 & 1.5493730 \\ \mathrm{H} & -3.6593330 & -3.8219990 & 0.3994970 \\ \mathrm{H} & -2.8466500 & -3.9045870 & 1.9778390 \\ \mathrm{O} & -2.0903700 & -3.9353910 & -1.3798080 \\ \mathrm{C} & -1.6428670 & 0.5025530 & -1.5429140 \\ \mathrm{C} & -1.8489010 & 1.9765280 & -1.9908940 \\ \mathrm{H} & -1.0795060 & 2.2187940 & -2.7299450 \\ \mathrm{~N} & -0.1069820 & -1.4030850 & 0.0718620 \\ \mathrm{C} & 3.4071860 & 0.0397090 & -0.0875220 \\ \mathrm{C} & 4.8992390 & 0.3590610 & 0.2179620 \\ \mathrm{O} & 2.8002070 & -0.7472720 & 0.6335900 \\ \mathrm{H} & 0.8606840 & -1.2303830 & 0.3725520 \\ \mathrm{O} & 2.9812490 & 0.6778560 & -1.1208000 \\ \mathrm{H} & 1.8798750 & 0.4297330 & -1.4135200 \\ \mathrm{C} & -1.2841830 & -0.8729790 & 2.3479150 \\ \mathrm{C} & -1.7747320 & -1.6417540 & 3.6019530 \\ \mathrm{H} & -1.8411050 & -0.9429930 & 4.4414470\end{array}$

$\begin{array}{cccc}\mathrm{H} & -2.7629850 & -2.0851370 & 3.4726690 \\ \mathrm{H} & -1.0759220 & -2.4362570 & 3.8855230 \\ \mathrm{C} & -2.3525200 & 0.1539910 & 1.9361310 \\ \mathrm{H} & -2.6803740 & 0.7086260 & 2.8211590 \\ \mathrm{H} & -3.2370520 & -0.3158910 & 1.4963470 \\ \mathrm{C} & 0.0130740 & -0.1390610 & 2.7604700 \\ \mathrm{H} & -0.2015190 & 0.5235850 & 3.6041690 \\ \mathrm{H} & 0.7894230 & -0.8430810 & 3.0791410 \\ \mathrm{H} & 0.4262610 & 0.4804700 & 1.9602840 \\ \mathrm{C} & 0.9654620 & -3.5774620 & -0.7004850 \\ \mathrm{H} & 0.8229600 & -4.3892160 & -1.4188470 \\ \mathrm{H} & 1.9450190 & -3.1264290 & -0.8775440 \\ \mathrm{H} & 0.9543080 & -4.0056420 & 0.3062650 \\ \mathrm{H} & -1.9680260 & 0.8924480 & 1.2312620 \\ \mathrm{~F} & 5.0901550 & 1.6891950 & 0.3787820 \\ \mathrm{~F} & 5.3261610 & -0.2526700 & 1.3365710 \\ \mathrm{~F} & 5.6939620 & -0.0465170 & -0.8012280 \\ \mathrm{H} & -2.3895060 & 0.2158170 & -0.8063690 \\ \mathrm{Cl} & -1.9580920 & -0.5586410 & -2.9935230 \\ \mathrm{H} & -2.8147910 & 2.0092790 & -2.5023940 \\ \mathrm{C} & -1.8410400 & 2.9932100 & -0.8638980 \\ \mathrm{C} & -0.6785860 & 3.7114480 & -0.5435760 \\ \mathrm{C} & -3.0125590 & 3.2529960 & -0.1351290 \\ \mathrm{C} & -0.6817280 & 4.6549510 & 0.4884900 \\ \mathrm{H} & 0.2330870 & 3.5407570 & -1.1105550 \\ \mathrm{C} & -3.0199280 & 4.1959990 & 0.8960860 \\ \mathrm{H} & -3.9269210 & 2.7183010 & -0.3806250 \\ \mathrm{C} & -1.8524550 & 4.8981170 & 1.2131800 \\ \mathrm{H} & 0.2270990 & 5.2027360 & 0.7201450 \\ \mathrm{H} & -3.9367790 & 4.3856130 & 1.4467150 \\ \mathrm{H} & -1.8573700 & 5.6334180 & 2.0124100\end{array}$

10.log

Potential Energy $=-1949.24828$

Zero-point Energy = -1948.78385

Free Energy = -1948.82750

Single-Point Energy B3LYP-D3(BJ)/6-311+G** PCM = -

1949.74475

Free Energy B3LYP-D3(BJ)/6-311+G** PCM (extrapolated

free energy from $q R R H O)=-1949.32397$

Nimag $=1\left(-533.9049 \mathrm{~cm}^{-1}\right)$

Charge $=0$ Multiplicity $=1$

$\begin{array}{lrrr}\mathrm{C} & 3.1105990 & -0.5891380 & -1.5764200 \\ \mathrm{C} & 2.7654150 & 0.5519640 & 0.4332690 \\ \mathrm{C} & 1.8510890 & 0.2519050 & -1.7318080 \\ \mathrm{H} & 3.2081330 & 1.5316230 & 0.1907390 \\ \mathrm{H} & 1.0685650 & -0.3165230 & -2.2351290 \\ \mathrm{C} & -0.1719290 & -0.6699280 & 0.0412660 \\ \mathrm{H} & -0.2702150 & -0.2611160 & 1.0504640 \\ \mathrm{O} & -0.9765110 & -0.2706630 & -0.8637940 \\ \mathrm{~N} & 3.5296740 & -0.4917140 & -0.2777750 \\ \mathrm{C} & 4.8716910 & -0.9379430 & 0.0842810 \\ \mathrm{H} & 4.8628760 & -1.5075260 & 1.0139780 \\ \mathrm{H} & 5.2222060 & -1.5831640 & -0.7214070 \\ \mathrm{H} & 5.5569860 & -0.0893320 & 0.1824370 \\ \mathrm{O} & 3.6680490 & -1.2123160 & -2.4783790 \\ \mathrm{C} & 0.2732070 & -2.1292750 & 0.0491580 \\ \mathrm{C} & -0.5983710 & -2.9795080 & 1.0136650 \\ \mathrm{~N} & 1.4601320 & 0.4828650 & -0.3113630 \\ \mathrm{C} & -1.5114290 & 2.8287570 & -0.3068340 \\ \mathrm{C} & -2.3983320 & 4.1040390 & -0.2070370 \\ \mathrm{O} & -0.2932690 & 2.9341030 & -0.1821090 \\ \mathrm{H} & 0.9598380 & 1.3768130 & -0.2222830 \\ \mathrm{O} & -2.2112050 & 1.7714220 & -0.5165380 \\ \mathrm{H} & -1.5742250 & 0.7824280 & -0.6540990 \\ \mathrm{C} & 2.6998910 & 0.5178630 & 1.9902780 \\ \mathrm{C} & 4.0708280 & 0.9658520 & 2.5595340 \\ \mathrm{H} & 3.9677790 & 1.1392610 & 3.6350500 \\ \mathrm{H} & 4.8542960 & 0.2183640 & 2.4270180 \\ \mathrm{H} & 4.4084910 & 1.9024080 & 2.1028020 \\ \mathrm{C} & 2.3334040 & -0.8523000 & 2.5883890 \\ \mathrm{H} & 2.4828780 & -0.8241270 & 3.6723240 \\ \mathrm{H} & 2.9519870 & -1.6622170 & 2.1916370 \\ \mathrm{C} & 1.6677230 & 1.5777290 & 2.4403850 \\ \mathrm{H} & 1.6666220 & 1.6379900 & 3.5327380 \\ \mathrm{H} & 1.9171260 & 2.5710430 & 2.0519490\end{array}$




$\begin{array}{crrr}\mathrm{H} & 0.6475580 & 1.3407670 & 2.1277560 \\ \mathrm{C} & 2.1282840 & 1.5362320 & -2.5289570 \\ \mathrm{H} & 2.4625090 & 1.2662080 & -3.5342340 \\ \mathrm{H} & 1.2130440 & 2.1272610 & -2.6151630 \\ \mathrm{H} & 2.9024710 & 2.1550150 & -2.0655210 \\ \mathrm{H} & 1.2852660 & -1.1081850 & 2.4251080 \\ \mathrm{~F} & -3.2878190 & 3.9949990 & 0.8085400 \\ \mathrm{~F} & -1.6677240 & 5.2123430 & 0.0088790 \\ \mathrm{~F} & -3.1036250 & 4.2935450 & -1.3472090 \\ \mathrm{H} & 1.3058380 & -2.2023560 & 0.3833770 \\ \mathrm{Cl} & 0.2691760 & -2.8510260 & -1.6231840 \\ \mathrm{H} & -0.1841790 & -3.9916060 & 0.9862640 \\ \mathrm{H} & -0.4090340 & -2.5950440 & 2.0225660 \\ \mathrm{C} & -2.0920220 & -3.0177510 & 0.7597090 \\ \mathrm{C} & -2.9518120 & -2.1143220 & 1.4040930 \\ \mathrm{C} & -2.6522870 & -3.9769510 & -0.0984570 \\ \mathrm{C} & -4.3313490 & -2.1522970 & 1.1819960 \\ \mathrm{H} & -2.5437270 & -1.3789350 & 2.0931600 \\ \mathrm{C} & -4.0313360 & -4.0183000 & -0.3234290 \\ \mathrm{H} & -2.0066350 & -4.6990220 & -0.5893570 \\ \mathrm{C} & -4.8755280 & -3.1031200 & 0.3132650 \\ \mathrm{H} & -4.9786820 & -1.4443730 & 1.6915200 \\ \mathrm{H} & -4.4453950 & -4.7689110 & -0.9905780 \\ \mathrm{H} & -5.9474310 & -3.1367670 & 0.1415870\end{array}$




\subsection{Ion Mobility-Mass Spectrometry with Aminals ( $2^{\text {nd }}$ Generation MacMillan Catalyst)}

Diastereomeric aminals were dissolved in acetonitrile to yield pure and 1:1 mixed solutions. Arrival time distributions (ATDs) and mass spectra of iminium ions were recorded on a home-built drift-tube ion mobility instrument which is described in detail elsewhere. ${ }^{30,31}$ Briefly, iminium ions in the gas phase were generated by nanoelectrospray ionization followed by in-source fragmentation of intact aminal precursors. Subsequently, the ions are injected to the drift tube $(161.2 \mathrm{~cm})$ using helium at ca. $4.00 \mathrm{mbar}$ as drift gas. Drift times were recorded at 14 different drift voltages from 2300 to $1000 \mathrm{~V}$ in $100 \mathrm{~V}$ steps. After transfer of the ions for mbar pressure to high vacuum, the ions are selected by their mass-tocharge ratio in a quadrupole mass filter. The ATDs are record using an electron multiplier detector which is on axis to the drift tube. For mass spectral analysis, the ions are pulsed off-axis to a Wiley-McLaren time-of-flight mass analyser. From the drift times, the instrument-independent collision cross sections (CCSs) can be derived using the Mason-Schamp equation. ${ }^{32,33}$ Arrival time distributions (ATDs) of iminium ions generated from both diastereomeric aminals and their mixture are shown below.<smiles>CN1C(=O)[C@@H](Br)N(C([C@H](Cl)Br)N2C(=O)CCC2=O)C1Br</smiles>

$(1 S, 2 R)-s y n-25$<smiles>CN1C(=O)[C@@H](Br)N([C@@H]([C@@H](Br)c2ccccc2)[C@H](Cl)Br)C1=O</smiles>

$(1 S, 2 S)$-anti-25
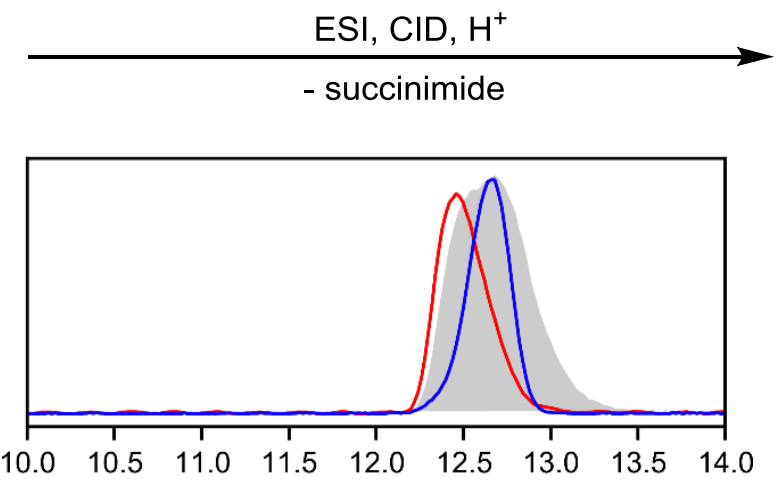

Drift Time [ms]

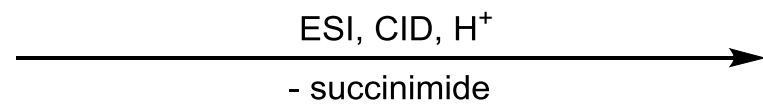<smiles>CN1C(=O)[C@H](Br)[N+](=CC(Cl)Br)[C@@H]1[13CH3]</smiles>

$E-(R)-26$

$127 \AA^{2}$<smiles>CN1C(=O)[C@H](Br)[C@H](Br)[N+]1=C[C@H](Cl)Br</smiles>

$E-(S)-26$

$124 \AA^{2}$

Figure SI-17. Ionization of aminals syn-25 and anti-25 and arrival time distributions of diastereomeric $E-(R)$ - (blue trace) and $E-(S)$-iminium ions (red trace). The trace of the mixture of both diastereomers is depicted in light grey. 


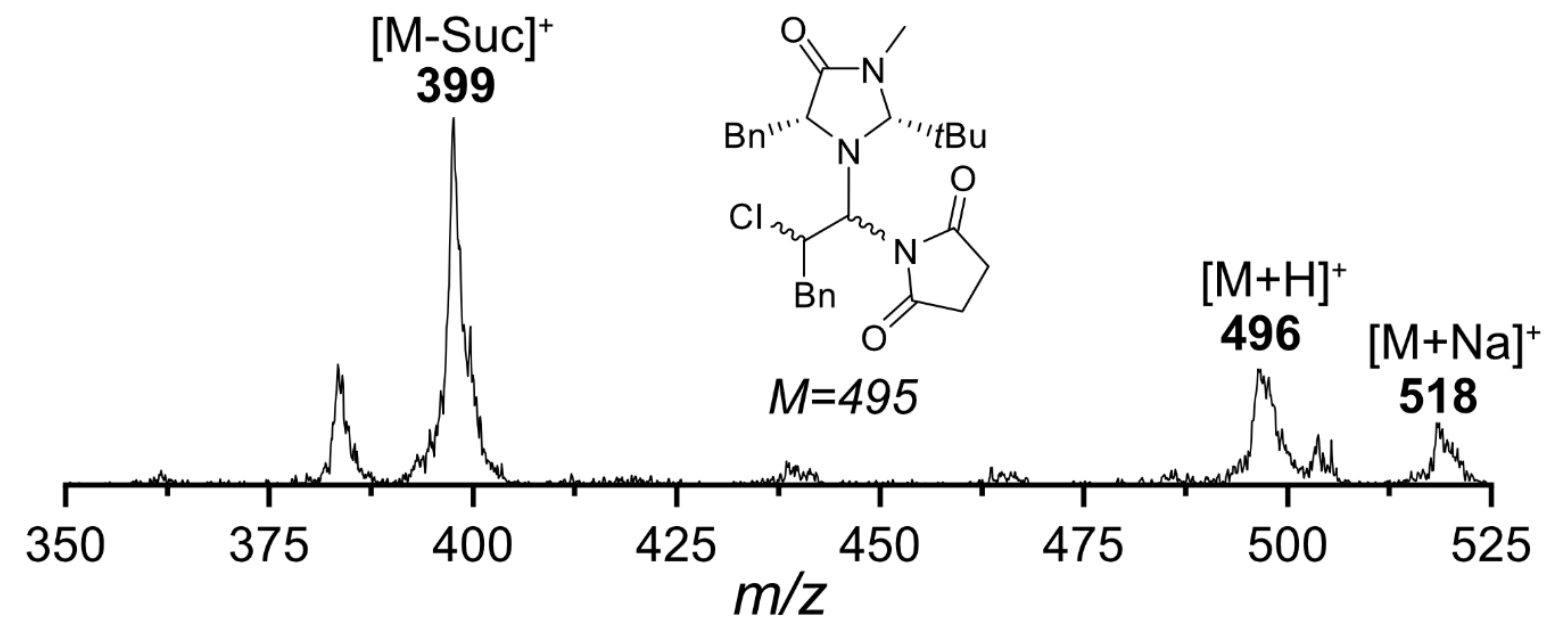

Figure SI-18. Mass spectra of iminium ions $(m / z=399)$ generated from aminal precursor ions $[\mathrm{M}+\mathrm{H}]^{+}$ $(m / z=496)$ and $[\mathrm{M}+\mathrm{Na}]^{+}(m / z=518)$.

\section{Computational Methods}

For each iminium ion $(E-(R), E-(S), Z-(R)$ and $Z-(S))$ the conformational space was sampled by using a genetic algorithm (GA) FAFOOM. ${ }^{34}$ In this case, all rotatable bonds were sampled, generating a pool of starting structures, which are automatically optimized with $\mathrm{FHI}$-aims ${ }^{35}$ (version 171221) at the dispersion corrected density functional PBE+vdW' ${ }^{\top S} 36,37$ level of theory using light basis set settings. For each type of isomers, ten GA runs were performed that yielded ca. 120 structures for each candidate. From that pool of structures, a certain number of distinct low-energy structures for each isomer were reoptimized, frequencies and Merz-Singh-Kollman ${ }^{38}$ charges calculated at the hybrid-DFT level PBE0+D3/6$311+G(d, p)^{39}$ using default settings in Gaussian 16, Revision A.03. ${ }^{14}$ The reoptimized structures and computed Merz-Singh-Kollman were used as input to compute theoretical CCS values using the trajectory method using the software hpccs. ${ }^{40}$ The calculation was carried out in helium as drift gas at 298.15 K. Default settings were used except for the number of points in velocity integration (60) and the number of points in Monte Carlo integrations of impact parameters and orientation (750). Computed energies plus zero-point vibrational energy, free energies at $298.15 \mathrm{~K}$ and computed CCSs are shown in Table SI-13. 3D-structures of each lowest-energy isomer are shown below and their xyz-coordinates can be found at the end of this chapter. 
Table SI-13. List of conformations reoptimized at PBE0+D3/6-311+G(d,p) level of theory. Energies $(\Delta E$, including zero-point-vibrational energy), free energies $(\Delta \mathrm{F})$ at $298.15 \mathrm{~K}$ and collision cross sections calculated with the trajectory method (CCS $\left.{ }^{\mathrm{TM}}\right)$ in He are assigned to each structure. The lowest-energy conformers for each isomer are labelled with an asterisk $\left({ }^{*}\right)$.

\begin{tabular}{|c|c|c|c|}
\hline \multirow[b]{2}{*}{ ID } & \multicolumn{3}{|c|}{$\Delta \mathrm{F}(\mathrm{PBE} 0+\mathrm{D} 3)$} \\
\hline & $\Delta \mathrm{E}(\mathrm{PBE} 0+\mathrm{D} 3)\left[\mathrm{kcal} \mathrm{mol}^{-1}\right]$ & {$\left[\mathrm{kcal} \mathrm{mol}^{-1}\right]$} & $\operatorname{CCS}^{\mathrm{TM}}\left[\AA^{2}\right]$ \\
\hline trans_S/conf_00* & 0.0 & 0.0 & 123 \\
\hline trans_S/conf_01 & 1.4 & 0.5 & 124 \\
\hline trans_S/conf_02 & 3.6 & 3.8 & 125 \\
\hline trans_S/conf_03 & 3.7 & 2.8 & 127 \\
\hline trans_S/conf_04 & 5.9 & 4.5 & 129 \\
\hline trans_S/conf_05 & 6.6 & 5.1 & 129 \\
\hline trans_R/conf_00* & 2.4 & 1.8 & 128 \\
\hline trans_R/conf_01 & 2.4 & 2.1 & 125 \\
\hline trans_R/conf_02 & 4.4 & 5.3 & 123 \\
\hline trans_R/conf_03 & 5.3 & 4.4 & 131 \\
\hline trans_R/conf_04 & 5.5 & 5.1 & 127 \\
\hline cis_S/conf_00* & 4.5 & 4.1 & 126 \\
\hline cis_S/conf_01 & 5.7 & 5.2 & 128 \\
\hline cis_S/conf_02 & 6.2 & 4.9 & 133 \\
\hline cis_S/conf_03 & 7.9 & 8.3 & 123 \\
\hline cis_S/conf_04 & 7.1 & 7.1 & 123 \\
\hline cis_R/conf_00* & 1.4 & 0.8 & 123 \\
\hline cis_R/conf_01 & 5.0 & 4.2 & 124 \\
\hline cis_R/conf_02 & 6.0 & 6.0 & 123 \\
\hline cis_R/conf_03 & 7.9 & 6.6 & 133 \\
\hline
\end{tabular}

a)

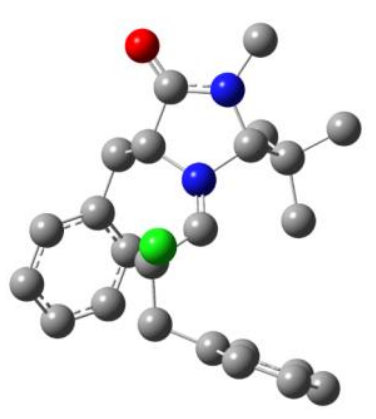

c)

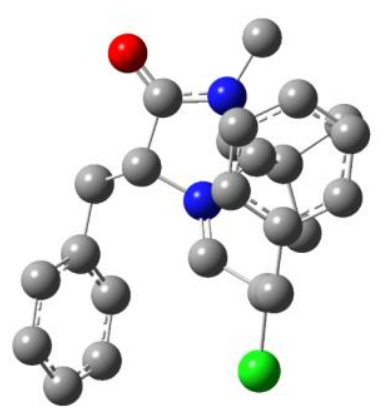

b)

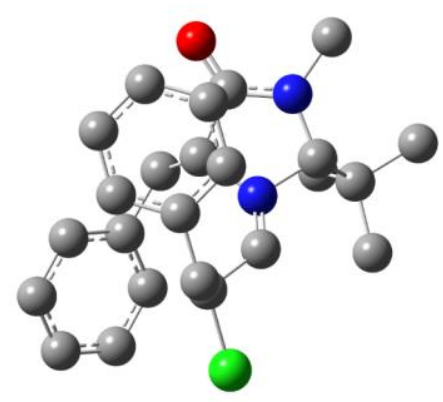

d)

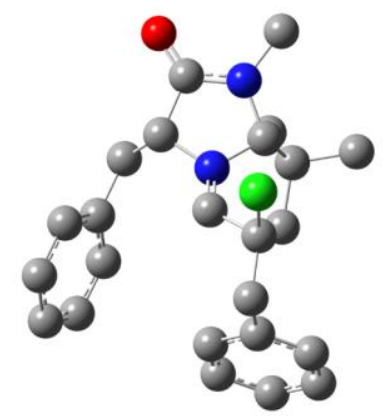

Figure SI-19. Computed lowest-energy structures for four distinct iminium ions: (a) $E-(R),(b) E-(S),(\mathrm{c})$ $Z-(R)$ and $(\mathrm{d}) Z-(S)$. Hydrogens are omitted for clarity. 
Table SI-14. xyz-Coordinates of reoptimized structures

MacMillan_Catalyst/trans_R/conf_00

Charge $=+1$,

C

C

C

C

$\mathrm{H}$

N

$\mathrm{N}$

$\mathrm{O}$

C

$\mathrm{H}$

$\mathrm{H}$

$\mathrm{H}$

C

C

$\mathrm{H}$

$\mathrm{H}$

$\mathrm{H}$

C

$\mathrm{H}$

$\mathrm{H}$

$\mathrm{H}$

C

$\mathrm{H}$

$\mathrm{H}$

$\mathrm{H}$

C

C

$\mathrm{H}$

C

$\mathrm{H}$

$\mathrm{H}$

C

C

C

C

$\mathrm{H}$

C

$\mathrm{H}$

C

$\mathrm{H}$

$\mathrm{H}$

$\mathrm{H}$

$\mathrm{Cl}$

C

C

$\mathrm{H}$

C

1.84003
1.87689
3.20941
0.57872
1.49436
1.02636
3.15318
4.16256
4.27626
4.90108
3.91977
4.88011
1.83570
2.49158
2.35714
3.56365
2.03039
0.38475
0.37340

$-0.18391$

$-0.14883$

2.57569

3.58578

2.66458

2.03820

$-0.22548$

$-1.10207$

$-0.65278$

$-2.50843$

$-2.44310$

$-3.14719$

$-3.06516$

$-3.00226$

$-3.60588$

$-3.45602$

$-2.61068$

$-4.05726$

$-3.68457$

$-3.97868$

$-3.41420$

$-4.48313$

$-4.34081$

$-1.08994$

$-0.14099$

$-1.35629$

0.26865

0.05139
0.99505

$-1.39314$

0.38309

2.65319

$-2.20115$

$-0.19435$

$-0.93582$

0.98874

$-1.81150$

$-1.31845$

$-2.76549$

$-1.98828$

$-1.89411$

$-3.28176$

$-3.72038$

$-3.24543$

$-3.96123$

$-2.05627$

$-2.48931$

$-2.73604$

$-1.10394$

$-0.96109$

$-0.73216$

$-1.43742$

$-0.02259$

$-0.31614$

0.71089

1.69874

0.78235

1.46510

1.26456

$-0.54151$

$-0.89306$

$-1.45076$

$-2.13359$

$-0.17809$

$-2.69272$

$-1.18389$

$-3.03872$

$-2.38775$

$-3.38799$

$-4.00463$

0.08438

2.19010

2.77053

1.39488

3.70581
Multiplicity $=+1$

0.73778

0.16100

0.98485

$-0.74446$

0.79753

0.46042

0.65539

1.40093

0.92437

1.66978

1.31794

0.03147

$-1.30880$

$-1.33395$

$-2.32549$

$-1.14025$

$-0.61011$

$-1.76311$

$-2.76622$

$-1.11920$

$-1.82123$

$-2.26105$

$-1.91160$

$-3.24034$

$-2.41211$

0.71021

1.32873

1.36399

0.73921

$-0.11296$

1.48379

0.29415

$-1.05507$

1.20434

$-1.48830$

$-1.77407$

0.77274

2.25304

$-0.57213$

$-2.54213$

1.48800

$-0.90682$

3.03342

$-1.84663$

$-2.19751$

$-2.46276$

0.00806 


$\begin{array}{lc}\mathrm{C} & -1.87497 \\ \mathrm{H} & -1.89064 \\ \mathrm{C} & -1.16902 \\ \mathrm{H} & 0.61337 \\ \mathrm{H} & -1.55961 \\ \mathrm{H} & -2.81795 \\ \mathrm{H} & -0.67395 \\ \mathrm{C} & 1.90178 \\ \mathrm{H} & 2.59058 \\ \mathrm{H} & 2.37666 \\ \mathrm{H} & 1.48721\end{array}$

$\begin{array}{cc}3.81130 & -1.43714 \\ 2.41892 & -3.07390 \\ 4.27651 & -0.33069 \\ 4.09914 & 0.85154 \\ 5.10148 & 0.25517 \\ 4.27042 & -1.71287 \\ -1.29503 & 0.55978 \\ 2.04431 & -0.38138 \\ 2.80653 & -0.00499 \\ 1.60581 & -1.25992 \\ 1.44767 & 1.66739\end{array}$

MacMillan_Catalyst/trans_S/conf_00

Charge $=+1$,

C

C

0.41962

2.26889

1.75472

$-1.58113$

2.54054

0.78370

2.72979

1.89818

4.07297

4.00882

4.47731

4.74258

2.75948

4.26519

4.60753

4.84539

4.50010

2.07124

2.49767

2.23377

0.99628

2.50604

2.87715

3.02445

1.44427

0.03497

$-1.42521$

05801

$-1.67126$

$-1.20116$

$-2.75031$

$-1.13248$

0.16780

$-1.88680$

0.71763

0.75041

0.36877

$-0.46636$

0.95658

Multiplicity $=+1$

0.95658
-0.95126
-0.09008

0.43457

$-1.38065$

$-1.78399$

$-0.30263$

1.42983

0.30796

0.44849

$-0.57849$

1.74227

$-2.28135$

0.99252

$-0.62465$

1.98443

1.07097

$-1.07374$

0.38337

$-1.93583$

0.38653

$-1.23482$

0.33659

$-1.93514$

0.63010

$-2.96758$

0.73142

$-1.48000$

$-0.17267$

$-1.41973$

1.56710

$-2.78487$

$-3.79052$

$-2.38976$

$-2.89864$

$-2.53977$

$-1.89740$

1.40757

1.39031

2.41690

1.23752

$-1.03969$

$-1.84304$

$-3.49834$

$-1.11770$

$-2.73809$

$-1.20398$

$-0.48273$

1.33093

$-0.23976$

1.45397

$-1.67239$

2.32485

1.05380

2.24405

0.98452

1.13186

3.22939

2.39888

2.21497

2.67500

1.45425

1.66643

2.77205

0.42037

3.64794

0.83910

2.27996

2.49572

3.74922

$-0.40351$ 


\begin{tabular}{cr}
2.43351 & 0.25816 \\
4.17713 & -0.20557 \\
4.00178 & 1.01519 \\
4.17772 & -1.20245 \\
4.92880 & -0.85710 \\
-0.20674 & 0.48701 \\
-0.06158 & -1.91293 \\
-0.45584 & -1.57791 \\
0.94368 & -2.28394 \\
-2.24765 & -1.33786 \\
-1.74859 & -1.11739 \\
0.23956 & -1.69048 \\
-2.64579 & -1.00697 \\
-2.95798 & -1.25564 \\
-3.65772 & -0.66190 \\
-2.05794 & -0.85962 \\
-0.81742 & 2.23759 \\
-0.48871 & -2.07474 \\
0.17170 & -2.94792 \\
-1.32801 & -2.30641 \\
1.19090 & -0.71267 \\
& \\
\hline
\end{tabular}

MacMillan_Catalyst/cis_R/conf_00 Charge $=+1$,

C

C

C

C

$\mathrm{H}$

N

N

$\mathrm{O}$

C

$\mathrm{H}$

$\mathrm{H}$

$\mathrm{H}$

C

C

$\mathrm{H}$

$\mathrm{H}$

$\mathrm{H}$

C

$\mathrm{H}$

$\mathrm{H}$

$\mathrm{H}$

C

$\mathrm{H}$

$\mathrm{H}$

$\mathrm{H}$

C

C

-0.34914
1.11752
0.93105
-2.82433
1.76623
-0.06297
1.72474
1.19576
3.10152
3.43423
3.71710
3.20982
0.77712
2.10648
1.89847
2.72409
2.68989
-0.09652
-0.35338
0.42715
-1.04017
0.07471
0.59086
0.04556
-0.96092
-0.63263
-0.23846

Multiplicity $=+1$ $-1.21055$ $-0.83267$ $-2.01645$ $-1.11879$ 0.03044 $-0.34109$ $-1.69737$ $-2.76155$ $-2.14299$ $-2.30245$ $-1.37308$ $-3.08369$

$-1.51543$

$-1.73471$

$-2.06654$

$-2.50062$ $-0.81060$ $-0.61756$

$-1.16520$ 0.28720

$-0.33622$

$-2.85627$

$-3.49816$

$-3.38732$

$-2.72838$

0.80074

1.91779
$-1.23991$

0.69123

$-1.34987$

$-0.96727$

0.87372

$-0.08195$

$-0.29445$

$-2.25902$

$-0.24262$

$-1.26897$

0.22477

0.30241

2.05075

2.78532

3.80540

2.31632

2.85272

2.92789

3.83791

3.25018

2.45209

1.85754

1.13934

2.81195

1.53568

0.03160

0.92622 


$\begin{array}{lc}\text { Cl } & -1.73447 \\ \mathrm{C} & 0.45283 \\ \mathrm{H} & -0.22503 \\ \mathrm{H} & 0.64435 \\ \mathrm{C} & 1.72832 \\ \mathrm{C} & 2.88929 \\ \mathrm{C} & 1.74938 \\ \mathrm{C} & 4.04439 \\ \mathrm{H} & 2.89522 \\ \mathrm{C} & 2.90079 \\ \mathrm{H} & 0.86418 \\ \mathrm{C} & 4.04882 \\ \mathrm{H} & 4.94623 \\ \mathrm{H} & 2.90654 \\ \mathrm{H} & 4.95200 \\ \mathrm{H} & 0.42086 \\ \mathrm{C} & -3.38150 \\ \mathrm{C} & -3.31429 \\ \mathrm{C} & -4.40352 \\ \mathrm{H} & -3.02461 \\ \mathrm{C} & -4.87400 \\ \mathrm{H} & -4.83216 \\ \mathrm{C} & -4.33170 \\ \mathrm{H} & -2.90648 \\ \mathrm{H} & -5.67011 \\ \mathrm{H} & -4.71125 \\ \mathrm{H} & -1.43727 \\ \mathrm{C} & -1.64331 \\ \mathrm{H} & -0.40159 \\ \mathrm{H} & -1.69932 \\ \mathrm{H} & -1.58009\end{array}$

$\begin{array}{cc}2.52456 & 1.68834 \\ 2.99433 & 0.07400 \\ 3.33234 & -0.71488 \\ 3.84757 & 0.72992 \\ 2.43297 & -0.49593 \\ 2.39682 & 0.27825 \\ 1.86739 & -1.77134 \\ 1.79708 & -0.21002 \\ 2.85834 & 1.26239 \\ 1.25969 & -2.25898 \\ 1.92465 & -2.40077 \\ 1.21951 & -1.47571 \\ 1.79319 & 0.39295 \\ 0.83126 & -3.25550 \\ 0.75798 & -1.85993 \\ 1.59985 & 1.72827 \\ -0.93287 & 0.29765 \\ -0.36597 & -2.03715 \\ -0.00920 & 0.49455 \\ -1.52475 & 1.13584 \\ 0.74421 & -0.57268 \\ 0.11883 & 1.48248 \\ 0.55958 & -1.84172 \\ -0.51564 & -3.03379 \\ 1.46473 & -0.42179 \\ 1.12989 & -2.68268 \\ 1.00664 & -0.67398 \\ -2.02644 & -1.15713 \\ -0.56442 & -2.11959 \\ -2.57349 & -2.10282 \\ -2.76935 & -0.36249\end{array}$

MacMillan_Catalyst/cis_S/conf_00 Charge $=+1$,

1.56613
-0.80711
1.28632
2.75200
-1.63748
0.20172
-0.06258
2.13246
-0.68646
0.07464
-1.50711
-1.06621
-1.36577
-2.79569
-3.23023
-2.83265
-3.43717

Multiplicity $=+1$

1.65003 2.05068

3.12435

$-0.46934$

2.09183

1.10968

3.28487

3.97689

4.58765

5.32400

4.64246

4.81662

1.66566

2.21408

2.00231

3.29639

1.74550
0.30206

$-0.09756$

0.03486

$-0.42391$

0.61480

0.45798

$-0.04360$

$-0.03395$

$-0.10740$

0.15167

0.61275

$-1.10607$

$-1.51148$

$-1.57549$

$-2.55523$

$-1.42703$

$-0.82166$ 


\begin{tabular}{|c|c|c|c|}
\hline C & 0.15098 & -1.40508 & -1.70647 \\
\hline $\mathrm{H}$ & -0.06810 & -1.84675 & -2.68126 \\
\hline $\mathrm{H}$ & -0.36403 & -2.02089 & -0.96558 \\
\hline $\mathrm{H}$ & -0.29958 & -0.40930 & -1.69125 \\
\hline C & 2.28422 & -0.53333 & -2.63445 \\
\hline $\mathrm{H}$ & 3.37386 & -0.50438 & -2.59051 \\
\hline $\mathrm{H}$ & 2.00620 & -0.97594 & -3.59393 \\
\hline $\mathrm{H}$ & 1.92347 & 0.49698 & -2.64169 \\
\hline C & 0.11389 & -0.04810 & 1.22494 \\
\hline C & -0.27294 & -1.37833 & 1.77425 \\
\hline $\mathrm{H}$ & 0.10325 & -2.21290 & 1.18635 \\
\hline C & -1.76381 & -1.51922 & 2.03905 \\
\hline $\mathrm{H}$ & -1.90885 & -2.40227 & 2.66538 \\
\hline $\mathrm{H}$ & -2.11789 & -0.65394 & 2.60766 \\
\hline C & -2.49464 & -1.66161 & 0.73224 \\
\hline C & -2.92618 & -0.53673 & 0.03142 \\
\hline C & -2.68352 & -2.92686 & 0.17552 \\
\hline C & -3.52849 & -0.67704 & -1.21363 \\
\hline $\mathrm{H}$ & -2.81286 & 0.45570 & 0.46037 \\
\hline C & -3.28785 & -3.06649 & -1.06687 \\
\hline $\mathrm{H}$ & -2.36762 & -3.81008 & 0.72431 \\
\hline C & -3.70636 & -1.93919 & -1.76645 \\
\hline $\mathrm{H}$ & -3.87035 & 0.20274 & -1.74667 \\
\hline $\mathrm{H}$ & -3.44123 & -4.05519 & -1.48542 \\
\hline $\mathrm{H}$ & -4.18490 & -2.04696 & -2.73370 \\
\hline $\mathrm{Cl}$ & 0.67223 & -1.36424 & 3.31884 \\
\hline C & -0.83474 & 3.30457 & 0.80724 \\
\hline C & -1.46090 & 2.52739 & -1.37630 \\
\hline C & -2.16157 & 3.61141 & 1.08146 \\
\hline $\mathrm{H}$ & -0.07663 & 3.53330 & 1.55265 \\
\hline C & -3.14247 & 3.38945 & 0.11886 \\
\hline $\mathrm{H}$ & -2.42739 & 4.04767 & 2.03831 \\
\hline C & -2.78617 & 2.85855 & -1.11368 \\
\hline $\mathrm{H}$ & -1.19158 & 2.11277 & -2.34349 \\
\hline $\mathrm{H}$ & -4.17578 & 3.64511 & 0.32570 \\
\hline $\mathrm{H}$ & -3.54109 & 2.70762 & -1.87794 \\
\hline $\mathrm{H}$ & -0.42278 & 0.81888 & 1.60795 \\
\hline C & 0.96808 & 2.43933 & -0.74439 \\
\hline $\mathrm{H}$ & 1.58953 & 2.05194 & 1.27860 \\
\hline $\mathrm{H}$ & 1.56995 & 3.35430 & -0.78664 \\
\hline $\mathrm{H}$ & 1.03645 & 1.97745 & -1.73064 \\
\hline
\end{tabular}

The combined approach (comparison of experimental and calculated CCSs) was successfully used to make a prediction whether $E$ - or $Z$ - iminium ions are formed in the decomposition process of the two diastereomeric aminals $(1 S, 2 R)$-syn-25 and $(1 S, 2 S)$-anti-25. The ATD traces of the selectively dissociated aminals suggest that the dissociation of each aminal leads to the corresponding $E$-iminium ion. The comparison of the computed and the experimental CCS value suggests the $E$-iminium to be formed for both epimers. 


\section{CHLORINATIONS WITH THE JØRGENSEN-HAYASHI-TYPE CATALYST}

\subsection{Aminals Derived from Isovaleraldehyde}

\subsubsection{Synthesis and Derivatization}

2-(1-((S)-2-(bis(3,5-bis(trifluoromethyl)phenyl)((trimethylsilyl)oxy)methyl)pyrrolidin-1-yl)-2chloro-3-methylbutyl)isoindoline-1,3-dione (14)

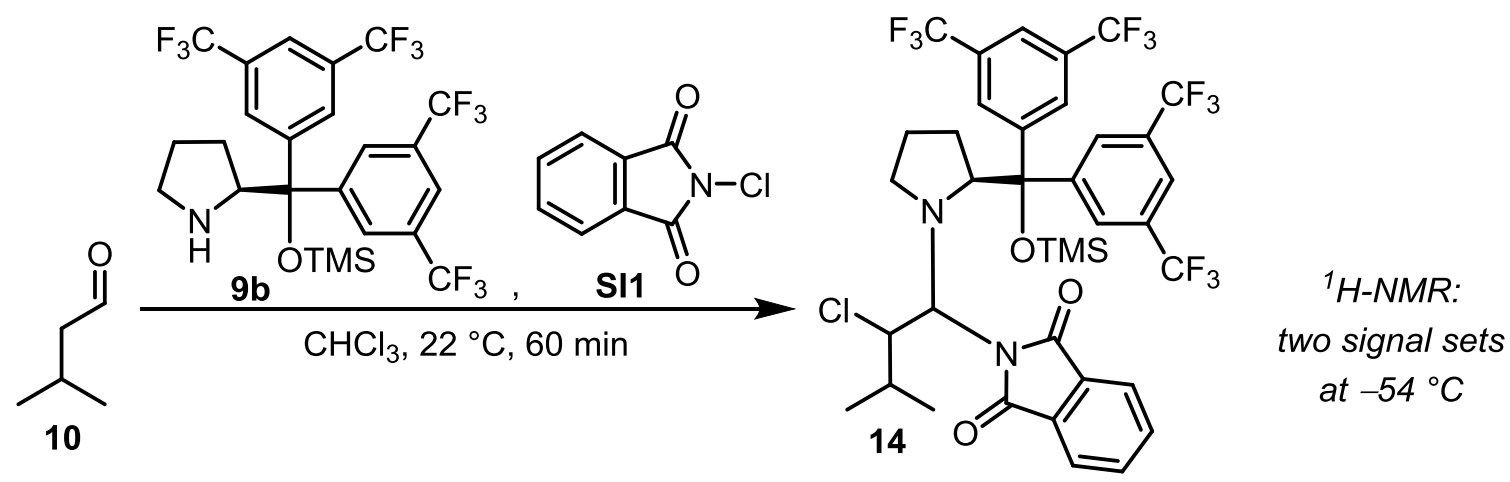

Catalysts $9 \mathrm{~b}(0.896 \mathrm{~g}, 1.50 \mathrm{mmol}, 50 \mathrm{~mol} \%)$ and NCP (0.272 g, $1.50 \mathrm{mmol}, 0.5$ equiv) were added successively to a solution of isovaleraldehyde $\left(0.323 \mathrm{~mL}, 3.00 \mathrm{mmol}, 1.0\right.$ equiv) in $\mathrm{CHCl}_{3}(14 \mathrm{~mL})$ at $22{ }^{\circ} \mathrm{C}$. After $60 \mathrm{~min}$ the solvent and unreacted isovaleraldehyde were removed under reduced pressure. The crude product was dissolved in pentane $(5 \mathrm{~mL})$ and the precipitating solids were filtered off. Pentane was removed under reduced pressure and the product was obtained as a foamy off-white solid $(1.04 \mathrm{~g}$, $1.23 \mathrm{mmol}, 82 \%$ ). Attempts to further purify the product by column chromatography (Silica, Alox) resulted in decomposition. ${ }^{1} \mathrm{H}$-NMR-spectra were recorded at $22{ }^{\circ} \mathrm{C}$ and $-54{ }^{\circ} \mathrm{C}$. Both spectra and the ratio of the two signal sets at $-54^{\circ} \mathrm{C}(80: 20)$ are in accordance with the spectra from the literature. ${ }^{28}$ (The aromatic signals were left out)

$m p=56^{\circ} \mathrm{C}$

IR (ATR): $\tilde{v}=3017,2970,1738,1366,1278,1217,1135,907,842,733 \mathrm{~cm}^{-1}$.

HRMS (ESI, pos. mode): $\mathrm{m} / z$ calculated for $\mathrm{C}_{37} \mathrm{H}_{35} \mathrm{ClF}_{12} \mathrm{~N}_{2} \mathrm{NaO}_{3} \mathrm{Si}[\mathrm{M}+\mathrm{Na}]^{+:}$869.1806, found 869.1766.

major species:

${ }^{1} \mathrm{H}-\mathrm{NMR}\left(\mathrm{CDCl}_{3}, 600 \mathrm{MHz},-54{ }^{\circ} \mathrm{C}\right) \delta=5.16(\mathrm{~d}, J=10.3 \mathrm{~Hz}, 1 \mathrm{H}), 4.99(\mathrm{~d}, J=10.5 \mathrm{~Hz}, 1 \mathrm{H}), 4.60(\mathrm{dd}, J$ $=7.3,4.8 \mathrm{~Hz}, 1 \mathrm{H}), 3.29(\mathrm{dt}, J=11.7,7.9 \mathrm{~Hz}, 1 \mathrm{H}), 2.16-2.10(\mathrm{~m}, 1 \mathrm{H}), 1.97(\mathrm{dt}, J=11.3,7.3 \mathrm{~Hz}, 1 \mathrm{H})$, $1.73-1.68(\mathrm{~m}, 2 \mathrm{H}), 1.19(\mathrm{dq}, J=13.1,6.6 \mathrm{~Hz}, 1 \mathrm{H}), 0.95(\mathrm{~d}, J=6.7 \mathrm{~Hz}, 3 \mathrm{H}), 0.14-0.05(\mathrm{~m}, 1 \mathrm{H}),-0.07$ $(\mathrm{d}, J=6.5 \mathrm{~Hz}, 3 \mathrm{H}),-0.22(\mathrm{~s}, 9 \mathrm{H}) \mathrm{ppm}$. 
${ }^{13} \mathrm{C}-\mathrm{NMR}\left(\mathrm{CDCl}_{3}, 151 \mathrm{MHz},-54{ }^{\circ} \mathrm{C}\right) \delta=171.2,170.3,84.0,76.1,68.5,65.4,48.6,29.1,27.5,24.0$, 21.2, 12.0, $1.6 \mathrm{ppm}$.

minor species:

${ }^{1} \mathrm{H}-\mathrm{NMR}\left(\mathrm{CDCl}_{3}, 600 \mathrm{MHz},-54^{\circ} \mathrm{C}\right) \delta=5.81(\mathrm{~d}, J=10.7 \mathrm{~Hz}, 1 \mathrm{H}), 5.05(\mathrm{~d}, J=10.8 \mathrm{~Hz}, 1 \mathrm{H}), 4.14(\mathrm{dd}, J$ $=9.9,4.0 \mathrm{~Hz}, 1 \mathrm{H}), 3.27-3.22(\mathrm{~m}, 1 \mathrm{H}), 2.39-2.32(\mathrm{~m}, 1 \mathrm{H}), 2.19-2.16(\mathrm{~m}, 1 \mathrm{H}), 1.87-1.80(\mathrm{~m}, 2 \mathrm{H})$, $1.42-1.35(\mathrm{~m}, 1 \mathrm{H}), 1.28(\mathrm{~d}, J=6.6 \mathrm{~Hz}, 3 \mathrm{H}), 1.06(\mathrm{~d}, J=6.9 \mathrm{~Hz}, 3 \mathrm{H}), 0.62(\mathrm{dp}, J=14.7,7.5 \mathrm{~Hz}, 1 \mathrm{H})$, $0.21(\mathrm{~s}, 9 \mathrm{H}) \mathrm{ppm}$.

${ }^{13} \mathrm{C}-\mathrm{NMR}\left(\mathrm{CDCl}_{3}, 151 \mathrm{MHz},-54{ }^{\circ} \mathrm{C}\right) \delta=170.7,170.8,82.2,75.9,68.7,66.5,48.2,29.2,28.8,23.9$, $21.3,15.5,1.8 \mathrm{ppm}$. 
The combination of aldehyde, Jørgensen-Hayashi-type catalyst $\mathbf{9 b}$, and NCP generates the corresponding aminal species almost quantitatively. The conversion of the aldehyde to the $\alpha-$ chloroaldehyde is always below $5 \%$. The low conversion in combination with the high volatility of chloroaldehyde 12 and alcohol SI5 makes the isolation and purification difficult. To overcome these problems, in-situ reduced chloroaldehyde 12 was directly converted into the non-volatile 3,5dinitrobenzoylester SI2.

\section{Enantiomeric Excess (Isovaleraldehyde, catalyst 9b and NCS)}

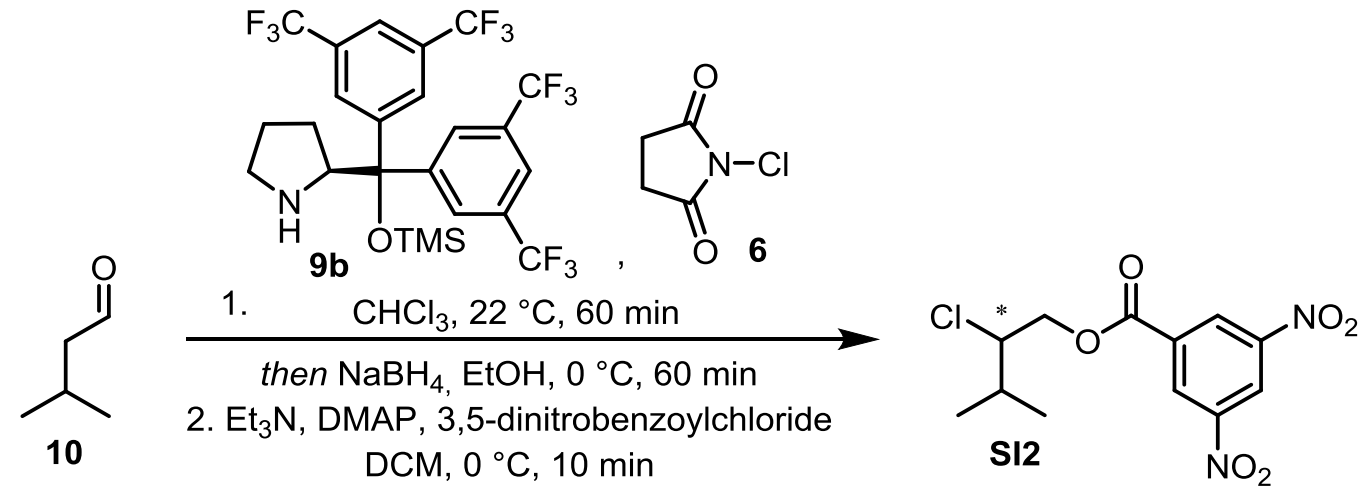

Isovaleraldehyde $\left(0.215 \mathrm{~mL}, 2.00 \mathrm{mmol}, 1.0\right.$ equiv) was dissolved in $\mathrm{CHCl}_{3}(4.0 \mathrm{~mL})$ at $22{ }^{\circ} \mathrm{C}$. Catalyst $9 \mathbf{b}(0.120 \mathrm{~g}, 0.200 \mathrm{mmol}, 10 \mathrm{~mol} \%)$ and NCS $(0.293 \mathrm{~g}, 2.20 \mathrm{mmol}, 1.1$ equiv) were added subsequently and the reaction mixture was stirred for $60 \mathrm{~min}$ at the same temperature. After the reaction was cooled to $0{ }^{\circ} \mathrm{C}, \mathrm{MeOH}(1.3 \mathrm{~mL})$ and $\mathrm{NaBH}_{4}(0.189 \mathrm{~g}, 5.00 \mathrm{mmol}, 2.5$ equiv) were added and the mixture was stirred for $60 \mathrm{~min}$ at the same temperature. The reaction was quenched with aqueous saturated $\mathrm{NH}_{4} \mathrm{Cl}$ $(5 \mathrm{~mL})$, the aqueous phase was extracted with dichloromethane $(3 \times 5 \mathrm{~mL})$ and the combined organic phases were dried over $\mathrm{NaSO}_{4}$. The solvent was removed under reduced pressure (product is volatile!) and the crude product was redissolved in dichloromethane $(4 \mathrm{~mL})$. The solution was cooled to $0{ }^{\circ} \mathrm{C}$ and DMAP (12.2 mg, $0.100 \mathrm{mmol}, 0.05$ equiv) and 3,5-dinitrobenzoylchloride ( $0.300 \mathrm{~g}, 1.30 \mathrm{mmol}, 0.7$ equiv) were added subsequently. $\mathrm{Et}_{3} \mathrm{~N}(0.236 \mathrm{~mL}, 1.70 \mathrm{mmol}, 0.9$ equiv) was added dropwise and the reaction mixture was stirred for $10 \mathrm{~min}$ at $0{ }^{\circ} \mathrm{C}$. The reaction was quenched with aqueous saturated $\mathrm{NaHCO}_{3}(5$ $\mathrm{mL})$, the aqueous phase was extracted with dichloromethane $(3 \times 5 \mathrm{~mL})$ and the combined organic phases were dried over $\mathrm{NaSO}_{4}$. The solvent was removed under reduced pressure and the crude product was purified by column chromatography (Silica, pentane/EtOAc 10:1). Ester SI2 (0.013 g, 41.0 $\mu \mathrm{mol}, 2 \%, 49 \%$ ee for the (S)-enantiomer) was obtained as a colorless solid.

${ }^{1} \mathrm{H}-\mathrm{NMR}\left(\mathrm{CDCl}_{3}, 600 \mathrm{MHz}\right) \delta=9.24(\mathrm{t}, J=2.2 \mathrm{~Hz}, 1 \mathrm{H}), 9.16(\mathrm{~d}, J=2.1 \mathrm{~Hz}, 2 \mathrm{H}), 4.66(\mathrm{dd}, J=11.7,4.7$ $\mathrm{Hz}, 1 \mathrm{H}$ ), 4.61 (dd, $J=11.7,7.9 \mathrm{~Hz}, 1 \mathrm{H}$ ), 4.18 (dt, $J=7.9,4.6 \mathrm{~Hz}, 1 \mathrm{H}$ ), 2.17 (heptd, $J=6.8,4.7 \mathrm{~Hz}, 1 \mathrm{H}$ ), $1.13(\mathrm{~d}, J=6.8 \mathrm{~Hz}, 3 \mathrm{H}), 1.09(\mathrm{~d}, J=6.7 \mathrm{~Hz}, 3 \mathrm{H}) \mathrm{ppm}$.

${ }^{13} \mathrm{C}-\mathrm{NMR}\left(\mathrm{CDCl}_{3}, 151 \mathrm{MHz}\right) \delta=162.3,148.9,133.5,129.7,122.8,68.5,65.0,31.7,20.0,17.5 \mathrm{ppm}$. 
(S)-2-chloro-3-methylbutyl-3,5-dinitrobenzoate ((S)-SI2)

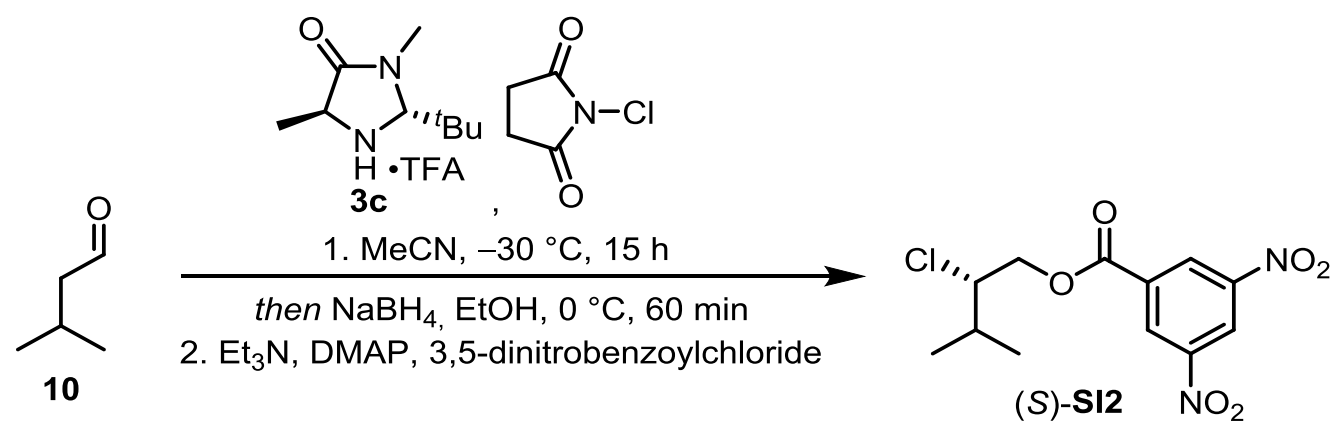

Isovaleraldehyde $(0.108 \mathrm{~mL}, 1.00 \mathrm{mmol}, 1.0$ equiv) was dissolved in $\mathrm{MeCN}(4.0 \mathrm{~mL})$ and cooled to $-30{ }^{\circ} \mathrm{C}$. Catalyst $3 \mathrm{c}(56.9 \mathrm{mg}, 0.200 \mathrm{mmol}, 20 \mathrm{~mol} \%)$ and NCS $(0.147 \mathrm{~g}, 1.10 \mathrm{mmol}, 1.1$ equiv) were added subsequently and the reaction mixture was stirred for $15 \mathrm{~h}$ at the same temperature. After the reaction was warmed to $0^{\circ} \mathrm{C}, \mathrm{MeOH}(1.3 \mathrm{~mL})$ and $\mathrm{NaBH}_{4}(94.6 \mathrm{mg}, 2.50 \mathrm{mmol}, 2.5$ equiv) were added and the mixture was stirred for $60 \mathrm{~min}$ at the same temperature. The reaction was quenched with aqueous saturated $\mathrm{NH}_{4} \mathrm{Cl}(10 \mathrm{~mL})$, the aqueous phase was extracted with dichloromethane $(3 \times 10 \mathrm{~mL})$ and the combined organic phases were dried over $\mathrm{NaSO}_{4}$. The solvent was removed under reduced pressure (product is volatile!) and the crude product was redissolved in dichloromethane $(4 \mathrm{~mL})$. The solution was cooled to $0{ }^{\circ} \mathrm{C}$ and DMAP (12.2 $\mathrm{mg}, 0.100 \mathrm{mmol}, 0.1$ equiv) and 3,5-dinitrobenzoylchloride ( $0.300 \mathrm{~g}, 1.30 \mathrm{mmol}, 1.3$ equiv) were added subsequently. $\mathrm{Et}_{3} \mathrm{~N}(0.237 \mathrm{~mL}, 1.70 \mathrm{mmol}, 1.7$ equiv) was added dropwise and the reaction mixture was stirred for $10 \mathrm{~min}$ at $0{ }^{\circ} \mathrm{C}$. The reaction was quenched with aqueous saturated $\mathrm{NaHCO}_{3}(10 \mathrm{~mL})$, the aqueous phase was extracted with dichloromethane $(3 \times 10$ $\mathrm{mL}$ ) and the combined organic phases were dried over $\mathrm{NaSO}_{4}$. The solvent was removed under reduced pressure and the crude product was purified by column chromatography (Silica, pentane/EtOAc 10:1). Ester (S)-SI2 (0.108 g, $0.341 \mathrm{mmol}, 34 \%, 96 \%$ ee) was obtained as colorless solid.

The ${ }^{1} \mathrm{H}-\mathrm{NMR}$ spectrum is in accordance with the previous experiment. 
(R)-2-chloro-3-methylbutyl-3,5-dinitrobenzoate $((R)$-SI2)

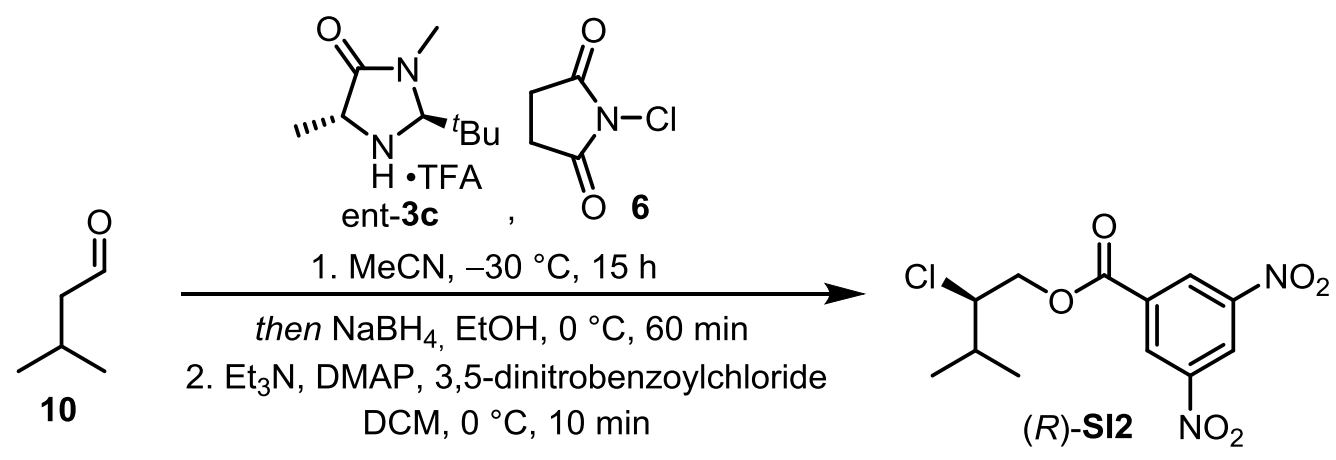

Isovaleraldehyde $(0.108 \mathrm{~mL}, 1.00 \mathrm{mmol}, 1.0$ equiv) was dissolved in $\mathrm{MeCN}(4.0 \mathrm{~mL})$ and cooled to $-30{ }^{\circ} \mathrm{C}$. Catalyst ent-3c $(56.9 \mathrm{mg}, 0.200 \mathrm{mmol}, 20 \mathrm{~mol} \%)$ and NCS $(0.147 \mathrm{~g}, 1.10 \mathrm{mmol}, 1.1$ equiv) were added subsequently and the reaction mixture was stirred for $15 \mathrm{~h}$ at the same temperature. After the reaction was warmed to $0^{\circ} \mathrm{C}, \mathrm{MeOH}(1.3 \mathrm{~mL})$ and $\mathrm{NaBH}_{4}(94.6 \mathrm{mg}, 2.50 \mathrm{mmol}, 2.5$ equiv) were added and the mixture was stirred for $60 \mathrm{~min}$ at the same temperature. The reaction was quenched with aqueous saturated $\mathrm{NH}_{4} \mathrm{Cl}(10 \mathrm{~mL})$, the aqueous phase was extracted with dichloromethane $(3 \times 10 \mathrm{~mL})$ and the combined organic phases were dried over $\mathrm{NaSO}_{4}$. The solvent was removed under reduced pressure (product is volatile!) and the crude product was redissolved in dichloromethane $(4 \mathrm{~mL})$. The solution was cooled to $0{ }^{\circ} \mathrm{C}$ and DMAP $(12.2 \mathrm{mg}, 0.100 \mathrm{mmol}, 0.1$ equiv) and 3,5-dinitrobenzoylchloride ( $0.300 \mathrm{~g}, 1.30 \mathrm{mmol}, 1.3$ equiv) were added subsequently. $\mathrm{Et}_{3} \mathrm{~N}(0.237 \mathrm{~mL}, 1.70 \mathrm{mmol}, 1.7$ equiv) was added dropwise and the reaction mixture was stirred for $10 \mathrm{~min}$ at $0{ }^{\circ} \mathrm{C}$. The reaction was quenched with aqueous saturated $\mathrm{NaHCO}_{3}(10 \mathrm{~mL})$, the aqueous phase was extracted with dichloromethane $(3 \times 10$ $\mathrm{mL}$ ) and the combined organic phases were dried over $\mathrm{NaSO}_{4}$. The solvent was removed under reduced pressure and the crude product was purified by column chromatography (Silica, pentane/EtOAc 10:1). Ester (R)-SI2 (97.0 mg, $0.306 \mathrm{mmol}, 31 \%, 95 \%$ ee) was obtained as colorless solid.

Spectroscopic data are in accordance with the previous experiment. 
In contrast to most of the aminals derived from the MacMillan-type catalysts, all attempts to purify or crystallize aminal $\mathbf{1 4}$ failed and led to decomposition. The reduction of $\mathrm{N}$-alkylphthalimides to amides is literature known ${ }^{22,41}$ and was anticipated to increase the stability of aminal $\mathbf{1 4}$ and enabled subsequent purification and crystallization.

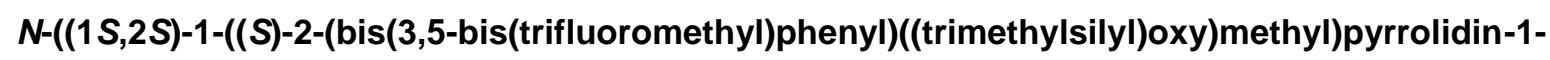
yl)-2-chloro-3-methylbutyl)-2-(hydroxymethyl)benzamide (SI3), 2-((1R,2S)-1-((S)-2-(bis(3,5-bis(trifluoromethyl)phenyl)((trimethylsilyl)oxy)methyl)pyrrolidin-1yl)-2-chloro-3-methylbutyl)-3-hydroxyisoindolin-1-one (SI4)

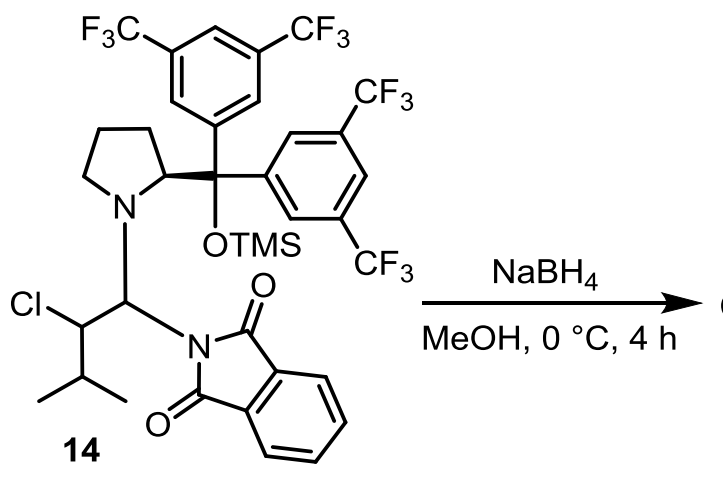<smiles>COC(c1cc(C(F)(F)F)cc(C(F)(F)F)c1)(c1cc(C(F)(F)F)cc(C(F)(F)F)c1)C1CCCN1[C@@H](NC(=O)c1ccccc1CO)[C@@H](Cl)C(C)C</smiles>

one signal set at $-60^{\circ} \mathrm{C}$ and $22{ }^{\circ} \mathrm{C}$<smiles>CC(C)[C@H](Cl)[C@@H](N1CCCC1C(O[Na])(c1cc(C(F)(F)F)cc(C(F)(F)F)c1)c1cc(C(F)(F)F)cc(C(F)(F)F)c1)N1C(=O)c2ccccc2C1O</smiles>

SI4 $\mathrm{NaBH}_{4}(67.0 \mathrm{mg}, 1.77 \mathrm{mmol}, 3.0$ equiv) was added to a solution of aminal $14(0.500 \mathrm{~g}, 0.590 \mathrm{mmol}$,
1.0 equiv) in dry $\mathrm{MeOH}(7.0 \mathrm{~mL})$ at $0{ }^{\circ} \mathrm{C}$. After $4 \mathrm{~h}$ at $0{ }^{\circ} \mathrm{C}$ the reaction was quenched by the addition of aqueous saturated $\mathrm{NaHCO}_{3}(5 \mathrm{~mL})$. The aqueous phase was extracted with dichloromethane $(3 \times 5 \mathrm{~mL})$, the combined organic phases were dried over $\mathrm{Na}_{2} \mathrm{SO}_{4}$ and the solvent was removed under reduced pressure. After purification by column chromatography $\left(\mathrm{SiO}_{2}\right.$, pentane/EtOAc 20:1 to 5:1) amide SI3 $(0.122 \mathrm{~g}, 0.143 \mathrm{mmol}, 24 \%)$ was obtained as an off-white foamy solid. The absolute configuration was determined by $x$-ray crystal structure analysis of the 3,5-dinitrobenzoyl-derivative. Hemiaminal SI4 (94.0 mg, $0.111 \mathrm{mmol}, 19 \%)$ was obtained as a pale yellow oil. The substance appears as a defined spot on the TLC. ${ }^{1} \mathrm{H}-\mathrm{NMR}$ of the hemiaminal SI4 shows extremely broadened signals for every hydrogen atom. A tentative structure proposal is based on the molecular mass and was supported by a further reduction process.

\section{Amide SI3:}

$\mathrm{mp}=76^{\circ} \mathrm{C}$

$[\alpha]_{D}^{26}=-99.8^{\circ}(c=3.00$, dichloromethane $)$

${ }^{1} \mathrm{H}-\mathrm{NMR}\left(\mathrm{CDCl}_{3}, 400 \mathrm{MHz}\right) \delta=8.31(\mathrm{~s}, 2 \mathrm{H}), 8.06(\mathrm{~s}, 2 \mathrm{H}), 7.94(\mathrm{~s}, 2 \mathrm{H}), 7.62(\mathrm{dd}, J=7.6,1.6 \mathrm{~Hz}, 1 \mathrm{H})$, $7.54-7.38(\mathrm{~m}, 3 \mathrm{H}), 7.03(\mathrm{~d}, J=8.9 \mathrm{~Hz}, 1 \mathrm{H}), 5.29(\mathrm{~s}, 1 \mathrm{H}), 4.95-4.86(\mathrm{~m}, 1 \mathrm{H}), 4.72(\mathrm{dd}, J=12.1,5.9$ $\mathrm{Hz}, 1 \mathrm{H}), 4.63(\mathrm{dd}, J=12.2,6.2 \mathrm{~Hz}, 1 \mathrm{H}), 4.24(\mathrm{t}, J=6.1 \mathrm{~Hz}, 1 \mathrm{H}), 3.76(\mathrm{~d}, J=7.4 \mathrm{~Hz}, 1 \mathrm{H}), 2.61$ (dd, $J=$ 
9.8, 7.8 Hz, 1H), $2.31(\mathrm{t}, J=8.6 \mathrm{~Hz}, 1 \mathrm{H}), 2.09-1.98(\mathrm{~m}, 1 \mathrm{H}), 1.86-1.73(\mathrm{~m}, 2 \mathrm{H}), 1.35(\mathrm{~s}, 1 \mathrm{H}), 0.91(\mathrm{~d}$, $J=6.6 \mathrm{~Hz}, 3 \mathrm{H}), 0.30(\mathrm{~s}, 3 \mathrm{H}), 0.07--0.10(\mathrm{~m}, 1 \mathrm{H}),-0.12(\mathrm{~s}, 9 \mathrm{H}) \mathrm{ppm}$.

${ }^{13} \mathrm{C}-\mathrm{NMR}\left(\mathrm{CDCl}_{3}, 176 \mathrm{MHz}\right) \delta=171.3,145.6,144.4,140.4,135.6,132.5-131.9(\mathrm{~m}), 131.9,131.3$, $131.2-130.7(\mathrm{~m}), 130.5,129.5,128.5,127.9,123.4(2 \times q, J=273.0,47.6 \mathrm{~Hz}), 122.3,121.7,83.1,71.6$, $70.5,68.9,64.9,47.2,31.2,28.1,24.2,20.5,14.4,1.7 \mathrm{ppm}$.

IR (ATR): $\tilde{v}=3276,2968,2924,1734,1636,1541,1372,1278,1173,1136,910,844,712 \mathrm{~cm}^{-1}$.

HRMS (ESI, pos. mode): $\mathrm{m} / z$ calculated for $\mathrm{C}_{37} \mathrm{H}_{40} \mathrm{ClF}_{12} \mathrm{~N}_{2} \mathrm{O}_{3} \mathrm{Si}[\mathrm{M}+\mathrm{H}]^{+}: 851.2300$, found 851,$2320 ; \mathrm{m} / \mathrm{z}$ calculated for $\mathrm{C}_{37} \mathrm{H}_{39} \mathrm{ClF}_{12} \mathrm{~N}_{2} \mathrm{NaO}_{3} \mathrm{Si}[\mathrm{M}+\mathrm{Na}]^{+}: 873.2119$, found $873.2157 ; \mathrm{m} / \mathrm{z}$ calculated for $\mathrm{C}_{37} \mathrm{H}_{39} \mathrm{ClF}_{12} \mathrm{KN}_{2} \mathrm{O}_{3} \mathrm{Si}[\mathrm{M}+\mathrm{K}]^{+}:$889.1859, found 889.1889 .

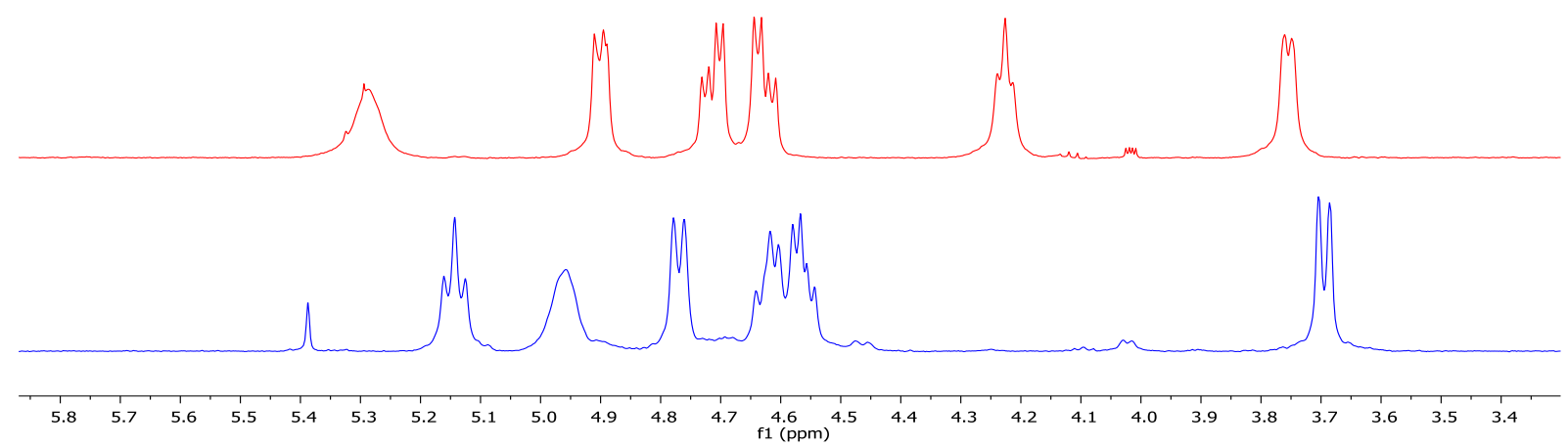

Figure SI-20. ${ }^{1} \mathrm{H}-\mathrm{NMR}$ spectra of amide $\mathrm{SI} 3$ at $23^{\circ} \mathrm{C}$ (red spectrum) and $-60^{\circ} \mathrm{C}$ (blue spectrum). We noted the absence of a second set of signals at $-60^{\circ} \mathrm{C}$.

\section{Hemiaminal S14:}

HRMS (ESI, pos. mode): $\mathrm{m} / z$ calculated for $\mathrm{C}_{37} \mathrm{H}_{37} \mathrm{ClF}_{12} \mathrm{~N}_{2} \mathrm{NaO}_{3} \mathrm{Si}[\mathrm{M}+\mathrm{Na}]^{+}:$871.1962, found 871.2005; $\mathrm{m} / \mathrm{z}$ calculated for $\mathrm{C}_{37} \mathrm{H}_{37} \mathrm{ClF}_{12} \mathrm{KN}_{2} \mathrm{O}_{3} \mathrm{Si}[\mathrm{M}+\mathrm{K}]^{+}:$887.1702, found 887.1733 . 
$N-((1 S, 2 S)-1-((S)-2-($ bis(3,5-bis(trifluoromethyl)phenyl)((trimethylsilyl)oxy)methyl)pyrrolidin-1yl)-2-chloro-3-methylbutyl)-2-(hydroxymethyl)benzamide (SI3) (reduction of SI4)
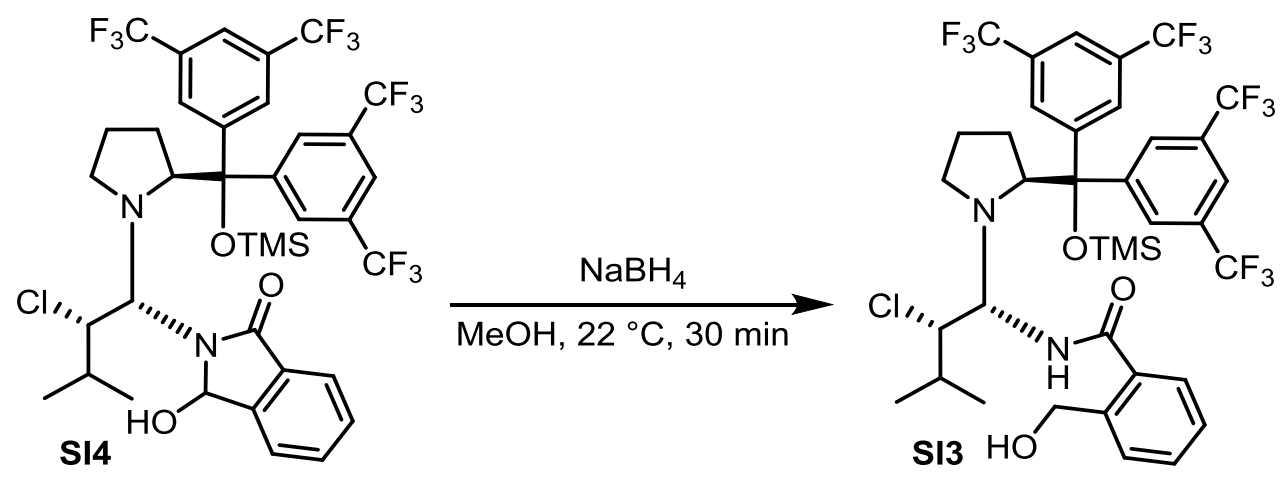

$\mathrm{NaBH}_{4}$ (13.4 mg, $0.353 \mathrm{mmol}, 5.0$ equiv) was added to a solution of hemiaminal SI4 (60 mg, $70.7 \mu \mathrm{mol}$, 1.0 equiv) in dry $\mathrm{MeOH}(0.8 \mathrm{~mL})$ at $22{ }^{\circ} \mathrm{C}$. After $30 \mathrm{~min}$ the reaction was quenched by the addition of aqueous saturated $\mathrm{NaHCO}_{3}(1 \mathrm{~mL})$. The aqueous phase was extracted with dichloromethane $(3 \times 1 \mathrm{~mL})$, the combined organic phases were dried over $\mathrm{Na}_{2} \mathrm{SO}_{4}$ and the solvent was removed under reduced pressure. After purification by column chromatography $\left(\mathrm{SiO}_{2}\right.$, pentane/EtOAc 10:1 to $\left.5: 1\right)$ amide SI3 (36.0 mg, $42.3 \mu \mathrm{mol}, 60 \%$ ) was obtained as an off-white foamy solid.

The spectroscopic data are in accordance with the previously isolated amide SI3. 
After the reduction of aminal $\mathbf{1 4}$ isolation and purification without notable decomposition was possible. Unfortunately, all attempts to isolate single crystals for X-ray crystal structure analysis failed. The esterification of the primary alcohol in amide SI3 with 3,5-dinitrobenzoyl chloride was anticipated to positively impact the crystallization behavior.

\section{2-(((1S,2S)-1-((S)-2-(bis(3,5-bis(trifluoromethyl)phenyl))((trimethylsilyl)oxy)methyl)pyrrolidin-1-} yl)-2-chloro-3-methylbutyl)carbamoyl)benzyl 3,5-dinitrobenzoate (15)<smiles>COC(c1cc(C(F)(F)F)cc(C(F)(F)F)c1)(c1cc(C(F)(F)F)cc(C(F)(F)F)c1)C1CCCN1[C@@H](C(=O)N[C@H](C(C)C)[C@H](Cl)C(C)C)C(F)(F)F</smiles><smiles>CCN(CC)C(=O)[N+](=O)[O-]</smiles><smiles>COC(c1cc(C(F)(F)F)cc(C(F)(F)F)c1)(c1cc(C(F)(F)F)cc(C(F)(F)F)c1)C1CCCN1[C@@H](NC(=O)c1ccccc1COC(=O)c1cc([N+](=O)[O-])cc([N+](=O)[O-])c1)[C@@H](Cl)C(C)C</smiles>

3,5-Dinitrobenzoylchlorid ( $16.5 \mathrm{mg}, 71.5 \mu \mathrm{mol}, 1.3$ equiv) and DMAP (1.00 mg, $8.25 \mu \mathrm{mol}, 0.15$ equiv) were added to an ice-cold solution of alcohol SI3 $(46.8 \mathrm{mg}, 55.0 \mu \mathrm{mol}, 1.0$ equiv) in dichloromethane $(0.30 \mathrm{~mL})$. Et $3 \mathrm{~N}\left(13.0 \mu \mathrm{L}, 93.5 \mu \mathrm{mol}, 1.7\right.$ equiv) was added slowly and the solution was warmed to $22^{\circ} \mathrm{C}$ over $10 \mathrm{~min}$. The reaction was quenched with aqueous saturated $\mathrm{NaHCO}_{3}(0.5 \mathrm{~mL})$, the aqueous phase was extracted with dichloromethane $(3 \times 0.5 \mathrm{~mL})$ and the combined organic phases were dried over $\mathrm{Na}_{2} \mathrm{SO}_{4}$. The solvent was removed under reduced pressure and the crude product was purified by column chromatography $\left(\mathrm{SiO}_{2}\right.$, pentane/EtOAc 6:1). Ester $15(39.0 \mathrm{mg}, 37.3 \mu \mathrm{mol}, 68 \%)$ was obtained as an off-white foamy solid. The absolute configuration was determined by X-ray crystal structure analysis.

$\mathbf{m p}=151^{\circ} \mathrm{C}$

$[\alpha]_{D}^{26}=-53.9^{\circ}(c=2.00$, dichloromethane $)$

${ }^{1} \mathrm{H}-\mathrm{NMR}\left(\mathrm{CDCl}_{3}, 700 \mathrm{MHz}\right) \delta=9.22(\mathrm{t}, J=2.1 \mathrm{~Hz}, 1 \mathrm{H}), 9.17(\mathrm{~d}, J=2.2 \mathrm{~Hz}, 2 \mathrm{H}), 8.30(\mathrm{~s}, 2 \mathrm{H}), 8.07(\mathrm{~s}$, 2H), $7.93(\mathrm{~d}, J=6.8 \mathrm{~Hz}, 2 \mathrm{H}$ ), 7.61 (ddd, $J=7.7,6.4,1.3 \mathrm{~Hz}, 2 \mathrm{H}$ ), 7.53 (td, $J=7.6,1.4 \mathrm{~Hz}, 1 \mathrm{H}$ ), 7.47 (td, $J=7.5,1.3 \mathrm{~Hz}, 1 \mathrm{H}), 6.94(\mathrm{~d}, J=9.0 \mathrm{~Hz}, 1 \mathrm{H}), 5.85(\mathrm{~d}, J=12.8 \mathrm{~Hz}, 1 \mathrm{H}), 5.75(\mathrm{~d}, J=13.1 \mathrm{~Hz}, 1 \mathrm{H}), 5.33$ (s, 1H), 4.98 (d, $J=9.4 \mathrm{~Hz}, 1 \mathrm{H}$ ), $3.79(\mathrm{~s}, 1 \mathrm{H}), 2.67$ (td, $J=9.6,7.3 \mathrm{~Hz}, 1 \mathrm{H}$ ), 2.33 (ddd, $J=9.9,7.9,2.7$ $\mathrm{Hz}, 1 \mathrm{H}$ ), $2.08(\mathrm{~s}, 1 \mathrm{H}), 1.93-1.86(\mathrm{~m}, 1 \mathrm{H}), 1.85-1.79(\mathrm{~m}, 1 \mathrm{H}), 1.40$ (dddd, $J=12.5,10.1,7.0,2.7 \mathrm{~Hz}$, $1 \mathrm{H}), 0.91(\mathrm{~d}, J=6.6 \mathrm{~Hz}, 3 \mathrm{H}), 0.32(\mathrm{~s}, 3 \mathrm{H}), 0.02(\mathrm{~s}, 1 \mathrm{H}),-0.12(\mathrm{~s}, 9 \mathrm{H}) \mathrm{ppm}$. 
${ }^{13} \mathrm{C}$-NMR $\left(\mathrm{CDCl}_{3}, 176 \mathrm{MHz}\right) \delta=169.9,162.8,148.8,145.6,144.5,136.1,133.9,133.7,132.0$ (q, $J=$ 33.5, 33.0 Hz), 131.2, $131.1-130.7(\mathrm{~m}), 130.6,130.2,129.7,129.6,129.3,127.5,123.4$ (qd, $J=273.0$, $40.2 \mathrm{~Hz}), 122.7,122.2,121.7,83.2,71.7,70.8,68.8,66.2,47.1,31.0,28.2,24.2,20.7,1.7$ ppm.

IR (ATR): $\tilde{v}=3353,3106,2964,1736,1666,1629,1548,1463,1371,1345,1276,1171,1133,910$ $843,722 \mathrm{~cm}^{-1}$.

HRMS (ESI, pos. mode): $\mathrm{m} / z$ calculated for $\mathrm{C}_{44} \mathrm{H}_{41} \mathrm{ClF}_{12} \mathrm{~N}_{4} \mathrm{NaO}_{8} \mathrm{Si}[\mathrm{M}+\mathrm{Na}]^{+}:$1067.2083, found 1067.2087; $\mathrm{m} / \mathrm{z}$ calculated for $\mathrm{C}_{44} \mathrm{H}_{41} \mathrm{ClF}_{12} \mathrm{KN}_{4} \mathrm{O}_{8} \mathrm{Si}[\mathrm{M}+\mathrm{K}]^{+}:$: 1083.1823 , found 1083.1816 . 
To avoid the oversight and potential loss of possible additional stereoisomers during the numerous purifications, an optimized synthesis protocol with a subsequent thorough isolation and purification process was developed.

\section{2-(((1S,2S)-1-((S)-2-(bis(3,5-bis(trifluoromethyl)phenyl))(trimethylsilyl)oxy)methyl)pyrrolidin-1-} yl)-2-chloro-3-methylbutyl)carbamoyl)benzyl 3,5-dinitrobenzoate (15) (optimized procedure)

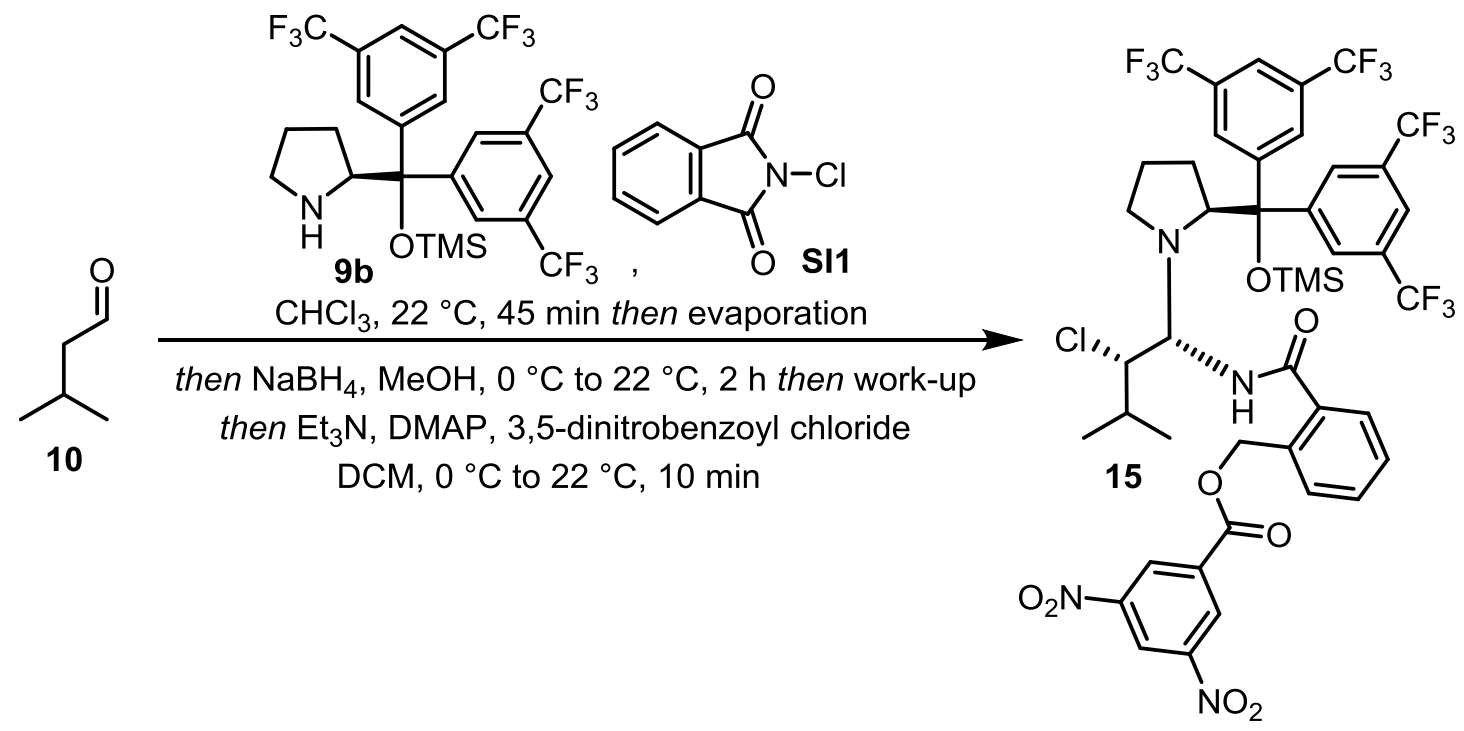

Catalyst 9b (2.39 g, $4.00 \mathrm{mmol}, 50 \mathrm{~mol} \%)$ and NCP $(0.726 \mathrm{~g}, 4.00 \mathrm{mmol}, 0.5$ equiv) were added successively to a solution of isovaleraldehyde $\left(0.861 \mathrm{~mL}, 8.00 \mathrm{mmol}, 1.0\right.$ equiv) in $\mathrm{CHCl}_{3}(36 \mathrm{~mL})$ at $22{ }^{\circ} \mathrm{C}$. After $45 \mathrm{~min}$ the solvent and unreacted isovaleraldehyde were removed under reduced pressure. The crude product was dissolved in dry $\mathrm{MeOH}(40 \mathrm{~mL})$ and cooled to $0{ }^{\circ} \mathrm{C}$. $\mathrm{NaBH}_{4}(1.34 \mathrm{~g}, 35.4 \mathrm{mmol}$, 10.0 equiv) was added in portions and the reaction was stirred for $2 \mathrm{~h}$ at $22{ }^{\circ} \mathrm{C}$. The reaction was quenched with aqueous saturated $\mathrm{NaHCO}_{3}(20 \mathrm{~mL})$, the aqueous phase was extracted with dichloromethane $(3 \times 20 \mathrm{~mL})$ and the combined organic phases were dried over $\mathrm{Na}_{2} \mathrm{SO}_{4}$. The solvent was removed under reduced pressure and the crude product was dissolved again in dichloromethane (14 mL). The reaction mixture was cooled to $0{ }^{\circ} \mathrm{C}$ and 3,5-dinitrobenzoyl chloride $(1.02 \mathrm{~g}, 4.41 \mathrm{mmol}$, 1.3 equiv) and DMAP ( $41.5 \mathrm{mg}, 0.340 \mathrm{mmol}, 10 \mathrm{~mol} \%)$ were added successively. $\mathrm{Et}_{3} \mathrm{~N}(0.804 \mathrm{~mL}, 5.77$ mmol, 1.7 equiv) was added dropwise and the reaction mixture was stirred for $10 \mathrm{~min}$ at $22{ }^{\circ} \mathrm{C}$. The reaction was quenched with aqueous saturated $\mathrm{NaHCO}_{3}(15 \mathrm{~mL})$, the aqueous phase was extracted with dichloromethane $(3 \times 20 \mathrm{~mL})$ and the combined organic phases were dried over $\mathrm{Na}_{2} \mathrm{SO}_{4}$. The solvent was removed under reduced pressure and the crude product was purified by column chromatography ( $\mathrm{SiO}_{2}$, pentane/EtOAc 10:1 to 0:1). Ester 15 (1.96 g, $1.87 \mathrm{mmol}, 47 \%$ over 3 steps, single diastereomer) was obtained as an off-white foamy solid. The spectroscopic data are in accordance with the previously isolated ester 15.

After a thorough analysis of all fractions from the column chromatographic, only a single diastereomer could be detected and isolated. This of course does not exclude the presence of additional, labile diastereomers, but it is the best we could do. 
$N-((1 S, 2 S)-1-((S)-2-($ bis(3,5-bis(trifluoromethyl)phenyl)((trimethylsilyl)oxy)methyl)pyrrolidin-1yl)-2-chloro-3-methylbutyl)-2-(hydroxymethyl)benzamide (SI3) (ester hydrolysis)<smiles>COC(c1cc(C(F)(F)F)cc(C(F)(F)F)c1)(c1cc(C(F)(F)F)cc(C(F)(F)F)c1)C1CCCN1[C@@H](NC(=O)c1ccccc1COC(=O)c1cc([N+](=O)[O-])cc([N+](=O)[O-])c1)[C@@H](Cl)C(C)C</smiles>

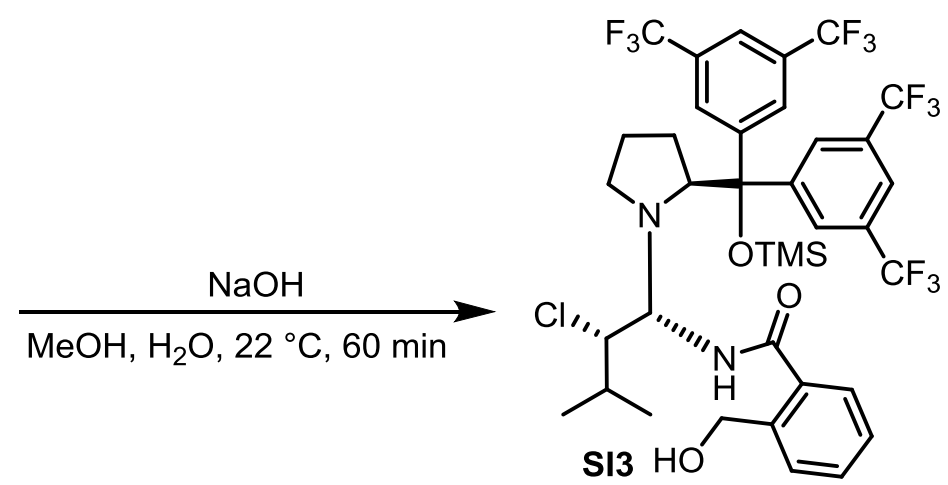

Three drops of aqueous $\mathrm{NaOH}(1 \mathrm{M})$ were added to a solution of ester $15(0.140 \mathrm{~g}, 0.134 \mathrm{mmol}$, 1.0 equiv) in $\mathrm{MeOH}(0.5 \mathrm{~mL})$ at $22^{\circ} \mathrm{C}$ and the reaction was stirred for $60 \mathrm{~min}$ at the same temperature. $\mathrm{H}_{2} \mathrm{O}(1 \mathrm{~mL})$ was added, the aqueous phase was extracted with dichloromethane $(3 \times 1 \mathrm{~mL})$, the combined organic phases were dried over $\mathrm{Na}_{2} \mathrm{SO}_{4}$ and the solvent was removed under reduced pressure. After column chromatography $\left(\mathrm{SiO}_{2}\right.$, pentane/EtOAc 10:1 to 5:1) pure alcohol SI3 (62.0 mg, $\left.72.8 \mu \mathrm{mol}, 54 \%\right)$ was obtained as an off-white foamy solid.

The spectroscopic data are in accordance with the previously synthesized alcohol SI3. 


\subsubsection{Structural Analysis}

\section{NOE and J-Coupling Analysis of Aminal 14}

To extend the characterization data above, a full assignment of all NMR resonances of the aminal 14 was attempted in $\mathrm{CDCl}_{3}$ at $219 \mathrm{~K}$ (see Table SI-15). At this temperature there is still severe overlap of the two species in the NMR spectra, particularly in the aromatic region, where most of the signals are additionally split by the ${ }^{19} \mathrm{~F}$ J-couplings (see Figures SI-21 to SI-22). Subsequently, aminal 14 was dissolved in $\mathrm{CD}_{2} \mathrm{Cl}_{2}$, showing almost the exact same chemical shifts and coupling patterns (see Table $\mathrm{SI}-16$ ), indicating that no major structural change is observed in this solvent. The solvent change however allows to further cool down to $190 \mathrm{~K}$, at which most resonances for the major species could be assigned. For the minor species, however, some aromatic signals (particularly quaternary carbons) remain unassigned as they could not be resolved.

Table SI-15. Assignment of aminal 14 in $\mathrm{CDCl}_{3}$ at $219 \mathrm{~K}$, shifts relative to $\mathrm{CHCl}_{3}$ at $7.26 \mathrm{ppm}$.

"a" on the same side as $\mathrm{H} 2$

"b" on the opposite side as $\mathrm{H} 2$

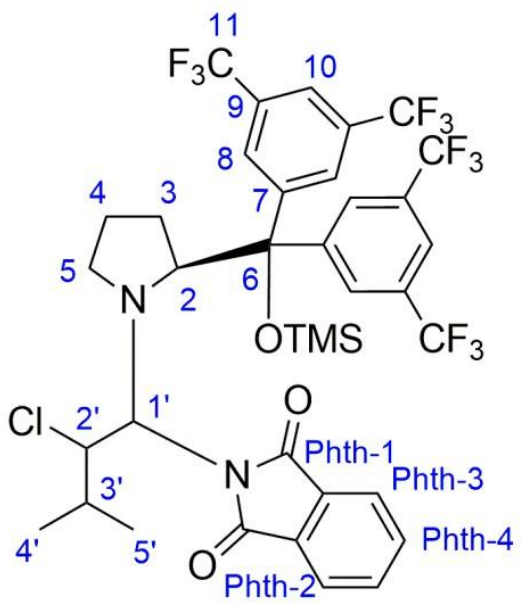

\begin{tabular}{|c|c|c|c|c|}
\hline & Major (80\%) & & Minor (20\%) & \\
\hline Position & $\delta^{13} C(p p m)$ & $\delta{ }^{1} H(p p m)$ & $\delta^{13} C(p p m)$ & $\delta{ }^{1} H(p p m)$ \\
\hline$\overline{2}$ & 68.6 & 4.60 & 68.6 & 4.15 \\
\hline $3 a$ & 27.7 & $1.71^{1}$ & 29.0 & 1.82 \\
\hline $3 b$ & 27.7 & $1.71^{1}$ & 29.0 & 1.38 \\
\hline $4 a$ & 24.3 & 1.20 & 24.1 & 1.28 \\
\hline $4 b$ & 24.3 & 0.09 & 24.1 & 0.63 \\
\hline $5 a$ & 48.8 & 3.29 & 48.5 & 3.27 \\
\hline $5 b$ & 48.8 & 1.97 & 48.5 & 2.16 \\
\hline 6 & 82.4 & & 84.2 & \\
\hline OTMS & 1.7 & -0.16 & 1.9 & -0.15 \\
\hline 1' & 76.3 & 5.16 & 76.1 & 5.81 \\
\hline $2^{\prime}$ & 65.6 & 4.98 & 66.8 & 5.05 \\
\hline 3' & 29.3 & 2.13 & 29.0 & 2.35 \\
\hline $4^{\prime}$ & 21.3 & 0.95 & 21.4 & 1.06 \\
\hline 5 & 12.2 & -0.01 & 15.7 & 1.28 \\
\hline Phth-12 & $170.5 / 171.4$ & & $170.8 / 170.9$ & \\
\hline
\end{tabular}

${ }^{1}$ diastereotopic protons strongly coupled

2 hindered rotation around $\mathrm{C}^{\prime}$ '-N-bond, no assignment Pth-1 vs Pth-1' etc. 
Table SI-16. Assignment of aminal 14 in $\mathrm{CD}_{2} \mathrm{Cl}_{2}$ at $190 \mathrm{~K}$, shifts relative to $\mathrm{CHDCl}_{2} @ 5.35 \mathrm{ppm}$.

"a" on the same side as $\mathrm{H} 2$

"b" on the opposite side as $\mathrm{H} 2$

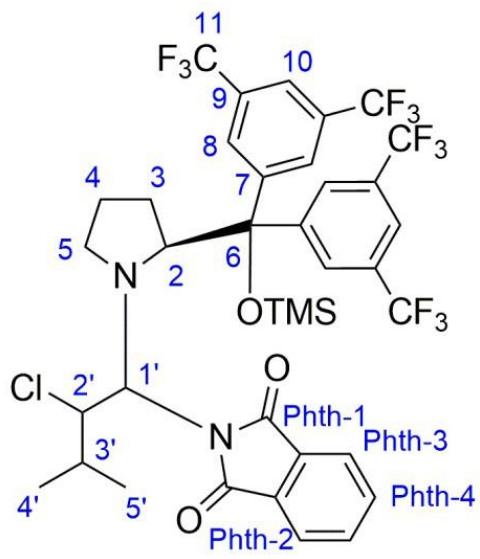

\begin{tabular}{|c|c|c|c|c|c|c|c|}
\hline \multirow[b]{2}{*}{ Position } & \multirow{2}{*}{$\begin{array}{l}\text { Major }(78 \%) \\
\qquad \delta{ }^{13} \mathrm{C}(\mathrm{ppm})\end{array}$} & \multicolumn{6}{|c|}{ Minor (22\%) } \\
\hline & & $\delta{ }^{1} H(p p m)$ & $J(H z)$ & $\delta^{13} C(p p m)$ & $\delta{ }^{1} H(p p m)$ & $J(H z)$ & \\
\hline$\overline{2}$ & 68.3 & 4.56 & ${ }^{3} J_{2,3 a / b}=7.6,4.7$ & 67.5 & 4.13 & ${ }^{3} J_{2,3 a / b}=9.8,2$. & \\
\hline $3 a$ & 27.5 & $1.68^{1}$ & 1 & 28.7 & 1.82 & $?$ & \\
\hline $3 b$ & 27.5 & $1.68^{1}$ & 1 & 28.7 & 1.38 & $?$ & \\
\hline $4 a$ & 24.1 & 1.15 & ${ }^{3} J_{4 a, 5 a}=8.0$ & 23.6 & 1.24 & ${ }^{2} J_{4 a, 4 b}=11.8$ & \\
\hline $4 b$ & 24.1 & 0.05 & ${ }^{3} J_{4 b, 5 a}=6.7$ & 23.6 & 0.36 & $\begin{array}{l}{ }^{2} J_{4 a, 4 b}= \\
{ }^{3} J_{4 b, 5 a}= \\
{ }^{3} J_{4 b, 5 b}=6.7\end{array}$ & $\begin{array}{r}11.8 \\
9.3\end{array}$ \\
\hline $5 a$ & 48.8 & 3.24 & $\begin{array}{l}{ }^{2} J_{5 a, 5 b}= \\
{ }^{3} J_{4 b, 5 a}= \\
{ }^{3} J_{4 a, 5 a}=8.0\end{array}$ & $\begin{array}{l}11.1,48.2 \\
6.7\end{array}$ & 3.12 & $\begin{array}{l}{ }^{2} J_{5 a, 5 b}= \\
{ }^{3} J_{4 b, 5 a}=9.3\end{array}$ & 9.8 \\
\hline $5 b$ & 48.8 & 1.94 & ${ }^{2} J_{5 a, 5 b}=11.1$ & 48.2 & 2.29 & $\begin{array}{l}{ }^{2} J_{5 a, 5 b}= \\
{ }^{3} J_{4 b, 5 b}=6.7\end{array}$ & 9.8 \\
\hline 6 & 82.2 & & & 84.3 & & & \\
\hline $7 A^{2}$ & 145.6 & & & 145.5 & & & \\
\hline $7 \mathrm{~B}^{2}$ & 143.9 & & & $?$ & & & \\
\hline $8 A^{2}$ & $130.4 / 131.4$ & $9.37 / 7.36$ & & $?$ & $?$ & & \\
\hline $8 \mathrm{~B}^{2}$ & $128.5 / 130.0$ & $8.20 / 7.68$ & & $129.2 / 132.1$ & $8.02 / 7.49$ & & \\
\hline $10 A^{2}$ & 122.7 & 8.02 & & $?$ & $?$ & & \\
\hline $10 B^{2}$ & 122.1 & 7.98 & & $?$ & $?$ & & \\
\hline $11 A^{2}$ & $123.9 / 124.4$ & & & $?$ & & & \\
\hline $11 \mathrm{~B}^{2}$ & $124.4 / 124.6$ & & & $?$ & & & \\
\hline OTMS & 1.3 & -0.24 & & 1.7 & -0.21 & & \\
\hline $1^{\prime}$ & 75.9 & 5.15 & ${ }^{3} J_{1,2 \prime}=10.3$ & 75.4 & 5.81 & ${ }^{3} J_{1,21}=10.9$ & \\
\hline $2^{\prime}$ & 65.8 & 5.02 & $\begin{array}{l}{ }^{3} J_{1,21}= \\
{ }^{3} J_{2 \prime, 31}<1.0\end{array}$ & $10.3,67.0$ & 5.07 & $\begin{array}{l}{ }^{3} J_{1,2 \prime}= \\
{ }^{3} J_{2 \prime, 3 \prime}<1.0\end{array}$ & 10.9, \\
\hline $3^{\prime}$ & 29.3 & 2.14 & ${ }^{3} J_{2 \prime, 3 \prime}<1.0$ & 29.3 & 2.38 & ${ }^{3} J_{2 \prime, 3 \prime}<1.0$ & \\
\hline $4^{\prime}$ & 21.2 & 0.93 & & 21.3 & 1.03 & & \\
\hline $5^{\prime}$ & 12.0 & -0.08 & & 15.7 & 1.27 & & \\
\hline Phth- $1^{3}$ & $170.2 / 171.7$ & & & $170.9 / 171.0$ & & & \\
\hline Phth- $2^{3}$ & $131.5 / ?$ & & & $?$ & & & \\
\hline
\end{tabular}

1 diastereotopic protons strongly coupled

2 hindered rotation around C2-C6-bond, no assignment of diastereotopic aromatic rings $A$ vs $B, N O E$ contacts hint at ring $\mathrm{B}$ being atop the pyrrolidine

${ }^{3}$ hindered rotation around $\mathrm{C} 1$ '-N-bond, no assignment Pth-1 vs Pth-1' etc.

? obfuscated by overlap / not determined 


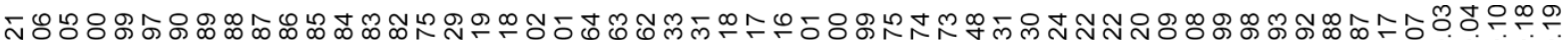

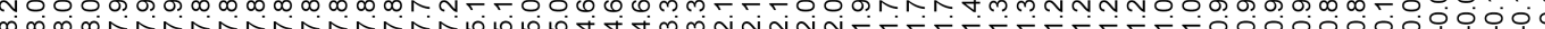

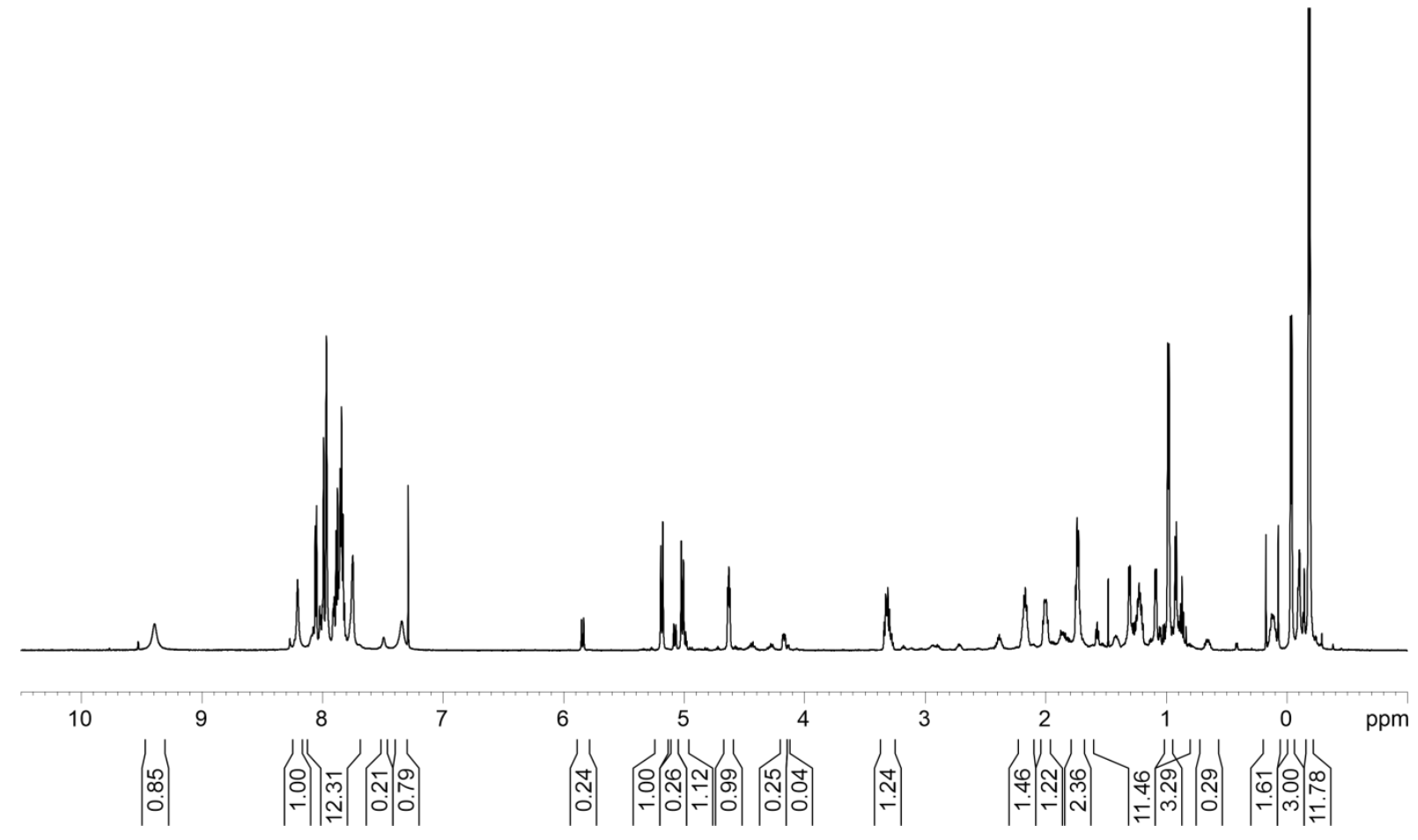

Figure SI-21. ${ }^{1} \mathrm{H}$ Spectrum of aminal 14 in $\mathrm{CDCl}_{3}$ at $219 \mathrm{~K}$.

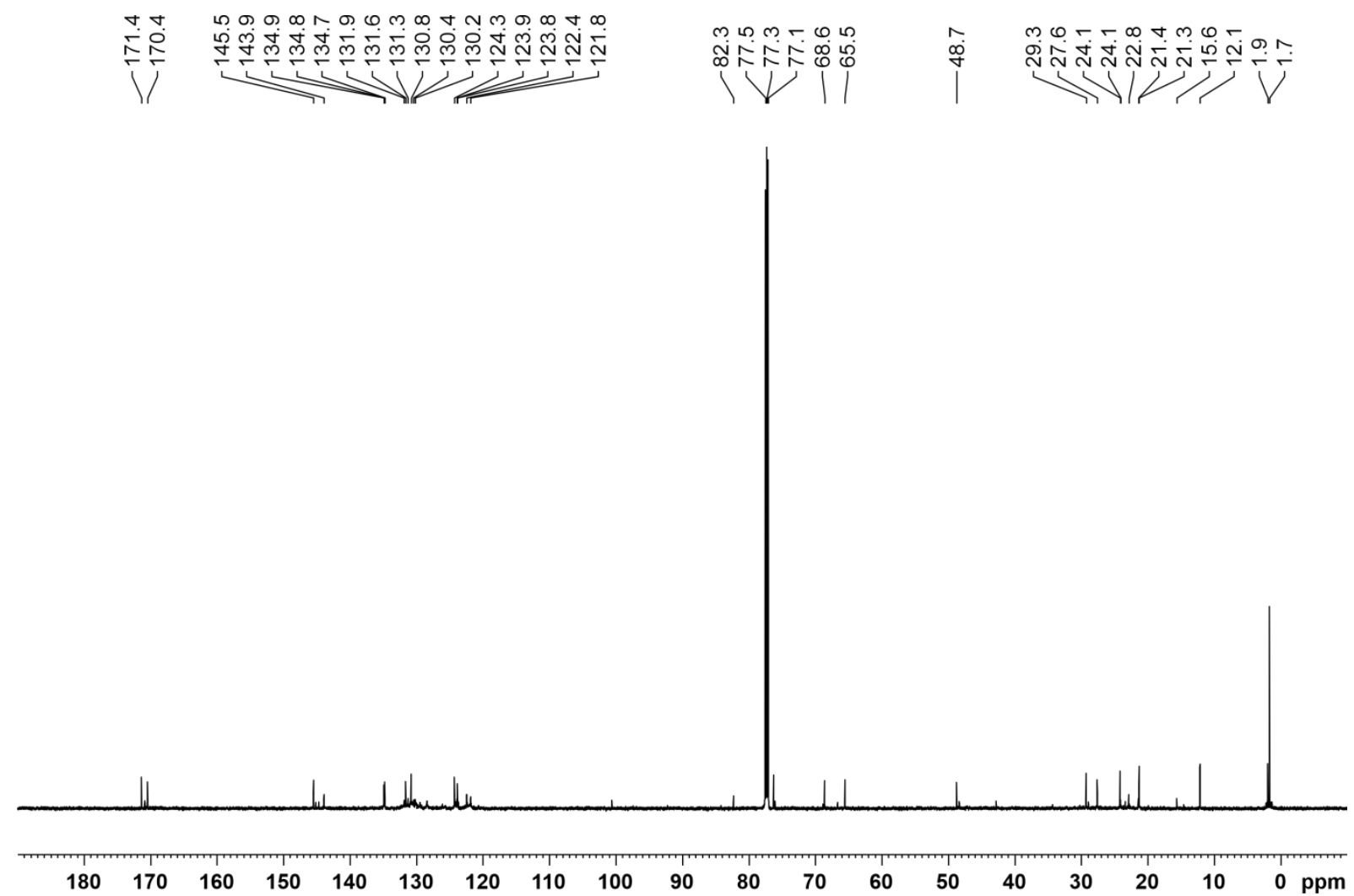

Figure SI-22. $\left\{{ }^{1} \mathrm{H}\right\}^{13} \mathrm{C}$ Spectrum of aminal 14 in $\mathrm{CDCl}_{3}$ at $219 \mathrm{~K}$. 


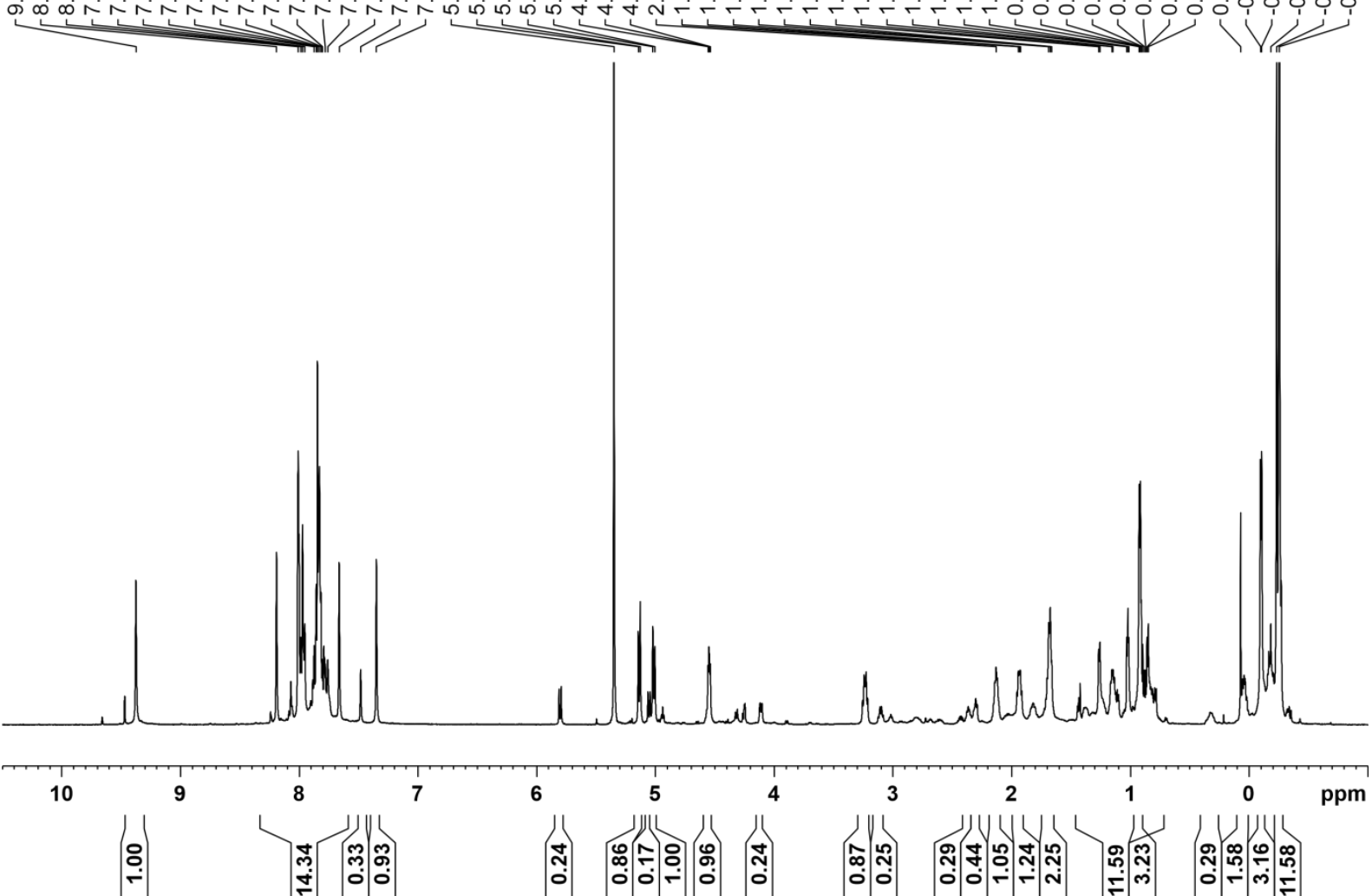

Figure SI-23. ${ }^{1} \mathrm{H}$ Spectrum of aminal 14 in $\mathrm{CD}_{2} \mathrm{Cl}_{2}$ at $190 \mathrm{~K}$.

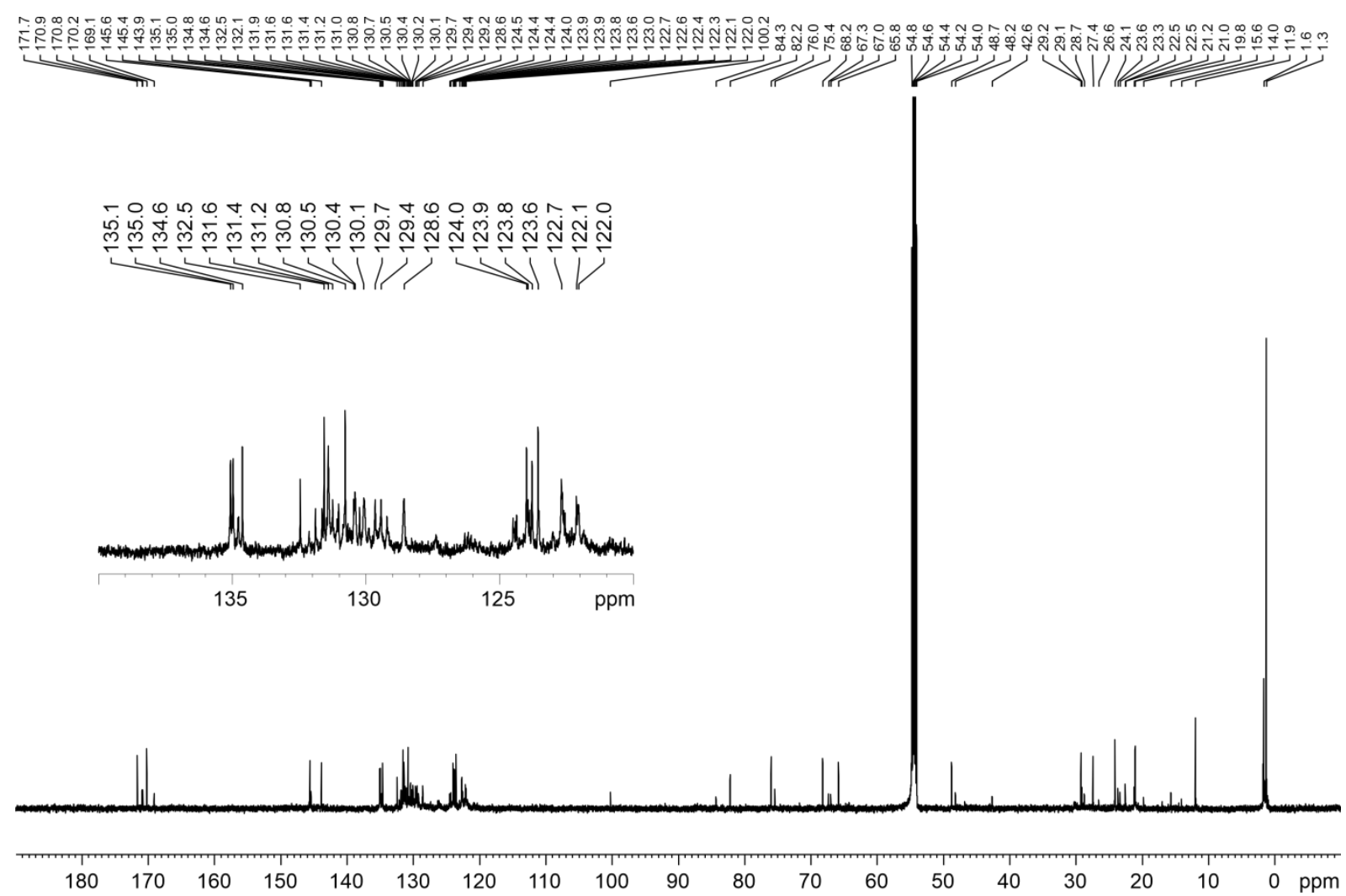

Figure SI-24. $\left\{{ }^{1} \mathrm{H}\right\}^{13} \mathrm{C}$ Spectrum of aminal 14 in $\mathrm{CD}_{2} \mathrm{Cl}_{2}$ at $190 \mathrm{~K}$. 


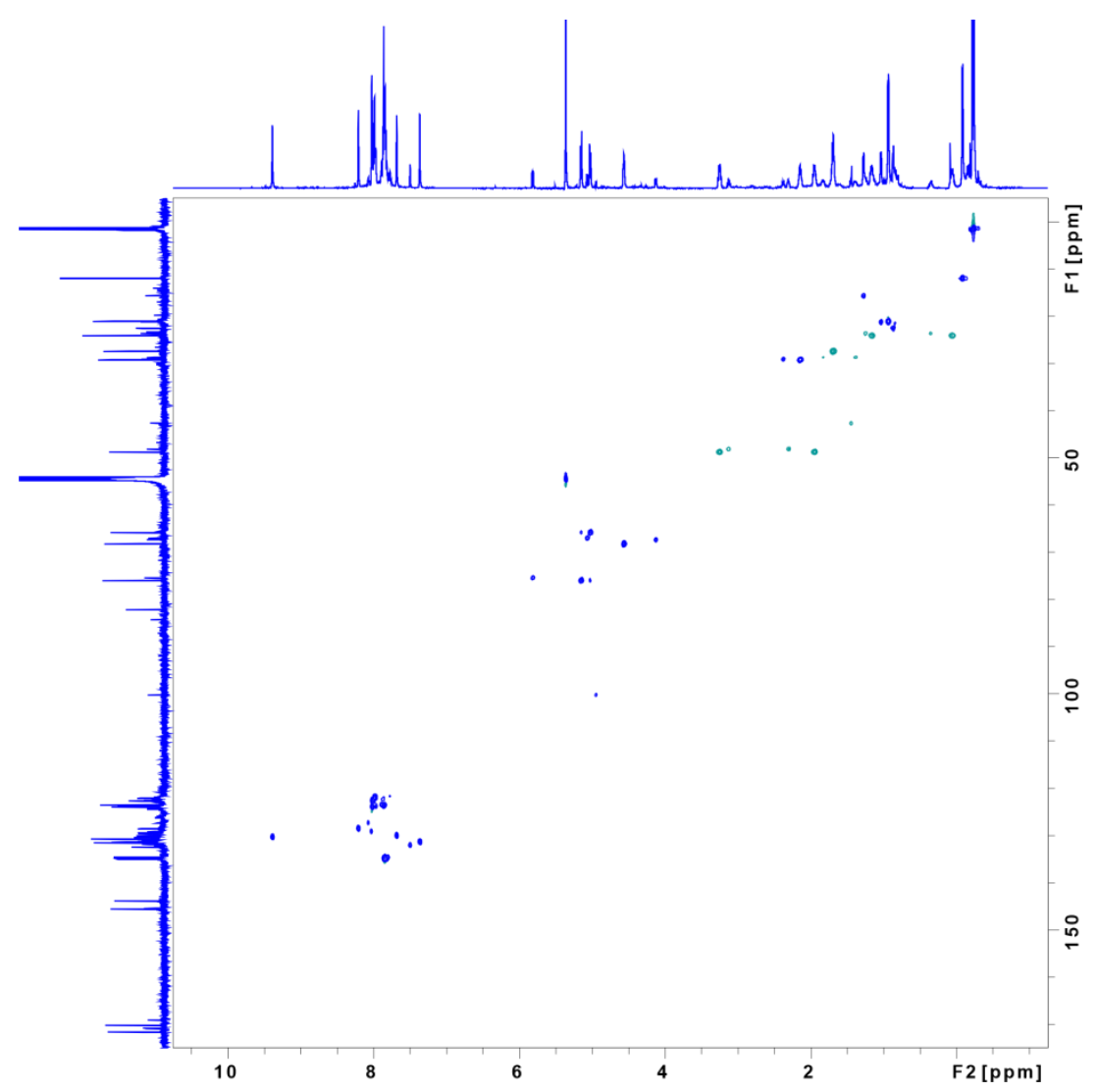

Figure SI-25. ${ }^{1} \mathrm{H}-{ }^{13} \mathrm{C} \mathrm{HSQC} \mathrm{Spectrum} \mathrm{of} \mathrm{aminal} 14$ in $\mathrm{CD}_{2} \mathrm{Cl}_{2}$ at $190 \mathrm{~K}$.

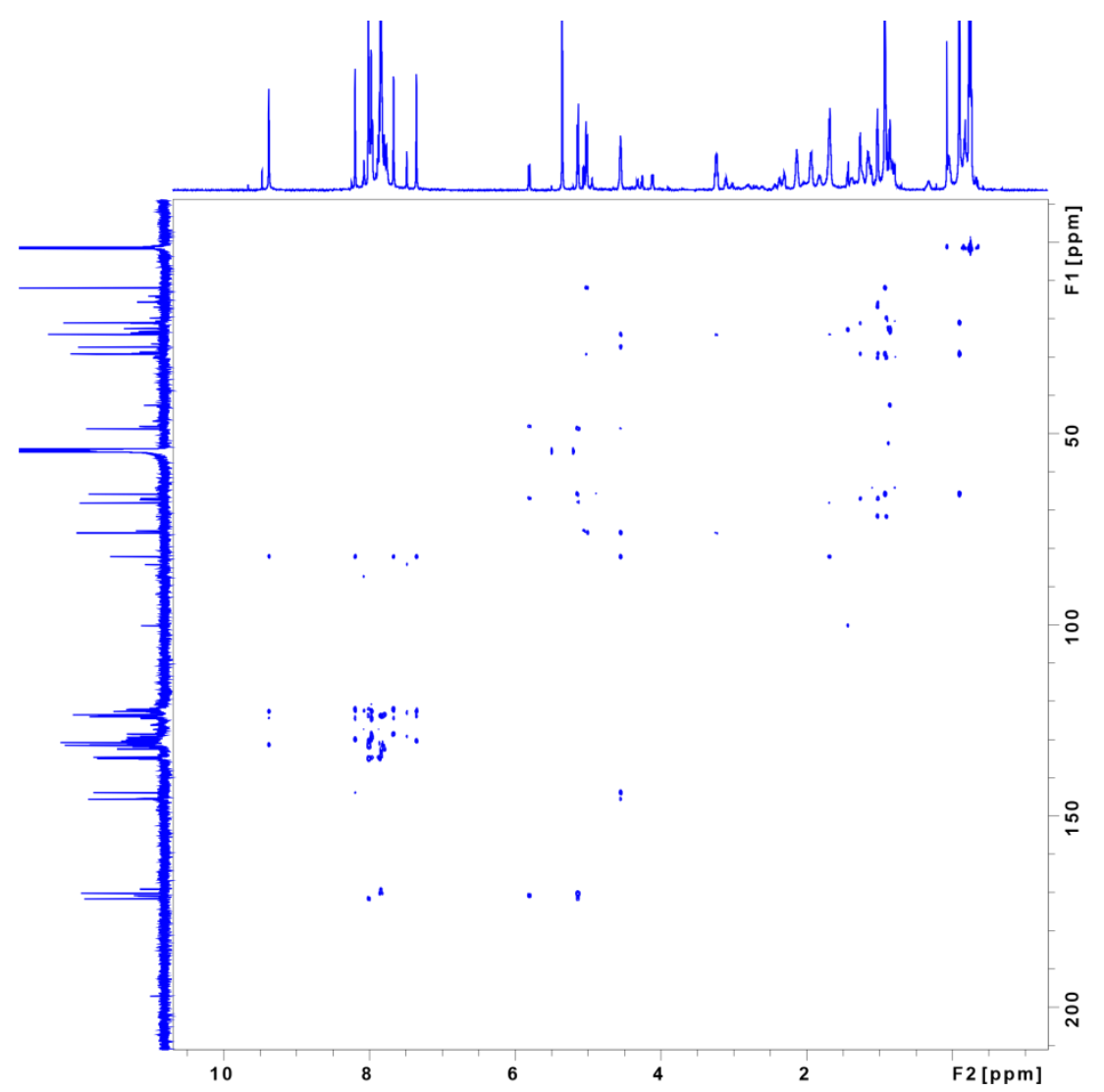

Figure SI-26. ${ }^{1} \mathrm{H}-{ }^{13} \mathrm{C}$ HMBC Spectrum of aminal 14 in $\mathrm{CD}_{2} \mathrm{Cl}_{2}$ at $190 \mathrm{~K}$. 
The experimental ${ }^{13} \mathrm{C}$ chemical shifts of major and minor species are fairly similar, while the observed ${ }^{1} \mathrm{H}$ chemical shifts as well as some - but notably not all - J-couplings show more marked differences between the two signal sets. The aminal proton $\mathrm{H}^{1}$ ' as well as the pyrrolidine proton $\mathrm{H} 2$ show the largest deviations in chemical shift between the major and the minor species, accompanied by a large difference for the resonance of the methyl group 5'. The $J$-couplings in the aminal part indicate an anti-periplanar arrangement of the protons $\mathrm{H}^{\prime}$ ' and $\mathrm{H} 2$ ' for both major and minor species. While not all $\mathrm{J}$-couplings along the pyrrolidine ring could be determined experimentally, the observed values apparently differ between the major and minor species, possibly indicating a difference in the (average) ring pucker.

From the EASY-ROESY spectra, the interconversion between the major and minor species is evident (see Figure SI-27). While the NOE/ROE contacts have the opposite phase to the diagonal, the crosspeaks generated by chemical exchange have the same phase. These exchange peaks become even more prevalent at higher temperatures ( $219 \mathrm{~K}$ and $240 \mathrm{~K}$, not shown here). Based on the exchange rate determined by the PANIC analysis, we estimate an energy difference for interconversion at $190 \mathrm{~K}$ of $\Delta \mathrm{G}^{\ddagger} \approx 12.4 \mathrm{kcal} \mathrm{mol}^{-1}$. The distinction between chemical exchange and NOE is not immediately obvious in the 1D NOE spectra, as the compound is in the slow motion regime, i.e. both effects show signals with the same phase as the selected (diagonal) signal. A representative mixing time series of selective 1D NOE spectra showing this behavior is given in Figure SI-28.

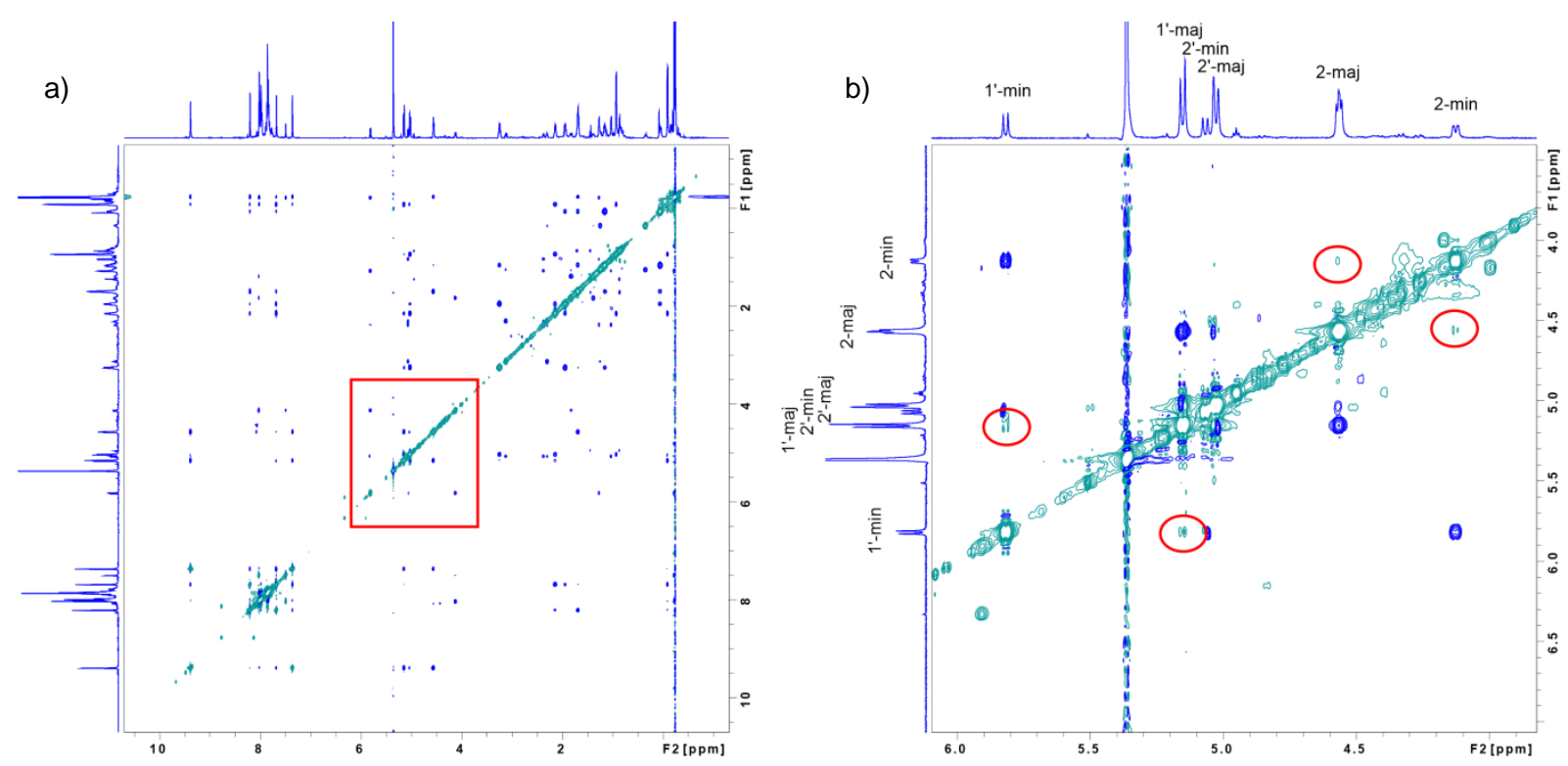

Figure SI-27. EASY-ROESY of aminal 14 in $\mathrm{CD}_{2} \mathrm{Cl}_{2}$ at $190 \mathrm{~K}$. Diagonal peaks and signals generated by chemical exchange are colored green, while $\mathrm{NOE} / \mathrm{ROE}$ cross-peaks are colored blue. a) full spectrum. b) zoom into the spectral region between 6 and $4 \mathrm{ppm}$. The highlighted signals show the interconversion of the major and minor species (negative phase, green cross-peaks) vs. the positive, blue cross-peaks for the NOE/ROE contacts. 

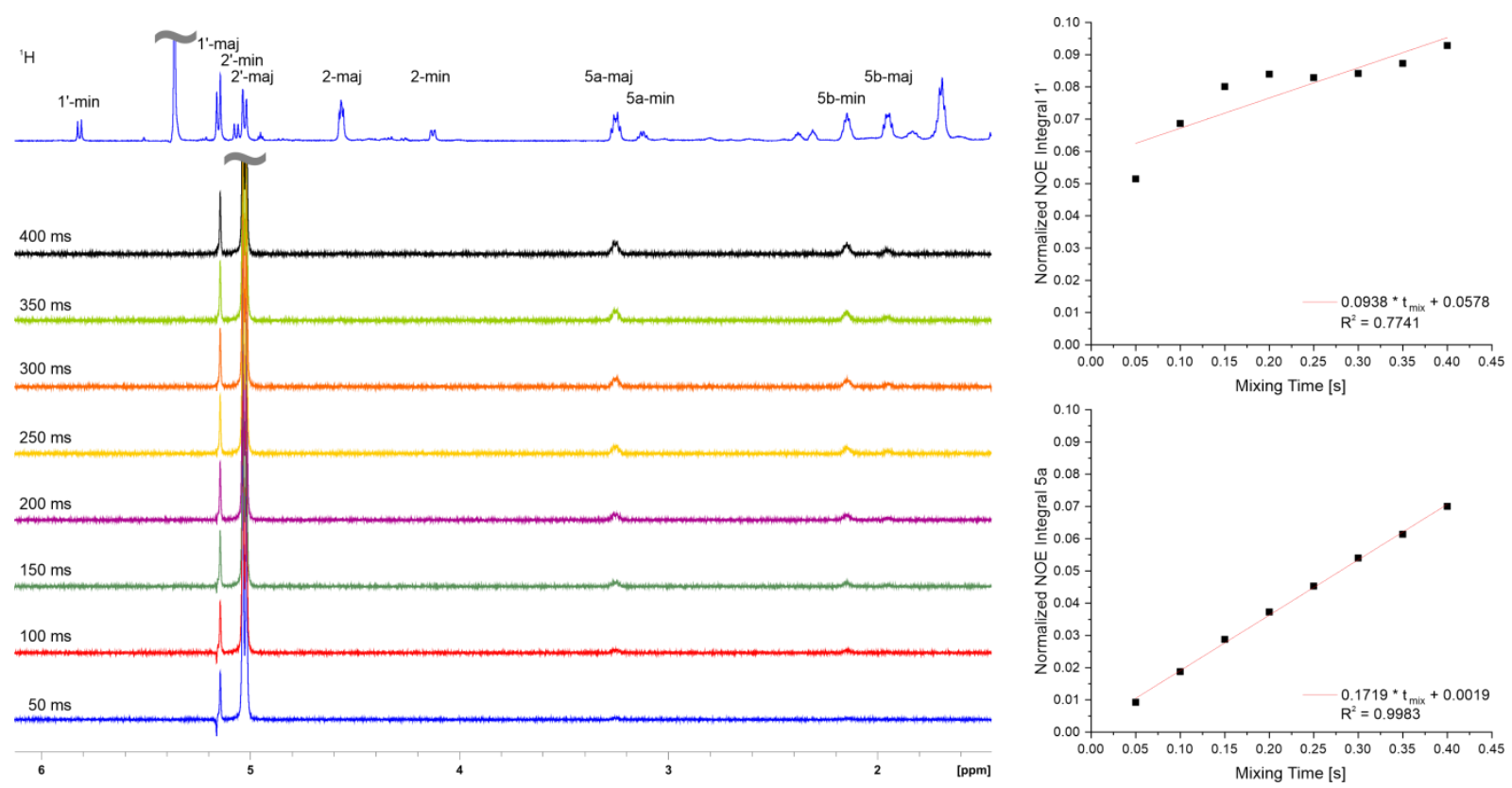

Figure SI-28. a) Example of a selective 1D NOE mixing time series of aminal 14 in $\mathrm{CD}_{2} \mathrm{Cl}_{2}$ at $190 \mathrm{~K}$, with the signal H2'-major selected. The mixing time is indicated with each spectrum. The NOE spectra are scaled to match the intensity of the selected signal for display purposes. The blue spectrum at the very top shows the corresponding part of the $1 \mathrm{D}{ }^{1} \mathrm{H}$ NMR spectrum. Note the distorted line shape of the $\mathrm{H} 1$ '-major signal at $5.15 \mathrm{ppm}$ which never reaches the expected doublet lineshape due to insufficient zero-quantum suppression. This leads to a larger error on the distance extracted from this peak (see below). All other signals show the expected undistorted linear build-up of the NOE cross-peak intensities with increasing mixing time. b) Selected PANIC plots for the normalized NOE integrals for the NOEs $\mathrm{H} 2$ '-major -> H1'-major (top panel) and H2'-major -> H5a-major. As expected from the lineshapes, the linear fit of the normalized integrals is fairly bad for the first NOE (H2'-major -> H1'-major) due to the $J$ coupling contributions, while the latter example shows the high-confidence linear fit $\left(R^{2}>0.99\right)$ observed for all other NOE contacts. The slope of the linear fit to the PANIC plot is used as crossrelaxation rate $\sigma$. Note that some "relayed" cross peaks are visible, which originate from magnetization transfer between the two species caused by chemical exchange and subsequent NOE (or vice versa).

The NOE derived distances calculated from the cross-relaxation rates by internal calibration with the cross-relaxation rate of the diastereotopic proton pair $\mathrm{H} 5 \mathrm{a} / \mathrm{b}$ are collected in Table SI-17. In cases where both signals could be selected with the selective pulse in the 1D NOE spectra, the distances corresponding to the individual cross-relaxation rates are averaged. While the set of distances for the minor species is smaller, the observed distances show very similar values for both species. Particularly the close distances from the protons $\mathrm{H}_{1}$ ' and $\mathrm{H} 2$ ' of the aminal to the pyrrolidine protons $\mathrm{H} 2$ and $\mathrm{H} 5 \mathrm{~b}$ indicate a similar geometry of both major and minor species with respect to the relative arrangement of the $\mathrm{Cl}$ and phthalimide substituents with respect to the pyrrolidine ring. The particularly interesting distance between the protons $\mathrm{H} 1$ ' and H2' in the aminal is difficult to address with these measurements. We used a variant of the 1D NOE pulse sequence already including a Thrippleton-Keeler element ${ }^{4}$ to suppress unwanted contributions by $J$-coupling of the two neighboring nuclei, however, the applied filter element was apparently not able to fully suppress these effects in the case of these two protons. Over 
the course of the mixing time series, the shape of the multiplet of the H2' signal changes over time (see Figure SI-28, the mixing time series for the $\mathrm{H} 1^{\prime}$ '-minor $->\mathrm{H} 2$ '-minor NOE shows the same effect but to a lesser extent), indicating $J$-coupling evolution alongside the desired NOE build-up. This effect results in an apparent oscillation in the normalized intensities of the PANIC plot (noted already by Kolmer et al. ${ }^{9}$ for such cases) and finally gives a reduced apparent NOE derived distance (see Table SI-17). Correcting for these effects would require extensive numerical simulations, which we did not undertake. We estimate the true experimental value for the H1'-H2' NOE distance to be around $2.9 \AA$, consistent with the anti-periplanar arrangement derived from the large $(>10 \mathrm{~Hz}){ }^{3} J_{H H}$ couplings.

Table SI-17. Experimental NOE derived distances for aminal 14 in $\mathrm{CDCl}_{3}$ at $219 \mathrm{~K}$ and $\mathrm{CD}_{2} \mathrm{Cl}_{2}$ at $190 \mathrm{~K}$.

\begin{tabular}{|c|c|c|c|c|c|c|c|}
\hline \multirow[b]{4}{*}{ Nucleus 1} & \multirow[b]{4}{*}{ Nucleus 2} & \multirow{2}{*}{\multicolumn{4}{|c|}{ Quantitative 1D selective NOE }} & \multicolumn{2}{|c|}{ Semi-quantitative } \\
\hline & & & & & & $2 \mathrm{D}$ & F1-PSYCHE- \\
\hline & & \multicolumn{2}{|c|}{$\mathrm{CDCl}_{3} @ 219 \mathrm{~K}$} & \multicolumn{2}{|c|}{$\mathrm{CD}_{2} \mathrm{Cl}_{2} @ 190 \mathrm{~K}$} & \multicolumn{2}{|c|}{$\mathrm{CD}_{2} \mathrm{Cl}_{2} @ 190 \mathrm{~K}$} \\
\hline & & $\operatorname{Major}(\AA ̊)$ & $\operatorname{Minor}(\AA)$ & Major $(\AA ̊)$ & Minor $(\AA ̊)$ & Major $(\AA ̊)$ & Minor $(\AA ̊)$ \\
\hline $1^{\prime}$ & 2 & 2.30 & 2.44 & $2.21(2)$ & $2.30(3)$ & 2.38 & 2.14 \\
\hline $1^{\prime}$ & $5 a$ & 3.10 & & $3.08(6)$ & & 2.29 & \\
\hline $1^{\prime}$ & $2^{\prime}$ & $2.58^{1}$ & $2.67^{1}$ & $2.39(27)^{1}$ & $2.79(5)^{1}$ & & \\
\hline $1^{\prime}$ & $3^{\prime}$ & & & $2.79(4)$ & & 2.44 & \\
\hline $1^{\prime}$ & 5 & & & & & 2.43 & 2.47 \\
\hline $2^{\prime}$ & 2 & & & $3.56(9)$ & & & \\
\hline $2^{\prime}$ & $5 a$ & 2.16 & 2.16 & $2.15(6)$ & $2.21(3)$ & 1.98 & 1.98 \\
\hline $2^{\prime}$ & $5 b$ & 2.22 & & $2.53(7)$ & & & \\
\hline $2^{\prime}$ & $3^{\prime}$ & & & $2.22(6)$ & & 2.22 & 2.01 \\
\hline $2^{\prime}$ & $4^{\prime}$ & & & $3.03(3)$ & & 2.72 & 2.74 \\
\hline $3^{\prime}$ & $5 a$ & & & $2.72(5)$ & & & \\
\hline $3^{\prime}$ & $5 b$ & & & $2.41(3)$ & & 2.28 & \\
\hline $3^{\prime}$ & $4^{\prime}$ & & & $2.90(3)$ & & 2.61 & 2.35 \\
\hline 3 & $5^{\prime}$ & & & & & 2.50 & 2.47 \\
\hline 4 & 5 & & & & & 3.05 & \\
\hline $5^{\prime}$ & $5 a$ & & & $3.47(5)$ & & & \\
\hline $5^{\prime}$ & $5 b$ & & & $3.28(4)$ & & & \\
\hline 2 & $5 a$ & & & $3.17(10)$ & & & \\
\hline $4 a$ & $4 b$ & & & & & 1.85 & 1.85 \\
\hline $4 a$ & $5 a$ & & & & & 2.46 & \\
\hline $4 b$ & $5 b$ & & & & & 2.31 & \\
\hline $5 a$ & $5 b$ & $1.76^{2}$ & 1.77 & $1.76^{2}$ & $1.83(1)$ & $1.76^{2}$ & 1.83 \\
\hline
\end{tabular}


1 insufficient zero-quantum suppression. Oscillation observed in PANIC fit.

${ }^{2}$ fixed reference distance.

Using the optimized geometries from the DFT calculations of chemical shifts enables the comparison of experimental distances to expected ones for different relative configurations of C1' and C2' as well as different conformations of the pyrrolidine ring and the bulky ligand. The syn geometries use the phthalimide substituent, while the anti geometries are taken from the corresponding succinimide calculations below. The values in Table SI-18 show an overall better agreement of experimental and calculated values for the syn arrangement of $\mathrm{Cl}$ and imide substituents at C2' and C1', respectively. Applying ensemble averaging with the populations used below in the calculation of chemical shifts improves the agreement with the experimental data slightly (see Table SI-19). From the geometry optimizations, particularly short distances are expected for $\mathrm{H}^{\prime}{ }^{\prime}-\mathrm{H} 2, \mathrm{H} 2{ }^{\prime}-\mathrm{H} 5 \mathrm{a}$ and $\mathrm{H} 3^{\prime}-\mathrm{H} 5 \mathrm{~b}$ for the syn diastereoisomers, while the latter distance is expected to be much larger in the anti diastereoisomers. Distances expected to be larger than $3 \AA$ from the DFT calculations are apparently underestimated by the experimental methods, which might be taken as indication for conformational flexibility (see below). Also distances involving the methyl groups 4' and 5' are underestimated. Interestingly, the experimental value for the H2'-H2 NOE distance is much shorter as compared to the expected value from the calculated ensembles. While this one particular value is well reproduced in the anti(S,S)/sc-exo geometry, several others (notably the short H3'-H5b distance) show much larger deviations from the experimental values. The observed deviations of NOEs involving methyl groups are a notorious issue in NOE analysis. The internal averaging of the internuclear interactions of the three methyl protons and another proton of interest are coupled to the overall tumbling of the molecule as well as any internal averaging related to conformational flexibility of the entire molecule. Furthermore the averaging depends on the angle the bond vectors (C-Me axis for the methyl group) of the two interacting partner take with respect to each other. ${ }^{10,42}$ The approximations taken herein, namely equal weighting of the three methyl proton distances (usually called "slow tumbling" or " $r^{-3}$ averaging", equation (2)) without considering corrections to angular contributions in addition to the " $r^{-6}$ averaging" of several geometries to form a conformer ensemble may not be applicable to the case here. Ignoring the methyl groups in the distance comparisons improves the agreement of experimental and calculated distances significantly, to $\sim 0.4 \AA$ RMSD for the favored syn models vs up to $1.0 \AA$ for the disfavored anti models of the major species. Thus the NOE data indicate that the major species is the syn-configured aminal. As the RMSD values are the root mean squared deviations normalized to the number of observations, they are expected to be larger for the major species, where four times the number of NOEs are experimentally observed than for the minor species. Thus the smaller value of the RMSD for the minor species does not indicate a better fit to any of the structures and no discrimination between structures is possible as all RMSD values for the minor species are (almost) within experimental error (at least $0.1 \AA$ ). The experimental NOE data therefore suggests that the major species is the syn diastereoisomer and not any of the anti models. With the reduced dataset for the minor species, however, no discrimination between the two configurations is possible based on NOEs alone. The NOEs indicate that the conformation of the pyrrolidine ring with respect to the rest of the compound (rotation around $\mathrm{N}-\mathrm{C} 1^{\prime}$ ' bond) is unchanged. Thus this can be excluded as a reason for the second signal set. ${ }^{43}$ As the large ${ }^{3} \mathrm{~J}$ coupling between $\mathrm{H} 1$ ' 
and $\mathrm{H}_{2}$ ' indicates that the antiperiplanar arrangement prevails, and the average ring-pucker is different in the two signal sets, we consider it unlikely that the minor signal set would be due to the antidiastereoisomer but consider a different conformation (sc-exo, see below and main text) more likely. Furthermore, quite distinct chemical shifts are observed. Thus we conducted calculations of the chemical shifts (see below).

Table SI-18. Comparison of experimentally derived NOE distances from quantitative 1D selective NOE spectra $\left(\mathrm{CD}_{2} \mathrm{Cl}_{2}, 190 \mathrm{~K}\right)$ with the internuclear distances of different structure models of aminal 14 . For the calculated geometries, the first row specifies the arrangement of the $\mathrm{Cl}$ and imide substituents at C2' and C1', while the second row specifies the orientation of the bulky ligand at C2. The last column shows a potential geometry where the pyrrolidine ring is rotated $180^{\circ}$ relative to the aminal.

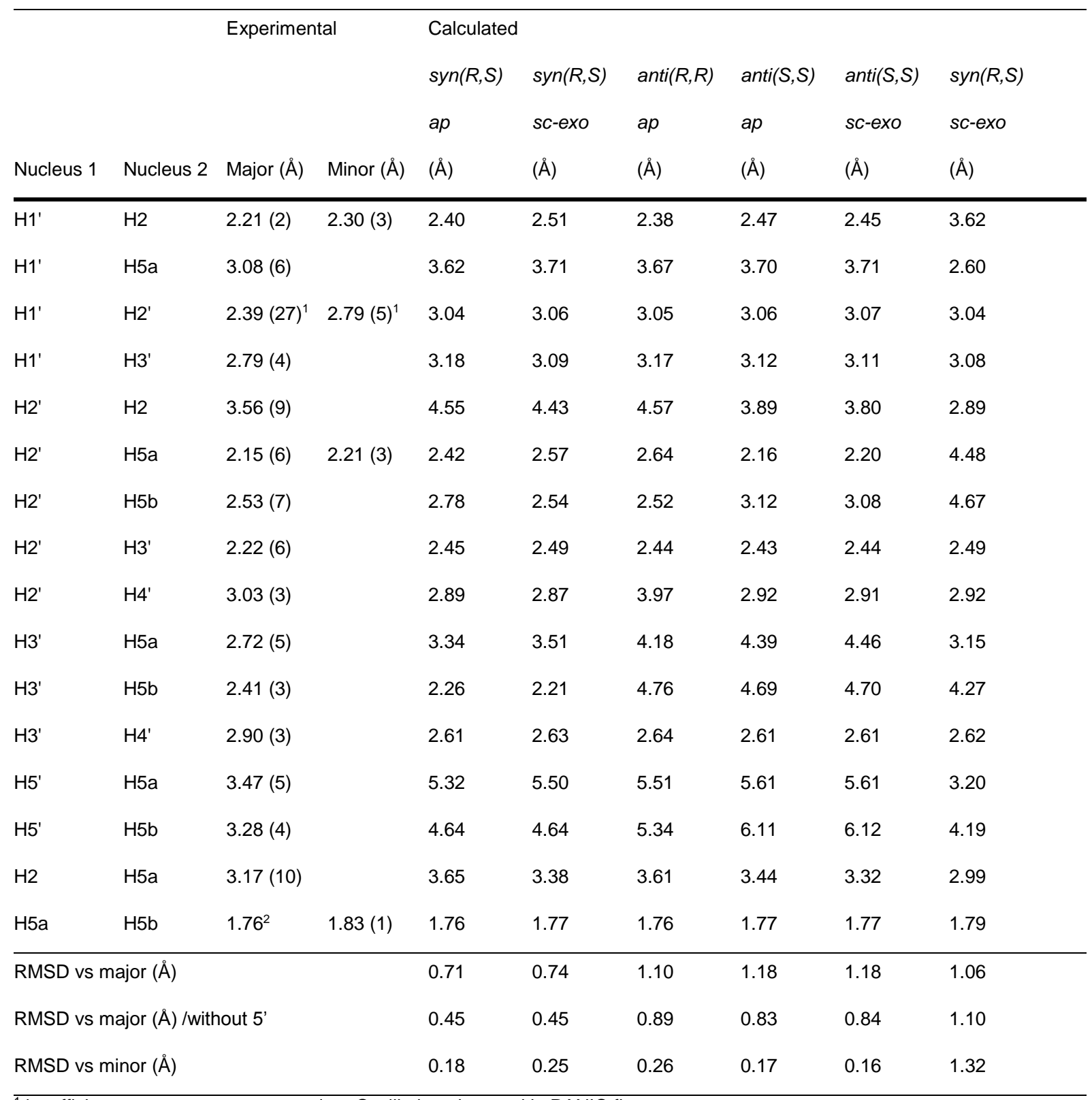

${ }^{1}$ insufficient zero-quantum suppression. Oscillation observed in PANIC fit.

2 fixed reference distance. 
Table SI-19. Comparison of experimentally derived NOE distances from quantitative 1D selective NOE spectra $\left(\mathrm{CD}_{2} \mathrm{Cl}_{2}, 190 \mathrm{~K}\right)$ with the internuclear distances of different averaged ensemble structure models of aminal 14. For the calculated ensembles, the first row specifies the arrangement of the $\mathrm{Cl}$ and imide substituents at C2' and C1', while the second row specifies the orientation of the bulky ligand at C2.

\begin{tabular}{|c|c|c|c|c|c|c|c|c|}
\hline \multirow[b]{4}{*}{ Nucleus 1} & \multirow[b]{4}{*}{ Nucleus 2} & \multicolumn{2}{|c|}{ Experimental } & \multicolumn{5}{|c|}{ Calculated averaged ensemble } \\
\hline & & \multirow[b]{3}{*}{$\operatorname{Major}(\AA ̊)$} & \multirow[b]{3}{*}{ Minor $(\AA)$} & \multirow{3}{*}{$\begin{array}{l}\operatorname{syn}(R, S) \\
a p \\
(\AA)\end{array}$} & \multirow{3}{*}{$\begin{array}{l}\operatorname{syn}(R, S) \\
\text { sc-exo } \\
(\AA)\end{array}$} & \multirow{3}{*}{$\begin{array}{l}\text { anti(R,R) } \\
\text { ap } \\
(\AA)\end{array}$} & \multirow{3}{*}{$\begin{array}{l}\text { anti }(S, S) \\
\text { ap } \\
(\AA)\end{array}$} & \multirow{3}{*}{$\begin{array}{l}\text { anti(S,S) } \\
\text { sc-exo } \\
(\AA)\end{array}$} \\
\hline & & & & & & & & \\
\hline & & & & & & & & \\
\hline $\mathrm{H} 1^{\prime}$ & $\mathrm{H} 2$ & $2.21(2)$ & $2.30(3)$ & 2.38 & 2.48 & 2.33 & 2.47 & 2.45 \\
\hline $\mathrm{H} 1^{\prime}$ & $\mathrm{H} 5 \mathrm{a}$ & $3.08(6)$ & & 3.62 & 3.68 & 3.64 & 3.58 & 3.71 \\
\hline $\mathrm{H} 1^{\prime}$ & $\mathrm{H}^{\prime}$ & $2.39(27)^{1}$ & $2.79(5)^{1}$ & 3.04 & 3.06 & 3.04 & 3.06 & 3.07 \\
\hline $\mathrm{H} 1^{\prime}$ & H3' & $2.79(4)$ & & 3.19 & 3.12 & 3.21 & 3.17 & 3.11 \\
\hline H2' & $\mathrm{H} 2$ & $3.56(9)$ & & 4.54 & 4.43 & 4.56 & 3.79 & 3.80 \\
\hline H2' & $\mathrm{H} 5 \mathrm{a}$ & $2.15(6)$ & $2.21(3)$ & 2.29 & 2.27 & 2.35 & 2.16 & 2.20 \\
\hline H2' & $\mathrm{H} 5 \mathrm{~b}$ & $2.53(7)$ & & 2.96 & 2.79 & 2.73 & 3.32 & 3.08 \\
\hline H2' & H3' & $2.22(6)$ & & 2.44 & 2.48 & 2.44 & 2.43 & 2.44 \\
\hline $\mathrm{H}^{\prime}$ & $\mathrm{H} 4^{\prime}$ & $3.03(3)$ & & 2.89 & 2.88 & 3.98 & 2.94 & 2.91 \\
\hline H3' & $\mathrm{H} 5 \mathrm{a}$ & $2.72(5)$ & & 3.04 & 2.99 & 3.99 & 4.20 & 4.46 \\
\hline H3' & $\mathrm{H} 5 \mathrm{~b}$ & $2.41(3)$ & & 2.38 & 2.35 & 4.97 & 4.88 & 4.70 \\
\hline H3' & $\mathrm{H}^{\prime}$ & 2.90 (3) & & 2.61 & 2.62 & 2.64 & 2.61 & 2.61 \\
\hline H5' & $\mathrm{H} 5 \mathrm{a}$ & $3.47(5)$ & & 5.15 & 5.17 & 5.23 & 5.58 & 5.61 \\
\hline H5' & $\mathrm{H} 5 \mathrm{~b}$ & $3.28(4)$ & & 4.73 & 4.72 & 5.63 & 6.20 & 6.12 \\
\hline $\mathrm{H} 2$ & $\mathrm{H} 5 \mathrm{a}$ & $3.17(10)$ & & 3.74 & 3.55 & 3.76 & 3.58 & 3.32 \\
\hline $\mathrm{H} 5 \mathrm{a}$ & $\mathrm{H} 5 \mathrm{~b}$ & $1.76^{2}$ & $1.83(1)$ & 1.77 & 1.77 & 1.76 & 1.77 & 1.77 \\
\hline \multicolumn{4}{|c|}{ RMSD vs major $(\AA ̊)$} & 0.69 & 0.67 & 1.12 & 1.20 & 1.18 \\
\hline \multicolumn{4}{|c|}{ RMSD vs major ( $\AA$ ) / without 5 ' } & 0.43 & 0.40 & 0.91 & 0.85 & 0.84 \\
\hline \multicolumn{4}{|c|}{ RMSD vs minor $(\AA)$} & 0.14 & 0.17 & 0.15 & 0.16 & 0.16 \\
\hline
\end{tabular}

${ }^{1}$ insufficient zero-quantum suppression. Oscillation observed in PANIC fit.

2 fixed reference distance. 


\section{Computational NMR Analysis of Aminals 11 (Succinimide Substituent) and 14 (Phthalimide Substituent)}

The calculated ${ }^{1} \mathrm{H}$ and ${ }^{13} \mathrm{C}$ chemical shifts for the syn, anti( $\left.\mathrm{C} 1^{\prime}\right)$, and anti( $\left.\mathrm{C} 2^{\prime}\right)$ isomers of 11 were compared to Blackmond and coworkers experimental shifts $\left(^{\ddagger}\right)^{28}$ (Tables SI-20-SI29). The weights, or percentages, for the conformers used in the calculations can be found in Table SI-40. The syn-11 diastereomer resulted in the lowest MAD of the ${ }^{1} \mathrm{H}$ and ${ }^{13} \mathrm{C}$ chemical shifts compared to their major product at $-54^{\circ} \mathrm{C}$. The calculated chemical shifts for the anti( $\left(\mathrm{C} 1^{\circ}\right)$ and anti( $\left.\mathrm{C} 2^{\prime}\right)$ isomers were compared to the minor product at $-54^{\circ} \mathrm{C}$, but did not result in good agreement with the experimental shifts. Continuation of the search for the minor product at $-54^{\circ} \mathrm{C}$, led to the separation of the calculated ap and exo-sc rotational conformer chemical shifts for syn-14. When the calculated chemical shifts were separated by this rotational classification, the ap and exo-sc weighted chemical shifts were in good agreement with major and minor chemical shifts respectively at $-54^{\circ} \mathrm{C}$ (Tables SI-30-SI-33). Using the same classification of the ap and exo-sc conformers for the succinimide version of the aminal (syn-11) the weighted ap conformers were in good agreement with major product chemical shifts (mean average deviations (MADs): $1.7 \mathrm{ppm}$ for ${ }^{13} \mathrm{C} ; 0.16 \mathrm{ppm}$ for ${ }^{1} \mathrm{H}$ ), while not all the exo-sc conformer chemical shifts were in good agreement with the minor product chemical shifts, such as the $\mathrm{C}\left(5^{\prime}\right) \mathrm{H}$ shift predicted to be $1.51 \mathrm{ppm}$, but which was reported to occur at $-0.13 \mathrm{ppm}$. Given the similarities between the calculated lowest energy conformers between the phthalimide (syn-14) and succinimide (syn-11) substituent aminals, and the good agreement between the exo-sc chemical shifts, further investigation may be needed to confirm all of the experimental minor product chemical shifts.

For NMR calculations with this level of theory: PCM(chloroform)-mPW1PW91/6-311+G(2d,p)//B3LYP$\mathrm{D} 3(\mathrm{BJ}) / 6-31 \mathrm{G}+(\mathrm{d}, \mathrm{p})$ expected absolute deviation for the ${ }^{1} \mathrm{H}$ chemical shifts is $<0.3 \mathrm{ppm}$ and $<7-8 \mathrm{ppm}$ for ${ }^{13} \mathrm{C}$ chemical shifts ${ }^{17,18}$ and are highlighted in red in the tables below. The $\mathrm{C} 2{ }^{\prime}{ }^{13} \mathrm{C}$ shift deviations for both major and minor products when compared to the ap and exo-sc calculated chemical shifts are around this upper limit of deviation. This is expected for carbons bonded to halogens due to relativistic effects. ${ }^{44}$ The ${ }^{1} \mathrm{H}$ shifts for the $\mathrm{H} 2$ ' carbon also had deviations $\sim 0.3 \mathrm{ppm}$ as well, suggesting minor variations in conformers or solvent interactions may be affecting this chemical shift. ${ }^{45}$ Chemical shifts that differ in the major and minor products are indicated $\left({ }^{*}\right)$.Chemical shift assignments which could be switched are assigned to minimize the mean average deviation (MAD and indicated ${ }^{* *}$ ).

The $J$-couplings for the syn-14 ap and exo-sc rotamers were also calculated for the pyrrolidine ring and compared to the calculated experimental J-couplings which were generally within $\sim 0.5 \mathrm{~Hz}$ of the experimental values (Tables SI-34-SI-37). Additionally, the theoretical chemical shifts for the iminium ion that forms from syn-11 were calculated to compare to unassigned peaks in the syn-11 spectra; however, no iminium ion peaks were able to be determined with this comparison (Tables SI-38 \& SI39). The barrier of rotation for the syn-14 ap and exo-sc rotamers was also calculated and found to be $13.9 \mathrm{kcal} \mathrm{mol}^{-1}$ at $-54^{\circ} \mathrm{C}$ with implicit chloroform when rotating the -OTMS group from the ap orientation towards the exo-sc orientation, similar to the experimental value of $12.8 \mathrm{kcal} \mathrm{mol}^{-1}$ (Figure SI-29). 
Table SI-20. Comparison of calculated and experiment major ${ }^{13} \mathrm{C}$ NMR $\delta$ 's for syn-11 (ap) at $-54^{\circ} \mathrm{C}$

\begin{tabular}{|c|c|c|c|c|c|c|}
\hline Exp. C\# & Comp. C\# & Comp. Isotropic & Comp. $\delta$ & Avg. Comp. $\delta$ & Syn. $\delta^{\ddagger}$ & Abs. Dev. \\
\hline C5 & $\mathrm{C} 1$ & 135.6845 & 48.6 & & 48.4 & 0.2 \\
\hline $\mathrm{C} 2$ & $\mathrm{C} 2$ & 116.4527 & 67.1 & & 67.8 & 0.7 \\
\hline C3 & C3 & 156.9831 & 28.2 & & 27.3 & 0.9 \\
\hline $\mathrm{C} 4$ & $\mathrm{C} 4$ & 159.9259 & 25.4 & & 23.8 & 1.6 \\
\hline${ }^{*} \mathrm{C} 11$ & C12 & 107.0240 & 76.1 & & 76.5 & 0.4 \\
\hline${ }^{*} \mathrm{C} 2$ & C14 & 111.0191 & 72.3 & & 64.3 & 8.0 \\
\hline C3' & C16 & 152.8208 & 32.2 & & 28.7 & 3.5 \\
\hline${ }^{*} \mathrm{C} 4{ }^{\prime}$ & C18 & 165.6695 & 19.9 & & 20.8 & 0.9 \\
\hline \multirow[t]{3}{*}{${ }^{\star} \mathrm{C} 5$} & C22 & 173.5483 & 12.3 & & 11.7 & 0.6 \\
\hline & $\mathrm{C} 27$ & 154.8925 & 30.2 & & & \\
\hline & C28 & 155.4406 & 29.7 & & & \\
\hline${ }^{* *} \mathrm{C} 5 "$ & C34 & -2.2923 & 181.0 & & 179.5 & 1.5 \\
\hline${ }^{* *} \mathrm{C} 2 "$ & C36 & -3.8047 & 182.5 & & 180.9 & 1.6 \\
\hline \multirow[t]{19}{*}{${ }^{*} \mathrm{C} 6$} & C38 & 100.1433 & 82.7 & & 81.9 & 0.8 \\
\hline & C39 & 34.6347 & 145.6 & & & \\
\hline & C40 & 50.0127 & 130.8 & & & \\
\hline & C41 & 48.8364 & 132.0 & & & \\
\hline & $\mathrm{C} 42$ & 50.6022 & 130.3 & & & \\
\hline & C44 & 51.8776 & 129.1 & & & \\
\hline & C46 & 59.7108 & 121.5 & & & \\
\hline & C48 & 33.1497 & 147.0 & & & \\
\hline & C49 & 48.2190 & 132.6 & & & \\
\hline & C50 & 46.7355 & 134.0 & & & \\
\hline & C51 & 48.3657 & 132.4 & & & \\
\hline & C53 & 49.2026 & 131.6 & & & \\
\hline & C55 & 58.2757 & 122.9 & & & \\
\hline & C57 & 52.3549 & 128.6 & & & \\
\hline & C58 & 52.4529 & 128.5 & & & \\
\hline & C59 & 51.8145 & 129.1 & & & \\
\hline & C60 & 51.9597 & 129.0 & & & \\
\hline & C62 & 184.8693 & 1.4 & & & \\
\hline & C66 & 186.5223 & -0.2 & & & \\
\hline \multirow[t]{2}{*}{ *OTMS } & C70 & 185.8696 & 0.5 & 0.6 & 1.3 & 0.7 \\
\hline & & & & & MAD: & 1.7 \\
\hline
\end{tabular}


Table SI-21. Comparison of calculated and experimental major ${ }^{1} \mathrm{H}$ NMR $\delta$ 's for syn-11 (ap) at $-54^{\circ} \mathrm{C}$.

\begin{tabular}{|c|c|c|c|c|c|c|c|}
\hline $\begin{array}{c}\text { Exp. } \\
\#\end{array}$ & $\begin{array}{c}\text { Comp. } \\
\text { C\# }\end{array}$ & Comp. H\# & Comp. Isotropic & Comp. $\delta$ & $\begin{array}{c}\text { Avg. } \\
\text { Comp. } \delta\end{array}$ & $\begin{array}{c}\text { Exp. } \\
\delta^{\ddagger}\end{array}$ & Abs. Dev. \\
\hline $\mathrm{C}(5) \mathrm{H}$ & 1 & H5 & 28.22 & 3.41 & 3.41 & 3.20 & 0.21 \\
\hline $\mathrm{C}(5) \mathrm{H}$ & 1 & $\mathrm{H} 6$ & 29.88 & 1.86 & 1.86 & 1.85 & 0.01 \\
\hline${ }^{*} \mathrm{C}(2) \mathrm{H}$ & 2 & $\mathrm{H} 7$ & 26.77 & 4.76 & 4.76 & 4.48 & 0.28 \\
\hline $\mathrm{C}(3) \mathrm{H}$ & 3 & $\mathrm{H} 8$ & 29.94 & 1.80 & & & \\
\hline $\mathrm{C}(4) \mathrm{H}$ & 4 & $\mathrm{H} 9$ & 31.64 & 0.21 & 0.21 & 0.13 & 0.08 \\
\hline $\mathrm{C}(4) \mathrm{H}$ & 4 & $\mathrm{H} 10$ & 30.57 & 1.21 & 1.21 & 1.15 & 0.06 \\
\hline${ }^{*} \mathrm{C}\left(1^{\prime}\right) \mathrm{H}$ & 12 & $\mathrm{H} 13$ & 26.35 & 5.16 & 5.16 & 5.00 & 0.16 \\
\hline${ }^{*} \mathrm{C}\left(2^{\prime}\right) \mathrm{H}$ & 14 & $\mathrm{H} 15$ & 26.29 & 5.21 & 5.21 & 4.90 & 0.31 \\
\hline \multirow[t]{3}{*}{$\mathrm{C}\left(3^{\prime}\right) \mathrm{H}$} & 16 & $\mathrm{H} 17$ & 29.39 & 2.32 & 2.32 & 2.05 & 0.27 \\
\hline & 18 & $\mathrm{H} 19$ & 30.65 & 1.14 & & & \\
\hline & 18 & $\mathrm{H} 20$ & 30.74 & 1.06 & & & \\
\hline \multirow[t]{3}{*}{${ }^{* *} \mathrm{C}\left(4^{\prime}\right) \mathrm{H}$} & 18 & $\mathrm{H} 21$ & 30.86 & 0.95 & 1.05 & 0.91 & 0.14 \\
\hline & 22 & $\mathrm{H} 23$ & 31.39 & 0.45 & & & \\
\hline & 22 & $\mathrm{H} 24$ & 31.83 & 0.04 & & & \\
\hline \multirow[t]{2}{*}{${ }^{* *} \mathrm{C}\left(5^{\prime}\right) \mathrm{H}$} & 22 & $\mathrm{H} 25$ & 32.15 & -0.26 & 0.08 & -0.13 & 0.21 \\
\hline & 27 & $\mathrm{H} 29$ & 29.07 & 2.62 & & & \\
\hline \multirow[t]{2}{*}{${ }^{* *} \mathrm{C}(3 " / \mathrm{C} 4 ") \mathrm{H}$} & 27 & $\mathrm{H} 30$ & 28.98 & 2.70 & 2.66 & 2.77 & 0.11 \\
\hline & 28 & H31 & 28.89 & 2.78 & & & \\
\hline \multirow[t]{15}{*}{${ }^{* *} \mathrm{C}(3 " / \mathrm{C} 4 ") \mathrm{H}$} & 28 & H32 & 28.89 & 2.79 & 2.79 & 2.94 & 0.15 \\
\hline & 40 & $\mathrm{H} 43$ & 22.84 & 8.43 & & & \\
\hline & 41 & H32 & 26.54 & 7.74 & & & \\
\hline & 46 & $\mathrm{H} 47$ & 23.36 & 7.94 & & & \\
\hline & 49 & H52 & 21.22 & 9.94 & & & \\
\hline & 50 & $\mathrm{H} 54$ & 46.39 & -13.55 & & & \\
\hline & 55 & H56 & 23.35 & 7.95 & & & \\
\hline & 62 & $\mathrm{H} 63$ & 31.37 & 0.47 & & & \\
\hline & 62 & $\mathrm{H} 64$ & 31.43 & 0.41 & & & \\
\hline & 62 & H65 & 31.66 & 0.20 & & & \\
\hline & 66 & $\mathrm{H} 67$ & 32.17 & -0.28 & & & \\
\hline & 66 & $\mathrm{H} 68$ & 32.39 & -0.48 & & & \\
\hline & 66 & $\mathrm{H} 69$ & 32.83 & -0.90 & & & \\
\hline & 70 & $\mathrm{H} 71$ & 31.85 & 0.02 & & & \\
\hline & 70 & $\mathrm{H} 72$ & 31.80 & 0.07 & & & \\
\hline *OTMS & 70 & H73 & 32.03 & -0.14 & -0.07 & -0.26 & 0.19 \\
\hline \multirow[t]{2}{*}{$\mathrm{C}(3) \mathrm{H}$} & 3 & $\mathrm{H} 87$ & 29.86 & 1.88 & 1.84 & 1.72 & 0.12 \\
\hline & & & & & & MAD & 0.16 \\
\hline
\end{tabular}


Table SI-22. Comparison of calculated and experimental minor ${ }^{13} \mathrm{C}$ NMR $\delta$ 's for syn-11 (exo-sc) at $-54^{\circ} \mathrm{C}$.

\begin{tabular}{|c|c|c|c|c|c|c|}
\hline Exp. C\# & Comp. C\# & Comp. Isotropic & Comp. $\delta$ & Avg. Comp. $\delta$ & Syn. $\delta^{\neq}$ & Abs. Dev \\
\hline C5 & C1 & 136.4713 & 47.9 & & 48.4 & 0.5 \\
\hline C2 & C2 & 117.7784 & 65.8 & & 70.7 & 4.9 \\
\hline C3 & C3 & 156.1918 & 28.9 & & 27.3 & 1.6 \\
\hline C4 & C4 & 160.7078 & 24.6 & & 23.8 & 0.8 \\
\hline${ }^{*} \mathrm{C} 1{ }^{\prime}$ & $\mathrm{C} 12$ & 107.8337 & 75.4 & & 76.5 & 1.1 \\
\hline${ }^{*} \mathrm{C} 2{ }^{\prime}$ & C14 & 109.9297 & 73.3 & & 65.4 & 7.9 \\
\hline $\mathrm{C}^{\prime}$ & C16 & 153.0082 & 32.0 & & 28.7 & 3.3 \\
\hline${ }^{*} \mathrm{C} 4{ }^{\prime}$ & C18 & 165.8347 & 19.7 & & 20.8 & 1.1 \\
\hline \multirow[t]{3}{*}{${ }^{*} \mathrm{C} 5{ }^{\prime}$} & C22 & 169.4198 & 16.3 & & 11.7 & 4.6 \\
\hline & C27 & 155.0574 & 30.0 & & & \\
\hline & C28 & 155.2203 & 29.9 & & & \\
\hline${ }^{* *} \mathrm{C} 5 "$ & C34 & -2.7958 & 181.5 & & 179 & 2.5 \\
\hline${ }^{* \star} \mathrm{C} 2 "$ & C36 & -2.1768 & 180.9 & & 180.1 & 0.8 \\
\hline \multirow[t]{19}{*}{${ }^{*} \mathrm{C} 6$} & C38 & 98.5761 & 84.2 & & 83.7 & 0.5 \\
\hline & С39 & 34.5114 & 145.7 & & & \\
\hline & $\mathrm{C} 40$ & 46.9006 & 133.8 & & & \\
\hline & C41 & 49.8154 & 131.0 & & & \\
\hline & C42 & 49.4106 & 131.4 & & & \\
\hline & C44 & 49.4941 & 131.3 & & & \\
\hline & C46 & 57.4787 & 123.7 & & & \\
\hline & C48 & 33.6336 & 146.6 & & & \\
\hline & C49 & 51.1595 & 129.7 & & & \\
\hline & C50 & 47.3518 & 133.4 & & & \\
\hline & C51 & 51.6159 & 129.3 & & & \\
\hline & C53 & 51.1792 & 129.7 & & & \\
\hline & C55 & 59.7931 & 121.5 & & & \\
\hline & C57 & 52.2149 & 128.7 & & & \\
\hline & C58 & 51.6628 & 129.3 & & & \\
\hline & C59 & 52.2399 & 128.7 & & & \\
\hline & $\mathrm{C} 60$ & 52.5823 & 128.4 & & & \\
\hline & C62 & 185.8896 & 0.4 & & & \\
\hline & C66 & 184.4579 & 1.8 & & & \\
\hline \multirow[t]{2}{*}{ *OTMS } & $\mathrm{C} 70$ & 184.2350 & 2.0 & 1.4 & 1.5 & 0.1 \\
\hline & & & & & MAD: & 2.3 \\
\hline
\end{tabular}


Table SI-23. Comparison of calculated and experimental minor ${ }^{1} \mathrm{H}$ NMR $\delta$ 's for syn-11 (exo-sc) at $-54^{\circ} \mathrm{C}$.

\begin{tabular}{|c|c|c|c|c|c|c|c|}
\hline $\begin{array}{c}\text { Exp. } \\
\text { C\# }\end{array}$ & $\begin{array}{c}\text { Comp. } \\
\text { C\# }\end{array}$ & Comp. H\# & $\begin{array}{c}\text { Comp. } \\
\text { Isotropic }\end{array}$ & Comp. $\delta$ & $\begin{array}{c}\text { Avg. } \\
\text { Comp. } \delta \\
\end{array}$ & $\begin{array}{c}\text { Exp. } \\
\delta^{f}\end{array}$ & Abs. Dev. \\
\hline $\mathrm{C}(5) \mathrm{H}$ & 1 & $\mathrm{H} 5$ & 28.34 & 3.29 & 3.29 & 3.2 & 0.09 \\
\hline $\mathrm{C}(5) \mathrm{H}$ & 1 & $\mathrm{H} 6$ & 29.45 & 2.26 & 2.26 & 1.85 & 0.41 \\
\hline${ }^{*} \mathrm{C}(2) \mathrm{H}$ & 2 & $\mathrm{H} 7$ & 27.34 & 4.23 & 4.23 & 4.08 & 0.15 \\
\hline $\mathrm{C}(3) \mathrm{H}$ & 3 & $\mathrm{H} 8$ & 29.88 & 1.86 & & & \\
\hline $\mathrm{C}(4) \mathrm{H}$ & 4 & H9 & 31.73 & 0.13 & 0.13 & 0.13 & 0.00 \\
\hline $\mathrm{C}(4) \mathrm{H}$ & 4 & $\mathrm{H} 10$ & 30.61 & 1.18 & 1.18 & 1.15 & 0.03 \\
\hline${ }^{*} \mathrm{C}\left(1^{\prime}\right) \mathrm{H}$ & 12 & $\mathrm{H} 13$ & 25.50 & 5.95 & 5.95 & 5.66 & 0.29 \\
\hline${ }^{*} \mathrm{C}\left(2^{\prime}\right) \mathrm{H}$ & 14 & $\mathrm{H} 15$ & 26.24 & 5.25 & 5.25 & 4.95 & 0.30 \\
\hline \multirow[t]{3}{*}{$\mathrm{C}\left(3^{\prime}\right) \mathrm{H}$} & 16 & $\mathrm{H} 17$ & 29.06 & 2.62 & 2.62 & 2.05 & 0.57 \\
\hline & 18 & $\mathrm{H} 19$ & 30.56 & 1.23 & & & \\
\hline & 18 & $\mathrm{H} 2 \mathrm{O}$ & 30.75 & 1.05 & & & \\
\hline \multirow[t]{3}{*}{${ }^{* *} \mathrm{C}\left(4^{\prime}\right) \mathrm{H}$} & 18 & $\mathrm{H} 21$ & 30.52 & 1.26 & 1.18 & 0.91 & 0.27 \\
\hline & 22 & $\mathrm{H} 23$ & 30.28 & 1.49 & & & \\
\hline & 22 & $\mathrm{H} 24$ & 30.50 & 1.28 & & & \\
\hline \multirow[t]{2}{*}{${ }^{* *} \mathrm{C}\left(5^{\prime}\right) \mathrm{H}$} & 22 & $\mathrm{H} 25$ & 29.97 & 1.77 & 1.51 & -0.13 & 1.64 \\
\hline & 27 & $\mathrm{H} 29$ & 29.02 & 2.66 & & & \\
\hline \multirow[t]{2}{*}{${ }^{* \star} \mathrm{C}(3 " / \mathrm{C} 4 ") \mathrm{H}$} & 27 & $\mathrm{H} 30$ & 28.98 & 2.70 & 2.68 & 2.77 & 0.09 \\
\hline & 28 & $\mathrm{H} 31$ & 28.85 & 2.82 & & & \\
\hline \multirow[t]{15}{*}{${ }^{* *} \mathrm{C}(3 " / \mathrm{C} 4 ") \mathrm{H}$} & 28 & H32 & 28.94 & 2.74 & 2.78 & 2.94 & 0.16 \\
\hline & 40 & $\mathrm{H} 43$ & 23.70 & 7.63 & & & \\
\hline & 41 & H32 & 26.54 & 8.05 & & & \\
\hline & 46 & $\mathrm{H} 47$ & 23.33 & 7.97 & & & \\
\hline & 49 & H52 & 24.19 & 7.17 & & & \\
\hline & 50 & $\mathrm{H} 54$ & 44.85 & -12.10 & & & \\
\hline & 55 & $\mathrm{H} 56$ & 23.36 & 7.94 & & & \\
\hline & 62 & $\mathrm{H} 63$ & 31.59 & 0.27 & & & \\
\hline & 62 & $\mathrm{H} 64$ & 33.66 & -1.67 & & & \\
\hline & 62 & $\mathrm{H} 65$ & 32.31 & -0.40 & & & \\
\hline & 66 & $\mathrm{H} 67$ & 31.11 & 0.71 & & & \\
\hline & 66 & $\mathrm{H} 68$ & 31.89 & -0.02 & & & \\
\hline & 66 & $\mathrm{H} 69$ & 31.69 & 0.17 & & & \\
\hline & 70 & $\mathrm{H} 71$ & 31.64 & 0.22 & & & \\
\hline & 70 & $\mathrm{H} 72$ & 31.75 & 0.12 & & & \\
\hline${ }^{*} \mathrm{OTMS}$ & 70 & $\mathrm{H} 73$ & 31.85 & 0.02 & -0.06 & -0.29 & 0.23 \\
\hline \multirow[t]{2}{*}{$\mathrm{C}(3) \mathrm{H}$} & 3 & H87 & 30.40 & 1.38 & 1.62 & 1.72 & 0.10 \\
\hline & & & & & & MAD & 0.31 \\
\hline
\end{tabular}


Table SI-24. Comparison of calculated and experimental minor ${ }^{13} \mathrm{C}$ NMR $\delta$ 's for anti(C1')-11 (ap) at $-54^{\circ} \mathrm{C}$.

\begin{tabular}{|c|c|c|c|c|c|c|}
\hline Exp. C\# & Comp. C\# & Comp. Isotropic & Comp. $\delta$ & Avg. Comp. $\delta$ & Syn. $\delta^{\neq}$ & Abs. Dev. \\
\hline C5 & $\mathrm{C} 1$ & 135.9947 & 48.3 & & 48.4 & 0.1 \\
\hline $\mathrm{C} 2$ & $\mathrm{C} 2$ & 115.7302 & 67.8 & & 70.7 & 2.9 \\
\hline C3 & C3 & 157.0734 & 28.1 & & 27.3 & 0.8 \\
\hline $\mathrm{C} 4$ & $\mathrm{C} 4$ & 160.2338 & 25.1 & & 23.8 & 1.3 \\
\hline${ }^{*} \mathrm{C} 1{ }^{\prime}$ & C12 & 114.5331 & 68.9 & & 76.5 & 7.6 \\
\hline${ }^{*} \mathrm{C} 2$ & C14 & 108.4440 & 74.8 & & 65.4 & 9.4 \\
\hline C3' & $\mathrm{C} 16$ & 151.6796 & 33.3 & & 28.7 & 4.6 \\
\hline${ }^{*} \mathrm{C} 4$ & C18 & 165.9822 & 19.6 & & 20.8 & 1.2 \\
\hline \multirow[t]{3}{*}{${ }^{*} \mathrm{C} 5$} & $\mathrm{C} 22$ & 171.6763 & 14.1 & & 11.7 & 2.4 \\
\hline & $\mathrm{C} 27$ & 155.5999 & 29.5 & & & \\
\hline & C28 & 155.5434 & 29.6 & & & \\
\hline${ }^{* *} \mathrm{C} 5 "$ & $\mathrm{C} 34$ & -1.1724 & 180.0 & & 179 & 1.0 \\
\hline${ }^{* *} \mathrm{C} 2 "$ & C36 & 0.6840 & 178.2 & & 180.1 & 1.9 \\
\hline \multirow[t]{19}{*}{${ }^{*} \mathrm{C} 6$} & C38 & 100.5030 & 82.4 & & 83.7 & 1.3 \\
\hline & C39 & 36.4178 & 143.9 & & & \\
\hline & $\mathrm{C} 40$ & 48.3516 & 132.4 & & & \\
\hline & C41 & 49.8264 & 131.0 & & & \\
\hline & C42 & 54.0621 & 127.0 & & & \\
\hline & C44 & 51.5846 & 129.3 & & & \\
\hline & $\mathrm{C} 46$ & 60.4671 & 120.8 & & & \\
\hline & C48 & 34.4201 & 145.8 & & & \\
\hline & C49 & 50.9172 & 130.0 & & & \\
\hline & C50 & 46.9404 & 133.8 & & & \\
\hline & C51 & 49.8517 & 131.0 & & & \\
\hline & C53 & 48.0804 & 132.7 & & & \\
\hline & C55 & 56.4885 & 124.6 & & & \\
\hline & C57 & 51.7504 & 129.2 & & & \\
\hline & C58 & 52.3672 & 128.6 & & & \\
\hline & C59 & 51.8414 & 129.1 & & & \\
\hline & $\mathrm{C} 60$ & 51.3528 & 129.6 & & & \\
\hline & C62 & 185.8755 & 0.5 & & & \\
\hline & C66 & 184.8698 & 1.4 & & & \\
\hline \multirow[t]{2}{*}{ *OTMS } & $\mathrm{C} 70$ & 186.2035 & 0.1 & 0.7 & 1.5 & 0.8 \\
\hline & & & & & MAD: & 2.7 \\
\hline
\end{tabular}


Table SI-25. Comparison of calculated and experimental minor ${ }^{1} \mathrm{H}$ NMR $\delta$ 's for anti(C1')-11 (ap) at $-54^{\circ} \mathrm{C}$.

\begin{tabular}{|c|c|c|c|c|c|c|c|}
\hline $\begin{array}{c}\text { Exp. } \\
\#\end{array}$ & $\begin{array}{c}\text { Comp. } \\
\text { C\# }\end{array}$ & Comp. H\# & Comp. Isotropic & Comp. $\delta$ & $\begin{array}{c}\text { Avg. } \\
\text { Comp. } \delta\end{array}$ & $\begin{array}{c}\text { Exp. } \\
\delta^{\ddagger}\end{array}$ & Abs. Dev. \\
\hline $\mathrm{C}(5) \mathrm{H}$ & 1 & H5 & 28.45 & 3.19 & 3.19 & 3.2 & 0.01 \\
\hline $\mathrm{C}(5) \mathrm{H}$ & 1 & $\mathrm{H} 6$ & 29.84 & 1.90 & 1.90 & 1.85 & 0.05 \\
\hline${ }^{*} \mathrm{C}(2) \mathrm{H}$ & 2 & $\mathrm{H} 7$ & 26.12 & 5.37 & 5.37 & 4.08 & 1.29 \\
\hline $\mathrm{C}(3) \mathrm{H}$ & 3 & H8 & 29.64 & 2.09 & & & \\
\hline $\mathrm{C}(4) \mathrm{H}$ & 4 & $\mathrm{H} 9$ & 31.73 & 0.13 & 0.13 & 0.13 & 0.00 \\
\hline $\mathrm{C}(4) \mathrm{H}$ & 4 & $\mathrm{H} 10$ & 30.34 & 1.43 & 1.43 & 1.15 & 0.28 \\
\hline${ }^{*} \mathrm{C}\left(1^{\prime}\right) \mathrm{H}$ & 12 & $\mathrm{H} 13$ & 26.40 & 5.10 & 5.10 & 5.66 & 0.56 \\
\hline${ }^{*} \mathrm{C}\left(2^{\prime}\right) \mathrm{H}$ & 14 & H15 & 25.93 & 5.54 & 5.54 & 4.95 & 0.59 \\
\hline \multirow[t]{3}{*}{$\mathrm{C}\left(3^{\prime}\right) \mathrm{H}$} & 16 & $\mathrm{H} 17$ & 30.19 & 1.57 & 1.57 & 2.05 & 0.48 \\
\hline & 18 & $\mathrm{H} 19$ & 30.78 & 1.02 & & & \\
\hline & 18 & $\mathrm{H} 20$ & 30.64 & 1.15 & & & \\
\hline \multirow[t]{3}{*}{${ }^{* *} \mathrm{C}\left(4^{\prime}\right) \mathrm{H}$} & 18 & $\mathrm{H} 21$ & 30.86 & 0.94 & 1.04 & 0.91 & 0.13 \\
\hline & 22 & $\mathrm{H} 23$ & 31.16 & 0.67 & & & \\
\hline & 22 & $\mathrm{H} 24$ & 31.15 & 0.67 & & & \\
\hline \multirow[t]{2}{*}{${ }^{* *} \mathrm{C}\left(5^{\prime}\right) \mathrm{H}$} & 22 & $\mathrm{H} 25$ & 30.59 & 1.19 & 0.85 & -0.13 & 0.98 \\
\hline & 27 & $\mathrm{H} 29$ & 29.28 & 2.42 & & & \\
\hline \multirow[t]{2}{*}{${ }^{* *} \mathrm{C}(3 " / \mathrm{C} 4 ") \mathrm{H}$} & 27 & $\mathrm{H} 30$ & 29.05 & 2.63 & 2.52 & 2.77 & 0.25 \\
\hline & 28 & H31 & 29.31 & 2.39 & & & \\
\hline \multirow[t]{15}{*}{${ }^{* *} \mathrm{C}(3 " / \mathrm{C} 4 ") \mathrm{H}$} & 28 & H32 & 28.94 & 2.74 & 2.57 & 2.94 & 0.37 \\
\hline & 40 & $\mathrm{H} 43$ & 23.98 & 7.36 & & & \\
\hline & 41 & $\mathrm{H} 32$ & 26.54 & 8.40 & & & \\
\hline & 46 & $\mathrm{H} 47$ & 23.40 & 7.91 & & & \\
\hline & 49 & H52 & 22.52 & 8.73 & & & \\
\hline & 50 & H54 & 24.02 & 7.32 & & & \\
\hline & 55 & H56 & 23.34 & 7.96 & & & \\
\hline & 62 & H63 & 32.02 & -0.13 & & & \\
\hline & 62 & $\mathrm{H} 64$ & 31.85 & 0.02 & & & \\
\hline & 62 & H65 & 32.28 & -0.38 & & & \\
\hline & 66 & $\mathrm{H} 67$ & 31.80 & 0.07 & & & \\
\hline & 66 & H68 & 31.33 & 0.51 & & & \\
\hline & 66 & H69 & 31.44 & 0.41 & & & \\
\hline & 70 & $\mathrm{H} 71$ & 32.57 & -0.65 & & & \\
\hline & 70 & $\mathrm{H} 72$ & 31.91 & -0.04 & & & \\
\hline *OTMS & 70 & $\mathrm{H} 73$ & 32.21 & -0.31 & -0.06 & -0.29 & 0.23 \\
\hline \multirow[t]{2}{*}{$\mathrm{C}(3) \mathrm{H}$} & 3 & H87 & 29.91 & 1.83 & 1.96 & 1.72 & 0.24 \\
\hline & & & & & & MAD & 0.39 \\
\hline
\end{tabular}


Table SI-26. Comparison of calculated and experimental minor ${ }^{13} \mathrm{C}$ NMR $\delta$ 's for anti(C1')-11 (exo-sC) at $-54^{\circ} \mathrm{C}$.

\begin{tabular}{|c|c|c|c|c|c|c|}
\hline Exp. C\# & Comp. C\# & Comp. Isotropic & Comp. $\delta$ & Avg. Comp. $\delta$ & Syn. $\delta^{\neq}$ & Abs. Dev \\
\hline C5 & C1 & 135.4575 & 48.8 & & 48.4 & 0.4 \\
\hline $\mathrm{C} 2$ & $\mathrm{C} 2$ & 110.9980 & 72.3 & & 70.7 & 1.6 \\
\hline C3 & C3 & 156.3759 & 28.8 & & 27.3 & 1.5 \\
\hline C4 & C4 & 160.0842 & 25.2 & & 23.8 & 1.4 \\
\hline${ }^{*} \mathrm{C} 1{ }^{\prime}$ & $\mathrm{C} 12$ & 113.3557 & 70.1 & & 76.5 & 6.4 \\
\hline${ }^{*} \mathrm{C} 2{ }^{\prime}$ & C14 & 109.9617 & 73.3 & & 65.4 & $7.9^{\ddagger}$ \\
\hline $\mathrm{C}^{\prime}$ & C16 & 151.9450 & 33.0 & & 28.7 & 4.3 \\
\hline${ }^{*} \mathrm{C} 4{ }^{\prime}$ & C18 & 165.7677 & 19.8 & & 20.8 & 1.0 \\
\hline \multirow[t]{3}{*}{${ }^{*} \mathrm{C} 5{ }^{\prime}$} & C22 & 171.3154 & 14.4 & & 11.7 & 2.7 \\
\hline & C27 & 155.0763 & 30.0 & & & \\
\hline & C28 & 154.6013 & 30.5 & & & \\
\hline${ }^{* *} \mathrm{C} 5 "$ & C34 & -2.1163 & 180.9 & & 179 & 1.9 \\
\hline${ }^{* \star} \mathrm{C} 2 "$ & C36 & -0.3893 & 179.2 & & 180.1 & 0.9 \\
\hline \multirow[t]{19}{*}{${ }^{*} \mathrm{C} 6$} & C38 & 96.1129 & 86.6 & & 83.7 & 2.9 \\
\hline & С39 & 33.8334 & 146.4 & & & \\
\hline & $\mathrm{C} 40$ & 48.5481 & 132.3 & & & \\
\hline & C41 & 48.0533 & 132.7 & & & \\
\hline & C42 & 49.9668 & 130.9 & & & \\
\hline & C44 & 48.8141 & 132.0 & & & \\
\hline & C46 & 58.6709 & 122.5 & & & \\
\hline & C48 & 34.4047 & 145.8 & & & \\
\hline & C49 & 51.4280 & 129.5 & & & \\
\hline & C50 & 46.5023 & 134.2 & & & \\
\hline & C51 & 51.3556 & 129.6 & & & \\
\hline & C53 & 52.2943 & 128.7 & & & \\
\hline & C55 & 59.7534 & 121.5 & & & \\
\hline & C57 & 52.1849 & 128.8 & & & \\
\hline & C58 & 51.1248 & 129.8 & & & \\
\hline & C59 & 52.2953 & 128.7 & & & \\
\hline & $\mathrm{C} 60$ & 52.5062 & 128.5 & & & \\
\hline & C62 & 185.1263 & 1.2 & & & \\
\hline & C66 & 185.3374 & 1.0 & & & \\
\hline \multirow[t]{2}{*}{ *OTMS } & $\mathrm{C} 70$ & 186.3819 & 0.0 & 0.7 & 1.5 & 0.8 \\
\hline & & & & & MAD: & 2.6 \\
\hline
\end{tabular}


Table SI-27. Comparison of calculated and experimental minor ${ }^{1} \mathrm{H}$ NMR $\delta$ 's for anti(C1')-11 (exo-sc) at $-54^{\circ} \mathrm{C}$.

\begin{tabular}{|c|c|c|c|c|c|c|c|}
\hline $\begin{array}{l}\text { Exp. } \\
\#\end{array}$ & $\begin{array}{c}\text { Comp. } \\
\text { C\# }\end{array}$ & Comp. H\# & $\begin{array}{l}\text { Comp. } \\
\text { Isotropic }\end{array}$ & Comp. $\delta$ & $\begin{array}{c}\text { Avg. } \\
\text { Comp. } \delta\end{array}$ & $\underset{\delta^{\ddagger}}{\operatorname{Exp} .}$ & Abs. Dev. \\
\hline $\mathrm{C}(5) \mathrm{H}$ & 1 & H5 & 28.55 & 3.10 & 3.10 & 3.2 & 0.10 \\
\hline $\mathrm{C}(5) \mathrm{H}$ & 1 & $\mathrm{H} 6$ & 29.35 & 2.35 & 2.35 & 1.85 & 0.50 \\
\hline${ }^{*} \mathrm{C}(2) \mathrm{H}$ & 2 & $\mathrm{H} 7$ & 26.17 & 5.32 & 5.32 & 4.08 & 1.24 \\
\hline $\mathrm{C}(3) \mathrm{H}$ & 3 & $\mathrm{H} 8$ & 29.54 & 2.17 & & & \\
\hline $\mathrm{C}(4) \mathrm{H}$ & 4 & $\mathrm{H} 9$ & 31.20 & 0.63 & 0.63 & 0.13 & 0.50 \\
\hline $\mathrm{C}(4) \mathrm{H}$ & 4 & $\mathrm{H} 10$ & 30.44 & 1.33 & 1.33 & 1.15 & 0.18 \\
\hline${ }^{*} \mathrm{C}\left(1^{\prime}\right) \mathrm{H}$ & 12 & $\mathrm{H} 13$ & 25.27 & 6.16 & 6.16 & 5.66 & 0.50 \\
\hline${ }^{*} \mathrm{C}\left(2^{\prime}\right) \mathrm{H}$ & 14 & $\mathrm{H} 15$ & 25.69 & 5.77 & 5.77 & 4.95 & 0.82 \\
\hline \multirow[t]{3}{*}{$\mathrm{C}\left(3^{\prime}\right) \mathrm{H}$} & 16 & $\mathrm{H} 17$ & 30.11 & 1.64 & 1.64 & 2.05 & 0.41 \\
\hline & 18 & $\mathrm{H} 19$ & 30.82 & 0.99 & & & \\
\hline & 18 & $\mathrm{H} 20$ & 30.90 & 0.91 & & & \\
\hline \multirow[t]{3}{*}{${ }^{* *} \mathrm{C}\left(4^{\prime}\right) \mathrm{H}$} & 18 & $\mathrm{H} 21$ & 30.49 & 1.29 & 1.06 & 0.91 & 0.15 \\
\hline & 22 & $\mathrm{H} 23$ & 30.62 & 1.17 & & & \\
\hline & 22 & $\mathrm{H} 24$ & 30.63 & 1.16 & & & \\
\hline \multirow[t]{2}{*}{${ }^{* *} \mathrm{C}\left(5^{\prime}\right) \mathrm{H}$} & 22 & $\mathrm{H} 25$ & 31.08 & 0.74 & 1.03 & -0.13 & 1.16 \\
\hline & 27 & $\mathrm{H} 29$ & 29.21 & 2.48 & & & \\
\hline \multirow[t]{2}{*}{${ }^{* *} \mathrm{C}(3 " / \mathrm{C} 4 ") \mathrm{H}$} & 27 & $\mathrm{H} 30$ & 29.12 & 2.57 & 2.52 & 2.77 & 0.25 \\
\hline & 28 & H31 & 29.04 & 2.64 & & & \\
\hline \multirow[t]{15}{*}{${ }^{* *} \mathrm{C}(3 " / \mathrm{C} 4 ") \mathrm{H}$} & 28 & $\mathrm{H} 32$ & 28.60 & 3.05 & 2.85 & 2.94 & 0.09 \\
\hline & 40 & $\mathrm{H} 43$ & 23.64 & 7.68 & & & \\
\hline & 41 & $\mathrm{H} 32$ & 26.54 & 8.23 & & & \\
\hline & 46 & $\mathrm{H} 47$ & 23.34 & 7.96 & & & \\
\hline & 49 & H52 & 23.93 & 7.41 & & & \\
\hline & 50 & $\mathrm{H} 54$ & 22.55 & 8.70 & & & \\
\hline & 55 & H56 & 23.42 & 7.89 & & & \\
\hline & 62 & $\mathrm{H} 63$ & 30.13 & 1.63 & & & \\
\hline & 62 & $\mathrm{H} 64$ & 31.48 & 0.37 & & & \\
\hline & 62 & $\mathrm{H} 65$ & 32.00 & -0.12 & & & \\
\hline & 66 & $\mathrm{H} 67$ & 31.55 & 0.31 & & & \\
\hline & 66 & $\mathrm{H} 68$ & 32.05 & -0.17 & & & \\
\hline & 66 & $\mathrm{H} 69$ & 32.11 & -0.22 & & & \\
\hline & 70 & $\mathrm{H} 71$ & 32.58 & -0.66 & & & \\
\hline & 70 & $\mathrm{H} 72$ & 32.48 & -0.56 & & & \\
\hline *OTMS & 70 & $\mathrm{H} 73$ & 33.84 & -1.84 & -0.14 & -0.29 & 0.15 \\
\hline \multirow[t]{2}{*}{$\mathrm{C}(3) \mathrm{H}$} & 3 & $\mathrm{H} 87$ & 30.32 & 1.45 & 1.81 & 1.72 & 0.09 \\
\hline & & & & & & MAD & 0.44 \\
\hline
\end{tabular}


Table SI-28. Comparison of calculated and experimental minor ${ }^{13} \mathrm{C}$ NMR $\delta$ 's for anti(C2')-11 (ap) at $-54^{\circ} \mathrm{C}$.

\begin{tabular}{|c|c|c|c|c|c|c|}
\hline Exp. C\# & Comp. C\# & $\begin{array}{c}\text { Comp. } \\
\text { Isotropic }\end{array}$ & Comp. $\delta$ & $\begin{array}{c}\text { Avg. } \\
\text { Comp. } \delta\end{array}$ & Syn. $\delta^{\ddagger}$ & Abs. Dev. \\
\hline C5 & $\mathrm{C} 1$ & 136.9004 & 47.5 & & 48.4 & 0.9 \\
\hline $\mathrm{C} 2$ & $\mathrm{C} 2$ & 115.5457 & 68.0 & & 70.7 & 2.7 \\
\hline C3 & C3 & 156.5798 & 28.6 & & 27.3 & 1.3 \\
\hline $\mathrm{C} 4$ & $\mathrm{C} 4$ & 160.0619 & 25.2 & & 23.8 & 1.4 \\
\hline $\mathrm{C} 1^{\prime}$ & C12 & 108.9728 & 74.3 & & 76.5 & 2.2 \\
\hline C2' & C14 & 108.1558 & 75.0 & & 65.4 & $9.6^{\ddagger}$ \\
\hline C3' & C16 & 152.3606 & 32.6 & & 28.7 & 3.9 \\
\hline C4' & C18 & 171.9968 & 13.8 & & 20.8 & 7.0 \\
\hline \multirow[t]{3}{*}{ C5' } & C22 & 165.6509 & 19.9 & & 11.7 & 8.2 \\
\hline & $\mathrm{C} 27$ & 155.1158 & 30.0 & & & \\
\hline & $\mathrm{C} 28$ & 154.7914 & 30.3 & & & \\
\hline C5" & C34 & -2.7252 & 181.5 & & 179 & 2.5 \\
\hline C2" & C36 & -2.8807 & 181.6 & & 180.1 & 1.5 \\
\hline \multirow[t]{21}{*}{ C6 } & C38 & 100.0367 & 82.8 & & 83.7 & 0.9 \\
\hline & C39 & 35.3661 & 144.9 & & & \\
\hline & C40 & 49.8824 & 131.0 & & & \\
\hline & C41 & 46.8657 & 133.9 & & & \\
\hline & C42 & 51.2183 & 129.7 & & & \\
\hline & C44 & 51.2936 & 129.6 & & & \\
\hline & C46 & 60.2623 & 121.0 & & & \\
\hline & C48 & 33.8848 & 146.3 & & & \\
\hline & C49 & 47.5167 & 133.2 & & & \\
\hline & C50 & 48.6472 & 132.2 & & & \\
\hline & C51 & 49.5448 & 131.3 & & & \\
\hline & C53 & 49.3588 & 131.5 & & & \\
\hline & C55 & 57.4194 & 123.7 & & & \\
\hline & C57 & 52.2395 & 128.7 & & & \\
\hline & C58 & 52.1519 & 128.8 & & & \\
\hline & C59 & 51.9835 & 129.0 & & & \\
\hline & C60 & 51.7189 & 129.2 & & & \\
\hline & C62 & 185.5768 & 0.7 & & & \\
\hline & C66 & 185.4877 & 0.8 & & & \\
\hline & $\mathrm{C} 70$ & 185.4005 & 0.9 & 0.8 & 1.5 & 0.7 \\
\hline & & & & & MAD: & 3.3 \\
\hline
\end{tabular}


Table SI-29. Comparison of calculated and experimental minor ${ }^{1} \mathrm{H}$ NMR $\delta$ 's for anti(C2')-11 (ap) at $-54^{\circ} \mathrm{C}$.

\begin{tabular}{|c|c|c|c|c|c|c|c|}
\hline $\begin{array}{c}\text { Exp. } \\
\#\end{array}$ & $\begin{array}{c}\text { Comp. } \\
\text { C\# }\end{array}$ & Comp. H\# & $\begin{array}{c}\text { Comp. } \\
\text { Isotropic }\end{array}$ & Comp. $\delta$ & $\begin{array}{c}\text { Avg. } \\
\text { Comp. } \delta \\
\end{array}$ & $\begin{array}{c}\text { Exp. } \\
\delta^{f}\end{array}$ & Abs. Dev. \\
\hline $\mathrm{C}(5) \mathrm{H}$ & 1 & $\mathrm{H} 5$ & 28.44 & 3.21 & 3.21 & 3.2 & 0.01 \\
\hline $\mathrm{C}(5) \mathrm{H}$ & 1 & $\mathrm{H} 6$ & 29.52 & 2.20 & 2.20 & 1.85 & 0.35 \\
\hline${ }^{*} \mathrm{C}(2) \mathrm{H}$ & 2 & $\mathrm{H} 7$ & 26.72 & 4.80 & 4.80 & 4.08 & 0.72 \\
\hline $\mathrm{C}(3) \mathrm{H}$ & 3 & $\mathrm{H} 8$ & 29.93 & 1.81 & & & \\
\hline $\mathrm{C}(4) \mathrm{H}$ & 4 & $\mathrm{H} 9$ & 31.73 & 0.13 & 0.13 & 0.13 & 0.00 \\
\hline $\mathrm{C}(4) \mathrm{H}$ & 4 & $\mathrm{H} 10$ & 30.66 & 1.14 & 1.14 & 1.15 & 0.01 \\
\hline${ }^{*} \mathrm{C}\left(1^{\prime}\right) \mathrm{H}$ & 12 & $\mathrm{H} 13$ & 26.37 & 5.13 & 5.13 & 5.66 & 0.53 \\
\hline${ }^{*} \mathrm{C}\left(2^{\prime}\right) \mathrm{H}$ & 14 & $\mathrm{H} 15$ & 26.69 & 4.83 & 4.83 & 4.95 & 0.12 \\
\hline \multirow[t]{3}{*}{$\mathrm{C}\left(3^{\prime}\right) \mathrm{H}$} & 16 & $\mathrm{H} 17$ & 30.02 & 1.73 & 1.73 & 2.05 & 0.32 \\
\hline & 18 & $\mathrm{H} 19$ & 31.16 & 0.66 & & & \\
\hline & 18 & $\mathrm{H} 2 \mathrm{O}$ & 31.07 & 0.75 & & & \\
\hline \multirow[t]{3}{*}{${ }^{* *} \mathrm{C}\left(4^{\prime}\right) \mathrm{H}$} & 18 & $\mathrm{H} 21$ & 31.18 & 0.64 & 0.68 & 0.91 & 0.23 \\
\hline & 22 & $\mathrm{H} 23$ & 30.82 & 0.98 & & & \\
\hline & 22 & $\mathrm{H} 24$ & 30.79 & 1.01 & & & \\
\hline \multirow[t]{2}{*}{${ }^{* *} \mathrm{C}\left(5^{\prime}\right) \mathrm{H}$} & 22 & $\mathrm{H} 25$ & 30.91 & 0.90 & 0.97 & -0.13 & 1.10 \\
\hline & 27 & $\mathrm{H} 29$ & 29.06 & 2.63 & & & \\
\hline \multirow[t]{2}{*}{${ }^{* *} \mathrm{C}(3 " / \mathrm{C} 4 ") \mathrm{H}$} & 27 & $\mathrm{H} 30$ & 29.11 & 2.58 & 2.60 & 2.77 & 0.17 \\
\hline & 28 & $\mathrm{H} 31$ & 28.87 & 2.80 & & & \\
\hline \multirow[t]{15}{*}{${ }^{\star \star} \mathrm{C}(3 " / \mathrm{C} 4 ") \mathrm{H}$} & 28 & H32 & 28.93 & 2.75 & 2.78 & 2.94 & 0.16 \\
\hline & 40 & $\mathrm{H} 43$ & 22.83 & 8.43 & & & \\
\hline & 41 & H32 & 26.54 & 8.77 & & & \\
\hline & 46 & $\mathrm{H} 47$ & 23.36 & 7.94 & & & \\
\hline & 49 & H52 & 23.34 & 7.96 & & & \\
\hline & 50 & H54 & 22.36 & 8.87 & & & \\
\hline & 55 & H56 & 23.38 & 7.92 & & & \\
\hline & 62 & $\mathrm{H} 63$ & 31.97 & -0.09 & & & \\
\hline & 62 & $\mathrm{H} 64$ & 32.08 & -0.19 & & & \\
\hline & 62 & $\mathrm{H} 65$ & 32.12 & -0.23 & & & \\
\hline & 66 & $\mathrm{H} 67$ & 31.50 & 0.34 & & & \\
\hline & 66 & $\mathrm{H} 68$ & 31.47 & 0.37 & & & \\
\hline & 66 & $\mathrm{H} 69$ & 31.42 & 0.43 & & & \\
\hline & 70 & $\mathrm{H} 71$ & 32.37 & -0.47 & & & \\
\hline & 70 & $\mathrm{H} 72$ & 32.35 & -0.44 & & & \\
\hline *OTMS & 70 & $\mathrm{H} 73$ & 32.07 & -0.18 & -0.05 & -0.29 & 0.24 \\
\hline \multirow[t]{2}{*}{$\mathrm{C}(3) \mathrm{H}$} & 3 & $\mathrm{H} 87$ & 30.06 & 1.69 & 1.75 & 1.72 & 0.03 \\
\hline & & & & & & MAD & 0.28 \\
\hline
\end{tabular}


Table SI-30. Comparison of calculated and experimental major ${ }^{13} \mathrm{C}$ NMR $\delta$ 's for syn-14 (ap) at $-54^{\circ} \mathrm{C}$.

\begin{tabular}{|c|c|c|c|c|c|c|}
\hline Exp. C\# & Comp. C\# & Comp. Isotropic & Comp. $\delta$ & Avg. Comp. $\delta$ & Syn. $\delta$ & Abs. Dev. \\
\hline C5 & C1 & 135.9388 & 48.4 & & 48.8 & 0.4 \\
\hline $\mathrm{C} 1^{\prime}$ & $\mathrm{C} 12$ & 107.9988 & 75.2 & & 76.3 & 1.1 \\
\hline C2' & C14 & 110.3959 & 72.9 & & 65.6 & $7.3^{\ddagger}$ \\
\hline C3' & C16 & 152.7793 & 32.2 & & 29.3 & 2.9 \\
\hline C4' & C18 & 165.6499 & 19.9 & & 21.3 & 1.4 \\
\hline $\mathrm{C} 2$ & $\mathrm{C} 2$ & 115.6540 & 67.9 & & 68.6 & 0.7 \\
\hline \multirow[t]{2}{*}{ C5' } & $\mathrm{C} 22$ & 173.5018 & 12.3 & & 12.2 & 0.1 \\
\hline & $\mathrm{C} 27$ & 49.2818 & 131.5 & & & \\
\hline C2"/C5" & $\mathrm{C} 29$ & 6.8274 & 172.3 & & 171.4 & 0.9 \\
\hline C3 & C3 & 156.9539 & 28.2 & & 27.7 & 0.5 \\
\hline C2"/C5" & C31 & 8.1297 & 171.0 & & 170.5 & 0.5 \\
\hline \multirow[t]{6}{*}{ C6 } & $\mathrm{C} 33$ & 100.1346 & 82.7 & & 82.4 & 0.3 \\
\hline & C34 & 35.4051 & 144.9 & & & \\
\hline & C35 & 49.7620 & 131.1 & & & \\
\hline & C36 & 48.7174 & 132.1 & & & \\
\hline & $\mathrm{C} 37$ & 50.5989 & 130.3 & & & \\
\hline & C39 & 51.8300 & 129.1 & & & \\
\hline \multirow[t]{12}{*}{$\mathrm{C} 4$} & $\mathrm{C} 4$ & 160.0826 & 25.2 & & 24.3 & 0.9 \\
\hline & $\mathrm{C} 41$ & 59.6637 & 121.6 & & & \\
\hline & $\mathrm{C} 43$ & 33.8341 & 146.4 & & & \\
\hline & $\mathrm{C} 44$ & 46.4848 & 134.2 & & & \\
\hline & C45 & 48.1872 & 132.6 & & & \\
\hline & $\mathrm{C} 46$ & 48.4947 & 132.3 & & & \\
\hline & C48 & 49.1247 & 131.7 & & & \\
\hline & C50 & 58.0782 & 123.1 & & & \\
\hline & C52 & 52.3296 & 128.6 & & & \\
\hline & C53 & 52.3279 & 128.6 & & & \\
\hline & C54 & 51.7803 & 129.2 & & & \\
\hline & C55 & 51.8710 & 129.1 & & & \\
\hline OTMS & C57 & 185.6073 & 0.7 & & & \\
\hline OTMS & C61 & 185.5508 & 0.8 & & & \\
\hline \multirow[t]{7}{*}{ OTMS } & C65 & 185.6881 & 0.6 & 0.7 & 1.7 & 1.0 \\
\hline & C83 & 49.9831 & 130.9 & & & \\
\hline & C84 & 57.2058 & 123.9 & & & \\
\hline & C85 & 57.5833 & 123.6 & & & \\
\hline & C86 & 45.7889 & 134.9 & & & \\
\hline & C88 & 45.6369 & 135.0 & & & \\
\hline & & & & & MAD: & 1.4 \\
\hline
\end{tabular}


Table SI-31. Comparison of calculated and experimental major ${ }^{1} \mathrm{H}$ NMR $\delta$ 's for syn-14 (ap) at $-54^{\circ} \mathrm{C}$.

\begin{tabular}{|c|c|c|c|c|c|c|c|}
\hline $\begin{array}{c}\text { Exp. } \\
\text { C\# }\end{array}$ & $\begin{array}{c}\text { Comp. } \\
\text { C\# }\end{array}$ & Comp. H\# & Comp. Isotropic & Comp. $\delta$ & $\begin{array}{c}\text { Avg. } \\
\text { Comp. } \delta\end{array}$ & $\begin{array}{c}\text { Exp. } \\
\delta\end{array}$ & Abs. Dev. \\
\hline $\mathrm{C} 4 \mathrm{a}$ & C4 & $\mathrm{H} 10$ & 30.61 & 1.18 & 1.18 & 1.20 & 0.02 \\
\hline$C 1^{\prime}$ & C12 & $\mathrm{H} 13$ & 26.26 & 5.24 & 5.24 & 5.16 & 0.08 \\
\hline C2' & C14 & $\mathrm{H} 15$ & 26.24 & 5.25 & 5.25 & 4.98 & 0.27 \\
\hline C3' & C16 & $\mathrm{H} 17$ & 29.31 & 2.39 & 2.39 & 2.13 & 0.26 \\
\hline C4' & C18 & $\mathrm{H} 19$ & 30.70 & 1.09 & & & \\
\hline C4' & C18 & $\mathrm{H} 2 \mathrm{O}$ & 30.75 & 1.05 & & & \\
\hline C4' & C18 & $\mathrm{H} 21$ & 30.74 & 1.06 & 1.07 & 0.95 & 0.12 \\
\hline C5' & $\mathrm{C} 22$ & $\mathrm{H} 23$ & 31.34 & 0.50 & & & \\
\hline C5' & $\mathrm{C} 22$ & $\mathrm{H} 24$ & 32.43 & -0.52 & & & \\
\hline \multirow[t]{6}{*}{ C5' } & $\mathrm{C} 22$ & $\mathrm{H} 25$ & 31.69 & 0.17 & 0.05 & -0.01 & 0.06 \\
\hline & C35 & $\mathrm{H} 38$ & 22.87 & 8.40 & & & \\
\hline & C36 & $\mathrm{H} 40$ & 23.44 & 7.87 & & & \\
\hline & C41 & $\mathrm{H} 42$ & 23.34 & 7.97 & & & \\
\hline & C44 & $\mathrm{H} 47$ & 22.28 & 8.95 & & & \\
\hline & C45 & $\mathrm{H} 49$ & 22.75 & 8.52 & & & \\
\hline \multirow[t]{2}{*}{ C5a } & $\mathrm{C} 1$ & $\mathrm{H} 5$ & 28.26 & 3.37 & 3.37 & 3.29 & 0.08 \\
\hline & C50 & H51 & 23.30 & 8.00 & & & \\
\hline OTMS & C57 & H58 & 31.77 & 0.10 & & & \\
\hline OTMS & C57 & H59 & 31.98 & -0.10 & & & \\
\hline C5b & C1 & $\mathrm{H} 6$ & 29.76 & 1.97 & 1.97 & 1.97 & 0.00 \\
\hline OTMS & C57 & H32 & 26.54 & -0.05 & & & \\
\hline OTMS & C61 & H62 & 31.54 & 0.31 & & & \\
\hline OTMS & C61 & $\mathrm{H} 63$ & 31.20 & 0.63 & & & \\
\hline OTMS & C61 & $\mathrm{H} 64$ & 31.83 & 0.04 & & & \\
\hline OTMS & C65 & $\mathrm{H} 66$ & 32.09 & -0.20 & & & \\
\hline OTMS & C65 & $\mathrm{H} 67$ & 32.15 & -0.26 & & & \\
\hline OTMS & C65 & H68 & 33.17 & -1.21 & -0.08 & -0.16 & 0.08 \\
\hline $\mathrm{C} 2$ & $\mathrm{C} 2$ & $\mathrm{H} 7$ & 26.73 & 4.80 & 4.80 & 4.60 & 0.20 \\
\hline C3a & C3 & $\mathrm{H} 8$ & 29.99 & 1.76 & 1.76 & 1.71 & 0.05 \\
\hline \multirow[t]{3}{*}{ C3b } & C3 & H82 & 29.94 & 1.80 & 1.80 & 1.71 & 0.09 \\
\hline & C84 & H87 & 23.36 & 7.94 & & & \\
\hline & C85 & H89 & 23.50 & 7.81 & & & \\
\hline \multirow[t]{4}{*}{ C4b } & C4 & H9 & 31.79 & 0.08 & 0.08 & 0.09 & 0.01 \\
\hline & C86 & H90 & 23.53 & 7.79 & & & \\
\hline & C88 & H91 & 23.55 & 7.76 & & & \\
\hline & & & & & & MAD & 0.10 \\
\hline
\end{tabular}


Table SI-32. Comparison of calculated and experimental minor ${ }^{13} \mathrm{C}$ NMR $\delta$ 's for syn-14 (exo-sc) at $-54^{\circ} \mathrm{C}$.

\begin{tabular}{|c|c|c|c|c|c|c|}
\hline Exp. C\# & Comp. C\# & Comp. Isotropic & Comp. $\delta$ & Avg. Comp. $\delta$ & Syn. $\delta$ & Abs. Dev. \\
\hline C5 & $\mathrm{C} 1$ & 136.4232 & 47.9 & & 48.5 & 0.6 \\
\hline $\mathrm{C} 1^{\prime}$ & C12 & 108.6580 & 74.6 & & 76.1 & 1.5 \\
\hline C2' & $\mathrm{C} 14$ & 109.1710 & 74.1 & & 66.8 & 7.3 \\
\hline C3' & $\mathrm{C} 16$ & 152.8625 & 32.1 & & 29.0 & 3.1 \\
\hline C4' & C18 & 165.8239 & 19.7 & & 21.4 & 1.7 \\
\hline $\mathrm{C} 2$ & $\mathrm{C} 2$ & 116.4201 & 67.1 & & 68.6 & 1.5 \\
\hline \multirow[t]{2}{*}{ C5' } & $\mathrm{C} 22$ & 169.4610 & 16.2 & & 15.7 & 0.5 \\
\hline & $\mathrm{C} 27$ & 48.8475 & 132.0 & & & \\
\hline C2"/C5" & C29 & 8.3013 & 170.9 & & 170.8 & 0.1 \\
\hline $\mathrm{C} 3$ & C3 & 155.8692 & 29.3 & & 29.0 & 0.3 \\
\hline C2"/C5" & C31 & 7.8370 & 171.3 & & 170.9 & 0.4 \\
\hline \multirow[t]{6}{*}{$\mathrm{C} 6$} & C33 & 98.5273 & 84.3 & & 84.2 & 0.1 \\
\hline & C34 & 34.5708 & 145.7 & & & \\
\hline & C35 & 47.0622 & 133.7 & & & \\
\hline & $\mathrm{C} 36$ & 49.5313 & 131.3 & & & \\
\hline & C37 & 49.5707 & 131.3 & & & \\
\hline & C39 & 49.6703 & 131.2 & & & \\
\hline \multirow[t]{12}{*}{$\mathrm{C} 4$} & C4 & 159.8652 & 25.4 & & 24.1 & 1.3 \\
\hline & C41 & 57.9075 & 123.3 & & & \\
\hline & $\mathrm{C} 43$ & 34.3743 & 145.9 & & & \\
\hline & C44 & 50.9557 & 129.9 & & & \\
\hline & C45 & 46.9211 & 133.8 & & & \\
\hline & C46 & 51.8629 & 129.1 & & & \\
\hline & C48 & 50.8591 & 130.0 & & & \\
\hline & C50 & 59.5760 & 121.7 & & & \\
\hline & C52 & 52.2178 & 128.7 & & & \\
\hline & C53 & 51.6846 & 129.2 & & & \\
\hline & C54 & 52.3688 & 128.6 & & & \\
\hline & C55 & 52.5147 & 128.4 & & & \\
\hline OTMS & $\mathrm{C} 57$ & 184.1522 & 2.1 & & & \\
\hline OTMS & C61 & 184.4262 & 1.9 & & & \\
\hline \multirow[t]{7}{*}{ OTMS } & $\mathrm{C} 65$ & 185.9906 & 0.4 & 1.4 & 1.9 & 0.5 \\
\hline & C83 & 49.5982 & 131.2 & & & \\
\hline & C84 & 57.5751 & 123.6 & & & \\
\hline & C85 & 57.9324 & 123.2 & & & \\
\hline & C86 & 46.5484 & 134.2 & & & \\
\hline & C88 & 46.3394 & 134.4 & & & \\
\hline & & & & & MAD: & 1.5 \\
\hline
\end{tabular}


Table SI-33. Comparison of calculated and experimental minor ${ }^{1} \mathrm{H}$ NMR $\delta$ 's for syn-14 (exo-sc) at $-54^{\circ} \mathrm{C}$.

\begin{tabular}{|c|c|c|c|c|c|c|c|}
\hline $\begin{array}{l}\text { Exp. } \\
\text { C\# }\end{array}$ & $\begin{array}{c}\text { Comp. } \\
\text { C\# }\end{array}$ & Comp. H\# & Comp. Isotropic & Comp. $\delta$ & $\begin{array}{c}\text { Avg. } \\
\text { Comp. } \delta\end{array}$ & $\begin{array}{c}\text { Exp. } \\
\delta\end{array}$ & Abs. Dev. \\
\hline $\mathrm{C} 4 \mathrm{a}$ & C4 & $\mathrm{H} 10$ & 30.53 & 1.25 & 1.25 & 1.28 & 0.03 \\
\hline $\mathrm{C} 1^{\prime}$ & C12 & $\mathrm{H} 13$ & 25.39 & 6.04 & 6.04 & 5.81 & 0.23 \\
\hline C2' & C14 & $\mathrm{H} 15$ & 26.21 & 5.28 & 5.28 & 5.05 & 0.23 \\
\hline C3' & C16 & $\mathrm{H} 17$ & 29.01 & 2.67 & 2.67 & 2.35 & 0.32 \\
\hline C4' & C18 & H19 & 30.54 & 1.25 & & & \\
\hline C4' & C18 & $\mathrm{H} 20$ & 30.64 & 1.15 & & & \\
\hline C4' & C18 & $\mathrm{H} 21$ & 30.59 & 1.20 & 1.20 & 1.06 & 0.14 \\
\hline C5' & $\mathrm{C} 22$ & $\mathrm{H} 23$ & 30.41 & 1.37 & & & \\
\hline C5' & $\mathrm{C} 22$ & $\mathrm{H} 24$ & 30.21 & 1.55 & & & \\
\hline \multirow[t]{6}{*}{ C5' } & $\mathrm{C} 22$ & $\mathrm{H} 25$ & 30.17 & 1.59 & 1.50 & 1.28 & 0.22 \\
\hline & C35 & H38 & 23.52 & 7.80 & & & \\
\hline & C36 & $\mathrm{H} 40$ & 23.29 & 8.00 & & & \\
\hline & C41 & $\mathrm{H} 42$ & 23.37 & 7.94 & & & \\
\hline & C44 & $\mathrm{H} 47$ & 24.09 & 7.27 & & & \\
\hline & C45 & $\mathrm{H} 49$ & 22.31 & 8.92 & & & \\
\hline \multirow[t]{2}{*}{$\mathrm{C} 5 \mathrm{a}$} & C1 & H5 & 28.18 & 3.44 & 3.44 & 3.27 & 0.17 \\
\hline & C50 & H51 & 23.33 & 7.97 & & & \\
\hline OTMS & C57 & H58 & 31.59 & 0.26 & & & \\
\hline OTMS & C57 & H59 & 31.09 & 0.73 & & & \\
\hline C5b & $\mathrm{C} 1$ & $\mathrm{H} 6$ & 29.56 & 2.16 & 2.16 & 2.16 & 0.00 \\
\hline OTMS & C57 & $\mathrm{H} 60$ & 26.54 & 0.43 & & & \\
\hline OTMS & C61 & H62 & 31.71 & 0.15 & & & \\
\hline OTMS & C61 & H63 & 31.76 & 0.10 & & & \\
\hline OTMS & C61 & $\mathrm{H} 64$ & 31.73 & 0.13 & & & \\
\hline OTMS & C65 & H66 & 31.56 & 0.30 & & & \\
\hline OTMS & C65 & $\mathrm{H} 67$ & 33.86 & -1.85 & & & \\
\hline OTMS & C65 & $\mathrm{H} 68$ & 32.27 & -0.37 & -0.01 & -0.15 & 0.14 \\
\hline $\mathrm{C} 2$ & $\mathrm{C} 2$ & $\mathrm{H} 7$ & 27.33 & 4.24 & 4.24 & 4.15 & 0.09 \\
\hline C3a & C3 & H8 & 29.94 & 1.81 & 1.81 & 1.82 & 0.01 \\
\hline \multirow[t]{3}{*}{$\mathrm{C} 3 \mathrm{~b}$} & C3 & H82 & 30.46 & 1.32 & 1.32 & 1.38 & 0.06 \\
\hline & C84 & $\mathrm{H} 87$ & 23.43 & 7.88 & & & \\
\hline & C85 & H89 & 23.46 & 7.85 & & & \\
\hline \multirow[t]{4}{*}{$C 4 b$} & $\mathrm{C} 4$ & H9 & 31.20 & 0.63 & 0.63 & 0.63 & 0.00 \\
\hline & C86 & $\mathrm{H} 90$ & 23.52 & 7.80 & & & \\
\hline & C88 & H91 & 23.53 & 7.79 & & & \\
\hline & & & & & & MAD & 0.13 \\
\hline
\end{tabular}


Table SI-34. Comparison of the experimental J-couplings for the syn-14 major product to the calculated ap conformers averaged according to Table SI-31.

\begin{tabular}{|c|c|c|c|c|c|}
\hline \multicolumn{2}{|c|}{ Scaling Factor: } & \multicolumn{4}{|l|}{0.9155} \\
\hline \multirow[b]{2}{*}{ Exp. H\# } & \multirow[b]{2}{*}{ Comp. H\# } & Comp. FC & Comp. FC & Syn. & \\
\hline & & $J(H z)$ & $J(H z)$ Scaled & J-coupling & Abs. Dev. \\
\hline $5 a-5 b$ & $5-6$ & -11.93 & -10.92 & & \\
\hline $5 a-4 b$ & $5-9$ & 6.87 & 6.29 & & \\
\hline $5 a-4 a$ & $5-10$ & 8.95 & 8.19 & & \\
\hline $5 b-4 a$ & $6-10$ & 8.67 & 7.94 & & \\
\hline $5 b-4 b$ & $6-9$ & 6.27 & 5.74 & & \\
\hline $4 a-4 b$ & $9-10$ & -13.68 & -12.52 & & \\
\hline $4 a-3 b$ & $10-82$ & 5.56 & 5.09 & & \\
\hline $2-3 a$ & $8-7$ & 9.56 & 8.75 & 7.6 & 1.15 \\
\hline $3 a-3 b$ & $8-82$ & -14.93 & -13.67 & & \\
\hline $2-3 b$ & $82-7$ & 3.49 & 3.19 & 4.7 & 1.51 \\
\hline $1^{\prime}-2^{\prime}$ & $13-15$ & 10.72 & 9.81 & 10.3 & 0.49 \\
\hline 2'-3' & $15-17$ & 1.89 & 1.73 & 1.0 & 0.73 \\
\hline
\end{tabular}

Table SI-35. Calculated J-couplings for the relevant syn-14 ap conformers.

\begin{tabular}{lllllll} 
& Weight of Conformer: & 0.1393 & 0.3362 & 0.0028 & 0.4677 & 0.0540 \\
\cline { 2 - 7 } Exp. H\# & Comp. H\# & $\mathbf{4}$ & $\mathbf{5 8}$ & $\mathbf{8}$ & $\mathbf{1 7}$ & $\mathbf{4 6}$ \\
\hline 5a-5b & $5-6$ & -11.03 & -13.22 & -11.06 & -11.13 & -13.19 \\
5a-4b & $5-9$ & 10.93 & 0.58 & 10.96 & 10.89 & 0.56 \\
5a-4a & $5-10$ & 9.34 & 8.31 & 9.30 & 9.36 & 8.22 \\
5b-4a & $6-10$ & 10.47 & 5.87 & 10.43 & 10.46 & 5.78 \\
5b-4b & $6-9$ & 1.42 & 13.84 & 1.40 & 1.43 & 13.85 \\
4a-4b & $9-10$ & -13.94 & -13.26 & -13.93 & -13.95 & -13.22 \\
4a-3b & $10-82$ & 0.58 & 13.35 & 0.58 & 0.57 & 13.41 \\
2-3a & $8-7$ & 9.70 & 9.41 & 9.73 & 9.67 & 9.18 \\
3a-3b & $8-82$ & -14.32 & -15.15 & -14.48 & -14.92 & -15.34 \\
2'-3b & $82-7$ & 0.69 & 7.80 & 0.70 & 0.73 & 7.90 \\
1'-2' & $13-15$ & 10.79 & 10.51 & 10.82 & 10.87 & 10.55 \\
2'-3' & $15-17$ & 1.94 & 1.84 & 1.96 & 1.92 & 1.84
\end{tabular}


Table SI-36. Comparison of the experimental J-couplings for the syn-14 minor product to the calculated exo-sc conformers averaged according to Table SI-33.

\begin{tabular}{|c|c|c|c|c|c|}
\hline \multicolumn{2}{|c|}{ Scaling Factor: } & \multicolumn{4}{|l|}{0.9155} \\
\hline \multirow[b]{2}{*}{ Exp. H\# } & \multirow[b]{2}{*}{ Comp. H\# } & \multirow{2}{*}{$\begin{array}{l}\text { Comp. FC } \\
\mathbf{J}(\mathrm{Hz})\end{array}$} & \multirow{2}{*}{$\begin{array}{l}\text { Comp. FC } \\
\text { J (Hz) Scaled }\end{array}$} & \multirow{2}{*}{$\begin{array}{l}\text { Syn. } \\
\text { J-coupling }\end{array}$} & \multirow[b]{2}{*}{ Abs. Dev } \\
\hline & & & & & \\
\hline $5 a-5 b$ & $5-6$ & -11.31 & -10.35 & & \\
\hline $5 a-4 b$ & $5-9$ & 6.45 & 5.90 & & \\
\hline $5 a-4 a$ & $5-10$ & 7.99 & 7.32 & & \\
\hline $5 b-4 a$ & $6-10$ & 7.19 & 6.59 & & \\
\hline $5 b-4 b$ & $6-9$ & 7.24 & 6.63 & & \\
\hline $4 a-4 b$ & $9-10$ & -13.41 & -12.28 & & \\
\hline $4 a-3 b$ & $10-82$ & 7.24 & 6.63 & & \\
\hline $2-3 a$ & $7-8$ & 9.84 & 9.00 & 9.8 & 0.80 \\
\hline $3 a-3 b$ & $8-82$ & -14.55 & -13.32 & & \\
\hline $2-3 b$ & $82-7$ & 5.73 & 5.25 & 2.4 & 2.85 \\
\hline $1^{\prime}-2^{\prime}$ & $13-15$ & 11.40 & 10.44 & 10.9 & 0.46 \\
\hline $2^{\prime}-3^{\prime}$ & $15-17$ & 1.37 & 1.25 & 1 & 0.25 \\
\hline
\end{tabular}

Table SI-37. Calculated J-couplings for the relevant syn-14 exo-sc conformers.

\begin{tabular}{lllll}
\cline { 2 - 5 } Exp. H\# & Weight of Conformer: & 0.4860 & 0.4150 & 0.0990 \\
\cline { 2 - 5 } & Comp. H\# & $\mathbf{3 3}$ & $\mathbf{6 2}$ & $\mathbf{2 1}$ \\
\hline 5a-5b & $5-6$ & -10.08 & -12.57 & -12.06 \\
5a-4b & $5-9$ & 12.68 & 0.56 & 0.53 \\
5a-4a & $5-10$ & 7.57 & 8.31 & 8.74 \\
5b-4a & $6-10$ & 8.93 & 5.53 & 5.68 \\
5b-4b & $6-9$ & 0.64 & 13.53 & 13.32 \\
4a-4b & $9-10$ & -13.67 & -13.18 & -13.07 \\
4a-3b & $10-82$ & 0.57 & 13.38 & 14.29 \\
2-3a & $8-7$ & 11.19 & 8.94 & 6.96 \\
3a-3b & $8-82$ & -14.75 & -14.53 & -13.62 \\
2'-3b & $82-7$ & 1.12 & 9.64 & 12.02 \\
1'-2' & $13-15$ & 11.63 & 11.29 & 10.79 \\
2'-3' & $15-17$ & 1.27 & 1.32 & 2.03
\end{tabular}


Table SI-38. Calculated ${ }^{13} \mathrm{C}$ NMR $\delta$ 's for the syn-11 iminium ion.

\begin{tabular}{|c|c|c|c|c|}
\hline Exp. \# & Comp \# & Comp. Isotropic & Comp. $\delta$ & Avg. Comp. $\delta$ \\
\hline $\mathrm{C} 5$ & $\mathrm{C} 1$ & 127.0736 & 56.9 & \\
\hline $\mathrm{C} 2$ & $\mathrm{C} 2$ & 100.6921 & 82.2 & \\
\hline $\mathrm{C} 3$ & C3 & 154.3676 & 30.7 & \\
\hline $\mathrm{C} 4$ & $\mathrm{C} 4$ & 157.6886 & 27.5 & \\
\hline$C 1^{\prime}$ & $\mathrm{C} 12$ & 4.3992 & 174.6 & \\
\hline C2' & $\mathrm{C} 14$ & 114.4977 & 69.0 & \\
\hline C3' & $\mathrm{C} 16$ & 146.7122 & 38.0 & \\
\hline C4' & C18 & 161.9306 & 23.4 & \\
\hline \multirow[t]{3}{*}{ C5' } & $\mathrm{C} 22$ & 165.5088 & 20.0 & \\
\hline & $\mathrm{C} 27$ & 98.2156 & 84.6 & \\
\hline & $\mathrm{C} 28$ & 39.8560 & 140.6 & \\
\hline C5" & C34 & 22.8471 & 156.9 & \\
\hline C2" & C36 & 22.5460 & 157.2 & \\
\hline \multirow[t]{20}{*}{ C6 } & C38 & 48.7216 & 132.1 & \\
\hline & C39 & 49.0893 & 131.7 & \\
\hline & $\mathrm{C} 40$ & 46.4822 & 134.2 & \\
\hline & $\mathrm{C} 41$ & 22.8941 & 156.9 & \\
\hline & $\mathrm{C} 42$ & 46.4378 & 134.3 & \\
\hline & $\mathrm{C} 44$ & 55.6195 & 125.5 & \\
\hline & $\mathrm{C} 46$ & 51.8209 & 129.1 & \\
\hline & C48 & 52.1416 & 128.8 & \\
\hline & C49 & 52.1917 & 128.8 & \\
\hline & C50 & 205.2978 & -18.2 & \\
\hline & C51 & 181.5245 & 4.6 & \\
\hline & C53 & 31.8869 & 148.2 & \\
\hline & C55 & 180.5291 & 5.6 & \\
\hline & C57 & 30.8771 & 149.2 & \\
\hline & C58 & 30.7102 & 149.4 & \\
\hline & C59 & 181.3023 & 4.9 & \\
\hline & $\mathrm{C} 60$ & 31.8039 & 148.3 & \\
\hline & C62 & 31.1726 & 148.9 & \\
\hline & C66 & 240.5816 & -52.0 & \\
\hline & C70 & 227.3965 & -39.4 & 19.2 \\
\hline
\end{tabular}


Table SI-39. Calculated ${ }^{1} \mathrm{H}$ NMR $\delta$ 's for the syn-11 iminium ion.

\begin{tabular}{|c|c|c|c|c|c|}
\hline Exp. \# & Comp. C \# & Comp \# & Comp. Isotropic & Comp. $\delta$ & Avg. Comp. $\delta$ \\
\hline $\mathrm{C}(5) \mathrm{H}$ & 1 & H5 & 27.1258 & 4.43 & 4.43 \\
\hline $\mathrm{C}(5) \mathrm{H}$ & 1 & $\mathrm{H} 6$ & 28.2451 & 3.38 & 3.38 \\
\hline $\mathrm{C}(2) \mathrm{H}$ & 2 & $\mathrm{H} 7$ & 25.7323 & 5.73 & 5.73 \\
\hline $\mathrm{C}(3) \mathrm{H}$ & 3 & $\mathrm{H} 8$ & 28.3500 & 3.29 & \\
\hline $\mathrm{C}(4) \mathrm{H}$ & 4 & $\mathrm{H} 9$ & 29.8048 & 1.93 & 1.93 \\
\hline $\mathrm{C}(4) \mathrm{H}$ & 4 & $\mathrm{H} 10$ & 29.0370 & 2.65 & 2.65 \\
\hline $\mathrm{C}\left(1^{\prime}\right) \mathrm{H}$ & 12 & $\mathrm{H} 13$ & 22.0869 & 9.13 & 9.13 \\
\hline $\mathrm{C}\left(2^{\prime}\right) \mathrm{H}$ & 14 & $\mathrm{H} 15$ & 26.5460 & 4.97 & 4.97 \\
\hline \multirow[t]{3}{*}{$\mathrm{C}\left(3^{\prime}\right) \mathrm{H}$} & 16 & $\mathrm{H} 17$ & 28.5227 & 3.13 & 3.13 \\
\hline & 18 & $\mathrm{H} 19$ & 29.6889 & 2.04 & \\
\hline & 18 & $\mathrm{H} 20$ & 29.6119 & 2.11 & \\
\hline \multirow[t]{3}{*}{$\mathrm{C}\left(4^{\prime}\right.$ or $\left.5^{\prime}\right) \mathrm{H}$} & 18 & $\mathrm{H} 21$ & 29.6133 & 2.11 & 2.09 \\
\hline & 22 & $\mathrm{H} 23$ & 29.9158 & 1.83 & \\
\hline & 22 & $\mathrm{H} 24$ & 29.5853 & 2.13 & \\
\hline \multirow[t]{2}{*}{$\mathrm{C}\left(4^{\prime}\right.$ or $\left.5^{\prime}\right) \mathrm{H}$} & 22 & $\mathrm{H} 25$ & 29.7018 & 2.03 & 2.00 \\
\hline & 27 & $\mathrm{H} 29$ & 47.6406 & -14.71 & \\
\hline \multirow[t]{2}{*}{$\mathrm{C}\left(3^{\prime \prime} / \mathrm{C} 4 "\right) \mathrm{H}$} & 27 & $\mathrm{H} 30$ & 48.4402 & -15.46 & -15.08 \\
\hline & 28 & H31 & 45.5225 & -12.73 & \\
\hline \multirow[t]{15}{*}{ C(3"/C4")H } & 28 & H32 & 22.8399 & 8.43 & -2.15 \\
\hline & 40 & $\mathrm{H} 43$ & 22.7776 & 8.49 & \\
\hline & 41 & H32 & 26.5383 & 8.70 & \\
\hline & 46 & $\mathrm{H} 47$ & 51.9737 & -18.75 & \\
\hline & 49 & H52 & 31.0776 & 0.74 & \\
\hline & 50 & H54 & 30.9380 & 0.87 & \\
\hline & 55 & H56 & 30.7511 & 1.05 & \\
\hline & 62 & H52 & 31.0776 & 0.74 & \\
\hline & 62 & $\mathrm{H} 53$ & 31.8869 & -0.01 & \\
\hline & 62 & H54 & 30.9380 & 0.87 & \\
\hline & 66 & H56 & 30.7511 & 1.05 & \\
\hline & 66 & $\mathrm{H} 57$ & 30.8771 & 0.93 & \\
\hline & 66 & H58 & 30.7102 & 1.09 & \\
\hline & 70 & $\mathrm{H} 60$ & 31.8039 & 0.06 & \\
\hline & 70 & $\mathrm{H} 61$ & 31.2639 & 0.57 & \\
\hline OTMS & 70 & $\mathrm{H} 62$ & 31.1726 & 0.65 & 0.66 \\
\hline $\mathrm{C}(3) \mathrm{H}$ & 3 & $\mathrm{H} 76$ & 28.8069 & 2.86 & 3.07 \\
\hline
\end{tabular}


Table SI-40. Weighted Averaging for the Calculated NMR and NBO calculations for syn-11, anti(C1')11, anti(C2')-11, and syn-14

syn-11 (ap) at $-54^{\circ} \mathrm{C}$

\begin{tabular}{|lll|}
\hline Filename & Computed Energy (H) & Conformer Weight \\
\hline crest_confs_1_h_2_nmr_2.log & -3561.5159 & 0.4707 \\
crest_confs_8_h_2_nmr_2.log & -3561.5156 & 0.1656 \\
crest_confs_5_h_2_nmr_2.log & -3561.5153 & 0.0668 \\
crest_confs_7_h_2_nmr.log & -3561.5153 & 0.1344 \\
crest_confs_21_h_7_nmr_2.log & -3561.5145 & 0.1625 \\
\hline
\end{tabular}

syn-11 (exo-sc) at $-54^{\circ} \mathrm{C}$

\begin{tabular}{|lll|}
\hline Filename & Computed Energy (H) & Conformer Weight \\
\hline crest_confs_11_h_2_nmr_2.log & -3561.5131 & 0.8337 \\
crest_confs_12_h_6_nmr_8.log & -3561.5130 & 0.1663 \\
\hline
\end{tabular}

anti(C1')-11 (ap) at $-54^{\circ} \mathrm{C}$

\begin{tabular}{|lll|}
\hline Filename & Computed Energy (H) & Conformer Weight \\
\hline crest_confs_2_h_5_nmr_2.log & -3561.5137 & 0.0994 \\
crest_confs_3_h_3_nmr_2.log & -3561.5133 & 0.4934 \\
crest_confs_39_h_2_nmr.log & -3561.5114 & 0.1949 \\
crest_confs_7_h_4_nmr_2.log & -3561.5113 & 0.1826 \\
crest_confs_10_h_5_nmr.log & -3561.5101 & 0.0105 \\
crest_confs_18_h_2_nmr.log & -3561.5101 & 0.0099 \\
crest_confs_12_h_5_nmr.log & -3561.5094 & 0.0093 \\
\hline
\end{tabular}

anti(C1')-11 (exo-sc) at $-54^{\circ} \mathrm{C}$

\begin{tabular}{|lll|}
\hline Filename & Computed Energy (H) & Conformer Weight \\
\hline crest_confs_44_h_4_nmr.log & -3561.5098 & 1 \\
\hline
\end{tabular}

anti(C2')-11 (ap) at $-54^{\circ} \mathrm{C}$

\begin{tabular}{|lll|}
\hline Filename & Computed Energy (H) & Conformer Weight \\
\hline crest_confs_55_h_3_low.log & -3561.5143 & 0.2927 \\
crest_confs_23_h_2_low.log & -3561.5134 & 0.1472 \\
crest_confs_6_h_3_low.log & -3561.5133 & 0.0620 \\
crest_confs_7_h_3_low.log & -3561.5129 & 0.3022 \\
crest_confs_9_h_3_low.log & -3561.5128 & 0.1959 \\
\hline
\end{tabular}


syn-14 (ap) at $-54^{\circ} \mathrm{C}$

\begin{tabular}{|lll|}
\hline Filename & Computed Energy (H) & Conformer Weight \\
\hline crest_confs_8_h_2_low.log & -3713.9415 & 0.0028 \\
crest_confs_4_h_2_low.log & -3713.9408 & 0.1393 \\
crest_confs_58_h_2_low.log & -3713.9398 & 0.3362 \\
crest_confs_17_h_3_low.log & -3713.9389 & 0.4677 \\
crest_confs_46_h_4_low.log & -3713.9379 & 0.0540 \\
\hline
\end{tabular}

syn-14 (exo-sc) at $-54^{\circ} \mathrm{C}$

\begin{tabular}{|lll|}
\hline Filename & Computed Energy (H) & Conformer Weight \\
\hline crest_confs_33_h_2_low.log & -3713.9381 & 0.4860 \\
crest_confs_21_h_2_low.log & -3713.9380 & 0.0990 \\
crest_confs_62_h_3_low.log & -3713.9375 & 0.4150 \\
\hline
\end{tabular}

\section{DFT NBO Analysis for 11 and 14}

NBO analysis was performed assess the $n_{\mathrm{N}(\text { cat })}-\sigma^{*} \mathrm{C}-\mathrm{N}$ (imide) interaction differences between the ap and exc-sc conformers for for $\mathbf{1 1}$ and 14, and between the syn and anti $\left(\mathrm{C} 1^{\prime}\right)-\mathbf{1 1}$ diastereomers The syn interactions were higher in energy when compared to the anti-conformers for 11, with a minor difference in the weighted energies between the ap and exo-sc products $\left(0.31 \mathrm{kcal} \mathrm{mol}^{-1}\right)$. The phthalimide aminal had a similar difference in energies between the two rotational isomers $\left(0.30 \mathrm{kcal} \mathrm{mol}^{-1}\right)$.

Table SI-41. DFT NBO Analysis

syn-11 (ap) Interaction: LP(1)N11-BD*(1)C12-N33

\begin{tabular}{lll} 
Conformer & E2 Stabilization Energy (kcal mol $\left.{ }^{-1}\right)$ & Conformer Weight \\
\hline 1 & 17.91 & 0.4707 \\
5 & 17.09 & 0.0668 \\
7 & 17.74 & 0.1344 \\
8 & 17.08 & 0.1656 \\
21 & 16.92 & 0.1625 \\
Weighted Energy: & 17.53 &
\end{tabular}

syn-11 (exo-sc) Interaction: LP(1)N11-BD*(1)C12-N33

Conformer

11

12

Weighted Energy:
E2 Stabilization Energy ( $\mathrm{kcal} \mathrm{mol}^{-1}$ ) Conformer Weight

$\begin{array}{ll}17.19 & 0.8337 \\ 17.36 & 0.1663\end{array}$

17.22 
anti(C1')-11 Interaction: $\quad$ LP(1)N11-BD*(1)C12-N33

Conformer

E2 Stabilization Energy (kcal mol-1) Orientation of OTMS

\begin{tabular}{lll}
\hline crest_confs_2_h_5_nmr_2.log & 7.95 & Ap \\
crest_confs_3_h_3_nmr_2.log & 7.68 & Ap \\
crest_confs_39_h_2_nmr.log & 7.84 & Ap \\
crest_confs_7_h_4_nmr_2.log & 7.51 & Ap \\
crest_confs_10_h_5_nmr.log & 8.51 & Ap \\
crest_confs_18_h_2_nmr.log & 8.42 & Ap \\
crest_confs_44_h_4_nmr.log & 7.48 & Exo-sc \\
crest_confs_12_h_5_nmr.log & 15.91 & Ap \\
& & \\
syn-14 (ap) Interaction: & LP(1)N11-BD*(1)C12-N28 & \\
Conformer & E2 Stabilization Energy (kcal mol-1) & Conformer Weight \\
\hline crest_confs_8_h_2_low.log & 16.65 & 0.0028 \\
crest_confs_4_h_2_low.log & 16.65 & 0.1393 \\
crest_confs_58_h_2_low.log & 15.99 & 0.3362 \\
crest_confs_17_h_3_low.log & 16.48 & 0.4677 \\
crest_confs_46_h_4_low.log & 15.86 & 0.0540 \\
Weighted Energy: & 16.31 & \\
& & \\
syn-14 (exo-sc) Interaction: & LP(1)N11-BD*(1)C12-N28 & \\
Conformer & E2 Stabilization Energy (kcal mol-1) & Conformer Weight \\
\hline crest_confs_33_h_2_low.log & 15.97 & 0.4860 \\
crest_confs_21_h_2_low.log & 15.52 & 0.0990 \\
crest_confs_62_h_3_low.log & 16.17 & 0.4150 \\
Weighted Energy: & 16.01 & \\
& &
\end{tabular}




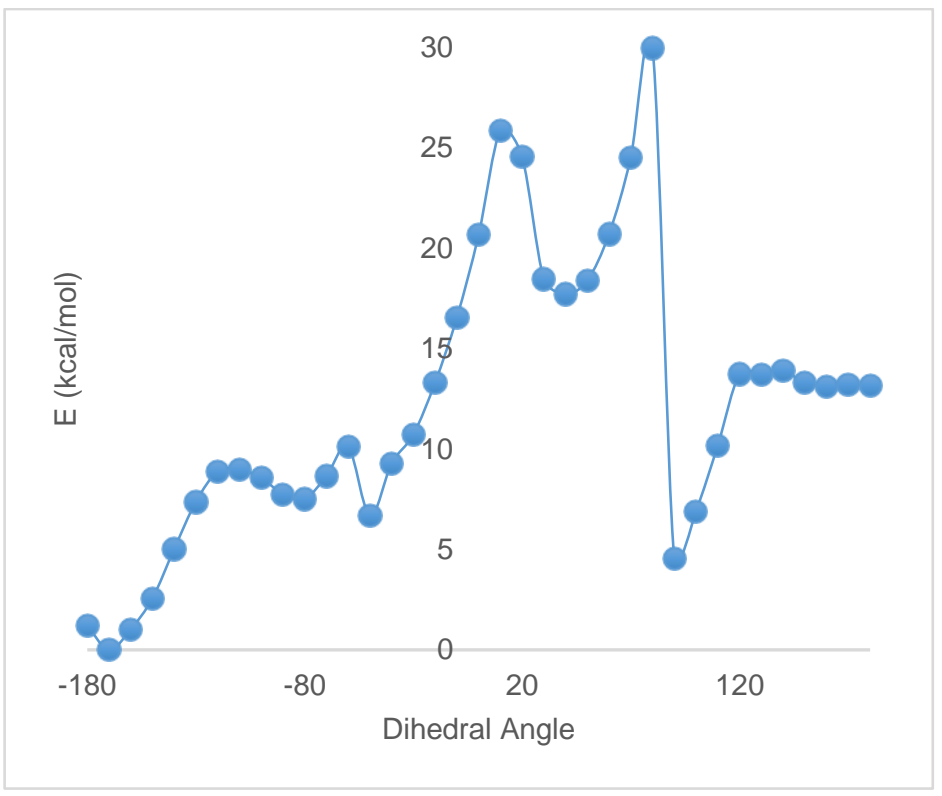

\begin{tabular}{|c|c|c|c|}
\hline Frame & $\begin{array}{l}\text { Diheral } \\
\text { Angle }\end{array}$ & $\begin{array}{l}\text { Relative } \\
\left(\text { kcal mol}^{-1}\right)\end{array}$ & Energy \\
\hline 1 & -180 & 1.24 & \\
\hline 2 & -170 & 0.00 & \\
\hline 3 & -160 & 1.02 & \\
\hline 4 & -150 & 2.57 & \\
\hline 5 & -140 & 5.03 & \\
\hline 6 & -130 & 7.38 & \\
\hline 7 & -120 & 8.88 & \\
\hline 8 & -110 & 8.98 & \\
\hline 9 & -100 & 8.58 & \\
\hline 10 & -90 & 7.75 & \\
\hline 11 & -80 & 7.52 & \\
\hline 12 & -70 & 8.65 & \\
\hline 13 & -60 & 10.12 & \\
\hline 14 & -50 & 6.68 & \\
\hline 15 & -40 & 9.28 & \\
\hline 16 & -30 & 10.73 & \\
\hline 17 & -20 & 13.31 & \\
\hline 18 & -10 & 16.54 & \\
\hline 19 & 0 & 20.66 & \\
\hline 20 & 10 & 25.84 & \\
\hline 21 & 20 & 24.55 & \\
\hline 22 & 30 & 18.48 & \\
\hline 23 & 40 & 17.73 & \\
\hline 24 & 50 & 18.40 & \\
\hline
\end{tabular}




$\begin{array}{lll}25 & 60 & 20.71 \\ 26 & 70 & 24.51 \\ 27 & 80 & 29.97 \\ 28 & 90 & 4.55 \\ 29 & 100 & 6.92 \\ 30 & 110 & 10.18 \\ 31 & 120 & 13.73 \\ 32 & 130 & 13.70 \\ 33 & 140 & 13.90 \\ 34 & 150 & 13.30 \\ 35 & 160 & 13.09 \\ 36 & 170 & 13.19 \\ 37 & 180 & 13.16\end{array}$

Figure SI-29. Dihedral angle scan to calculate the ap to exo-sc rotational barrier for syn-14. 


\section{Structure Search in the CCDC Data Base}

A structure search in the CCDC data base for Jørgensen-Hayashi catalysts $9 b$ and $9 c$ with the pyrrolidine nitrogen bound to a stereogenic $\mathrm{sp}^{3}$ hybridized carbon reveals striking similarities concerning the catalyst structure. Each structure in the database had the pyrrolidine ring in an envelope conformation with the $\mathrm{y}$-carbon tilted out of plane toward the diarylsilylether moiety and the nitrogen lone pair (down conformation). For the orientation of the diarylsilylether group, two distinct conformational minima can be observed. In 7/10 crystal structures, the $\mathrm{N}$ - and $\mathrm{O}$-substituent at the exocyclic $\mathrm{C}-\mathrm{C}$ bond of the catalyst possess an antiperiplanar $(a p)$ relationship whereas in three cases an exo-synclinal (exosc) conformation was adopted.
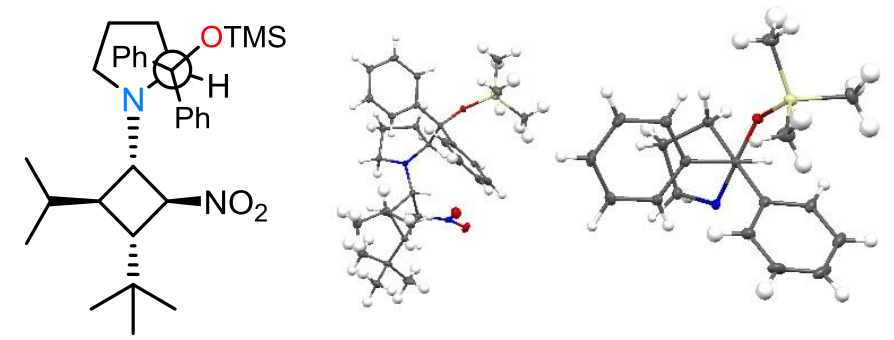

Figure SI-30. 883666 $6^{46,47}(a p)$
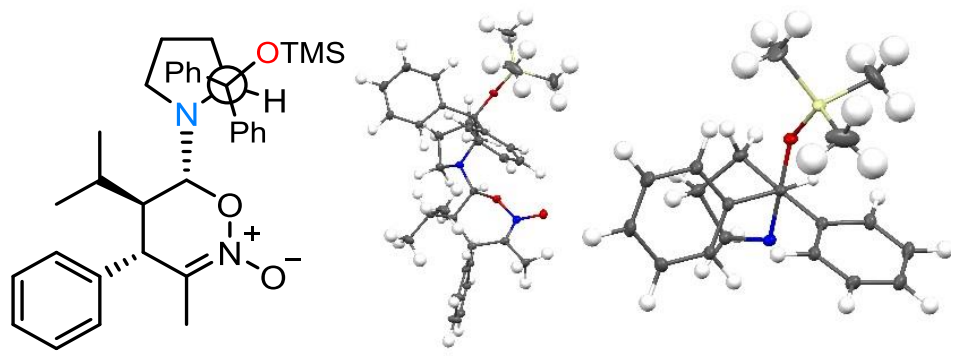

Figure SI-31. $883667^{46,47}(a p)$
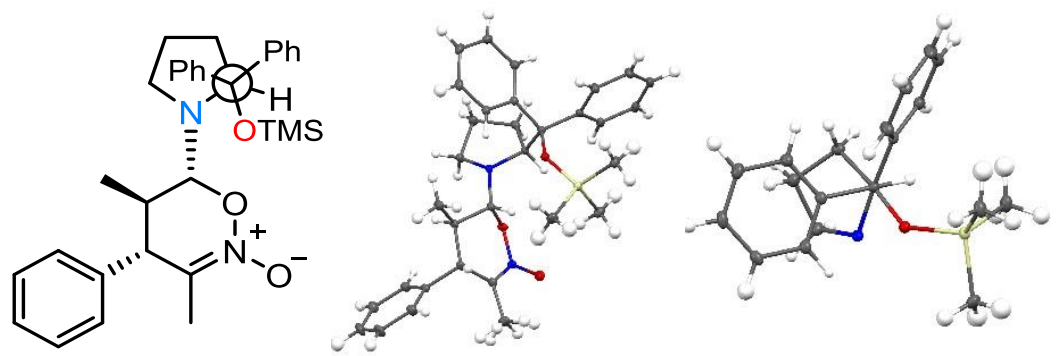

Figure SI-32. $883668^{46,47}$ (sc-exo)

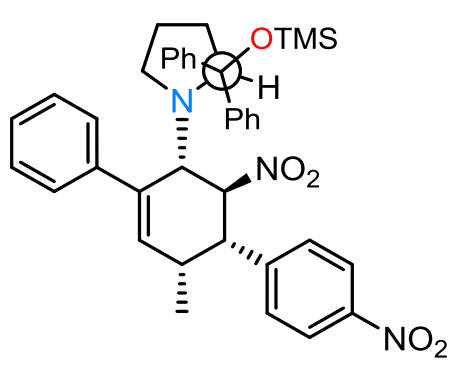
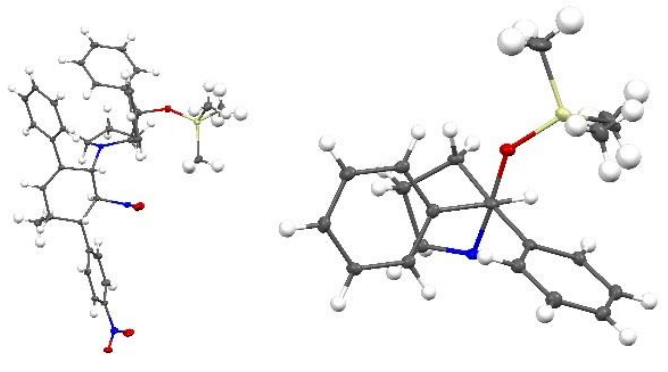

Figure SI-33. $885695^{48}$ (ap) 

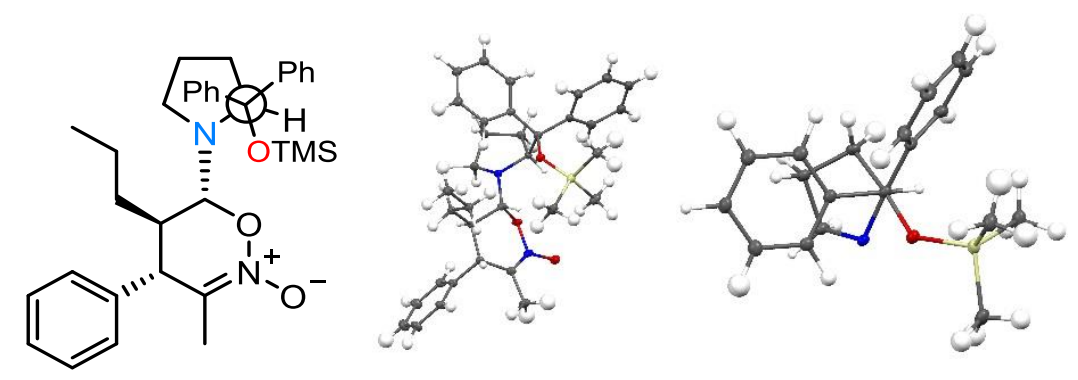

Figure SI-34. $919662^{47}$ (sc-exo)
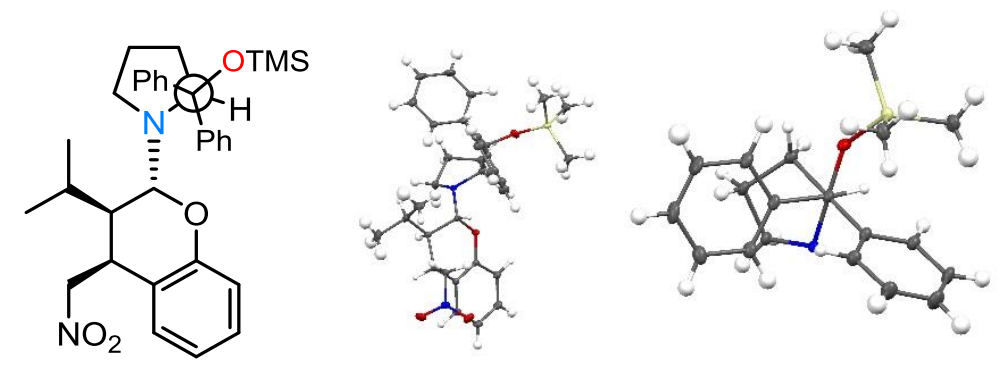

Figure SI-35. $919664^{47}(a p)$
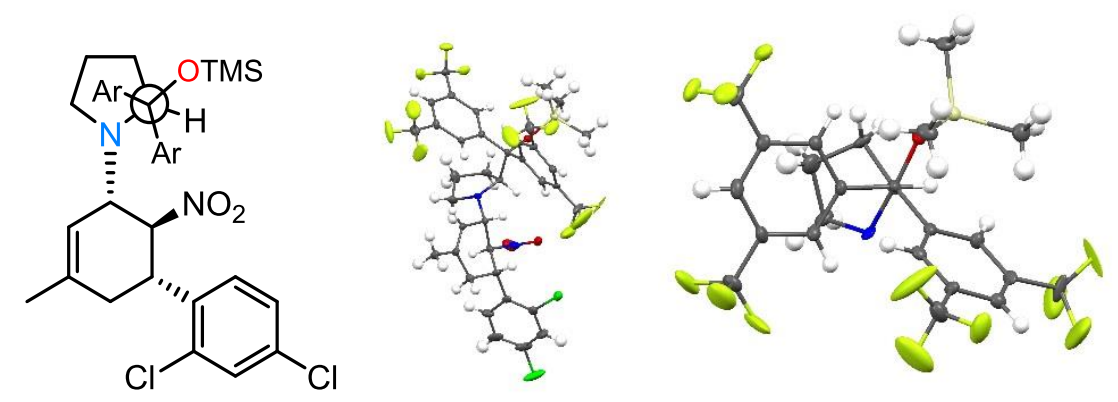

Figure SI-36. $973119^{49}(a p)$
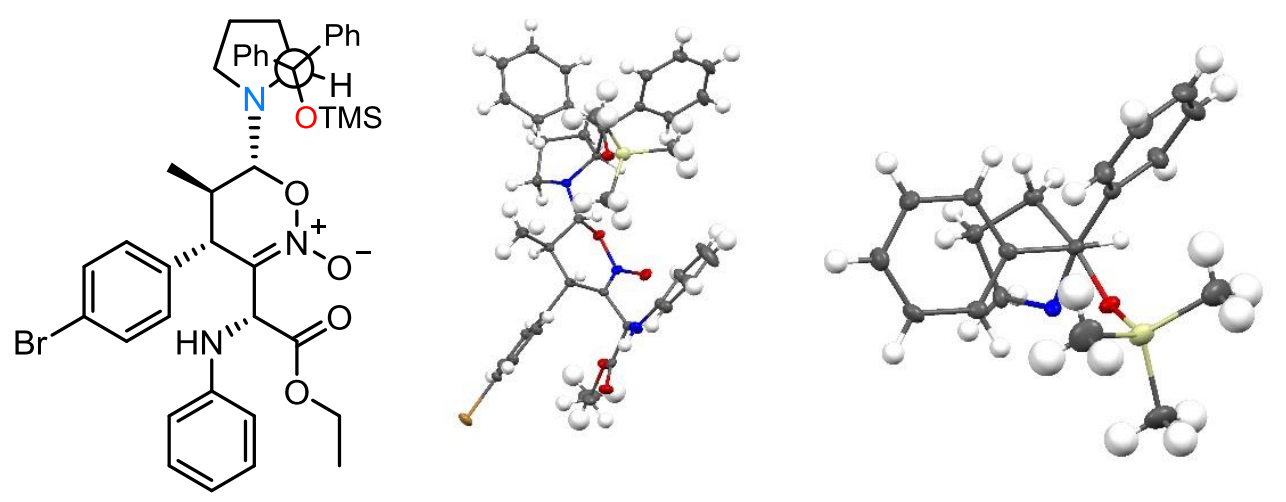

Figure SI-37. $1434063^{50}$ (sc-exo) 


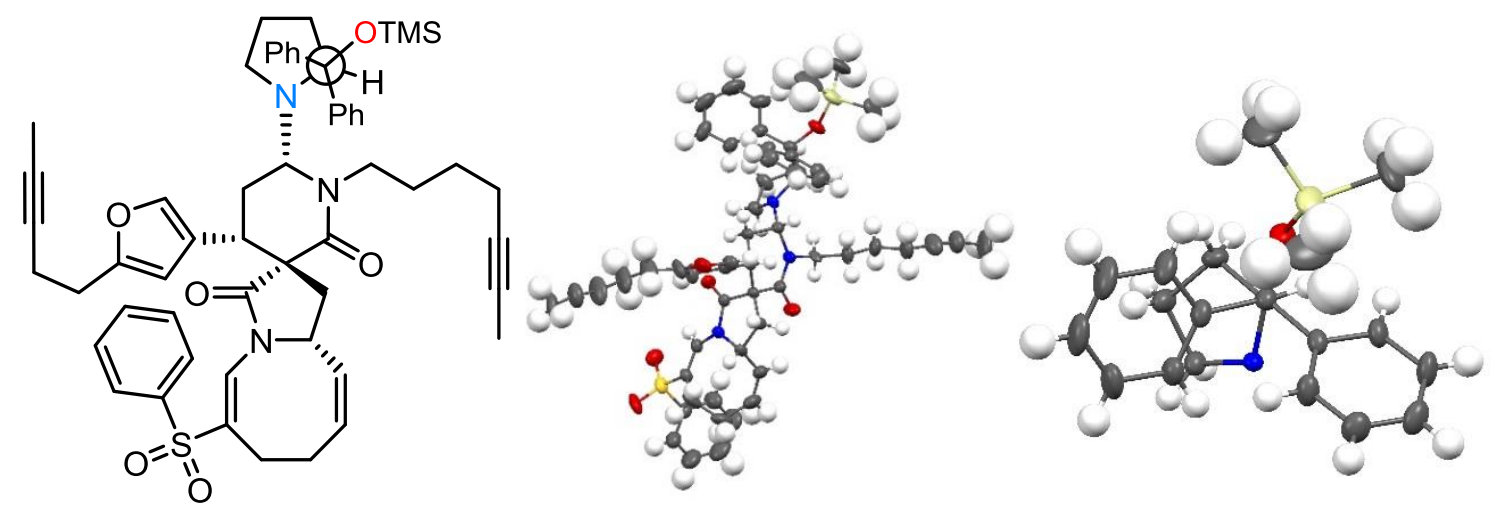

Figure SI-38. $1521504^{51}(a p)$

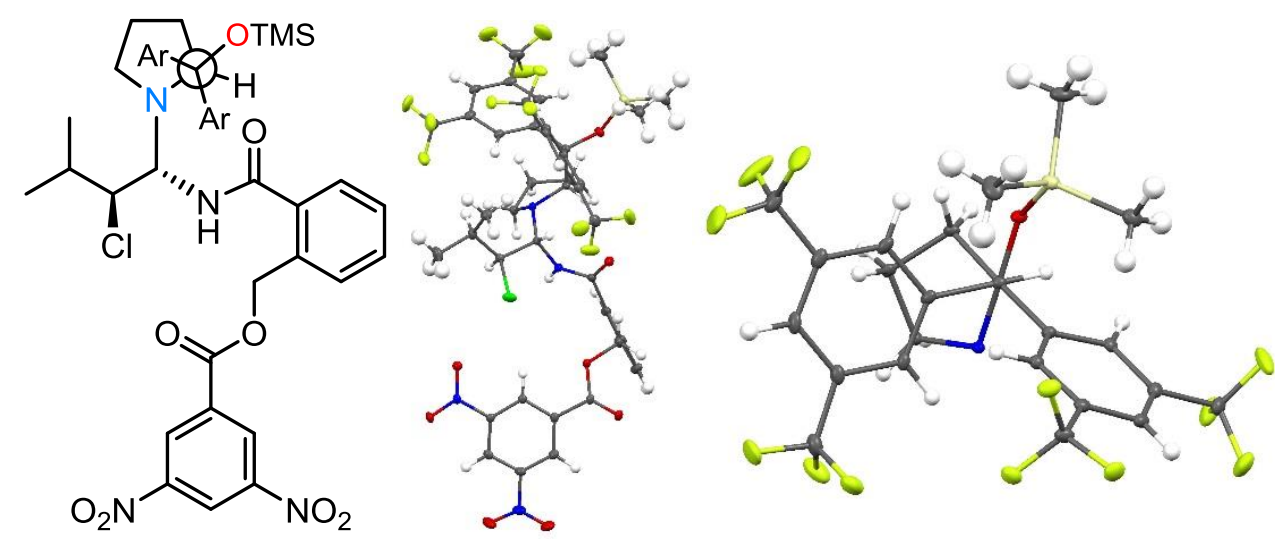

Figure SI-39. 2045387 (ap)

(This work) 


\subsubsection{Decomposition and Deuterium Incorporation Studies}

Decomposition and deuterium incorporation experiments were envisioned to shed more light on the decomposition mechanism of aminal $\mathbf{1 4}$ and should allow the distinction between E2-elimination (via chloroenamine $)^{28}$ and hydrolysis via chloroiminium ion.

\section{Decomposition of Aminal 14 (1)}<smiles>COC(c1cc(C(F)(F)F)cc(C(F)(F)F)c1)(c1cc(C(F)(F)F)cc(C(F)(F)F)c1)C1CCCN1C(C(Cl)C(C)C)N1C(=O)c2ccccc2C1=O</smiles>

$\underset{\text { then } \mathrm{NaBH}_{4}, \mathrm{EtOH}, 0^{\circ} \mathrm{C}, 60 \text { min }}{\mathrm{CHCl}_{3} \mathrm{O} \text { (2 equiv), } 22^{\circ} \mathrm{C}}$<smiles>CC(C)[C@]([O])(Cl)CO</smiles>

$\mathrm{H} / \mathrm{D}-(\mathrm{S})-\mathrm{S} 15 \quad \mathrm{H} / \mathrm{D}-(R)-\mathrm{S} I 5$

$\mathrm{D}_{2} \mathrm{O}\left(36.1 \mu \mathrm{L}, 2.00 \mathrm{mmol}, 2.0\right.$ equiv) and TFAd $_{1}(154 \mu \mathrm{L}, 2.00 \mathrm{mmol}, 2.0$ equiv) were added subsequently to a solution of aminal $14\left(0.847 \mathrm{~g}, 1.00 \mathrm{mmol}, 1.0\right.$ equiv) in $\mathrm{CHCl}_{3}(50 \mathrm{~mL})$ at $22{ }^{\circ} \mathrm{C}$. Aliquots $(5 \mathrm{~mL})$ were taken from the reaction mixture after defined points of time (10 min, $30 \mathrm{~min}, 60$ min, 2 h, 4 h, 22 h, 70 h) and added to a solution of $\mathrm{NaBH}_{4}(18.9 \mathrm{mg}, 500 \mu \mathrm{mol}, 5.0$ equiv) in $\mathrm{EtOH}(2.0$ $\mathrm{mL}$ ). After $30 \mathrm{~min}$ the reactions were quenched with aqueous saturated $\mathrm{NH}_{4} \mathrm{Cl}$. The aqueous phase was extracted with EtOAc ( $3 \times 5 \mathrm{~mL})$, the combined organic phases were dried over $\mathrm{Na}_{2} \mathrm{SO}_{4}$ and the solvent was removed under reduced pressure. The crude products were purified by column chromatography $\left(\mathrm{SiO}_{2}\right.$, pentane/EtOAc 10:1). Deuterium incorporation was quantified by ${ }^{1} \mathrm{H}-\mathrm{NMR}$ spectra analysis and the enantiomeric excess was determined by chiral GC.

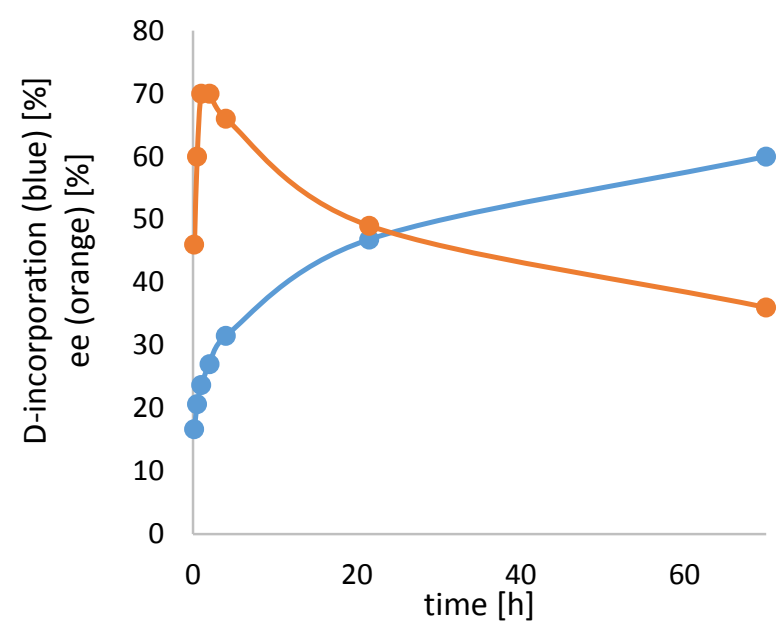

\begin{tabular}{|c|c|c|c|c|c|}
\hline \multirow{2}{*}{$\begin{array}{c}\mathrm{t} \\
{[\mathrm{min}]}\end{array}$} & \multirow[b]{2}{*}{$\mathrm{t}[\mathrm{h}]$} & \multicolumn{2}{|c|}{ integration } & \multirow{2}{*}{$\begin{array}{c}\text { deuterium } \\
\text { incorp. } \\
\text { [\%] }\end{array}$} & \\
\hline & & $3.93 \mathrm{ppm}$ & $3.81 \mathrm{ppm}$ & & [\%] \\
\hline 10 & 0,2 & 1,00 & 1,20 & 16,67 & 46 \\
\hline 30 & 0,5 & 1,00 & 1,26 & 20,63 & 60 \\
\hline 60 & 1,0 & 1,00 & 1,31 & 23,66 & 70 \\
\hline 120 & 2,0 & 1,00 & 1,37 & 27,01 & 70 \\
\hline 240 & 4,0 & 1,00 & 1,46 & 31,51 & 66 \\
\hline 1290 & 21,5 & 1,00 & 1,88 & 46,81 & 49 \\
\hline 4200 & 70,0 & 1,00 & 2,50 & 60,00 & 36 \\
\hline
\end{tabular}

Figure SI-40. Monitoring of the decomposition of aminal 14 with deuterium incorporation and ee over time. 


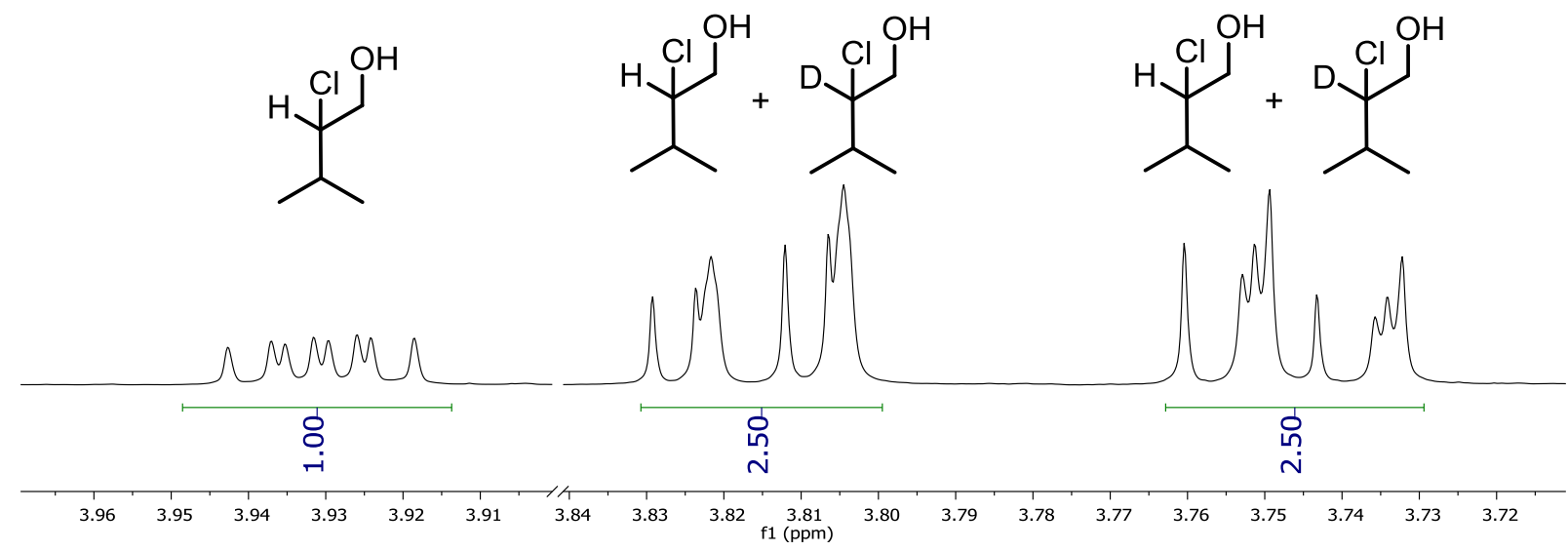

Figure SI-41. Exemplary ${ }^{1} \mathrm{H}-\mathrm{NMR}$ spectrum of a reduced sample $(70 \mathrm{~h})$.

When synthesizing aminal $\mathbf{1 4}$ it was not possible to remove all chloroaldehyde residues under reduced pressure. At the beginning of the decomposition experiment, these residues are present in higher concentrations than the chloroaldehydes formed by the decomposition of the aminal. As a consequence, the ee of the first sample ( $t=10 \mathrm{~min}$; ee $=46 \%$ ) corresponds roughly with the ee of the products formed from the a-chlorination reaction (ee $=49 \%$ with NCS). Over time, the proportion of chloroaldehyde originating from the decomposition of the aminal increases compared to the chloroaldehyde originating from the a-chlorination reaction. Since the aminal decomposes exclusively to the $(S)$-chloroaldehyde (see decomposition with the aminal SI6 derived from propanal), the ee increases over time. The acidcatalyzed (TFA- $d_{1}$ ) racemization of the chloroaldehydes subsequently leads to a continuous decrease of the ee over time. A comparison with the decomposition experiment of the propanal-derived aminal (aminal could be obtained without chloroaldehyde impurities) helps to rationalize this experiment. 


\section{Decomposition of Aminal 14 (2)}<smiles>[Z20]C(C)C([2H])(Cl)C(C)C</smiles>

$0.60 \mathrm{~mL}$ of a stock solution $\left(\mathrm{CDCl}_{3}(6.0 \mathrm{~mL}), \mathrm{D}_{2} \mathrm{O}(4.73 \mu \mathrm{L})\right.$ and $\left.\operatorname{TFAd}_{1}(9.11 \mu \mathrm{L})\right)$ was added to a NMRtube containing aminal 14 (10.0 mg, $11.8 \mu \mathrm{mol}, 1.0$ equiv). The NMR-tube was tightly sealed and ${ }^{1} \mathrm{H}-$ NMR spectra were recorded every 5 min over a period of $10 \mathrm{~h}$. Two additional spectra were recorded after $24 \mathrm{~h}$ and $43 \mathrm{~h}$. Conversion was quantified by ${ }^{1} \mathrm{H}-\mathrm{NMR}$ spectra analysis with $\mathrm{CDCl}_{3}$ as the internal standard. Deuterium incorporation was qualitatively evaluated by ${ }^{1} \mathrm{H}-\mathrm{NMR}$ spectroscopy.

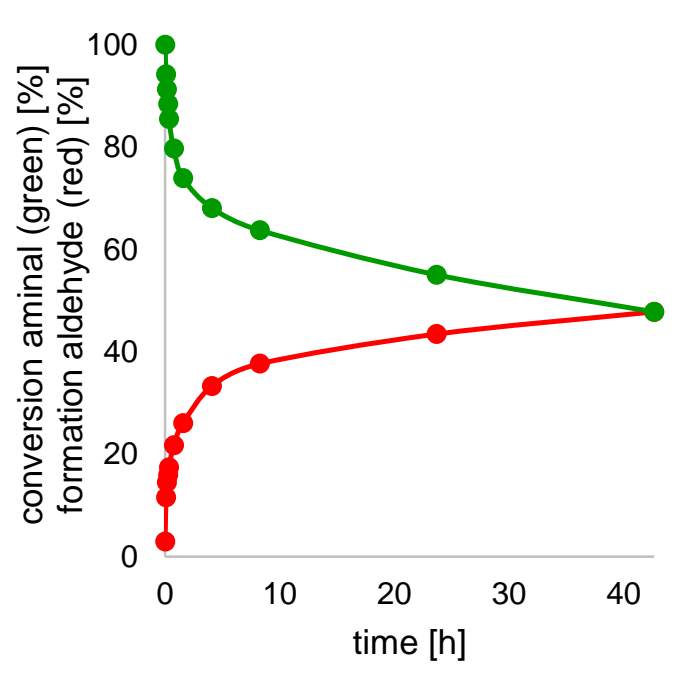

\begin{tabular}{cc|cc|cc}
\begin{tabular}{cc|c} 
time \\
{$[\mathrm{min}]$}
\end{tabular} & $\begin{array}{c}\text { time } \\
{[\mathrm{h}]}\end{array}$ & $\begin{array}{c}\text { integration } \\
\text { aldehyde } \\
9.52 \mathrm{ppm}\end{array}$ & $\begin{array}{c}\text { conversion } \\
\text { aldehyde } \\
{[\%]}\end{array}$ & $\begin{array}{c}\text { integration } \\
\text { aminal } \\
5.03 \mathrm{ppm}\end{array}$ & $\begin{array}{c}\text { conversion } \\
\text { aminal } \\
{[\%]}\end{array}$ \\
\hline 0 & 0,0 & 0,02 & 3 & 0,69 & 100 \\
5 & 0,1 & 0,08 & 12 & 0,65 & 94 \\
10 & 0,2 & 0,1 & 14 & 0,63 & 91 \\
15 & 0,3 & 0,11 & 16 & 0,61 & 88 \\
20 & 0,3 & 0,12 & 17 & 0,59 & 85 \\
45 & 0,8 & 0,15 & 22 & 0,55 & 80 \\
95 & 1,6 & 0,18 & 26 & 0,51 & 74 \\
245 & 4,1 & 0,23 & 33 & 0,47 & 68 \\
495 & 8,3 & 0,26 & 38 & 0,44 & 64 \\
1420 & 23,7 & 0,3 & 43 & 0,38 & 55 \\
2560 & 42,7 & 0,33 & 48 & 0,33 & 48
\end{tabular}

Figure SI-42. Monitoring of the decomposition of aminal 14. 
<smiles>CC(C)C(Cl)C=O</smiles>

$\mathrm{H}-12$<smiles>[2H]C(Cl)(Cl)C(=O)C(C)C</smiles>

D-12

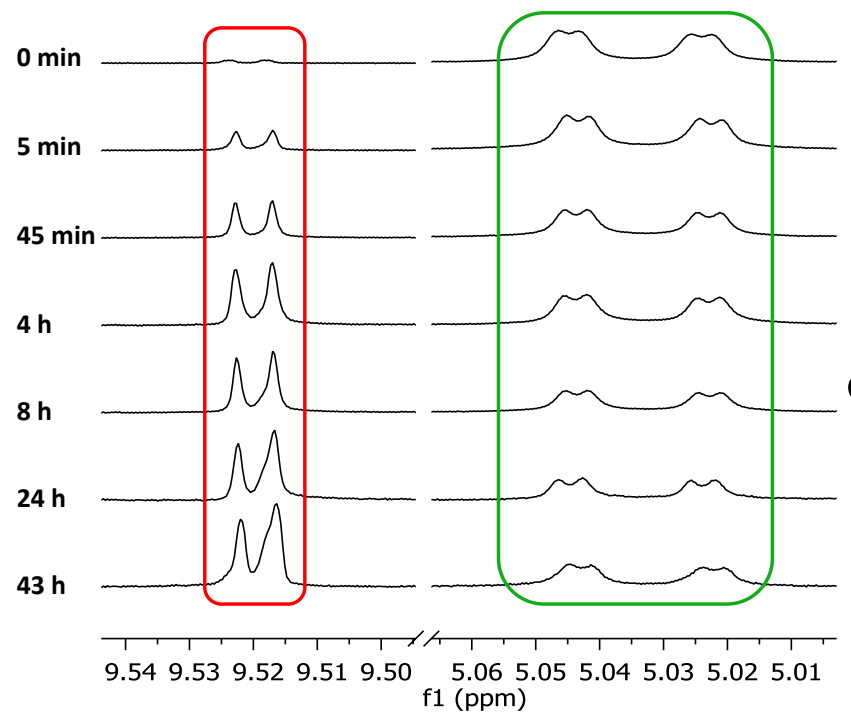

Figure SI-43. ${ }^{1} \mathrm{H}-\mathrm{NMR}$ spectra of the ongoing decomposition reaction (2 equiv of $\mathrm{D}_{2} \mathrm{O}, 1$ equiv of TFAd 1 ) of aminal 14 allows a qualitative evaluation of the deuterium incorporation into the $\alpha$-chloroaldehydes.

The analysis of the aldehyde- $\mathrm{H}$ signals in the ${ }^{1} \mathrm{H}-\mathrm{NMR}$ spectra allows a qualitative evaluation of the deuterium incorporation into the $\alpha$-chloroaldehydes. Almost no deuterium incorporation was observed during the first hours. Downstream incorporation of deuterium into the chloroaldehyde can be explained by acid-catalyzed enolization or enamine formation (doublet at $9.52 \mathrm{ppm}(\alpha-\mathrm{H}$-chloroaldehyde) $->$ singlet at $9.52 \mathrm{ppm}$ ( $\alpha$-D-chloroaldehyde)). 


\section{Decomposition of Aminal 14 (3)}

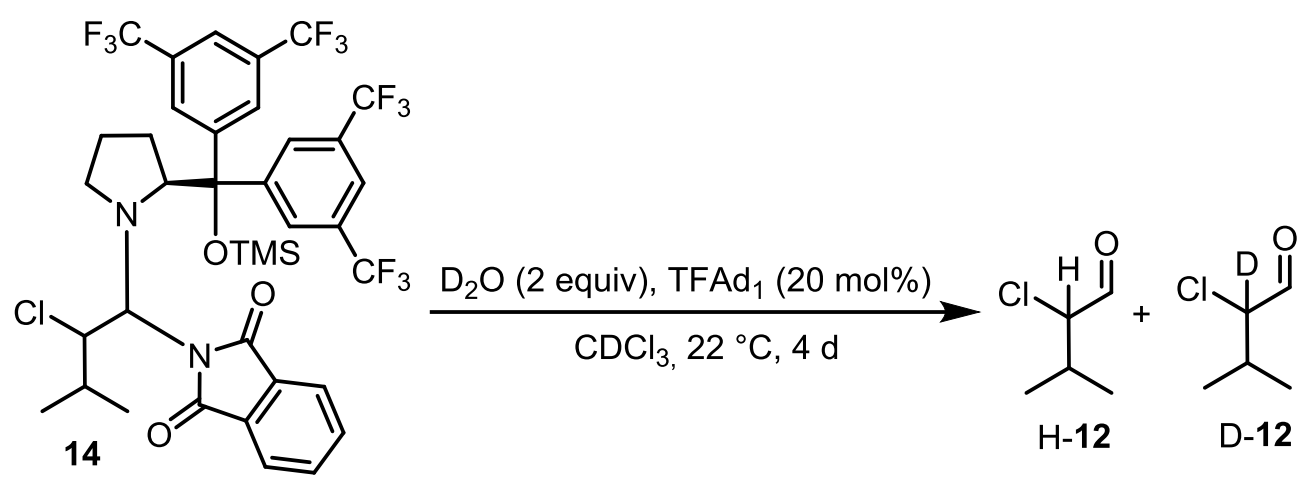

$0.60 \mathrm{~mL}$ of a stock solution $\left(\mathrm{CDCl}_{3}(6.0 \mathrm{~mL}), \mathrm{D}_{2} \mathrm{O}(4.73 \mu \mathrm{L})\right.$ and $\left.\operatorname{TFAd}_{1}(1.82 \mu \mathrm{L})\right)$ was added to a NMRtube containing aminal 14 (10.0 mg, $11.8 \mu \mathrm{mol}, 1.0$ equiv). The NMR-tube was tightly sealed and ${ }^{1} \mathrm{H}-$ NMR spectra were recorded over a period of 4 days. Deuterium incorporation and conversion was qualitatively evaluated by ${ }^{1} \mathrm{H}-\mathrm{NMR}$ spectroscopy.

D-12
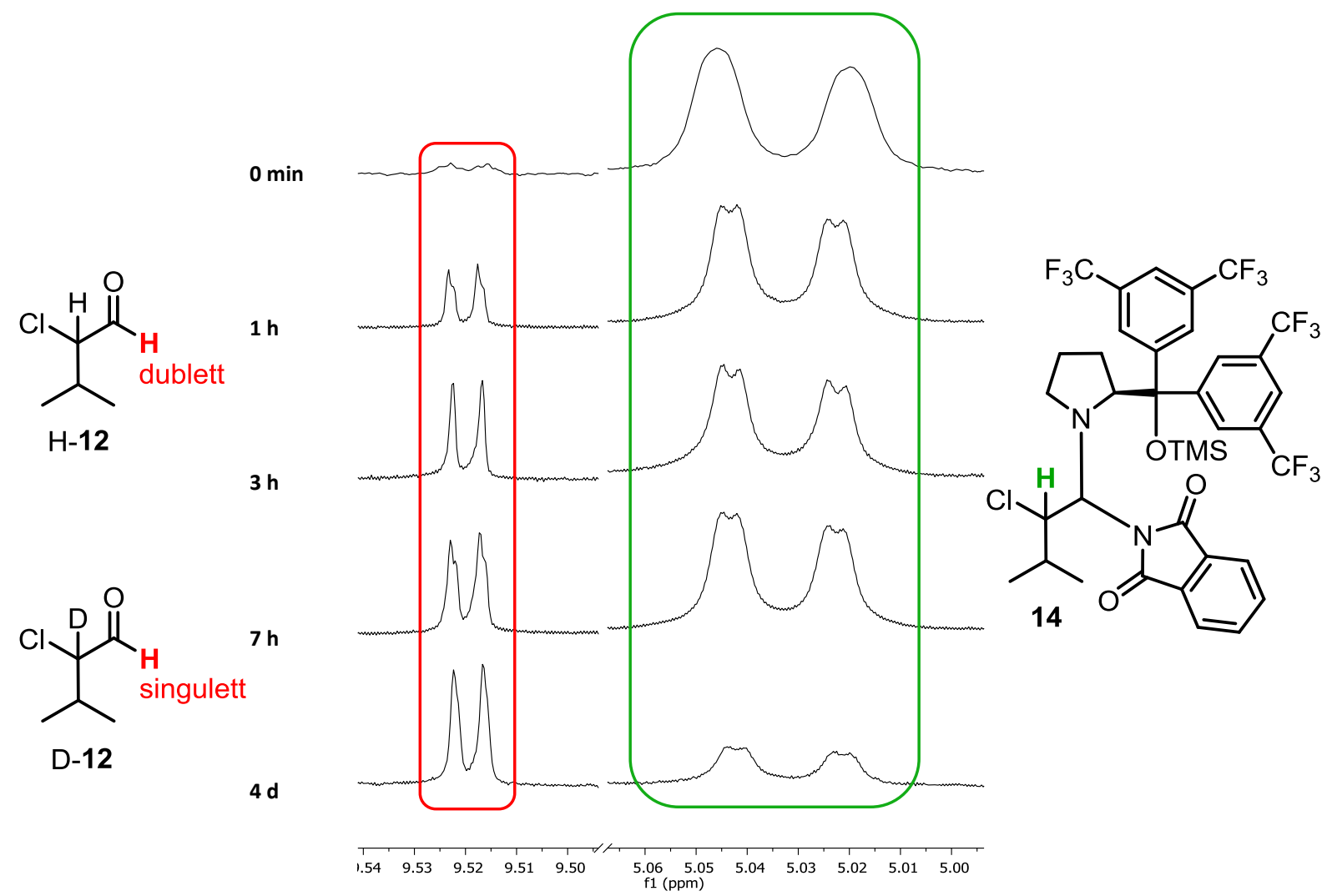

$4 \mathrm{~d}$

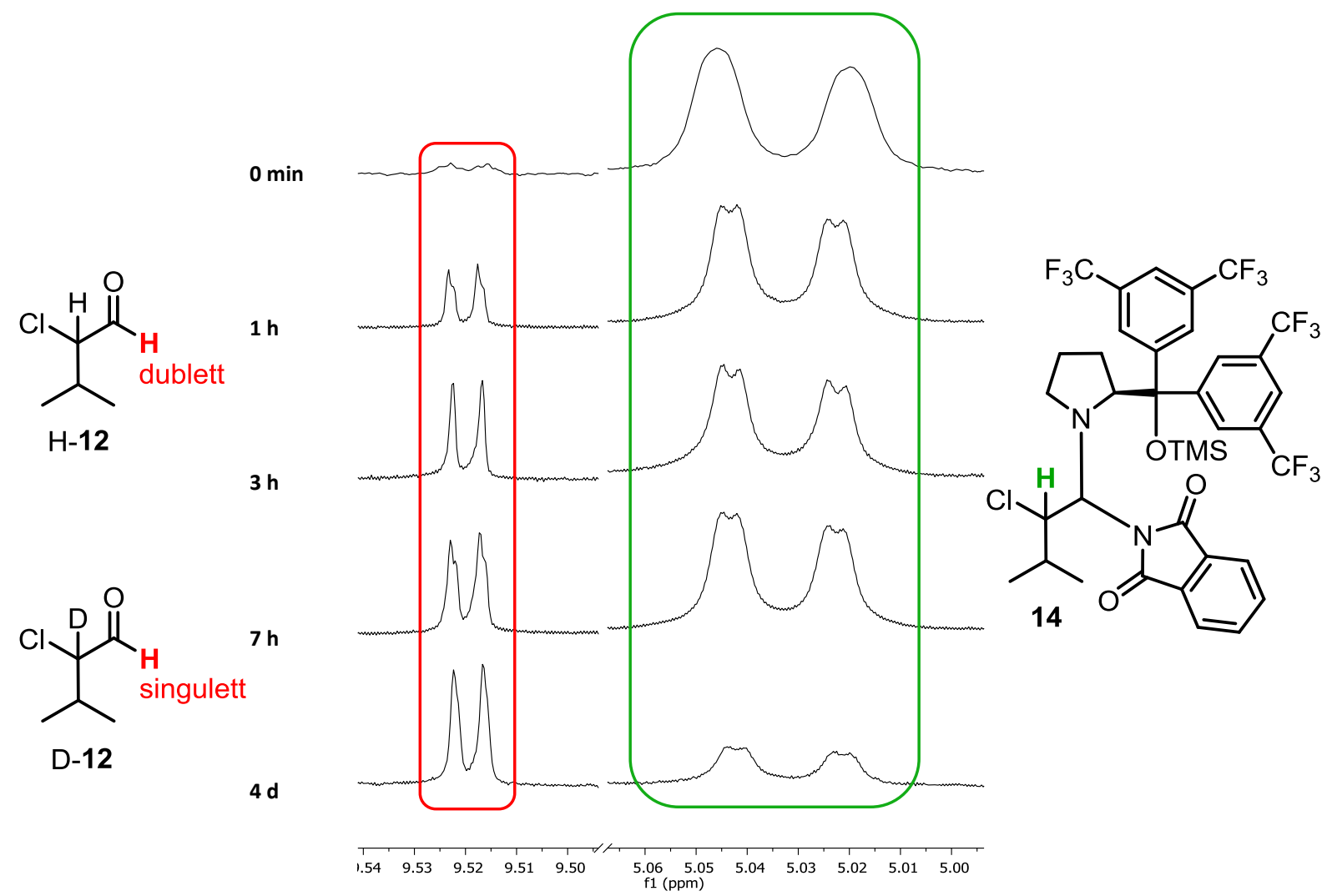

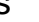

Figure SI-44. ${ }^{1} \mathrm{H}-\mathrm{NMR}$ spectra of the ongoing decomposition reaction (2 equiv of $\mathrm{D}_{2} \mathrm{O}, 20 \mathrm{~mol} \%$ of $\operatorname{TFAd}_{1}$ ) of aminal $\mathbf{1 4}$ allows a qualitative evaluation of the deuterium incorporation into the $\alpha$ chloroaldehydes.

Lowering the amount of TFA-d (from to 2 equiv to 20 mol\%) leads to a slower decomposition of aminal 14. Deuterium incorporation into the $\alpha$-position of the aldehyde can be almost completely suppressed when using 20 mol\% TFA- $d_{1}$ (clean doublet for the aldehyde-proton). 


\section{Decomposition of Aminal 14 (4)}

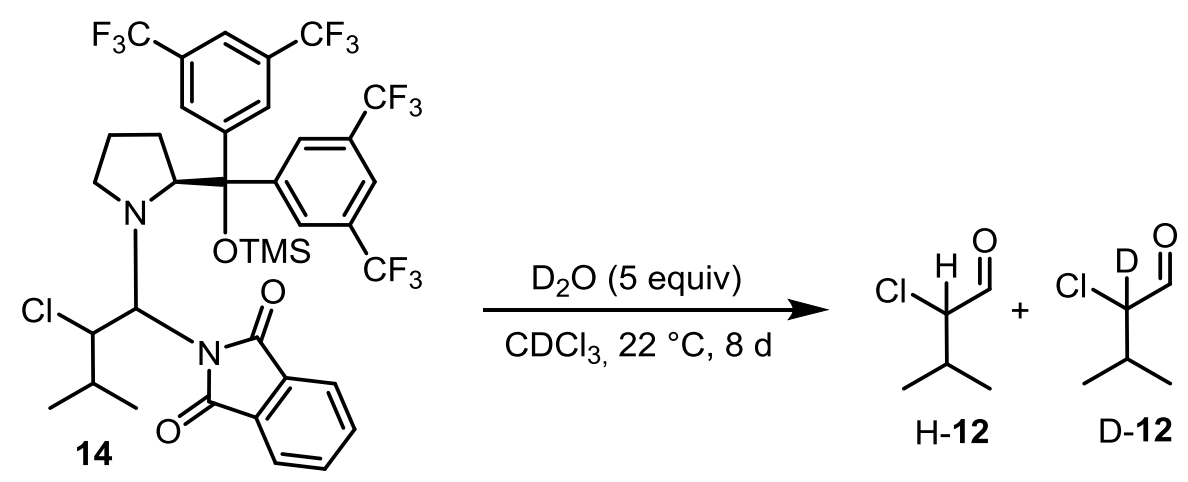

$0.60 \mathrm{~mL}$ of a stock solution $\left(\mathrm{CDCl}_{3}(6.0 \mathrm{~mL}), \mathrm{D}_{2} \mathrm{O}(11.8 \mu \mathrm{L})\right)$ was added to a NMR-tube containing aminal 14 (10.0 mg, $11.8 \mu \mathrm{mol}, 1.0$ equiv). The NMR-tube was tightly sealed and ${ }^{1} \mathrm{H}$-NMR spectra were recorded over a period of 8 days. Deuterium incorporation and conversion was qualitatively and quantitatively evaluated by ${ }^{1} \mathrm{H}-\mathrm{NMR}$ spectroscopy.

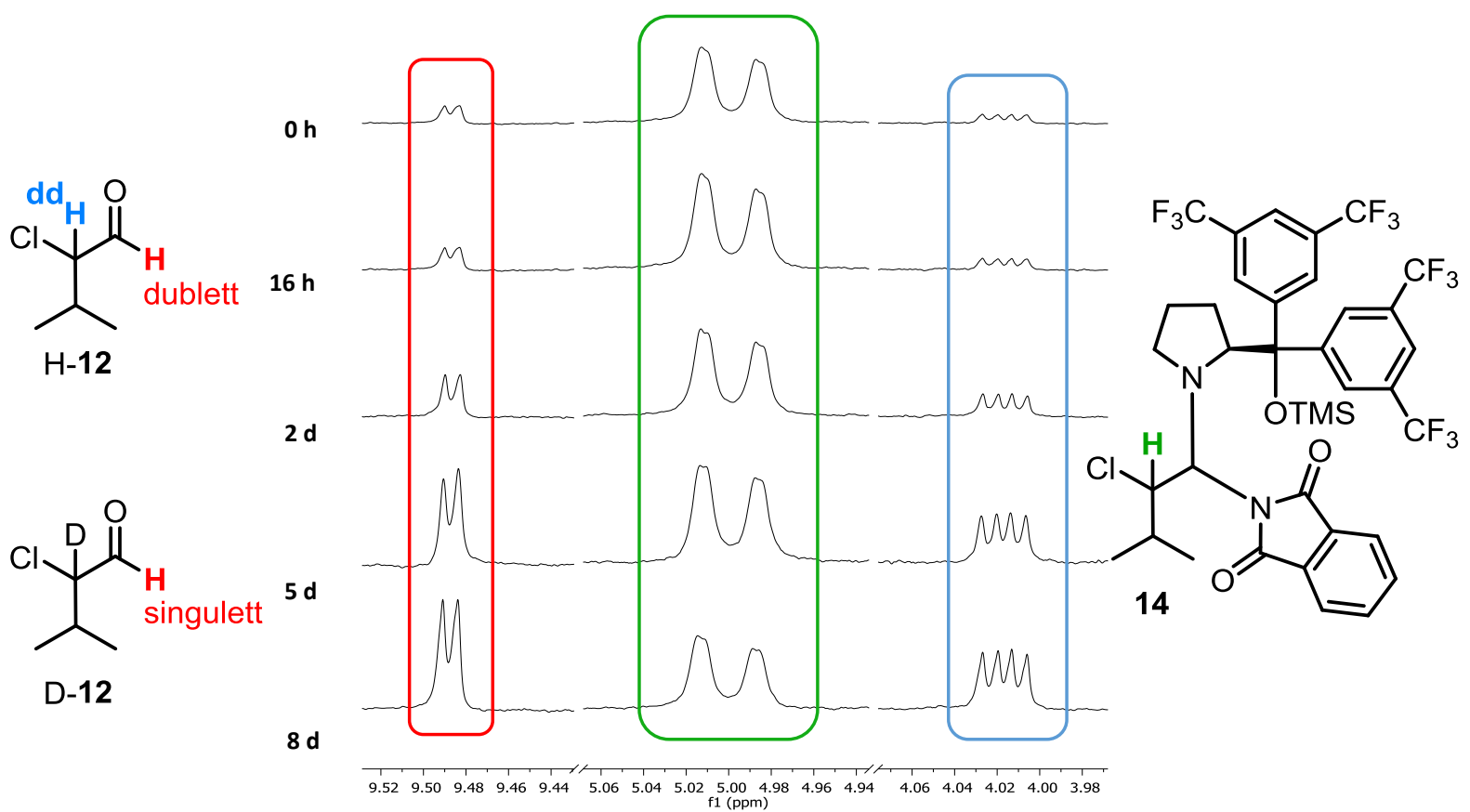

Figure SI-45. ${ }^{1} \mathrm{H}-\mathrm{NMR}$ spectra of the ongoing decomposition reaction (5 equiv of $\mathrm{D}_{2} \mathrm{O}$ ) of aminal 14 allows a qualitative evaluation of the deuterium incorporation into the a-chloroaldehydes.
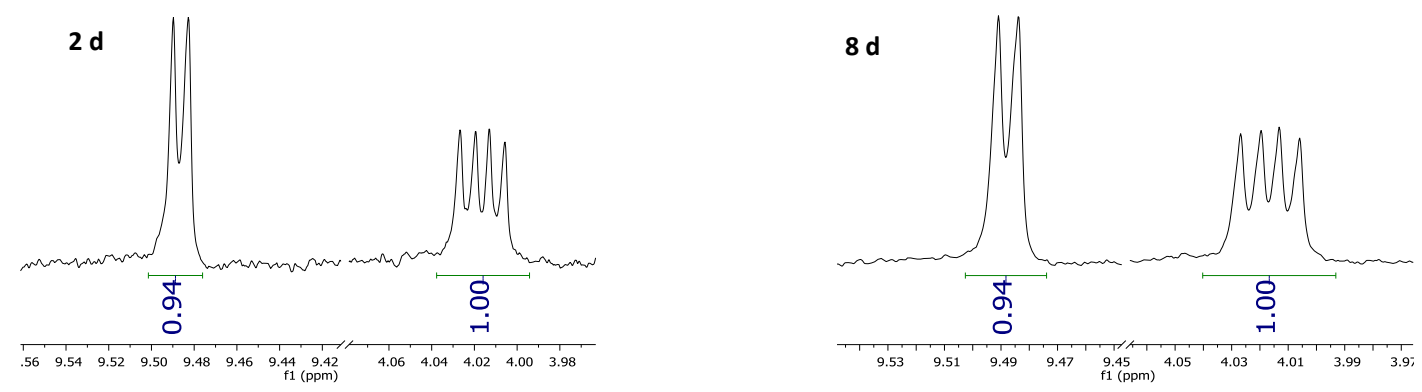

Figure SI-46. Exemplary ${ }^{1} \mathrm{H}$-NMR spectra (after $2 \mathrm{~d}$ and $8 \mathrm{~d}$ ) of the ongoing decomposition reaction (5 equiv of $\mathrm{D}_{2} \mathrm{O}$ ) of aminal $\mathbf{1 4}$ allows a quantification of the deuterium incorporation into the a-position. 
Omitting acidic conditions further decreases the rate of aminal hydrolysis. Nevertheless, only $\mathrm{D}_{2} \mathrm{O}$ can mediate the decomposition effectively. As shown in the figure above, aminal hydrolysis under non acidic conditions takes place without any detectable incorporation of deuterium in the a-position of the aldehyde over long term ( 8 days). The sharp doublet of the aldehyde proton (red) and the 1:1 ratio between the aldehyde proton (red) and the $\alpha$-proton of the aldehyde (orange) prove this issue. E2elimination of the aminal with generation of chloroenamines, as proposed for the Curtin-Hammettscenario cannot explain the complete absence of deuterium incorporation. Whereas the "classical" hydrolysis pathway via chloroiminium ion with an intact $\alpha$-position is in line with the absence of deuterium incorporation. 


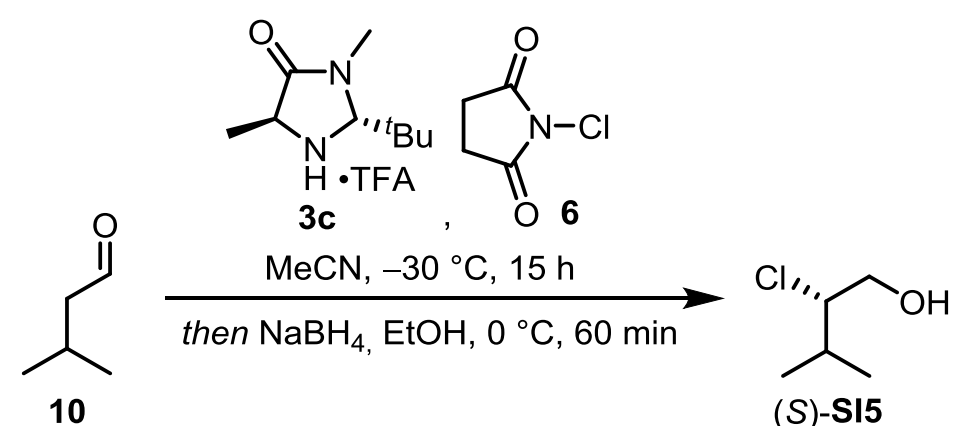

Isovaleraldehyde $(0.215 \mathrm{~mL}, 2.00 \mathrm{mmol}, 1.0$ equiv) was dissolved in $\mathrm{MeCN}(8.0 \mathrm{~mL})$ and cooled to $-30^{\circ} \mathrm{C}$. Catalyst $3 \mathrm{c}(0.114 \mathrm{~g}, 0.400 \mathrm{mmol}, 20 \mathrm{~mol} \%)$ and NCS $(0.320 \mathrm{~g}, 2.40 \mathrm{mmol}, 1.2$ equiv) were added subsequently and the reaction mixture was stirred for $15 \mathrm{~h}$ at the same temperature. After the reaction was warmed to $0^{\circ} \mathrm{C}, \mathrm{MeOH}(3.0 \mathrm{~mL})$ and $\mathrm{NaBH}_{4}(0.189 \mathrm{~g}, 5.00 \mathrm{mmol}, 2.5$ equiv) were added and the mixture was stirred for $60 \mathrm{~min}$ at the same temperature. The reaction was quenched with aqueous saturated $\mathrm{NH}_{4} \mathrm{Cl}(10 \mathrm{~mL})$, the aqueous phase was extracted with dichloromethane $(3 \times 15 \mathrm{~mL})$ and the combined organic phases were dried over $\mathrm{NaSO}_{4}$. The solvent was removed under reduced pressure and the crude product was purified by column chromatography (Silica, pentane/EtOAc 10:1). Pure chloroalcohol (S)-SI5 $(0.111 \mathrm{~g}, 0.905 \mathrm{mmol}, 45 \%, 89 \%$ ee) was obtained as a colorless oil.

${ }^{1} \mathrm{H}-\mathrm{NMR}\left(\mathrm{CDCl}_{3}, 400 \mathrm{MHz}\right) \delta=3.92(\mathrm{ddd}, J=7.8,5.2,3.8 \mathrm{~Hz}, 1 \mathrm{H}), 3.81(\mathrm{dd}, J=12.0,3.8 \mathrm{~Hz}, 1 \mathrm{H}), 3.73$ (dd, $J=12.0,7.8 \mathrm{~Hz}, 1 \mathrm{H}), 2.12-2.03(\mathrm{~m}, 1 \mathrm{H}), 2.02(\mathrm{~s}, 1 \mathrm{H}), 1.03(\mathrm{~d}, J=6.8 \mathrm{~Hz}, 3 \mathrm{H}), 1.01(\mathrm{~d}, J=6.7$ $\mathrm{Hz}, 3 \mathrm{H}) \mathrm{ppm}$. 


\section{(R)-2-chloro-3-methylbutan-1-ol ((R)-SI5)}

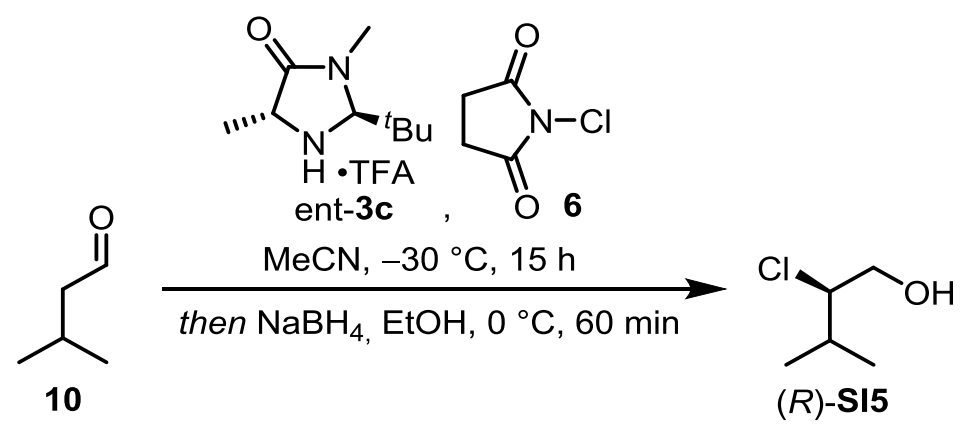

Isovaleraldehyde $(0.215 \mathrm{~mL}, 2.00 \mathrm{mmol}, 1.0$ equiv) was dissolved in $\mathrm{MeCN}(8.0 \mathrm{~mL})$ and cooled to $-30{ }^{\circ} \mathrm{C}$. Catalyst ent-3c $(0.114 \mathrm{~g}, 0.400 \mathrm{mmol}, 20 \mathrm{~mol} \%)$ and NCS $(0.320 \mathrm{~g}, 2.40 \mathrm{mmol}, 1.2$ equiv) were added subsequently and the reaction mixture was stirred for $15 \mathrm{~h}$ at the same temperature. After the reaction was warmed to $0{ }^{\circ} \mathrm{C}, \mathrm{MeOH}(3.0 \mathrm{~mL})$ and $\mathrm{NaBH}_{4}(0.189 \mathrm{~g}, 5.00 \mathrm{mmol}, 2.5$ equiv) were added and the mixture was stirred for $60 \mathrm{~min}$ at the same temperature. The reaction was quenched with aqueous saturated $\mathrm{NH}_{4} \mathrm{Cl}(10 \mathrm{~mL})$, the aqueous phase was extracted with dichloromethane $(3 \times 15 \mathrm{~mL})$ and the combined organic phases were dried over $\mathrm{NaSO}_{4}$. The solvent was removed under reduced pressure and the crude product was purified by column chromatography (Silica, pentane/EtOAc 10:1). Pure chloroalcohol $(R)$-SI5 (0.102 g, $0.832 \mathrm{mmol}, 42 \%, 95 \%$ ee) was obtained as a colorless oil.

Spectroscopic data are in accordance with the previous experiment. 


\subsection{Aminals Derived from Propanal}

\subsubsection{Synthesis}

When synthesizing aminal 14, it was not possible to remove all impurities of isovaleraldehyde (10) and 2-chloro-3-methylbutanal (12) under reduced pressure. Due to the fact, that a pure and aldehyde-free aminal is crucial for some follow-up experiments we changed from isovaleraldehyde to the more volatile propanal. As a result, all aldehyde-impurities could be removed under reduced pressure.

2-(1-((S)-2-(bis(3,5-bis(trifluoromethyl)phenyl)((trimethylsilyl)oxy)methyl)pyrrolidin-1-yl)-2chloropropyl)isoindoline-1,3-dione (SI6)

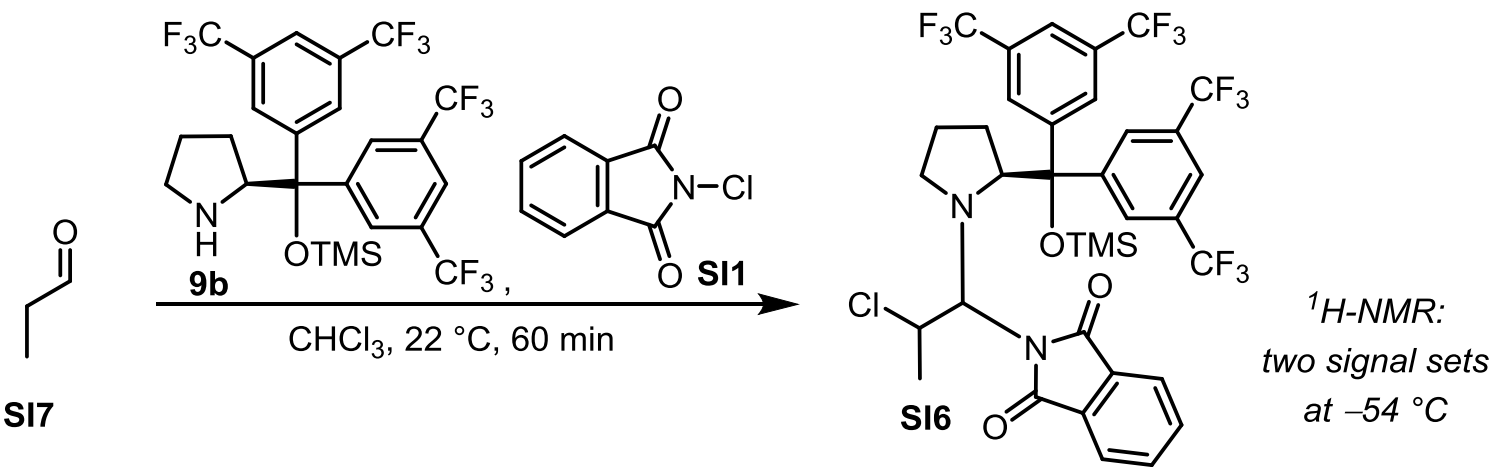

Catalysts $9 \mathrm{~b}(2.91 \mathrm{~g}, 4.87 \mathrm{mmol}, 50 \mathrm{~mol} \%)$ and NCP (0.884 g, $4.87 \mathrm{mmol}, 0.5$ equiv) were added successively to a solution of propanal $\left(0.698 \mathrm{~mL}, 9.74 \mathrm{mmol}, 1.0\right.$ equiv) in $\mathrm{CHCl}_{3}(44 \mathrm{~mL})$ at $22{ }^{\circ} \mathrm{C}$. After $60 \mathrm{~min}$ the solvent and aldehydes were removed under reduced pressure. The crude product was dissolved in pentane $(20 \mathrm{~mL})$ and the precipitating solids were filtered off. Pentane was removed under reduced pressure and the aminal SI6 was obtained as a foamy off-white solid (3.54 g, $4.32 \mathrm{mmol}, 89 \%$ ). ${ }^{1} \mathrm{H}-\mathrm{NMR}$ spectroscopy at $-54{ }^{\circ} \mathrm{C}$ shows two signal sets with a ratio of $68: 32$.

$\mathbf{m p}=65^{\circ} \mathrm{C}$

IR (ATR): $\tilde{v}=3055,2988,2307,1713,1280,1264,1176,1139,844,733,704 \mathrm{~cm}^{-1}$

HRMS (ESI, pos. mode): $\mathrm{m} / z$ calculated for $\mathrm{C}_{35} \mathrm{H}_{31} \mathrm{ClF}_{12} \mathrm{~N}_{2} \mathrm{NaO}_{3} \mathrm{Si}[\mathrm{M}+\mathrm{Na}]^{+}: 841.1493$, found 841.1487; $\mathrm{m} / \mathrm{z}$ calculated for $\mathrm{C}_{35} \mathrm{H}_{31} \mathrm{ClF}_{12} \mathrm{KN}_{2} \mathrm{O}_{3} \mathrm{Si}[\mathrm{M}+\mathrm{K}]^{+}:$857.1233, found 857.1207.

\section{major stereoisomer:}

${ }^{1} \mathrm{H}-\mathrm{NMR}\left(\mathrm{CDCl}_{3}, 600 \mathrm{MHz},-54{ }^{\circ} \mathrm{C}\right) \delta=5.88(\mathrm{~d}, J=10.5 \mathrm{~Hz}, 1 \mathrm{H}), 5.19(\mathrm{dq}, J=13.1,6.7 \mathrm{~Hz}, 1 \mathrm{H}), 4.18$ (dd, $J=10.2,3.2 \mathrm{~Hz}, 1 \mathrm{H}$ ), 3.00 (td, $J=9.5,6.5 \mathrm{~Hz}, 1 \mathrm{H}$ ), 2.46 (ddd, $J=9.9,6.8,3.4 \mathrm{~Hz}, 1 \mathrm{H}$ ), $1.86-1.81$ $(\mathrm{m}, 1 \mathrm{H}), 1.79(\mathrm{~d}, J=6.8 \mathrm{~Hz}, 3 \mathrm{H}), 1.40-1.34(\mathrm{~m}, 1 \mathrm{H}), 1.32-1.26(\mathrm{~m}, 1 \mathrm{H}), 0.41-0.31(\mathrm{~m}, 1 \mathrm{H}),-0.18$ $(\mathrm{s}, 9 \mathrm{H}) \mathrm{ppm}$. 
${ }^{13} \mathrm{C}-\mathrm{NMR}\left(\mathrm{CDCl}_{3}, 151 \mathrm{MHz},-54{ }^{\circ} \mathrm{C}\right) \delta=171.0,170.9,83.7,77.9,66.8,55.0,47.6,27.9,23.8,23.3,1.5$ ppm.

\section{minor stereoisomer:}

${ }^{1} \mathrm{H}-\mathrm{NMR}\left(\mathrm{CDCl}_{3}, 600 \mathrm{MHz},-54{ }^{\circ} \mathrm{C}\right) \delta=5.01-4.92(\mathrm{~m}, 1 \mathrm{H}), 4.91-4.87(\mathrm{~m}, 1 \mathrm{H}), 4.58(\mathrm{~d}, J=8.1 \mathrm{~Hz}$, $1 \mathrm{H}), 3.12(\mathrm{q}, J=8.3 \mathrm{~Hz}, 1 \mathrm{H}), 2.28-2.23(\mathrm{~m}, 1 \mathrm{H}), 1.73-1.67(\mathrm{~m}, 1 \mathrm{H}), 1.64-1.58(\mathrm{~m}, 1 \mathrm{H}), 1.24-1.20$ (m, 1H), $0.99(\mathrm{~d}, J=6.3 \mathrm{~Hz}, 3 \mathrm{H}),-0.15(\mathrm{~s}, 9 \mathrm{H}) \mathrm{ppm}$.

${ }^{13} \mathrm{C}-\mathrm{NMR}\left(\mathrm{CDCl}_{3}, 151 \mathrm{MHz},-54{ }^{\circ} \mathrm{C}\right) \delta=171.1,170.2,78.5,70.0,53.8,48.0,27.4,24.1,22.3,1.7 \mathrm{ppm}$.

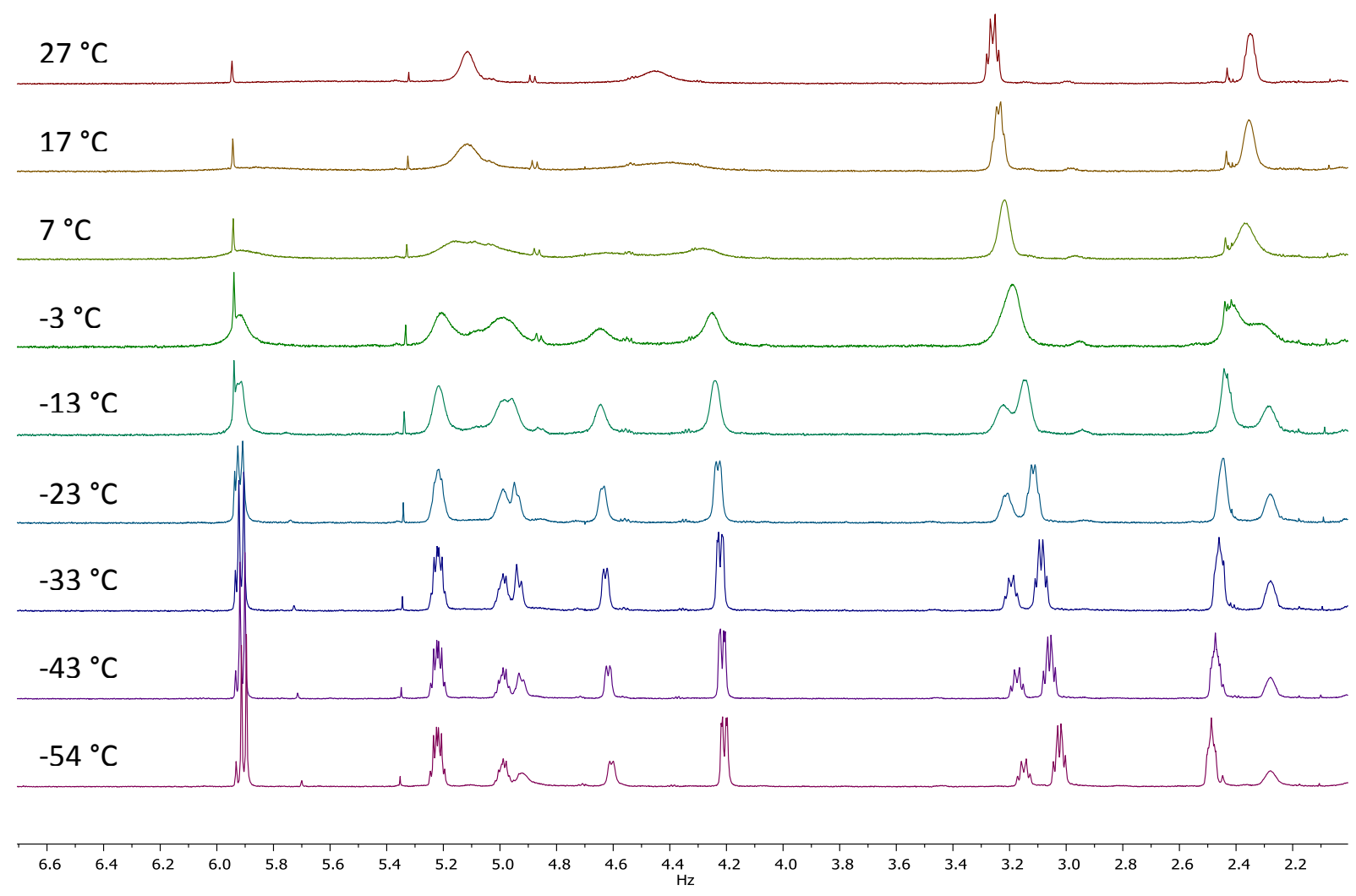

Figure SI-47. ${ }^{1} \mathrm{H}-\mathrm{NMR}$ spectra of aminal SI6 at different temperatures shows the emergence of two signal sets at low temperatures.

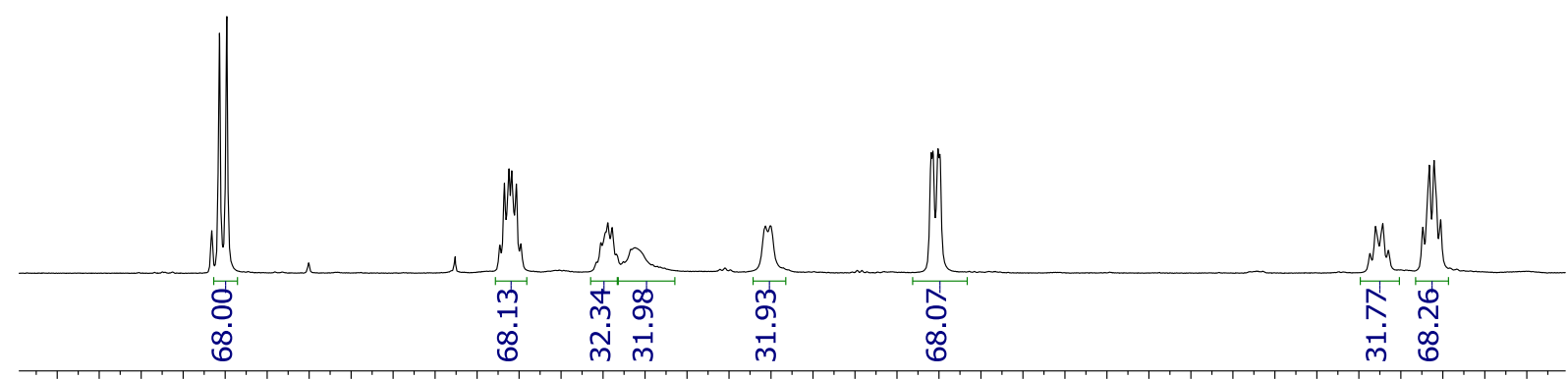

$\begin{array}{llllllllllllllllllllllllllllllllllllllllllllllllllllll}6.3 & 6.2 & 6.1 & 6.0 & 5.9 & 5.8 & 5.7 & 5.6 & 5.5 & 5.4 & 5.3 & 5.2 & 5.1 & 5.0 & 4.9 & 4.8 & 4.7 & 4.6 & 4.5 & 4.4 & 4.3 & 4.2 & 4.1 & 4.0 & 3.9 & 3.8 & 3.7 & 3.6 & 3.5 & 3.4 & 3.3 & 3.2 & 3.1 & 3.0 & 2.9 & 2.8\end{array}$

Figure SI-48. The isomeric ratio (68:32) was assigned at $-54^{\circ} \mathrm{C}$. 
The combination of aldehyde, Jørgensen-Hayashi-type catalyst $\mathbf{9 b}$, and NCP generates almost quantitatively the corresponding aminal species. As a consequence, the conversion of the aldehyde to the $\alpha$-chloroaldehyde is always below $5 \%$. The low conversion in combination with the high volatility of aldehyde SI8 and alcohol SI9 makes the isolation and purification difficult. To overcome these problems, the in-situ reduced chloroaldehyde SI8 was directly transformed into the non-volatile 3,5dinitrobenzoylester SI10.

\section{Enantiomeric Excess (Propanal, Catalyst 9b and NCS/NCP)}

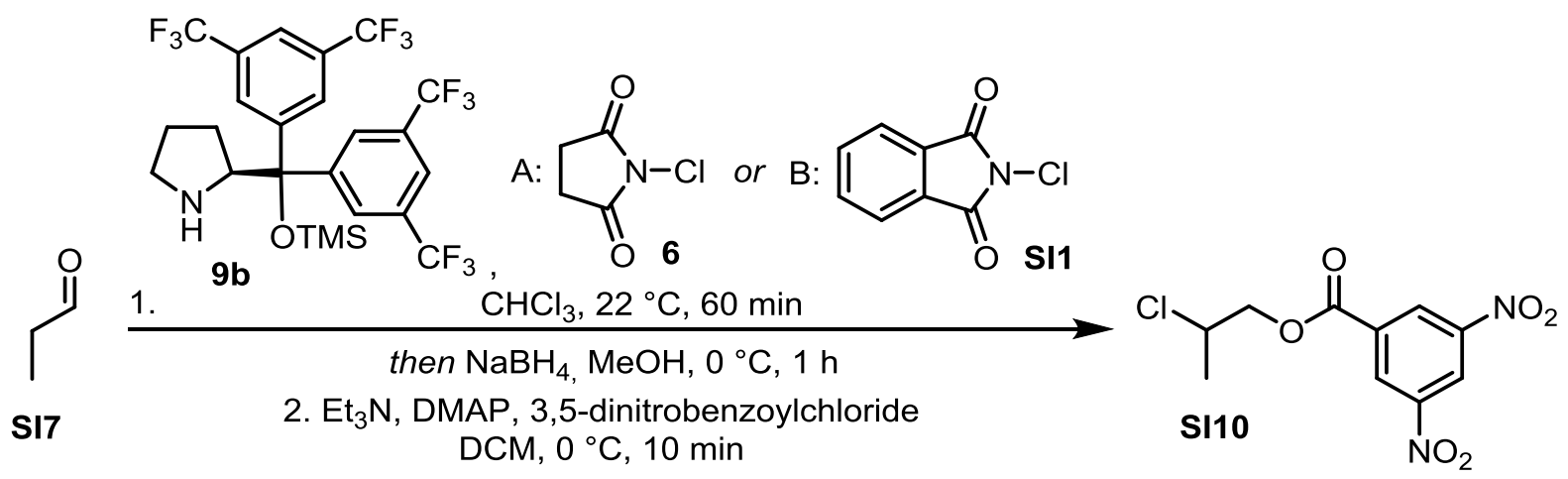

Propanal $\left(0.359 \mathrm{~mL}, 5.00 \mathrm{mmol}, 1.0\right.$ equiv) was dissolved in $\mathrm{CHCl}_{3}(10.0 \mathrm{~mL})$ at $22{ }^{\circ} \mathrm{C}$. Catalyst $9 \mathbf{b}$ $(0.299 \mathrm{~g}, 0.500 \mathrm{mmol}, 10 \mathrm{~mol} \%)$ and A: NCS $(0.734 \mathrm{~g}, 5.50 \mathrm{mmol}, 1.1$ equiv) or B: NCP (0.999 g, $5.50 \mathrm{mmol}, 1.1$ equiv) were added subsequently and the reaction mixture was stirred for $60 \mathrm{~min}$ at the same temperature. After the reaction was cooled to $0{ }^{\circ} \mathrm{C}, \mathrm{MeOH}(3.3 \mathrm{~mL})$ and $\mathrm{NaBH}_{4}(0.473 \mathrm{~g}$, $12.5 \mathrm{mmol}, 2.5$ equiv) were added and the mixture was stirred for $60 \mathrm{~min}$ at the same temperature. The reaction was quenched with aqueous saturated $\mathrm{NH}_{4} \mathrm{Cl}(10 \mathrm{~mL})$, the aqueous phase was extracted with dichloromethane $(3 \times 15 \mathrm{~mL})$ and the combined organic phases were dried over $\mathrm{NaSO}_{4}$. The solvent was removed under reduced pressure (product is volatile!) and the crude product was dissolved in dichloromethane $(10 \mathrm{~mL})$. The solution was cooled to $0^{\circ} \mathrm{C}$ and DMAP $(30.5 \mathrm{mg}, 0.250 \mathrm{mmol}, 0.1$ equiv) and 3,5-dinitrobenzoylchloride $\left(0.749 \mathrm{~g}, 3.25 \mathrm{mmol}, 1.3\right.$ equiv) were added subsequently. $\mathrm{Et}_{3} \mathrm{~N}(0.589$ $\mathrm{mL}, 4.25 \mathrm{mmol}, 1.7$ equiv) was added dropwise and the reaction mixture was stirred for $10 \mathrm{~min}$ at $0^{\circ} \mathrm{C}$. The reaction was quenched with aqueous saturated $\mathrm{NaHCO}_{3}(10 \mathrm{~mL})$, the aqueous phase was extracted with dichloromethane $(3 \times 15 \mathrm{~mL})$ and the combined organic phases were dried over $\mathrm{NaSO}_{4}$. The solvent was removed under reduced pressure and the crude product was purified by column chromatography (Silica, pentane/EtOAc 10:1).

A: Ester SI10 ( $0.073 \mathrm{~g}, 0.253 \mathrm{mmol}, 5 \%, 5 \%$ ee for the $(R)$-enantiomer) was obtained as a yellow oil. B: Ester SI10 (0.050 g, $0.173 \mathrm{mmol}, 3 \%, 14 \%$ ee for the $(R)$-enantiomer) was obtained as a yellow oil.

${ }^{1} \mathrm{H}-\mathrm{NMR}\left(\mathrm{CDCl}_{3}, 600 \mathrm{MHz}\right) \delta=9.23(\mathrm{t}, J=2.1 \mathrm{~Hz}, 1 \mathrm{H}), 9.16(\mathrm{~d}, J=2.1 \mathrm{~Hz}, 2 \mathrm{H}), 4.57(\mathrm{dd}, J=11.6,5.1$ $\mathrm{Hz}, 1 \mathrm{H}), 4.53(\mathrm{dd}, J=11.6,7.0 \mathrm{~Hz}, 1 \mathrm{H}), 4.44-4.31(\mathrm{~m}, 1 \mathrm{H}), 1.64(\mathrm{~d}, J=6.7 \mathrm{~Hz}, 3) \mathrm{ppm}$.

${ }^{13} \mathrm{C}-\mathrm{NMR}\left(\mathrm{CDCl}_{3}, 151 \mathrm{MHz}\right) \delta=162.2,148.8,133.4,129.6,122.8,70.5,53.6,21.5 . \mathrm{ppm}$. 
The low ee and the inverted absolute configuration (compared to isovaleraldehyde) for propanal as a substrate may be due to the lower energy barrier for the rotation around the $\mathrm{C}-\mathrm{N}$-bond of the enamine compared to other substrates carrying more bulky alkyl-substituents (isovaleraldehyde). ${ }^{52}$

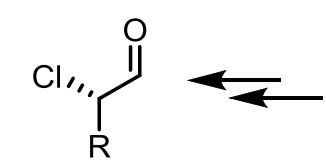

(S)-chloroaldehyde

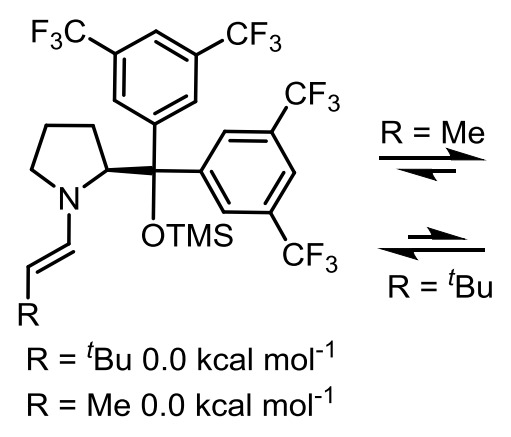

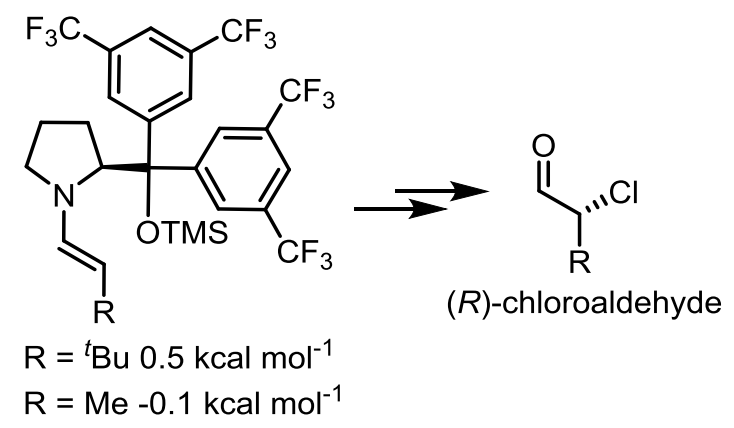

Figure SI-49. Relative free energies $(\Delta G)$ of isovaleralydehyde- and propanal-derived enamines according to Jørgensen et al. ${ }^{52}$

By simply replacing the substrate (isovaleraldehyde $\rightarrow$ propanal) we demonstrated that the correlation between the ratio of interconverting aminals $(68: 32 ; 36 \%$ excess) and the enantiomeric ratio of the chloroaldehyde product $(57: 43 ; 14 \%$ excess) is no longer present. 
(S)-2-chloropropyl-3,5-dinitrobenzoate ((S)-SI10)

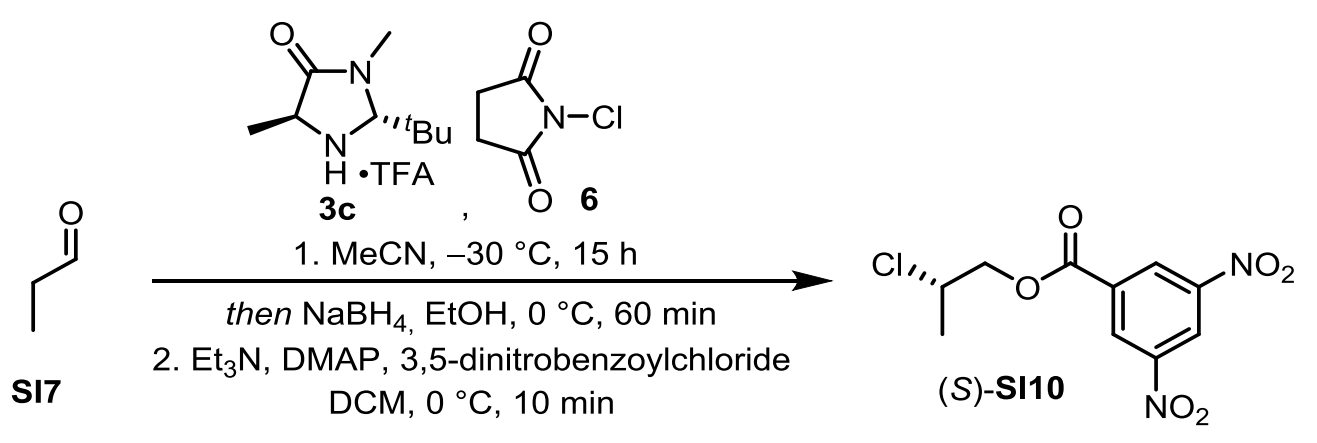

Propanal ( $0.143 \mathrm{~mL}, 2.00 \mathrm{mmol}, 1.0$ equiv) was dissolved in $\mathrm{MeCN}(8.0 \mathrm{~mL})$ and cooled to $-30{ }^{\circ} \mathrm{C}$. Catalyst 3c·TFA ( $0.114 \mathrm{~g}, 0.400 \mathrm{mmol}, 20 \mathrm{~mol} \%)$ and NCS $(0.320 \mathrm{~g}, 2.40 \mathrm{mmol}, 1.2$ equiv) were added subsequently and the reaction mixture was stirred for $15 \mathrm{~h}$ at the same temperature. After the reaction was warmed to $0{ }^{\circ} \mathrm{C}, \mathrm{MeOH}(3.0 \mathrm{~mL})$ and $\mathrm{NaBH}_{4}(0.189 \mathrm{~g}, 5.00 \mathrm{mmol}, 2.5$ equiv) were added and the mixture was stirred for $60 \mathrm{~min}$ at the same temperature. The reaction was quenched with aqueous saturated $\mathrm{NH}_{4} \mathrm{Cl}(10 \mathrm{~mL})$, the aqueous phase was extracted with dichloromethane $(3 \times 15 \mathrm{~mL})$ and the combined organic phases were dried over $\mathrm{NaSO}_{4}$. The solvent was removed under reduced pressure (product is volatile!) and the crude product was dissolved in dichloromethane (8 $\mathrm{mL})$. The solution was cooled to $0^{\circ} \mathrm{C}$ and DMAP (24.4 mg, $0.200 \mathrm{mmol}, 0.1$ equiv) and 3,5-dinitrobenzoylchloride $(0.599 \mathrm{~g}$, $2.60 \mathrm{mmol}, 1.3$ equiv) were added subsequently. $\mathrm{Et}_{3} \mathrm{~N}(0.474 \mathrm{~mL}, 3.40 \mathrm{mmol}, 1.7$ equiv) was added dropwise and the reaction mixture was stirred for $10 \mathrm{~min}$ at $0{ }^{\circ} \mathrm{C}$. The reaction was quenched with aqueous saturated $\mathrm{NaHCO}_{3}(10 \mathrm{~mL})$, the aqueous phase was extracted with dichloromethane $(3 \times 10$ $\mathrm{mL}$ ) and the combined organic phases were dried over $\mathrm{NaSO}_{4}$. The solvent was removed under reduced pressure and the crude product was purified by column chromatography (Silica, pentane/EtOAc 10:1). Ester (S)-SI10 (0.108 g, $0.374 \mathrm{mmol}, 19 \%, 94 \% \mathrm{ee})$ was obtained as a yellowish oil.

The ${ }^{1} \mathrm{H}-\mathrm{NMR}$ spectrum is in accordance with the previous experiment. 
(R)-2-chloropropyl-3,5-dinitrobenzoate ((R)-SI10)

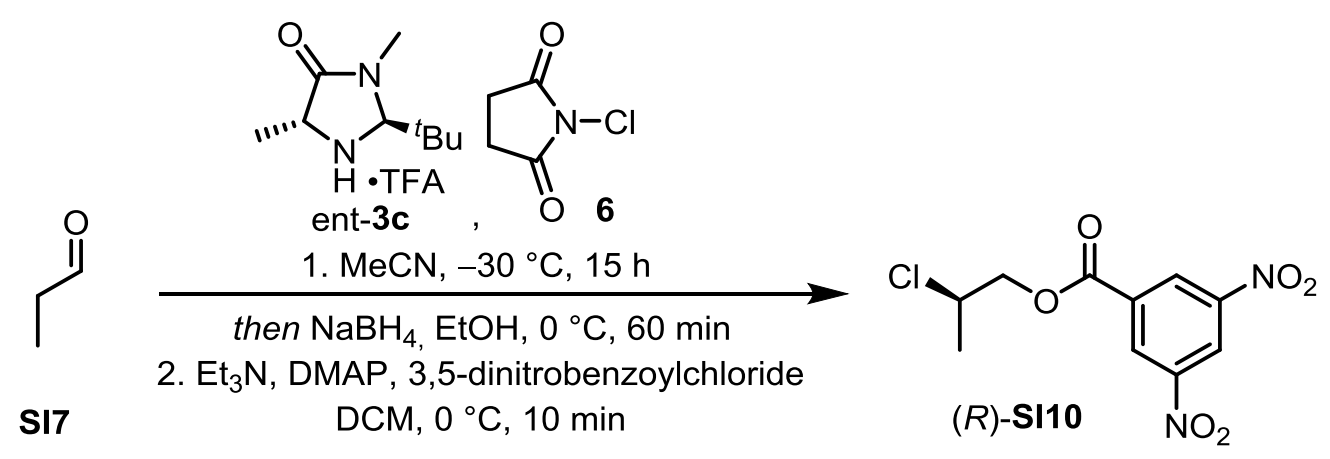

Propanal $\left(0.143 \mathrm{~mL}, 2.00 \mathrm{mmol}, 1.0\right.$ equiv) was dissolved in $\mathrm{MeCN}(8.0 \mathrm{~mL})$ and cooled to $-30{ }^{\circ} \mathrm{C}$. Catalyst ent-3c·TFA $(0.114 \mathrm{~g}, 0.400 \mathrm{mmol}, 20 \mathrm{~mol} \%)$ and NCS $(0.320 \mathrm{~g}, 2.40 \mathrm{mmol}, 1.2$ equiv) were added subsequently and the reaction mixture was stirred for $15 \mathrm{~h}$ at the same temperature. After the reaction was warmed to $0^{\circ} \mathrm{C}, \mathrm{MeOH}(3.0 \mathrm{~mL})$ and $\mathrm{NaBH}_{4}(0.189 \mathrm{~g}, 5.00 \mathrm{mmol}, 2.5$ equiv) were added and the mixture was stirred for $60 \mathrm{~min}$ at the same temperature. The reaction was quenched with aqueous saturated $\mathrm{NH}_{4} \mathrm{Cl}(10 \mathrm{~mL})$, the aqueous phase was extracted with dichloromethane $(3 \times 15 \mathrm{~mL})$ and the combined organic phases were dried over $\mathrm{NaSO}_{4}$. The solvent was removed under reduced pressure (product is volatile!) and the crude product was redissolved in dichloromethane $(8 \mathrm{~mL})$. The solution was cooled to $0{ }^{\circ} \mathrm{C}$ and DMAP $(24.4 \mathrm{mg}, 0.200 \mathrm{mmol}, 0.1$ equiv) and 3,5-dinitrobenzoylchloride ( $0.599 \mathrm{~g}, 2.60 \mathrm{mmol}, 1.3$ equiv) were added subsequently. $\mathrm{Et}_{3} \mathrm{~N}(0.474 \mathrm{~mL}, 3.40 \mathrm{mmol}, 1.7$ equiv) was added dropwise and the reaction mixture was stirred for $10 \mathrm{~min}$ at $0{ }^{\circ} \mathrm{C}$. The reaction was quenched with aqueous saturated $\mathrm{NaHCO}_{3}(10 \mathrm{~mL})$, the aqueous phase was extracted with dichloromethane $(3 \times 10$ $\mathrm{mL}$ ) and the combined organic phases were dried over $\mathrm{NaSO}_{4}$. The solvent was removed under reduced pressure and the crude product was purified by column chromatography (Silica, pentane/EtOAc 10:1). Ester $(R)$-SI10 (0.109 g, $0.378 \mathrm{mmol}, 19 \%, 94 \%$ ee) was obtained as a yellowish oil.

The ${ }^{1} \mathrm{H}-\mathrm{NMR}$ spectrum is in accordance with the previous experiment. 


\subsubsection{Decomposition and Deuterium Incorporation Studies}

\section{Decomposition of Aminal SI6 (1)}

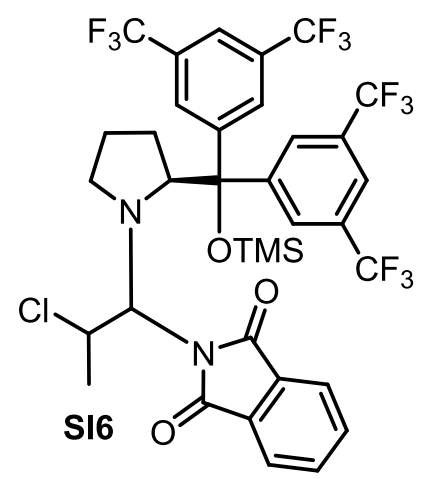

$$
\underset{\text { then } \mathrm{NaBH}_{4}, \mathrm{MeOH}, 0^{\circ} \mathrm{C}, 60 \text { min }}{\stackrel{\mathrm{H}_{2} \mathrm{O} \text { (2 equiv), TFA (2 equiv) }}{\mathrm{CHCl}_{3}, 22^{\circ} \mathrm{C}}}
$$

$\mathrm{H}_{2} \mathrm{O}(72.1 \mu \mathrm{L}, 4.00 \mathrm{mmol}, 2.0$ equiv) and TFA ( $308 \mu \mathrm{L}, 4.00 \mathrm{mmol}, 2.0$ equiv) were added subsequently to a solution of aminal $\mathbf{S I 6}\left(1.64 \mathrm{~g}, 2.00 \mathrm{mmol}, 1.0\right.$ equiv) in $\mathrm{CHCl}_{3}(100 \mathrm{~mL})$ at $22{ }^{\circ} \mathrm{C}$. Aliquots $(10 \mathrm{~mL})$ were taken from the reaction mixture after defined points of time $(1 \mathrm{~min}, 5 \mathrm{~min}, 10 \mathrm{~min}, 20 \mathrm{~min}, 30 \mathrm{~min}$, $60 \mathrm{~min}, 2 \mathrm{~h}, 98 \mathrm{~h}$ ) and added to a solution of $\mathrm{NaBH}_{4}(75.7 \mathrm{mg}, 2.00 \mathrm{mmol}, 10.0$ equiv) in $\mathrm{MeOH}(4.0 \mathrm{~mL})$. After 60 min the reactions were quenched with aqueous saturated $\mathrm{NH}_{4} \mathrm{Cl}$. The aqueous phase was extracted with dichloromethane $(3 \times 5 \mathrm{~mL})$, the combined organic phases were dried over $\mathrm{Na}_{2} \mathrm{SO}_{4}$ and the solvent was partially removed under reduced pressure (product is volatile!). The enantiomeric excess of the crude products was determined by chiral GC.

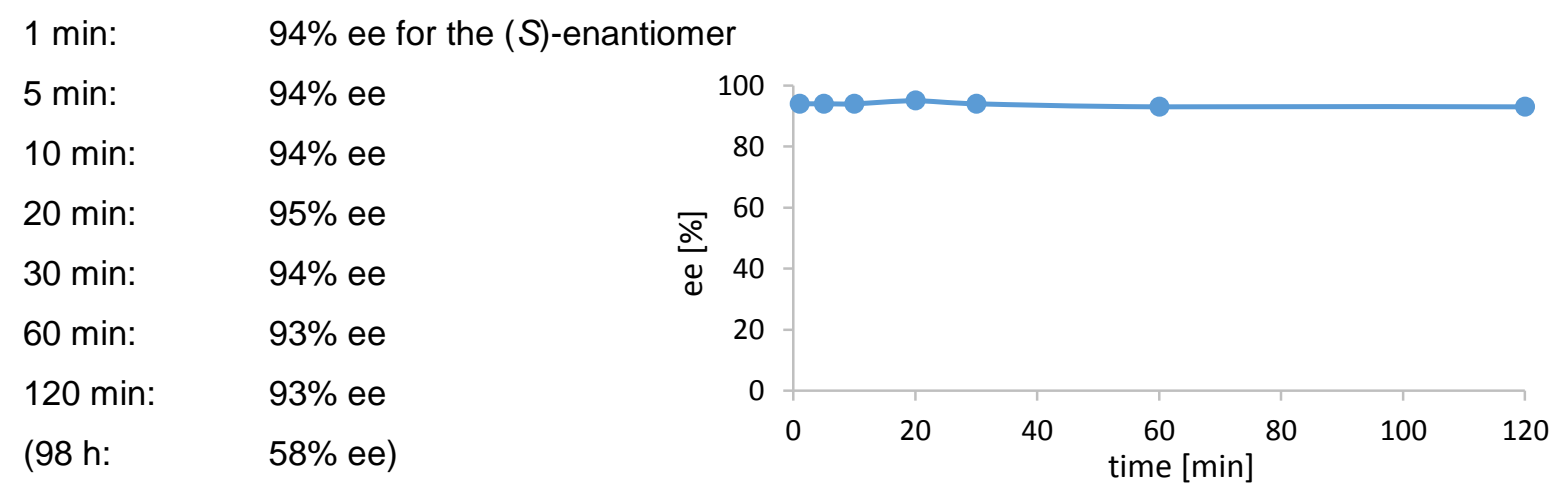

Figure SI-50. The acid-catalyzed decomposition of aminal SI6 generates almost enantiopure (S)chloroaldehyde product.

As mentioned before (decomposition of aminal 14, synthesis of aminal SI6) it was possible to obtain aminal SI6 without any detectable aldehyde or chloroaldehyde impurities. The decomposition experiment shows that aminal SI6 decomposes to almost enantiopure chloroaldehyde (S)-SI8. In contrast, the mechanism of the Curtin-Hammett scenario predicts the existence of both enantiomers in the same ratio as the interconverting species (ratio of signal sets at $-54^{\circ} \mathrm{C}=68: 32 \rightarrow 36 \%$ ee). 


\section{2-Chloropropan-1-ol (rac-SI9)}

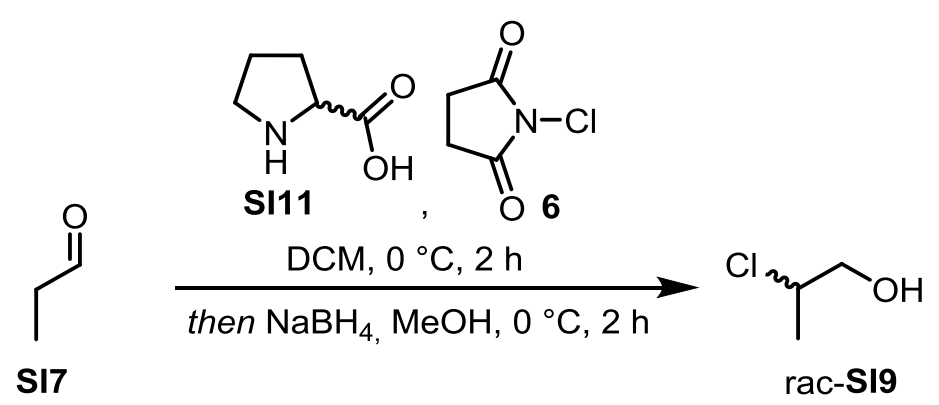

Racemic proline (SI11) (0.104 g, $0.900 \mathrm{mmol}, 0.3$ equiv) and NCS ( $0.401 \mathrm{~g}, 3.00 \mathrm{mmol}, 1.0$ equiv) were added successively to an ice-cold solution of propanal $(0.215 \mathrm{~mL}, 3.00 \mathrm{mmol}, 1.0$ equiv) in dichloromethane $(8.1 \mathrm{~mL})$. After stirring for $2 \mathrm{~h}$ at $0{ }^{\circ} \mathrm{C}, \mathrm{MeOH}(4.1 \mathrm{~mL})$ and $\mathrm{NaBH}_{4}(0.284 \mathrm{~g}, 7.50 \mathrm{mmol}$, 2.5 equiv) were added and the solution was stirred for another $2 \mathrm{~h}$ at $0^{\circ} \mathrm{C}$. The reaction was quenched with aqueous saturated $\mathrm{NH}_{4} \mathrm{Cl}(5 \mathrm{~mL})$, the aqueous phase was extracted with dichloromethane $(3 \times 10$ $\mathrm{mL}$ ) and the combined organic phases were dried over $\mathrm{NaSO}_{4}$. The solvent was removed under reduced pressure (product is volatile!) and the crude product was purified by column chromatography (Silica, pentane/Et $\left.{ }_{2} \mathrm{O} 3: 1\right)$. Alcohol rac-SI9 (35.0 mg, $0.370 \mathrm{mmol}, 12 \%$; product is volatile!) was obtained as a colorless oil.

The ${ }^{1} \mathrm{H}-\mathrm{NMR}$ spectrum is in accordance with the literature. ${ }^{53}$

${ }^{1} \mathrm{H}-\mathrm{NMR}\left(\mathrm{CD}_{2} \mathrm{Cl}_{2}, 400 \mathrm{MHz}\right) \delta=4.13(\mathrm{pdd}, J=6.8,4.2,1.0 \mathrm{~Hz}, 1 \mathrm{H}), 3.71(\mathrm{dd}, J=11.9,4.0 \mathrm{~Hz}, 1 \mathrm{H})$, $3.60(\mathrm{dd}, J=12.0,7.0 \mathrm{~Hz}, 1 \mathrm{H}), 2.29(\mathrm{~s}, 1 \mathrm{H}), 1.48(\mathrm{~d}, J=6.7 \mathrm{~Hz}, 3 \mathrm{H}) \mathrm{ppm}$. 


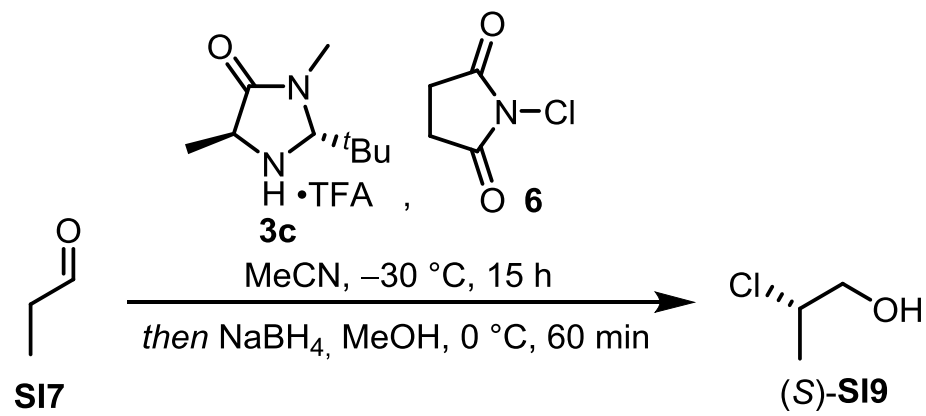

Propanal $\left(0.143 \mathrm{~mL}, 2.00 \mathrm{mmol}, 1.0\right.$ equiv) was dissolved in $\mathrm{MeCN}(8.0 \mathrm{~mL})$ and cooled to $-30^{\circ} \mathrm{C}$. Catalyst 3c·TFA ( $0.114 \mathrm{~g}, 0.400 \mathrm{mmol}, 20 \mathrm{~mol} \%)$ and NCS ( $0.320 \mathrm{~g}, 2.40 \mathrm{mmol}, 1.2$ equiv) were added subsequently and the reaction mixture was stirred for $15 \mathrm{~h}$ at the same temperature. After the reaction was warmed to $0{ }^{\circ} \mathrm{C}, \mathrm{MeOH}(3.0 \mathrm{~mL})$ and $\mathrm{NaBH}_{4}(0.189 \mathrm{~g}, 5.00 \mathrm{mmol}, 2.5$ equiv) were added and the mixture was stirred for $60 \mathrm{~min}$ at the same temperature. The reaction was quenched with aqueous saturated $\mathrm{NH}_{4} \mathrm{Cl}(10 \mathrm{~mL})$, the aqueous phase was extracted with dichloromethane $(3 \times 15 \mathrm{~mL})$ and the combined organic phases were dried over $\mathrm{NaSO}_{4}$. The solvent was removed under reduced pressure (product is volatile!) and the crude product ( $99 \%$ ee; determined by chiral GC) was used without further purification.

(R)-2-chloropropan-1-ol ((R)-SI9)

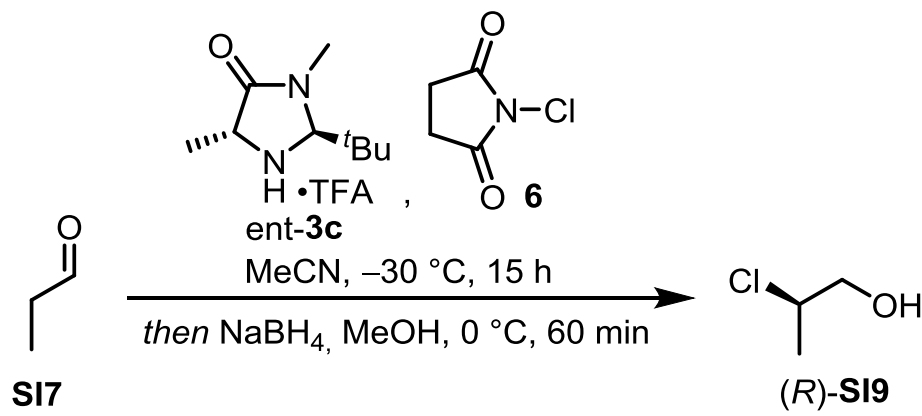

Propanal $\left(0.143 \mathrm{~mL}, 2.00 \mathrm{mmol}, 1.0\right.$ equiv) was dissolved in $\mathrm{MeCN}(8.0 \mathrm{~mL})$ and cooled to $-30{ }^{\circ} \mathrm{C}$. Catalyst ent-3c·TFA $(0.114 \mathrm{~g}, 0.400 \mathrm{mmol}, 20 \mathrm{~mol} \%)$ and NCS $(0.320 \mathrm{~g}, 2.40 \mathrm{mmol}, 1.2$ equiv) were added subsequently and the reaction mixture was stirred for $15 \mathrm{~h}$ at the same temperature. After the reaction was warmed to $0^{\circ} \mathrm{C}, \mathrm{MeOH}(3.0 \mathrm{~mL})$ and $\mathrm{NaBH}_{4}(0.189 \mathrm{~g}, 5.00 \mathrm{mmol}, 2.5$ equiv) were added and the mixture was stirred for $60 \mathrm{~min}$ at the same temperature. The reaction was quenched with aqueous saturated $\mathrm{NH}_{4} \mathrm{Cl}(10 \mathrm{~mL})$, the aqueous phase was extracted with dichloromethane $(3 \times 15 \mathrm{~mL})$ and the combined organic phases were dried over $\mathrm{NaSO}_{4}$. The solvent was removed under reduced pressure (product is volatile!) and the crude product ( $>99 \%$ ee; determined by chiral GC) was used without further purification. 
Decomposition of Aminal SI6 (2)

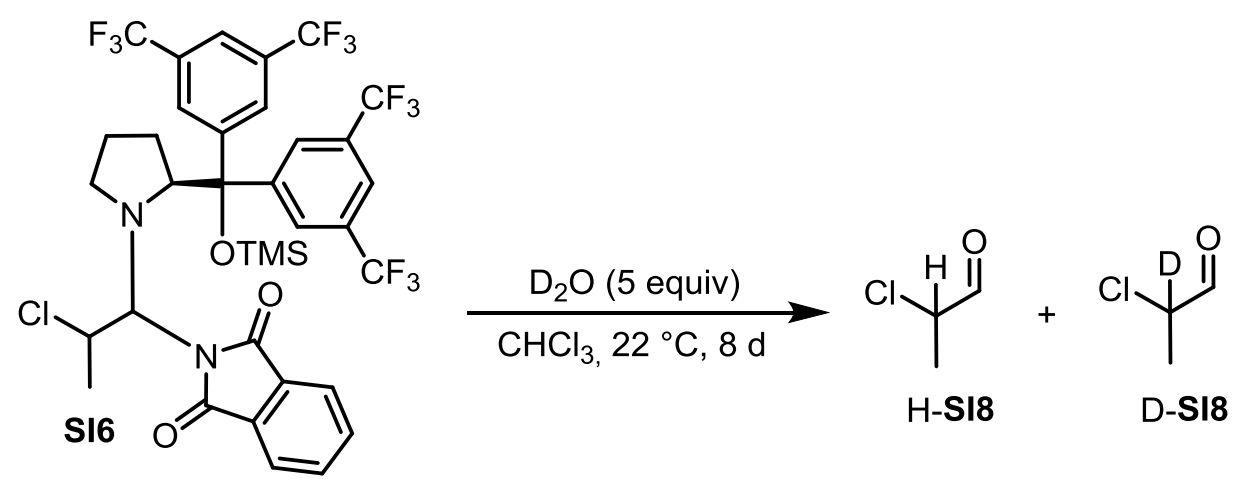

$0.60 \mathrm{~mL}$ of a stock solution $\left(\mathrm{CDCl}_{3}(6.0 \mathrm{~mL}), \mathrm{D}_{2} \mathrm{O}(11.8 \mu \mathrm{L})\right.$ was added to a NMR-tube containing aminal SI6 (9.67 mg, $11.8 \mu \mathrm{mol}, 1.0$ equiv). The NMR-tube was tightly sealed and ${ }^{1} \mathrm{H}$-NMR spectra were recorded over a period of 8 days. Deuterium incorporation and conversion was qualitatively evaluated by ${ }^{1} \mathrm{H}-\mathrm{NMR}$ spectroscopy.

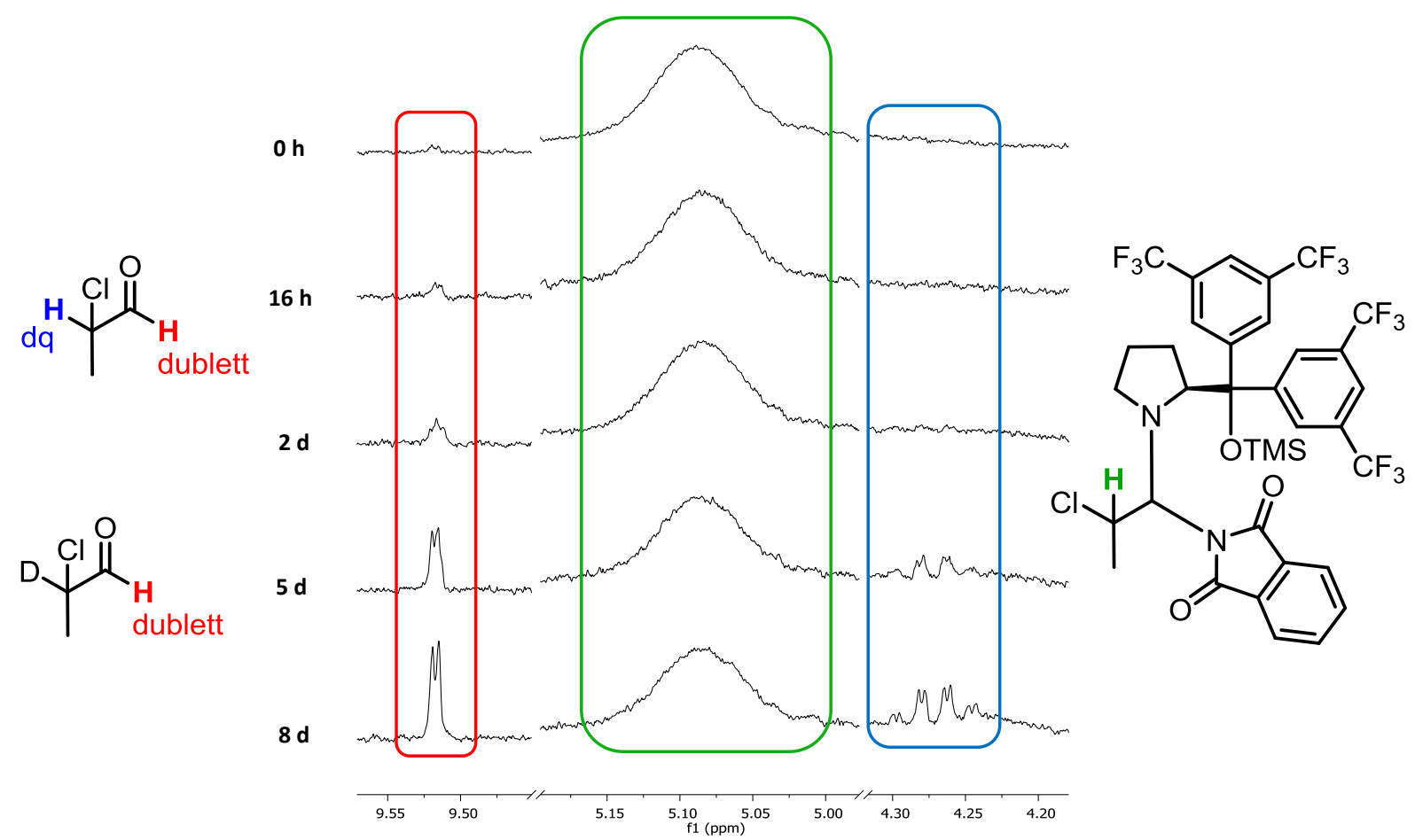

Figure SI-51. ${ }^{1} \mathrm{H}-\mathrm{NMR}$ spectra of the ongoing decomposition reaction ( 5 equiv of $\mathrm{D}_{2} \mathrm{O}$ ) of aminal SI6 allows a qualitative evaluation of the deuterium incorporation into the $\alpha$-chloroaldehydes.

In accordance to the previous $\mathrm{D}_{2} \mathrm{O}$-mediated hydrolysis experiment of aminal 14 , significant deuterium incorporation cannot be detected. 


\section{FURTHER RESULTS}

\section{Deuterium incorporation experiment}<smiles>[2H]C(Cl)(CO)C([2H])(Cl)CO</smiles>

$\mathrm{D}_{2} \mathrm{O}(98.0 \mu \mathrm{L}, 6.02 \mathrm{mmol}, 2$ equiv), TFAd 1 ( $464 \mu \mathrm{L}, 6.02 \mathrm{mmol}, 2.0$ equiv), the catalyst $(0.602 \mathrm{mmol}$, $20 \mathrm{~mol} \%)$ and NCS ( $0.442 \mathrm{~g}, 3.31 \mathrm{mmol}, 1.1$ equiv) were added subsequently to a solution of distilled hydrocinnamic aldehyde $(0.400 \mathrm{~mL}, 3.01 \mathrm{mmol}, 1.0$ equiv) in $\mathrm{MeCN}$ (catalyst $3 \mathbf{c}$ and ent-3b) or dichloromethane (catalyst $9 \mathrm{c})\left(12 \mathrm{~mL}\right.$ ) at $20^{\circ} \mathrm{C}$ or $-30^{\circ} \mathrm{C}$. After $18 \mathrm{~h}$ the reaction was adjusted to $0{ }^{\circ} \mathrm{C}$. $\mathrm{EtOH}(4.00 \mathrm{~mL})$ and $\mathrm{NaBH}_{4}(0.569 \mathrm{~g}, 15.0 \mathrm{mmol}, 5.0$ equiv) were added and the reaction mixture was stirred for $60 \mathrm{~min}$ at the same temperature. The reaction was quenched with aqueous saturated $\mathrm{NH}_{4} \mathrm{Cl}$, extracted with EtOAc $(3 \times 10 \mathrm{~mL})$ and the combined organic phases were dried over $\mathrm{Na}_{2} \mathrm{SO}_{4}$. The solvent was removed under reduced pressure and the crude product was purified by column chromatography (Silica, 10:1 to 5:1 pentane/EtOAc). Deuterium incorporation was qualitatively evaluated by comparison of the ${ }^{13} \mathrm{C}-\mathrm{NMR}$ spectra and quantified by ${ }^{1} \mathrm{H}-\mathrm{NMR}$ spectroscopy

Example:
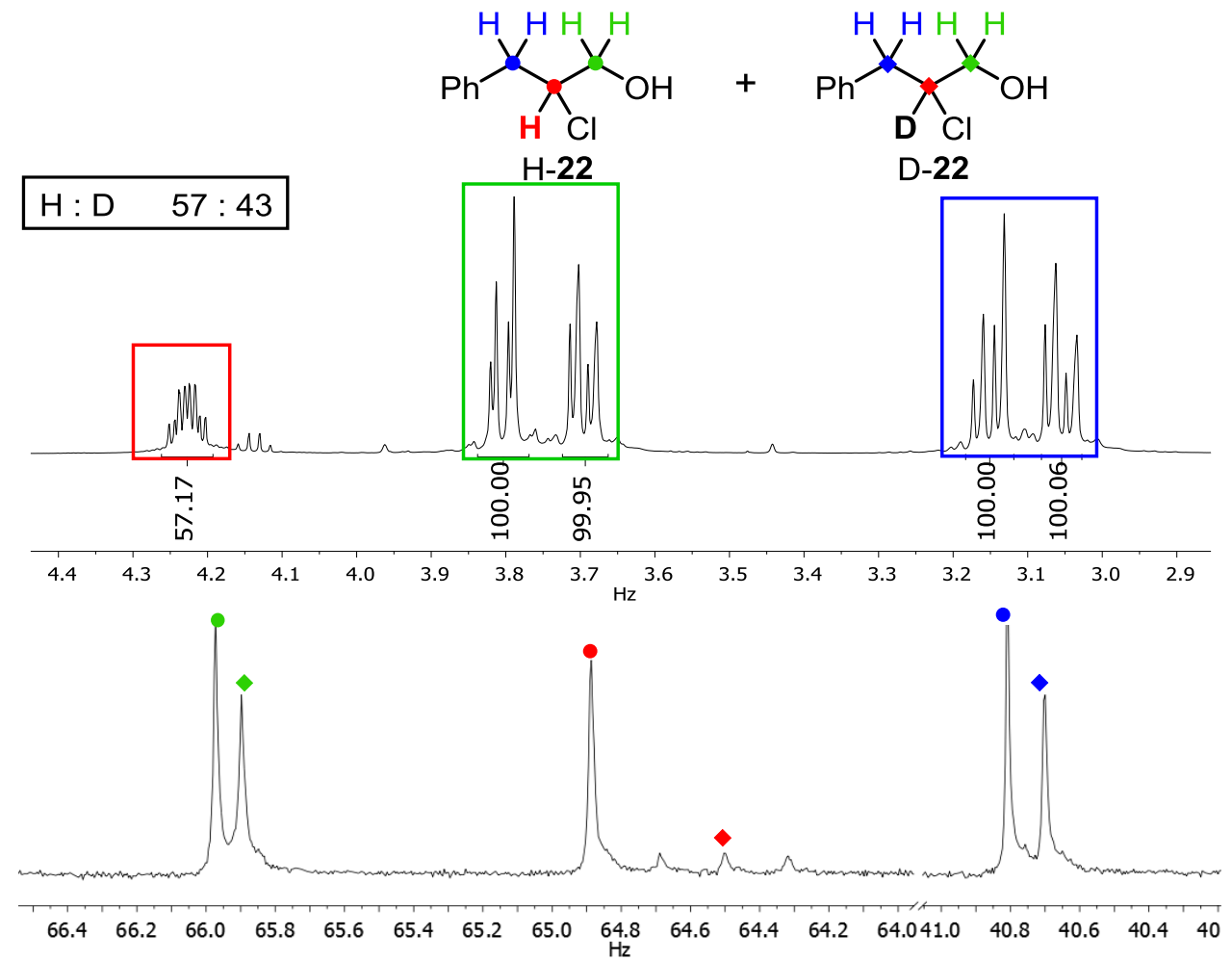

Figure SI-52. Exemplary ${ }^{1} \mathrm{H}$ - (top) and ${ }^{13} \mathrm{C}-\mathrm{NMR}$ (bottom) spectrum for the evaluation of deuterium incorporation. 
Table SI-41. Quantification of deuterium incorporation under various conditions

\begin{tabular}{l|c|c|c|c} 
& solvent & T [ $\left.{ }^{\circ} \mathbf{C}\right]$ & H : D & t \\
\hline & MeCN & 20 & $90: 10$ & $18 \mathrm{~h}$ \\
\cline { 2 - 5 } & MeCN & -30 & $100: 0$ & $18 \mathrm{~h}$ \\
\hline
\end{tabular}

[a] no TFA- $d_{1}, 12 \%$ conversion, $59 \%$ ee 


\section{Comparison of Different Catalysts}

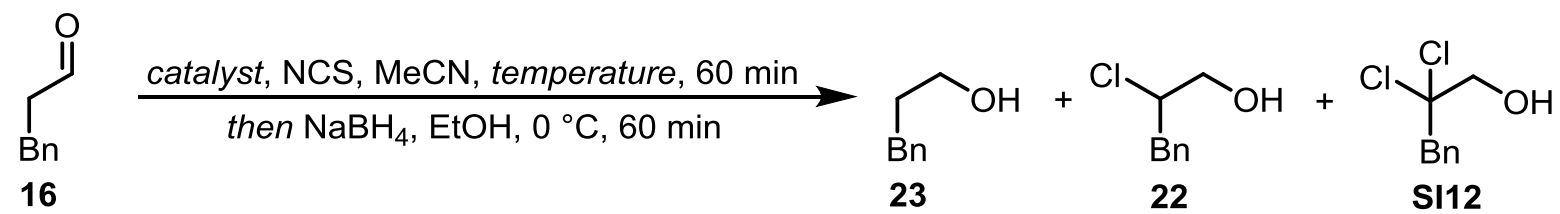

The catalyst $(0.150 \mathrm{mmol}, 20 \mathrm{~mol} \%)$ and NCS $(0.111 \mathrm{~g}, 0.828 \mathrm{mmol}, 1.1$ equiv) were added successively to a tempered $\left(22^{\circ} \mathrm{C}\right.$ or $\left.0{ }^{\circ} \mathrm{C}\right)$ solution of hydrocinnamic aldehyde $(0.100 \mathrm{~mL}, 0.752 \mathrm{mmol}, 1.0$ equiv) and $\mathrm{MeCN}(3.0 \mathrm{~mL})$. The reaction was stirred for $60 \mathrm{~min}$ at the indicated temperature and then cooled to $0{ }^{\circ} \mathrm{C}$. EtOH $(1.0 \mathrm{~mL})$ and $\mathrm{NaBH}_{4}(71.2 \mathrm{mg}, 1.88 \mathrm{mmol}, 2.5$ equiv) were added and the reaction mixture was stirred for further $60 \mathrm{~min}$ at $0{ }^{\circ} \mathrm{C}$. The reaction was quenched with aqueous saturated $\mathrm{NH}_{4} \mathrm{Cl}$, the aqueous phase was extracted with EtOAc $(3 \times 5 \mathrm{~mL})$, the combined organic phases were dried over $\mathrm{Na}_{2} \mathrm{SO}_{4}$ and the solvent was removed under reduced pressure. The crude product was purified by column chromatography ( $\mathrm{SiO}_{2}$, pentane/EtOAc 10:1 to 5:1) and the pure alcohols 23, 22 and Sl12 were obtained as colorless oils. The enantiomeric excesses of the chloroalcohols $\mathbf{2 2}$ were determined by chiral HPLC.

Table SI-42. Comparison of different pyrrolidine- and imidazolidinone-based organocatalysts in the $\alpha$ chlorination of 16

\begin{tabular}{|c|c|c|c|c|c|c|}
\hline & temperature & $23[\%]$ & $22[\%]$ & SI12 [\%] & sum [\%] & ee (22) [\%] \\
\hline \multirow[t]{2}{*}{ pyrrolidine (SI13) } & $22^{\circ} \mathrm{C}$ & 26 & 17 & 19 & 62 & 0 \\
\hline & $0^{\circ} \mathrm{C}$ & 42 & 16 & 10 & 68 & 0 \\
\hline \multirow[t]{2}{*}{ L-proline $((S)-$ SI11) } & $22^{\circ} \mathrm{C}$ & 26 & 32 & 0 & 58 & 1 \\
\hline & $0^{\circ} \mathrm{C}$ & 49 & 14 & 0 & 63 & 9 \\
\hline \multirow[t]{2}{*}{ L-prolinamide (SI14) } & $22^{\circ} \mathrm{C}$ & 37 & 20 & 0 & 57 & 29 \\
\hline & $0^{\circ} \mathrm{C}$ & 46 & 8 & 0 & 54 & 44 \\
\hline \multirow[t]{2}{*}{ Jørgensen-Hayashi catalyst (9c) } & $22^{\circ} \mathrm{C}$ & 0 & 3 & 43 & 46 & 11 \\
\hline & $0^{\circ} \mathrm{C}$ & 0 & 17 & 25 & 42 & 16 \\
\hline in dichloromethane & $0^{\circ} \mathrm{C}$ & 0 & 33 & 13 & 46 & 69 \\
\hline \multirow[t]{2}{*}{ diphenylyrrolidine catalyst (SI15) } & $22^{\circ} \mathrm{C}$ & 0 & 18 & 22 & 40 & 97 \\
\hline & $0^{\circ} \mathrm{C}$ & 0 & 24 & 8 & 32 & 98 \\
\hline \multirow[t]{2}{*}{$1^{\text {st }}$ gen. MacMillan catalyst $(\mathbf{3 a})$} & $22^{\circ} \mathrm{C}$ & 0 & 51 & 23 & 74 & 36 \\
\hline & $0^{\circ} \mathrm{C}$ & 17 & 48 & 18 & 83 & 70 \\
\hline \multirow[t]{2}{*}{$2^{\text {nd }}$ gen. MacMillan catalyst (ent-3b) } & $22^{\circ} \mathrm{C}$ & 39 & 26 & 9 & 74 & 28 \\
\hline & $0^{\circ} \mathrm{C}$ & 53 & 27 & 2 & 82 & 48 \\
\hline \multirow[t]{2}{*}{$3^{\text {rd }}$ gen. MacMillan catalyst $(\mathbf{3 c})$} & $22^{\circ} \mathrm{C}$ & 2 & 64 & 0 & 66 & 91 \\
\hline & $0^{\circ} \mathrm{C}$ & 24 & 54 & 0 & 78 & 96 \\
\hline
\end{tabular}




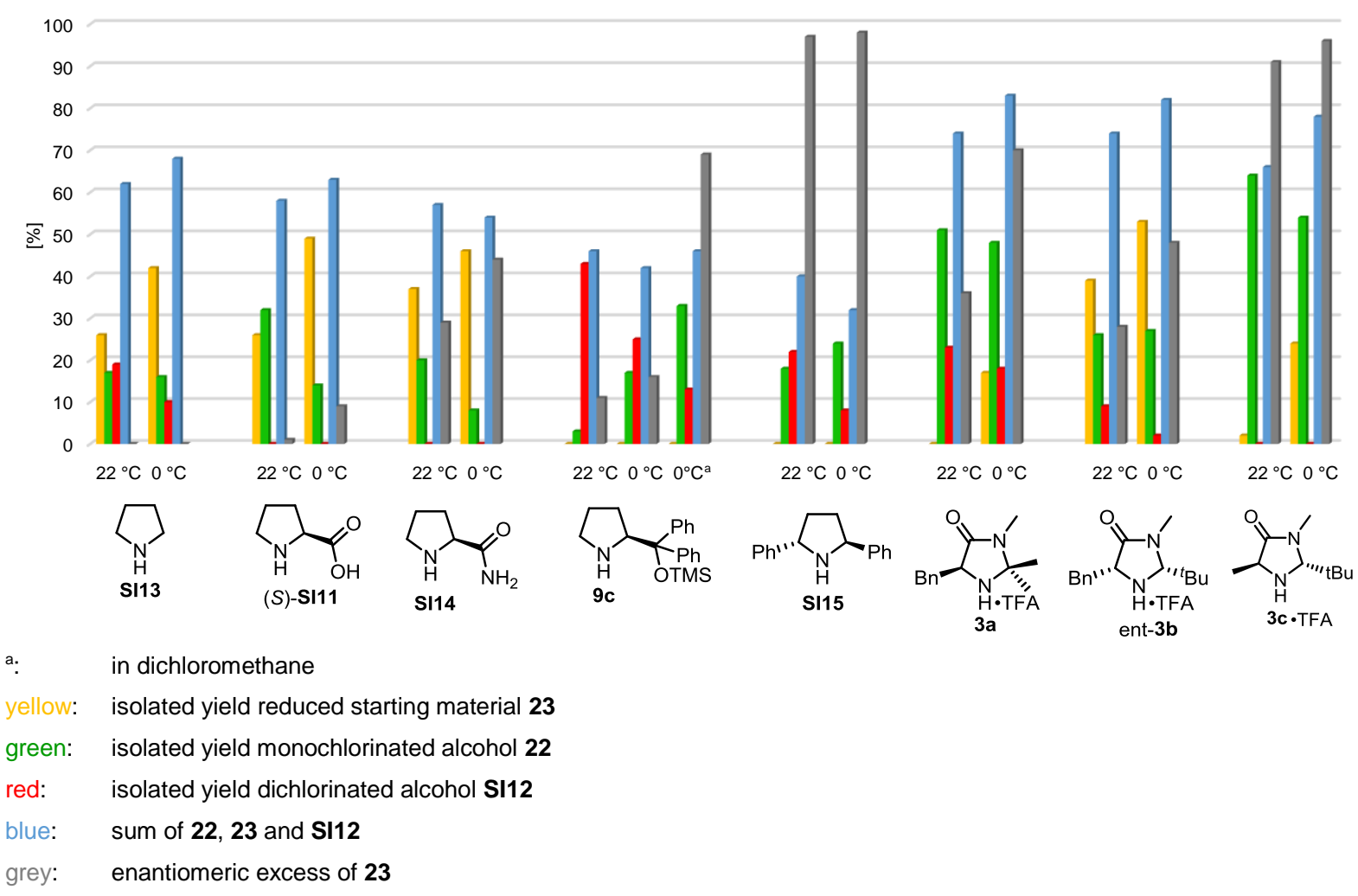

Figure SI-53. Comparison of the catalytic performance between numerous pyrrolidine- and imidazolidinone-based organocatalysts.

The comparison of different pyrrolidine- and imidazolidinone-based organocatalysts reveals some major differences in the catalytic performance. Dichlorination can be completely suppressed when catalysts (S)-SI11, SI14 and 3c were used. Raising the temperature enhances the amount of dichlorinated byproduct for all other catalysts. Excellent enantioselectivities under these conditions were exclusively achieved with catalyst SI15 and 3c. The C2 or C2-like symmetry of both catalysts might play a role here. The sum of 23, 22 and SI12 can be considered as a parameter for the selectivity of the catalytic system. Low values are indicative for ongoing side-reactions, whereas high values reflect a "clean" reaction with less side products. Concerning the formation of side products, imidazolidinone-based catalysts perform better than pyrrolidine-based catalysts under these conditions. 


\section{REFERENCES}

(1) Bodenhausen, G.; Ruben, D. J. Chem. Phys. Lett. 1980, 69, 185-189.

(2) Bax, A.; Summers, M. F. J. Am. Chem. Soc. 1986, 108, 2093-2094.

(3) Thiele, C. M.; Petzold, K.; Schleucher, J. Chem. Eur. J. 2009, 15, 585-588.

(4) Thrippleton, M. J.; Keeler, J. Angew. Chem. Int. Ed. 2003, 42, 3938-3941.

(5) Kupče, E.; Boyd, J.; Campbell, I. D. J. Magn. Reson. B 1995, 106, 300-303.

(6) Ilgen, J.; Nowag, J.; Kaltschnee, L.; Schmidts, V.; Thiele, C. M. J. Magn. Reson. 2021, 324, 106900.

(7) Foroozandeh, M.; Adams, R. W.; Meharry, N. J.; Jeannerat, D.; Nilsson, M.; Morris, G. A. Angew. Chem. Int. Ed. 2014, 53, 6990-6992.

(8) Hu, H.; Krishnamurthy, K. J. Magn. Reson. 2006, 182, 173-177.

(9) Kolmer, A.; Edwards, L. J.; Kuprov, I.; Thiele, C. M. J. Magn. Reson. 2015, 261, 101-109.

(10) Neuhaus, D.; Williamson, M. P. The Nuclear Overhauser Effect in Structural and Conformational Analysis, $2^{\text {nd }}$ Ed., Wiley, Chichester, 2000.

(11) Kolmer, A.; Edwards, L. J.; Kuprov, I.; Thiele, C. M. J. Magn. Reson. 2015, 261, 101-109.

(12) Sinnaeve, D.; Foroozandeh, M.; Nilsson, M.; Morris, G. A. Angew. Chem. Int. Ed. 2016, 55, 1090-1093.

(13) a) https://gaussian.com/scrf/; b) Tomasi, J.; Mennucci, B.; Cammi, R. Chem. Rev. 2005, 105, 2999-3093; c)_London, F. J. Phys. Radium, 1937, 8, 397-409; d) McWeeny, R. Phys. Rev. 1962, 126, 1028-1034; e) Ditchfield, R. Mol. Phys. 1974, 27, 789-807; f) Wolinski, K.; Hinton, J. F.; Pulay, P. J. Am. Chem. Soc. 1990, 112, 8251-8260; g) Cheeseman, J. R.; Trucks, G. W.; Keith, T. A.; Frisch, M. J. J. Chem. Phys. 1996, 104, 5497-5509; h) Towns, J.; Cockerill, T.; Dahan, M.; Foster, I.; Gaither, K.; Grimshaw, A.; Hazlewood, V.; Lathrop, S.; Lifka, D.; Peterson, G. D.; Roskies, R.; Scott, J. R.; Wilkins-Diehr, N. Comput. Sci. Eng. 2014, 16, 62 74; i) Grimme, S.; Antony, J.; Ehrlich, S.; Krieg, H. J. Chem. Phys. 2010, 132, 154104; j) Grimme, S.; Ehrlich, S.; Goerigk, L. J. Comput. Chem. 2011, 32, 1456-1465.

(14) Gaussian 16, Revision C.01: Frisch, M. J.; Trucks, G. W.; Schlegel, H. B.; Scuseria, G. E.; Robb, M. A.; Cheeseman, J. R.; Scalmani, G.; Barone, V.; Petersson, G. A.; Nakatsuji, H.; Li, X.; Caricato, M.; Marenich, A. V.; Bloino, J.; Janesko, B. G.; Gomperts, R.; Mennucci, B.; Hratchian, H. P.; Ortiz, J. V.; Izmaylov, A. F.; Sonnenberg, J. L.; Williams-Young, D.; Ding, F.; Lipparini, F.; Egidi, F.; Goings, J.; Peng, B.; Petrone, A.; Henderson, T.; Ranasinghe, D.; Zakrzewski, V. G.; Gao, J.; Rega, N.; Zheng, G.; Liang, W.; Hada, M.; Ehara, M.; Toyota, K.; Fukuda, R.; Hasegawa, J.; Ishida, M.; Nakajima, T.; Honda, Y.; Kitao, O.; Nakai, H.; Vreven, T.; Throssell, K.; Montgomery, J. A., Jr.; Peralta, J. E.; Ogliaro, F.; Bearpark, M. J.; Heyd, J. J.; Brothers, E. N.; Kudin, K. N.; Staroverov, V. N.; Keith, T. A.; Kobayashi, R.; Normand, J.; Raghavachari, K.; Rendell, A. P.; Burant, J. C.; Iyengar, S. S.; Tomasi, J.; Cossi, M.; Millam, J. M.; Klene, M.; Adamo, C.; Cammi, R.; Ochterski, J. W.; Martin, R. L.; Morokuma, K.; Farkas, O.; Foresman, J. B.; Fox, D. J. Gaussian, Inc., Wallingford CT, 2016.

(15) Pracht, P.; Bohle, F.; Grimme, S. Phys. Chem. Chem. Phys. 2020, 22, 7169-7192.

(16) Grimme, S. J. Chem. Theory Comput. 2019, 15, 2847-2862.

(17) Lodewyk, M. W.; Siebert, M. R.; Tantillo, D. J. Chem. Rev. 2012, 112, 1839-1862.

(18) CHESHIRE, Chemical Shift Repository with Coupling Constants Added Too. http://cheshirenmr.info

(19) a) Foster, J. P.; Weinhold, F. J. Am. Chem. Soc. 1980,102, 7211-7218; b) Reed, A. E.; Weinhold, F. J. Chem. Phys. 1983, 78, 4066-4073; c) Reed, A. E.; Weinstock, R. B.; Weinhold, F. J. Chem. Phys. 1985, 83, 735-746; d) Reed, A. E.; Weinhold, F. J. Chem. Phys. 1985, 83, 1736-1740; e) J. E. Carpenter, Extension of Lewis structure concepts to open-shell and excited-state molecular species, Ph.D. thesis, University of Wisconsin, Madison, WI, 1987; f) Carpenter, J. E.; Weinhold, F. J. Mol. Struct. (Theochem) 1988, 139, 41-62; g) Reed, A. E.; Curtiss, L. A.; Weinhold, F. Chem. Rev. 1988, 88, 899-926; h) Weinhold, F. and Carpenter, J. E. in The Structure of Small Molecules and lons, Naaman, E. R.; Vager, Z. Plenum, 1988, 227-236.

(20) Legault, C. Y. CYLview, 1.0b. Université de Sherbrooke. 2009.

(21) Álvarez-Moreno, M.; de Graaf, C.; López, N.; Maseras, F.; Poblet, J. M.; Bo, C. J. Chem. Inf. Model. 2015, 55, 95-103. 
(22) Ponath, S.; Menger, M.; Grothues, L.; Weber, M.; Lentz, D.; Strohmann, C.; Christmann, M. Angew. Chem. Int. Ed. 2018, 57, 11683-11687.

(23) Burés, J.; Armstrong, A.; Blackmond, D. G. Acc. Chem. Res. 2016, 49, 214-222.

(24) Burés, J.; Dingwall, P.; Armstrong, A.; Blackmond, D. G. Angew. Chem. Int. Ed. 2014, 53, 8700-8704.

(25) Wiest, J. The Role of Charged and Uncharged Intermediates in Organocatalysis, MS Thesis, 2012, Universität Basel (conducted in the Blackmond group at The Scripps Research Institute)

(26) Brochu, M. P.; Brown, S. P.; MacMillan, D. W. C. J. Am. Chem. Soc. 2004, 126, 4108-4109.

(27) Amatore, M.; Beeson, T. D.; Brown, S. P.; MacMillan, D. W. C. Angew. Chem. Int. Ed. 2009, 48, 5121-5124.

(28) Burés, J.; Armstrong, A.; Blackmond, D. G. J. Am. Chem. Soc. 2012, 134, 6741-6750.

(29) Chen, G.; Fu, C.; Ma, S. Tetrahedron 2006, 62, 4444-4452.

(30) Warnke, S.; von Helden, G.; Pagel, K. Proteomics 2015, 15, 2804-2812.

(31) Hoffmann, W.; Langenhan, J.; Huhmann, S.; Moschner, J.; Chang, R.; Accorsi, M.; Seo, J.; Rademann, J.; Meijer, G.; Koksch, B.; Bowers, M. T.; von Helden, G.; Pagel, K. Angew. Chem. Int. Ed. 2019, 58, 8216-8220.

(32) Revercomb, H. E.; Mason, E. A. Anal. Chem. 1975, 47, 970-983.

(33) Gabelica, V.; Shvartsburg, A. A.; Afonso, C.; Barran, P.; Benesch, J. L. P.; Bleiholder, C.; Bowers, M. T.; Bilbao, A.; Bush, M. F.; Campbell, J. L.; Campuzano, I. D. G.; Causon, T.; Clowers, B. H.; Creaser, C. S.; De Pauw, E.; Far, J.; Fernandez-Lima, F.; Fjeldsted, J. C.; Giles, K.; Groessl, M.; Hogan, C. J., Jr.; Hann, S.; Kim, H. I.; Kurulugama, R. T.; May, J. C.; McLean, J. A.; Pagel, K.; Richardson, K.; Ridgeway, M. E.; Rosu, F.; Sobott, F.; Thalassinos, K.; Valentine, S. J.; Wyttenbach, T. Mass Spectrom. Rev. 2019, 38, 291-320.

(34) Supady, A.; Blum, V.; Baldauf, C. J. Chem. Inf. Model. 2015, 55, 2338-2348.

(35) Blum, V.; Gehrke, R.; Hanke, F.; Havu, P.; Havu, V.; Ren, X.; Reuter, K.; Scheffler, M. Comput. Phys. Commun. 2009, 180, 2175-2196.

(36) Perdew, J. P.; Burke, K.; Ernzerhof, M. Phys. Rev. Lett. 1996, 77, 3865-3868.

(37) Tkatchenko, A.; Scheffler, M. Phys. Rev. Lett. 2009, 102, 073005.

(38) Singh, U. C.; Kollman, P. A. J. Comput. Chem. 1984, 5, 129-145.

(39) Adamo, C.; Barone, V. J. Chem. Phys. 1999, 110, 6158-6170.

(40) Zanotto, L.; Heerdt, G.; Souza, P. C. T.; Araujo, G.; Skaf, M. S. J. Comput. Chem. 2018, 39, $1675-1681$.

(41) Horii, Z-I.; Iwata, C.; Tamura, Y. J. Org. Chem. 1961, 26, 2273-2276.

(42) Thiele, C. M.; Kolmer A. J. Magn. Reson. 2016, 266, 69-72.

(43) a) Schmid, M. B.; Zeitler, K.; Gschwind, R. M. Angew. Chemie. Int. Ed. 2010, 49, 4997-5003; b) Schmid, M. B.; Zeitler, K.; Gschwind, R. M. Chem. Sci. 2011, 2, 1793-1803; c) Renzi, P.; Hioe, J.; Gschwind, R. M. Acc. Chem. Res. 2017, 50, 2936-2948.

(44) Iron, M. A. J. Chem. Theory Comput. 2017, 13, 5798-5819.

(45) Merrill, A.T.; Tantillo, D. J. Magn. Reson. Chem. 2020, 58, 576-583.

(46) Seebach, D.; Sun, X.; Sparr, C.; Ebert, M.-O.; Schweizer, W. B.; Beck, A. K. Helv. Chim. Acta 2012, 95, 1064-1078.

(47) Seebach, D.; Sun, X.; Ebert, M.-O.; W. Schweizer, W. B.; Purkayastha, N.; Beck, A. K.; Duschmalé, J.; Wennemers, H.; Mukaiyama, T.; Benohoud, M.; Hayashi, Y.; Reiher, M. Helv. Chim. Acta 2013, 96, 799-852.

(48) Cassani, C.; Melchiorre, P. Org. Lett. 2012, 14, 5590-5593.

(49) Weber, A. K.; Schachtner, J.; Fichtler, R.; Leermann, T. M.; Neudörfl, J. M.; von Wangelin, A. J. Org. Biomol. Chem. 2014, 12, 5267-5277.

(50) Gurubrahamam, R.; Chen, Y. M.; Huang, W.-Y.; Chan, Y.-T.; Chang, H.-K.; Tsai, M.-K.; Chen, K. Org. Lett. 2016, 18, 3046-3049.

(51) Boeckman Junior, R. K.; Wang, H.; Rugg, K. W.; Genung, N. E.; Chen, K.; Ryder, T. R. Org. Lett. 2016, 18, 6136-6139.

(52) Dinér, P.; Kjærsgaard, A.; Lie, M.; Jørgensen, K. Chem. Eur. J. 2008, 14, 122-127.

(53) Marathias, V. M.; Goljer, I.; Bach II, A. C. Magn. Reson. Chem. 2005, 43, 512-519. 


\section{APPENDIX}

\subsection{NMR-Spectra}

1-((1S,2R)-1-((R)-5-benzyl-2,2,3-trimethyl-4-oxoimidazolidin-1-yl)-2-chloro-3methylbutyl)pyrrolidine-2,5-dione (syn-20)<smiles>CC(C)C(Cl)[C@H](N1C(=O)CCC1=O)N1[C@H](Br)C(=O)N(C)C1(C)C</smiles>

syn-20
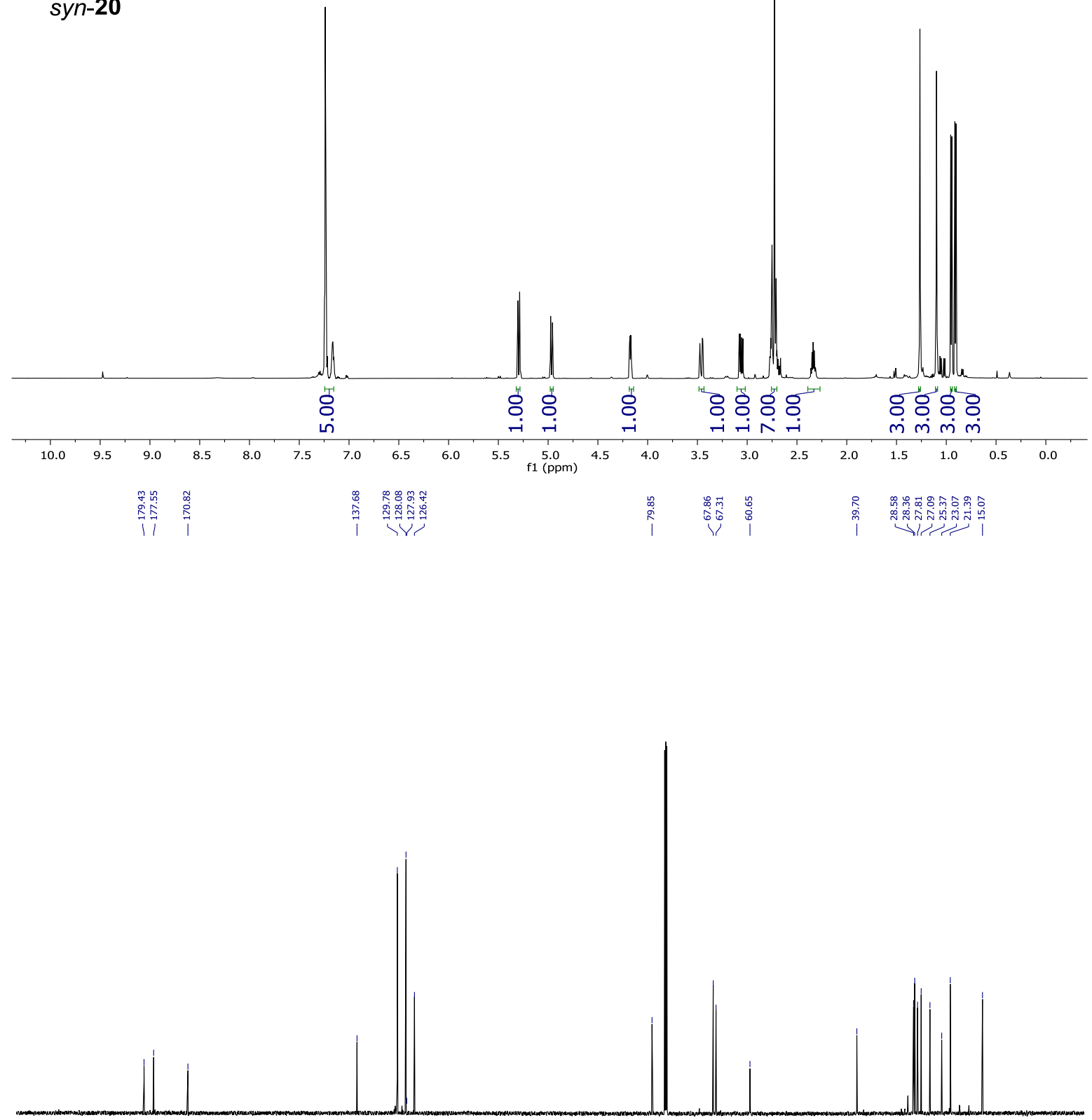

$\begin{array}{lllllllllll}200 & 190 & 180 & 170 & 160 & 150 & 140 & 130 & 120 & 110 & 100 \\ & & & & & & & & & & 100\end{array}$ 
1-((S)-1-((R)-5-benzyl-2,2,3-trimethyl-4-oxoimidazolidin-1-yl)-2,2-dichloro-3-

methylbutyl)pyrrolidine-2,5-dione (24a)<smiles>[Z16]C(C)C(Cl)(Cl)[C@H](N1C(=O)CCC1=O)N1[C@H](Br)C(=O)N(C)C1(C)C</smiles>

24a
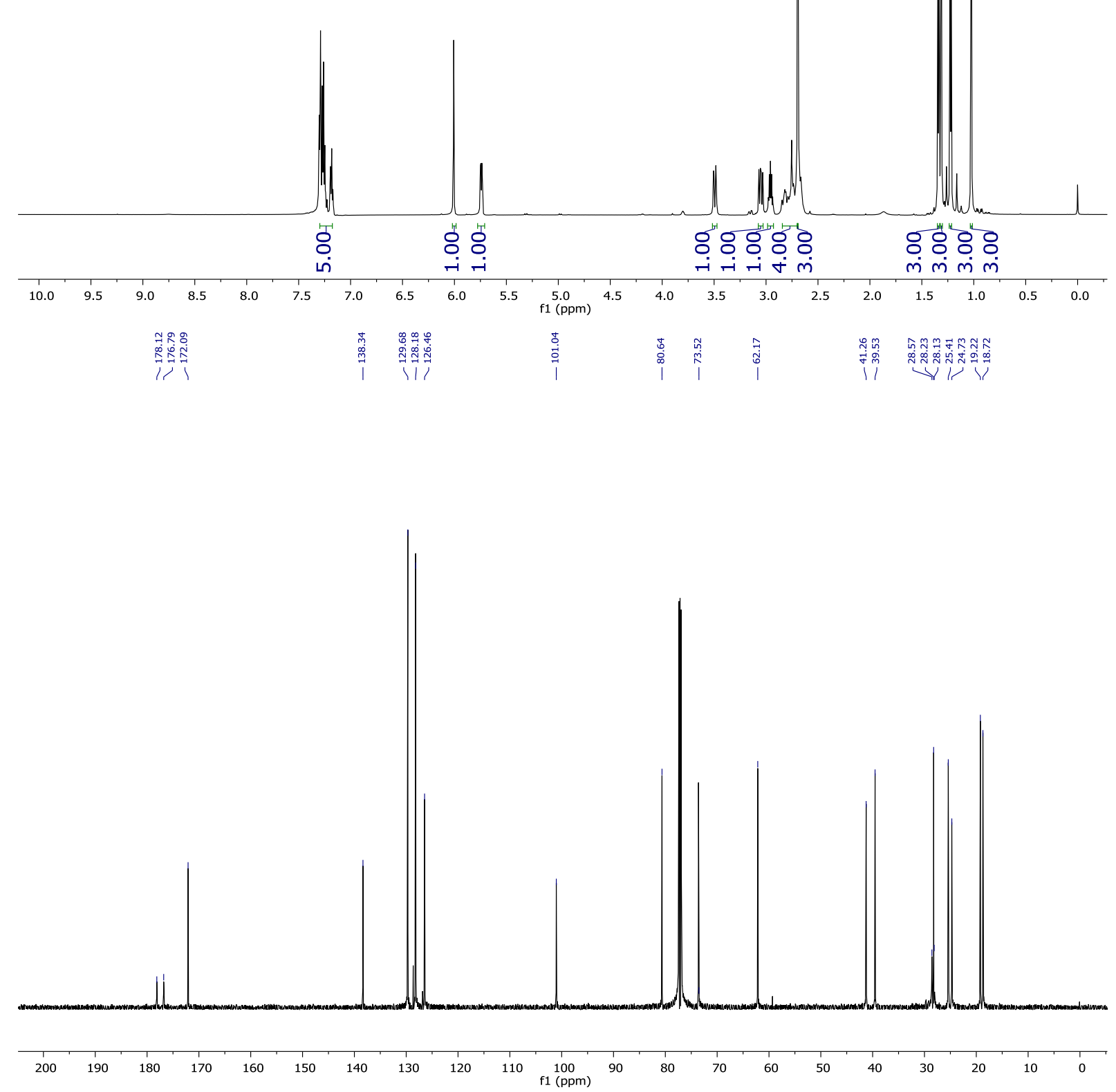
1-((1S,2R)-1-((2R,5R)-5-benzyl-2-(tert-butyl)-3-methyl-4-oxoimidazolidin-1-yl)-2-chloro-3methylbutyl)pyrrolidine-2,5-dione ((1S,2R)-syn-21)<smiles>CC(C)C(Cl)C(N1C(=O)CCC1=O)N1[C@H](Br)C(=O)N(C)[C@H]1Br</smiles>

$(1 S, 2 R)-s y n-21$
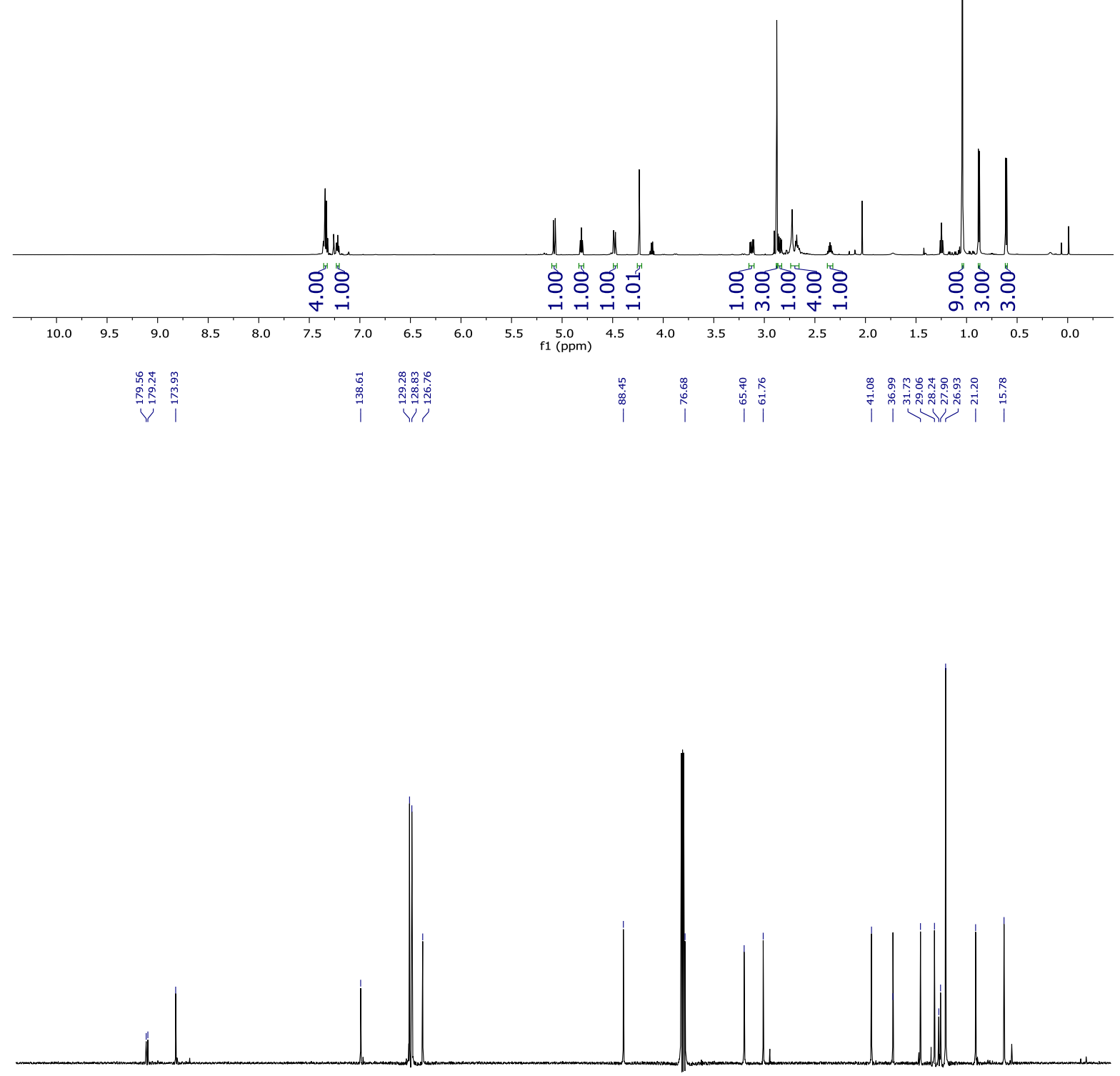

$\begin{array}{lllllllllll}200 & 190 & 180 & 170 & 160 & 150 & 140 & 130 & 120 & 110 & 100 \\ \mathrm{f} 1(\mathrm{ppm})\end{array}$

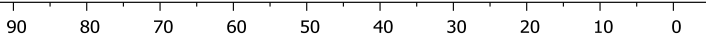


1-((1S,2S)-1-((2R,5R)-5-benzyl-2-(tert-butyl)-3-methyl-4-oxoimidazolidin-1-yl)-2-chloro-3methylbutyl)pyrrolidine-2,5-dione ((1S,2S)-anti-21)<smiles>CC(C)[C@H](Cl)N1C(=O)CCC1=O</smiles>

$(1 S, 2 S)$-anti-21

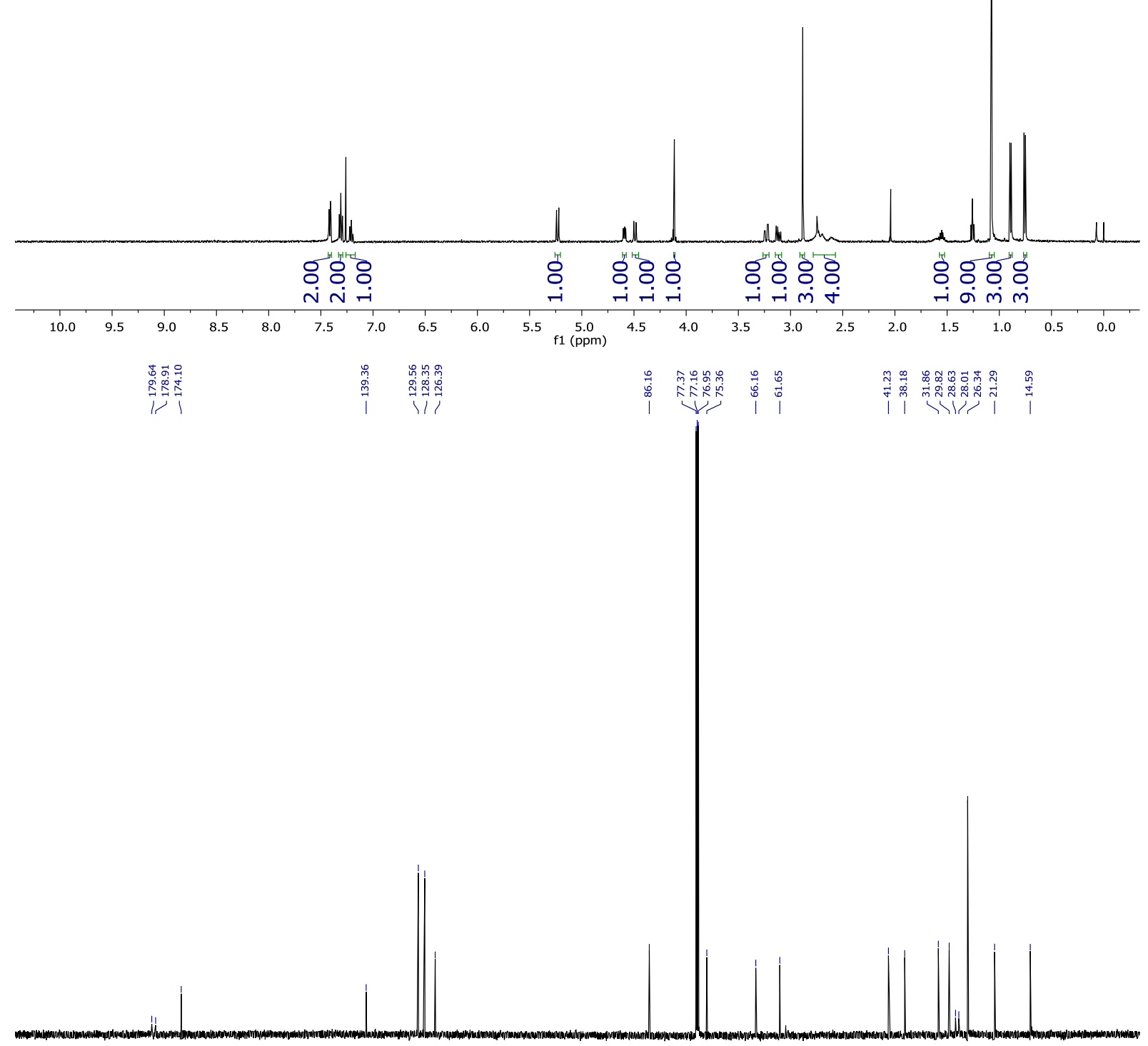

$\begin{array}{lllllllllllllllllllllll} & 1 & 1 & 1 & 180 & 170 & 160 & 150 & 140 & 130 & 120 & 110 & 100 & 90 & 80 & 70 & 60 & 50 & 40 & 30 & 20 & 10 & 0\end{array}$ 
1-((1R,2S)-1-((2R,5R)-5-benzyl-2-(tert-butyl)-3-methyl-4-oxoimidazolidin-1-yl)-2-chloro-3methylbutyl)pyrrolidine-2,5-dione ((1R,2S)-syn-21)<smiles>CC(C)[C@H](Cl)[C@H](N1C(=O)CCC1=O)N1C([13CH3])[C@@H](Br)C(=O)N1C</smiles>

(1R,2S)-syn-21

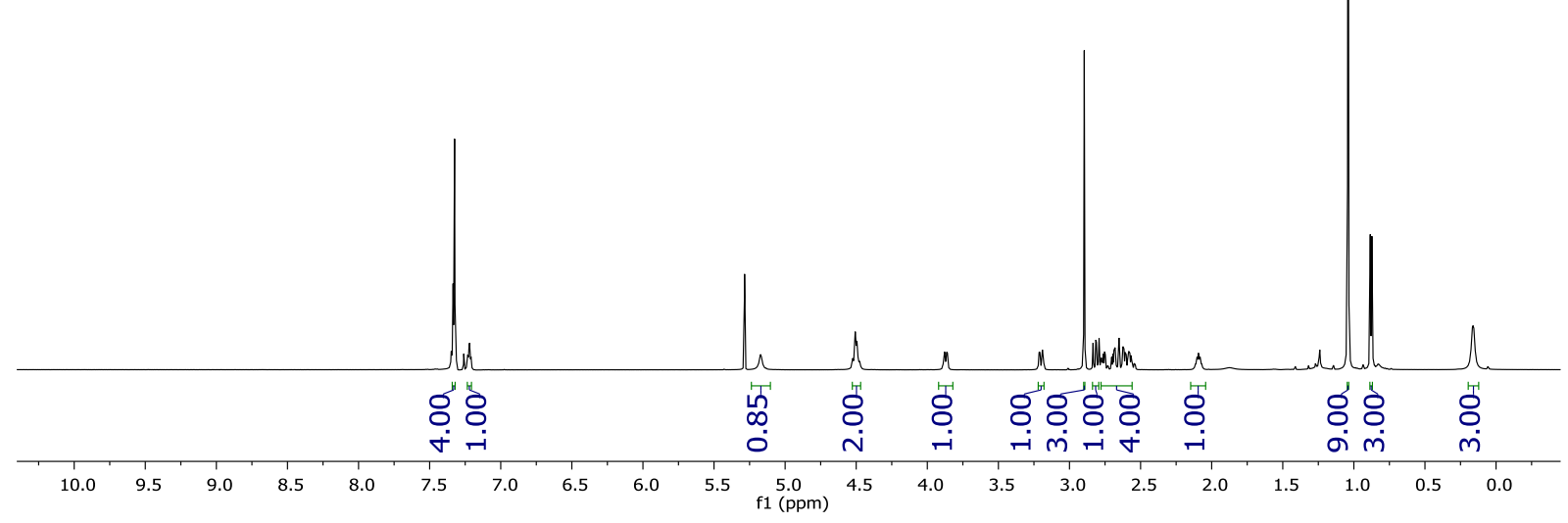

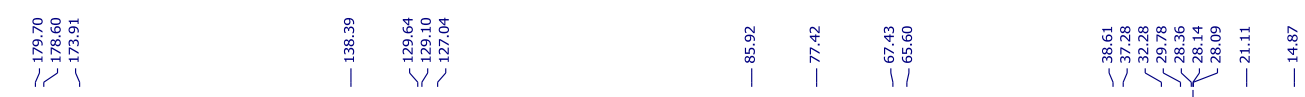

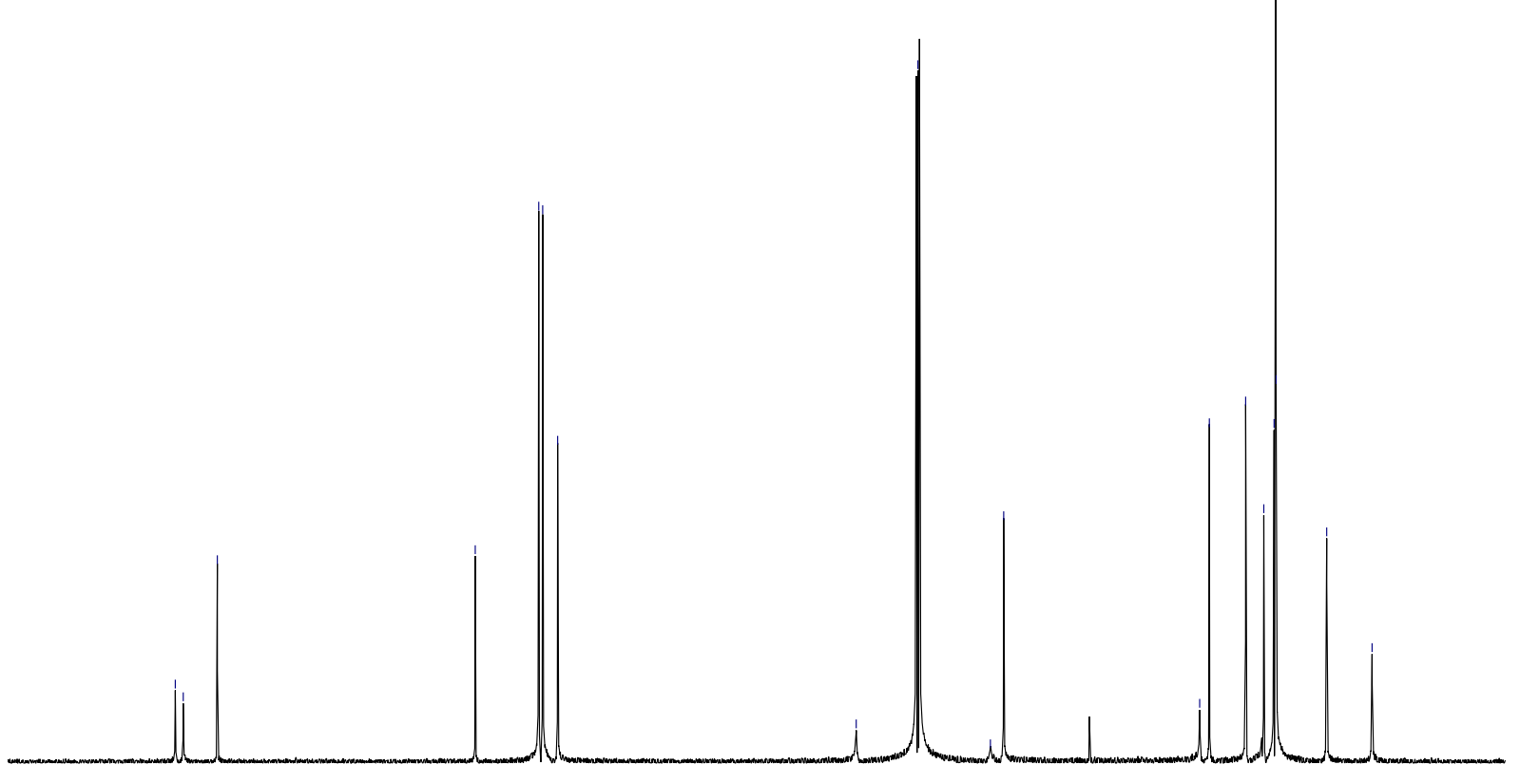

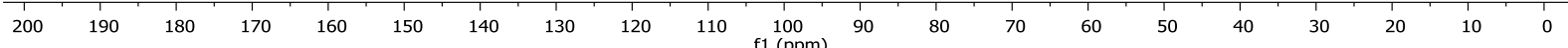


1-((1S,2R)-1-((2R,5R)-5-benzyl-2-(tert-butyl)-3-methyl-4-oxoimidazolidin-1-yl)-2-chloro-3phenylpropyl)pyrrolidine-2,5-dione ((1S,2R)-syn-25)<smiles>CN1C(=O)[C@@H](Br)N([C@H](C(Cl)Br)N2C(=O)CCC2=O)C1[13CH3]</smiles>

$(1 S, 2 R)-$ syn-25

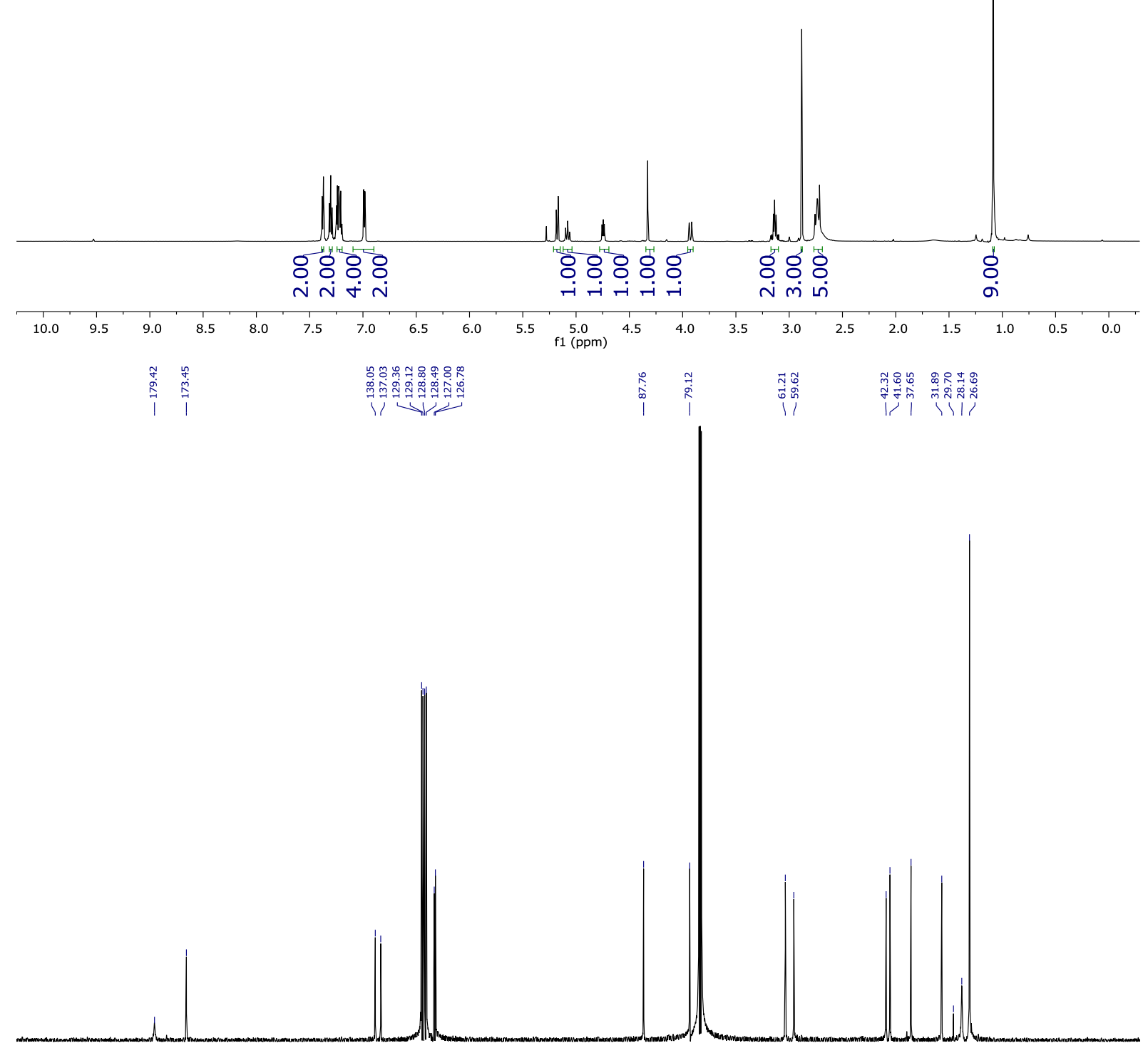

$\begin{array}{llllllllll}200 & 190 & 180 & 170 & 160 & 150 & 140 & 130 & 120 & 110 \\ \mathrm{f} 1(\mathrm{ppm})\end{array}$ 
1-((1S,2S)-1-((2R,5R)-5-benzyl-2-(tert-butyl)-3-methyl-4-oxoimidazolidin-1-yl)-2-chloro-3phenylpropyl)pyrrolidine-2,5-dione ((1S,2S)-anti-25)

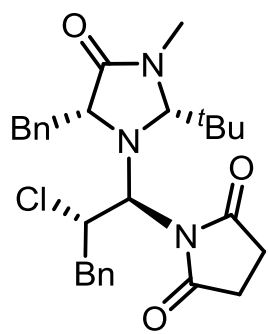

$(1 S, 2 S)$-anti-25

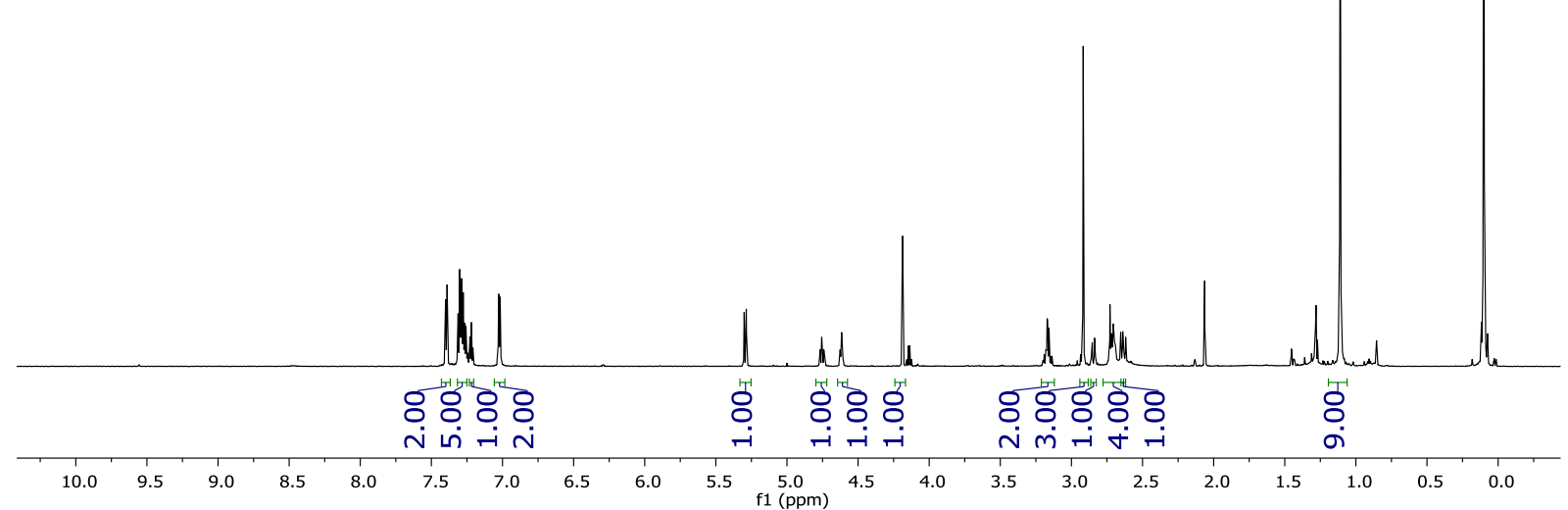

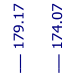

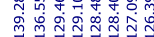
1

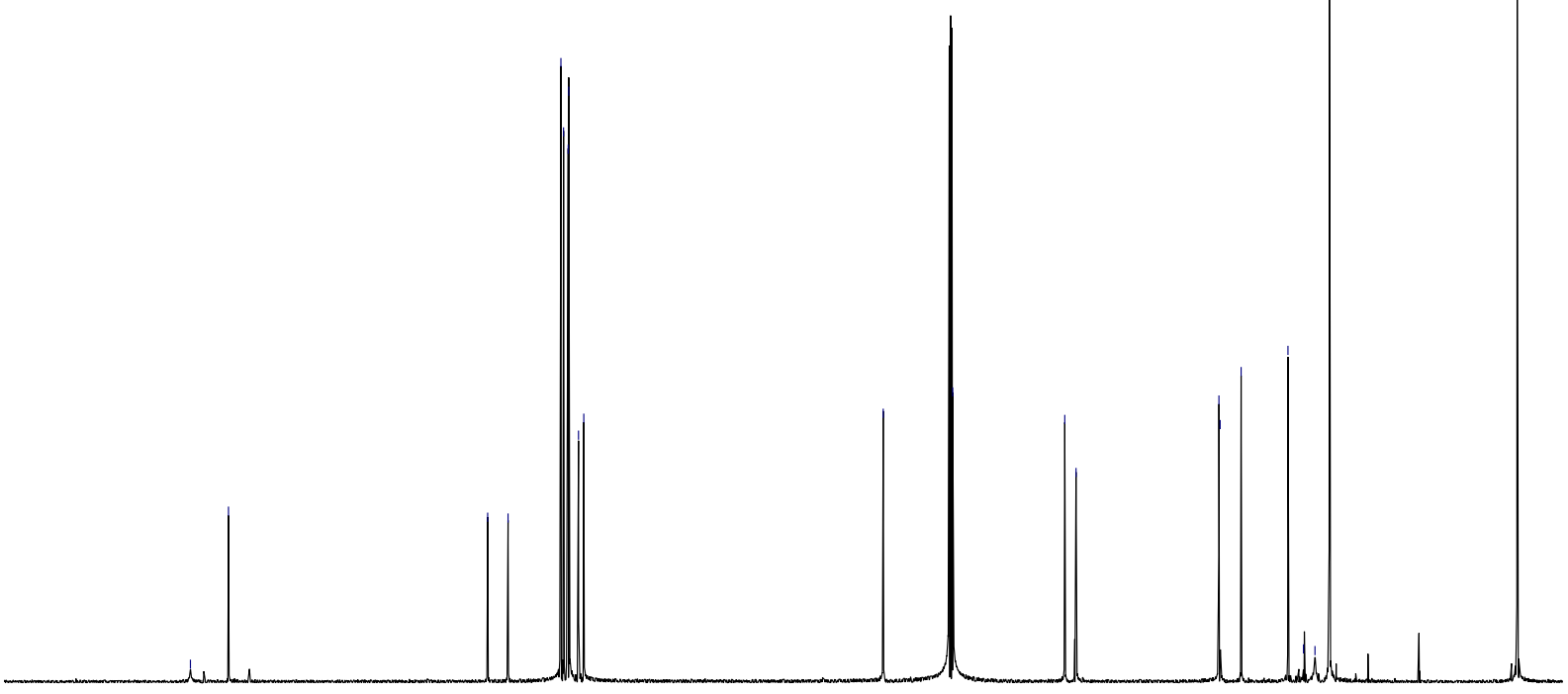

200 $\begin{array}{lllll}190 & 180 & 170 & 160 & 150\end{array}$ 
1-((1R,2S)-1-((2R,5R)-5-benzyl-2-(tert-butyl)-3-methyl-4-oxoimidazolidin-1-yl)-2-chloro-3phenylpropyl)pyrrolidine-2,5-dione ((1R,2S)-syn-25)<smiles></smiles>

$(1 R, 2 S)-s y n-25$

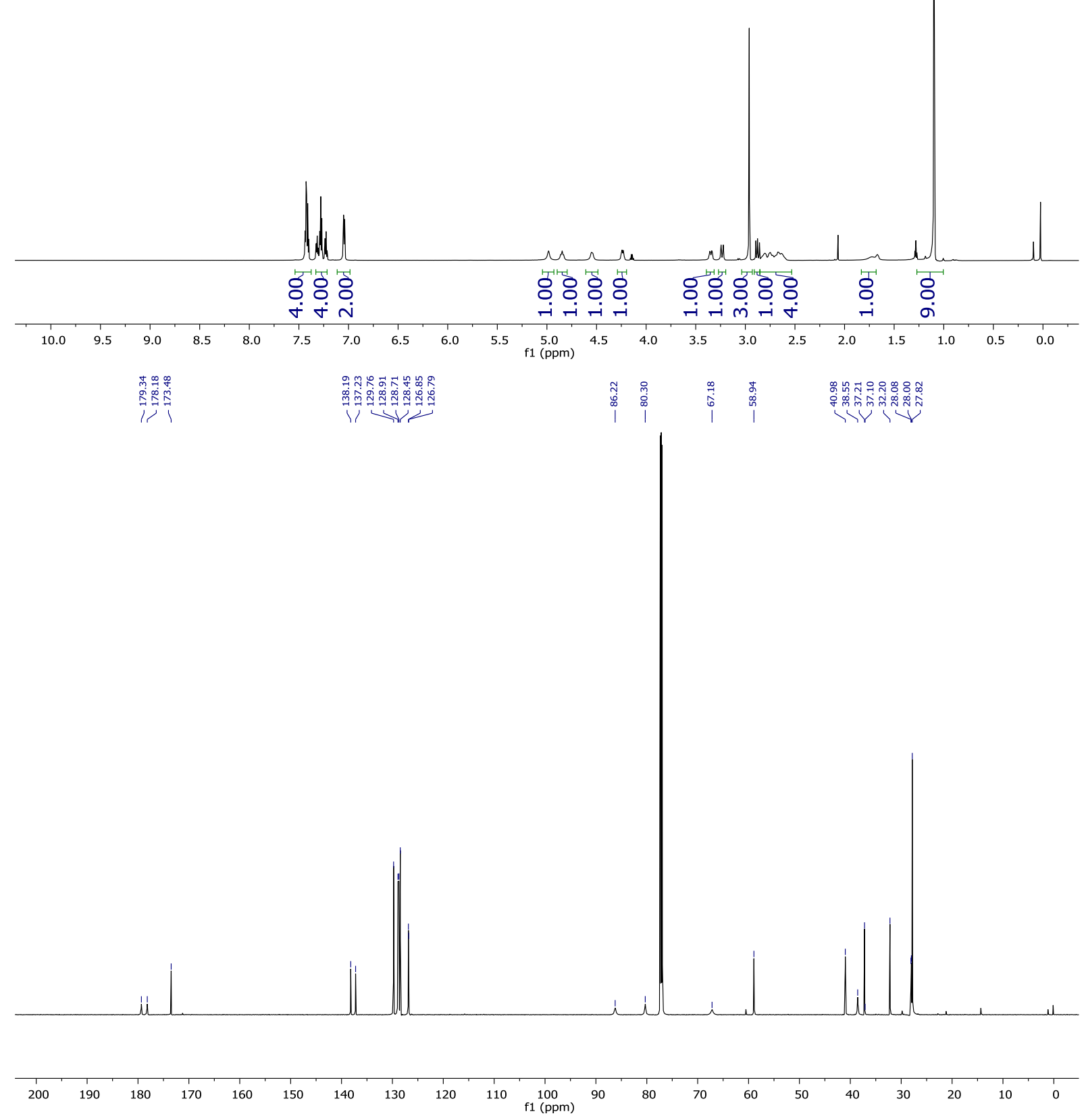


Decomposition of Aminal syn-18b

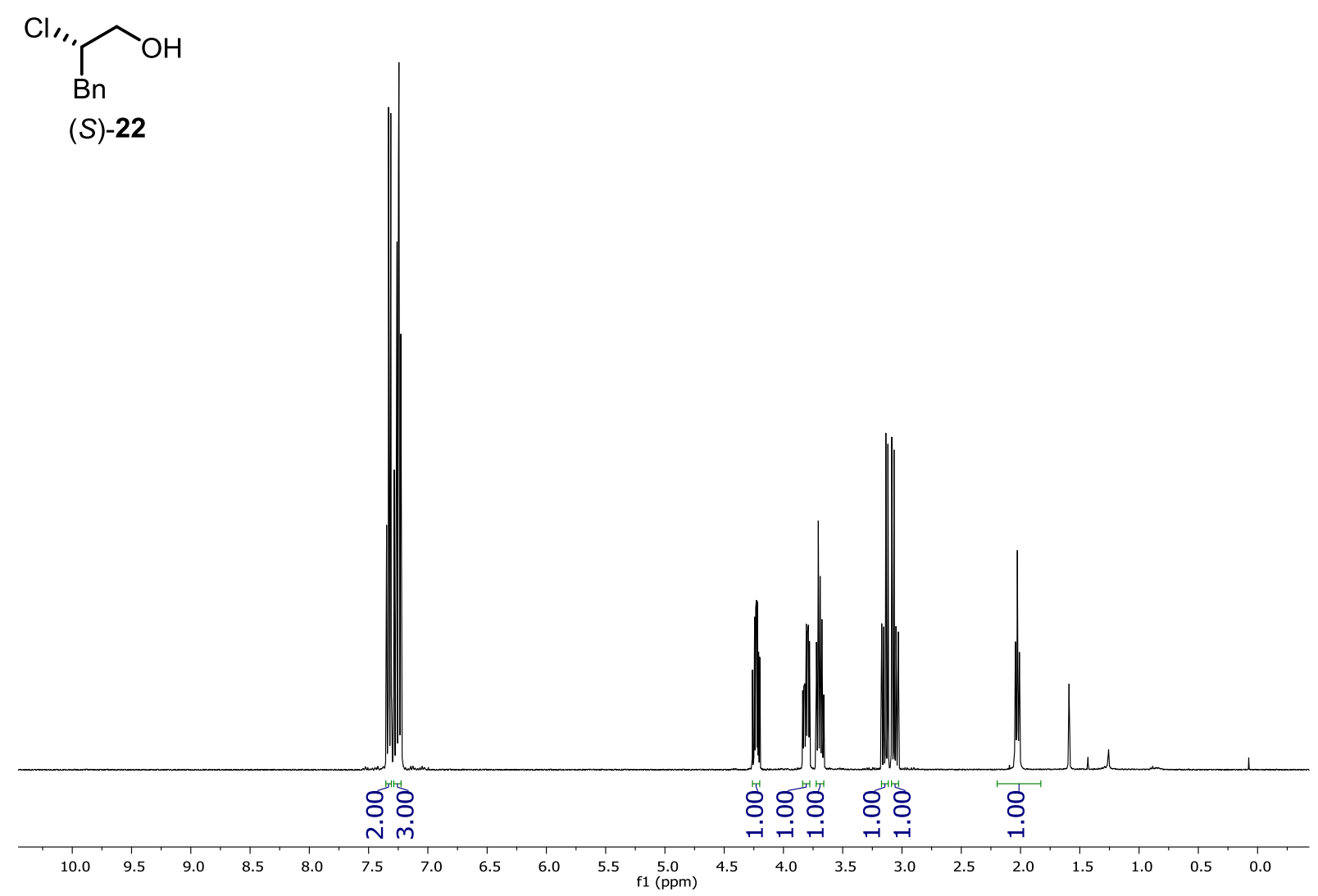




\section{KIE-experiment (1)}

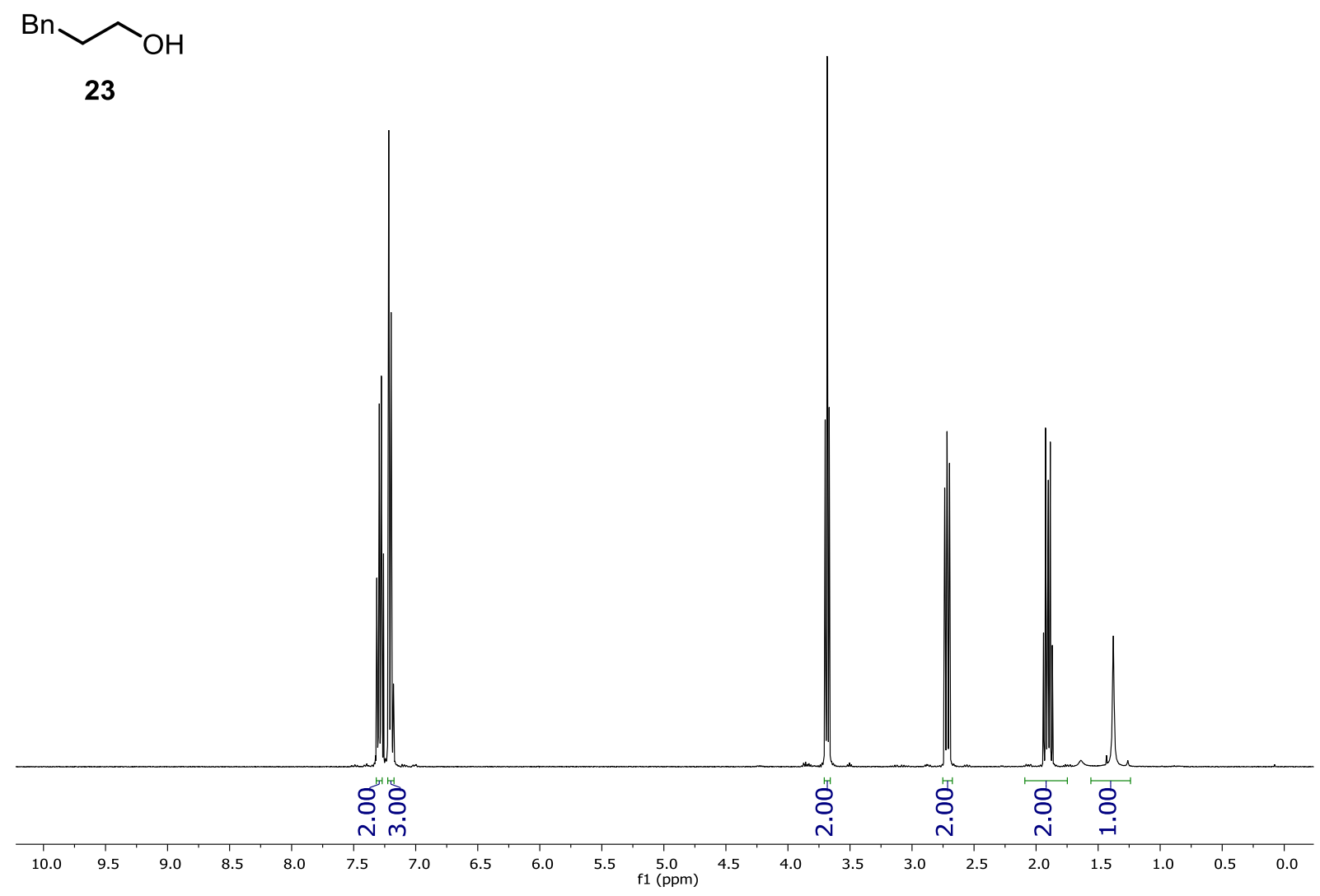

${ }^{1} \mathrm{H}-\mathrm{NMR}$ (crude) after reductive work-up:
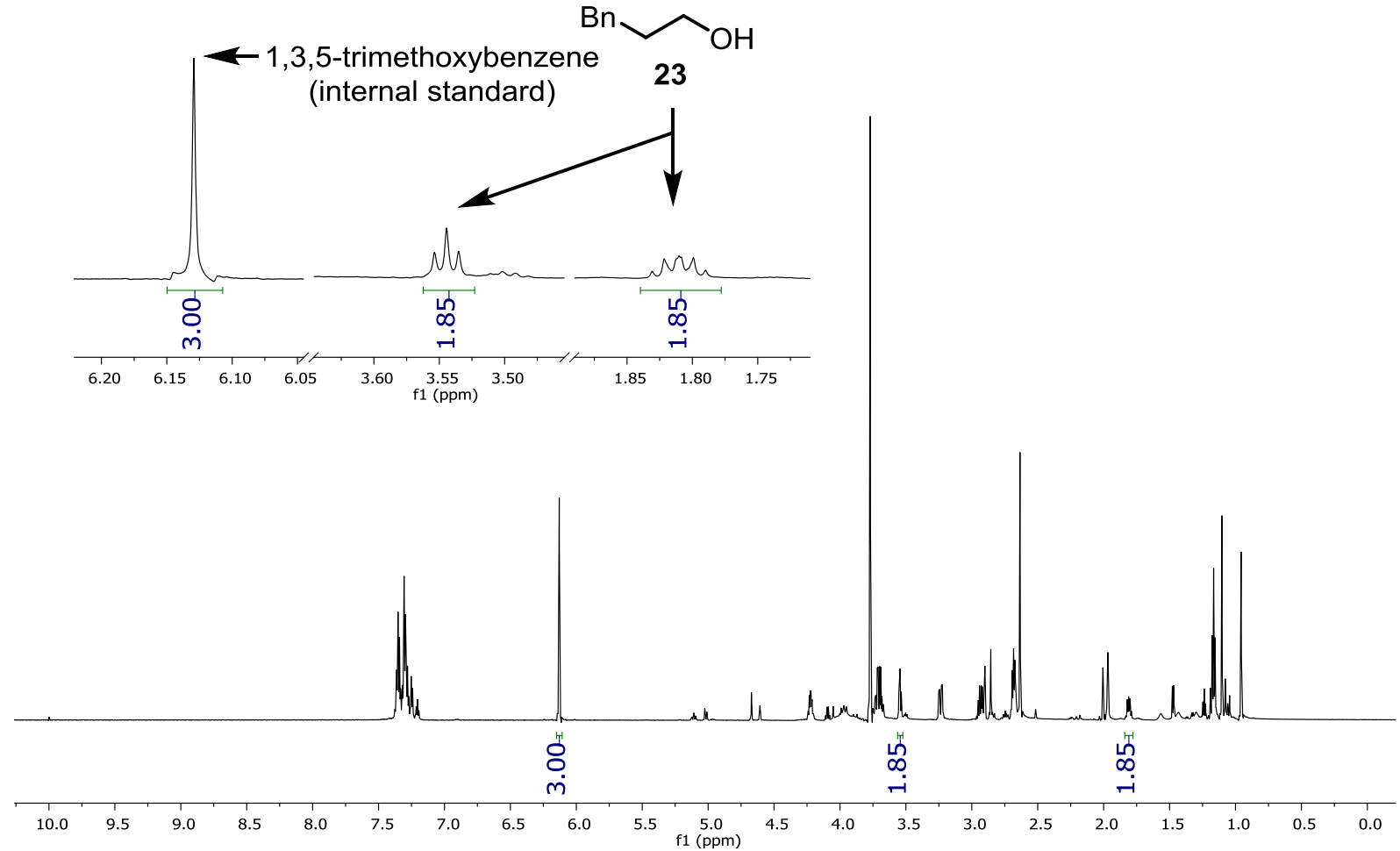
2-(1-((S)-2-(bis(3,5-bis(trifluoromethyl)phenyl)((trimethylsilyl)oxy)methyl)pyrrolidin-1-yl)-2chloro-3-methylbutyl)isoindoline-1,3-dione (14)<smiles>COC(c1cc(C(F)(F)F)cc(C(F)(F)F)c1)(c1cc(C(F)(F)F)cc(C(F)(F)F)c1)C1CCCN1C(C(Cl)C(C)C)N1C(=O)c2ccccc2C1=O</smiles>
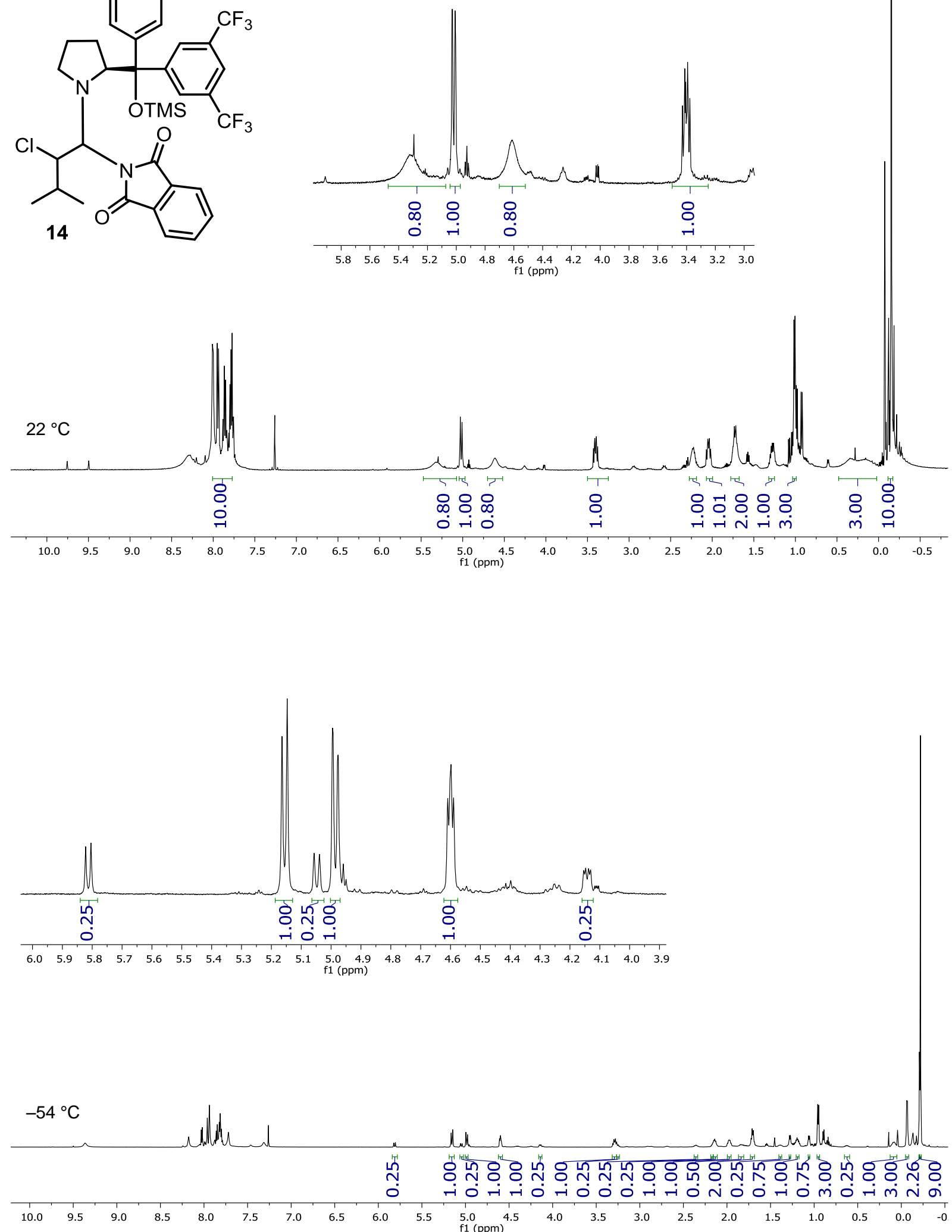


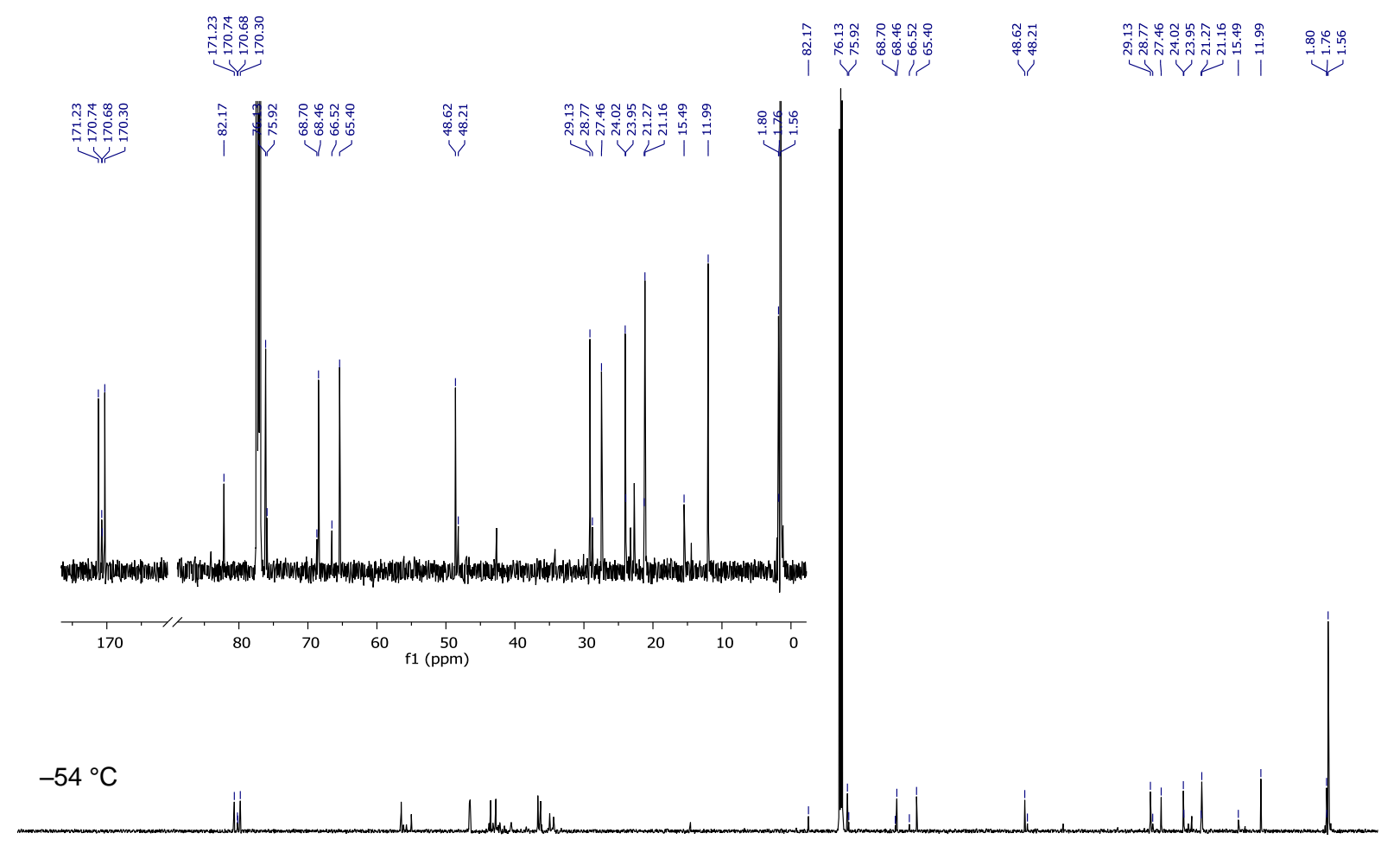

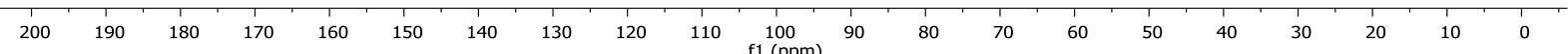


(S)-2-chloro-3-methylbutyl 3,5-dinitrobenzoate ((S)-SI2)
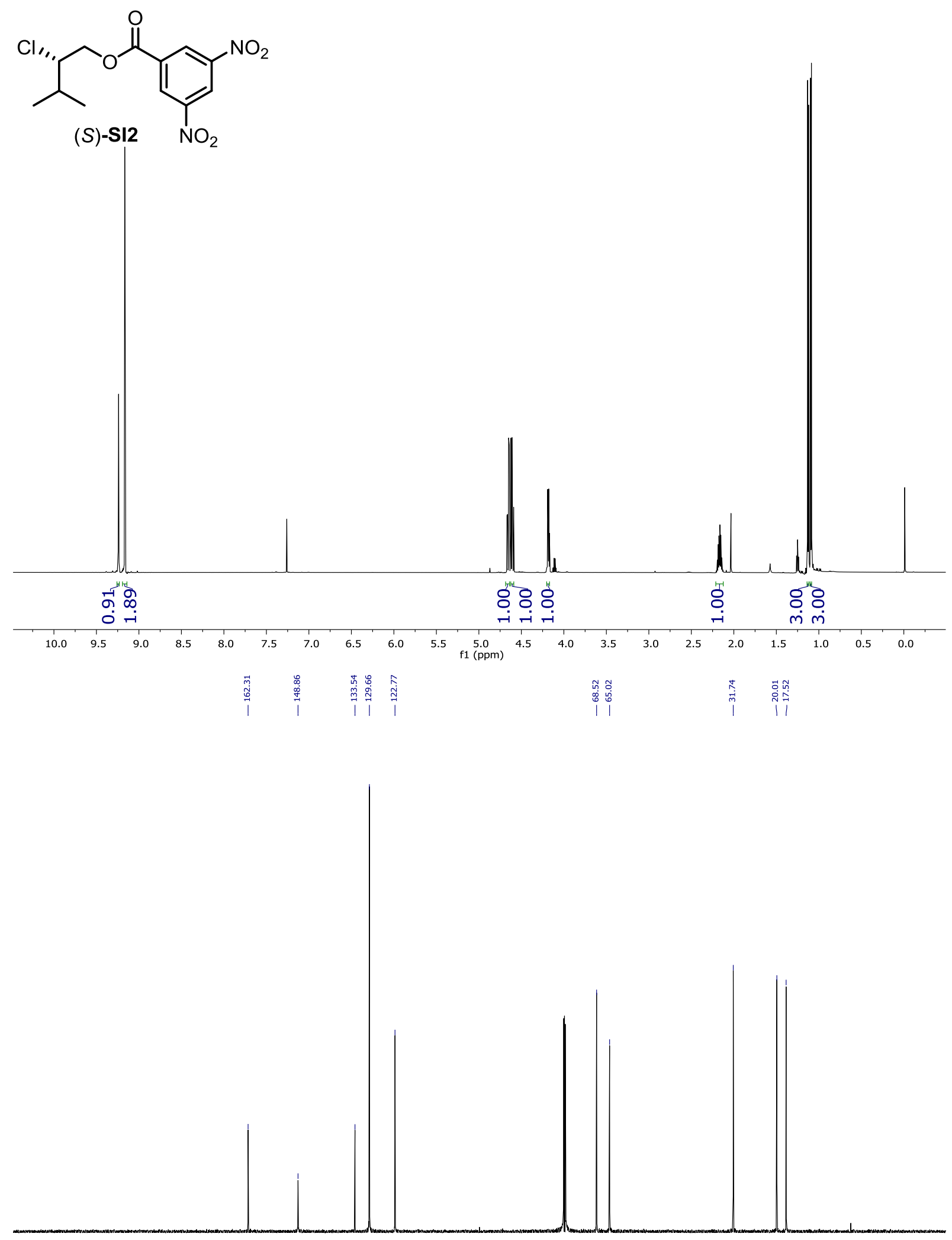

$\begin{array}{llllllllllllllllllllllllllllll}220 & 210 & 200 & 190 & 180 & 170 & 160 & 150 & 140 & 130 & 120 & 110 & 100 & 90 & 80 & 70 & 60 & 50 & 40 & 30 & 20 & 10 & 0 & -10 & -20\end{array}$ 


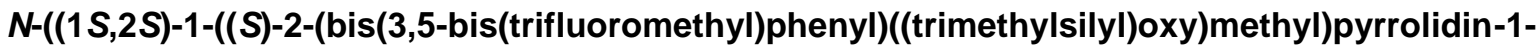
yl)-2-chloro-3-methylbutyl)-2-(hydroxymethyl)benzamide (SI3)<smiles>CC(C)OC(c1cc(C(F)(F)F)cc(C(F)(F)F)c1)(c1cc(C(F)(F)F)cc(C(F)(F)F)c1)N1CCCC1[C@@H](NC(=O)c1ccccc1CO)[C@@H](Cl)C(C)C</smiles>
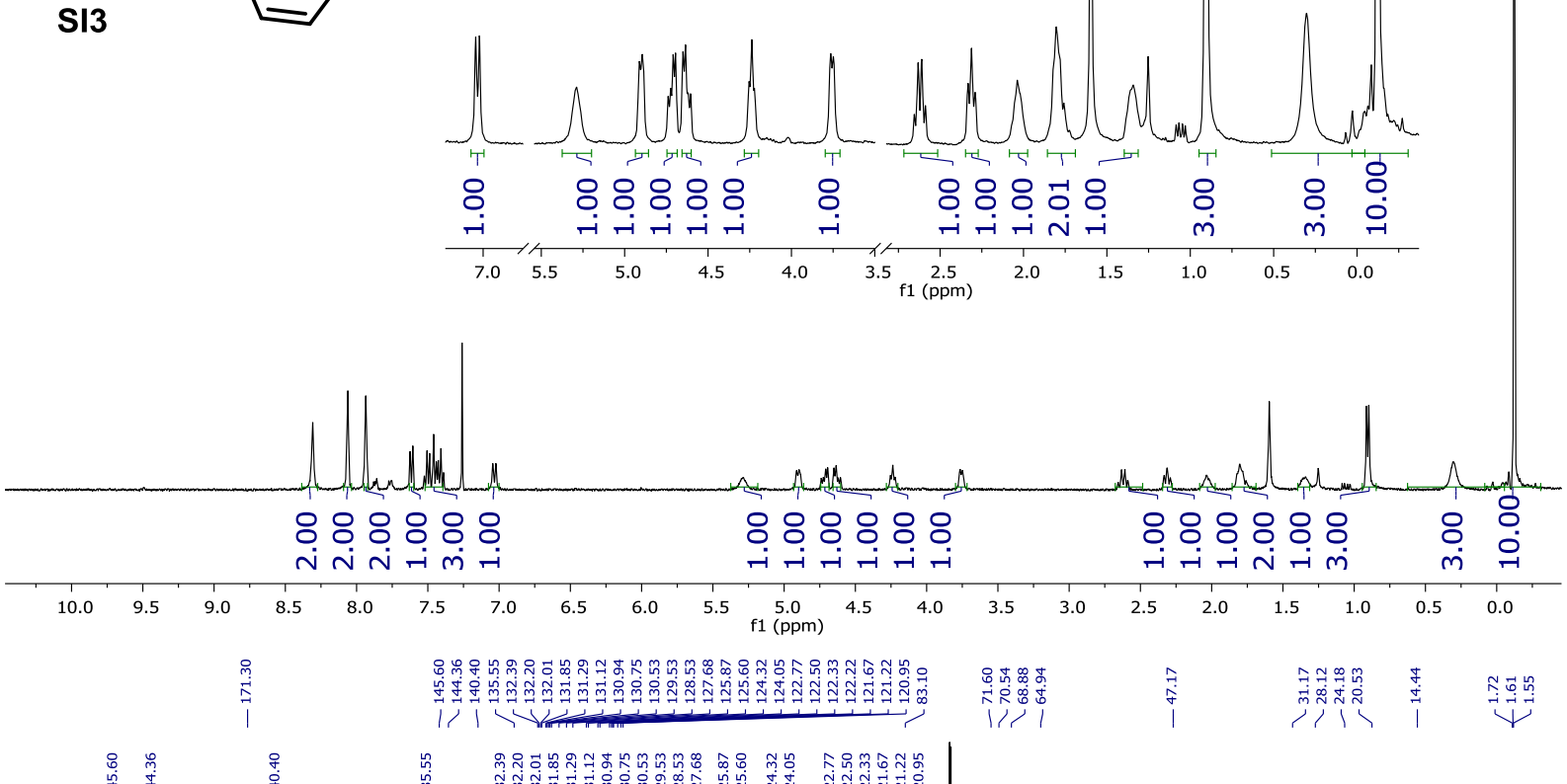

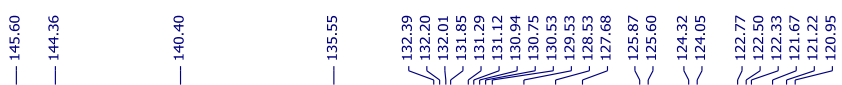

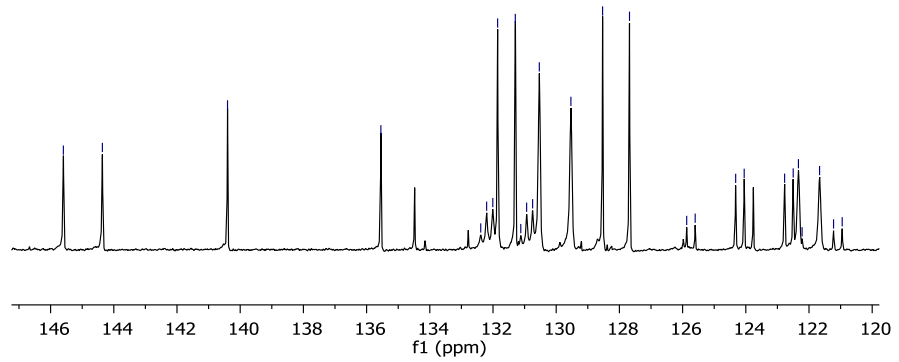

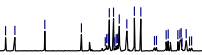

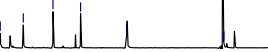

$\begin{array}{lllllllllllllllllllll}200 & 190 & 180 & 170 & 160 & 150 & 140 & 130 & 120 & 110 & 100 & 90 & 80 & 70 & 60 & 50 & 40 & 30 & 20 & 10 & 0\end{array}$ 
2-(((1S,2S)-1-((S)-2-(bis(3,5-bis(trifluoromethyl)phenyl)((trimethylsilyl)oxy)methyl)pyrrolidin-1yl)-2-chloro-3-methylbutyl)carbamoyl)benzyl 3,5-dinitrobenzoate (15)<smiles>[M]OC(c1cc(C(F)(F)F)cc(C(F)(F)F)c1)(c1cc(C(F)(F)F)cc(C(F)(F)F)c1)C1CCNC1[C@@H](NC(=O)c1ccccc1COC(=O)c1cc([N+](=O)[O-])cc([N+](=O)[O-])c1)[C@@H](Cl)C(C)C</smiles>
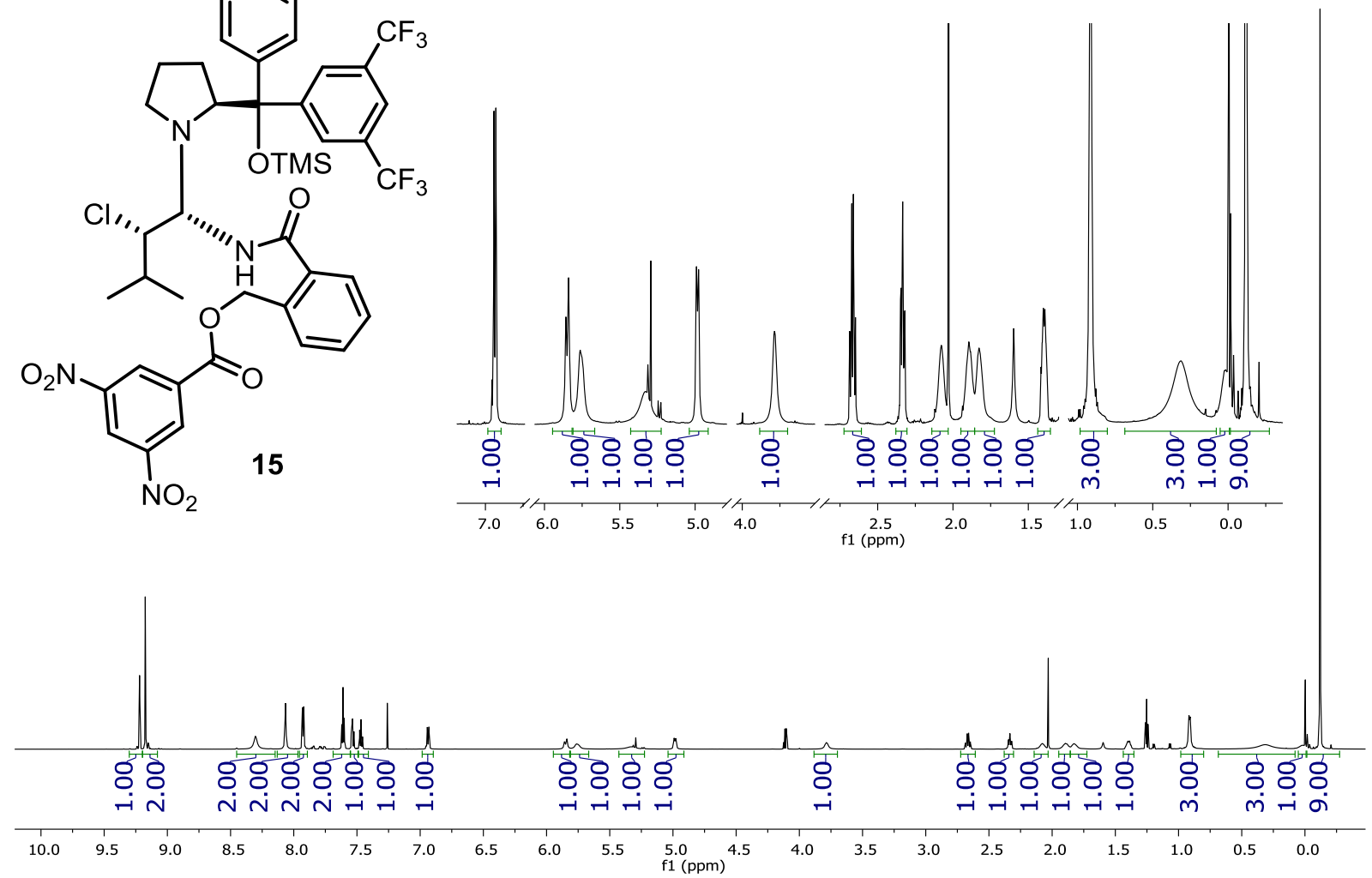

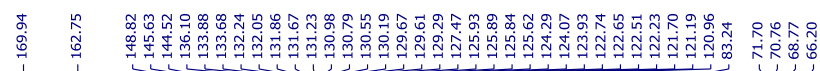

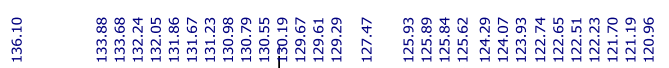
|

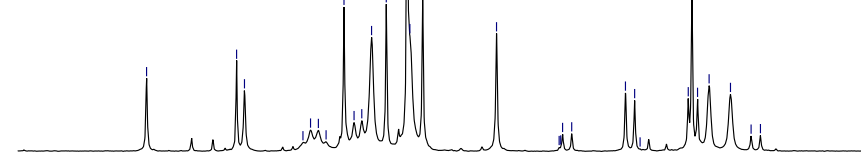

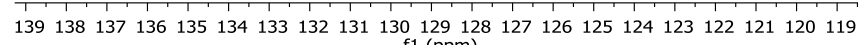

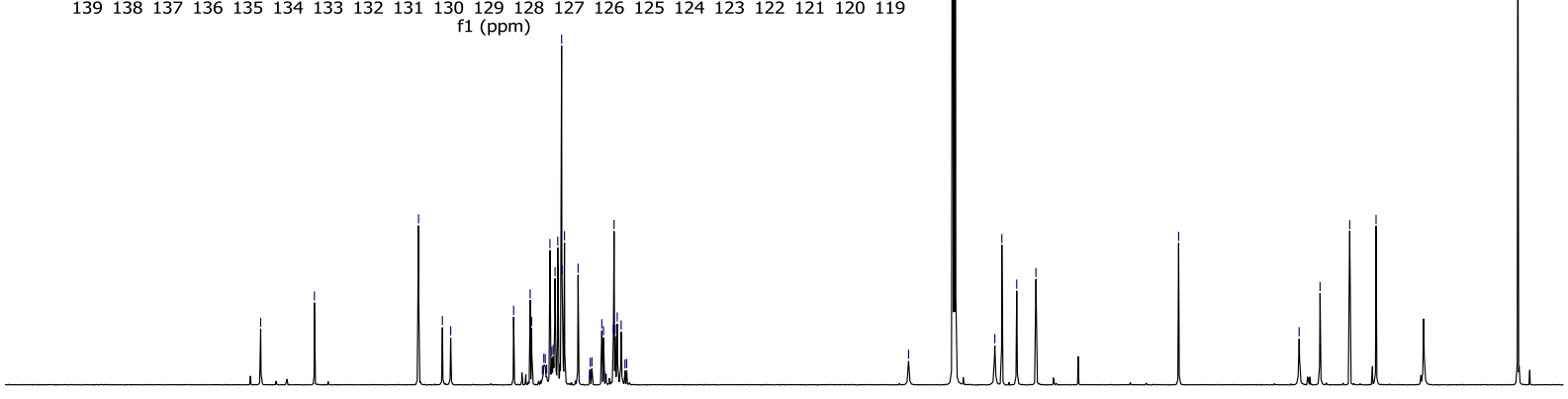

200

$170 \quad 160 \quad 150$

$140 \quad 130$

$120 \quad 110 \quad 100$

$90 \quad 80$

1
$70 \quad 60$

50

$\begin{array}{llll}30 & 20 & 10 & 1\end{array}$ 
Decomposition of Aminal 14 (1) Last sample (70 h, EtOAc impurities)

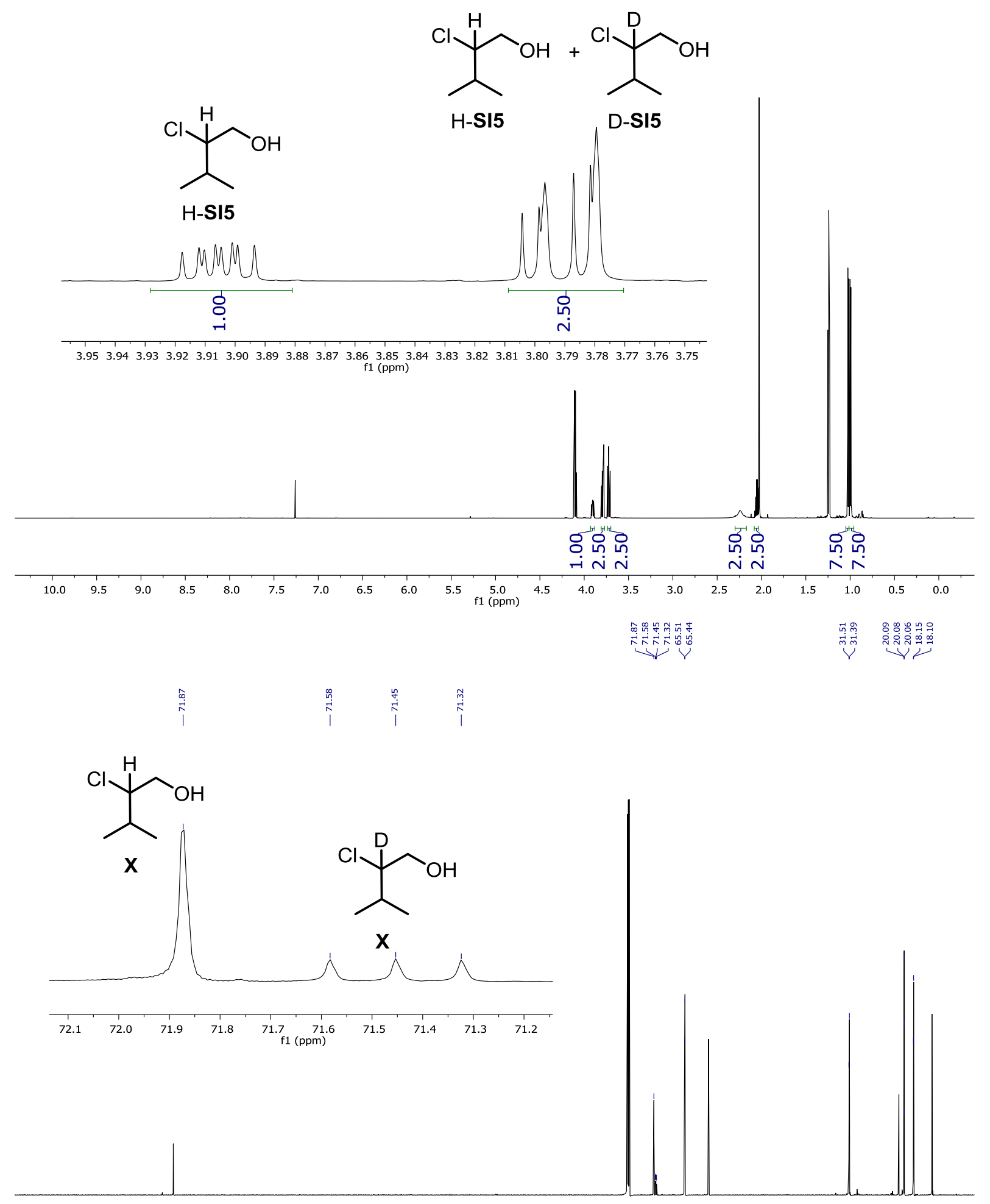

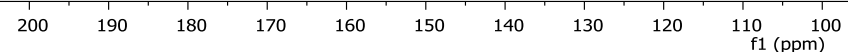


(S)-2-chloro-3-methylbutan-1-ol ((S)-SI5)<smiles>CC(C)[C](Cl)CO</smiles>

(S)-SI5

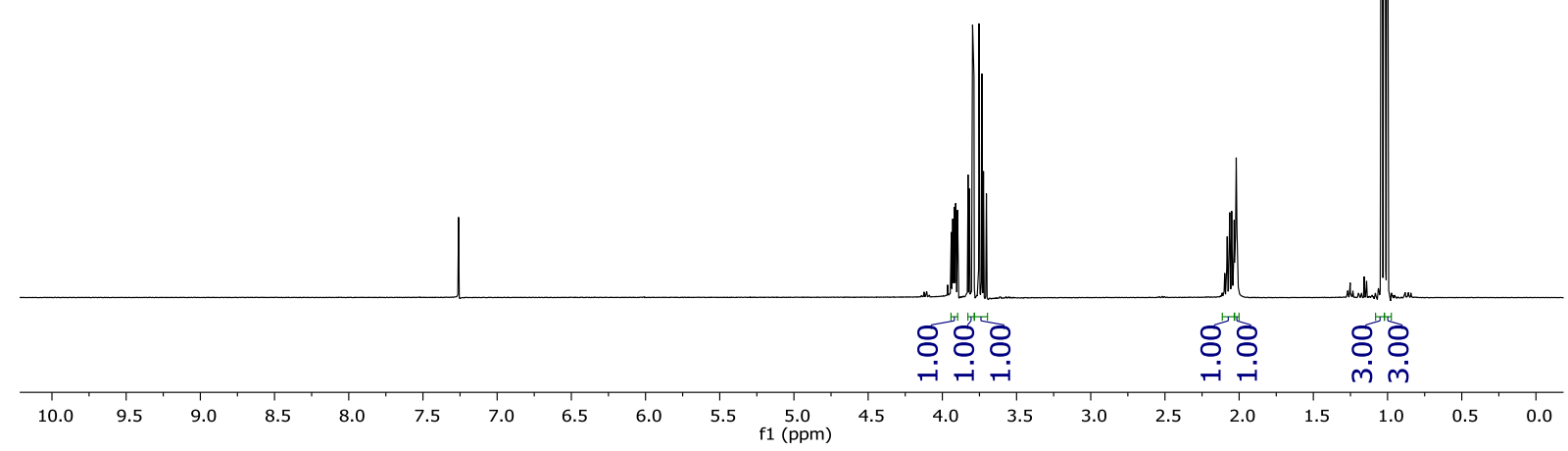


2-(1-((S)-2-(bis(3,5-bis(trifluoromethyl)phenyl)((trimethylsilyl)oxy)methyl)pyrrolidin-1-yl)-2chloropropyl)isoindoline-1,3-dione (SI6)<smiles>COC(c1cc(C(F)(F)F)cc(C(F)(F)F)c1)(c1cc(C(F)(F)F)cc(C(F)(F)F)c1)C1CCCN1C(C(C)Cl)N1C(=O)c2ccccc2C1=O</smiles>
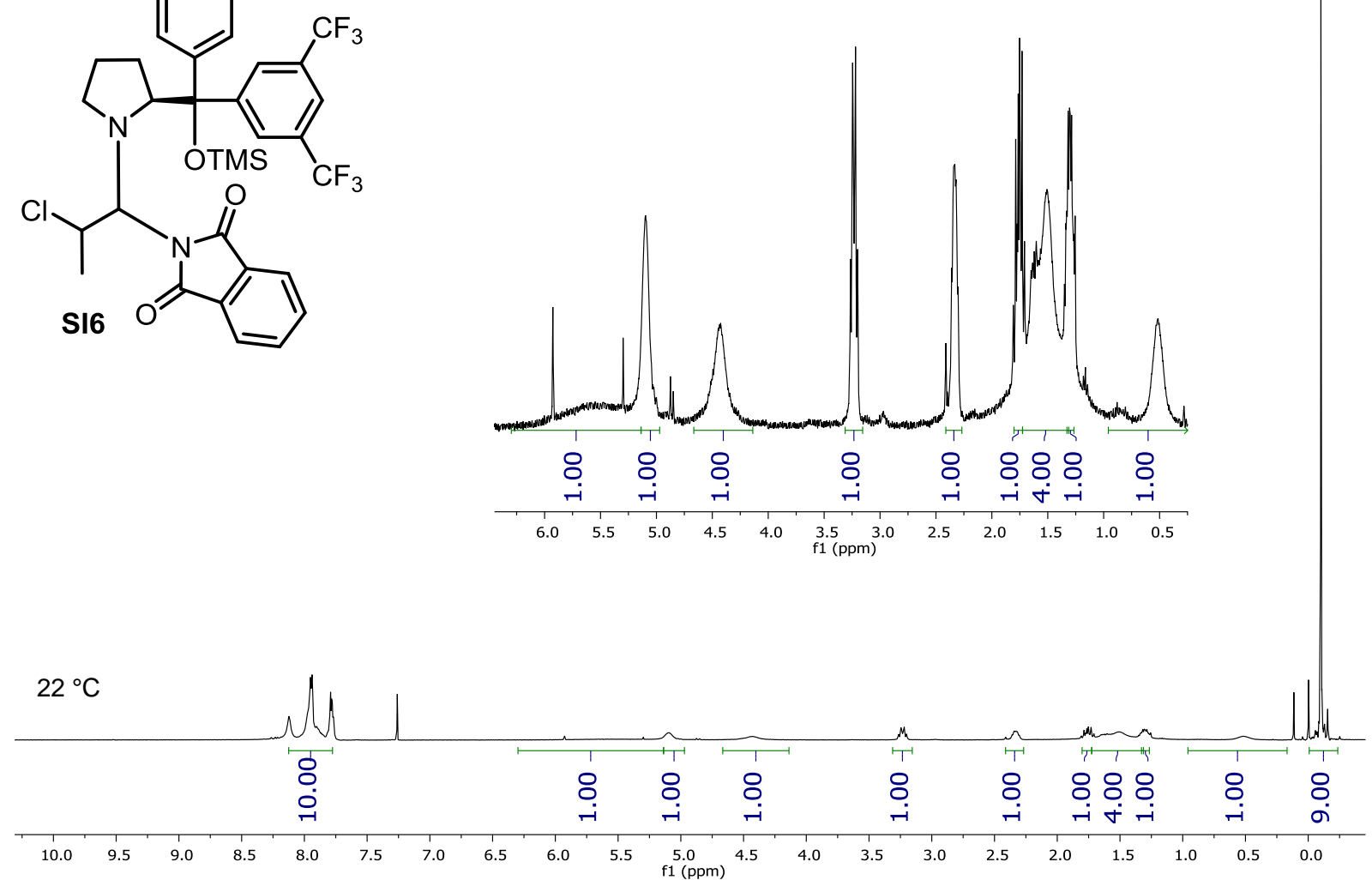
2-(1-((S)-2-(bis(3,5-bis(trifluoromethyl)phenyl)((trimethylsilyl)oxy)methyl)pyrrolidin-1-yl)-2chloropropyl)isoindoline-1,3-dione (SI6)
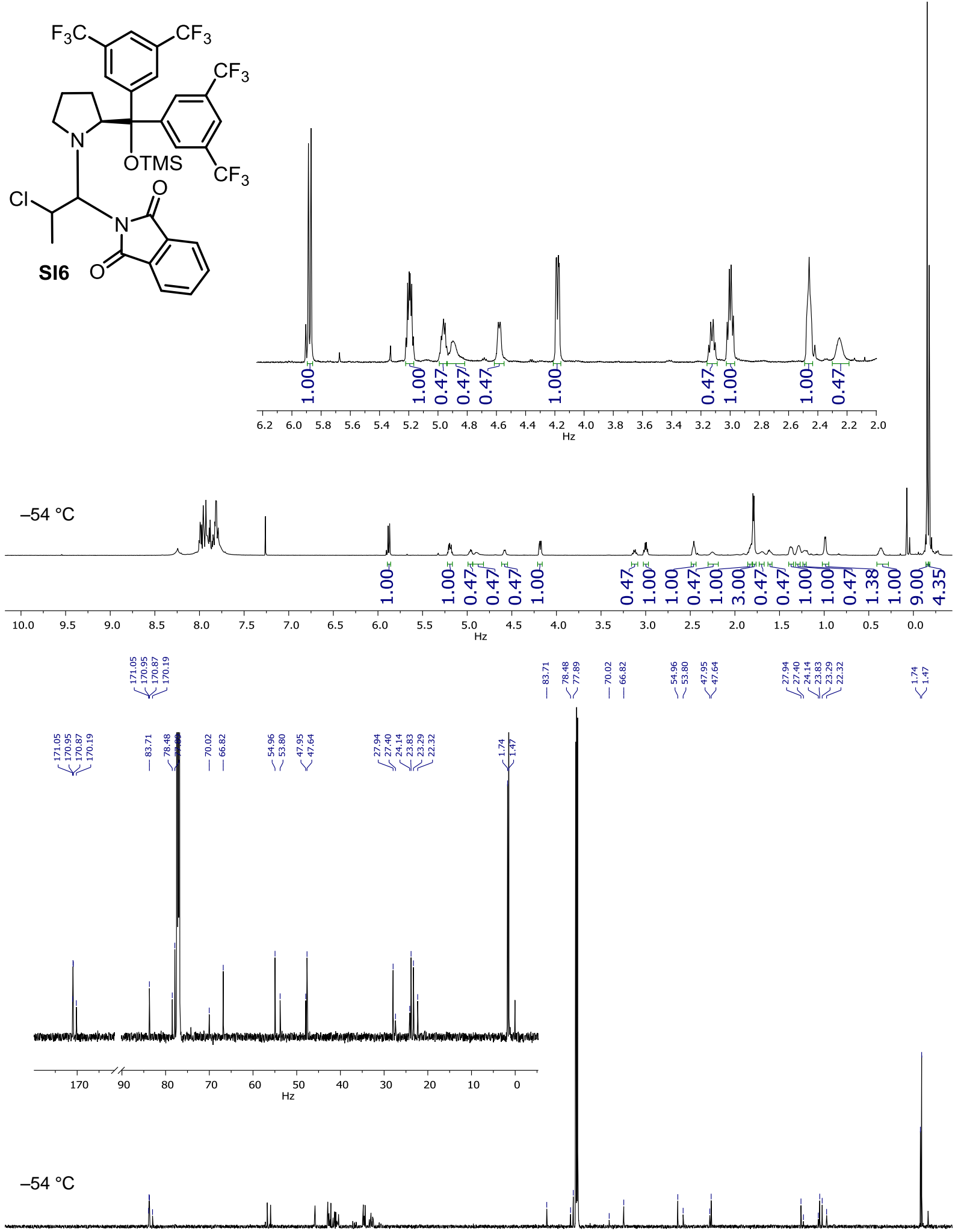

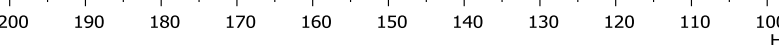


(S)-2-chloropropyl 3,5-dinitrobenzoate ((S)-SI10)<smiles>C[C@H](Cl)COC(=O)c1cc([N+](=O)[O-])cc([N+](=O)[O-])c1</smiles>
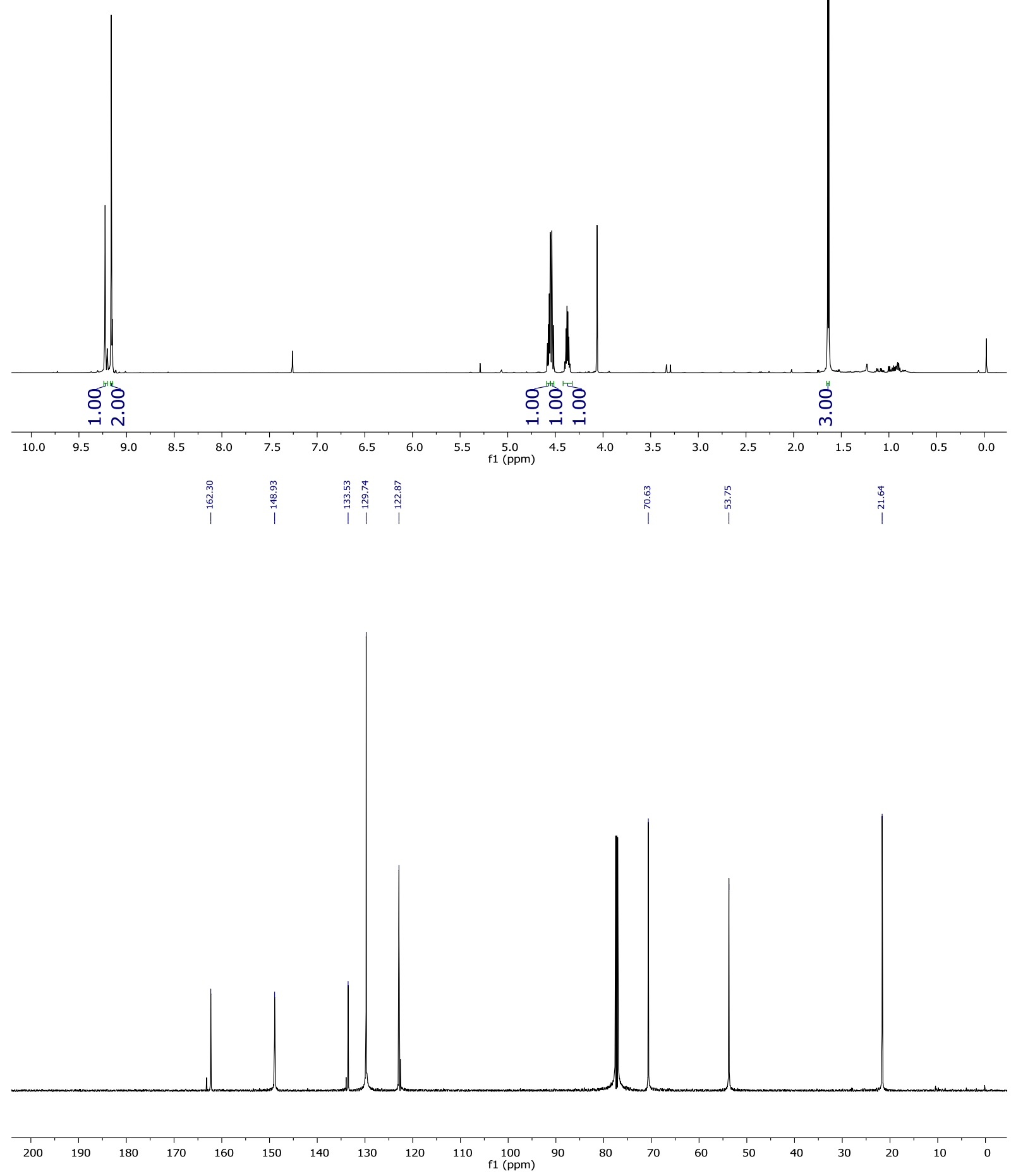
2-chloropropan-1-ol (rac-SI9)<smiles>OC[C@H](Cl)[GeH3]</smiles>

rac-SI9

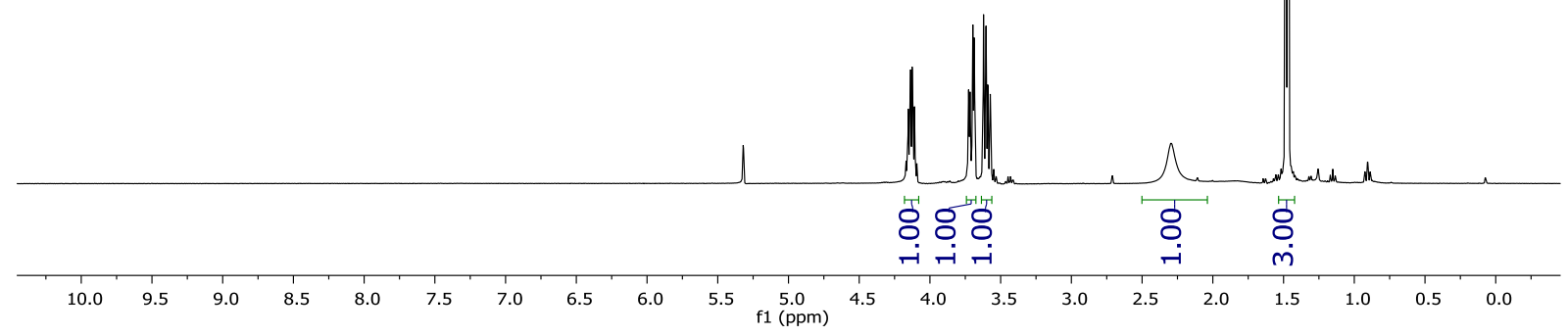


<smiles>OCC(Cl)Br</smiles>

H-22<smiles>[2H]C(Cl)(Br)CO</smiles>

D-22<smiles>CC1NC([18F])N(C)C1=O</smiles>
$3 c$ $20{ }^{\circ} \mathrm{C}$
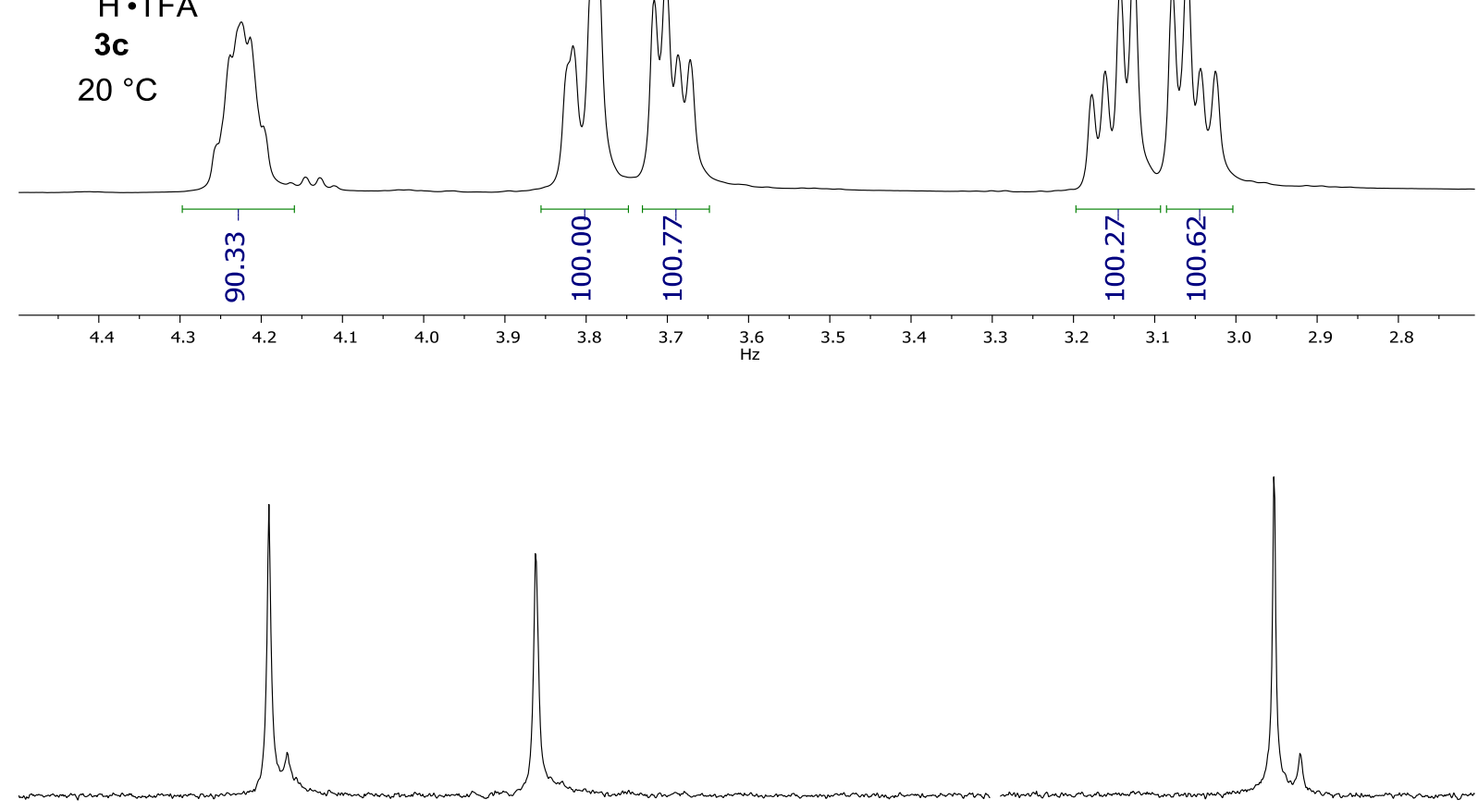

\begin{tabular}{llllllllllllllllllllllllllllllllllllllllllll}
\hline 7.0 & 66.8 & 66.6 & 66.4 & 66.2 & 66.0 & 65.8 & 65.6 & 65.4 & 65.2 & 65.0 & 64.8 & 64.6 & 64.4 & 64.2 & 64.0 & 63.8 & 63.6 & 63.4 & 63.2 & 41.8 & 41.6 & 41.4 & 41.2 & 41.0 & 40.8 & 40.6 & 40.4 & 40.2 & 40
\end{tabular}
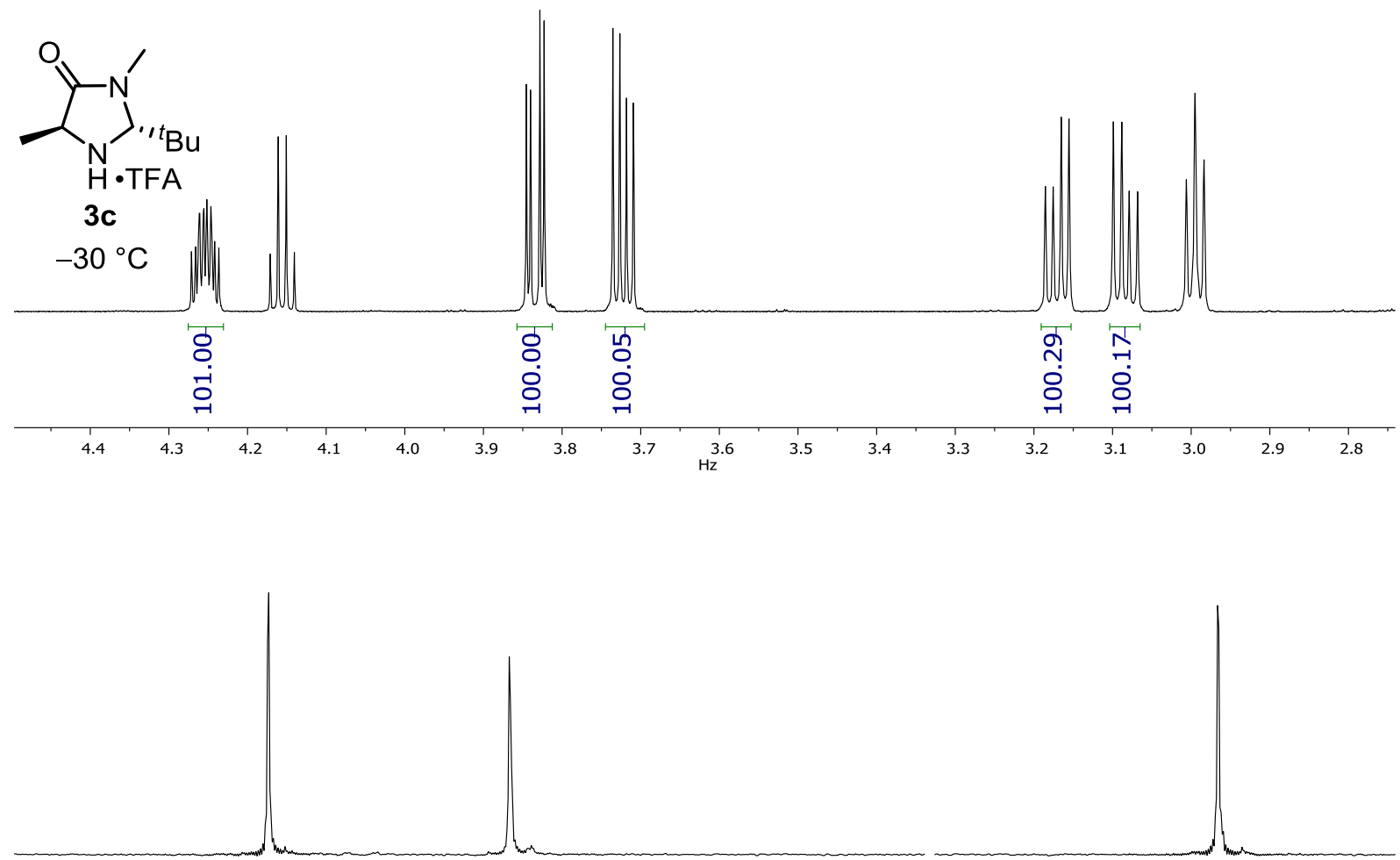

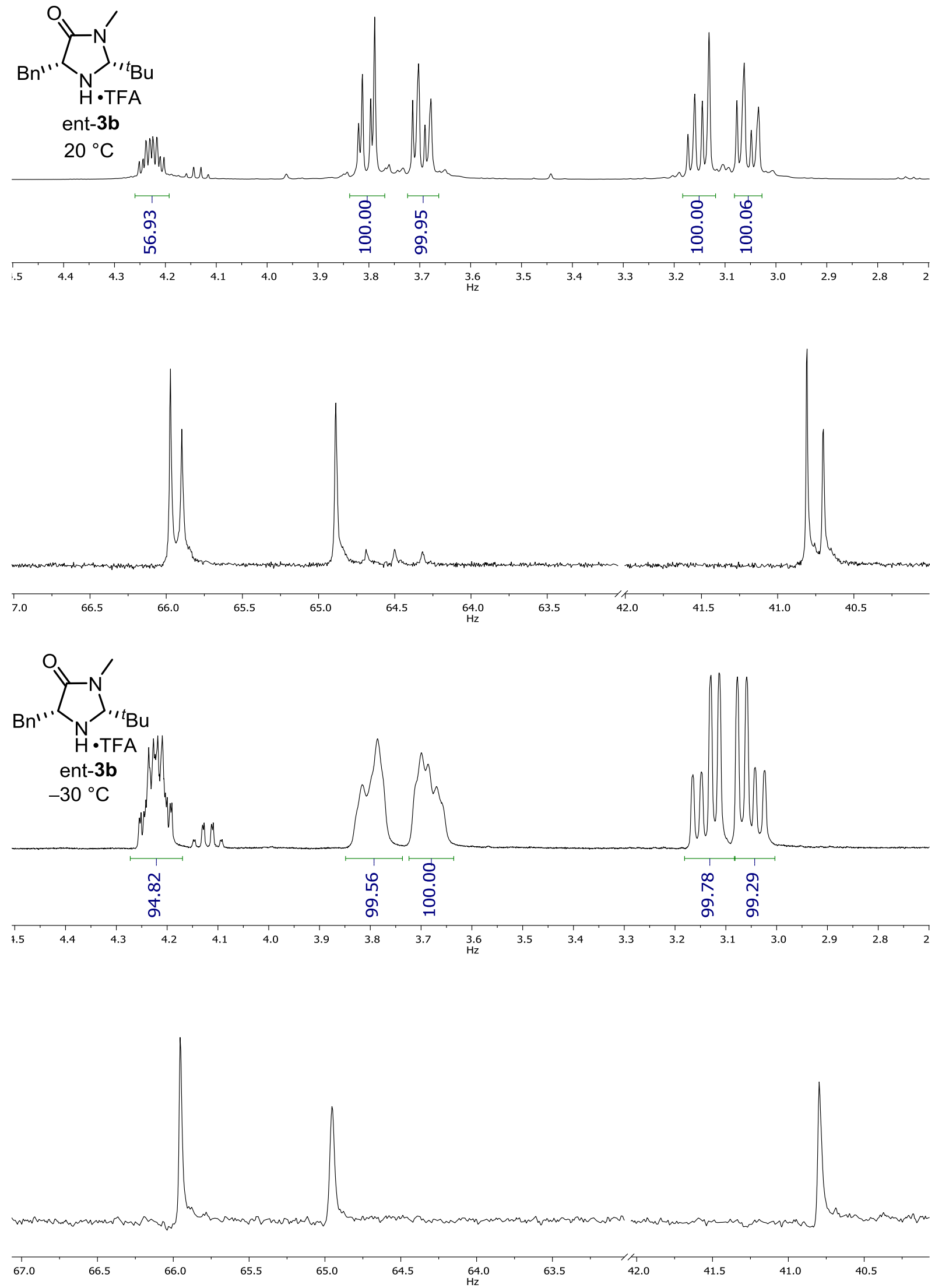

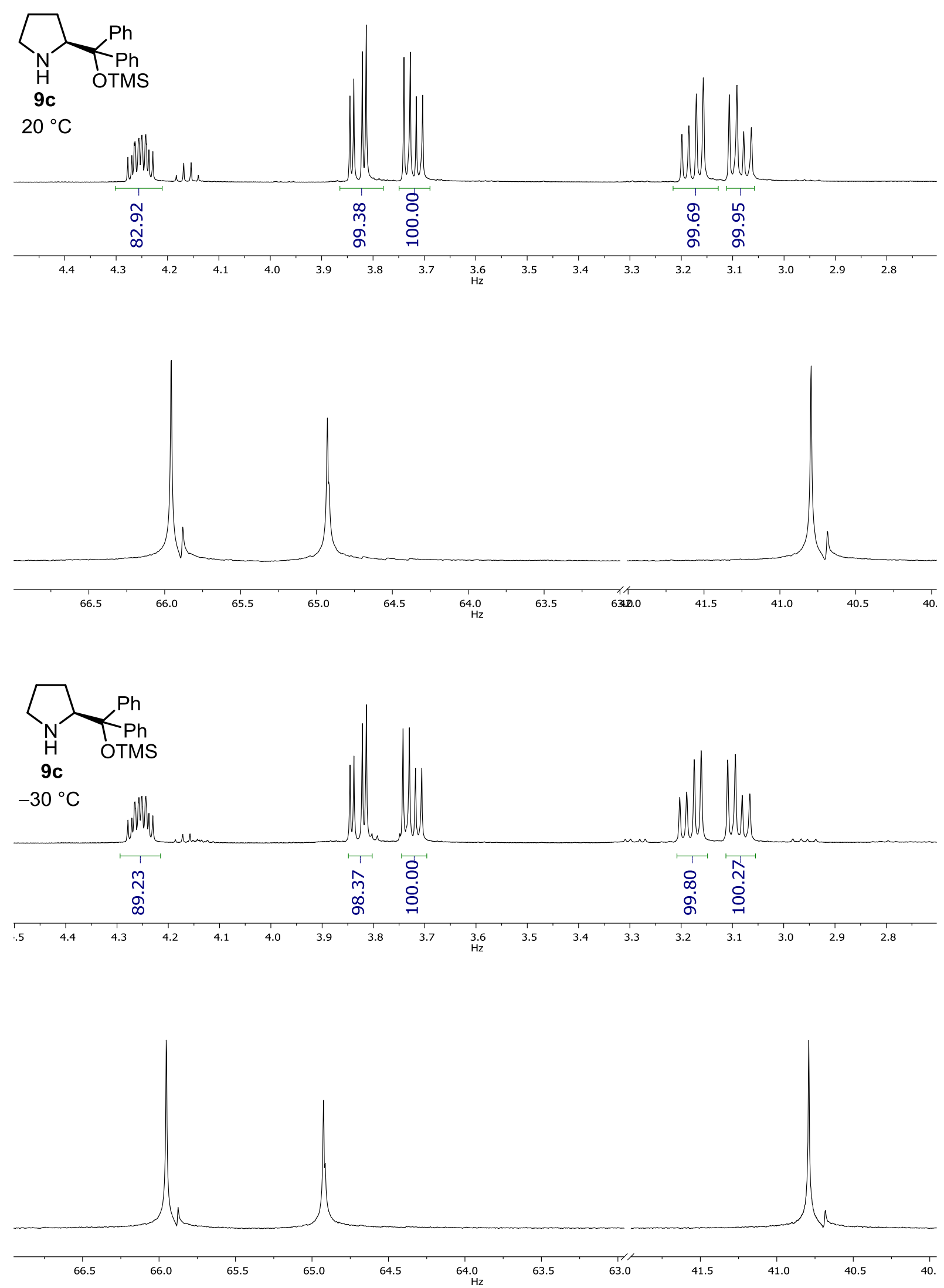

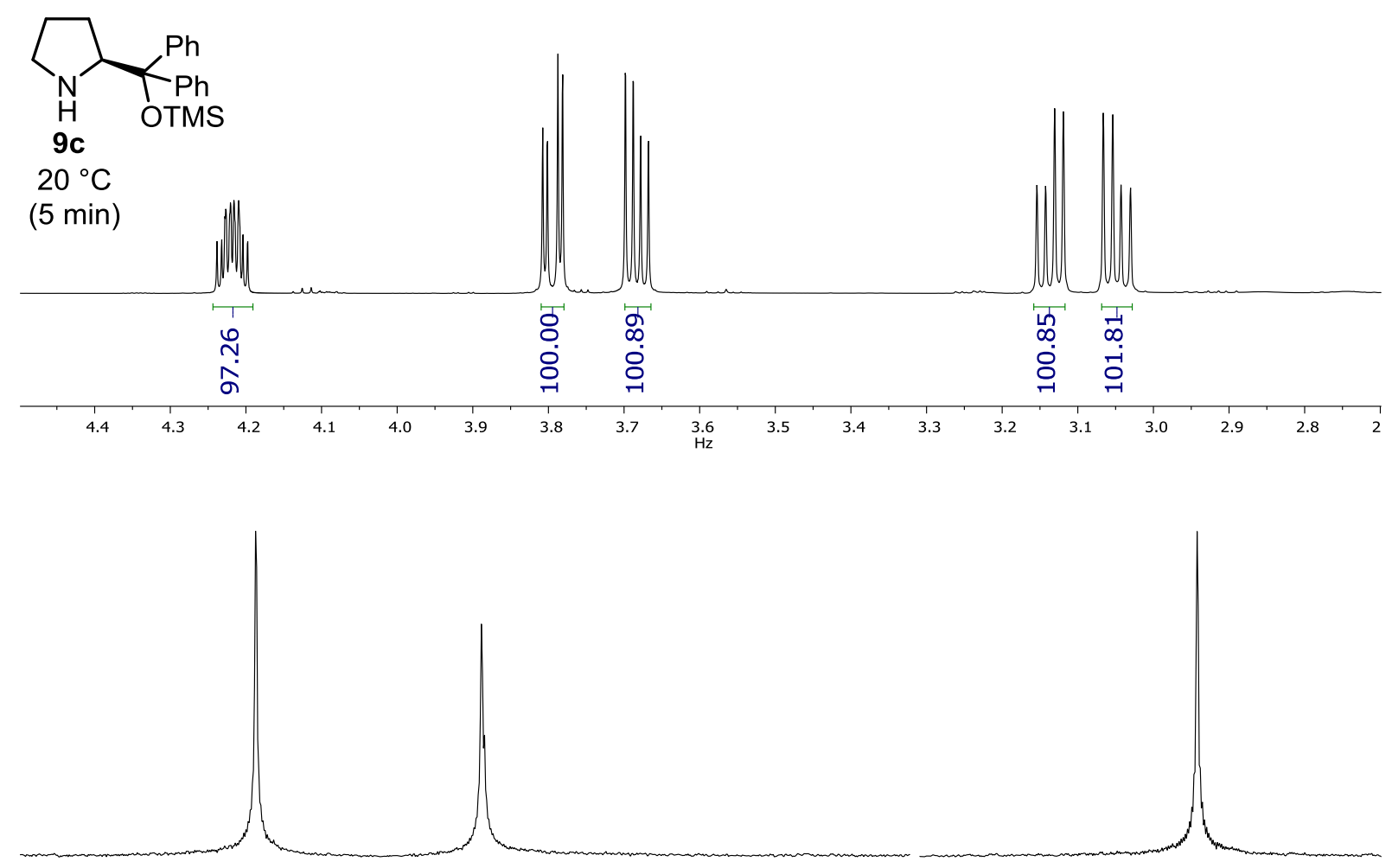

$\begin{array}{lllllllllllllllllllllllllllllllllllllllllll}7.0 & 66.8 & 66.6 & 66.4 & 66.2 & 66.0 & 65.8 & 65.6 & 65.4 & 65.2 & 65.0 & 64.8 & 64.6 & 64.4 & 64.2 & 64.0 & 63.8 & 63.6 & 63.4 & 63.2 & 42.0 & 41.8 & 41.6 & 41.4 & 41.2 & 41.0 & 40.8 & 40.6 & 40.4 & 40.2 & 40 .\end{array}$ 
2,2-dichloro-3-phenylpropan-1-ol (SI12)

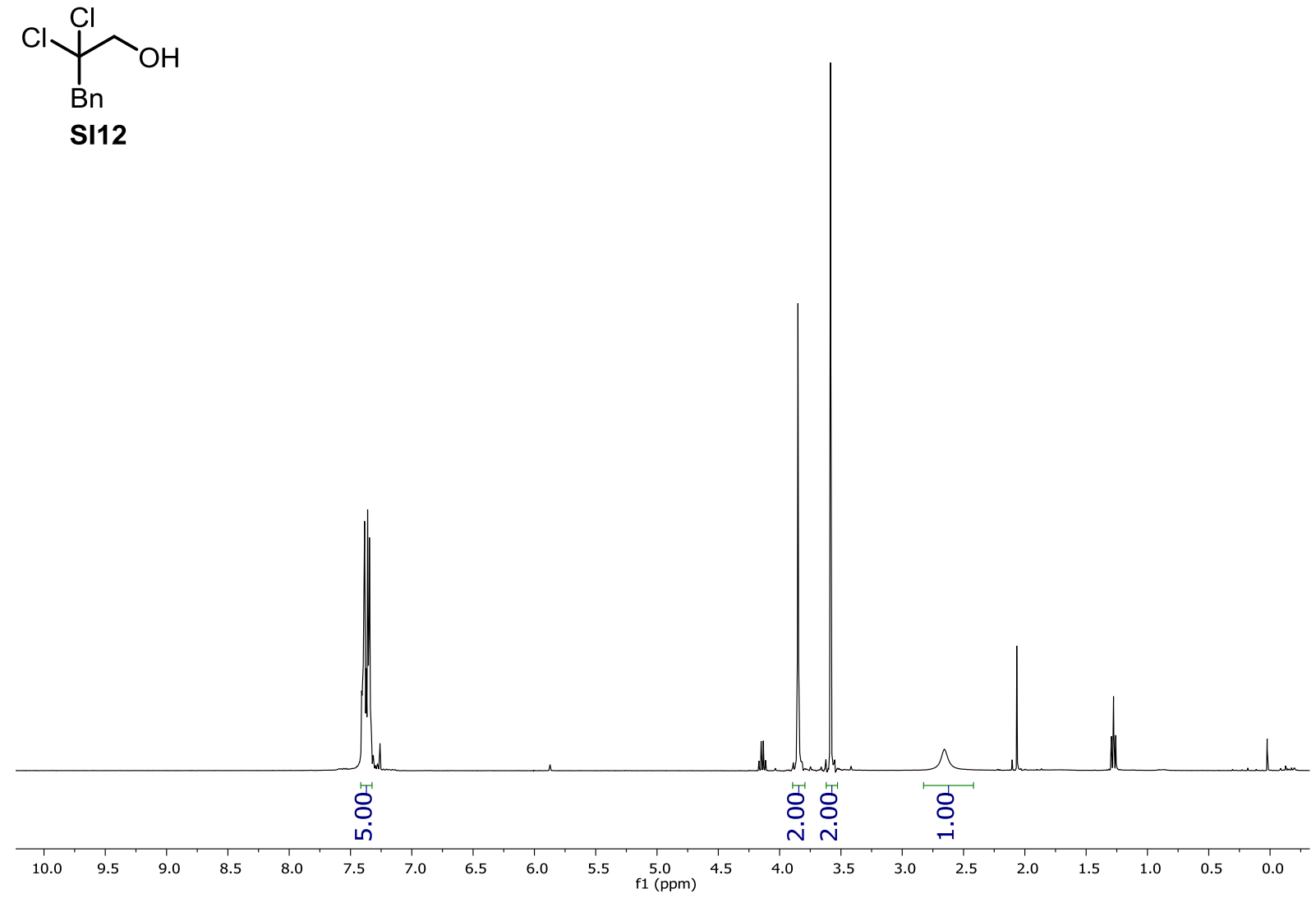


3-phenylpropanal-2,2- $d_{2}\left(D_{2}-16\right)$<smiles>[2H]C([2H])(Br)C=O</smiles>

$D_{2}-16$

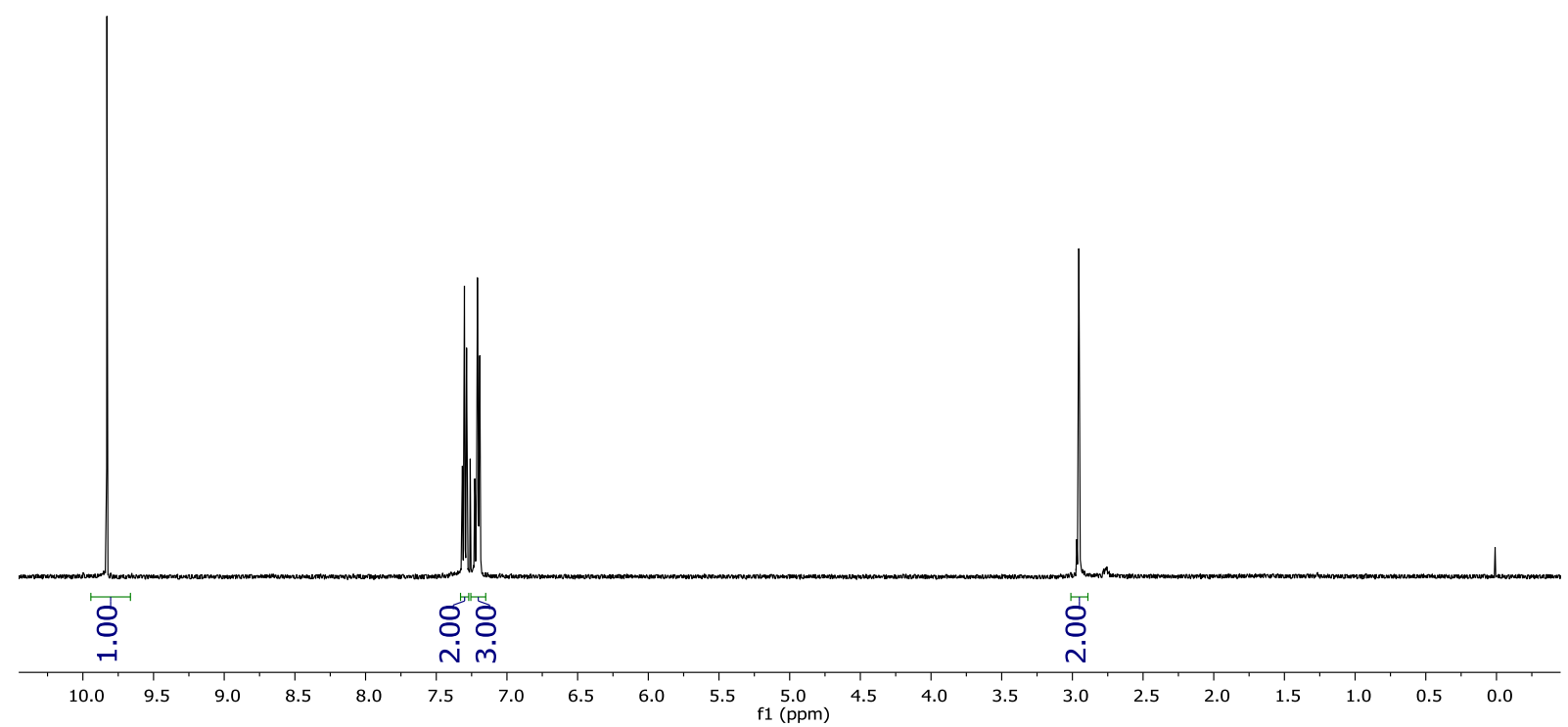


Isotopic competition experiment<smiles>OCC(Cl)Br</smiles>

$\mathrm{H}-22$<smiles>[2H]C(Cl)(Br)CO</smiles>

D-22

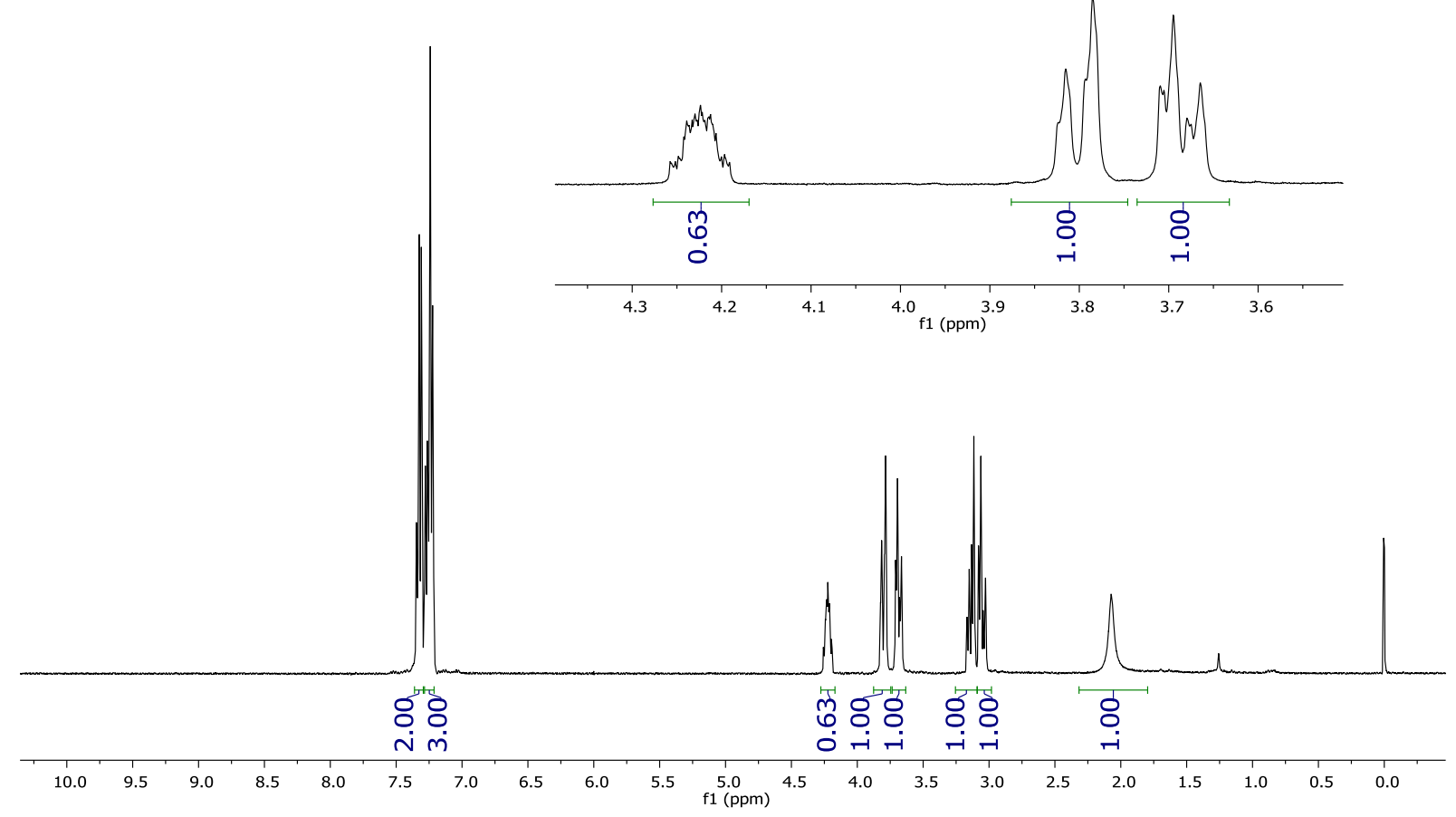




\subsection{HPLC /GC Traces}

1-((1S,2R)-1-((2R,5R)-5-benzyl-2-(tert-butyl)-3-methyl-4-oxoimidazolidin-1-yl)-2-chloro-3methylbutyl)pyrrolidine-2,5-dione ((1S,2R)-syn-21), 1-((1S,2S)-1-((2R,5R)-5-benzyl-2-(tert-butyl)3-methyl-4-oxoimidazolidin-1-yl)-2-chloro-3-methylbutyl)pyrrolidine-2,5-dione ((1S,2S)-anti-21), 1-((1R,2S)-1-((2R,5R)-5-benzyl-2-(tert-butyl)-3-methyl-4-oxoimidazolidin-1-yl)-2-chloro-3methylbutyl)pyrrolidine-2,5-dione ((1R,2S)-syn-21)

HPLC: $1.0 \mathrm{ml} / \mathrm{min}, 25 \% \mathrm{PrOH} /$ hexane, Nucleosil 50-5 $(0.4 \times 25 \mathrm{~cm})$

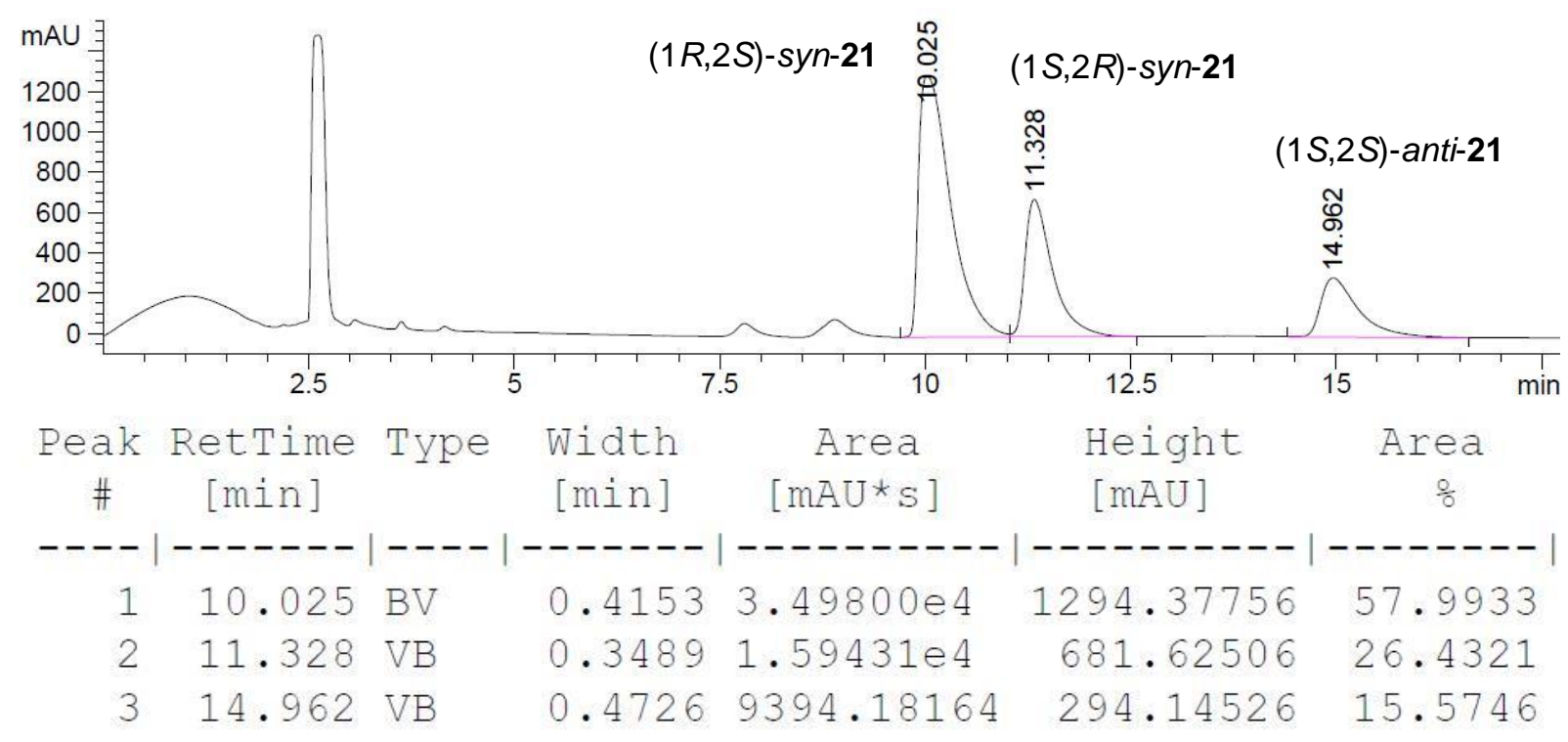<smiles>CC(C)C(Cl)C(N1C(=O)CCC1=O)N1C(=O)[C@@H](Br)C(=O)N1C</smiles>

$(1 S, 2 R)-s y n-21$<smiles>CC(C)[C@H](Cl)[C@H](N1C(=O)CCC1=O)N1C([13CH3])N(C)C(=O)[C@H]1Br</smiles>

$(1 S, 2 S)-a n t i-21$<smiles>CC(C)[C@H](Cl)[C@@H](N1C(=O)CCC1=O)N1C(=O)N(C)C(=O)[C@H]1Br</smiles>

$(1 R, 2 S)-s y n-21$ 
1-((1S,2R)-1-((2R,5R)-5-benzyl-2-(tert-butyl)-3-methyl-4-oxoimidazolidin-1-yl)-2-chloro-3phenylpropyl)pyrrolidine-2,5-dione $\quad((1 S, 2 R)$-syn-25), $\quad$ 1-((1S,2S)-1-((2R,5R)-5-benzyl-2-(tertbutyl)-3-methyl-4-oxoimidazolidin-1-yl)-2-chloro-3-phenylpropyl)pyrrolidine-2,5-dione ((1S,2S)anti-25), 1-((1R,2S)-1-((2R,5R)-5-benzyl-2-(tert-butyl)-3-methyl-4-oxoimidazolidin-1-yl)-2-chloro3-phenylpropyl)pyrrolidine-2,5-dione ((1R,2S)-syn-25)

HPLC: $1.0 \mathrm{ml} / \mathrm{min}, 20 \% \mathrm{PrOH} /$ hexane, Nucleosil 50-5 $(0.4 \times 25 \mathrm{~cm})$

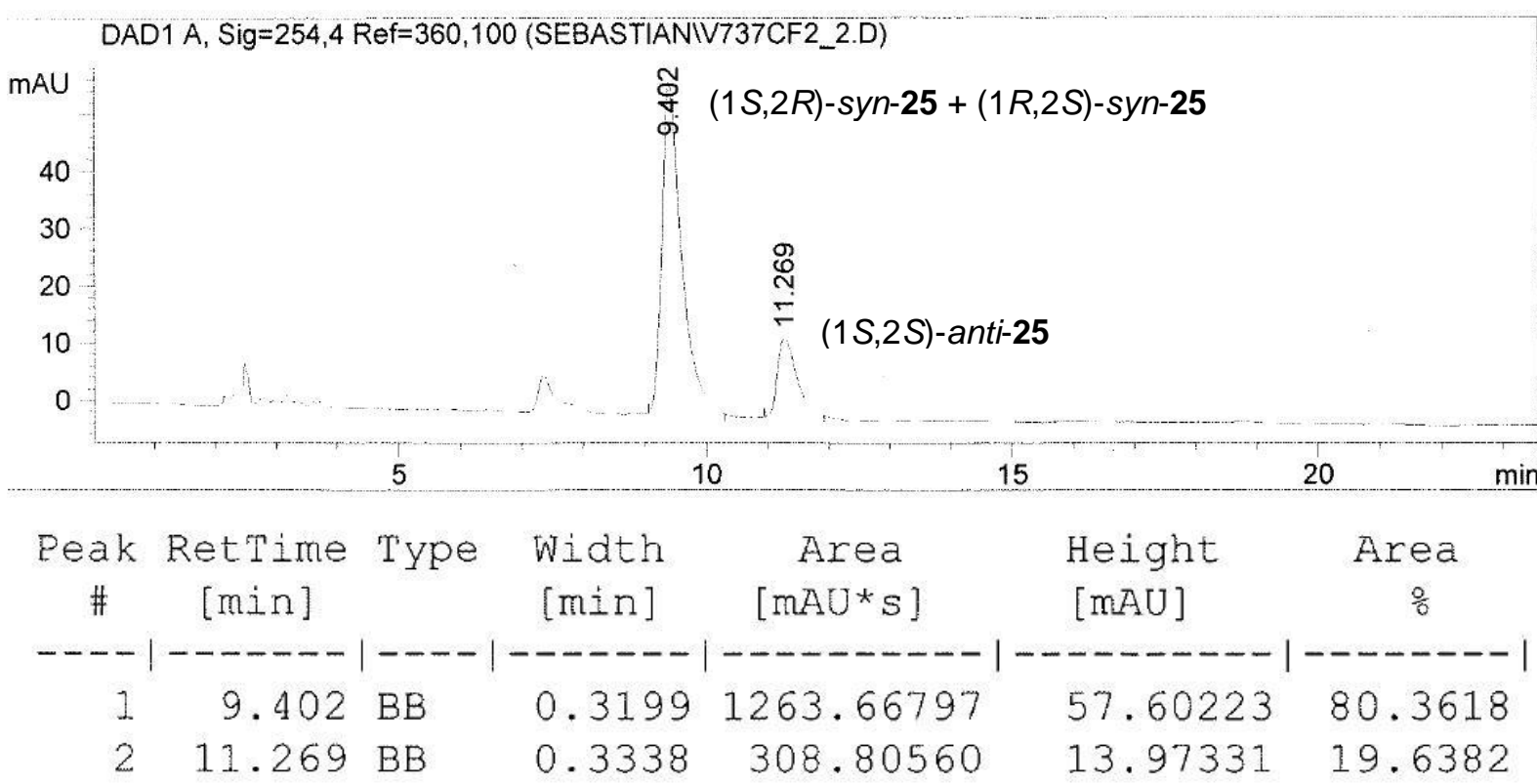

HPLC: $20.0 \mathrm{ml} / \mathrm{min}, 5 \% \mathrm{EtOH} /$ hexane, Nucleosil 50-5 (1.6 x $25 \mathrm{~cm})$

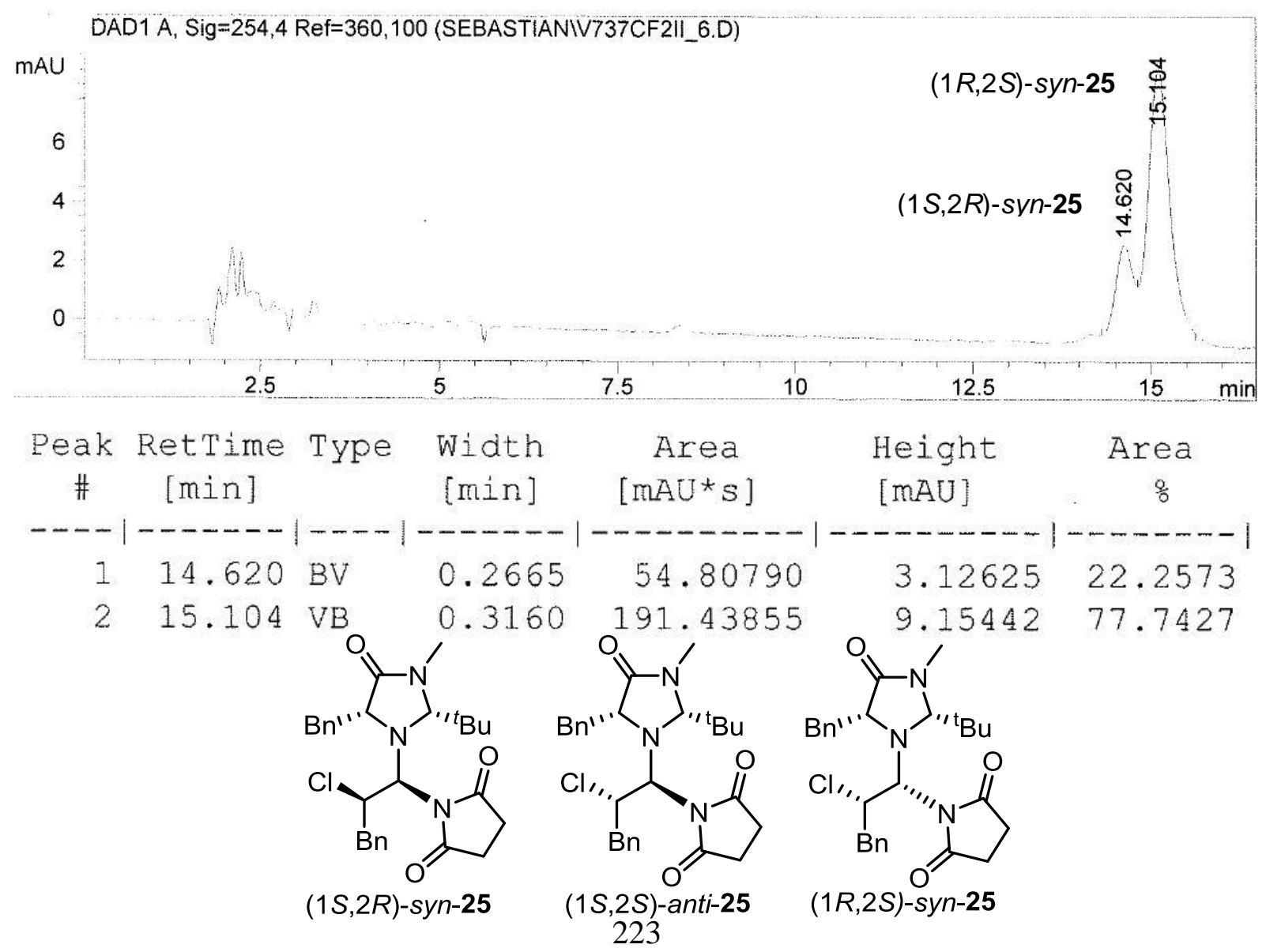




\section{Decomposition of Aminal syn-18b}

HPLC: $0.8 \mathrm{ml} / \mathrm{min}, 5 \% \mathrm{EtOH} /$ hexane, Chiralpak IA $(0.46 \times 25 \mathrm{~cm})$

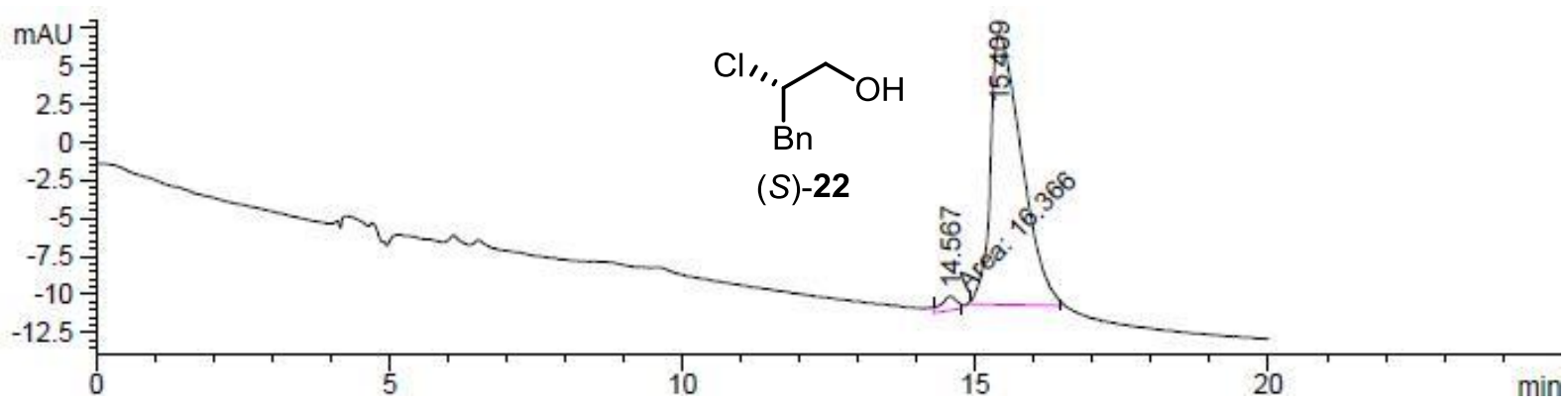

\begin{tabular}{|c|c|c|c|c|c|c|}
\hline $\begin{array}{c}\text { Peak } \\
\#\end{array}$ & $\begin{array}{c}\text { Retrime } \\
\text { [min] }\end{array}$ & Type & $\begin{array}{l}\text { Width } \\
\text { [min] }\end{array}$ & $\begin{array}{c}\text { Area } \\
{\left[\mathrm{mAU}{ }^{\star} s\right]}\end{array}$ & $\begin{array}{l}\text { Height } \\
\text { [mAU] }\end{array}$ & $\begin{array}{c}\text { Area } \\
\&\end{array}$ \\
\hline & & & & -------- & |----1---- & ----- \\
\hline 1 & 14.567 & MM & 0.2986 & 16.36597 & $9.13470=-1$ & 2.4270 \\
\hline 2 & 15.409 & BB & 0.5419 & 657.95312 & 17.69229 & 97.5730 \\
\hline
\end{tabular}

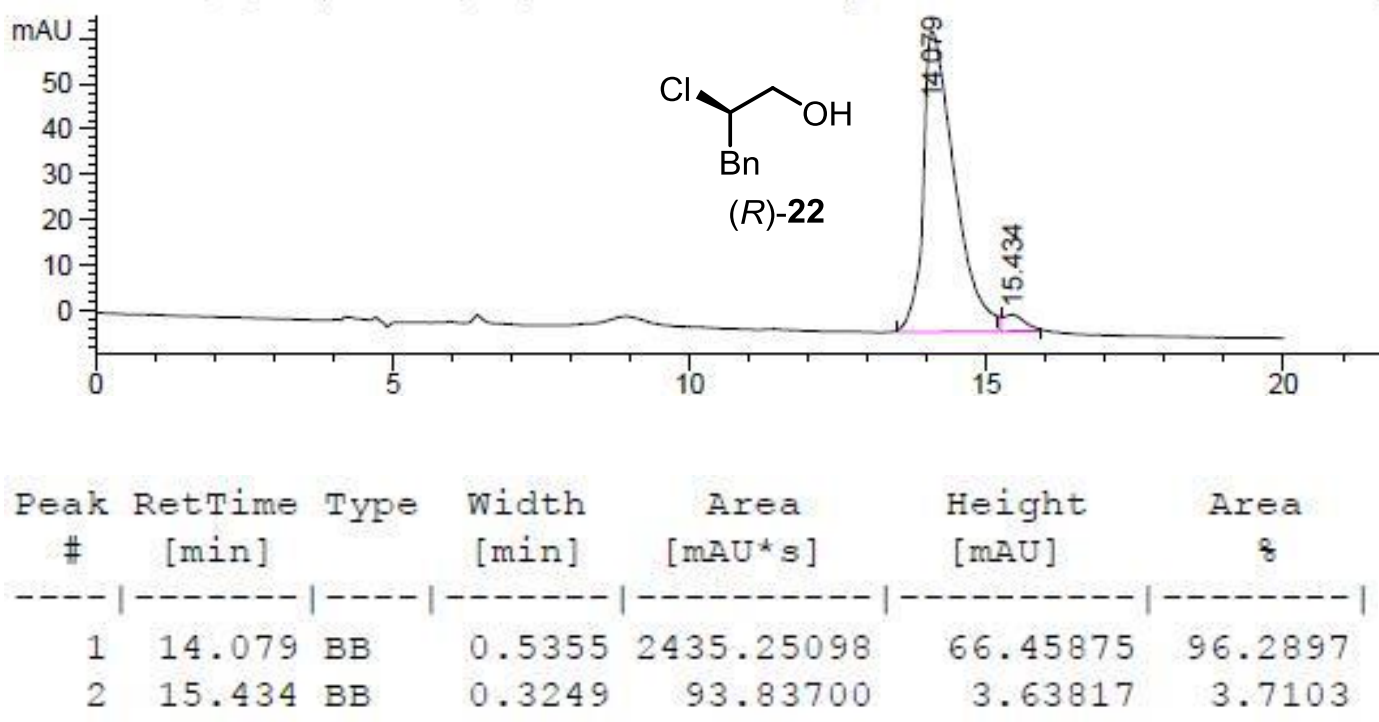



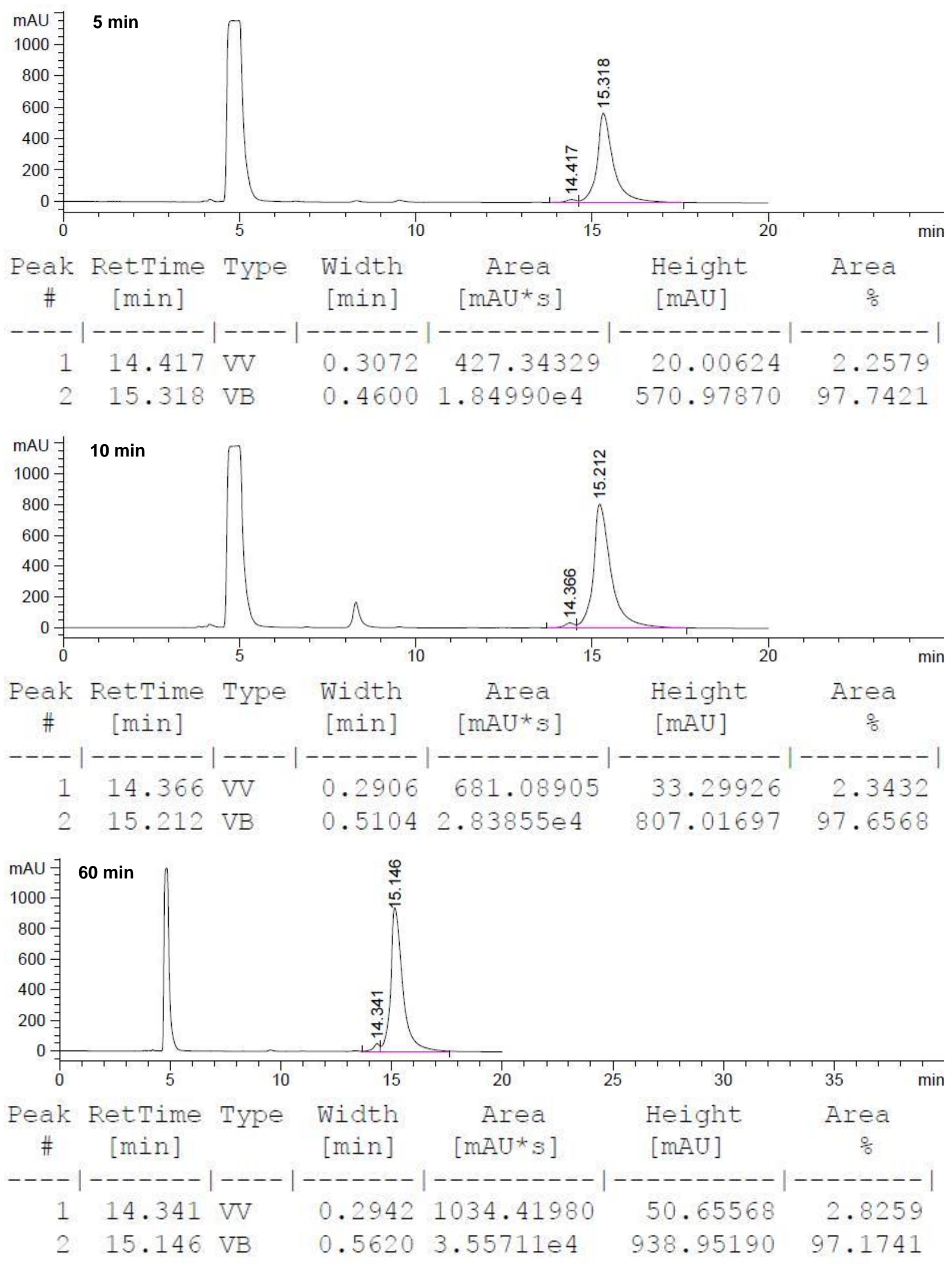


\section{Enantiomeric excess (Isovaleraldehyde, Catalyst $9 b$ and NCS)}

HPLC: $0.8 \mathrm{ml} / \mathrm{min}, 10 \% \mathrm{EtOH} /$ hexane, Chiralpak IA $(0.46 \times 25 \mathrm{~cm})$
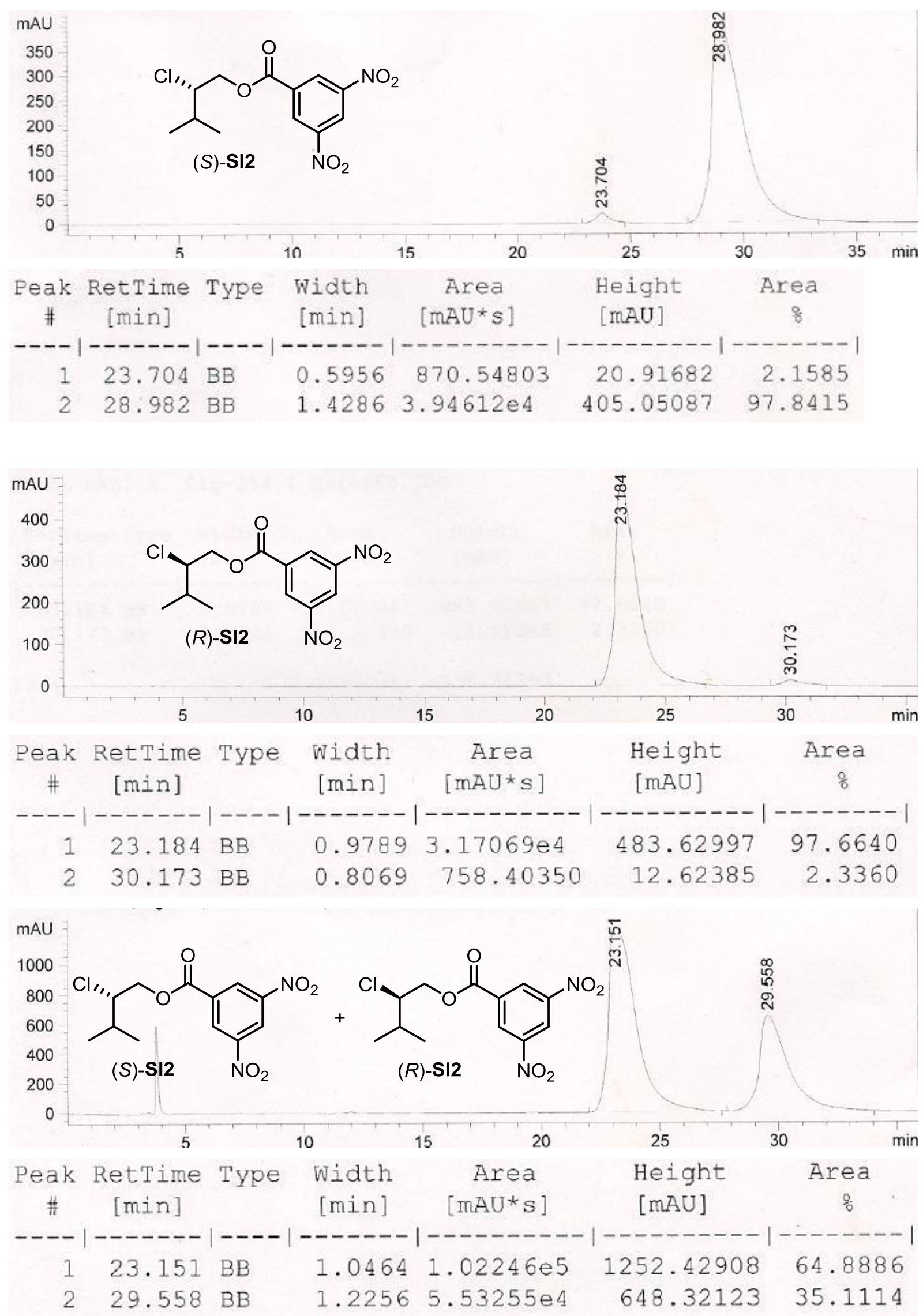


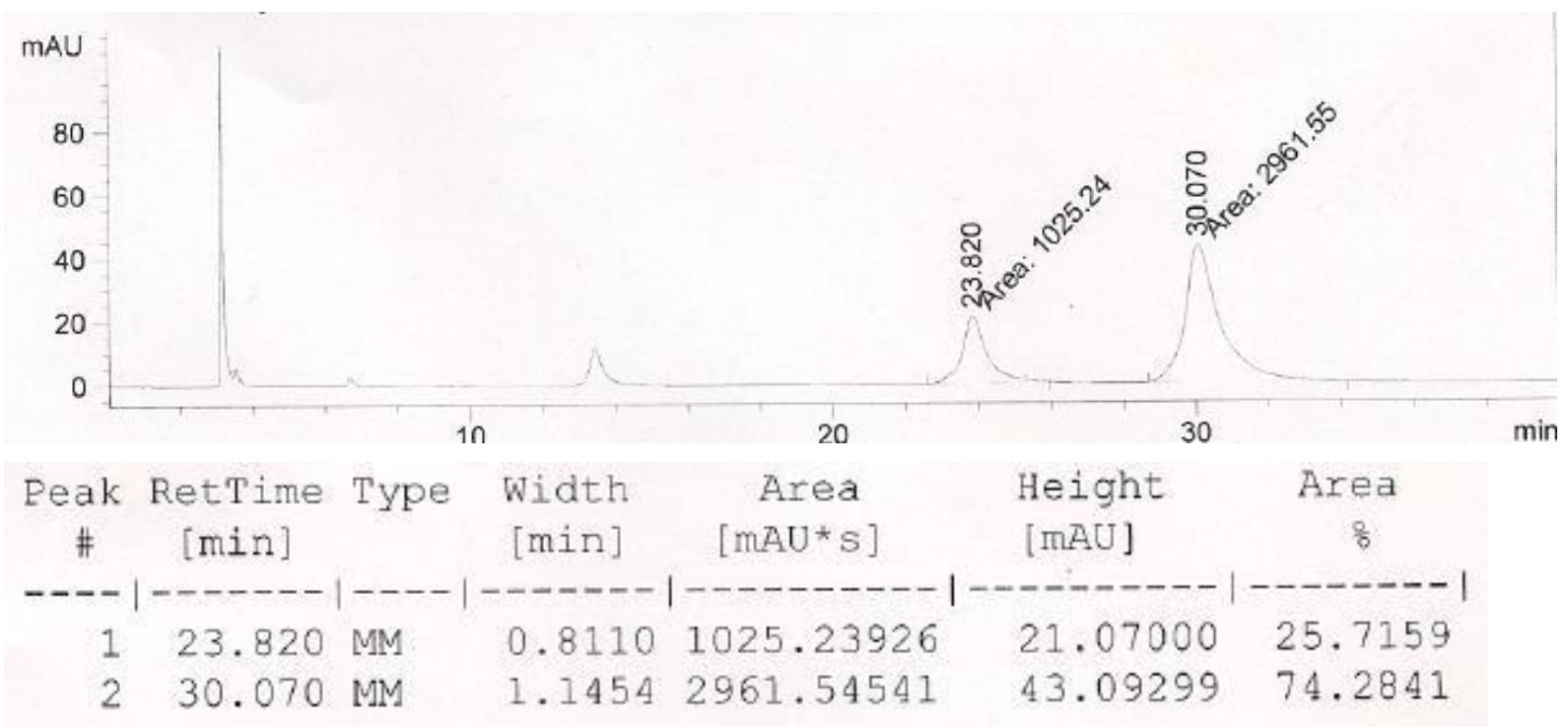




\section{Enantiomeric Excess (Propanal, Catalyst 9b and NCP or NCS)}

HPLC: $1.0 \mathrm{ml} / \mathrm{min}, 20 \% \mathrm{EtOH} /$ hexane, Chiralpak IA (0.46 x $25 \mathrm{~cm})$
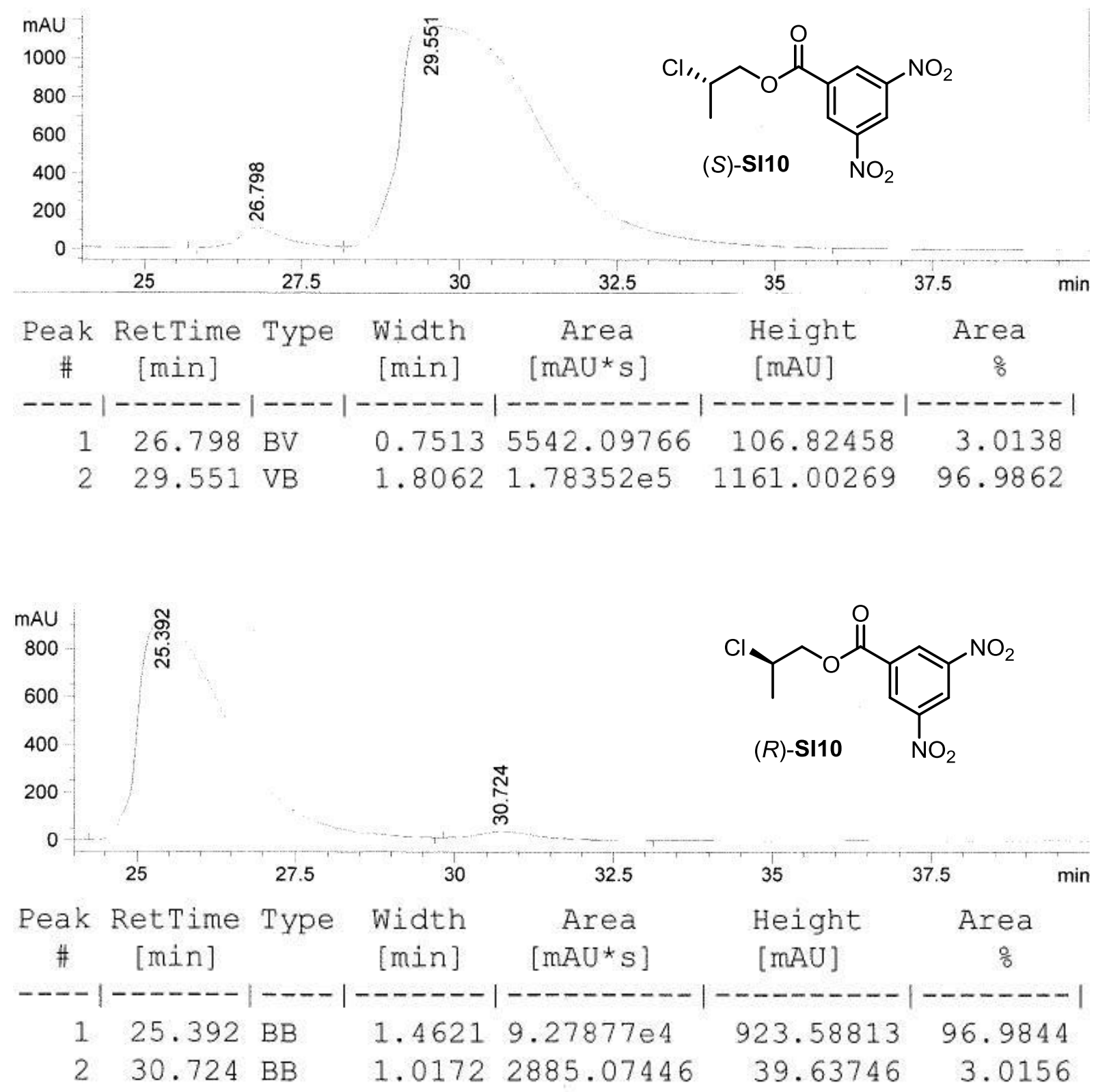


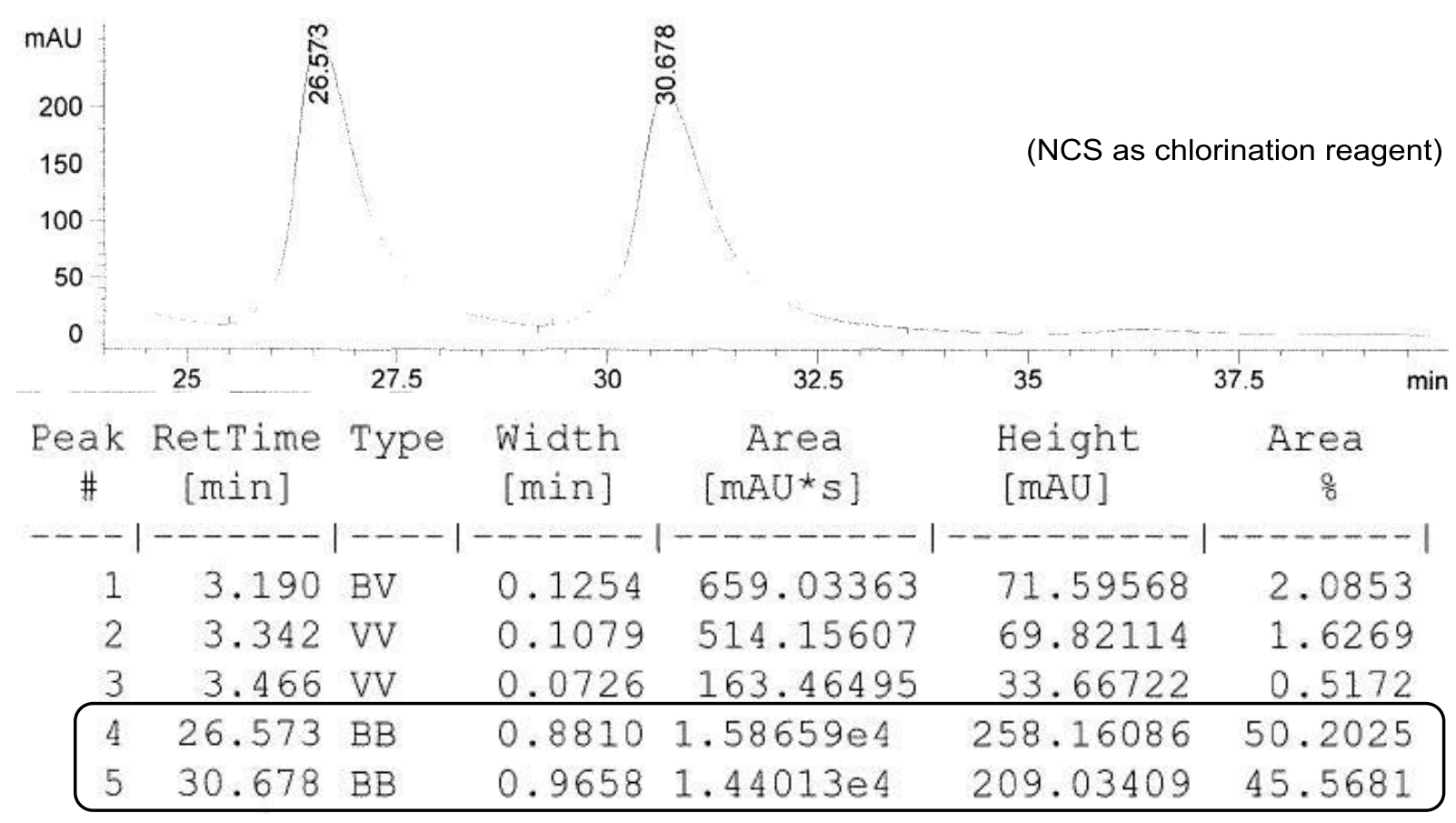

corrected: $\mathrm{t}=26.573$ area: $52.4195 \% ; \mathrm{t}=30.678$ area: $47.5805 \%$

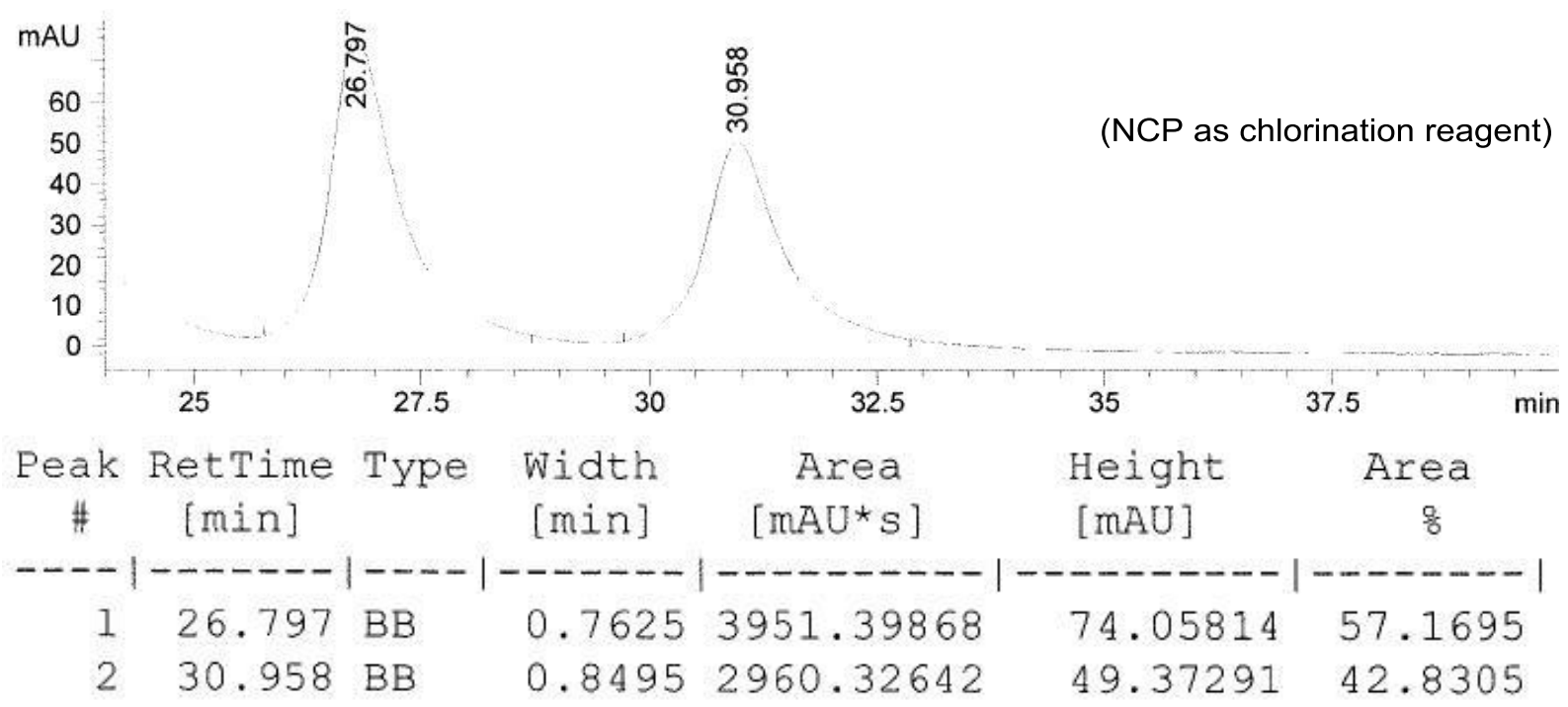


Decomposition of aminal 14 (1)

GC: Lipodex-E, $70^{\circ} \mathrm{C}, 1,1 \mathrm{ml} / \mathrm{min} \mathrm{He}$; FID $300^{\circ} \mathrm{C}$, Split 50:1
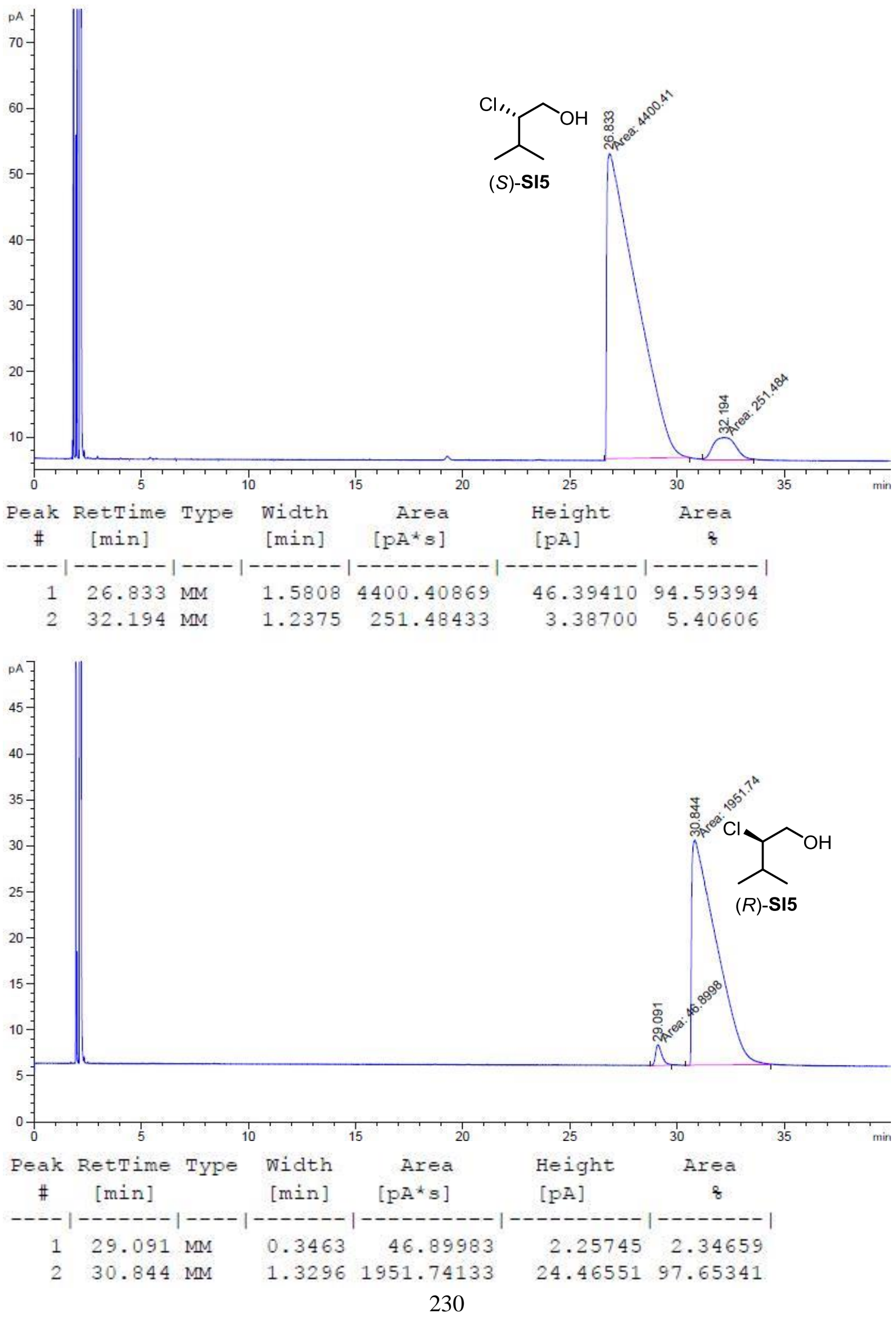

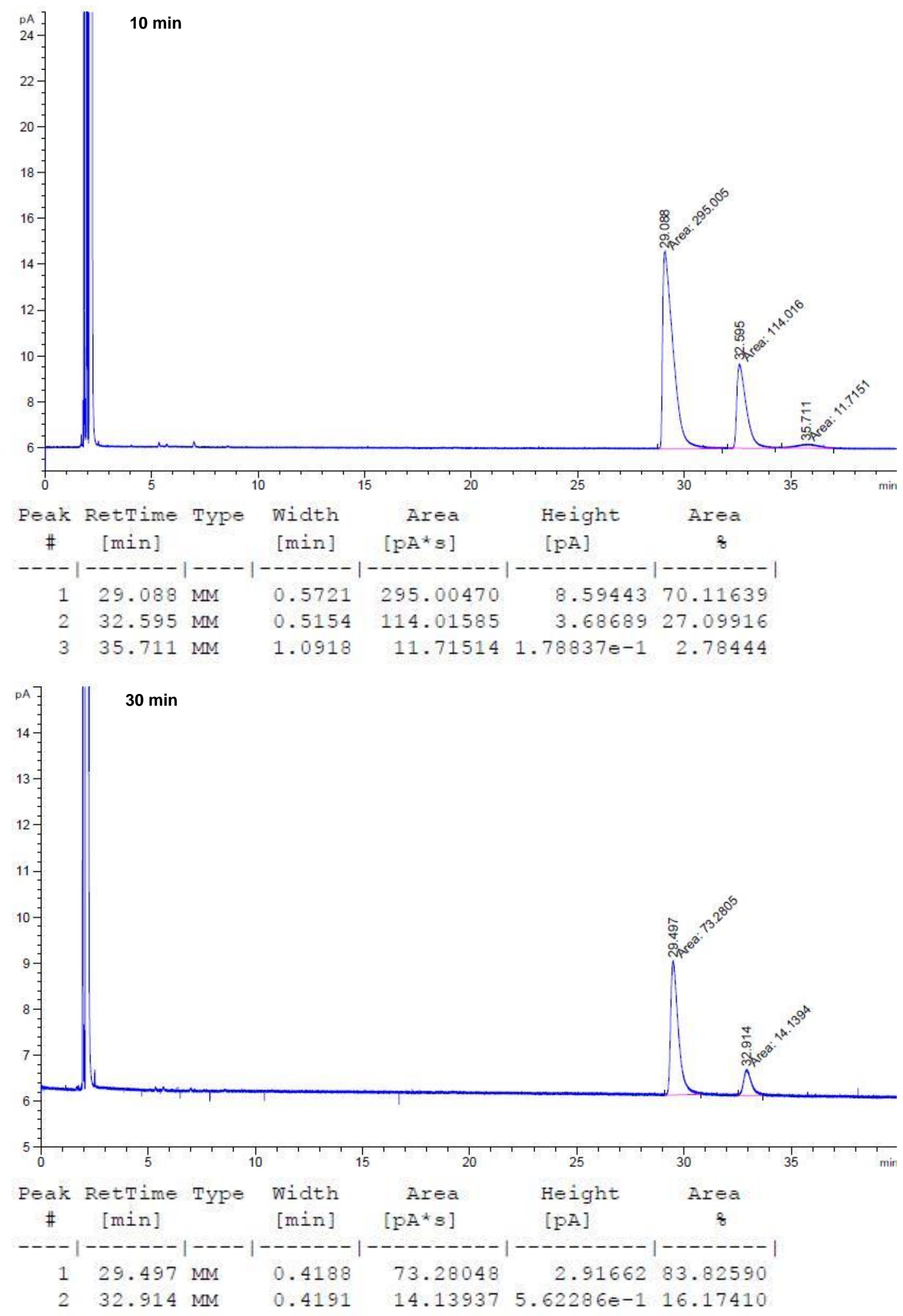

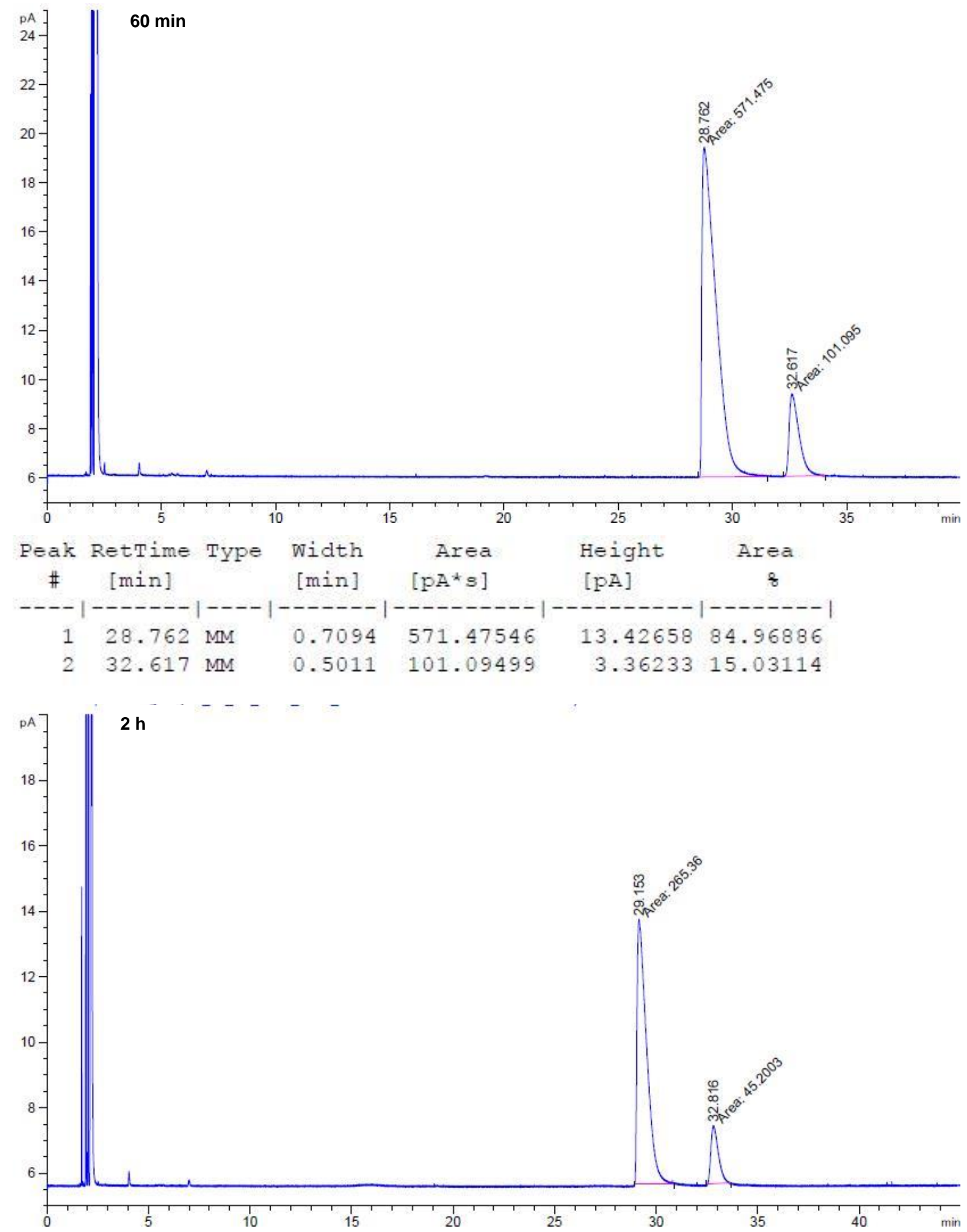

\begin{tabular}{|c|c|c|c|c|c|c|}
\hline $\begin{array}{c}\text { Peak } \\
\#\end{array}$ & $\begin{array}{c}\text { RetTime } \\
\text { [min] }\end{array}$ & Type & $\begin{array}{l}\text { Width } \\
\text { [min] }\end{array}$ & $\begin{array}{r}\text { Area } \\
{\left[\mathrm{pA}^{\star} s\right]}\end{array}$ & $\begin{array}{l}\text { Height } \\
{[\mathrm{pA}]}\end{array}$ & $\begin{array}{c}\text { Area } \\
\&\end{array}$ \\
\hline 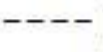 & 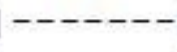 & & - & -------- & - & - \\
\hline 1 & 29.153 & MM & 0.5473 & 265.36035 & 8.08067 & 85.44559 \\
\hline 2 & 32.816 & MM & 0.4243 & 45.20028 & 1.77552 & 14.55441 \\
\hline
\end{tabular}



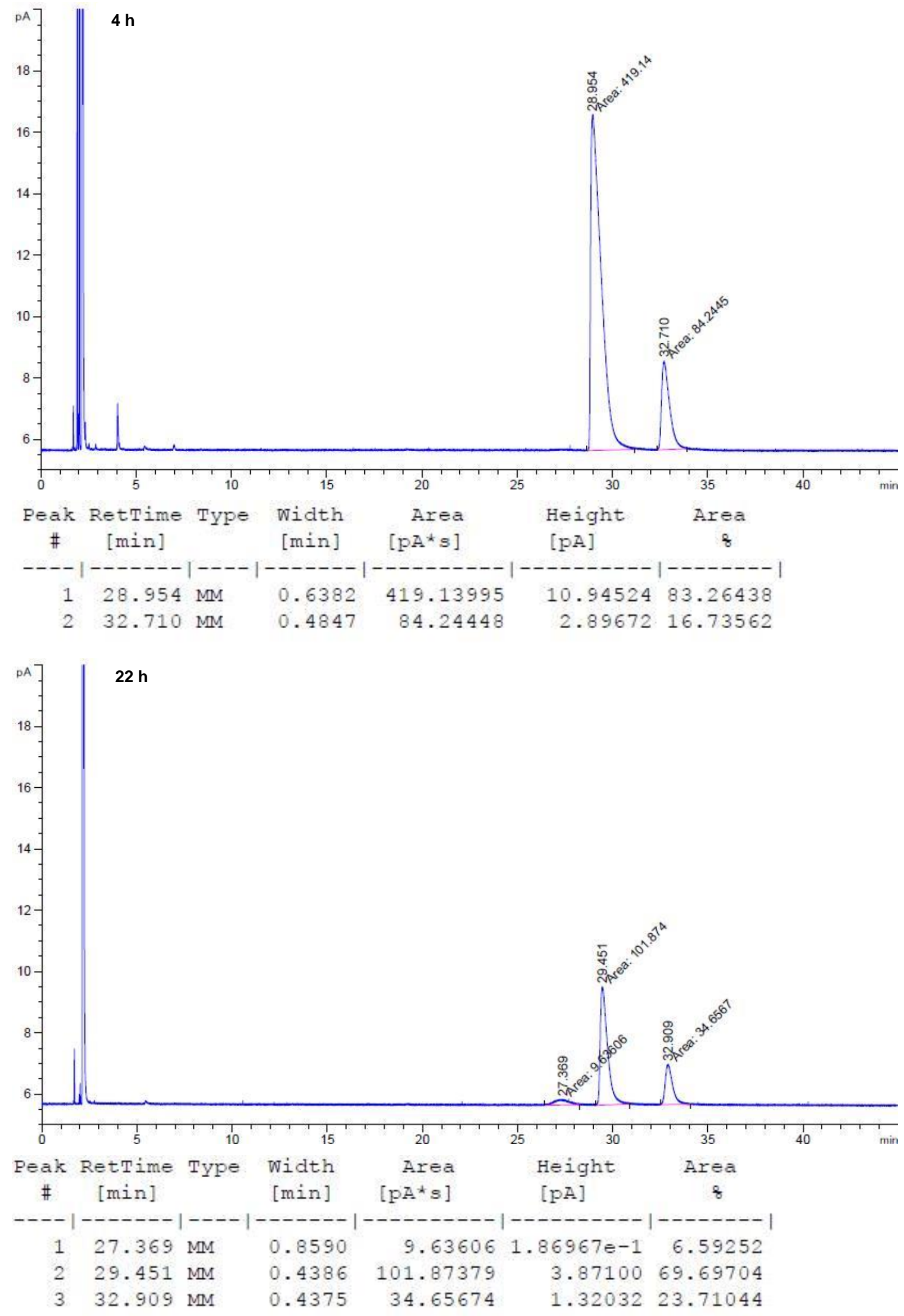


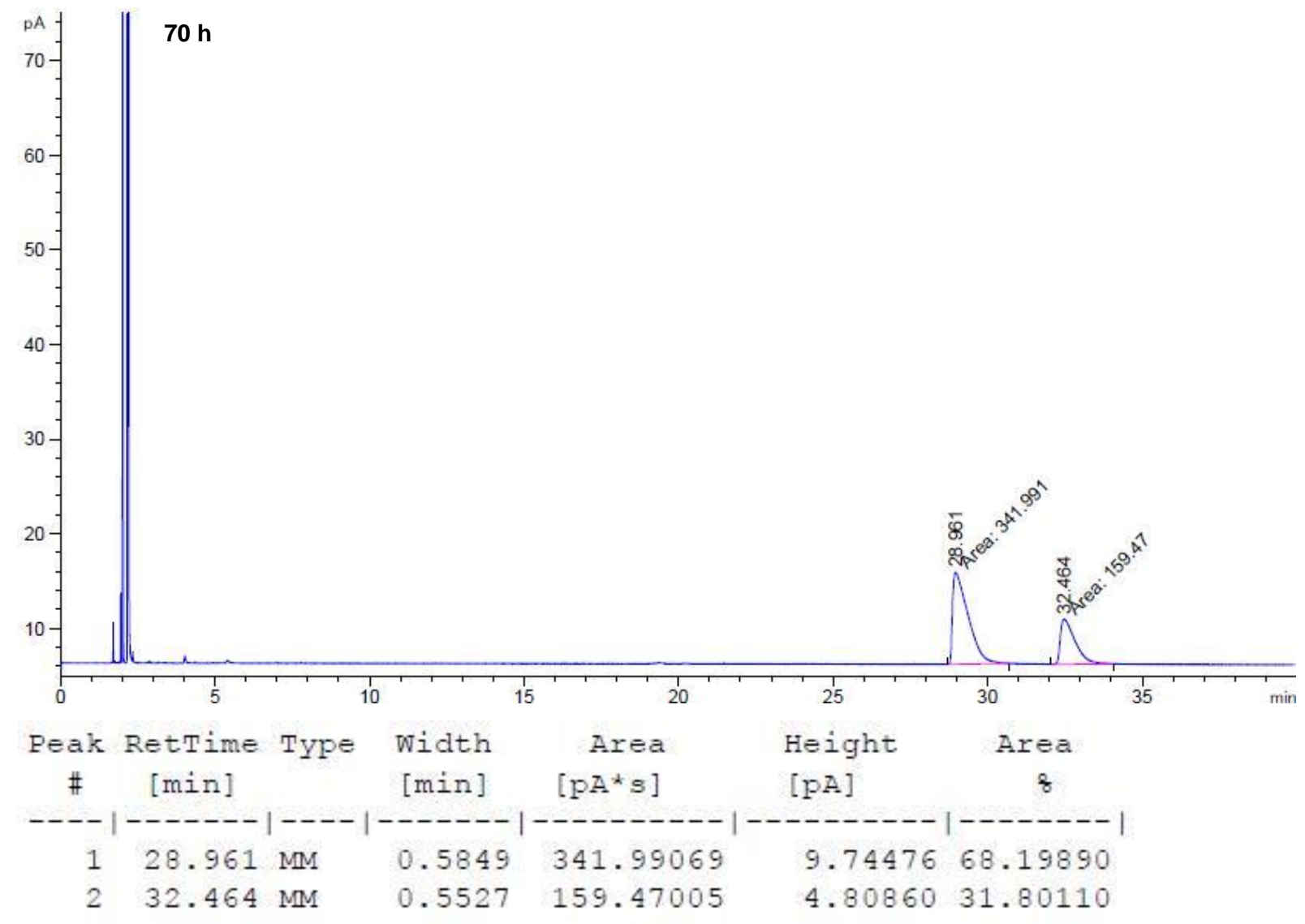




\section{Decomposition of aminal SI6 (1)}

GC: Lipodex-E, $40-70^{\circ} \mathrm{C}\left(1.5^{\circ} \mathrm{C} / \mathrm{min}\right), 1,1 \mathrm{ml} / \mathrm{min} \mathrm{He}$; FID $300^{\circ} \mathrm{C}$, Split $50: 1$
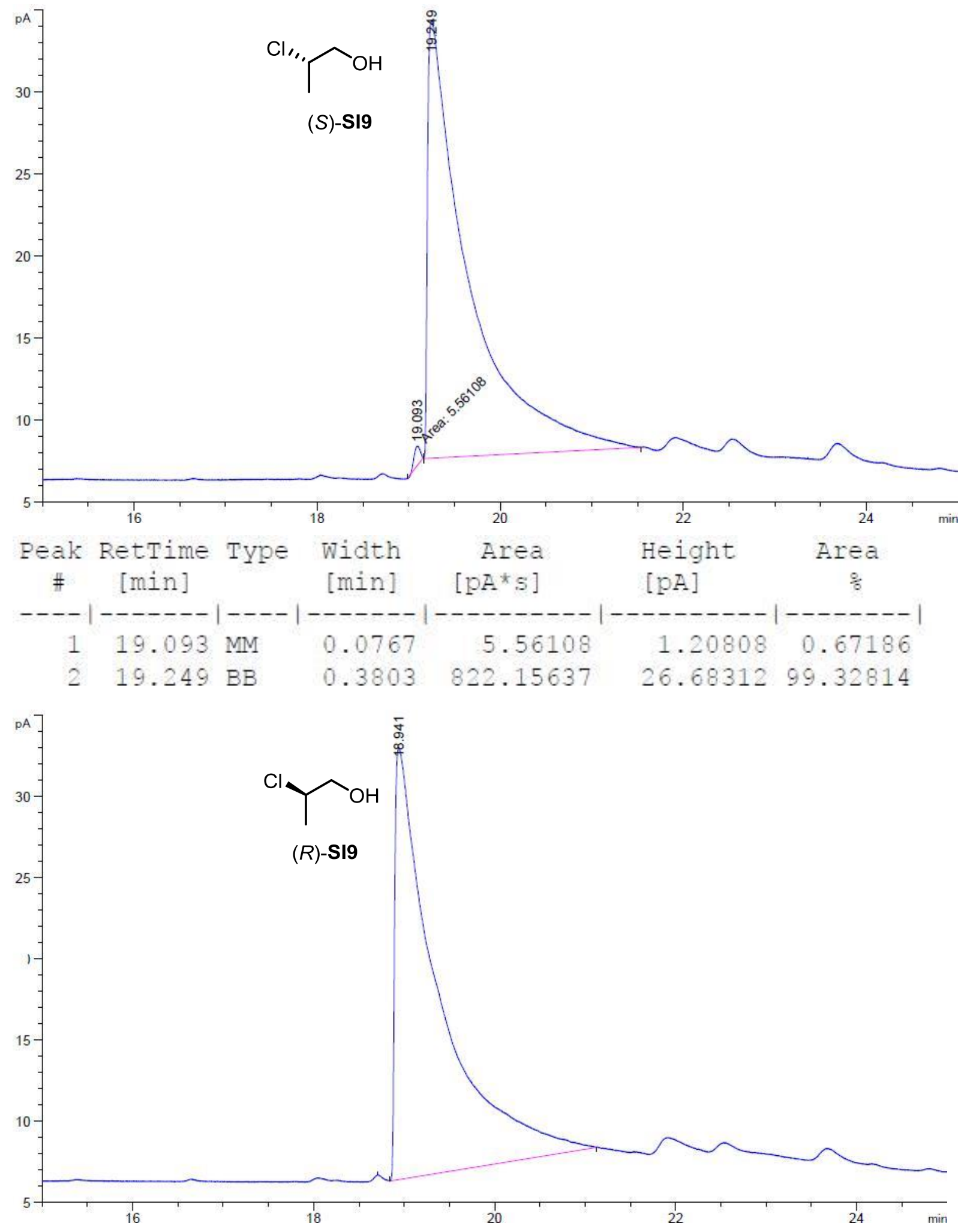

Peak RetTime Type width Area Height Area

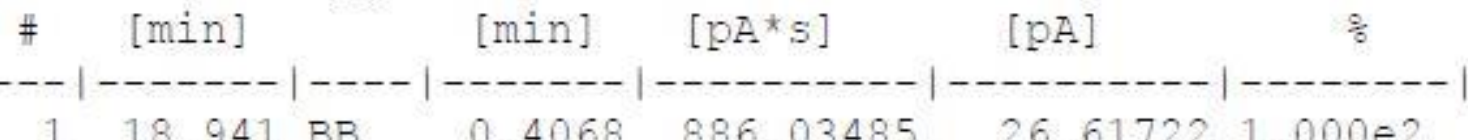

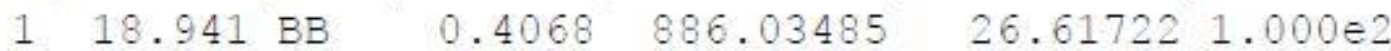



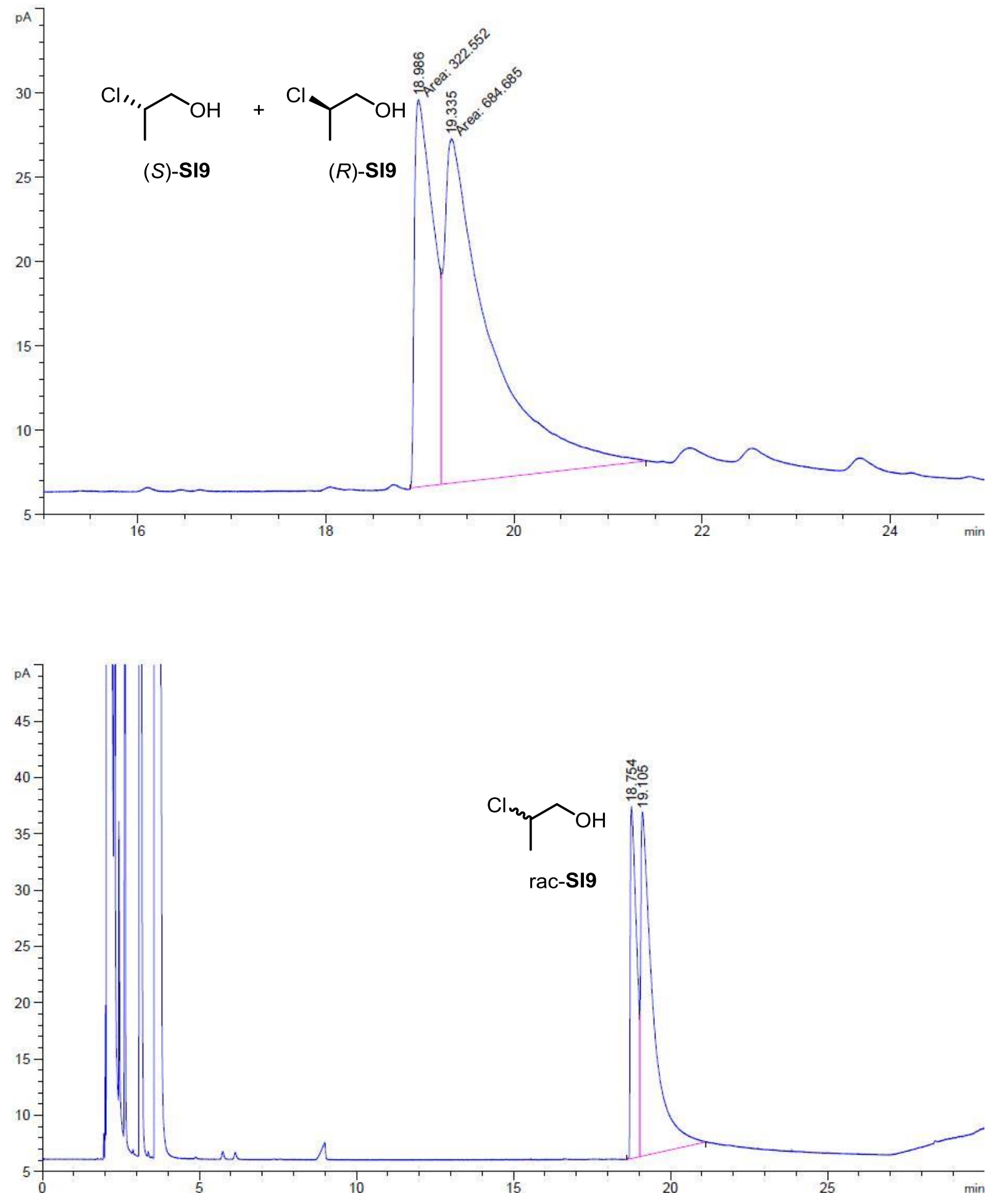

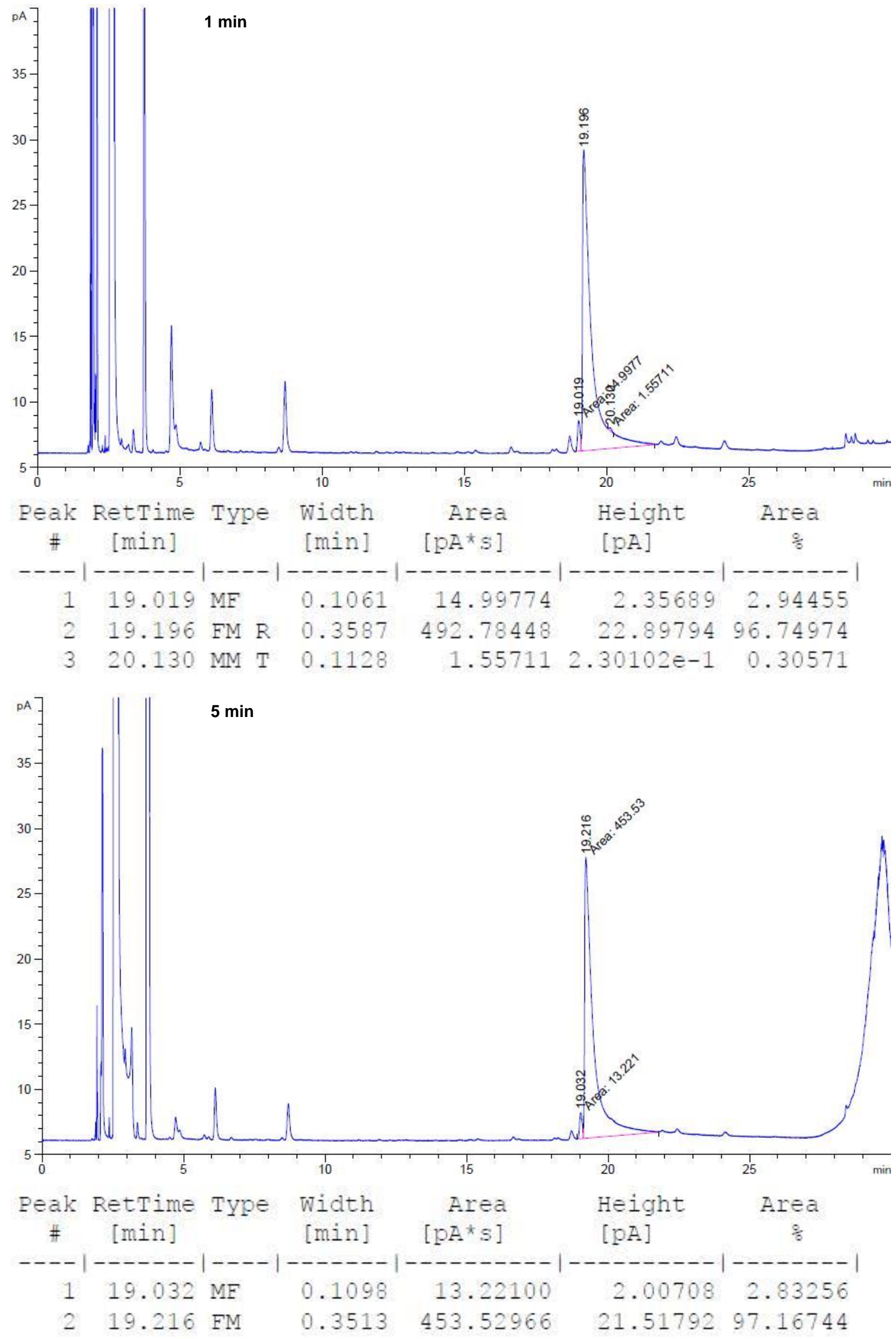

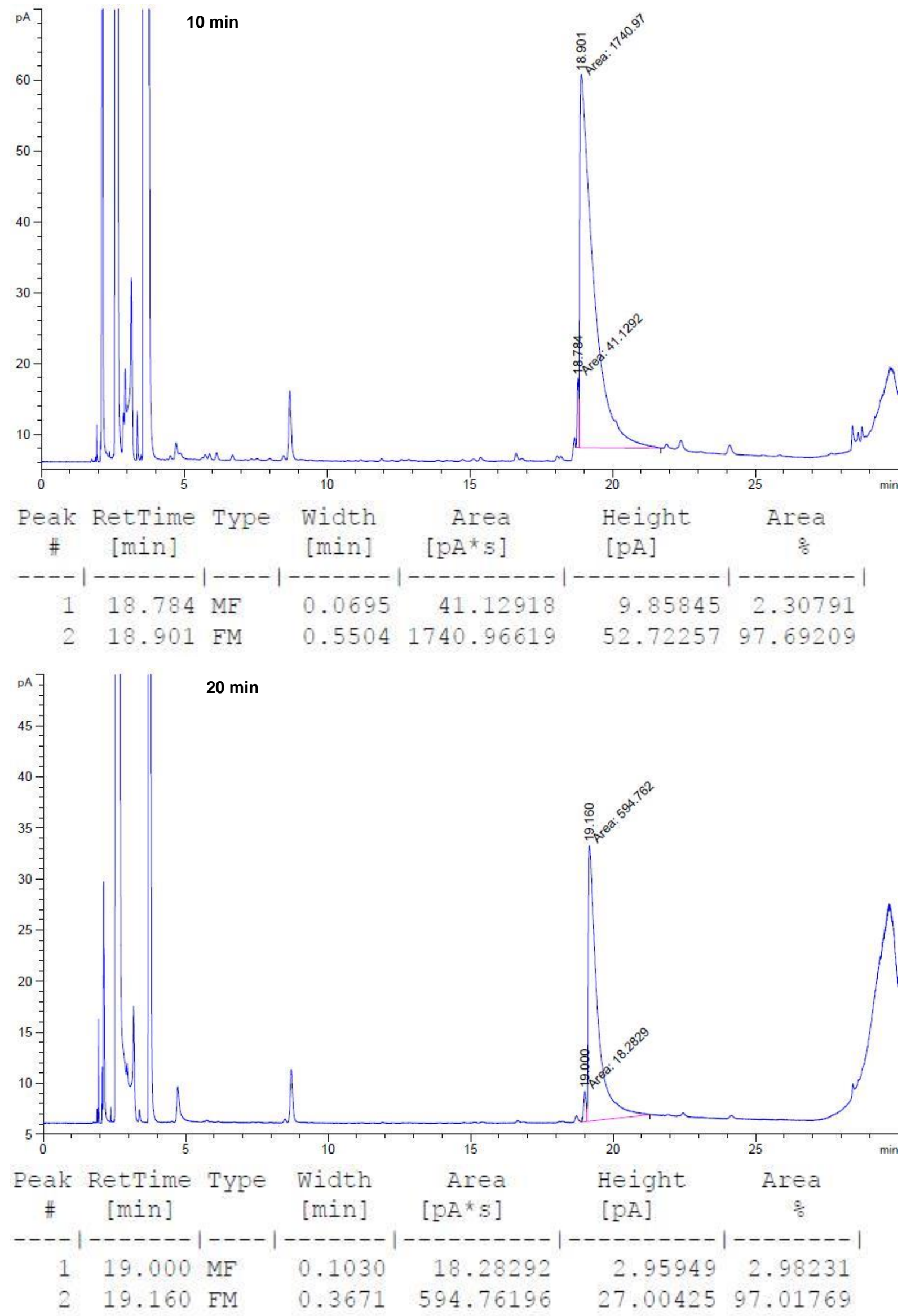

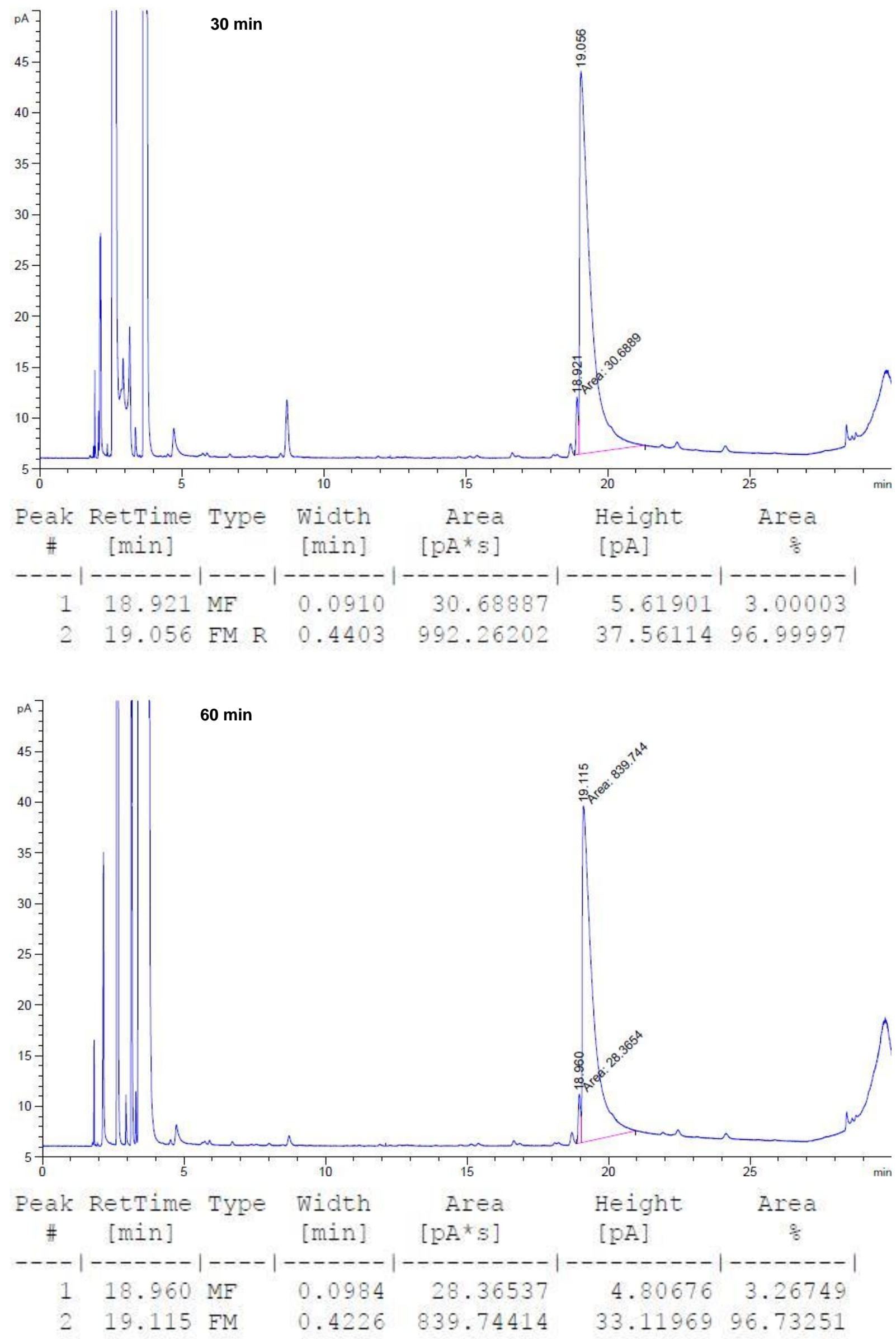

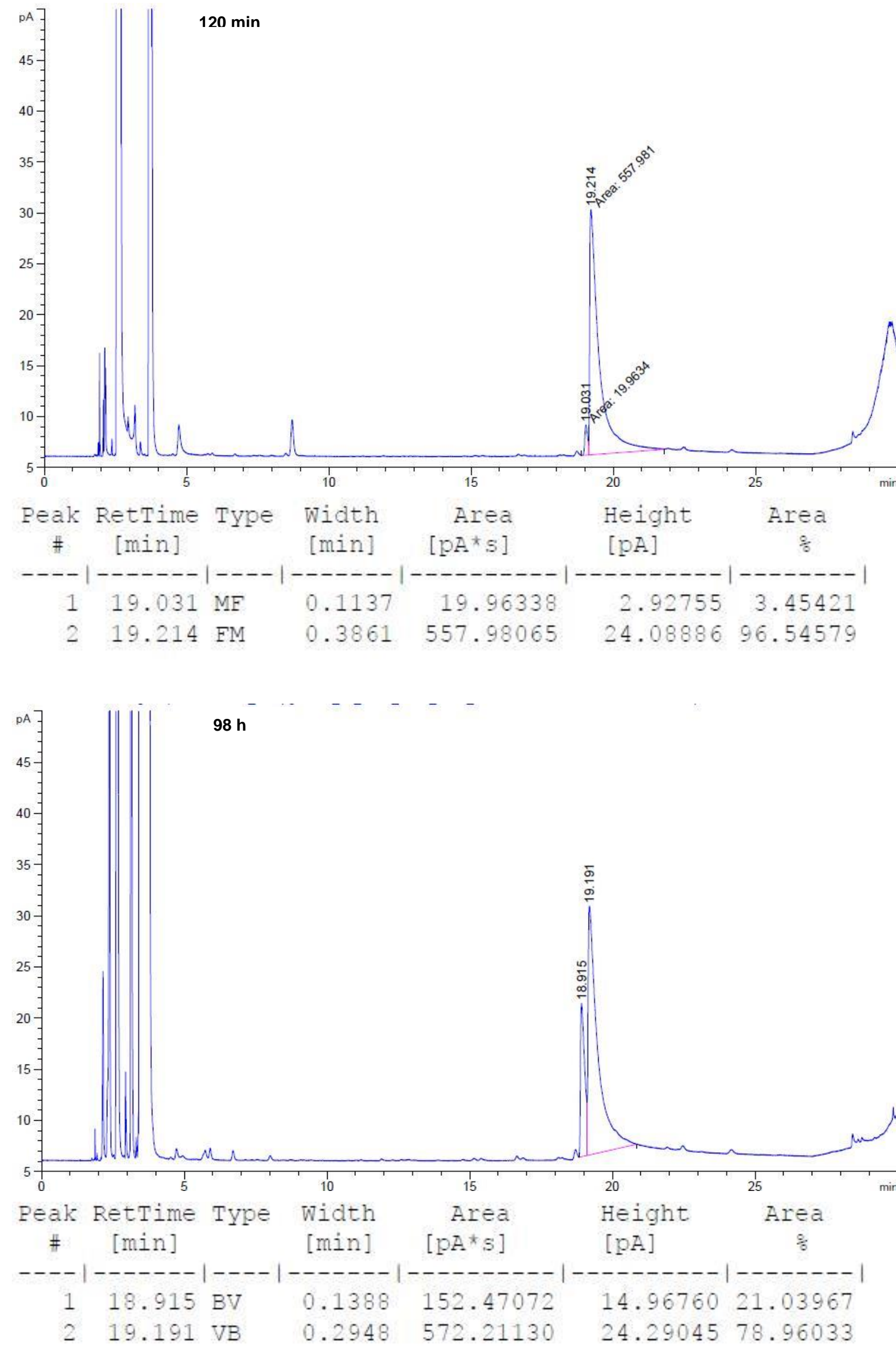


\section{Deuterium incorporation experiment}

HPLC: $0.8 \mathrm{ml} / \mathrm{min}, 5 \% \mathrm{EtOH} /$ hexane, Chiralpak IA $(0.46 \times 25 \mathrm{~cm})$

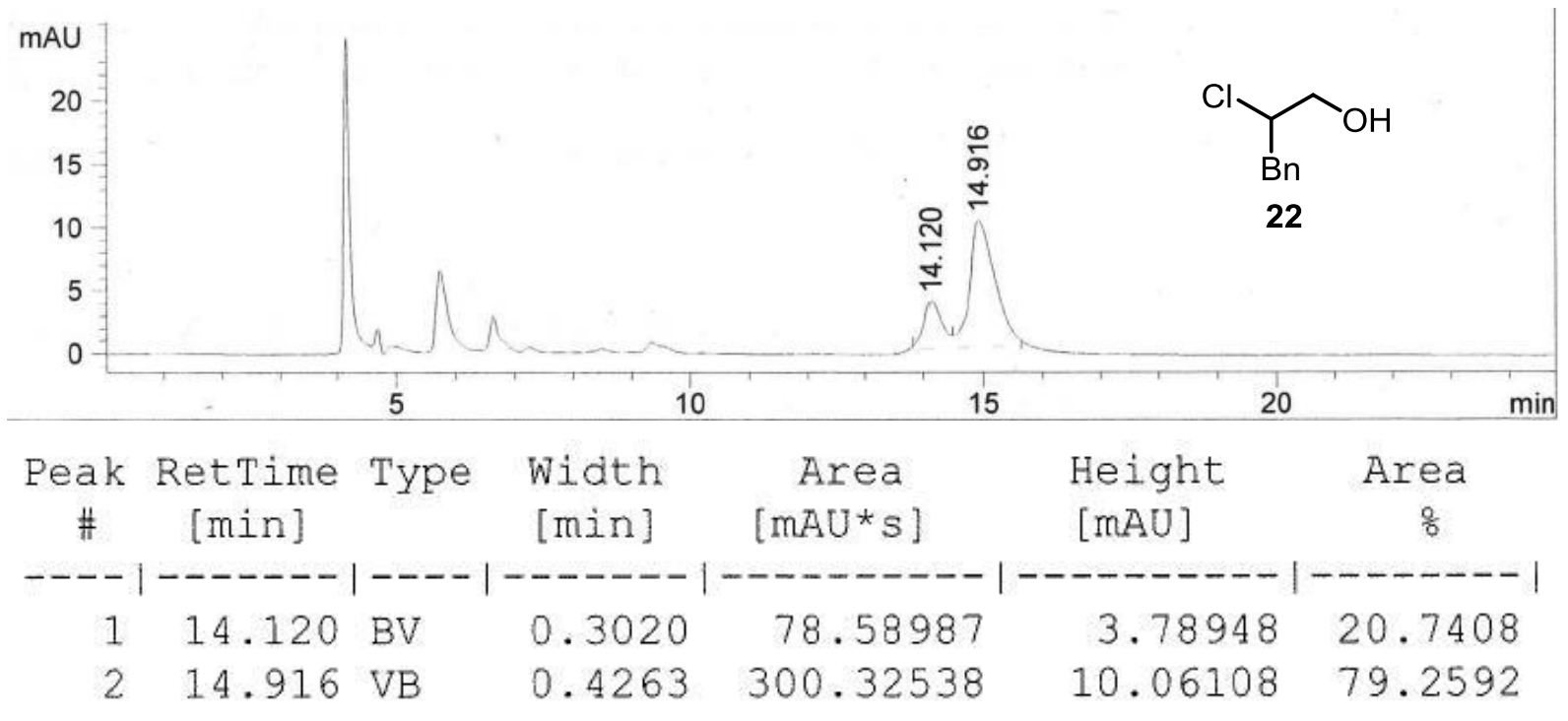




\subsection{Crystallographic Data}

1-((1S,2R)-1-((R)-5-benzyl-2,2,3-trimethyl-4-oxoimidazolidin-1-yl)-2-chloro-3methylbutyl)pyrrolidine-2,5-dione (syn-20)<smiles>CC(C)[C@H](Cl)[C@@H](N1C(=O)CCC1=O)N1[C@H](Br)C(=O)N(C)C1(C)C</smiles>

Deposition number: 2041073

Bond precision: C-C $=0.0022 \AA$

Wavelength $=1.54178$

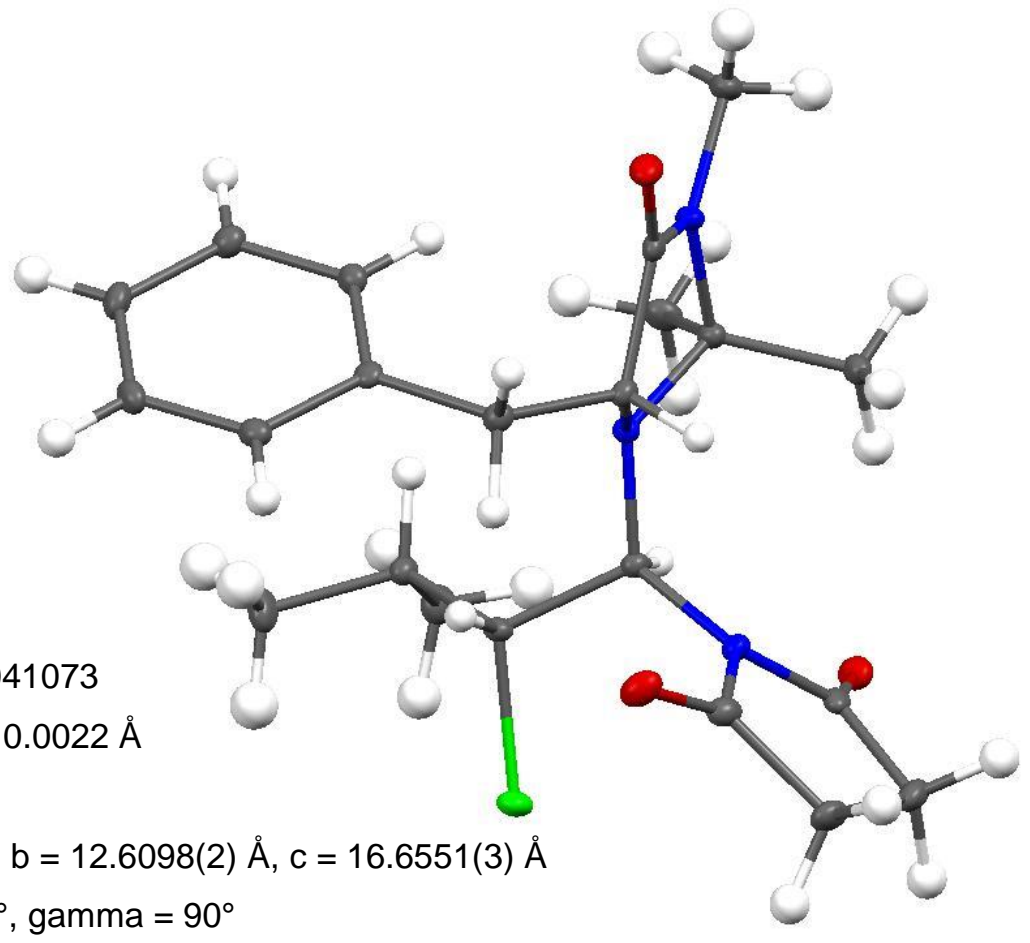

Cell: $a=10.1851(2) \AA, b=12.6098(2) \AA, c=16.6551(3) \AA$

alpha $=90^{\circ}$, beta $=90^{\circ}$, gamma $=90^{\circ}$

Temperature: $100 \mathrm{~K}$

Volume: $2139.05(7) \AA^{3}$

Space group: P 212121

Hall group: P 2ac 2ab

Dx,g cm-3: 1.304

Z: 4

$\mathrm{Mu}(\mathrm{mm}-1): 1.808$

F000: 896.0

F000': 899.88

h,k,Imax: 12, 15, 20

Nref: 3871

Tmin,Tmax: 0.588, 0.753

Tmin': 0.384

Reported T Limits: Tmin $=0.588$, Tmax $=0.753$

AbsCorr $=$ MULTI-SCAN

Data completeness $=1.73 / 0.99$

Theta $(\max )=68.353$

$\mathrm{R}($ reflections $)=0.0214$ (3831)

$\mathrm{wR} 2$ (reflections) $=0.0556(3871)$

$S=1.045$

Npar $=268$ 
1-((S)-1-((R)-5-benzyl-2,2,3-trimethyl-4-oxoimidazolidin-1-yl)-2,2-dichloro-3-

methylbutyl)pyrrolidine-2,5-dione (24a)<smiles>[Z16]C(C)C(Cl)(Cl)[C@H](N1C(=O)CCC1=O)N1[C@H](Br)C(=O)N(C)C1(C)C</smiles>

Deposition number: 2049431

Bond precision: $\mathrm{C}-\mathrm{C}=0.0032 \AA$

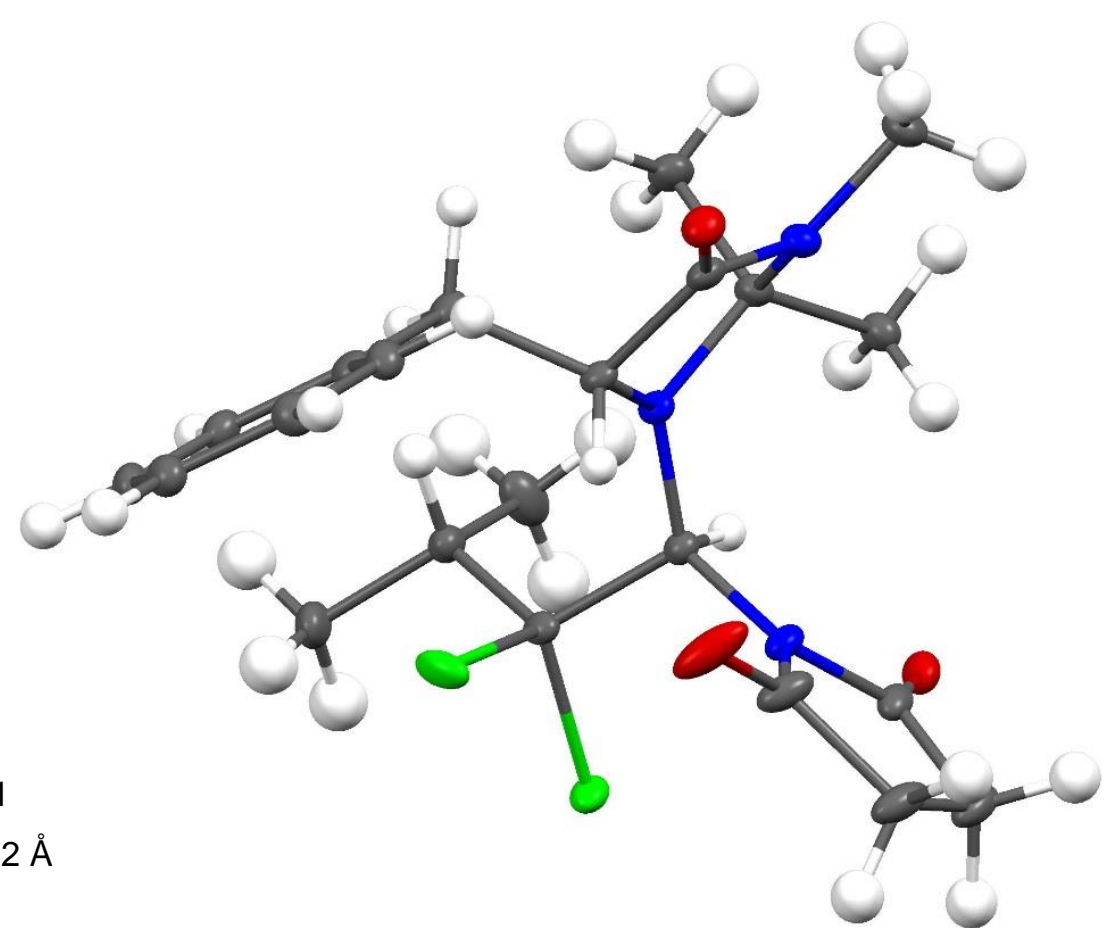

Wavelength $=1.54178$

Cell: $a=10.72160(7) \AA, b=9.17122(6) \AA, c=22.72408(14) \AA$

alpha $=90^{\circ}$, beta $=91.1666(3)^{\circ}$, gamma $=90^{\circ}$

Temperature: $100 \mathrm{~K}$

Volume: 2234.00(2) $\AA^{3}$

Space group: $P 21$

Hall group: $\mathrm{P} 2 \mathrm{yb}$

Dx,g cm-3: 1.351

Z: 4

$\mathrm{Mu}(\mathrm{mm}-1): 2.848$

F000: 960.0

F000': 965.35

h,k,Imax: 12, 11, 27

Nref: 8056

Tmin,Tmax: 0.540, 0.693

Tmin': 0.417

Reported T Limits: Tmin $=0.540$, Tmax $=0.693$

AbsCorr $=$ MULTI-SCAN

Data completeness $=1.85 / 0.99$

Theta $(\max )=68.262$

$\mathrm{R}($ reflections $)=0.0231$ (7929)

$\mathrm{wR} 2$ (reflections) $=0.0615$ (8056)

$\mathrm{S}=1.040$

Npar $=551$ 
<smiles>CC1OC(=O)N([C@@H]([C@H](Cl)Br)N2C(=O)CCC2=O)[C@H](Br)C1=O</smiles>

(1S,2R)-syn-21

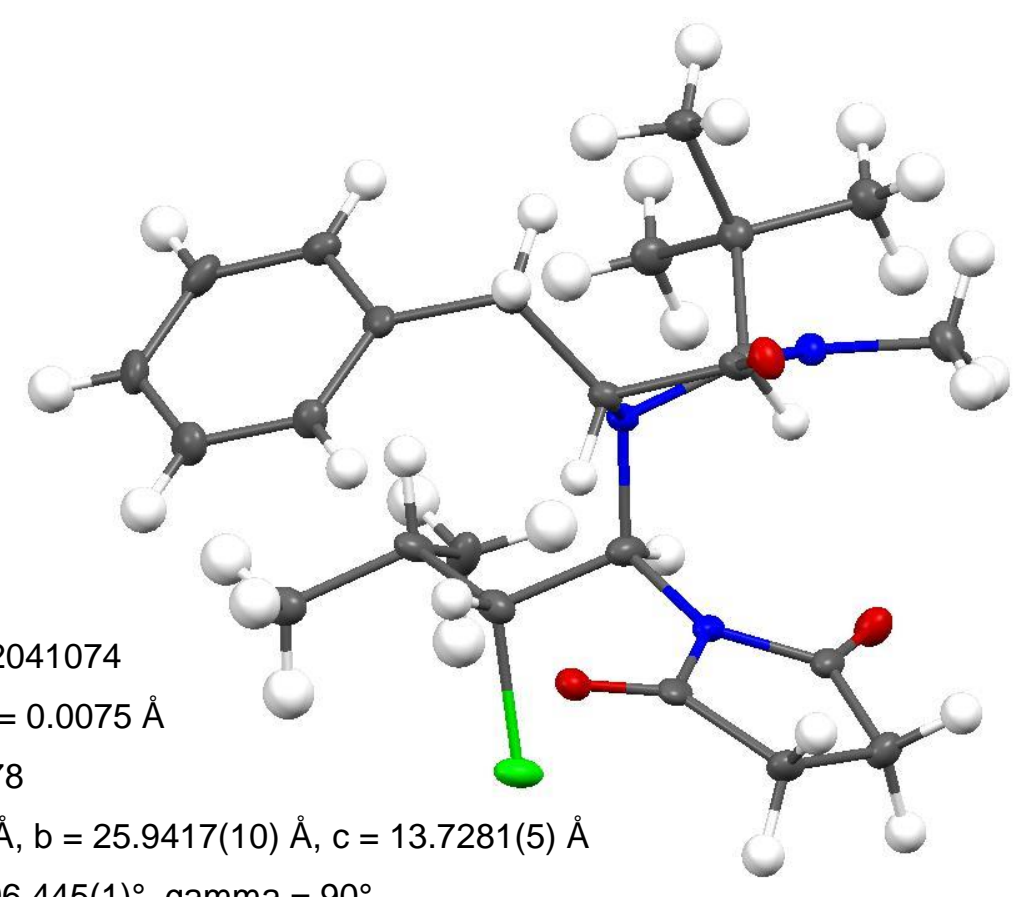

Deposition number: 2041074

Bond precision: $\mathrm{C}-\mathrm{C}=0.0075 \AA$

Wavelength $=1.54178$

Cell: $a=10.4883(4) \AA, b=25.9417(10) \AA, c=13.7281(5) \AA$

Alpha $=90^{\circ}$, beta $=96.445(1)^{\circ}$, gamma $=90^{\circ}$

Temperature: $101 \mathrm{~K}$

Volume: 3711.6(2) $\AA^{3}$

Space group: P $21 \mathrm{P} 1211$

Hall group: P 2yb P 2yb

$\mathrm{Dx}, \mathrm{g} \mathrm{cm}-3=1.203$

Z: 6

$\mathrm{Mu}(\mathrm{mm}-1): 1.592$

F000: 1440.0

F000': 1446.02

h,k,Imax: 12, 31, 16

Nref: 13347

Tmin,Tmax: 0.638,0.728

Tmin': 0.548

Reported T Limits: Tmin $=0.638$, Tmax $=0.728$

AbsCorr $=$ MULTI-SCAN

Data completeness $=1.89 / 0.97$

Theta $(\max )=68.874$

$\mathrm{R}$ (reflections) $=0.0548(13035)$

$\mathrm{wR} 2$ (reflections) $=0.1294(13347)$

$S=1.024$

Npar $=857$ 
<smiles>CC(C)[C@H](Cl)[C@@H](N1C(=O)CCC1=O)N1C(=O)N(C)C(=O)[C@H]1Br</smiles>

$(1 S, 2 S)$-anti-21

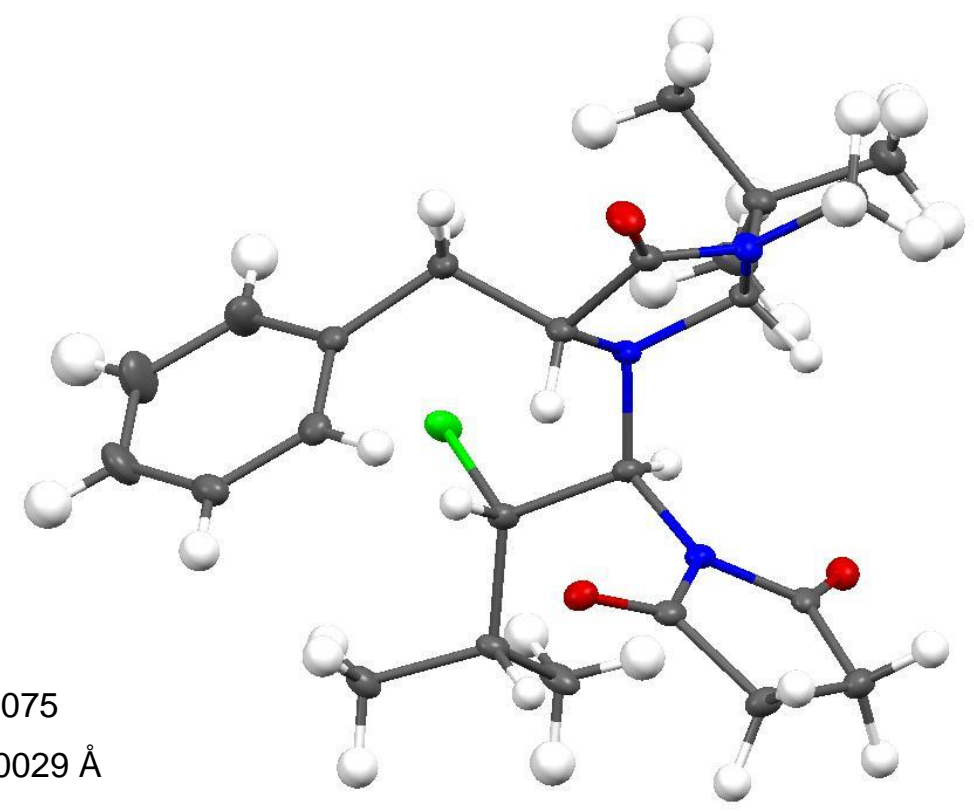

Deposition number: 2041075

Bond precision: $\mathrm{C}-\mathrm{C}=0.0029 \AA$

Wavelength $=1.54178$

Cell: $a=24.00236(15) \AA, b=9.35451(6) \AA, c=10.74110(7) \AA$

alpha $=90^{\circ}$, beta $=93.6494(1)^{\circ}$, gamma $=90^{\circ}$

Temperature: $100 \mathrm{~K}$

Volume: 2406.81(3) $\AA^{3}$

Space group: C 2 C 2

Hall group: C 2y C 2y

Dx,g cm-3: 1.236

Z: 4

$\mathrm{Mu}(\mathrm{mm}-1): 1.637$

F000: 960.0

F000': 964.01

h,k,Imax: 28, 11, 12

Nref: 4089

Tmin,Tmax: 0.464, 0.563

Tmin': 0.365

Reported T Limits: Tmin $=0.464$, Tmax $=0.563$

AbsCorr $=$ MULTI-SCAN

Data completeness $=1.74 / 0.93$

Theta $(\max )=68.276$

$\mathrm{R}$ (reflections) $=0.0236(4085)$

wR2(reflections) $=0.0581$ (4089)

$S=1.079$

Npar $=287$ 
<smiles>CC(C)[C@@H](Cl)[C@H](N1C(=O)CCC1=O)N1C([13CH3])[C@@H](Br)C(=O)N1C</smiles>

(1R,2S)-syn-21

Deposition number: 2041077

Bond precision: $\mathrm{C}-\mathrm{C}=0.0320 \AA$

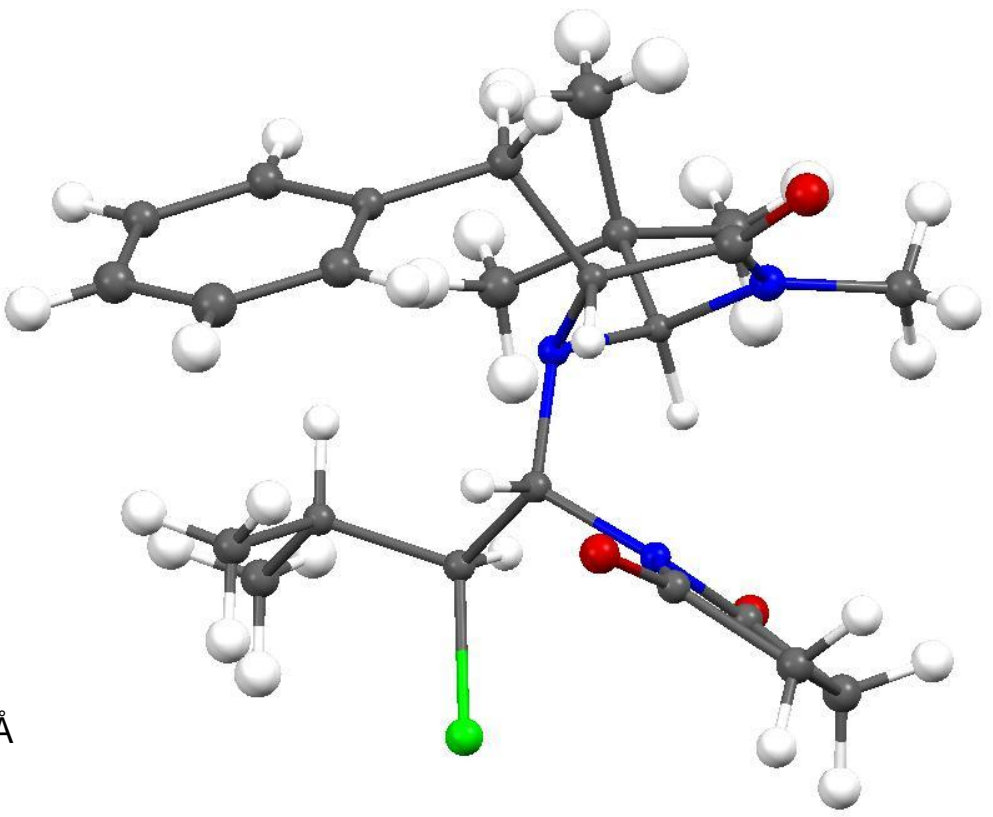

Wavelength $=1.54178$

Cell: $a=10.2163(4) \AA, b=25.1458(11) \AA, c=14.4592(6) \AA$

alpha $=90^{\circ}$, beta $=94.880(2)^{\circ}$, gamma $=90^{\circ}$

Temperature: $100 \mathrm{~K}$

Volume: 3701.1(3) $\AA^{3}$

Space group: P $21 \mathrm{P} 1211$

Hall group: P 2yb P 2yb

Moiety formula

Dx,g cm-3: 1.207

Z: 6

$\mathrm{Mu}(\mathrm{mm}-1): 1.597$

F000: 1442.0

F000': 1448.02

h,k,Imax: 12, 30, 17

Nref: 11279

Tmin,Tmax: 0.557, 0.753

Tmin': 0.270

Reported T Limits: Tmin $=0.557$, Tmax $=0.753$

AbsCorr $=$ MULTI-SCAN

Data completeness $=1.62 / 0.83$

Theta $(\max )=68.367$

$\mathrm{R}$ (reflections) $=0.1344(10576)$

wR2 (reflections) $=0.3992(11279)$

$S=1.382$

Npar $=392$ 
1-((1S,2R)-1-((2R,5R)-5-benzyl-2-(tert-butyl)-3-methyl-4-oxoimidazolidin-1-yl)-2-chloro-3phenylpropyl)pyrrolidine-2,5-dione ((1S,2R)-syn-25)

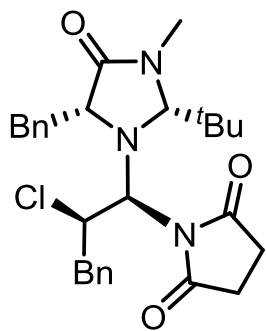

$(1 S, 2 R)-s y n-25$

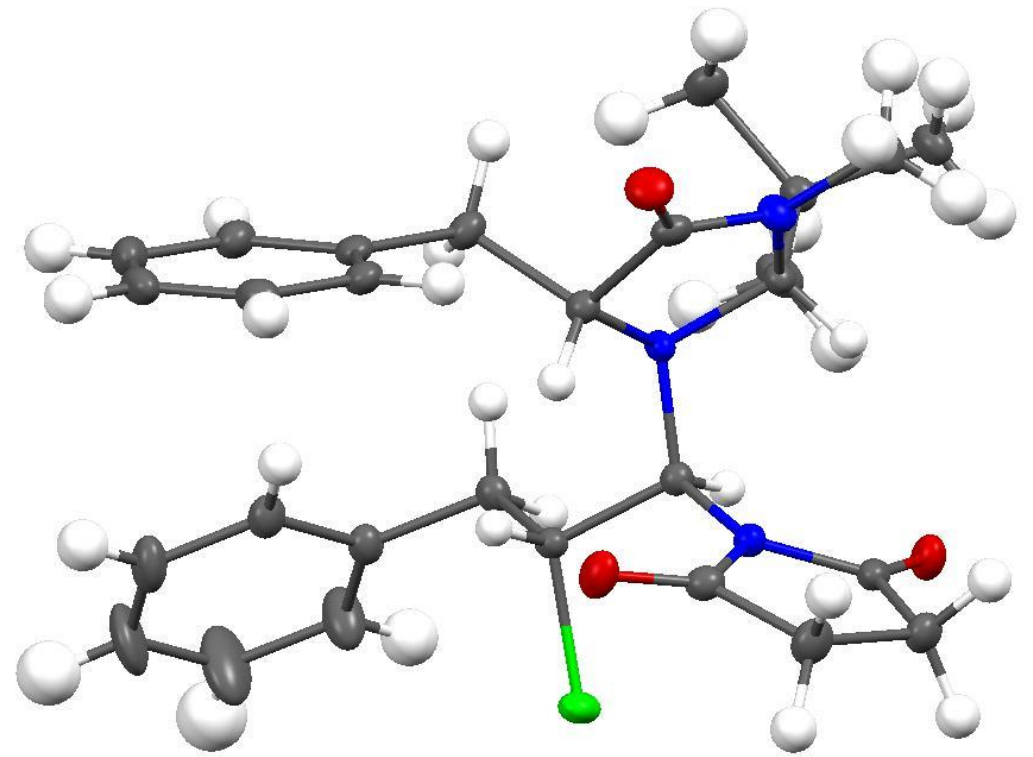

Deposition number: 2041078

Bond precision: $\mathrm{C}-\mathrm{C}=0.0024 \AA$

Wavelength $=1.54178$

Cell: $a=10.52726(7) \AA, b=13.34540(9) \AA, c=18.82390(12) \AA$

alpha $=90^{\circ}$, beta $=90^{\circ}$, gamma $=90^{\circ}$

Temperature: $100 \mathrm{~K}$

Volume: $2644.58(3) \AA^{3}$

Space group: P 212121

Hall group: $P 2 \mathrm{ac} 2 \mathrm{ab}$

Dx,g cm-3: 1.246

Z: 4

$\mathrm{Mu}(\mathrm{mm}-1): 1.544$

F000: 1056.0

F000': 1060.29

h,k,Imax: 12, 16, 22

Nref: 4815

Tmin,Tmax: 0.480, 0.714

Tmin': 0.420

Reported T Limits: $\operatorname{Tmin}=0.480, \mathrm{Tmax}=0.714$

AbsCorr $=$ MULTI-SCAN

Data completeness $=1.75 / 0.99$

Theta $(\max )=68.453$

$\mathrm{R}$ (reflections) $=0.0227(4763)$

wR2 $($ reflections $)=0.0586(4815)$

$S=1.046$

Npar $=320$ 
<smiles>CN1C(=O)[C@H](Br)N([C@@H](Cc2ccccc2)C(Cl)Cl)[C@H]1N1C(=O)CCC1=O</smiles>

$(1 S, 2 S)-a n t i-25$

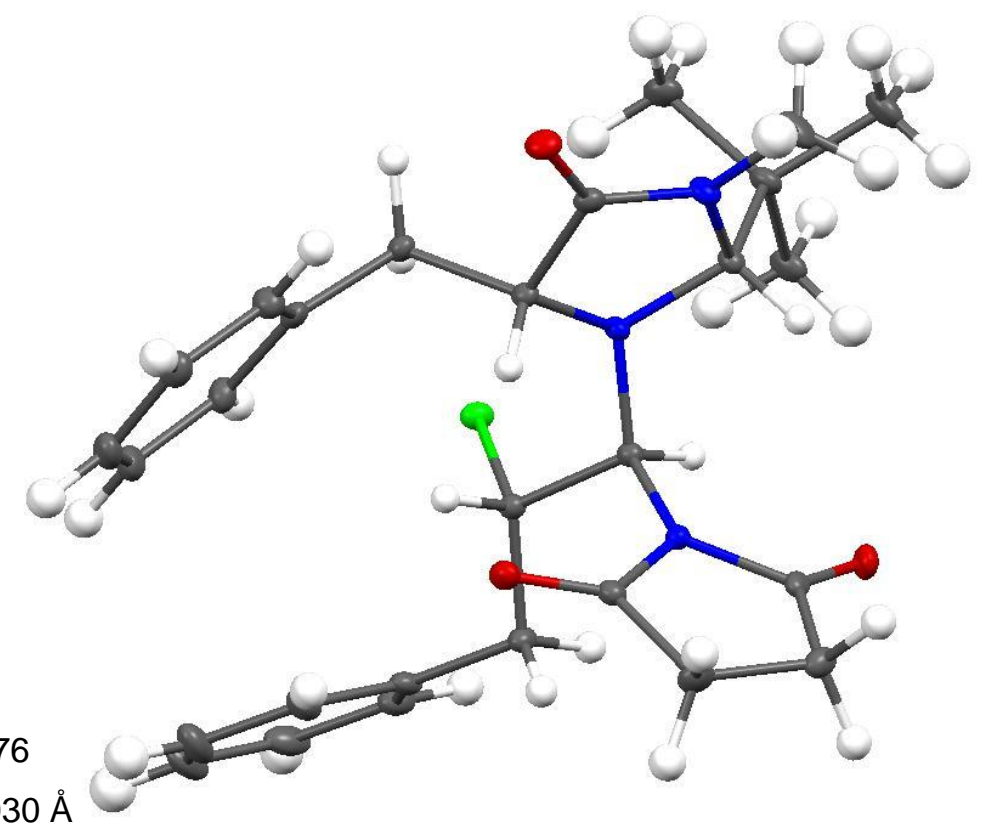

Deposition number: 2041076

Bond precision: $\mathrm{C}-\mathrm{C}=0.0030 \AA$

Wavelength $=1.54178$

Cell: $a=9.0086(4) \AA, b=10.9058(6) \AA, c=13.4651(6) \AA$

alpha $=90^{\circ}$, beta $=97.426(2)^{\circ}$, gamma $=90^{\circ}$

Temperature: $100 \mathrm{~K}$

Volume: $1311.80(11) \AA^{3}$

Space group: P 1211

Hall group: $P 2 y b$

Dx,g cm-3: 1.256

Z: 2

$\mathrm{Mu}(\mathrm{mm}-1): 1.557$

F000: 528.0

F000': 530.14

h,k,Imax: 10,13,16

Nref: 4722

Tmin,Tmax: 0.583, 0.753

Tmin': 0.388

Reported T Limits: $\operatorname{Tmin}=0.583, \mathrm{Tmax}=0.753$

AbsCorr $=$ MULTI-SCAN

Data completeness $=1.85 / 0.98$

Theta $(\max )=68.467$

$\mathrm{R}$ (reflections $)=0.0250(4685)$

wR2(reflections) $=0.0624(4722)$

$\mathrm{S}=1.032$

Npar $=321$ 
1-((1R,2S)-1-((2R,5R)-5-benzyl-2-(tert-butyl)-3-methyl-4-oxoimidazolidin-1-yl)-2-chloro-3phenylpropyl)pyrrolidine-2,5-dione ((1R,2S)-syn-25)<smiles>C[13CH2]N1[C@H](Br)C(=O)N(C)[C@H]1[C@H](Cc1ccccc1)N1C(=O)CCC1=O</smiles>

$(1 R, 2 S)-s y n-25$

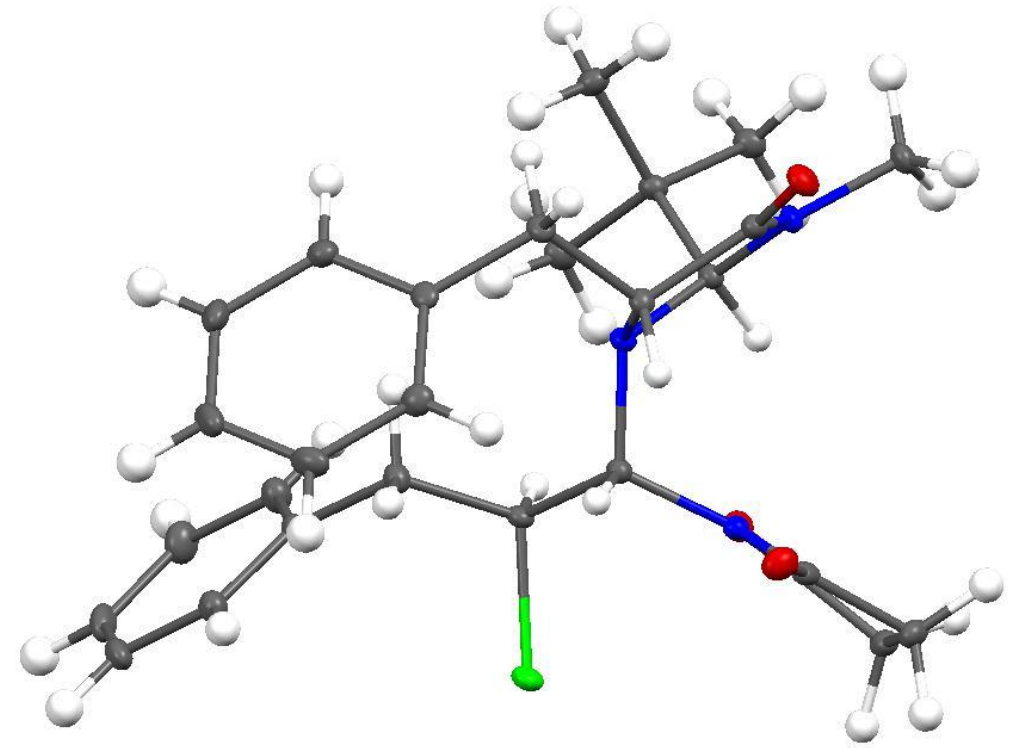

Deposition number: 2043370

Bond precision: $\mathrm{C}-\mathrm{C}=0.0028 \AA$

Wavelength $=0.71073$

Cell: $a=8.9226(2) \AA, b=15.9789(3) \AA, c=17.9095(3) \AA$

alpha $=90^{\circ}$, beta $=90^{\circ}$, gamma $=90^{\circ}$

Temperature: $100 \mathrm{~K}$

Volume: $2553.42(9) \AA^{3}$

Space group: P 212121

Hall group: $P 2 a c 2 a b$

Dx,g cm-3: 1.290

Z: 4

$\mathrm{Mu}(\mathrm{mm}-1): 0.184$

F000: 1056.0

F000': 1057.00

h,k,Imax: 10,19,21

Nref: 4853

Tmin,Tmax: 0.780,0.836

Tmin': 0.941

Reported T Limits: Tmin $=0.780$, Tmax $=0.836$

AbsCorr $=$ MULTI-SCAN

Data completeness $=1.76 / 1.00$

Theta $(\max )=25.702$

$\mathrm{R}($ reflections $)=0.0247(4738)$

$\mathrm{wR} 2$ (reflections) $=0.0603(4853)$

$S=1.075$

$\mathrm{Npar}=320$ 

yl)-2-chloro-3-methylbutyl)carbamoyl)benzyl 3,5-dinitrobenzoate (15)<smiles>COC(c1cc(C(F)(F)F)cc(C(F)(F)F)c1)(c1cc(C(F)(F)F)cc(C(F)(F)F)c1)C1CCCN1[C@@H](C(=O)N[C@@H](C(C)C)C(Cl)C(F)(F)F)c1ccccc1COC(=O)c1cc([N+](=O)[O-])cc([N+](=O)[O-])c1</smiles>

Deposition number: 2045387

Bond precision: $\mathrm{C}-\mathrm{C}=0.0060 \AA$ Wavelength $=1.54178$

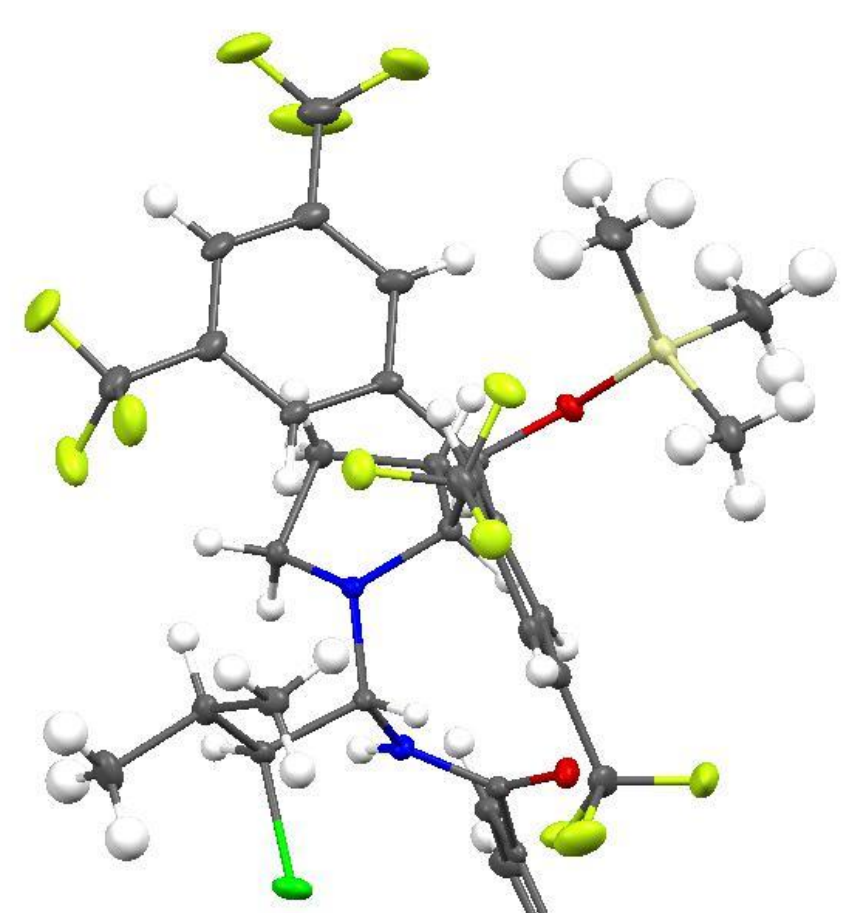

Cell: $a=9.87405(7) \AA, b=19.31855(13) \AA, c=25.10775(17) \AA$ alpha $=90^{\circ}$, beta $=96.9297(3)^{\circ}$, gamma $=90^{\circ}$ Temperature: $100 \mathrm{~K}$ Volume: 4754.37(6) $\AA^{3}$

Space group: $P 21$ Hall group: $P 2 \mathrm{yb}$ Dx,g cm-3: 1.460 Z: 4

$\mathrm{Mu}(\mathrm{mm}-1): 1.876$

F000: 2144.0

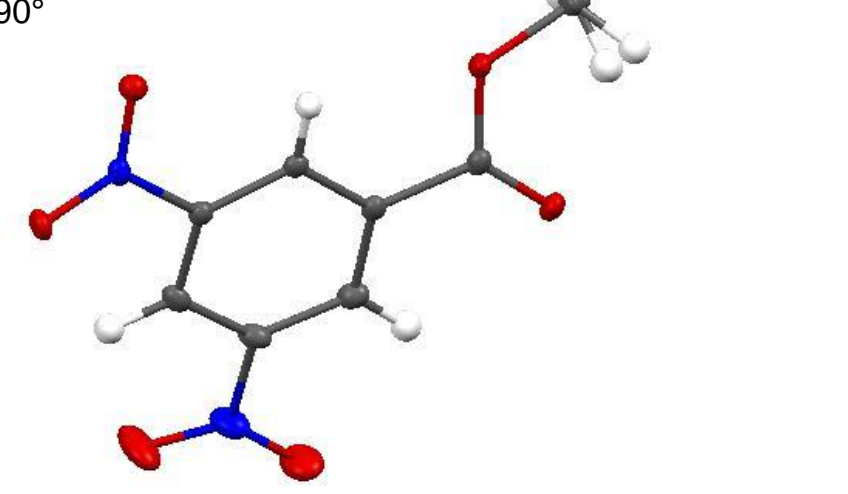

F000': 2154.95

h,k,Imax: $11,23,30$

Nref: 16869

Tmin,Tmax: 0.502,0.705

Tmin': 0.394

Reported T Limits: Tmin $=0.502$, Tmax $=0.705$

AbsCorr $=$ MULTI-SCAN

Data completeness $=1.88 / 0.97$

Theta $(\max )=68.297$

$\mathrm{R}$ (reflections) $=0.0435$ (16690)

$\mathrm{wR} 2$ (reflections) $=0.1138(16869)$

$S=1.111$

Npar $=1272$ 